\title{
A STRUCTURAL ANALYSIS OF A FACTORY HALL WITH A TWO-STORY OFFICE BUILDING AS A PRECAST AND PRESTRESSED CONCRETE CONSTRUCTION
}

\author{
By \\ Olesja Befus \\ B.Eng, University of Applied Sciences Saarbrücken, Germany, 2015 \\ A project \\ presented to Ryerson University and \\ Karlsruhe University of Applied Sciences \\ in partial fulfillment of the \\ requirements for the degree of \\ Master of Engineering \\ in the program of \\ Civil Engineering
}

Karlsruhe, Germany, 2018

COOlesja Befus, 2018 


\section{AUTHOR'S DECLARATION FOR ELECTRONIC SUBMISSION OF A MRP}

I hereby declare that I am the sole author of this MRP. This is a true copy of the MRP, including any required final revisions.

I authorize Ryerson University to lend this MRP to other institutions or individuals for the purpose of scholarly research.

I further authorize Ryerson University to reproduce this MRP by photocopying or by other means, in total or part, at the request of other institutions or individuals for the purpose of scholarly research.

I understand that my MRP may be electronically available to the public. 
A STRUCTURAL ANALYSIS OF A FACTORY HALL WITH A TWO-STORY OFFICE BUILDING

AS A PRECAST AND PRESTRESSED

CONCRETE CONSTRUCTION

Master of Engineering, 2018

\title{
Olesja Befus
}

Civil Engineering

Ryerson University and

Karlsruhe University of Applied Sciences

\begin{abstract}
The objective of this thesis is to develop a precast and prestressed concrete design for a factory hall, which was initially planned as a steel structure. Furthermore, a structural analysis is conducted on several chosen structural elements according to the European Standards and the German Annexes respectively. The analysis is done both by manual calculation and software calculation for comparison and includes the ultimate limit state design, the serviceability limit state design and the design for the state of transportation and assembly of the precast members. Lastly, to illustrate the results of the analysis, an overview drawing with the new concrete design as well as formwork and reinforcement drawings for each of the analyzed structural members are developed.
\end{abstract}




\section{ACKNOWLEDGEMENTS}

I would like to express my gratitude to my supervisor at HsKA, Dr. Christian Enderle, for his persistent support and guidance. His valuable experience and professional expertise was of great help throughout this endeavour. Furthermore, I thank him for always being patient and understanding throughout the process of crafting this thesis.

I would also like to thank my supervisor at Ryerson University, Dr. Reza Kianoush, for his guidance and intriguing lectures in the field of reinforced concrete design throughout my studies in Toronto. After attending his lecture on Advanced Concrete Design, I decided to write my Directed Studies about Strut-and-Tie Modelling under his supervision and I am grateful that he agreed to supervise my thesis.

Furthermore, I am thankful for my family for constantly supporting me during my studies and for my friends and fellow students who shared in this wonderful German/Canadian exchange experience and kept me motivated all the way through. A special thank you to my boyfriend for patiently proofreading and editing my thesis. 


\section{TABLE OF CONTENTS}

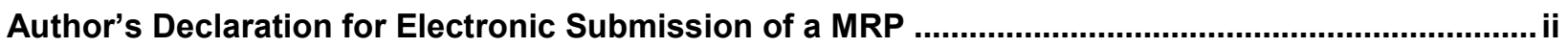

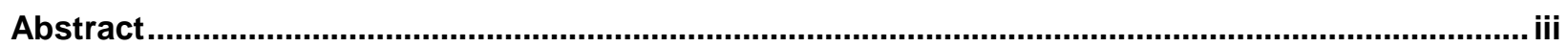

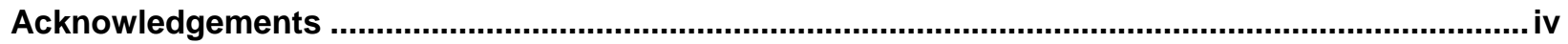

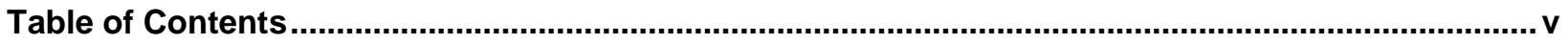

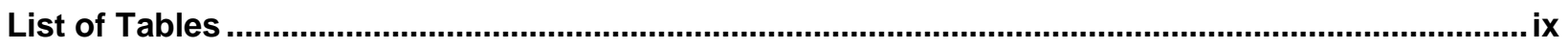

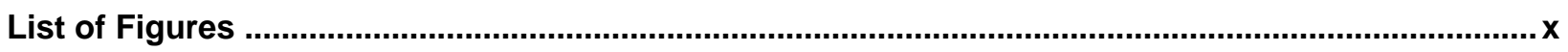

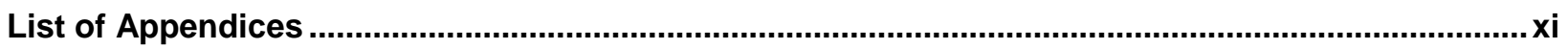

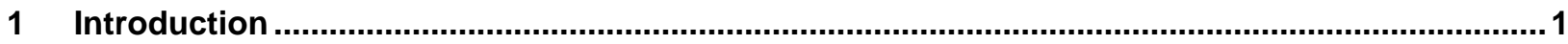

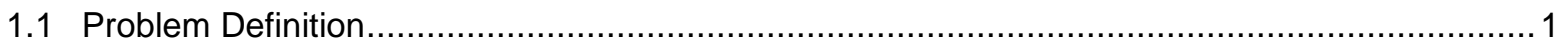

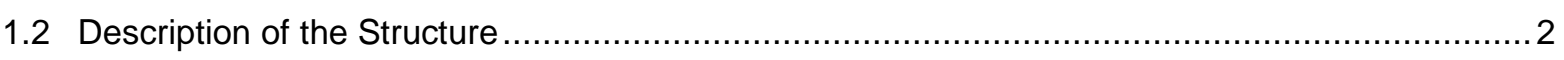

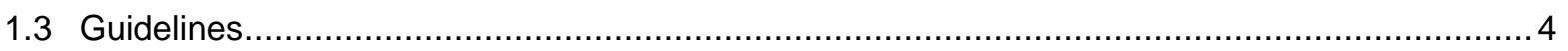

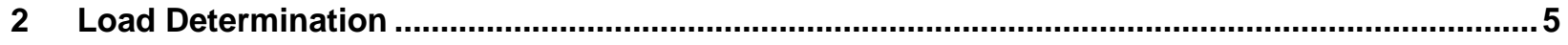

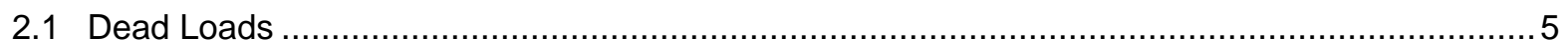

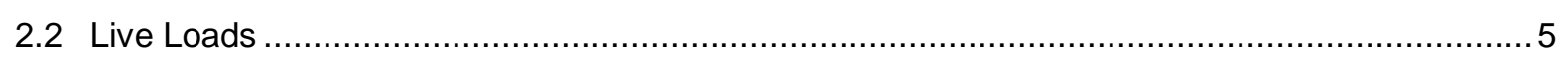

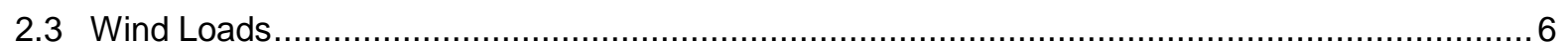

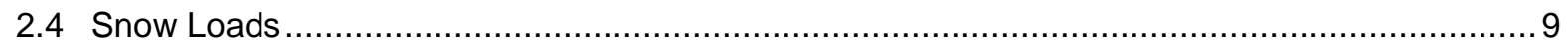

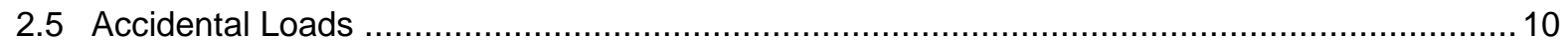

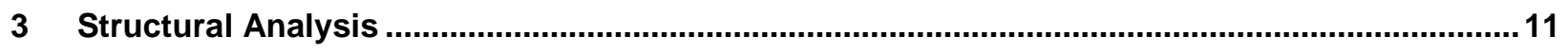

3.1 Action Combinations and Factored Loads ................................................................... 11

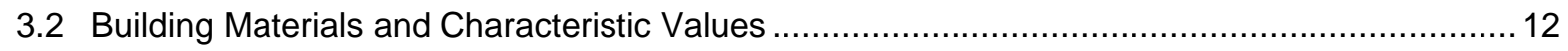

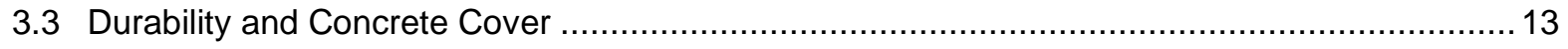

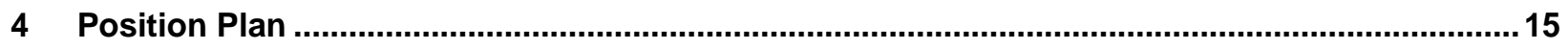

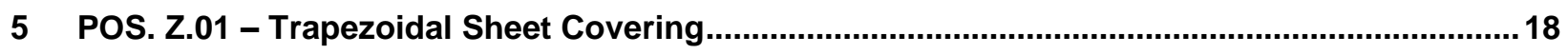

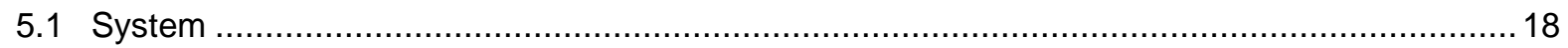

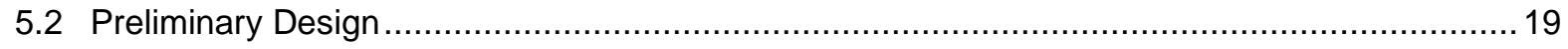

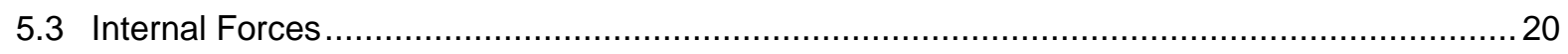

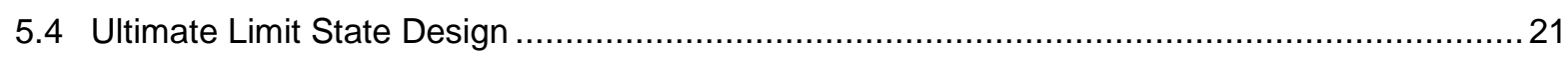

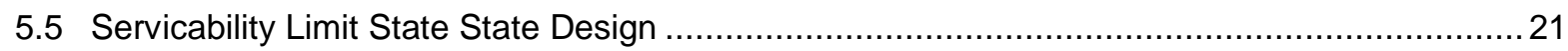

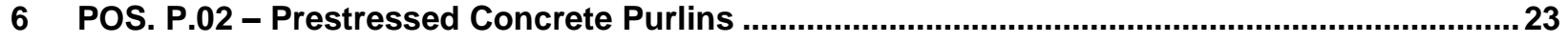

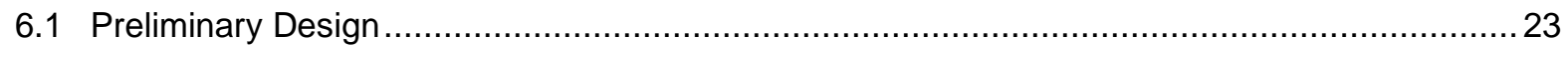

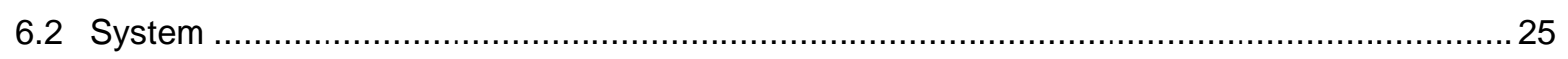

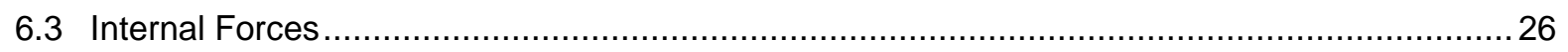

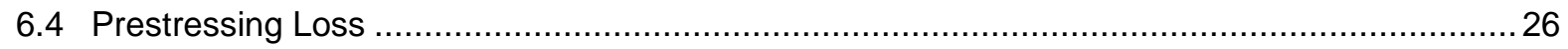

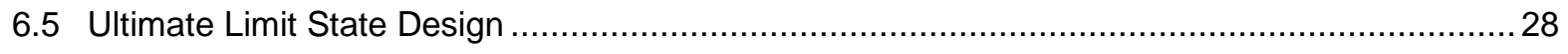

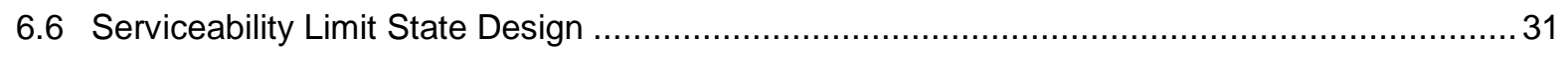




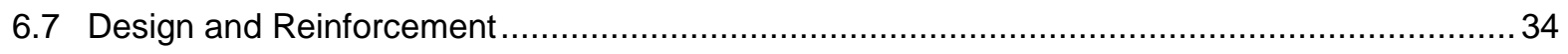

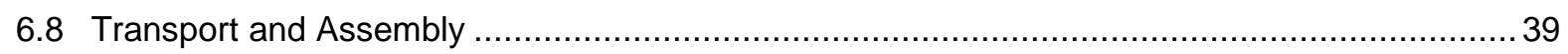

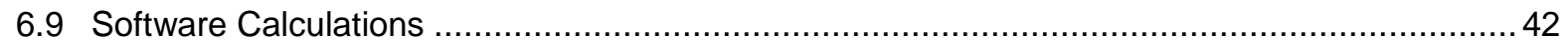

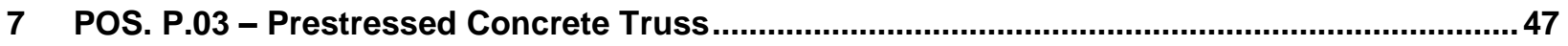

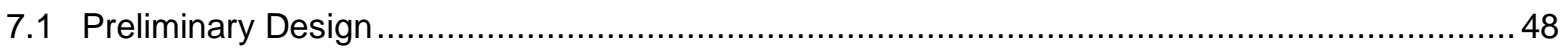

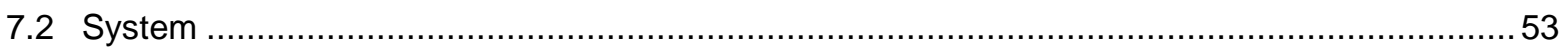

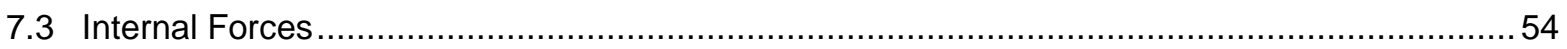

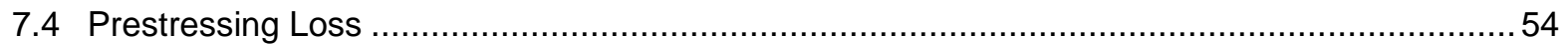

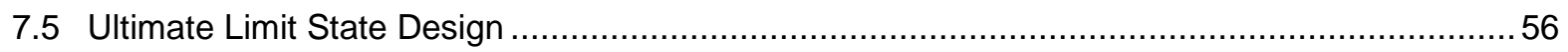

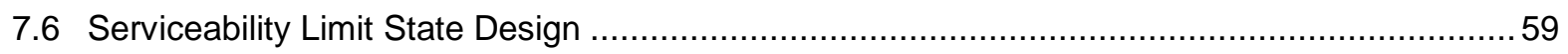

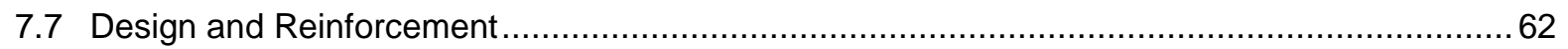

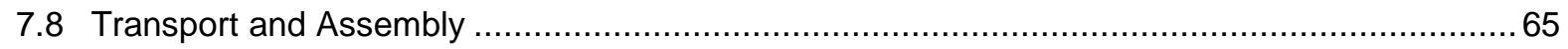

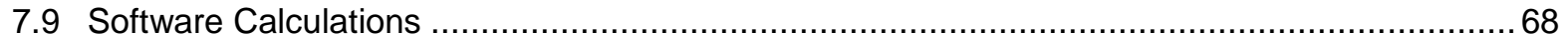

8 POS. C.04 - Precast Concrete Column With Biaxial Wind Loads .............................................73

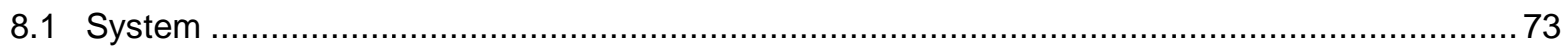

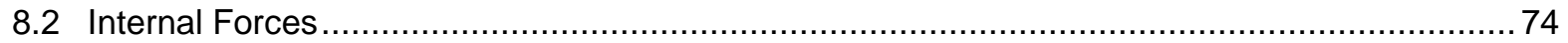

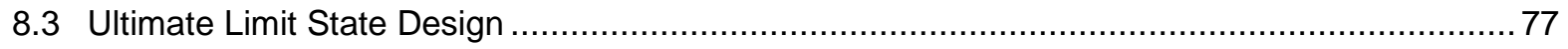

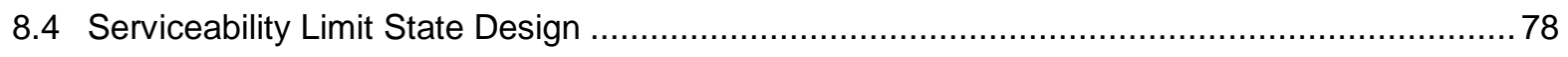

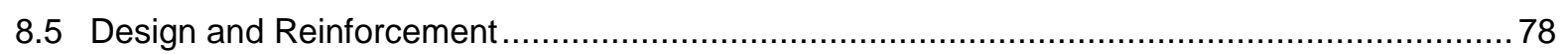

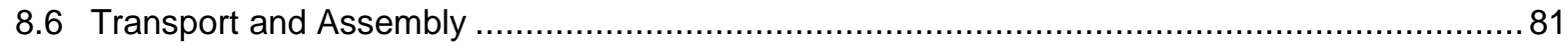

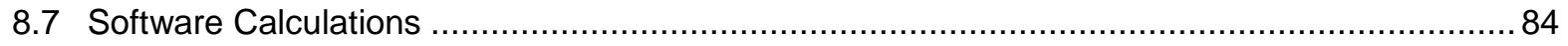

9 POS. C.05 - Precast Concrete Column With Maximum Vertical Load .......................................86

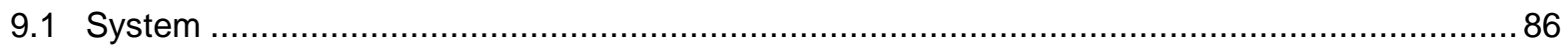

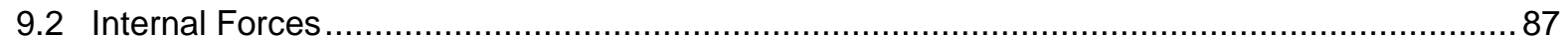

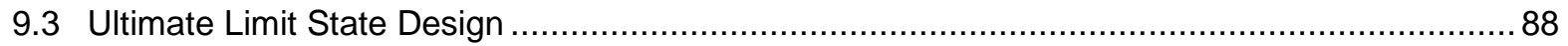

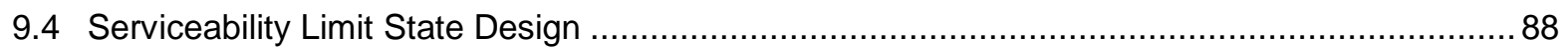

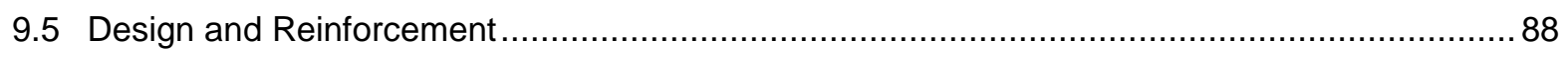

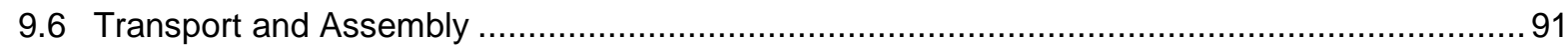

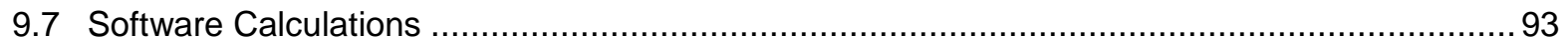

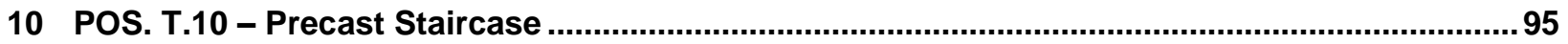

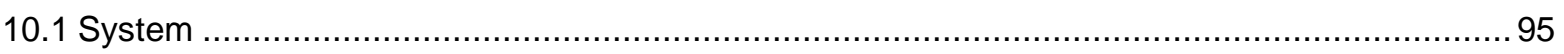

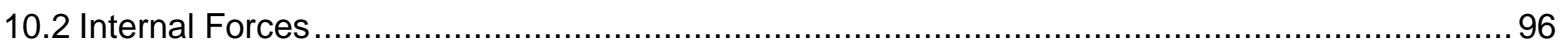

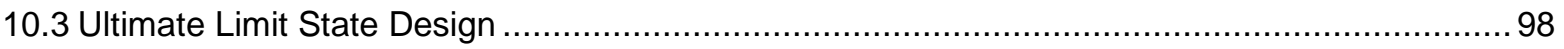

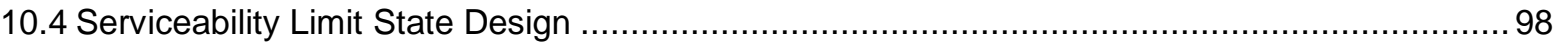

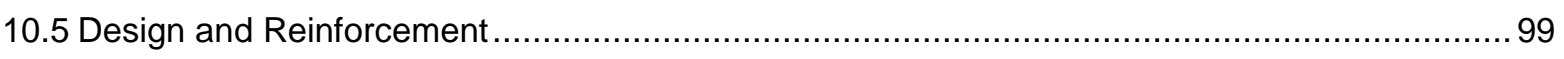

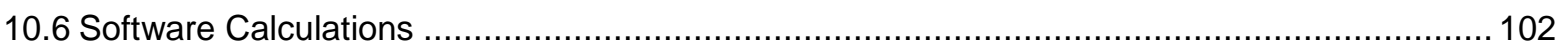

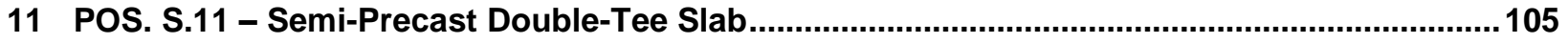




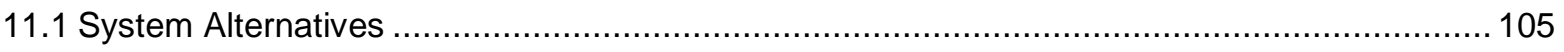

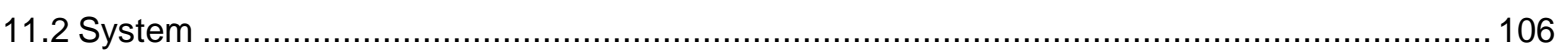

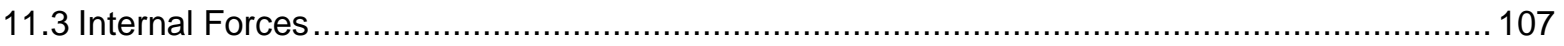

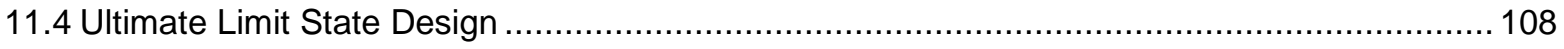

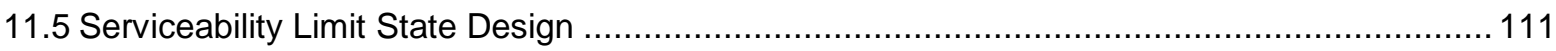

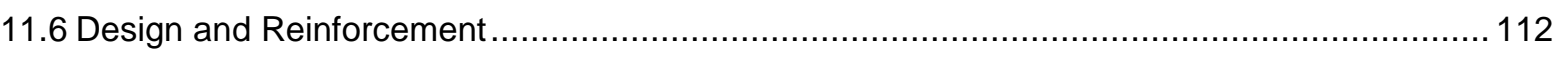

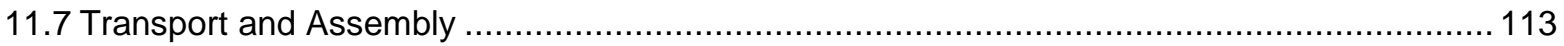

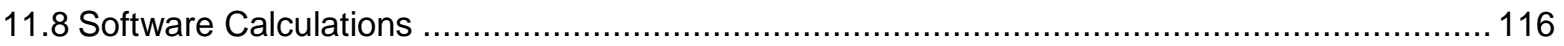

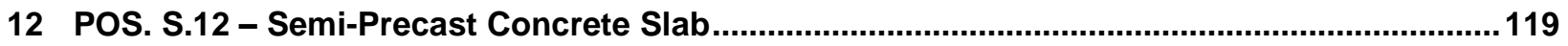

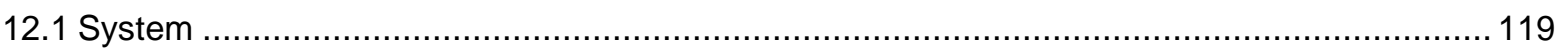

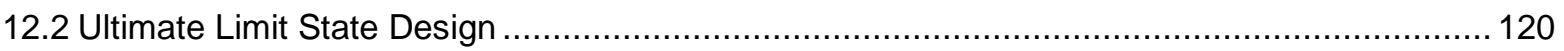

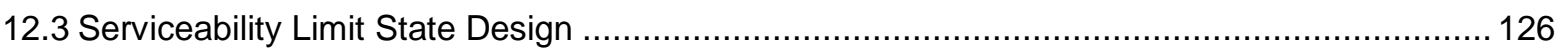

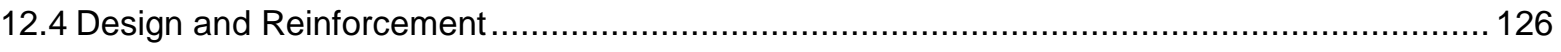

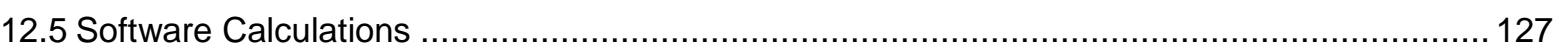

13 POS. B.13 - Semi-Precast Concrete Beam ........................................................................ 134

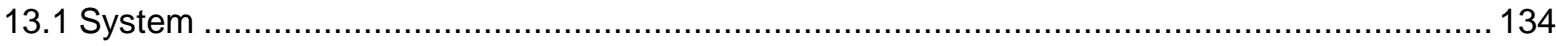

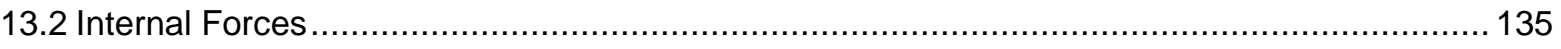

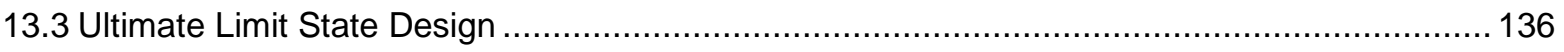

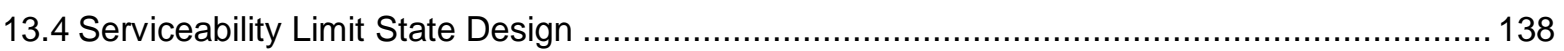

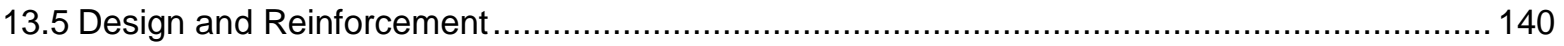

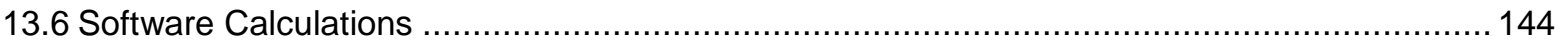

14 POS. W.14 - Load Bearing Precast Concrete Wall Panel ...................................................... 148

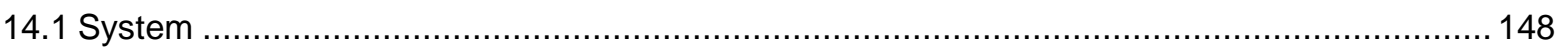

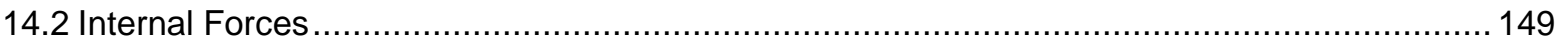

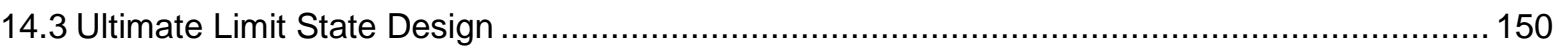

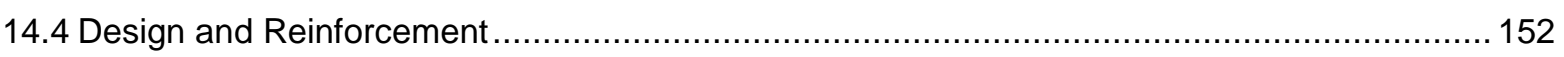

15 POS. W.14 a - Strut-And-Tie Model of the Concrete Wall ....................................................152

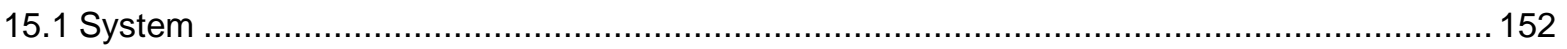

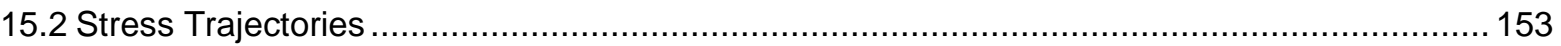

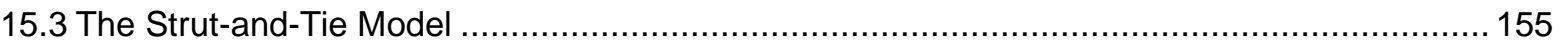

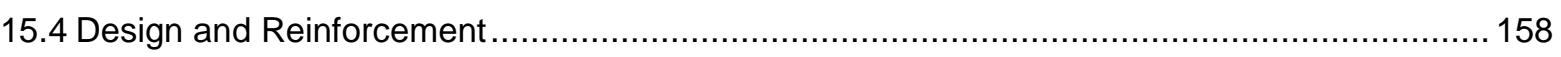

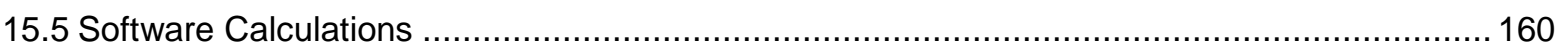

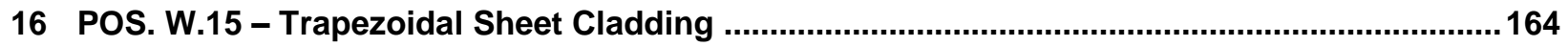

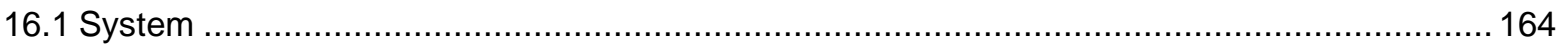

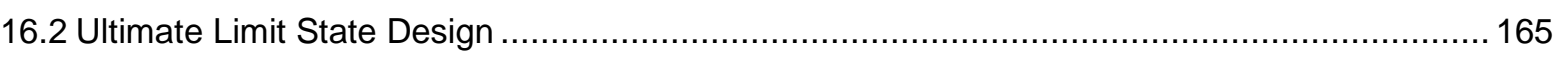

17 POS. F.21 - Pocket Foundation of Column C.04 ….......................................................... 166

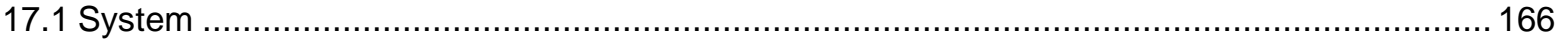




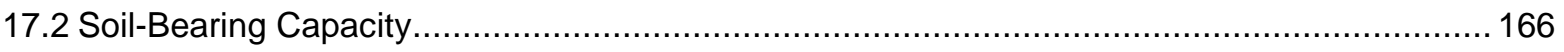

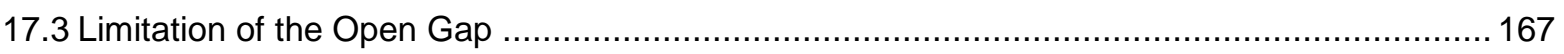

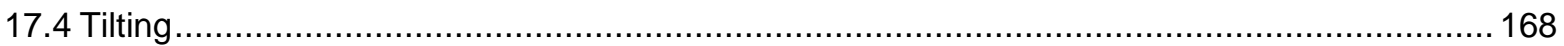

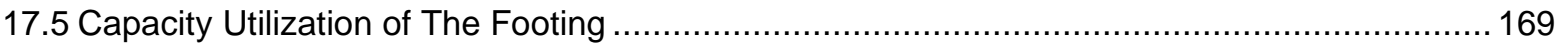

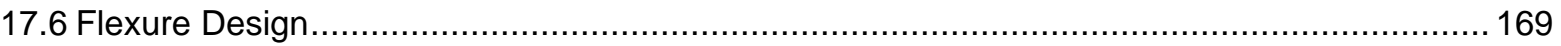

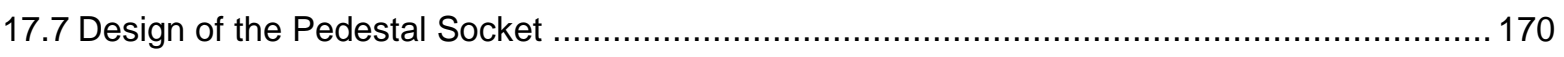

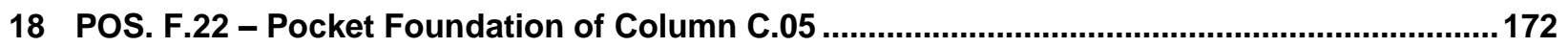

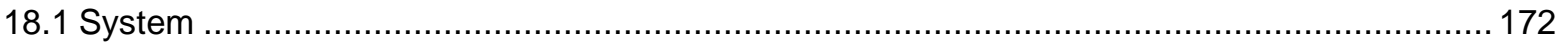

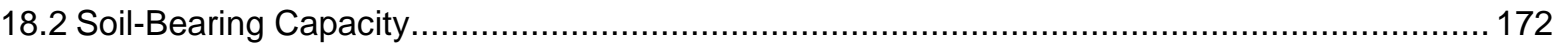

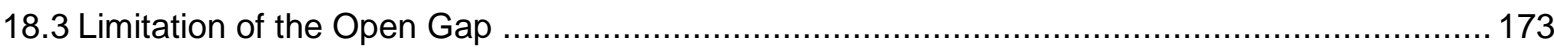

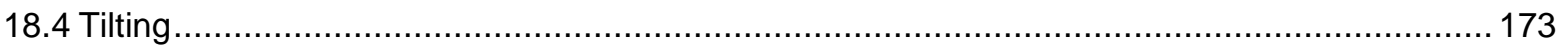

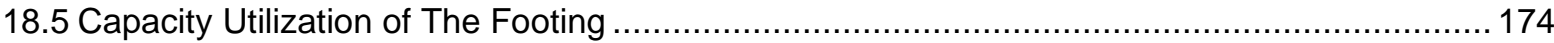

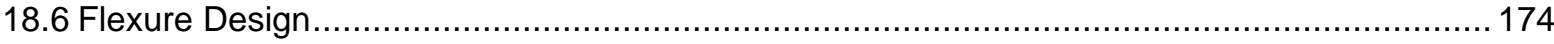

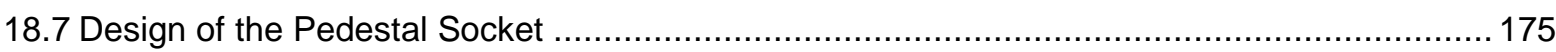

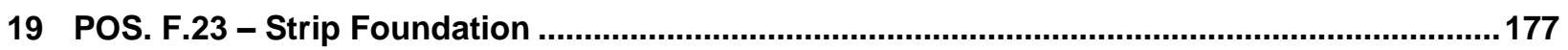

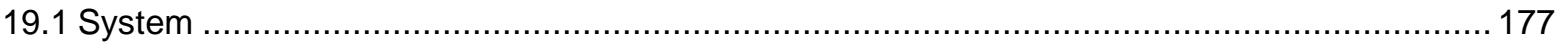

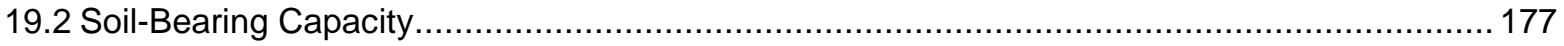

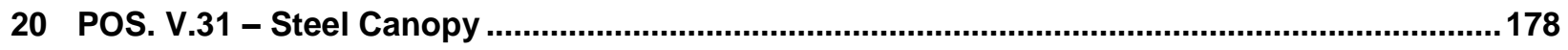

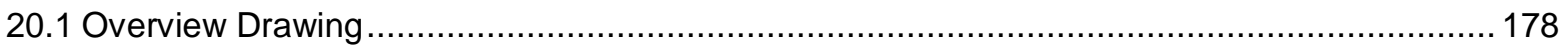

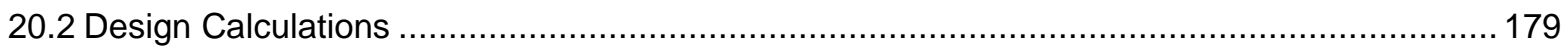

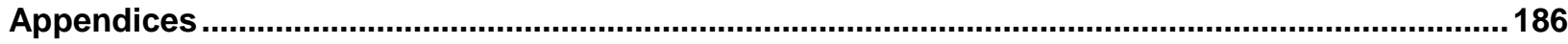

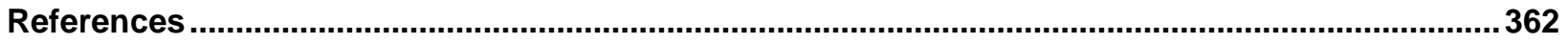

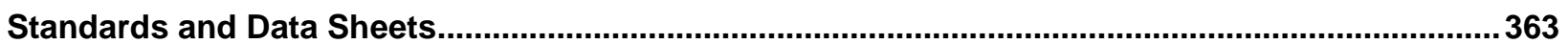




\section{LIST OF TABLES}

Table 1 - Dead Loads from the Floor Structure ........................................................................ 5

Table 2 - Dead Loads from the Roof Structure ....................................................................... 5

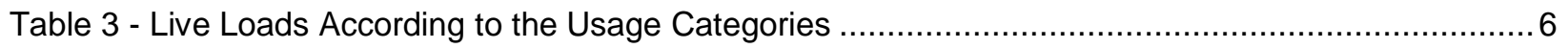

Table 4 - Wind Pressure Coefficients and Wind Pressure on different Areas of the Roof ....................... 7

Table 5 - Wind Pressure Coefficients and Wind Pressure on different Areas of the Exterior Walls ............ 8

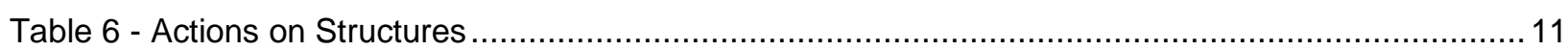

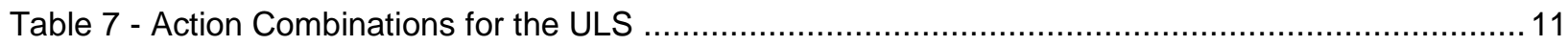

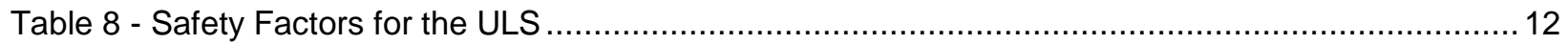

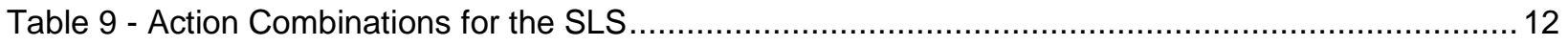

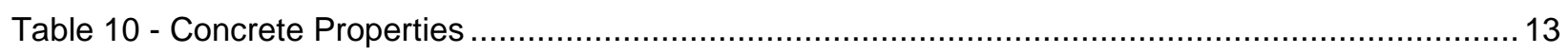

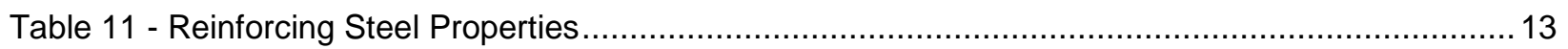

Table 12 - Minimum Concrete Cover $\mathrm{C}_{\text {min,dur }}$ in Relation to the Exposure Class ................................... 14

Table 13 - Sizing Table for the T135.1 as a Three-Span System with a Pressing Load......................... 19

Table 14 - Sizing Table for the T135.1 as a Three-Span System with a Lifting Load ............................. 19

Table 15 - Cross Section Values for Hoesch T 135.1 Positive Position .................................................... 22

Table 16 - Sizing Table for 'HALFEN DEHA Lifting Anchor Systems' ............................................. 41

Table 17 - Sizing Table for 'HALFEN DEHA Lifting Anchor Systems' ................................................ 67

Table 18 - Sizing table for 'HALFEN DEHA Lifting Anchor Systems'.................................................. 83

Table 19 - Sizing Table for 'HALFEN DEHA Lifting Anchor Systems' ................................................ 92

Table 20 - Sizing Table for the Schöck Tronsole® Type T ......................................................... 100

Table 21 - Sizing Table for 'HALFEN DEHA Lifting Anchor Systems' ............................................. 115

Table 22 - Resulting Forces of the Strut-and-Tie Model........................................................... 158 


\section{LIST OF FIGURES}

Figure 1 - Layout Drawing of the Factory Hall by Schöne + Seeburger ............................................ 2

Figure 2 - Layout Drawing of the Ground Floor of the Office Section of the Building .............................. 3

Figure 3 - Layout Drawing of the Second Floor of the Office Section by Schöne + Seeburger.................. 3

Figure 4 - Section B-B of the Hall by Schöne + Seeburger ........................................................ 3

Figure 5 - Section E-E of the hall by Schöne + Seeburger ......................................................... 3

Figure 6 - Section C-C of the Hall by Schöne + Seeburger .................................................... 4

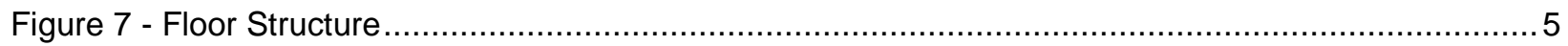

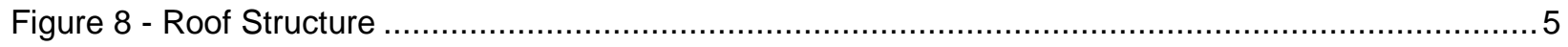

Figure 9 - Wind Zone Map for Germany taken form DIN EN 1991-1-4 NA ........................................ 6

Figure 10 - Definition of Areas on the Roof with different Wind Pressure Coefficients ............................. 7

Figure 11 - Wind Pressure Distribution on the Exterior Walls (left: $\theta=0^{\circ}$, right: $\left.\theta=90^{\circ}\right) \ldots \ldots \ldots \ldots \ldots \ldots \ldots . . . \ldots$

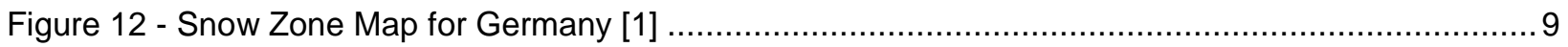

Figure 13 - Snow Loading Arrangement for Saw-Tooth Roofs taken from DIN EN 1991-1-3 Bild 5.4 _..... 10

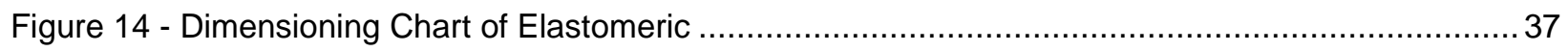

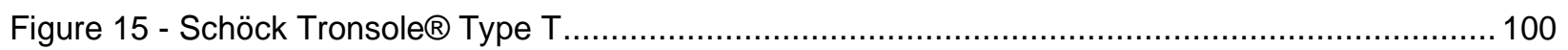

Figure 16 - Proposal of a Combination of a Slab-Beam System and a Flat Slab .............................. 105

Figure 17 - Proposal of a Combination of a Double-Tee Slab System and A Two-Way Slab................ 106

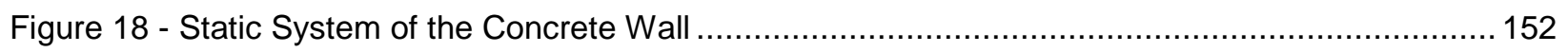

Figure 19 - Stress Trajectories for a Single-Span Deep Beam .......................................................... 153

Figure 20 - Stress Trajectories for a Double-Span Deep Beam ................................................... 154

Figure 21 - Stress Trajectories for a Deep Beam with a Continuous Support ...................................... 154

Figure 22 - Static System to Calculate the Reaction Forces of the Concrete Wall .............................. 155

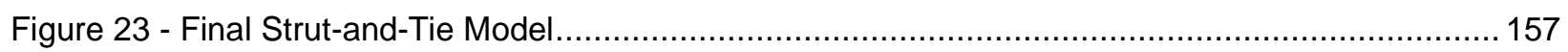

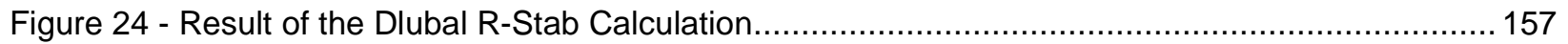

Figure 25 - Hoesch Thermowand https://hoesch-bau.com/wand/ipn-kern/hoeschR-thermowand.html .. 164

Figure 26 - Sizing Table of the Sheet Cladding 'Hoesch Thermowand VL 80' for Wind Suction............. 165

Figure 27 - Sizing Table of the Sheet Cladding 'Hoesch Thermowand VL 80' for Wind Pressure .......... 165

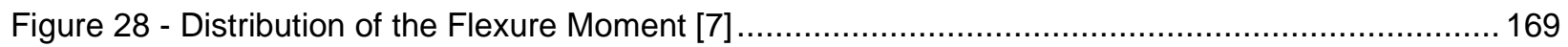

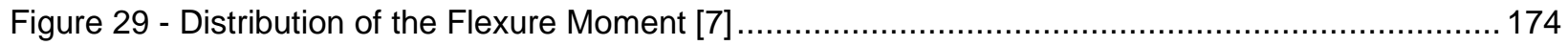

Figure 30 - Buckling Length for a Clamped Column with Attached Hinged Columns .......................... 183 


\section{LIST OF APPENDICES}

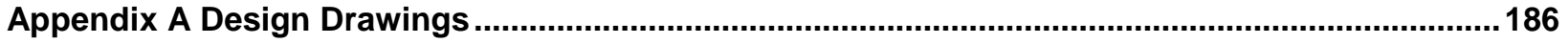

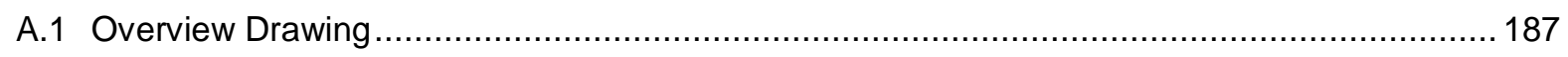

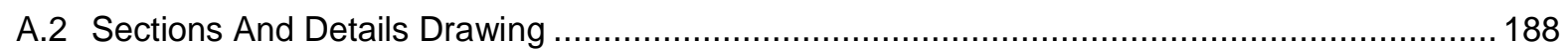

A.3 Formwork and Reinforcement Plan of a Prestressed Concrete Truss ................................ 189

A.4 Formwork and Reinforcement Plan of a Column ............................................................. 190

A.5 Formwork and Reinforcement Plan of a Double-Tee Slab................................................ 191

A.6 Formwork and Reinforcement Plan of Part of the Slab.................................................... 192

A.7 Formwork and Reinforcement Plan of a Concrete Beam ................................................ 193

A.8 Formwork and Reinforcement Plan of a Column Footing .................................................. 194

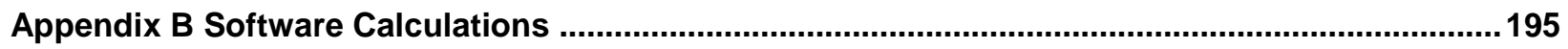

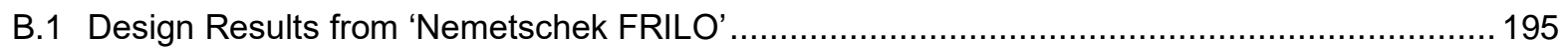

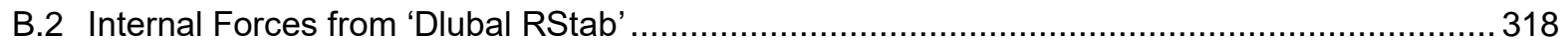




\section{$1 \quad$ INTRODUCTION}

\subsection{PROBLEM DEFINITION}

The objective of this MRP is to develop an alternative proposal for the design of a pre-existing factory hall built in Betzweiler-Wälde, Germany, and planned by the company Schöne + Seeburger / Schwäbisch Gemünd. The original design of the factory hall was a steel structure and the given layout drawings are based on a steel design. As an alternative proposal, the hall will be designed as a precast and prestressed concrete structure. A structural analysis must be conducted in a form that a test engineer could approve of. Due to the large scale of this building and the number of different structural members, only an example of each of the main structural parts of the building are to be designed.

This includes the design of:

- the roof covering

- a prestressed concrete purlin

- a prestressed concrete truss

- the main concrete columns and the supports of the truss

- the exterior walls

- the concrete slab and one beam of the office section

- a staircase

- the main single and strip foundations.

Additionally, a rough design of a steel canopy for the entry of the building will be completed. All calculations must be done according to the European Standards, the Eurocodes, and respectively the German national annexes.

In addition, several layout and reinforcement drawings must be made based on the results of the design calculations.

Following drawings must be included:

- A layout drawing of the roof of the factory hall

- A sections and details drawing of the factory hall

- A formwork and reinforcement plan of the main roof truss

- A formwork and reinforcement plan of one column

- A formwork and reinforcement plan of one beam

- A formwork and reinforcement plan of an excerpt of the slab

- A formwork and reinforcement plan of one individual foundation. 


\subsection{DESCRIPTION OF THE STRUCTURE}

Figure 1 shows the layout of the building, which is divided into two parts. The main part is the factory hall, which functions as a large office furniture factory. The smaller part in the lower right corner is a two-story office section which is framed by the large hall structure.

The hall structure measures about 120 metres in length and 91 metres in width with a maximum height of 10.65 meter. The office section is 98 metres long and 15 metres wide. For an easier overview, the layout of the building is divided into a grid. The short side of the building is divided by an alphabetical axis every 30 meters and the long side is divided by a numbered axis every 15 metres. The main columns are arranged at each intersection of this grid.

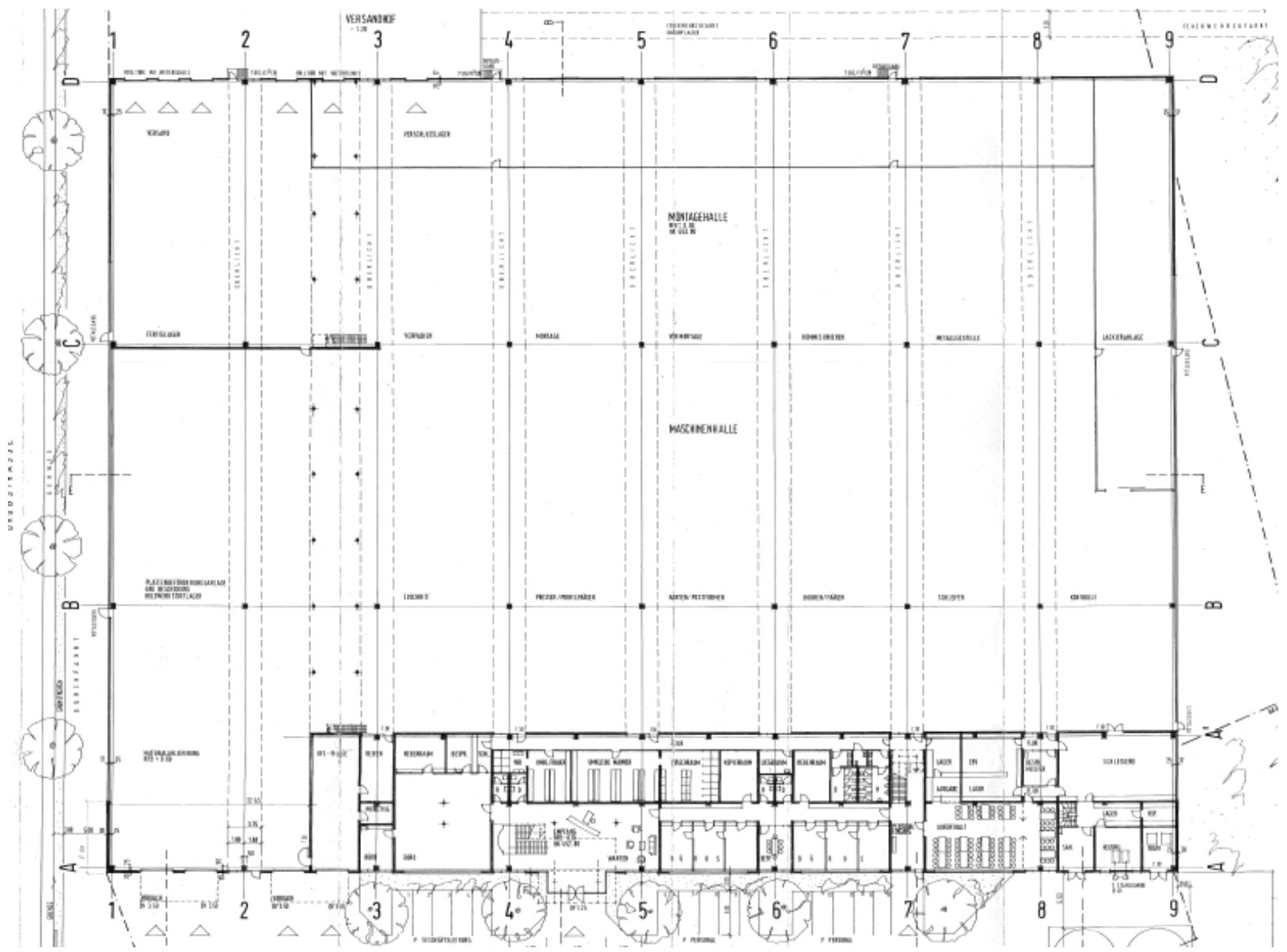

Figure 1 - Layout Drawing of the Factory Hall by Schöne + Seeburger

Figure 2 and Figure 3 show the layout of the first and second floor of the office section, respectively. In addition to the axis of the hall, another axis ( $\mathrm{A}^{\prime}$ between $\mathrm{A}$ and $\mathrm{B}$ ) was added. Along this axis, an additional column row for the office floor is provided, as well as additional columns along the middle axis of the office building. The entry of the office building is between axis 4 and 5 , and as it is planned to be a big open space, the slab between the first and second floor is cut out above the entrance area. A large staircase leads to the second floor. 


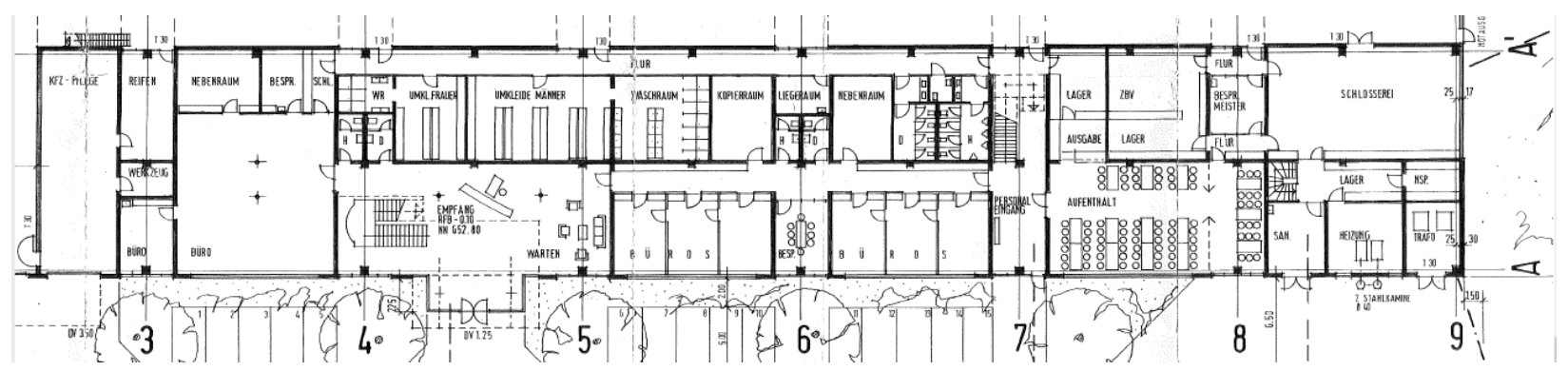

Figure 2 - Layout Drawing of the Ground Floor of the Office Section of the Building by Schöne + Seeburger

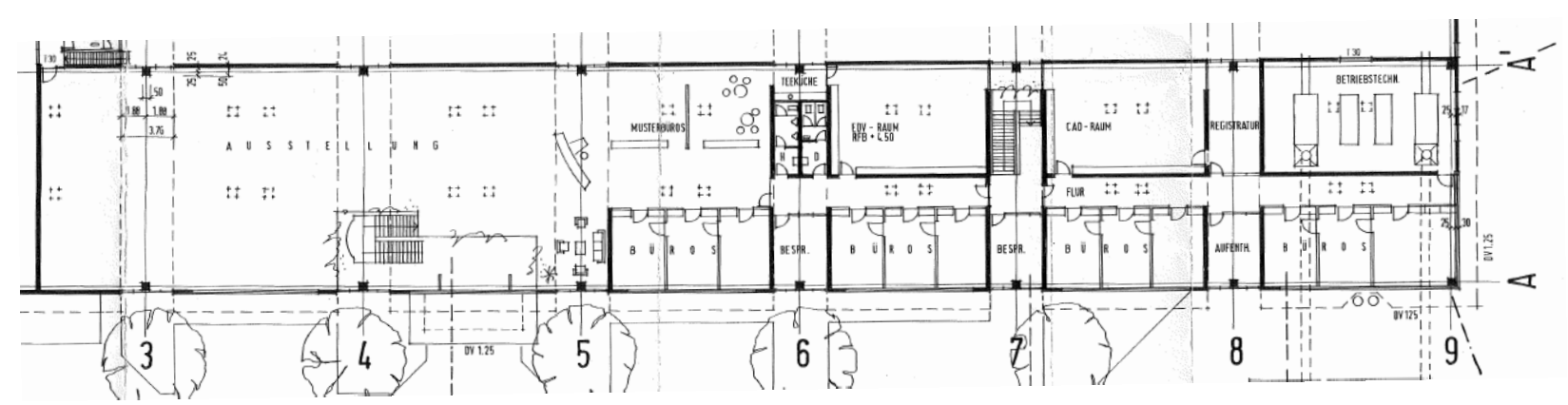

Figure 3 - Layout Drawing of the Second Floor of the Office Section by Schöne + Seeburger

Figure 4 to Figure 6 show different section drawing of the factory hall. Section B-B (Figure 4) cuts along the short side of the building in between axis 4 and 5 . As mentioned previously, these diagrams demonstrate the open structure of the entrance to the office building and also shows how the office is framed by the large structure of the hall instead of being added to it as a separate structure.

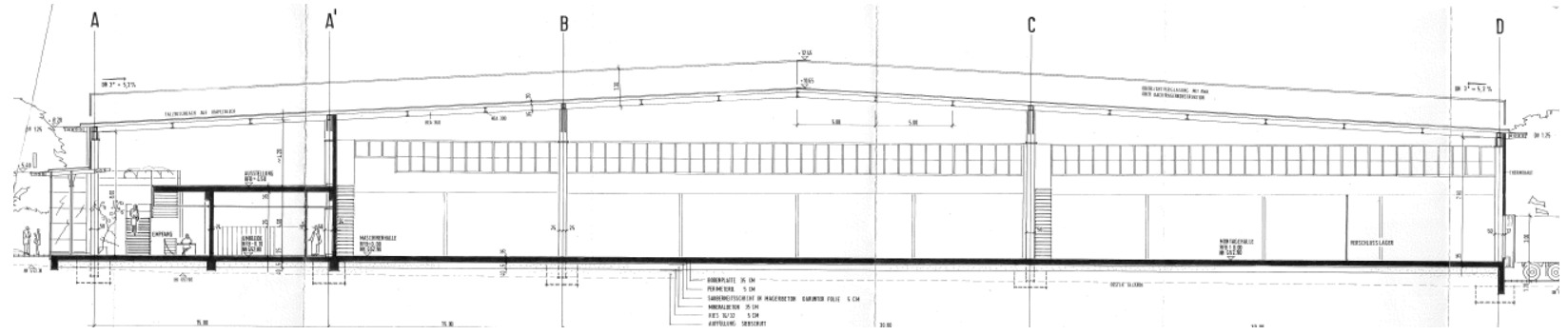

Figure 4 - Section B-B of the Hall by Schöne + Seeburger

Section E-E (Figure 5) is a cut along the long side of the building an shows the big factory hall.

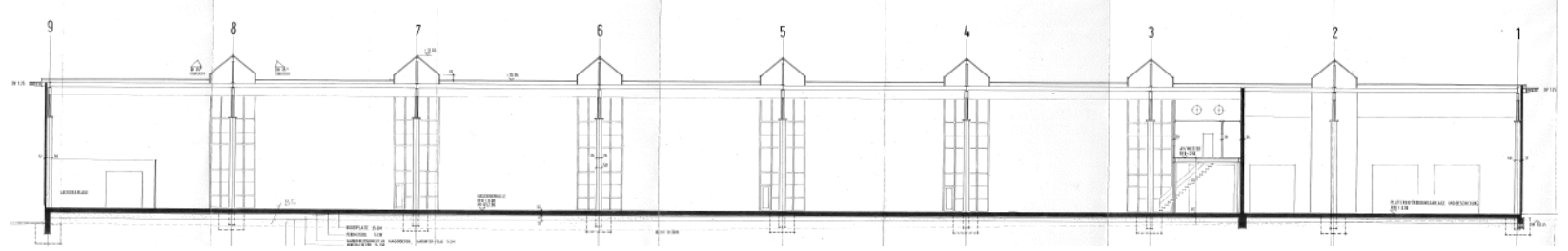

Figure 5 - Section E-E of the hall by Schöne + Seeburger 
Section C-C (Figure 6) is another cut through the office building and shows the second staircase.

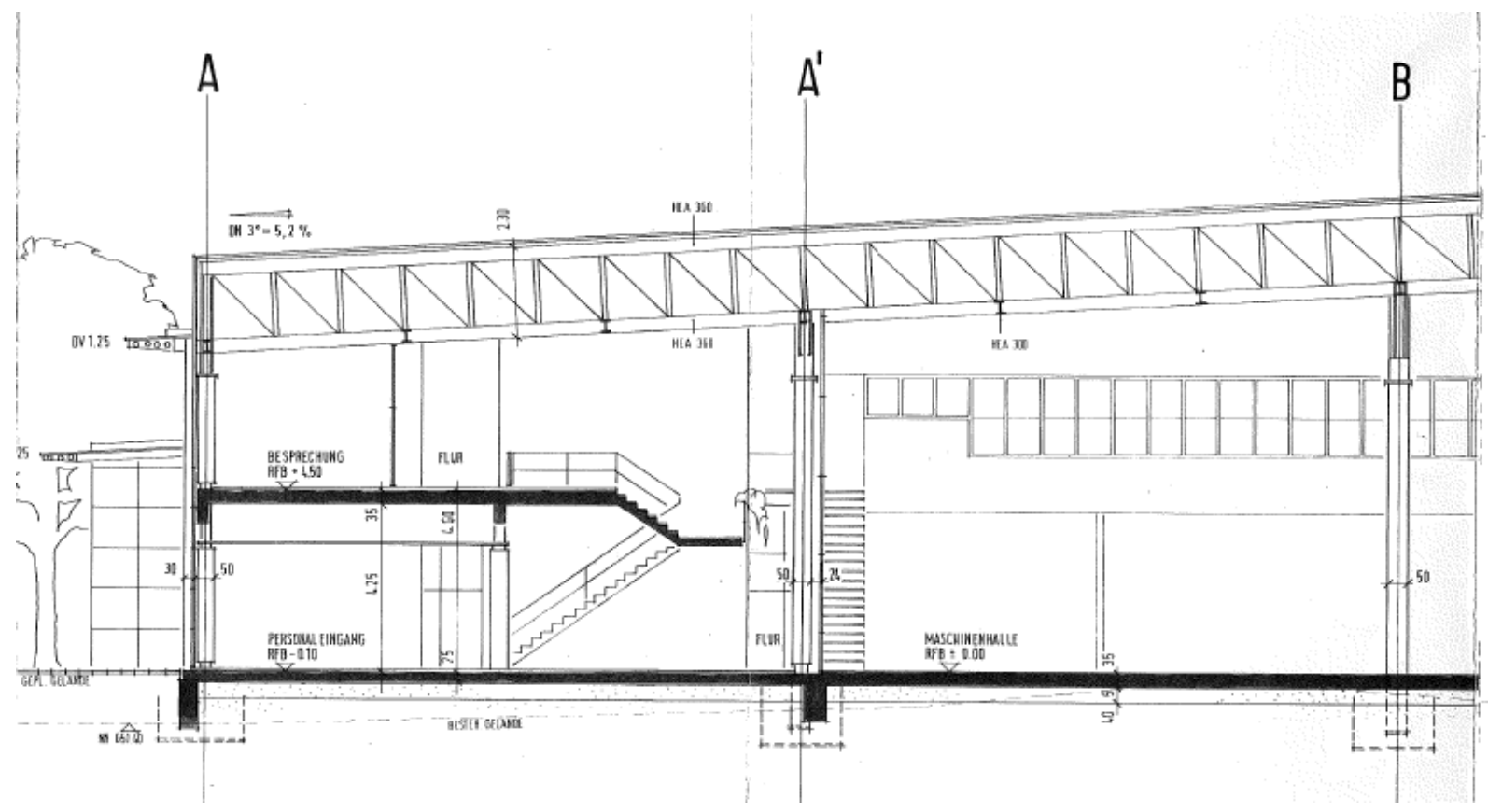

Figure 6 - Section C-C of the Hall by Schöne + Seeburger

\subsection{GUIDELINES}

Apart from the measurements and the geometry given in the drawings, certain guidelines for the new design of the building as a concrete structure (instead of a steel structure) must be met.

\subsubsection{HALL STRUCTURE}

The hall structure must consist of:

- Trapezoidal sheets with insulation as roof covering

- Prestressed concrete purlins with a length of approximately 15 metres and in a of 5 metres between each other

- Prestressed concrete trusses with a length of about 30 metres and in a distance of 15 metres between each other

- Clamped concrete columns which are intended to brace the hall structure against wind

- Trapezoidal sheet cladding for the façade.

\subsubsection{OFFICE BUILDING}

The office building must consist of:

- Trapezoidal sheets with insulation as roof covering

- A composite plank floor with precast concrete beams

- Clamped concrete columns which are intended to brace the hall structure against wind

- Concrete wall panels. 


\section{LOAD DETERMINATION}

\subsection{DEAD LOADS}

The dead loads mainly result from the roof and floor structure.

\subsubsection{FLOOR STRUCTURE}

The composition of the dead loads resulting from the floor structure is summarized in Table 1.

Table 1 - Dead Loads from the Floor Structure

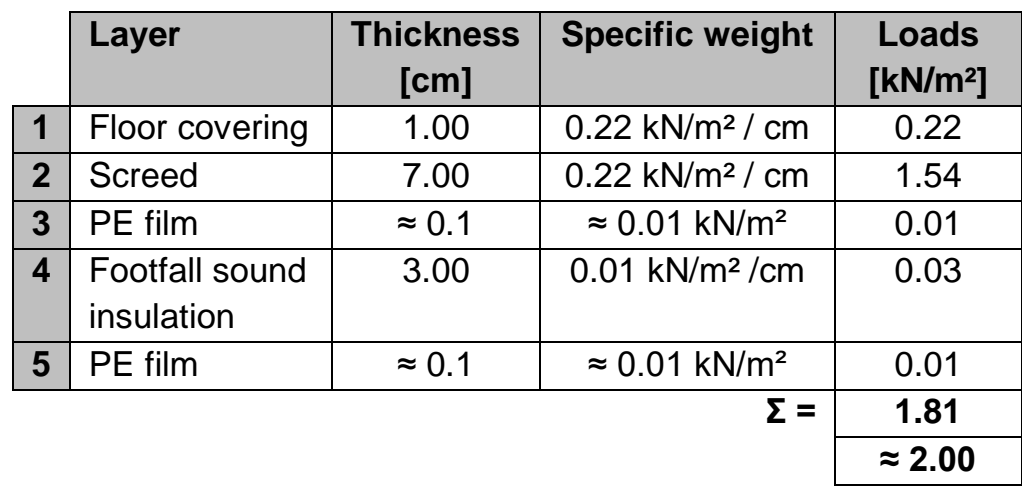

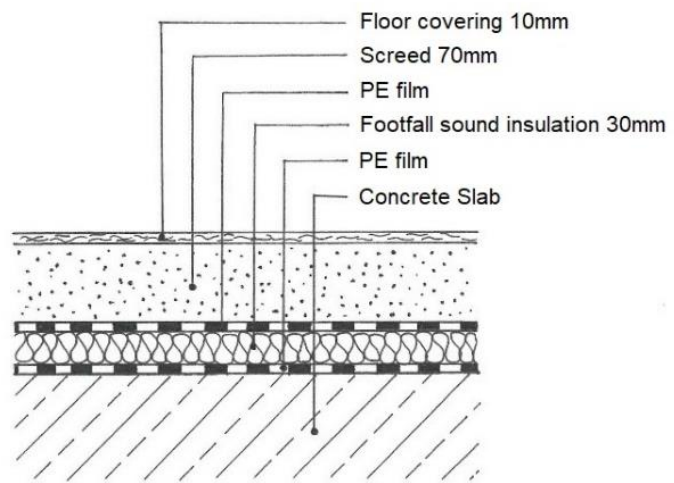

\subsubsection{ROOF STRUCTURE}

The composition of the dead loads resulting from the roof structure is summarized in Table 2.

Table 2 - Dead Loads from the Roof Structure

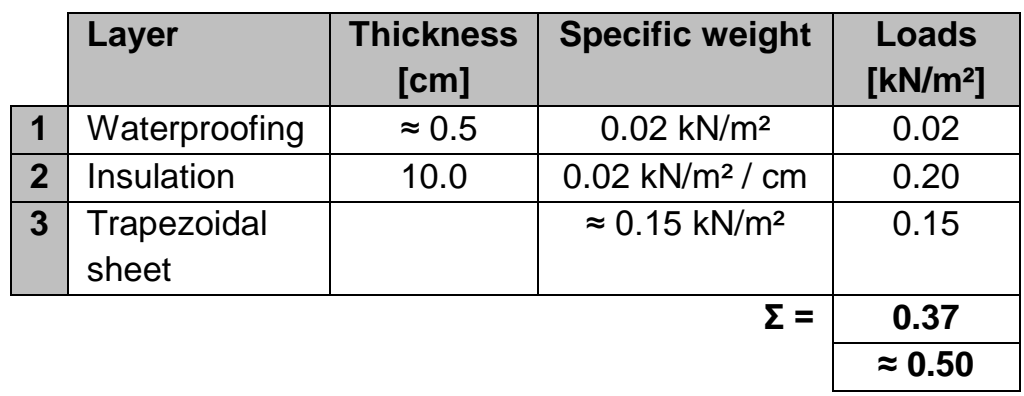

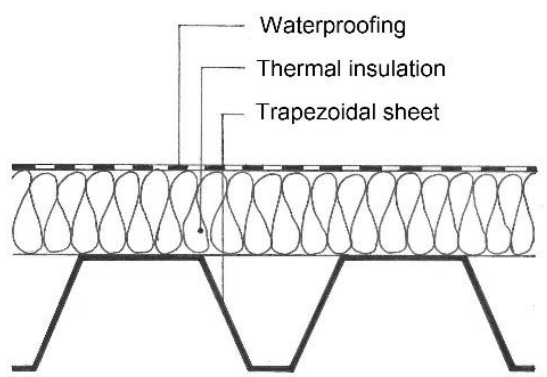

\subsection{LIVE LOADS}

As per DIN EN 1991-1-1 and DIN EN 1991-1-1/NA the usage category of the building must be defined with the respective live load which has to be considered in the structural analysis. Since the building is of mixed usage, with office areas on one side and storage areas on the other, different usage categories must be observed. They can be seen in Table 3. 
Table 3 - Live Loads According to the Usage Categories

\begin{tabular}{|c|c|c|c|c|}
\hline \multicolumn{2}{|c|}{ Category* } & Usage & $\begin{array}{c}q_{k} \\
{\left[k N / m^{2}\right]}\end{array}$ & $\begin{array}{c}Q_{k} \\
{[k N]}\end{array}$ \\
\hline \multicolumn{2}{|l|}{$\mathrm{B} 1$} & Office areas & 2.0 & 2.0 \\
\hline \multicolumn{2}{|l|}{ E1 } & Warehouses and workshops without forklift traffic & 5.0 & 4.0 \\
\hline \multicolumn{2}{|l|}{ E2.5 } & Warehouses with forklift traffic of the category FL4 ${ }^{\star *}$ & 20.0 & $2 \times 90.0$ \\
\hline \multicolumn{2}{|l|}{$\mathrm{T} 1$} & Stair cases and -landings in office buildings & 3.0 & 2.0 \\
\hline $\begin{array}{l} \\
\star * \\
q_{k} \\
Q_{k}\end{array}$ & $\begin{array}{l}\text { Accorc } \\
\text { Accorc } \\
\text { Chara } \\
\text { Chara }\end{array}$ & $\begin{array}{l}\text { ing to DIN EN 1991-1-1/NA Tab. 6.1DE } \\
\text { ing to DIN EN 1991-1-1 Tab. } 6.5 \\
\text { teristic distributed live load } \\
\text { teristic concentrated live load }\end{array}$ & & \\
\hline
\end{tabular}

\subsection{WIND LOADS}

The wind loads are determined according to DIN EN 1991-1-4. The value depends on the location and the height of the building.

Location oft he Building: Betzweiler-Wälde, Baden-Württemberg, Germany

Wind zone (Figure 9): $\quad$ Wind zone 1

Terrain category: Mixed profile "Inland"

Height of the building: $\quad z=10.65 \mathrm{~m}$

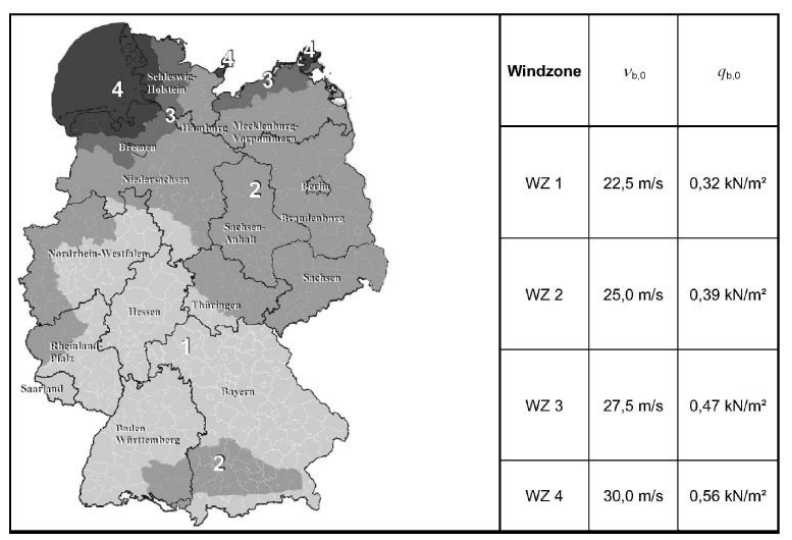

Figure 9 - Wind Zone Map for Germany taken form DIN EN 1991-1-4 NA

The velocity pressure for buildings with a height above $7 \mathrm{~m}$ and in the category above is calculated according to following formula:

$q_{p}(z)=1.7 \cdot q_{b, 0} \cdot\left(\frac{z}{10}\right)^{0,37} \quad$ for $7 \mathrm{~m}<\mathrm{z} \leq 50 \mathrm{~m}$

With $\quad \mathrm{q}_{\mathrm{p}}$ : Velocity Pressure

$\mathrm{z}$ : Height of the building

$\mathrm{q}_{\mathrm{b}, 0}$ : $\quad$ Basis velocity pressure.

With this formula the velocity pressure can be calculated as: 
$q_{p}(10,65)=1.7 \cdot 0.32 \cdot\left(\frac{10.65}{10}\right)^{0.37}=0.56 \mathrm{kN} / \mathrm{m}^{2}$

\subsubsection{WIND PRESSURE ON THE ROOF}

In accordance with DIN EN 1991-1-4 the coefficients for the wind pressure on the roof can be divided into five different areas $\mathrm{F}$ to $\mathrm{J}$ as it is shown in Figure 10.
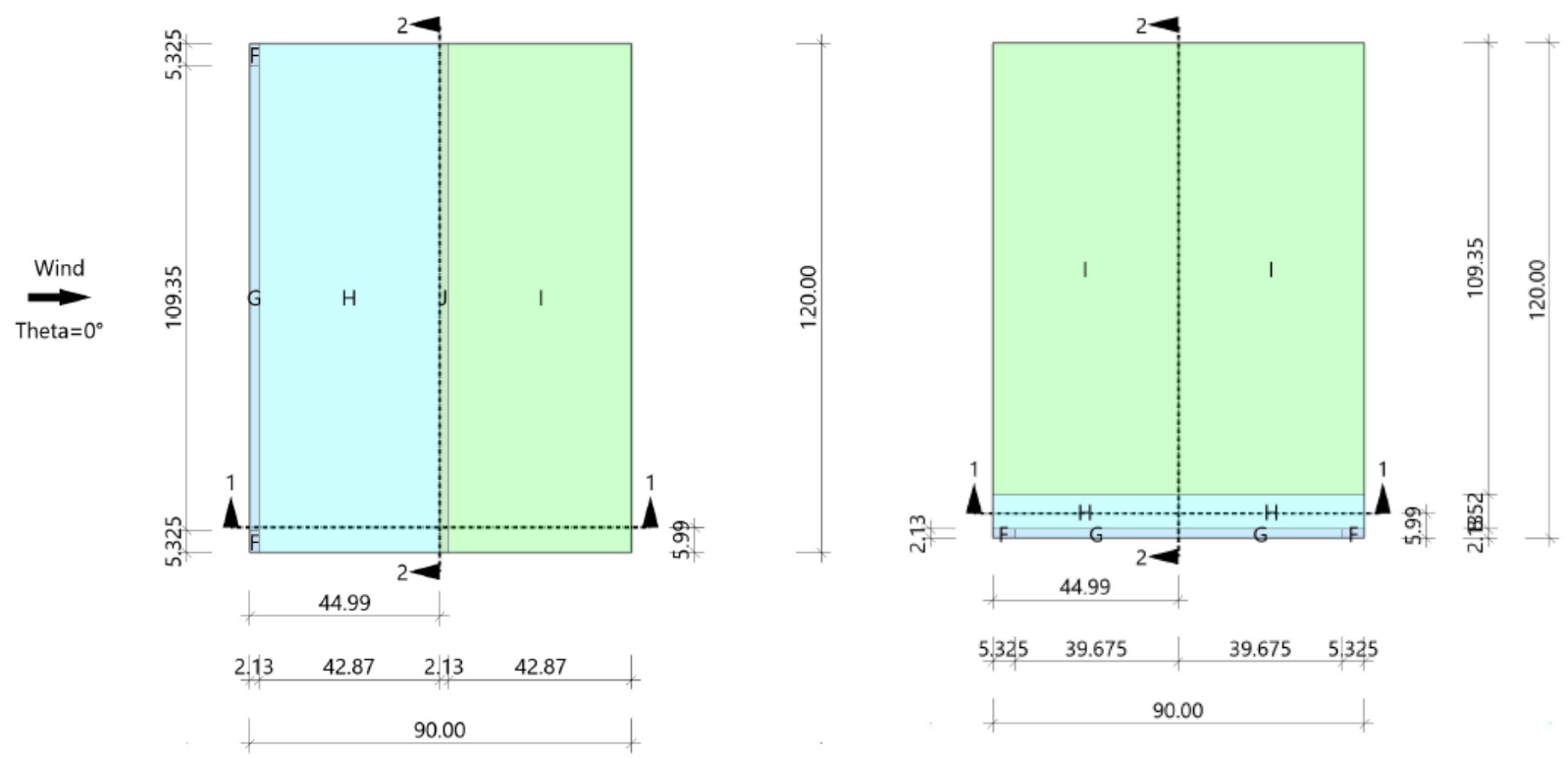

Figure 10 - Definition of Areas on the Roof with different Wind Pressure Coefficients

The following table sums up the different wind pressure coefficients which must be taken into account in the determination of the decisive wind pressure on the roof of the building.

Table 4 - Wind Pressure Coefficients and Wind Pressure on different Areas of the Roof

\begin{tabular}{|c|c|c|c|c|c|c|c|c|}
\hline & \multicolumn{4}{|c|}{ Inflow Direction $\theta=0^{\circ}$} & \multicolumn{4}{|c|}{ Inflow Direction $\theta=90^{\circ}$} \\
\hline Area & $\begin{array}{c}C_{p e, 10} \\
{[-]}\end{array}$ & $\begin{array}{c}C_{p e, 1} \\
{[-]}\end{array}$ & $\begin{array}{c}w_{e, 10} \\
{\left[k N / m^{2}\right]}\end{array}$ & $\begin{array}{c}W_{e, 1} \\
{\left[k N / m^{2}\right]}\end{array}$ & $\begin{array}{c}C_{p e, 10} \\
{[-]}\end{array}$ & $\begin{array}{c}C_{p e, 1} \\
{[-]}\end{array}$ & $\begin{array}{c}w_{e, 10} \\
{\left[k N / m^{2}\right]}\end{array}$ & $\begin{array}{c}\mathrm{w}_{\mathrm{e}, 1} \\
{\left[\mathrm{kN} / \mathrm{m}^{2}\right]}\end{array}$ \\
\hline $\mathrm{F}$ & -1.80 & -2.50 & -1.00 & -1.39 & -1.80 & -2.50 & -1.00 & -1.39 \\
\hline $\bar{G}$ & -1.20 & -2.00 & -0.67 & -1.11 & -1.20 & -2.00 & -0.67 & -1.11 \\
\hline$\overline{\mathrm{H}}$ & -0.70 & -1.20 & -0.39 & -0.67 & -0.70 & -1.20 & -0.39 & -0.67 \\
\hline I & $\begin{array}{l}+0.20 \\
-0.60\end{array}$ & $\begin{array}{l}+0.20 \\
-0.60\end{array}$ & $\begin{array}{l}+0.11 \\
-0.33\end{array}$ & $\begin{array}{l}+0.11 \\
-0.33\end{array}$ & $\begin{array}{l}+0.20 \\
-0.60\end{array}$ & $\begin{array}{l}+0.20 \\
-0.60\end{array}$ & $\begin{array}{l}+0.11 \\
-0.33\end{array}$ & $\begin{array}{l}+0.11 \\
-0.33\end{array}$ \\
\hline J & $\begin{array}{l}+0.20 \\
-0.60\end{array}$ & $\begin{array}{l}+0.20 \\
-0.60\end{array}$ & $\begin{array}{l}+0.11 \\
-0.33\end{array}$ & $\begin{array}{l}+0.11 \\
-0.33\end{array}$ & - & - & - & - \\
\hline $\begin{array}{l}\mathrm{C}_{\mathrm{pe}, 10} \\
\mathrm{C}_{\mathrm{pe}, 1} \\
\mathrm{w}_{\mathrm{e}, 10} \\
\mathrm{w}_{\mathrm{e}, 1} \\
\end{array}$ & $\begin{array}{l}\text { Wind Pressure } \\
\text { Wind Pressure } \\
\text { Wind Pressure } \\
\text { Wind Pressure }\end{array}$ & $\begin{array}{l}\text { ficient for } \\
\text { ficient for } \\
\text { n Area A } \\
\text { n Area 1 }\end{array}$ & $\begin{array}{l}\text { Area } A>10 \\
\text { Area } 1 \mathrm{~m}^{2}< \\
\mathrm{m}^{2} \\
\mathrm{~A} \leq 10 \mathrm{~m}^{2}\end{array}$ & $10 \mathrm{~m}^{2}$ & & & & \\
\hline
\end{tabular}




\subsubsection{WIND PRESSURE ON THE EXTERIOR WALLS}

Like the wind pressure on the roof, the pressure on the walls must be divided into five different areas, $A$ to $\mathrm{E}$, with the respective coefficients. The wind pressure distribution on the walls can be seen in Figure 11 .
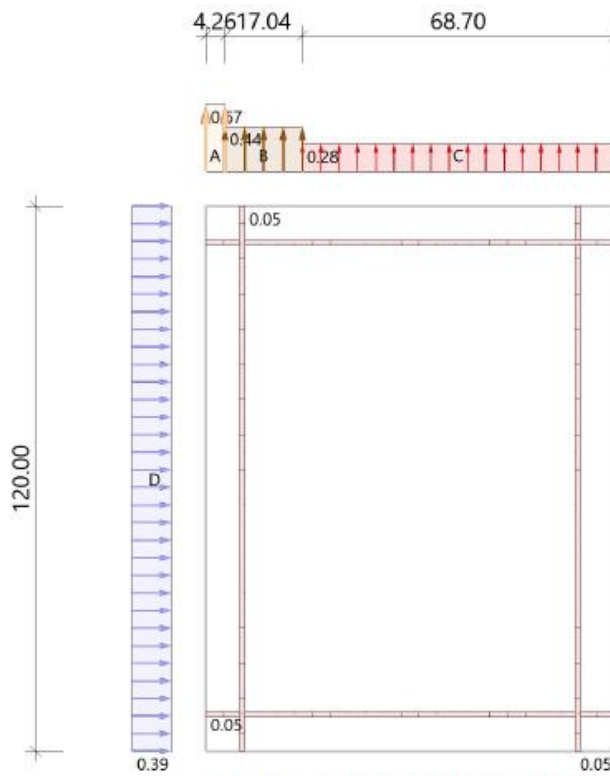
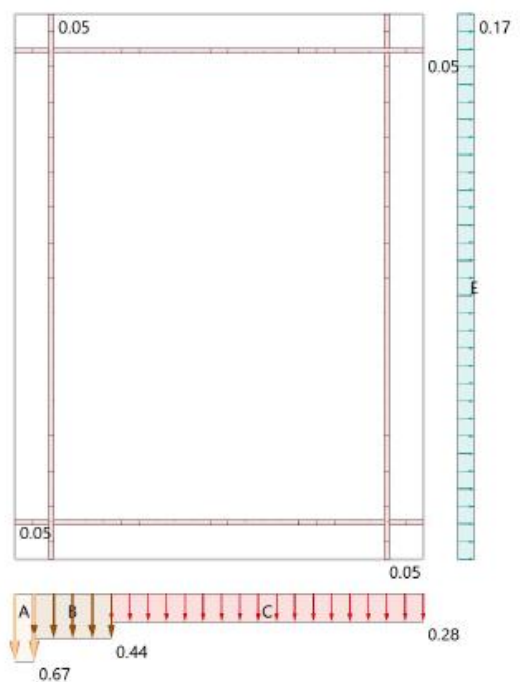

4.2617 .04

68.70
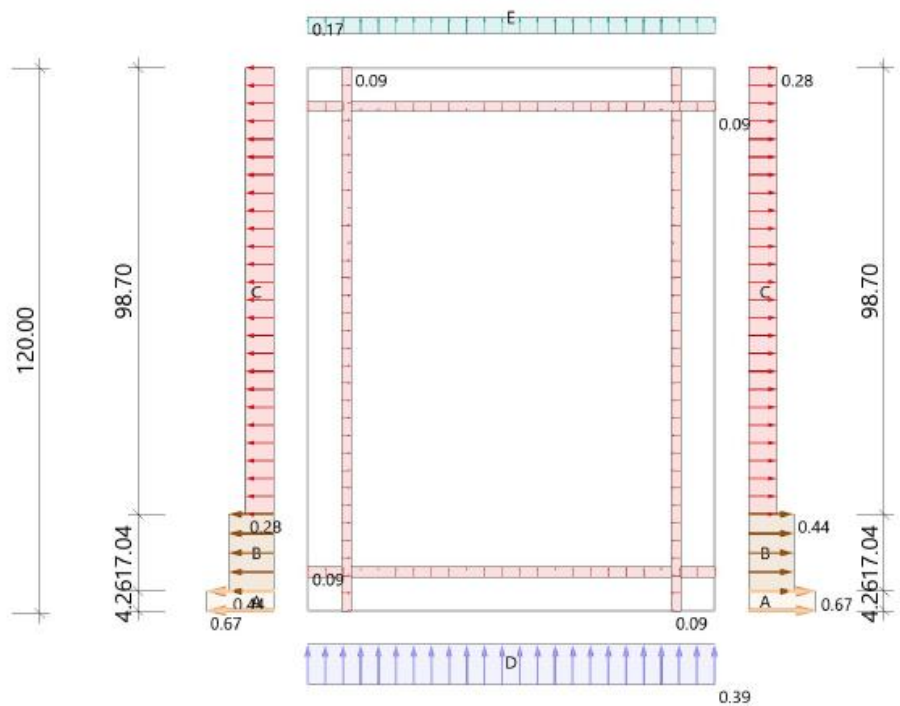

90.00

Figure 11 - Wind Pressure Distribution on the Exterior Walls (left: $\theta=0^{\circ}$, right: $\theta=90^{\circ}$ )

Wind pressure coefficients and wind pressure distributions are summed up in following table.

Table 5 - Wind Pressure Coefficients and Wind Pressure on different Areas of the Exterior Walls

\begin{tabular}{|c|c|c|c|c|c|c|c|c|}
\hline & \multicolumn{4}{|c|}{ Inflow Direction $\theta=0^{\circ}$} & \multicolumn{4}{|c|}{ Inflow Direction $\theta=90^{\circ}$} \\
\hline Area & $\begin{array}{c}C_{p e, 10} \\
{[-]}\end{array}$ & $\begin{array}{c}C_{p e, 1} \\
{[-]}\end{array}$ & $\begin{array}{c}w_{e, 10} \\
{\left[k N / m^{2}\right]}\end{array}$ & $\begin{array}{c}W_{\mathrm{e}, 1} \\
{\left[\mathrm{kN} / \mathrm{m}^{2}\right]}\end{array}$ & $\begin{array}{c}\text { Cpe,10 } \\
{[-]}\end{array}$ & $\begin{array}{c}C_{p e, 1} \\
{[-]}\end{array}$ & $\begin{array}{c}w_{e, 10} \\
{\left[k N / m^{2}\right]}\end{array}$ & $\begin{array}{c}W_{e, 1} \\
{\left[k N / m^{2}\right]}\end{array}$ \\
\hline A & -1.20 & -1.40 & -0.67 & -0.78 & -1.20 & -1.40 & -0.67 & -0.78 \\
\hline$B$ & -0.80 & -1.10 & -0.44 & -0.61 & -0.80 & -1.10 & -0.44 & -0.61 \\
\hline C & -0.50 & -0.50 & -0.28 & -0.28 & -0.50 & -0.50 & -0.28 & -0.28 \\
\hline $\mathrm{D}$ & +0.70 & +1.00 & +0.39 & +0.56 & +0.70 & +1.00 & +0.39 & +0.56 \\
\hline $\mathrm{E}$ & -0.30 & -0.50 & -0.17 & -0.28 & -0.30 & -0.50 & -0.17 & -0.28 \\
\hline $\begin{array}{l}C_{p e, 10} \\
C_{p e, 1} \\
W_{e, 10} \\
W_{e, 1}\end{array}$ & $\begin{array}{l}\text { Wind Pres } \\
\text { Wind Pres } \\
\text { Wind Pres } \\
\text { Wind Pres }\end{array}$ & $\begin{array}{l}\text { Coefficier } \\
\text { Coefficier } \\
\text { for an Are } \\
\text { for an Are }\end{array}$ & $\begin{array}{l}\text { an Area } A \\
\text { an Area } 1 \\
>10 \mathrm{~m}^{2} \\
\mathrm{~m}^{2}<\mathrm{A} \leq 10\end{array}$ & $\begin{array}{l}0 \mathrm{~m}^{2} \\
<\mathrm{A} \leq 10 \mathrm{~m}^{2}\end{array}$ & & & & \\
\hline
\end{tabular}




\subsection{SNOW LOADS}

The snow loads are determined according to DIN EN 1991-1-2 and similar to the wind loads, the snow loads depend on location and height above sea level of the building.

Snow zone: $\quad 2 a$

Height above sea level: $\quad A=660.00 \mathrm{~m}$

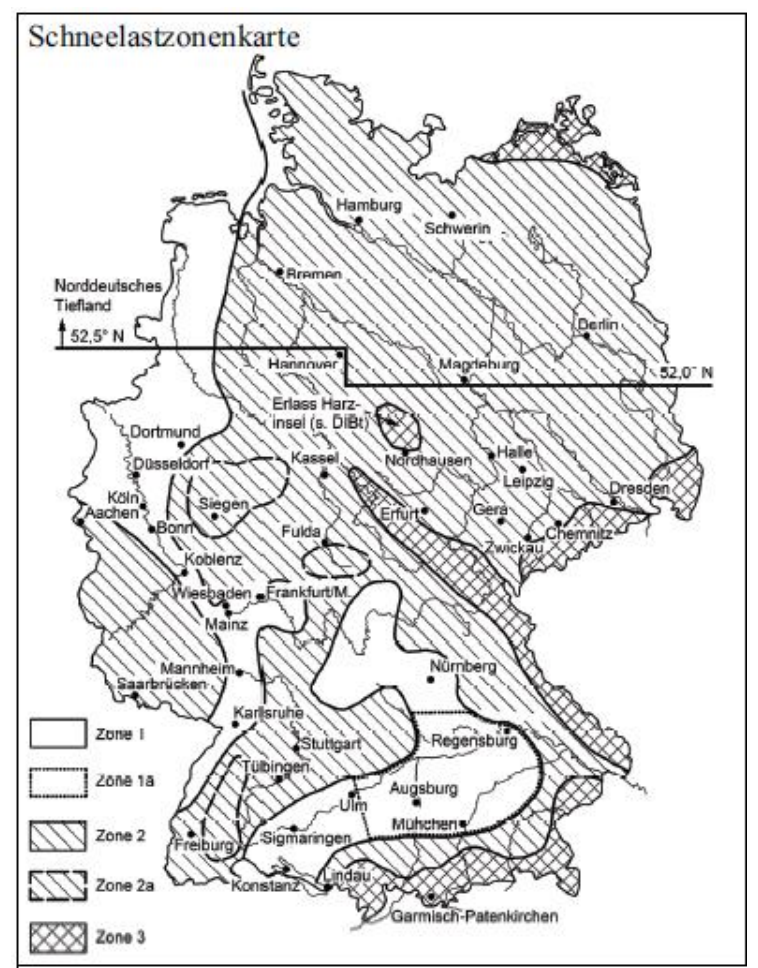

Figure 12 - Snow Zone Map for Germany [1]

The general snow load for snow zone $2 a$ is calculated according to following formula:

$s_{k}=1.25 \cdot\left[0.25+1.91 \cdot\left(\frac{A+140}{760}\right)^{2}\right] \geq 1.06$

with $\quad$ A: Height above sea level.

Using this formula, the snow load is:

$s_{k}=1.25 \cdot\left[0.25+1.91 \cdot\left(\frac{660+140}{760}\right)^{2}\right]=2.96 \mathrm{kN} / \mathrm{m}^{2}>1.06$.

The shape of the roof of the building is considered with a snow coefficient $\mu$ which is depends on the roof inclination $\alpha$. In the case of a saw-tooth roof, two different coefficients must be defined to take snow drifts into consideration, as is illustrated in Figure 13. 


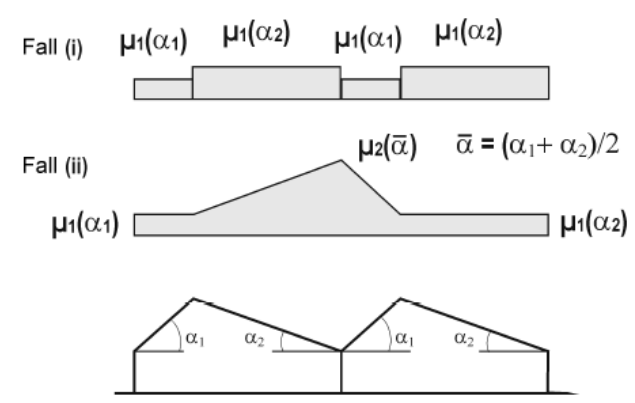

Figure 13 - Snow Loading Arrangement for Saw-Tooth Roofs taken from DIN EN 1991-1-3 Bild 5.4

Since $\alpha_{1}=\alpha_{2}=3,1^{\circ}$ the coefficients and respectively the snow loads are:

$\mu_{1}=0.8$

$\rightarrow s_{k}=\mu_{1} \cdot s_{k, 0}=0.8 \cdot 2.96 \mathrm{kN} / \mathrm{m}^{2}=2.37 \mathrm{kN} / \mathrm{m}^{2}$

$\mu_{2}=0.8+0.8 \cdot \alpha / 30^{\circ}=0.88$

$\rightarrow s_{k}=\mu_{2} \cdot s_{k, 0}=0.88 \cdot 2.96 \mathrm{kN} / \mathrm{m}^{2}=2.60 \mathrm{kN} / \mathrm{m}^{2}$

\subsection{ACCIDENTAL LOADS}

In addition to the imposed loads above, there are accidental loads to be considered because of expected forklift traffic in the building. This causes not only additional vertical loads but also horizontal impact loads on the columns of the hall resulting from a possible collision of the forklift trucks with the columns.

In this case the forklifts have a permissible total weight of $7.5 \mathrm{t}$, which is $75 \mathrm{kN}$. According to DIN EN 199111 Tab. 6.5, the forklifts can be classified in the category FL4 with a maximum total weight (including self-weight and lifting capacity) of $100 \mathrm{kN}$.

DIN EN 1991-1-7 says that the horizontal impact load caused by forklift collision must be considered with an equivalent load $F=5 \mathrm{~W}$, with $\mathrm{W}$ being the permissible total weight of the forklift. The load must be positioned in a height of $0.75 \mathrm{~m}$ above ground.

$F=5 \mathrm{~W}=5 \cdot 75 \mathrm{kN}=375 \mathrm{kN}$

This means that the equivalent load for a forklift collision for this building amounts to $375 \mathrm{kN}$. 


\section{STRUCTURAL ANALYSIS}

The following chapter gives a quick overview over the main design guidelines and requirements given in the European Standards, the Eurocodes. Most significant for the design of concrete structures are the Eurocodes 0 to 2, and respectively their national annexes, per the german annex.

Eurocode 0 (ECO) describes the basis of structural design and must be applied through all types of designs. It includes the standards for load and resistance factors as well as action combinations. The different types of actions on structures and load cases, like imposed loads for buildings, wind loads, snow loads and others are included in the Eurocode 1 (EC1). Finally, the Eurocode 2 (EC2) regulates the design and construction of reinforced and prestressed concrete structures.

\subsection{ACTION COMBINATIONS AND FACTORED LOADS}

As mentioned above, the different action combinations for the ultimate limit state design and the serviceability limit state design are introduced in the EC1. The different actions and their formula symbols that are described in this chapter and used throughout this MRP are summarized in Table 6.

Table 6 - Actions on Structures

\begin{tabular}{|l|c|l|c|}
\hline \multicolumn{2}{|l|}{ Permanent Loads } & \multicolumn{2}{|l|}{ Variable Loads } \\
\hline Dead load & $G_{k}$ & Live load & $Q_{k}$ \\
\hline Prestressing load & $P_{k}$ & Snow load & $S_{k}, Q_{k, s}$ \\
\cline { 3 - 4 } & & Wind load & $W_{k}, Q_{k, W}$ \\
\hline \multicolumn{2}{|l}{ Accidental load } & & $A_{d}$ \\
\hline
\end{tabular}

\subsubsection{ULTIMATE LIMIT STATE (ULS)}

The different action combinations for the ultimate limit sate can be seen in Table 7, where

$G_{k} \quad$ Characteristic dead load

$Q_{k} \quad$ Characteristic live load / variable load

$\gamma \quad$ Safety factor (see Table 8 or DIN EN 1990, Appendix A, Tab. NA.A. 1.2 (B))

$\psi_{i} \quad$ Combination coefficient (see DIN EN 1990, Appendix A, Tab. A.1.1).

Table 7 - Action Combinations for the ULS

\begin{tabular}{|lr|l|}
\hline Loading Situation & & Action Combinations ${ }^{1)}$ \\
\hline Permanent and temporary loads & $Q_{\mathrm{Ed}}=$ & $\sum_{j \geq 1} \gamma_{G, j} \cdot G_{k, j} \oplus \gamma_{Q, 1} \cdot Q_{k, 1} \oplus \sum_{i>1} \gamma_{Q, i} \cdot \psi_{0, i} \cdot Q_{k, i}$ \\
\hline Accidental loads & $Q_{A d}=$ & $\sum_{j \geq 1} \gamma_{G A, j} \cdot G_{k, j} \oplus A_{d} \oplus \gamma_{Q A, 1} \cdot \psi_{1,1} \cdot Q_{k, i} \oplus \sum_{i>1} \gamma_{Q A, i} \cdot \psi_{2, i} \cdot Q_{k, i}$ \\
\hline 1) According to DIN EN 1990, 6.4.3 & & \\
\hline
\end{tabular}


The safety factors used above are shown in Table 8.

Table 8 - Safety Factors for the ULS

\begin{tabular}{|c|c|c|c|}
\hline Actions & & Final State & State of Construction \\
\hline Permanent & $\gamma_{G}=$ & $1.35(1.00)^{1)}$ & \multirow{2}{*}{$1.15^{2)}$} \\
\hline Variable & $\gamma_{Q}=$ & $1.50(0)^{1)}$ & \\
\hline Prestressing & $\gamma_{P}=$ & \multicolumn{2}{|c|}{$1.00^{3)}$} \\
\hline $\begin{array}{l}\text { 1) According to } D \\
\text { 2) According to } D \\
\text { 3) According to } D\end{array}$ & $\begin{array}{l}\text {, Appen } \\
2-1-1,1 \\
2-1-1,2 .\end{array}$ & A. 1.2 (B) & \\
\hline
\end{tabular}

\subsubsection{SERVICEABILITY LIMIT STATE (SLS)}

The action combinations for the serviceability limit state are described in Table 9.

Table 9 - Action Combinations for the SLS

\begin{tabular}{|ll|l|}
\hline Loading situation & & Action combinations ${ }^{1)}$ \\
\hline Rare situation & $Q_{\mathrm{Ed}, \text { rare }}=$ & $\sum_{j \geq 1} G_{k, j} \oplus Q_{k, 1} \oplus \sum_{i>1} \psi_{0, i} \cdot Q_{k, i}$ \\
\hline Frequent situation & $Q_{\mathrm{Ed}, \text { frequ }}=$ & $\sum_{j \geq 1} G_{k, j} \oplus \psi_{1,1} \cdot Q_{k, 1} \oplus \sum_{i>1} \psi_{2, i} \cdot Q_{k, i}$ \\
\hline Permanent situation & $Q_{\mathrm{Ed}, \text { perm }}=$ & $\sum_{j \geq 1} G_{k, j} \oplus \sum_{i>1} \psi_{2, i} \cdot Q_{k, i}$ \\
\hline 1) According to DIN EN 1990, 6.5.3 & & \\
\hline
\end{tabular}

\subsection{BUILDING MATERIALS AND CHARACTERISTIC VALUES}

The building materials used for the structure are concrete, reinforcing steel and prestressing steel. The property values of these materials are defined in the EC 2 and the following chapter will give a short overview of the main properties needed for the structural design.

\subsubsection{CONCRETE}

The EC 2 distinguishes 15 different concrete strength classes starting at C1215 and ending with C100/115. The first 9 classes and the most relevant for the following structural design are listed in Table 10. The concrete strength classes describe the compressive strength of the concrete based on their characteristic cylinder strength, $f_{c k}$, and their cube strength, $f_{c k, c u b e}$, which are determined at a concrete age of 28 days. The value $f_{c d}$ is the factored cylinder strength with a safety factor of $y_{c}=1.5$ and another reduction factor $\alpha_{\mathrm{cc}}=0.85$. 
Table 10 - Concrete Properties

\begin{tabular}{|l|c|c|c|c|c|c|c|c|c|}
\hline$\left[\mathbf{N} / \mathbf{m m}^{2}\right]^{*}$ & $\mathbf{C 1 2 / 1 5}$ & $\mathbf{C 1 6 / 2 0}$ & $\mathbf{C 2 0 / 2 5}$ & $\mathbf{C 2 5 / 3 0}$ & $\mathbf{C 3 0 / 3 7}$ & $\mathbf{C 3 5 / 4 5}$ & $\mathbf{C 4 0 / 5 0}$ & $\mathbf{C 4 5 / 5 5}$ & $\mathbf{C 5 0 / 6 0}$ \\
\hline $\mathbf{f}_{\mathrm{ck}}$ & 12 & 16 & 20 & 25 & 30 & 35 & 40 & 45 & 50 \\
\hline $\mathbf{f}_{\mathrm{ck}, \text { cube }}$ & 15 & 20 & 25 & 30 & 37 & 45 & 50 & 55 & 60 \\
\hline $\mathbf{f}_{\mathrm{cm}}$ & 20 & 24 & 28 & 33 & 38 & 43 & 48 & 53 & 58 \\
\hline $\mathbf{f}_{\mathrm{cd}}$ & 6.8 & 9.1 & 11.3 & 14.2 & 17.0 & 19.8 & 22.7 & 25.5 & 28.3 \\
\hline $\mathbf{f}_{\mathrm{ctm}}$ & 1.6 & 1.9 & 2.2 & 2.6 & 2.9 & 3.2 & 3.5 & 3.8 & 4.1 \\
\hline $\mathbf{E}_{\mathrm{cm}}$ & 27,000 & 29,000 & 30,000 & 31,000 & 33,000 & 34,000 & 35,000 & 36,000 & 37,000 \\
\hline
\end{tabular}

\subsubsection{REINFORCING STEEL}

The EC2 applies to ribbed and weldable reinforcement and it specifies its requirements. The most important properties of reinforcing steel are a high tensile strength, good bond characteristics and a high ductility. Following table describes the properties of the reinforcing steel used in the structural design.

Table 11 - Reinforcing Steel Properties

\begin{tabular}{|c|c|c|c|c|c|c|}
\hline Type & Ductility & $f_{\mathrm{yk}}\left[\mathrm{Nmm}^{2}\right]^{*}$ & $f_{y d}\left[\mathrm{~N} / \mathrm{mm}^{2}\right]$ & $\mathrm{ftkcal}_{\mathrm{t}}\left[\mathrm{N} / \mathrm{mm}^{2}\right]$ & $\varepsilon_{\text {ud }}[\%]$ & $E_{s}\left[\mathrm{~N} / \mathrm{mm}^{2}\right]$ \\
\hline B500A & regular & 500 & 435 & 525 & 25 & 200,000 \\
\hline $\begin{array}{ll}* \\
f_{y k} \\
f_{y d}\end{array}$ & $\begin{array}{l}\text { a } \\
\text { strength }\end{array}$ & & $\begin{array}{l}\mathrm{f}_{\mathrm{t} \text {,cal }} \\
\varepsilon_{\text {ud }} \\
\mathrm{E}_{\mathrm{s}}\end{array}$ & $\begin{array}{l}\text { tensile strength } \\
\text { strain limit } \\
\text { modulus of elastici }\end{array}$ & & \\
\hline
\end{tabular}

\subsubsection{PRESTRESSING STEEL}

The prestressing steel that is used for this design is a seven-wire strand and the properties can be taken from the Building Control Certification Nr. Z-12.3-107 from the German Institute for Structural Engineering.

\subsection{DURABILITY AND CONCRETE COVER}

In order to ensure the bond between the concrete and reinforcing steel, as well as the prestressing steel and to protect the steel against corrosion and fire, a minimum distance between the surface of the concrete and the first layer of reinforcement bars is required. These requirements are defined in DIN EN 1992-1-1 and the national appendix, respectively.

The total concrete cover $\mathrm{C}_{\text {nom }}$ is composed of two values. The minimum concrete cover $\mathrm{C}_{\min }$ and an allowance $\Delta c_{\text {dev }}$ which takes possible deviations in the construction process into account.

$c_{\text {nom }}=c_{\text {min }}+\Delta c_{d e v}$

with $\quad c_{\min }=\max \left\{\begin{array}{l}c_{\min , b}(\text { bond }) \\ c_{\min , \text { dur }}(\text { durability) } \\ 10 \mathrm{~mm}\end{array} \quad\right.$ and $\quad \Delta c_{\text {dev }}=15 \mathrm{~mm}$ (for XC1 only $10 \mathrm{~mm}$ ). 
In case of precast concrete, $\Delta c_{\text {dev }}$ can be reduced by $5 \mathrm{~mm}$ if a high production quality is ensured and the concrete cover is regularly tested.

The values for $\mathrm{C}_{\min , \mathrm{b}}$ are defined as follows:

For reinforcing steel: $\quad c_{\min , b}=\varnothing_{s}$ where $\varnothing_{s}$ is the diameter of the reinforcement bar.

For prestressing steel: $\quad c_{\min , b}=2.5 \varnothing_{P}$ (in case of immediate bonding).

The value for $\mathrm{C}_{\min , \mathrm{dur}}$ depends on the exposure class of the concrete and can be taken from Table 12 . Exposure classes describe different corrosive environments, which may cause damage to the concrete and the reinforcement, and therefore are a decisive factor in choosing the thickness of the concrete cover. The three exposure classes which are significant for the value of $c_{\text {min,dur }}$ and which are distinguished in Table 12 are XC1 to XC4 - concrete at risk of carbonation, XD1 to XD3 - concrete exposed to de-icing salt or other chlorides, and XS1 to XS3 - concrete in sea water environments.

Table 12 - Minimum Concrete Cover Cmin,dur in Relation to the Exposure Class

\begin{tabular}{|l|c|c|c|c|}
\cline { 2 - 5 } \multicolumn{1}{c|}{} & \multicolumn{4}{c|}{ Cmin,dur in [mm] } \\
\hline Exposure Class & XC1 & $\begin{array}{l}\text { XC2 } \\
\text { XC3 }\end{array}$ & XC4 & $\begin{array}{c}\text { XD1, XD2, XD3 } \\
\text { XS1, XS2, XS3 }\end{array}$ \\
\hline Reinforcing Steel & 10 & 20 & 25 & 40 \\
\hline Prestressing Steel & 20 & 30 & 35 & 50 \\
\hline
\end{tabular}




\section{$4 \quad$ POSITION PLAN}

The drawings on the next two pages will give an overview of the different structural elements, the structural positions, which will be analyzed and designed in this paper. Each position is indicated by a letter and a two-digit number. The letters indicate the type of structural member and are assigned as follows:

$$
\begin{array}{ll}
\mathbf{Z} & =\text { Trapezoidal sheet covering } \\
\mathbf{P} & =\text { Prestressed member } \\
\mathbf{C} & =\text { Column } \\
\mathbf{S} & =\text { Slab } \\
\mathbf{B} & =\text { Beam } \\
\mathbf{T} & =\text { Staircase } \\
\mathbf{F} & =\text { Footing } \\
\mathbf{W} & =\text { Wall } \\
\mathbf{V} & =\text { Steel Structures }
\end{array}
$$

The first of the two-digit number stands for different levels or parts of the building. The hall structure is indicated by 0 , the office building by 1 , the foundation of the building by 2 and any additional structures, like the steel canopy, are indicated by the number 3 . 


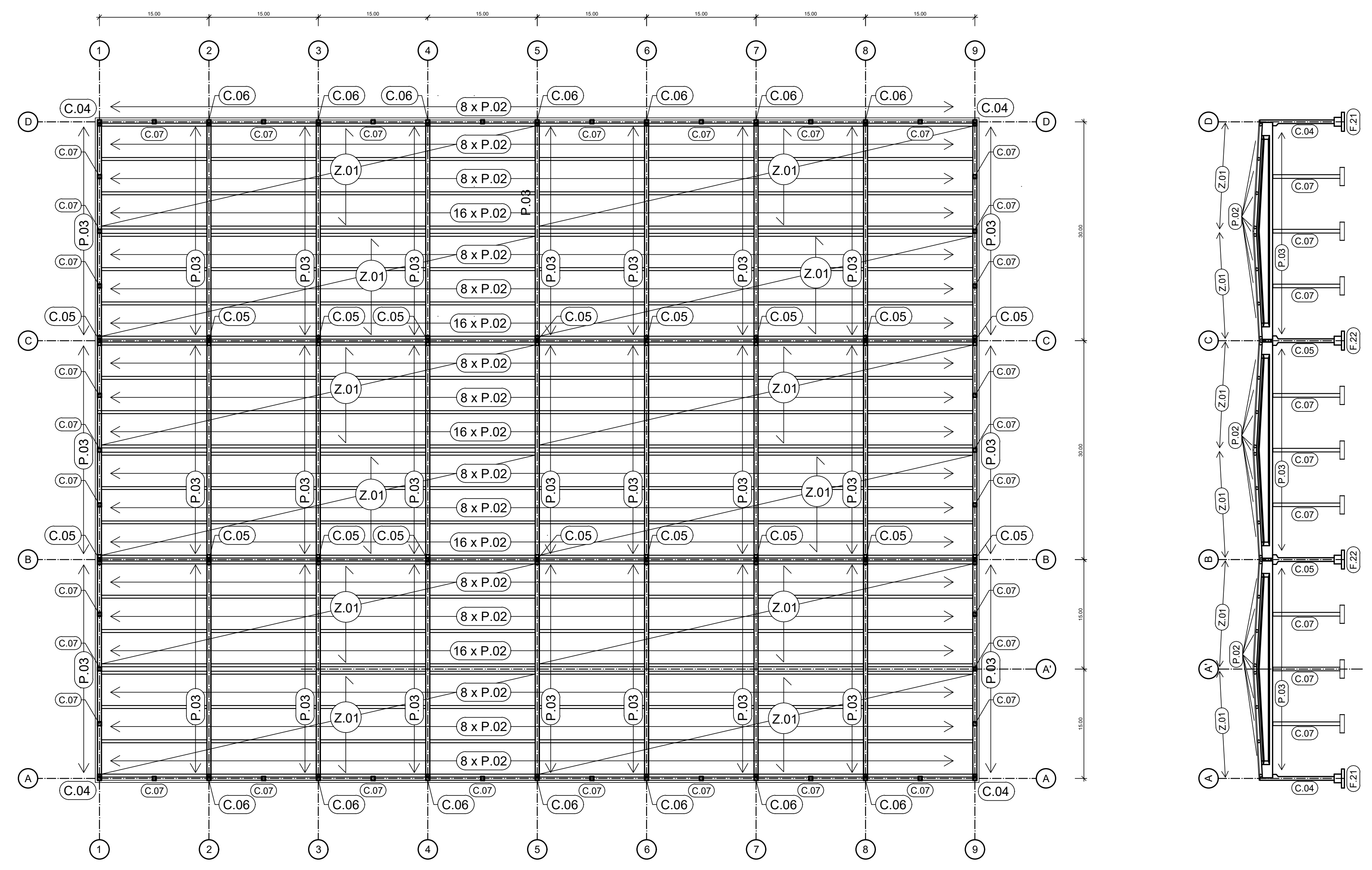




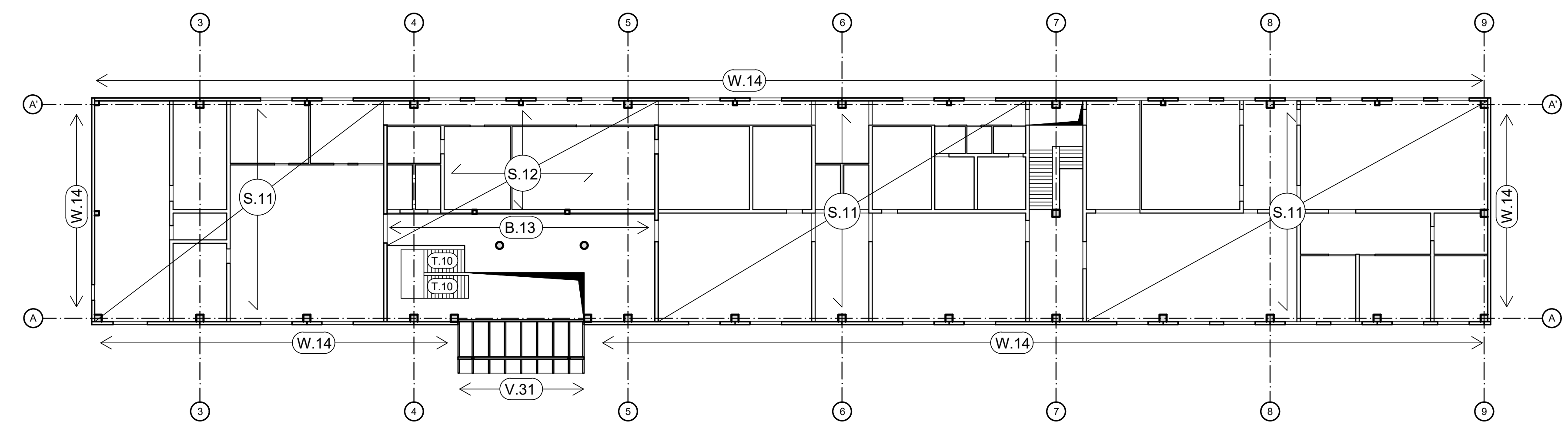


5 POS. Z.01 - TRAPEZOIDAL SHEET COVERING

\subsection{SYSTEM}

System

Profile

Characteristic Loads

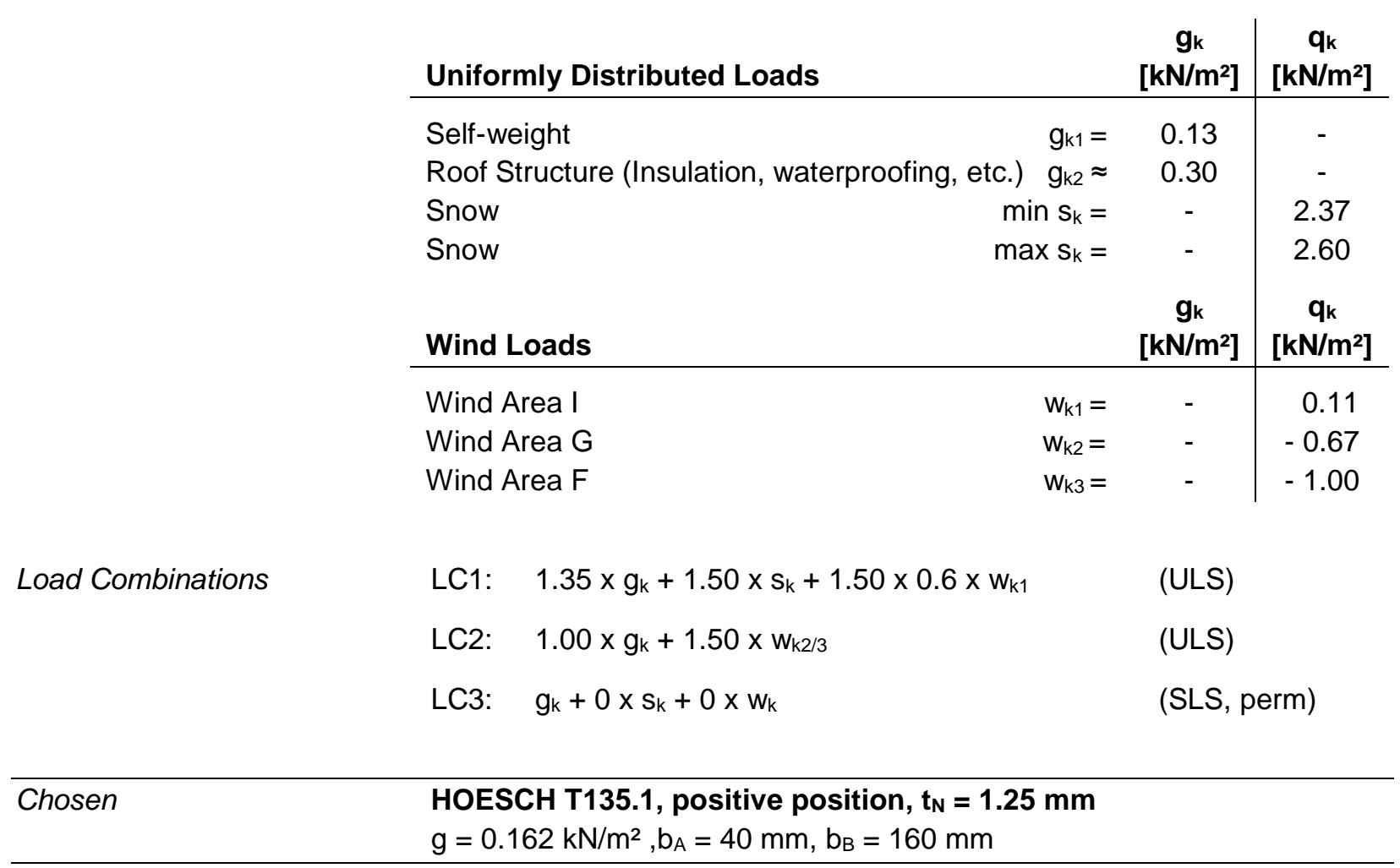

1) Wind Pressure

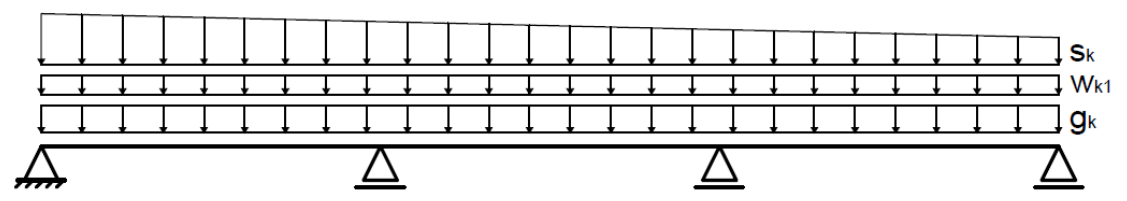

2) Wind Suction

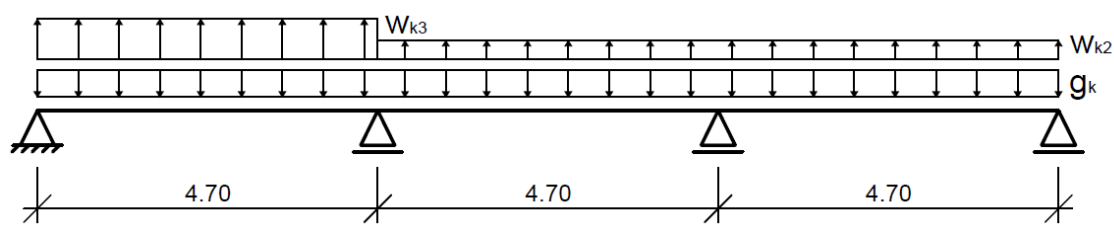

Hoesch T 135.1, positive position, $\mathrm{t}_{\mathrm{N}}=1.00 \mathrm{~mm}, \mathrm{f}_{\mathrm{yk}}=320 \mathrm{~N} / \mathrm{mm}^{2}$

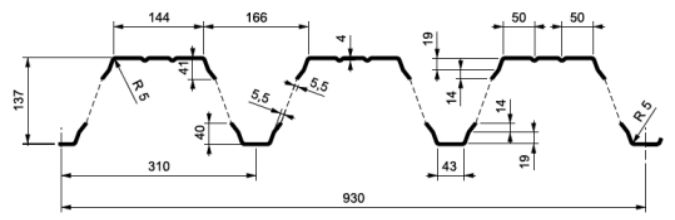




\subsection{PRELIMINARY DESIGN}

Design Loads

1) Wind PRESSURE: $\quad q_{k}=0.30 \times 1.35 / 1.5+2.49+0.11=2.87 \mathrm{kN} / \mathrm{m}^{2}$

2) Wind SUCTION: $\quad q_{k}=0.30 \times 1.0 / 1.5-0.67=-0.47 \mathrm{kN} / \mathrm{m}^{2}$

The preliminary design of the trapezoidal sheets can be done according to

Table 13 and Table 14 provided by the manufacturer HOESCH. The trapezoidal sheets must be designed for a pressing load as well as for a lifting load resulting from wind suction.

Table 13 - Sizing Table for the T135.1 as a Three-Span System with a Pressing Load

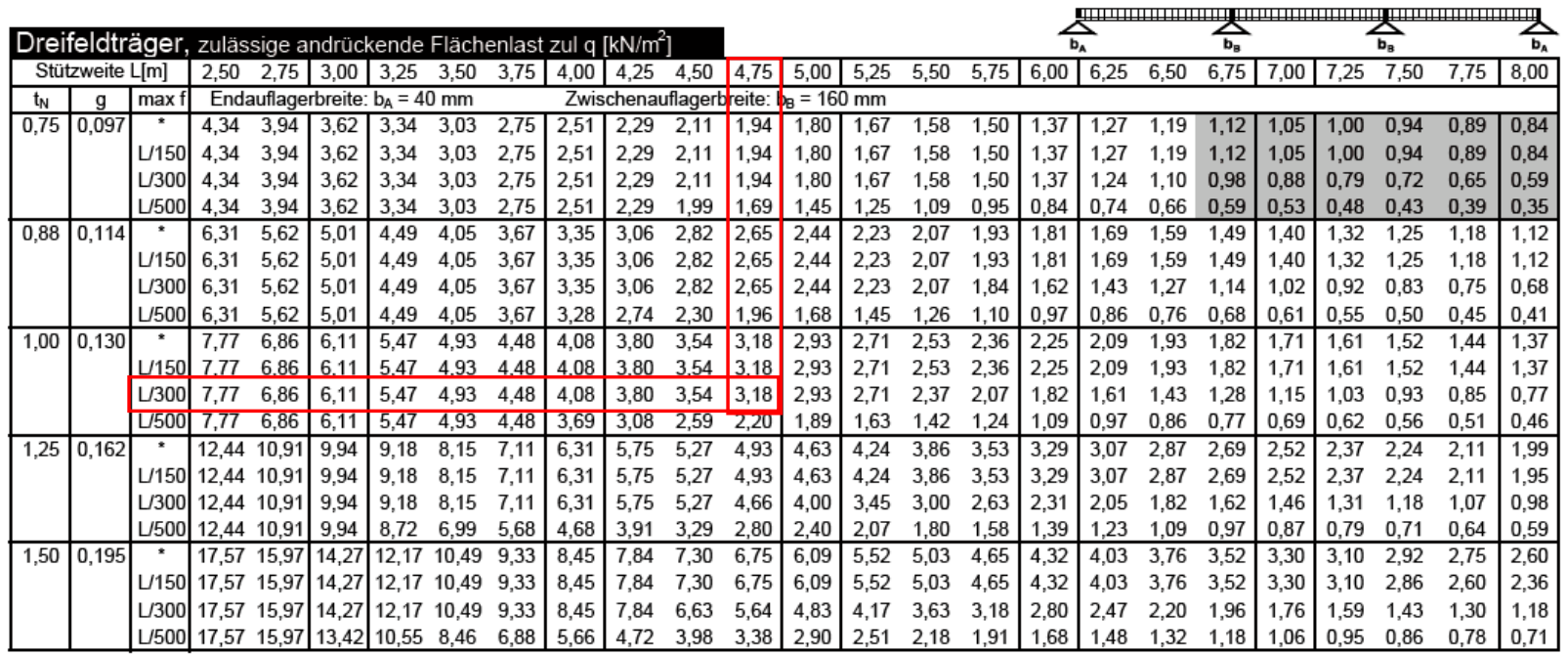

Table 14 - Sizing Table for the T135.1 as a Three-Span System with a Lifting Load

\begin{tabular}{|c|c|c|c|c|c|c|c|c|c|c|c|c|c|c|c|c|c|c|c|c|c|c|c|c|c|c|}
\hline & & & & & & & & & & & & & & & & & & & & & & & & & & \\
\hline Dre & eldt & aje & rul & si & & & & & & & & & & & & & & b & & & $b_{8}$ & & & $\widehat{b_{3}}$ & & $\widehat{b_{A}}$ \\
\hline Stüt & eite & L[m] & 2,50 & 2,75 & 3,00 & 3,25 & 3,50 & 3,75 & 4,00 & 4,25 & 4,50 & 4,75 & 5,00 & 5,25 & 5,50 & 5,75 & 6,00 & 6,2 & & & 6,75 & 7,00 & 7,25 & 7,50 & 7,75 & 8,00 \\
\hline$t_{N}$ & g & $\operatorname{maxf}$ & & & & & & & & & & & & & & & & & & & & & & & & \\
\hline 0,75 & 0,097 & $*$ & 62 & 6,58 & 5,72 & 4,99 & 4,36 & 3,83 & 3,36 & 2,98 & 2,66 & & 15 & 95 & 1,78 & 1,63 & 49 & ,3 & 12 & & 1,18 & 1,10 & 1,02 & 0,96 & 0,90 & 0,84 \\
\hline & & L150 & 62 & 6,58 & 5,72 & 99 & 36 & 3,83 & 3,36 & 2,98 & 2,66 & 38 & 2 & 1,95 & 1,78 & 1,63 & 1,49 & 1,3 & 1,2 & & 1,18 & 1,10 & 1,02 & 0,96 & 0,90 & 0,84 \\
\hline & & L/300 & 62 & 6,58 & 5,72 & 4,99 & 4,36 & 3,83 & 3,36 & 2,98 & 2,66 & 38 & 2,15 & 1,95 & 1,78 & 1,59 & 1,40 & 1,2 & 1, & & 0,98 & 0,88 & 0,79 & 0,72 & 0,65 & 0,59 \\
\hline & & ப500 & 7,62 & 6,58 & 5,72 & 4,99 & 4,23 & 3,44 & 2,83 & 2,36 & 1,99 & 69 & 1,45 & 1,25 & 1,09 & 0,95 & 0,84 & 0,7 & 0,6 & & 0,59 & 0,53 & 0,48 & 0,43 & 0,39 & 0,35 \\
\hline 0,88 & 0,114 & ${ }^{*}$ &, 67 & 9,00 & 7,61 & 6,48 & 5,59 & 4,87 & 4,28 & 3,79 & 3,38 & 03 & 2,74 & 2,48 & 2,27 & 2,07 & 1,90 & 1,7 & $\overline{1,6}$ & & 1,50 & 1,40 & 1,30 & 1,22 & 1,14 & 1,07 \\
\hline & & L150 & 67 & 9,00 & 7,61 & 6,48 & 5,59 & 4,87 & 4,28 & 3,79 & 3,38 & 03 & 2,74 & 2,48 & 2,27 & 2,07 & 1,90 & 1,7 & 1,6 & & 1,50 & 1,40 & 1,30 & 1,22 & 1,14 & 1,07 \\
\hline & & L/300 & 67 & 9,00 & 7,61 & 6,48 & 5,59 & 4,87 & 4,28 & 3,79 & 3,38 & 03 & 2,74 & 2,42 & 2,10 & 1,84 & 1,62 & 1,4 & 1,2 & & 1,14 & 1,02 & 0,92 & 0,83 & 0,75 & 0,68 \\
\hline & & ᄂ500 &, 67 & 9,00 & 7,61 & 6,12 & 4,90 & 3, & 3,28 & 2,74 & & & 1,6 & 1,45 & 1,26 & 10 & 0, & 0,8 & & & 0,68 & 0,61 & 0,55 & 0,50 &, 45 & 0,41 \\
\hline 1,00 & ,130 & * & 13,27 & 10,97 & 9,23 & 7,86 & 6,78 & 5,91 & $\overline{5,19}$ & 4,60 & 4,10 & 3,68 & 3,3 & 3,01 & 2,75 & 2,51 & 2,3 & 2,1 & & & 1,82 & 1,7 & 1,58 & 1,48 & 88 & 1,30 \\
\hline & & $L / 150$ & 3.27 & 10.97 & 9.23 & 7.86 & 6.78 & 5.91 & 5.19 & 4.60 & 4.10 & 3.68 & & 3,01 & 2,75 & 2,51 & & 2,1 & & & 1,82 & 1, & 1,58 & 1,48 & & 1,30 \\
\hline & & L/300 & 3,27 & 10,97 & 9,23 & 7,86 & 6,78 & 5,91 & 5,1 & 4,60 & & 36 & & 2,72 & 2,36 & 2,0 & & 1,6 & & & 1,28 & & 1,03 & 0,93 & 35 & 0,77 \\
\hline & & L500 &, 27 & 10,97 & 8,75 & 6,88 & 51 & 4,48 & 3,69 & 3,08 &, 59 & 2,20 & 1,89 & 1,63 & 1,42 & 1,24 & 1,09 & 0,9 & 0,0 & & 0,77 & 0,69 & 0,62 & 0,56 & 0,51 & 0,46 \\
\hline 1,25 & 0,162 & \begin{tabular}{|l|}
$*$ \\
\end{tabular} & 92 & 14,83 & 12,46 & 10,62 & 9,16 & 7,97 & 7,00 & 6,21 & & 4,97 & 4,4 & 4,07 & 3,71 & 3,39 & 3,1 & 2,8 & 2,6 & & 2,46 & 2,2 & 2,13 & 2,00 & 1,87 & 1,75 \\
\hline & & L150 & 92 & 14,83 & 12,46 & 0,62 & 9,16 & 7,97 & 7,00 & 6,21 & 5,5 & 4,97 & 4 & 4,07 & 3,71 & 3,3 & 3,1 & 2,8 & 2,6 & & 2,46 & 29 &, 13 &, 00 & 37 & 1,75 \\
\hline & & L/300 & 7,92 & 14,83 & 12,46 & 10,62 & 9,16 & 7,97 & 7,00 & 6,21 & & 4,66 & 4,0 & 3,45 & 3,00 & 2,6 & 2,3 & 2,0 & 1,8 & & 1, & & 1,31 & 1,18 & 77 & 0,98 \\
\hline & & $\mathrm{L} 500$ &, 92 & 14,41 & 11,09 & 8,72 & 6,99 & 5,68 & 4,68 & 3,90 & & 2,79 & $2,4 \mathrm{C}$ & 2,07 & 1,80 & 1,58 & 1,3 & 1,2 & & & 0,97 & 0,87 &, 79 & 0,71 &, 64 & 0,59 \\
\hline 1,50 & 0,195 & ${ }^{*}$ & 22,21 & 18,35 & 15,43 & 13,14 & 11,33 & 9,87 & 8,67 & 7,68 & 6,85 & 6,15 & $\overline{5,5}$ & 5,03 & 4,59 & 4,20 & 3,8 & 3,5 & 3, & & 3,05 & 2,83 & 2,64 & 2,47 & 2,31 & 2,17 \\
\hline & & L150 & 21 & 18,35 & 15,43 &, 14 & 11,3 & 9,87 & 8,67 & 7,68 & 6,85 & 6,15 & 5,5 & 5,03 & 4,59 & 4,20 & 3,85 & 3,5 & 3 , & & 3,05 & 2,83 & 2,64 & 2,47 & 2,31 & 2,17 \\
\hline & & & 21 & 18, & & & & 9,87 & 8,67 & 7,68 & 6,6 & 5,6 & 4,8 & 4,18 & 3,63 & 3,18 & 2,8 & 2, & & & 1,96 & 1,76 & 1,59 & 1,43 & 1,30 & 1,18 \\
\hline & & & 22,21 & 17,44 & & 10,55 & 8,46 & 6,87 & 5,66 & 4,72 & 3,98 & 3,38 & 2,90 & 2,51 & 2,18 & 1,91 & 1,68 & 1,4 & & & 1,18 & 1,06 & 0,95 & 0,86 & 0,78 & 0,71 \\
\hline
\end{tabular}




\subsection{INTERNAL FORCES}

Internal forces

\begin{tabular}{|l|c|c|c|c|c|}
\cline { 2 - 6 } \multicolumn{1}{c|}{} & $\begin{array}{c}\max \mathbf{M}_{\mathbf{y d}, \mathbf{F}} \\
{[\mathbf{k N m} / \mathbf{m}]}\end{array}$ & $\begin{array}{c}\max \mathbf{M}_{\mathbf{y d}, \mathbf{S}} \\
{[\mathbf{k N m} / \mathbf{m}]}\end{array}$ & $\begin{array}{c}\max \mathbf{V}_{\mathbf{z d}} \\
{[\mathbf{k N} / \mathbf{m}]}\end{array}$ & $\begin{array}{c}\max \mathbf{R}_{\mathbf{z d}, \mathbf{A}} \\
{[\mathbf{k N} / \mathbf{m}]}\end{array}$ & $\begin{array}{c}\max \mathbf{R}_{\mathbf{z d}, \mathbf{B}} \\
{[\mathbf{k N} / \mathbf{m}]}\end{array}$ \\
\hline LC1 & 8.19 & -10.16 & 12.96 & 8.73 & 23.68 \\
\hline LC2 & -1.91 & 1.84 & 2.74 & -1.96 & -4.12 \\
\hline
\end{tabular}

Internal Force Diagrams

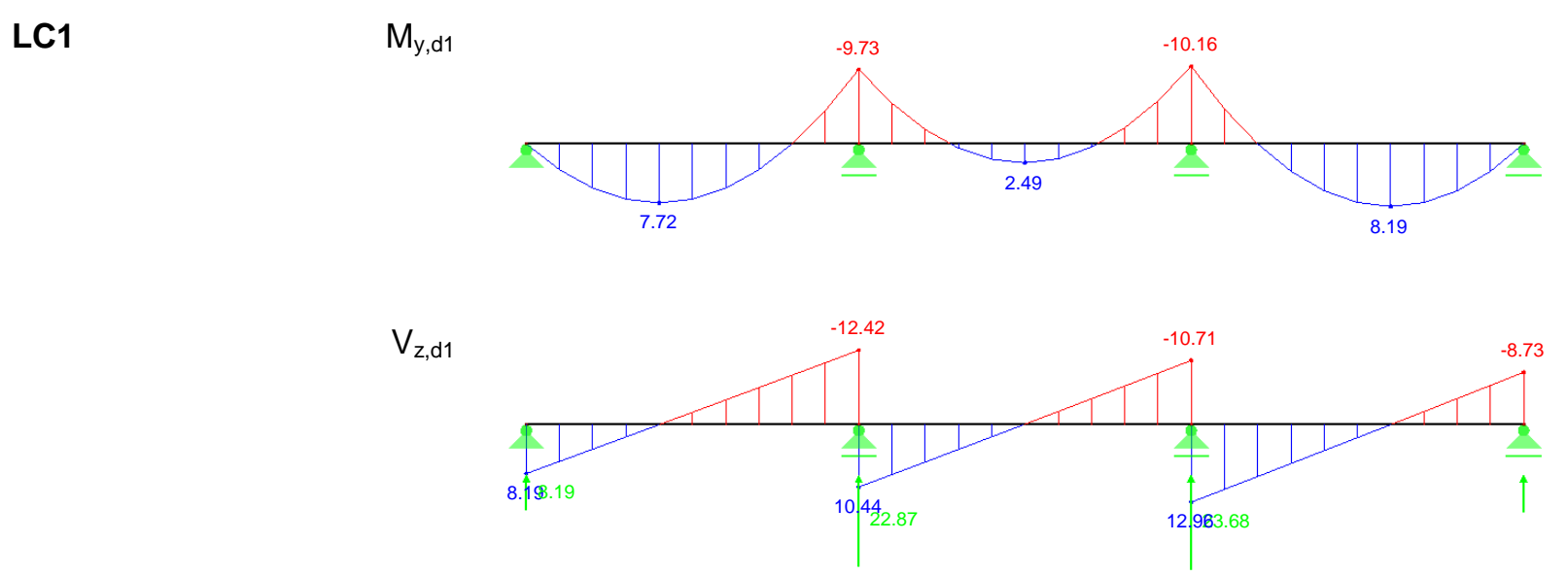

LC2

$M_{y, d 2}$

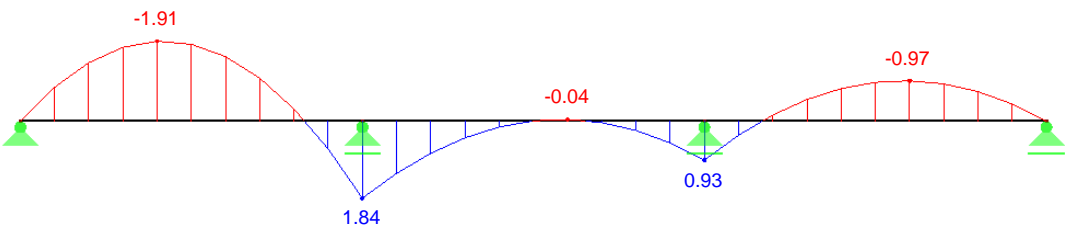

$\mathrm{V}_{\mathrm{z}, \mathrm{d} 2}$

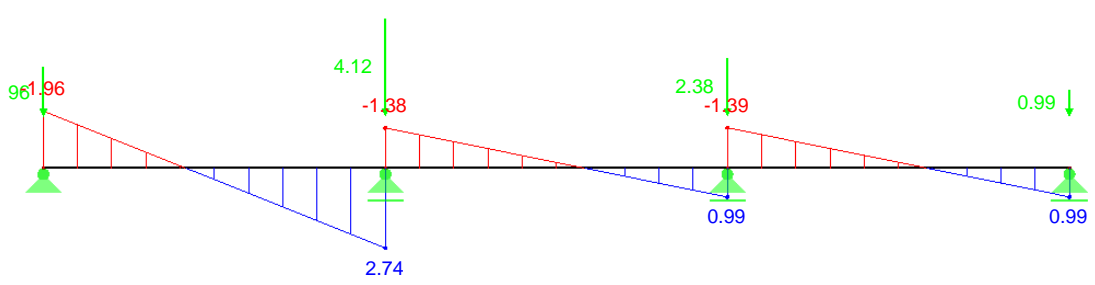




\subsection{ULTIMATE LIMIT STATE DESIGN}

The cross-section values are taken from Table 15 and the static proof is done according to DIN EN 1993- 3, DIN 18807 and DIN 18800.

End support

Intermediate support

Field moment

Support moment

Interaction

Interaction $\left(t_{N=1.25)}\right.$

Span limit

$$
\begin{aligned}
& R_{A, E d} \leq R_{A, F k} / \gamma_{M} \rightarrow 8.73 \leq 13.30 / 1.25=10.64 \checkmark \\
& R_{B, E d} \leq R_{B, F k}^{\omega} / \gamma_{M} \rightarrow 23.68 \leq 37.00 / 1.25=29.60 \checkmark \\
& M_{F, E d} \leq M_{F, F K}^{c} / \gamma_{M} \rightarrow 8.19 \leq 14.80 / 1.25=11.84 \checkmark \\
& M_{B, E d} \leq M_{B, F k}^{c} / \gamma_{M} \rightarrow 10.16 \leq 15.60 / 1.25=12.48 \checkmark \\
& \frac{M_{B, E d}}{M_{B, B k}^{0} / \gamma_{M}}+\left(\frac{R_{B, E d}}{R_{B, R K}^{0} / \gamma_{M}}\right)^{2} \leq 1.00 \\
& \frac{10.16}{17.40 / 1.25}+\left(\frac{23.68}{47.97 / 1.25}\right)^{2}=1.11>1.00 \text { 々 The design is not fulfilled. }
\end{aligned}
$$

The sheet thickness must be adjusted to $t_{N}=1.25 \mathrm{~mm}$ !

$$
\frac{10.16}{24.0 / 1.25}+\left(\frac{23.68}{82.79 / 1.25}\right)^{2}=0.66<1.00 \checkmark
$$

$L_{\max }=18.00 \mathrm{~m}>L=4.50 \mathrm{~m} \checkmark$

\subsection{SERVICABILITY LIMIT STATE STATE DESIGN}

Deflection of the trapezoidal sheet:

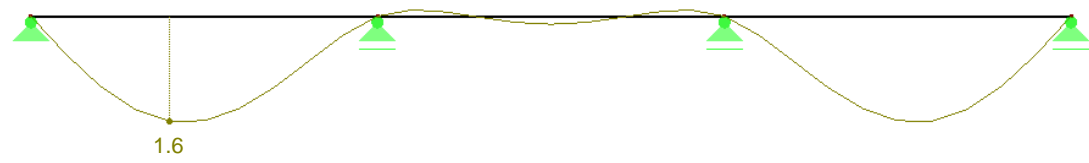

It must b

$\max f=1.60 \mathrm{~mm}$

Here

$$
\max f \leq L / 300
$$

$L / 300=450 / 300=1.50 \mathrm{~cm}=15 \mathrm{~mm}>\max f=1.60 \mathrm{~mm} \checkmark$ 
Table 15 - Cross Section Values for Hoesch T 135.1 Positive Position
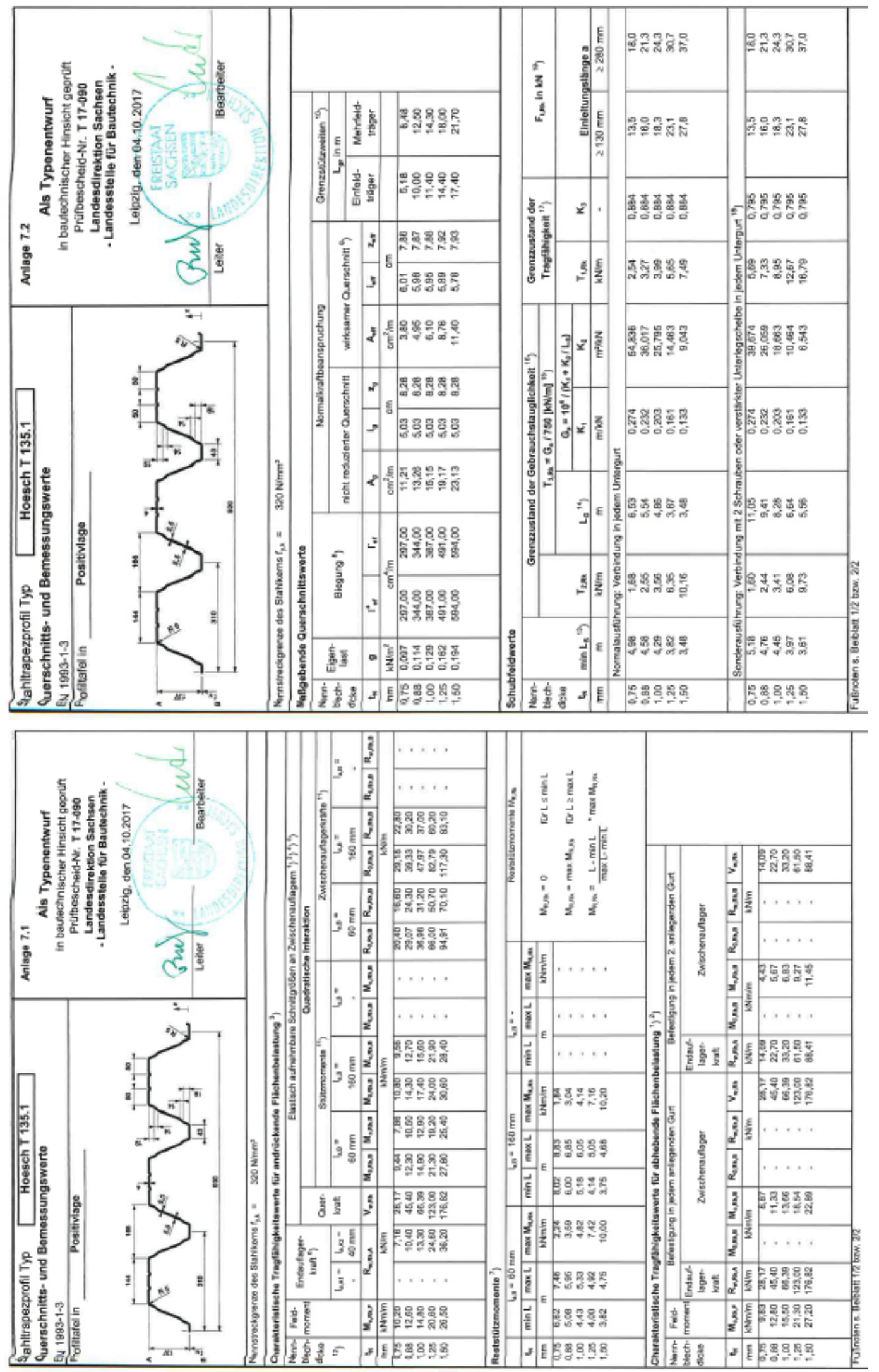
6 POS. P.02 - PRESTRESSED CONCRETE PURLINS

\subsection{PRELIMINARY DESIGN}

System

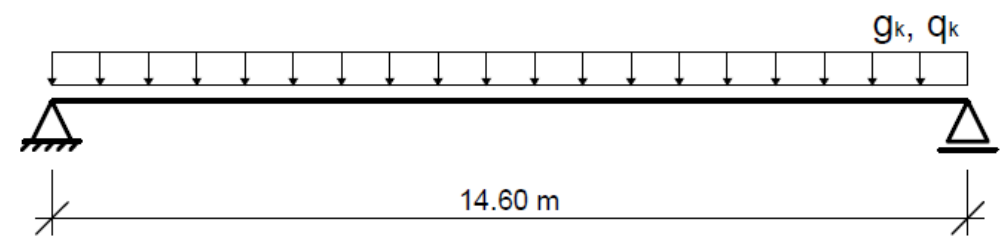

Cross-section

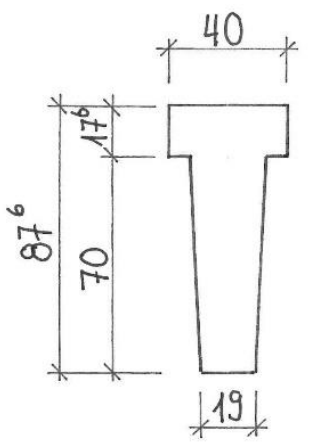

Building materials

C $30 / 37$ | St 1570/1770 | B 500A

Characteristic loads

\begin{tabular}{|c|c|c|c|}
\hline \multicolumn{2}{|l|}{ Uniformly Distributed Loads } & \multirow{2}{*}{$\begin{array}{c}\begin{array}{c}\mathbf{g}_{\mathbf{k}} \\
{[\mathrm{kN} / \mathrm{m}]}\end{array} \\
5.74\end{array}$} & \multirow{2}{*}{$\begin{array}{c}\begin{array}{c}\mathbf{q}_{\mathbf{k}} \\
{[\mathrm{kN} / \mathrm{m}]}\end{array} \\
-\end{array}$} \\
\hline $\mathrm{g}_{\mathrm{k} 1}=0.23 \mathrm{~cm}^{2} \times 25 \mathrm{k}$ & $/ \mathrm{m}^{3}=$ & & \\
\hline Roof Structure - Reaction Force from TR.01 & $g_{\mathrm{k} 2}=$ & 2.59 & - \\
\hline Snow - Reaction Force from TR.01 & $\mathrm{q}_{\mathrm{k} 1}=$ & - & 13.12 \\
\hline \multirow[t]{2}{*}{ Wind - Reaction Force form TR.01 } & $\mathrm{q}_{\mathrm{k} 2}=$ & - & 0.57 \\
\hline & & 8.33 & $\begin{array}{r}13.12 \\
0.57\end{array}$ \\
\hline
\end{tabular}

Characteristic bending moment

\begin{tabular}{|c|c|c|c|}
\hline $\mathbf{M}_{\mathrm{Gk} 1}[\mathbf{k N m}]$ & $\mathbf{M}_{\mathrm{Gk} 2}[\mathbf{k N m}]$ & $\mathbf{M}_{\mathbf{Q k} 1}[\mathbf{k N m}]$ & $\mathbf{M}_{\mathbf{Q k 2}}[\mathbf{k N m}]$ \\
\hline 153.0 & 69.0 & 349.6 & 15.2 \\
\hline
\end{tabular}

Assumptions

$\begin{array}{ll}\text { Prestressing } & \sigma_{m, t=0} \approx 1000 \mathrm{~N} / \mathrm{mm}^{2} \\ \text { Prestressing loss } & \approx 15 \%\end{array}$


Prestressing with immediate bond

Cross-section Properties

$Z_{c u}=49.76 \mathrm{~cm}$ (Position of the center of gravity)

$A_{c}=2296.5 \mathrm{~cm}^{2}$

Moment of inertia

$I_{y c}=1,518,675 \mathrm{~cm}^{4}$

Section modulus

$W_{c u}=\frac{l_{y c}}{Z_{c u}}=\frac{1,518,675 \mathrm{~cm}^{4}}{49.76 \mathrm{~cm}}=30,520 \mathrm{~cm}^{3}$

Bending Compression Zone

Effective depth

$d=87.6 \mathrm{~cm}-10 \mathrm{~cm}=77.6 \mathrm{~cm}$

$\delta=h_{f} / d=17.6 / 77.6=0.227$

$\beta=b_{w} / b_{\text {eff }}=19 \mathrm{~cm} / 40 \mathrm{~cm}=0.475$

$\rightarrow \eta=\frac{1}{(1-\beta) \cdot(1-0.5 \delta) \cdot \delta+0.32 \beta}=\underline{\underline{3.88}}$

ULS

$M_{E d}^{G+Q}=1.35 \cdot(153.0+69.0)+1.50 \cdot 349.6+1.5 \cdot 0.6 \cdot 15.2=837.7 \mathrm{kNm}$

$b_{\text {eff, req }} \geq \frac{M_{E d}}{f_{c d}} \cdot \eta=\frac{0.8377 \mathrm{MNm}}{17 \mathrm{MN} / \mathrm{m}^{2}} \cdot 3.88 / d^{2}=\underline{\underline{0.115 m}}<b_{\text {eff,prov }}=0.40 \mathrm{~m} \checkmark$

$\rightarrow$ The provided bending compression zone is sufficient.

Required Prestressing Steel

SLS

$M_{\text {Ed,frequ }}=(153.0+69.0)+0.2 \cdot 349.6+0 \cdot 15.2=291.9 \mathrm{kNm}$

$r_{\text {inf }}=0.95, \alpha_{\text {loss }}=0.15, \sigma_{p m, t=0}=1000 \mathrm{~N} / \mathrm{mm}^{2}$

$z_{c p}=49.76 \mathrm{~cm}-10 \mathrm{~cm}=39.76 \mathrm{~cm}$

Required prestressing steel

$$
\begin{aligned}
A_{P, \text { req }} \geq \frac{M_{E d}}{r_{\text {inf }} \cdot\left(1-\alpha_{\text {loss }}\right) \cdot \sigma_{p m, t=0} \cdot\left(\frac{W_{c u}}{A_{c}}+z_{c p}\right)} \\
=\frac{29190 \mathrm{kNcm}}{0.95 \cdot(1-0.15) \cdot 100 \mathrm{kN} / \mathrm{cm}^{2} \cdot\left(\frac{30520}{2296.5}+39.76\right)}=\underline{\underline{6.81 \mathrm{~cm}^{2}}}
\end{aligned}
$$

$\rightarrow$ The required cross-section of the pre-stressing steel is $6.81 \mathrm{~cm}^{2}$.

Tension Zone

$\min M_{G k}=153.0 \mathrm{kNm} \quad M_{E d, \text { requ }}=291.9 \mathrm{kNm}$
$W_{\text {cu,req }} \geq \frac{\frac{M_{E d}}{r_{\text {inf }} \cdot\left(1-\alpha_{\text {loss }}\right)}-\min M_{G k}}{0.6 \cdot f_{c k}}=\frac{\frac{29190}{0.95 \cdot(1-0.15)}-15300}{0.6 \cdot 3.0}=\underline{\underline{11,584 \mathrm{~cm}^{3}}}$


$W_{\text {cu,prov }}=30,520 \mathrm{~cm}^{3}>W_{\text {cu,req }}=11,427 \mathrm{~cm}^{3} \checkmark$

$\rightarrow$ The tension zone of the purlin is sufficient.

Chosen

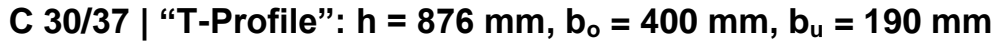
St $1570 / 1770 \mid 8 \varnothing_{\mathrm{P}}=12.5 \mathrm{~mm}=8 \times 0.93 \mathrm{~cm}^{2}=7.44 \mathrm{~cm}^{2}$

\subsection{SYSTEM}

System

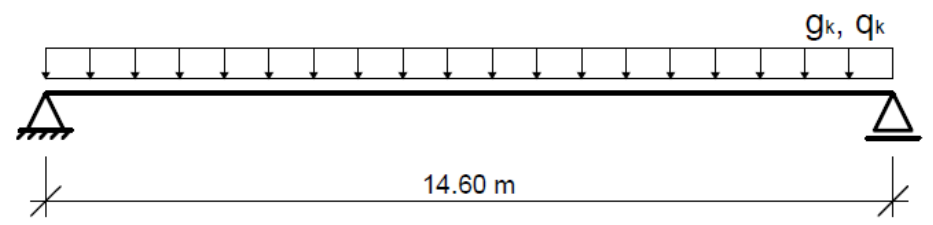

Cross-section

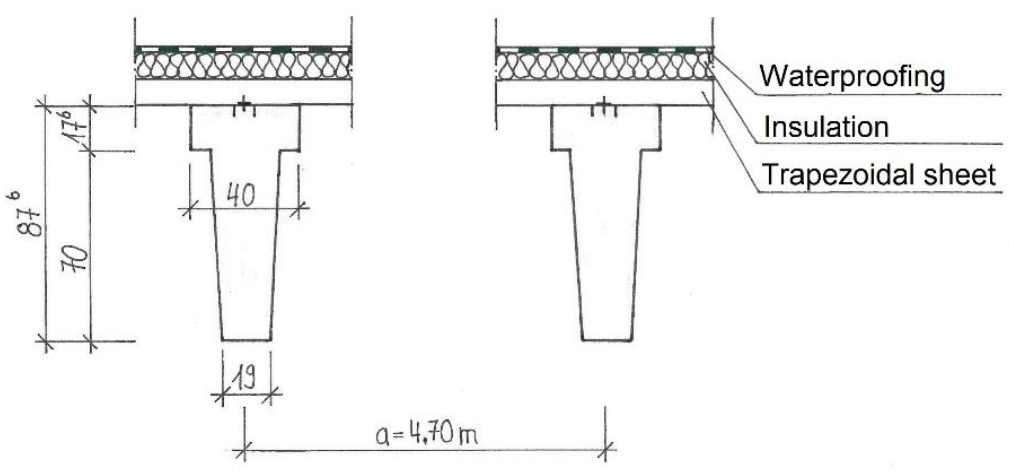

\begin{tabular}{|c|c|c|c|c|c|}
\hline $\mathbf{A}_{\mathbf{c}}\left[\mathbf{c m}^{2}\right]$ & $\mathbf{U}[\mathbf{c m}]$ & $\mathbf{X}_{\mathbf{s}, \mathrm{u}}[\mathbf{c m}]$ & $\mathbf{X}_{\mathbf{s}, \mathrm{o}}[\mathbf{c m}]$ & $\mathbf{I}_{\mathbf{y}}\left[\mathbf{c m}^{4}\right]$ & $\mathbf{W}_{\mathbf{u}}\left[\mathrm{cm}^{3}\right]$ \\
\hline 2297 & 248 & 49.8 & 37.8 & $1,518,675$ & 30,520 \\
\hline
\end{tabular}

Building materials

Exposure class

Reinforcing steel

Prestressing steel

\section{30/37 | St 1570/1770 | B 500A}

XC1, WO

$\mathrm{C}_{\mathrm{nom}, \mathrm{w}}=\mathrm{c}_{\text {min }}+\Delta \mathrm{c}_{\mathrm{dev}}=10 \mathrm{~mm}+10 \mathrm{~mm}=20 \mathrm{~mm}$

$A_{s}=2 \varnothing_{s} 16=4.02 \mathrm{~cm}^{2}$

$\mathrm{a}_{\mathrm{u}}=4.0 \mathrm{~cm}$

$\mathrm{C}_{\mathrm{nom}}=2.5 \times 12.5 \mathrm{~mm}+10 \mathrm{~mm}=42 \mathrm{~mm}$

1. Layer: $A_{p}=3 \varnothing_{p} 12.5=3 \times 0.93 \mathrm{~cm}^{2}$

$a_{p}=7.5 \mathrm{~cm}$

2. Layer: $A_{p}=3 \varnothing_{p} 12.5=3 \times 0.93 \mathrm{~cm}^{2}$

$a_{p}=11.7 \mathrm{~cm}$

3. Layer: $A_{p}=2 \varnothing_{p} 12.5=2 \times 0.93 \mathrm{~cm}^{2}$

$a_{p}=15.9 \mathrm{~cm}$ 
Characteristic loads

\begin{tabular}{|c|c|c|c|}
\hline \multicolumn{2}{|l|}{ Uniformly Distributed Loads } & $\begin{array}{c}\mathbf{g}_{k} \\
{[k N / m]}\end{array}$ & $\begin{array}{c}q_{k} \\
{[k N / m]}\end{array}$ \\
\hline$g_{\mathrm{k} 1}=0.23 \mathrm{~cm}^{2} \times 25 \mathrm{k}$ & $\mathrm{g}_{\mathrm{k} 1}=0.23 \mathrm{~cm}^{2} \times 25 \mathrm{kN} / \mathrm{m}^{3}=$ & 5.74 & - \\
\hline Roof Structure - Reaction Force from TR.01 & $g_{\mathrm{k} 2}=$ & 2.59 & - \\
\hline Snow - Reaction Force from TR.01 & $q_{k 1}=$ & - & 13.12 \\
\hline \multirow[t]{2}{*}{ Wind - Reaction Force form TR.01 } & $\mathrm{q}_{\mathrm{k} 2}=$ & - & 0.57 \\
\hline & & 8.33 & $\begin{array}{r}13.12 \\
0.57\end{array}$ \\
\hline
\end{tabular}

\subsection{INTERNAL FORCES}

Characteristic

(at $\mathrm{x}=\mathrm{L} / 2=7.35 \mathrm{~m}$ )

\begin{tabular}{|c|c|c|c|}
\hline $\mathbf{M}_{\mathbf{g k} 1}[\mathbf{k N m}]$ & $\mathbf{M}_{\mathbf{g k} 2}[\mathbf{k N m}]$ & $\mathbf{M}_{\mathbf{q k} 1}[\mathbf{k N m}]$ & $\mathbf{M}_{\mathbf{q k} 2}[\mathbf{k N m}]$ \\
\hline 153.0 & 69.0 & 349.5 & 15.2 \\
\hline
\end{tabular}

\begin{tabular}{|c|c|c|c|}
\hline $\mathbf{V}_{\mathbf{g k} 1}[\mathbf{k N}]$ & $\mathbf{V}_{\mathbf{g k} \mathbf{2}}[\mathbf{k N}]$ & $\mathbf{V}_{\mathbf{q k} 1}[\mathbf{k N}]$ & $\mathbf{V}_{\mathbf{q k} 2}[\mathbf{k N}]$ \\
\hline 41.9 & 18.9 & 95.8 & 4.2 \\
\hline
\end{tabular}

Design

\begin{tabular}{|c|c|c|c|}
\hline ULS & \multicolumn{3}{|c|}{ SLS } \\
\hline $\mathbf{M}_{\mathrm{Ed}}[\mathrm{kNm}]$ & $\mathbf{M}_{\text {rare }}[\mathrm{kNm}]$ & $\mathbf{M}_{\text {frequ }}[\mathbf{k N m}]$ & $\mathbf{M}_{\text {perm }}[\mathbf{k N m}]$ \\
\hline 837.7 & 580.7 & 291.9 & 222.0 \\
\hline
\end{tabular}

\begin{tabular}{|c|c|c|c|}
\hline ULS & \multicolumn{3}{|c|}{ SLS } \\
\hline $\mathbf{V}_{\text {Ed }}[\mathbf{k N}]$ & $\mathbf{V}_{\text {rare }}[\mathbf{k N}]$ & $\mathbf{V}_{\text {frequ }}[\mathbf{k N}]$ & $\mathbf{V}_{\text {perm }}[\mathbf{k N}]$ \\
\hline 229.5 & 159.1 & 80.0 & 60.8 \\
\hline
\end{tabular}

\subsection{PRESTRESSING LOSS}

Maximum prestressing force

(1) $P_{m 0, \max }=0.75 \cdot A_{p} \cdot f_{p k}=0.75 \cdot 8 \cdot 0.934 \cdot 177 \mathrm{kN} / \mathrm{cm}^{2}=991.9 \mathrm{kN}$

(2) $P_{m 0, \max }=0.85 \cdot A_{p} \cdot f_{p 0,1 \mathrm{k}}=0.85 \cdot 8 \cdot 0.934 \cdot 150 \mathrm{kN} / \mathrm{cm}^{2}=\underline{\underline{952.7 \mathrm{kN}}}$

$\rightarrow P_{m, t=0}=747.2 \mathrm{kN}<P_{m 0, \max }=952.7 \mathrm{kN} \checkmark$

Effective thickness

$h_{0}=\frac{2 \cdot A_{c}}{U}=\frac{2 \cdot 2296.5}{247.9}=18.53 \mathrm{~cm}$

Drying shrinkage

$\varepsilon_{c d}(\infty)=k_{h} \cdot \varepsilon_{c d, 0}=0.872 \cdot 0.67 \%=0.584 \%$

Autogenous shrinkage:

$\varepsilon_{c a}(\infty)=2.5 \cdot\left(f_{c k}-10\right) \cdot 10^{-6}=2.5 \cdot(30-10) \cdot 10^{-6}=0.050 \%$ o

Total shrinkage

$\varepsilon_{c s}(\infty)=\varepsilon_{c d}(\infty)+\varepsilon_{c a}(\infty)=0.585 \%$ o $+0.050 \%$ o $=0.634 \%$ 
Final creep ratio $\quad$ for $t_{0}=5$ day: $\quad \varphi\left(\infty, t_{0}\right)=2.899$

Prestressing loss due to relaxation

$$
\begin{aligned}
& \sigma_{p, \text { perm }}=\frac{E_{p}}{E_{c m}} \cdot\left[\frac{M_{E d, \text { perm }}}{I_{c}} \cdot z_{p}\right]=\frac{196}{33} \cdot\left[\frac{0.222}{0.01518675} \cdot 0.386\right]=33.5 \mathrm{MN} / \mathrm{m}^{2} \\
& N_{p, t=0}=-P_{0, t=0}=-747 \mathrm{kN} \quad M_{p, t=0}=N_{p, t=0} \cdot z_{p}=-747 \cdot 0.386=-288 \mathrm{kNm} \\
& \sigma_{p, t=0}=\frac{P_{0, t=0}}{A_{p}}+\frac{E_{p}}{E_{c m}} \cdot\left[\frac{N_{p, t=0}}{A_{c}}+\frac{M_{p, t=0}}{I_{c}} \cdot z_{p}\right] \\
& =\frac{747}{8 \cdot 0.934}+\frac{196}{33} \cdot\left[\frac{-747}{2297}+\frac{-28800}{1,518,675} \cdot 38.6\right]=937.2 \mathrm{MN} / \mathrm{m}^{2} \\
& R_{i} / R_{m}=\frac{\sigma_{p, p e r m}+\sigma_{p, t=0}}{f_{p k}}=\frac{33.5+937.2}{1770}=0.5484 \quad \rightarrow \kappa_{p}=1.194 \% \\
& \Delta \sigma_{p r}=\kappa \cdot\left(\sigma_{p, p e r m}+\sigma_{p, t=0}\right)=0.01194 \cdot(33.5+937.2)=11.6 \mathrm{MN} / \mathrm{m}^{2}
\end{aligned}
$$

Total Prestressing Loss

$$
\begin{aligned}
& \sigma_{c p, \text { perm }}=\frac{M_{E d, p e r m}}{I_{c}} \cdot z_{p}=\frac{22200}{1,518,675} \cdot 38.6=5.64 M N / m^{2} \\
& \sigma_{c p, t=0}=\frac{N_{p, t=0}}{A_{c}}+\frac{M_{p, t=0}}{l_{c}} \cdot z_{p}=\frac{-747}{2297}+\frac{-28800}{1,518,675} \cdot 38.6=-10.6 \mathrm{MN} / \mathrm{m}^{2} \\
& \alpha_{p}=\frac{E_{p}}{E_{c m}}=\frac{196,000}{33,000}=5.94 \\
& \Delta \sigma_{p, c+s+r}=\frac{\varepsilon_{c s}(t) \cdot E_{p}+0.8 \cdot \Delta \sigma_{p r}+\alpha_{p} \cdot \varphi\left(\infty, t_{0}\right) \cdot\left(\sigma_{c p, p e r m}+\sigma_{c p, t=0}\right)}{1+\alpha_{p} \cdot \frac{A_{p}}{A_{c}} \cdot\left(1+\frac{A_{c}}{I_{c}} \cdot z_{p}^{2}\right) \cdot\left[1+0.8 \cdot \varphi\left(\infty, t_{0}\right)\right]} \\
& =-102.9-7.7-70.4=-180.9 \mathrm{MN} / \mathrm{m}^{2}
\end{aligned}
$$

Shrinkage

Relaxation

Creep
$-102.9 \mathrm{MN} / \mathrm{m}^{2} \rightarrow 56.9 \%$

- $\quad 7.7 \mathrm{MN} / \mathrm{m}^{2} \rightarrow 4.2 \%$

- $70.4 \mathrm{MN} / \mathrm{m}^{2} \rightarrow 38.9 \%$

$$
\begin{array}{ll}
\Delta P_{c+s+r}=\Delta \sigma_{p, c+s+r} \cdot A_{p}=-180.9 \cdot 8 \cdot 0.934 \cdot 10^{-4}=-135.2 \mathrm{KN} \\
\frac{\Delta P_{c+s+r}}{P_{m, t=0}}=\frac{135.2}{747}=0.142 \quad \rightarrow \alpha_{\text {loss }}=14.2 \%<15 \% \checkmark \\
P_{m, t=\infty}=747-135.2=\underline{\underline{612 \mathrm{kN}}} \rightarrow \underline{\underline{77 \mathrm{kN} / \text { strand }}}
\end{array}
$$




\subsection{ULTIMATE LIMIT STATE DESIGN}

\subsubsection{FLEXURE DESIGN}

\section{Compression Zone}

The neutral axis lies below the compression flange; therefore the effective width of the flange must be adjusted according to [2].

$$
\begin{aligned}
& b_{i}=\lambda_{b} \cdot b_{\text {eff }}=0.864 \cdot 40=34.6 \mathrm{~cm} \\
& d=78.9 \mathrm{~cm} \quad z_{r}=d-x_{s o}=41.1 \mathrm{~cm} \quad z_{p}=x_{s u}-a_{p}=38.6 \mathrm{~cm} \\
& M_{E d r}=M_{E d}+P_{m, t=\infty} \cdot\left(z_{r}-z_{p}\right)=837.27+612 \cdot(0.411-0.386)=853 \mathrm{kNm} \\
& \mu_{E d r}=\frac{M_{E d r}}{b_{e f f} \cdot d^{2} \cdot f_{c d}}=\frac{85300 \mathrm{kNcm}}{34.5 \cdot 78.9^{2} \cdot 1.7 \mathrm{kN} / \mathrm{cm}^{2}}=0.233<\mu_{E d \text {, max }}=0.40 \checkmark \\
& \rightarrow \text { The compression zone is sufficient. }
\end{aligned}
$$

Tension Zone

Using the design table from [3] following values for the height of the compression zone $\mathrm{x}$ and the lever arm of the internal forces $z$ can be determined:

Lever arm z

Compression zone

Rebar strain

Prestressing steel strain

Total strain

Permitted strain

Required reinforcement

Chosen

$$
\begin{array}{lr}
\zeta=0.859 \quad \rightarrow z=\zeta \cdot d=0.859 \cdot 78.9=67.8 \mathrm{~cm} \\
\begin{array}{l}
\xi=0.339 \\
\varepsilon_{s}=\Delta \varepsilon_{p}=6.81 \% 0>2.17 \% \mathrm{o}
\end{array} \rightarrow \sigma_{s d}=f_{y d}=435 \mathrm{MN} / \mathrm{m}^{2}
\end{array}
$$

$$
\varepsilon_{p}^{(0)}=\frac{P_{m, t=0}}{A_{p} \cdot E_{p}}=\frac{612 \mathrm{kN}}{8 \cdot 0.934 \mathrm{~cm}^{2} \cdot 19,600 \mathrm{kN} / \mathrm{cm}^{2}}=4.18 \%
$$

$$
\varepsilon_{p}=\varepsilon_{p}^{(0)}+\Delta \varepsilon_{p}=4.18 \% \mathrm{o}+6.81 \% \mathrm{o}=10.99 \%
$$

$$
f_{p d}=\frac{f_{p 0,1, k}}{\gamma_{p}}=\frac{1500 \mathrm{~N} / \mathrm{mm}^{2}}{1.15}=1304 \mathrm{~N} / \mathrm{mm}^{2}
$$

$$
\begin{aligned}
\varepsilon_{p 0,1 d} & =\frac{f_{p d}}{E_{p}}=\frac{1304 \mathrm{~N} / \mathrm{mm}^{2}}{196,000 \mathrm{~N} / \mathrm{mm}^{2}}=6.7 \% 0 \\
\varepsilon_{p 0,1 d} & =6.7 \% \circ<\varepsilon_{p}=10.99 \% \mathrm{o} \quad \rightarrow \sigma_{p d}=f_{p d}=1304 \mathrm{~N} / \mathrm{mm}^{2}
\end{aligned}
$$

The required reinforcement is higher than the previously estimated reinforcement of $A_{s}=2 \varnothing_{s} 16=4.02 \mathrm{~cm}^{2}$. A new reinforcement of $A_{s}=3 \varnothing_{s} 20=9.42 \mathrm{~cm}^{2}$ is chosen. Which means the static height as well as the height of the compression zone change: 


$$
\begin{array}{ll}
d=87.6 \mathrm{~cm}-7.17 \mathrm{~cm}= & 80.4 \mathrm{~cm} \\
\zeta=0.864 & \rightarrow z=\zeta \cdot d=0.864 \cdot 80.4=69.5 \mathrm{~m} \\
\xi=0.327 & \rightarrow x=\xi \cdot d=0.327 \cdot 70.4=26.3 \mathrm{~cm}
\end{array}
$$

Precompressed Tension Zone

$$
N_{\rho, t=0}=-747 k N \quad M_{p, t=0}=-288 k N m
$$

Design normal force

Effective depth

Design bending moment

Required reinforcement

$$
\begin{aligned}
& N_{E d}=\gamma_{p} \cdot N_{p, t=0}=1.0 \cdot(-747 \mathrm{kN})=-747 \mathrm{kN} \\
& d \approx 87.6-4=83.6 \mathrm{~cm} \quad \rightarrow z_{s 2}=d-x_{s u}=83.6-49.8=33.8 \mathrm{~cm}
\end{aligned}
$$$$
M_{E d}=\gamma_{G} \cdot \min M_{G}+\gamma_{P} \cdot M_{P, t=0}=1.0 \cdot 153.0+1.0 \cdot(-288)=-135.3 \mathrm{kNm}
$$$$
M_{E d, s 2}=M_{E d}-N_{E d} \cdot z_{s 2}=-135.3-0.338 \cdot(-747)=388 \mathrm{kNm}
$$$$
\mu_{E d s 2}=\frac{M_{E d s 2}}{b_{\text {eff }} \cdot d^{2} \cdot f_{c d}}+\frac{N_{E d}}{f_{y d}}=0.095 \quad \rightarrow \omega_{2}=0.099
$$

$$
A_{s 2 \text {,req }}=\omega_{2} \cdot \frac{b_{\text {eff }} \cdot d}{f_{y d} / f_{c d}}+\frac{N_{E d}}{f_{y d}}=0.099 \cdot \frac{34.6 \cdot 83.6}{43.5 / 1.7}+\frac{-747}{43.5}=-6.00<0
$$

No reinforcement in the compression zone is required.

\subsubsection{SHEAR DESIGN}

Design location

$$
\begin{aligned}
& x_{1}=a_{i}+d=0.2+0.804=1.00 m \quad \text { (direct support) } \\
& x_{2}=a_{i}=0.2 m
\end{aligned}
$$

Shear force at $x$

$$
\begin{aligned}
& V_{E d, 1}=198.1 \mathrm{kN} \\
& V_{E d, 2}=223.2 \mathrm{kN}
\end{aligned}
$$

Shear Force Resistance

$$
\begin{aligned}
& a_{r}=7.17 \mathrm{~cm} \quad \rightarrow d=87.6-7.17=80.4 \mathrm{~cm} \\
& k=1+\sqrt{\frac{20}{d}}=1+\sqrt{\frac{20}{80.4}}=1.50<2.00
\end{aligned}
$$

Longitudinal reinforcement

$$
A_{s l}=A_{s}+A_{p}=9.42+7.47=16.89 \mathrm{~cm}^{2}
$$

Percentage of reinforcement

$$
\rho_{1}=\frac{A_{s /}}{b_{w} \cdot d}=\frac{16.89}{19 \cdot 80.4}=0.0111 \leq 0.02
$$

Compressive stress

$$
\begin{aligned}
& \sigma_{c p}= \frac{N_{E d}}{A_{c}}=\frac{0.612}{0.2297}=2.67 \mathrm{MN} / \mathrm{m}^{2}<0.2 \cdot f_{c d}=3.40 \mathrm{MN} / \mathrm{m}^{2} \\
& V_{R d, c}= {\left[0.10 \cdot \mathrm{k} \cdot\left(100 \cdot \rho_{l} \cdot f_{c k}\right)^{1 / 3}+0.12 \cdot \sigma_{c p}\right] \cdot b_{w} \cdot d } \\
& V_{R d, c}= {\left[0.10 \cdot 1.5 \cdot(100 \cdot 0.0111 \cdot 3,0)^{1 / 3}+0.12 \cdot 2.67\right] \cdot 0.19 \cdot 0.804 } \\
&= 122.5 \mathrm{kN} \\
& d=80.4 \mathrm{~cm} \geq 80 \mathrm{~cm} \rightarrow v_{\min }=0.025 \cdot 1.5^{3 / 2} \cdot(30)^{1 / 2}=0.251 \mathrm{MN} / \mathrm{m}^{2}
\end{aligned}
$$




$$
\begin{aligned}
& V_{R d, c, \min }=\left[v_{\min }+0.12 \cdot \sigma_{c p}\right] \cdot b_{w} \cdot d=[0.251+0.12 \cdot 2.67] \cdot 0.19 \cdot 0.804 \\
& =87.3 \mathrm{kN} \\
& \text { Shear force resistance } \quad V_{R d, c}=\underline{\underline{122.5 \mathrm{kN}}}>V_{R d, c, \min }=87.3 \mathrm{kN} \\
& V_{E d}=198.1 \mathrm{kN}>V_{R d, c}=122.5 \mathrm{kN} \rightarrow \text { Shear force reinforcement is needed! }
\end{aligned}
$$

Friction force

$$
\begin{aligned}
& V_{R d, c c}=0.24 \cdot\left(f_{c k}\right)^{1 / 3} \cdot\left(1-1.2 \cdot \frac{\sigma_{c p}}{f_{c d}}\right) \cdot b_{w} \cdot z \\
& V_{R d, c c}=0.24 \cdot(3.0)^{1 / 3} \cdot\left(1-1.2 \cdot \frac{0.267}{1.7}\right) \cdot 19 \cdot 69.5=79.9 \mathrm{kN} \\
& 1.0 \leq \cot \Theta=\frac{1.2+1.4 \cdot \sigma_{c p} / f_{c d}}{1-V_{R d, c c} / V_{E d}}=\frac{1.2+1.4 \cdot 0.267 / 1.7}{1-79.9 / 122.5}=2.38 \leq 3.0
\end{aligned}
$$

Inclination

Required Shear Force Reinforcement

$$
\begin{aligned}
& \text { Vertical stirrups } \quad \alpha=90^{\circ} \\
& a_{s w, r e q}=\frac{V_{E d}}{f_{y d} \cdot z \cdot(\cot \theta+\cot \alpha) \cdot \sin \alpha} \\
& =\frac{198.1 \mathrm{kN}}{43.5 \cdot 69.5 \cdot(2.38+0) \cdot 1}=2.75 \mathrm{~cm}^{2} / \mathrm{m}
\end{aligned}
$$

Chosen

$\varnothing 8 / 25=4.02 \mathrm{~cm}^{2} / \mathrm{m}$

stirrups

Resistance of the Compression Struts

$$
\begin{aligned}
V_{R d, \max } & =0.75 \cdot b_{w} \cdot z \cdot f_{c d} \cdot \frac{\cot \theta+\cot \alpha}{1+\cot ^{2} \theta}=0.75 \cdot 19 \cdot 69.5 \cdot 1.7 \cdot \frac{2.38}{1+2.38^{2}} \\
& =601.1 \mathrm{kN} \\
V_{E d, 2}= & 223 \mathrm{kN}<V_{R d, \max }=601 \mathrm{kN} \quad \rightarrow \text { The compression strut is ok. }
\end{aligned}
$$

Minimum Shear Force Reinforcement

$$
\begin{aligned}
& \min a_{s w}=\rho \cdot b_{w} \cdot \sin \alpha \quad \text { and } \quad \rho=0.16 \cdot f_{c t m} / f_{y k}=0.16 \cdot 2.9 / 500=9.3 \% o \\
& \min a_{s w}=0.00093 \cdot 0.19 \cdot \sin 90^{\circ}=1.77 \cdot 10^{-4} \mathrm{~m}^{2} / \mathrm{m}=\underline{\underline{1.77 \mathrm{~cm}^{2} / \mathrm{m}}}
\end{aligned}
$$




\subsubsection{TILTING}

According to DIN EN 1992-1-1, 5.9 a simplified proof of the safety against tilting can be conducted with following formula:

$$
\begin{aligned}
& b_{\text {req }} \geq \sqrt[4]{\left(\frac{I_{0}}{50}\right)^{3} \cdot h} \quad \text { and } \quad \frac{h}{b} \leq 5.0 \\
& b_{\text {req }} \geq \sqrt[4]{\left(\frac{14.6}{50}\right)^{3} \cdot 0.876}=0.384 m<b_{\text {prov }}=0.40 m \quad \text { and } \quad \frac{h}{b}=\frac{87.6}{40}=2.19<5.0
\end{aligned}
$$

This means that the purlin is safe against tilting and no further analysis is necessary. The simplified proof is sufficient.

\subsection{SERVICEABILITY LIMIT STATE DESIGN}

\subsubsection{STRESS LIMITS}

First or second order?

$$
\begin{aligned}
& \min P_{k, t}=r_{\text {inf }} \cdot P_{m, t=\infty}=0.95 \cdot 612 \mathrm{kN}=581.4 \mathrm{kN} \\
& N_{E d, \text { rare }}=-\min P_{k, t}=-581.4 \mathrm{kN} \\
& M_{E d, \text { rare }}=M_{E d, \text { rare }}^{G+Q}-\min P_{k, t} \cdot z_{p}=580.7-581.4 \cdot 0.386=356.3 \mathrm{kNm} \\
& \sigma_{c, \text { rare }}=\frac{N_{E d, \text { rare }}}{A_{c}}+\frac{M_{E d, \text { rare }}}{l_{c}} \cdot z_{u} \\
& \sigma_{c, \text { rare }}=\frac{-581.4}{2297}+\frac{35630}{1,518,675} \cdot 49.8=0.91 \mathrm{kN} / \mathrm{cm}^{2}=9.1 \mathrm{MN} / \mathrm{m}^{2} \\
& \sigma_{c, \text { rare }}=9.1 \mathrm{MN} / \mathrm{m}^{2}>f_{c t m}=2.90 \mathrm{MN} / \mathrm{m}^{2} \\
& \rightarrow \text { second order must be considered }
\end{aligned}
$$

\section{Concrete Compression Stresses}

$$
\begin{aligned}
& \frac{2 \cdot A_{c} \cdot z_{p}}{z \cdot x \cdot b_{\text {eff }}}=\frac{2 \cdot 2297 \cdot 38.6}{69.5 \cdot 26.3 \cdot 34.5}=2.81>1.0 \quad \rightarrow \min P_{k, t}=581.4 \mathrm{kN} \\
& N_{E d, \text { perm }}=-581.4 \mathrm{kN} \\
& M_{E d, \text { perm }}=M_{E d, \text { perm }}^{G+Q}-\min P_{k, t} \cdot z_{p}=222.0-581 \cdot 0.386=-2.36 \mathrm{kNm} \\
& \sigma_{c, \text { perm }}=\frac{N_{E d, p e r m}}{A_{c}}+\frac{2 M_{E d, p e r m}}{z \cdot x \cdot b_{\text {eff }}} \leq 0.45 f_{c k} \\
& \sigma_{c, \text { perm }}=\frac{-581}{2297}+\frac{2 \cdot(-236)}{69.5 \cdot 26.3 \cdot 0.345}=-0.246 \mathrm{kN} / \mathrm{cm}^{2}=-2.46 \mathrm{MN} / \mathrm{m}^{2} \\
& \left|\sigma_{c, \text { perm }}\right|=2.46 \mathrm{MN} / \mathrm{m}^{2}<0.45 f_{c k}=0.45 \cdot 30=13.5 \mathrm{MN} / \mathrm{m}^{2} \checkmark
\end{aligned}
$$


Stresses in the Reinforcement

$$
\begin{aligned}
N_{E d, \text { rare }}= & -581.4 \mathrm{kN} \\
M_{E d, \text { rare }} & =M_{E d, \text { rare }}^{G+Q}-\min P_{k, t} \cdot\left(z_{r}-z_{p}\right) \\
& =581-581.4 \cdot(0.426-0.386)=604 \mathrm{kNm} \\
\sigma_{s, \text { rare }}= & {\left[\frac{M_{E d, \text { rare }}}{z}+N_{E d, \text { rare }}\right] \cdot \frac{1}{A_{s 1}+A_{p}} \leq 0.8 f_{y k} } \\
\sigma_{s, \text { rare }}= & {\left[\frac{60400}{69.5}-581.4\right] \cdot \frac{1}{9.42+7.47} \cdot 10^{-1}=170.5 \mathrm{MN} / \mathrm{m}^{2} } \\
\leq & 0.8 \cdot 500=400 \mathrm{MN} / \mathrm{m}^{2} \checkmark
\end{aligned}
$$

Stresses in the Prestressing Steel

$$
\begin{aligned}
\min P_{k, t} & =P_{m, t=\infty}=612 k N \quad N_{E d, p e r m}=-\min P_{k, t}=-612 k N \\
M_{E d, p e r m} & =M_{E d, \text { perm }}^{G+Q}-\min P_{k, t} \cdot z_{p}=222.0-612 \cdot 0.386=246.5 \mathrm{kNm} \\
\sigma_{p, p e r m} & =\frac{P_{m, t=\infty}}{A_{p}}\left[\frac{M_{E d, p e r m}}{z}+N_{E d, p e r m}\right] \cdot \frac{1}{A_{s 1}+A_{p}} \leq 0.65 f_{p k} \\
\sigma_{p, \text { perm }} & =\frac{612}{7.47}\left[\frac{24650}{69.5}-612\right] \cdot \frac{1}{9.42+7.47} \cdot 10^{-1}=667 \mathrm{MN} / \mathrm{m}^{2} \\
& \leq 0.65 \cdot 1770=1150 \mathrm{MN} / \mathrm{m}^{2} \checkmark
\end{aligned}
$$

\subsubsection{MINIMUM REINFORCEMENT AGAINST CRACKING}

Reinforcement needed?

$$
\sigma_{c, \text { rare }}=9.1 \mathrm{MN} / \mathrm{m}^{2}>0
$$

$\rightarrow$ a minimum reinforcement to prevent cracking is necessary

Area of the Tension Zone

$$
\begin{aligned}
& \sigma_{l}=f_{c t m}=\sigma_{l}^{N}+\sigma_{l}^{M}=2.9 \mathrm{MN} / \mathrm{m}^{2}=\frac{-0.581}{0.2297}+\sigma_{l}^{M} \\
& \sigma_{l}^{M}=5.43 \mathrm{MN} / \mathrm{m}^{2} \quad \rightarrow \sigma_{u}^{M}=-5.43 \mathrm{MN} / \mathrm{m}^{2} \\
& \sigma_{u}=\sigma_{u}{ }^{N}+\sigma_{u}{ }^{M}=\frac{-0.581}{0.2297}-5.43=-7.96 \mathrm{MN} / \mathrm{m}^{2} \\
& h_{t}=\frac{2.9}{2.9+7.96} \cdot 87.6 \mathrm{~cm}=23.4 \mathrm{~cm} \quad \rightarrow A_{c t}=19.0 \cdot 23.4=\underline{\underline{444.3 \mathrm{~cm}^{2}}}
\end{aligned}
$$

Area of tension zone

Minimum Required Cracking Reinforcement

Tensile strength

$$
\begin{array}{ll}
f_{c t, \text { eff }}=f_{c t m} \geq 2.9 \mathrm{MN} / \mathrm{m}^{2} & \rightarrow f_{c t, \text { eff }}=2.9 \mathrm{MN} / \mathrm{m}^{2} \geq \underline{\underline{2.9 \mathrm{MN} / \mathrm{m}^{2}}} \\
h^{\star}=h \leq 1.0 \mathrm{~m} & \rightarrow h^{*}=0.19 \mathrm{~m} \\
\text { for } h<30 \mathrm{~cm} & \rightarrow k=0.8
\end{array}
$$




$$
\begin{aligned}
& k_{1}=1.5 \\
& k_{c}=0.4 \cdot\left[1-\frac{\sigma_{c}}{k_{1} \cdot h / h^{*} \cdot f_{c t, e f f}}\right]=0.4 \cdot\left[1-\frac{581.4 / 2297}{1.5 \cdot 1.0 \cdot 0.29}\right]=0.167 \leq 1.0
\end{aligned}
$$

Crack width limit

$$
w_{k}=0.2 \mathrm{~mm}
$$

Limit diameter

$$
\begin{aligned}
& \text { (according to DIN EN 1992-1-1, Tab. NA.7.1.) } \\
& 1.0=20 \mathrm{~mm}
\end{aligned}
$$

Reinforcement stresses

$$
\sigma_{s}=188.0 \mathrm{MN} / \mathrm{m}^{2} \quad \text { (according to DIN EN 1992-1-1, Tab. NA.7.2.) }
$$

Required reinforcement

$$
\begin{aligned}
A_{s, \text { min }, \text { req }} & =k_{c} \cdot k \cdot f_{c t, \text { eff }} \cdot \frac{A_{c t}}{\sigma_{s}}=0.167 \cdot 0.8 \cdot 2.9 \cdot \frac{444.3}{188.0}=0.92 \mathrm{~cm}^{2} \\
& <A_{s, \text { prov }}=9.42 \mathrm{~cm}^{2} \rightarrow \text { The provided reinforcement is sufficient! }
\end{aligned}
$$

\subsubsection{LIMITATION OF THE CRACK WIDTH}

\section{Percentage of Reinforcement}

$$
\begin{aligned}
& h / d_{1}=21.9 \rightarrow h_{c, \text { eff }} / d_{1}=4.19 \\
& h_{c, \text { eff }}=4.19 \cdot 4.0 \mathrm{~cm}=16.8 \mathrm{~cm} \\
& A_{c, \text { eff }}=19.0 \cdot 16.8=318.4 \mathrm{~cm}^{2} \\
& \xi=0.6 \quad \varnothing_{s}=20 \mathrm{~mm} \quad \varnothing_{p}=7.2 \mathrm{~mm} / h_{\mathrm{c}, \text { ef }} / d_{1} \\
& \xi=\sqrt{\xi \cdot \phi_{s} / \phi_{p}}=\sqrt{0.6 \cdot 20 / 7.2}=1.29 \\
& \rho_{\text {tot }}=\left(A_{s}+A_{p}\right) / A_{c, \text { eff }}=(9.42+7.47) / 318.4=0.0530 \\
& \rho_{p, \text { eff }}=\left(A_{s}+\xi_{1}^{2} A_{p}\right) / A_{c, \text { eff }}=\left(9.42+1.29^{2} \cdot 7.47\right) / 318.4=0.0687
\end{aligned}
$$

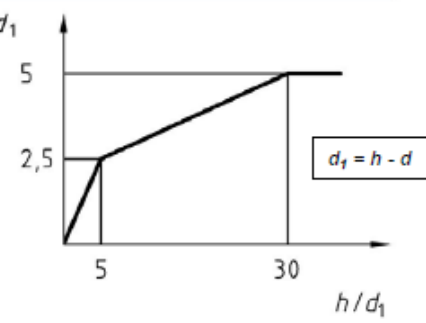

Reinforcement Stresses

$$
\begin{aligned}
& \sigma_{s}=\left[\frac{M_{E d r}}{z}+N_{E d}\right] \cdot \frac{1}{A_{s}+A_{\rho}}+0.4 \cdot f_{c t, e f f} \cdot\left[\frac{1}{\rho_{p, \text { eff }}}-\frac{1}{\rho_{\text {tot }}}\right] \\
& \sigma_{s}=\left[\frac{31520}{69.5}-581.4\right] \cdot \frac{1}{9.42+7.47}+0.4 \cdot 2.9 \cdot\left[\frac{1}{0.053}-\frac{1}{0.0687}\right]=-8.08 \mathrm{kN} / \mathrm{cm}^{2} \\
& \rightarrow \sigma_{s}=-80.8 \mathrm{MN} / \mathrm{m}^{2}<0
\end{aligned}
$$

The stress is negative which means the cross section is under compression so there won't be any cracking. 


\subsection{DESIGN AND REINFORCEMENT}

\subsubsection{ANCHORAGE OF TENDONS}

Transmission of the Stresses

Transmission length

$$
\begin{array}{ll}
l_{p t}=\alpha_{1} \cdot \alpha_{2} \cdot \varnothing_{p} \cdot \sigma_{p m 0} / f_{b p t} & \\
\alpha_{1}=1.25 & \text { no wire stripping } \\
\alpha_{2}=0.19 & \text { for strands } \\
\varnothing_{p}=12.5 \mathrm{~mm} & \\
f_{b p t}=3.3 \mathrm{MN} / \mathrm{m}^{2} & \text { for C30/37 and good bond } \\
\sigma_{p m 0}=1000 \mathrm{MN} / \mathrm{m}^{2} & \\
l_{p t}=1.25 \cdot 0.19 \cdot 1.25 \cdot 1000 / 3.3=90.0 \mathrm{~cm}
\end{array}
$$

Modified transmission length $\quad I_{p t d}=0.8 \cdot I_{p t}=0.8 \cdot 90.0=72.0 \mathrm{~cm}$

Dispersion length $\quad I_{\text {disp }}=\sqrt{I_{p t d}^{2}+d_{p}^{2}}=\sqrt{72.0^{2}+76.4^{2}}=105.0 \mathrm{~cm}$

Splitting tensile force

$$
\begin{aligned}
& F_{t d}=P_{m, 0}-\left|\sigma_{c, m}\right| \cdot A_{c, u} \\
& N_{E d}^{P}=-P_{m, t=0}=-747 \mathrm{kN} \quad M_{E d}^{P}=N_{E d}^{P} \cdot z_{p}=-747 \cdot 0.386=-288 \mathrm{kNm} \\
& A_{c, u}=a_{p} \cdot b=(15.9-1.25 / 2) \cdot 19=314 \mathrm{~cm}^{2} \\
& z_{c, u}=z_{s, u}-a_{p} / 2=49.8-16.5 / 2=41.5 \mathrm{~cm} \\
& \sigma_{c, m}\left(x=I_{\text {disp }}\right)=\frac{N_{E d}^{P}}{A_{c}}+\frac{M_{E d}^{P}}{I_{c}} \cdot z_{c, u}=\frac{-747}{2297}+\frac{-28800}{1,518,675} \cdot 41.5 \cdot 10^{-1} \\
& =-11.1 \mathrm{MN} / \mathrm{m}^{2} \\
& F_{t d}=747-1.11 \cdot 314=\underline{\underline{398 k N}}
\end{aligned}
$$

Required reinforcement

$$
A_{s p, r e q}=\frac{\gamma_{p, s p}}{3} \cdot \frac{F_{t d}}{f_{y d}}=\frac{1.35}{3} \cdot \frac{398}{43.5}=4.18 \mathrm{~cm}^{2}
$$

The reinforcement is to be distributed over the length of

$0.75 \cdot I_{\text {disp }}=0.75 \cdot 105=78.8 \mathrm{~cm}$

$a_{s p, r e q}=4.18 / 0.788=\underline{\underline{5.31 \mathrm{~cm}^{2} / \mathrm{m}}}$

\section{Chosen}

Tensile forces at the edge $\quad Z_{E d}^{P}=P_{m 0} \cdot\left[\frac{e}{h}-\frac{1}{6}\right]=747 \cdot\left[\frac{38.6}{100}-\frac{1}{6}\right]=163.9 \mathrm{kN}$ 
Required reinforcement

Chosen

$$
A_{s z, r e q}=Z_{E d} / f_{y d}=163.9 / 43.5=\underline{\underline{3.77 \mathrm{~cm}^{2}}}
$$

$3 \varnothing 14=4.62 \mathrm{~cm}^{2}$

\section{U-bars}

Anchorage of Tendons in the Ultimate Limit State

Anchorage length

$$
\begin{aligned}
& I_{b p d}=I_{p t 2}+\alpha_{2} \cdot \varnothing_{P} \cdot \frac{\sigma_{p d}-\sigma_{p m, t=\infty}}{f_{b p d}} \\
& I_{p t 2}=1.2 \cdot I_{p t}=1.2 \cdot 90.0=108.0 \mathrm{~cm} \\
& \sigma_{p d}=f_{p, 01 k} / \gamma_{p}=1500 / 1.15=1304 \mathrm{MN} / \mathrm{m}^{2} \\
& \sigma_{p m, t=\infty}=P_{m, t=\infty} / A_{p}=612 / 7.47=81.9 \mathrm{kN} / \mathrm{cm}^{2} \\
& I_{b p d}=108+0.19 \cdot 1.25 \cdot \frac{1304-81.9}{3.3}=\underline{\underline{143 \mathrm{~cm}}}
\end{aligned}
$$

Internal forces at $x$

$$
\begin{aligned}
& N_{P, t=\infty}=-P_{m, t=\infty}=-612 \mathrm{kN} \quad M_{P, t=\infty}=-612 \cdot 0.386=-237 \mathrm{kNm} \\
& q_{E d}=1.35 \cdot(5.74+2.59)+1.5 \cdot 13.1+0.9 \cdot 0.6=31.4 \mathrm{kN} / \mathrm{m} \\
& x=I_{\text {disp }}+a_{i}=143+21.5=164.5 \mathrm{~cm} \\
& M_{E d}(x=165)=31.4 \cdot 14.6 \cdot 1.65 / 2+31.4 \cdot 1.65^{2} / 2=336 \mathrm{kNm} \\
& M_{E d}=M_{E d}(x)+M_{E d}^{P}=336-236=99 \mathrm{kNm}
\end{aligned}
$$

Concrete stresses

$$
\begin{aligned}
& \sigma_{c, u}=\frac{N_{E d}}{A_{c}}+\frac{M_{E d}}{I_{c}} \cdot z_{u}=\frac{-612}{2297}+\frac{9900}{1,518,675} \cdot 49.8=0.059 \mathrm{kN} / \mathrm{m}^{2} \\
& \sigma_{c, u}=0.59 \mathrm{MN} / \mathrm{m}^{2}<0.7 \cdot f_{c t m}=0.7 \cdot 2.9=2.03 \mathrm{MN} / \mathrm{m}^{2}
\end{aligned}
$$

\subsubsection{MINIMUM REINFORCEMENT TO ENSURE ROBUSTNESS}

$$
\begin{aligned}
& M_{c r}=f_{c t m} \cdot W_{c u} \quad W_{c u}=30,520 \mathrm{~cm}^{3} \\
& M_{c r}=2.9 \cdot 30,520 \cdot 10^{-3}=88.5 \mathrm{kNm} \\
& A_{s, \text { min }}=\frac{1}{f_{y k}} \cdot \frac{M_{c r}}{z}=\frac{1}{50.0} \cdot \frac{8850}{69.5}=2.55 \mathrm{~cm}^{2}<A_{s, \text { prov }}=9.42 \mathrm{~cm}^{2}
\end{aligned}
$$

\subsubsection{DESIGN OF THE SUPPORT}

Width of the Joints

The width of the joints between two purlins facing each other and between the purlin and the concrete truss can be estimated according to [4] and [5]. The width of the joints is the result of the sum of the different possible dimensional deviations during the process of the production and assembly of the beams.

$\delta_{\text {comb }}=\delta_{\max }+\sqrt{\sum \delta_{i}^{2}} \quad$ with $\quad \delta_{\text {comb }} \quad$ Total allowed dimensional deviation 


$$
\begin{array}{ll}
\delta_{\max } & \text { Maximum dimensional deviation } \\
\delta_{i} & \text { Other possible dimensional deviations. }
\end{array}
$$

The different dimensional deviations can be taken from DIN 18202 and DIN 18203.

Here it is:

$$
\begin{aligned}
& \delta_{\text {comb }}=\delta_{\max }+\sqrt{\sum \delta_{i}^{2}}=\frac{1}{2} \cdot\left(24+\sqrt{16^{2}+16^{2}+24^{2}}\right)=28.5 \mathrm{~mm} \\
& \text { Chosen } \quad \delta=30 \mathrm{~mm}
\end{aligned}
$$

\section{Dimensions of the Bearing}

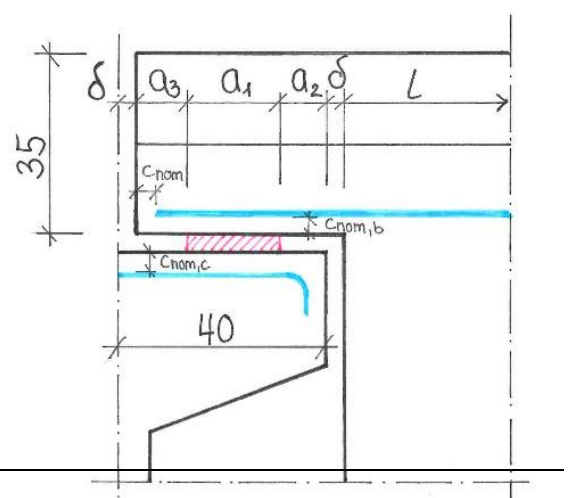

The width of the elastomeric bearing is chosen to $b e b=200 \mathrm{~mm}$. The maximum length of the bearing can be calculated as per DIN EN 1992-1-1, 10.9.5.2 with following formula:

$$
\begin{aligned}
& a=a_{1}+a_{2}+a_{3}+\sqrt{\Delta a_{2}^{2}+\Delta a_{3}^{2}} \quad \text { with } \quad \begin{array}{ll}
a_{1} & \text { Length of the elastomeric bearing, } \\
& a_{1}>\text { min } a_{1} \text { (DIN EN 1992-1-1, Tab. 10.2) } \\
& a_{2} \quad \text { Distance of the bearing to the edge of the support } \\
a_{3} \quad \text { Distance of the bearing to the edge of the beam } \\
\Delta a \quad \text { Dimensional deviations. }
\end{array}
\end{aligned}
$$

Here:

$$
\begin{aligned}
& \text { Assumption } \quad a_{1} \approx 250 \mathrm{~mm} \rightarrow \sigma_{E d}=V_{E d} / a_{1} \cdot b_{1}=230 \mathrm{kN} / 25 \cdot 20=0.46 \mathrm{kN} / \mathrm{cm}^{2} \\
& \min a_{1}=70 \mathrm{~mm} \text { for } 0.15<\sigma_{E d} / f_{c d}=0.46 / 1.7=0.27<0.40 \\
& \text { (DIN EN 1992-1-1, Tab.10.2) } \\
& a_{2}=c_{\text {nom }}+d_{s} / 2+c_{\text {nom }, c} / 2=20+10 / 2+20 / 2=35 \mathrm{~mm}>\min a_{2}=15 \mathrm{~mm} \\
& \text { (DIN EN 1992-1-1, Tab.10.3) } \\
& a_{3}=c_{n o m}+d_{s} / 2+c_{n o m, b} / 2=20+12 / 2+20 / 2=36 m m>\min a_{3}=15 m m \\
& \text { (DIN EN 1992-1-1, Tab.10.4) } \\
& \Delta a_{2}=/ / 1200=14700 / 1200=12.25 \mathrm{~mm} \\
& \text { (DIN EN 1992-1-1, Tab.10.5) } \\
& \Delta a_{3}=/ / 2500=14700 / 2500=5.88 \mathrm{~mm} \\
& \rightarrow a_{\text {req }}=250+35+36+\sqrt{12.25^{2}+5.88^{2}}=334.6 m m<a_{\text {prov }}=400-30=\underline{\underline{370 m m}} \checkmark
\end{aligned}
$$


Elastomeric Bearing

The elastomeric bearing that is used here is by the company Calenberg Ingenieure and is chosen in accordance with the technical information and dimensioning tables of the bearings [6].

Size of the bearing

Bearing compression

Form factor $\mathrm{l} \times \mathrm{b} \times \mathrm{t}=280 \times 200 \times 15 \mathrm{~mm}$

$$
\sigma_{m, k}=\frac{V_{E d}}{a_{1} \cdot b_{1}}=\frac{230}{28 \cdot 20}=0.41 \mathrm{kN} / \mathrm{cm}^{2}=4.1 \mathrm{MN} / \mathrm{m}^{2}
$$

$S=\frac{58}{15}=3.87$

\section{Chosen}

\section{'Compressionslager'}

elastomeric bearing

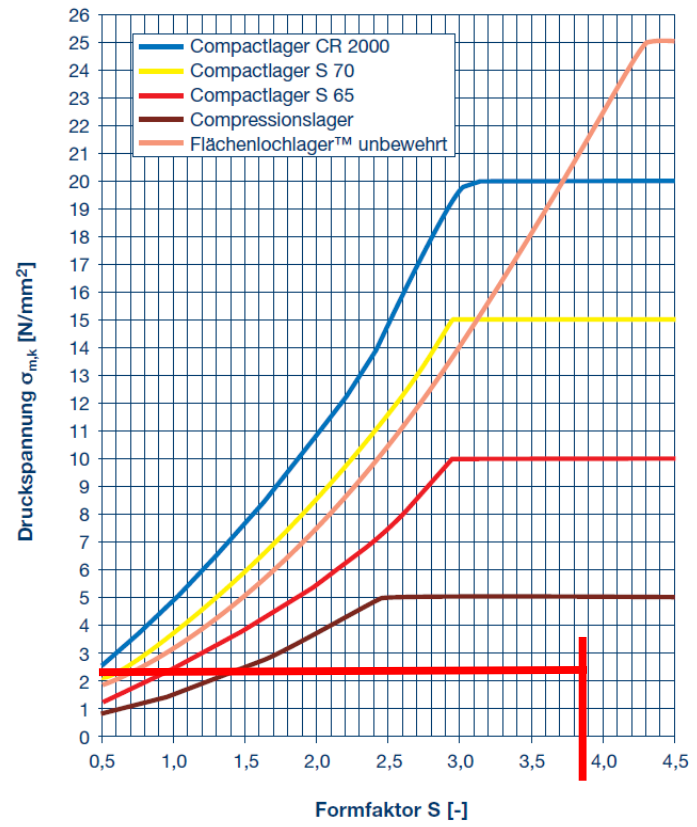

\subsubsection{DESIGN OF THE NOTCH}

Vertical Stirrups

$$
\begin{aligned}
& Z_{E d}^{V}=V_{E d}=230 \mathrm{kN} \\
& A_{s, \text { req }}^{V}=Z_{E d}^{V} / f_{y d}=230 / 43.5=5.28 \mathrm{~cm}^{2}
\end{aligned}
$$

\section{Chosen \\ $6 \varnothing 8 / \mathrm{e}=5 \mathrm{~cm}$}

$I_{b, \text { ind }}=5 \cdot 5+2 \cdot 0.8 / 2=26 \mathrm{~cm}$

$e=18.5+3+2+26 / 2=36.5$

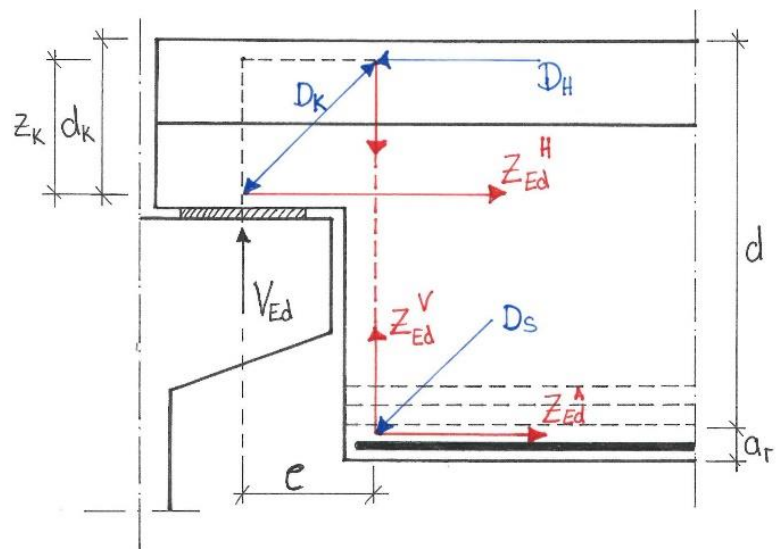


Design of the Console

Minimum static height

$\min d_{k}=\frac{3.4 \cdot V_{E d}}{b \cdot f_{c d}}=\frac{3.4 \cdot 230}{0.24 \cdot 1.7}=19.2 \mathrm{~cm}$

$d_{k, \text { prov }} \approx 30 \mathrm{~cm}>\min d_{k}=19.2 \mathrm{~cm}$

Lever arm of the internal forces

$z_{k} \approx 0.9 \cdot d_{k}=0.9 \cdot 31=27 \mathrm{~cm}$

Horizontal force at support

$Z_{E d}^{H}=V_{E d} \cdot \frac{e}{Z_{k}}=230 \cdot \frac{36.5}{27}=311 \mathrm{kN}$

Required reinforcement

$A_{s, r e q}=\frac{Z_{E d}^{H}}{f_{y d}}=\frac{311}{43.5}=7.2 \mathrm{~cm}^{2}$

Chosen

$4 \varnothing 12=9.05 \mathrm{~cm}^{2}$

reinforcement loops

Static height

$$
d_{k, \text { prov }}=35-2-1-1.2-2 / 2=29.8 \mathrm{~cm} \approx d_{k, e s t}=30 \mathrm{~cm} \checkmark
$$

Anchorage of the Reinforcement Loops at the Direct Support

$$
\begin{aligned}
& I_{b, e q}=\alpha_{1} \cdot \frac{A_{s, \text { req }}}{A_{s, \text { prov }}} \cdot I_{b, \text { rqd }}>I_{b, \text { min }} \quad I_{b, \text { rqd }}=43 \mathrm{~cm} \quad \text { for } \varnothing=12 \mathrm{~mm}, \text { C30/37 and good bond } \\
& I_{b, e q}=0.7 \cdot \frac{7.2}{9.05} \cdot 43=23.9 \mathrm{~cm} \quad I_{b, \min }=0.3 \cdot \alpha_{1} \cdot I_{b, \text { rad }}=0.3 \cdot 0.7 \cdot 43=9 \mathrm{~cm}>10 \cdot 1.2=\underline{\underline{12 \mathrm{~cm}}} \\
& I_{b, e q}=23.9 \mathrm{~cm}>I_{b, \text { min }}=12 \mathrm{~cm} \quad \rightarrow \quad I_{b, \text { dir }}=\frac{2}{3} \cdot I_{b, e q}=\frac{2}{3} \cdot 23.9=\underline{\underline{15.9 \mathrm{~cm}}}>6.7 \cdot 1.2=8.0 \mathrm{~cm} \\
& I_{b, \text { dir, prov }}=32.5-2-1.2-2 / 2=28.3 \mathrm{~cm}>I_{b, \text { dir }, \text { req }}=15.9 \mathrm{~cm} \checkmark
\end{aligned}
$$

Anchorage of the Reinforcement Loops at the Indirect Support

$$
\begin{aligned}
& I_{b, e q}=\alpha_{1} \cdot \frac{A_{s, \text { req }}}{A_{s, \text { prov }}} \cdot I_{b, \text { rqd }}>I_{b, \text { min }} \\
& I_{b, r q d}=61 \mathrm{~cm} \quad \text { for } \varnothing=12 \mathrm{~mm}, C 30 / 37 \text { and moderate bond } \\
& I_{b, e q}=1.0 \cdot \frac{7.2}{9.05} \cdot 61=48.5 \mathrm{~cm} \\
& I_{b, \min }=0.3 \cdot \alpha_{1} \cdot I_{b, r q d}=0.3 \cdot 61=\underline{\underline{18.3 \mathrm{~cm}}}>10 \cdot 1.2=12 \mathrm{~cm} \\
& I_{b, e q}=48.5 \mathrm{~cm}>I_{b, \min }=18.3 \mathrm{~cm} \\
& \rightarrow \quad I_{b, \text { ind }}=\underline{\underline{48.5 \mathrm{~cm}}} \\
& \min />I_{b, \text { dir }}+a+b+c_{\text {nom }}+I_{b, \text { ind }} / 2+\left(\left(d-d_{k}\right) / \tan \Theta\right)+I_{b, \text { ind, }} / 2 \\
& \min />28+4.5+3+2+26 / 2+((80.4-30) / \tan 40)+48.5 / 2 \\
& =\underline{\underline{135 \mathrm{~cm}}}
\end{aligned}
$$




\subsubsection{REINFORCEMENT DRAWING}
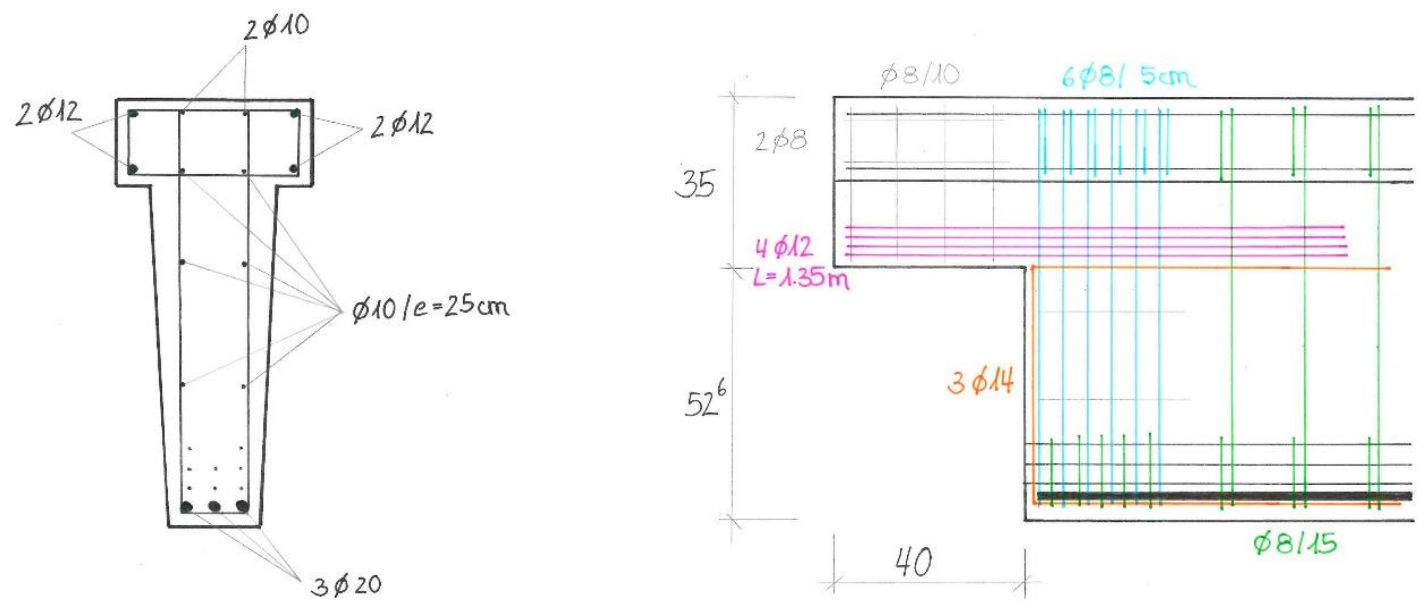

\subsection{TRANSPORT AND ASSEMBLY}

\subsubsection{TRANSPORT AND LIFTING ON THE CONSTRUCTION SITE}

The beam will be suspended with the help of anchors at around 1/5 of the length of the beam. Which might cause tension over the anchors and might require upper reinforcement.

In the following the static system during transportation and assembly can be seen.
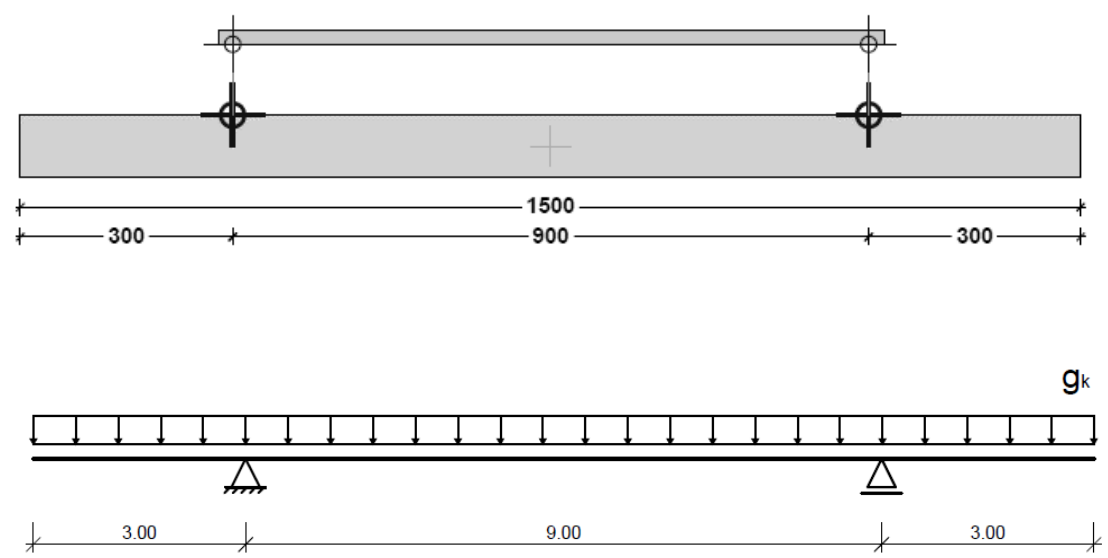

Lifting out of the Formwork

$\begin{array}{ll}\text { Lifting loads } & \text { self-weight } \quad g_{k}=5.74 \mathrm{kN} / \mathrm{m} \\ \text { Bending moment } & M_{E d}^{G}=1.15 \cdot(-5.74) \cdot 3.0^{2} / 2=-29.7 \mathrm{kNm} \\ & M_{E d s}=M_{E d}^{G}-N_{E d} \cdot z_{s 1}=29.7-(-747.0) \cdot(0.378-0.04)=282 \mathrm{kNm}\end{array}$

Time of first lifting

approximately after 5 days $(t=5 d)$ 
Compressive strength at time $t f_{c m}(t)=\beta_{c c}(t) \cdot f_{c m} \quad \beta_{c c}(t=5 d)=e^{s(1-\sqrt{28 / t})}=e^{0.2(1-\sqrt{28 / 5})}=0.76$

$$
\begin{aligned}
& f_{c m}(t=5 d)=0.76 \cdot 38=28.88 \mathrm{~N} / \mathrm{mm}^{2} \\
& f_{c k}(t=5 d)=f_{c m}(t)-8=28.88-8=20.88 \mathrm{~N} / \mathrm{mm}^{2} \\
& f_{c d}(t=5 d)=0.85 \cdot f_{c k}(t) / 1.5=0.85 \cdot 20.88 / 1.5=\underline{\underline{11.8 \mathrm{~N} / \mathrm{mm}^{2}}}
\end{aligned}
$$

Required reinforcement

$$
\begin{aligned}
\mu_{E d s}= & \frac{M_{E d s}}{b \cdot d^{2} \cdot f_{c d}}=\frac{28200}{19 \cdot 83.6^{2} \cdot 1.18}=0.18 \quad \rightarrow \omega=0.2007 \\
A_{s 2, \text { req }} & =\left(\omega \cdot b \cdot d \cdot f_{c d}+N_{E d}\right) / \sigma_{s d} \\
& =(0.2007 \cdot 19.0 \cdot 83.6 \cdot 1.18-747) / 43.5=\underline{\underline{-8.5 \mathrm{~cm}^{2}}}<0
\end{aligned}
$$

No additional upper reinforcement is required!

\subsubsection{TILTING}

According to DIN EN 1992-1-1, 5.9 a simplified proof of the safety against tilting can be conducted with following formula:

$$
\begin{aligned}
& b_{\text {req }} \geq \sqrt[4]{\left(\frac{I_{0}}{70}\right)^{3} \cdot h} \quad \text { and } \quad \frac{h}{b} \leq 5.0 \quad \text { for tilting during transport and assembly of the beam. } \\
& b_{\text {req }} \geq \sqrt[4]{\left(\frac{9.0}{70}\right)^{3} \cdot 0.876}=0.21 m<b_{\text {prov }}=0.40 m \text { and } \quad \frac{h}{b}=\frac{87.6}{40}=2.19<5.0
\end{aligned}
$$

This means that the purlin is safe against tilting during transport and assembly and no further analysis is necessary. The simplified proof is sufficient.

\subsubsection{TRANSPORT ANCHORS}

In order to lift the beam out of its formwork as well as to be able to transport and assemble it on site, transport anchors are needed. Those are installed during the production of the beam and must be designed in accordance with the VDI/BV-BS-Richtlinie 6205. For this project the anchors of the company Halfen are chosen.

When choosing the right anchors two different cases must be distinguished. For lifting the beam out of the formwork, a traverse is used which results in only vertical tensile forces on the anchor. For the lifting on site, a suspension gear is used which causes inclined tensile forces.

Lifting out of the Formwork

$\begin{array}{ll}\text { Self-weight } & F_{G}=5.74 \cdot 15=86.1 \mathrm{kN} \\ \text { Formwork adhesion } & F_{\text {adh }}=q_{a d h} \cdot A_{t} \quad \rightarrow \text { no adhesion here because of hinged formwork! } \\ \text { Dynamic factor } & \psi_{d y n}=1.3 \\ \text { Factor for inclination } & z=1.0 \text { for } \beta=90^{\circ}\end{array}$


Tensile force $\quad F_{z}=\frac{F_{G} \cdot \psi_{d y n} \cdot z}{n}=\frac{86.1 \cdot 1.3 \cdot 1.0}{2}=\underline{\underline{56.0 \mathrm{kN}}}$

Lifting on Site

Dynamic factor

Factor for inclination

Tensile force

Chosen

Load capacity of the anchor $\psi_{d y n}=1.3$

and $\quad z=1.16$ for $\beta=30^{\circ}$

$F_{z}=\frac{F_{G} \cdot \psi_{d y n} \cdot z}{n}=\frac{86.1 \cdot 1.3 \cdot 1.16}{2}=\underline{\underline{64.9 \mathrm{kN}}}$

HALFEN DEHA Spherical head anchor 6000-7,5-0300

Table 16 - Sizing Table for 'HALFEN DEHA Lifting Anchor Systems'

\begin{tabular}{|c|c|c|c|c|c|c|c|c|c|}
\hline \multirow{3}{*}{$\begin{array}{l}\text { Load } \\
\text { class }\end{array}$} & \multirow{3}{*}{ Article number } & \multirow{3}{*}{$\begin{array}{l}\text { Anchor } \\
\text { length } \\
\text { । } \\
\text { [mm] }\end{array}$} & \multirow{3}{*}{$\begin{array}{c}\text { Minimum height } \\
\text { of beams } \\
B_{1} \\
{[\mathrm{~mm}]}\end{array}$} & \multirow{3}{*}{$\begin{array}{c}\text { Wall } \\
\text { thickness } \\
2 \times \mathrm{e}_{\mathrm{r}} \\
\text { [mm] }\end{array}$} & \multicolumn{4}{|c|}{ Load capacity $[\mathrm{kN}]$ at concrete strength $\mathrm{f}_{\mathrm{ci}}$ for } & \multirow{3}{*}{$\begin{array}{c}\text { Axial spacing of } \\
\text { anchors } \\
\mathrm{e}_{\mathrm{z}} \\
{[\mathrm{mm}]}\end{array}$} \\
\hline & & & & & $\begin{array}{c}\text { Axial pull } \\
\text { up to } 30^{\circ}[\beta]\end{array}$ & $\begin{array}{l}\text { Diagonal pull } \\
\text { up to } 60^{\circ}[\beta]\end{array}$ & $\begin{array}{l}\text { Axial pull and } \\
\text { diagonal pull } \\
\text { up to } 60^{\circ}[\beta]\end{array}$ & $\begin{array}{l}\text { Axial pull and } \\
\text { diagonal pull } \\
\text { up to } 60^{\circ}[\beta]\end{array}$ & \\
\hline & & & & & $15 \mathrm{~N} / \mathrm{mm}^{2}$ & $15 \mathrm{~N} / \mathrm{mm}^{2}$ & $25 \mathrm{~N} / \mathrm{mm}^{2}$ & $35 \mathrm{~N} / \mathrm{mm}^{2}$ & \\
\hline \multirow{9}{*}{7,5} & \multirow{3}{*}{$6000-7,5-0200$} & \multirow{3}{*}{200} & \multirow{3}{*}{410} & 240 & 45.1 & 36.0 & 58.2 & 68.8 & \multirow{3}{*}{610} \\
\hline & & & & 260 & 47.8 & 38.3 & 61.8 & 73.1 & \\
\hline & & & & 280 & 50.6 & 40.5 & 65.3 & 75.0 & \\
\hline & \multirow{3}{*}{$6000-7,5-0300$} & \multirow{3}{*}{300} & \multirow{3}{*}{610} & 200 & 54.1 & 43.3 & 69.9 & \multirow{3}{*}{75.0} & \multirow{3}{*}{910} \\
\hline & & & & 220 & 58.1 & 46.5 & 750 & & \\
\hline & & & & 240 & 62.2 & 49.7 & 15.0 & & \\
\hline & \multirow{3}{*}{$6000-7,5-0540$} & \multirow{3}{*}{540} & \multirow{3}{*}{1090} & 160 & 63.2 & 58.4 & \multirow{3}{*}{75.0} & \multirow{3}{*}{75.0} & \multirow{3}{*}{1630} \\
\hline & & & & 180 & 71.1 & 63.8 & & & \\
\hline & & & & 200 & 75.0 & 69.1 & & & \\
\hline
\end{tabular}

$\mathrm{f}_{\mathrm{ci}}=$ concrete cube strength at time of lifting

$$
F_{z, \text { Rd }}=69.9 \mathrm{kN}>F_{z, E d}=64.9 \mathrm{kN} \checkmark \quad(\text { see Table 16) }
$$




\subsection{SOFTWARE CALCULATIONS}

\section{Demo Frilo Nemetschek}

\section{Position: P.02 - Prestressed Concrete Purlin}

Spannbettbinder B8 01/2018 (Frilo R-2018-1/P12)
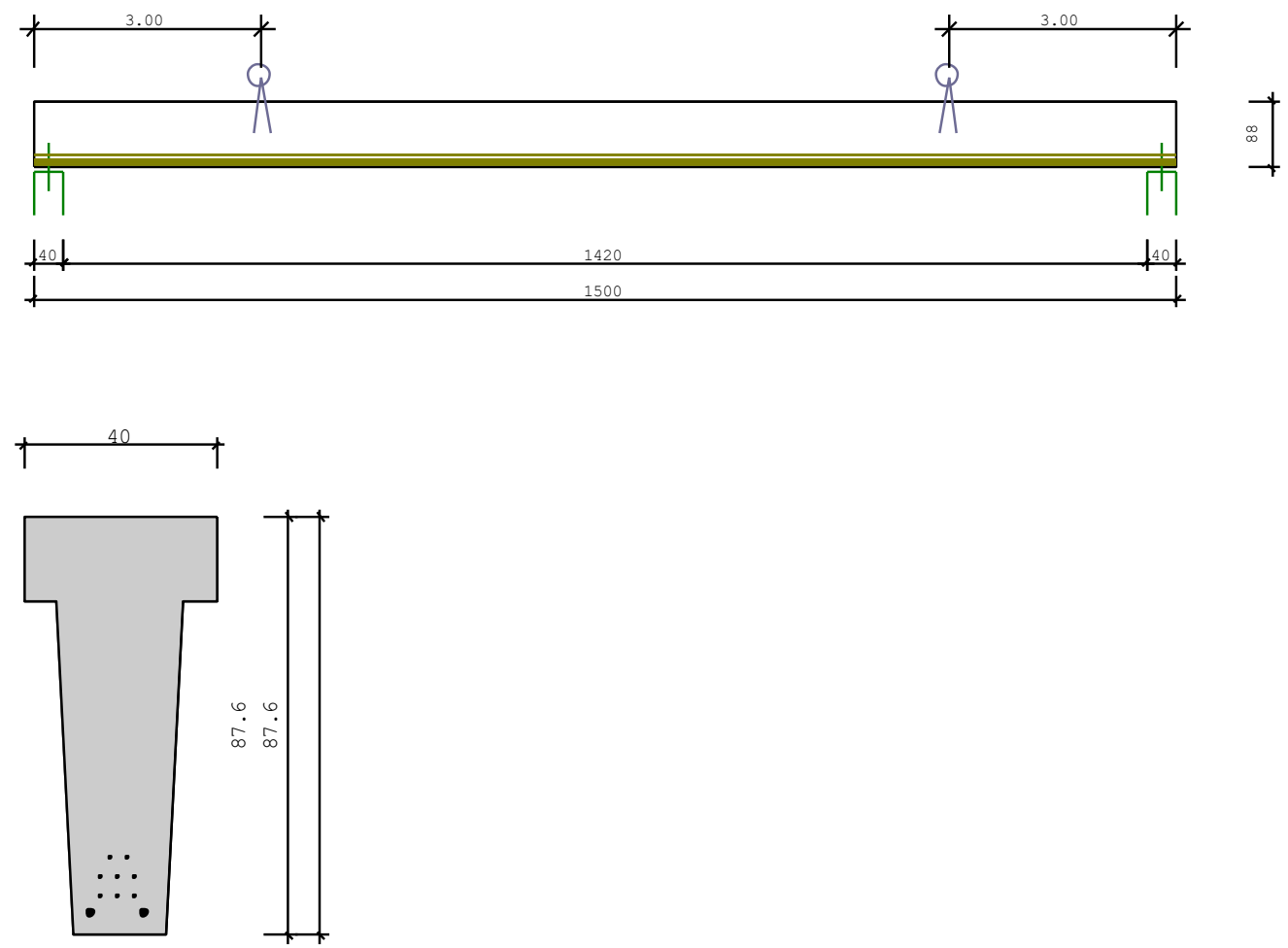

$+19+$

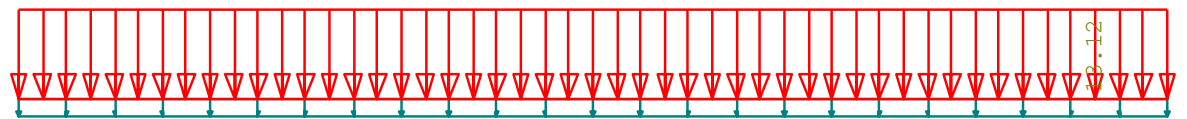

Eigengewicht Fertigteil nicht dargestellt

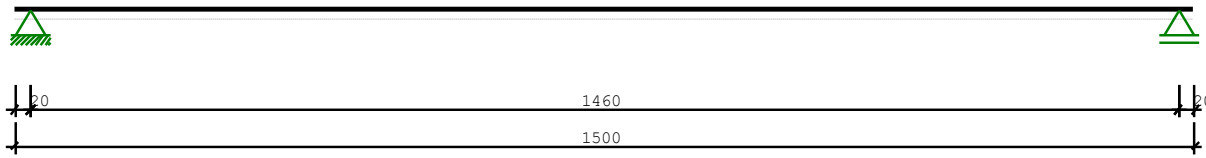

Advices:

No continuous reinforcement to $10 \mathrm{~cm}$ below UE found! 


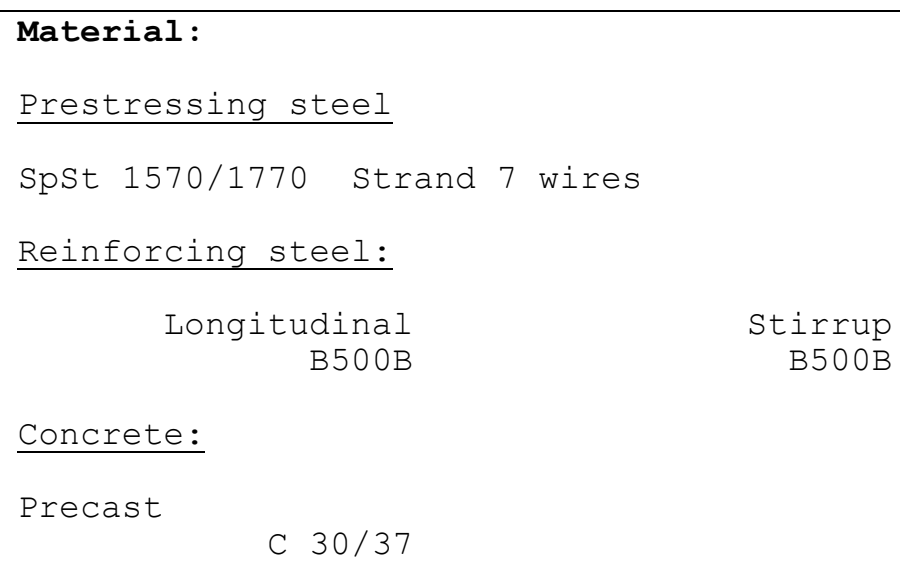

Loads :

Self weight

$\begin{array}{llll}\text { Beam beginning } & \mathrm{g} 11 & =5.70 \mathrm{kN} / \mathrm{m} \\ \text { Beam end } & \mathrm{g} 12 & =5.70 \mathrm{kN} / \mathrm{m} \\ & & & \\ \text { Total } & \mathrm{G} & = & 85.5 \mathrm{kN} \\ \text { Volume } & \mathrm{V} & =3.42 \mathrm{~m}^{3} \\ \text { Surf. } & \mathrm{A} & =31.68 \mathrm{~m}^{2}\end{array}$

Live loads

Units: Single load[kN] Single moment[kNm] line load[kN/m] span type gle qle Dist. a gri qri Length Fact Act. Sim. Pos. $[\mathrm{m}]$

$\begin{array}{rrrrr}1 & 1 & 2.59 & 13.12 & 1.00\end{array}$

Load types: 1 = uniformly distr., 2 = single load at a, 3 = single moment at a

4 = trapezoidal load from a, 5 = triangle load over L

Actions:

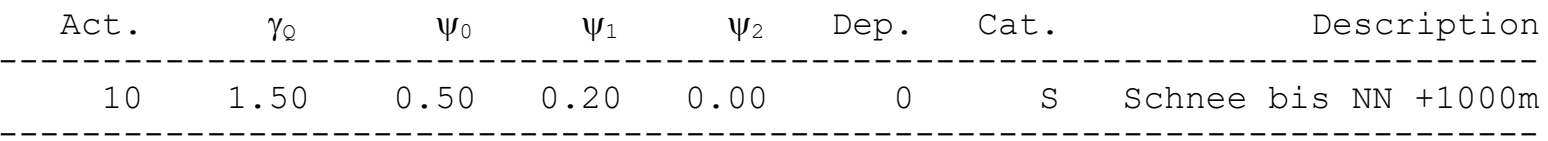

Tendons:

Dist (LE) $>4.8 \mathrm{~cm}$ axis horizontal $>4.3 \mathrm{~cm}$ vertical $>3.7 \mathrm{~cm}$

lay. num- area Dist.LE Prestressing <--- Isolations --->

No. ber Ap Yp $\quad \sigma_{p}(0)$ Count to $x 1$ from $x 2$

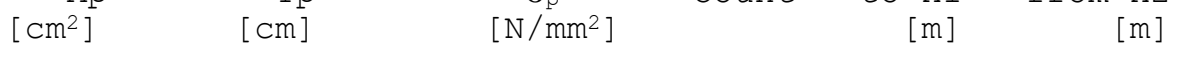

-------------------------------------------------------------------------

\begin{tabular}{|c|c|c|c|c|}
\hline 1 & 3 & 2.79 & 7.5 & 1000 \\
\hline 2 & 3 & 2.79 & 11.7 & 1000 \\
\hline 3 & 2 & 1.86 & 15.9 & 1000 \\
\hline
\end{tabular}


$\mathrm{x} 1$ and $\mathrm{x} 2$ with respect to the left beginning from joint

The calculation of the losses due to creep, shrinkage and relaxation

following the method from Abelein

\section{RESULTS ( summary)}

Reaction forces $(t=$ infinitely):

Units: all [kN] G:perm., Q:variable., V: Sum

\begin{tabular}{|c|c|c|c|c|c|}
\hline Support point & G & $\min Q$ & $\begin{array}{c}\text { value- } \\
\max Q\end{array}$ & $\begin{array}{l}<--U L \\
\min V\end{array}$ & $\max V$ \\
\hline $\begin{array}{l}---------1 \\
\text { A }(l e f t)\end{array}$ & 61.66 & 0.00 & 95.78 & 61.66 & 226.90 \\
\hline B (right) & 61.66 & 0.00 & 95.78 & 61.66 & 226.90 \\
\hline
\end{tabular}

max. bending moment in erection state(char. value):

$$
\mathrm{MF}==570.36 \mathrm{kNm} \text { at } \mathrm{x}=7.50 \mathrm{~m}
$$

Required shear reinforcement:

Column A: asw $=2.73 \mathrm{~cm}^{2} / \mathrm{m}$

Column B: asw $=2.73 \mathrm{~cm}^{2} / \mathrm{m}$

Bursting reinforcement

$\begin{array}{rlrl}\text { left Laying length } & =0.95 \mathrm{~m} & \\ & & 0.00 \mathrm{~m} & \text { As } \\ \text { from } & = & 0.95 \mathrm{~m} & \end{array}$

Check of anchorage

left: Tensile force resistance in anchoring area util = 1.02 additional reinforcement necessary

right: Tensile force resistance in anchoring area Util = 1.02 additional reinforcement necessary

Overview crit. sections

Selected basic grid: 20 Sections

\begin{tabular}{|c|c|c|c|c|}
\hline Checkvalue & & Extrem & Utilisation & $\mathrm{x} \quad[\mathrm{m}]$ \\
\hline Flexural capacity bottom & $\eta=$ & 1.02 & 0.98 & 7.50 \\
\hline Flexural capacity top & $\eta=$ & 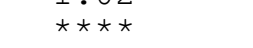 & 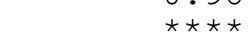 & 0.59 \\
\hline Resisting tens force top & $\eta=$ & $\star \star \star \star$ & $\star \star \star \star ~$ & 0.59 \\
\hline Prc.:Compr.stress $\quad \mathrm{CC}$ & $\sigma c=$ & $-17.20 \mathrm{~N} / \mathrm{mm}^{2}$ & 0.96 & 3.00 \\
\hline Tension prestress. steel & $\sigma \mathrm{p}, \mathrm{QC}=$ & $963.2 \mathrm{~N} / \mathrm{mm}^{2}$ & 0.84 & 7.50 \\
\hline Stress in prestress.steel & $\sigma \mathrm{p}, \mathrm{Cc}_{\mathrm{c}}=$ & $1110.9 \mathrm{~N} / \mathrm{mm}^{2}$ & 0.79 & 7.50 \\
\hline Stress in rebars & $\sigma s=$ & $156.8 \mathrm{~N} / \mathrm{mm}^{2}$ & 0.39 & 7.50 \\
\hline Crack MinAs+AsDuc bottom & AsMin $=$ & 2.4 & 0.37 & 0.02 \\
\hline Crack MinAs+AsDuc top & AsMin $=$ & $\star \star \star \star \star \mathrm{cm}^{2}$ & 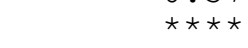 & 0.02 \\
\hline Crack width bottom & $\mathrm{wk}=$ & $0.00 \mathrm{~mm}$ & 0.01 & 7.50 \\
\hline Crack width & $\mathrm{wk}=$ & $\star \star \star \star \mathrm{mm}$ & $\star \star \star \star ~$ & 0.47 \\
\hline Sagging & fo $=$ & $-1.0 \mathrm{~cm}$ & 0.17 & 7.11 \\
\hline Sagging & $\mathrm{fu}=$ & $0.1 \mathrm{~cm}$ & 0.01 & 0.00 \\
\hline
\end{tabular}




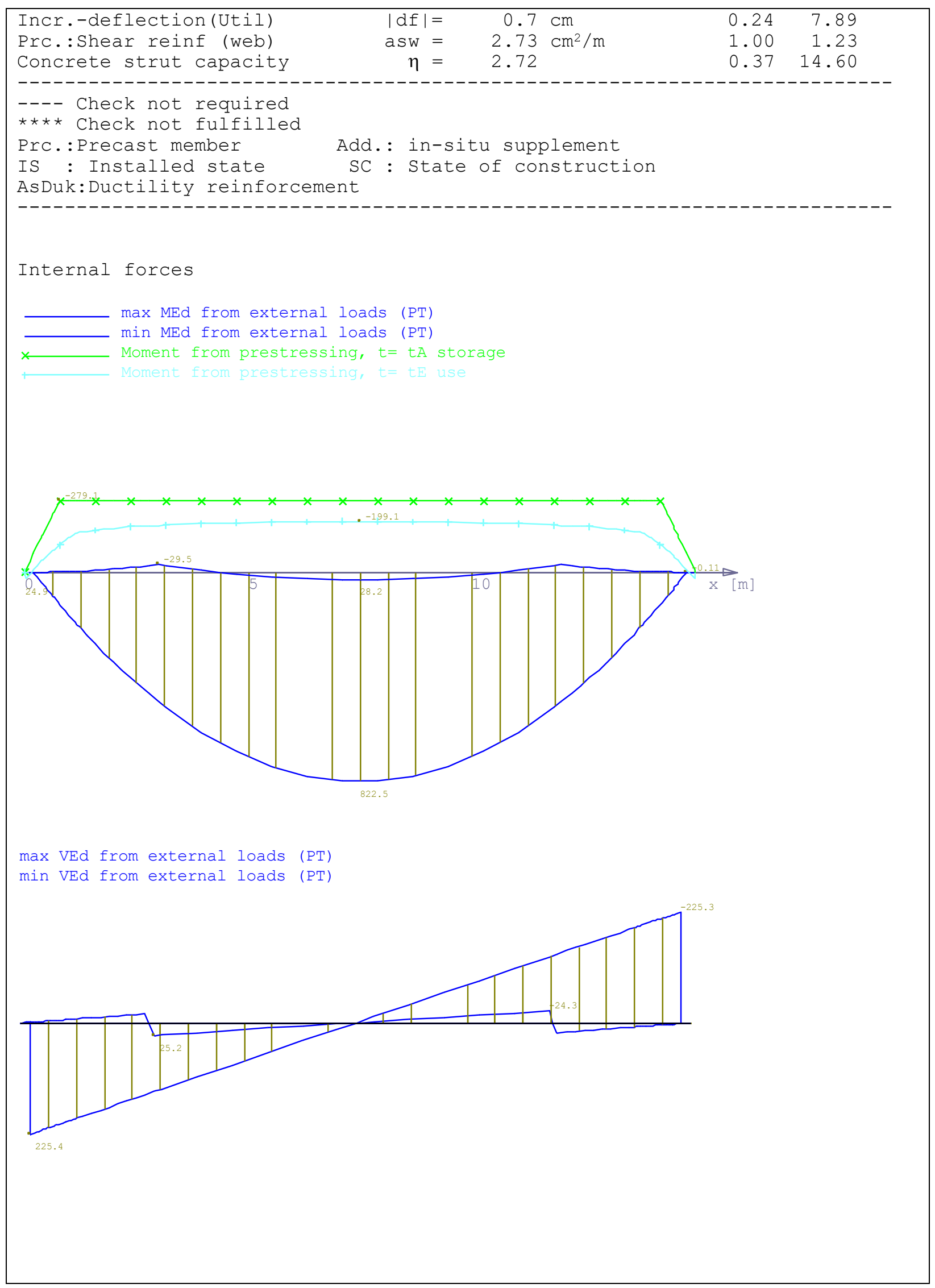




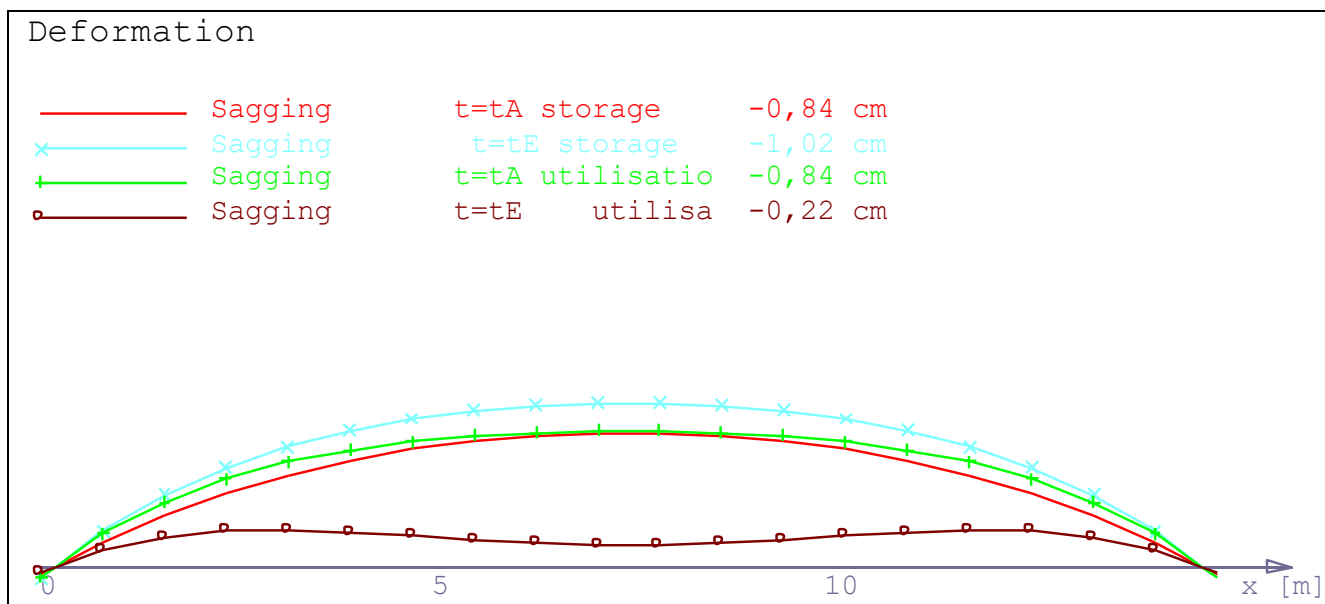




\section{POS. P.03 - PRESTRESSED CONCRETE TRUSS}

In the following the pre-stressed concrete truss will be designed. To ensure a roof drainage a tapered beam with straight lower chord is chosen with a slope of $3.1^{\circ}$. The design will be made according to following formwork layout.

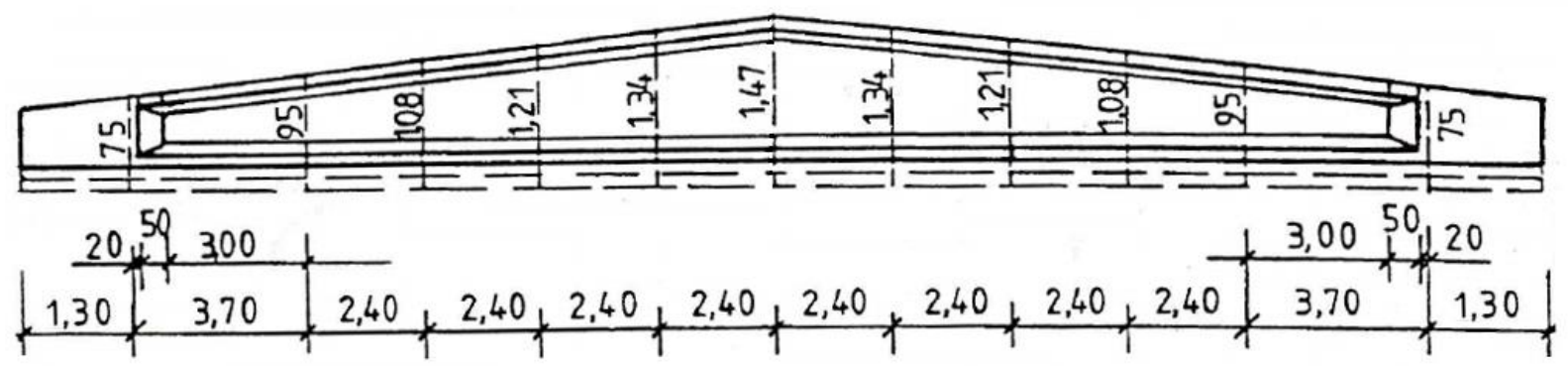

The respective cross section is shown in the drawing.

The height of the lower chord as well as the height of the web can be adjusted according to the static analysis and the constructional needs. Though the maximum height of the web cannot exceed $1.13 \mathrm{~m}$.

Each truss will span between two columns that are arranged at the intersections of the numerical and alphabetical axes of the building resulting in a span length of approximately $29.2 \mathrm{~m}$. This means that in each numerical axis in total three pre-stressed concrete trusses are positioned.

To be able to approximate the needed dimensions of the truss and the amount of pre-stressing steel a preliminary design must be made.

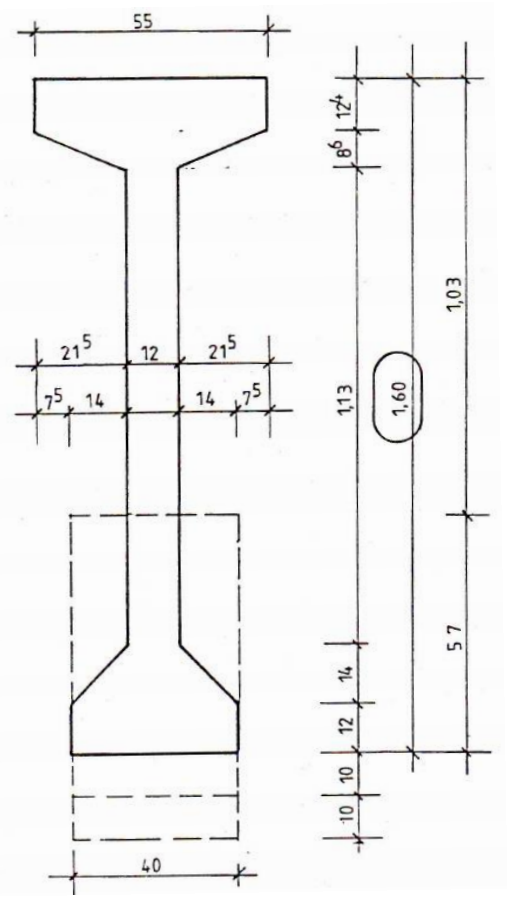




\subsection{PRELIMINARY DESIGN}

System

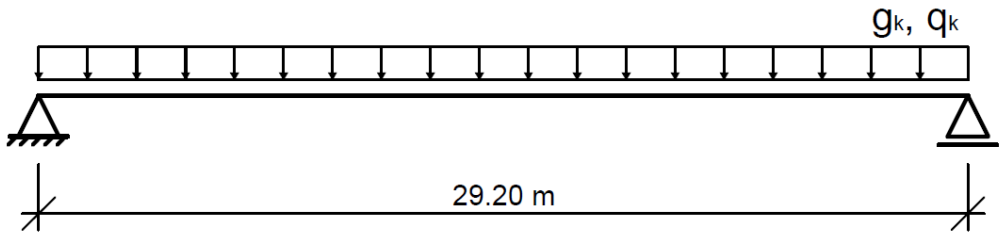

Span $\mathrm{L}=29.2 \mathrm{~m}$, Distance $\mathrm{a}=15 \mathrm{~m}$

Cross-section

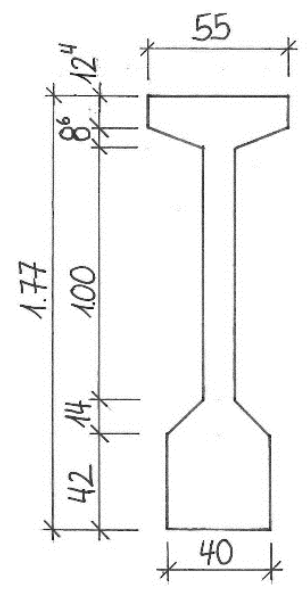

Building materials

C $45 / 55$ | St 1570/1770 | B 500A

Exposure class

XC1, WO

Characteristic loads

\begin{tabular}{|c|c|c|c|}
\hline Uniformly Distri & d Loads & $\begin{array}{c}\mathbf{g}_{\mathrm{k}} \\
{[\mathrm{kN} / \mathrm{m}]}\end{array}$ & $\begin{array}{c}\mathbf{q}_{k} \\
{[\mathrm{kN} / \mathrm{m}]}\end{array}$ \\
\hline Self-weight & & & \\
\hline Max. self-weight & $\mathrm{g}_{\mathrm{k} 1, \max }=0.42 \mathrm{~cm}^{2} \times 25 \mathrm{kN} / \mathrm{m}^{3}=$ & 10.50 & - \\
\hline Min. self-weight & $\mathrm{g}_{\mathrm{k} 1, \min }=0.33 \mathrm{~cm}^{2} \times 25 \mathrm{kN} / \mathrm{m}^{3}=$ & 8.25 & - \\
\hline & $g_{k, \text { mean }}=(10.50+8.25) / 2=$ & 9.40 & \\
\hline Purlin+Roof Strt & & & \\
\hline$g_{\mathrm{k} 2}=(4 \times 8.69+$ & .69) $\mathrm{kN} / \mathrm{m} \times 14.70 \mathrm{~m} / 29.20 \mathrm{~m}=$ & 31.00 & - \\
\hline Snow & & & \\
\hline$q_{\mathrm{k} 1}=(4 \times 12.25+$ & 4.46) $\mathrm{kN} / \mathrm{m} \times 14.7 \mathrm{~m} / 29.20 \mathrm{~m}=$ & - & 34.20 \\
\hline Wind (Area I) & & & \\
\hline$q_{\mathrm{k} 2}=(4 \times 0.57+$ & .21) $\mathrm{kN} / \mathrm{m} \times 14.7 \mathrm{~m} / 29.20$ & - & 1.60 \\
\hline
\end{tabular}


Design location

Bending moments

$$
\begin{aligned}
& a=d_{a} / \tan \alpha=0.98 / \tan \left(3.1^{\circ}\right)=18.08 m \\
& x=-a+\sqrt{a^{2}+a \cdot L}=-18.08+\sqrt{18.08^{2}+18.08 \cdot 29.2}=11.16 m
\end{aligned}
$$

At the design location $x=11.16 \mathrm{~m}$ :

\begin{tabular}{|c|c|c|c|}
\hline $\mathbf{M}_{\mathbf{g k 1}}[\mathbf{k N m}]$ & $\mathbf{M}_{\mathbf{G k 2}}[\mathbf{k N m}]$ & $\mathbf{M}_{\mathbf{Q k} 1}[\mathbf{k N m}]$ & $\mathbf{M}_{\mathbf{Q k 2}}[\mathbf{k N m}]$ \\
\hline 946.2 & 3120.0 & 3442.4 & 161.1 \\
\hline
\end{tabular}

Assumptions

Prestressing

$\sigma_{m, t=0} \approx 1000 \mathrm{~N} / \mathrm{mm}^{2}$

Prestressing loss

$\approx 15 \%$

Prestressing with immediate bond

Cross-section Properties

At $x=11.16 m$

$h_{x=11.16}=158 \mathrm{~cm}$

Center of gravity

$z_{\text {cu }}=73 \mathrm{~cm}$

$A_{c}=3986.1 \mathrm{~cm}^{2}$

$I_{y c}=11,720,979 \mathrm{~cm}^{4}$

$W_{c u}=\frac{l_{y c}}{Z_{c u}}=\frac{11,720,979 \mathrm{~cm}^{4}}{73 \mathrm{~cm}}=160,577 \mathrm{~cm}^{3}$

Bending Compression Zone

Effective depth

$d=158 \mathrm{~cm}-15 \mathrm{~cm}=143 \mathrm{~cm}$

$\delta=h_{f} / d=16.7 / 143=0.1168$

$\beta=b_{w} / b_{\text {eff }}=12 \mathrm{~cm} / 55 \mathrm{~cm}=0.218$

$\rightarrow \eta=\frac{1}{(1-\beta) \cdot(1-0.5 \delta) \cdot \delta+0.32 \beta}=\underline{\underline{6.42}}$

$M_{E d}^{G+Q}=1.35 \cdot(946.2+3120.3)+1.50 \cdot 3442.4+1.5 \cdot 0.6 \cdot 161.1=10,940 \mathrm{kNm}$

$b_{\text {eff,req }} \geq \frac{M_{E d}}{f_{c d}} \cdot \eta / d^{2}=\frac{10.94 M N m}{25.5 M N / m^{2}} \cdot 6.42 / 1.43^{2}=1.35 m$

$b_{\text {eff,prov }}=55 \mathrm{~cm}<b_{\text {eff,req }}=135 \mathrm{~cm}$ 々

$\rightarrow$ The provided bending compression zone is NOT sufficient.

A different cross-section has to be chosen or the compression zone must be increased by increasing the height or the length of the compression flange. 


\subsubsection{CONCRETE TRUSS ALTERNATIVE 1}

To strengthen the compression zone, the height of compression flange can be increased. Additional $30 \mathrm{~cm}$ of height will be added to the compression flange and $20 \mathrm{~cm}$ will be added to the tension zone. So, the maximum height of the truss is $227 \mathrm{~cm}$.

Cross-section

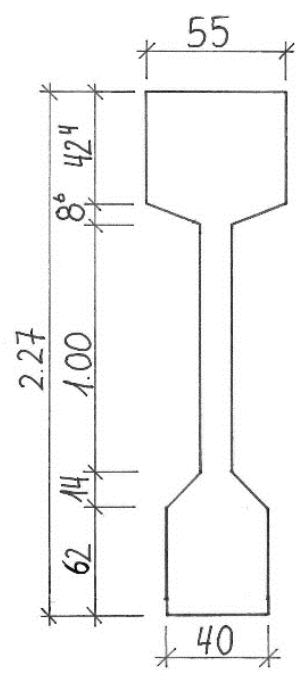

Building materials

Exposure class

Characteristic loads

Design location

Bending moment

\section{45/55 | St 1570/1770 | B 500A}

XC1, WO

$\Delta \mathrm{g}_{\mathrm{k}} \approx(0.40 \times 0.20+0.55 \times 0.30) \times 25 \mathrm{kN} / \mathrm{m}^{3}=6.10 \mathrm{kN} / \mathrm{m}$

$\mathrm{gk}_{1, \text { new }}=9.40+6.10=15.5 \mathrm{kN} / \mathrm{m}$

$a=d_{a} / \tan \alpha=1.48 / \tan \left(3.1^{\circ}\right)=27.31 m$

$x=-a+\sqrt{a^{2}+a \cdot L}=11.97 m$

At the design location $x=11.97 \mathrm{~m}$ :

\begin{tabular}{|c|c|c|c|}
\hline $\mathbf{M}_{\mathbf{g k 1} 1}[\mathbf{k N m}]$ & $\mathbf{M}_{\mathbf{G k 2}}[\mathbf{k N m}]$ & $\mathbf{M}_{\mathbf{Q k} 1}[\mathbf{k N m}]$ & $\mathbf{M}_{\mathbf{Q k 2} 2}[\mathbf{k N m}]$ \\
\hline 1598.6 & 3197.2 & 3527.2 & 165.0 \\
\hline
\end{tabular}

Cross-section Properties

At $x=11.97 m$

Center of gravity

$$
\begin{aligned}
& h_{x=11.97}=213 \mathrm{~cm} \\
& Z_{c u}=113 \mathrm{~cm} \\
& A_{c}=6218 \mathrm{~cm}^{2} \quad I_{y c}=33,899,542 \mathrm{~cm}^{4} \\
& W_{c u}=\frac{I_{y c}}{Z_{c u}}=\frac{33,899,542 \mathrm{~cm}^{4}}{113 \mathrm{~cm}}=272,902 \mathrm{~cm}^{3}
\end{aligned}
$$


Bending Compression Zone

\begin{tabular}{|c|c|}
\hline Effective depth & $d=213 \mathrm{~cm}-15 \mathrm{~cm}=198 \mathrm{~cm}$ \\
\hline & $\delta=h_{f} / d=46.2 / 198=0.2361$ \\
\hline & $\beta=b_{w} / b_{\text {eff }}=12 \mathrm{~cm} / 55 \mathrm{~cm}=0.218$ \\
\hline & $\rightarrow \eta=\frac{1}{(1-\beta) \cdot(1-0.5 \delta) \cdot \delta+0.32 \beta}=\underline{\underline{4.30}}$ \\
\hline ULS & $M_{E d}^{G+Q}=1.35 \cdot M_{G}+1.50 \cdot M_{Q 1}+1.5 \cdot 0.6 \cdot M_{Q 2}=12,154 \mathrm{kNm}$ \\
\hline & $b_{\text {eff }, \text { req }} \geq \frac{M_{E d}}{f_{c d}} \cdot \eta / d^{2}=\frac{12.154 \mathrm{MNm}}{25.5 \mathrm{MN} / \mathrm{m}^{2}} \cdot 4.30 / 1.98^{2}=0.523 \mathrm{~m}$ \\
\hline & $b_{\text {eff }, \text { prov }}=55 \mathrm{~cm}>b_{\text {eff,req }}=52.3 \mathrm{~cm} \checkmark$ \\
\hline & $\rightarrow$ The provided bending compression zone is sufficient. \\
\hline
\end{tabular}

Required Prestressing Steel

SLS

$M_{\text {Ed,frequ }}=M_{G}+0.2 \cdot M_{Q 1}+0 \cdot M_{Q 2}=5501.2 \mathrm{kNm}$

$r_{\text {inf }}=0.95, \alpha_{\text {loss }}=0.15, \sigma_{p m, t=0}=1000 \mathrm{~N} / \mathrm{mm}^{2}$

$z_{c p}=113 \mathrm{~cm}-15 \mathrm{~cm}=98 \mathrm{~cm}$

Required prestressing steel

$$
\begin{aligned}
A_{P, \text { req }} & \geq \frac{M_{E d}}{r_{\text {inf }} \cdot\left(1-\alpha_{\text {loss }}\right) \cdot \sigma_{p m, t=0} \cdot\left(\frac{W_{c u}}{A_{c}}+z_{c p}\right)} \\
& =\frac{550,120 \mathrm{kNcm}}{0.95 \cdot(1-0.15) \cdot 100 \mathrm{kN} / \mathrm{cm}^{2} \cdot\left(\frac{300,176}{6218}+98.0\right)}=\underline{\underline{46.60 \mathrm{~cm}^{2}}}
\end{aligned}
$$

$\rightarrow$ The required cross-section of the pre-stressing steel is $46.60 \mathrm{~cm}^{2}$.

Tension Zone

$\begin{aligned} S L S \quad & \min M_{G k}=1599 \mathrm{kNm} \quad M_{E d, \text { frequ }}=5501.2 \mathrm{kNm} \\ & W_{c u, \text { req }} \geq \frac{\frac{M_{E d}}{r_{\text {inf }} \cdot\left(1-\alpha_{\text {loss }}\right)}-\min M_{G k}}{0.6 \cdot f_{c k}}=\frac{\frac{550,120}{0.95 \cdot(1-0.15)}-159,900}{0.6 \cdot 4.5}=193,113 \mathrm{~cm}^{3} \\ & W_{c u, \text { prov }}=193,113 \mathrm{~cm}^{3}>W_{c u, \text { req }}=300,176 \mathrm{~cm}^{3} \checkmark \\ & \rightarrow \text { The tension zone of the purlin is sufficient. }\end{aligned}$




\subsubsection{CONCRETE TRUSS ALTERNATIVE 2}

Another option is to use a higher truss with a wider compression flange.

Cross-section

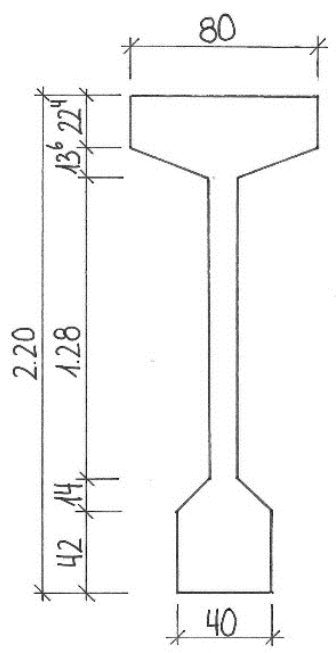

Building materials

Design location

Cross-section Properties

At $x=11.89 m$ :

Center of gravity

$h_{x=11.89}=205 \mathrm{~cm}$

$z_{c u}=114.1 \mathrm{~cm}$

$A_{c}=5818 \mathrm{~cm}^{2}$

$I_{y c}=31,841,471 \mathrm{~cm}^{4}$

$W_{c u}=\frac{I_{y c}}{z_{c u}}=\frac{31,841,471 \mathrm{~cm}^{4}}{114.1 \mathrm{~cm}}=279,176 \mathrm{~cm}^{3}$

Bending Compression Zone

$b_{\text {eff, req }}=0.689 \mathrm{~m}<b_{\text {eff,prov }}=80 \mathrm{~cm} \checkmark$

$\rightarrow$ The provided bending compression zone is sufficient.

Required Prestressing Steel

$A_{P, \text { req }}=\underline{\underline{45.94 \mathrm{~cm}^{2}}}$

$\rightarrow$ The required cross-section of the pre-stressing steel is $45,94 \mathrm{~cm}^{2}$.

Tension Zone

$W_{\text {cu,prov }}=279,177 \mathrm{~cm}^{3}>W_{\text {cu,req }}=172,009 \mathrm{~cm}^{3} \checkmark$

$\rightarrow$ The tension zone of the purlin is sufficient. 
Alternative 2 has a smaller cross-section than alternative 1 that is why it is chosen here.

Chosen

C 50/60 | "T-Profile": $h=220 \mathrm{~mm}, b_{o}=800 \mathrm{~mm}, b_{u}=400 \mathrm{~mm}$ St $1570 / 1770 \mid 50 \varnothing_{P}=12.5 \mathrm{~mm}=50 \times 0.934 \mathrm{~cm}^{2}=46.70 \mathrm{~cm}^{2}$

\subsection{SYSTEM}

System

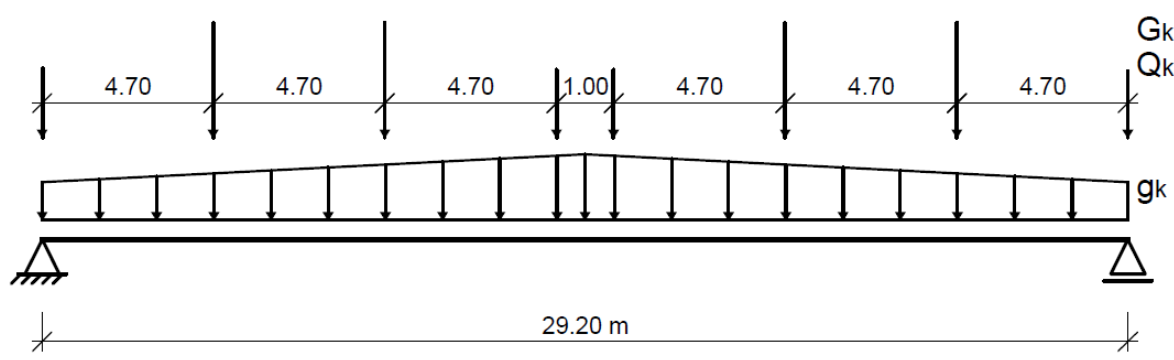

Cross-section

Building materials

Exposure class

Reinforcing steel

Prestressing steel
See Chapter 7.1.2

\section{50/60 | St 1570/1770 | B 500A}

XC1, WO

$$
\begin{aligned}
& \mathrm{C}_{\text {nom }, \mathrm{w}}=\mathrm{c}_{\text {min }}+\Delta \mathrm{c}_{\text {dev }}=10 \mathrm{~mm}+10 \mathrm{~mm}=20 \mathrm{~mm} \\
& \mathrm{C}_{\text {nom }, \mathrm{s}}=\mathrm{c}_{\text {min }}+\Delta \mathrm{c}_{\text {dev }}=20 \mathrm{~mm}+10 \mathrm{~mm}=30 \mathrm{~mm} \rightarrow \mathrm{c}_{\mathrm{v}, \mathrm{w}, \text { min }}=20 \mathrm{~mm} \\
& \text { Chosen } \quad \mathrm{c}_{\mathrm{v}}=20 \mathrm{~mm}
\end{aligned}
$$

$$
A_{s}=4 \varnothing_{s} 16=8.04 \mathrm{~cm}^{2} \quad a_{u}=4.0 \mathrm{~cm}
$$

$\mathrm{C}_{\mathrm{nom}}=2.5 \times 12.5 \mathrm{~mm}+10 \mathrm{~mm}=42 \mathrm{~mm}$
1. Layer:
$A_{p}=8 \varnothing_{p} 12.5$
$a_{p}=7.5 \mathrm{~cm}$
2. Layer:
$A_{p}=8 \varnothing_{p} 12.5$
$a_{p}=11.7 \mathrm{~cm}$
3. Layer:
$A_{p}=8 \varnothing_{p} 12.5$
$a_{p}=15.9 \mathrm{~cm}$
4. Layer:
$A_{p}=8 \varnothing_{p} 12.5$
$a_{p}=20.1 \mathrm{~cm}$
5. Layer:
$A_{p}=8 \varnothing_{p} 12.5$
$a_{p}=24.3 \mathrm{~cm}$
6. Layer:
$A_{p}=8 \varnothing_{p} 12.5$
$a_{p}=28.5 \mathrm{~cm}$
7. Layer:
$A_{p}=2 \varnothing_{p} 12.5$
$a_{p}=32.7 \mathrm{~cm}$
$\Sigma$
$A_{p}=50 \varnothing_{p} 12.5=46.7 \mathrm{~cm}^{2}$ 
Characteristic loads

\begin{tabular}{|c|c|c|c|}
\hline \multicolumn{2}{|l|}{ Uniformly Distributed Loads } & $\begin{array}{c}\mathbf{g}_{k} \\
{[\mathrm{kN} / \mathrm{m}]}\end{array}$ & $\begin{array}{c}\mathbf{q}_{k} \\
{[k N / m]}\end{array}$ \\
\hline \multicolumn{3}{|l|}{ Self-weight } & \\
\hline \multicolumn{2}{|c|}{$\mathrm{g}_{\mathrm{k} 1, \max }=0.60 \mathrm{~cm}^{2} \times 25 \mathrm{kN} / \mathrm{m}^{3}=$} & 15.0 & \\
\hline \multicolumn{2}{|c|}{$\mathrm{g}_{\mathrm{k} 1, \min }=0.56 \mathrm{~cm}^{2} \times 25 \mathrm{kN} / \mathrm{m}^{3}=$} & 14.0 & - \\
\hline \multicolumn{2}{|l|}{ Concentrated Loads } & $\begin{array}{c}\mathbf{G}_{\mathbf{k}} \\
{[\mathbf{k N}]}\end{array}$ & $\begin{array}{c}\mathbf{Q}_{\mathbf{k}} \\
{[\mathrm{kN}]}\end{array}$ \\
\hline \multicolumn{2}{|l|}{ Purlin - Reaction Force from P.02 } & 83.8 & - \\
\hline \multirow{2}{*}{ Roof Structure - Reaction Force from P.02 } & $\operatorname{Min}=$ & 5.0 & - \\
\hline & $\operatorname{Max}=$ & 37.8 & - \\
\hline \multirow[t]{2}{*}{ Snow - Reaction Force from P.02 } & $\operatorname{Min}=$ & - & 65.6 \\
\hline & $\operatorname{Max}=$ & - & 183.7 \\
\hline \multirow[t]{2}{*}{ Wind - Reaction Force from P.02 } & $\operatorname{Min}=$ & - & 3.1 \\
\hline & $\operatorname{Max}=$ & - & 8.3 \\
\hline
\end{tabular}

\subsection{INTERNAL FORCES}

Characteristic

(at $x=11.89)$

\begin{tabular}{|c|c|c|c|}
\hline $\mathbf{M}_{\mathbf{g k} 1}[\mathbf{k N m}]$ & $\mathbf{M}_{\mathbf{g k} 2}[\mathbf{k N m}]$ & $\mathbf{M}_{\mathbf{q k} 1}[\mathbf{k N m}]$ & $\mathbf{M}_{\mathbf{q k} 2}[\mathbf{k N m}]$ \\
\hline 1508.0 & 2790.0 & 3393.0 & 155.3 \\
\hline
\end{tabular}

\begin{tabular}{|c|c|c|c|}
\hline $\mathbf{V}_{\mathbf{g k} \mathbf{1}}[\mathbf{k N}]$ & $\mathbf{V}_{\mathbf{g k} \mathbf{2}}[\mathbf{k N}]$ & $\mathbf{V}_{\mathbf{q k} \mathbf{1}}[\mathbf{k N}]$ & $\mathbf{V}_{\mathbf{q k} \mathbf{2}}[\mathbf{k N}]$ \\
\hline 211.7 & 423.8 & 502.0 & 23.0 \\
\hline
\end{tabular}

Design

\begin{tabular}{|c|c|c|c|}
\hline ULS & \multicolumn{3}{|c|}{ SLS } \\
\hline $\mathrm{M}_{\mathrm{Ed}}[\mathrm{kNm}]$ & $M_{\text {rare }}[\mathrm{kNm}]$ & $M_{\text {frequ }}[\mathrm{kNm}]$ & $\mathrm{M}_{\text {perm }}[\mathrm{kNm}]$ \\
\hline $11,031.6$ & $7,784.2$ & $4,976.6$ & $4,298.0$ \\
\hline ULS & \multicolumn{3}{|c|}{ SLS } \\
\hline $\mathrm{V}_{\mathrm{Ed}}[\mathrm{kN}]$ & $\mathrm{V}_{\text {rare }}[\mathrm{kN}]$ & $\mathrm{V}_{\text {frequ }}[\mathrm{kN}]$ & $\mathbf{V}_{\text {perm }}[\mathbf{k N}]$ \\
\hline $1,631.6$ & 1151.3 & 735.9 & 635.5 \\
\hline
\end{tabular}

\subsection{PRESTRESSING LOSS}
Maximum prestressing force
(1) $P_{m 0, \max }=0.75 \cdot A_{p} \cdot f_{p k}=0.75 \cdot 50 \cdot 0.934 \cdot 177 \mathrm{kN} / \mathrm{cm}^{2}=6199 \mathrm{kN}$
(2) $P_{\text {mo,max }}=0.85 \cdot A_{p} \cdot f_{p 0,1 \mathrm{k}}=0.85 \cdot 50 \cdot 0.934 \cdot 150 \mathrm{kN} / \mathrm{cm}^{2}=\underline{\underline{5954 \mathrm{kN}}}$
$\rightarrow P_{m, t=0}=4670 k N<P_{m 0, \max }=5954 k N \checkmark$ 
Effective thickness $\quad h_{0}=\frac{2 \cdot A_{c}}{U}=\frac{2 \cdot 5818}{588}=19.8 \mathrm{~cm}$

Drying shrinkage $\quad \varepsilon_{c d}(\infty)=k_{h} \cdot \varepsilon_{c d, 0}=0.853 \cdot 0.54 \%=0.461 \%$

Autogenous shrinkage: $\quad \varepsilon_{c a}(\infty)=2.5 \cdot\left(f_{c k}-10\right) \cdot 10^{-6}=2.5 \cdot(50-10) \cdot 10^{-6}=0.100 \%$ o

Total shrinkage

$\varepsilon_{c s}(\infty)=\varepsilon_{c d}(\infty)+\varepsilon_{c a}(\infty)=0.461 \% 0+0.100 \%=0.561 \%$ o

Final creep ratio $\quad$ for to $=5$ day: $\quad \varphi\left(\infty, t_{0}\right)=1.89$

Prestressing loss due to relaxation

$$
\begin{aligned}
& \sigma_{p, p e r m}=\frac{E_{p}}{E_{c m}} \cdot\left[\frac{M_{E d, p e r m}}{I_{c}} \cdot z_{p}\right]=\frac{196}{33} \cdot\left[\frac{4.298}{0.31,841,471} \cdot 0.955\right]=68.3 \mathrm{MN} / \mathrm{m}^{2} \\
& N_{p, t=0}=-P_{0, t=0}=-4670 \mathrm{kN}, \\
& M_{p, t=0}=N_{p, t=0} \cdot z_{p}=-4670 \cdot 0.955=-4458 \mathrm{kNm} \\
& \sigma_{p, t=0}=\frac{P_{0, t=0}}{A_{p}}+\frac{E_{p}}{E_{c m}} \cdot\left[\frac{N_{p, t=0}}{A_{c}}+\frac{M_{p, t=0}}{I_{c}} \cdot z_{p}\right]=886.7 \mathrm{MN} / \mathrm{m}^{2} \\
& R_{i} / R_{m}=\frac{\sigma_{p, p e r m}+\sigma_{p, t=0}}{f_{p k}}=\frac{68.3+886.7}{1770}=0.5395 \quad \rightarrow \kappa_{p}=1.158 \% \\
& \Delta \sigma_{p r}=\kappa \cdot\left(\sigma_{p, p e r m}+\sigma_{p, t=0}\right)=0.01158 \cdot(68.3+886.7)=11.1 \mathrm{MN} / \mathrm{m}^{2}
\end{aligned}
$$

Total Prestressing Loss

Shrinkage

Relaxation

Creep

$$
\begin{aligned}
& \sigma_{c p, p e r m}= \frac{M_{E d, p e r m}}{I_{c}} \cdot z_{p}=\frac{429800}{31,841,471} \cdot 95.5=12.9 \mathrm{MN} / \mathrm{m}^{2} \\
& \sigma_{c p, t=0}=\frac{N_{p, t=0}}{A_{c}}+\frac{M_{p, t=0}}{I_{c}} \cdot z_{p}=\frac{-4760}{5818}+\frac{-429800}{31,841,471} \cdot 95.5=-21.4 \mathrm{MN} / \mathrm{m}^{2} \\
& \alpha_{p}=\frac{E_{p}}{E_{c m}}=5.30 \\
& \begin{aligned}
\Delta \sigma_{p, c+S+r}= & \frac{\varepsilon_{c s}(t) \cdot E_{p}+0.8 \cdot \Delta \sigma_{p r}+\alpha_{p} \cdot \varphi\left(\infty, t_{0}\right) \cdot\left(\sigma_{c p, p e r m}+\sigma_{c p, t=0}\right)}{A_{p}} \cdot\left(1+\frac{A_{c}}{I_{c}} \cdot z_{p}^{2}\right) \cdot\left[1+0.8 \cdot \varphi\left(\infty, t_{0}\right)\right] \\
& =-85.5-6.9-66.3=-158.7 \mathrm{MN} / \mathrm{m}^{2}
\end{aligned}
\end{aligned}
$$

$-85.5 \mathrm{MN} / \mathrm{m}^{2} \rightarrow 53.9 \%$

- $6.9 \mathrm{MN} / \mathrm{m}^{2} \rightarrow 4.3 \%$

- $66.3 \mathrm{MN} / \mathrm{m}^{2} \rightarrow 41.8 \%$

$$
\Delta P_{c+s+r}=\Delta \sigma_{p, c+s+r} \cdot A_{p}=-158.7 \cdot 50 \cdot 0.934 \cdot 10^{-4}=-741.2 K N
$$




$$
\begin{array}{ll}
\frac{\Delta P_{c+s+r}}{P_{m, t=0}}=\frac{741.2}{4760}=0.124 & \rightarrow \alpha_{\text {loss }}=12.4 \%<15 \% \\
P_{m, t=\infty}=4760-741=\underline{\underline{3929 \mathrm{kN}}} \rightarrow \underline{\underline{98 \mathrm{kN} / \text { strand }}}
\end{array}
$$

\subsection{ULTIMATE LIMIT STATE DESIGN}

\subsubsection{FLEXURE DESIGN}

Compression Zone

$b_{\text {eff }} / b_{w}=80 / 12=6.67>5.0 \rightarrow$ Slim cross-section, it can be assumed that the compression zone stays within the flange of the beam

Effective depth

$$
\begin{aligned}
& d=188.6 \mathrm{~cm} \quad z_{r}=d-x_{s o}=97.6 \mathrm{~cm} \\
& z_{p}=x_{\text {su }}-a_{p}=95.5 \mathrm{~cm} \\
& M_{E d r}=M_{E d}+P_{m, t=\infty} \cdot\left(z_{r}-z_{p}\right)=11,032+3929 \cdot(0.976-0.955) \\
& =11,116 \mathrm{kNm} \\
& \mu_{E d r}=\frac{M_{E d r}}{b_{e f f} \cdot d^{2} \cdot f_{c d}}=\frac{1111600 \mathrm{kNcm}}{80.0 \cdot 188.6^{2} \cdot 2.83 \mathrm{kN} / \mathrm{cm}^{2}}=0.138<\mu_{E d \text { max }}=0.40 \checkmark \\
& \rightarrow \text { The compression zone is sufficient. }
\end{aligned}
$$

Tension Zone

Using the design table from [3] following values for the height of the compression zone $x$ and the lever arm of the internal forces $z$ can be determined:

$$
\begin{aligned}
& \text { Lever arm } z \\
& \zeta=0.923 \quad \rightarrow z=\zeta \cdot d=0.923 \cdot 188.6=174.0 \mathrm{~cm} \\
& \text { Compression zone } \\
& \xi=0.185 \\
& \rightarrow x=\xi \cdot d=0.185 \cdot 177=34.9 \mathrm{~cm} \\
& \text { Rebar strain } \\
& \varepsilon_{s}=\Delta \varepsilon_{p}=15.47 \%>2.17 \% \circ \quad \rightarrow \sigma_{s d}=f_{y d}=435 \mathrm{MN} / \mathrm{m}^{2} \\
& \varepsilon_{p}^{(0)}=\frac{P_{m, t=\infty}}{A_{p} \cdot E_{p}}=\frac{3929 \mathrm{kN}}{50 \cdot 0.934 \mathrm{~cm}^{2} \cdot 19,600 \mathrm{kN} / \mathrm{cm}^{2}}=4.29 \% \\
& \varepsilon_{p}=\varepsilon_{p}^{(0)}+\Delta \varepsilon_{p}=4.29 \%+15.47 \% \text { o }=19.76 \% \\
& f_{p d}=\frac{f_{p 0,1, k}}{\gamma_{p}}=\frac{1500 \mathrm{~N} / \mathrm{mm}^{2}}{1.15}=1304 \mathrm{~N} / \mathrm{mm}^{2} \\
& \varepsilon_{p 0,1 d}=\frac{f_{p d}}{E_{p}}=\frac{1304 \mathrm{~N} / \mathrm{mm}^{2}}{196,000 \mathrm{~N} / \mathrm{mm}^{2}}=6.7 \% \\
& \varepsilon_{p 0,1 d}=6.7 \% \circ<\varepsilon_{p}=19.76 \% \circ \quad \rightarrow \sigma_{p d}=f_{p d}=1304 \mathrm{~N} / \mathrm{mm}^{2} \\
& A_{s 1, r e q}=\left(\frac{M_{E d r}}{z}-A_{p} \cdot \sigma_{p d}\right) \cdot \frac{1}{\sigma_{s d}}=\left(\frac{1,111,600}{174}-46.7 \cdot 130.4\right) \cdot \frac{1}{43.5} \\
& =6.80 \mathrm{~cm}^{2}
\end{aligned}
$$




\section{Chosen}

Precompressed Tension Zone

\section{lower reinforcement}

\begin{tabular}{ll}
\hline & $N_{p, t=0}=-4670 \mathrm{kN} \quad M_{p, t=0}=-4458 \mathrm{kNm}$ \\
Design normal force & $N_{E d}=\gamma_{p} \cdot N_{p, t=0}=1.0 \cdot(-4670 \mathrm{kN})=-4670 \mathrm{kN}$ \\
Effective depth & $d \approx 205-4=201 \mathrm{~cm} \quad \rightarrow \quad z_{s 2}=d-x_{s u}=201-114.1=89.9 \mathrm{~cm}$ \\
& $M_{E d}=\gamma_{G} \cdot \min M_{G}+\gamma_{P} \cdot M_{P, t=0}=1.0 \cdot 1508.0+1.0 \cdot(-4458)=-2950 \mathrm{Nm}$ \\
Design bending moment & $M_{E d, s 2}=M_{E d}-N_{E d} \cdot z_{s 2}=-2950-0.899 \cdot(-4670)=7011 \mathrm{kNm}$ \\
& $\mu_{E d s 2}=\frac{M_{E d s 2}}{b_{e f f} \cdot d^{2} \cdot f_{c d}}+\frac{N_{E d}}{f_{y d}}=0.077 \quad \rightarrow \quad \omega_{2}=0.0804$ \\
Required reinforcement & $A_{s 2, \text { req }}=\omega_{2} \cdot \frac{b_{e f f} \cdot d}{f_{y d} / f_{c d}}+\frac{N_{E d}}{f_{y d}}=0.0804 \cdot \frac{80 \cdot 201}{43.5 / 1.7}+\frac{-4760}{43.5}=-23.3<0$
\end{tabular}

No reinforcement in the compression zone is required.

\subsubsection{SHEAR DESIGN}

Design location (Direct support)

$$
\begin{aligned}
& x_{1}=1.40 \mathrm{~m} \\
& x_{2}=a_{i}=0.25 \mathrm{~m} \\
& x_{3}=1.50 \mathrm{~m} \\
& V_{E d, 1}=1374 \mathrm{kN} \\
& V_{E d, 2}=1396 \mathrm{kN} \\
& V_{E d, 3}=1372 \mathrm{kN}
\end{aligned}
$$

( $\approx$ end of dispersion length) (face of support) (transition of web width)

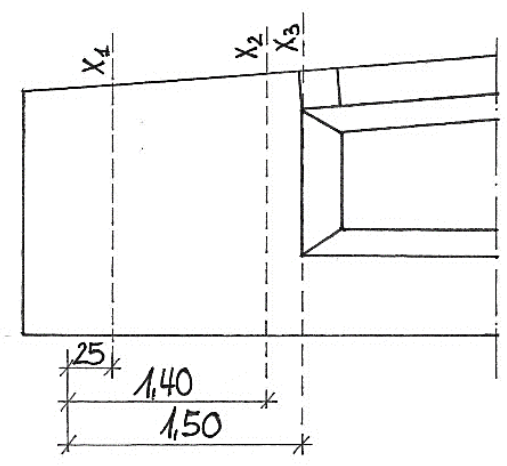

Shear Force Resistance

\begin{tabular}{ll}
\hline & $a_{r}=16.5 \mathrm{~cm} \quad \rightarrow d=205-16.5=188.5 \mathrm{~cm}$ \\
& $k=1+\sqrt{\frac{20}{d}}=1+\sqrt{\frac{20}{188.5}}=1.39<2.00$ \\
Longitudinal reinforcement & $A_{s l}=A_{s}+A_{p}=8.04+46.70=54.7 \mathrm{~cm}^{2}$ \\
Percentage of reinforcement & $\rho_{1}=\frac{A_{s l}}{b_{w} \cdot d}=\frac{54.7}{40 \cdot 134}=0.0102 \leq 0.02$
\end{tabular}


Compressive stress

$$
\begin{aligned}
& \sigma_{c p}=\frac{N_{E d}}{A_{c}}=\frac{0.3929}{40 \cdot 149} \cdot 10=6.59 \mathrm{MN} / \mathrm{m}^{2} \\
& <0.2 \cdot f_{c d}=0.2 \cdot 28.3 \mathrm{MN} / \mathrm{m}^{2}=5.66 \mathrm{MN} / \mathrm{m}^{2} \\
& V_{R d, c}=\left[0.10 \cdot 1.39 \cdot(100 \cdot 0.0102 \cdot 50)^{1 / 3}+0.12 \cdot 6.59\right] \cdot 0.40 \cdot 1.34 \cdot 10^{3} \\
& =701 \mathrm{kN} \\
& d=134 \mathrm{~cm} \geq 80 \mathrm{~cm} \rightarrow v_{\min }=0.025 \cdot 1.39^{3 / 2} \cdot(50)^{1 / 2}=0.290 \mathrm{MN} / \mathrm{m}^{2} \\
& V_{R d, c, \min }=[0.290+0.12 \cdot 6.59] \cdot 0.40 \cdot 1.34 \cdot 10^{3}=580 \mathrm{kN}
\end{aligned}
$$

Shear force resistance

$$
\begin{aligned}
& V_{R d, c}=\underline{\underline{701 \mathrm{kN}}}>V_{R d, c, \min }=580 \mathrm{kN} \\
& V_{E d}=1374 \mathrm{kN}>V_{R d, c}=701 \mathrm{kN} \rightarrow \text { Shear force reinforcement is needed! }
\end{aligned}
$$

Inclination of Concrete Compression Struts

Friction force

$$
V_{R d, c c}=0.24 \cdot(50)^{1 / 3} \cdot\left(1-1.2 \cdot \frac{6.59}{28.3}\right) \cdot 0.4 \cdot 1.21=308 \mathrm{kN}
$$

Inclination

$$
1.0 \leq \cot \theta=\frac{1.2+1.4 \cdot \sigma_{c p} / f_{c d}}{1-V_{R d, c c} / V_{E d}}=\frac{1.2+1.4 \cdot 6.59 / 28.3}{1-308 / 1374}=1.97 \leq 3.0
$$

Required Shear Force Reinforcement

Vertical stirrups $\quad \alpha=90^{\circ}$

Lever arm of the internal forces $z \approx 0.9 \cdot d=0.9 \cdot 134=121 \mathrm{~cm}$

Required reinforcement $\quad a_{s w, r e q}=\frac{1374 k N}{43.5 \cdot 1.21 \cdot(1.97+0) \cdot 1}=13.3 \mathrm{~cm}^{2} / \mathrm{m}$

\section{Chosen $\quad \varnothing 10 / 10=15.71 \mathrm{~cm}^{2} / \mathrm{m} \quad$ stirrups}

Resistance of the Compression Struts

At $x_{2}=0.25 \mathrm{~m}$

$$
\begin{aligned}
& V_{R d, \text { max }}=0.75 \cdot 40 \cdot 121 \cdot 2.83 \cdot \frac{1.97}{1+1.97^{2}}=4146 \mathrm{kN} \\
& V_{E d, 2}=1396 \mathrm{kN}<V_{R d, \text { max }}=4146 \mathrm{kN} \quad \rightarrow \text { The compression strut is ok. }
\end{aligned}
$$

At $x_{3}=1.50 \mathrm{~m}$

$$
\text { Simplified assumption: } b_{w}=0.12 m
$$

$$
\begin{aligned}
& \cot \theta=1.68 \\
& V_{R d, \text { max }}=0.75 \cdot 12 \cdot 121 \cdot 2.83 \cdot \frac{1.68}{1+1.68^{2}}=1355 \mathrm{kN} \\
& V_{E d, 3} / V_{R d, \text { max }}=1372 \mathrm{kN} / 1355 \mathrm{kN}=1.01 \approx 1.00
\end{aligned}
$$

An exceedance of $1 \%$ is acceptable given that the real web width at $1.50 \mathrm{~m}$ is bigger than the assumed $12 \mathrm{~cm}$. 
Minimum Shear Force Reinforcement

$\min a_{s w}=\rho \cdot b_{w} \cdot \sin \alpha \quad$ and $\quad \rho=0.16 \cdot f_{c t m} / f_{y k}=0.16 \cdot 4.1 / 500=1.31 \%$

$\min a_{s w}=0.00131 \cdot 0.40 \cdot \sin 90^{\circ}=5.24 \cdot 10^{-4} \mathrm{~m}^{2} / \mathrm{m}=\underline{\underline{5.24 \mathrm{~cm}^{2} / \mathrm{m}}}$

\subsubsection{TILTING}

According to DIN EN 1992-1-1, 5.9 a simplified proof of the safety against tilting can be conducted with following formula:

$$
\begin{aligned}
& b_{\text {req }} \geq \sqrt[4]{\left(\frac{I_{0}}{50}\right)^{3} \cdot h} \quad \text { and } \quad \frac{h}{b} \leq 5.0 \\
& b_{\text {req }} \geq \sqrt[4]{\left(\frac{29.2}{50}\right)^{3} \cdot 2.20}=0.81 m \cong b_{\text {prov }}=0.80 m \text { and } \quad \frac{h}{b}=\frac{220}{80}=2.75<5.0
\end{aligned}
$$

This means that the beam is safe against tilting and no further analysis is necessary. The simplified proof is sufficient.

\subsection{SERVICEABILITY LIMIT STATE DESIGN}

\subsubsection{STRESS LIMITS}

Second or first order?

$$
\begin{aligned}
& \min P_{k, t}=r_{\text {inf }} \cdot P_{m, t=\infty}=0.95 \cdot 3929 \mathrm{kN}=3732 \mathrm{kN} \\
& N_{E d, \text { rare }}=-\min P_{k, t}=-3732 \mathrm{kN} \\
& M_{E d, \text { rare }}=M_{E d, \text { rare }}^{G+Q}-\min P_{k, t} \cdot z_{p}=7784-3732 \cdot 0.0 .955=4221 \mathrm{kNm} \\
& \sigma_{c, \text { rare }}=\frac{-3732}{5818}+\frac{422100}{31841471} \cdot 114 \cdot 10=8.70 \mathrm{MN} / \mathrm{m}^{2} \\
& \sigma_{c, \text { rare }}=8.7 \mathrm{MN} / \mathrm{m}^{2}>f_{c t m}=4.1 \mathrm{MN} / \mathrm{m}^{2} \\
& \rightarrow \text { Second order must be considered }
\end{aligned}
$$

\section{Concrete Compression Stresses}

$$
\begin{aligned}
& \frac{2 \cdot A_{c} \cdot z_{p}}{Z \cdot x \cdot b_{\text {eff }}}=\frac{2 \cdot 5818 \cdot 95.5}{174 \cdot 34.9 \cdot 80}=2.29>1.0 \rightarrow \min P_{k, t}=3732 \mathrm{kN} \\
& N_{E d, p e r m}=-3732 \mathrm{kN} \\
& M_{E d, p e r m}=M_{E d, p e r m}^{G+Q}-\min P_{k, t} \cdot z_{p}=4298-3732 \cdot 0.955=735 \mathrm{kNm} \\
& \sigma_{c, \text { perm }}=\frac{N_{E d, \text { perm }}}{A_{c}}+\frac{2 M_{E d, p e r m}}{z \cdot x \cdot b_{\text {eff }}} \leq 0.45 f_{c k} \\
& \sigma_{c, \text { perm }}=\frac{-3732}{5818}+\frac{2 \cdot 73500}{174 \cdot 34.9 \cdot 0.80} \cdot 10=-9.44 \mathrm{MN} / \mathrm{m}^{2}
\end{aligned}
$$




$$
\left|\sigma_{c, \text { perm }}\right|=9.44 M N / m^{2}<0.45 f_{c k}=0.45 \cdot 50=22.5 M N / m^{2} \checkmark
$$

Stresses in the Reinforcement

$$
\begin{aligned}
N_{E d, \text { rare }} & =-3732 \mathrm{kN} \\
M_{E d, \text { rare }} & =7784-3732 \cdot(0.976-0.955)=7864 \mathrm{kNm} \\
\sigma_{s, \text { rare }} & =\left[\frac{M_{E d, \text { rare }}}{z}+N_{E d, \text { rare }}\right] \cdot \frac{1}{A_{s 1}+A_{p}} \leq 0.8 f_{y k} \\
\sigma_{s, \text { rare }} & =\left[\frac{786400}{174}-3732\right] \cdot \frac{1}{54.7} \cdot 10=143.7 \mathrm{MN} / \mathrm{m}^{2} \\
\leq & 0.8 \cdot 500=400 \mathrm{MN} / \mathrm{m}^{2}
\end{aligned}
$$

Stresses in the Prestressing Steel

$$
\begin{aligned}
\min P_{k, t} & =P_{m, t=\infty}=3929 \mathrm{kN} \quad N_{E d, p e r m}=-\min P_{k, t}=-3929 \mathrm{kN} \\
M_{E d, p e r m} & =M_{E d, p e r m}^{G+Q}-\min P_{k, t} \cdot z_{p}=4298-3929 \cdot 0.955=4382 \mathrm{kNm} \\
\sigma_{p, \text { perm }} & =\frac{P_{m, t=\infty}}{A_{p}}\left[\frac{M_{E d, p e r m}}{z}+N_{E d, p e r m}\right] \cdot \frac{1}{A_{s 1}+A_{p}} \leq 0.65 f_{p k} \\
\sigma_{p, \text { perm }} & =\frac{3929}{46.7}\left[\frac{438200}{174}-3929\right] \cdot \frac{1}{54.7} \cdot 10=583.6 \mathrm{MN} / \mathrm{m}^{2} \\
& \leq 0.65 \cdot 1770=1150 \mathrm{MN} / \mathrm{m}^{2} \checkmark
\end{aligned}
$$

\begin{tabular}{|c|c|c|}
\hline \multirow[t]{2}{*}{ Tensile strength } & $f_{c t, e f f}=f_{c t m} \geq 2.9 \mathrm{MN} / \mathrm{m}^{2}$ & $\rightarrow f_{c t, e f f}=4.1 \mathrm{MN} / \mathrm{m}^{2} \geq 2.9 \mathrm{MN} / \mathrm{m}^{2}$ \\
\hline & $h=2.05 m$ & $\rightarrow h^{\star}=1.0 m$ \\
\hline
\end{tabular}

\subsubsection{MINIMUM REINFORCEMENT AGAINST CRACKING}

Reinforcement needed?

$$
\sigma_{c, \text { rare }}=8.70 \mathrm{MN} / \mathrm{m}^{2}>0
$$

$\rightarrow$ a minimum reinforcement to prevent cracking is necessary

Area of the Tension Zone

Area of tension zone

$$
\begin{aligned}
& \sigma_{I}=f_{c t m}=\sigma_{I}^{N}+\sigma_{I}^{M}=4.1 \mathrm{MN} / \mathrm{m}^{2}=\frac{-3.732}{0.5818}+\sigma_{I}^{M} \\
& \sigma_{I}^{M}=10.51 \mathrm{MN} / \mathrm{m}^{2} \quad \rightarrow \sigma_{u}^{M}=-10.51 \mathrm{MN} / \mathrm{m}^{2} \\
& \sigma_{u}=\sigma_{u}{ }^{N}+\sigma_{u}{ }^{M}=\frac{-3.732}{0.5818}-10.51=-16.9 \mathrm{MN} / \mathrm{m}^{2}
\end{aligned}
$$

$$
h_{t}=\frac{4.1}{4.1+16.9} \cdot 205 \mathrm{~cm}=40 \mathrm{~cm} \rightarrow A_{c t}=40 \cdot 19=\underline{\underline{480 \mathrm{~cm}^{2}}}
$$

Minimum Required Cracking Reinforcement 


$$
\begin{array}{ll} 
& \begin{array}{l}
\text { for } h>80 \mathrm{~cm} \\
k_{1}=1.5
\end{array} \\
& k_{c}=0.4 \cdot\left[1-\frac{\sigma_{c}}{k_{1} \cdot h / h^{*} \cdot f_{c t, \text { eff }}}\right]=0.4 \cdot\left[1-\frac{3.732 / 0.5818}{1.5 \cdot 2.05 / 1.0 \cdot 0.41}\right]=0.197 \leq 1.0 \\
\text { Crack width limit } & w_{k}=0.2 \mathrm{~mm} \quad(\text { according to DIN EN 1992-1-1, Tab. NA.7.1.) } \\
\text { Limit diameter } & \phi_{s}{ }^{*}=\phi_{s} \cdot \frac{2.9}{f_{c t, e f f}}=20 \mathrm{~mm} \cdot \frac{2.9}{4.1}=14.15 \mathrm{~mm}^{1} \\
\text { Reinforcement stresses } & \sigma_{s}=223 \mathrm{MN} / \mathrm{m}^{2} \quad(\text { according to DIN EN 1992-1-1, Tab. NA.7.2.) } \\
\text { Required Reinforcement } & A_{s, \text { min, req }}=k_{c} \cdot k \cdot f_{c t, \text { eff }} \cdot \frac{A_{c t}}{\sigma_{s}}=0.197 \cdot 0.5 \cdot 4.1 \cdot \frac{480}{223}=0.87 \mathrm{~cm}^{2} \\
& \rightarrow \quad \text { The provided reinforcement is sufficient! }
\end{array}
$$

\subsubsection{LIMITATION OF THE CRACK WIDTH}

Percentage of Reinforcement

$$
\begin{aligned}
& h / d_{1}=51.25 \rightarrow h_{c, \text { eff }} / d_{1}=5.0 \\
& h_{c, \text { eff }}=5.0 \cdot 4.0 \mathrm{~cm}=20 \mathrm{~cm} \\
& A_{c, \text { eff }}=12.0 \cdot 20=240 \mathrm{~cm}^{2} \\
& \xi=0.6 \varnothing_{s}=20 \mathrm{~mm}^{1} \quad \varnothing_{p}=7.2 \mathrm{~mm} \\
& \xi_{1}=\sqrt{\xi \cdot \phi_{s} / \phi_{p}}=\sqrt{0.6 \cdot 20 / 7.2}=1.29 \\
& \rho_{\text {tot }}=\left(A_{s}+A_{p}\right) / A_{c, \text { eff }}=63.3 / 240=0.2600 \\
& \rho_{p, \text { eff }}=\left(A_{s}+\xi_{1}^{2} A_{p}\right) / A_{c, \text { eff }}=\left(15.7+1.29^{2} \cdot 47.6\right) / 240=0.3897
\end{aligned}
$$

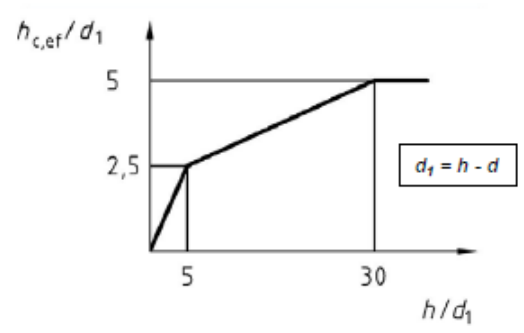

Reinforcement Stresses

$$
\begin{aligned}
& \sigma_{s}=\left[\frac{M_{E d r}}{z}+N_{E d}\right] \cdot \frac{1}{A_{s}+A_{p}}+0.4 \cdot f_{c t, e f f} \cdot\left[\frac{1}{\rho_{p, \text { eff }}}-\frac{1}{\rho_{\text {tot }}}\right] \\
& \sigma_{s}=\left[\frac{509300}{174}-\right] \cdot \frac{1}{63.3}+0.4 \cdot 4.1 \cdot\left[\frac{1}{0.26}-\frac{1}{0.39}\right]=-135.3 \mathrm{kN} / \mathrm{cm}^{2} \\
& \rightarrow \sigma_{s}=-135.3 \mathrm{MN} / \mathrm{m}^{2}<0
\end{aligned}
$$

The stress is negative which means the cross section is under compression so there won't be any cracking.

\footnotetext{
${ }^{1}$ The diameter of the reinforcement has to be changed to $20 \mathrm{~mm}$ due to the required minimum reinforcement (see chapter 6.8)
} 


\subsection{DESIGN AND REINFORCEMENT}

\subsubsection{ANCHORAGE OF TENDONS}

Transmission of the Stresses

Transmission length

$$
\begin{array}{ll}
I_{p t}=\alpha_{1} \cdot \alpha_{2} \cdot \varnothing_{p} \cdot \sigma_{p m 0} / f_{b p t} & \\
\alpha_{1}=1.25 & \text { no wire stripping } \\
\alpha_{2}=0.19 & \text { for strands } \\
\varnothing_{p}=12.5 \mathrm{~mm} & \text { for C50/60 and good bond } \\
f_{b p t}=4.6 \mathrm{MN} / \mathrm{m}^{2} & \\
\sigma_{p m 0}=1000 \mathrm{MN} / \mathrm{m}^{2} & \\
l_{p t}=1.25 \cdot 0.19 \cdot 1.25 \cdot 1000 / 4.6=64.5 \mathrm{~cm}
\end{array}
$$

Modified transmission length $\quad I_{p t d}=0.8 \cdot l_{p t}=0.8 \cdot 64.5=51.6 \mathrm{~cm}$

Dispersion length

$I_{\text {disp }}=\sqrt{I_{p t d}^{2}+d_{p}^{2}}=\sqrt{51.6^{2}+130.4^{2}}=140 \mathrm{~cm} \quad d_{p}=130.4 \mathrm{~cm}$ at $x=1.40 \mathrm{~m}$

Splitting tensile force

$$
\begin{aligned}
& F_{t d}=P_{m, 0}-\left|\sigma_{c, m}\right| \cdot A_{c, u} \\
& N_{E d}^{P}=-P_{m, t=0}=-4760 \mathrm{kN} \quad M_{E d}^{P}=-4760 \cdot(0.745-0.186)=-2611 \mathrm{kNm} \\
& A_{c, u}=a_{p} \cdot b=(32.7-1.25 / 2) \cdot 40=33.325 \cdot 40=1333 \mathrm{~cm}^{2} \\
& z_{c, u}=z_{s, u}-a_{p} / 2=74.5-33.33 / 2=57.8 \mathrm{~cm} \\
& \sigma_{c, m}\left(x=I_{\text {disp }}\right)=\frac{N_{E d}^{P}}{A_{c}}+\frac{M_{E d}^{P}}{I_{c}} \cdot z_{c, u}=\frac{-4670}{149 \cdot 40}+\frac{-261100}{40 \cdot 149^{3} / 12} \cdot 57.8 \cdot 10 \\
& \quad=-21.5 \mathrm{MN} / \mathrm{m}^{2} \\
& F_{t d}=4670-2.15 \cdot 1333=\underline{\underline{1804 \mathrm{kN}}}
\end{aligned}
$$

Required reinforcement

$A_{s p, r e q}=\frac{\gamma_{p, s p}}{3} \cdot \frac{F_{t d}}{f_{y d}}=\frac{1.35}{3} \cdot \frac{1804}{43.5}=18.7 \mathrm{~cm}^{2}$

The reinforcement is to be distributed over the length of

$0.75 \cdot I_{\text {disp }}=0.75 \cdot 140=105 \mathrm{~cm}$

$a_{\text {sp,req }}=18.7 / 1.05=\underline{\underline{17.8 \mathrm{~cm}^{2} / \mathrm{m}}}$

\section{Chosen}

$\varnothing 10 / 7.5=20.9 \mathrm{~cm}^{2} / \mathrm{m}$

stirrups

Tensile forces at the edge $\quad Z_{E d}^{P}=P_{m 0} \cdot\left[\frac{e}{h}-\frac{1}{6}\right]=4670 \cdot\left[\frac{55.9}{149}-\frac{1}{6}\right]=974 \mathrm{kN}$ 
Required reinforcement

Chosen
$A_{s z, r e q}=Z_{E d} / f_{y d}=974 / 43.5=\underline{\underline{22.4 \mathrm{~cm}^{2}}}$

$10 \varnothing 12=22.6 \mathrm{~cm}^{2}$

reinforcement loops

Anchorage of Tendons in the Ultimate Limit State

Anchorage length

$$
\begin{aligned}
& I_{b p d}=I_{p t 2}+\alpha_{2} \cdot \varnothing_{P} \cdot \frac{\sigma_{p d}-\sigma_{p m, t=\infty}}{f_{b p d}} \\
& I_{p t 2}=1.2 \cdot I_{p t}=1.2 \cdot 64.5=77.4 \mathrm{~cm} \\
& \sigma_{p d}=f_{p, 01 k} / \gamma_{p}=1500 / 1.15=1304 \mathrm{MN} / \mathrm{m}^{2} \\
& \sigma_{p m, t=\infty}=P_{m, t=\infty} / A_{p}=3929 / 46.7=84.1 \mathrm{kN} / \mathrm{cm}^{2} \\
& I_{b p d}=77.4+0.19 \cdot 1.25 \cdot \frac{1304-841}{4.6}=\underline{\underline{101 \mathrm{~cm}}}
\end{aligned}
$$

Internal forces at $x$

$$
\begin{aligned}
& N_{P, t=\infty}=-P_{m, t=\infty}=-3929 \mathrm{kN} \quad N_{P, t=\infty}=-P_{m, t=\infty}=-3929 \mathrm{kN} \\
& x=I_{b p d}-U=101+25=76 \mathrm{~cm} \\
& M_{E d}(x=76)=1064 \mathrm{kNm} \\
& M_{E d}=M_{E d}(x)+M_{E d}^{P}=1064-2118=-1054 \mathrm{kNm}
\end{aligned}
$$

Concrete stresses

$$
\begin{aligned}
& \sigma_{c, u}=\frac{N_{E d}}{A_{c}}+\frac{M_{E d}}{I_{c}} \cdot z_{u}=\frac{-3929}{145 \cdot 40}+\frac{-105400}{40 \cdot 145^{3} / 12} \cdot 72.5=-0.687 \mathrm{kN} / \mathrm{m}^{2} \\
& \sigma_{c, u}=-6.9 \mathrm{MN} / \mathrm{m}^{2}<<0.7 \cdot f_{c t m}=0.7 \cdot 4.1=2.87 \mathrm{MN} / \mathrm{m}^{2}
\end{aligned}
$$

\subsubsection{MINIMUM REINFORCEMENT TO ENSURE ROBUSTNESS}

$$
\begin{aligned}
& M_{c r}=f_{c t m} \cdot W_{c u} \quad W_{c u}=279,176 \mathrm{~cm}^{3} \\
& M_{c r}=4.1 \cdot 279.176 \cdot 10^{-3}=1145 \mathrm{kNm} \\
& A_{s, \min }=\frac{1}{f_{y k}} \cdot \frac{M_{c r}}{z}=\frac{1}{50.0} \cdot \frac{114500}{174}=15.2 \mathrm{~cm}^{2}>A_{s, \text { prov }}=8.04 \mathrm{~cm}^{2} \text { 々 }
\end{aligned}
$$

The provided reinforcement is not enough to cover the minimum reinforcement which is needed to ensure the robustness of the beam, therefore a new reinforcement is chosen.

\section{Chosen}

$$
5 \varnothing 20=15.7 \mathrm{~cm}^{2}
$$

\section{lower longitudinal reinforcement}

\subsubsection{STIRRUPS AT THE RIDGE}

Flexure moment

Lever arm of the forces

$$
\begin{aligned}
& M_{E d}=11,533 \mathrm{kNm} \\
& z=0.9 \cdot d=0.9 \cdot(220-16.5)=183 \mathrm{~cm}
\end{aligned}
$$


Required Reinforcement $\quad A_{s, r e q}=\frac{2 \cdot M_{E d} \cdot \sin \alpha}{z \cdot f_{y d}}=\frac{2 \cdot 1153300 \cdot \sin 3.1^{\circ}}{183 \cdot 43.5}=\underline{\underline{15,7 \mathrm{~cm}^{2}}}$

Chosen

$7 \varnothing 12=15.84 \mathrm{~cm}^{2}$

stirrups

\subsubsection{DESIGN OF THE SUPPORT}

Width of the Joints

The width of the joints between a concrete truss and a column can be estimated according to [4] and [5]. The width of the joints is the result of the sum of the different possible dimensional deviations during the process of the production and assembly of the beams.

$$
\begin{array}{ccl}
\delta_{\text {comb }}=\delta_{\max }+\sqrt{\sum \delta_{i}^{2}} \quad \text { with } & \delta_{\text {comb }} & \text { Total allowed dimensional deviation } \\
& \delta_{\max } & \text { Maximum dimensional deviation } \\
& \delta_{i} & \text { Other possible dimensional deviations. }
\end{array}
$$

The different dimensional deviations can be taken from DIN 18202 and DIN 18203.

Here it is:

$\delta_{\text {comb }}=\delta_{\max }+\sqrt{\sum \delta_{i}^{2}}=\frac{1}{2} \cdot\left(30+\sqrt{30^{2}+25^{2}+25^{2}}\right)=38.2 \mathrm{~mm}$

Chosen $\quad \delta=40 \mathrm{~mm}$

Dimensions of the Bearing

The width of the elastomeric bearing is chosen to $b e b=200 \mathrm{~mm}$. The maximum length of the bearing can be calculated as per DIN EN 1992-1-1, 10.9.5.2 with following formula:

$a=a_{1}+a_{2}+a_{3}+\sqrt{\Delta a_{2}^{2}+\Delta a_{3}^{2}} \quad$ with $\quad a_{1} \quad$ Length of the elastomeric bearing, $a_{1}>\min _{1}$ (DIN EN 1992-1-1, Tab. 10.2)

$a_{2} \quad$ Distance of the bearing to the edge of the support

$a_{3} \quad$ Distance of the bearing to the edge of the beam

$\Delta a \quad$ Dimensional deviations.

Here:

Assumption $\quad a_{1} \approx 500 \mathrm{~mm}, b_{1}=350 \mathrm{~mm} \quad \rightarrow \sigma_{E d}=V_{E d} / a_{1} \cdot b_{1}=1632 \mathrm{kN} / 50 \cdot 35=0.93 \mathrm{kN} / \mathrm{cm}^{2}$

$\min a_{1}=70 \mathrm{~mm}$ for $0.15<\sigma_{E d} / f_{c d}=0.93 / 2.83=0.33<0.40$

(DIN EN 1992-1-1, Tab.10.2)

$a_{2}=c_{\text {nom }}+d_{s} / 2+c_{\text {nom, }} / 2=20+10 / 2+20 / 2=35 m m>m i n a_{2}=15 m m \quad$ (DIN EN 1992-1-1, Tab.10.3)

$a_{3}=c_{\text {nom }}+d_{s} / 2+c_{\text {nom, } b} / 2=20+12 / 2+20 / 2=36 m m>\min _{3}=15 m m \quad$ (DIN EN 1992-1-1, Tab.10.4) 
$\Delta a_{2}=/ / 1200=29500 / 1200=24.6 m m$

(DIN EN 1992-1-1, Tab.10.5)

$\Delta a_{3}=/ / 2500=29500 / 2500=11.8 m m$

$\rightarrow a_{\text {req }}=500+35+36+\sqrt{24.6^{2}+11.8^{2}}=598 m m<a_{\text {prov }}=650-40=\underline{\underline{610 m m}}$

\section{Chosen}

$$
a_{1}=500 \mathrm{~mm} / b_{1}=350 \mathrm{~mm}
$$

size of the bearing

Elastomeric Bearing

The elastomeric bearing that is used here is by the company Calenberg Ingenieure and is chosen in accordance with the technical information and dimensioning tables of the bearings [6].

Size of the bearing

$\mathrm{l} \times \mathrm{b} \times \mathrm{t}=500 \times 350 \times 20 \mathrm{~mm}$

Bearing compression

$$
\sigma_{m, k}=\frac{V_{E d}}{a_{1} \cdot b_{1}}=\frac{1632}{50 \cdot 35}=0.93 \mathrm{kN} / \mathrm{cm}^{2}=9.3 \mathrm{MN} / \mathrm{m}^{2}
$$

Form factor

$$
S=\frac{100}{20}=5
$$

Chosen

\section{'Compactlager S65'}

\subsubsection{REINFORCEMENT DRAWING}

See Appendix A.3, page 189.

\subsection{TRANSPORT AND ASSEMBLY}

\subsubsection{TRANSPORT AND LIFTING ON THE CONSTRUCTION SITE}

The beam will be suspended with the help of two anchors on each side above the center of gravity at around $1 / 6$ of the length of the beam. Which might cause tension over the anchors and might require upper reinforcement.

In the following the static system during transportation and assembly can be seen.

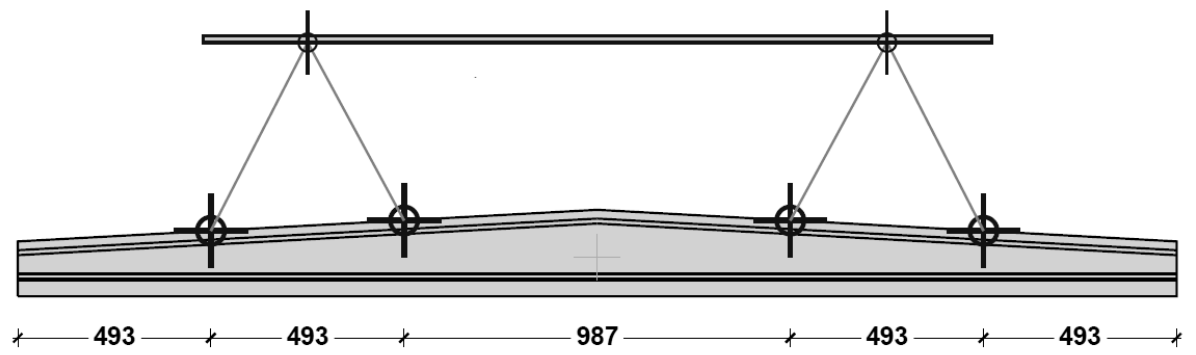


Simplified static system:

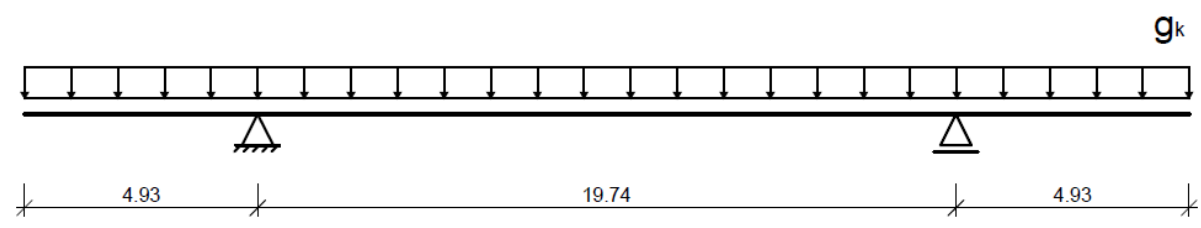

Lifting out of the Formwork

$\begin{array}{lll}\text { Lifting loads } & \text { self-weight } & g_{k}=14.5 \mathrm{kN} / \mathrm{m} \\ \text { Height of the beam at } 4.93 \mathrm{~m} & h=1.67 \mathrm{~m} & X_{s o}=73.1 \mathrm{~cm} \\ \text { Bending Moment } & M_{E d}^{G}=1.15 \cdot(-14.5) \cdot 4.93^{2} / 2=-203 \mathrm{kNm} \\ & M_{E d s}=M_{E d}^{G}-N_{E d} \cdot z_{s 1}=203-(-4760) \cdot(0.731-0.04)=3492 \mathrm{kNm}\end{array}$

Time of first lifting approximately after 5 days $(t=5 d)$

Compressive strength at time $t$

$$
\begin{aligned}
& f_{c m}(t)=\beta_{c c}(t) \cdot f_{c m} \quad \rightarrow \quad \beta_{c c}(t=5 d)=e^{s(1-\sqrt{28 / t})}=e^{0.2(1-\sqrt{28 / 5})}=0.76 \\
& f_{c m}(t=5 d)=0.76 \cdot 58=44.1 \mathrm{~N} / \mathrm{mm}^{2} \\
& f_{c k}(t=5 d)=f_{c m}(t)-8=44.1-8=36.1 \mathrm{~N} / \mathrm{mm}^{2} \\
& f_{c d}(t=5 d)=0.85 \cdot f_{c k}(t) / 1.5=0.85 \cdot 36.1 / 1.5=\underline{\underline{17.9 \mathrm{~N} / \mathrm{mm}^{2}}}
\end{aligned}
$$

Required Reinforcement $\quad \mu_{E d s}=\frac{M_{E d s}}{b \cdot d^{2} \cdot f_{c d}}=\frac{349200}{40 \cdot 163^{2} \cdot 1.79}=0.18 \quad \rightarrow \omega=0.2007$

$$
\begin{aligned}
A_{s 2, \text { req }} & =\left(\omega \cdot b \cdot d \cdot f_{c d}+N_{E d}\right) / \sigma_{s d} \\
& =(0.2007 \cdot 40 \cdot 163 \cdot 1.79-4760) / 43.5=\underline{\underline{-55.6 \mathrm{~cm}^{2}}}<0
\end{aligned}
$$

No additional upper reinforcement is required!

\subsubsection{TILTING}

According to DIN EN 1992-1-1, 5.9 a simplified proof of the safety against tilting can be conducted with following formula:

$$
\begin{aligned}
& b_{\text {req }} \geq \sqrt[4]{\left(\frac{I_{0}}{70}\right)^{3} \cdot h} \quad \text { and } \quad \frac{h}{b} \leq 5.0 \quad \text { for tilting during transport and assembly of the beam. } \\
& b_{\text {req }} \geq \sqrt[4]{\left(\frac{9.87}{70}\right)^{3} \cdot 2.2}=0.28 m<b_{\text {prov }}=0.80 m \quad \text { and } \quad \frac{h}{b}=\frac{220}{80}=2.75<5.0
\end{aligned}
$$


This means that the beam is safe against tilting during transport and assembly and no further analysis is necessary. The simplified proof is sufficient.

\subsubsection{TRANSPORT ANCHORS}

The transport anchors are designed in accordance with the VDI/BV-BS-Richtlinie 6205. For this project the anchors of the company HALFEN are chosen (see Chapter 6.8.3).

Due to the length and the weight of the concrete truss four anchors are needed and a lifting beam is used for both lifting out of the formwork as well as transport and lifting on site.

Maximum Lifting Load on the Anchors

$\begin{array}{ll}\text { Self-weight } & F_{G}=14.5 \cdot 29.6=429 \mathrm{kN} \\ \text { Formwork adhesion } & F_{\text {adh }}=q_{\text {adh }} \cdot A_{f} \quad \rightarrow \text { no adhesion here because of hinged formwork! } \\ \text { Dynamic factor } & \psi_{\text {dyn }}=1.3 \\ \text { Factor for inclination } & z=1.16 \mathrm{for} \beta=30^{\circ} \\ \text { Lifting load } & F_{z}=\frac{\left(F_{G}+F_{\text {adh }}\right) \cdot \psi_{\text {dyn }} \cdot z}{n}=\frac{429 \cdot 1.3 \cdot 1.16}{4}=162 \mathrm{kN}\end{array}$

Chosen

HALFEN DEHA Double-headed lifting anchor 6000-32,0-0700D WB

Load capacity

$$
F_{z, \text { Rd }}=189 \mathrm{kN}>F_{z, E d}=162 \mathrm{kN} \checkmark \quad \text { (see Table 17) }
$$

Table 17 - Sizing Table for 'HALFEN DEHA Lifting Anchor Systems'

\begin{tabular}{|c|c|c|c|c|c|}
\hline \multicolumn{6}{|c|}{ Load capacities for axial pull and diagonal pull up to $60^{\circ}[B]$} \\
\hline \multirow[t]{2}{*}{ Load class } & \multirow[t]{2}{*}{ Article number } & \multirow{2}{*}{$\begin{array}{c}\text { Min. web } \\
\text { thickness } \\
2 \times \mathrm{e}_{\mathrm{r}} \\
{[\mathrm{mm}]}\end{array}$} & \multirow{2}{*}{$\begin{array}{c}\text { Axial spacing } \\
\text { of anchors } \\
\mathrm{e}_{\mathrm{z}} \\
{[\mathrm{mm}]}\end{array}$} & \multicolumn{2}{|c|}{$\begin{array}{c}\text { Axial pull and diagonal pull up to } 60^{\circ}[\beta] \\
\text { Load capacity }[\mathrm{kN}] \\
\text { concrete strength } \mathrm{f}_{\mathrm{ci}}\end{array}$} \\
\hline & & & & $45 \mathrm{~N} / \mathrm{mm}^{2}$ & $55 \mathrm{~N} / \mathrm{mm}^{2}$ \\
\hline \multirow{2}{*}{10,0} & \multirow{2}{*}{$6000-10,0-0340 D$} & 120 & \multirow{2}{*}{$\geq 1360$} & 88.0 & 98.0 \\
\hline & & 140 & & 100.0 & 100.0 \\
\hline \multirow{2}{*}{15,0} & \multirow{2}{*}{$6000-15,0-0400 D$} & 120 & \multirow{2}{*}{$\geq 1600$} & 130.0 & 145.0 \\
\hline & & 140 & & 150.0 & 150.0 \\
\hline \multirow{3}{*}{20,0} & \multirow{3}{*}{$6000-20,0-0500 D$} & 120 & \multirow{3}{*}{$\geq 2000$} & 136.0 & 151.0 \\
\hline & & 140 & & 173.0 & 192.0 \\
\hline & & 160 & & 197.0 & 200.0 \\
\hline \multirow{4}{*}{32,0} & \multirow{4}{*}{$6000-32,0-0700 \mathrm{D}$} & 120 & \multirow{4}{*}{$\geq 2800$} & 189.0 & 210.0 \\
\hline & & 140 & & 220.0 & 245.0 \\
\hline & & 160 & & 251.0 & 280.0 \\
\hline & & 180 & & 282.0 & 315.0 \\
\hline
\end{tabular}




\subsection{SOFTWARE CALCULATIONS}

\section{Demo Frilo Nemetschek}

\section{Position: P.03 - Prestressed Concrete Purlin}

Spannbettbinder B8 01/2018 (Frilo R-2018-1/P12)
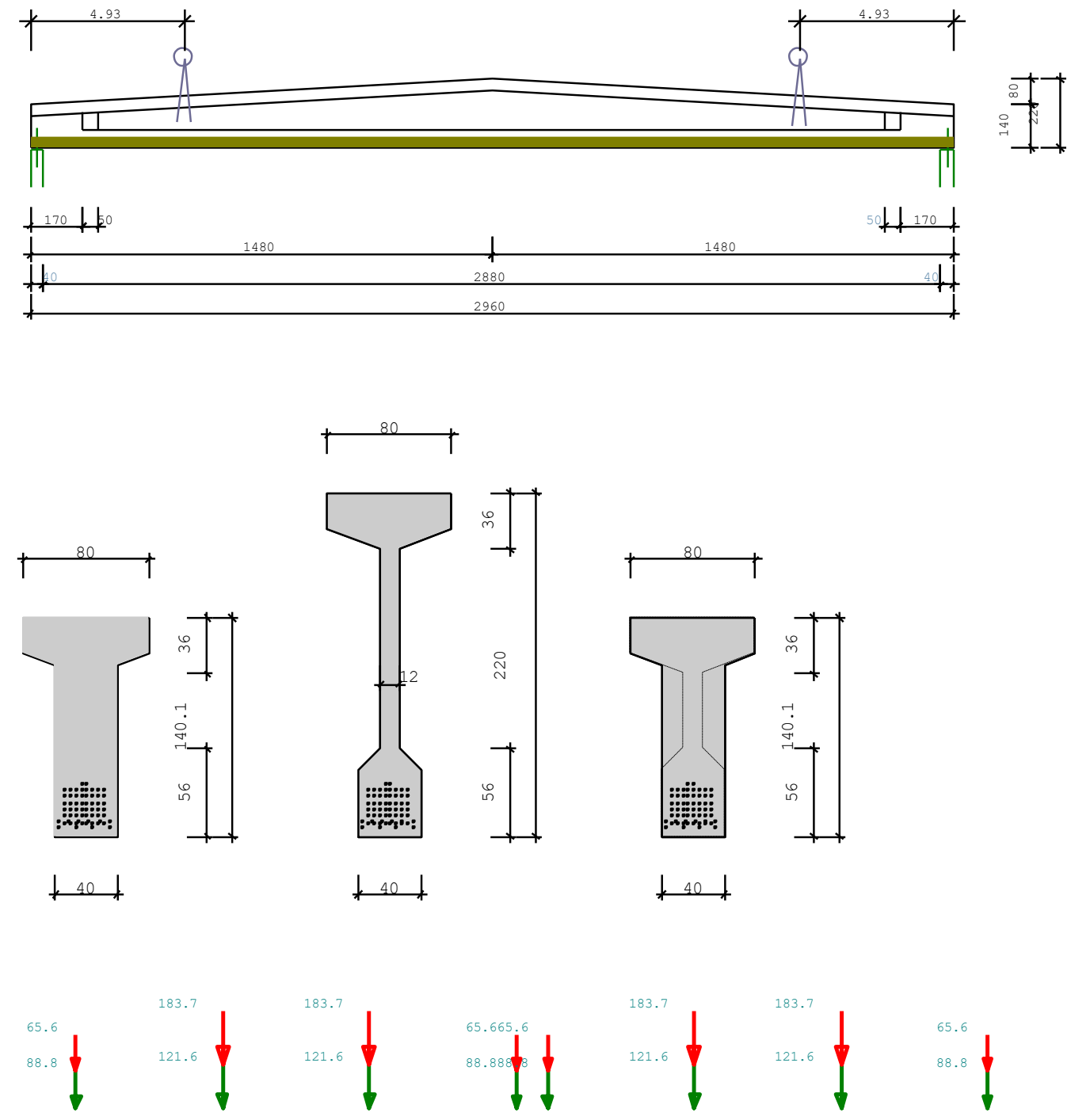

Eigengewicht Fertigteil nicht dargestellt

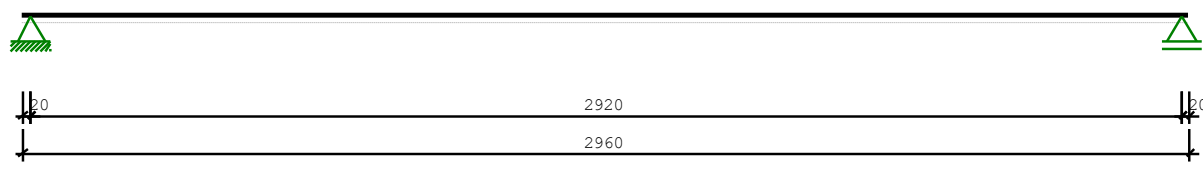

Advices:

No continuous reinforcement to $10 \mathrm{~cm}$ below UE found! 


\section{System:}

Double-pitch roof

\section{Material:}

Prestressing steel Spst $1500 / 1770$ Strand 7 wires

Reinforcing steel:

$\begin{aligned} \text { Longitudinal } & \text { Stirrup } \\ \text { B } 500 \text { B } & \text { B } 500 \text { B }\end{aligned}$

Concrete:

Precast

C $50 / 60$

Loads :

Self weight

Total

Volume

$\mathrm{G}=425.4 \mathrm{kN}$

Surf.

$\mathrm{V}=17.01 \mathrm{~m}^{3}$

Live loads

Units: Single load[kN] Single moment [kNm] line load $[\mathrm{kN} / \mathrm{m}]$

span type gle qle Dist. a gri qri Length Fact Act. Sim. Pos. $[\mathrm{m}]$ [m]

\begin{tabular}{|c|c|c|c|c|c|c|c|}
\hline 1 & 2 & 88.80 & 65.60 & 0.00 & 1.00 & 10 & 0 \\
\hline 1 & 2 & 88.80 & 65.60 & 14.10 & 1.00 & 10 & 0 \\
\hline 1 & 2 & 88.80 & 65.60 & 15.10 & 1.00 & 10 & 0 \\
\hline 1 & 2 & 88.80 & 65.60 & 29.20 & 1.00 & 10 & 0 \\
\hline 1 & 2 & 121.60 & 183.70 & 4.70 & 1.00 & 10 & 0 \\
\hline 1 & 2 & 121.60 & 183.70 & 9.40 & 1.00 & 10 & 0 \\
\hline 1 & 2 & 121.60 & 183.70 & 19.80 & 1.00 & 10 & 0 \\
\hline 1 & 2 & 121.60 & 183.70 & 24.50 & 1.00 & 10 & 0 \\
\hline
\end{tabular}

Load types: 1 = uniformly distr., 2 = single load at a, 3 = single moment at a

4 = trapezoidal load from a, 5 = triangle load over $\mathrm{L}$

Actions:

Act. $\gamma_{Q} \quad \psi_{0} \quad \psi_{1} \quad \psi_{2} \quad$ Dep. Cat. $\quad$ Description

$\begin{array}{llllll}10 & 1.50 & 0.50 & 0.20 & 0.00 & 0\end{array}$ S Schnee bis $\mathrm{NN}+1000 \mathrm{~m}$




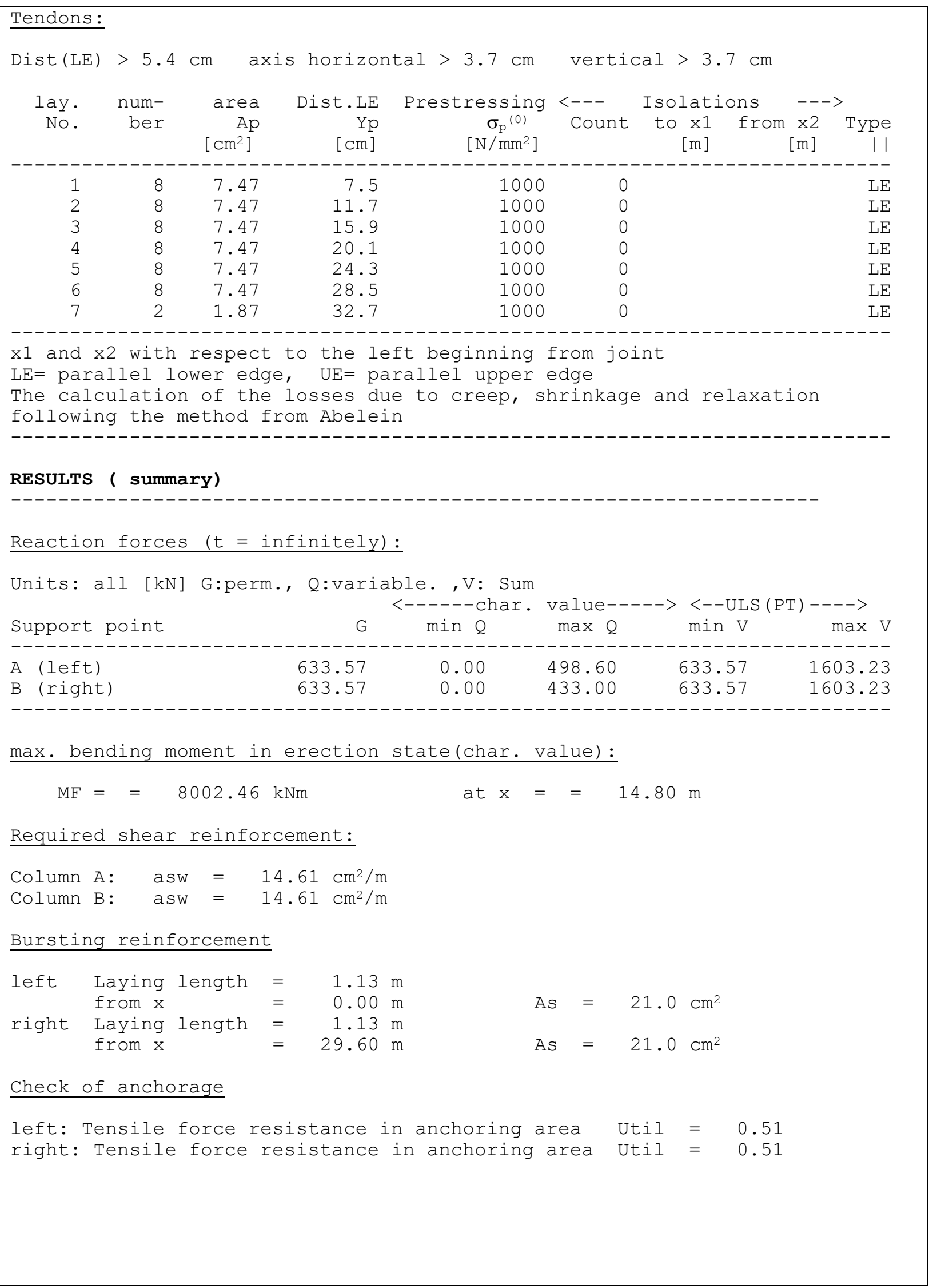


Overview crit. sections

Selected basic grid: 10 Sections

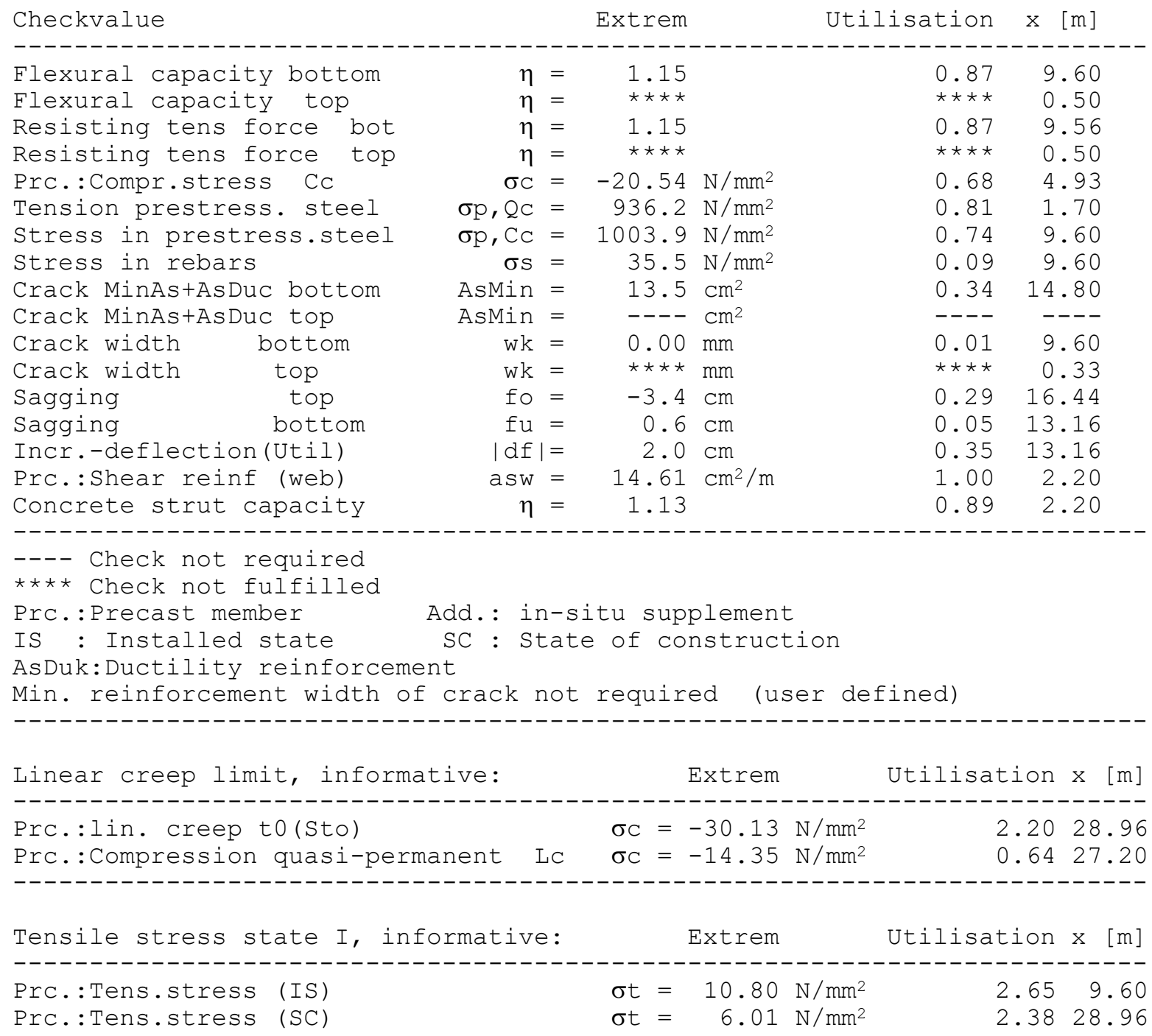




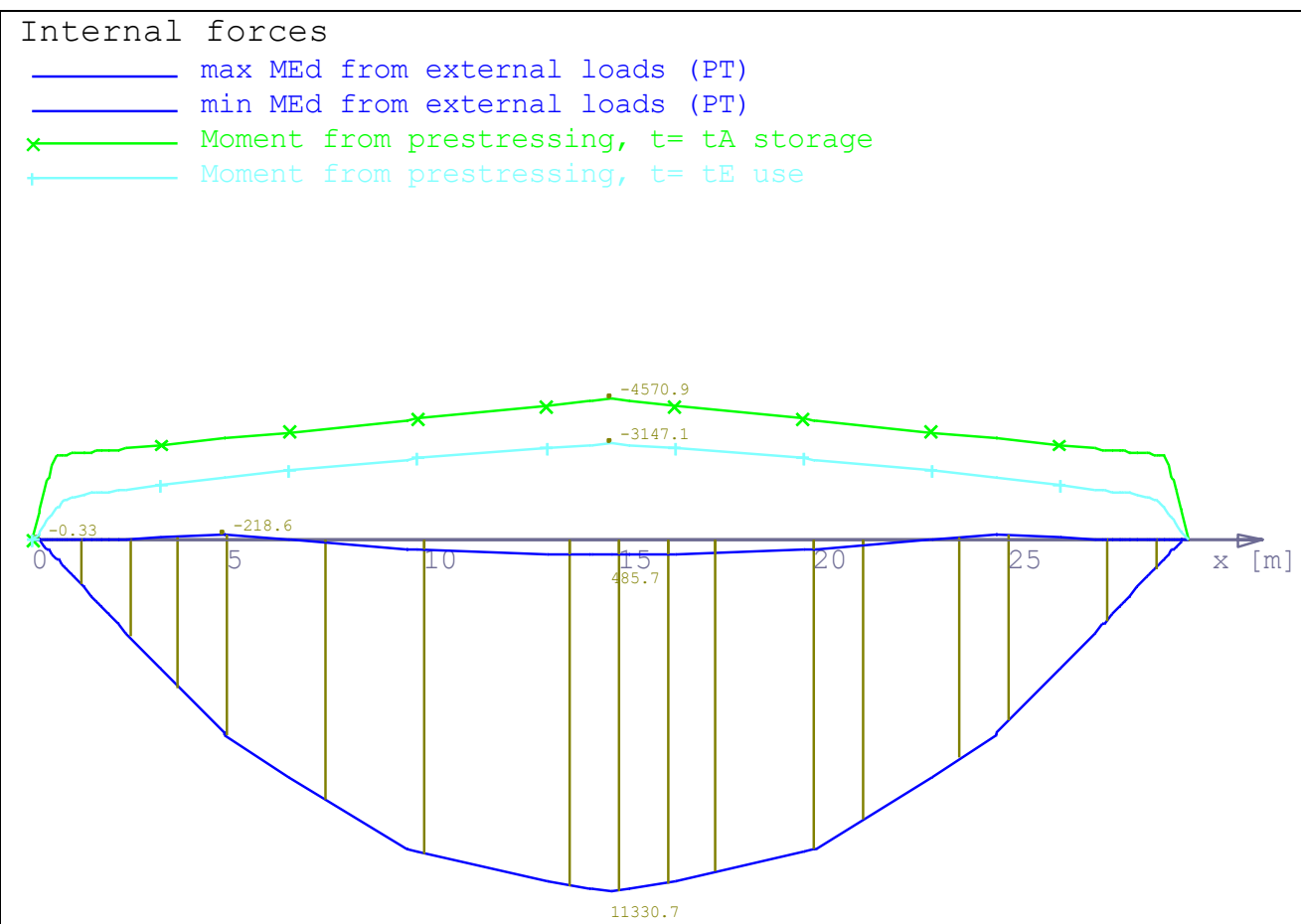

max VEd from external loads (PT)

min VEd from external loads (PT)

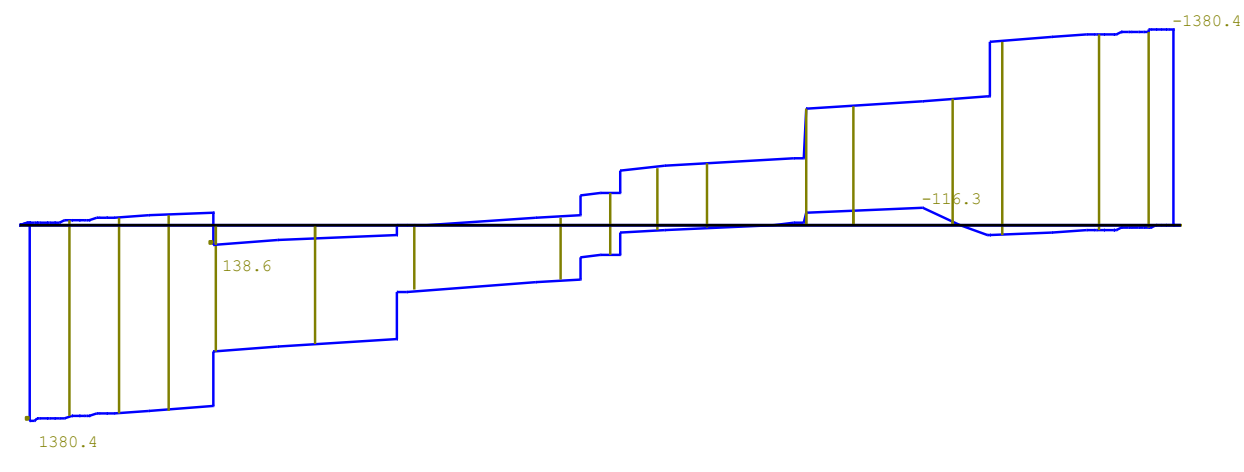

Deformation

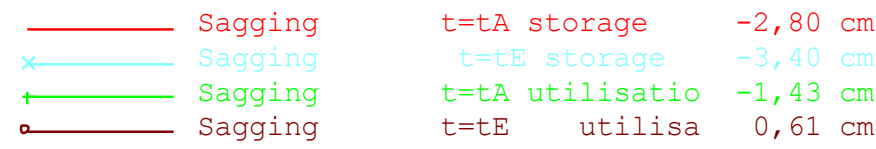

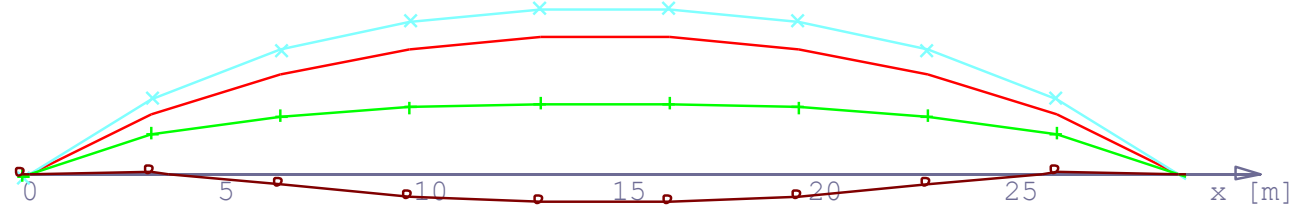


8 POS. C.04 - PRECAST CONCRETE COLUMN WITH BIAXIAL WIND LOADS

\subsection{SYSTEM}
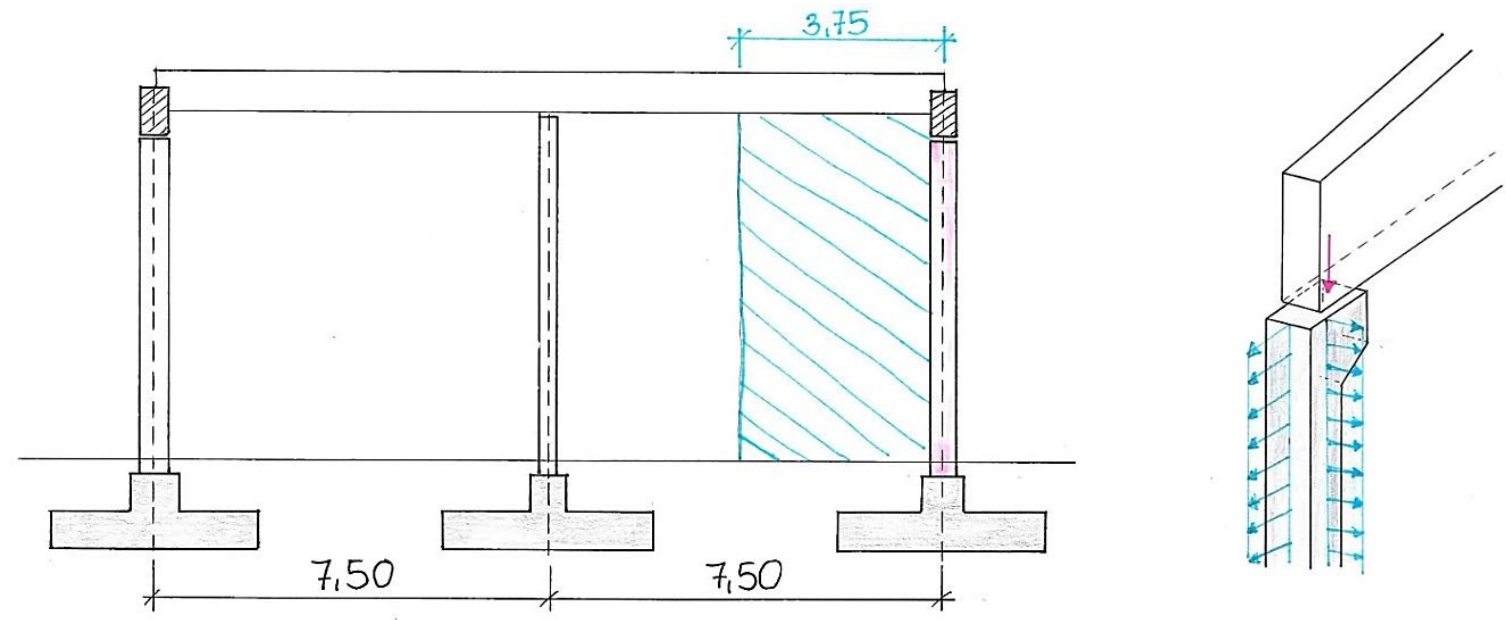

System

Cross-section

Building materials

Exposure class

Reinforcing Steel
Load Case I

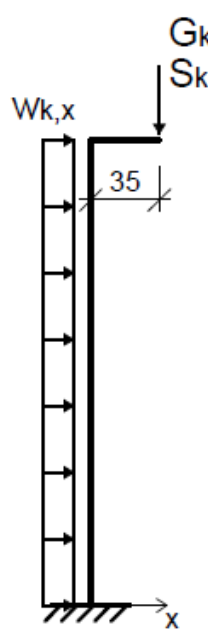

$\mathrm{b} / \mathrm{h}=50 / 50 \mathrm{~cm}$

\section{35/45 | B 500A}

XC1, wO

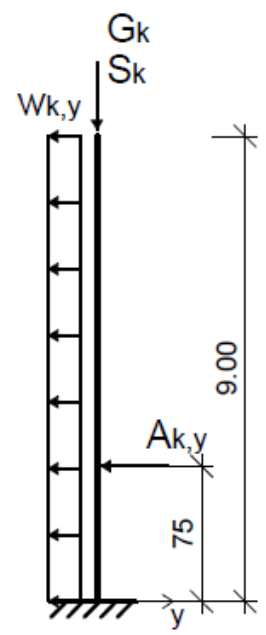

Load Case II

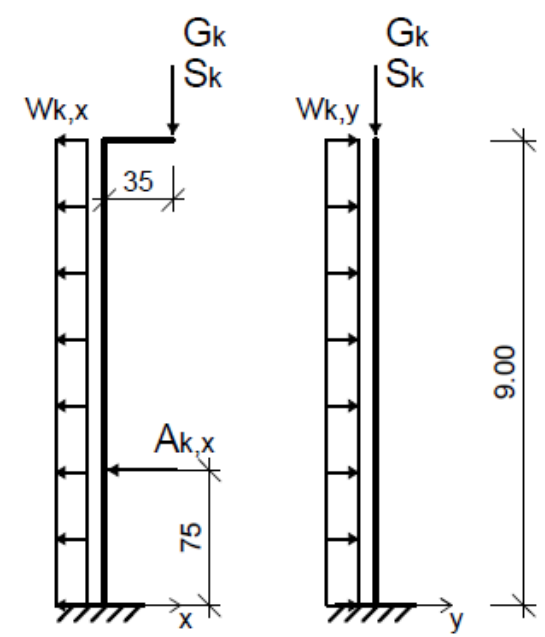

$\mathrm{c}_{\mathrm{nom}, \mathrm{w}}=\mathrm{c}_{\min }+\Delta \mathrm{c}_{\mathrm{dev}}=10 \mathrm{~mm}+10 \mathrm{~mm}=20 \mathrm{~mm}$

$\mathrm{c}_{\mathrm{nom}, \mathrm{s}}=\mathrm{c}_{\text {min }}+\Delta \mathrm{c}_{\mathrm{dev}}=25 \mathrm{~mm}+10 \mathrm{~mm}=35 \mathrm{~mm} \quad \rightarrow \mathrm{c}_{\mathrm{v}, \mathrm{w}, \text { min }}=25 \mathrm{~mm}$

Chosen $\quad c_{v}=25 \mathrm{~mm}$ 
Characteristic Loads

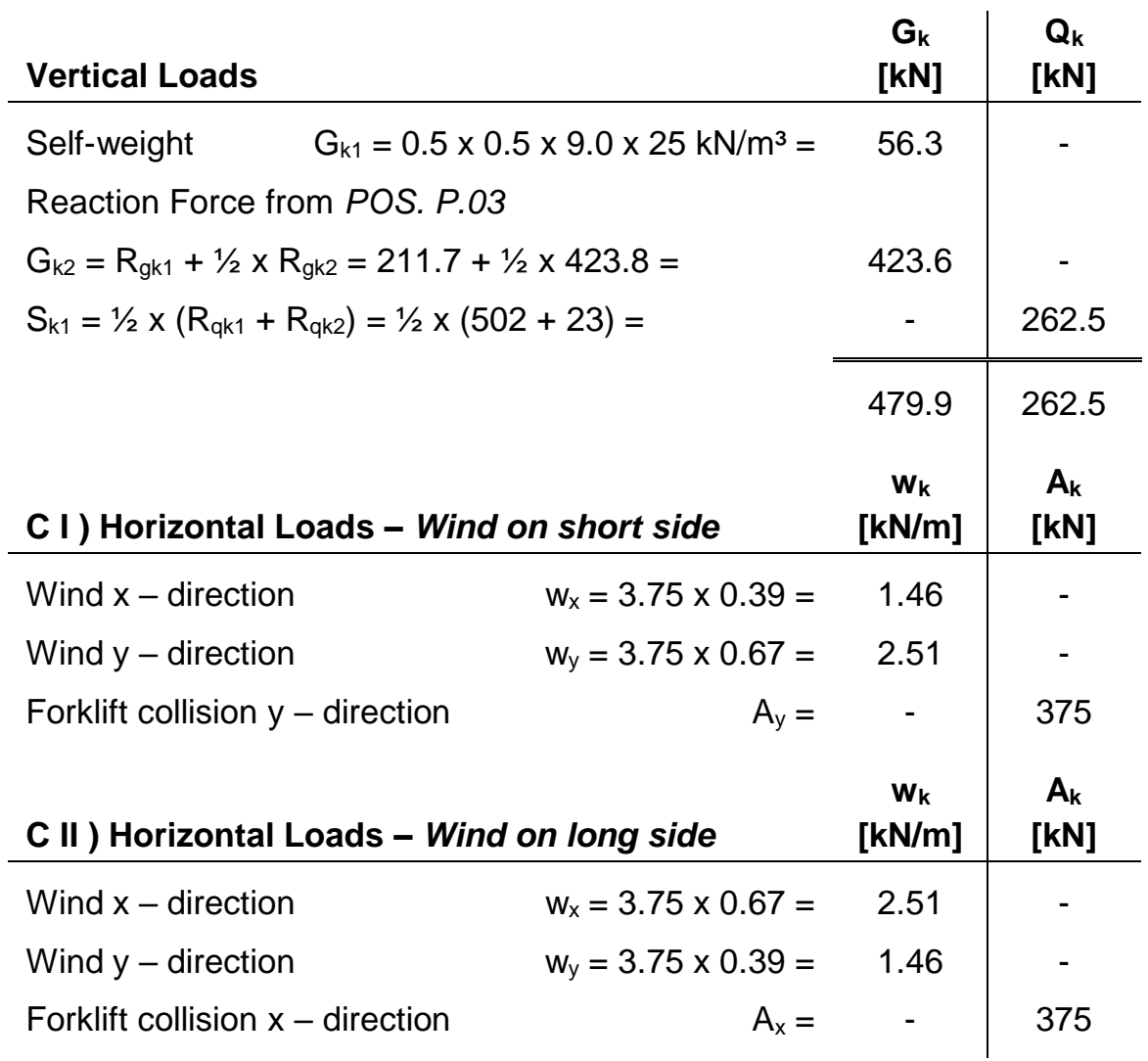

\subsection{INTERNAL FORCES}

\subsubsection{LOAD COMBINATIONS}

\begin{tabular}{|l|l|l|l|}
\hline \multicolumn{2}{|c|}{ Load combinations in the ultimate limit state } & $\psi$ \\
\hline LC1 & Maximum vertical load & $\begin{array}{l}Q_{E d}=1.35 \cdot G_{k} \oplus 1.5 \cdot S_{k} \\
q_{E d}=1.5 \cdot \psi_{0} \cdot w_{k}\end{array}$ & $\psi_{0}=0.6$ \\
\hline LC2 & Maximum horizontal load & $\begin{array}{l}Q_{E d}=1.35 \cdot G_{k} \oplus 1.5 \cdot \psi_{0} \cdot S_{k} \\
q_{E d}=1.5 \cdot w_{k}\end{array}$ & $\psi_{0}=0.5$ \\
& & $\begin{array}{l}Q_{E d}=1.0 \cdot G_{k} \oplus 0 \cdot S_{k} \\
q_{E d}=1.5 \cdot w_{k}\end{array}$ & \\
\hline LC3 & Minimum vertical load & $\begin{array}{l}Q_{E d}=1.0 \cdot G_{k} \oplus 1.0 A_{k} \oplus 1.5 \cdot \psi_{1} \cdot S_{k} \\
q_{E d}=1.5 \cdot \psi_{2} \cdot w_{k}\end{array}$ & $\begin{array}{l}\psi_{1}=0.2 \\
\psi_{2}=0\end{array}$ \\
\hline LC4 & Maximum vertical load with collision load & $\begin{array}{l}Q_{E d}=1.0 \cdot G_{k} \oplus 1.0 A_{k} \oplus 1.5 \cdot \psi_{2} \cdot S_{k} \\
q_{E d}=1.5 \cdot \psi_{1} \cdot w_{k}\end{array}$ & $\psi_{2}=0$ \\
& & Maximum horizontal load with collision load & $\psi_{1}=0.2$ \\
\hline LC6 & Minimum vertical load with collision load & $\begin{array}{l}Q_{E d}=1.0 \cdot G_{k} \oplus 1.0 A_{k} \\
q_{E d}=1.5 \cdot \psi_{1} \cdot w_{k}\end{array}$ & $\psi_{1}=0.2$ \\
\hline
\end{tabular}




\subsubsection{ECCENTRICITY}

Slenderness of the column $\quad i=0.289 \cdot h=0.289 \cdot 50=14.45 \mathrm{~cm} \quad \lambda=\frac{I_{0}}{i}=\frac{2 \cdot 900}{14.45}=125$

$80<\lambda=125<160 \rightarrow$ Slender column!

Limit slenderness

$\left|n_{E d}\right|=\frac{N_{E d}}{A_{c} \cdot f_{c d}}=\frac{1042}{50 \cdot 50 \cdot 1.98}=0.21<0.41 \quad \lambda_{\text {lim }}=\frac{16}{\sqrt{\left|n_{E d}\right|}}=\frac{16}{\sqrt{0.21}}=34.9$

$\lambda=125>\lambda_{\lim }=34.9 \rightarrow$ Second order effect must be considered

Eccentricity

$$
\begin{aligned}
& e_{t o t}=e_{i}+e_{2} \\
& e_{i}=\theta_{i} \cdot \frac{I_{0}}{2}=\frac{1}{100 \cdot \sqrt{l}} \cdot \frac{I_{0}}{2}=\frac{1}{100 \cdot \sqrt{9}} \cdot \frac{18}{2}=0.03 \mathrm{~m}=3 \mathrm{~cm} \\
& e_{2}=K_{1} \cdot \frac{l_{0}^{2}}{10} \cdot \frac{1}{r}=1.0 \cdot \frac{18^{2}}{207 \cdot 0.45 \cdot 10}=0.35 \mathrm{~m}=35 \mathrm{~cm} \\
& e_{\text {tot }}=e_{i}+e_{2}=3+35=38 \mathrm{~cm}
\end{aligned}
$$

\subsubsection{CHARACTERISTIC FORCES AT THE SUPPORT}

\begin{tabular}{|c|c|c|c|c|c|}
\cline { 2 - 6 } \multicolumn{1}{c|}{} & $\mathbf{N}_{\mathbf{k}}[\mathbf{k N}]$ & $\mathbf{M}_{\mathbf{y}, \mathbf{k}}[\mathbf{k N m}]$ & $\mathbf{V}_{\mathbf{x}, \mathbf{k}}[\mathbf{k N}]$ & $\mathbf{M}_{\mathbf{x}, \mathbf{k}}[\mathbf{k N m}]$ & $\mathbf{V}_{\mathbf{y}, \mathbf{k}}[\mathbf{k N}]$ \\
\hline $\mathbf{G}_{\mathbf{k}}$ & -479.9 & -168.0 & 0.0 & 0.0 & 0.0 \\
\hline $\mathbf{S}_{\mathbf{k}}$ & -262.5 & -91.9 & 0.0 & 0.0 & 0.0 \\
\hline $\mathbf{w}_{\mathbf{x}, \mathbf{k}}$ & 0.0 & $-15.8 /+27.1$ & $+3.5 /-6.0$ & 0.0 & 0.0 \\
\hline $\mathbf{w}_{\mathbf{y}, \mathbf{k}}$ & 0.0 & 0.0 & 0.0 & $+15.8 /-27.1$ & $+3.5 /-6.0$ \\
\hline $\mathbf{A}_{\mathbf{x}, \mathbf{k}}$ & 0.0 & +393.8 & -375.0 & 0.0 & 0.0 \\
\hline $\mathbf{A}_{\mathbf{y}, \mathbf{k}}$ & 0.0 & -393.8 & +375.0 & 0.0 & 0.0 \\
\hline
\end{tabular}

\subsubsection{DESIGN FORCES AT THE SUPPORT}

\begin{tabular}{|c|c|c|c|c|c|c|}
\cline { 2 - 7 } \multicolumn{2}{c|}{} & N $_{\text {Ed }}$ & M $_{\text {y,Ed }}$ & $\mathbf{V}_{\mathbf{x}, \text { Ed }}$ & $\mathbf{M}_{\text {x,Ed }}$ & $\mathbf{V}_{\mathbf{y}, \text { Ed }}$ \\
\hline \multirow{4}{*}{ C I } & LC1 & -1041.6 & -527.9 & 4.9 & -47.9 & -7.2 \\
\cline { 2 - 7 } & LC2 & -844.7 & -416.9 & 6.7 & -61.1 & -10.5 \\
\cline { 2 - 7 } & LC3 & -479.9 & -224.6 & 6.1 & -51.4 & -9.9 \\
\cline { 2 - 7 } & LC4 & -558.6 & -237.1 & 0.9 & -410.3 & -376.0 \\
\cline { 2 - 7 } & LC5 & -480.0 & -204.3 & 1.9 & -416.4 & -377.7 \\
\cline { 2 - 7 } & LC6 & -479.9 & -204.3 & 1.9 & -416.4 & -377.7 \\
\hline \multirow{2}{*}{ C II } & LC1 & -1041.6 & -444.1 & -7.2 & 35.9 & 4.9 \\
\cline { 2 - 7 } & LC2 & -844.7 & -314.1 & -10.5 & 41.8 & 6.7 \\
\hline
\end{tabular}




\begin{tabular}{|c|c|c|c|c|c|c|}
\hline LC3 & -479.9 & -140.0 & -9.9 & 33.2 & 6.1 \\
\cline { 2 - 6 } LC4 & -558.6 & 182.4 & -376.0 & 9.3 & 0.9 \\
\cline { 2 - 6 } LC5 & -479.9 & 225.1 & -377.7 & 12.9 & 1.9 \\
\cline { 2 - 6 } & LC6 & -479.9 & 225.1 & -377.7 & 12.9 & 1.9 \\
\hline
\end{tabular}

\subsubsection{MAXIMUM AND MINIMUM INTERNAL FORCES}

$\mathrm{Cl}-$ Wind on Short Side of the Building

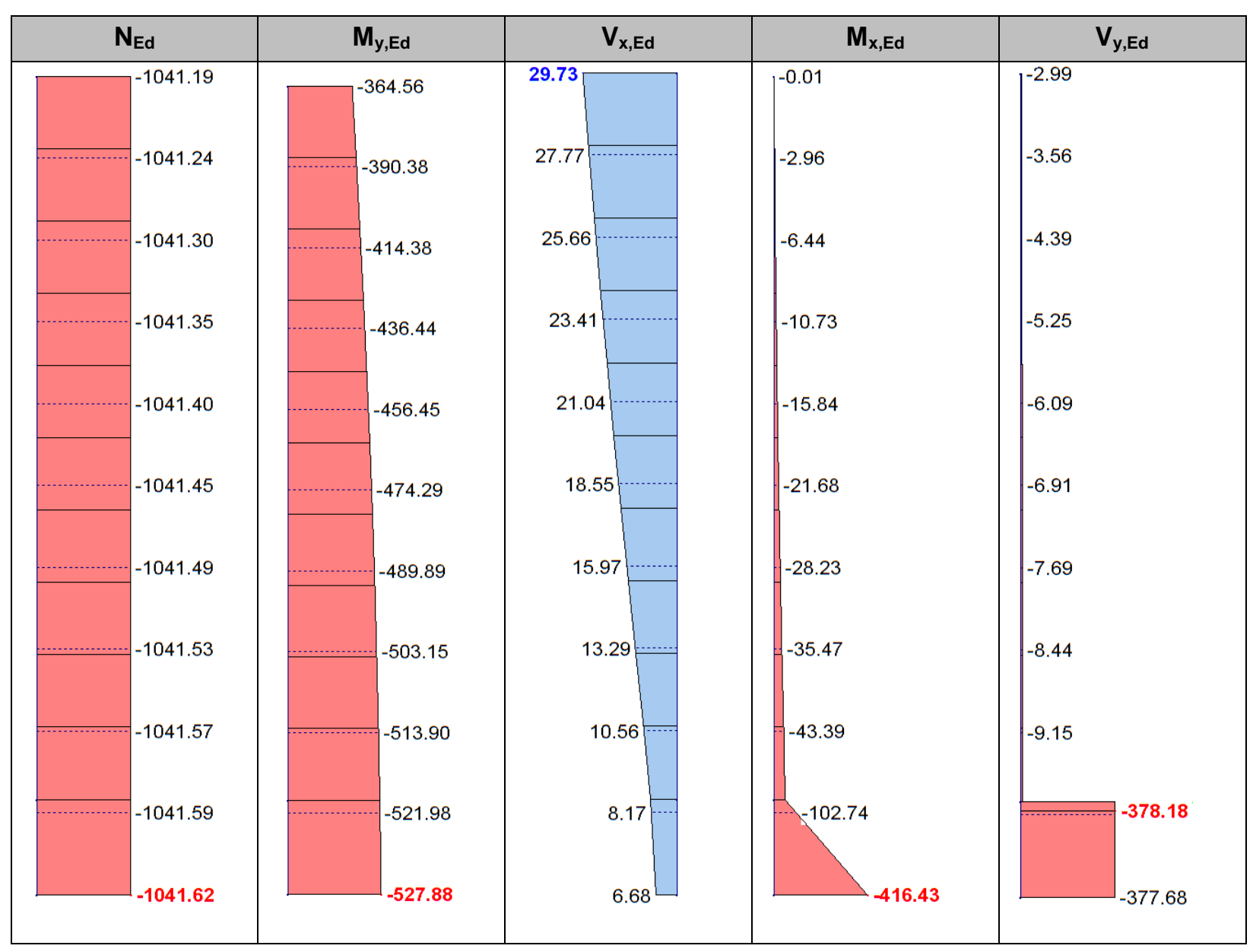


C II - Wind on Long Side of the Building

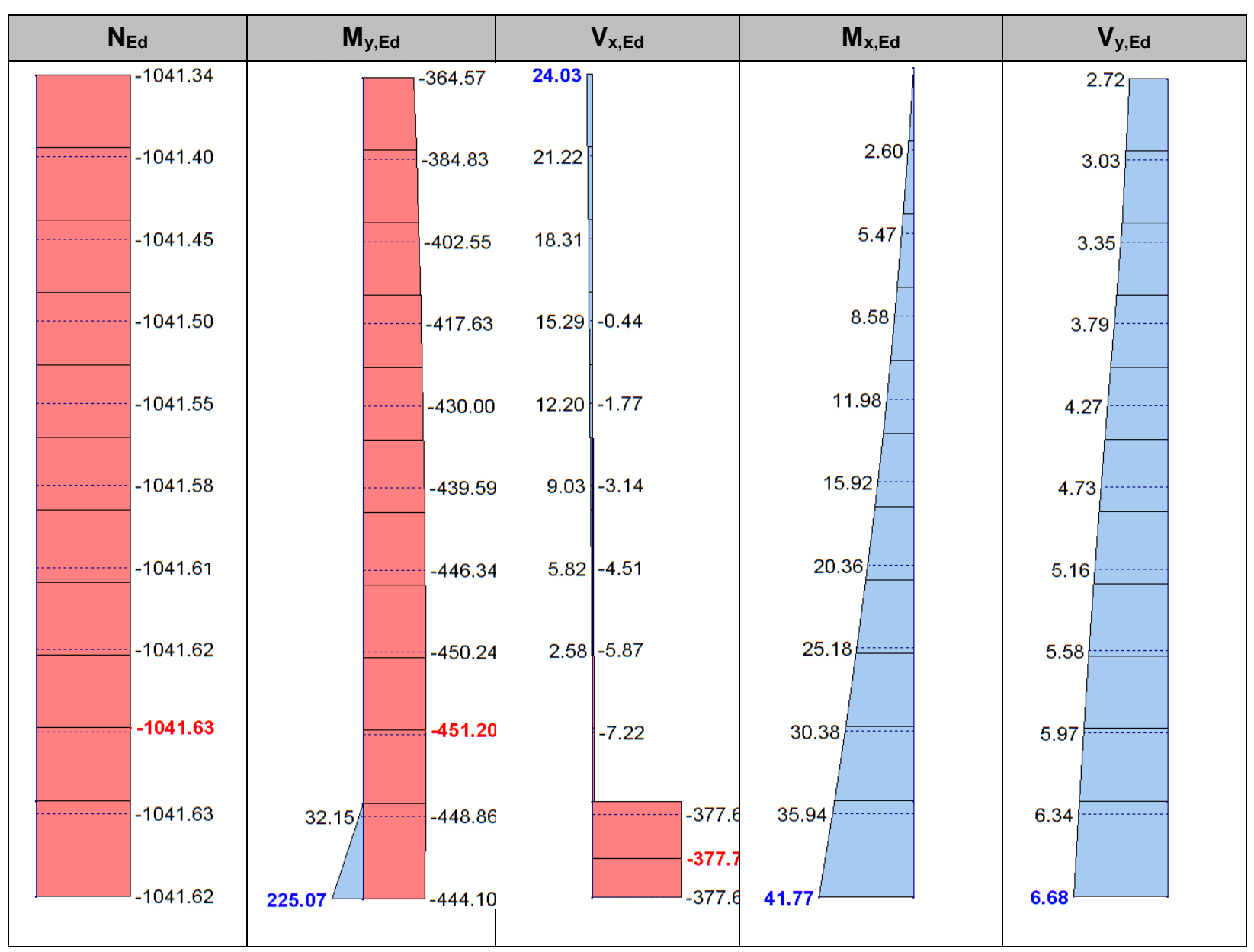

\subsection{ULTIMATE LIMIT STATE DESIGN}

$$
\begin{array}{ll}
\mu_{E d y}=\frac{M_{E d y}}{b \cdot h^{2} \cdot f_{c d}} & \mu_{E d x}=\frac{M_{E d x}}{b^{2} \cdot h \cdot f_{c d}} \quad \rightarrow \mu_{1}=\max \left\{\mu_{E d, y}, \mu_{E d, x}\right\} ; \mu_{2}=\min \left\{\mu_{E d, y}, \mu_{E d, x}\right\} \\
v=v_{E d}=\frac{N_{E d}}{b \cdot h \cdot f_{c d}} \quad \rightarrow \omega_{t o t}
\end{array}
$$

\begin{tabular}{|c|c|c|c|c|c|c|c|c|c|}
\hline & & $\mu_{\mathrm{Ed}, \mathrm{y}}$ & $\mu_{\mathrm{Ed}, \mathbf{x}}$ & $\mathbf{v}$ & $\mu_{\mathrm{Ed}, 1}$ & $\boldsymbol{\mu}_{\mathrm{Ed}, 2}$ & $\omega_{\text {tot }}$ & $A_{s, r e q}$ & $1 / 4 A_{s, \text { req }}$ \\
\hline \multirow{4}{*}{ C I } & LC1 & 0.213 & 0.019 & -0.210 & 0.213 & 0.019 & 0.8 & 91.03 & 22.76 \\
\hline & LC2 & 0.168 & 0.025 & -0.171 & 0.168 & 0.025 & 0.3 & 34.14 & 8.53 \\
\hline & LC3 & 0.091 & 0.021 & -0.097 & 0.091 & 0.021 & 0.175 & 19.91 & 4.98 \\
\hline & LC4 & 0.096 & 0.166 & -0.113 & 0.166 & 0.096 & 0.45 & 51.21 & 12.80 \\
\hline
\end{tabular}$$
A_{s, \text { req }}=\omega_{\text {tot }} \cdot \frac{b \cdot h}{f_{y d} / f_{c d}} \quad \text { on each side: } \frac{1}{4} A_{s, \text { req }}
$$ 


\begin{tabular}{|c|c|c|c|c|c|c|c|c|c|}
\hline \multirow{7}{*}{ LC5 } & 0.083 & 0.168 & -0.097 & 0.168 & 0.083 & 0.45 & $\mathbf{5 1 . 2 1}$ & $\mathbf{1 2 . 8 0}$ \\
\cline { 2 - 9 } & LC6 & 0.083 & 0.168 & -0.097 & 0.168 & 0.083 & 0.45 & $\mathbf{5 1 . 2 1}$ & $\mathbf{1 2 . 8 0}$ \\
\hline \multirow{4}{*}{ C II } & LC1 & 0.179 & 0.015 & -0.210 & 0.179 & 0.015 & 0.4 & $\mathbf{4 5 . 5 2}$ & $\mathbf{1 1 . 3 8}$ \\
\cline { 2 - 9 } & LC2 & 0.127 & 0.017 & -0.171 & 0.127 & 0.017 & 0.3 & $\mathbf{3 4 . 1 4}$ & $\mathbf{8 . 5 3}$ \\
\cline { 2 - 9 } & LC3 & 0.057 & 0.013 & -0.097 & 0.057 & 0.013 & 0.05 & $\mathbf{5 . 6 9}$ & $\mathbf{1 . 4 2}$ \\
\cline { 2 - 9 } & LC4 & 0.074 & 0.004 & -0.113 & 0.074 & 0.004 & 0.01 & $\mathbf{1 . 1 4}$ & $\mathbf{0 . 2 8}$ \\
\cline { 2 - 9 } & LC5 & 0.091 & 0.005 & -0.097 & 0.091 & 0.005 & 0.01 & $\mathbf{1 . 1 4}$ & $\mathbf{0 . 2 8}$ \\
\cline { 2 - 10 } & LC6 & 0.091 & 0.005 & -0.097 & 0.091 & 0.005 & 0.01 & $\mathbf{1 . 1 4}$ & $\mathbf{0 . 2 8}$ \\
\hline
\end{tabular}

Chosen

$4 \times 5 \varnothing 25=98.2 \mathrm{~cm}^{2}$

(5 Ø 25 on each side)

\subsection{SERVICEABILITY LIMIT STATE DESIGN}

According to DIN-EN-1992-1-1 no design in the serviceability limit state has to be done. No cracks are expected for the columns at serviceability limit state.

\subsection{DESIGN AND REINFORCEMENT}

\subsubsection{MINIMUM AND MAXIMUM LONGITUDINAL REINFORCEMENT}

Maximum reinforcement

Minimum reinforcement

$$
A_{s, \max }=\frac{1}{2} \cdot 0.09 \cdot A_{c}=\frac{1}{2} \cdot 0.09 \cdot 50 \cdot 50=112.5 \mathrm{~cm}^{2}
$$$$
A_{s, \min }=\frac{0.15 \cdot\left|N_{E d}\right|}{f_{y d}}=\frac{0.15 \cdot 1042}{43.5}=3.59 \mathrm{~cm}^{2}
$$

\subsubsection{TRANSVERSE REINFORCEMENT}

Minimum diameter

Maximum distance

$$
\min d_{s, b u ̈} \geq\left\{\begin{array}{l}
6 \mathrm{~mm} \\
0.25 \cdot \max d_{s, l}=0.25 \cdot 25=\underline{\underline{6.25 \mathrm{~mm}}}
\end{array}\right.
$$

$$
\min s_{w} \leq\left\{\begin{array}{l}
12 \cdot \min d_{s, l}=12 \cdot 25=300 \mathrm{~mm} \\
h_{\min }=500 \mathrm{~mm} \\
\underline{\underline{300 \mathrm{~mm}}}
\end{array}\right.
$$




\subsubsection{DESIGN OF THE CORBEL}

Forces on the corbel

$$
\begin{aligned}
& V_{E d}=1042 \mathrm{kN} \\
& T_{E d}=V_{E d} \cdot l_{\text {eff }} / 300=1042 \cdot 29.2 / 300=101 \mathrm{kNm} \\
& H_{E d, y}^{T}=T_{E d} / z_{H E D}=101 \mathrm{kNm} / 1.32 \mathrm{~m}=76.5 \mathrm{kN} \\
& H_{E d, y}^{W}=3 \mathrm{kN} \quad H_{E d, x}^{w}=30 \mathrm{kN}
\end{aligned}
$$

\begin{tabular}{|c|c|c|}
\hline Bending moment & $M_{E d}=101 \mathrm{kNm}$ & \\
\hline Static height & $d=50-2-1-1=46 \mathrm{~cm}$ & \\
\hline Flexure design & $\mu_{E d s}=\frac{M_{E d s}}{b \cdot d^{2} \cdot f_{c d}}=\frac{10100}{30 \cdot 46^{2} \cdot 1.98}=0.08$ & $\rightarrow \omega=0.0836$ \\
\hline Reinforcement & $A_{s, \text { req }}=\omega \cdot b \cdot d \cdot f_{c d} / \sigma_{s d}=0.0836 \cdot 30 \cdot 46$ & $435=5.25 \mathrm{~cm}^{2}$ \\
\hline Chosen & $2 \varnothing 20=6.28 \mathrm{~cm}^{2}$ & dinal reinforc \\
\hline
\end{tabular}

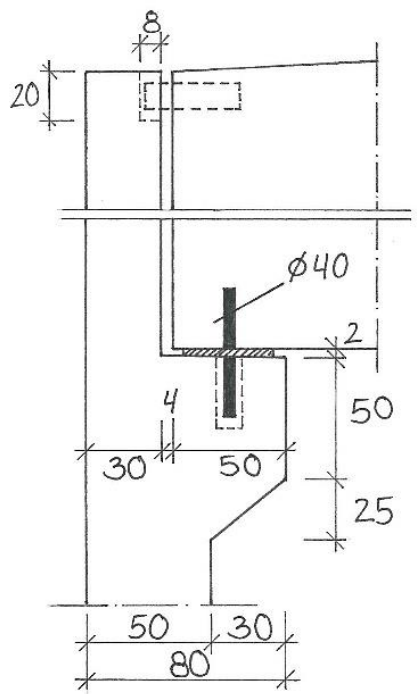

Flexure Design of the Column

Shear Design of the Column

Shear force

Shear force resistance

Reinforcement longitudinal reinforcement

Supplementary Reinforcement

$$
H_{E d, y}=76.5 k N+3 k N=79.5 k N
$$

$$
V_{R d \text { max }}=0.75 \cdot 30 \cdot 0.9 \cdot 46 \cdot 1.98 \cdot \frac{1.2}{1+1.2^{2}}=458 \mathrm{kN} \quad(\cot \theta \approx 1.2)
$$$$
V_{E d}=H_{E d, y}=79.5 \mathrm{kN}<V_{R d \text { max }}=458 \mathrm{kN} \rightarrow \text { The compression strut is ok. }
$$

$$
a_{s w, r e q}=\frac{V_{E d}}{f_{y d} \cdot z \cdot \cot \theta}=\frac{79.5 \mathrm{kN}}{43.5 \cdot 0.9 \cdot 0.46 \cdot 1.2}=3.68 \mathrm{~cm}^{2} / \mathrm{m}
$$

$$
A_{s, \text { req }}=H_{E d}^{T} / f_{y d}=79.5 / 43.5=1.83 \mathrm{~cm}^{2}
$$

Bearing Load 
Compression stresses $\quad \sigma_{E d}=\frac{V_{E d}}{a_{L} \cdot b_{L}}=\frac{1042 \mathrm{kN}}{35 \cdot 35} \cdot 10=8.5 \mathrm{MN} / \mathrm{m}^{2} \leq 0.85 \cdot f_{c d}=0.85 \cdot 19.8=16.8 \mathrm{MN} / \mathrm{m}^{2} \checkmark$

Main Reinforcement of the Corbel

Static height

Tensile force

Reinforcement
Chosen

Provided static height

Reinforcement

Anchorage at the support

Overlap length

$$
\begin{aligned}
& I_{b, r q d}=81 \mathrm{~cm} \quad(\varnothing 25) \\
& I_{b d}=\alpha_{1} \cdot I_{b, \text { rqd }} \cdot\left(A_{s, \text { req }} / A_{s, \text { prov }}\right)=1.0 \cdot 81 \cdot(20.5 / 24.6)=67.5 \mathrm{~cm} \\
& I_{0}=\alpha_{6} \cdot I_{b d}=2.0 \cdot 67.5=\underline{\underline{135 \mathrm{~cm}}}>I_{0, \min } \\
& I_{0, \text { min }}=0.3 \cdot \alpha_{6} \cdot I_{b, \text { rqd }}=0.3 \cdot 2.0 \cdot 81=\underline{\underline{48.6 \mathrm{~cm}}} \geq\left\{\begin{array}{l}
15 \cdot 2.5=37.5 \mathrm{~cm} \\
20 \mathrm{~cm}
\end{array}\right.
\end{aligned}
$$$$
\rightarrow I_{0}=135 \mathrm{~cm}
$$

reinforcement loop (3 layers)

$d_{\text {prov }}=75-2-1-1.4-2-1.4 / 2=75-7.1=67.9 \mathrm{~cm}<d_{\text {est }}=69 \mathrm{~cm}$

$F_{\text {sd }}=\frac{1.1 \cdot 27}{0.85 \cdot 67.9} \cdot 1040+30=565 \mathrm{kN}$

$A_{s, \text { req }}=\frac{F_{s d}}{f_{y d}}=\frac{565}{43.5}=13.0 \mathrm{~cm}^{2}<A_{s, p r o v}=18.48 \mathrm{~cm}^{2}$

for C35/45 and moderate bond

4 $(20.5 / 24.6)=37.2 \mathrm{~cm}$

$>I_{b, \min }=0.3 \cdot 0.7 \cdot 64=13.4 \mathrm{~cm}>10 \phi_{s}=\underline{\underline{14.0 \mathrm{~cm}}}$

$I_{b, \text { dir }}=2 / 3 \cdot I_{b d}=2 / 3 \cdot 37.2=\underline{\underline{24.8 \mathrm{~cm}}}>6.7 \phi_{s}=9.4 \mathrm{~cm}$

$I_{b, \text { dir }, \text { prov }}==23+17.5-2-1.4-1=36.1 \mathrm{~cm}>I_{b, \text { dir }, \text { req }}=24.8 \mathrm{~cm} \checkmark$

Secondary Reinforcement

Crosswise reinforcement

$A_{s, \text { net,req }}=0.5 \cdot A_{s}=0.5 \cdot 13.0=7.5 \mathrm{~cm}^{2}$

Chosen

$5 \varnothing 10=7.85 \mathrm{~cm}^{2}$ 
Design of the Shear Force Dowel

Horizontal force

$H_{E d}=\sqrt{30^{2}+79.5^{2}}=85 \mathrm{kN}$

Shear force resistance

$W_{\text {dowel }}=r^{3} \cdot \pi / 4=2.0^{3} \cdot \pi / 4=6.28 \mathrm{~cm}^{3}$

$H_{R d}=1.25 \cdot f_{y d} \cdot \frac{W_{\text {dowel }}}{t / 2 \cdot d_{\text {dowel }}}=1.25 \cdot 43.5 \cdot \frac{6.28}{1.0+4.0}=68.3 \mathrm{kN}$ (per dowel)

2 dowels $\rightarrow H_{R d}=2 \cdot 68.3 k N=137 k N>H_{E d}=85 k N \checkmark$

Chosen

Distance to the edge

\section{2 dowels $\varnothing 40$}

$x$ - direction: $\quad 3 \cdot d_{s}=12 \mathrm{~cm}<u_{x}=23 \mathrm{~cm}<8 \cdot d_{s}=32 \mathrm{~cm}$

Supplementary reinforcement necessary:

$A_{s, x, \text { req }}=H_{E d} / f_{y d}=30.0 / 43.5=0.7 \mathrm{~cm}^{2}$

$y$-direction: $\quad 3 \cdot d_{s}=12 \mathrm{~cm}<u_{y}=17 \mathrm{~cm}<8 \cdot d_{s}=32 \mathrm{~cm}$

Supplementary reinforcement necessary:

$A_{s, x, r e q}=H_{E d} / f_{y d}=79.5 / 43.5=1.83 \mathrm{~cm}^{2}$

\subsection{TRANSPORT AND ASSEMBLY}

\subsubsection{TRANSPORT AND LIFTING ON THE CONSTRUCTION SITE}

The beam will be suspended with the help of two anchors on each side above the center of gravity at around $1 / 6$ of the length of the beam. Which might cause tension over the anchors and might require upper reinforcement.

System

In the following the static system during transportation and assembly can be seen.

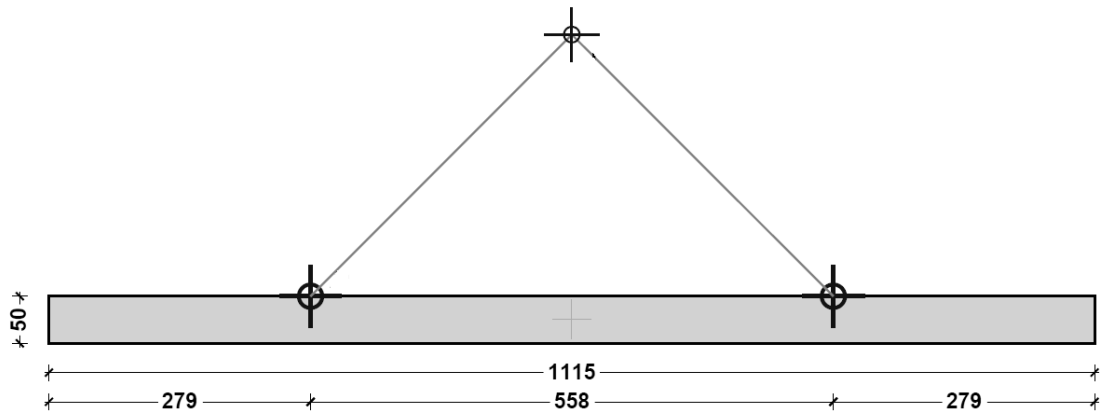


Simplified static system:

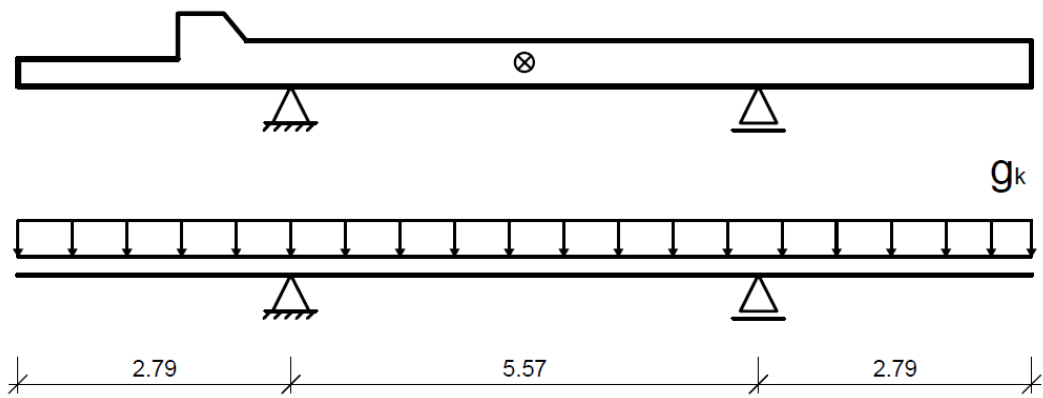

Design

\begin{tabular}{|c|c|c|}
\hline \multirow[t]{2}{*}{ Lifting loads } & $A=5.65 \mathrm{~m}^{2}$ & $V=5.65 \times 0.50=2.825 \mathrm{~m}^{3}$ \\
\hline & self-weight & $g_{k}=2.825 m^{3} \times 25 \mathrm{kN} / \mathrm{m}^{3} / 11.15 \mathrm{~m}=6.33 \mathrm{kN} / \mathrm{m}$ \\
\hline
\end{tabular}

Bending moment $\quad M_{E d}^{G}=1.15 \cdot(-6.33) \cdot 2.79^{2} / 2=-28.3 \mathrm{kNm}$

Time of first lifting approximately after 5 days $(t=5 d)$

Compressive strength at time $t$

$$
\begin{aligned}
& f_{c m}(t)=\beta_{c c}(t) \cdot f_{c m} \quad \rightarrow \quad \beta_{c c}(t=5 d)=e^{s(1-\sqrt{28 / t})}=e^{0.2(1-\sqrt{28 / 5})}=0.76 \\
& f_{c m}(t=5 d)=0.76 \cdot 43=32.68 \mathrm{~N} / \mathrm{mm}^{2} \\
& f_{c k}(t=5 d)=f_{c m}(t)-8=32.68-8=24.68 \mathrm{~N} / \mathrm{mm}^{2} \\
& f_{c c}(t=5 d)=0.85 \cdot f_{c k}(t) / 1.5=0.85 \cdot 24.68 / 1.5=\underline{\underline{14.0 \mathrm{~N} / \mathrm{mm}^{2}}}
\end{aligned}
$$

Required reinforcement

$$
\begin{aligned}
\mu_{E d s}= & \frac{M_{E d s}}{b \cdot d^{2} \cdot f_{c d}}=\frac{2830}{50 \cdot 45^{2} \cdot 1.4}=0.02 \rightarrow \omega=0.0203 \\
A_{s 2, \text { req }} & =\left(\omega \cdot b \cdot d \cdot f_{c d}+N_{E d}\right) / \sigma_{s d} \\
& =0.0203 \cdot 50 \cdot 45 \cdot 1.40 / 43.5=\underline{\underline{1.47 \mathrm{~cm}^{2}}}<A_{s, \text { prov }}=24.55 \mathrm{~cm}^{2}
\end{aligned}
$$

No additional reinforcement is required!

\subsubsection{TRANSPORT ANCHORS}

In order to lift the beam out of its formwork as well as to be able to transport and assemble it on site, transport anchors are needed. Those are installed during the production of the beam and must be designed in accordance with the VDI/BV-BS-Richtlinie 6205. For this project the anchors of the company Halfen are chosen.

Self-weight

$$
F_{G}=6.33 \mathrm{kN} / \mathrm{m} \cdot 11.15 \mathrm{~m}=70.6 \mathrm{kN}
$$


Formwork adhesion

Dynamic factor

Factor for inclination

Tensile force

\section{Chosen}

Load capacity of the anchor

$$
F_{a d h}=q_{a d h} \cdot A_{t} \quad \rightarrow \text { no adhesion here because of hinged formwork! }
$$$$
\psi_{\text {dyn }}=1.3
$$

$$
z=1.41 \text { for } \beta=45^{\circ}
$$

$$
F_{z}=\frac{F_{G} \cdot \psi_{d y n} \cdot z}{n}=\frac{70.6 \cdot 1.3 \cdot 1.41}{2}=\underline{\underline{64.7 \mathrm{kN}}}
$$

\begin{tabular}{|c|c|c|c|c|c|c|c|c|c|}
\hline \multirow{3}{*}{$\begin{array}{l}\text { Load } \\
\text { class }\end{array}$} & \multirow{3}{*}{ Article number } & \multirow{3}{*}{$\begin{array}{l}\text { Anchor } \\
\text { length } \\
\text { । } \\
{[\mathrm{mm}]}\end{array}$} & \multirow{3}{*}{$\begin{array}{c}\text { Minimum height } \\
\text { of beams } \\
B_{1} \\
{[\mathrm{~mm}]}\end{array}$} & \multirow{3}{*}{$\begin{array}{c}\begin{array}{c}\text { Wall } \\
\text { thickness }\end{array} \\
2 \times \mathrm{e}_{\mathrm{r}} \\
{[\mathrm{mm}]}\end{array}$} & \multicolumn{4}{|c|}{ Load capacity $[\mathrm{kN}]$ at concrete strength $\mathrm{f}_{\mathrm{ci}}$ for } & \multirow{3}{*}{$\begin{array}{c}\text { Axial spacing of } \\
\text { anchors } \\
\mathrm{e}_{\mathrm{z}} \\
{[\mathrm{mm}]}\end{array}$} \\
\hline & & & & & $\begin{array}{c}\text { Axial pull } \\
\text { up to } 30^{\circ}[\beta]\end{array}$ & $\begin{array}{l}\text { Diagonal pull } \\
\text { up to } 60^{\circ}[\beta]\end{array}$ & $\begin{array}{l}\text { Axial pull and } \\
\text { diagonal pull } \\
\text { up to } 60^{\circ}[\beta]\end{array}$ & $\begin{array}{l}\text { Axial pull and } \\
\text { diagonal pull } \\
\text { up to } 60^{\circ}[\beta]\end{array}$ & \\
\hline & & & & & $15 \mathrm{~N} / \mathrm{mm}^{2}$ & $15 \mathrm{~N} / \mathrm{mm}^{2}$ & $25 \mathrm{~N} / \mathrm{mm}^{2}$ & $35 \mathrm{~N} / \mathrm{mm}^{2}$ & \\
\hline \multirow{9}{*}{10,0} & \multirow{3}{*}{$6000-10,0-0170$} & \multirow{3}{*}{170} & \multirow{3}{*}{340} & 300 & 46.4 & 37.2 & 60.0 & 70.9 & \multirow{3}{*}{520} \\
\hline & & & & 350 & 52.1 & 41.7 & 67.3 & 79.6 & \\
\hline & & & & 400 & 57.6 & 46.1 & 74.4 & 88.0 & \\
\hline & \multirow{3}{*}{$6000-10,0-0340$} & \multirow{3}{*}{340} & \multirow{3}{*}{680} & 280 & 76.6 & 61.3 & 98.9 & \multirow{3}{*}{100.0} & \multirow{3}{*}{1030} \\
\hline & & & & 300 & 80.7 & 64.5 & 1000 & & \\
\hline & & & & 320 & 84.7 & 67.7 & 100.0 & & \\
\hline & \multirow{3}{*}{$6000-10,0-0680$} & \multirow{3}{*}{680} & \multirow{3}{*}{1360} & 160 & 73.7 & 70.0 & 95.2 & \multirow{3}{*}{100.0} & \multirow{3}{*}{2050} \\
\hline & & & & 180 & 83.0 & 76.5 & \multirow{2}{*}{100.0} & & \\
\hline & & & & 200 & 92.2 & 82.8 & & & \\
\hline
\end{tabular}

\section{HALFEN DEHA Spherical head anchor 6000-10,0-0170}

$$
F_{z, R d}=67.3 k N>F_{z, E d}=64.7 k N \checkmark \quad \text { (see Table 18) }
$$

Table 18 - Sizing table for 'HALFEN DEHA Lifting Anchor Systems' 


\subsection{SOFTWARE CALCULATIONS}

\section{Demo Frilo Nemetschek}

\section{Position: C.04 - Precast Concrete Column with Biaxial Wind Loads}

Reinforced Concrete Column B5 01/2018 (Frilo R-2018-1/P12)

CANTILEVER COLUMN, Rectangle, 2-axial strained

Calculation base: DIN EN 1992-1-1/NA/A1:2015-12

$\mathrm{E}=34000 \mathrm{~N} / \mathrm{mm} 2 \quad \rho=2500 \mathrm{~kg} / \mathrm{m} 3$

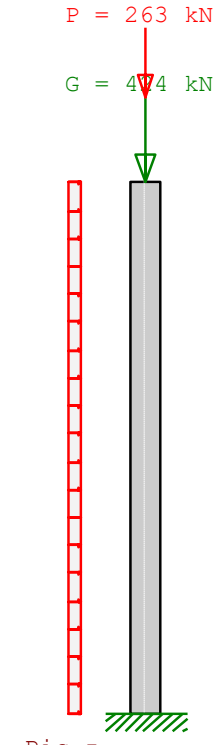

$\stackrel{\text { Ric }}{\longrightarrow}$

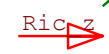

$1 \quad$ Mery $=$

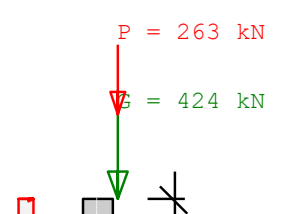

C $35 / 45$ B500A

$=2.01$

Reinf. along perimeter 1/4 Per side

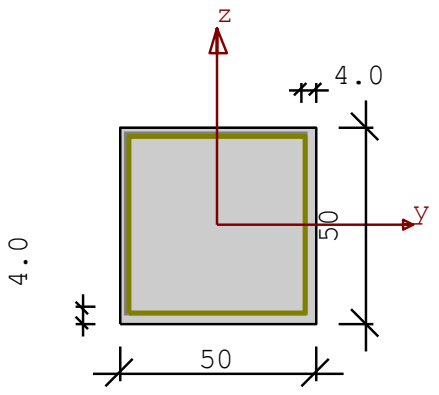

thns,

$66.87 \mathrm{kNm} \quad \operatorname{Mcrz}=66.87 \mathrm{kNm}$

NODES - LOADS :

LCNo. KNo. $\quad \mathrm{V} \quad$ ey $\quad$ ez $\quad$ Py $\quad$ Pz $\quad$ My $\quad$ Mz act con alt

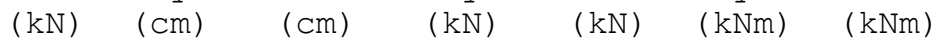

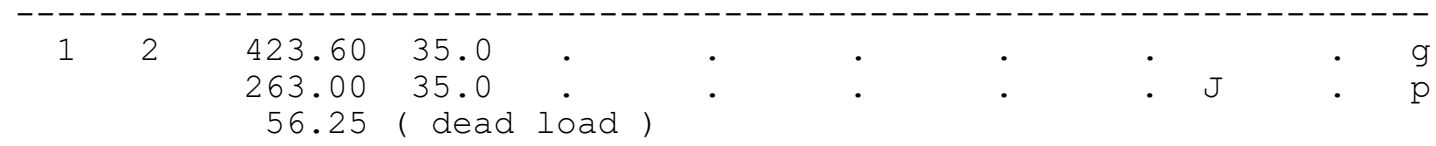

MEMBER - LOADS :

LCNo MNo type dir g1 g2 dist length actGrp con alt

$(\mathrm{kN} / \mathrm{m}, \mathrm{kN}) \quad(\mathrm{m}) \quad(\mathrm{m})$

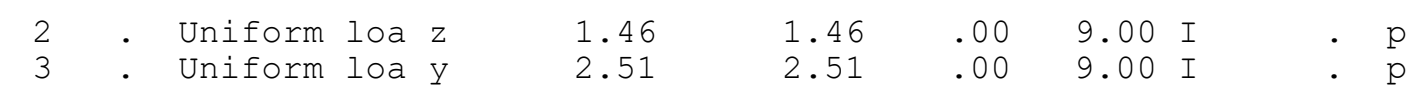




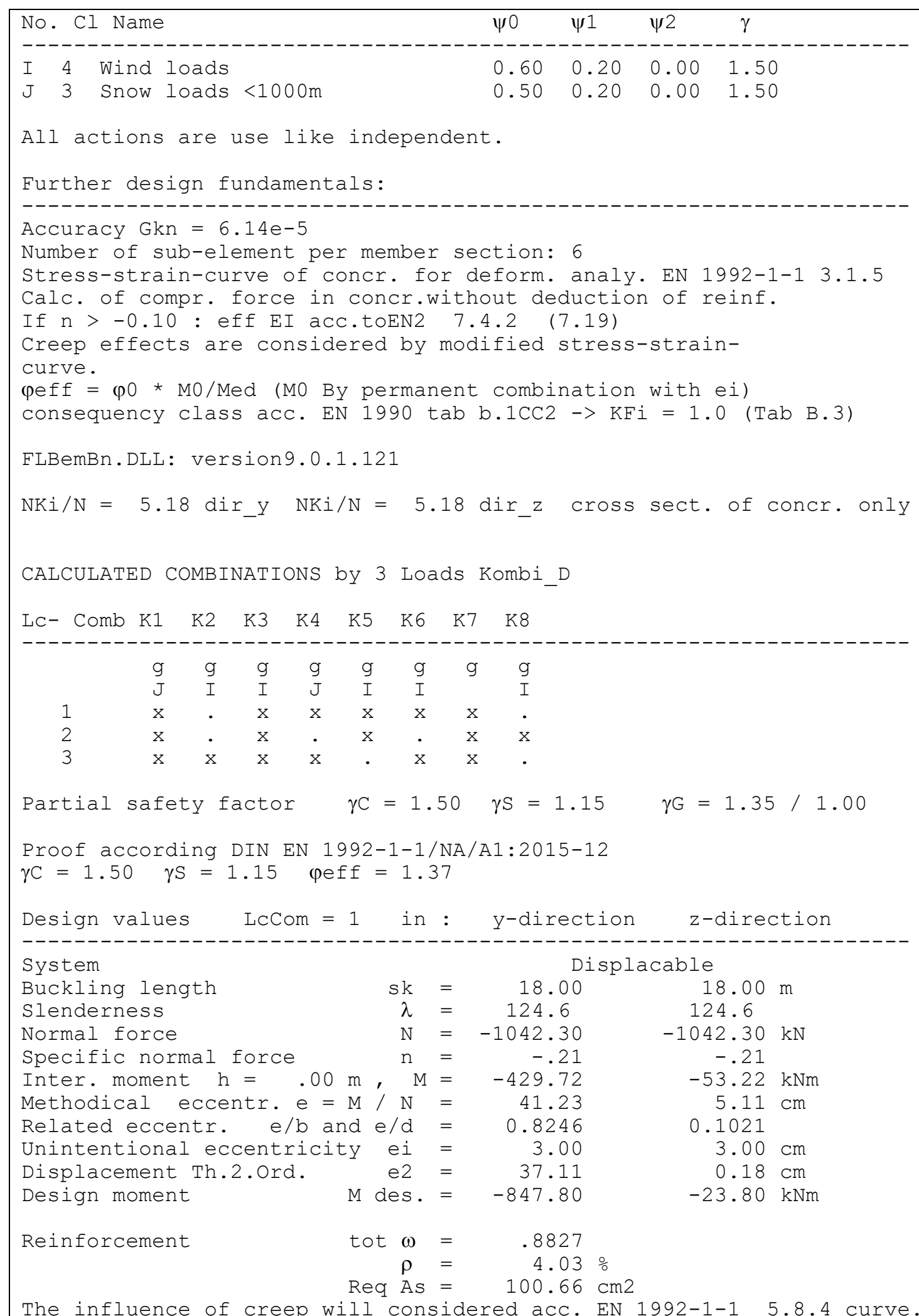

The influence of creep will considered acc. EN 1992-1-1 5.8.4 curve. 


\section{POS. C.05 - PRECAST CONCRETE COLUMN WITH MAXIMUM VERTICAL LOAD}

\subsection{SYSTEM}

System

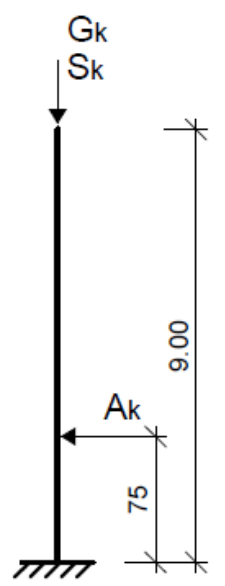

Cross-section

$b / h=50 / 50 \mathrm{~cm}$

Building materials

C 35/45 | B 500A

Exposure class

XC1, WO

Reinforcing steel

$$
\begin{aligned}
& \mathrm{c}_{\mathrm{nom}, \mathrm{w}}=\mathrm{c}_{\text {min }}+\Delta \mathrm{c}_{\text {dev }}=10 \mathrm{~mm}+10 \mathrm{~mm}=20 \mathrm{~mm} \\
& \mathrm{c}_{\text {nom }, \mathrm{s}}=\mathrm{c}_{\text {min }}+\Delta \mathrm{c}_{\mathrm{dev}}=25 \mathrm{~mm}+10 \mathrm{~mm}=35 \mathrm{~mm} \quad \rightarrow \mathrm{c}_{\mathrm{v}, \mathrm{w}, \text { min }}=25 \mathrm{~mm} \\
& \text { Chosen } \quad \mathrm{c}_{\mathrm{nom}}=25 \mathrm{~mm}
\end{aligned}
$$

\begin{tabular}{|c|c|c|c|}
\hline Vertical Loads & & $\begin{array}{c}G_{k} \\
{[k N]}\end{array}$ & $\begin{array}{c}Q_{k} \\
{[k N]}\end{array}$ \\
\hline Self-weight & $\mathrm{G}_{\mathrm{k} 1}=0.5 \times 0.5 \times 9.0 \times 25 \mathrm{kN} / \mathrm{m}^{3}=$ & 56.3 & - \\
\hline \multicolumn{4}{|c|}{ Reaction Force from POS. P.03 } \\
\hline & $G_{k 2}=2 \times(211.7+423.8)=$ & 1271.0 & - \\
\hline & $S_{k}=2 \times(502+23)=$ & - & 1050.0 \\
\hline & & 1327.3 & 1050.0 \\
\hline \multicolumn{3}{|l|}{ Horizontal Loads } & $\begin{array}{c}A_{k} \\
{[k N]}\end{array}$ \\
\hline Forklift collision & $A_{k}=$ & - & 375.0 \\
\hline
\end{tabular}

Characteristic loads 


\subsection{INTERNAL FORCES}

\subsubsection{LOAD COMBINATIONS}

\begin{tabular}{|l|l|l|c|}
\hline \multicolumn{2}{|c|}{ Load combinations in the ultimate limit state } & $\psi$ \\
\hline LC1 & Maximum vertical load & $Q_{E d}=1.35 \cdot G_{k} \oplus 1.5 \cdot S_{k}$ & \\
\hline LC2 & Vertical load with collision load & $Q_{E d}=1.0 \cdot G_{k} \oplus 1.0 A_{k} \oplus 1.5 \cdot \psi_{1} \cdot S_{k}$ & $\psi_{1}=0.2$ \\
\hline LC3 & Minimum vertical load with collision load & $Q_{E d}=1.0 \cdot G_{k} \oplus 1.0 A_{k}$ & \\
\hline
\end{tabular}

\subsubsection{ECCENTRICITY}

Slenderness of the column

$$
i=0.289 \cdot h=0.289 \cdot 50=14.45 \mathrm{~cm}
$$$$
\lambda=\frac{I_{0}}{i}=\frac{2 \cdot 900}{14.45}=125
$$$$
80<\lambda=125<160 \rightarrow \text { Slender column! }
$$

Limit slenderness

$$
\begin{aligned}
& \left|n_{E d}\right|=\frac{N_{E d}}{A_{c} \cdot f_{c d}}=\frac{3367}{50 \cdot 50 \cdot 1.98}=0.68>0.41 \quad \lambda_{\text {lim }}=25 \\
& \lambda=125>\lambda_{\text {lim }}=25 \quad \rightarrow \text { Second order effect must be considered! }
\end{aligned}
$$

Eccentricity

$$
\begin{aligned}
& e_{i}=\theta_{i} \cdot \frac{I_{0}}{2}=\frac{1}{100 \cdot \sqrt{l}} \cdot \frac{I_{0}}{2}=\frac{1}{100 \cdot \sqrt{9}} \cdot \frac{18}{2}=0.03 \mathrm{~m}=3 \mathrm{~cm} \\
& e_{2}=K_{1} \cdot \frac{l_{0}^{2}}{10} \cdot \frac{1}{r}=1.0 \cdot \frac{18^{2}}{207 \cdot 0.45 \cdot 10}=0.35 \mathrm{~m}=35 \mathrm{~cm} \\
& e_{\text {tot }}=e_{i}+e_{2}=3+35=38 \mathrm{~cm}
\end{aligned}
$$

\subsubsection{CHARACTERISTIC FORCES AT THE SUPPORT}

\begin{tabular}{|c|c|c|c|}
\cline { 2 - 4 } \multicolumn{1}{c|}{} & $\mathbf{N}_{\mathbf{k}}[\mathbf{k N}]$ & $\mathbf{M}_{\mathbf{k}}[\mathbf{k N m}]$ & $\mathbf{V}_{\mathbf{k}}[\mathbf{k N}]$ \\
\hline $\mathbf{G}_{\mathbf{k}}$ & -1327.3 & 0.0 & 0.0 \\
\hline $\mathbf{S}_{\mathbf{k}}$ & -1050.0 & 0.0 & 0.0 \\
\hline $\mathbf{A}_{\mathbf{k}}$ & 0.0 & -393.8 & +375.0 \\
\hline
\end{tabular}

\subsubsection{DESIGN FORCES AT THE SUPPORT}

\begin{tabular}{|c|c|c|c|}
\cline { 2 - 4 } \multicolumn{1}{c|}{} & $\mathbf{N}_{\mathrm{Ed}}$ & $\mathbf{M}_{\mathrm{Ed}}$ & $\mathbf{V}_{\mathrm{Ed}}$ \\
\hline LC1 & -3366.9 & -151.7 & +5.6 \\
\hline LC2 & -1537.4 & -451.0 & +380.4 \\
\hline LC3 & -1327.3 & -440.5 & +379.6 \\
\hline
\end{tabular}




\subsection{ULTIMATE LIMIT STATE DESIGN}

$$
\begin{array}{ll}
\mu_{E d}=\frac{M_{E d}}{b \cdot h^{2} \cdot f_{c d}} & v=v_{E d}=\frac{N_{E d}}{b \cdot h \cdot f_{c d}}
\end{array} \quad \rightarrow \omega_{t o t}
$$

\begin{tabular}{|c|c|c|c|c|c|c|c|c|c|}
\hline & & $\mu_{\mathrm{Ed}, \mathrm{y}}$ & $\boldsymbol{\mu}_{\mathrm{Ed}, \mathbf{x}}$ & $\mathbf{v}$ & $\mu_{\mathrm{Ed}, 1}$ & $\mu_{\mathrm{Ed}, 2}$ & $\omega_{\text {tot }}$ & $A_{s, \text { req }}$ & $1 / 4 A_{s, \text { req }}$ \\
\hline \multirow{3}{*}{ CI } & LC1 & -3366.9 & -151.7 & 5.6 & 0.061 & -0.680 & 0.00 & 0.00 & 0.00 \\
\hline & LC2 & -1537.4 & -451.0 & 380.4 & 0.182 & -0.311 & 0.30 & 34.14 & 8.53 \\
\hline & LC3 & -1327.3 & -440.5 & 379.6 & 0.178 & -0.268 & 0.25 & 28.45 & 7.11 \\
\hline
\end{tabular}

\subsection{SERVICEABILITY LIMIT STATE DESIGN}

According to DIN-EN-1992-1-1 no design in the serviceability limit state has to be done. No cracks are expected for the columns at serviceability limit state.

\subsection{DESIGN AND REINFORCEMENT}

\subsubsection{MINIMUM AND MAXIMUM LONGITUDINAL REINFORCEMENT}

Maximum reinforcement

$$
A_{s, \max }=\frac{1}{2} \cdot 0.09 \cdot A_{c}=\frac{1}{2} \cdot 0.09 \cdot 50 \cdot 50=112.5 \mathrm{~cm}^{2}
$$

Minimum reinforcement

$$
A_{s, \min }=\frac{0.15 \cdot\left|N_{E d}\right|}{f_{y d}}=\frac{0.15 \cdot 3367}{43.5}=11.6 \mathrm{~cm}^{2}
$$

\subsubsection{TRANSVERSE REINFORCEMENT}

Minimum diameter

Maximum distance

Chosen $\min d_{s, b u ̈} \geq\left\{\begin{array}{l}6 \mathrm{~mm} \\ 0.25 \cdot \max d_{s, l}=0.25 \cdot 25=\underline{\underline{6.25 \mathrm{~mm}}}\end{array}\right.$

$$
\min s_{w} \leq\left\{\begin{array}{l}
12 \cdot \min d_{s, l}=12 \cdot 25=300 \mathrm{~mm} \\
h_{\min }=500 \mathrm{~mm} \\
\underline{\underline{300 \mathrm{~mm}}}
\end{array}\right.
$$

$\varnothing 8 / 25=4.02 \mathrm{~cm}^{2} / \mathrm{m}$ stirrups 


\subsubsection{DESIGN OF THE CORBEL}

Forces on the corbel

$$
\begin{aligned}
& V_{E d}=\frac{1}{2} \cdot 3291 \mathrm{kN}=1646 \mathrm{kN} \\
& T_{E d}=V_{E d} \cdot l_{\text {eff }} / 300=1646 \cdot 29.2 / 300=160 \mathrm{kNm} \\
& H_{E d, y}^{T}=T_{E d} / z_{H E D}=160 \mathrm{kNm} / 1.32 \mathrm{~m}=121 \mathrm{kN}
\end{aligned}
$$

\begin{tabular}{|c|c|}
\hline Bending moment & $M_{E d}=160 \mathrm{kNm}$ \\
\hline Static height & $d=50-2-1-1=46 \mathrm{~cm}$ \\
\hline Flexure design & $\mu_{E d s}=\frac{M_{E d s}}{b \cdot d^{2} \cdot f_{c d}}=\frac{16000}{30 \cdot 46^{2} \cdot 1.98}=0.13$ \\
\hline Reinforcement & $A_{s, \text { req }}=\omega \cdot b \cdot d \cdot f_{c d} / \sigma_{s d}=0.1404 \cdot 30 \cdot 46 \cdot 19.8 / 435=8.80 \mathrm{~cm}^{2}$ \\
\hline Chosen & longitudinal reinforcement \\
\hline
\end{tabular}

Flexure Design of the Column

Shear Design of the Column

Shear force

$H_{E d, y}=121 k N$

Shear force resistance

$V_{R d \text { max }}=0.75 \cdot 30 \cdot 0.9 \cdot 46 \cdot 1.98 \cdot \frac{1.2}{1+1.2^{2}}=458 \mathrm{kN} \quad(\cot \theta \approx 1.2)$

$V_{E d}=H_{E d, y}=121 \mathrm{kN}<V_{R d, \max }=458 \mathrm{kN} \rightarrow$ The compression strut is ok.

Reinforcement

$a_{s w, r e q}=\frac{V_{E d}}{f_{y d} \cdot z \cdot \cot \theta}=\frac{121 \mathrm{kN}}{43.5 \cdot 0.9 \cdot 0.46 \cdot 1.2}=5.6 \mathrm{~cm}^{2} / \mathrm{m}$

Chosen

$\varnothing 8 / 10=10.05 \mathrm{~cm}^{2} / \mathrm{m}$

Bearing Load

Size of the support

$350 \times 350 \times 2 \mathrm{~mm}$

Compression stresses

$\sigma_{E d}=\frac{V_{E d}}{a_{L} \cdot b_{L}}=\frac{1646 \mathrm{kN}}{35 \cdot 35} \cdot 10=13.4 \mathrm{MN} / \mathrm{m}^{2} \leq 0.85 \cdot f_{c d}=0.85 \cdot 19.8=16.8 \mathrm{MN} / \mathrm{m}^{2} \checkmark$

Main Reinforcement of the Corbel

Static height

Tensile force

Reinforcement

Chosen

$$
\begin{aligned}
& d \approx 75-9=66 \mathrm{~cm} \\
& F_{s d}=\frac{1.1 \cdot a}{0.85 \cdot d} \cdot V_{E d}+H_{E d}>V_{E d} / 2 \\
& F_{s d}=\frac{1.1 \cdot 27}{0.85 \cdot 66} \cdot 1646=871 \mathrm{kN}>\frac{1646}{2}=823 \mathrm{kN}
\end{aligned}
$$

$$
A_{s, r e q}=\frac{F_{s d}}{f_{y d}}=\frac{871}{43.5}=20.0 \mathrm{~cm}^{2}
$$

$8 \varnothing 14=24.64 \mathrm{~cm}^{2}$ 
Provided static height

Anchorage at the support

Overlap length$$
I_{b, r q d}=64 \mathrm{~cm} \quad(\varnothing 20)
$$$$
I_{b d}=\alpha_{1} \cdot I_{b, \text { rad }} \cdot\left(A_{s, \text { req }} / A_{s, \text { prov }}\right)=1.0 \cdot 64 \cdot(20 / 24.6)=52 \mathrm{~cm}
$$$$
I_{0}=\alpha_{6} \cdot I_{b d}=2.0 \cdot 52=\underline{\underline{104 \mathrm{~cm}}}>I_{0, \mathrm{~min}}
$$$$
I_{0, \min }=0.3 \cdot \alpha_{6} \cdot I_{b, r q d}=0.3 \cdot 2.0 \cdot 64=\underline{\underline{38.4 \mathrm{~cm}}} \geq\left\{\begin{array}{l}
15 \cdot 2=30 \mathrm{~cm} \\
20 \mathrm{~cm}
\end{array}\right.
$$$$
\rightarrow I_{0}=104 \mathrm{~cm}
$$

Secondary Reinforcement

Crosswise reinforcement

Chosen

$$
A_{s, \text { net,req }}=0.5 \cdot A_{s}=0.5 \cdot 20=10 \mathrm{~cm}^{2}
$$

$5 \varnothing 12=11.3 \mathrm{~cm}^{2}$

Design of the Shear Force Dowel

Horizontal force

Shear force resistance

\section{Chosen}

Distance to the edge

$$
\begin{aligned}
& H_{E d}=121 \mathrm{kN} \\
& W_{\text {dowel }}=r^{3} \cdot \pi / 4=2.0^{30} \cdot \pi / 4=6.28 \mathrm{~cm}^{3} \\
& H_{R d}=1.25 \cdot f_{y d} \cdot \frac{W_{\text {dowel }}}{t / 2 \cdot d_{\text {dowel }}}=1.25 \cdot 43.5 \cdot \frac{6.28}{1.0+4.0}=68.3 \mathrm{kN} \text { (per dowel) } \\
& 2 \text { dowels } \rightarrow H_{R d}=2 \cdot 68.3 \mathrm{kN}=137 \mathrm{kN}>H_{E d}=121 \mathrm{kN}
\end{aligned}
$$

\section{2 dowels $\varnothing 40$}

$x$ - direction: $\quad 3 \cdot d_{s}=12 \mathrm{~cm}<u_{x}=23 \mathrm{~cm}<8 \cdot d_{s}=32 \mathrm{~cm}$

$y$-direction: $3 \cdot d_{s}=12 \mathrm{~cm}<u_{y}=17 \mathrm{~cm}<8 \cdot d_{s}=32 \mathrm{~cm}$

Supplementary reinforcement necessary:

$A_{s, \text { req }}=H_{E d} / f_{y d}=121 / 43.5=2.78 \mathrm{~cm}^{2}$ 


\subsubsection{REINFORCEMENT DRAWING}

See Appendix A.4, page 190.

\subsection{TRANSPORT AND ASSEMBLY}

\subsubsection{TRANSPORT AND LIFTING ON THE CONSTRUCTION SITE}

The beam will be suspended with the help of two anchors on each side above the center of gravity at around $1 / 6$ of the length of the beam. Which might cause tension over the anchors and might require upper reinforcement.

System

In the following the static system during transportation and assembly can be seen.
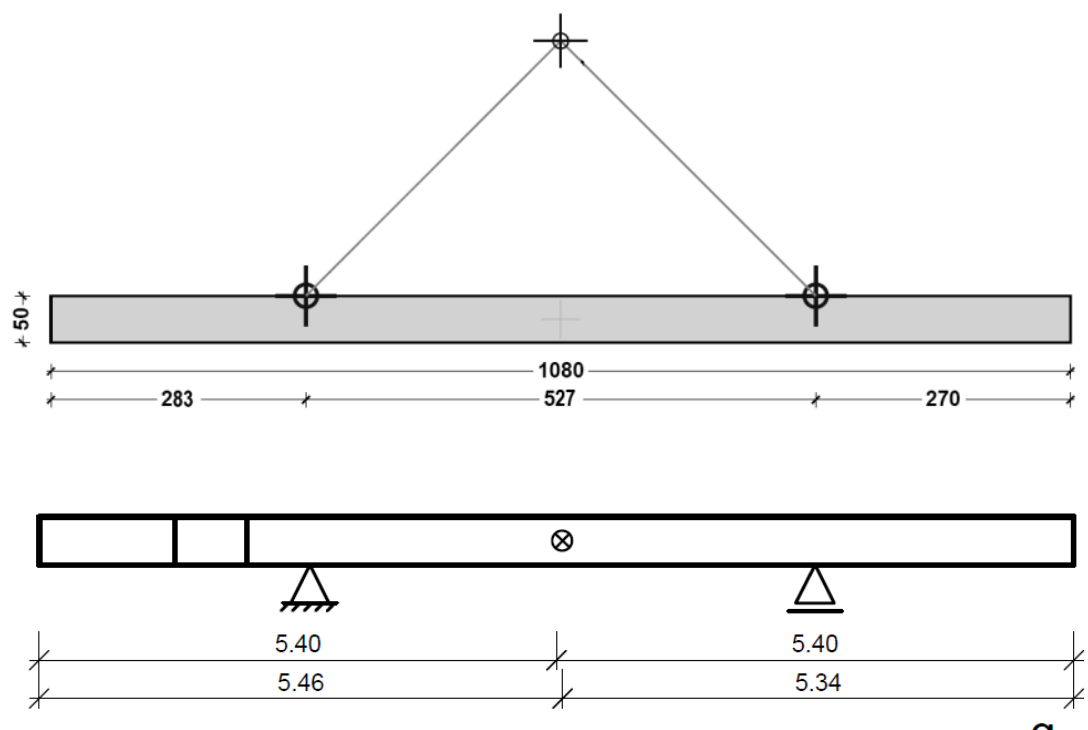

$g_{k}$

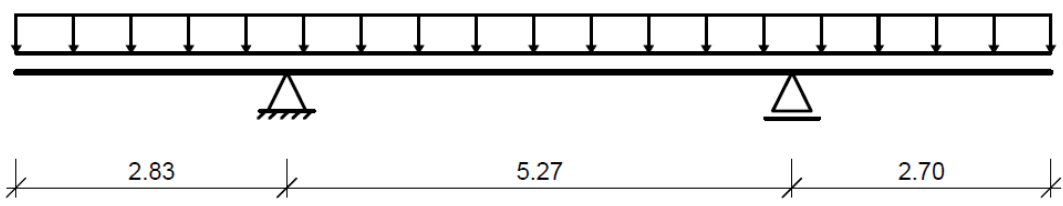

Design

Lifting loads

Bending Moment

Time of first lifting

$$
\begin{array}{ll}
A=5.65 \mathrm{~m}^{2} & V=5.65 \times 0.50=2.825 \mathrm{~m}^{3} \\
\text { self-weight } & g_{k}=2.825 \mathrm{~m}^{3} \times 25 \mathrm{kN} / \mathrm{m}^{3} / 11.15 \mathrm{~m}=6.33 \mathrm{kN} / \mathrm{m}
\end{array}
$$


Compressive strength at time $t$

Required reinforcement

$$
\begin{aligned}
& f_{c m}(t)=\beta_{c c}(t) \cdot f_{c m} \quad \rightarrow \quad \beta_{c c}(t=5 d)=e^{s(1-\sqrt{28 / t})}=e^{0.2(1-\sqrt{28 / 5})}=0.76 \\
& f_{c m}(t=5 d)=0.76 \cdot 43=32.68 \mathrm{~N} / \mathrm{mm}^{2} \\
& f_{c k}(t=5 d)=f_{c m}(t)-8=32.68-8=24.68 \mathrm{~N} / \mathrm{mm}^{2} \\
& f_{c d}(t=5 d)=0.85 \cdot f_{c k}(t) / 1.5=0.85 \cdot 24.68 / 1.5=\underline{\underline{14.0 \mathrm{~N} / \mathrm{mm}^{2}}}
\end{aligned}
$$$$
\mu_{E d s}=\frac{M_{E d s}}{b \cdot d^{2} \cdot f_{c d}}=\frac{2920}{50 \cdot 45^{2} \cdot 1.4}=0.021 \rightarrow \omega=0.0203
$$$$
A_{s 2, \text { req }}=\left(\omega \cdot b \cdot d \cdot f_{c d}+N_{E d}\right) / \sigma_{s d}
$$$$
=0.0203 \cdot 50 \cdot 45 \cdot 1.40 / 43.5=\underline{\underline{1.47 \mathrm{~cm}^{2}}}<A_{s, \text { prov }}=9.42 \mathrm{~cm}^{2}
$$

No additional reinforcement is required!

\subsubsection{TRANSPORT ANCHORS}

In order to lift the beam out of its formwork as well as to be able to transport and assemble it on site, transport anchors are needed. Those are installed during the production of the beam and must be designed in accordance with the VDI/BV-BS-Richtlinie 6205. For this project the anchors of the company Halfen are chosen.

Self-weight

Formwork adhesion

Dynamic factor

Factor for inclination

Tensile force

\section{Chosen}

Load capacity of the anchor

$$
\begin{aligned}
& F_{G}=6.33 \mathrm{kN} / \mathrm{m} \cdot 10.8 \mathrm{~m}=68.4 \mathrm{kN} \\
& F_{a d h}=q_{a d h} \cdot A_{f} \quad \rightarrow \text { no adhesion here because of hinged formwork! } \\
& \psi_{d y n}=1.3 \\
& z=1.41 \mathrm{for} \beta=45^{\circ} \\
& F_{z}=\frac{F_{G} \cdot \psi_{d y n} \cdot z}{n}=\frac{68.4 \cdot 1.3 \cdot 1.41}{2}=\underline{\underline{62.7 \mathrm{kN}}}
\end{aligned}
$$

\begin{tabular}{|c|c|c|c|c|c|c|c|c|c|}
\hline \multirow{3}{*}{$\begin{array}{l}\text { Load } \\
\text { class }\end{array}$} & \multirow{3}{*}{ Article number } & \multirow{3}{*}{$\begin{array}{c}\text { Anchor } \\
\text { length } \\
\text { । } \\
\text { [mm] }\end{array}$} & \multirow{3}{*}{$\begin{array}{c}\text { Minimum height } \\
\text { of beams } \\
B_{1} \\
{[\mathrm{~mm}]}\end{array}$} & \multirow{3}{*}{$\begin{array}{c}\text { Wall } \\
\text { thickness } \\
2 \times \mathrm{e}_{\mathrm{r}} \\
{[\mathrm{mm}]}\end{array}$} & \multicolumn{4}{|c|}{ Load capacity $[k N]$ at concrete strength $f_{\mathrm{c}}$ for } & \multirow{3}{*}{$\begin{array}{c}\text { Axial spacing of } \\
\text { anchors } \\
e_{z} \\
{[\mathrm{~mm}]}\end{array}$} \\
\hline & & & & & $\begin{array}{c}\text { Axial pull } \\
\text { up to } 30^{\circ}[\beta]\end{array}$ & $\begin{array}{l}\text { Diagonal pull } \\
\text { up to } 60^{\circ}[\beta]\end{array}$ & $\begin{array}{l}\text { Axial pull and } \\
\text { diagonal pull } \\
\text { up to } 60^{\circ}[\beta]\end{array}$ & $\begin{array}{l}\text { Axial pull and } \\
\text { diagonal pull } \\
\text { up to } 60^{\circ}[\beta]\end{array}$ & \\
\hline & & & & & $15 \mathrm{~N} / \mathrm{mm}^{2}$ & $15 \mathrm{~N} / \mathrm{mm}^{2}$ & $25 \mathrm{~N} / \mathrm{mm}^{2}$ & $35 \mathrm{~N} / \mathrm{mm}^{2}$ & \\
\hline \multirow{9}{*}{7,5} & \multirow{3}{*}{$6000-7,5-0200$} & \multirow{3}{*}{200} & \multirow{3}{*}{410} & 240 & 45.1 & 36.0 & 58.2 & 68.8 & \multirow{3}{*}{610} \\
\hline & & & & 260 & 47.8 & 38.3 & 618 & 73.1 & \\
\hline & & & & 280 & 50.6 & 40.5 & 65.3 & 75.0 & \\
\hline & \multirow{3}{*}{$6000-7,5-0300$} & \multirow{3}{*}{300} & \multirow{3}{*}{610} & 200 & 54.1 & 43.3 & 69.9 & \multirow{3}{*}{75.0} & \multirow{3}{*}{910} \\
\hline & & & & 220 & 58.1 & 46.5 & & & \\
\hline & & & & 240 & 62.2 & 49.7 & 75.0 & & \\
\hline & \multirow{3}{*}{$6000-7,5-0540$} & \multirow{3}{*}{540} & \multirow{3}{*}{1090} & 160 & 63.2 & 58.4 & \multirow{3}{*}{75.0} & \multirow{3}{*}{75.0} & \multirow{3}{*}{1630} \\
\hline & & & & 180 & 71.1 & 63.8 & & & \\
\hline & & & & 200 & 75.0 & 69.1 & & & \\
\hline
\end{tabular}

HALFEN DEHA Spherical head anchor 6000-7,5-0200

$$
F_{z, \text { Rd }}=65.3 \mathrm{kN}>F_{z, E d}=62.7 \mathrm{kN} \checkmark \quad(\text { see Table 19) }
$$

Table 19 - Sizing Table for 'HALFEN DEHA Lifting Anchor Systems' 


\subsection{SOFTWARE CALCULATIONS}

\section{Demo Frilo Nemetschek}

\section{Position: C.05 - Precast Concrete Column with Maximum Vertical Load}

\section{Reinforced Concrete Column B5 01/2018 (Frilo R-2018-1/P12)}

CANTILEVER COLUMN, Rectangle, 2-axial strained

Calculation base: DIN EN 1992-1-1/NA/A1:2015-12

$\mathrm{E}=34000 \mathrm{~N} / \mathrm{mm} 2 \quad \rho=2500 \mathrm{~kg} / \mathrm{m} 3$
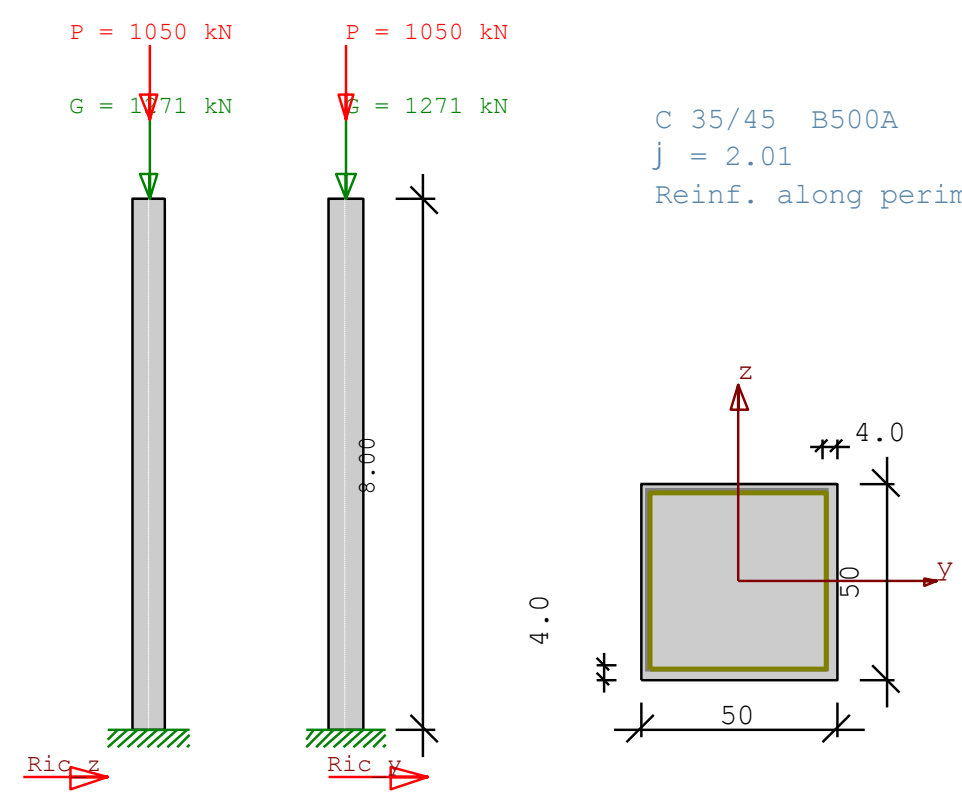

$$
1 \operatorname{Mcry}=66.87 \mathrm{kNm} \quad \operatorname{Mcrz}=66.87 \mathrm{kNm}
$$

NODES - LOADS :

LcNo. KNo. $\mathrm{V}$ ey ez $\quad \mathrm{Py} \quad \mathrm{Pz} \quad \mathrm{My}$ Mz act con alt

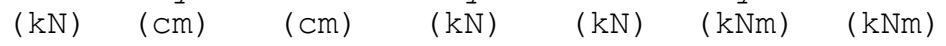

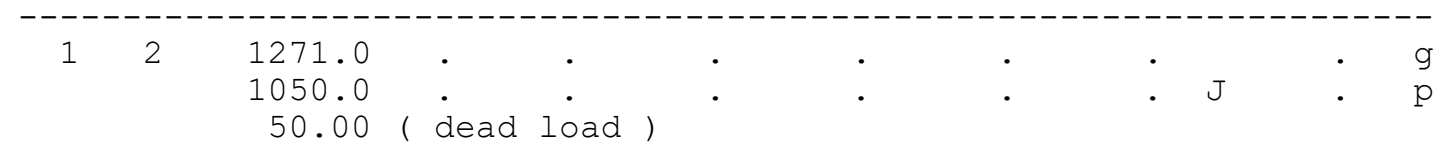

Actions:

No. Cl Name $\psi$ \% $\psi 1 \quad \psi 2 \quad \gamma$
J 3 Snow loads $<1000 \mathrm{~m}$
$0.50 \quad 0.20 \quad 0.00 \quad 1.50$

Further design fundamentals:

Accuracy Gkn $=8.48 e-5$

Number of sub-element per member section: 6

Stress-strain-curve of concr. for deform. analy. EN 1992-1-1 3.1.5 


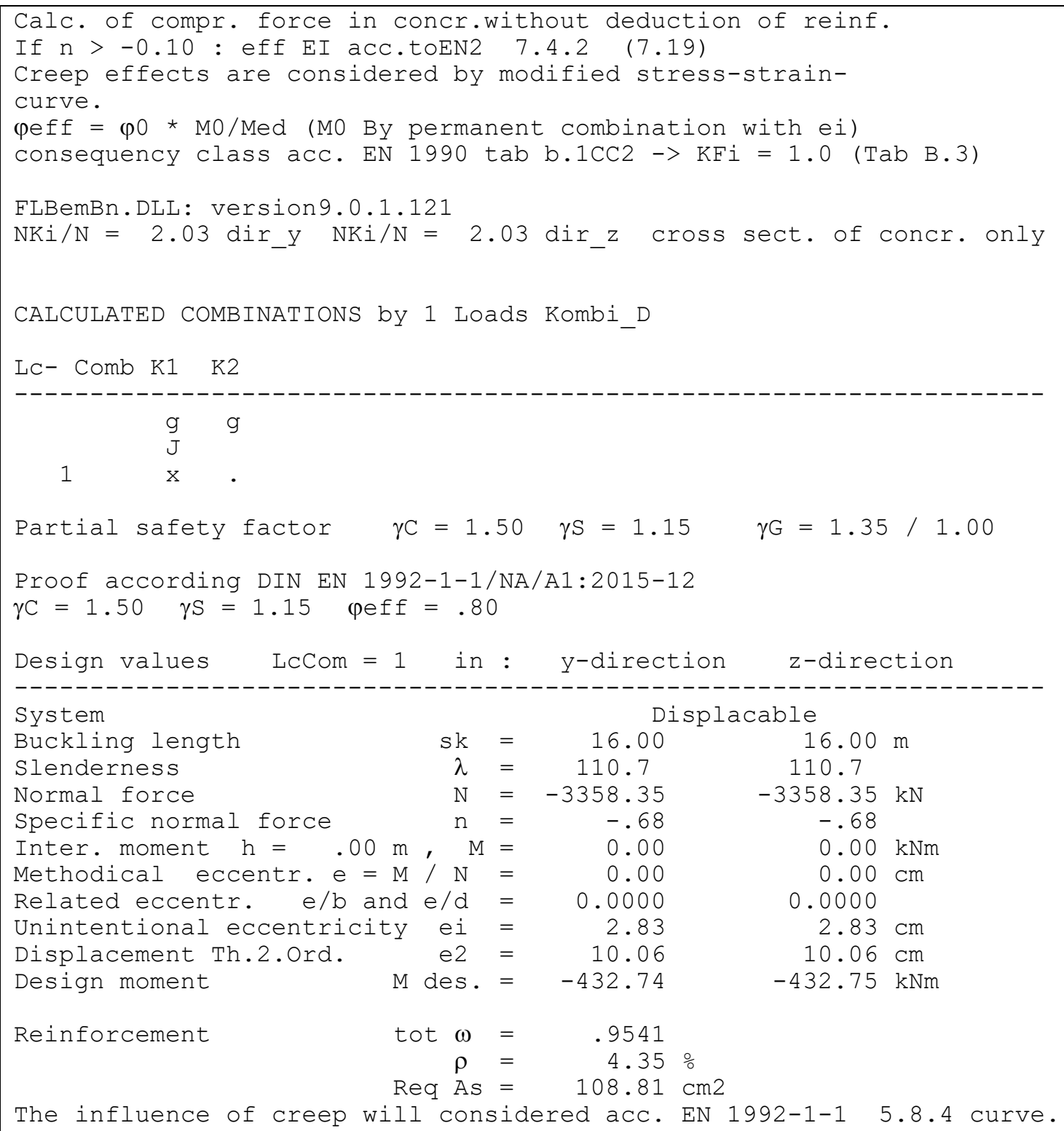


10 POS. T.10 - PRECAST STAIRCASE

\subsection{SYSTEM}

\section{System}
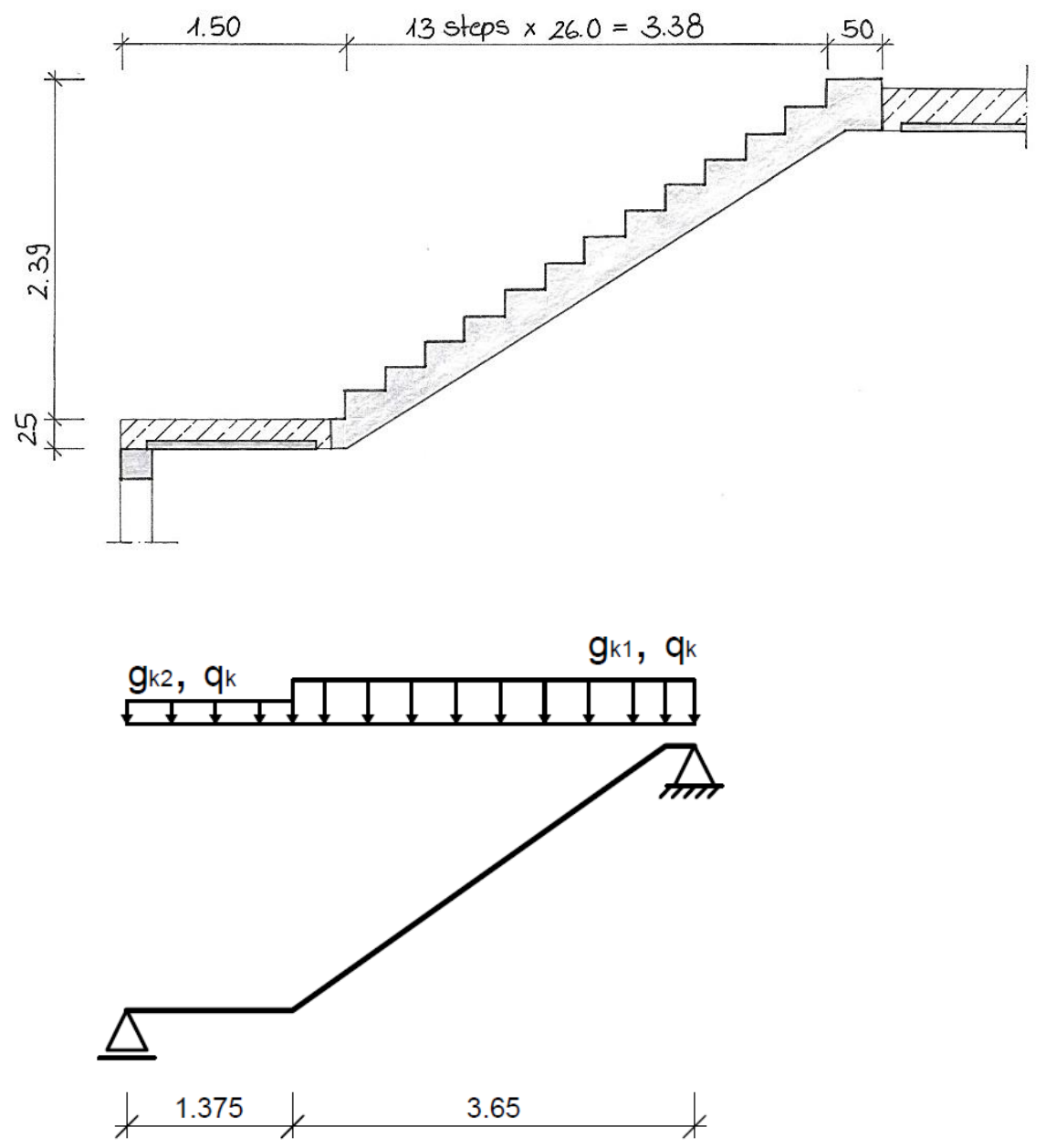

Cross-section

13 steps $\times 18.4 / 26 \mathrm{~cm}, \mathrm{t}=20 \mathrm{~cm}$

Building materials

C 30/37 | B 500A

Exposure class

XC1, WO

Reinforcing steel

$$
C_{\text {nom }}=10 \mathrm{~mm}+10 \mathrm{~mm}=20 \mathrm{~mm}
$$

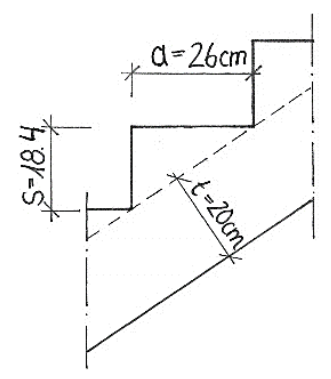


Characteristic Loads

\begin{tabular}{|c|c|c|c|}
\hline \multicolumn{2}{|c|}{ Uniformly Distributed Loads } & \multirow{2}{*}{$\begin{array}{c}\begin{array}{c}\mathbf{g}_{\mathbf{k}} \\
{[\mathbf{k N} / \mathbf{m}]}\end{array} \\
2.30\end{array}$} & \multirow{2}{*}{$\frac{\begin{array}{c}\mathbf{q}_{\mathrm{k}} \\
{[\mathrm{kN} / \mathrm{m}]}\end{array}}{-}$} \\
\hline Self-weight steps & $g_{\mathrm{k} 1,1}=1 / 2 \times 0.184 \times 25 \mathrm{kN} / \mathrm{m}^{3}=$ & & \\
\hline Self-weight slab & $\mathrm{g}_{\mathrm{k} 1,2}=0.20 \times 25 \mathrm{kN} / \mathrm{m}^{3} / \cos \left(35^{\circ}\right)=$ & 6.10 & - \\
\hline Self-weight landing & $g \quad g_{k, 2}=0.25 \times 25 \mathrm{kN} / \mathrm{m}^{3}=$ & 6.25 & \\
\hline Floor structure & $g_{k 1+2}=$ & 1.50 & - \\
\hline \multirow[t]{2}{*}{ Live load (Cat. T1 } & - Stair cases) & - & 3.00 \\
\hline & $\begin{array}{l}g_{k 1}= \\
g_{k 2}=\end{array}$ & $\begin{array}{l}8.40 \\
7.75\end{array}$ & 3.00 \\
\hline
\end{tabular}

Design Loads

$$
\begin{array}{ll}
q_{E d}=1.35 \cdot g_{k}+1.5 \cdot q_{k} & \\
q_{p e r m}=1.0 \cdot g_{k}+1.0 \cdot \psi_{2} \cdot q_{k} & \psi_{2}=0.5 \text { (Cat. T1) }
\end{array}
$$

\subsection{INTERNAL FORCES}

Flexure moment

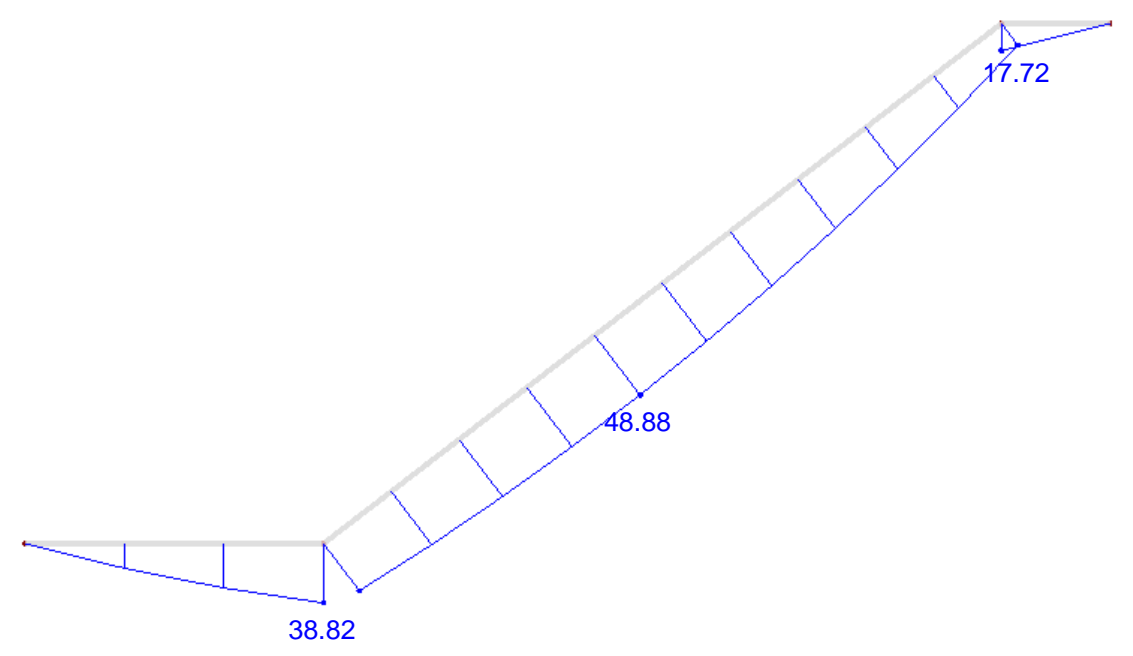

\begin{tabular}{|c|c|c|c|c|}
\cline { 2 - 5 } \multicolumn{1}{c|}{} & $\begin{array}{c}\mathbf{m}_{\mathbf{g k}} \\
{[\mathbf{k N m} / \mathbf{m}]}\end{array}$ & $\begin{array}{c}\mathbf{m}_{\mathbf{q k}} \\
{[\mathbf{k N m} / \mathbf{m}]}\end{array}$ & $\begin{array}{c}\mathbf{m}_{\mathrm{Ed}} \\
{[\mathbf{U} \mathbf{S}]}\end{array}$ & $\begin{array}{c}\mathbf{m}_{\text {perm }} \\
{[\mathbf{S L S}]}\end{array}$ \\
\hline $\mathbf{m}_{\mathbf{L}}$ & 20.5 & 7.5 & 38.8 & 24.2 \\
\hline $\mathbf{m}_{\mathbf{S}}$ & 25.8 & 9.3 & 48.9 & 30.5 \\
\hline
\end{tabular}




\section{Shear force}

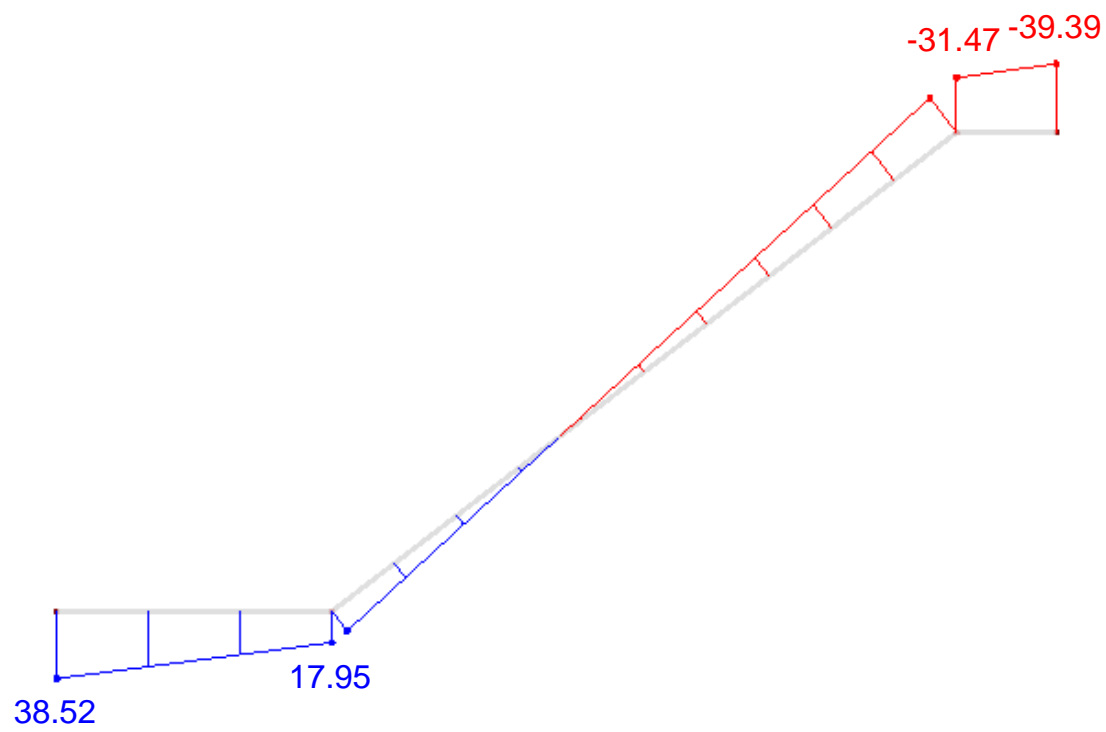

\begin{tabular}{|c|c|c|c|c|}
\cline { 2 - 5 } \multicolumn{1}{c|}{} & $\mathbf{v}_{\mathbf{g k}}[\mathbf{k N} / \mathbf{m}]$ & $\mathbf{V}_{\mathbf{q k}}[\mathbf{k N} / \mathbf{m}]$ & $\mathbf{V}_{\mathrm{Ed}}[\mathrm{ULS}]$ & $\mathbf{V}_{\mathrm{Ed}}[\mathbf{S L S}]$ \\
\hline $\mathbf{V}_{\max }$ & 20.9 & 7.5 & 39.4 & 24.6 \\
\hline
\end{tabular}

\section{Normal force}

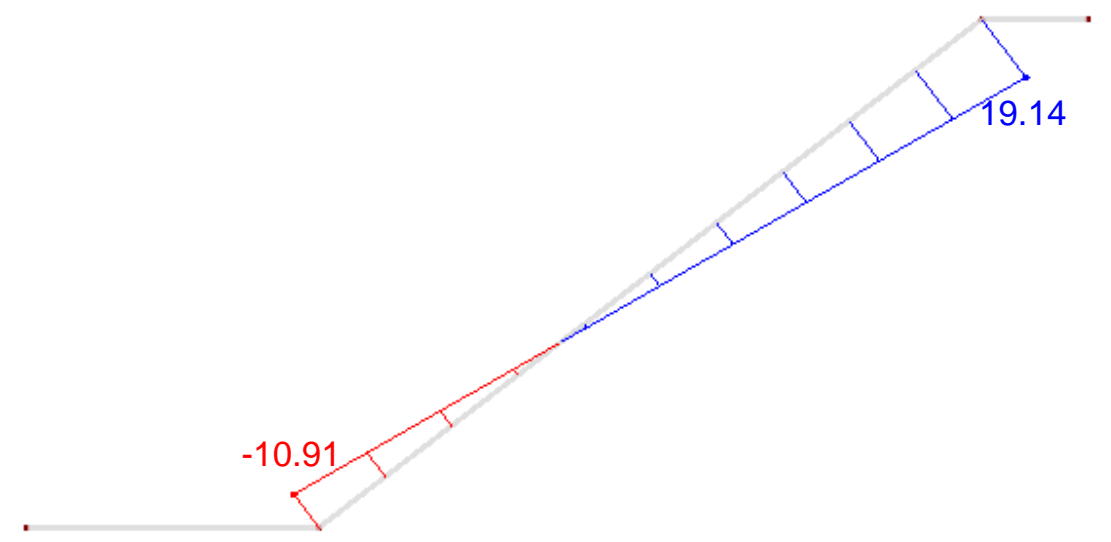

\begin{tabular}{|c|c|c|c|c|}
\cline { 2 - 5 } \multicolumn{1}{c|}{} & $\mathbf{n}_{\mathbf{g k}}[\mathbf{k N} / \mathbf{m}]$ & $\mathbf{n}_{\mathbf{q k}}[\mathbf{k N} / \mathbf{m}]$ & $\mathbf{n}_{\mathrm{Ed}}[\mathrm{ULS}]$ & $\mathbf{n}_{\mathrm{Ed}}[\mathrm{SLS}]$ \\
\hline $\mathbf{n}_{\mathbf{L}}$ & -5.8 & -2.1 & -10.9 & -6.8 \\
\hline $\mathbf{n}_{\mathbf{R}}$ & 10.1 & 3.6 & 19.1 & 12.0 \\
\hline
\end{tabular}




\subsection{ULTIMATE LIMIT STATE DESIGN}

\subsubsection{FLEXURE DESIGN}

Effective depth

$d=h-c_{\text {nom }}-d_{s, q u e r}-d_{s, \text { längs }} / 2=20-2.0-1.0-1.0 / 2=16.5 \mathrm{~cm}$

Reinforcement in the stairway

$\mu_{E d s}=\frac{m_{E d s}}{b_{e f f} \cdot d^{2} \cdot f_{c d}}=\frac{4890 \mathrm{kNcm}}{100 \cdot 16.5^{2} \cdot 1.7 \mathrm{kN} / \mathrm{cm}^{2}}=0.11 \quad \rightarrow \omega=0.0728$

Longitudinal reinforcement

$a_{s l, \text { req }}=\omega \cdot b \cdot d \cdot f_{c d} \cdot \frac{1}{\sigma_{s d}}=0.1170 \cdot 100 \cdot 16.5 \cdot 1.7 \cdot \frac{1}{43.5}=7.54 \mathrm{~cm}^{2} / \mathrm{m}$

Chosen

$\varnothing 10 / 10=7.85 \mathrm{~cm}^{2} / \mathrm{m}$

longitudinal reinforcement

Transverse reinforcement

$a_{s q, r e q}=0.2 \cdot a_{s l}=0.2 \cdot 4.69=0.94 \mathrm{~cm}^{2} / \mathrm{m}$

Chosen

$\varnothing 8 / 25=2.01 \mathrm{~cm}^{2} / \mathrm{m}$

transverse reinforcement

10.3.2 SHEAR DESIGN

Scale factor

$k=1+\sqrt{\frac{20}{d}}=1+\sqrt{\frac{20}{16.5}}=2.10<\underline{\underline{2.00}}$

Percentage of reinforcement

$\rho_{1}=\frac{a_{s l}}{b_{w} \cdot d}=\frac{7.85}{100 \cdot 16.5}=0.0048 \leq 0.02$

Shear force resistance

$v_{R d, c}=\left[0.10 \cdot k \cdot\left(100 \cdot \rho_{l} \cdot f_{c k}\right)^{1 / 3}+0.12 \cdot \sigma_{c p}\right] \cdot b_{w} \cdot d$

$V_{R d, c}=\left[0.10 \cdot 2.0 \cdot(100 \cdot 0.0048 \cdot 30)^{1 / 3}\right] \cdot 1.0 \cdot 0.165 \cdot 10^{3}=80.3 \mathrm{kN} / \mathrm{m}$

$d=16.5 \mathrm{~cm}<60 \mathrm{~cm} \rightarrow v_{\min }=0.035 \cdot 2.00^{3 / 2} \cdot(30)^{1 / 2}=0.542 \mathrm{MN} / \mathrm{m}^{2}$

$v_{R d, c, \min }=0.542 \cdot 1.00 \cdot 0.165 \cdot 10^{3}=\underline{\underline{89.4 \mathrm{kN}}} / \mathrm{m}>v_{R d, c}=80.3 \mathrm{kN} / \mathrm{m}$

$v_{E d}=39.4 \mathrm{kN} / m<v_{R d, c}=89.4 \mathrm{kN} / \mathrm{m} \checkmark$

No further shear force reinforcement is needed!

\subsection{SERVICEABILITY LIMIT STATE DESIGN}

\subsubsection{LIMITATION OF THE CRACK WIDTH}

Maximum diameter of the reinforcement

Crack width

Lever arm of the forces

Reinforcement stresses

$$
\mathrm{w}_{\mathrm{k}}=0.4 \mathrm{~mm}
$$$$
\zeta=0.940
$$

(according to DIN EN 1992-1-1, Tab. NA.7.1.)

$\rightarrow z=0.940 \cdot 16.5=15.5 \mathrm{~cm}$

$\sigma_{s}=\frac{m_{E d s}}{z \cdot a_{s l}}=\frac{3050 \mathrm{kNcm} / \mathrm{m}}{15.5 \cdot 7.85 \mathrm{~cm}^{2} / \mathrm{m}}=25.1 \mathrm{kN} / \mathrm{cm}^{2}=251 \mathrm{~N} / \mathrm{mm}^{2}$ 
Limit diameter

$$
\begin{aligned}
& d_{s}^{*}=22.35 \\
& d_{s}=d_{s}^{*} \cdot f_{c t, \text { eff }} / 2.9=22.35 \cdot 1.0=22.35 \mathrm{~mm}>d_{s, \text { prov }}=10 \mathrm{~mm}
\end{aligned}
$$

Minimum Reinforcement to Prevent Cracking

$\begin{array}{ll}\text { Tensile strength } & \rightarrow f_{c t, \text { eff }}=2.9 \mathrm{MN} / \mathrm{m}^{2}<\underline{\underline{3.0 \mathrm{MN}} / \mathrm{m}^{2}} \\ \text { for } h<30 \mathrm{~cm} & \rightarrow k=0.80 \\ \text { for flexure } & \rightarrow k_{c}=0.4\end{array}$

Tensile stress area

$A_{c t}=b \cdot h / 2=100 \cdot 20 / 2=1000 \mathrm{~cm}^{2}$

Reinforcement stresses

$$
\sigma_{s}=380 M N / m^{2}
$$$$
\left(w_{k}=0.4 m m, d_{s}=10 \mathrm{~mm}\right)
$$

Required Reinforcement

$$
\begin{aligned}
a_{s, \text { min, req }} & =k_{c} \cdot k \cdot f_{c t, \text { eff }} \cdot \frac{A_{c t}}{\sigma_{s}}=0.4 \cdot 0.8 \cdot 3.0 \cdot \frac{1000}{380}=2.53 \mathrm{~cm}^{2} / \mathrm{m} \\
& <a_{s, \text { prov }}=7.85 \mathrm{~cm}^{2} / \mathrm{m} \checkmark
\end{aligned}
$$

The provided reinforcement is sufficient!

\subsubsection{LIMITATION OF THE DEFORMATION}

$$
\begin{aligned}
& l / d \leq K \cdot 35=1.0 \cdot 35 \\
& I / d_{\text {prov }}=503 / 16.5=30.5<35
\end{aligned}
$$

The provided slab thickness is sufficient!

\subsection{DESIGN AND REINFORCEMENT}

10.5.1 MINIMUM REINFORCEMENT TO ENSURE ROBUSTNESS

$$
\begin{aligned}
& m_{c r}=f_{c t m} \cdot W_{c u} \quad W_{c u}=100 \cdot 20^{2} / 6=6667 \mathrm{~cm}^{3} \\
& m_{c r}=2.9 \cdot 6667 \cdot 10^{-3}=19.33 \mathrm{kNm} / \mathrm{m} \\
& a_{s, \text { min }}=\frac{1}{f_{y k}} \cdot \frac{m_{c r}}{z}=\frac{1}{50.0} \cdot \frac{1933}{15.9}=2.43 \mathrm{~cm}^{2} / \mathrm{m}>a_{s, \text { prov }}=7.85 \mathrm{~cm}^{2} / \mathrm{m}
\end{aligned}
$$

The provided reinforcement is sufficient!

\subsubsection{ANCHORAGE LENGTH AT THE SUPPORT}

Global offset

$$
\begin{aligned}
& a_{l}=1.0 \cdot d=16.5 \mathrm{~cm} \\
& F_{E d}=\frac{V_{E d} \cdot a_{l}}{z}=\frac{39.4 \cdot 16.5}{15.5}=41.9 \mathrm{kN} \\
& a_{s, \text { req }}=\frac{F_{E d}}{f_{y d}}=\frac{41.9}{43.5}=0.96 \mathrm{~cm}^{2} / \mathrm{m}
\end{aligned}
$$

Anchorage length

$$
I_{b, r a d}=36 \mathrm{~cm}
$$

for C30/37 and good bond 


$$
\begin{aligned}
I_{b d} & =\alpha_{1} \cdot \alpha_{4} \cdot \alpha_{5} \cdot I_{b, \text { rad }} \cdot\left(a_{s, \text { req }} / a_{s, \text { prov }}\right)=1.0 \cdot 36 \cdot(0.96 / 7.85)=4.4 \mathrm{~cm} \\
& >I_{b, \text { min }}=0.3 \cdot 1.0 \cdot 36=\underline{\underline{10.8 \mathrm{~cm}}}>10 \phi_{s}=10.0 \mathrm{~cm} \\
I_{b, \text { dir }} & =2 / 3 \cdot I_{b d}=2 / 3 \cdot 10.8=\underline{\underline{7.2 \mathrm{~cm}}}>6.7 \phi_{s}=6.7 \mathrm{~cm}
\end{aligned}
$$

\subsubsection{OVERLAP LENGTH}

$$
\begin{aligned}
& I_{0}=\alpha_{1} \cdot \alpha_{5} \cdot \alpha_{6} \cdot I_{b, \text { rqd }} \cdot\left(a_{s, \text { req }} / a_{s, \text { prov }}\right)=1.0 \cdot 1.4 \cdot(0.96 / 7.85) \cdot 36=5.3 \mathrm{~cm} \\
& I_{0, \text { min }}=0.3 \cdot \alpha_{1} \cdot \alpha_{6} \cdot I_{b, \text { rqd }}=0.3 \cdot 1.0 \cdot 1.4 \cdot 36=15.1 \mathrm{~cm}>\left\{\begin{array}{l}
15 \phi_{s}=15 \mathrm{~cm} \\
\underline{\underline{20 \mathrm{~cm}}}
\end{array}\right. \\
& \Rightarrow I_{0}=20 \mathrm{~cm}
\end{aligned}
$$

\begin{tabular}{|c|c|c|c|c|c|}
\hline Schöck Tronsole Typ & $\mathrm{T}-\mathrm{V} 2-\mathrm{NF}$ & T-V4-NF & T-V6-NF & T-V7-NF & T-V8-NF \\
\hline Bemessungswerte bei & \multicolumn{5}{|c|}{ Betonfestigkeit Podest $\geq$ C20/25, Treppenlauf $\geq$ C30/37 } \\
\hline Tronsole ${ }^{\oplus}$-Höhe H [mm] & \multicolumn{5}{|c|}{$\mathrm{V}_{\mathrm{Rd}, 2}[\mathrm{kN} /$ Element $]$} \\
\hline $160-170\left(\mathrm{~h}_{\mathrm{A}} \geq 180 \mathrm{~mm}\right)$ & 14,3 & 28,6 & 42,9 & 50,1 & 57,2 \\
\hline \multirow[t]{2}{*}{$180-320$} & 17,4 & 34,8 & 52,2 & 60,9 & 69,6 \\
\hline & \multicolumn{5}{|c|}{$\mathrm{V}_{\mathrm{Rd}, y}[\mathrm{kN} /$ Element] } \\
\hline $160-320$ & $\pm 1,6$ & $\pm 3,3$ & $\pm 5,0$ & $\pm 5,8$ & $\pm 6,6$ \\
\hline
\end{tabular}

\subsubsection{DESIGN OF THE SUPPORT TRONSOLE}

To improve the sound insulation of the stairs a the Tronsole ${ }^{\circledR}$ of the manufacturer Schöck has to be installed at the connection between the stairway and the upper slab. The sound insulating Tronsole ${ }^{\circledR}$ of the type $T$ as can be seen in Figure 15.

Table 20 - Sizing Table for the Schöck Tronsole® Type T

Shear force at the support $\quad v_{E d}=39.4 \mathrm{kN} / \mathrm{m}$

\section{Chosen}

\section{Schöck Tronsole ${ }^{\circledR}$ type T-V6-NF-H320-L1000}

Maximum shear force

$$
v_{R d}=52.2 \mathrm{kN} / m>v_{E d}=39.4 \mathrm{kN} / m \checkmark
$$

(see Table 20)
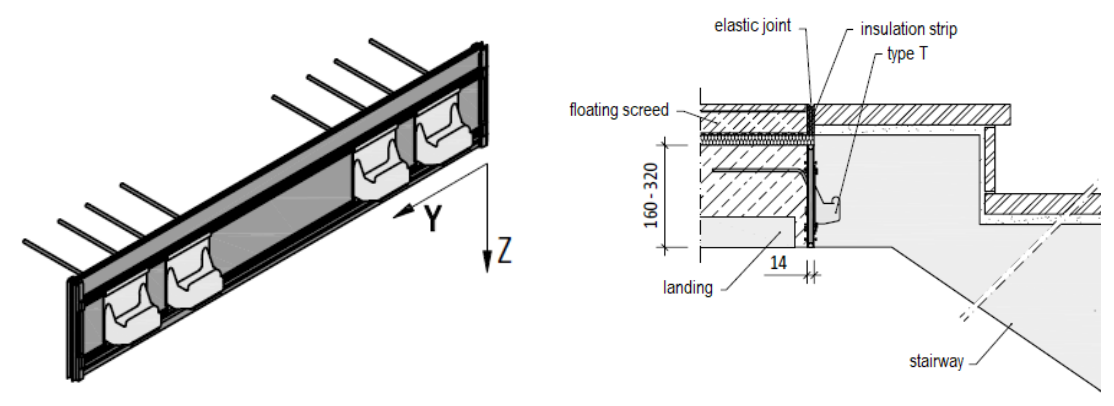

Figure 15 - Schöck Tronsole® Type T 


\subsubsection{REINFORCEMENT DRAWING}

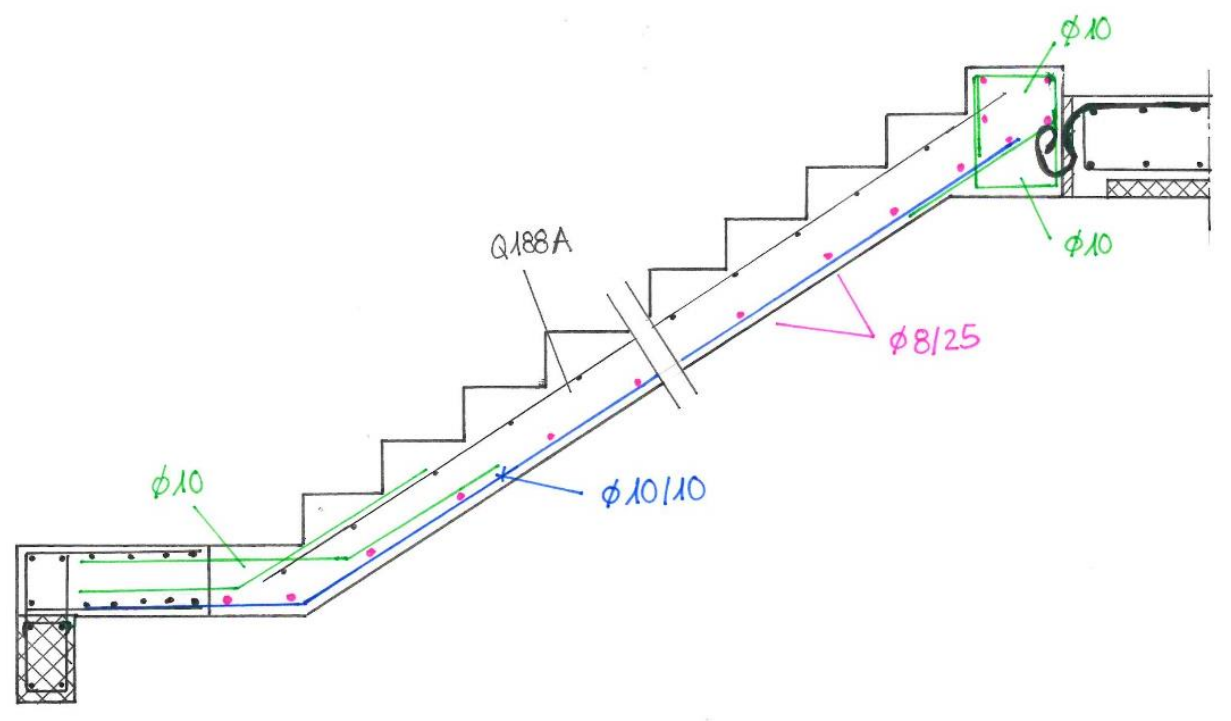




\subsection{SOFTWARE CALCULATIONS}

\section{Demo Frilo Nemetschek}

\section{Position: T.10 - Precast Staircase}

Treppenlauf B7+ 01/18A 01/2018 (Frilo R-2018-1/P12)

System

System graphics
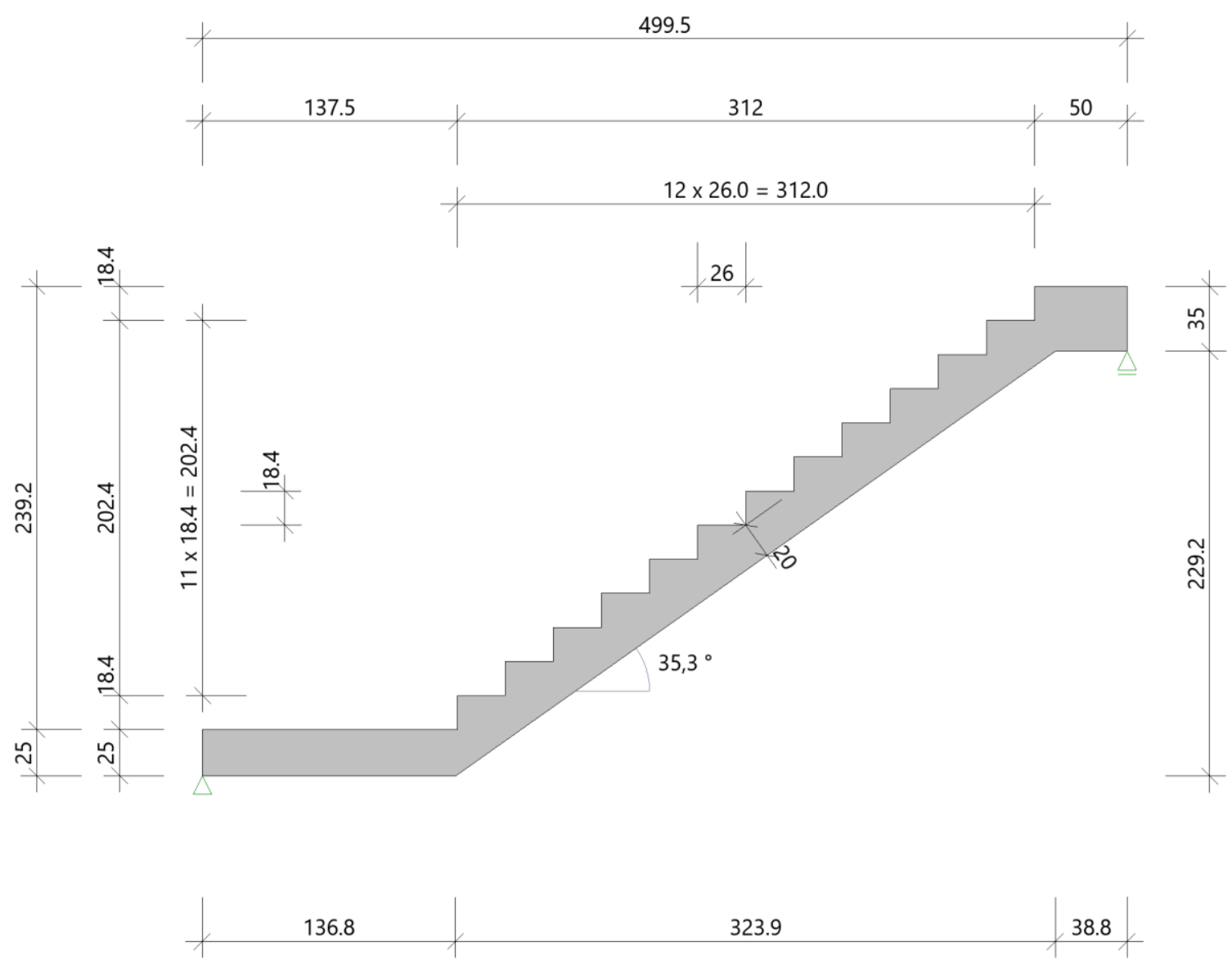

\section{Loads}

\begin{tabular}{|c|c|c|c|}
\hline $\begin{array}{l}\text { Location } \\
\text { - }\end{array}$ & $\begin{array}{l}\text { Type } \\
-\end{array}$ & $\mathrm{kN} / \mathrm{m}^{\frac{9}{2}}$ & $\mathrm{kN} / \mathrm{m}^{2}$ \\
\hline Stairway & $\begin{array}{l}\text { Covering } \\
\text { Live load }\end{array}$ & $\begin{array}{r}1.50 \\
-\end{array}$ & 3.00 \\
\hline Landing/console bottom & $\begin{array}{l}\text { Covering } \\
\text { Live load }\end{array}$ & $\begin{array}{r}1.50 \\
-\end{array}$ & 3.00 \\
\hline Landing/console top & $\begin{array}{l}\text { Covering } \\
\text { Live load }\end{array}$ & $\begin{array}{r}1.50 \\
-\end{array}$ & 3.00 \\
\hline
\end{tabular}




\begin{tabular}{|c|c|c|c|}
\hline $\begin{array}{l}\text { Location } \\
\text { - }\end{array}$ & $\begin{array}{l}\text { Type } \\
-\end{array}$ & $\mathrm{kN} / \mathrm{m}^{\mathrm{g}}$ & $\mathrm{kN} / \mathrm{m}^{2}$ \\
\hline Stairway & $\begin{array}{l}\text { Self weight } \\
\text { Covering } \\
\text { Live load } \\
\text { Total }\end{array}$ & $\begin{array}{r}8.43 \\
1.50 \\
- \\
9.93\end{array}$ & $\begin{array}{r}- \\
3.00 \\
3.00\end{array}$ \\
\hline Landing/console bottom & $\begin{array}{l}\text { Self weight } \\
\text { Covering } \\
\text { Live load } \\
\text { Total }\end{array}$ & $\begin{array}{r}6.25 \\
1.50 \\
- \\
7.75\end{array}$ & $\begin{array}{r}- \\
3.00 \\
3.00\end{array}$ \\
\hline Landing/console top & $\begin{array}{l}\text { Self weight } \\
\text { Covering } \\
\text { Live load } \\
\text { Total }\end{array}$ & $\begin{array}{r}8.75 \\
1.50 \\
- \\
10.25\end{array}$ & $\begin{array}{r}- \\
- \\
3.00 \\
3.00\end{array}$ \\
\hline
\end{tabular}

The dead weight is with gamma $=25.00 \mathrm{kN} / \mathrm{m}^{3}$ considered.

\section{Results}

Bending design

All design results per $m$ stair width!

Flexural reinforcement

Location $\quad d \quad M_{E d} N_{E d}$ req. asb req. ast \&About

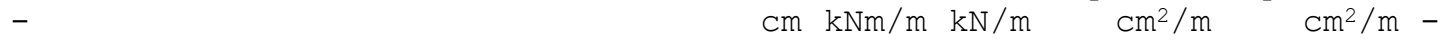

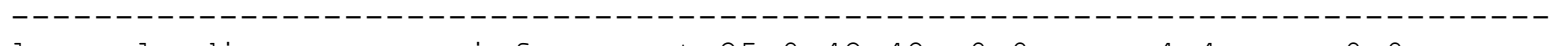

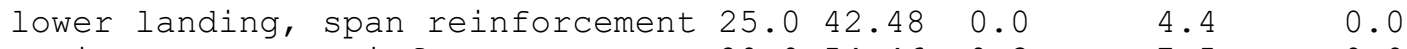

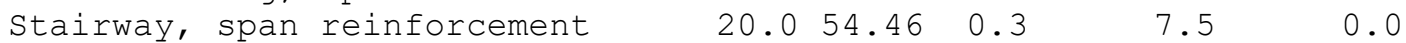

upper landing, span reinforcement $\begin{array}{rrrrr}35.0 & 6.73 & 0.0 & 4.1 & 0.0\end{array}$ *)

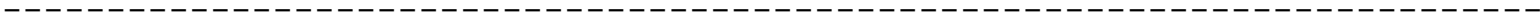

*) Minimum longitudinal reinforcement is decisive

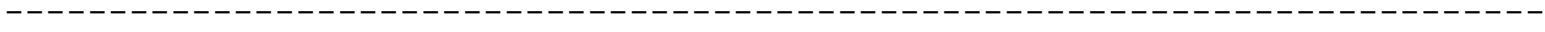

exist. reinforcement

Stairway, span reinforcement $7 \varnothing 12 / 15.0 \mathrm{~cm}$ (Suggestion from program for number $\varnothing$ )

exist. a sbtm $=7.54 \mathrm{~cm}^{2} / \mathrm{m}$ 
$\underline{\text { Shear design }}$

Shear reinforcement B500A

Location $\quad V_{E d} \quad N_{E d} \quad k_{z} \quad \theta \quad a_{S I} V_{R d, c} V_{R d, m a x}$ req. asstir \&About

- $\mathrm{kN} / \mathrm{m} \quad \mathrm{kN} / \mathrm{m} \quad$ - Degree $\mathrm{cm}^{2} / \mathrm{m} \quad \mathrm{kN} / \mathrm{m} \quad \mathrm{kN} / \mathrm{m} \mathrm{cm}^{2} / \mathrm{m}^{2} \quad$

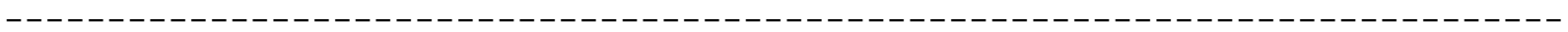

lower landing left $\left.\begin{array}{llllllllll}41.2 & 0.0 & 0.82 & 18.4 & 0.0 & 115.1 & 688.5 & 9.3 & *\end{array}\right)$

$\left.\begin{array}{lllllllll}\text { lower landing right } 20.7 & 0.0 & 0.82 & 18.4 & 4.4 & 115.1 & 688.5 & 9.3 & *\end{array}\right)$

Stairway left $\quad 16.9-12.00 .76$

Stairway right $\quad-31.4 \quad 22.3 \quad 0.76$

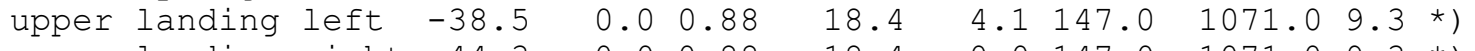

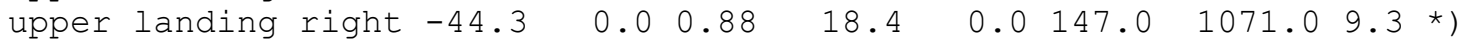

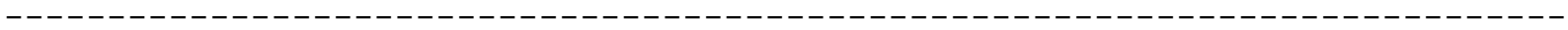

*) Minimum shear reinforcement is decisive

----------------------------------------------------------

crack width verification

The check is carried out with the quasi-permanent action combination

Crack width limitation stairs:

Location $\quad d M_{E d} N_{E d}$ exist. Asb exist. Ast Env.Cl $d_{s}$, exist $d_{s, l i m i t}$

exist. $W$ perm. $W$

$\begin{array}{lllllllllll}- & \mathrm{cm} & \mathrm{kNm} & \mathrm{kN} & \mathrm{cm}^{2} & \mathrm{~cm}^{2} & - & \mathrm{mm}\end{array}$

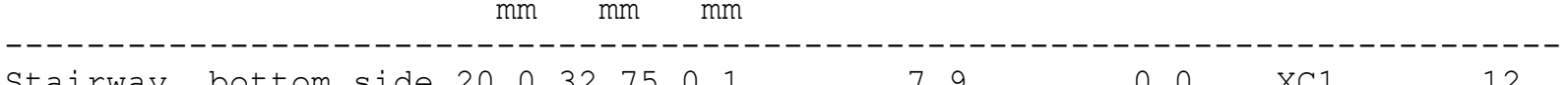

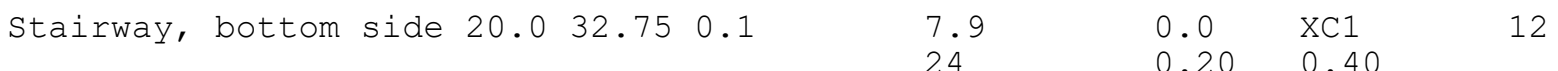

-

Deformation

The calculation will be done with quasi permanent Action combination at state $I\left(E_{\mathrm{cm}}=33000 \mathrm{~N} / \mathrm{mm}^{2}\right)$.

max. $f=0.4 \mathrm{~cm}$ (in staircase at $\mathrm{x}=1.45 \mathrm{~m}$ )

Note: The deflection value is to be understood perpendicular to the corresponding member axis. The $x$-value refers to the beginning of the member (beginning lower platform, staircase or upper platform) and runs in the direction of the member axis. 


\section{POS. S.11 - SEMI-PRECAST DOUBLE-TEE SLAB}

\subsection{SYSTEM ALTERNATIVES}

For the design of the slab of the office, a few possible solutions have been developed. The biggest issues of this slab are the large span length, 7.50 metres and 15.0 metres between the axes, as well as the opening in the slab above the entrance area. The two final possible solutions are described in the following chapters.

\subsubsection{COMBINATION OF A SLAB-BEAM SYSTEM AND A FLAT SLAB}

The first proposal is a combination of a slab-beam system and a flat slab between the axes 4 and 5 , where the large opening is located. The original layout of the office includes additional columns at every 7.50 metres in axis $A^{\prime}$ as well as additional columns in the middle axis between $A$ and $A^{\prime}$. Beams span between each column along axis $A, A^{\prime}$ and the middle column row with span lengths of $7.50 \mathrm{~m}$ and 15.00 metres as can be seen in Figure 16. The slab spans between those beams creating a double-span system with a span length of $7.50 \mathrm{~m}$. Because of the opening over the entrance, the beam system in axis $A$ has to be interrupted. The walls next to axis 4 and 5 are used as additional support for the flat slab in-between. This system would require beams with fairly large cross-sections and a slab with a minimum thickness of $25 \mathrm{~cm}$.

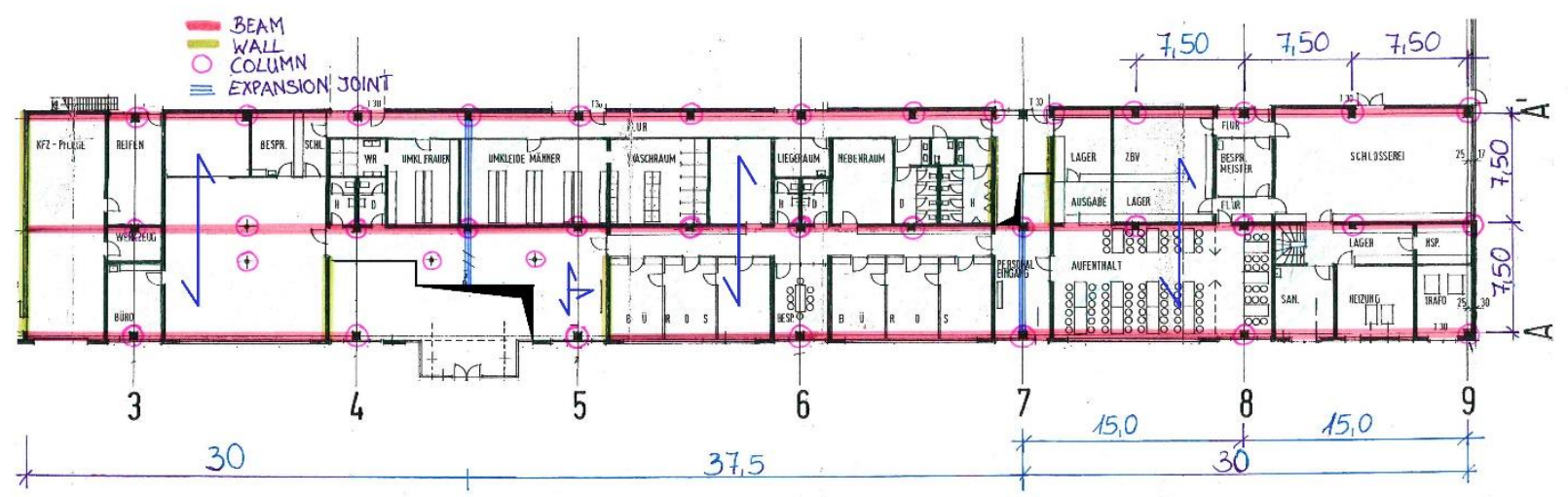

Figure 16 - Proposal of a Combination of a Slab-Beam System and a Flat Slab

\subsubsection{COMBINATION OF A DOUBLE-TEE BEAM SLAB AND A TWO-WAY SLAB}

Another possible solution is a combination of a double-tee beam system and a two-way slab as illustrated in Figure 17. The double-tee beams span between axis $A$ and $A^{\prime}$. That way large span lengths are possible and the column row in the middle, as well as the beams, are no longer necessary. Which creates more space and more flexibility on the ground floor of the office. In addition, instead of beams the concrete wall panels around the office are used as supports for the beams. Between axis 4 and 5, a two-way slab is used supported by the two load bearing walls next to the axes, a beam between those two and again the concrete wall panel in axis A'. The advantage of this system is that almost no columns and beams are needed inside the office area. Moreover, compared to the previous proposal, the construction of this slab system is easier 
and faster due to the high degree of prefabricated members. Therefore this system is chosen and will be analyzed in the following chapters.

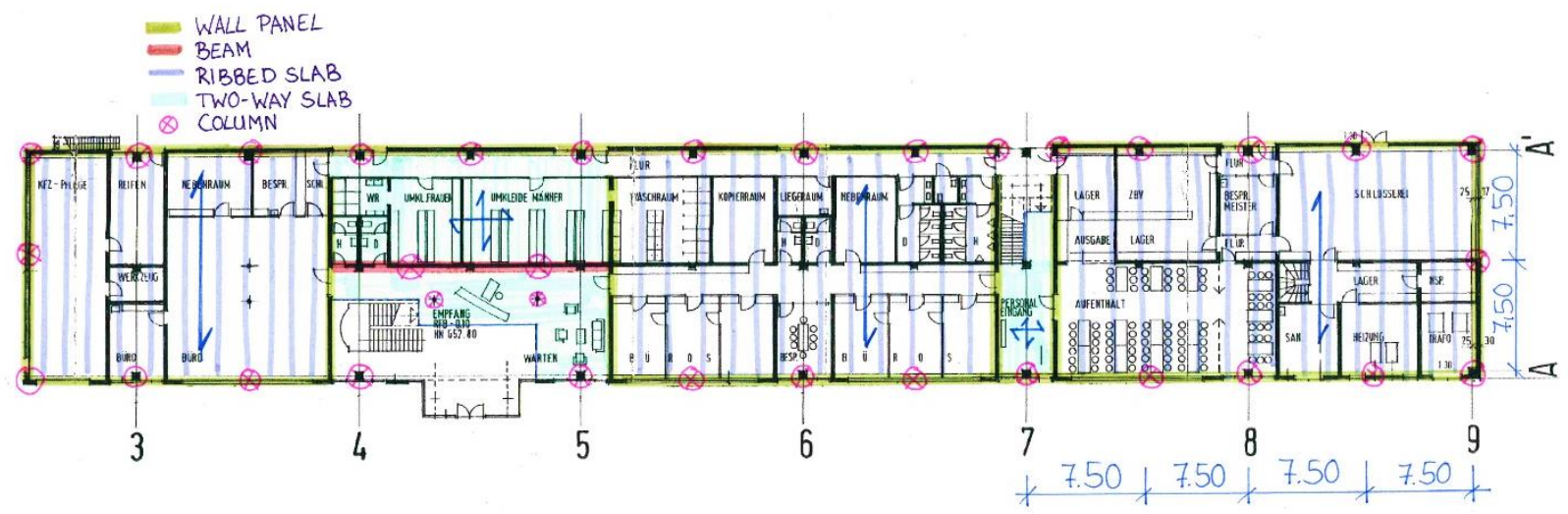

Figure 17 - Proposal of a Combination of a Double-Tee Slab System and A Two-Way Slab

\subsection{SYSTEM}

Longitudinal system

Transverse system

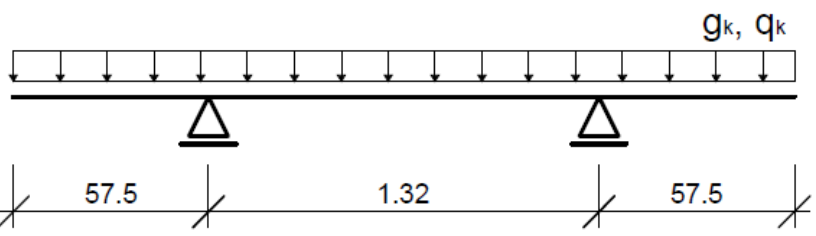

Cross-section

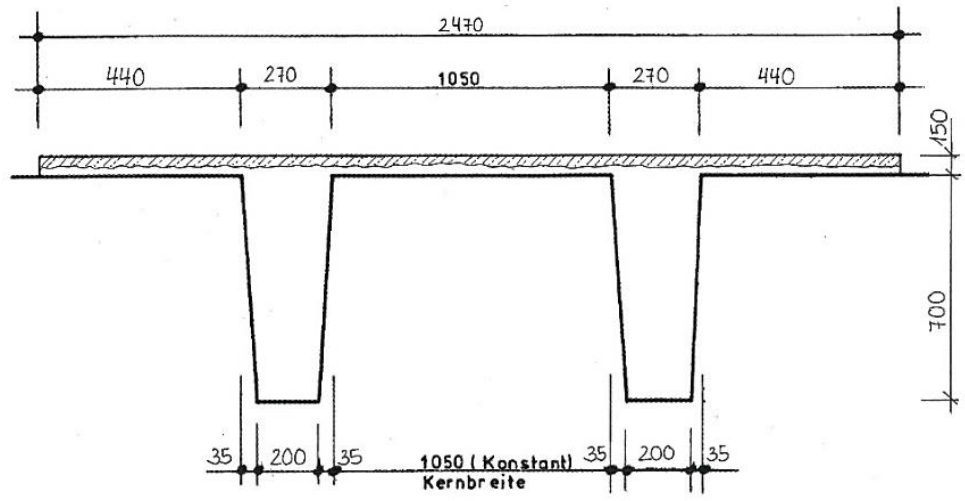

\begin{tabular}{|c|c|c|c|c|c|}
\hline $\mathbf{A}_{\mathbf{c}}\left[\mathbf{c m}^{2}\right]$ & $\mathbf{W}_{\mathrm{c}, \mathrm{u}}\left[\mathbf{c m}^{3}\right]$ & $\mathbf{I}_{\mathbf{y}}\left[\mathbf{c m}^{4}\right]$ & $\mathbf{z}_{\mathrm{s}, \mathrm{u}}[\mathbf{c m}]$ & $\mathbf{z}_{\mathrm{s}, \mathrm{o}}[\mathbf{c m}]$ & $\mathbf{d}[\mathbf{c m}]$ \\
\hline 6,995 & 73,694 & $4,298,400$ & 58.3 & 26.7 & 77.5 \\
\hline
\end{tabular}


Building materials

Exposure class

Reinforcing steel

Characteristic loads

\section{35/45 | B 500A}

$\mathrm{XC1}$, WO

$C_{\text {nom }}=10 \mathrm{~mm}+10 \mathrm{~mm}=20 \mathrm{~mm}$
Chosen: $\quad \mathrm{c}_{\mathrm{nom}}=25 \mathrm{~mm}$

\begin{tabular}{|c|c|c|c|}
\hline \multicolumn{2}{|c|}{ Uniformly Distributed Loads } & \multirow{2}{*}{$\frac{\begin{array}{c}\mathbf{g}_{\mathbf{k}} \\
{\left[\mathbf{k N} / \mathbf{m}^{2}\right]}\end{array}}{17.5}$} & \multirow{2}{*}{$\begin{array}{c}\mathbf{q}_{\mathbf{k}} \\
{\left[\mathrm{kN} / \mathrm{m}^{2}\right]} \\
-\end{array}$} \\
\hline Self-weight & $\mathrm{g}_{\mathrm{k} 1}=0.6995 \mathrm{~m}^{2} \times 25 \mathrm{kN} / \mathrm{m}^{3}=$ & & \\
\hline Floor structure & $g_{\mathrm{k} 2}=$ & 2.0 & - \\
\hline Live load (Cat. B & ffice Building) & - & 2.0 \\
\hline \multirow{2}{*}{\multicolumn{2}{|c|}{ Load from partition walls }} & & 1.2 \\
\hline & & 19.5 & 3.2 \\
\hline
\end{tabular}

\subsection{INTERNAL FORCES}

\subsubsection{LONGITUDINAL DIRECTION}

Loads in longitudinal direction

$$
\begin{aligned}
& g_{k}=17.5+2.47 \mathrm{~m} \cdot 2.0=22.5 \mathrm{kN} / \mathrm{m} \\
& q_{k}=2.47 \mathrm{~m} \cdot 3.2=7.9 \mathrm{kN} / \mathrm{m} \\
& q_{E d}=1.35 \cdot g_{k}+1.50 \cdot q_{k}=42.2 \mathrm{kN} / \mathrm{m} \\
& q_{p e r m}=g_{k}+0.3 \cdot q_{k}=24.9 \mathrm{kN} / \mathrm{m}
\end{aligned}
$$

Flexure moment

\begin{tabular}{|c|c|c|c|}
\hline $\mathbf{M}_{\mathbf{g k}}[\mathrm{kNm}]$ & $\mathbf{M}_{\mathbf{q k}}[\mathbf{k N m}]$ & $\mathbf{M}_{\mathrm{Ed}}[\mathrm{ULS}]$ & $\mathbf{M}_{\text {perm }}[\mathbf{S L S}]$ \\
\hline 684.5 & 240.3 & 1283.7 & 757.5 \\
\hline
\end{tabular}

Shear force

\begin{tabular}{|c|c|c|c|}
\hline $\mathbf{V}_{\mathbf{g k}}[\mathbf{k N}]$ & $\mathbf{V}_{\mathbf{q k}}[\mathbf{k N}]$ & $\mathbf{V}_{\mathrm{Ed}}[\mathbf{U L S}]$ & $\mathbf{V}_{\mathbf{p e r m}}[\mathbf{S L S}]$ \\
\hline 175.5 & 61.6 & 329.2 & 194.2 \\
\hline
\end{tabular}

\subsubsection{TRANSVERSE DIRECTION}

Loads in transverse direction

$$
\begin{aligned}
& g_{k}=0.15 \cdot 25 \mathrm{kN} / \mathrm{m}^{3}+2.0=5.75 \mathrm{kNm} / \mathrm{m} \\
& q_{k}=3.20 \mathrm{kNm} / \mathrm{m} \\
& q_{E d}=1.35 \cdot g_{k}+1.50 \cdot q_{k}=12.6 \mathrm{kN} / \mathrm{m} \\
& q_{\text {perm }}=g_{k}+0.3 \cdot q_{k}=6.7 \mathrm{kN} / \mathrm{m}
\end{aligned}
$$


Flexure moment

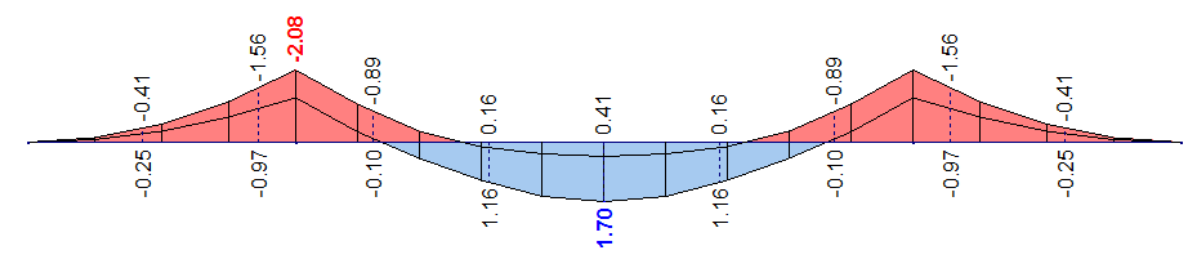

\begin{tabular}{|c|c|c|c|c|}
\cline { 2 - 5 } \multicolumn{1}{c|}{} & $\begin{array}{c}\mathbf{m}_{\mathbf{g k}} \\
{[\mathbf{k N m} / \mathbf{m}]}\end{array}$ & $\begin{array}{c}\mathbf{m}_{\mathbf{q k}} \\
{[\mathbf{k N m} / \mathbf{m}]}\end{array}$ & $\begin{array}{c}\mathbf{m}_{\mathbf{E d}} \\
{[\mathbf{U L S}]}\end{array}$ & $\begin{array}{c}\mathbf{M}_{\text {perm }} \\
{[\mathbf{S L S}]}\end{array}$ \\
\hline Midspan & 0.30 & 0.70 & 1.70 & 0.51 \\
\hline Support & -0.95 & -0.53 & -2.08 & -1.11 \\
\hline
\end{tabular}

Shear force

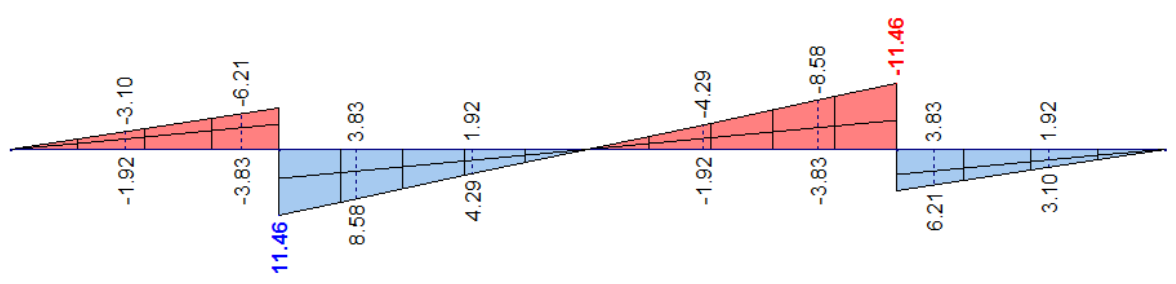

\begin{tabular}{|c|c|c|c|}
\hline $\begin{array}{c}\mathbf{V}_{\mathbf{g k}} \\
{[\mathbf{k N} / \mathbf{m}]}\end{array}$ & $\begin{array}{c}\mathbf{V}_{\mathbf{q k}} \\
{[\mathbf{k N} / \mathbf{m}]}\end{array}$ & $\begin{array}{c}\mathbf{V}_{\text {Ed }} \\
{[\mathrm{ULS}]}\end{array}$ & $\begin{array}{c}\mathbf{V}_{\text {perm }} \\
{[\mathbf{S L S}]}\end{array}$ \\
\hline 3.79 & 2.11 & 11.46 & 4.42 \\
\hline
\end{tabular}

\subsection{ULTIMATE LIMIT STATE DESIGN}

\subsubsection{FLEXURE DESIGN}

Longitudinal direction - Design of the T-Beam

Effective width

$$
\begin{aligned}
b_{\text {eff }, 1}=0.2 \cdot 44+0.1 \cdot 1560=164.8 \mathrm{~cm} & <0.2 \cdot 1560=312 \mathrm{~cm} \\
& <b_{1}=\underline{\underline{44 \mathrm{~cm}}} \\
b_{\text {eff }, 2}=0.2 \cdot 52.5+0.1 \cdot 1560=160.5 \mathrm{~cm} & <0.2 \cdot 1560=312 \mathrm{~cm} \\
& <b_{2}=\underline{\underline{52.5 \mathrm{~cm}}} \\
b_{\text {eff }}=2 \cdot\left(b_{\text {eff }, 1}+b_{w}+b_{\text {eff }, 2}\right)=\underline{\underline{.47 m}} &
\end{aligned}
$$

Longitudinal reinforcement Compression zone at the height of the in-situ concrete $\rightarrow$ C 20/25!

$$
\begin{aligned}
& \mu_{E d s}=\frac{M_{E d s}}{b_{\text {eff }} \cdot d^{2} \cdot f_{c d}}=\frac{128370 \mathrm{kNcm}}{247 \cdot 77.5^{2} \cdot 1.13 \mathrm{kN} / \mathrm{cm}^{2}}=0.08 \rightarrow \omega=0.0836 \\
& \xi=0.107 \rightarrow x=0.107 \cdot 77.5=8.3 \mathrm{~cm}<h_{f}=15 \mathrm{~cm} \\
& A_{s l, r e q}=\omega \cdot b \cdot d \cdot f_{c d} \cdot \frac{1}{\sigma_{s d}}=0.0836 \cdot 247 \cdot 77.5 \cdot 1.13 \cdot \frac{1}{43.5}=41.6 \mathrm{~cm}^{2}
\end{aligned}
$$

Chosen 
New static height

$$
d=h-c_{\text {nom }}-d_{s, b u ̈}-d_{s, l a ̈ l} / 2=85-2.0-1.0-3.25=78.75 \mathrm{~cm}
$$

Transverse direction - Design of the slab

Static height

$$
d=h-c_{\text {nom }}-d_{s, \text { quer }}-d_{s, \text { längs }} / 2=15-2.0-1.0-1.0 / 2=11.5 \mathrm{~cm}
$$

Reinforcement at Support

$$
\begin{aligned}
& \mu_{E d s}=\frac{m_{E d s}}{b_{\text {eff }} \cdot d^{2} \cdot f_{c d}}=\frac{208 \mathrm{kNcm}}{100 \cdot 11.5^{2} \cdot 1.98 \mathrm{kN} / \mathrm{cm}^{2}}=0.008 \rightarrow \omega=0.0101 \\
& a_{s o, \text { req }}=\omega \cdot b \cdot d \cdot f_{c d} \cdot \frac{1}{\sigma_{s d}}=0.0101 \cdot 100 \cdot 11.5 \cdot 1.98 \cdot \frac{1}{43.5}=0.53 \mathrm{~cm}^{2} / \mathrm{m}
\end{aligned}
$$

Reinforcement at Midspan

Compression zone at the height of the in-situ concrete $\rightarrow C 20 / 25$ !

$$
\begin{aligned}
& \mu_{E d s}=\frac{m_{E d s}}{b_{\text {eff }} \cdot d^{2} \cdot f_{c d}}=\frac{170 \mathrm{kNcm}}{100 \cdot 11.5^{2} \cdot 1.13 \mathrm{kN} / \mathrm{cm}^{2}}=0.011 \rightarrow \omega=0.0101 \\
& a_{s o, r e q}=\omega \cdot b \cdot d \cdot f_{c d} \cdot \frac{1}{\sigma_{s d}}=0.0101 \cdot 100 \cdot 11.5 \cdot 1.98 \cdot \frac{1}{43.5}=0.53 \mathrm{~cm}^{2} / \mathrm{m}
\end{aligned}
$$

Chosen

Q188A

Q188A upper transverse reinforcement lower transverse reinforcement

\subsubsection{SHEAR DESIGN}

Longitudinal direction - Design of the T-Beam

Design location

Shear force at design location

Scale factor

Percentage of reinforcement

Shear force resistance

$$
x=a_{i}+d=0.1+78.8=79.8 \mathrm{~cm}
$$

$V_{E d, x}=329.2-0.798 \cdot 42.2=295.5 \mathrm{kN}$

$$
k=1+\sqrt{\frac{20}{d}}=1+\sqrt{\frac{20}{78.8}}=1.50<\underline{\underline{2.00}}
$$

$\rho_{1}=\frac{A_{s l}}{b_{w} \cdot d}=\frac{49.1}{40 \cdot 78.8}=0.016 \leq \underline{\underline{0.02}}$

$$
\begin{aligned}
& V_{R d, c}=\left[0.10 \cdot \mathrm{k} \cdot\left(100 \cdot \rho_{l} \cdot f_{c k}\right)^{1 / 3}+0.12 \cdot \sigma_{c p}\right] \cdot b_{w} \cdot d \\
& V_{R d, c}=\left[0.10 \cdot 1.5 \cdot(100 \cdot 0.016 \cdot 35)^{1 / 3}\right] \cdot 0.78 \cdot 0.40 \cdot 10^{3}=179 \mathrm{kN} \\
& d=78.8 \mathrm{~cm}>80 \mathrm{~cm} \rightarrow v_{\min }=0.025 \cdot 1.50^{3 / 2} \cdot(35)^{1 / 2}=0.272 \mathrm{MN} / \mathrm{m}^{2} \\
& V_{R d, c, \min }=0.272 \cdot 0.40 \cdot 0.788 \cdot 10^{3}=85.7 \mathrm{kN}<V_{R d, c}=179 \mathrm{kN} \\
& V_{E d}=295.5 \mathrm{kN}>V_{R d, c}=179 \mathrm{kN}
\end{aligned}
$$

$\rightarrow$ Shear reinforcement is needed!

Lever arm of the internal forces $z=\zeta \cdot d=0.956 \cdot 78.8=75.3 \mathrm{~cm}$ 
Friction Force:

Inclination:

Vertical stirrups

Required reinforcement

Chosen

$$
V_{R d, c c}=0.24 \cdot(35)^{1 / 3} \cdot 0.4 \cdot 0.753 \cdot 10^{3}=236.5 \mathrm{kN}
$$

$1.0 \leq \cot \theta=\frac{1.2}{1-V_{R d, c c} / V_{E d}}=\frac{1.2}{1-236.5 / 295.5}=6.0 \leq \underline{\underline{3.0}}$

$$
\alpha=90^{\circ}
$$

$a_{s w, r e q}=\frac{295.5 \mathrm{kN}}{43.5 \cdot 0.753 \cdot 3.0}=3.0 \mathrm{~cm}^{2} / \mathrm{m}$

\section{$\varnothing 8 / 20=5.03 \mathrm{~cm}^{2} / \mathrm{m}$}

stirrups

Resistance of the Compression Struts

$$
\begin{aligned}
& V_{R d \text {,max }}=0.75 \cdot 40 \cdot 75.3 \cdot 1.98 \cdot \frac{3.00}{1+3.00^{2}}=1342 \mathrm{kN} \\
& V_{E d, \text { max }}=329.2 \mathrm{kN}<V_{R d, \text { max }}=1342 \mathrm{kN} \rightarrow \text { The compression strut is ok. }
\end{aligned}
$$

Minimum shear reinforcement $\quad \min a_{s w}=\rho \cdot b_{w} \cdot \sin \alpha \quad$ and $\quad \rho=0.16 \cdot f_{c t m} / f_{y k}=0.16 \cdot 3.2 / 500=1.02 \%$

$$
\begin{aligned}
& \min a_{s w}=0.00102 \cdot 0.40 \cdot \sin 90^{\circ}=4.08 \cdot 10^{-4} \mathrm{~m}^{2} / \mathrm{m}=\underline{\underline{4.08 \mathrm{~cm}^{2} / \mathrm{m}}} \\
& \min a_{s w}=4.08 \mathrm{~cm}^{2} / \mathrm{m}<a_{s w, p r o v}=5.03 \mathrm{~cm}^{2} / \mathrm{m}^{\checkmark}
\end{aligned}
$$

Transverse direction - Design of the slab (C20/25)

Scale factor

$$
k=1+\sqrt{\frac{20}{d}}=1+\sqrt{\frac{20}{11.5}}=2.32<\underline{\underline{2.00}}
$$

Percentage of reinforcement

$$
\rho_{1}=\frac{a_{s l}}{b_{w} \cdot d}=\frac{2.01}{100 \cdot 11.5}=0.0017 \leq 0.02
$$

Shear force resistance

$$
\begin{aligned}
& v_{R d, c}=\left[0.10 \cdot \mathrm{k} \cdot\left(100 \cdot \rho_{l} \cdot f_{c k}\right)^{1 / 3}+0.12 \cdot \sigma_{c p}\right] \cdot b_{w} \cdot d \\
& v_{R d, c}=\left[0.10 \cdot 2.0 \cdot(100 \cdot 0.0017 \cdot 20)^{1 / 3}\right] \cdot 1.0 \cdot 0.115 \cdot 10^{3}=34.6 \mathrm{kN} / \mathrm{m} \\
& d=11.5 \mathrm{~cm}<60 \mathrm{~cm} \rightarrow v_{\min }=0.035 \cdot 2.00^{3 / 2} \cdot(20)^{1 / 2}=0.443 \mathrm{MN} / \mathrm{m}^{2} \\
& v_{R d, c, \min }=0.443 \cdot 1.00 \cdot 0.115 \cdot 10^{3}=\underline{\underline{50.9 \mathrm{kN}}} / \mathrm{m}>v_{R d, c}=34.6 \mathrm{kN} / \mathrm{m} \\
& v_{E d}=11.5 \mathrm{kN} / \mathrm{m}<v_{R d, c}=50.9 \mathrm{kN} / \mathrm{m} \checkmark
\end{aligned}
$$

No further shear force reinforcement is needed! 


\subsection{SERVICEABILITY LIMIT STATE DESIGN}

\subsubsection{LIMITATION OF THE CRACK WIDTH}

Maximum diameter of the reinforcement

Crack width

Lever arm of the forces

Reinforcement stresses $\mathrm{w}_{\mathrm{k}}=0.4 \mathrm{~mm}$ (according to DIN EN 1992-1-1, Tab. NA.7.1.)

$z=\zeta \cdot d=0.956 \cdot 78.8=75.3 \mathrm{~cm}$

$\sigma_{s}=\frac{M_{p e r m}}{z \cdot A_{s l}}=\frac{75750 \mathrm{kNcm}}{75.3 \cdot 49.1 \mathrm{~cm}^{2}}=20.5 \mathrm{kN} / \mathrm{cm}^{2}=205 \mathrm{~N} / \mathrm{mm}^{2}$

Limit diameter

$$
\begin{aligned}
& d_{s}^{*}=33.63 \\
& d_{s}=d_{s}^{*} \cdot f_{c t, e f f} / 2.9=33.63 \cdot 3.2 / 2.9=37.1 \mathrm{~mm}>d_{s, p r o v}=25 \mathrm{~mm}
\end{aligned}
$$

\begin{tabular}{|c|c|c|}
\hline \multirow[t]{3}{*}{ Tensile strength } & $f_{c t, e f f}=f_{c t m} \geq 3.0 \mathrm{MN} / \mathrm{m}^{2}$ & $\rightarrow f_{c t, \text { eff }}=3.2 \mathrm{MN} / \mathrm{m}^{2}>3.0 \mathrm{MN} / \mathrm{m}^{2}$ \\
\hline & for $h>80 \mathrm{~cm}$ & $\rightarrow k=0.50$ \\
\hline & for flexure & $\rightarrow k_{c}=0.4$ \\
\hline
\end{tabular}

Minimum reinforcement to prevent cracking

Tensile stress area

Reinforcement stresses

Required Reinforcement
$A_{c t}=b \cdot h / 2=40 \cdot 85 / 2=1700 \mathrm{~cm}^{2}$

$$
\sigma_{s}=236 M N / m^{2} \quad\left(\mathrm{w}_{\mathrm{k}}=0.4 \mathrm{~mm}, \mathrm{~d}_{\mathrm{s}}=25 \mathrm{~mm}\right)
$$

$$
A_{s, \text { min, req }}=k_{c} \cdot k \cdot f_{c t, \text { eff }} \cdot \frac{A_{c t}}{\sigma_{s}}=0.4 \cdot 0.5 \cdot 3.2 \cdot \frac{1700}{236}=4.6 \mathrm{~cm}^{2}
$$

$$
<A_{s, \text { prov }}=49.1 \mathrm{~cm}^{2} \checkmark
$$

The provided reinforcement is sufficient!

\subsubsection{DEFORMATION OF THE SLAB}

Deformation of the Slab under $q_{p e r m}$

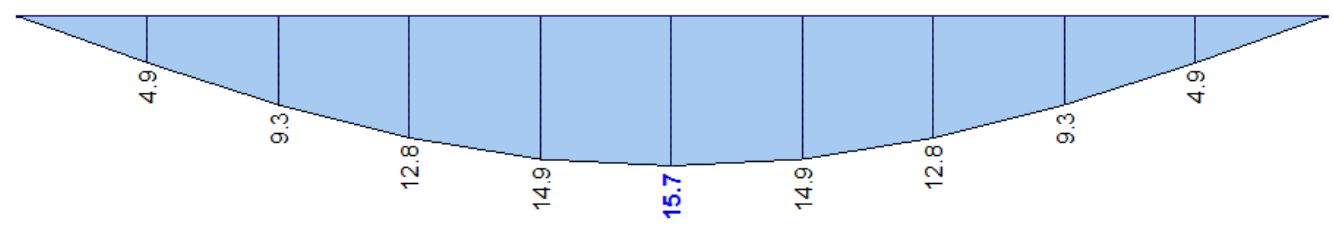

$f_{\max }=15.7 m m<I / 250=15600 / 200=62.4 m m \checkmark$

The deformation does not exceed the maximum allowed deformation! 


\subsection{DESIGN AND REINFORCEMENT}

\subsubsection{MINIMUM REINFORCEMANT TO ENSURE ROBUSTNESS}

Longitudinal direction - Design of the T-Beam

$$
\begin{aligned}
& M_{c r}=f_{c t m} \cdot W_{c u} \quad W_{c u}=73,694 \mathrm{~cm}^{3} \\
& M_{c r}=3.2 \cdot 73,694 \cdot 10^{-3}=235.8 \mathrm{kNm} \\
& A_{s, \text { in }}=\frac{1}{f_{y k}} \cdot \frac{M_{c r}}{z}=\frac{1}{50.0} \cdot \frac{23580}{75.3}=6.3 \mathrm{~cm}^{2}<A_{s, \text { rov }}=49.1 \mathrm{~cm}^{2}
\end{aligned}
$$

Transverse direction - Design of the slab

$$
\begin{aligned}
& M_{c r}=f_{c t m} \cdot W_{c u} \quad W_{c u}=100 \cdot 15^{2} / 6=3750 \mathrm{~cm}^{3} \\
& M_{c r}=2.2 \cdot 3750 \cdot 10^{-3}=8.25 \mathrm{kNm} / \mathrm{m} \\
& a_{s, \min }=\frac{1}{f_{y k}} \cdot \frac{M_{c r}}{z}=\frac{1}{50.0} \cdot \frac{8.25}{11.4}=0.02 \mathrm{~cm}^{2} / m<a_{s, p r o v}=2.01 \mathrm{~cm}^{2} / \mathrm{m}
\end{aligned}
$$

\subsubsection{DESIGN OF THE INTERCONNECTING JOINT}

Design location

Shear force

Rough joint

Maximum shear force

$$
\begin{aligned}
& x=0.1+0.115-0.09=0.125 \mathrm{~m} \\
& V_{E d, x}=329.2-0.125 \cdot 42.2=323.9 \mathrm{kN} \\
& v_{E d, i}=\frac{323.9}{247 \cdot 11.4}=0.115 \mathrm{kN} / \mathrm{cm}^{2}
\end{aligned}
$$

$$
\begin{array}{ccc}
c=0.4 \quad \mu=0.7 \quad v=0.5 \\
v_{R d i, c}=c \cdot 0.12 \cdot\left(f_{c k}\right)^{2 / 3}=0.4 \cdot 0.12 \cdot(20)^{2 / 3} \cdot 10=3.54 \mathrm{kN} / \mathrm{cm}^{2} \\
>v_{E d, i}=0.115 \mathrm{kN} / \mathrm{cm}^{2} \checkmark
\end{array}
$$

No reinforcement at the interconnection joint necessary!

\subsubsection{CONNECTION OF THE COMPRESSION FLANGE}

Design location

Flexure moment

Bond force in the joint

Shear force resistance

$$
\begin{aligned}
& \Delta x=1 / 2 \cdot l / 2=15.6 / 4=3.90 \mathrm{~m} \\
& M_{E d}(x=3.9)=329.2 \cdot 3.9-42.2 \cdot 3.92 / 2=962.9 \mathrm{kNm}=\Delta M_{E d} \\
& v_{E d}=\frac{\Delta M_{E d}}{z \cdot h_{f} \cdot \Delta x} \cdot \frac{b_{a}}{b_{\text {eff }}}=\frac{962.9}{0.735 \cdot 0.15 \cdot 3.90} \cdot \frac{97.5}{247}=884 \mathrm{kN} / \mathrm{m}^{2} \\
& \cot \theta=1.2 \\
& v_{R d, \max }=\frac{0.75 \cdot f_{c d}}{\cot \theta+\tan \theta}=\frac{0.75 \cdot 11.3}{1.2+1 / 1.2} \cdot 10^{3}=4170 \mathrm{kN} / \mathrm{m}^{2}>v_{E d}=884 \mathrm{kN} / \mathrm{m}^{2}
\end{aligned}
$$


Transverse reinforcement $\quad a_{s w, r e q}=\frac{v_{E d} \cdot h_{f}}{f_{y d} \cdot \cot \theta}=\frac{884 \cdot 0.15}{43.5 \cdot 1.2}=2.54 \mathrm{~cm}^{2} / \mathrm{m}$

Chosen

Q188A

Q188 A upper reinforcement

lower reinforcement

\subsubsection{REINFORCEMENT DRAWING}

See Appendix A.5, page 191.

\subsection{TRANSPORT AND ASSEMBLY}

\subsubsection{TRANSPORT TO THE SITE}

Each Double-Tee Slab will be suspended with the help of four anchors evenly placed across from each other.

System
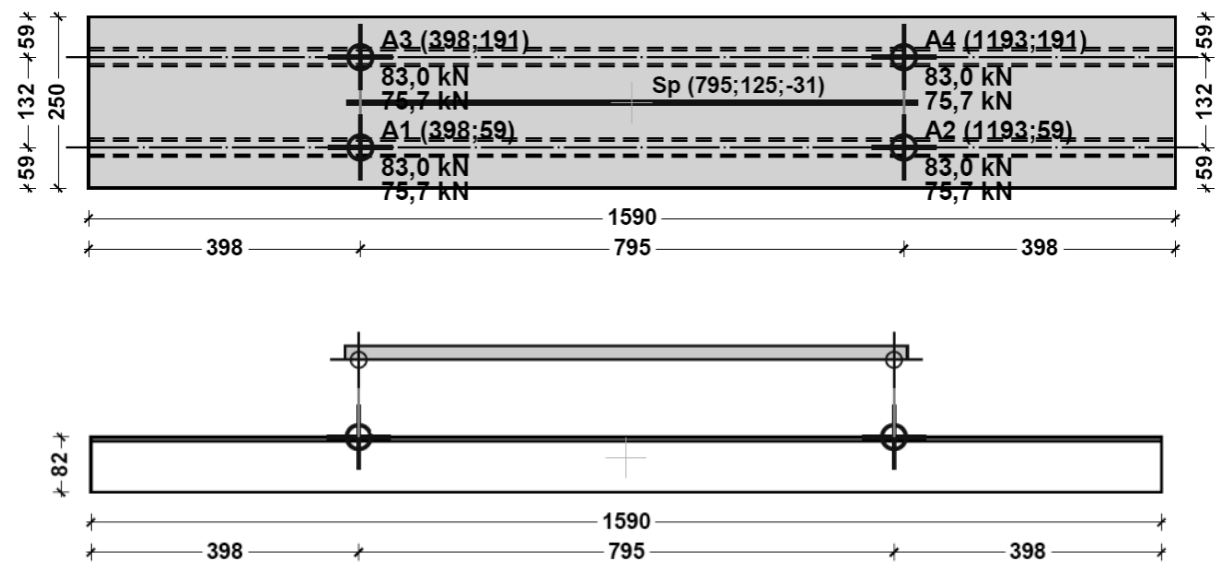

Static System Longitudinal Direction

$g_{k}$

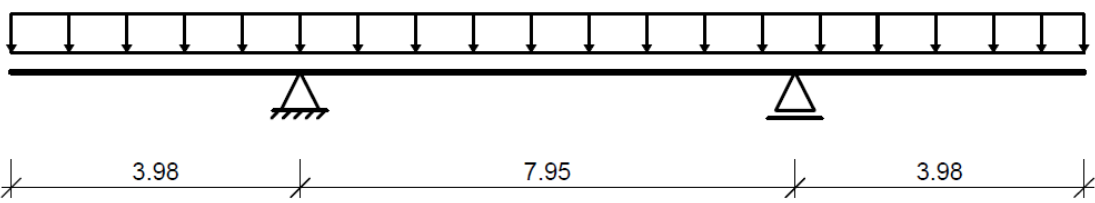

Static System Transverse Direction

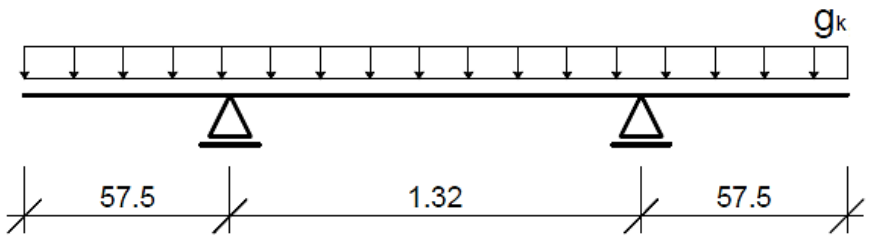


Design in Longitudinal Direction

Self-weight $\begin{aligned} & V=0.70 \mathrm{~m}^{3} / \mathrm{m} \\ & g_{k}=0.70 \mathrm{~m}^{3} \times 25 \mathrm{kN} / \mathrm{m}^{3}=17.5 \mathrm{kN} / \mathrm{m}\end{aligned}$

Flexure Moment

$M_{E d}^{G}=1.15 \cdot(-17.5) \cdot 3.98^{2} / 2=-159.4 k N m$

Time of first lifting

approximately after 5 days $(t=5 d)$

Compressive strength at time $t$

\[ \begin{aligned} & f_{c m}(t)=\beta_{c c}(t) \cdot f_{c m} \rightarrow \quad \beta_{c c}(t=5 d)=e^{s(1-\sqrt{28 / t})}=e^{0.2(1-\sqrt{28 / 5})}=0.76 \\ & f_{c m}(t=5 d)=0.76 \cdot 43=32.68 \mathrm{~N} / \mathrm{mm}^{2}\end{aligned} \]
$f_{c k}(t=5 d)=f_{c m}(t)-8=32.68-8=24.68 \mathrm{~N} / \mathrm{mm}^{2}$
$f_{c d}(t=5 d)=0.85 \cdot f_{c k}(t) / 1.5=0.85 \cdot 24.68 / 1.5=\underline{\underline{14.0 \mathrm{~N} / \mathrm{mm}^{2}}}$
$d \approx 76-5=71 \mathrm{~cm}$
Effective depth $\begin{aligned} \mu_{E d s} & =\frac{M_{E d s}}{b \cdot d^{2} \cdot f_{c d}}=\frac{15940}{248 \cdot 71^{2} \cdot 1.4}=0.01 \quad \rightarrow \omega=0.0101 \\ \text { Required Reinforcement } & =0.0101 \cdot 248 \cdot 71 \cdot 1.40 / 43.5=\underline{\underline{5.72 c m^{2}}} \\ A_{s 2, r e q} & =\left(\omega \cdot b \cdot d \cdot f_{c d}+N_{E d}\right) / \sigma_{s d} \\ \text { Chosen } & \text { upper reinforcement }\end{aligned}$
Design in Transverse Direction

Load on flange

Flexure moment

Effective depth

Required reinforcement

$$
g_{k}=0.15 \mathrm{~m} \times 25 \mathrm{kN} / \mathrm{m}^{3}=3.75 \mathrm{kN} / \mathrm{m} / \mathrm{m}
$$

$$
M_{E d}^{G}=1.15 \cdot(-3.75) \cdot 0.575^{2} / 2=-0.71 \mathrm{kNm} / \mathrm{m}
$$

$d \approx 4 \mathrm{~cm}$

$$
\begin{aligned}
\mu_{E d s}= & \frac{M_{E d s}}{b \cdot d^{2} \cdot f_{c d}}=\frac{71}{100 \cdot 4^{2} \cdot 1.4}=0.03 \rightarrow \omega=0.0306 \\
a_{s 2, r e q} & \left(\omega \cdot b \cdot d \cdot f_{c d}+N_{E d}\right) / \sigma_{s d} \\
& =0.0306 \cdot 100 \cdot 4 \cdot 1.40 / 43.5=\underline{\underline{0.39 \mathrm{~cm}^{2}}} / \mathrm{m}
\end{aligned}
$$

\subsubsection{TRANSPORT ANCHORS}

In order to lift the double-tee slab out of its formwork as well as to be able to transport and assemble it on site, transport anchors are needed. Those are installed during the production of the beam and must be designed in accordance with the VDI/BV-BS-Richtlinie 6205. For this project the anchors of the company Halfen are chosen.

Self-weight

Formwork adhesion

$$
F_{G}=\left(17.5 \mathrm{kN} / m-0.08 \cdot 25 \mathrm{kN} / \mathrm{m}^{3}\right) \cdot 15.9 m=246.5 \mathrm{kN}
$$$$
F_{\text {adh }}=q_{\text {adh }} \cdot A_{f} \quad \rightarrow \text { no adhesion here because of hinged formwork! }
$$ 
Dynamic factor

Factor for inclination

Tensile force

$$
\begin{aligned}
& \psi_{d y n}=1.3 \\
& Z=1.16 \text { for } \beta=30^{\circ} \\
& F_{z}=\frac{F_{G} \cdot \psi_{d y n} \cdot z}{n}=\frac{246.5 \cdot 1.3 \cdot 1.16}{4}=\underline{\underline{92.9 \mathrm{kN}}}
\end{aligned}
$$

\section{HALFEN DEHA Spherical head anchor 6000-10,0-0340}

\begin{tabular}{|c|c|c|c|c|c|c|c|c|c|}
\hline \multirow[b]{2}{*}{$\begin{array}{l}\text { Load } \\
\text { class }\end{array}$} & \multirow[b]{2}{*}{ Article number } & \multirow{2}{*}{$\begin{array}{l}\text { Anchor } \\
\text { length } \\
\text { I } \\
{[\mathrm{mm}]}\end{array}$} & \multirow{2}{*}{$\begin{array}{c}\text { Minimum height } \\
\text { of beams } \\
B_{1} \\
{[\mathrm{~mm}]}\end{array}$} & \multirow{2}{*}{$\begin{array}{l}\text { Wall } \\
\text { thickness } \\
2 \times \mathrm{e}_{\mathrm{r}} \\
{[\mathrm{mm}]}\end{array}$} & \multicolumn{4}{|c|}{ Load capacity $[\mathrm{kN}]$ at concrete strength $\mathrm{f}_{\mathrm{ci}}$ for } & \multirow{2}{*}{$\begin{array}{c}\text { Axial spacing of } \\
\text { anchors } \\
e_{z} \\
{[\mathrm{~mm}]}\end{array}$} \\
\hline & & & & & $\begin{array}{l}\text { Axial pull } \\
\text { up to } 30^{\circ}[\beta] \\
15 \mathrm{~N} / \mathrm{mm}^{2}\end{array}$ & $\begin{array}{l}\text { Diagonal pull } \\
\text { up to } 60^{\circ}[\beta] \\
15 \mathrm{~N} / \mathrm{mm}^{2}\end{array}$ & $\begin{array}{c}\text { Axial pull and } \\
\text { diagonal pull } \\
\text { up to } 60^{\circ}[\beta] \\
25 \mathrm{~N} / \mathrm{mm}^{2}\end{array}$ & $\begin{array}{c}\text { Axial pull and } \\
\text { diagonal pull } \\
\text { up to } 60^{\circ}[\beta] \\
35 \mathrm{~N} / \mathrm{mm}^{2}\end{array}$ & \\
\hline \multirow{9}{*}{10,0} & \multirow{3}{*}{$6000-10,0-0170$} & \multirow{3}{*}{170} & \multirow{3}{*}{340} & 300 & 46.4 & 37.2 & 60.0 & 70.9 & \multirow{3}{*}{520} \\
\hline & & & & 350 & 52.1 & 41.7 & 67.3 & 79.6 & \\
\hline & & & & 400 & 57.6 & 46.1 & 74.4 & 88.0 & \\
\hline & \multirow{3}{*}{$6000-10,0-0340$} & \multirow{3}{*}{340} & \multirow{3}{*}{680} & 280 & 76.6 & 61.3 & 98.9 & \multirow{3}{*}{100.0} & \multirow{3}{*}{1030} \\
\hline & & & & 300 & 80.7 & 64.5 & 1000 & & \\
\hline & & & & 320 & 84.7 & 67.7 & 100.0 & & \\
\hline & \multirow{3}{*}{$6000-10,0-0680$} & \multirow{3}{*}{680} & \multirow{3}{*}{1360} & 160 & 73.7 & 70.0 & 95.2 & \multirow{3}{*}{100.0} & \multirow{3}{*}{2050} \\
\hline & & & & 180 & 83.0 & 76.5 & \multirow{2}{*}{100.0} & & \\
\hline & & & & 200 & 92.2 & 82.8 & & & \\
\hline
\end{tabular}

$$
F_{z, \text { Rd }}=92.9 k N>F_{z, E d}=98.9 k N \checkmark \quad \text { (see Table 21) }
$$

Table 21 - Sizing Table for 'HALFEN DEHA Lifting Anchor Systems' 


\subsection{SOFTWARE CALCULATIONS}

\section{Demo Frilo Nemetschek}

\section{Position: S.11 - Semi-Precast Double-Tee Slab}

Durchlaufträger DLT10 01/2018 (Frilo R-2018-1/P12)

\section{Scale $1: 125$}
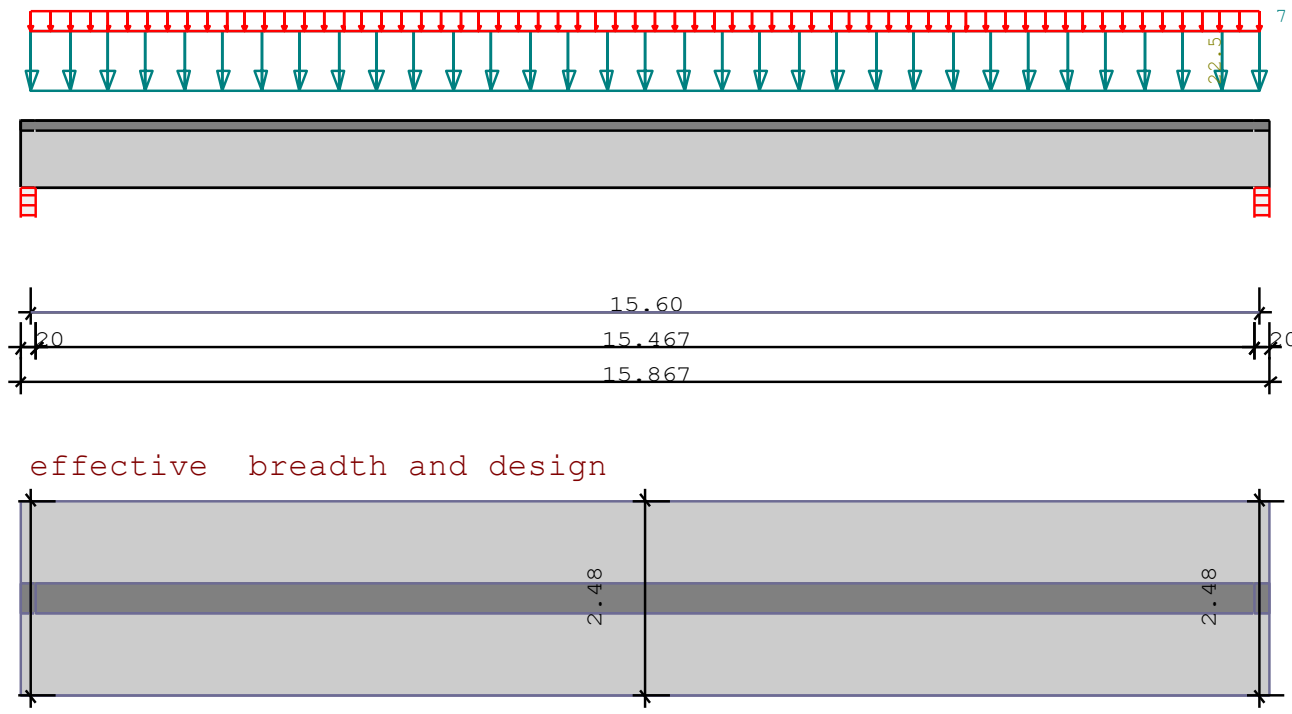

Results for 1-times loads

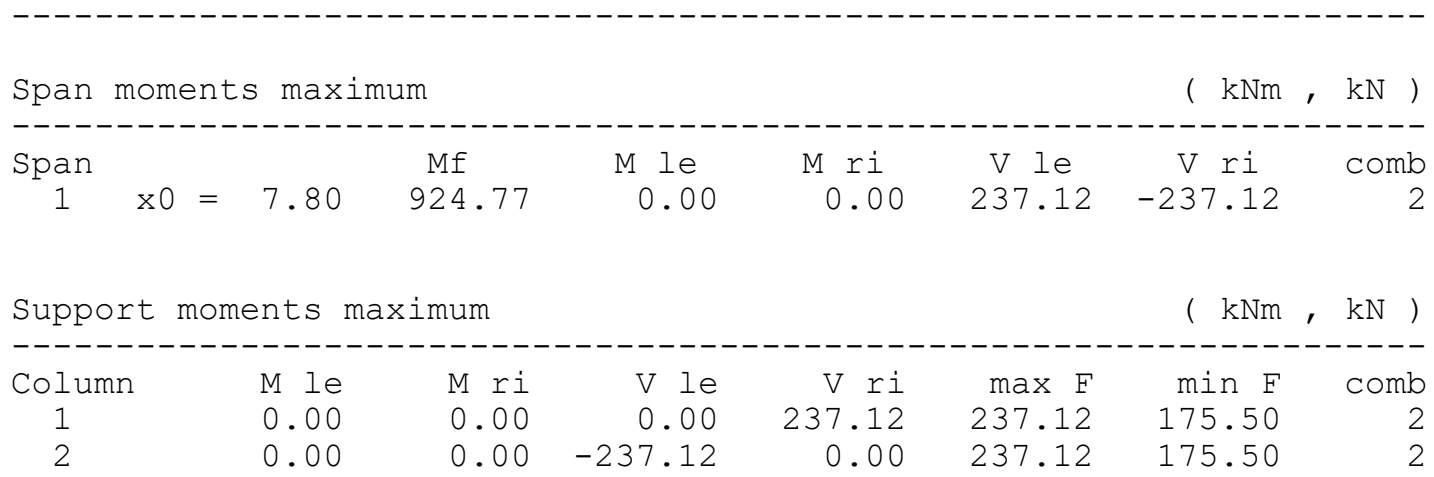

Moment boundary diagram

--------------------------------------------------------------------

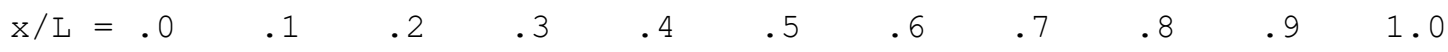
Span

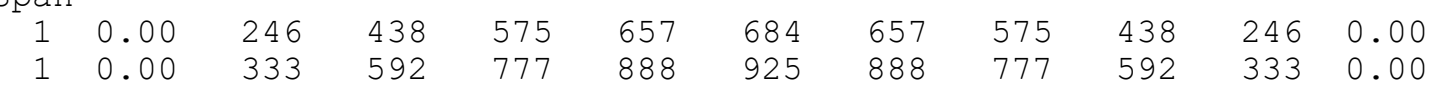

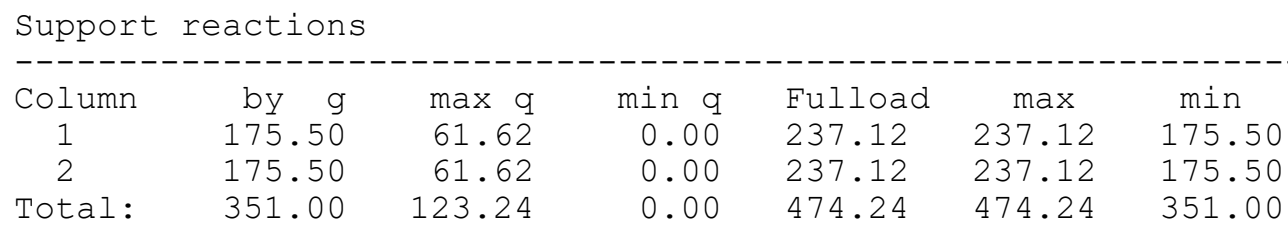




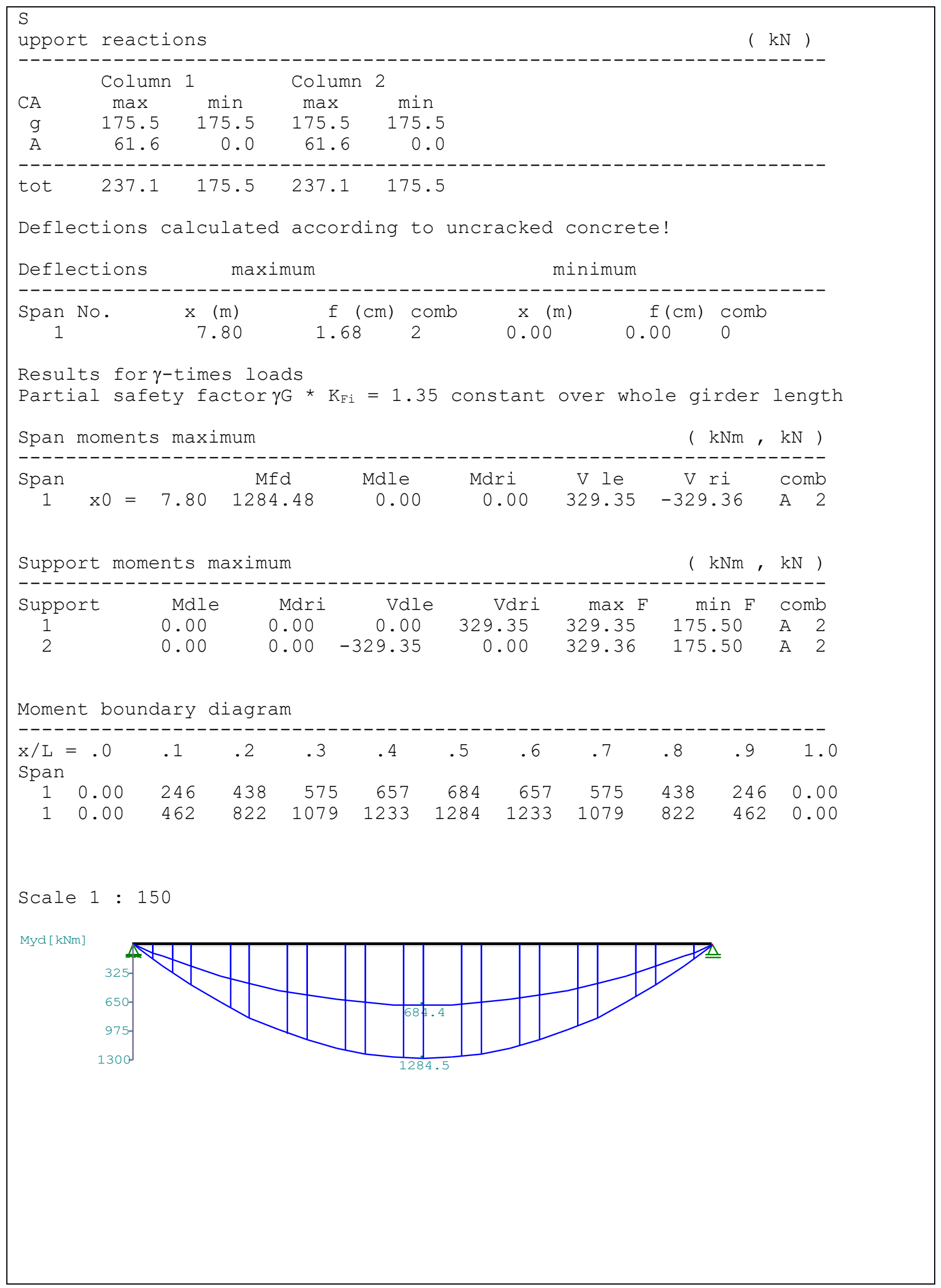




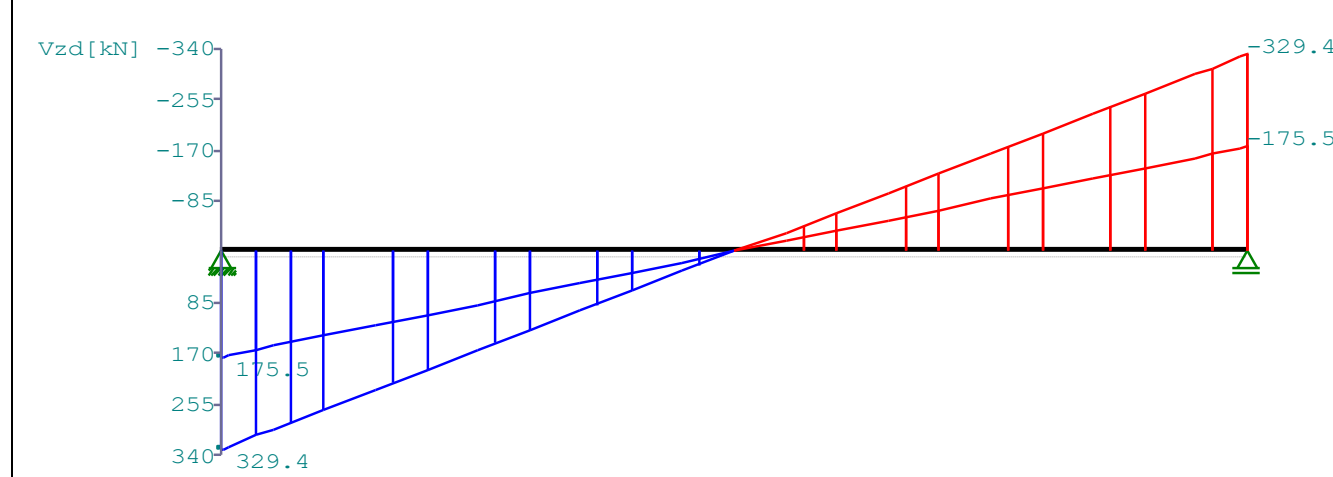

Minimum reinforcement EN2 9.2.1.1 (9.1) fctm $=3.21 \mathrm{~N} / \mathrm{mm} 2$

Calculating $\mathrm{Wy}$, breadth of slab is limited to $2 * \mathrm{~b} 0$.

Q.No. min $\mathrm{Mb}$ req As min Mo req As

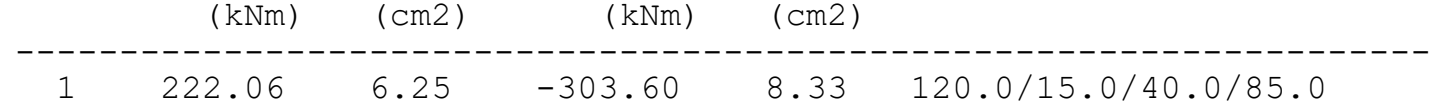

Span reinforcement

\begin{tabular}{|c|c|c|c|c|c|c|c|}
\hline $\begin{array}{l}\text { Span } \\
\text { No. }\end{array}$ & $\begin{array}{c}x \\
(m)\end{array}$ & $\begin{array}{l}\text { Myd } \\
(\mathrm{kNm})\end{array}$ & $\begin{array}{c}\min \text { Myd } \\
(\mathrm{kNm})\end{array}$ & $\begin{array}{l}d \\
(\mathrm{~cm})\end{array}$ & $\mathrm{kx}$ & $\begin{array}{c}\text { Asb Ast } \\
(\mathrm{cm} 2)\end{array}$ & comb \\
\hline 1 & 7.80 & 1284.5 & & 79.0 & 0.07 & $\begin{array}{ll}36.5 & 0.0\end{array}$ & A 2 \\
\hline
\end{tabular}

On first support are at least $11.3 \mathrm{~cm} 2$ to be anchored.

On last support are at least $11.3 \mathrm{~cm} 2$ to be anchored.

Shear force VK-support is with $\mathrm{F}=\mathrm{V}$, Ed * Cot(Theta) / 2 considered.

shear force reinforcement B500A DIN EN 1992-1-1/NA/A1:2015-12 6.2

column dist kz VEd $\Theta \quad$ VRd, C VRd, max a max asw

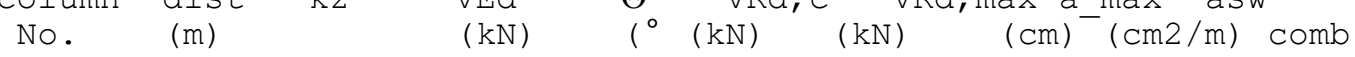

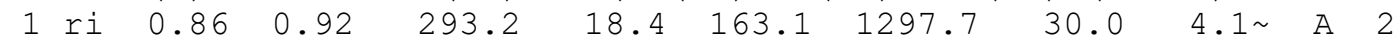

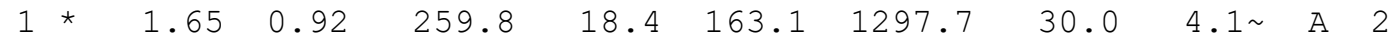

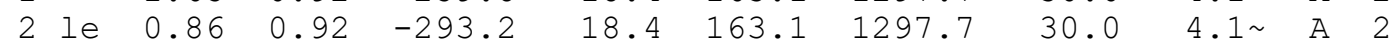

2 * $\quad 1.65 \quad 0.92 \quad-259.8 \quad 18.4 \quad 163.1 \quad 1297.7 \quad 30.0 \quad 4.1 \sim$ A 2

$\sim$ at the end of line: Minimum stirrup reinforcement

max distance of stirrups will with $\Theta>=40^{\circ}$ investigated (paper DAfStb $525)$.

shoulder shear

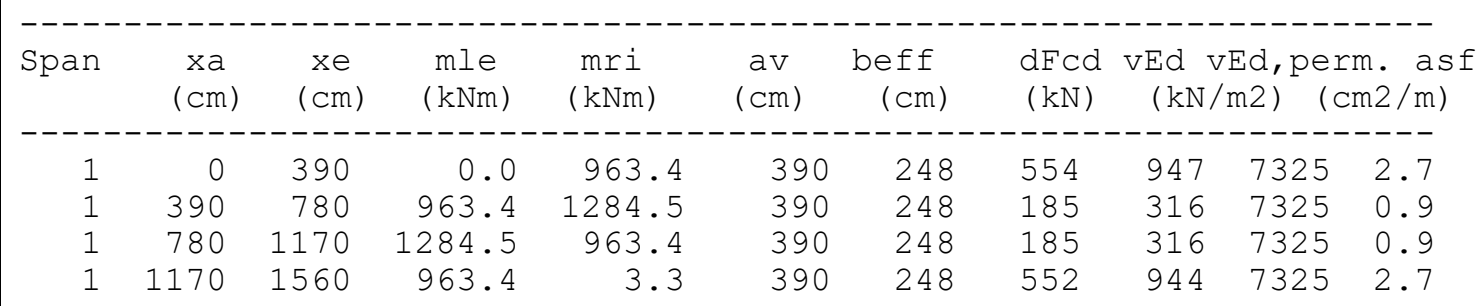


12 POS. S.12 - SEMI-PRECAST CONCRETE SLAB

\subsection{SYSTEM}

System

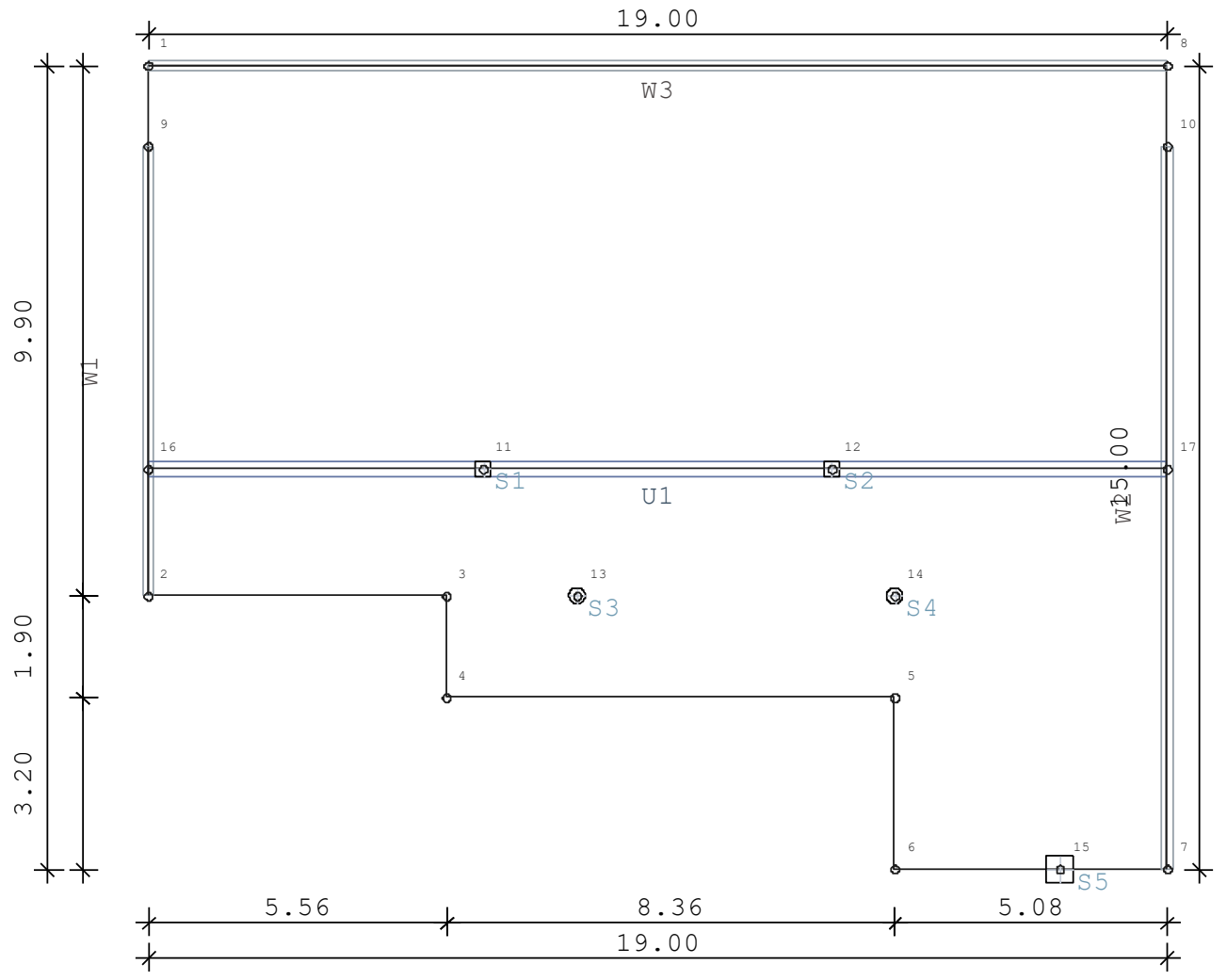

Cross-section

$\mathrm{b} / \mathrm{h} / \mathrm{d}=100 / 35 \mathrm{~cm}$

Building materials

C 30/37 | B 500A

Exposure class

XC1, WO

Reinforcing steel

$C_{n o m}=10 \mathrm{~mm}+10 \mathrm{~mm}=20 \mathrm{~mm}$

Characteristic loads

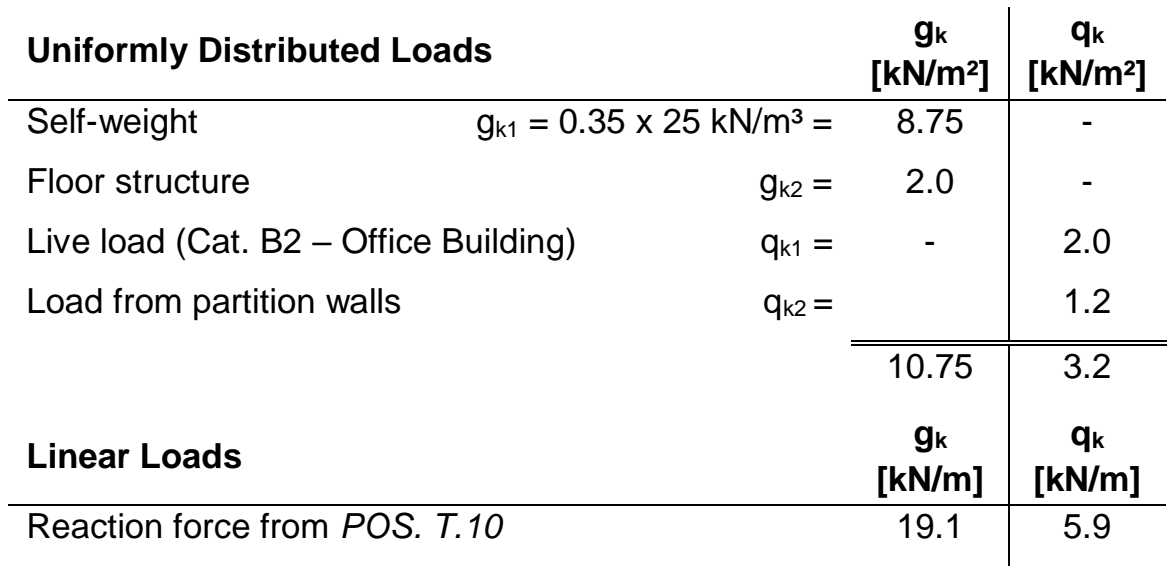




\subsection{ULTIMATE LIMIT STATE DESIGN}

\subsubsection{FLEXURE DESIGN}

Lower Design Bending Moment mB - 2

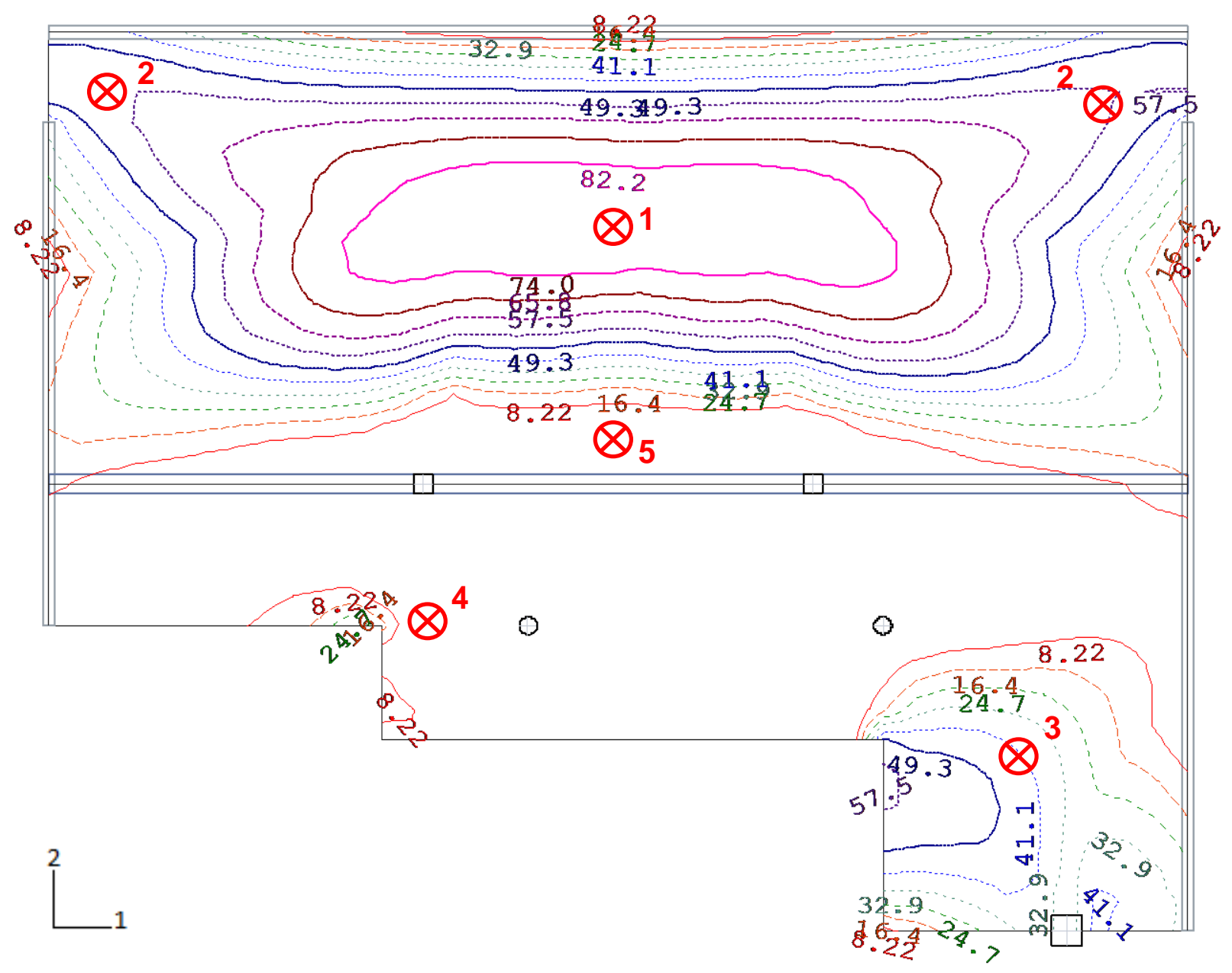

$d_{1}=h-c_{\text {nom }}-d_{s} / 2=35-2.0-1.0 / 2=32.5 \mathrm{~cm}$

(Reinforcement in the precast concrete)

$\mu_{E d s}=\frac{m_{E d, I_{-} 2}}{100 \cdot 32.5^{2} \cdot 1.98}=4.78 \cdot 10^{-6} \cdot m_{E d, I_{-} 2} \quad \rightarrow \omega \quad \rightarrow a_{s, r e q}=\omega \cdot b \cdot d \cdot f_{c d} \cdot \frac{1}{\sigma_{s d}}=\omega \cdot 147.9 \mathrm{~cm}^{2}$

Chosen

Lattice girder KT 800 (lower rebars $2 \varnothing 10$, distance $\mathrm{s}_{\mathrm{T}}=45 \mathrm{~cm}, \mathrm{a}_{\mathrm{s}}=3.49 \mathrm{~cm}^{2} / \mathrm{m}$ )

\begin{tabular}{|c|c|c|c|c|c|c|c|}
\hline & \multirow{2}{*}{$\begin{array}{c}m_{E d, I 2} 2 \\
{[k N m / m]}\end{array}$} & \multirow{2}{*}{$\begin{array}{l}\mu_{\mathrm{Ed}} \\
{[-]}\end{array}$} & \multirow{2}{*}{$\begin{array}{c}\omega \\
{[-]}\end{array}$} & \multirow{2}{*}{$\begin{array}{c}a_{\mathrm{s}, \mathrm{I} \_2, \mathrm{req}} \\
{\left[\mathrm{cm}^{2} / \mathrm{m}\right]}\end{array}$} & \multicolumn{2}{|c|}{ Chosen } & \multirow{2}{*}{$\begin{array}{l}a_{\mathrm{s}, \mathrm{l} \_2, \text { prov }} \\
{\left[\mathrm{cm}^{2} / \mathrm{m}\right]}\end{array}$} \\
\hline & & & & & KT 800 & Additional & \\
\hline 1 & 82.20 & 0.0458 & 0.04625 & 5.78 & $2 \varnothing 10 / 45$ & $\varnothing 8 / 20$ & 6.00 \\
\hline 2 & 57.50 & 0.0320 & 0.0306 & 3.83 & $2 \varnothing 10 / 45$ & $\varnothing 8 / 25$ & 5.50 \\
\hline 3 & 49.30 & 0.0275 & 0.0306 & 3.83 & $2 \varnothing 10 / 45$ & $\varnothing 8 / 25$ & 5.50 \\
\hline 4 & 24.70 & 0.0138 & 0.0152 & 1.90 & $2 \varnothing 10 / 45$ & $\varnothing 8 / 25$ & 5.50 \\
\hline 5 & 8.22 & 0.0046 & 0.0101 & 1.26 & $2 \varnothing 10 / 45$ & $\varnothing 8 / 25$ & 5.50 \\
\hline
\end{tabular}


Lower Design Bending Moment mB - 1

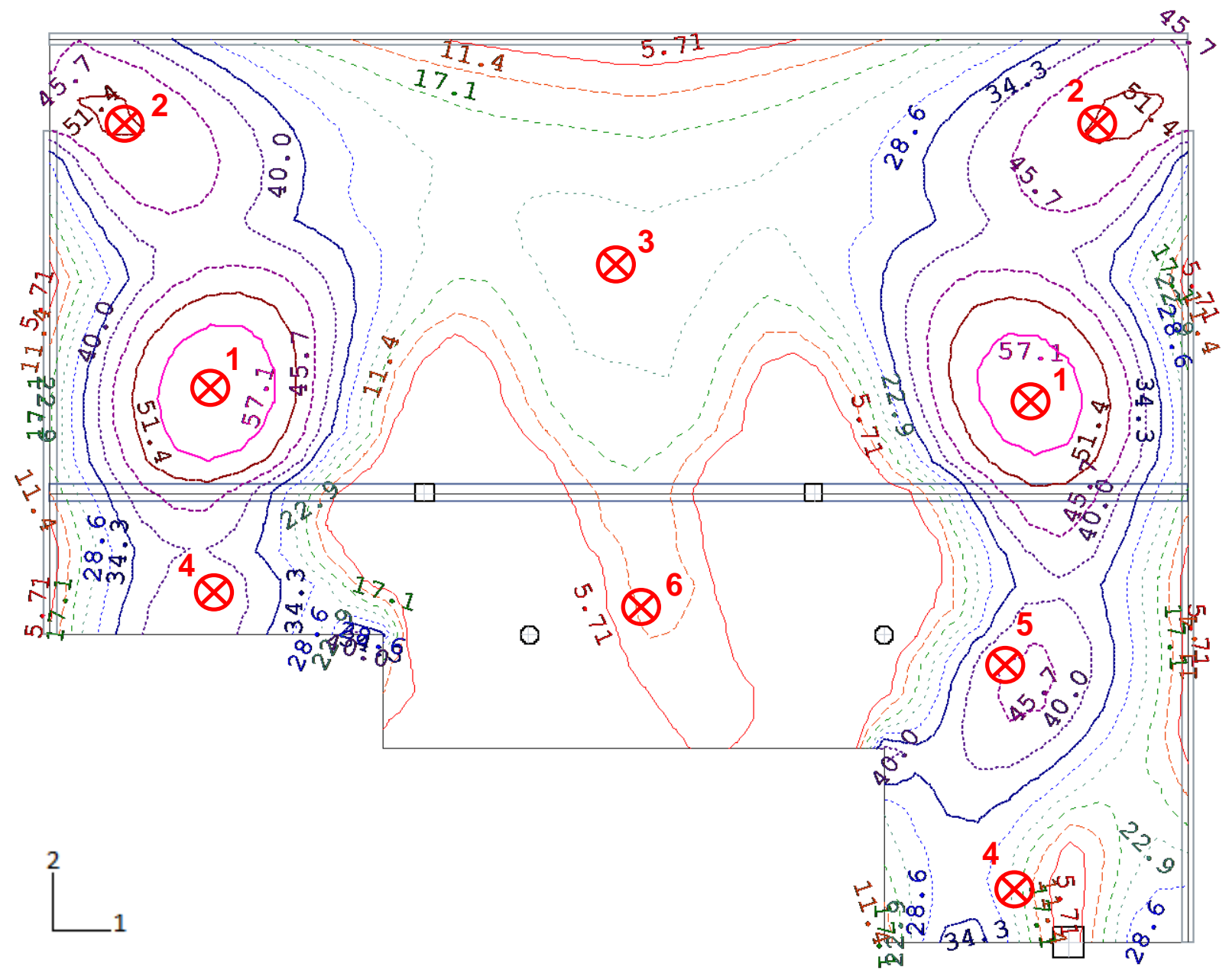

$d_{1}=h-h_{F}-d_{s} / 2=35-4.0-1.0 / 2=30.5 \mathrm{~cm}$

(Reinforcement in the in-situ concrete)

$\mu_{E d s}=\frac{m_{E d, I_{-} 1}}{100 \cdot 30.5^{2} \cdot 1.98}=4.12 \cdot 10^{-6} \cdot m_{E d, I_{-} 1} \quad \rightarrow \omega \quad \rightarrow a_{s, r e q}=\omega \cdot b \cdot d \cdot f_{c d} \cdot \frac{1}{\sigma_{s d}}=\omega \cdot 159.31 \mathrm{~cm}^{2}$

\begin{tabular}{|c|c|c|c|c|c|c|}
\cline { 2 - 7 } \multicolumn{1}{c|}{} & $\begin{array}{c}\mathbf{m}_{\mathrm{Ed}, \mathbf{I} \_\mathbf{1}} \\
{[\mathbf{k N m} / \mathbf{m}]}\end{array}$ & $\begin{array}{c}\boldsymbol{\mu}_{\mathrm{Ed}} \\
{[-]}\end{array}$ & $\begin{array}{c}\boldsymbol{\omega} \\
{[-]}\end{array}$ & $\begin{array}{c}\mathbf{a}_{\mathbf{s}, \mathbf{I} \mathbf{1}, \mathbf{r e q}} \\
{\left[\mathbf{c m}^{2} / \mathbf{m}\right]}\end{array}$ & $\begin{array}{c}\text { Chosen } \\
\text { Reinforcement }\end{array}$ & $\begin{array}{c}\mathbf{a}_{\mathbf{s}, \mathbf{I} \mathbf{1}, \mathbf{p r o v}} \\
{\left[\mathbf{c m}^{2} / \mathbf{m}\right]}\end{array}$ \\
\hline $\mathbf{1}$ & 57.10 & 0.0361 & 0.0358 & 4.48 & $\varnothing 8 / 10$ & 5.03 \\
\hline $\mathbf{2}$ & 51.40 & 0.0325 & 0.0306 & 3.83 & $\varnothing 8 / 10^{2}$ & 5.03 \\
\hline $\mathbf{3}$ & 22.90 & 0.0145 & 0.0152 & 1.90 & $\varnothing 8 / 10^{2}$ & 5.03 \\
\hline $\mathbf{4}$ & 34.30 & 0.0217 & 0.0203 & 2.54 & $\varnothing 8 / 10^{2}$ & 5.03 \\
\hline $\mathbf{5}$ & 45.70 & 0.0289 & 0.0306 & 3.83 & $\varnothing 8 / 10^{2}$ & 5.03 \\
\hline $\mathbf{6}$ & 5.71 & 0.0036 & 0.0101 & 1.26 & $\varnothing 8 / 10^{2}$ & 5.03 \\
\hline
\end{tabular}

${ }^{2}$ Chosen due to a necessary minimum reinforcement (see Chapter 11.4.1) 
Upper Design Bending Moment mB - 2

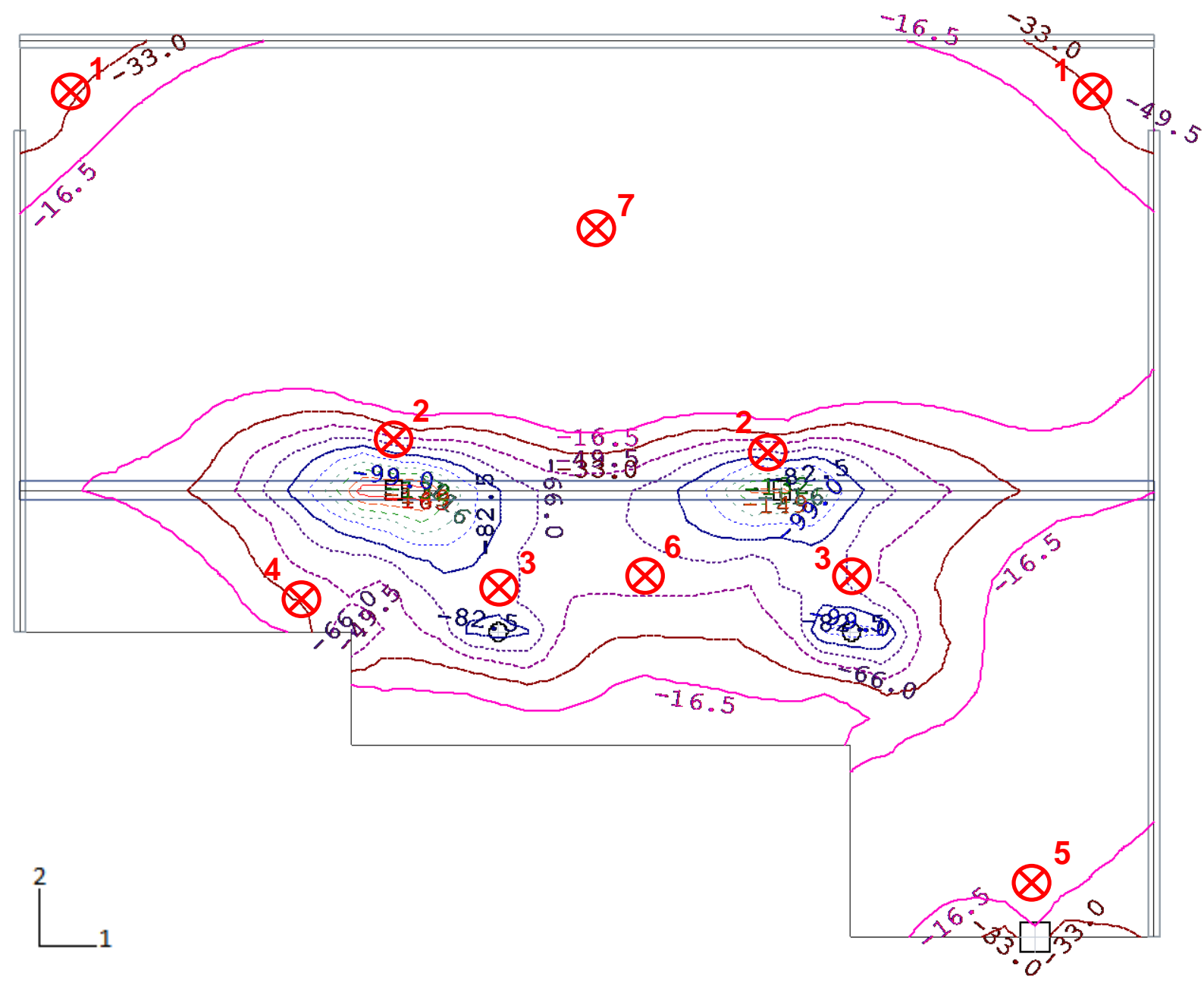

$d_{1}=h-c_{c o m}-d_{s} / 2=35-2.0-1.0 / 2=32.5 \mathrm{~cm}$

(Reinforcement in the in-situ concrete)

$\mu_{E d s}=\frac{m_{E d, u_{-} 2}}{100 \cdot 32.5^{2} \cdot 1.98}=4.78 \cdot 10^{-6} \cdot m_{E d, u_{-} 2} \quad \rightarrow \omega \quad \rightarrow a_{s, r e q}=\omega \cdot b \cdot d \cdot f_{c d} \cdot \frac{1}{\sigma_{s d}}=\omega \cdot 147.9 \mathrm{~cm}^{2}$

\begin{tabular}{|c|c|c|c|c|c|c|c|}
\hline & \multirow{2}{*}{$\begin{array}{c}\mathrm{m}_{\mathrm{Ed}, \mathrm{I} \_2} \\
{[\mathrm{kNm} / \mathrm{m}]}\end{array}$} & \multirow{2}{*}{$\begin{array}{c}\mu_{\mathrm{Ed}} \\
{[-]}\end{array}$} & \multirow{2}{*}{$\begin{array}{l}\omega \\
{[-]}\end{array}$} & \multirow{2}{*}{$\begin{array}{c}a_{s, 1 \_2, \text { req }} \\
{\left[\mathrm{cm}^{2} / \mathrm{m}\right]}\end{array}$} & \multicolumn{2}{|c|}{ Chosen Reinforcement } & \multirow{2}{*}{$\begin{array}{l}a_{\mathrm{s}, \mathrm{l} \_2, \text { pro }} \\
{\left[\mathrm{cm}^{2} / \mathrm{m}\right]}\end{array}$} \\
\hline & & & & & Mesh & Additional & \\
\hline 1 & 33.00 & 0.0184 & 0.0203 & 2.54 & Q $335 \mathrm{~A}$ & - & 3.35 \\
\hline 2 & 149.00 & 0.0830 & 0.0891 & 11.14 & Q $335 \mathrm{~A}$ & $\varnothing 10 / 7.5$ & 13.82 \\
\hline 3 & 82.50 & 0.0459 & 0.04625 & 5.78 & Q $524 A$ & $\varnothing 8 / 25$ & 7.25 \\
\hline 4 & 66.00 & 0.0368 & 0.0358 & 4.48 & Q $524 \mathrm{~A}$ & - & 5.24 \\
\hline 5 & 33.00 & 0.0184 & 0.0203 & 2.54 & Q $524 \mathrm{~A}$ & - & 5.24 \\
\hline 6 & 49.50 & 0.0276 & 0.0306 & 3.83 & Q $524 \mathrm{~A}$ & - & 5.24 \\
\hline 7 & 16.50 & 0.0092 & 0.0101 & 1.26 & Q $335 \mathrm{~A}$ & - & 3.35 \\
\hline
\end{tabular}


Upper Design Bending Moment mB - 1

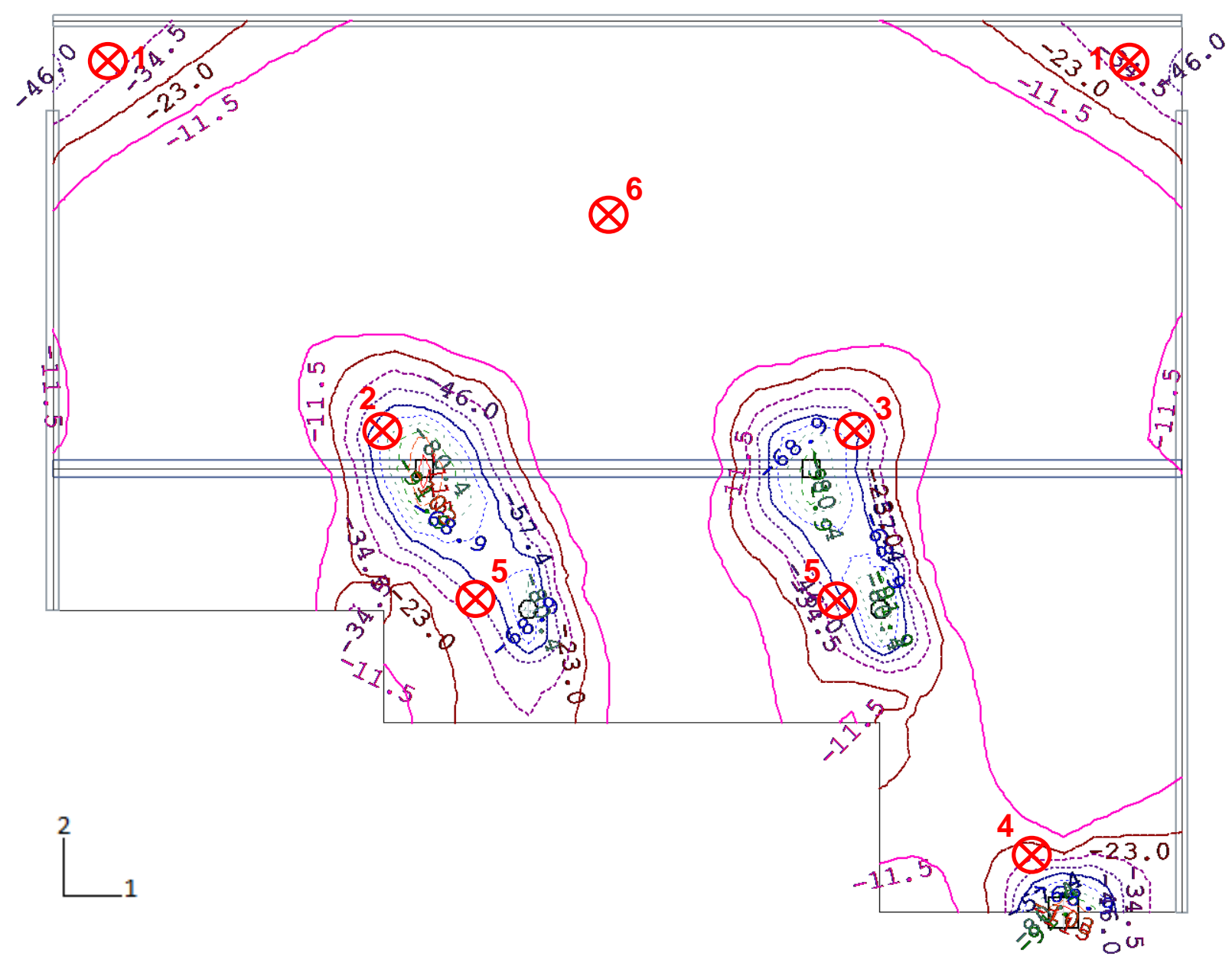

$d_{1}=h-c_{c o m}-d_{s, 2}-d_{s, 1} / 2=35-2.0-1.0-1.0 / 2=31.5 \mathrm{~cm} \quad$ (Reinforcement in the in-situ concrete)

$\mu_{E d s}=\frac{m_{E d, u_{-} 1}}{100 \cdot 31.5^{2} \cdot 1.98}=5.09 \cdot 10^{-6} \cdot m_{E d, u_{-} 1} \quad \rightarrow \omega \quad \rightarrow a_{s, r e q}=\omega \cdot b \cdot d \cdot f_{c d} \cdot \frac{1}{\sigma_{s d}}=\omega \cdot 143.4 \mathrm{~cm}^{2}$

\begin{tabular}{|c|c|c|c|c|c|c|c|}
\hline & \multirow{2}{*}{$\begin{array}{c}\mathrm{m}_{\mathrm{Ed}, \mathrm{u} \_1} \\
{[\mathrm{kNm} / \mathrm{m}]}\end{array}$} & \multirow{2}{*}{$\begin{array}{c}\mu_{\mathrm{Ed}} \\
{[-]}\end{array}$} & \multirow{2}{*}{$\begin{array}{l}\omega \\
{[-]}\end{array}$} & \multirow{2}{*}{$\begin{array}{l}a_{s, u_{1} 1, \text { req }} \\
{\left[\mathrm{cm}^{2} / \mathrm{m}\right]}\end{array}$} & \multicolumn{2}{|c|}{ Chosen Reinforcement } & \multirow{2}{*}{$\begin{array}{l}a_{s, u_{1} 1, p r o v} \\
{\left[\mathrm{~cm}^{2} / \mathrm{m}\right]}\end{array}$} \\
\hline & & & & & Mesh & Additional & \\
\hline 1 & 46.00 & 0.0273 & 0.0306 & 3.83 & Q $335 \mathrm{~A}$ & $4 \varnothing 10 / 10$ & 6.49 \\
\hline 2 & 103.00 & 0.0611 & 0.06745 & 8.44 & Q $335 \mathrm{~A}$ & $\varnothing 10 / 15$ & 8.59 \\
\hline 3 & 80.40 & 0.0477 & 0.0515 & 6.44 & Q $335 \mathrm{~A}$ & $\varnothing 10 / 15$ & 8.59 \\
\hline 4 & 91.90 & 0.0545 & 0.0568 & 7.10 & Q 524A & $3 \varnothing 10 / 10$ & 7.60 \\
\hline 5 & 68.90 & 0.0408 & 0.041 & 5.13 & Q $524 \mathrm{~A}$ & - & 5.24 \\
\hline 6 & 11.50 & 0.0068 & 0.0101 & 1.26 & Q $335 \mathrm{~A}$ & - & 3.35 \\
\hline
\end{tabular}




\subsubsection{SHEAR DESIGN}
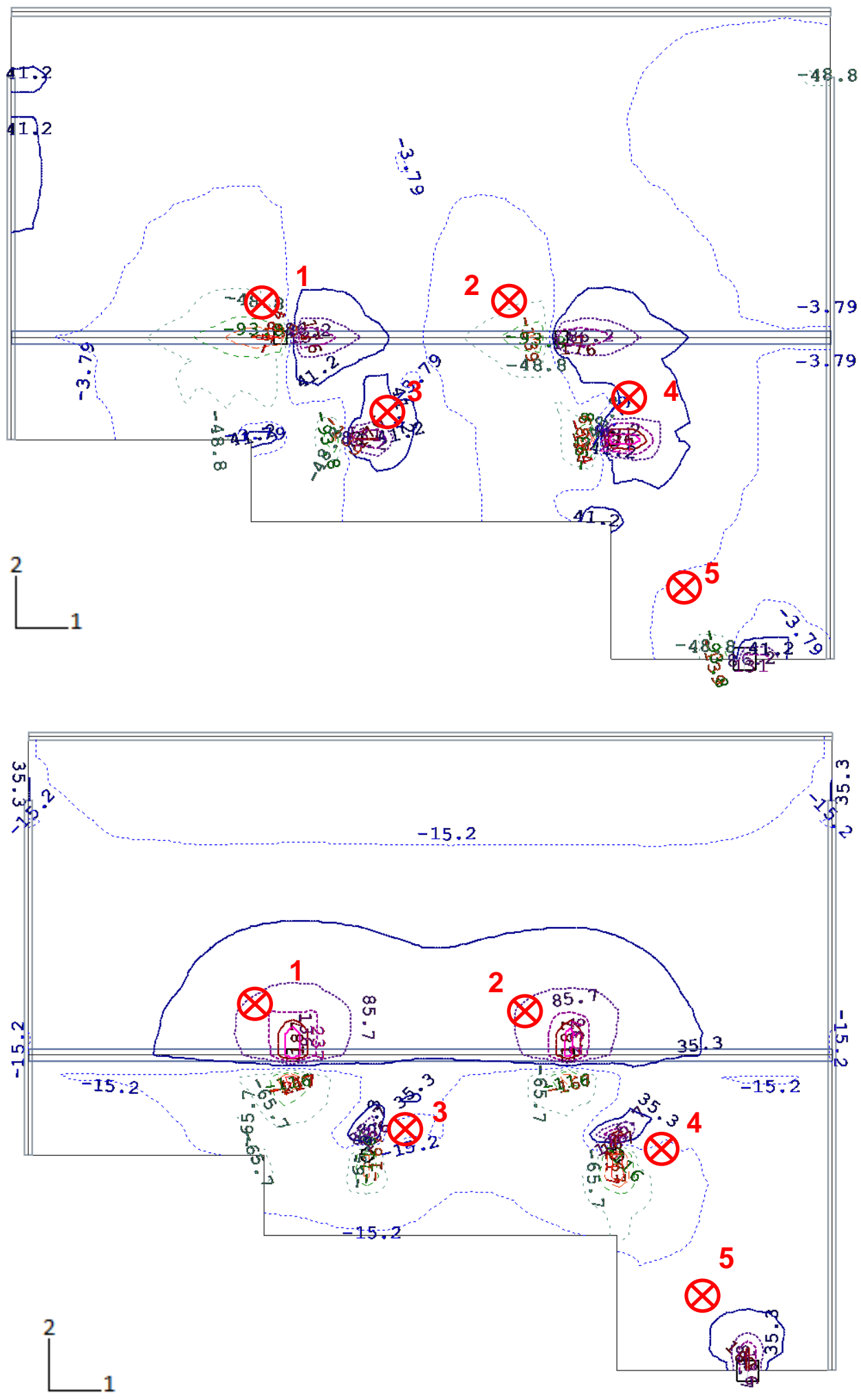


\begin{tabular}{|c|c|c|c|c|c|}
\cline { 2 - 6 } \multicolumn{1}{c|}{} & $\mathbf{1}$ & $\mathbf{2}$ & $\mathbf{3}$ & $\mathbf{4}$ & $\mathbf{5}$ \\
\hline $\mathbf{V}_{\mathrm{Ed}, \mathbf{1}}[\mathbf{k N} / \mathbf{m}]$ & 93.6 & 93.6 & 86.1 & 48.9 & 48.9 \\
\hline $\mathbf{V}_{\mathrm{Ed}, 2}[\mathbf{k N} / \mathbf{m}]$ & 136.0 & 136.0 & 65.7 & 65.7 & 35.3 \\
\hline
\end{tabular}

Shear force at design location $v_{E d, 2}=136.0 \mathrm{kN} / \mathrm{m}$

Scale factor $\quad k=1+\sqrt{\frac{20}{d}}=1+\sqrt{\frac{20}{31}}=1.80<\underline{\underline{2.00}}$

Percentage of reinforcement $\quad \rho_{1}=\frac{A_{s l}}{b_{w} \cdot d}=\frac{3.35 \mathrm{~cm}^{2} / \mathrm{m}}{100 \cdot 31}=0.0011 \leq \underline{\underline{0.02}}$

Shear force resistance $\quad V_{R d, c}=\left[0.10 \cdot k \cdot\left(100 \cdot \rho_{l} \cdot f_{c k}\right)^{1 / 3}+0.12 \cdot \sigma_{c p}\right] \cdot b_{w} \cdot d$

$V_{R d, c}=\left[0.10 \cdot 1.8 \cdot(100 \cdot 0.0011 \cdot 30)^{1 / 3}\right] \cdot 0.31 \cdot 1.0 \cdot 10^{3}=83.1 \mathrm{kN} / \mathrm{m}$

$d=31 \mathrm{~cm}<60 \mathrm{~cm} \rightarrow v_{\min }=0.035 \cdot 1.80^{3 / 2} \cdot(30)^{1 / 2}=0.463 \mathrm{MN} / \mathrm{m}^{2}$

$v_{R d, c, \min }=0.463 \cdot 1.00 \cdot 0.31 \cdot 10^{3}=\underline{\underline{143.5 \mathrm{kN} / m}}>v_{R d, c}=83.1 \mathrm{kN} / \mathrm{m}$

$v_{E d}=136 \mathrm{kN} / m<v_{R d, c}=143.5 \mathrm{kN} / \mathrm{m}$

$\rightarrow$ No shear reinforcement is needed!

Minimum shear reinforcement $\quad \min a_{s w}=\rho \cdot b_{w} \cdot \sin \alpha \quad$ and $\quad \rho=0.16 \cdot f_{c t m} / f_{y k}=0.16 \cdot 3.2 / 500=1.02 \%$ $\min a_{s w}=0.00102 \cdot 0.40 \cdot \sin 90^{\circ}=4.08 \cdot 10^{-4} \mathrm{~m}^{2} / \mathrm{m}=\underline{\underline{4.08 \mathrm{~cm}^{2} / \mathrm{m}}}$ $\min a_{s w}=4.08 \mathrm{~cm}^{2} / m<a_{s w, p r o v}=5.03 \mathrm{~cm}^{2} / \mathrm{m} \checkmark$ 


\subsection{SERVICEABILITY LIMIT STATE DESIGN}

\section{Deformation of the Slab under $q_{p e r m}$}

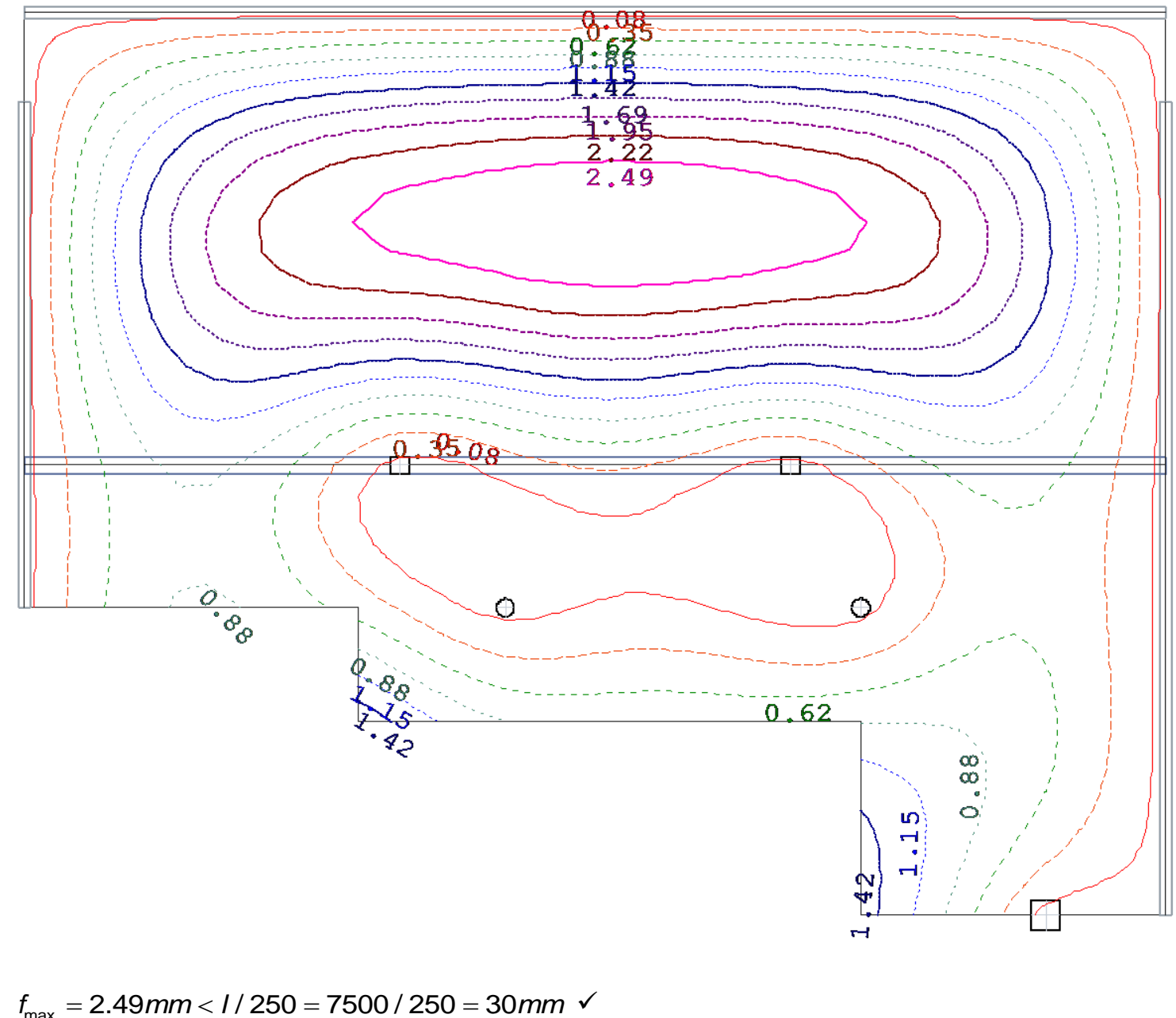

The deformation does not exceed the maximum allowed deformation!

\subsection{DESIGN AND REINFORCEMENT}

\subsubsection{MINIMUM REINFORCEMANT TO ENSURE ROBUSTNESS}

$$
\begin{aligned}
& M_{c r}=f_{c t m} \cdot W_{c u} \quad W_{c u}=100 \cdot 35^{2} / 6=20,417 \mathrm{~cm}^{3} \\
& M_{c r}=2.9 \cdot 20,417 \cdot 10^{-3}=59.2 \mathrm{kNm}
\end{aligned}
$$

Direction of the longer span:

$a_{s, \min , 1}=\frac{1}{f_{y k}} \cdot \frac{M_{c r}}{z}=\frac{1}{50.0} \cdot \frac{5920}{0.9 \cdot 30.5}=4.31 \mathrm{~cm}^{2} / \mathrm{m}<a_{s, 1, p r o v}=5.5 \mathrm{~cm}^{2} / \mathrm{m}^{\checkmark}$

Direction of the shorter span:

$$
a_{s, \min , 2}=\frac{1}{f_{y k}} \cdot \frac{M_{c r}}{z}=\frac{1}{50.0} \cdot \frac{5920}{0.9 \cdot 32.5}=4.04 \mathrm{~cm}^{2} / \mathrm{m}<a_{s, 2, \text { prov }}=5.03 \mathrm{~cm}^{2} / \mathrm{m}^{\checkmark}
$$




\subsection{SOFTWARE CALCULATIONS}

\section{Demo Frilo Nemetschek}

\section{Position: S.12 - Semi-Precast Concrete Slab}

Plates by Finite Elements PLT 01/2018 (Frilo R-2018-1/P12)

System: Floor Plan with FE Mesh

Scale 1 : 150

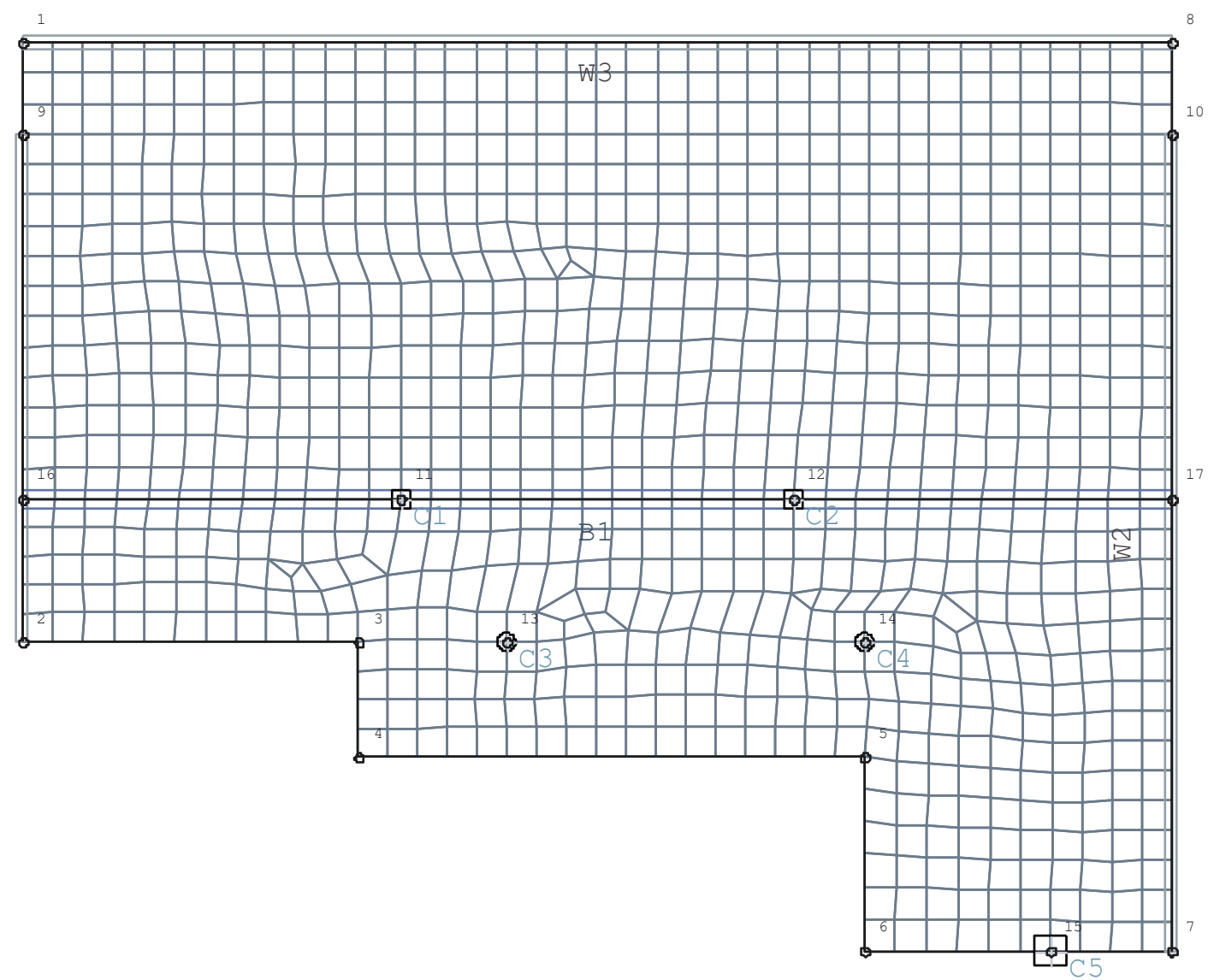




\section{LOADCASE 1 "Self-Weight"}

Type:

Dead loads due to

permanent

Plate, beams and parapets

are included:

Action:

Partial safety factor action:

Partial safety factor concrete:

Partial safety factor steel:

Load points:

Point loads:

Line loads:

Area loads:

Temperature loads:

Total of input loads:

(portion on the plate)

Dead loads due to

plate and beams:

Total of all loads:

Total of all support reactions:

\section{Load case 1 "Self-Weight"}

Area Loads

Scale $1: 150$

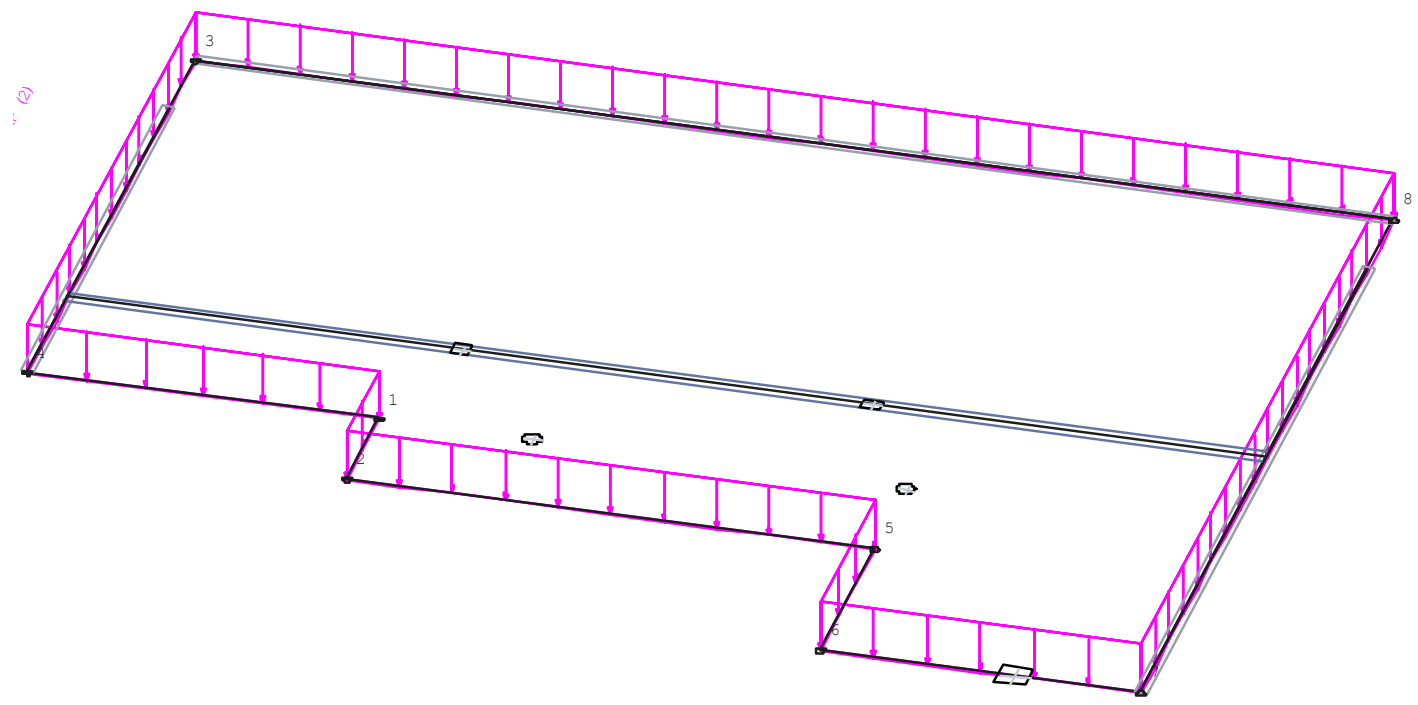




\section{LOADCASE 2 "Live Load"}

Type:

Dead loads due to

non permanent

Plate, beams and parapets

are included:

Action:

Partial safety factor action:

Partial safety factor concrete:

Partial safety factor steel:

Load points:

Point loads:

Line loads:

Area loads:

Temperature loads:

Total of input loads:

(portion on the plate)

Total of all support reactions:

NO

Büros

1.50

1.50

1.15

8

0

1

1

$747[\mathrm{kN}]$

$747[\mathrm{kN}]$

Load case 2 "Live Load"

Area Loads

Scale 1 : 150

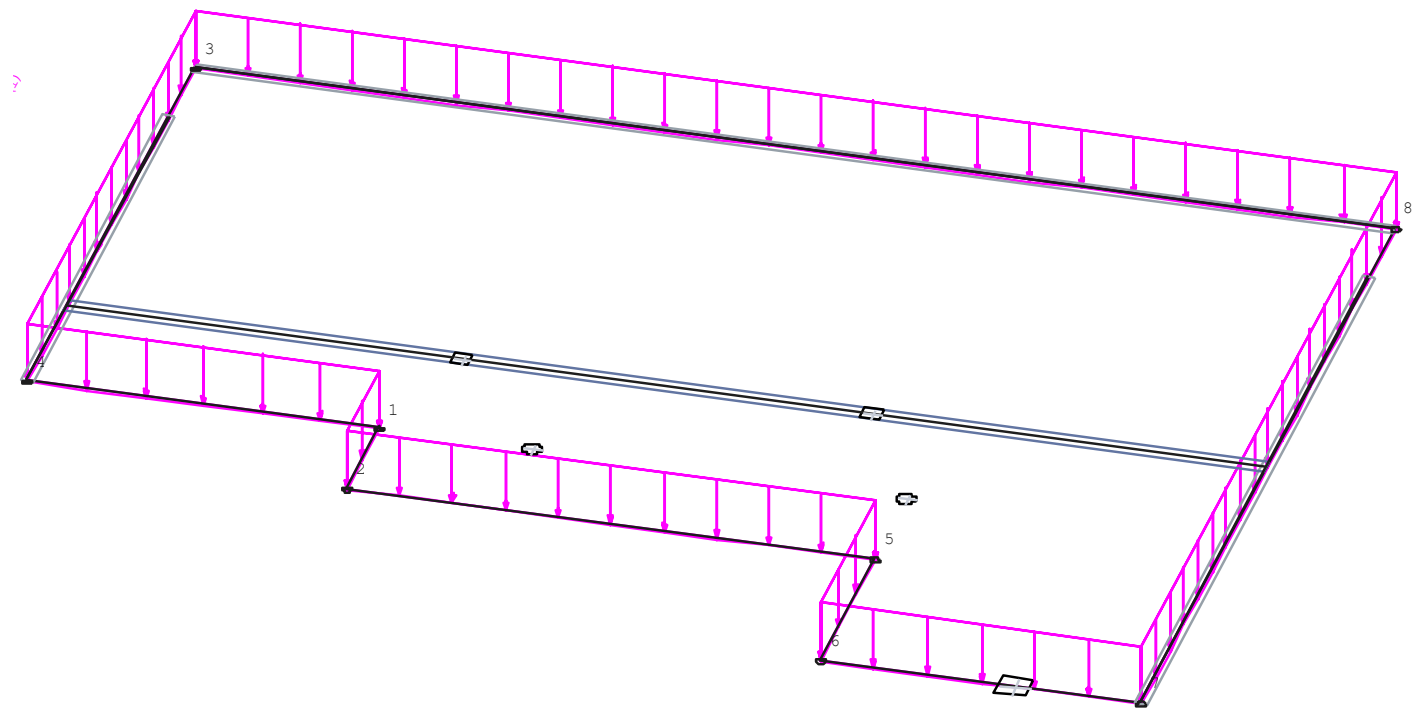




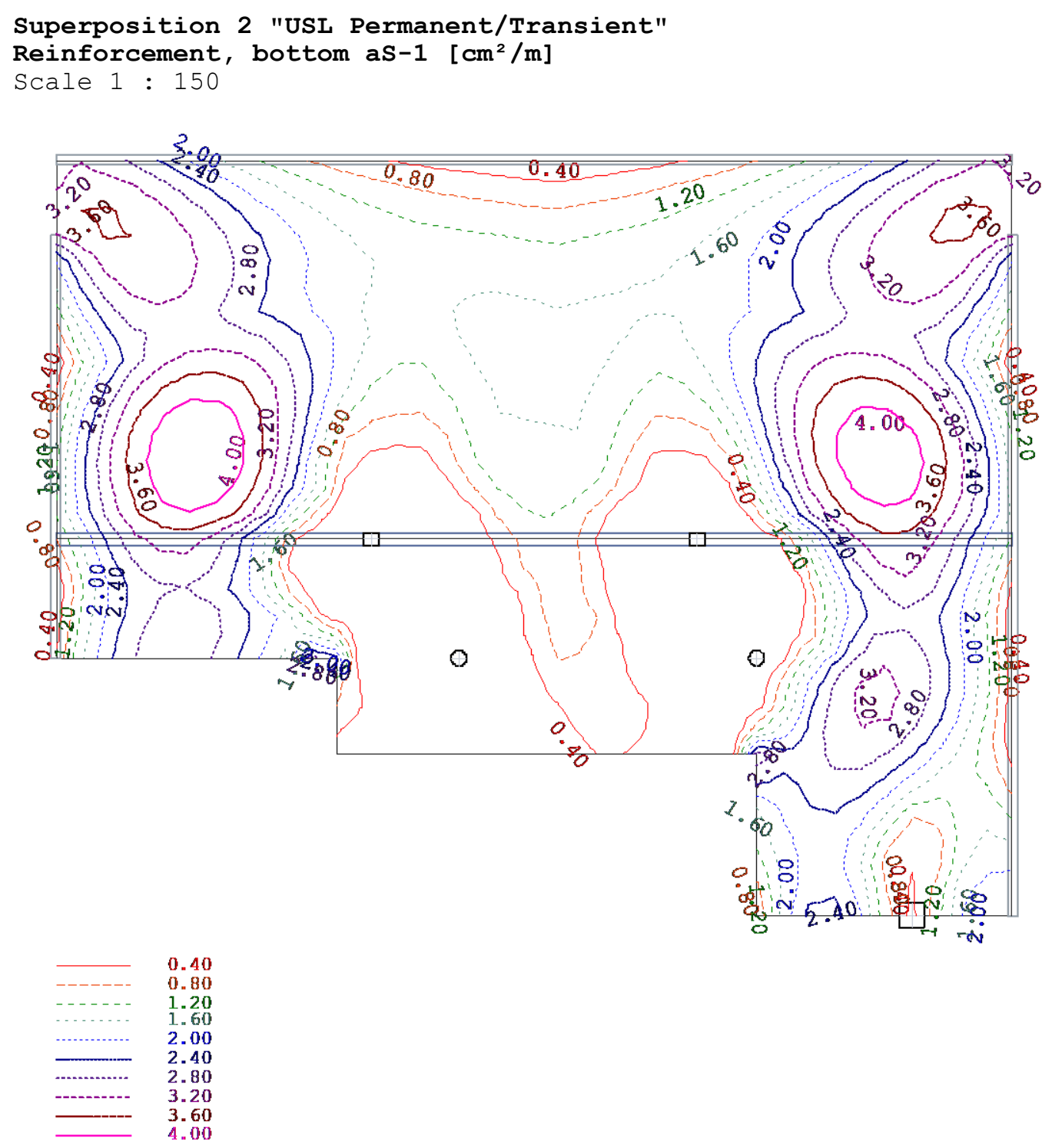




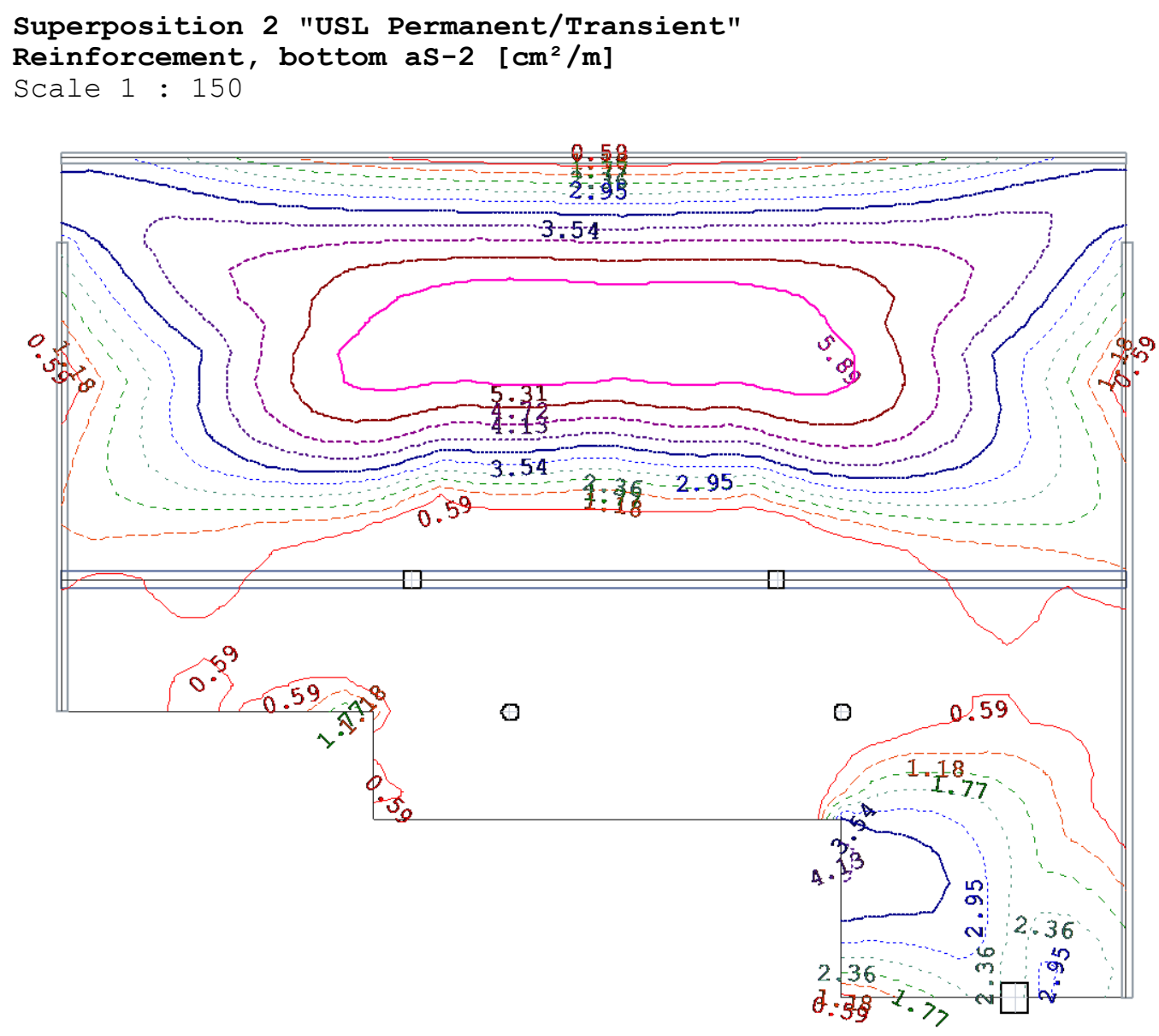

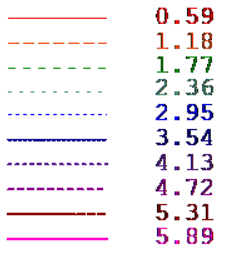


Superposition 2 "USL Permanent/Transient"

Reinforcement, top as-1 $\left[\mathrm{cm}^{2} / \mathrm{m}\right]$

Scale 1 : 150

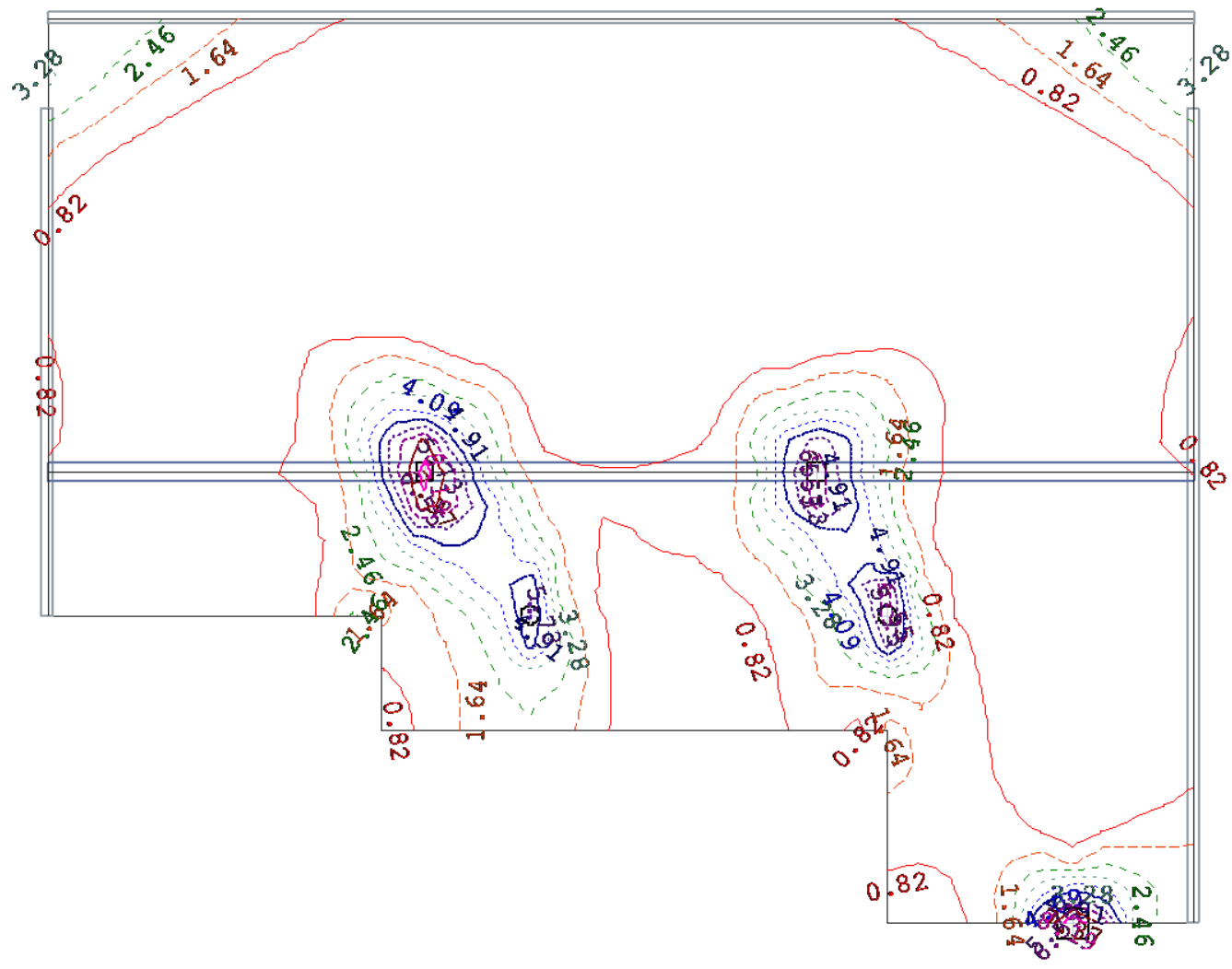

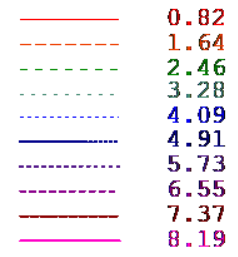


Superposition 2 "USL Permanent/Transient"

Reinforcement, top as-2 [ $\left.\mathrm{cm}^{2} / \mathrm{m}\right]$

Scale 1 : 125

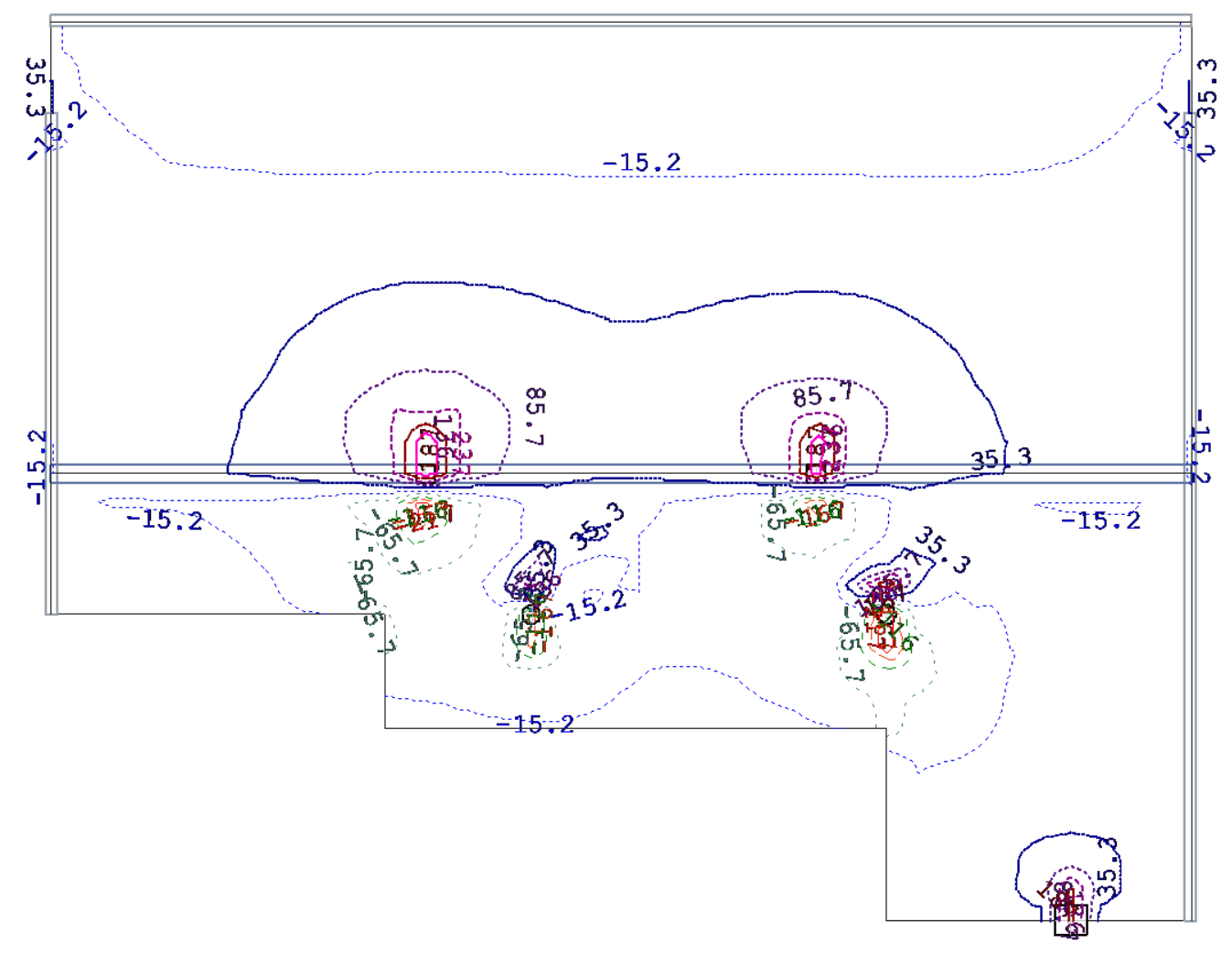

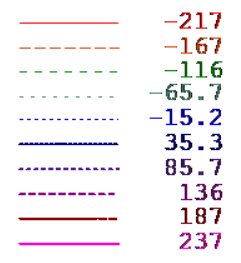




\section{POS. B.13 - SEMI-PRECAST CONCRETE BEAM}

\subsection{SYSTEM}

System

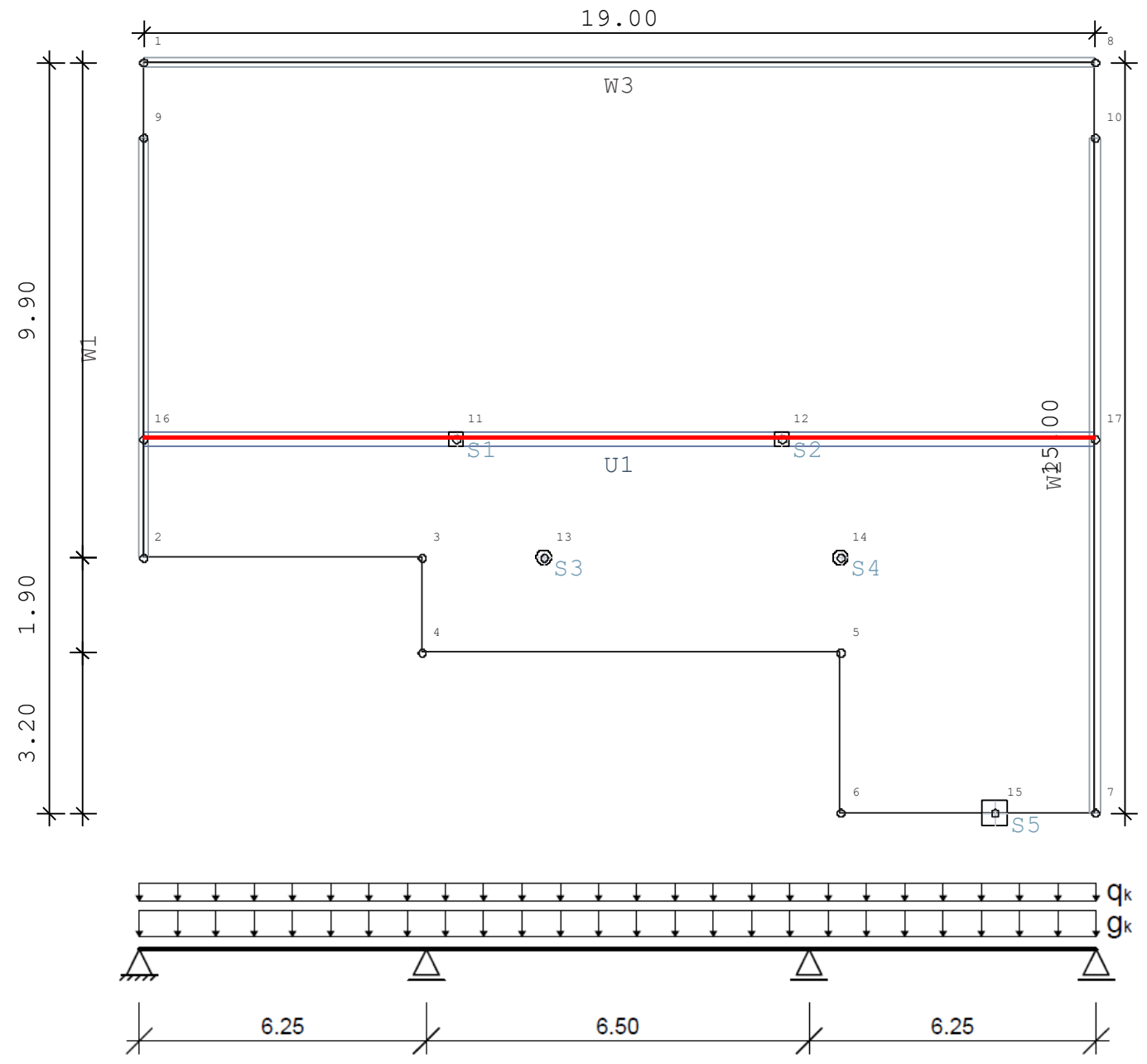

Cross-section

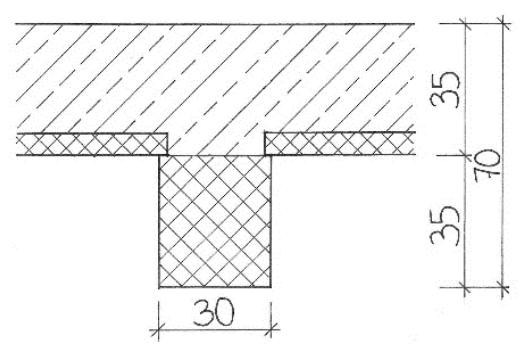

Building materials

Exposure class

Reinforcing steel

\section{30/37 | B 500A}

XC1, WO

$\mathrm{C}_{\mathrm{nom}}=10 \mathrm{~mm}+10 \mathrm{~mm}=20 \mathrm{~mm}$

Chosen:

$\mathrm{C}_{\text {nom }}=25 \mathrm{~mm}$ 
Characteristic loads

\begin{tabular}{lc|c} 
Uniformly Distributed Loads & $\begin{array}{c}\mathbf{g}_{\mathbf{k}} \\
{[\mathbf{k N} / \mathbf{m}]}\end{array}$ & $\begin{array}{c}\mathbf{q}_{\mathbf{k}} \\
{[\mathbf{k N} / \mathbf{m}]}\end{array}$ \\
\hline see POS. S.12 & - & -
\end{tabular}

\subsection{INTERNAL FORCES}

Design Forces

\begin{tabular}{|c|c|c|c|c|c|}
\hline & Span 1 & Support B & Span 2 & Support C & Span 3 \\
\hline $\max \mathrm{M}_{\mathrm{Ed}}[\mathrm{kNm}]$ & 184 & -364 & 109 & -324 & 161 \\
\hline
\end{tabular}

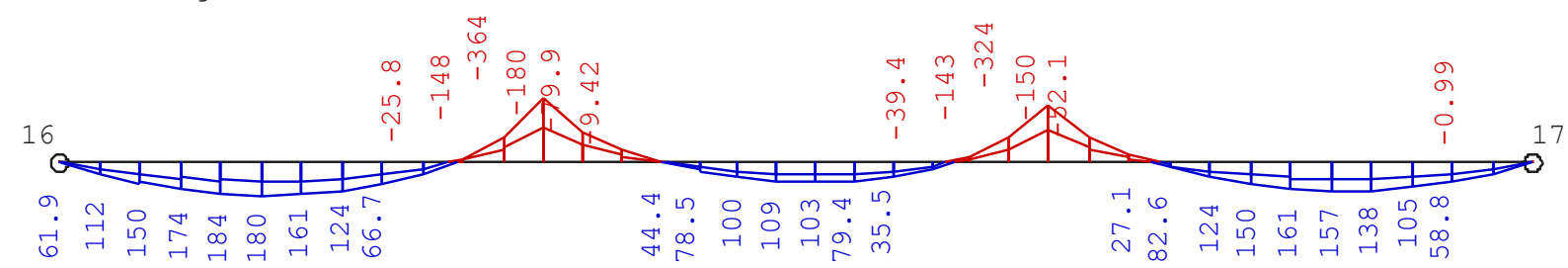

Shear force

\begin{tabular}{|c|c|c|c|c|c|c|}
\hline & Support A & Support $B_{L}$ & Support $B_{R}$ & Support $C_{L}$ & Support $C_{R}$ & Support D \\
\hline $\max V_{E d}[k N]$ & 121 & -414 & 366 & -362 & 330 & -115 \\
\hline
\end{tabular}

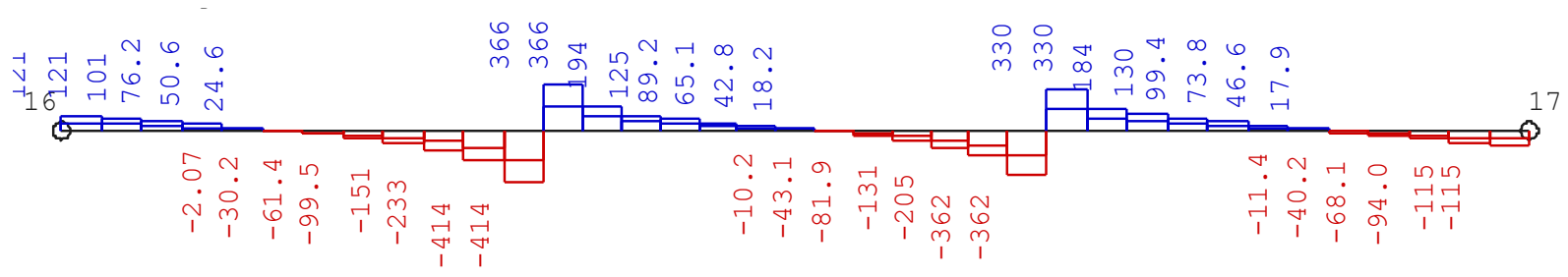

Characteristic forces

\section{Bending moment}

\begin{tabular}{|l|c|c|c|c|c|}
\cline { 2 - 5 } \multicolumn{1}{c|}{} & Span 1 & Support B & Span 2 & Support C & Span 3 \\
\hline $\mathrm{M}_{\mathrm{gk}}[\mathrm{kNm}]$ & 103 & -204 & 61.3 & -182 & 90.6 \\
\hline $\mathrm{M}_{\mathrm{qk}}[\mathrm{kNm}]$ & 29.7 & -58.7 & 17.6 & -52.2 & 26.0 \\
\hline $\mathrm{M}_{\text {perm }}[\mathrm{kNm}]$ & 111.9 & -221.6 & 66.6 & -197.7 & 98.1 \\
\hline
\end{tabular}


Bending moment Mgk

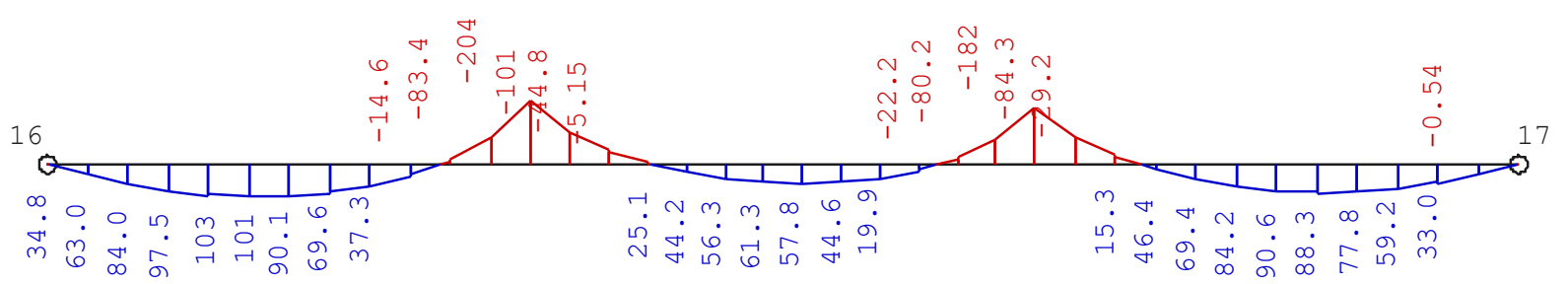

Bending moment Mqk

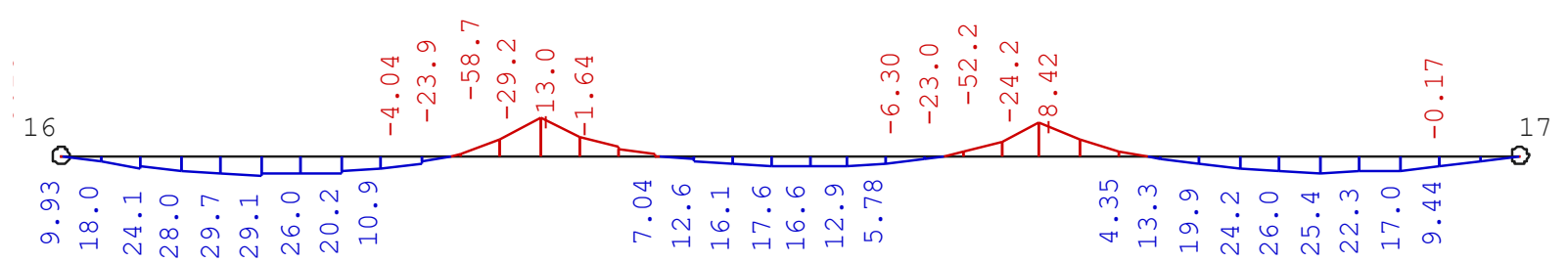

\subsection{ULTIMATE LIMIT STATE DESIGN}

\subsubsection{FLEXURE DESIGN}

Lower reinforcement at span 1

Effective width

Lower reinforcement

Chosen

New static height

$$
\begin{aligned}
& b_{\text {eff }, 1}=0.2 \cdot 360+0.1 \cdot 0.85 \cdot 625=125 \mathrm{~cm}<\left\{\begin{array}{l}
0.2 \cdot 0.85 \cdot 625=\underline{106 \mathrm{~cm}} \\
b_{1}=360 \mathrm{~cm}
\end{array}\right. \\
& b_{e f f, 2}=0.2 \cdot 220+0.1 \cdot 0.85 \cdot 625=\underline{\underline{97 \mathrm{~cm}}}<\left\{\begin{array}{l}
106 \mathrm{~cm} \\
b_{2}=220 \mathrm{~cm}
\end{array}\right. \\
& b_{\text {eff }}=b_{\text {eff }, 1}+b_{w}+b_{\text {eff }, 2}=1.06+0.30+0.97=\underline{\underline{.33 m}}
\end{aligned}
$$

$$
\begin{aligned}
& \mu_{E d s}=\frac{M_{E d s}}{b_{\text {eff }} \cdot d^{2} \cdot f_{c d}}=\frac{18400 \mathrm{kNcm}}{233 \cdot 65^{2} \cdot 1.70 \mathrm{kN} / \mathrm{cm}^{2}}=0.011 \rightarrow \omega=0.0101 \\
& \xi=0.030 \rightarrow x=0.030 \cdot 65=1.95 \mathrm{~cm}<h_{f}=35 \mathrm{~cm} \\
& A_{s l, r e q}=\omega \cdot b \cdot d \cdot f_{c d} \cdot \frac{1}{\sigma_{s d}}=0.0101 \cdot 233 \cdot 65 \cdot 1.70 \cdot \frac{1}{43.5}=5.98 \mathrm{~cm}^{2}
\end{aligned}
$$

$$
\begin{aligned}
d & =h-c_{\text {nот }}-d_{s, b u ̈}-d_{s, l a} / 2=70-2.0-1.0-1.6 / 2=66.2 \mathrm{~cm} \\
& >d_{e s t}=65 \mathrm{~cm} \checkmark
\end{aligned}
$$


Lower Reinforcement at Span 2 and Span 3

\begin{tabular}{|l|c|c|c|c|c|c|c|c|c|c|}
\cline { 2 - 11 } \multicolumn{1}{c|}{} & $\begin{array}{c}\mathbf{M}_{\mathrm{Ed}} \\
{[\mathbf{k N m}]}\end{array}$ & $\begin{array}{c}\mathbf{b}_{\text {eff }} \\
{[\mathbf{c m}]}\end{array}$ & $\begin{array}{c}\mathbf{d} \\
{[\mathbf{c m}]}\end{array}$ & $\begin{array}{c}\boldsymbol{\mu}_{\mathrm{Ed}} \\
{[-]}\end{array}$ & $\begin{array}{c}\boldsymbol{\omega} \\
{[-]}\end{array}$ & $\begin{array}{c}\mathbf{X} \\
{[\mathbf{c m}]}\end{array}$ & $\begin{array}{c}\mathbf{z} \\
{[\mathbf{c m}]}\end{array}$ & $\begin{array}{c}\mathbf{A}_{\mathbf{s}, \text { req }} \\
{\left[\mathbf{c m}^{2}\right]}\end{array}$ & Chosen & $\begin{array}{c}\mathbf{A}_{\mathbf{s}, \text { prov }} \\
{\left[\mathbf{c m}^{2}\right]}\end{array}$ \\
\hline Span 2 & 109.0 & 189 & 65 & 0.0080 & 0.0081 & 1.56 & 51.48 & 3.88 & $\mathbf{4 \varnothing 1 4}$ & $\mathbf{6 . 1 6}$ \\
\hline Span 3 & 161.0 & 211 & 65 & 0.0106 & 0.0107 & 2.00 & 64.34 & 5.74 & $\mathbf{4 \varnothing 1 4}$ & $\mathbf{6 . 1 6}$ \\
\hline
\end{tabular}

Upper Reinforcement at Support B

Upper reinforcement

$$
\begin{aligned}
& \mu_{E d s}=\frac{M_{E d s}}{b_{w} \cdot d^{2} \cdot f_{c d}}=\frac{36400 \mathrm{kNcm}}{30 \cdot 65^{2} \cdot 1.70 \mathrm{kN} / \mathrm{cm}^{2}}=0.169 \quad \rightarrow \omega=0.1882 \\
& A_{s l, \text { req }}=\omega \cdot b \cdot d \cdot f_{c d} \cdot \frac{1}{\sigma_{s d}}=0.1882 \cdot 30 \cdot 65 \cdot 1.70 \cdot \frac{1}{43.5}=14.3 \mathrm{~cm}^{2}
\end{aligned}
$$

Chosen

New static height upper reinforcement

$$
\begin{aligned}
d & =h-c_{\text {nom }}-d_{s, l}-d_{s, q}-d_{s, b} / 2=70-2.0-1.0-2.0 / 2=65 \mathrm{~cm} \\
& >d_{\text {est }}=65 \mathrm{~cm} \checkmark
\end{aligned}
$$

Upper Reinforcement at Support C

\begin{tabular}{|c|c|c|c|c|c|c|c|c|c|c|}
\cline { 2 - 10 } \multicolumn{1}{c|}{} & $\begin{array}{c}\mathbf{M}_{\mathrm{Ed}} \\
{[\mathbf{k N m}]}\end{array}$ & $\begin{array}{c}\mathbf{b}_{\mathrm{w}} \\
{[\mathbf{c m}]}\end{array}$ & $\begin{array}{c}\mathbf{d} \\
{[\mathbf{c m}]}\end{array}$ & $\begin{array}{c}\boldsymbol{\mu}_{\mathrm{Ed}} \\
{[-]}\end{array}$ & $\begin{array}{c}\boldsymbol{\omega} \\
{[-]}\end{array}$ & $\begin{array}{c}\mathbf{X} \\
{[\mathbf{c m}]}\end{array}$ & $\begin{array}{c}\mathbf{z} \\
{[\mathbf{c m}]}\end{array}$ & $\begin{array}{c}\mathbf{A}_{\mathbf{s}, \text { req }} \\
{\left[\mathbf{c m}^{2}\right]}\end{array}$ & Chosen & $\begin{array}{c}\mathbf{A}_{\mathbf{s}, \text { prov }} \\
{\left[\mathbf{c m}^{2}\right]}\end{array}$ \\
\hline Supp C & -324.0 & 30 & 65 & 0.1504 & 0.1638 & 13.13 & 59.54 & 12.48 & $\mathbf{5 ~ Ø ~ 2 0}$ & $\mathbf{1 5 . 7 0}$ \\
\hline
\end{tabular}

\subsubsection{SHEAR DESIGN}

Shear Design Calculations at Support $\mathrm{B}_{\mathrm{L}}$

$$
\begin{array}{ll}
\text { Shear force } & V_{E d}=414 \mathrm{kN} \\
\text { Scale factor } & k=1+\sqrt{\frac{20}{d}}=1+\sqrt{\frac{20}{66}}=1.55<\underline{\underline{2.00}} \\
\text { Percentage of reinforcement } & \rho_{1}=\frac{A_{s l}}{b_{w} \cdot d}=\frac{15.7}{30 \cdot 65}=0.0081 \leq \underline{\underline{0.02}} \\
\text { Shear force resistance } & V_{R d, c}=\left[0.10 \cdot \mathrm{k} \cdot\left(100 \cdot \rho_{l} \cdot f_{c k}\right)^{1 / 3}+0.12 \cdot \sigma_{c p}\right] \cdot b_{w} \cdot d \\
& V_{R d, c}=\left[0.10 \cdot 1.55 \cdot(100 \cdot 0.0081 \cdot 30)^{1 / 3}\right] \cdot 0.65 \cdot 0.30 \cdot 10^{3}=87.6 \mathrm{kN} \\
& d=65 \mathrm{~cm}>60 \mathrm{~cm} \quad \rightarrow v_{\text {min }}=0.0325 \cdot 1.55^{3 / 2} \cdot(30)^{1 / 2}=0.345 \mathrm{MN} / \mathrm{m}^{2} \\
& V_{R d, c, \text { min }}=0.345 \cdot 0.30 \cdot 0.65 \cdot 10^{3}=67.3 \mathrm{kN}<V_{R d, c}=87.6 \mathrm{kN} \\
& V_{E d}=414 \mathrm{kN}>V_{R d, c}=87.6 \mathrm{kN} \rightarrow \text { Shear reinforcement is needed! }
\end{array}
$$

Lever arm of the internal forces $z=\zeta \cdot d=0.903 \cdot 65=58.7 \mathrm{~cm}$

Friction Force:

$$
V_{R d, c c}=0.24 \cdot(30)^{1 / 3} \cdot 0.3 \cdot 0.587 \cdot 10^{3}=131.3 \mathrm{kN}
$$


Inclination:

$$
1.0 \leq \cot \theta=\frac{1.2}{1-V_{R d, c c} / V_{E d}}=\frac{1.2}{1-131.3 / 414}=1.76 \leq \underline{\underline{3.0}}
$$

Vertical stirrups

$$
\alpha=90^{\circ}
$$

Required reinforcement

$$
a_{s w, r e q}=\frac{414 k N}{43.5 \cdot 0.587 \cdot 1.75}=9.3 \mathrm{~cm}^{2} / \mathrm{m}
$$

\section{Chosen}

\section{stirrups}

Resistance of the compression struts

$$
\begin{aligned}
& V_{R d \text {,max }}=0.75 \cdot 30 \cdot 58.7 \cdot 1.7 \cdot \frac{1.76}{1+1.76^{2}}=965 \mathrm{kN} \\
& V_{E d, \text { max }}=414 \mathrm{kN}<V_{R d, \text { max }}=965 \mathrm{kN} \quad \rightarrow \text { The compression strut is ok. }
\end{aligned}
$$

\begin{tabular}{|c|c|c|c|c|c|c|c|c|}
\hline & $\begin{array}{l}V_{E d} \\
{[k N]}\end{array}$ & $\begin{array}{l}V_{\text {Rd,c }} \\
{[k N]}\end{array}$ & $\begin{array}{l}\mathrm{V}_{\mathrm{Rd}, \mathrm{cc}} \\
{[\mathrm{kN}]}\end{array}$ & $\begin{array}{c}\cot \theta \\
{[-]}\end{array}$ & $\begin{array}{c}a_{\text {sw,req }} \\
{\left[\mathrm{cm}^{2} / \mathrm{m}\right]}\end{array}$ & Chosen & $\begin{array}{c}a_{\text {sw,prov }} \\
{\left[\mathrm{cm}^{2} / \mathrm{m}\right]}\end{array}$ & $\begin{array}{c}\mathbf{V}_{\text {Rd,max }} \\
{[\mathrm{kN}]}\end{array}$ \\
\hline Supp $A_{R}$ & 121 & 67.29 & 130.88 & 1.200 & 3.96 & $\varnothing 8 / 20$ & 5.03 & 1100.5 \\
\hline Supp $B_{L}$ & -414 & 87.63 & 131.31 & 1.757 & 9.23 & $\varnothing 8 / 10$ & 10.05 & 965.0 \\
\hline Supp $B_{R}$ & 366 & 87.63 & 131.31 & 1.871 & 7.66 & $\varnothing 8 / 12.5$ & 8.04 & 933.2 \\
\hline Supp $C_{L}$ & -362 & 87.63 & 133.20 & 1.899 & 7.36 & $\varnothing 8 / 12.5$ & 8.04 & 939.0 \\
\hline Supp $C_{R}$ & 330 & 87.63 & 133.20 & 2.012 & 6.33 & $\varnothing 8 / 15$ & 6.71 & 907.6 \\
\hline Supp $D_{L}$ & -115 & 67.29 & 130.88 & 1.200 & 3.77 & $\varnothing 8 / 20$ & 5.03 & 1100.5 \\
\hline
\end{tabular}

Minimum shear reinforcement $\quad \min a_{s w}=\rho \cdot b_{w} \cdot \sin \alpha \quad$ and $\quad \rho=0.16 \cdot f_{c t m} / f_{y k}=0.16 \cdot 2.9 / 500=0.93 \%$

$$
\begin{aligned}
& \min a_{s w}=0.0093 \cdot 0.30 \cdot \sin 90^{\circ}=0.28 \cdot 10^{-4} \mathrm{~m}^{2} / \mathrm{m}=\underline{\underline{0.28 \mathrm{~cm}^{2} / \mathrm{m}}} \\
& \min a_{s w}=0.28 \mathrm{~cm}^{2} / \mathrm{m}<a_{s w, \text { prov }}=10.48 \mathrm{~cm}^{2} / \mathrm{m} \checkmark
\end{aligned}
$$

Shear Design Calculations at other Supports

\subsection{SERVICEABILITY LIMIT STATE DESIGN}

\subsubsection{LIMITATION OF THE CRACK WIDTH}

Maximum Diameter of the Reinforcement

$\begin{array}{lll}\text { Crack width } & \mathrm{w}_{\mathrm{k}}=0.4 \mathrm{~mm} & \text { (according to DIN EN 1992-1-1, Tab. NA.7.1.) } \\ \text { Reinforcement stresses } & \sigma_{s}=\frac{M_{\text {perm }}}{z \cdot A_{s l}} \rightarrow d_{s}^{*} \\ \text { Limit diameter } & d_{s}=d_{s}^{*} \cdot f_{c t, \text { eff }} / 2.9>d_{s, \text { prov }}\end{array}$




\begin{tabular}{|l|c|c|c|c|c|c|c|}
\cline { 2 - 8 } \multicolumn{1}{c|}{} & $\begin{array}{c}\mathbf{M}_{\text {perm }} \\
{[\mathbf{k N m}]}\end{array}$ & $\begin{array}{c}\mathbf{A}_{\mathbf{s}, \text { prov }} \\
{\left[\mathbf{c m}^{2}\right]}\end{array}$ & $\begin{array}{c}\mathbf{z} \\
{[\mathbf{c m}]}\end{array}$ & $\begin{array}{c}\mathbf{\sigma}_{\mathbf{s}} \\
{\left[\mathbf{N} / \mathbf{m m}^{2}\right]}\end{array}$ & $\begin{array}{c}\mathbf{d}_{\mathbf{s}}{ }^{*} \\
{[\mathbf{m m}]}\end{array}$ & $\begin{array}{c}\mathbf{m a x ~ d}_{\mathbf{s}} \\
{[\mathbf{m m}]}\end{array}$ & $\begin{array}{c}\text { prov d }_{\mathbf{s}} \\
{[\mathbf{m m}]}\end{array}$ \\
\hline Span 1 & 111.9 & 6.16 & 64.35 & 282.29 & 17.77 & $\mathbf{1 7 . 7 7}$ & $>\mathbf{1 4}$ \\
\hline Supp B & -221.6 & 15.7 & 58.70 & 240.47 & 24.00 & $\mathbf{2 4 . 0 0}$ & $>\mathbf{2 0}$ \\
\hline Span 2 & 66.6 & 6.16 & 51.48 & 210.02 & 32.25 & $\mathbf{3 2 . 2 5}$ & $>14$ \\
\hline Supp C & -197.7 & 15.7 & 59.54 & 211.49 & 31.84 & $\mathbf{3 1 . 8 4}$ & $>\mathbf{2 0}$ \\
\hline Span 3 & 98.1 & 6.16 & 64.35 & 247.49 & 16.88 & $\mathbf{1 6 . 8 8}$ & $>\mathbf{1 4}$ \\
\hline
\end{tabular}

\subsubsection{MINIMUM REINFORCEMENT TO PREVENT CRACKING (SUPPORT B)}

Minimum Reinforcement of the Flange

Tensile strength $\quad f_{c t, \text { eff }}=f_{c t m} \geq 3.0 \mathrm{MN} / \mathrm{m}^{2} \quad \rightarrow f_{c t, \text { eff }}=2.9 \mathrm{MN} / \mathrm{m}^{2}>\underline{\underline{3.0 \mathrm{MN} / \mathrm{m}^{2}}}$

Tensile stresses at the flange

$$
\begin{aligned}
& \sigma_{c}=-\frac{(21.5-17.5)}{21.5} \cdot 3.0=-0.558 \mathrm{MN} / \mathrm{m}^{2} \\
& h=35 \mathrm{~cm} \quad \rightarrow h^{*}=35 \mathrm{~cm} \\
& k_{1}=1.5 \\
& k_{c}=0.4 \cdot\left[1-\frac{\sigma_{c}}{k_{1} \cdot h / h^{*} \cdot f_{c t, e f f}}\right]=0.4 \cdot\left[1-\frac{-0.558}{1.5 \cdot 3.0}\right]=0.45 \leq 1.0 \\
& \text { for } h=35 \mathrm{~cm}>30 \mathrm{~cm} \quad \rightarrow k=0.77
\end{aligned}
$$

Tensile stress area

$A_{c t}=0.215 \cdot 1.0=0.215 \mathrm{~m}^{2} / \mathrm{m}$

Reinforcement stresses

$$
\begin{aligned}
& d_{s}=10 \mathrm{~mm} \rightarrow d_{s}^{*}=\frac{2.9}{f_{c t, e f f}} \cdot d_{s}=\frac{2.9}{3.0} \cdot 10=9.7 \\
& \sigma_{s}=386 \mathrm{MN} / \mathrm{m}^{2} \quad\left(\mathrm{w}_{\mathrm{k}}=0.4 \mathrm{~mm}, d_{\mathrm{s}}=9.7 \mathrm{~mm}\right)
\end{aligned}
$$

Required reinforcement

$$
\begin{aligned}
& a_{s, \text { min, req }}=k_{c} \cdot k \cdot f_{c t, \text { eff }} \cdot \frac{A_{c t}}{\sigma_{s}}=0.45 \cdot 0.77 \cdot 3.0 \cdot \frac{0.215}{386} \cdot 10^{4}=5.79 \mathrm{~cm}^{2} / \mathrm{m} \\
& <a_{s, \text { prov }}=8.59 \mathrm{~cm}^{2} / \mathrm{m} \quad(Q 335 A+\varnothing 10 / 15)^{3}
\end{aligned}
$$

Minimum Reinforcement of the Web

Compression stresses at the web

$$
\begin{aligned}
& \sigma_{c}=-3.0+\frac{35.0}{21.5} \cdot 3.0=+1.883 \mathrm{MN} / \mathrm{m}^{2} \\
& k_{1}=1.5
\end{aligned}
$$

${ }^{3}$ See Chapter 12.2.1 (Upper reinforcement of the slab in direction 1) 


$$
\begin{gathered}
k_{c}=0.4 \cdot\left[1-\frac{\sigma_{c}}{k_{1} \cdot h / h^{*} \cdot f_{c t, e f f}}\right]=0.4 \cdot\left[1-\frac{1.883}{1.5 \cdot 3.0}\right]=0.23 \leq 1.0 \\
\text { for } h=70 \mathrm{~cm}>30 \mathrm{~cm} \quad \rightarrow k=0.56
\end{gathered}
$$

Tensile stress area

Reinforcement stresses

Required reinforcement

$$
A_{c t}=0.215 \cdot 0.3=0.0645 m^{2}
$$$$
d_{s}=20 \mathrm{~mm} \rightarrow d_{s}^{*}=\frac{2.9}{f_{c t, e f f}} \cdot d_{s}=\frac{2.9}{3.0} \cdot 20=19.33
$$

$\sigma_{s}=271 M N / m^{2}$ $\left(w_{k}=0.4 \mathrm{~mm}, d_{\mathrm{s}}=19.33 \mathrm{~mm}\right)$

$A_{s, \text { min, req }}=k_{c} \cdot k \cdot f_{c t, \text { eff }} \cdot \frac{A_{c t}}{\sigma_{s}}=0.23 \cdot 0.56 \cdot 3.0 \cdot \frac{0.0645}{271} \cdot 10^{4}=0.92 \mathrm{~cm}^{2}$

$<A_{s, \text { prov }}=15.7 \mathrm{~cm}^{2} \quad(5 \varnothing 20)^{4}$

\subsubsection{DEFORMATION OF THE SLAB}

Deformation of the slab under $q_{p e r m}$

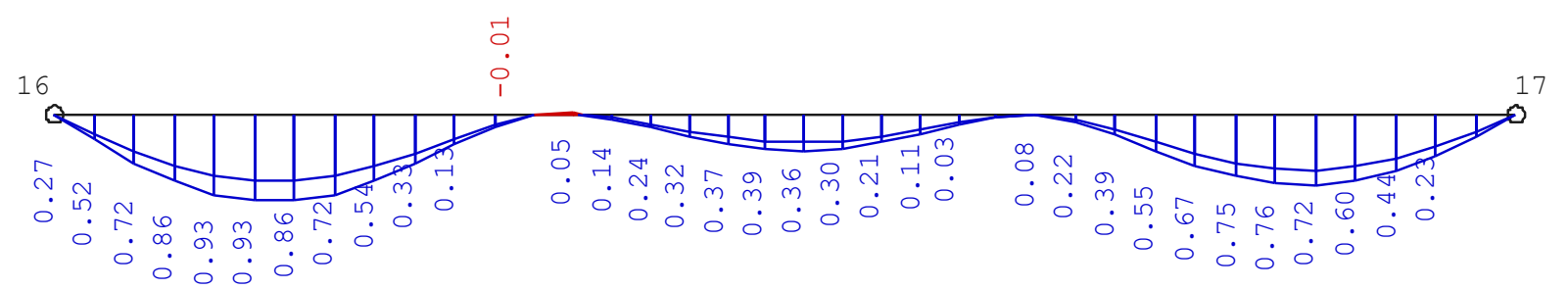

$f_{\max }=0.93 \mathrm{~mm}<I / 250=6250 / 200=31.25 \mathrm{~mm} \checkmark$

The deformation does not exceed the maximum allowed deformation!

\subsection{DESIGN AND REINFORCEMENT}

\subsubsection{MINIMUM REINFORCEMANT TO ENSURE ROBUSTNESS}

$$
M_{c r}=f_{c t m} \cdot W_{c u} \quad W_{c u}=I_{y} / z_{s} \quad A_{s, \min }=\frac{1}{f_{y k}} \cdot \frac{M_{c r}}{z}
$$

\begin{tabular}{|l|c|c|c|c|c|c|c|c|}
\cline { 2 - 9 } \multicolumn{1}{c|}{} & $\begin{array}{c}\mathbf{b}_{\text {eff }} \\
{[\mathbf{c m}]}\end{array}$ & $\begin{array}{c}\mathbf{z}_{\mathbf{s}, \mathbf{u}} \\
{[\mathbf{c m}]}\end{array}$ & $\begin{array}{c}\mathbf{I}_{\mathbf{y}} \\
{\left[\mathbf{c m}^{4}\right]}\end{array}$ & $\begin{array}{c}\mathbf{W}_{\mathbf{u}} \\
{\left[\mathbf{c m}^{3}\right]}\end{array}$ & $\begin{array}{c}\mathbf{W}_{\mathbf{o}} \\
{\left[\mathbf{c m}^{3}\right]}\end{array}$ & $\begin{array}{c}\mathbf{M}_{\text {cr }} \\
{[\mathbf{k N c m}]}\end{array}$ & $\begin{array}{c}\mathbf{A}_{\mathbf{s}, \mathbf{m i n}, \text { req }} \\
{\left[\mathbf{c m}^{2}\right]}\end{array}$ & $\begin{array}{c}\mathbf{A}_{\mathbf{s}, \text { prov }} \\
{\left[\mathbf{c m}^{2}\right]}\end{array}$ \\
\hline Span 1 & 233 & 48.51 & 2079207 & 42864 & - & 12430 & $\mathbf{3 . 8 6}$ & $\mathbf{6 . 1 6}$ \\
\hline Supp B & 106 & 44.78 & 1488435 & - & 59017 & 17115 & $\mathbf{5 . 8 3}$ & $\mathbf{1 5 . 7 0}$ \\
\hline Span 2 & 189 & 47.71 & 1892520 & 39671 & - & 11505 & $\mathbf{4 . 4 7}$ & $\mathbf{6 . 1 6}$ \\
\hline Supp C & 106 & 44.78 & 1488435 & - & 59017 & 17115 & $\mathbf{5 . 7 5}$ & $\mathbf{1 5 . 7 0}$ \\
\hline Span 3 & 211 & 48.14 & 1987209 & 41277 & - & 11970 & $\mathbf{3 . 7 2}$ & $\mathbf{6 . 1 6}$ \\
\hline
\end{tabular}

${ }^{4}$ See Chapter 13.3.1 (Upper reinforcement of the beam at support B) 


\subsubsection{DESIGN OF THE INTERCONNECTING JOINT}

Design location

$x=\frac{0.15}{2}+0.65-0.29=0.435 m$

Shear force

$V_{E d, x} \approx 414 k N$

$v_{E d, i}=\frac{F_{c d j}}{F_{c d}} \cdot \frac{V_{E d}}{z \cdot b_{F}}=1.0 \cdot \frac{414}{0.26 \cdot 0.587}=2713 \mathrm{kN} / \mathrm{m}^{2}$

Rough joint

$$
c=0.4 \quad \mu=0.7 \quad v=0.5
$$

Maximum shear force

$$
\begin{aligned}
v_{R d i, c} & =c \cdot 0.12 \cdot\left(f_{c k}\right)^{2 / 3}=0.4 \cdot 0.12 \cdot(30)^{2 / 3} \cdot 10^{3}=463 \mathrm{kN} / \mathrm{m}^{2} \\
& <v_{E d, i}=2713 \mathrm{kN} / \mathrm{m}^{2} \checkmark
\end{aligned}
$$

Reinforcement at the interconnection joint necessary!

Reinforcement

$a_{s, F}=\frac{\left(v_{E d i}-v_{R d i, c}\right) \cdot b_{F}}{f_{y d} \cdot 1.2 \cdot \mu}=\frac{(2713-463) \cdot 0.26}{43.5 \cdot 1.2 \cdot 0.7}=\underline{\underline{16.0 \mathrm{~cm}^{2} / \mathrm{m}}}$

Maximum shear force

$$
v_{\text {Rdi, max }}=0.5 \cdot v \cdot f_{c d}=0.5 \cdot 0.5 \cdot 17 \cdot 10^{3}=4250 \mathrm{kN} / \mathrm{m}^{2}>v_{E d i}=2713 \mathrm{kN} / \mathrm{m}^{2}
$$

\subsubsection{CONNECTION OF THE COMPRESSION FLANGE (SPAN 1)}

Design location

Flexure moment

Bond force in the joint

Shear force resistance

Transverse reinforcement

$$
\Delta x=1.30 m
$$

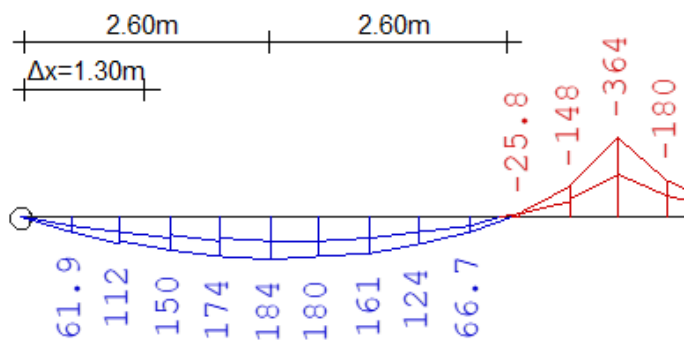

$$
M_{E d}(x=1.30) \approx 140 \mathrm{kNm}=\Delta M_{E d}
$$

$v_{E d}=\frac{\Delta M_{E d}}{z \cdot h_{f} \cdot \Delta x} \cdot \frac{b_{a}}{b_{\text {eff }}}=\frac{140}{0.644 \cdot 0.35 \cdot 1.30} \cdot \frac{97}{233}=199 \mathrm{kN} / \mathrm{m}^{2}$

$\cot \theta=1.2$

$v_{R d, \max }=\frac{0.75 \cdot f_{c d}}{\cot \theta+\tan \theta}=\frac{0.75 \cdot 17}{1.2+1 / 1.2} \cdot 10^{3}=6271 \mathrm{kN} / \mathrm{m}^{2}>v_{E d}=199 \mathrm{kN} / \mathrm{m}^{2}$

$a_{s w, r e q}=\frac{v_{E d} \cdot h_{f}}{f_{y d} \cdot \cot \theta}=\frac{199 \cdot 0.35}{43.5 \cdot 1.2}=1.33 \mathrm{~cm}^{2} / \mathrm{m}$

$a_{s, u_{-} \text {prov }}=3.35 \mathrm{~cm}^{2} / \mathrm{m}>a_{s, u_{-} \text {req }}=1.33 / 2=0.67 \mathrm{~cm}^{2} / \mathrm{m} \checkmark$

$a_{s, I_{\perp} \text { prov }}=5.5 \mathrm{~cm}^{2} / \mathrm{m}>a_{s, I_{\text {req }}}=1.33 / 2=0.67 \mathrm{~cm}^{2} / \mathrm{m} \checkmark$ 


\subsubsection{CONNECTION OF THE TENSION FLANGE}

Design location

Flexure moment

Bond force in the joint

Shear force resistance

Transverse reinforcement

$$
\Delta x=1.05 / 2=0.525 m
$$

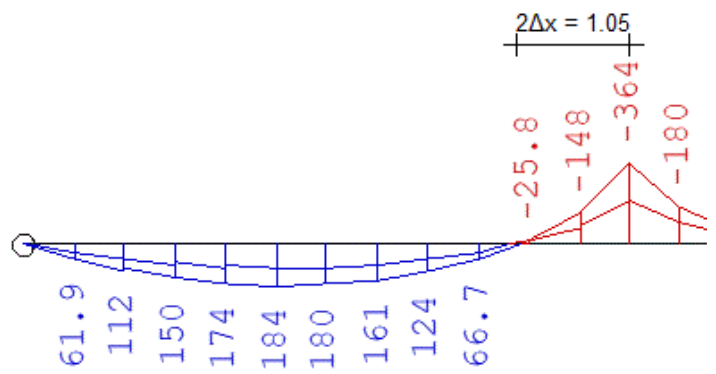

$$
\Delta M_{E d}=364-148=216 k N m
$$

$v_{E d}=\frac{\Delta M_{E d}}{z \cdot h_{f} \cdot \Delta x} \cdot \frac{A_{s a}}{A_{s}}=\frac{216}{0.587 \cdot 0.35 \cdot 0.525} \cdot \frac{6.28}{15.7}=801 \mathrm{kN} / \mathrm{m}^{2}$

$\cot \theta=1.0$

$v_{R d, \text { max }}=\frac{0.75 \cdot f_{c d}}{\cot \theta+\tan \theta}=\frac{0.75 \cdot 17}{1.0+1 / 1.0} \cdot 10^{3}=6375 \mathrm{kN} / \mathrm{m}^{2}>v_{E d}=801 \mathrm{kN} / \mathrm{m}^{2}$

$a_{s w, r e q}=\frac{v_{E d} \cdot h_{f}}{f_{y d} \cdot \cot \theta}=\frac{801 \cdot 0.35}{43.5 \cdot 1.0}=6.44 \mathrm{~cm}^{2} / \mathrm{m}$

$a_{s, u_{-} \text {prov }}=3.35 \mathrm{~cm}^{2} / \mathrm{m}>a_{s, u_{-} \text {req }}=6.44 / 2=3.22 \mathrm{~cm}^{2} / \mathrm{m} \checkmark$

$a_{s, I_{\perp} \text { prov }}=5.5 \mathrm{~cm}^{2} / \mathrm{m}>a_{s, I_{-} \text {req }}=6.44 / 2=3.22 \mathrm{~cm}^{2} / \mathrm{m} \checkmark$

\subsubsection{ANCHORAGE LENGTH AT THE SUPPORT}

End Support A and D

Global offset

$a_{l}=0.9 \cdot 65 \cdot 1.2 / 2=35.1 \mathrm{~cm} \quad F_{E d}=\frac{V_{E d} \cdot a_{l}}{z}=\frac{121 \cdot 35.1}{0.9 \cdot 65}=72.6 \mathrm{kN}$

$$
A_{s, \text { req }}=\frac{F_{E d}}{f_{y d}}=\frac{72.6}{43.5}=1.67 \mathrm{~cm}^{2}
$$

Anchorage length

$$
\begin{aligned}
& I_{b, \text { rad }}=36 \mathrm{~cm} \quad \text { for C30/37 and good bond } \\
& I_{b d}=\alpha_{1} \cdot I_{b, \text { rad }} \cdot\left(A_{s, \text { req }} / A_{s, \text { prov }}\right)=0.7 \cdot 50 \cdot(5.98 / 6.16)=33.98 \mathrm{~cm} \\
& >I_{b, \text { min }}=0.3 \cdot 0.7 \cdot 50=10.5 \mathrm{~cm}>10 \phi_{s}=14.0 \mathrm{~cm} \\
& I_{b, \text { dir }}=2 / 3 \cdot I_{b d}=2 / 3 \cdot 33.98=\underline{\underline{22.7 \mathrm{~cm}}}>6.7 \phi_{s}=9.4 \mathrm{~cm}
\end{aligned}
$$

Intermediate Support B and C

$$
I_{b d}=6 \cdot \phi=6 \cdot 1.4=8.4 \mathrm{~cm}
$$


13.5.6 OVERLAP LENGTH

$$
\begin{array}{ll}
\varnothing_{s}=14 \quad I_{0} & =\alpha_{1} \cdot \alpha_{6} \cdot I_{b, \text { rqd }} \cdot\left(A_{s, \text { req }} / A_{s, \text { prov }}\right)=1.0 \cdot 1.4 \cdot 50 \cdot(5.98 / 6.16)=68 \mathrm{~cm} \\
& >I_{0, \text { min }}=21 \mathrm{~cm} \\
\varnothing_{s}=20 \quad & I_{0} \\
& \alpha_{1} \cdot \alpha_{6} \cdot I_{b, \text { rqd }} \cdot\left(A_{s, \text { req }} / A_{s, \text { prov }}\right)=1.0 \cdot 2.0 \cdot 89 \cdot(14.34 / 15.7)=163 \mathrm{~cm} \\
& >I_{0, \text { min }}=53 \mathrm{~cm}
\end{array}
$$

\subsubsection{REINFORCEMENT DRAWING}

See Appendix A.7, page 193. 


\subsection{SOFTWARE CALCULATIONS}

\section{Demo Frilo Nemetschek}

\section{Position: B.13 - Semi-Precast Concrete Beam}

Durchlaufträger DLT10 01/2018 (Frilo R-2018-1/P12)

Scale $1: 150$
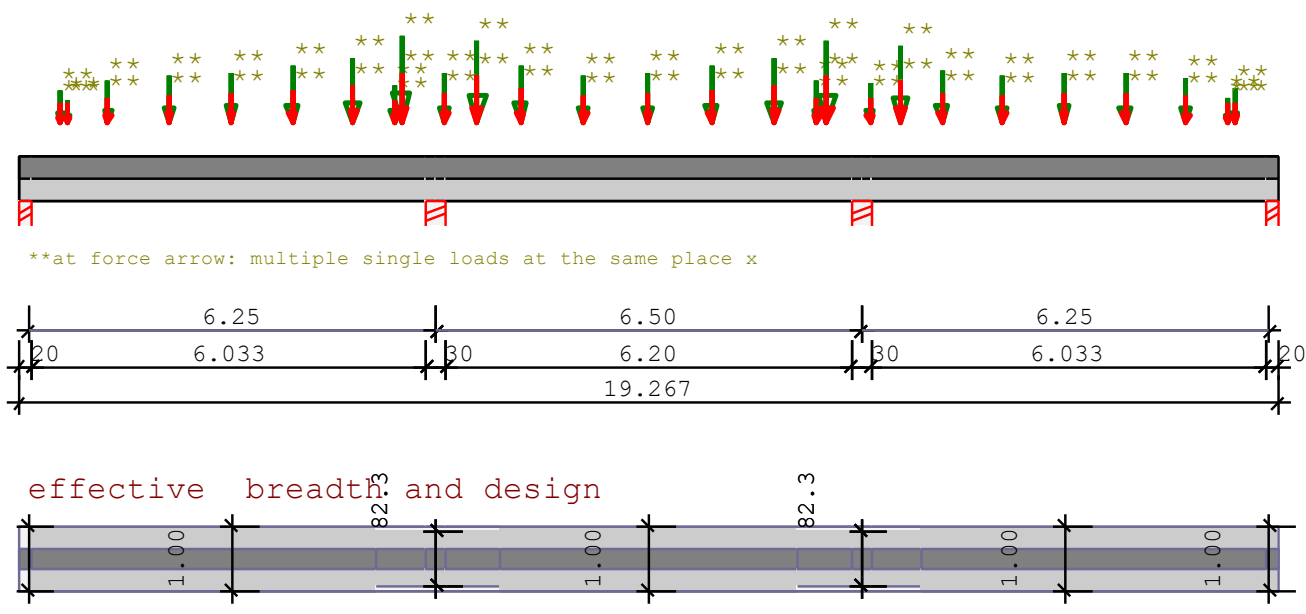

Reinforced concrete girder over 3 Spans C30/37 E = $33000 \mathrm{~N} / \mathrm{mm} 2$ DIN EN 1992-1-1/NA/A1:2015-12

Decke über NG (Normalgeschoss) von Gebäudemodell

\begin{tabular}{|c|c|c|c|c|c|c|c|c|}
\hline System & lengtl & & cross-s & tion & lues & & & \\
\hline Span & 11 & m) & bt & ht & bo & ho & bb & $h b$ \\
\hline 1 & 6.25 & constant & 100.0 & 35.0 & 30.0 & 70.0 & & \\
\hline 2 & 6.50 & constant & 100.0 & 35.0 & 30.0 & 70.0 & & \\
\hline 3 & 6.25 & constant & 100.0 & 35.0 & 30.0 & 70.0 & & \\
\hline
\end{tabular}

Cross-sections with eff. active breadth

\begin{tabular}{|c|c|c|c|c|c|c|c|c|}
\hline $\begin{array}{c}x \\
(m)\end{array}$ & $\begin{array}{c}\mathrm{bt} \\
(\mathrm{cm})\end{array}$ & $\begin{array}{l}\text { ht } \\
(\mathrm{cm})\end{array}$ & $\begin{array}{c}\mathrm{b} 0 \\
(\mathrm{~cm})\end{array}$ & $\begin{array}{l}\mathrm{h} 0 \\
(\mathrm{~cm})\end{array}$ & $\begin{array}{c}\mathrm{bb} \\
(\mathrm{cm})\end{array}$ & $\begin{array}{l}\text { hb } \\
(\mathrm{cm})\end{array}$ & $\begin{array}{l}\text { Wyb } \\
\text { (m3) }\end{array}$ & $\begin{array}{l}\text { Wyt } \\
\text { (m3) }\end{array}$ \\
\hline 0.00 & 100.0 & 35.0 & 30.0 & 70.0 & & & 0.0327 & 0.0568 \\
\hline 5.31 & 100.0 & 35.0 & 30.0 & 70.0 & & & 0.0327 & 0.0568 \\
\hline 5.31 & 82.3 & 35.0 & 30.0 & 70.0 & & & 0.0311 & 0.050 \\
\hline 6.25 & 82.3 & 35.0 & 30.0 & 70.0 & & & 0.0311 & 0.050 \\
\hline 7.22 & 82.3 & 35.0 & 30.0 & 70.0 & & & 0.0311 & 0.050 \\
\hline 7.23 & 100.0 & 35.0 & 30.0 & 70.0 & & & 0.0327 & 0.056 \\
\hline 11.77 & 100.0 & 35.0 & 30.0 & 70.0 & & & 0.0327 & 0.056 \\
\hline 11.78 & 82.3 & 35.0 & 30.0 & 70.0 & & & 0.0311 & 0.050 \\
\hline 12.75 & 82.3 & 35.0 & 30.0 & 70.0 & & & 0.0311 & 0.050 \\
\hline 13.69 & 82.3 & 35.0 & 30.0 & 70.0 & & & 0.0311 & 0.050 \\
\hline 13.69 & 100.0 & 35.0 & 30.0 & 70.0 & & & 0.0327 & \\
\hline 19.00 & 100.0 & 35.0 & 30.0 & 70.0 & & & 0.0327 & 0.0568 \\
\hline
\end{tabular}




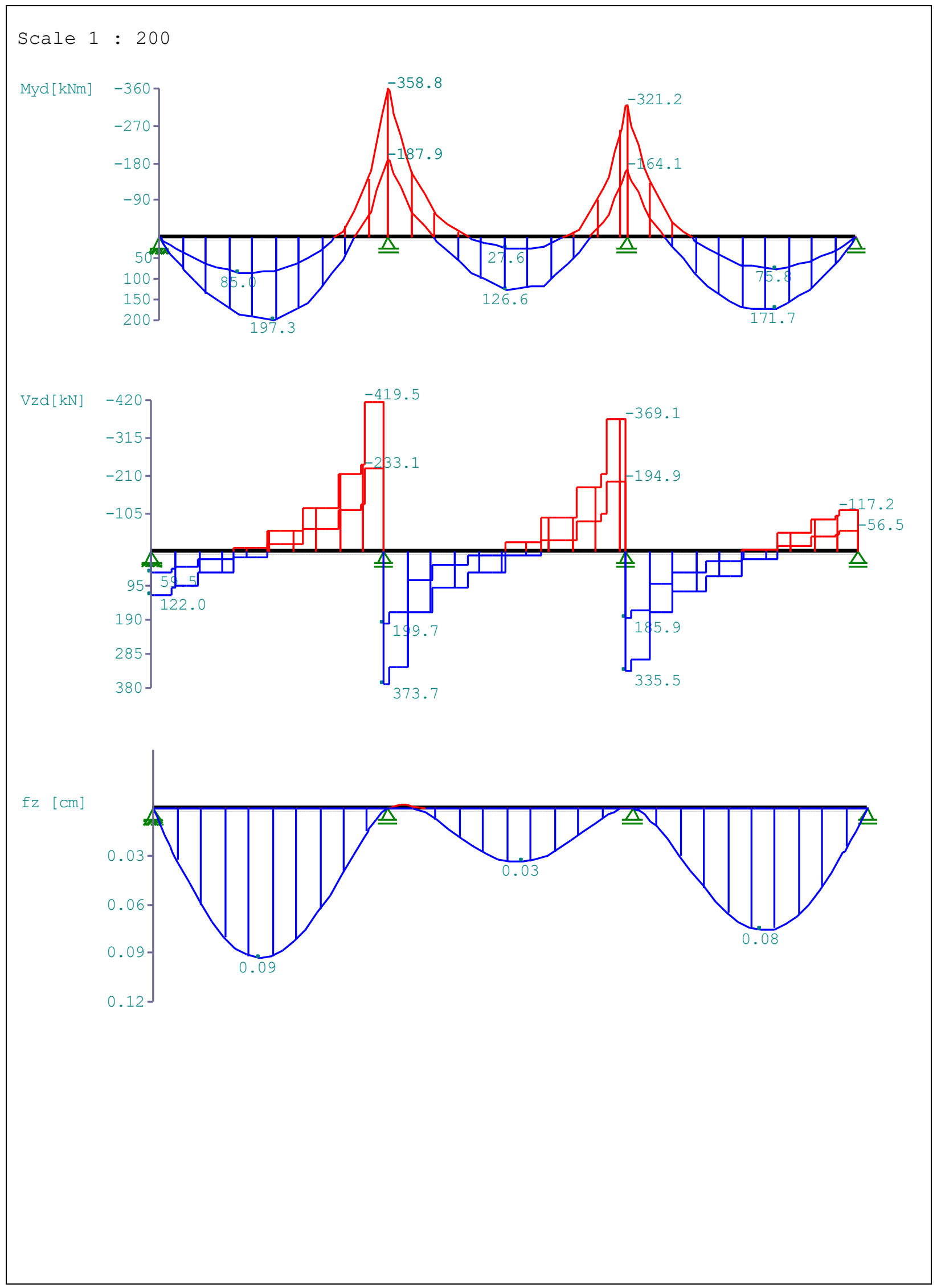


Design DIN EN 1992-1-1/NA/A1:2015-12

FLBemBn.DLL: Version 9.0.1.121 (1)

C30/37 B500A normaly ductil

Minimum reinforcement EN2 9.2.1.1 (9.1) fctm $=2.90 \mathrm{~N} / \mathrm{mm} 2$

Calculating $W y$, breadth of slab is limited to $2 * b 0$.

Q.No. min $\mathrm{Mb}$ req As min Mo req As

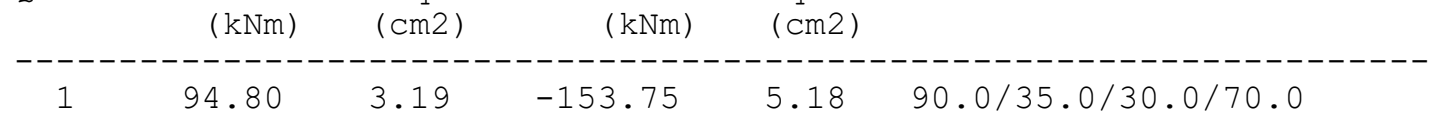

Span reinforcement

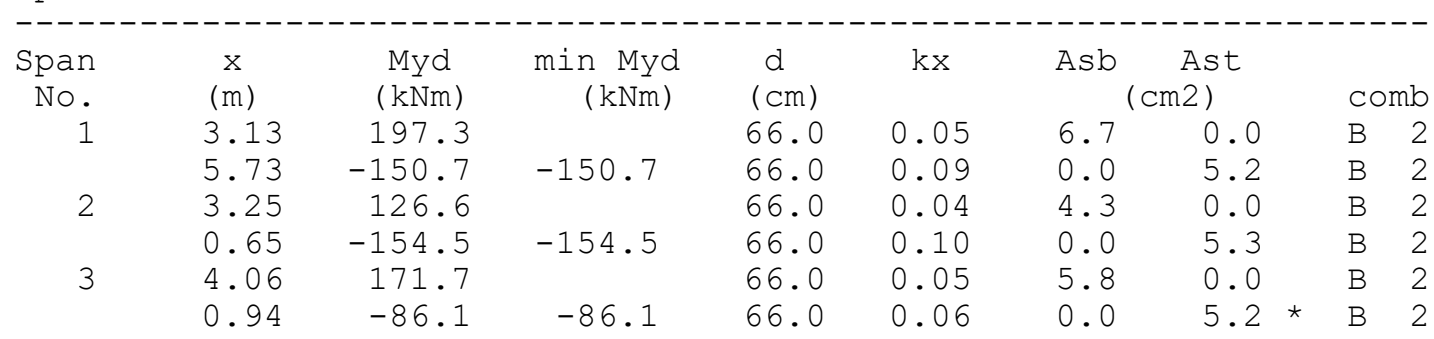

* Minimum reinforcement acc.to DIN EN 1992-1 9.2.1.1 (1)

On first support are at least $4.2 \mathrm{~cm} 2$ to be anchored.

On last support are at least $4.0 \mathrm{~cm} 2$ to be anchored.

Shear force VK-support is with $F=V$, Ed * Cot(Theta) / 2 considered.

Support reinforcement DIN EN 1992:2015 5.5

\begin{tabular}{|c|c|c|c|c|c|c|c|c|c|}
\hline Column & $\begin{array}{l}x \\
(m) \\
0\end{array}$ & $\begin{array}{l}\text { Myd } \\
(\mathrm{kNm})\end{array}$ & $\begin{array}{c}\text { des... Myd } \\
(\mathrm{kNm})\end{array}$ & $\begin{array}{c}d \\
(\mathrm{~cm})\end{array}$ & $\mathrm{kx}$ & \multicolumn{2}{|c|}{$(\mathrm{cm} 2)$} & \multicolumn{2}{|c|}{ comb } \\
\hline $21 e$ & 0.15 & -350.1 & -236.2 & 66.0 & 0.14 & 0.0 & 8.4 & B & 2 \\
\hline ri & 0.15 & -350.1 & -244.5 & 66.0 & 0.14 & 0.0 & 8.7 & B & 2 \\
\hline le & 0.15 & -310.7 & -211.7 & 66.0 & 0.12 & 0.0 & 7.4 & B & 2 \\
\hline $3 r i$ & 0.15 & -310.7 & -215.2 & 66.0 & 0.13 & 0.0 & 7.5 & B & 2 \\
\hline $41 e$ & 0.00 & 0.0 & & & & & & & 1 \\
\hline
\end{tabular}

shear force reinforcement B500A DIN EN 1992-1-1/NA/A1:2015-12 6.2

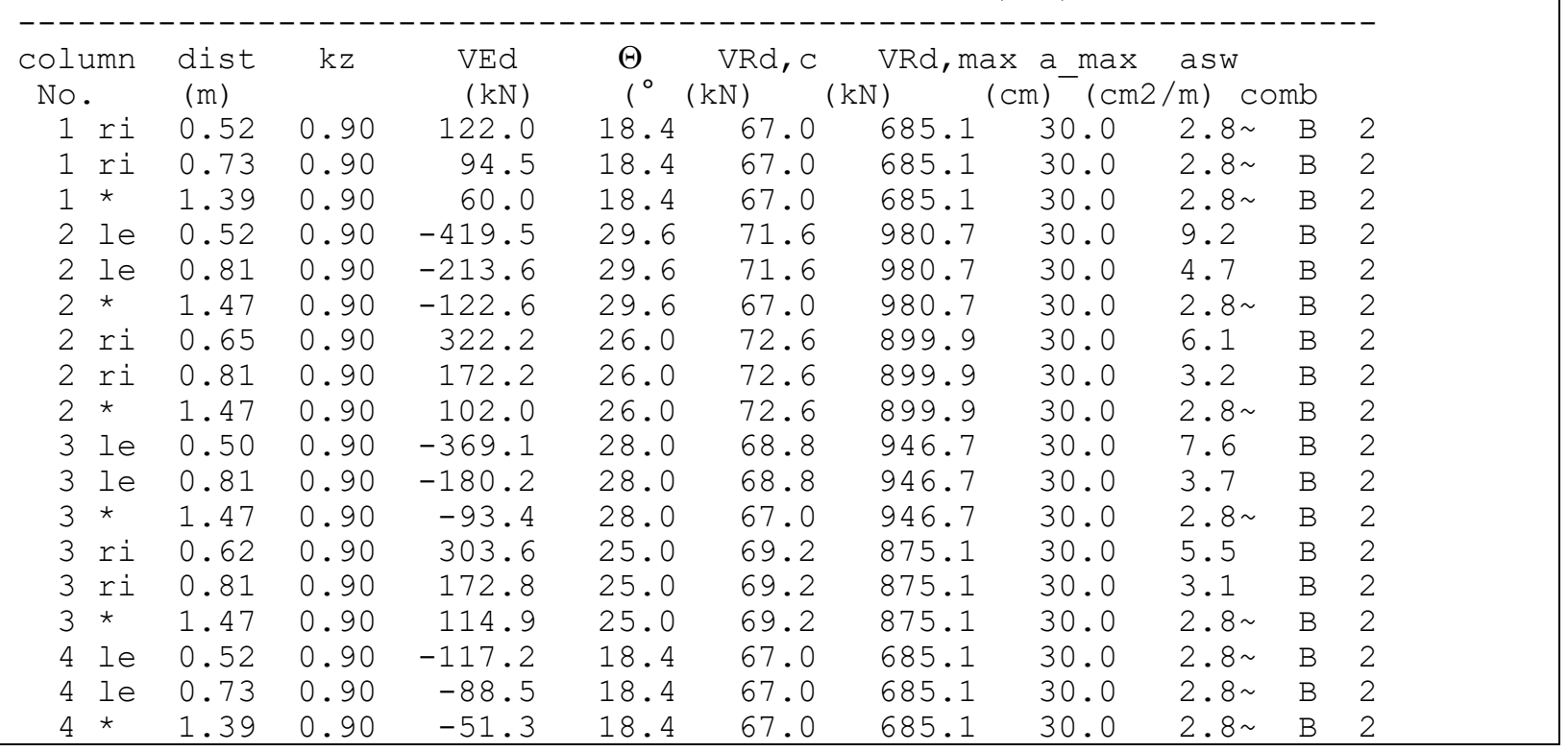




\begin{tabular}{|c|c|c|c|c|c|c|c|c|c|c|}
\hline \multicolumn{11}{|c|}{$\begin{array}{l}\sim \text { at the end of li } \\
\text { max distance of st } \\
525) \text {. } \\
\text { reduction of singl } \\
\text { shoulder shear }\end{array}$} \\
\hline Span & $\begin{array}{c}\mathrm{xa} \\
(\mathrm{cm})\end{array}$ & $\begin{array}{c}\text { xe } \\
(\mathrm{cm})\end{array}$ & $\begin{array}{c}\text { mle } \\
(\mathrm{kNm})\end{array}$ & $\begin{array}{c}\text { mri } \\
(\mathrm{kNm})\end{array}$ & $\begin{array}{l}\mathrm{av} \\
(\mathrm{cm})\end{array}$ & $\begin{array}{l}\text { beff } \\
\quad(\mathrm{cm})\end{array}$ & $\begin{array}{c}d F C d \\
(k N)\end{array}$ & $\begin{array}{l}\mathrm{vEd} \\
\quad(\mathrm{kN}\end{array}$ & $\begin{array}{l}\text { ted, per } \\
\text { m2) }\end{array}$ & $\begin{array}{l}m \text { asf } \\
m 2 / m)\end{array}$ \\
\hline 1 & 0 & 156 & 0.0 & 150.8 & 156 & 100 & 89 & 162 & 6278 & 11 \\
\hline 1 & 156 & 313 & 150.8 & 197.3 & 156 & 100 & 27 & 50 & 6278 & 0.3 \\
\hline 1 & 313 & 422 & 197.3 & 139.3 & 110 & 100 & 34 & 89 & 6278 & 0.6 \\
\hline 1 & 422 & 532 & 139.3 & -0.1 & 110 & 100 & 82 & 214 & 6278 & 1.4 \\
\hline 2 & 125 & 225 & 1.1 & 84.0 & 100 & 100 & 49 & 139 & 6278 & 0.9 \\
\hline 2 & 225 & 325 & 84.0 & 126.6 & 100 & 100 & 25 & 71 & 6278 & 0.5 \\
\hline 2 & 325 & 437 & 126.6 & 103.9 & 112 & 100 & 13 & 34 & 6278 & 0.2 \\
\hline 2 & 437 & 549 & 103.9 & -0.2 & 112 & 100 & 61 & 156 & 6278 & 1.0 \\
\hline 3 & 107 & 257 & 0.2 & 137.8 & 150 & 100 & 81 & 155 & 6278 & 1.0 \\
\hline 3 & 257 & 406 & 137.8 & 171.7 & 150 & 100 & 20 & 38 & 6278 & 0.3 \\
\hline 3 & 406 & 516 & 171.7 & 112.2 & 109 & 100 & 35 & 91 & 6278 & 0.6 \\
\hline 3 & 516 & 625 & 112.2 & 1.2 & 109 & 100 & 65 & 171 & 6278 & 1.1 \\
\hline 3 & 516 & 625 & 112.2 & 1.2 & 109 & 100 & 65 & 171 & 6278 & 1.1 \\
\hline 3 & 516 & 625 & 112.2 & 1.2 & 109 & 100 & 65 & 171 & 6278 & 1.1 \\
\hline
\end{tabular}




\section{POS. W.14 - LOAD BEARING PRECAST CONCRETE WALL PANEL}

\subsection{SYSTEM}

The concrete wall panel functions as the support of the double-tee slab and has to carry the resulting vertical loads as well as horizontal wind loads. For the design of the wall panel a simplified system as shown below is sufficient to describe the load paths and calculate the required reinforcement. A more in-depth analysis of the concrete wall will be done in the following chapters.

System

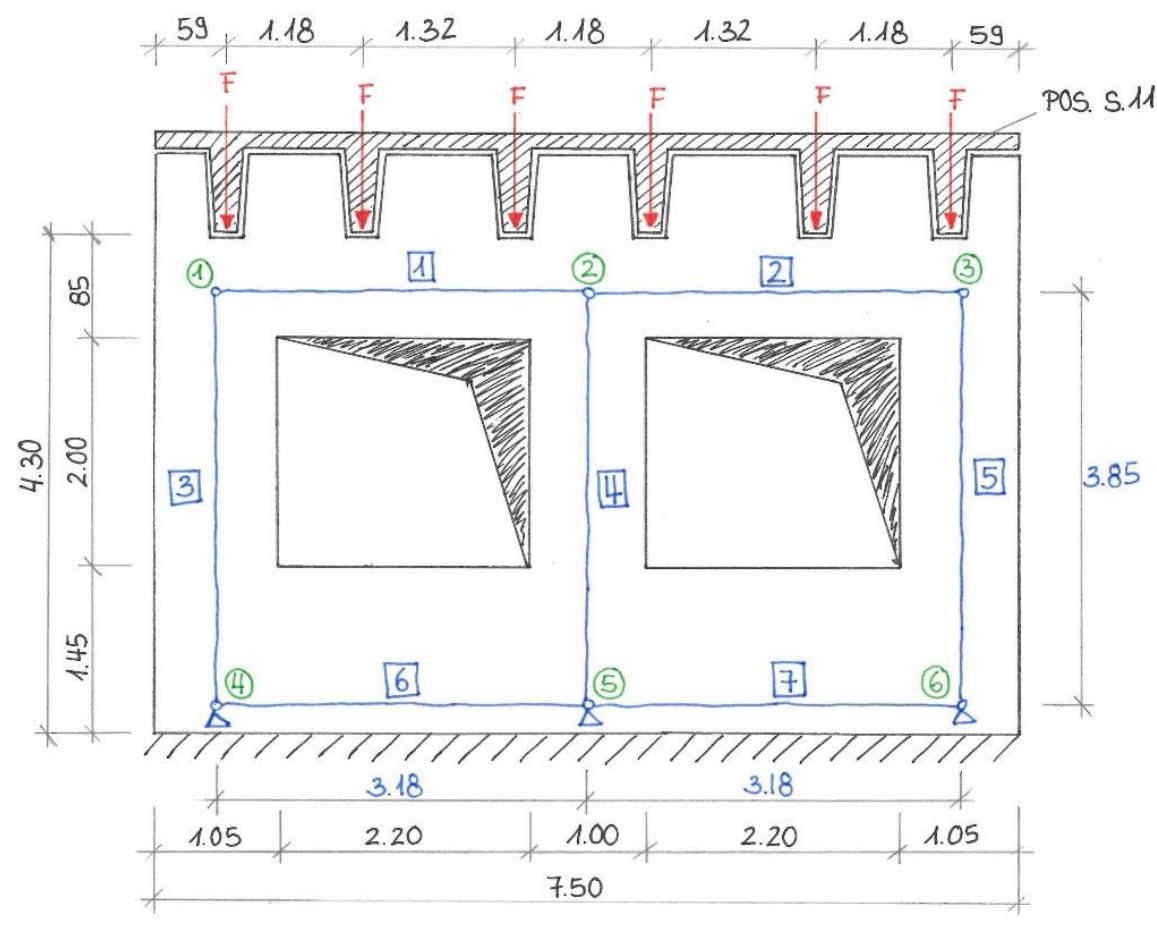

Cross-section

Building materials

Exposure class

Reinforcing steel

Characteristic loads $\mathrm{h}=20 \mathrm{~cm}$

\section{35/45 | B 500A}

XC1, WO

$\mathrm{C}_{\mathrm{nom}}=10 \mathrm{~mm}+10 \mathrm{~mm}=20 \mathrm{~mm}$

Chosen:

$\mathrm{C}_{\text {nom }}=25 \mathrm{~mm}$

\begin{tabular}{c|c}
$\begin{array}{c}\mathbf{G}_{\mathbf{k}} \\
{[\mathbf{k N}]}\end{array}$ & $\begin{array}{c}\mathbf{Q}_{\mathbf{k}} \\
{[\mathbf{k N}]}\end{array}$ \\
\hline- & - \\
88.0 & 31.0
\end{tabular}




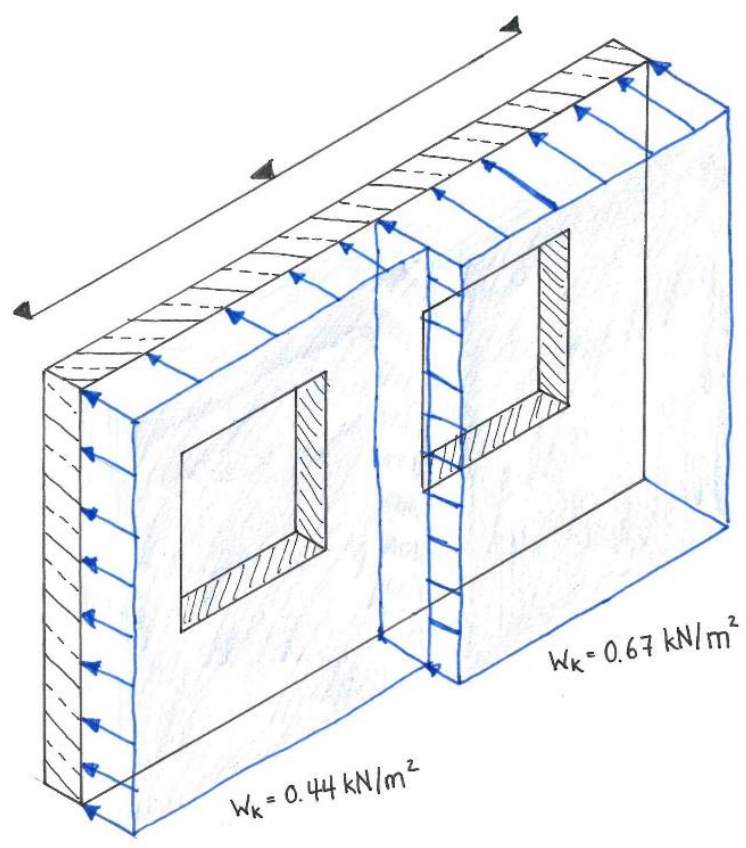

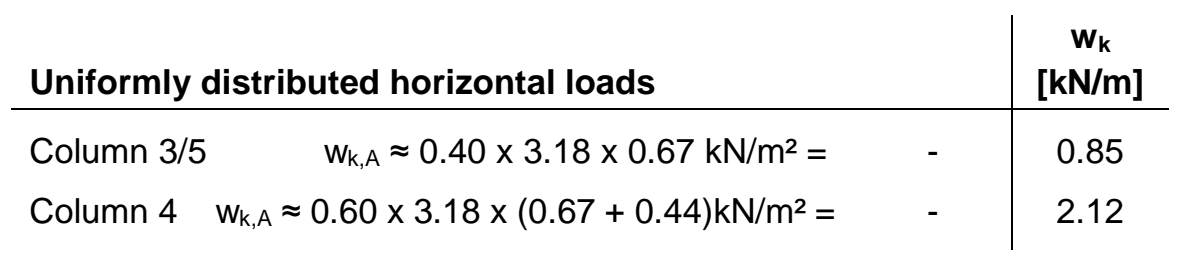

\subsection{INTERNAL FORCES}

\subsubsection{INTERNAL FORCES RESULTING FROM VERTICAL LOADS}

Flexure moment

\begin{tabular}{|l|c|c|c|}
\cline { 2 - 4 } \multicolumn{1}{c|}{} & $\mathbf{M}_{\mathrm{Gk}}[\mathbf{k N m}]$ & $\mathbf{M}_{\mathrm{Qk}}[\mathbf{k N m}]$ & $\mathbf{M}_{\mathrm{Ed}}[\mathbf{k N m}]$ \\
\hline Truss 1 and 2 & 64.2 & 21.4 & 118.7 \\
\hline Node 2 & -79.8 & -26.6 & -147.7 \\
\hline Truss 6 and 7 & 4.8 & 0.0 & 6.45 \\
\hline Node 5 & -7.4 & 0.0 & -10.0 \\
\hline
\end{tabular}

Shear force

\begin{tabular}{|l|c|c|c|}
\cline { 2 - 4 } \multicolumn{1}{c|}{} & $\mathbf{V}_{\mathrm{Gk}}[\mathrm{kN}]$ & $\mathbf{V}_{\mathrm{Qk}}[\mathbf{k N}]$ & $\mathbf{V}_{\mathrm{Ed}}[\mathrm{kN}]$ \\
\hline Node 1 and 3 & 50.9 & 16.0 & 92.8 \\
\hline Node 2 & -138.6 & -46.0 & -256.1 \\
\hline Node 4 and 6 & 9.7 & 0.0 & 13.1 \\
\hline Node 5 & -13.3 & 0.0 & -18.0 \\
\hline
\end{tabular}


Normal force

\begin{tabular}{|l|c|c|c|}
\cline { 2 - 4 } \multicolumn{1}{c|}{} & $\mathbf{N}_{\mathrm{Gk}}[\mathrm{kNm}]$ & $\mathbf{N}_{\mathrm{Qk}}[\mathbf{k N m}]$ & $\mathbf{N}_{\mathrm{Ed}}[\mathrm{kNm}]$ \\
\hline Truss 3 and 5 & -138.9 & -47.0 & -258.1 \\
\hline Truss 4 & -277.2 & -91.9 & -512.1 \\
\hline
\end{tabular}

\subsubsection{INTERNAL FORCES RESULTING FROM HORIZONTAL LOADS}

Flexure moment

\begin{tabular}{|l|c|c|}
\cline { 2 - 3 } \multicolumn{1}{c|}{} & $\mathbf{M}_{\mathbf{w k}}[\mathrm{kNm}]$ & $\mathbf{M}_{\mathrm{Ed}}[\mathbf{k N m}]$ \\
\hline Column A / C & 2.0 & 2.9 \\
\hline Column B & 4.9 & 7.4 \\
\hline
\end{tabular}

Shear force

\begin{tabular}{|l|c|c|}
\cline { 2 - 3 } \multicolumn{1}{c|}{} & $\mathbf{V}_{\mathbf{w k}}[\mathbf{k N}]$ & $\mathbf{V}_{\mathrm{Ed}}[\mathbf{k N}]$ \\
\hline Column A / C & 1.8 & 2.8 \\
\hline Column B & 4.6 & 6.8 \\
\hline
\end{tabular}

\subsection{ULTIMATE LIMIT STATE DESIGN}

\subsubsection{FLEXURE DESIGN OF TRUSS 1 AND 2}

Lower Reinforcement Truss 1 and 2

$\begin{array}{ll}\text { Cross-section } & b / h=20 / 85 \mathrm{~cm} \\ \text { Effective depth } & d=85-2.5-1-2.0 / 2=80.5 \mathrm{~cm} \\ \text { Lower reinforcement } & \mu_{E d s}=\frac{M_{E d s}}{b \cdot d^{2} \cdot f_{c d}}=\frac{11870 \mathrm{kNcm}}{20 \cdot 80.5^{2} \cdot 1.98 \mathrm{kN} / \mathrm{cm}^{2}}=0.046 \quad \rightarrow \omega=0.0476 \\ & A_{s l, \text { req }}=\omega \cdot b \cdot d \cdot f_{c d} \cdot \frac{1}{\sigma_{s d}}=0.0476 \cdot 20 \cdot 80.5 \cdot 1.98 \cdot \frac{1}{43.5}=3.5 \mathrm{~cm}^{2}\end{array}$

Chosen

$2 \varnothing 16=4.02 \mathrm{~cm}^{2}$

lower reinforcement

Upper Reinforcement Node 2

Cross-section
Effective depth
Lower reinforcement

Chosen $b / h=20 / 85 \mathrm{~cm}$

$d=85-2.5-1-2.0 / 2=80.5 \mathrm{~cm}$

$\mu_{E d s}=\frac{M_{E d s}}{b \cdot d^{2} \cdot f_{c d}}=\frac{14770 \mathrm{kNcm}}{20 \cdot 80.5^{2} \cdot 1.98 \mathrm{kN} / \mathrm{cm}^{2}}=0.058 \quad \rightarrow \omega=0.060$

$A_{s l, \text { req }}=\omega \cdot b \cdot d \cdot f_{c d} \cdot \frac{1}{\sigma_{s d}}=0.060 \cdot 20 \cdot 80.5 \cdot 1.98 \cdot \frac{1}{43.5}=4.40 \mathrm{~cm}^{2}$

$2 \varnothing 20=6.28 \mathrm{~cm}^{2}$ upper reinforcement 


\subsubsection{DESIGN OF COLUMN A/C AND B}

The design of column B is decisive for the structure due to its smaller cross-section and higher loads.

\section{Column B}

Cross-section

$$
b / h=100 / 20 \mathrm{~cm}
$$

Slenderness of the column

$$
\beta=1.0 \quad I_{0}=1.0 \cdot 4.30=4.30 m
$$

$$
i=0.289 \cdot h=0.289 \cdot 20=5.78 \mathrm{~cm} \quad \lambda=\frac{I_{0}}{i}=\frac{430}{5.78}=74.4
$$

Limit slenderness

$$
\begin{aligned}
& \left|n_{E d}\right|=\frac{N_{E d}}{A_{c} \cdot f_{c d}}=\frac{512.1}{100 \cdot 20 \cdot 1.98}=0.13<0.41 \\
& \lambda_{\lim }=\frac{16}{\sqrt{\left|n_{E d}\right|}}=\frac{16}{\sqrt{0.13}}=44.4 \\
& \lambda=74.4>\lambda_{\text {lim }}=44.4 \quad \rightarrow \text { second order effects must be considered! }
\end{aligned}
$$

Eccentricity due to imperfections

$$
\begin{aligned}
& \alpha_{h}=\frac{2}{\sqrt{I}}=\frac{2}{\sqrt{4.30}}=0.96 \quad \theta_{i}=\frac{1}{200} \cdot \alpha_{h}=\frac{1}{200} \cdot 0.96=4.82 \cdot 10^{-3} \\
& e_{i}=\theta_{i} \cdot \frac{I_{0}}{2}=4.82 \cdot 10^{-3} \cdot \frac{4.30}{2}=0.01 \mathrm{~m}
\end{aligned}
$$

Eccentricity due to second order effects

$$
e_{2}=K_{1} \cdot \frac{l_{0}^{2}}{10} \cdot \frac{1}{r}=1.0 \cdot \frac{4.30^{2} \cdot 0.0022}{10 \cdot 0.45 \cdot 0.155}=0.06 \mathrm{~m}
$$

Total eccentricity

Load combinations

Flexure moment

Design $e_{t o t}=e_{i}+e_{2}=0.01+0.06=0.07 m$

LC1: $\quad 1.35 \cdot G_{k} \oplus 1.50 \cdot Q_{k} \oplus 1.50 \cdot \psi_{0} \cdot w_{k}$ $\psi_{0}=0.6$

LC2: $\quad 1.0 \cdot G_{K} \oplus 1.5 \cdot w_{k}$

$M_{E d s}=M_{E d}+N_{E d} \cdot e_{t o t}$

LC1: $\quad M_{E d s}=0.6 \cdot 7.4+0.07 \cdot 512.1=40.3 \mathrm{kNm}$

LC2: $\quad M_{E d s}=7.4+0.07 \cdot 1.35 \cdot 277.2=33.6 \mathrm{kNm}$

The flexure Moment is very small and can be neglected for the design of the column.

$N_{E d}=512.1 \mathrm{kN}<\max N_{E d}=A_{c} \cdot f_{c d}=100 \cdot 20 \cdot 1.98=3960 \mathrm{kN} \checkmark$ 


\subsection{DESIGN AND REINFORCEMENT}

\subsubsection{MINIMUM REINFORCEMANT FOR CONCRETE WALLS}

Normal force

$N_{E d}=512.1 \mathrm{kN}<0.3 \cdot f_{c d} \cdot A_{c}=0.3 \cdot 1.98 \cdot 100 \cdot 20=1188 \mathrm{kN}$

Minimum reinforcement

$$
\begin{aligned}
a_{s, v, \text { min }}= & 0.15 \cdot \frac{\left|N_{E d}\right|}{f_{y d}}=0.15 \cdot \frac{512.1}{43.5}=1.77 \mathrm{~cm}^{2} / \mathrm{m} \text { vertical reinforcement } \\
& \geq 0.003 \cdot A_{c}=0.003 \cdot 20=0.06 \mathrm{~cm}^{2} / \mathrm{m} \\
a_{s, h, \text { min }} & \geq 0.5 \cdot a_{s, v}=0.5 \cdot 1.77=0.885 \mathrm{~cm}^{2} / \mathrm{m} \quad \text { horizontal reinforcement }
\end{aligned}
$$

\section{POS. W.14 A - STRUT-AND-TIE MODEL OF THE CONCRETE WALL}

In Chapter 14 the design calculations for the concrete wall panel are made based on a very simple static system. For comparison a more detailed analysis based on a strut and tie model is made in the following chapter. Finite element analysis is used to generate the stress trajectories of the wall and in accordance with these a strut and tie model is developed which can be used to design the reinforcement of the wall.

\subsection{SYSTEM}

Further see Chapter 14.1.

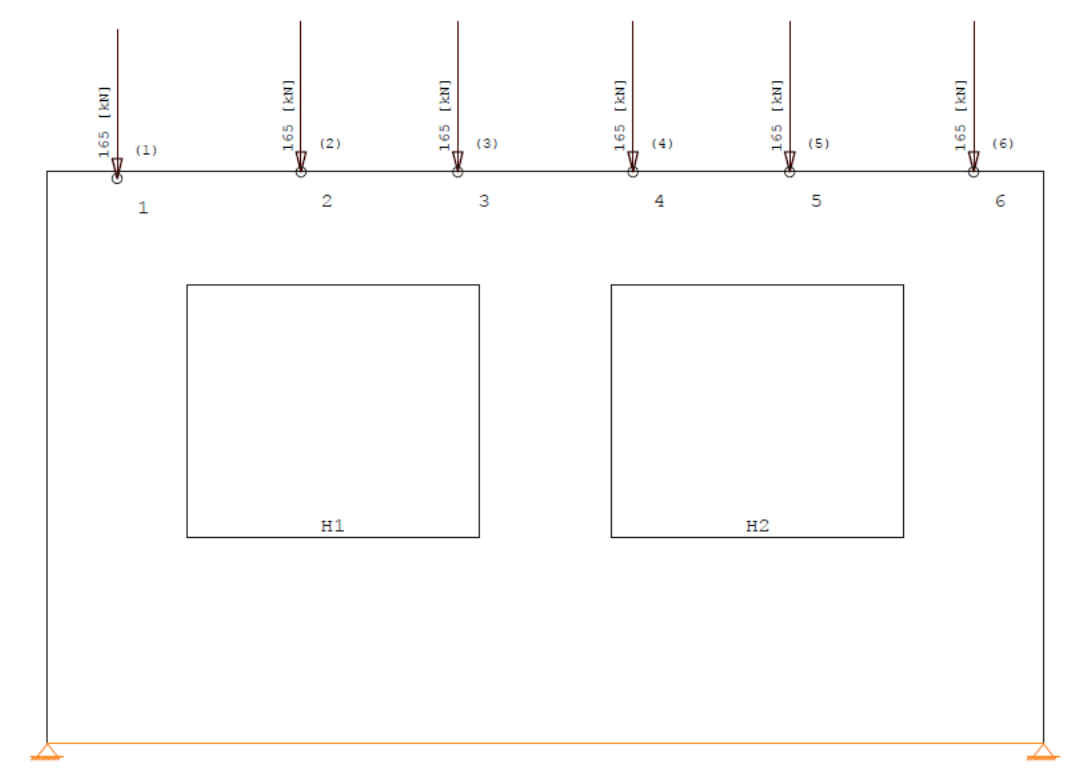

Figure 18 - Static System of the Concrete Wall 


\subsection{STRESS TRAJECTORIES}

The stress trajectories are computed with the program Nemetschek FRILO. To find the best matching stress trajectories and therefore strut and tie model, three different systems are analyzed and at the end one is chosen to be used for the design calculations. The three system that are analyzed are a single-span deep beam, a double-span deep beam and a wall with a line support.

Due to their low impact, the wind loads are neglected in this analysis and only the concentrated loads from the double-tee slab are regarded.

\subsubsection{SINGLE-SPAN DEEP BEAM}

Figure 19 shows the stress trajectories for the single-span deep beam.

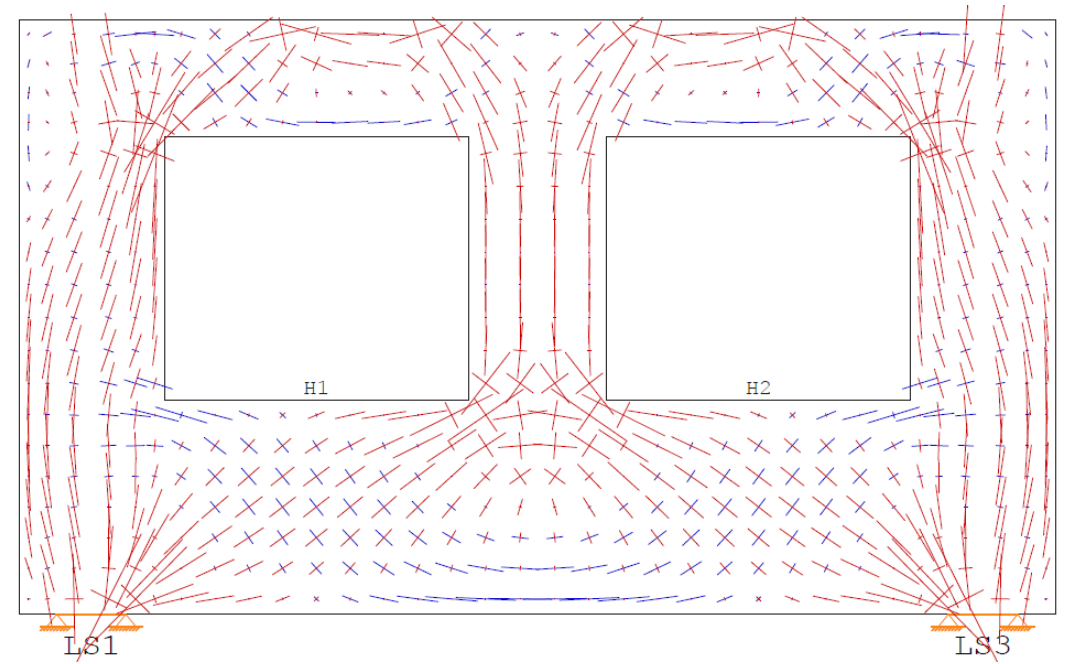

Figure 19 - Stress Trajectories for a Single-Span Deep Beam

The stresses above the window holes look similar to the stress trajectories of a double span beam. The 'columns' between the window holes act as supports for the beam and tension ties form above them. Another thing that must be noted is the development of a big tension tie at the lower edge of the wall between the two supports. The bottle-shape of the two compression struts coming from the intermediate column leading to the two supports can be seen clearly. 


\subsubsection{DOUBLE-SPAN DEEP BEAM}

In Figure 20 an intermediate support is added, making it a double-span deep beam.

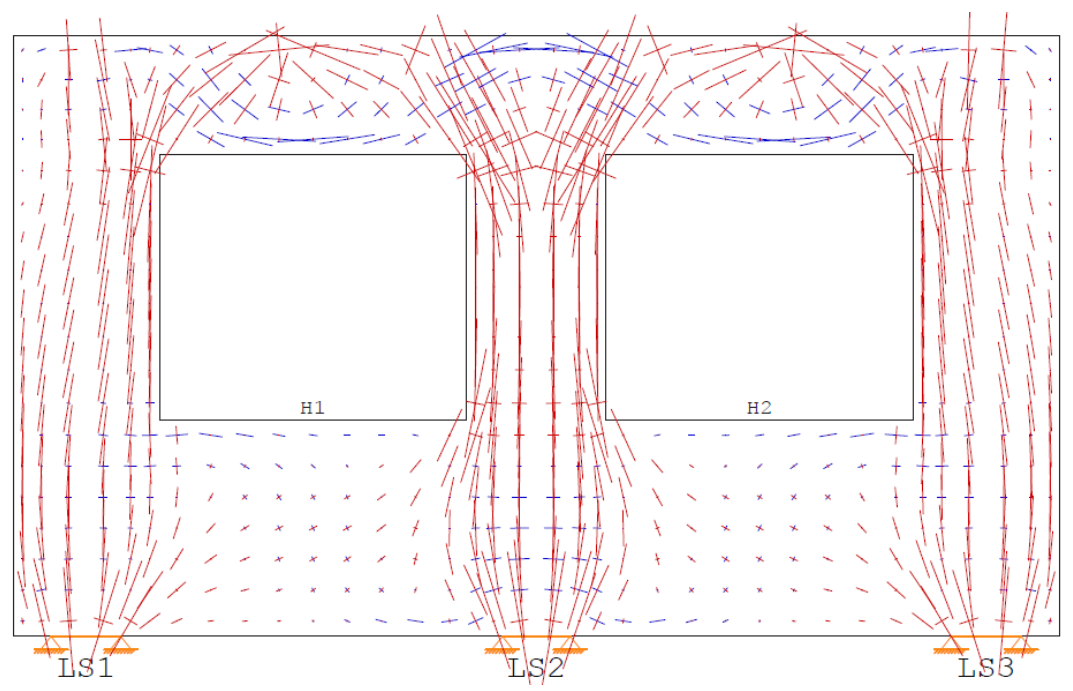

Figure 20 - Stress Trajectories for a Double-Span Deep Beam

The stress trajectories above the windows are very similar to the ones of the single-span deep beam. The part above the windows acts like a regular double-span beam. The compression struts go directly to the support and there is almost no tension at the lower edge of the wall. Most of the tension results from the transverse tension of the compression struts due to their bottle-shape.

\subsubsection{CONTINUOUS SUPPORT}

Finally, the third system can be seen in Figure 21 - a deep beam with a continuous support.

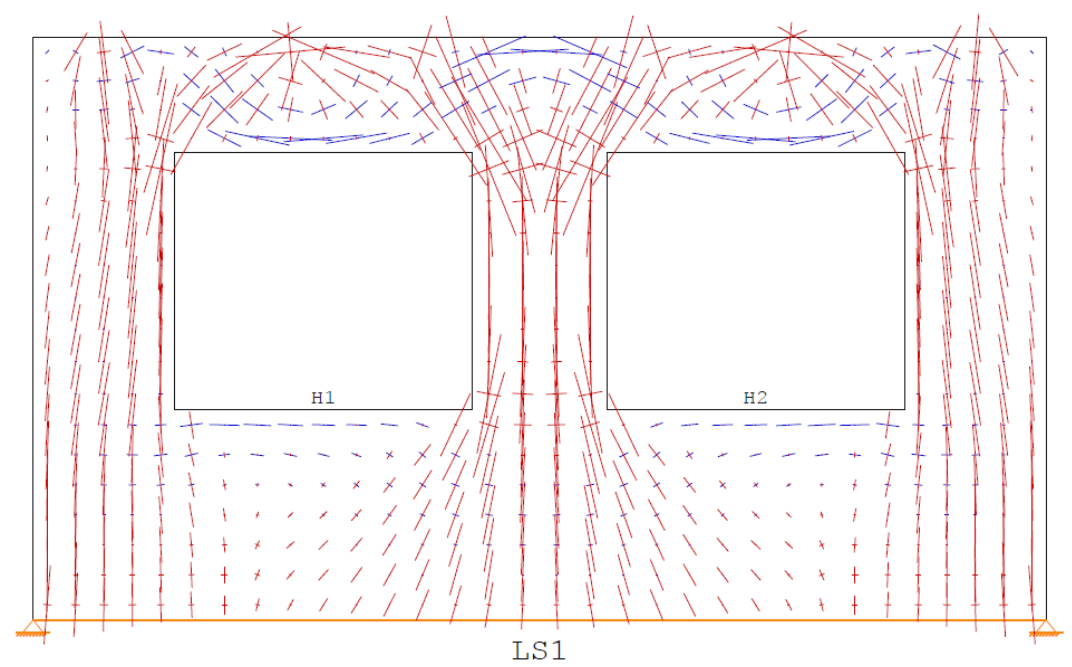

Figure 21 - Stress Trajectories for a Deep Beam with a Continuous Support

Again, the stresses above the windows resemble the previous two systems. The outer compression struts go to the support in a straight line and so is the compression strut in the middle for the most part. The 
compression stresses fan out closer to the lower edge of the wall. Tension ties develop horizontally under the window holes.

\subsubsection{CONCLUSION}

The stresses above the window resemble each other in all three systems and act like a double-span beam so for that part the path of the loads is very clear. System two and three clearly show that the compression struts will then follow along the 'columns' between the holes in the wall and directly lead to the support. A development seen in system one is unlikely to occur since the loads will follow the shortest path to the supports. Therefore, system two (Figure 20) seems to depict the load paths and stress trajectories of the wall the best and will be used to develop a strut and tie model and to design the reinforcement of the wall.

\subsection{THE STRUT-AND-TIE MODEL}

\subsubsection{REACTION FORCES AT THE SUPPORT}

The reaction forces can be assumed to be the mean value between the reaction forces of a double-span beam and two single-span beams.

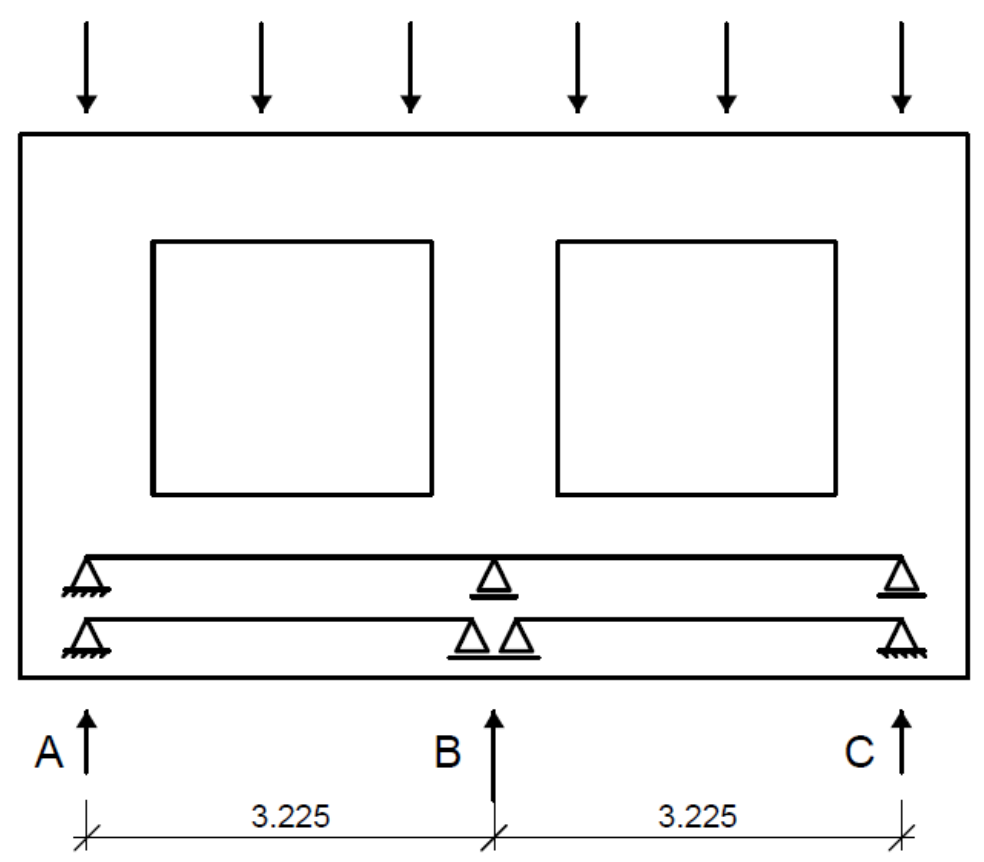

Figure 22 - Static System to Calculate the Reaction Forces of the Concrete Wall 
Reaction forces for a double-span beam

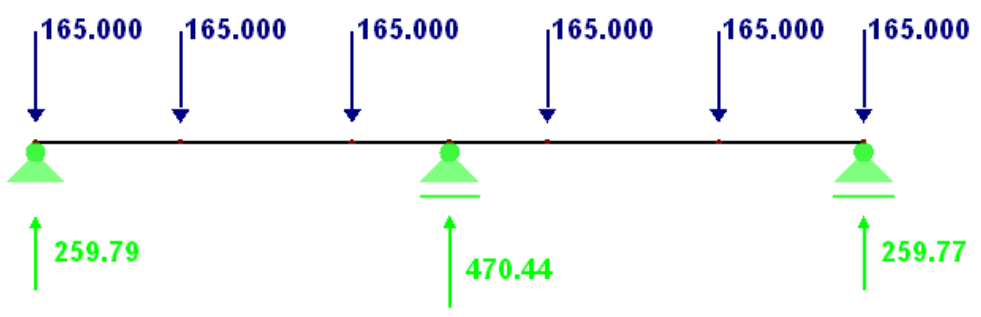

Reaction forces for a single-span beam

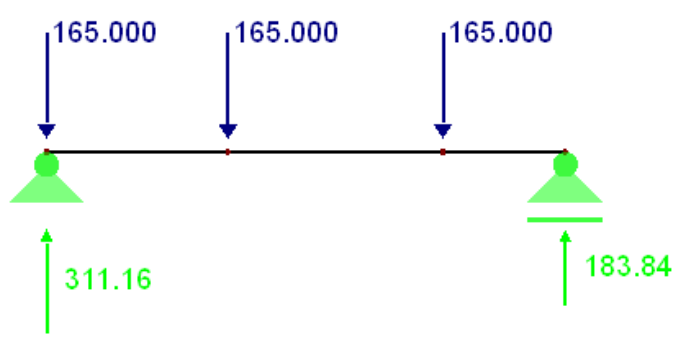

Reaction forces of the wall

\begin{tabular}{|l|c|c|c|}
\cline { 2 - 4 } \multicolumn{1}{c|}{} & A & B & C \\
\hline Double-Span & $259.8 \mathrm{kN}$ & $470.4 \mathrm{kN}$ & $259.8 \mathrm{kN}$ \\
\hline $\mathbf{2}$ x Single-Span & $311.2 \mathrm{kN}$ & $367.7 \mathrm{kN}$ & $311.2 \mathrm{kN}$ \\
\hline Average & $\mathbf{2 8 5 . 5} \mathbf{~ k N}$ & $\mathbf{4 1 9 . 1} \mathbf{~ k N}$ & $\mathbf{2 8 5 . 5} \mathbf{~ k N}$ \\
\hline
\end{tabular}

\subsubsection{STRUT-AND-TIE MODEL}

The final strut and tie model can be seen in Figure 23. Dashed lines illustrate the compression struts and solid lines illustrate the tension ties.

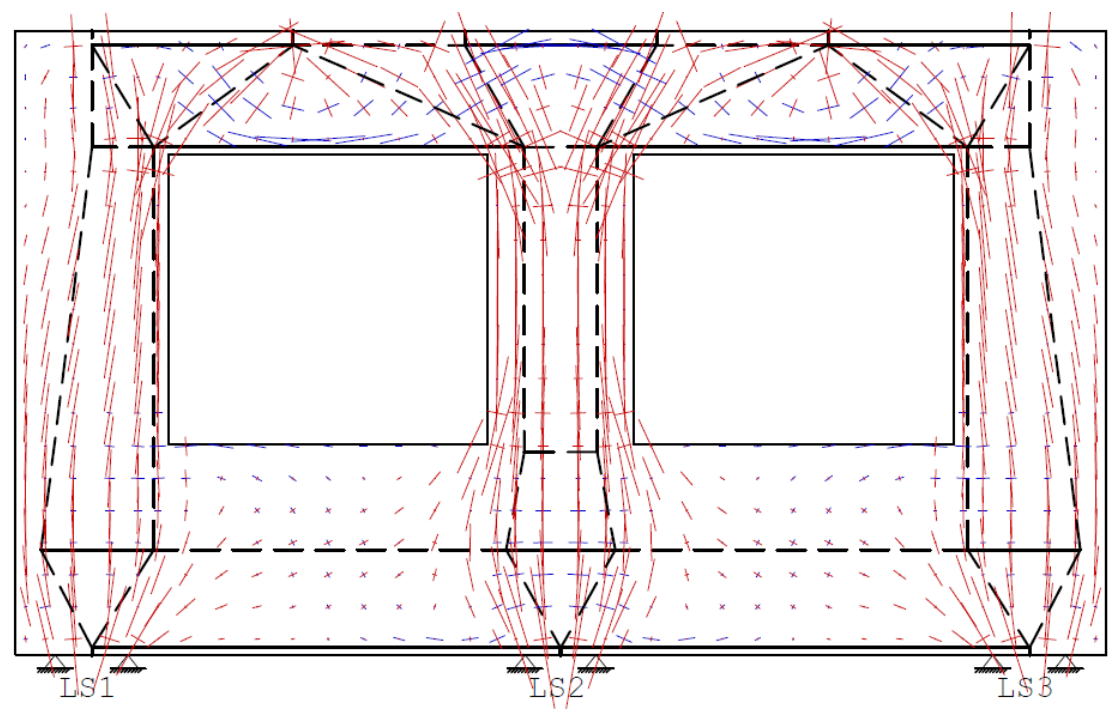




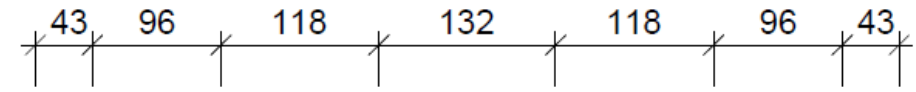

\section{(1)}

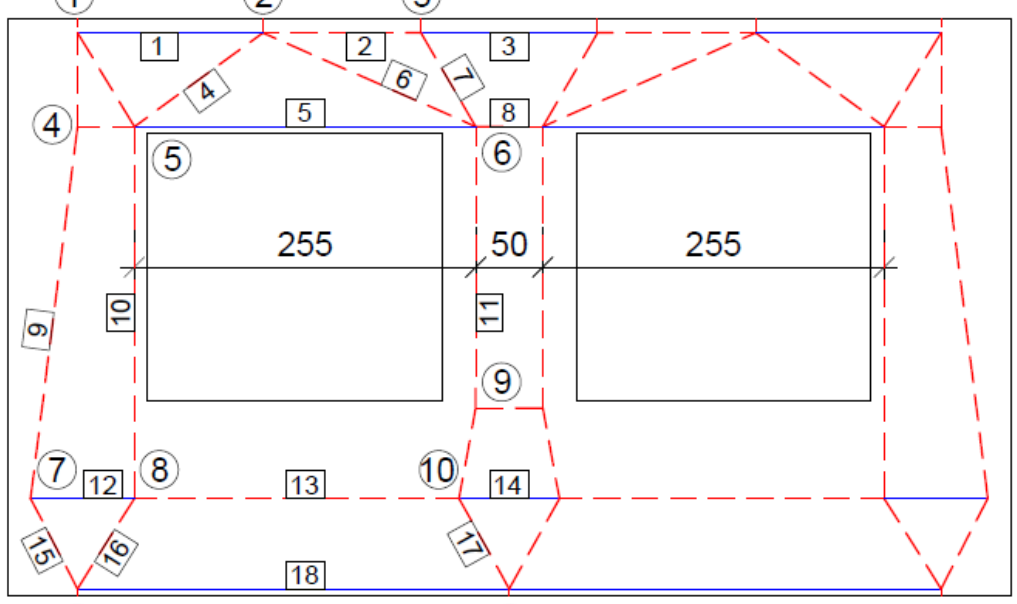

(11) $35|43|$ $243 \quad 38$

Figure 23 - Final Strut-and-Tie Model

\subsubsection{RESULTING FORCES}

The resulting forces in the struts and ties are calculated with the program Dlubal R-Stab and are summarized in Table 22. Compression struts are shown in red and with a negative sign and the tension ties are shown in blue and with a positive sign.

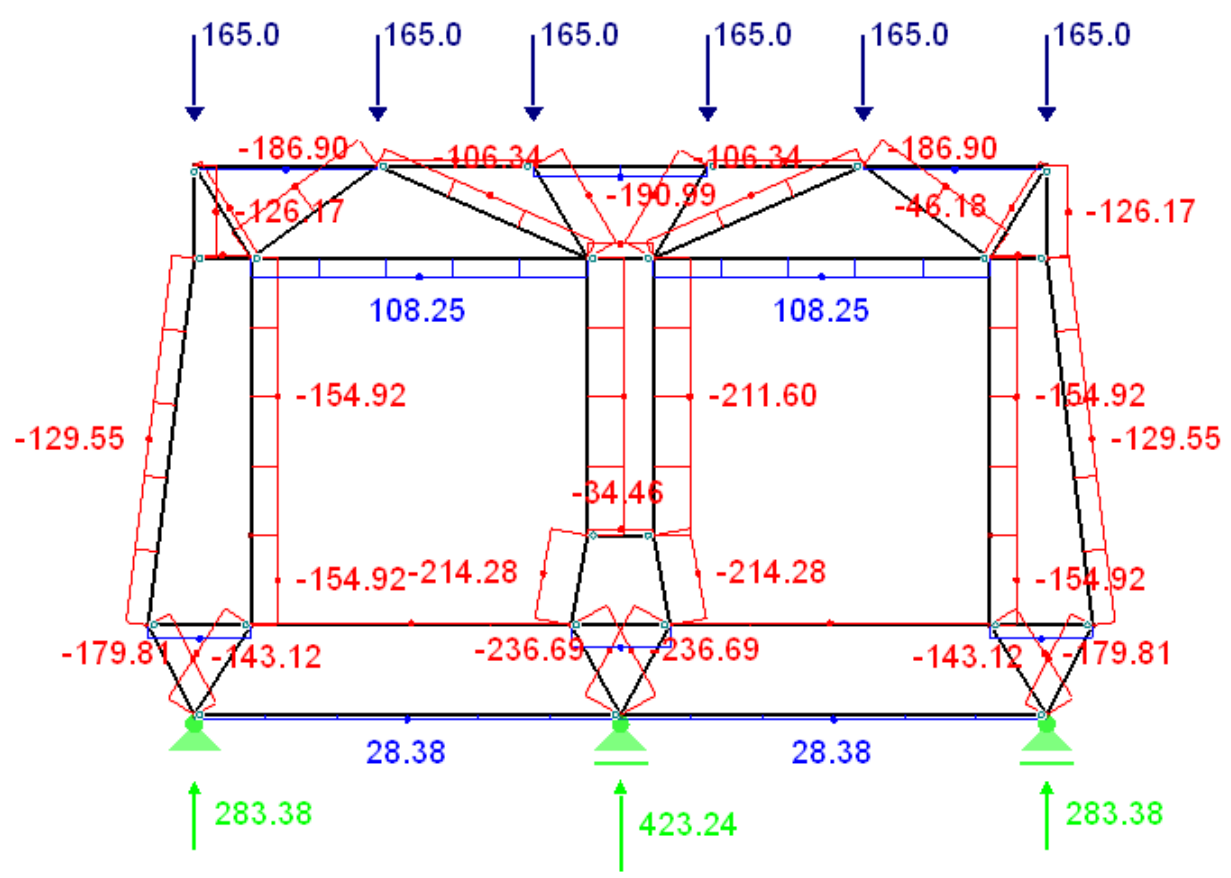

Figure 24 - Result of the Dlubal R-Stab Calculation 
Table 22 - Resulting Forces of the Strut-and-Tie Model

\begin{tabular}{|c|c|c|c|c|c|c|c|c|c|}
\hline Truss & 1 & 2 & 3 & 4 & 5 & 6 & 7 & 8 & 9 \\
\hline Force [kN] & 21.2 & -31.0 & 65.2 & -186.9 & 108.3 & -106.3 & -191.0 & -83.0 & -129.6 \\
\hline & 10 & 11 & 12 & 13 & 14 & 15 & 16 & 17 & 18 \\
\hline & -154.9 & -211.6 & 79.7 & -10.2 & 130.3 & -143.1 & -179.8 & -236.7 & 28.4 \\
\hline
\end{tabular}

\subsection{DESIGN AND REINFORCEMENT}

\subsubsection{TENSION TIES}

Truss 1

Truss 3

Truss 5

Truss 12

Truss 14

Truss 18

$$
A_{s, r e q}=\frac{F_{E d}}{f_{y d}}=\frac{21.2}{43.5}=0.49 \mathrm{~cm}^{2} \quad \rightarrow 2 \varnothing 8=1.01 \mathrm{~cm}^{2}
$$$$
A_{s, \text { req }}=\frac{F_{E d}}{f_{y d}}=\frac{65.2}{43.5}=1.50 \mathrm{~cm}^{2} \quad \rightarrow 2 \varnothing 10=1.57 \mathrm{~cm}^{2}
$$

$$
A_{s, r e q}=\frac{F_{E d}}{f_{y d}}=\frac{108.3}{43.5}=2.49 \mathrm{~cm}^{2} \quad \rightarrow 2 \times 2 \varnothing 10=3.14 \mathrm{~cm}^{2}
$$

$$
A_{s, \text { req }}=\frac{F_{E d}}{f_{y d}}=\frac{79.7}{43.5}=1.83 \mathrm{~cm}^{2} \quad \rightarrow 2 \times 2 \varnothing 10=3.14 \mathrm{~cm}^{2}
$$

$$
A_{s, \text { req }}=\frac{F_{E d}}{f_{y d}}=\frac{130.3}{43.5}=3.00 \mathrm{~cm}^{2} \quad \rightarrow 2 \times 2 \varnothing 10=3.14 \mathrm{~cm}^{2}
$$

$$
A_{s, \text { req }}=\frac{F_{E d}}{f_{y d}}=\frac{28.4}{43.5}=0.65 \mathrm{~cm}^{2} \quad \rightarrow 2 \varnothing 8=1.01 \mathrm{~cm}^{2}
$$

15.4.2 MINIMUM REINFORCEMENT

$$
a_{s, \min }=\max \left\{\begin{array}{l}
0.75 \cdot 10^{-3} \cdot a_{c}=0.75 \cdot 10^{-3} \cdot 20 \cdot 100=1.5 \mathrm{~cm}^{2} / \mathrm{m} \\
1.5 \mathrm{~cm}^{2} / \mathrm{m}
\end{array}\right.
$$


15.4.3 REINFORCEMENT DRAWING

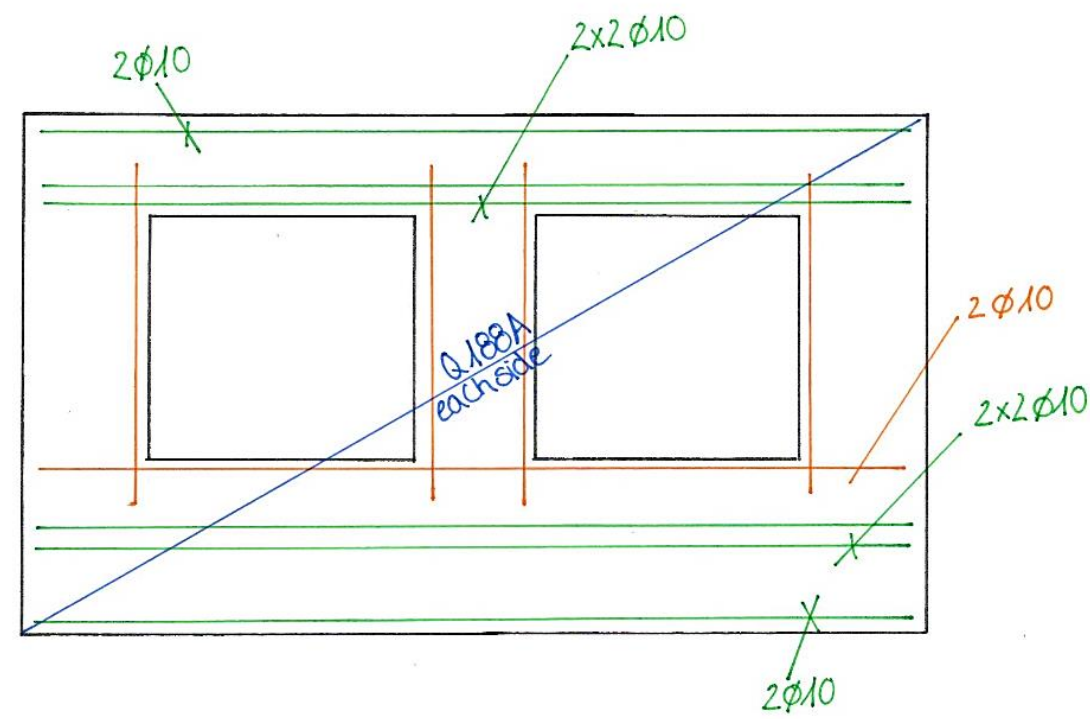




\subsection{SOFTWARE CALCULATIONS}

\section{Demo Frilo Nemetschek}

\section{Position: W.14a - Load Bearing Concrete Wall}

Panels by Finite Elements SCN 01/2018 (Frilo R-2018-1/P12)

\section{System}

Scale $1: 75$

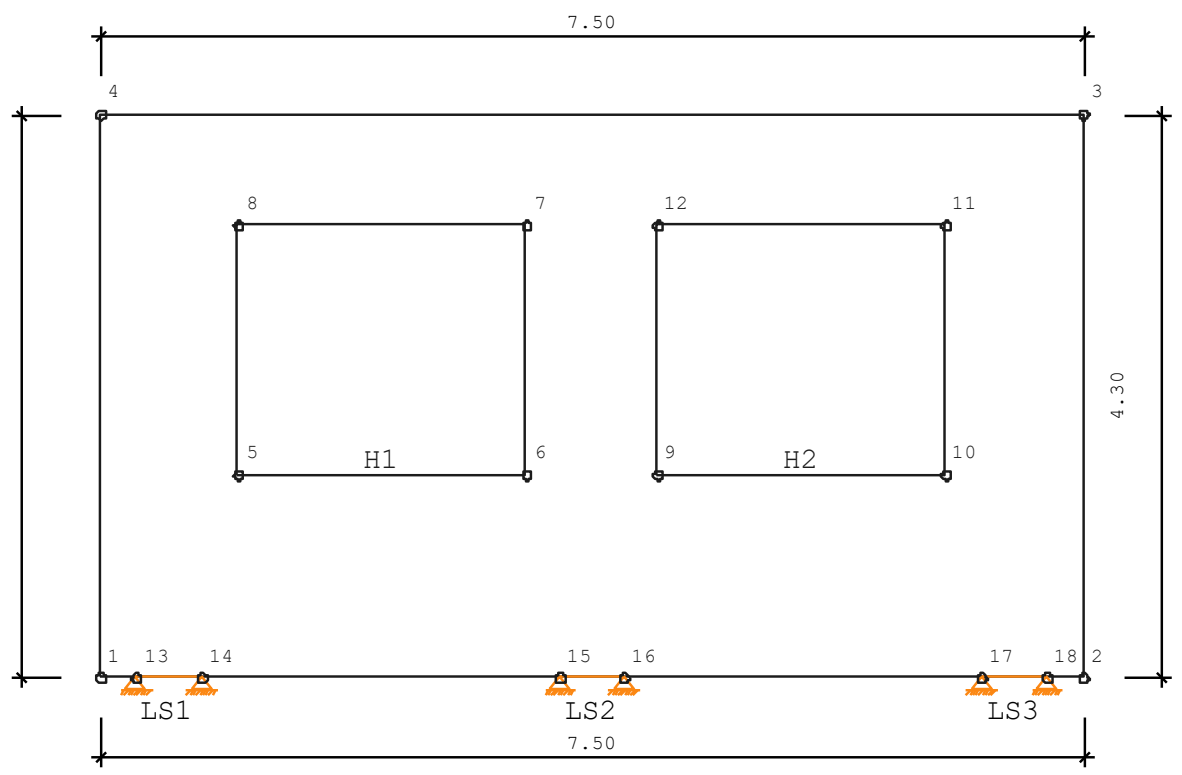

Load case 1 "PoS. S.11 - Dead Load"

Point loads

Scale 1 : 75

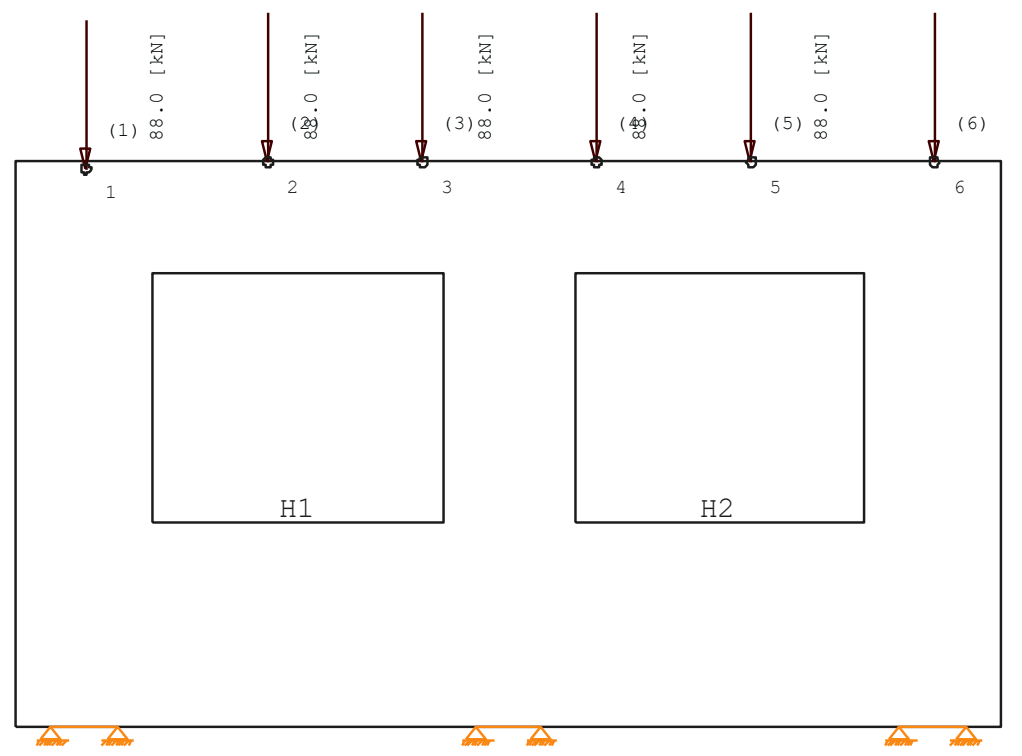




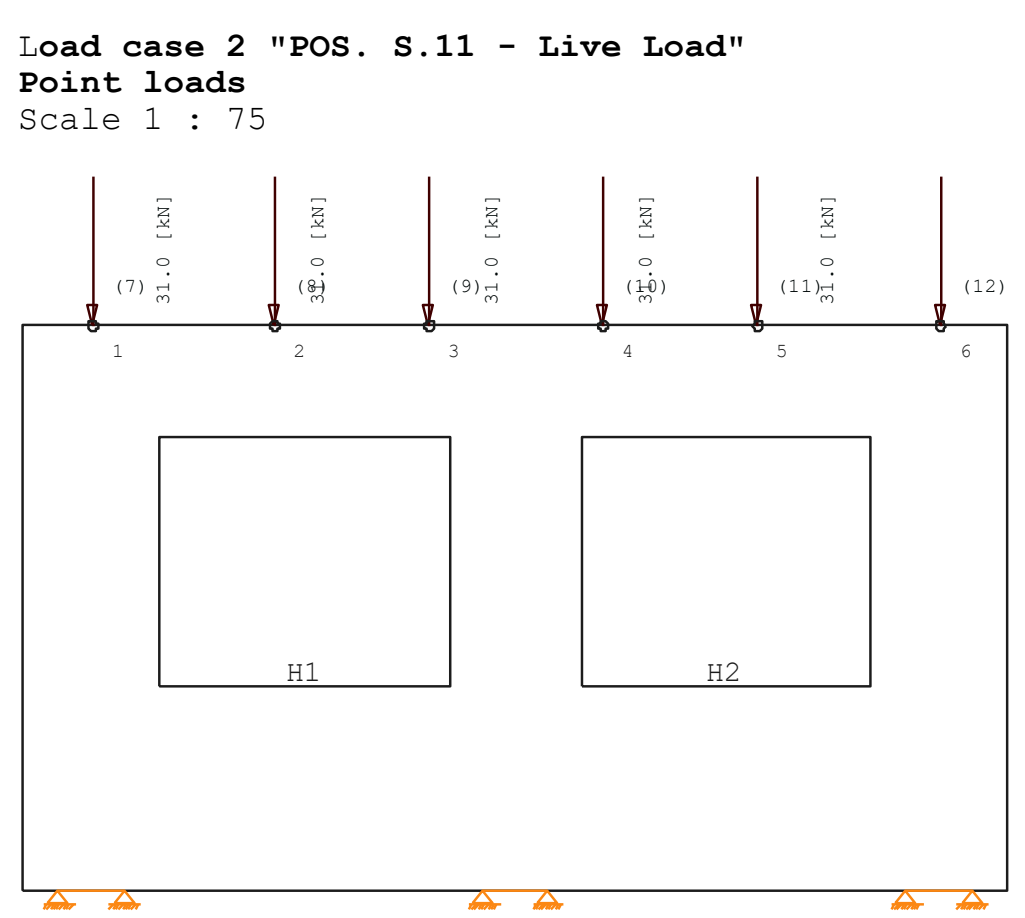

SUPERPOSITION 1 "Characteristic"

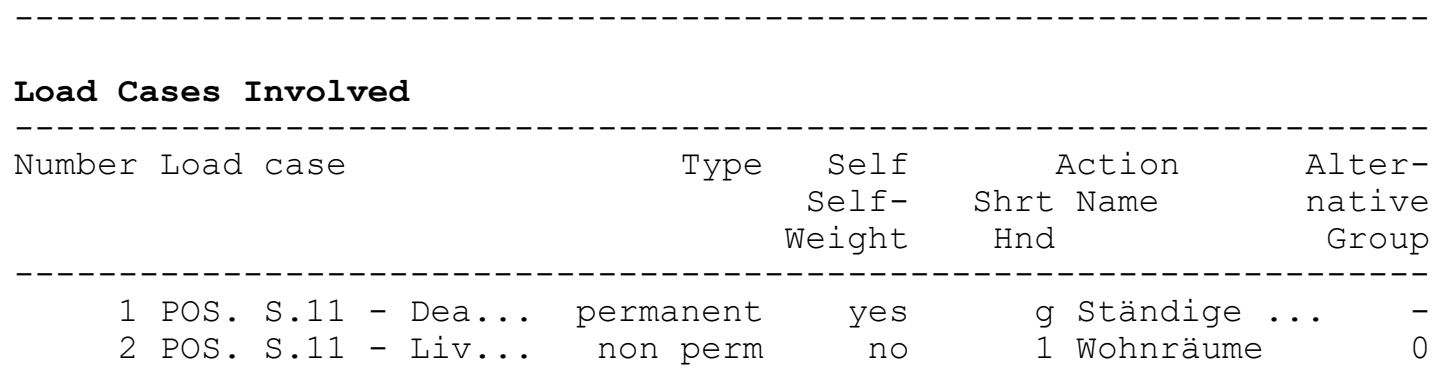

\section{Action}

Number Shrt Name Type Hnd

$\begin{array}{rlll}1 & 1 & \text { Wohnräume } & \text { non perm } \\ 2 & 9 & \text { Ständige Lasten } & \text { permanent }\end{array}$

SUPERPOSITION 2 "ULS Permanent/Transient"

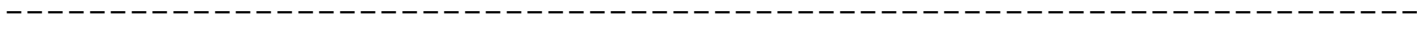

Load Cases Involved

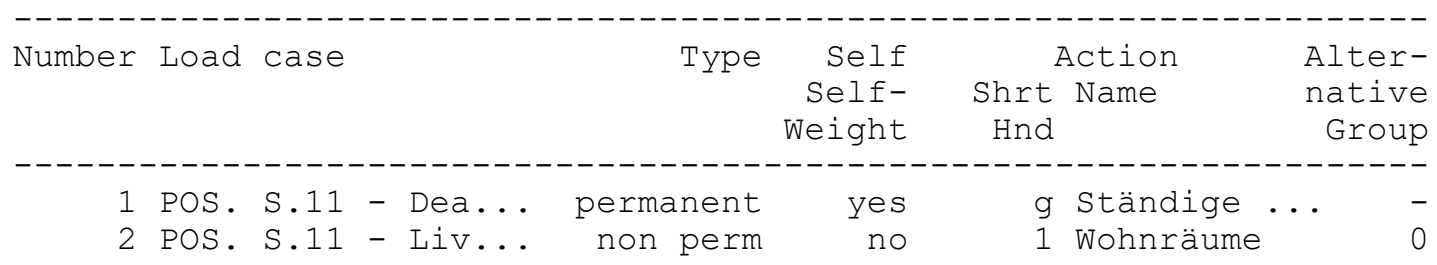




\begin{tabular}{|c|c|c|c|c|c|}
\hline Action & & & & & \\
\hline Number & $\begin{array}{l}\text { Shrt Name } \\
\text { Hnd }\end{array}$ & Type & $\begin{array}{r}\text { Partial } \mathrm{S} \\
\text { sup }\end{array}$ & $\begin{array}{l}\text { afety } \\
\text { inf }\end{array}$ & $\begin{array}{l}\text { Combination } \\
\text { dom ndo }\end{array}$ \\
\hline 1 & 1 Wohnräume & non perm & 1.50 & 0.00 & 1.00 \\
\hline 2 & g Ständige Lasten & permanent & 1.35 & 1.00 & 1.001 .00 \\
\hline
\end{tabular}

\section{NOTE: Design Values}

All results of a superposition of load cases

Include both partial safety and combination

factors: DIN EN 1990/NA:2010-12

\section{NOTE : Combination Factors}

With the combination of independent, variable actions

the individual dominant action is determined for both each

location and for each action quantity.

In geneneral, the dominant actions differ with each

location and each quantity

The individually found dominant action receives the

conbination factor 1.00. In case of only one variable action

this action is considered dominant.

Superposition 2 "ULS Permanent/Transient"

Concrete Load (Compressive) uC-1, uC-2 [\%]

Scale 1 : 50

\begin{tabular}{|c|c|c|c|c|c|c|c|c|c|c|c|c|c|c|}
\hline 8 & 8 & 9 & 21 & 21 & 10 & 20 & 3 & 20 & 10 & 21 & 21 & 9 & 10 & 11 \\
\hline 13 & 21 & 9 & 14 & 14 & 10 & 20 & 3 & 20 & 10 & 14 & 14 & 9 & 33 & 26 \\
\hline 3 & 4 & 15 & 11 & 10 & 9 & 21 & 8 & 21 & 9 & 10 & 11 & 15 & 4 & \\
\hline 8 & 19 & 24 & 16 & 10 & 9 & 29 & 12 & 29 & 9 & 10 & 16 & 24 & 15 & 9 \\
\hline 2 & 16 & $\overline{I I}$ & & & & 24 & 9 & $2 \longdiv { 4 }$ & & & & $\overline{I 2}$ & 16 & 2 \\
\hline 6 & 24 & 25 & & & & 28 & 16 & 28 & & & & 25 & 24 & 6 \\
\hline 2 & 2 & & & & & & 1 & 7 & & & & & 2 & 2 \\
\hline 10 & 19 & 19 & & & & 17 & 17 & 17 & & & & 19 & 19 & 10 \\
\hline 11 & 16 & 16 & & & & 17 & 17 & 17 & & & & 16 & 16 & 11 \\
\hline 12 & $\begin{array}{c}1 \\
12\end{array}$ & 13 & & & $\begin{array}{l}1 \\
1 \\
\end{array}$ & \begin{tabular}{l|l}
1 & 0 \\
1 & 9 \\
\end{tabular} & $\begin{array}{c}3 \\
15\end{array}$ & \begin{tabular}{l|l}
1 & 0 \\
2 & 0
\end{tabular} & $\begin{array}{l}1 \\
1 \\
\end{array}$ & & & 13 & $\begin{array}{c}1 \\
12\end{array}$ & 12 \\
\hline 12 & 12 & $\begin{array}{l}1 \\
8\end{array}$ & $\begin{array}{l}2 \\
2\end{array}$ & $\begin{array}{l}1 \\
1\end{array}$ & $\begin{array}{l}2 \\
3\end{array}$ & $\begin{array}{c}9 \\
18\end{array}$ & $\begin{array}{c}2 \\
14\end{array}$ & $\begin{array}{c}9 \\
18\end{array}$ & $\begin{array}{l}2 \\
3\end{array}$ & $\begin{array}{l}1 \\
1\end{array}$ & $\begin{array}{l}2 \\
2\end{array}$ & $\begin{array}{l}1 \\
8\end{array}$ & 11 & 12 \\
\hline 14 & 13 & $\begin{array}{l}3 \\
6\end{array}$ & $\begin{array}{l}3 \\
2\end{array}$ & $\begin{array}{l}2 \\
2\end{array}$ & $\begin{array}{l}2 \\
3\end{array}$ & $\begin{array}{c}2 \\
14\end{array}$ & 17 & $\begin{array}{c}2 \\
14\end{array}$ & $\begin{array}{l}2 \\
3\end{array}$ & $\begin{array}{l}2 \\
2\end{array}$ & $\begin{array}{l}3 \\
2\end{array}$ & $\begin{array}{l}3 \\
6\end{array}$ & 13 & 14 \\
\hline $\begin{array}{c}7 \\
23\end{array}$ & $\begin{array}{l}14 \\
24\end{array}$ & $\begin{array}{l}5 \\
5\end{array}$ & 2 & $\begin{array}{l}2 \\
2\end{array}$ & $\begin{array}{l}2 \\
2\end{array}$ & $\begin{array}{c}9 \\
20\end{array}$ & $\begin{array}{l}13 \\
31\end{array}$ & $\begin{array}{c}9 \\
20\end{array}$ & $\begin{array}{l}2 \\
2\end{array}$ & $\begin{array}{l}2 \\
2\end{array}$ & 2 & $\begin{array}{l}5 \\
6\end{array}$ & $\begin{array}{l}14 \\
24\end{array}$ & $\begin{array}{c}7 \\
23\end{array}$ \\
\hline
\end{tabular}

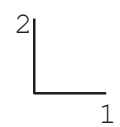




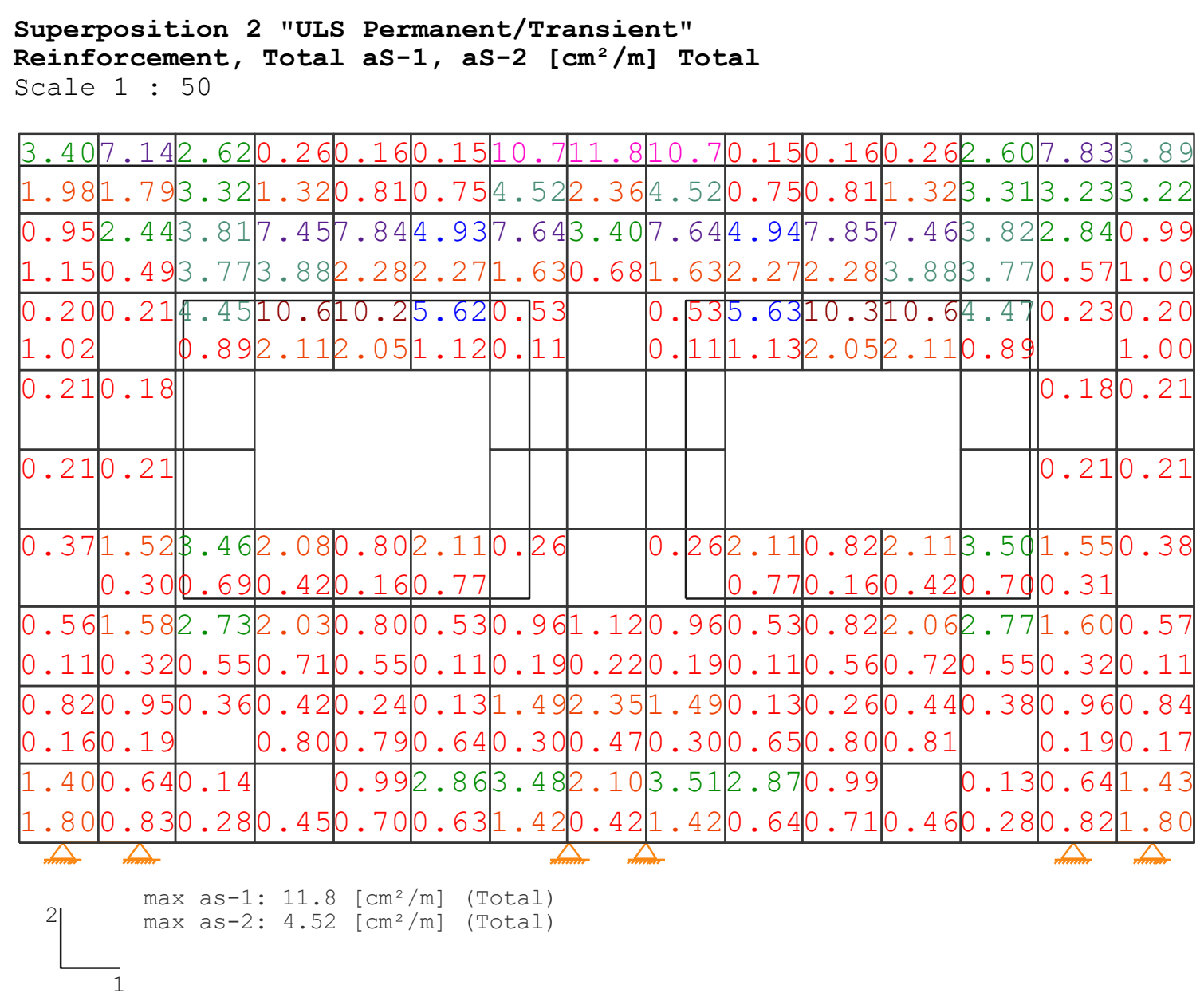




\section{POS. W.15 - TRAPEZOIDAL SHEET CLADDING}

\subsection{SYSTEM}

System

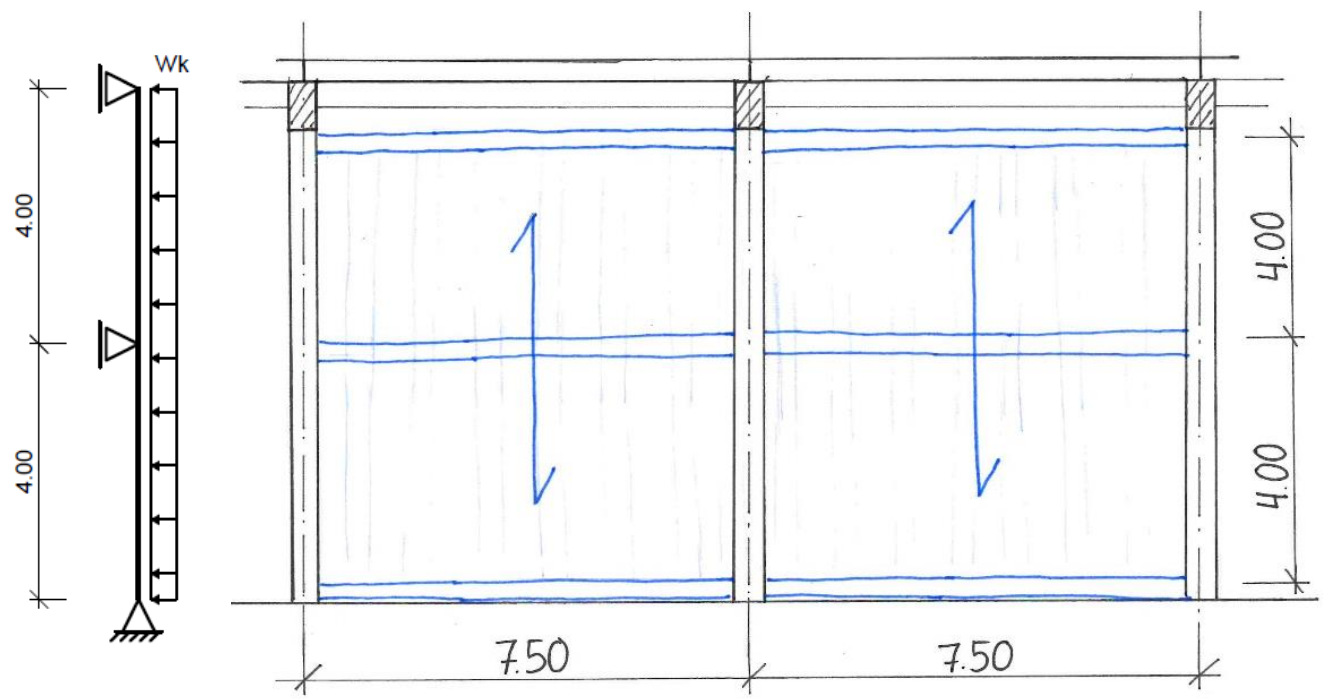

Profile

Hoesch Thermowand VL 80

$$
\begin{array}{ll}
\mathrm{t}_{\mathrm{N}, \mathrm{a}}=0.75 \mathrm{~mm} & \text { (external sheet thickness) } \\
\mathrm{t}_{\mathrm{N}, \mathrm{i}}=0.50 \mathrm{~mm} & \text { (internal sheet thickness) }
\end{array}
$$

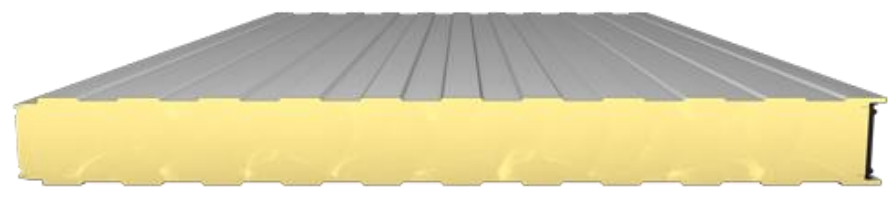

Materials

$f_{y k}=320 \mathrm{~N} / \mathrm{mm}^{2}$

Characteristic loads

\begin{tabular}{lllc|c} 
& & & $\begin{array}{c}\mathbf{g}_{\mathbf{k}} \\
{\left[\mathbf{k N} / \mathbf{m}^{2}\right]}\end{array}$ & $\begin{array}{c}\mathbf{q}_{\mathbf{k}} \\
{\left[\mathbf{k N} / \mathbf{m}^{2}\right]}\end{array}$ \\
\hline Uniformly Distributed Loads & & $\mathrm{w}_{\mathrm{k}, \mathrm{A}}=$ & - & -0.67 \\
Wind Area A & (wind suction) & $\mathrm{w}_{\mathrm{k}, \mathrm{D}}=$ & - & +0.39
\end{tabular}




\subsection{ULTIMATE LIMIT STATE DESIGN}

\subsubsection{WIND SUCTION}

The design of the sheet cladding is done according to the sizing table of the producer HOESCH.

$w_{k, A}=-0.67 k N / m^{2}<w_{k, \max }=-1.21 k N / m^{2} \checkmark$

\begin{tabular}{|c|c|c|c|c|c|c|c|c|c|c|c|c|c|c|c|c|c|c|c|c|c|c|c|}
\hline \multicolumn{2}{|c|}{ Stützweite $L[\mathrm{~m}]$} & & & Do & 2,25 & & & & 3,25 & & & & 25 & 50 & & 00 & 5.25 & 5,5 & 5,75 & 6,00 & 25 & 75 & 7,00 \\
\hline We, 10 & & 5,52 & 4,64 & 3,99 & 3,51 & 3,09 & 2,56 & 2,15 & 1,83 & 1,58 & 1,38 & 1,21 & 1,07 & 0,96 & 0,86 & 0,77 & 0,70 & 0,64 & 0,58 & 0,54 & 0,50 & 0,46 & \\
\hline $\begin{array}{l}n_{A} \leq 3^{3} \\
W e, 1 \\
n_{B} \leq 6\end{array}$ & 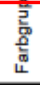 & $\begin{array}{c}3 \\
5,52 \\
6 \\
\end{array}$ & $\begin{array}{c}3 \\
4,64 \\
6 \\
\end{array}$ & $\begin{array}{c}3 \\
3,99 \\
6\end{array}$ & $\begin{array}{c}3 \\
3,51 \\
6\end{array}$ & $\begin{array}{c}3 \\
3,12 \\
6\end{array}$ & $\begin{array}{c}3 \\
2,82 \\
6\end{array}$ & $\begin{array}{c}3 \\
2,57 \\
6\end{array}$ & $\begin{array}{c}3 \\
2,36 \\
6\end{array}$ & $\begin{array}{c}3 \\
2,18 \\
6\end{array}$ & $\begin{array}{c}3 \\
2,03 \\
6\end{array}$ & $\begin{array}{c}3 \\
1,81 \\
6\end{array}$ & $\begin{array}{c}3 \\
1,61 \\
6\end{array}$ & $\begin{array}{c}3 \\
1,43 \\
6\end{array}$ & $\begin{array}{c}3 \\
1,29 \\
5\end{array}$ & $\begin{array}{c}3 \\
1,16 \\
5\end{array}$ & $\begin{array}{c}3 \\
1,05 \\
5\end{array}$ & $\begin{array}{c}3 \\
0,96 \\
5\end{array}$ & $\begin{array}{c}3 \\
0,88 \\
5\end{array}$ & $\begin{array}{c}3 \\
0,81 \\
4\end{array}$ & $\begin{array}{c}3 \\
0,74 \\
4\end{array}$ & $\begin{array}{c}3 \\
0,69 \\
4\end{array}$ & \\
\hline We,10 & $\overline{\bar{g}}$ & 5,38 & 4,52 & 3,89 & 3,42 & 3,04 & 2,56 & 2,15 & 1,83 & 1,58 & 1,38 & 1,21 & 1,07 & 0,96 & 0,86 & 0,77 & 0,70 & 0,64 & 0,58 & 0,54 & 0,50 & 0,46 & \\
\hline $\begin{array}{l}n_{\mathrm{A}} \leq 3 \\
\mathrm{We}, 1 \\
n_{\mathrm{B}} \leq 6\end{array}$ & 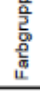 & $\begin{array}{c}3 \\
5,38 \\
6 \\
\end{array}$ & $\begin{array}{c}3 \\
4,52 \\
6 \\
\end{array}$ & $\begin{array}{c}3 \\
3,89 \\
6 \\
\end{array}$ & $\begin{array}{c}3 \\
3,42 \\
6 \\
\end{array}$ & $\begin{array}{c}3 \\
3,04 \\
6 \\
\end{array}$ & $\begin{array}{c}3 \\
2,75 \\
6 \\
\end{array}$ & $\begin{array}{c}3 \\
2,50 \\
6 \\
\end{array}$ & $\begin{array}{c}3 \\
2,30 \\
6 \\
\end{array}$ & $\begin{array}{c}3 \\
2,13 \\
6\end{array}$ & $\begin{array}{c}3 \\
1,99 \\
6 \\
\end{array}$ & $\begin{array}{c}3 \\
1,81 \\
6 \\
\end{array}$ & $\begin{array}{c}3 \\
1,61 \\
6 \\
\end{array}$ & $\begin{array}{c}3 \\
1,43 \\
6 \\
\end{array}$ & $\begin{array}{c}3 \\
1,29 \\
6 \\
\end{array}$ & $\begin{array}{c}3 \\
1,16 \\
5 \\
\end{array}$ & $\begin{array}{c}3 \\
1,05 \\
5 \\
\end{array}$ & $\begin{array}{c}3 \\
0,96 \\
5 \\
\end{array}$ & $\begin{array}{c}3 \\
0,88 \\
5 \\
\end{array}$ & $\begin{array}{c}3 \\
0,81 \\
5 \\
\end{array}$ & $\begin{array}{c}3 \\
0,74 \\
4 \\
\end{array}$ & $\begin{array}{c}3 \\
0,69 \\
4 \\
\end{array}$ & \\
\hline We,10 & & 5,17 & 4,34 & 3,73 & 3,28 & 2,92 & 2,56 & 2,15 & 1,83 & 1,58 & 1,38 & 1,21 & 1,07 & 0,96 & 0,86 & 0,77 & 0,70 & 0,64 & 0,58 & 0,54 & 0,50 & 0,46 & \\
\hline $\begin{array}{l}n_{A} \leq 3 \\
W e, 1 \\
n_{\Omega} \leq 6\end{array}$ & 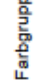 & $\begin{array}{c}3 \\
5,17 \\
6\end{array}$ & $\begin{array}{c}3 \\
4,34 \\
6\end{array}$ & $\begin{array}{c}3 \\
3,73 \\
6\end{array}$ & $\begin{array}{c}3 \\
3,28 \\
6\end{array}$ & $\begin{array}{c}3 \\
2,92 \\
6\end{array}$ & $\begin{array}{c}3 \\
2,64 \\
6\end{array}$ & $\begin{array}{c}3 \\
2,41 \\
6\end{array}$ & $\begin{array}{c}3 \\
2,22 \\
6\end{array}$ & $\begin{array}{c}3 \\
2,06\end{array}$ & $\begin{array}{c}3 \\
1,92 \\
6\end{array}$ & $\begin{array}{c}3 \\
1,80\end{array}$ & $\begin{array}{c}3 \\
1,61 \\
6\end{array}$ & $\begin{array}{c}3 \\
1,43 \\
6\end{array}$ & $\begin{array}{c}3 \\
1,29\end{array}$ & $\begin{array}{c}3 \\
1,16 \\
5\end{array}$ & $\begin{array}{c}3 \\
1,05 \\
5\end{array}$ & $\begin{array}{c}3 \\
0,96 \\
5\end{array}$ & $\begin{array}{c}3 \\
0,88 \\
5\end{array}$ & $\begin{array}{c}3 \\
0,81 \\
5\end{array}$ & $\begin{array}{c}3 \\
0,74 \\
5\end{array}$ & $\begin{array}{c}3 \\
0,69 \\
4\end{array}$ & \\
\hline
\end{tabular}

Figure 26 - Sizing Table of the Sheet Cladding 'Hoesch Thermowand VL 80' for Wind Suction

\subsubsection{WIND PRESSURE}

The design of the sheet cladding is done according to the sizing table of the producer HOESCH.

$w_{k, A}=+0.39 \mathrm{kN} / \mathrm{m}^{2}<w_{k, \max }=+0.56 \mathrm{kN} / \mathrm{m}^{2} \checkmark$

\begin{tabular}{|c|c|c|c|c|c|c|c|c|c|c|c|c|c|c|c|c|c|c|c|c|c|c|}
\hline Stützweite L[m] & 1,50 & 1,75 & 2,00 & 2,25 & 2,50 & 2,75 & 3,00 & 3,25 & 3,50 & 3,75 & 4,00 & 4,25 & 4,50 & 4,75 & 5,00 & 5,25 & 5,50 & 5,75 & 6,00 & 6,25 & $6,50 \quad 6,75$ & 7,00 \\
\hline Breite $b_{A}=40[\mathrm{~mm}]$ & 40 & 40 & 40 & 40 & 40. & 40 & 40 & 40 & 40 & 40 & 40 & 40 & 40 & 40 & 40 & 40 & 40 & 40 & 40 & 40 & 40 & \\
\hline We,10 & 1,50 & 1,29 & 1,13 & 1,00 & 0,90 & 0,82 & 0,75 & 0,69 & 0,64 & 0,60 & 0,56 & 0,53 & 0,50 & 0,47 & 0,45 & 0,43 & 0,41 & 0,39 & 0,38 & 0,33 & 0,29 & \\
\hline Breite $b_{8}{ }^{3}=60[\mathrm{~mm}]$ & 60 & 60 & 60 & 60 & 60 & 60 & 60 & 60 & 60 & 60 & 60 & 60 & 60 & 60 & 60 & 60 & 60 & 60 & 60 & 60 & 60 & \\
\hline Breite $b_{A} \leq 60[\mathrm{~mm}]$ & 40 & 40 & 40 & 40 & 40 & 40 & 40 & 40 & 40 & 40 & 40 & 40 & 40 & 40 & 40 & 40 & 40 & 40 & 40 & 40 & 40 & \\
\hline We,10 & 2,00 & 1,72 & 1,50 & 1,33 & 1,20 & 1,09 & 1,00 & 0,92 & 0,86 & 0,80 & 0,75 & 0,71 & 0,67 & 0,63 & 0,60 & 0,57 & 0,51 & 0,44 & 0,38 & 0,33 & 0,29 & \\
\hline Breite $b_{B} \leq 80[\mathrm{~mm}]$ & 80 & 80 & 80 & 80 & 80 & 80 & 80 & 80 & 80 & 80 & 80 & 80 & 80 & 80 & 80 & 80 & 74 & 67 & 61 & 60 & 60 & \\
\hline Breite $b_{\mathrm{A}} \leq 80[\mathrm{~mm}]$ & 63 & 62 & 62 & 63 & 62 & 63 & 63 & 62 & 63 & 62 & 62 & 63 & 60 & 53 & 47 & 41 & 40 & 40 & 40 & 40 & 40 & \\
\hline We,10 & 3,13 & 2,68 & 2,35 & 2,09 & 1,88 & 1,71 & 1,57 & 1,44 & 1,34 & 1,25 & 1,17 & 1,11 & 1,01 & 0,84 & 0,70 & 0,59 & 0,51 & 0,44 & 0,38 & 0,33 & 0,29 & \\
\hline Breite $b_{B} \leq 125[\mathrm{~mm}]$ & 125 & 125 & 125 & 125 & 125 & 125 & 125 & 125 & 125 & 125 & 125 & 125 & 121 & 108 & 93 & 83 & 74 & 67 & 61 & 60 & 60 & \\
\hline
\end{tabular}

Figure 27 - Sizing Table of the Sheet Cladding 'Hoesch Thermowand VL 80' for Wind Pressure 


\section{POS. F.21 - POCKET FOUNDATION OF COLUMN C.04}

\subsection{SYSTEM}

System

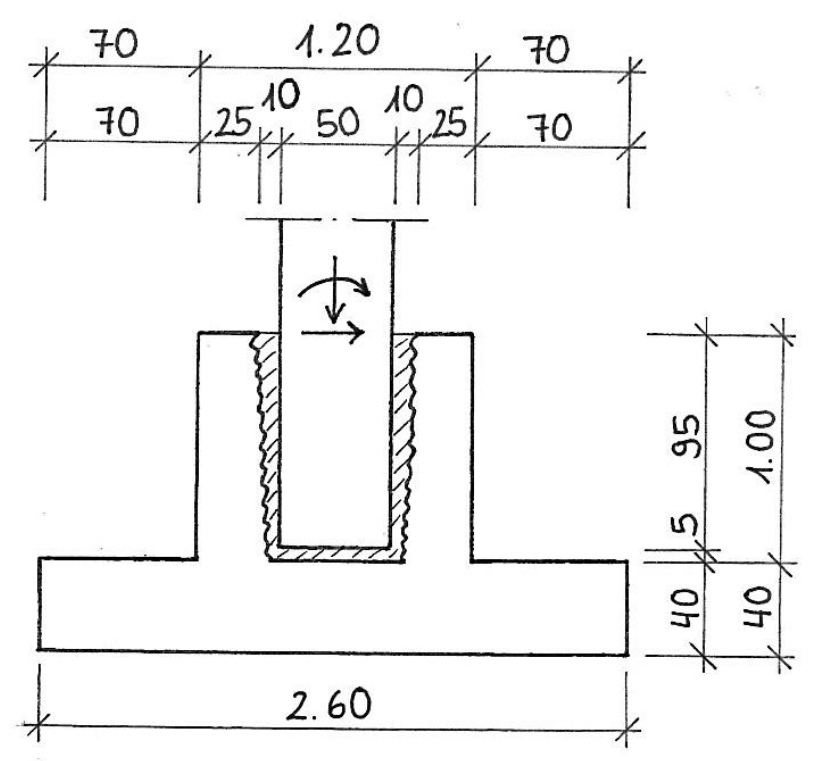

Cross-section $\quad b_{x} \times b_{y} \times h=2.60 \times 2.60 \times 0.40 m$

Building materials

C 35/45 | B 500A

Exposure class

XC2

Concrete cover

$\mathrm{C}_{\mathrm{nom}}=20 \mathrm{~mm}+15 \mathrm{~mm}=35 \mathrm{~mm}$

Characteristic loads

Reaction forces from POS. C.04- see Chapter 8.2.4

\subsection{SOIL-BEARING CAPACITY}

The maximum characteristic bearing capacity of the soil is $\sigma_{R, k}=400 \mathrm{kN} / \mathrm{m}^{2}$. The size of the shallow foundation has to be chosen so the stresses caused by the load on the footing do not exceed the maximum bearing capacity, which means

$$
\begin{array}{ll}
\sigma_{E d}= & \frac{N_{E d, s}}{A^{\prime}} \leq \sigma_{R d}=1.4 \cdot \sigma_{R k}=1.4 \cdot 400=560 \mathrm{kN} / \mathrm{m}^{2} \text { and } \quad A^{\prime}=\left(b_{x}-2 e_{x}\right)\left(b_{y}-2 e_{y}\right) \\
N_{E d, s}= & N_{E d}+1.0 / 1.35 \cdot G_{k} \\
M_{E d, s}=M_{E d}+h \cdot V_{E d} & G_{k}=2.6 \cdot 2.6 \cdot 0.4 \cdot 25 \mathrm{kN} / \mathrm{m}^{3}=67.6 \mathrm{kN} \quad \text { (self-weight of the footing) } \\
& e=\frac{M_{E d, s}}{N_{E d, s}}
\end{array}
$$




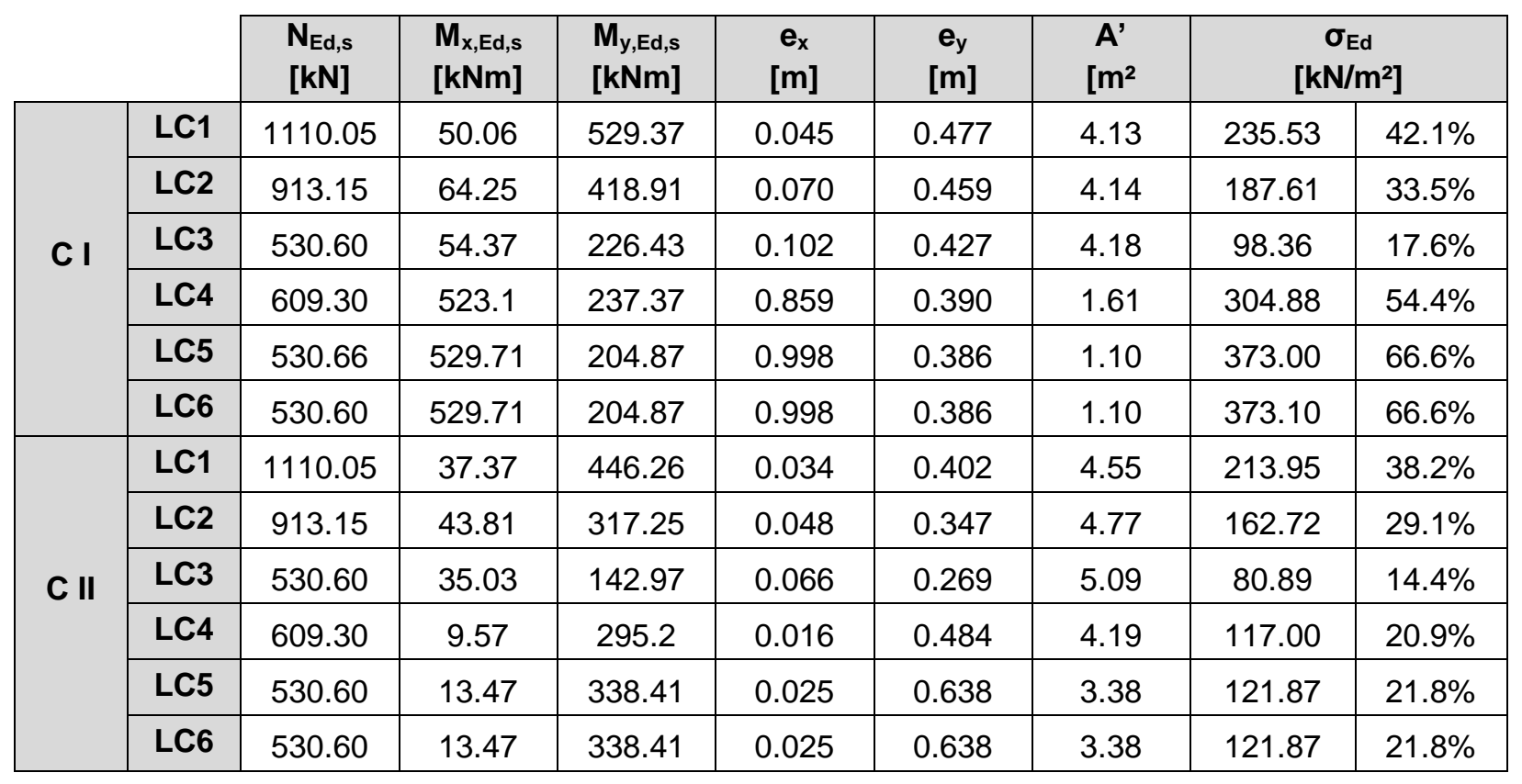

\subsection{LIMITATION OF THE OPEN GAP}

It has to be ensured that no rotation in the shallow foundation occurs due to horizontal loads and bending moments. A simplified and conservative approach is to proof that the eccentricities caused by those loads will not exceed a certain value. These limitations can be described with following formulas for rectangular footings [7]:

(1) $\frac{e_{x, k}}{b_{x}}+\frac{e_{y, k}}{b_{y}} \leq \frac{1}{6}$

with $\quad e_{x, k}, e_{y, k} \quad$ Eccentricities caused by horizontal characteristic dead loads

$b_{x}, b_{y} \quad$ Width of the shallow foundation

(2) $\left(\frac{e_{x, k}}{b_{x}}\right)^{2}+\left(\frac{e_{y, k}}{b_{y}}\right)^{2} \leq \frac{1}{9}$

with $\quad e_{x, k}, e_{y, k} \quad$ Eccentricities caused by horizontal characteristic dead and live loads

$b_{x}, b_{y} \quad$ Width of the shallow foundation 


$$
N_{k, s}=N_{k}+G_{k} \quad \quad M_{k, s}=M_{k}+h \cdot V_{k} \quad e=\frac{M_{E d, s}}{N_{E d, s}}
$$

\begin{tabular}{|l|c|c|c|c|c|}
\cline { 2 - 6 } \multicolumn{1}{c|}{} & \multicolumn{3}{c|}{ Characteristic forces } & \multicolumn{2}{c|}{ Eccentricities } \\
\cline { 2 - 6 } \multicolumn{1}{c|}{} & $\mathbf{N}_{\mathbf{k}, \mathbf{s}}[\mathbf{k N}]$ & $\mathbf{M}_{\mathbf{x}, \mathbf{k}, \mathbf{s}}[\mathbf{k N m}]$ & $\mathbf{M}_{\mathbf{y}, \mathbf{k}, \mathbf{s}}[\mathbf{k N m}]$ & $\mathbf{e}_{\mathbf{x}, \mathbf{k}}[\mathbf{m}]$ & $\mathbf{e}_{\mathbf{y}, \mathbf{k}}[\mathbf{m}]$ \\
\hline $\mathbf{G}_{\mathbf{k}}$ & 530.6 & 0.0 & 168.0 & 0 & 0.317 \\
\hline $\mathbf{G}_{\mathbf{k}}+\mathbf{S}_{\mathbf{k}}+\mathbf{W}_{\mathbf{k}}+\mathbf{A}_{\mathbf{k}}$ & 793.1 & 28.9 & 670.6 & 0.036 & 0.845 \\
\hline
\end{tabular}

(1) $\frac{e_{x, k}}{b_{x}}+\frac{e_{y, k}}{b_{y}}=\frac{0}{2.6}+\frac{0.317}{2.6}=0.122 \leq \frac{1}{6}=0.17 \checkmark \quad \rightarrow 73.1 \%$

(2) $\left(\frac{e_{x, k}}{b_{x}}\right)^{2}+\left(\frac{e_{y, k}}{b_{y}}\right)^{2}=\left(\frac{0.036}{2.6}\right)^{2}+\left(\frac{0.845}{2.6}\right)^{2}=0.106 \leq \frac{1}{9}=0.111 \checkmark \quad \rightarrow 95.3 \%$

\subsection{TILTING}

Shallow foundations must be prevented from tilting. In order to proof that the foundations is safe from tilting again a conservative approach is to limit the eccentricities that occur from the factored horizontal loads. This can be done by meeting following conditions for rectangular footings [7]:

(1) $\frac{e_{x, d}}{b_{x}} \leq \frac{1}{2}$

(2) $\frac{e_{y, d}}{b_{y}} \leq \frac{1}{2} \quad$ with $\quad e_{x, k}, e_{y, k} \quad$ Eccentricities caused by horizontal factored loads.

\begin{tabular}{|c|c|c|c|c|c|c|c|c|c|c|}
\hline & \multirow{2}{*}{$\begin{array}{c}\begin{array}{c}\mathbf{N}_{\mathrm{Ed}, \mathbf{s}} \\
{[\mathrm{kN}]}\end{array} \\
1110.05\end{array}$} & \multirow{2}{*}{$\begin{array}{c}\mathbf{M}_{\mathbf{x}, \mathrm{Ed}, \mathbf{s}} \\
{[\mathrm{kNm}]}\end{array}$} & \multirow{2}{*}{$\begin{array}{l}\mathbf{M}_{\mathbf{y}, \mathrm{Ed}, \mathbf{s}} \\
{[\mathbf{k N m}]}\end{array}$} & \multirow{2}{*}{$\begin{array}{c}\begin{array}{c}\mathbf{e}_{\mathbf{x}, \mathbf{d}} \\
{[\mathbf{m}]}\end{array} \\
0.045\end{array}$} & \multirow{2}{*}{$\begin{array}{c}\begin{array}{c}e_{y, d} \\
{[\mathbf{m}]}\end{array} \\
0.477\end{array}$} & \multicolumn{2}{|c|}{$\begin{array}{c}\mathbf{e}_{\mathbf{x}, \mathbf{d}} / \mathbf{b}_{\mathbf{x}} \\
{[-]}\end{array}$} & \multicolumn{2}{|c|}{$\begin{array}{c}\mathbf{e}_{\mathrm{y}, \mathrm{d}} / \mathbf{b}_{\mathrm{y}} \\
{[-]}\end{array}$} \\
\hline \multirow{6}{*}{ C I } & LC1 & & & & & & 0.017 & $3.5 \%$ & 0.183 & $36.7 \%$ \\
\hline & LC2 & 913.15 & 64.25 & 418.91 & 0.070 & 0.459 & 0.027 & $5.4 \%$ & 0.176 & $35.3 \%$ \\
\hline & LC3 & 530.60 & 54.37 & 226.43 & 0.102 & 0.427 & 0.039 & $7.9 \%$ & 0.164 & $32.8 \%$ \\
\hline & LC4 & 609.30 & 523.1 & 237.37 & 0.859 & 0.390 & 0.330 & $66.0 \%$ & 0.150 & $30.0 \%$ \\
\hline & LC5 & 530.66 & 529.71 & 204.87 & 0.998 & 0.386 & 0.384 & $76.8 \%$ & 0.148 & $29.7 \%$ \\
\hline & LC6 & 530.60 & 529.71 & 204.87 & 0.998 & 0.386 & 0.384 & $76.8 \%$ & 0.149 & $29.7 \%$ \\
\hline \multirow{6}{*}{ C II } & LC1 & 1110.05 & 37.37 & 446.26 & 0.034 & 0.402 & 0.013 & $2.6 \%$ & 0.155 & $30.9 \%$ \\
\hline & LC2 & 913.15 & 43.81 & 317.25 & 0.048 & 0.347 & 0.018 & $3.7 \%$ & 0.134 & $26.7 \%$ \\
\hline & LC3 & 530.60 & 35.03 & 142.97 & 0.066 & 0.269 & 0.025 & $5.1 \%$ & 0.104 & $20.7 \%$ \\
\hline & LC4 & 609.30 & 9.57 & 295.2 & 0.016 & 0.484 & 0.006 & $1.2 \%$ & 0.186 & $37.3 \%$ \\
\hline & LC5 & 530.60 & 13.47 & 338.41 & 0.025 & 0.638 & 0.010 & $2.0 \%$ & 0.245 & $49.1 \%$ \\
\hline & LC6 & 530.60 & 13.47 & 338.41 & 0.025 & 0.638 & 0.010 & $2.0 \%$ & 0.245 & $49.1 \%$ \\
\hline
\end{tabular}




\subsection{CAPACITY UTILIZATION OF THE FOOTING}

\begin{tabular}{|l|c|}
\cline { 2 - 2 } \multicolumn{1}{c|}{} & Maximum utilization [\%] \\
\hline Bearing Capacity & $66.6 \%$ \\
\hline Open Gap & $95.3 \%$ \\
\hline Tilting & $37.3 \%$ \\
\hline
\end{tabular}

As can be seen in the table above the limitation of the open gap is the decisive criteria for choosing the size of the footing.

\subsection{FLEXURE DESIGN}

The flexure design of the footing is done according to [7].

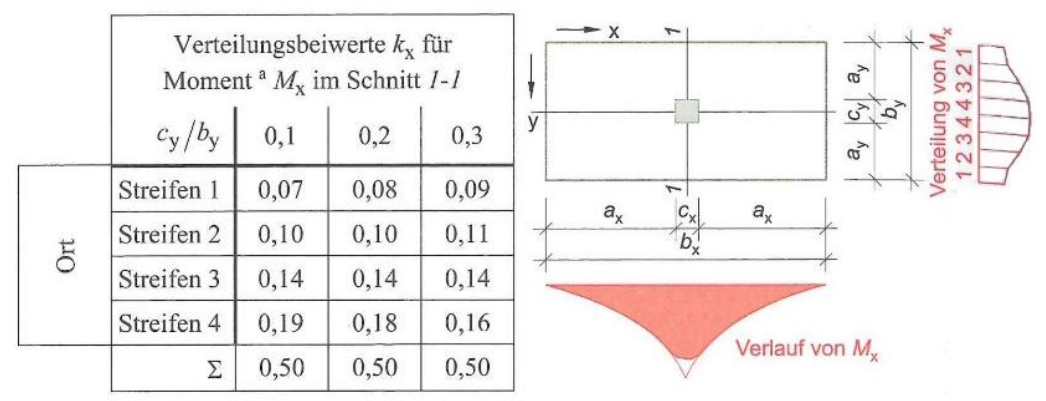

Figure 28 - Distribution of the Flexure Moment [7]

\subsubsection{FLEXURE DESIGN}

Maximum soil stress

$$
\sigma_{E d, \max }=373.0 \mathrm{kN} / \mathrm{m}^{2} \quad \text { (see Chapter 17.2) }
$$

Flexure moment

$$
M_{E d}=\sigma_{E d} \cdot b_{y} \cdot \frac{b_{x}^{2}}{8}=373.0 \cdot 2.6 \cdot \frac{2.6^{2}}{8}=819.5 \mathrm{kNm}
$$

Distribution of the flexure moment

$$
\begin{aligned}
& \frac{c}{b}=\frac{50}{260}=0.192 \approx 0.2 \quad \rightarrow k_{4}=0.18 \\
& \Delta M_{E d}=k_{4} \cdot M_{E d}=0.18 \cdot 819.5=147.5 \mathrm{kNm} \\
& d_{\text {est }}=h-c_{\text {nom }}-d_{s} / 2=40-3.5-2.0-2.0 / 2=33 \mathrm{~cm}
\end{aligned}
$$

Required reinforcement

$$
\mu_{E d s}=\frac{8 \cdot \Delta M_{E d x}}{b \cdot d^{2} \cdot f_{c d}}=\frac{8 \cdot 14750}{260 \cdot 33^{2} \cdot 1.98}=0.21 \quad \rightarrow \omega=0.2395
$$

$$
A_{s 4, \text { req }}=0.2395 \cdot 260 \cdot 33 \cdot 19.8 / 8 \cdot 441=11.53 \mathrm{~cm}^{2}
$$

$$
\begin{aligned}
& A_{s 3, r \text { eq }}=\frac{k_{x i}}{k_{x 4}} \cdot A_{s \times 4}=\frac{0.14}{0.18} \cdot 11.53 \mathrm{~cm}^{2}=8.97 \mathrm{~cm}^{2} \\
& A_{s 2, \text { req }}=\frac{k_{x i}}{k_{x 4}} \cdot A_{s \times 4}=\frac{0.10}{0.18} \cdot 11.53 \mathrm{~cm}^{2}=6.41 \mathrm{~cm}^{2}
\end{aligned}
$$




$$
A_{s 1, r e q}=\frac{k_{x i}}{k_{x 4}} \cdot A_{s \times 4}=\frac{0.08}{0.18} \cdot 11.53 \mathrm{~cm}^{2}=5.12 \mathrm{~cm}^{2}
$$

Chosen

$\begin{array}{ll}\varnothing 16 / 5=13.07 \mathrm{~cm}^{2} / \text { strip } & \text { strip 4 } \\ \varnothing 16 / 7=9.33 \mathrm{~cm}^{2} / \text { strip } & \text { strip 3 } \\ \varnothing 16 / 9=7.25 \mathrm{~cm}^{2} / \text { strip } & \text { strip 2 } \\ \varnothing 16 / 12.5=5.23 \mathrm{~cm}^{2} / \text { strip } & \text { strip 1 }\end{array}$

\subsection{DESIGN OF THE PEDESTAL SOCKET}

\subsubsection{DEPTH OF THE SOCKET}

Design forces

Minimum depth

Total depth of the socket
The decisive load for the depth of the socket is Case I Load Combination 6 with a minimum normal force and a maximum bending moment. ${ }^{5}$

\begin{tabular}{|c|c|c|c|c|}
\hline $\begin{array}{c}\mathbf{N}_{\mathrm{Ed}} \\
{[\mathbf{k N}]}\end{array}$ & $\begin{array}{c}\mathbf{M}_{\mathbf{y}, \mathrm{Ed}} \\
{[\mathbf{k N m}]}\end{array}$ & $\begin{array}{c}\mathbf{V}_{\mathbf{x}, \mathrm{Ed}} \\
{[\mathbf{k N}]}\end{array}$ & $\begin{array}{c}\mathbf{M}_{\mathbf{x}, \mathrm{Ed}} \\
{[\mathbf{k N m}]}\end{array}$ & $\begin{array}{c}\mathbf{V}_{\mathbf{y}, \mathrm{Ed}} \\
{[\mathbf{k N}]}\end{array}$ \\
\hline-479.9 & -204.3 & 1.9 & -416.4 & -377.7 \\
\hline
\end{tabular}

According to [8] the depth of the socket can be estimated using following formulas:

$$
\begin{array}{ll}
\frac{M_{E d}}{N_{E d} \cdot h} \leq 0.15 & \rightarrow t \geq 1.2 \cdot h \quad \frac{M_{E d}}{N_{E d} \cdot h}=2.0 \quad \rightarrow t \geq 2.0 \cdot h \\
\text { But } & t_{\min }=1.5 \cdot h \\
\text { Here: } & \frac{M_{E d}}{N_{E d} \cdot h}=\frac{416.4}{479.9 \cdot 0.5}=1.74 \quad \rightarrow t \geq 1.9 \cdot 50=95 \mathrm{~cm}
\end{array}
$$

$$
h=95+5=\underline{\underline{100 \mathrm{~cm}}} \quad(+5 \mathrm{~cm} \text { for levelling shims })
$$

\subsubsection{DESIGN OF THE WALLS}

$$
\min d_{k}=\frac{W}{3}=\frac{10+50+10}{3}=23.3 \mathrm{~cm}>20 \mathrm{~cm}
$$

Chosen

$$
d_{k}=25 \mathrm{~cm}
$$

\subsubsection{REINFORCEMENT OF THE SOCKET}

Maximum Horizontal Forces

Upper horizontal force $\quad H_{E d}^{U}=\frac{6}{5} \cdot \frac{M_{E d}}{t}+\frac{6}{5} \cdot H_{E d}=\frac{6}{5} \cdot \frac{416.4}{0.95}+\frac{6}{5} \cdot 377.7=979.2 \mathrm{kN}$

${ }^{5}$ See Chapter 8.2.4 
Lower horizontal force $\quad H_{E d}^{L}=\frac{6}{5} \cdot \frac{M_{E d}}{t}+\frac{1}{5} \cdot H_{E d}=\frac{6}{5} \cdot \frac{416.4}{0.95}+\frac{1}{5} \cdot 377.7=658.4 \mathrm{kN}$

Upper Reinforcement

$$
A_{s, r e q}^{U}=\frac{H_{E d}^{U}}{2} \cdot \frac{1}{f_{y d}}=\frac{979.2}{2} \cdot \frac{1}{43.5}=11.3 \mathrm{~cm}^{2}
$$

Chosen

Lap length
4 reinforcement loops $\varnothing 14=12.32 \mathrm{~cm}^{2}$

$\varnothing_{s}=10$ and moderate bond

$I_{0}=\alpha_{1} \cdot \alpha_{6} \cdot I_{b, \text { rqd }} \cdot\left(A_{s, \text { req }} / A_{s, \text { prov }}\right)=1.0 \cdot 1.4 \cdot 58 \cdot(11.3 / 12.64)=50.1 \mathrm{~cm}$

$$
>I_{0, \min }=20 \mathrm{~cm}
$$

per wall
Vertical Reinforcement

Vertical tension force

$$
F_{s d}^{V}=H_{E d}^{U} \cdot \frac{f_{k}}{g_{k}}=979.2 \cdot \frac{84.2}{91.4}=902.1 \mathrm{kN}
$$$$
f_{k}=h_{k}-\frac{t}{6}=100-\frac{95}{6}=84.2 \mathrm{~cm} ; g_{k}=0.85 \cdot\left(b_{k}-\frac{d_{k}}{2}\right)=0.85 \cdot\left(120-\frac{25}{2}\right)=91.4 \mathrm{~cm}
$$

Vertical reinforcement

$$
A_{s, \text { req }}^{v}=\frac{F_{s d}^{V}}{2} \cdot \frac{1}{f_{y d}}=\frac{902.1}{2} \cdot \frac{1}{43.5}=10.4 \mathrm{~cm}^{2}
$$

\section{Chosen}

Lap length
4 vertical stirrups $\varnothing 14=12.32 \mathrm{~cm}^{2}$

$\varnothing_{s}=10$ and good bond

$$
\begin{aligned}
I_{0} & =\alpha_{1} \cdot \alpha_{6} \cdot I_{b, \text { rqd }} \cdot\left(A_{s, \text { req }} / A_{s, \text { prov }}\right)=1.0 \cdot 1.4 \cdot 40 \cdot(10.4 / 11.06)=53 \mathrm{~cm} \\
& >I_{0, \min }=20 \mathrm{~cm}
\end{aligned}
$$

\subsubsection{REINFORCEMENT DRAWING}

See Appendix A.8, page 194. 


\section{POS. F.22 - POCKET FOUNDATION OF COLUMN C.05}

\subsection{SYSTEM}

System:

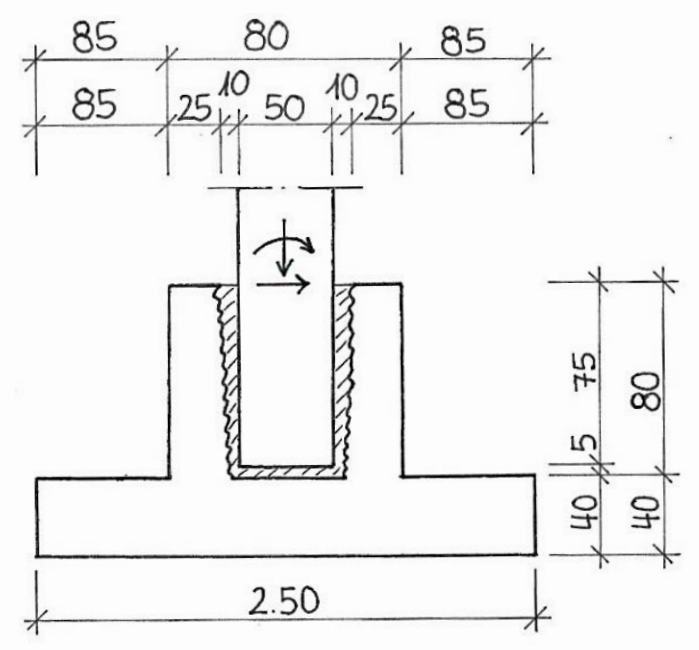

Cross-section $\quad b_{x} \times b_{y} \times h=2.50 \times 2.50 \times 0.40 m$

Building materials $\quad$ C 35/45 | B 500A

Exposure class $\quad$ XC2

Reinforcing steel $\quad \mathrm{C}_{\mathrm{nom}}=20 \mathrm{~mm}+15 \mathrm{~mm}=35 \mathrm{~mm}$

Characteristic loads $\quad$ Reaction forces from POS. C.04- see Chapter 8.2.4

\subsection{SOIL-BEARING CAPACITY}

The maximum characteristic bearing capacity of the soil is $\sigma_{R, k}=400 \mathrm{kN} / \mathrm{m}^{2}$. The size of the shallow foundation has to be chosen so the stresses caused by the load on the footing do not exceed the maximum bearing capacity, which means

$$
\begin{array}{ll}
\sigma_{E d}= & \frac{N_{E d, s}}{A^{\prime}} \leq \sigma_{R d}=1.4 \cdot \sigma_{R k}=1.4 \cdot 400=560 \mathrm{kN} / \mathrm{m}^{2} \quad \text { and } \quad A^{\prime}=\left(b_{x}-2 e_{x}\right)\left(b_{y}-2 e_{y}\right) \\
N_{E d, s}=N_{E d}+1.0 / 1.35 \cdot G_{k} & G_{k}=2.5 \cdot 2.5 \cdot 0.4 \cdot 25 \mathrm{kN} / \mathrm{m}^{3}=62.5 \mathrm{kN} \quad \text { (self-weight of the footing) } \\
M_{E d, s}=M_{E d}+h \cdot V_{E d} & e=\frac{M_{E d, s}}{N_{E d, s}}
\end{array}
$$

The decisive load combination for the soil-bearing capacity is LC1 (see Chapter 9.2.4). 


\begin{tabular}{|c|c|c|c|c|c|c|}
\cline { 2 - 7 } \multicolumn{1}{c|}{} & $\begin{array}{c}\mathbf{N}_{\mathrm{Ed}, \mathbf{s}} \\
{[\mathbf{k N}]}\end{array}$ & $\begin{array}{c}\mathbf{M}_{\mathrm{Ed}, \mathbf{s}} \\
{[\mathbf{k N m}]}\end{array}$ & $\begin{array}{c}\mathbf{e}_{\mathbf{x}} \\
{[\mathbf{m}]}\end{array}$ & $\begin{array}{c}\mathbf{A}^{\prime} \\
{\left[\mathbf{m}^{2}\right.}\end{array}$ & \multicolumn{2}{c|}{$\begin{array}{c}\boldsymbol{\sigma}_{\mathrm{Ed}} \\
{\left[\mathbf{k N} / \mathbf{m}^{2}\right]}\end{array}$} \\
\hline LC1 & 3451.28 & 153.94 & 0.045 & 6.03 & 544.64 & $97.3 \%$ \\
\hline
\end{tabular}

\subsection{LIMITATION OF THE OPEN GAP}

It has to be ensured that no rotation in the shallow foundation occurs due to horizontal loads and bending moments. A simplified and conservative approach is to proof that the eccentricities caused by those loads will not exceed a certain value. These limitations can be described with following formulas for rectangular footings [7]:

$\begin{array}{lll}\text { (1) } \frac{e}{b} \leq \frac{1}{6} & \text { for dead loads } & \text { (2) } \frac{e}{b} \leq \frac{1}{3}\end{array}$

$N_{k, s}=N_{k}+G_{k} \quad M_{k, s}=M_{k}+h \cdot V_{k} \quad e=\frac{M_{E d, s}}{N_{E d, s}}$

\begin{tabular}{|l|c|c|c|}
\cline { 2 - 4 } \multicolumn{1}{c|}{} & \multicolumn{2}{c|}{ Characteristic forces } & Eccentricities \\
\cline { 2 - 4 } \multicolumn{1}{c|}{} & $\mathbf{N}_{\mathbf{k}, \mathbf{s}}[\mathbf{k N}]$ & $\mathbf{M}_{\mathbf{k}, \mathbf{s}}[\mathbf{k N m}]$ & $\mathbf{e}_{\mathbf{k}}[\mathbf{m}]$ \\
\hline $\mathbf{G}_{\mathbf{k}}$ & 1389.8 & 0.0 & 0 \\
\hline $\mathbf{G}_{\mathbf{k}}+\mathbf{S}_{\mathbf{k}}+\mathbf{A}_{\mathbf{k}}$ & 2439.8 & 543.8 & 0.223 \\
\hline
\end{tabular}

(1) $\frac{e_{k}}{b}=0 \leq \frac{1}{6}=0.17 \checkmark \rightarrow 0.0 \%$

(2) $\frac{e_{k}}{b}=\frac{0.223}{2.50}=0.008 \leq \frac{1}{3}=0.33 \checkmark \quad \rightarrow 7.2 \%$

\subsection{TILTING}

Shallow foundations must be prevented from tilting. In order to proof that the foundations is safe from tilting again a conservative approach is to limit the eccentricities that occur from the factored horizontal loads. This can be done by meeting following conditions for rectangular footings [7]:

(3) $\frac{e_{d}}{b} \leq \frac{1}{2}$

\begin{tabular}{|c|c|c|c|c|c|}
\cline { 2 - 6 } \multicolumn{1}{c|}{} & $\begin{array}{c}\mathbf{N}_{\text {Ed,s }} \\
{[\mathbf{k N}]}\end{array}$ & $\begin{array}{c}\mathbf{M}_{\text {Ed,s }} \\
{[\mathbf{k N m}]}\end{array}$ & $\begin{array}{c}\mathbf{e}_{\mathrm{d}} \\
{[\mathrm{m}]}\end{array}$ & \multicolumn{2}{c|}{$\begin{array}{c}\mathbf{e}_{\mathrm{d}} / \mathbf{b} \\
{[-]}\end{array}$} \\
\hline LC1 & 3451.28 & 153.94 & 0.045 & 0.018 & $3.6 \%$ \\
\hline LC2 & 1599.90 & 603.16 & 0.377 & 0.151 & $30.2 \%$ \\
\hline LC3 & 1389.80 & 592.34 & 0.426 & 0.170 & $34.1 \%$ \\
\hline
\end{tabular}




\subsection{CAPACITY UTILIZATION OF THE FOOTING}

\begin{tabular}{|l|c|}
\cline { 2 - 2 } \multicolumn{1}{c|}{} & Maximum utilization [\%] \\
\hline Bearing Capacity & $97.3 \%$ \\
\hline Open Gap & $7.2 \%$ \\
\hline Tilting & $34.1 \%$ \\
\hline
\end{tabular}

As can be seen in the table above the bearing capacity is the decisive criteria for choosing the size of the footing.

\subsection{FLEXURE DESIGN}

The flexure design of the footing is done according to [7].

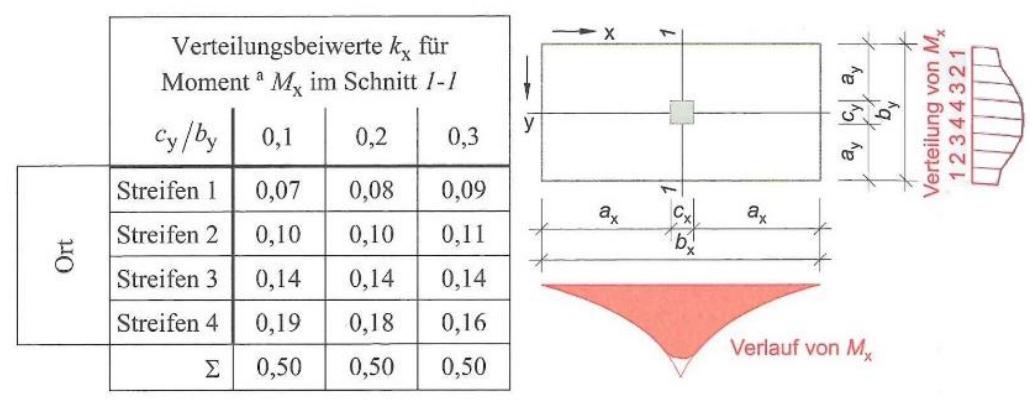

Figure 29 - Distribution of the Flexure Moment [7]

\subsubsection{FLEXURE DESIGN}

Maximum soil stress

$$
\sigma_{E d, \max }=544.6 \mathrm{kN} / \mathrm{m}^{2} \quad \text { (see Chapter 17.2) }
$$

Flexure moment

$$
M_{E d}=\sigma_{E d} \cdot b_{y} \cdot \frac{b_{x}^{2}}{8}=554.6 \cdot 2.5 \cdot \frac{2.5^{2}}{8}=1083 \mathrm{kNm}
$$

Distribution of the flexure moment

Required reinforcement

$$
\begin{aligned}
& \frac{c}{b}=\frac{50}{250}=0.2 \quad \rightarrow k_{4}=0.18 \\
& \Delta M_{E d}=k_{4} \cdot M_{E d}=0.18 \cdot 1083=194.9 \mathrm{kNm} \\
& d_{\text {est }}=h-c_{\text {nom }}-d_{s} / 2=40-3.5-2.0-2.0 / 2=33 \mathrm{~cm}
\end{aligned}
$$

$$
\mu_{E d s}=\frac{8 \cdot \Delta M_{E d x}}{b \cdot d^{2} \cdot f_{c d}}=\frac{8 \cdot 19490}{250 \cdot 33^{2} \cdot 1.98}=0.29 \rightarrow \omega=0.3546
$$$$
A_{s 4, \text { req }}=0.3546 \cdot 250 \cdot 33 \cdot 19.8 / 8 \cdot 437=16.6 \mathrm{~cm}^{2}
$$$$
A_{s 3, r e q}=\frac{k_{x i}}{k_{x 4}} \cdot A_{s \times 4}=\frac{0.14}{0.18} \cdot 16.6 \mathrm{~cm}^{2}=12.9 \mathrm{~cm}^{2}
$$$$
A_{s 2, \text { req }}=\frac{k_{x i}}{k_{x 4}} \cdot A_{s \times 4}=\frac{0.10}{0.18} \cdot 16.6 \mathrm{~cm}^{2}=9.2 \mathrm{~cm}^{2}
$$ 


$$
A_{s 1, \text { eq }}=\frac{k_{x i}}{k_{x 4}} \cdot A_{s \times 4}=\frac{0.08}{0.18} \cdot 16.6 \mathrm{~cm}^{2}=7.4 \mathrm{~cm}^{2}
$$

Chosen

$\begin{array}{ll}\varnothing 20 / 5=19.6 \mathrm{~cm}^{2} / \text { strip } & \text { strip 4 } \\ \varnothing 20 / 7.5=13.1 \mathrm{~cm}^{2} / \text { strip } & \text { strip 3 } \\ \varnothing 20 / 10=9.8 \mathrm{~cm}^{2} / \text { strip } & \text { strip 2 } \\ \varnothing 20 / 12.5=7.9 \mathrm{~cm}^{2} / \text { strip } & \text { strip 1 }\end{array}$

\subsection{DESIGN OF THE PEDESTAL SOCKET}

\subsubsection{DEPTH OF THE SOCKET}

Design forces

Minimum depth

Total depth of the socket
The decisive load for the depth of the socket is LC 5 with a minimum normal force and a maximum bending moment (see Chapter 9.2.4).

\begin{tabular}{|c|c|c|}
\hline $\begin{array}{c}\mathbf{N}_{\mathbf{E d}} \\
{[\mathbf{k N}]}\end{array}$ & $\begin{array}{c}\mathbf{M}_{\mathbf{y}, \mathrm{Ed}} \\
{[\mathbf{k N m}]}\end{array}$ & $\begin{array}{c}\mathbf{V}_{\mathbf{x}, \mathrm{Ed}} \\
{[\mathbf{k N}]}\end{array}$ \\
\hline-1327.3 & -440.5 & 379.6 \\
\hline
\end{tabular}

According to [8] the depth of the socket can be estimated using following formulas:

$$
\begin{array}{ll}
\frac{M_{E d}}{N_{E d} \cdot h} \leq 0.15 & \rightarrow t \geq 1.2 \cdot h \quad \frac{M_{E d}}{N_{E d} \cdot h}=2.0 \quad \rightarrow t \geq 2.0 \cdot h \\
\text { But } & t_{\min }=1.5 \cdot h \\
\text { Here: } & \frac{M_{E d}}{N_{E d} \cdot h}=\frac{440.5}{1327.3 \cdot 0.5}=0.66 \quad \rightarrow t \geq 1.5 \cdot 50=75 \mathrm{~cm}
\end{array}
$$

$$
h=75+5=\underline{\underline{80 \mathrm{~cm}}} \quad(+5 \mathrm{~cm} \text { for levelling shims })
$$

18.7.2 DESIGN OF THE WALLS
Chosen

$$
\min d_{k}=\frac{W}{3}=\frac{10+50+10}{3}=23.3 \mathrm{~cm}>20 \mathrm{~cm}
$$

\subsubsection{REINFORCEMENT OF THE SOCKET}

Maximum Horizontal Forces

$\begin{array}{ll}\text { Upper horizontal force } & H_{E d}^{U}=\frac{6}{5} \cdot \frac{M_{E d}}{t}+\frac{6}{5} \cdot H_{E d}=\frac{6}{5} \cdot \frac{440.5}{0.75}+\frac{6}{5} \cdot 379.6=1160 \mathrm{kN} \\ \text { Lower horizontal force } & H_{E d}^{L}=\frac{6}{5} \cdot \frac{M_{E d}}{t}+\frac{1}{5} \cdot H_{E d}=\frac{6}{5} \cdot \frac{440.5}{0.75}+\frac{1}{5} \cdot 379.6=781 \mathrm{kN}\end{array}$


Upper Reinforcement

$$
A_{s, \text { req }}^{U}=\frac{H_{E d}^{U}}{2} \cdot \frac{1}{f_{y d}}=\frac{1160}{2} \cdot \frac{1}{43.5}=13.3 \mathrm{~cm}^{2}
$$

\section{Chosen}

Lap length per wall

$$
\begin{aligned}
& \emptyset_{s}=10 \text { and moderate bond } \\
& \begin{aligned}
I_{0} & =\alpha_{1} \cdot \alpha_{6} \cdot I_{b, \text { rqd }} \cdot\left(A_{s, \text { req }} / A_{s, \text { prov }}\right)=1.0 \cdot 1.4 \cdot 58 \cdot(11.3 / 12.64)=50.1 \mathrm{~cm} \\
& >I_{0, \min }=20 \mathrm{~cm}
\end{aligned}
\end{aligned}
$$

Vertical reinforcement

Vertical tension force

$$
\begin{aligned}
& F_{s d}^{v}=H_{E d}^{v} \cdot \frac{f_{k}}{g_{k}}=1160 \cdot \frac{67.5}{91.4}=857 \mathrm{kN} \\
& f_{k}=h_{k}-\frac{t}{6}=80-\frac{75}{6}=67.5 \mathrm{~cm} ; g_{k}=0.85 \cdot\left(b_{k}-\frac{d_{k}}{2}\right)=0.85 \cdot\left(120-\frac{25}{2}\right)=91.4 \mathrm{~cm}
\end{aligned}
$$

Vertical reinforcement

$$
A_{s, \text { req }}^{v}=\frac{F_{s d}^{v}}{2} \cdot \frac{1}{f_{y d}}=\frac{857}{2} \cdot \frac{1}{43.5}=9.9 \mathrm{~cm}^{2}
$$

\section{Chosen}

\section{7 vertical stirrups $\varnothing 10 \mathrm{~m}=11.06 \mathrm{~cm}^{2}$}

\section{per corner}

Lap length $\varnothing_{s}=10$ and good bond

$$
\begin{aligned}
I_{0} & =\alpha_{1} \cdot \alpha_{6} \cdot I_{b, \text { rqd }} \cdot\left(A_{s, \text { req }} / A_{s, \text { prov }}\right)=1.0 \cdot 1.4 \cdot 40 \cdot(10.4 / 11.06)=53 \mathrm{~cm} \\
& >I_{0, \min }=20 \mathrm{~cm}
\end{aligned}
$$


19 POS. F.23-STRIP FOUNDATION

\subsection{SYSTEM}

Cross-section

Building materials

Exposition class

Reinforcing steel

Characteristic loads $\mathrm{b} / \mathrm{h}=50 / 80 \mathrm{~cm}$

\section{30/37 | B 500A}

XC2

$c_{n o m}=20 m m+15 m m=35 m m$

\begin{tabular}{lc|c} 
Uniformly Distributed Loads & $\begin{array}{c}\mathbf{g}_{\mathbf{k}} \\
{[\mathbf{k N} / \mathbf{m}]}\end{array}$ & $\begin{array}{c}\mathbf{q}_{\mathbf{k}} \\
{[\mathbf{k N} / \mathbf{m}]}\end{array}$ \\
\hline $\begin{array}{l}\text { Self-weight } \mathrm{g}_{\mathrm{k} 1}=0.3 \times 0.8 \times 25 \mathrm{kN} / \mathrm{m}^{3} \\
\text { Reaction Force from POS. W.14 }\end{array}$ & 6.00 & - \\
$\mathrm{g}_{\mathrm{k} 2=4.30 \times 0.2 \times 25 \mathrm{kN} / \mathrm{m}^{3}+6 \times 88.0 \mathrm{kN} / 7.50 \mathrm{~m}=}$ & 91.9 & - \\
$\qquad \mathrm{q}_{\mathrm{k}}=6 \times 31.0 \mathrm{kN} / 7.50 \mathrm{~m}=$ & - & 24.8 \\
& $=97.9$ & 24.8
\end{tabular}

\subsection{SOIL-BEARING CAPACITY}

Factored load

Soil-bearing capacity
$N_{E d}=1.35 \cdot 97.9+1.50 \cdot 24.8=169.4 k N$

$\sigma_{E d}=\frac{N_{E d}}{A}=\frac{169.4}{0.5 \cdot 1.0}=338.8 \mathrm{kN} / \mathrm{m}^{2} \leq \sigma_{R d}=1.4 \cdot \sigma_{R k}=1.4 \cdot 400=560 \mathrm{kN} / \mathrm{m}^{2}$ 
20 POS. V.31 - STEEL CANOPY

\subsection{OVERVIEW DRAWING}

\subsubsection{SKETCH}
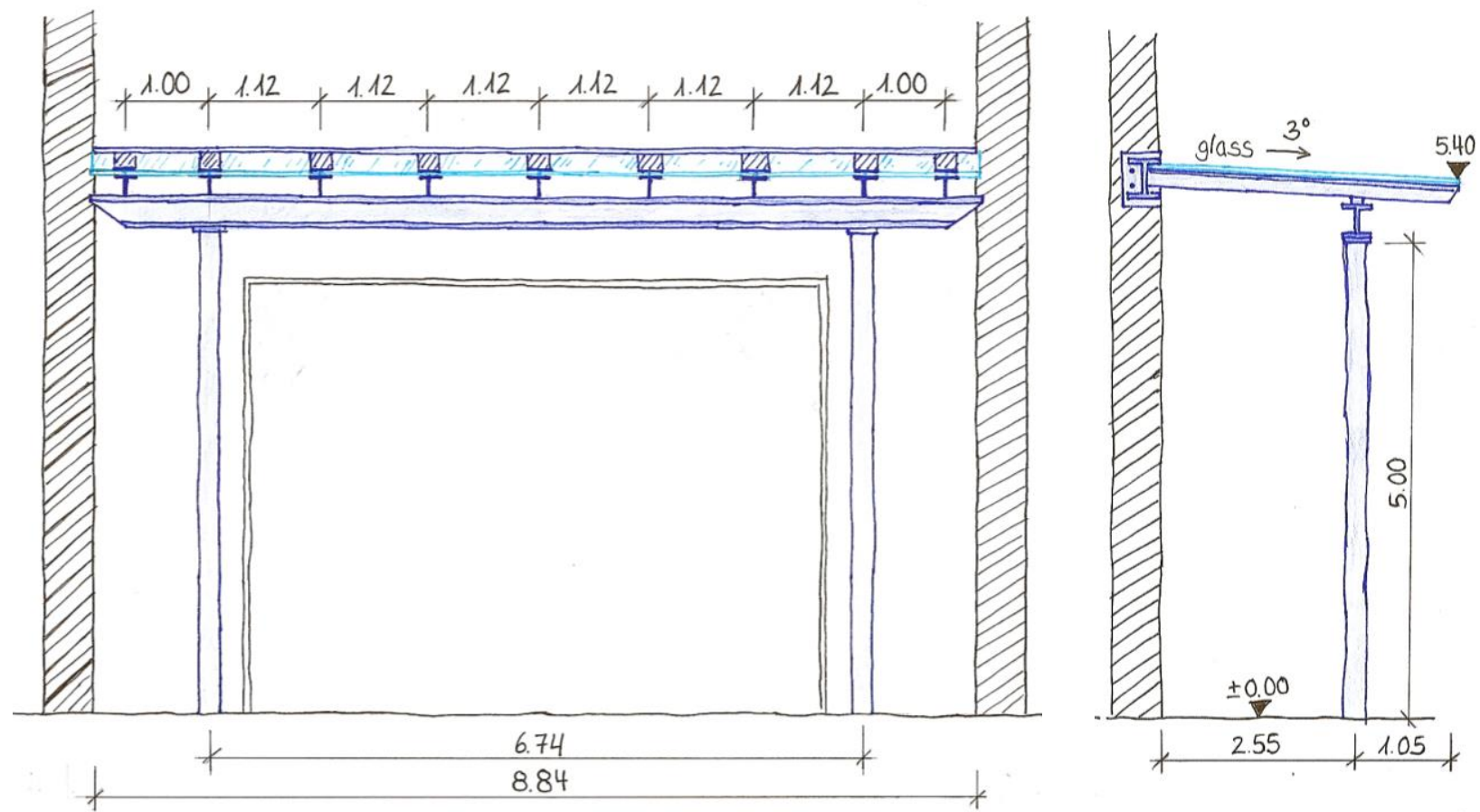

\subsubsection{POSITION PLAN}

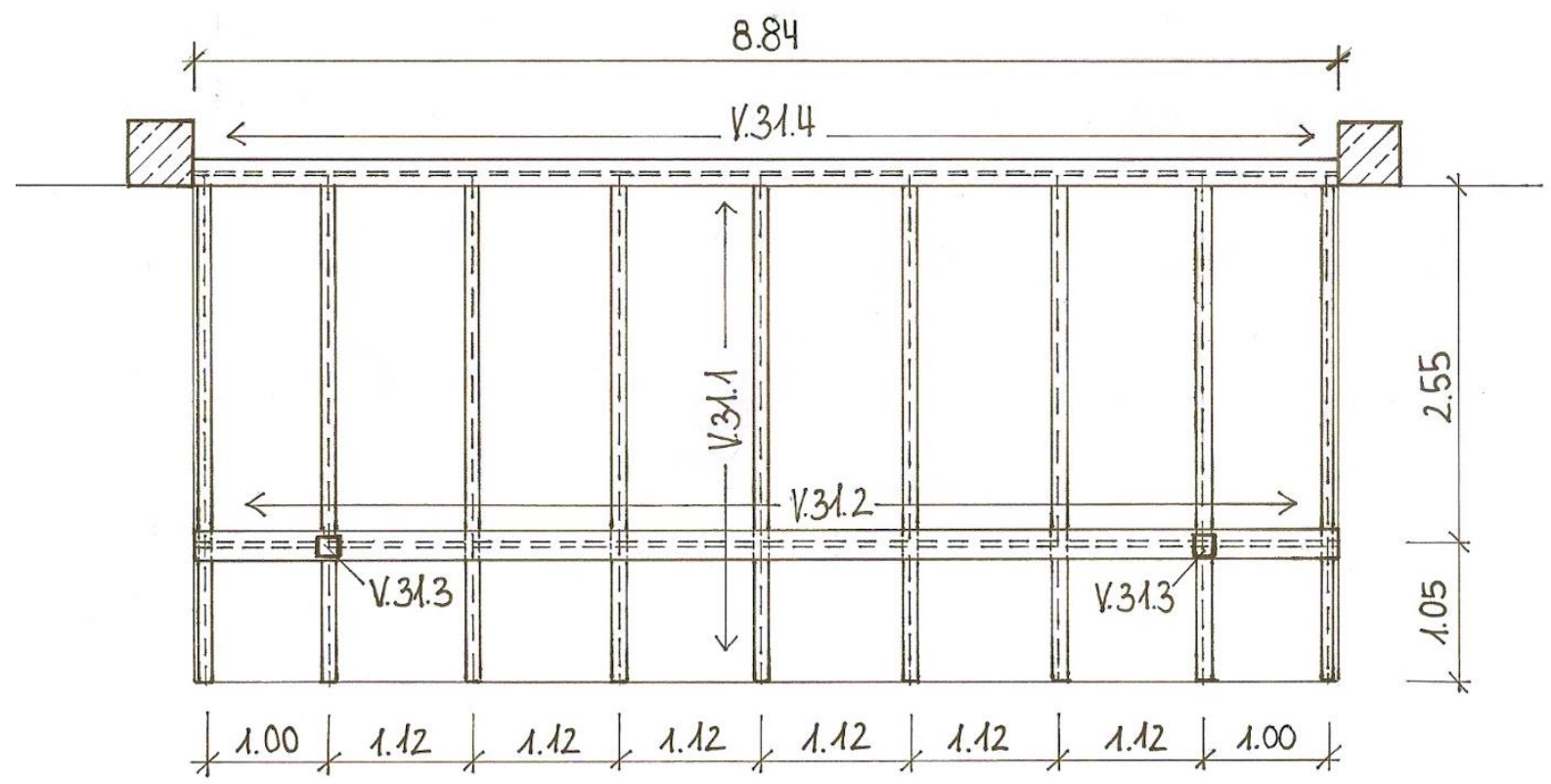




\subsection{DESIGN CALCULATIONS}

\subsubsection{POSITION V.31.1}

System

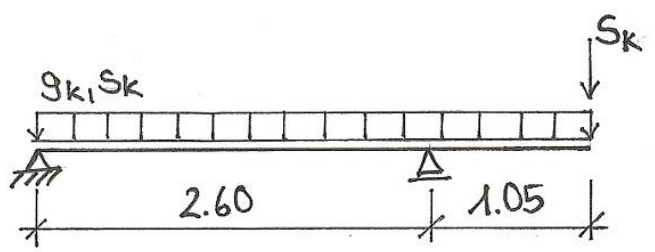

Cross-section

$2 \times$ L100x50x6 (S 235)

\begin{tabular}{|c|c|c|c|c|c|c|c|}
\hline $\begin{array}{c}\mathbf{a} \\
{[\mathbf{m m}]}\end{array}$ & $\begin{array}{c}\mathbf{b} \\
{[\mathbf{m m}]}\end{array}$ & $\begin{array}{c}\mathbf{t} \\
{[\mathbf{m m}]}\end{array}$ & $\begin{array}{c}\mathbf{r} \\
{[\mathbf{m m}]}\end{array}$ & $\begin{array}{c}\mathbf{A} \\
{\left[\mathbf{c m}^{2}\right]}\end{array}$ & $\begin{array}{c}\mathbf{I}_{\mathbf{y}} \\
{\left[\mathbf{c m}^{3}\right]}\end{array}$ & $\begin{array}{c}\mathbf{W}_{\mathrm{el}, \mathbf{y}} \\
{\left[\mathbf{c m}^{3}\right]}\end{array}$ & $\begin{array}{c}\mathbf{9} \mathbf{k} \\
{[\mathbf{k N} / \mathbf{m}]}\end{array}$ \\
\hline 100 & 50 & 6 & 8 & 8.71 & 89.9 & 13.8 & 0.0684 \\
\hline
\end{tabular}

Characteristic loads

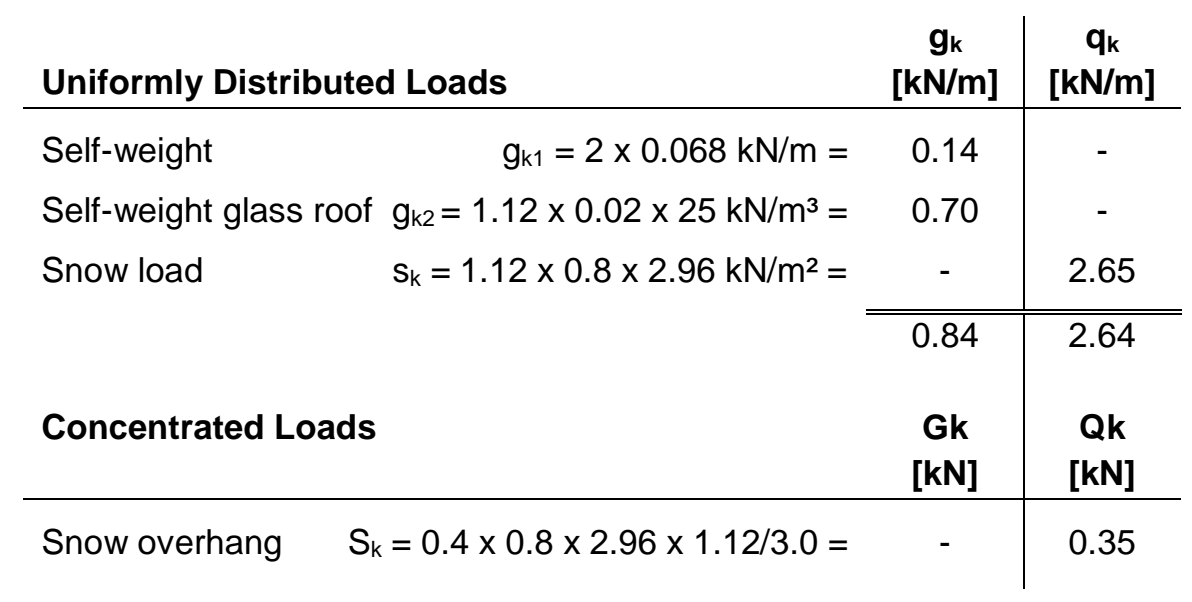

Design loads

LC1: $\quad q_{E d}=1.35 \cdot g_{k}+1.50 \cdot s_{k}=1.35 \cdot 0.84+1.50 \cdot 2.64=5.09 \mathrm{kN} / \mathrm{m}$ $Q_{E d}=1.50 \cdot S_{k}=1.50 \cdot 0.35=0.53 \mathrm{kN}$

LC2: $\quad q_{E d}=1.00 \cdot g_{k}+0 \cdot s_{k}=1.00 \cdot 0.84=0.84 \mathrm{kN} / \mathrm{m}$

$Q_{E d}=0 \cdot S_{k}=0 \mathrm{kN}$ 
Internal Forces

\section{Flexure moment}

\begin{tabular}{|lc|c|c|c|}
\cline { 2 - 5 } \multicolumn{1}{c|}{} & $\begin{array}{c}\mathbf{M}_{\mathbf{g k}} \\
{[\mathbf{k N m}]}\end{array}$ & $\begin{array}{c}\mathbf{M}_{\mathbf{s k}} \\
{[\mathbf{k N m}]}\end{array}$ & $\begin{array}{c}\min \mathbf{M}_{\mathrm{Ed}} \\
{[\mathbf{k N m}]}\end{array}$ & $\begin{array}{c}\max \mathbf{M}_{\mathrm{Ed}} \\
{[\mathbf{k N m}]}\end{array}$ \\
\hline Span & 0.50 & 1.42 & 2.80 & 4.09 \\
\hline Cantilever & -0.46 & -1.83 & -0.46 & -3.37 \\
\hline
\end{tabular}

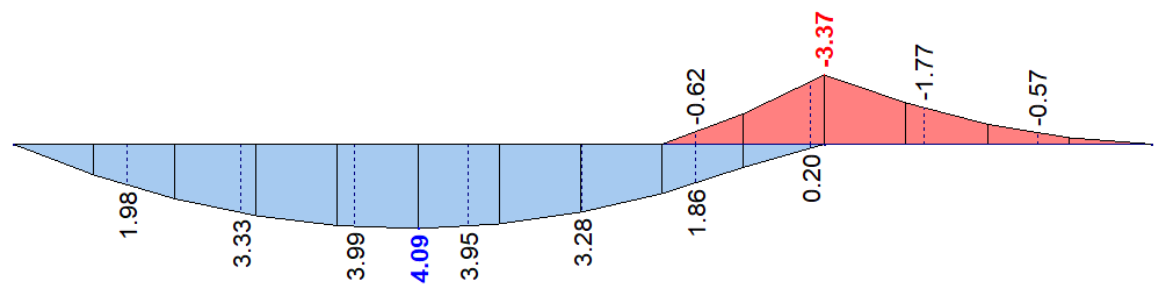

Shear force

\begin{tabular}{|l|c|c|c|c|}
\cline { 2 - 5 } \multicolumn{1}{c|}{} & $\begin{array}{c}\mathbf{V}_{\mathbf{g k}} \\
{[\mathbf{k N}]}\end{array}$ & $\begin{array}{c}\mathbf{V}_{\mathbf{s k}} \\
{[\mathbf{k N}]}\end{array}$ & $\begin{array}{c}\min \mathbf{V}_{\mathbf{E d}} \\
{[\mathbf{k N}]}\end{array}$ & $\begin{array}{c}\max \mathbf{V M}_{\mathbf{E d}} \\
{[\mathbf{k N}]}\end{array}$ \\
\hline Support A & 0.91 & 2.74 & 5.35 & 6.46 \\
\hline Support $\mathrm{B}_{\mathbf{L}}$ & -1.27 & -4.15 & -6.82 & -7.94 \\
\hline Support $\mathrm{B}_{\mathbf{R}}$ & 0.88 & 3.13 & 0.88 & 5.89 \\
\hline
\end{tabular}

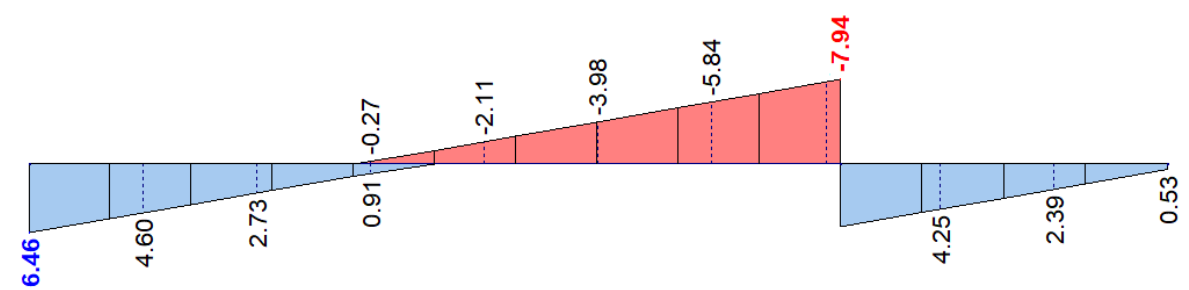

Ultimate Limit State Design

Plastic internal forces

$N_{p l}=205 \mathrm{kN} \quad M_{p l, y, R d}=6.00 \mathrm{kNm} \quad V_{p l, z, R d}=66.0 \mathrm{kN}$

Maximum flexure moment

$$
\frac{M_{E d}}{M_{p l, R d}}=\frac{4.09}{6.00}=0.68<1.00 \checkmark
$$

Maximum shear force

$$
\frac{V_{E d}}{V_{p l, z, R d}}=\frac{7.94 \mathrm{kN}}{66.0}=0.12<1.00<0.50 \checkmark \rightarrow \text { No M-N-interaction necessary! }
$$

Maximum Deflection

Span

$\max f=5.0 \mathrm{~mm}<L / 300=2600 / 300=8.7 \mathrm{~mm} \rightarrow \eta=0.57$

Cantilever

$$
\max f=5.7 \mathrm{~mm}<L / 150=1050 / 150=7.0 \mathrm{~mm} \rightarrow \eta=0.81
$$




\subsubsection{POSITION V.31.2}

System

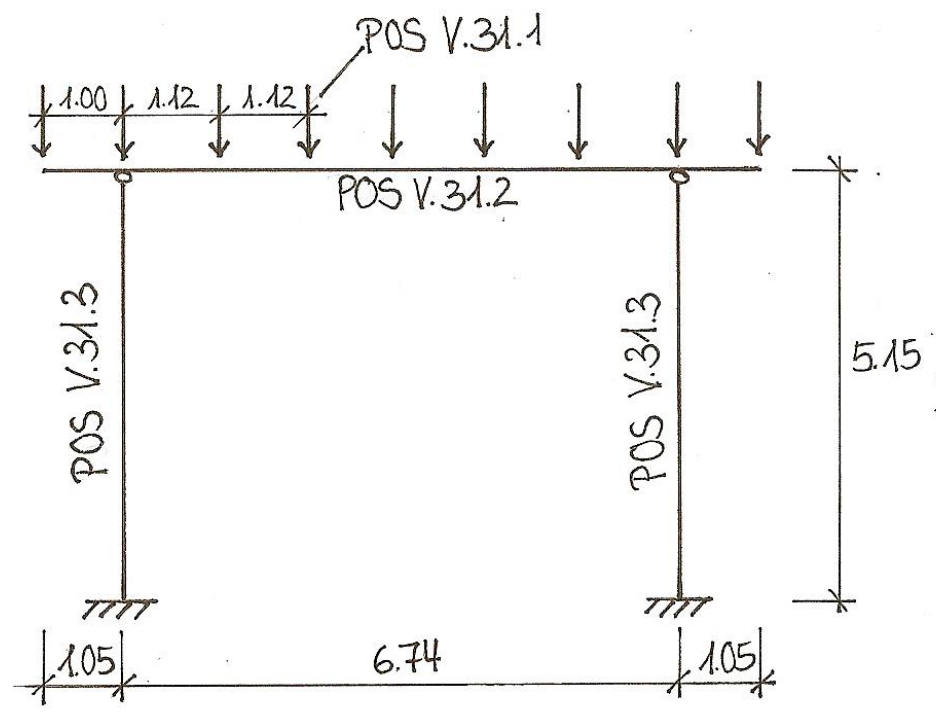

Cross-section

IPE 300 (S235)

\begin{tabular}{|c|c|c|c|c|c|c|c|}
\hline $\begin{array}{c}\mathbf{h} \\
{[\mathbf{m m}]}\end{array}$ & $\begin{array}{c}\mathbf{b} \\
{[\mathbf{m m}]}\end{array}$ & $\begin{array}{c}\mathbf{t}_{\mathbf{w}} \\
{[\mathbf{m m}]}\end{array}$ & $\begin{array}{c}\mathbf{t}_{\mathbf{f}} \\
{[\mathbf{m m}]}\end{array}$ & $\begin{array}{c}\mathbf{A} \\
{\left[\mathbf{c m}^{2}\right]}\end{array}$ & $\begin{array}{c}\mathbf{I}_{\mathbf{y}} \\
{\left[\mathbf{c m}^{3}\right]}\end{array}$ & $\begin{array}{c}\mathbf{W}_{\mathrm{el}, \mathbf{y}} \\
{\left[\mathbf{c m}^{3}\right]}\end{array}$ & $\begin{array}{c}\mathbf{g}_{\mathbf{k}} \\
{[\mathbf{k N} / \mathbf{m}]}\end{array}$ \\
\hline 300 & 150 & 7.1 & 10.7 & 53.8 & 8360 & 557 & 0.422 \\
\hline
\end{tabular}

Characteristic loads

\begin{tabular}{lc|c} 
Uniformly Distributed Loads & $\begin{array}{c}\mathbf{g}_{\mathbf{k}} \\
{[\mathbf{k N} / \mathbf{m}]}\end{array}$ & $\begin{array}{c}\mathbf{q}_{\mathbf{k}} \\
{[\mathbf{k N} / \mathbf{m}]}\end{array}$ \\
\hline Self-weight & - & - \\
& & \\
& (generated by the software) & \\
Concentrated Loads & $\begin{array}{c}\mathbf{G k} \\
{[\mathbf{k N}]}\end{array}$ & $\begin{array}{c}\mathbf{Q k} \\
{[\mathbf{k N}]}\end{array}$ \\
\hline Reaction forces from POS. V.31.1 & 2.15 & 7.28
\end{tabular}

Design loads

$Q_{E d}=1.35 \cdot G_{k}+1.50 \cdot Q_{k}=1.35 \cdot 2.15+1.50 \cdot 7.28=14.42 \mathrm{kN}$ 
Internal Forces

\section{Flexure moment}

\begin{tabular}{|l|c|c|c|}
\cline { 2 - 4 } \multicolumn{1}{c|}{} & $\mathbf{M}_{\mathbf{g k}}[\mathbf{k N m}]$ & $\mathbf{M}_{\mathbf{s k}}[\mathbf{k N m}]$ & $\mathbf{M}_{\mathrm{Ed}}[\mathbf{k N m}]$ \\
\hline Span & 10.78 & 29.16 & 58.28 \\
\hline Cantilever & -2.49 & -7.64 & -14.83 \\
\hline
\end{tabular}

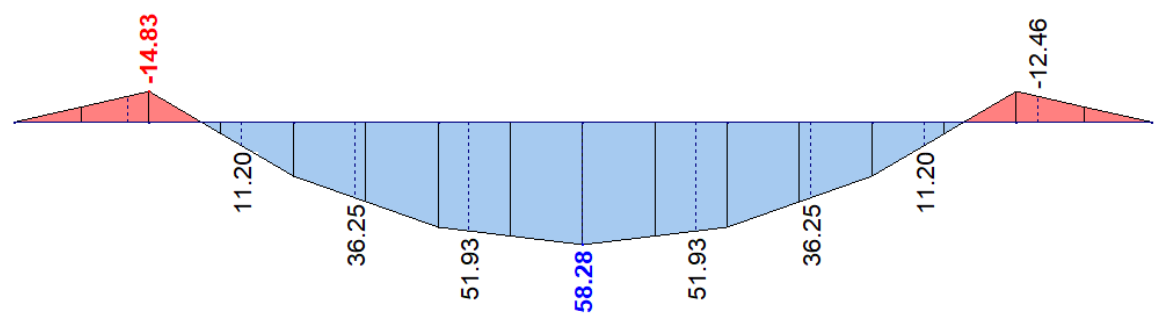

Shear force

\begin{tabular}{|l|c|c|c|}
\cline { 2 - 4 } \multicolumn{1}{c|}{} & $\mathbf{V}_{\mathbf{g k}}[\mathbf{k N}]$ & $\mathbf{V}_{\text {sk }}[\mathbf{k N}]$ & $\mathbf{V}_{\mathrm{Ed}}[\mathbf{k N}]$ \\
\hline Support $\mathbf{A}_{\mathbf{L}}$ & -2.59 & -7.28 & -14.33 \\
\hline Support $\mathbf{A}_{\mathbf{R}}$ & 6.80 & 18.20 & 36.48 \\
\hline
\end{tabular}

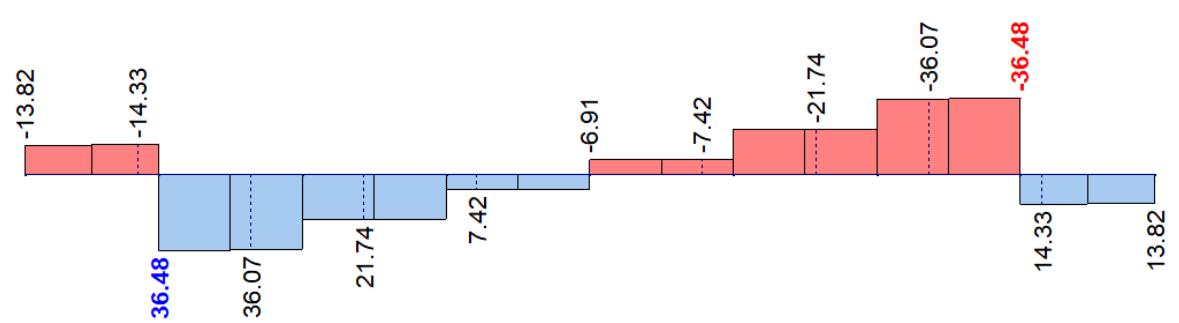

Ultimate Limit State Design

Plastic internal forces

$$
N_{p l}=2656 k N \quad M_{p l, y, R d}=325 k N m \quad V_{p l, z, R d}=512 k N
$$

Maximum flexure moment

$$
\frac{M_{E d}}{M_{p l, R d}}=\frac{58.3 \mathrm{kNm}}{325 \mathrm{kNm}}=0.18<1.00^{\checkmark}
$$

Maximum shear force

$$
\frac{V_{E d}}{V_{p l, z, R d}}=\frac{36.5 \mathrm{kN}}{512 \mathrm{kN}}=0.07<1.00<0.50 \checkmark \rightarrow \text { No M-N-interaction necessary! }
$$

Maximum Deflection

$$
\max f=15.6 \mathrm{~mm}<L / 300=6740 / 300=22.5 \mathrm{~mm} \rightarrow \eta=0.69
$$




\subsubsection{POSITION V.31.3}

System

See Chapter 20.2.2

Cross-section QRO 100x5 (S235)

\begin{tabular}{|c|c|c|c|c|c|}
\hline $\begin{array}{c}\mathbf{b} \\
{[\mathbf{m m}]}\end{array}$ & $\begin{array}{c}\mathbf{t} \\
{[\mathbf{m m}]}\end{array}$ & $\begin{array}{c}\mathbf{A} \\
{\left[\mathbf{c m}^{2}\right]}\end{array}$ & $\begin{array}{c}\mathbf{I}_{\mathbf{y}} \\
{\left[\mathrm{cm}^{3}\right]}\end{array}$ & $\begin{array}{c}\mathbf{i} \\
{[\mathbf{c m}]}\end{array}$ & $\begin{array}{c}\mathbf{9 k} \\
{[\mathbf{k N} / \mathbf{m}]}\end{array}$ \\
\hline 100 & 5 & 18.4 & 271 & 3.84 & 0.144 \\
\hline
\end{tabular}

Internal Forces

Normal force

\begin{tabular}{|c|c|c|}
\hline $\mathbf{N}_{\mathbf{g k}}[\mathbf{k N}]$ & $\mathbf{N}_{\mathbf{s k}}[\mathbf{k N}]$ & $\mathbf{N}_{\mathrm{Ed}}[\mathbf{k N}]$ \\
\hline-12.46 & -32.76 & -69.95 \\
\hline
\end{tabular}

Ultimate Limit State Design

Buckling length

Reduction coefficient

Maximum normal force

Utilisation
Simplified assumption: $\beta \approx 2.7$ (see Figure 30 )

$L_{c r}=\beta \cdot L_{0}=2.7 \cdot 5.15=13.9 m$

$\bar{\lambda}=\frac{L_{c r}}{93.9 \cdot i}=\frac{13.9 m}{93.9 \cdot 3.84}=0.039$

Imperfection coefficient $\alpha=0.49$

$\phi=0.5 \cdot\left[1+\alpha \cdot(\bar{\lambda}-0.2)+\bar{\lambda}^{2}\right]$

$=0.5 \cdot\left[1+0.49 \cdot(0.039-0.2)+0.039^{2}\right]$

$=0.46$

$\chi=\frac{1}{\phi+\sqrt{\phi^{2}-\bar{\lambda}^{2}}}$

$=\frac{1}{0.46+\sqrt{0.46^{2}-0.039^{2}}}=1.09 \leq \underline{\underline{1.0}}$

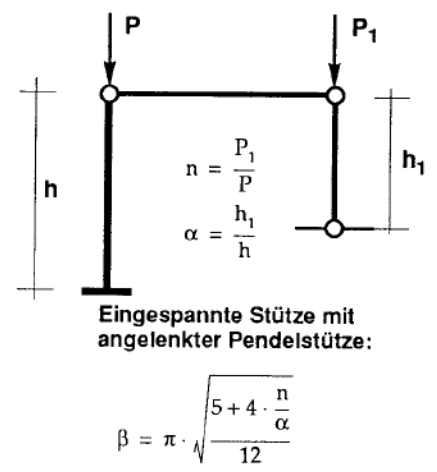

Sonderfall: $P_{1}=P$ und $h_{1}=h->B=2,7$

$N_{c r, R d}=\chi \cdot N_{\rho l, R d}=1.0 \cdot 18.4 \cdot 23.5=432.4 \mathrm{kN}$

$\eta=\frac{N_{E d}}{N_{c r, R d}}=\frac{69.95}{432.4}=0.16<1.00 \checkmark$ 


\subsubsection{POSITION V.31.4}

System

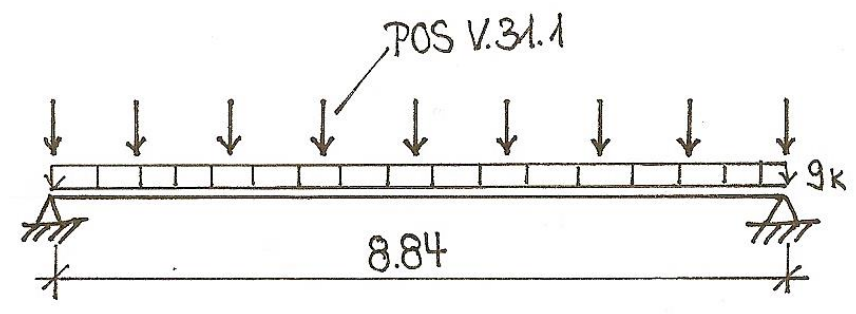

Cross-section

IPE270 (S235)

\begin{tabular}{|c|c|c|c|c|c|c|c|}
\hline $\begin{array}{c}\mathbf{h} \\
{[\mathbf{m m}]}\end{array}$ & $\begin{array}{c}\mathbf{b} \\
{[\mathbf{m m}]}\end{array}$ & $\begin{array}{c}\mathbf{t}_{\mathbf{w}} \\
{[\mathbf{m m}]}\end{array}$ & $\begin{array}{c}\mathbf{t}_{\mathbf{f}} \\
{[\mathbf{m m}]}\end{array}$ & $\begin{array}{c}\mathbf{A} \\
{\left[\mathbf{c m}^{2}\right]}\end{array}$ & $\begin{array}{c}\mathbf{I}_{\mathbf{y}} \\
{\left[\mathbf{c m}^{3}\right]}\end{array}$ & $\begin{array}{c}\mathbf{W}_{\text {el,y }} \\
{\left[\mathbf{c m}^{3}\right]}\end{array}$ & $\begin{array}{c}\mathbf{g}_{\mathbf{k}} \\
{[\mathbf{k N} / \mathbf{m}]}\end{array}$ \\
\hline 270 & 135 & 6.6 & 10.2 & 45.9 & 5790 & 429 & 0.361 \\
\hline
\end{tabular}

Characteristic loads

\begin{tabular}{lc|c} 
Uniformly Distributed Loads & $\begin{array}{c}\mathbf{g}_{\mathbf{k}} \\
{[\mathbf{k N} / \mathbf{m}]}\end{array}$ & $\begin{array}{c}\mathbf{q}_{\mathbf{k}} \\
{[\mathbf{k N} / \mathbf{m}]}\end{array}$ \\
\hline Self-weight & - & - \\
& & \\
Concentrated Loads & $\begin{array}{c}\mathbf{G k} \\
{[\mathbf{k N}]}\end{array}$ & $\begin{array}{c}\mathbf{Q k} \\
{[\mathbf{k N}]}\end{array}$ \\
\hline Reaction forces from POS. V.31.1 & 0.91 & 2.74
\end{tabular}

Design loads

$$
Q_{E d}=1.35 \cdot G_{k}+1.50 \cdot S_{k}=1.35 \cdot 0.91+1.50 \cdot 2.74=5.34 k N
$$

Internal Forces

\section{Flexure moment}

\begin{tabular}{|c|c|c|}
\hline $\mathbf{M}_{\mathbf{g k}}[\mathbf{k N m}]$ & $\mathbf{M}_{\mathbf{s k}}[\mathbf{k N m}]$ & $\mathbf{M}_{\mathrm{Ed}}[\mathbf{k N m}]$ \\
\hline 11.46 & 23.92 & 51.36 \\
\hline
\end{tabular}

Shear force

\begin{tabular}{|c|c|c|}
\hline $\mathbf{V}_{\mathbf{g k}}[\mathbf{k N}]$ & $\mathbf{V}_{\mathbf{s k}}[\mathbf{k N}]$ & $\mathbf{V}_{\mathrm{Ed}}[\mathbf{k N}]$ \\
\hline 4.78 & 9.59 & 20.32 \\
\hline
\end{tabular}


Ultimate Limit State Design

Plastic internal forces $\quad N_{p l}=1079 \mathrm{kN} \quad M_{p l, y, R d}=113.7 \mathrm{kNm} \quad V_{p l, z, R d}=299.8 \mathrm{kN}$

Maximum flexure moment $\quad \frac{M_{E d}}{M_{p l, R d}}=\frac{51.36 \mathrm{kNm}}{113.7 \mathrm{kNm}}=0.45<1.00 \checkmark$

Maximum shear force

$$
\frac{V_{E d}}{V_{p l, z, R d}}=\frac{20.32 \mathrm{kN}}{229.8 \mathrm{kN}}=0.07<1.00<0.50 \checkmark \rightarrow \text { No M-N-interaction necessary! }
$$

Maximum Deflection

$$
\max f=23.7 \mathrm{~mm}<L / 300=8840 / 300=29.5 \mathrm{~mm} \rightarrow \eta=0.80
$$




\section{APPENDICES}

\section{APPENDIX A DESIGN DRAWINGS}

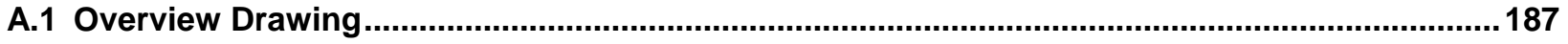

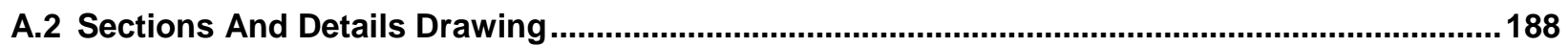

A.3 Formwork and Reinforcement Plan of a Prestressed Concrete Truss .................................... 189

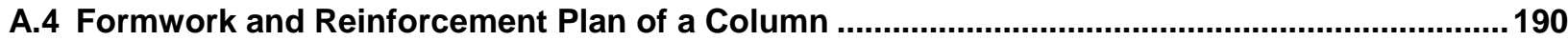

A.5 Formwork and Reinforcement Plan of a Double-Tee Slab ...................................................191

A.6 Formwork and Reinforcement Plan of Part of the Slab ....................................................... 192

A.7 Formwork and Reinforcement Plan of a Concrete Beam .....................................................193

A.8 Formwork and Reinforcement Plan of a Column Footing ........................................................ 194 


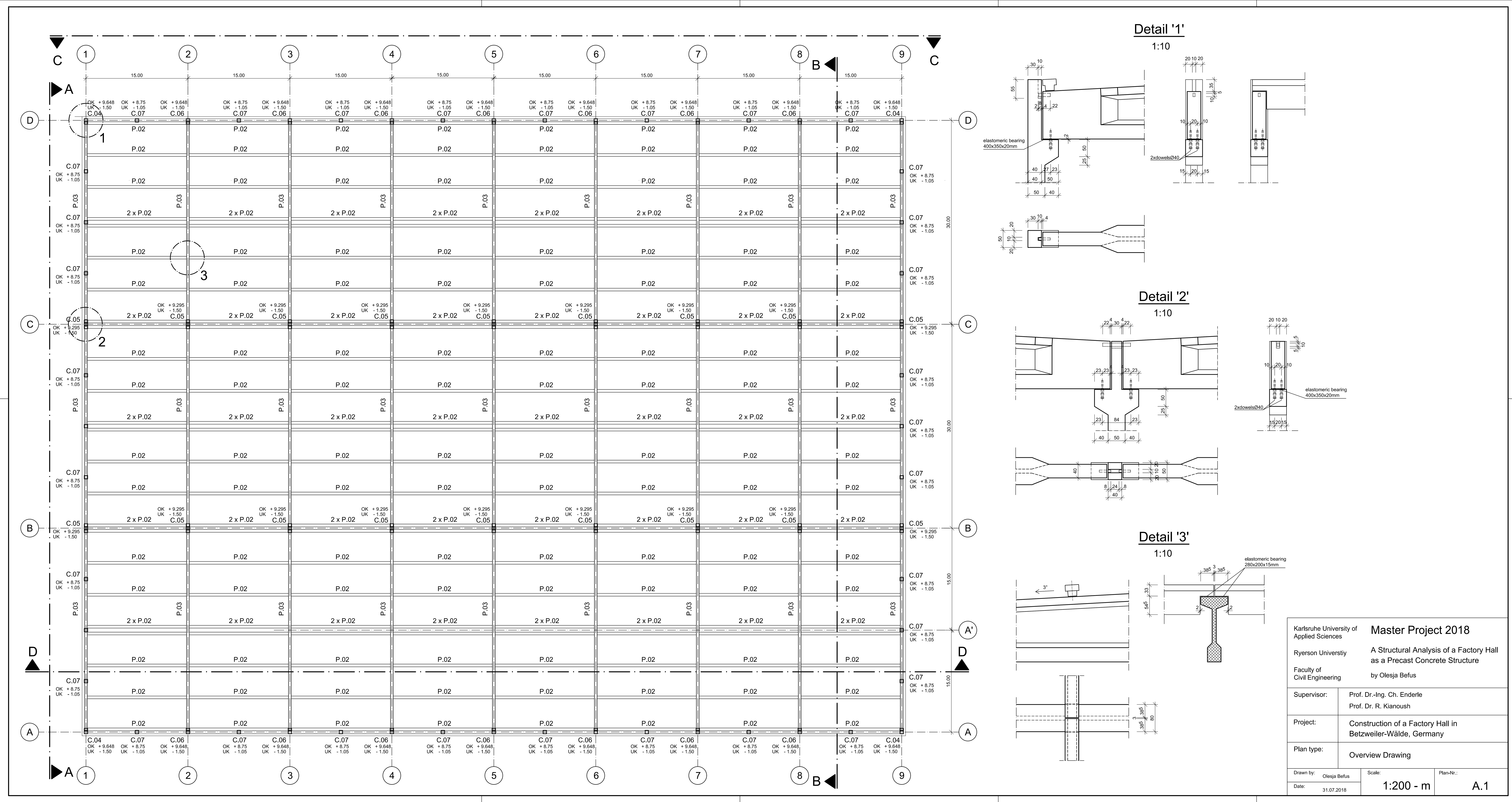




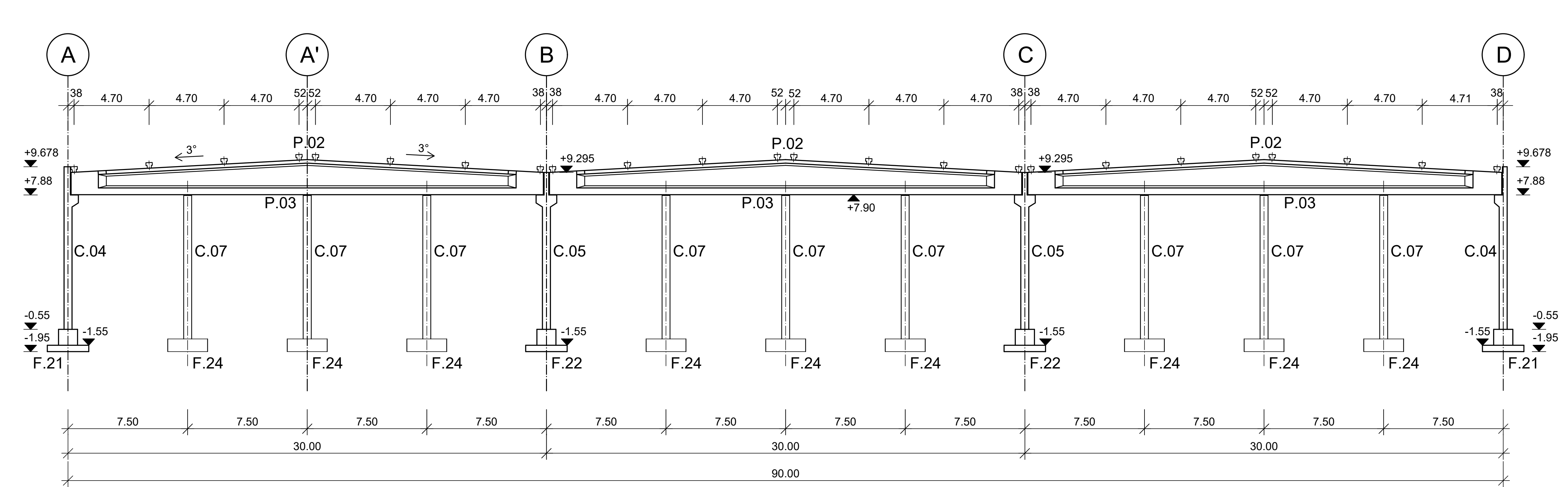

View C-C

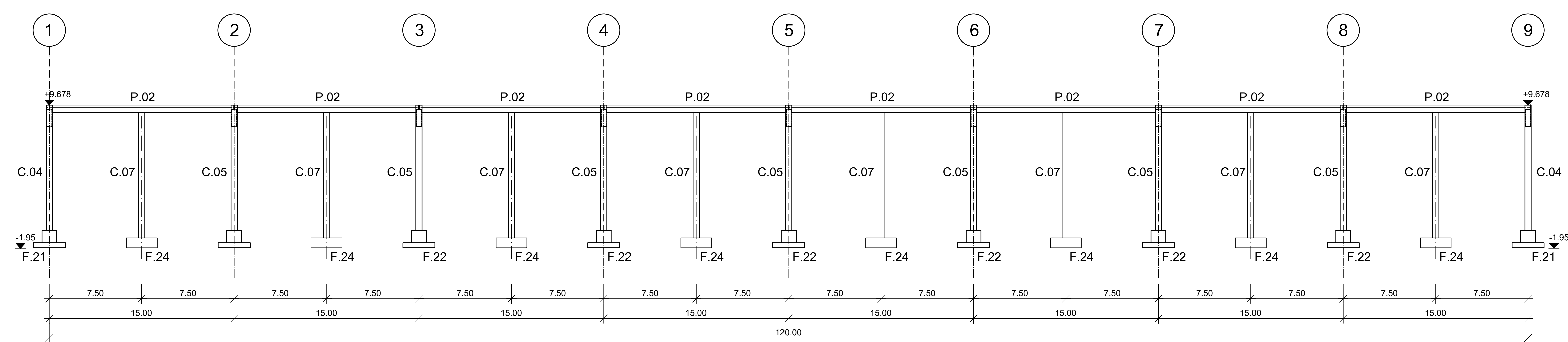

Section D-D

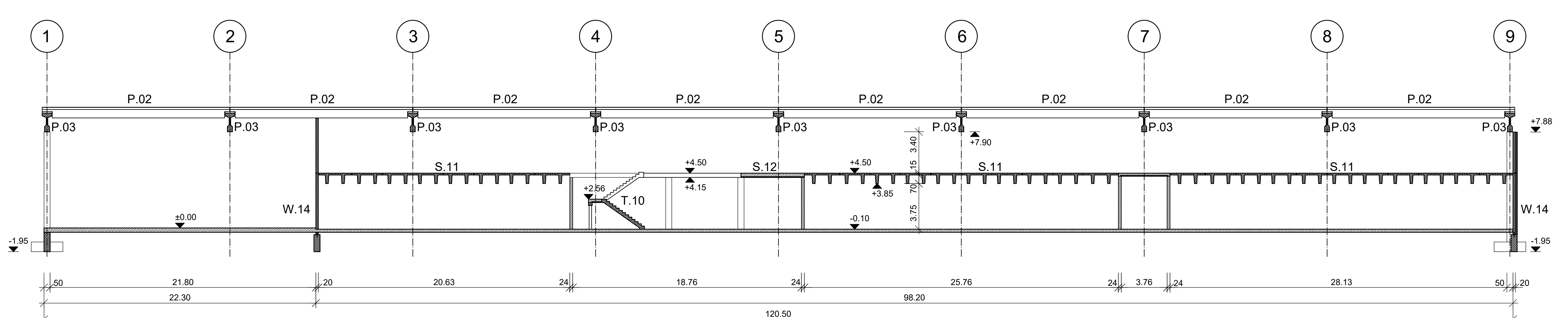

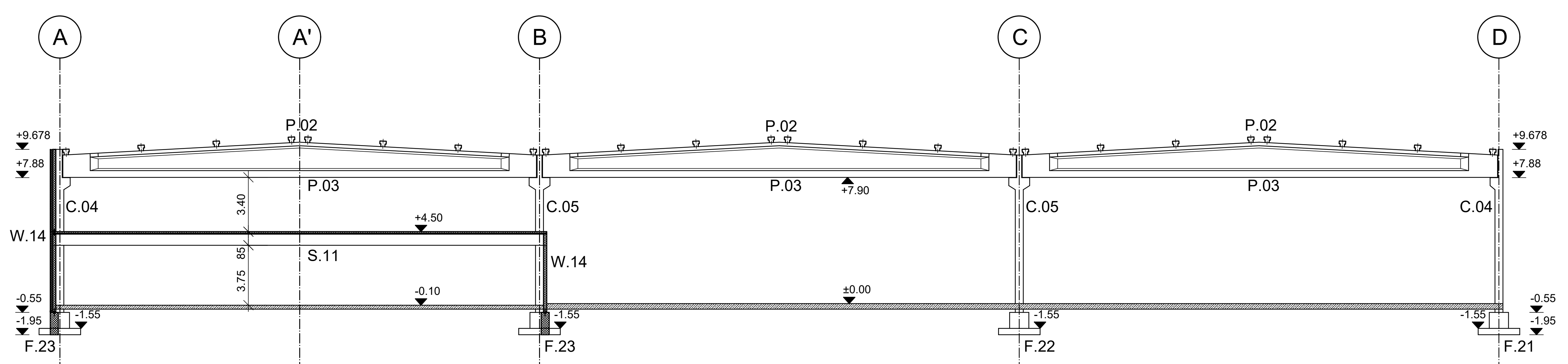

Overview Drawing see Plan Nr. A.1

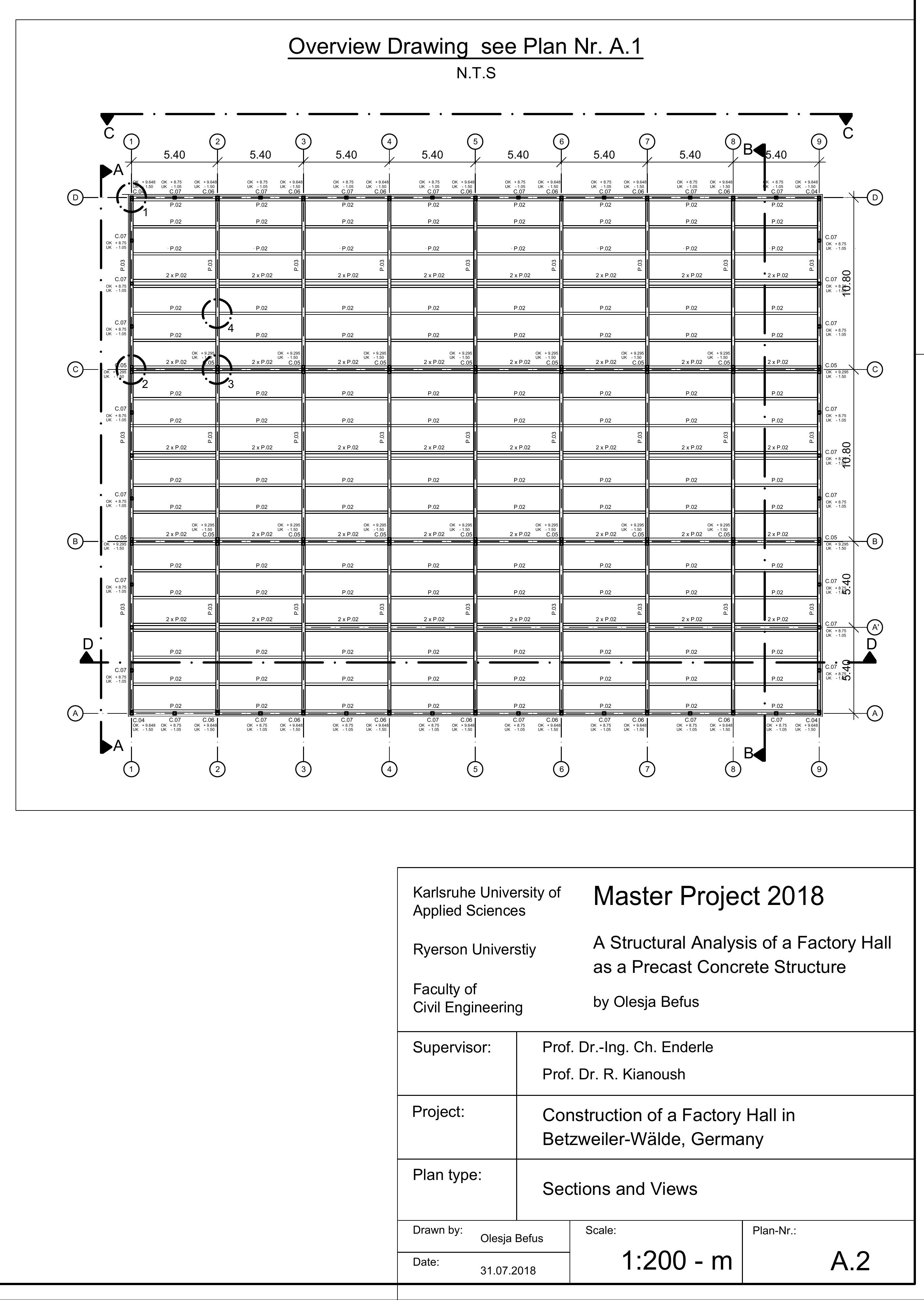



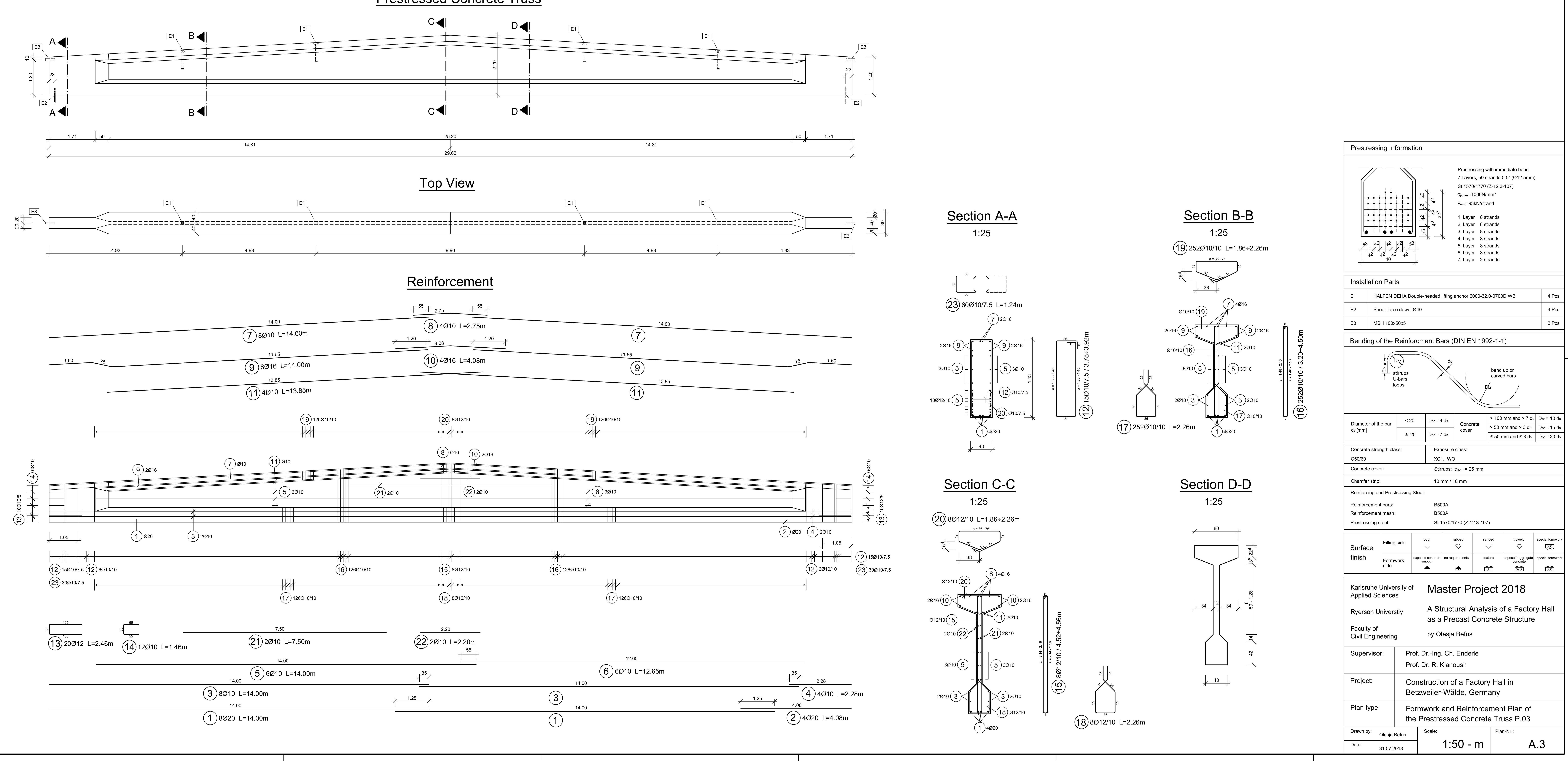
Column C.05

${ }_{141}^{216} y^{50} y^{25} t^{2}$

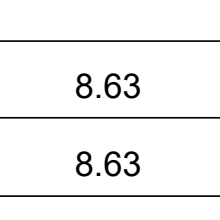

$\sqrt{2+1}$
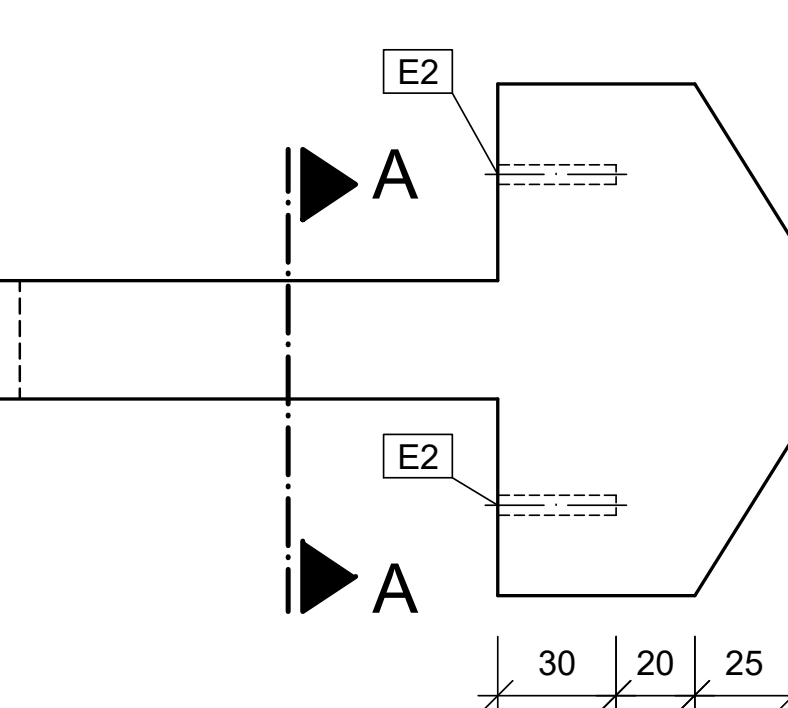

$\overbrace{8}^{\frac{8}{8}}$
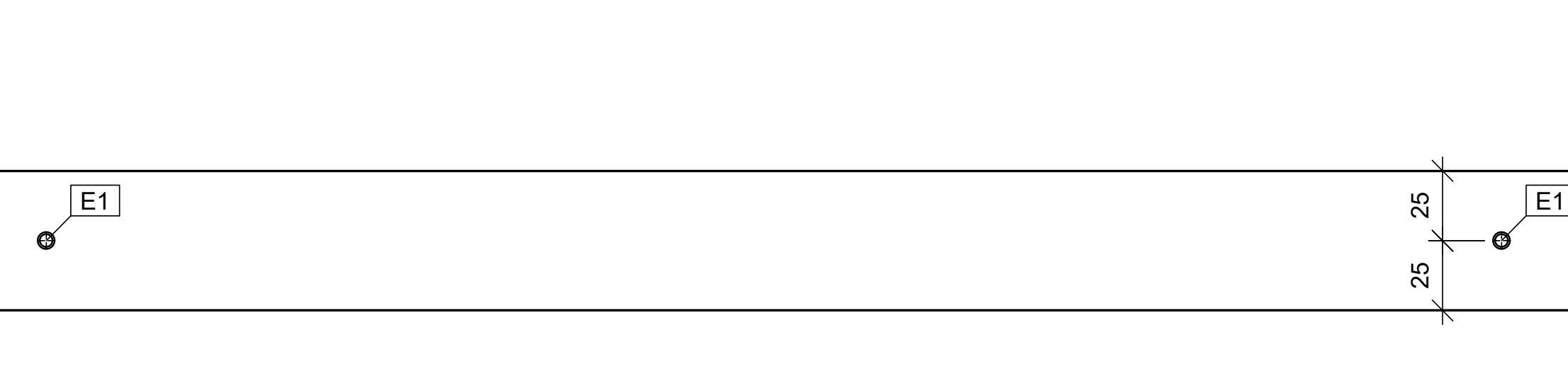

$\rightarrow x^{\text {En }}$

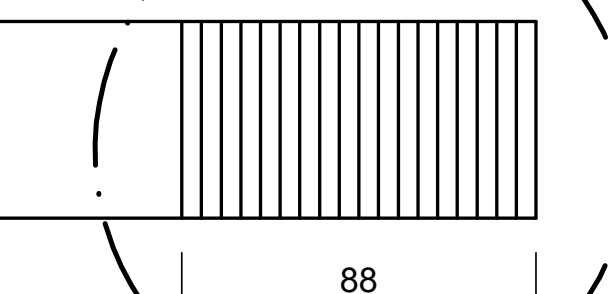

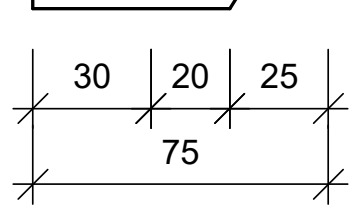

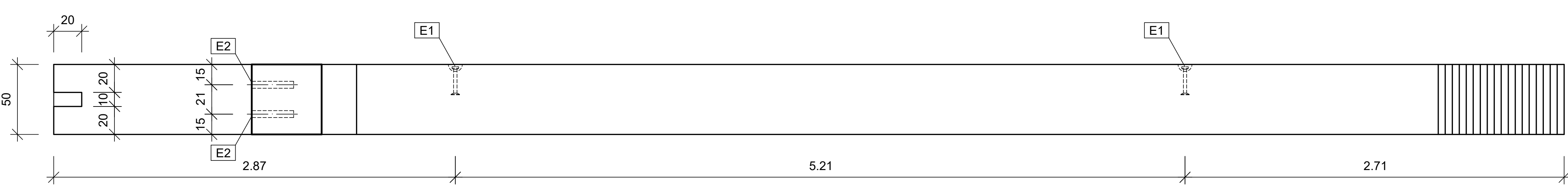

Reinforcement
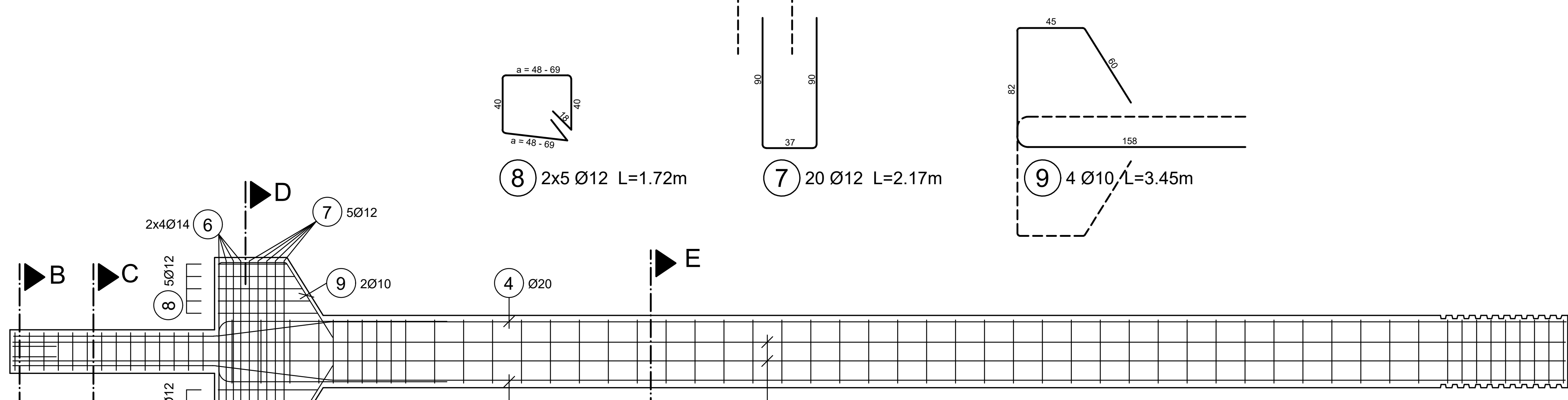

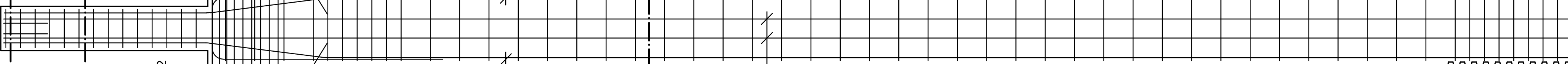

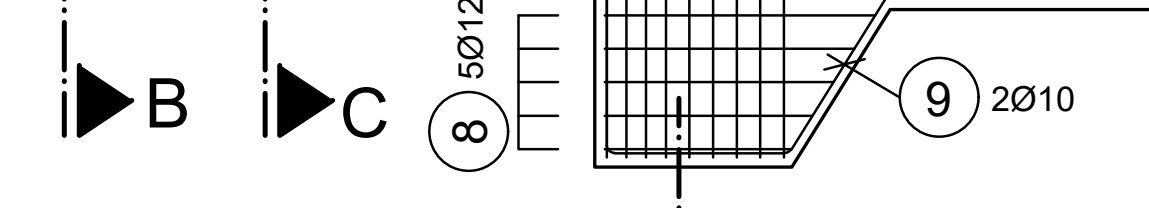
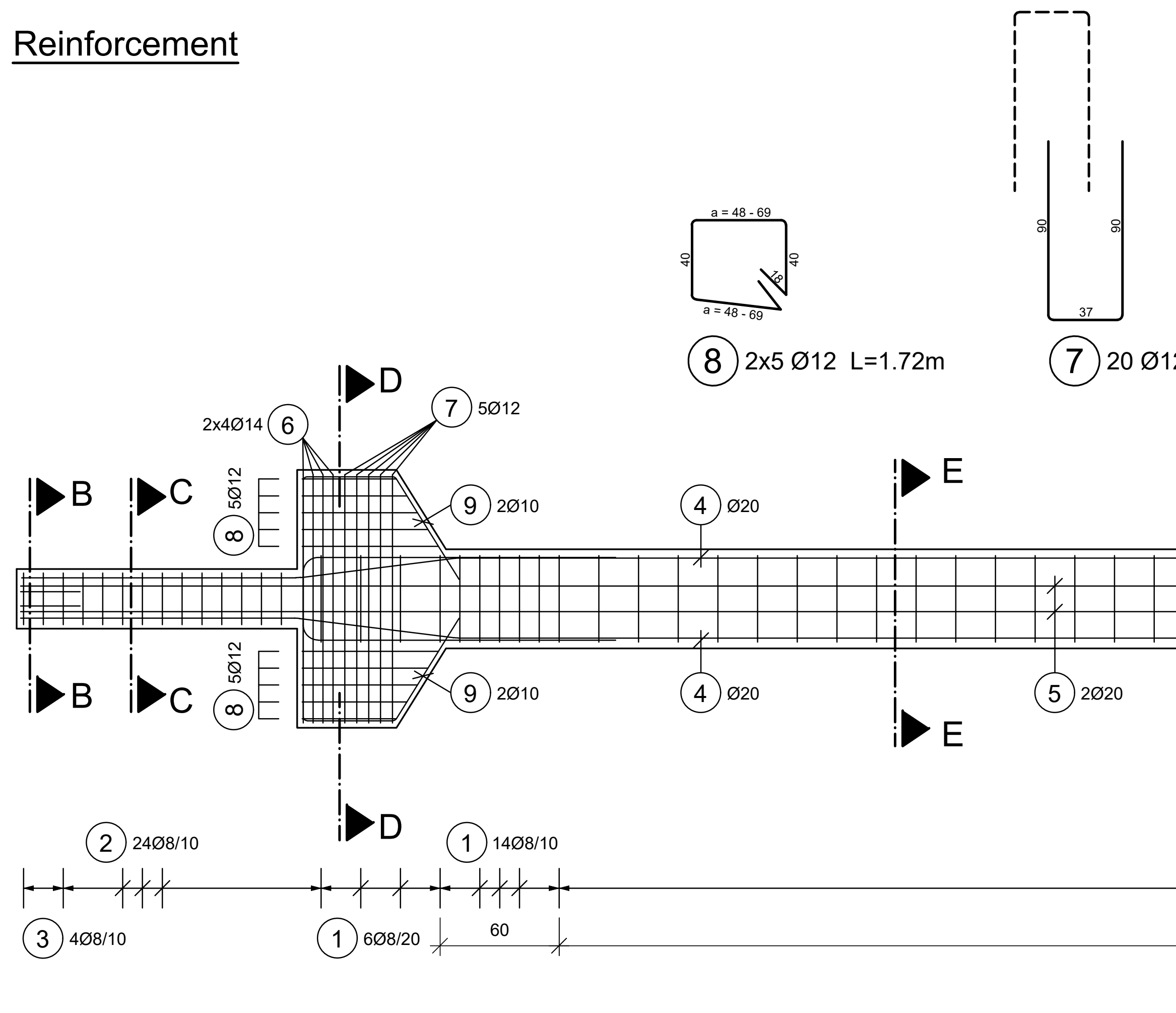

(3) 488110

(1) 180810

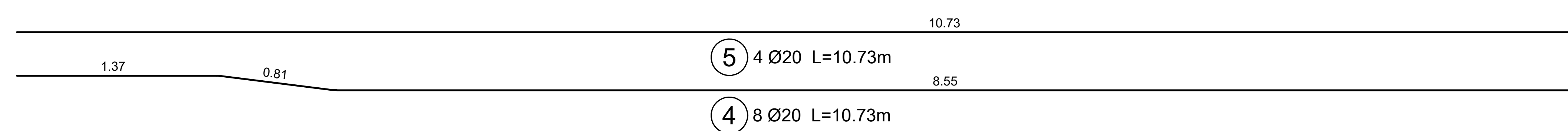

Section A-A
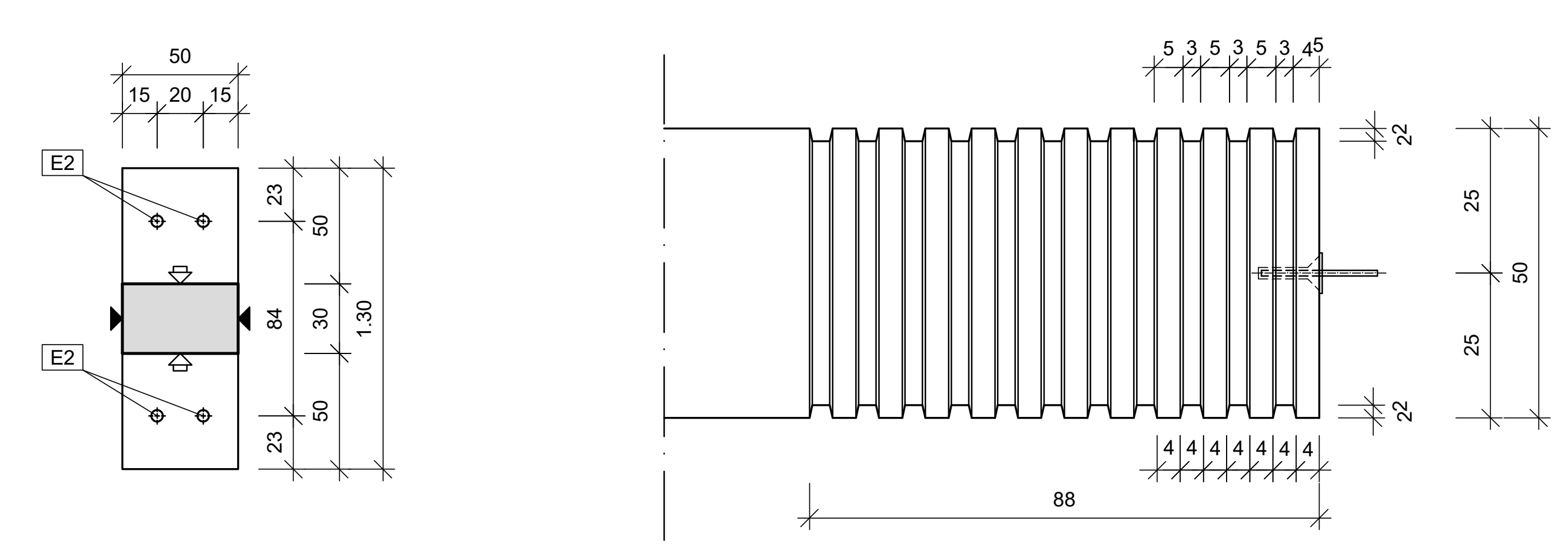

$$
\frac{\text { Section B-B }}{\text { (3) } 8.88110 \mathrm{~L}=1.04 \mathrm{~m}}
$$

Section D-D

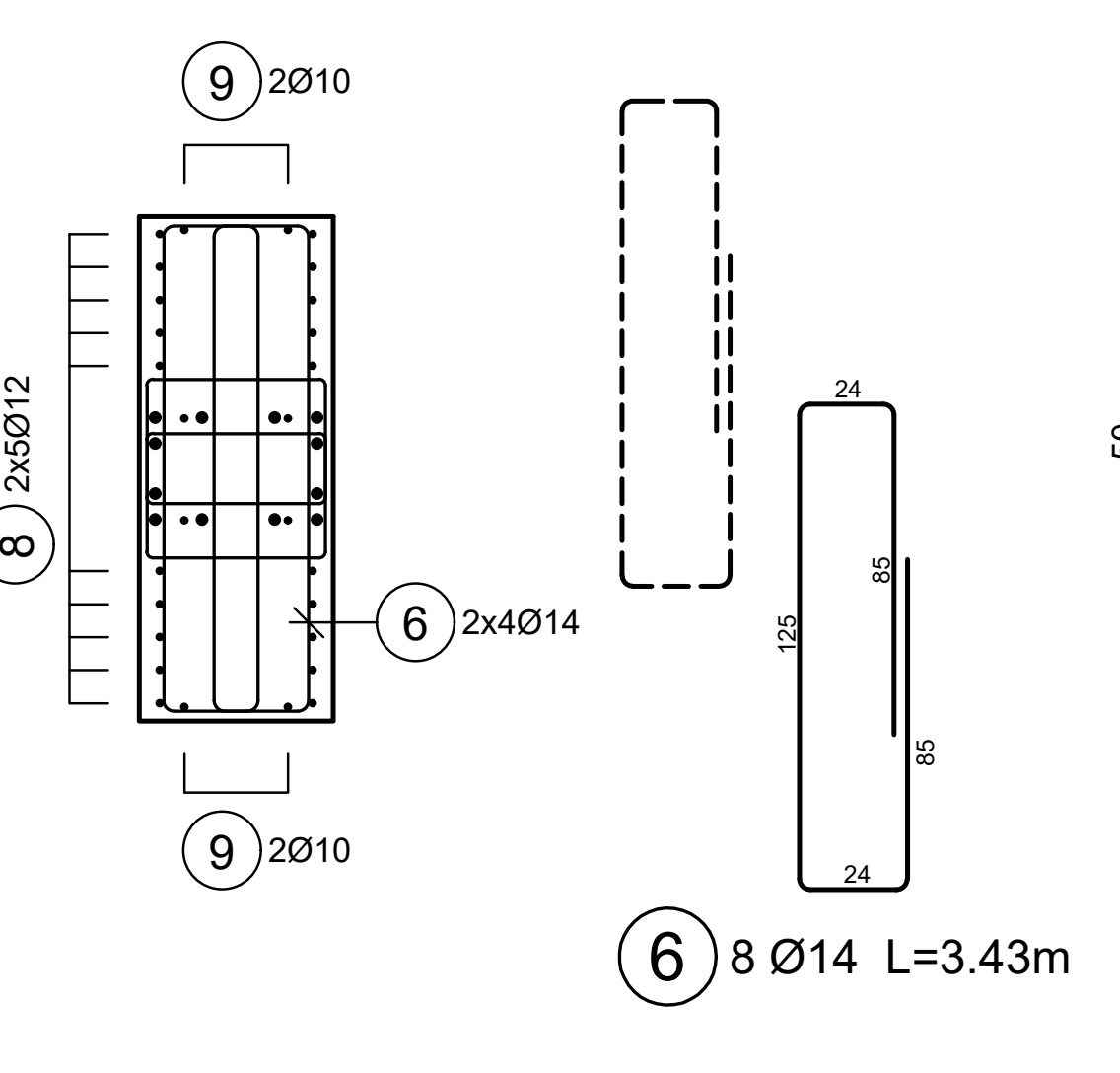

$\underline{\text { Section C-C }}$

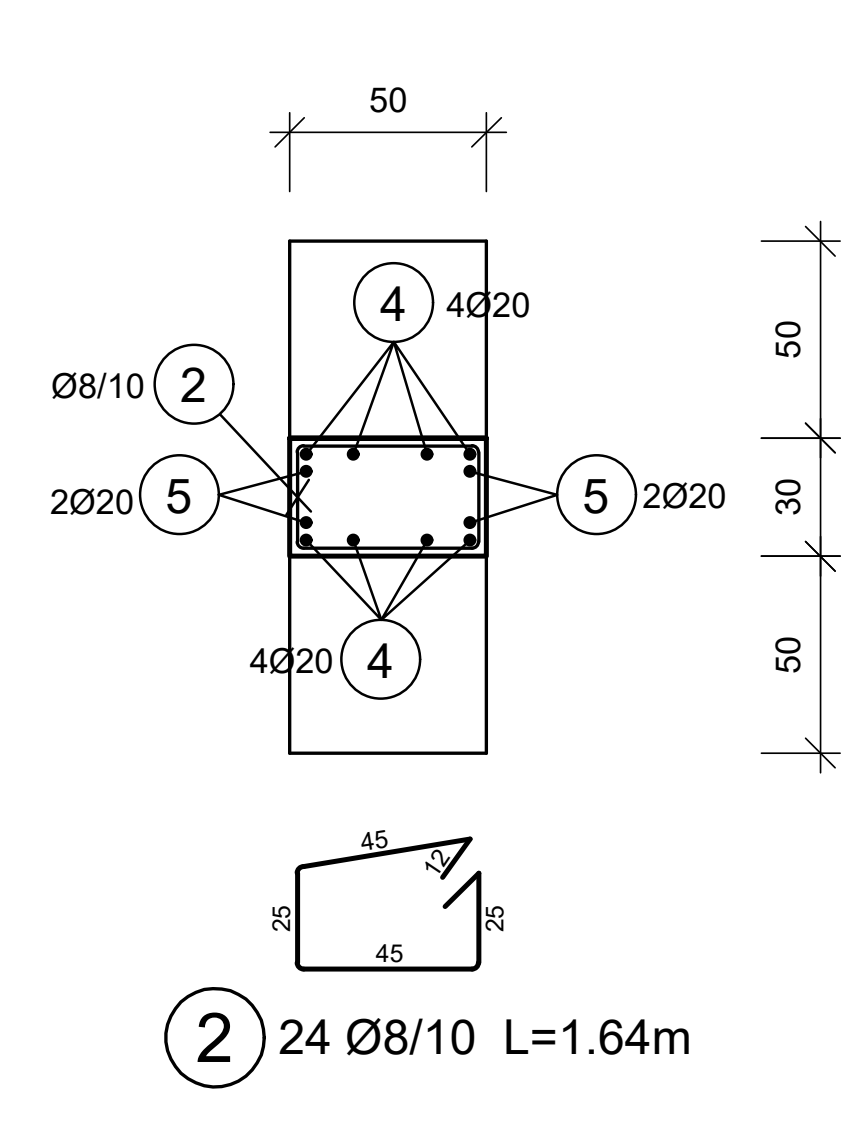

Section E-E

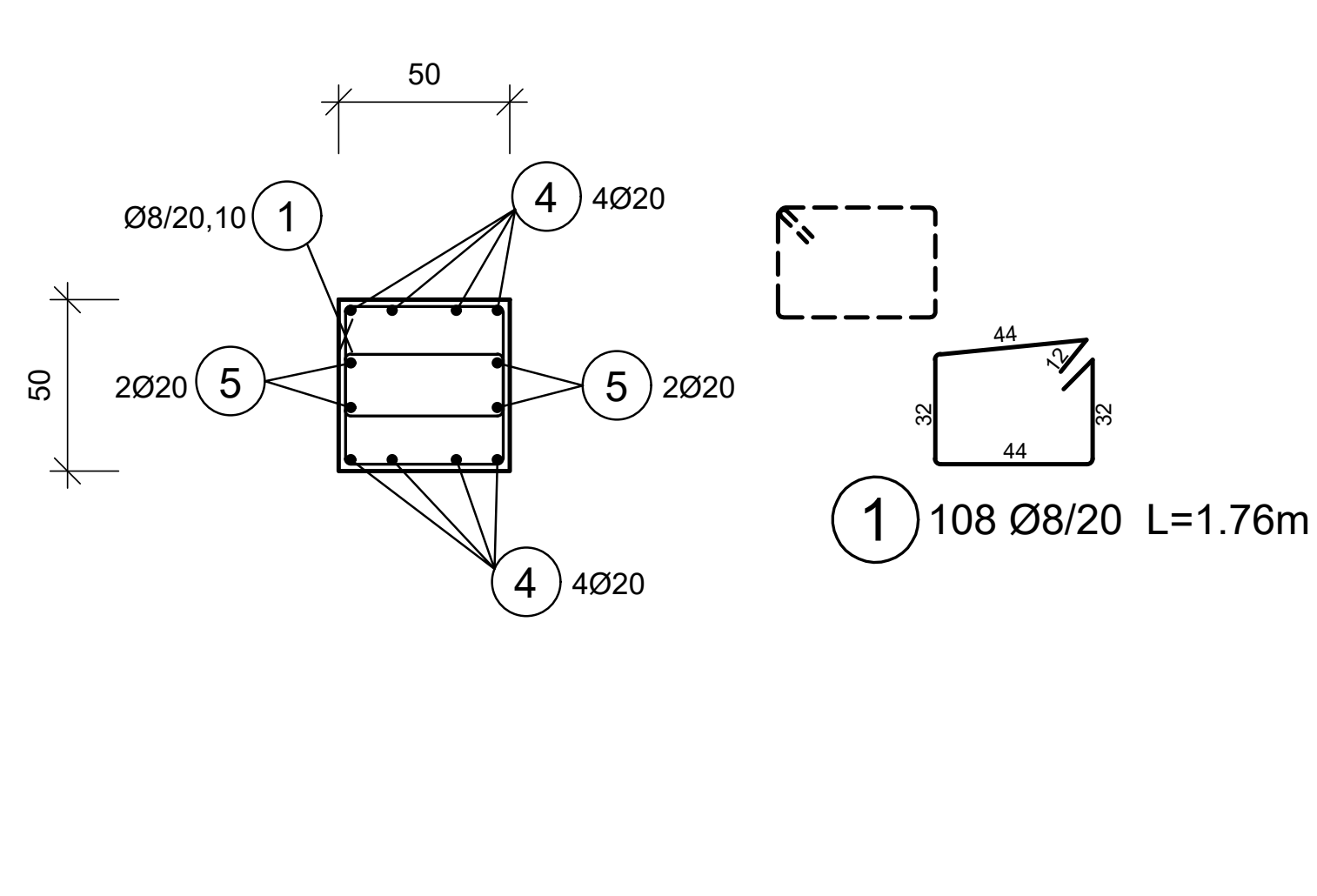

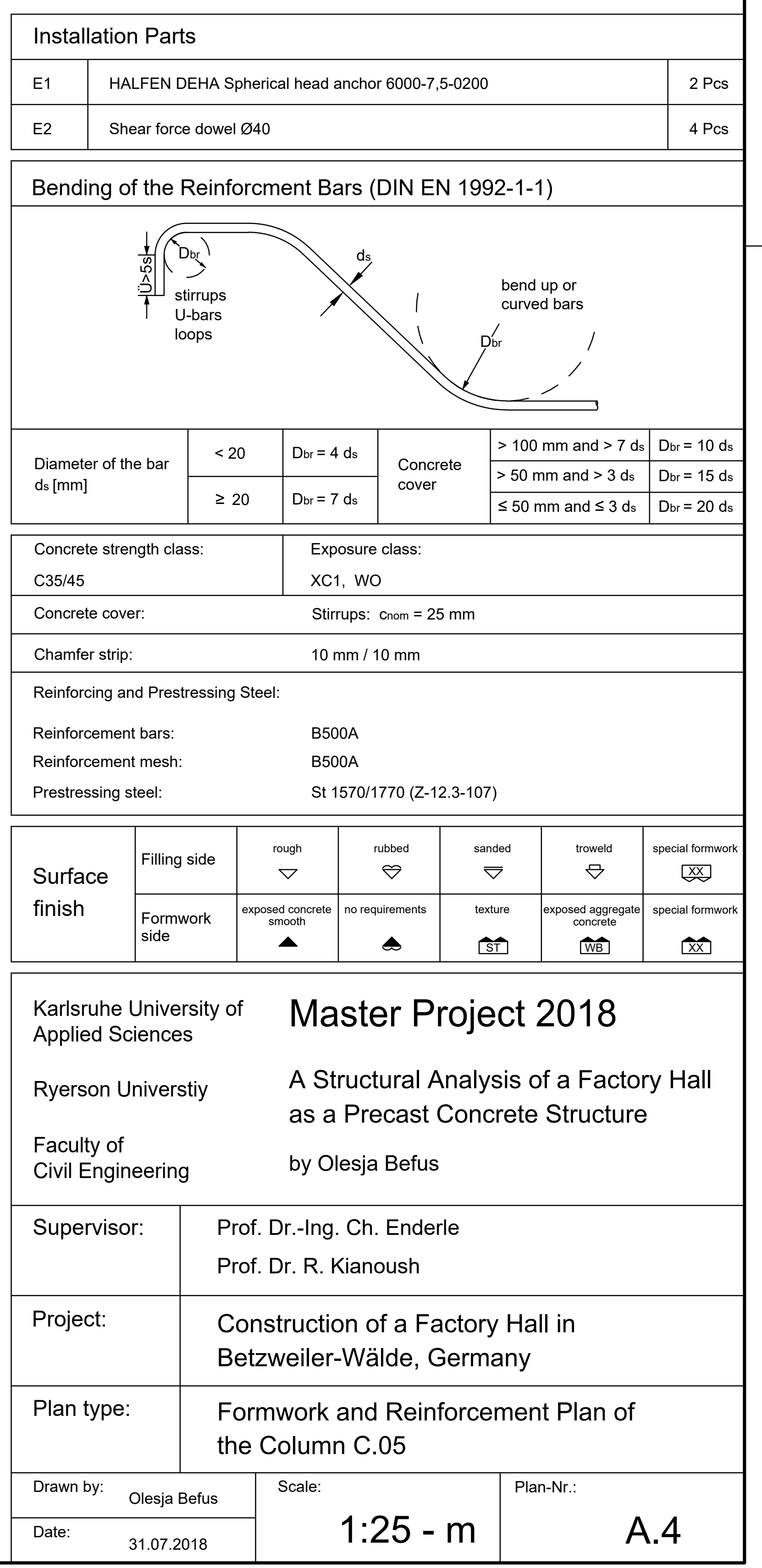


3.98

7.94
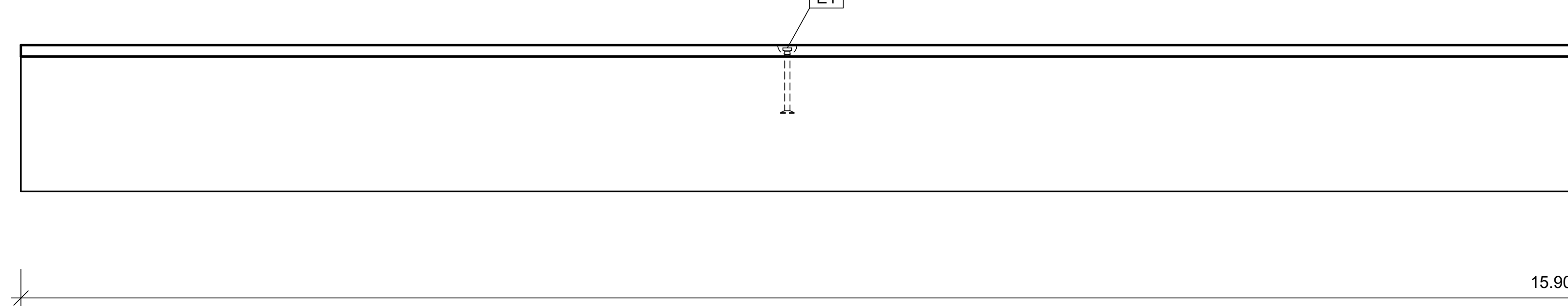

1550

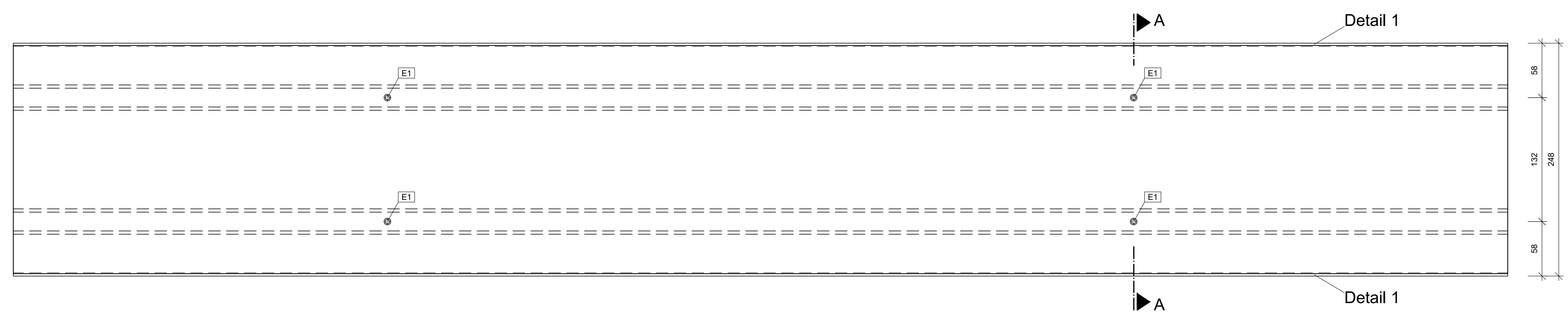

$\underline{\text { Reinforcement }}$

(1) 10608115

(2010 (5:

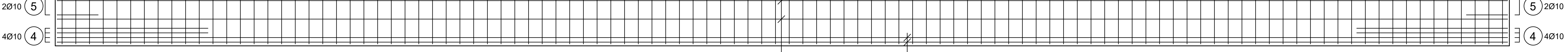
(3) 2010

(2) 2023

(3) $8 \varnothing 10$ L $=15.84 \mathrm{~m}$

(2) $10.625 \mathrm{~L}=15.84 \mathrm{~m}$

$=\longdiv { \text { (4) } 8 . 8 1 0 \mathrm { L } = 3 . 3 . 4 6 \mathrm { m } } \quad$ (5) $4.010 \mathrm{L=1.07 \textrm {m }}$
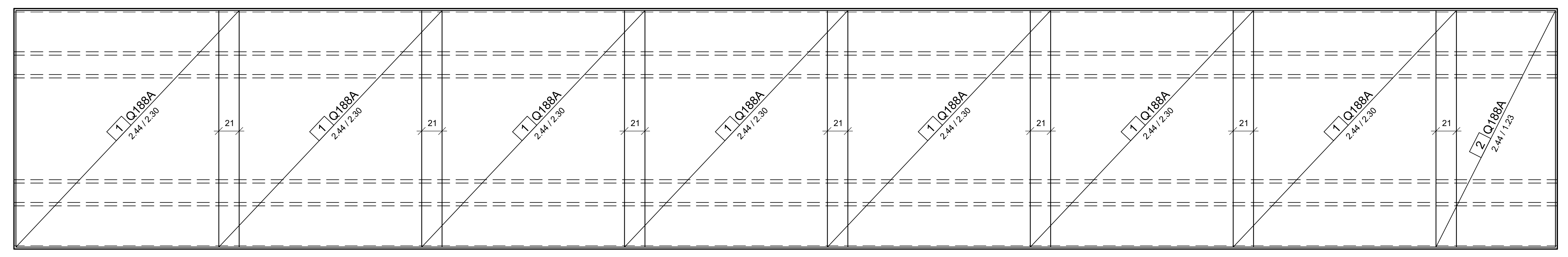

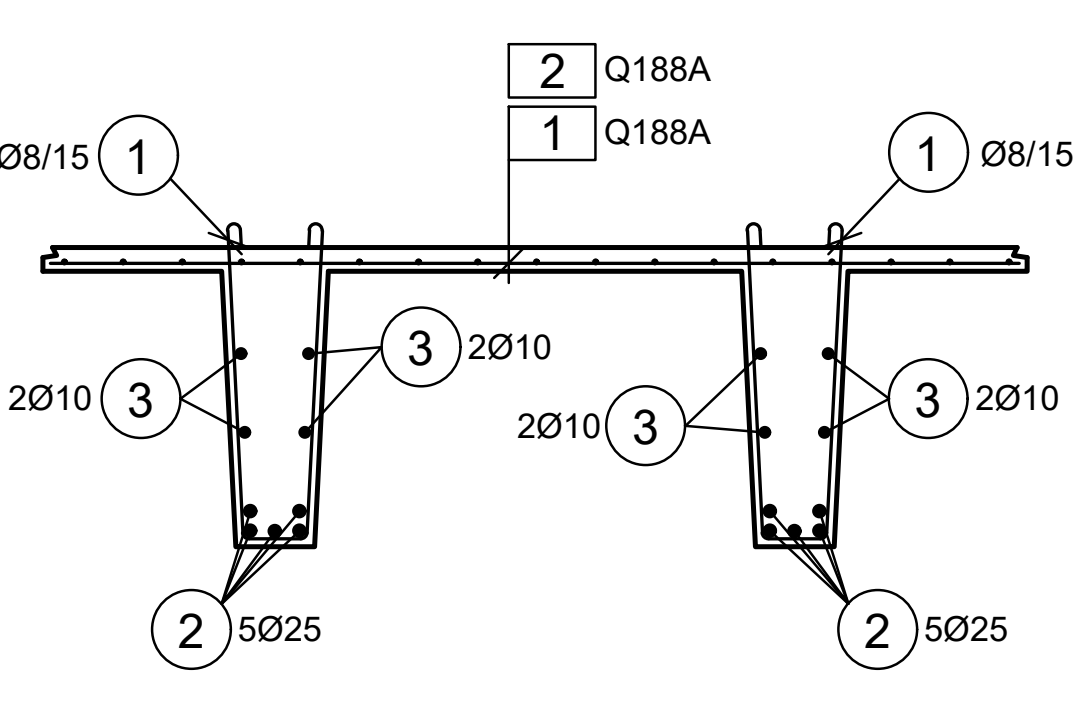

$\{=$

(1) $108.88 / 15 \mathrm{~L}=1.90 \mathrm{~m}$
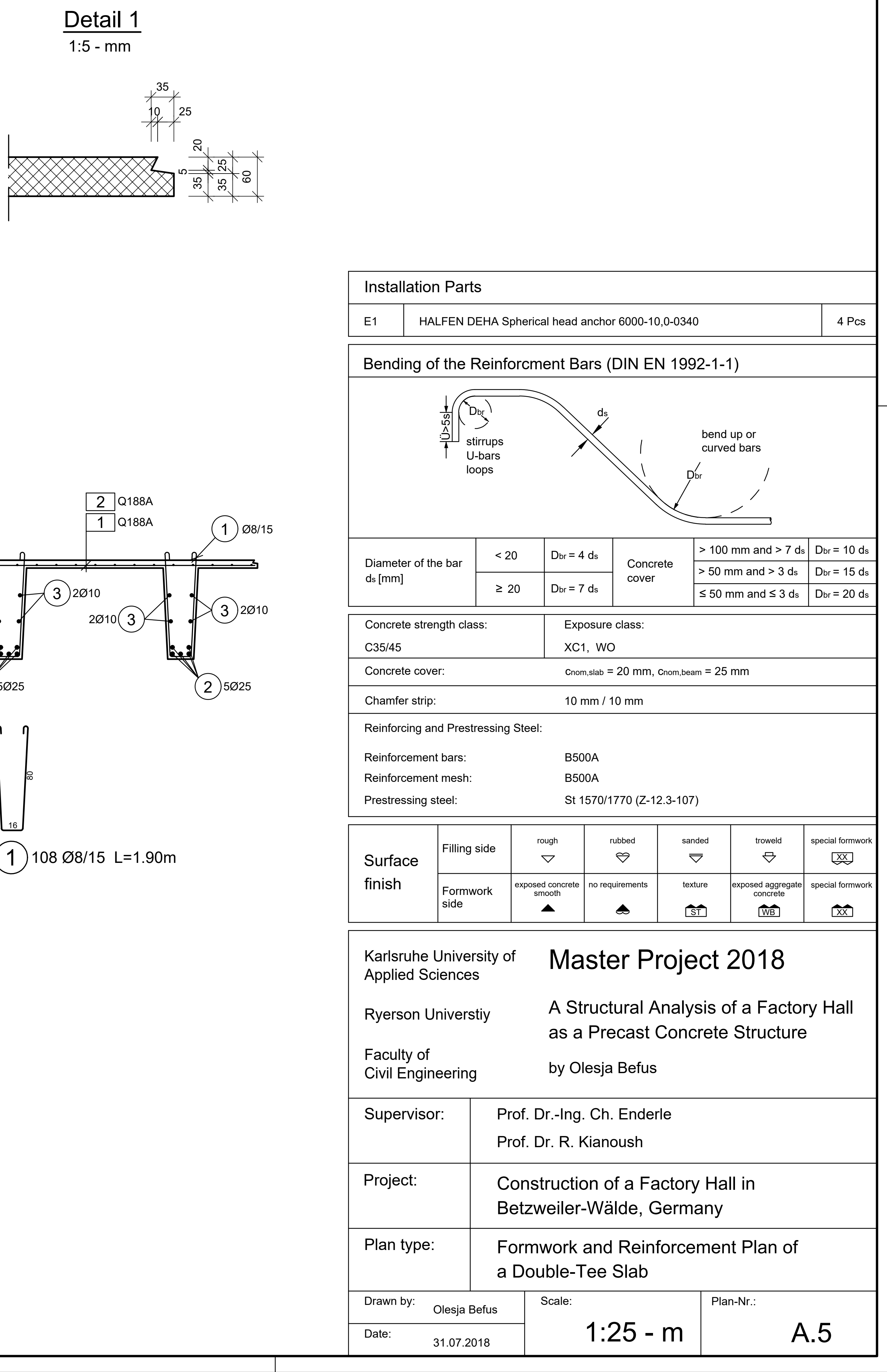

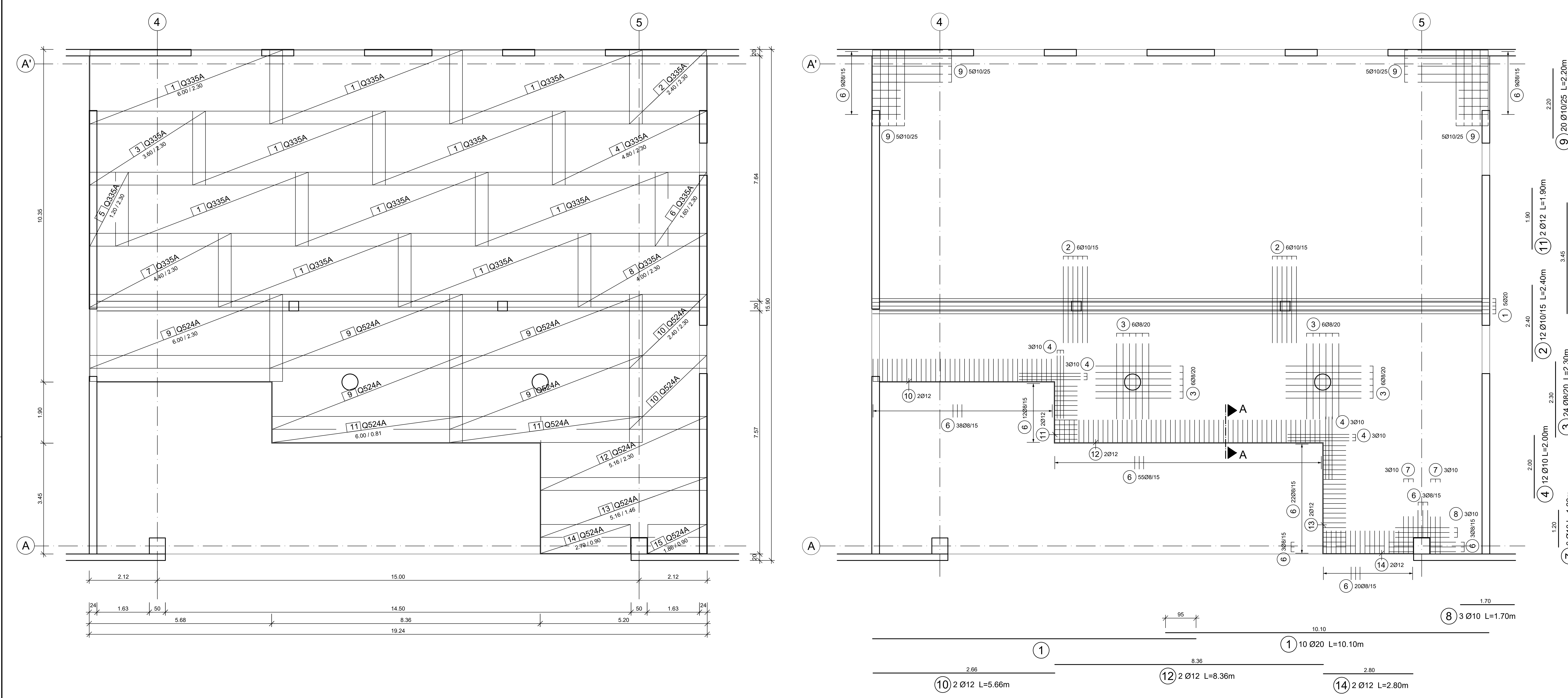

$\frac{\text { Section A-A }}{1: 25}$
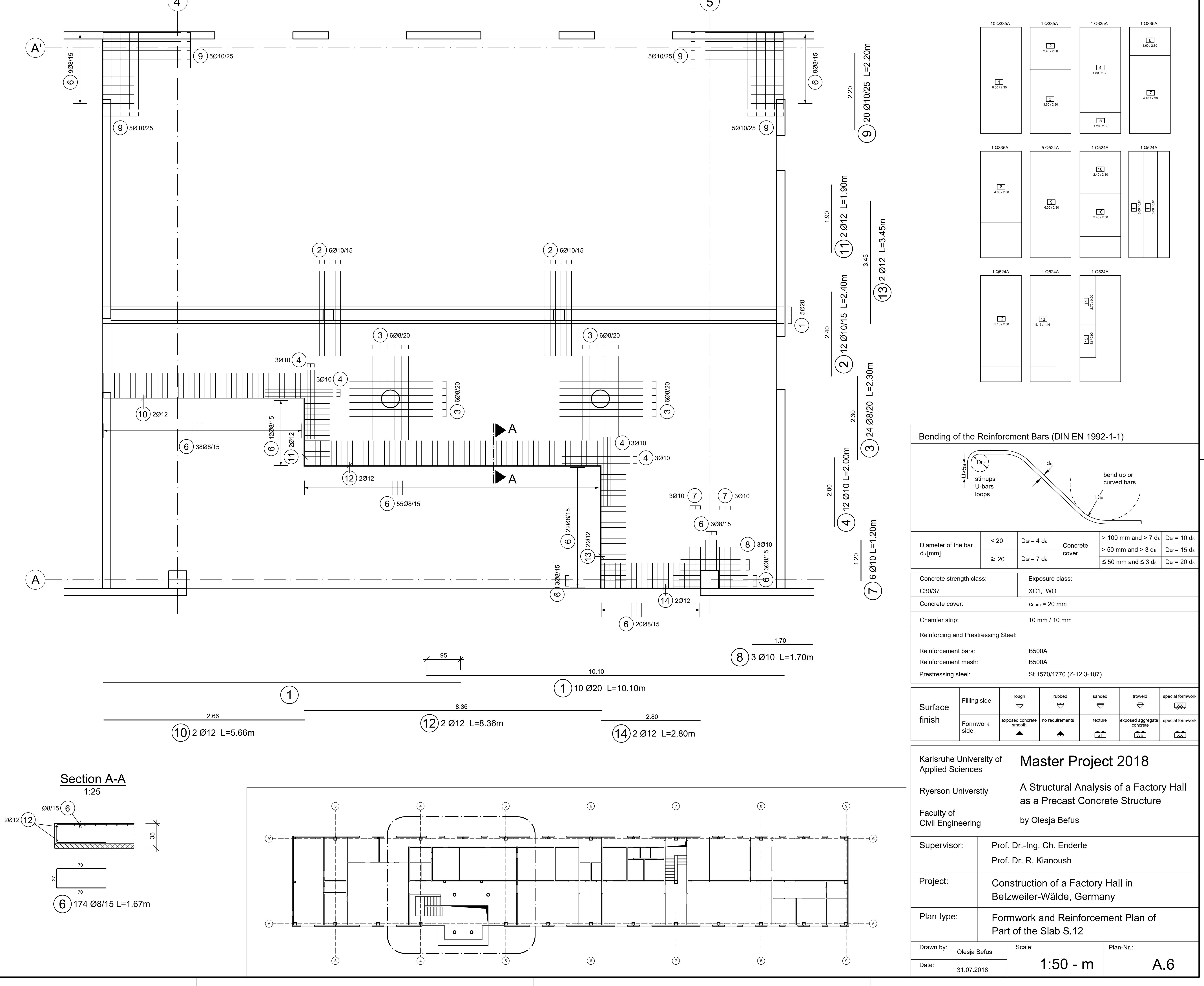


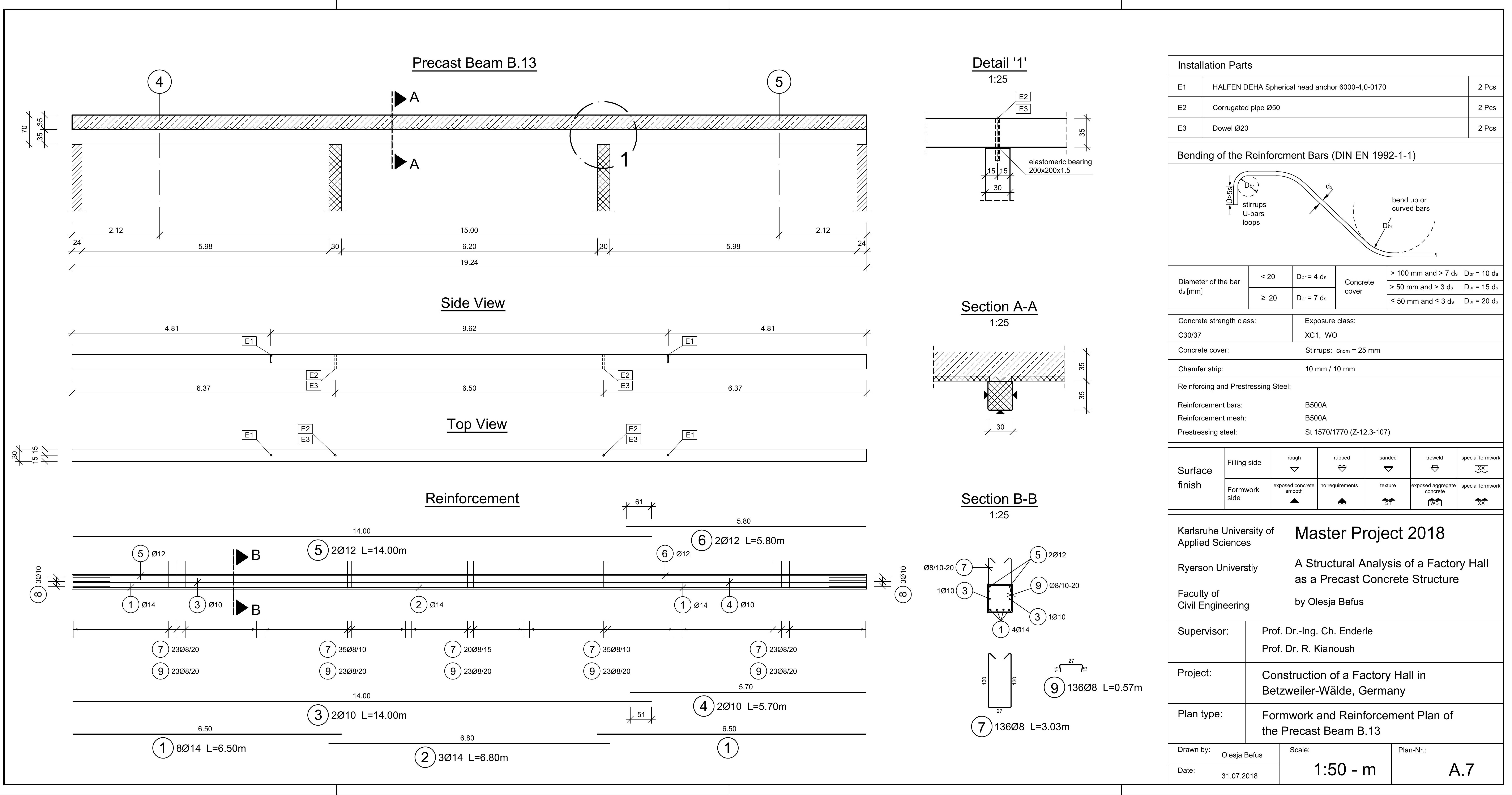


Pocket Foundation F.21

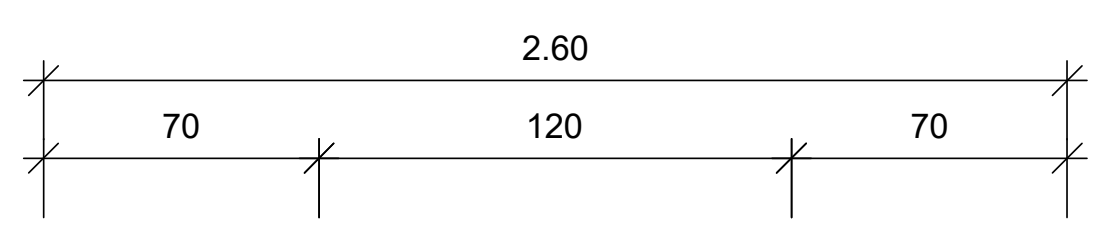

$\psi^{25} \stackrel{5}{1} 60 \quad 7^{525} \%$

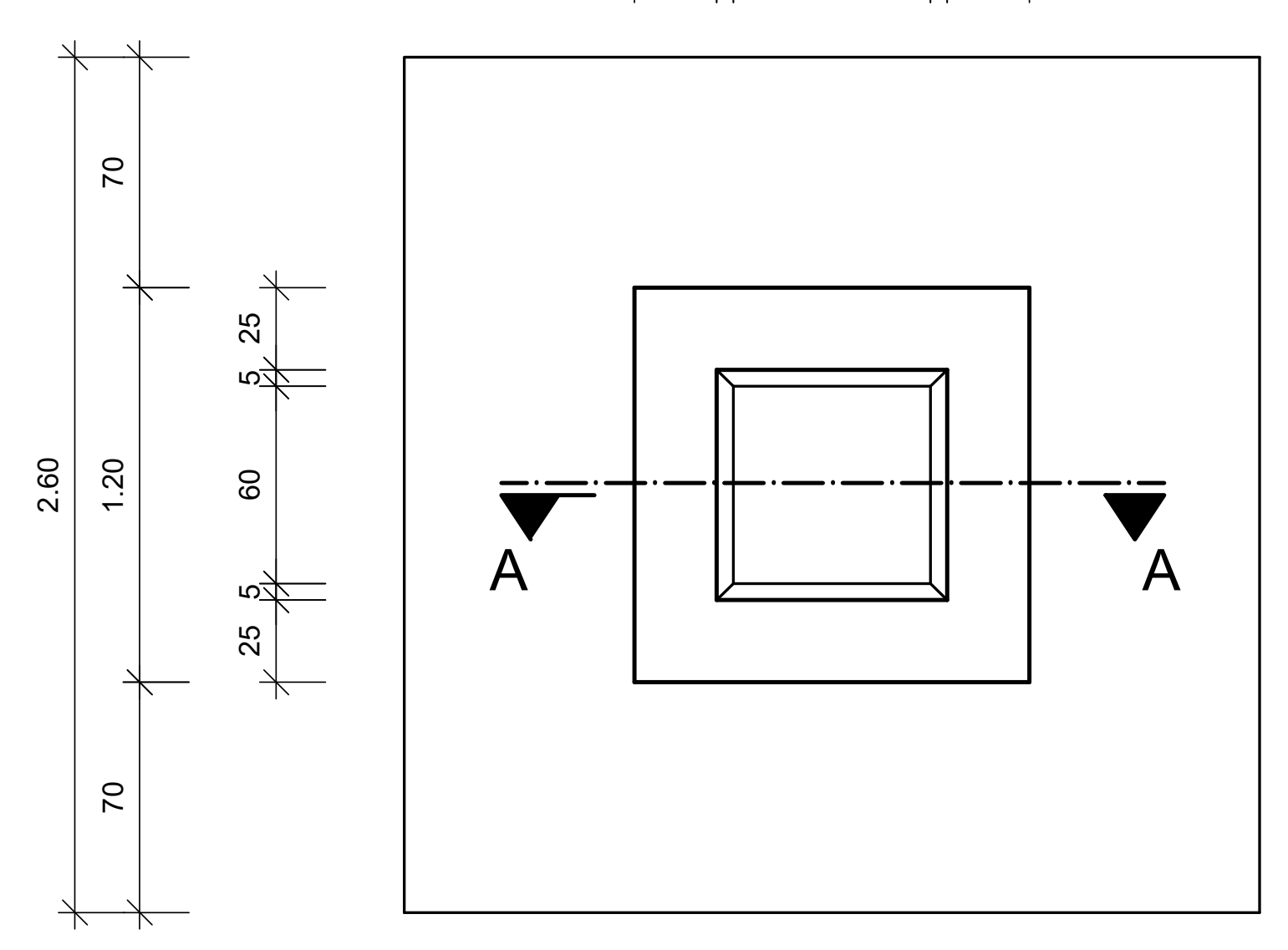

Section A-A

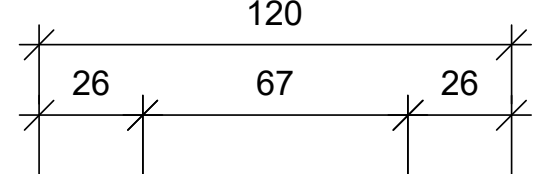
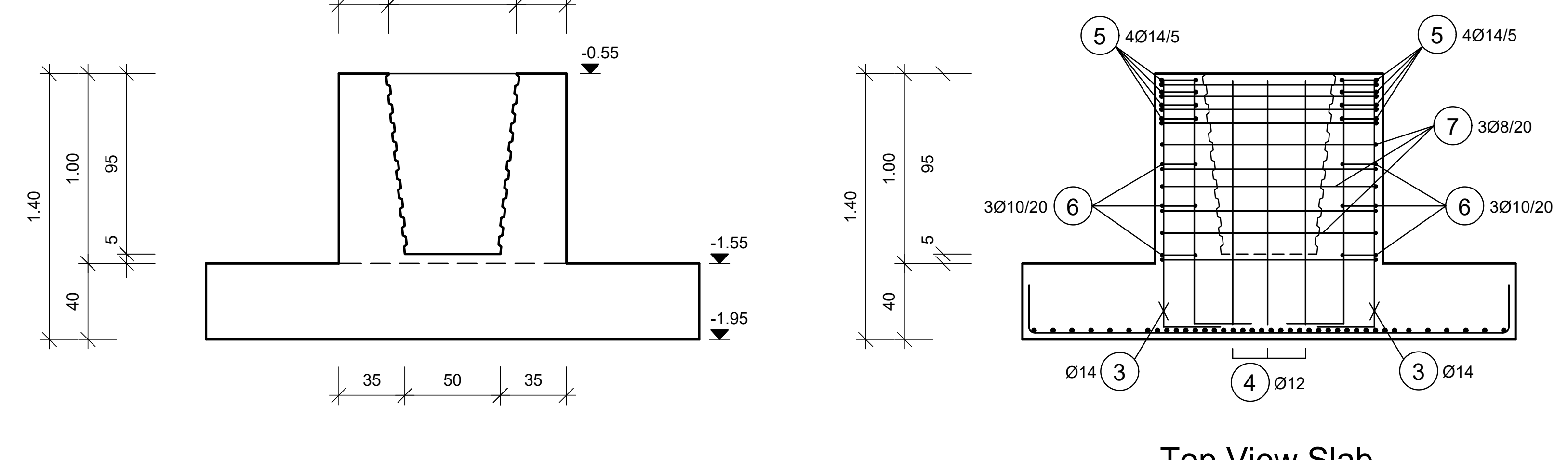

Top View Slab

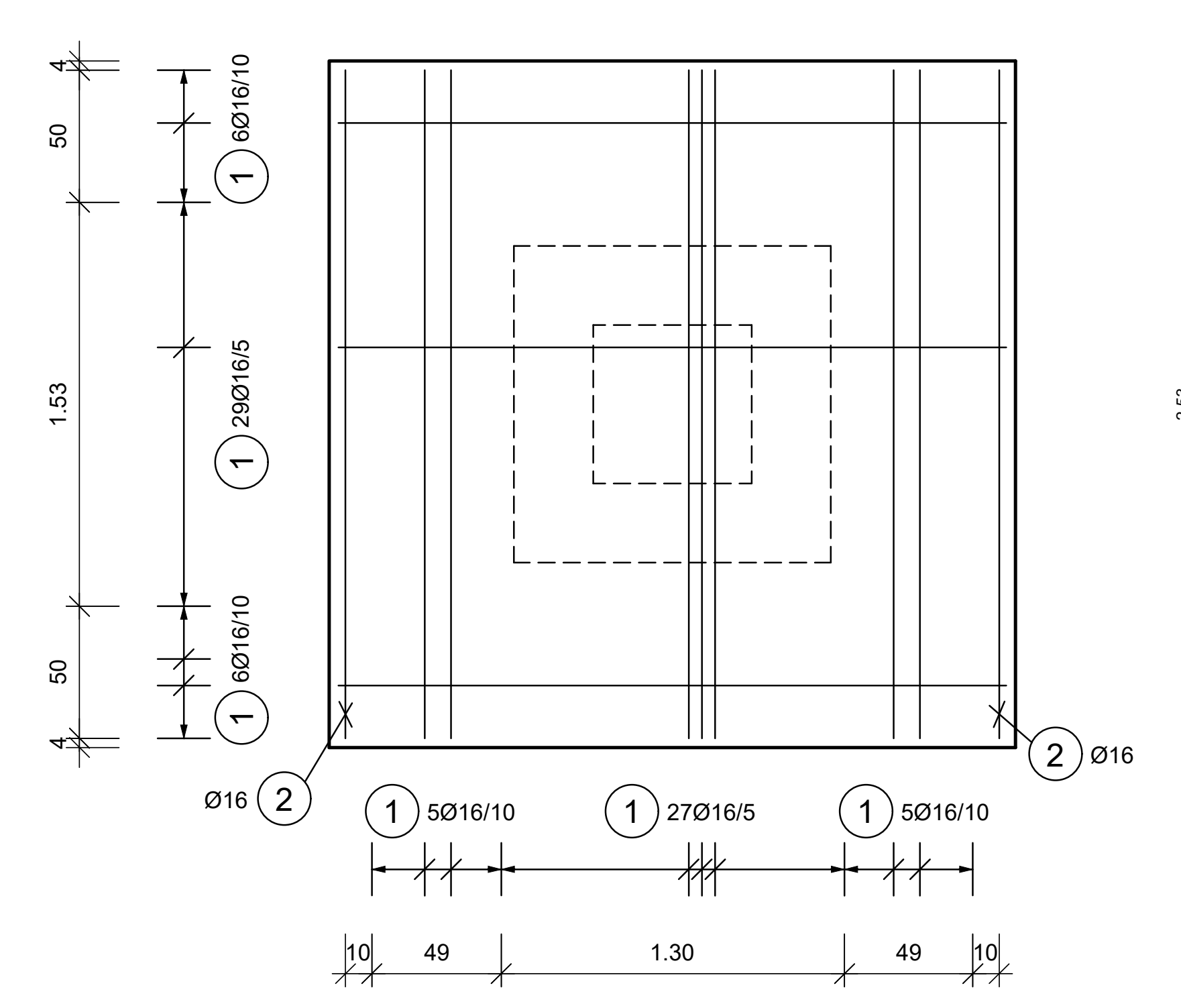

Section C-C
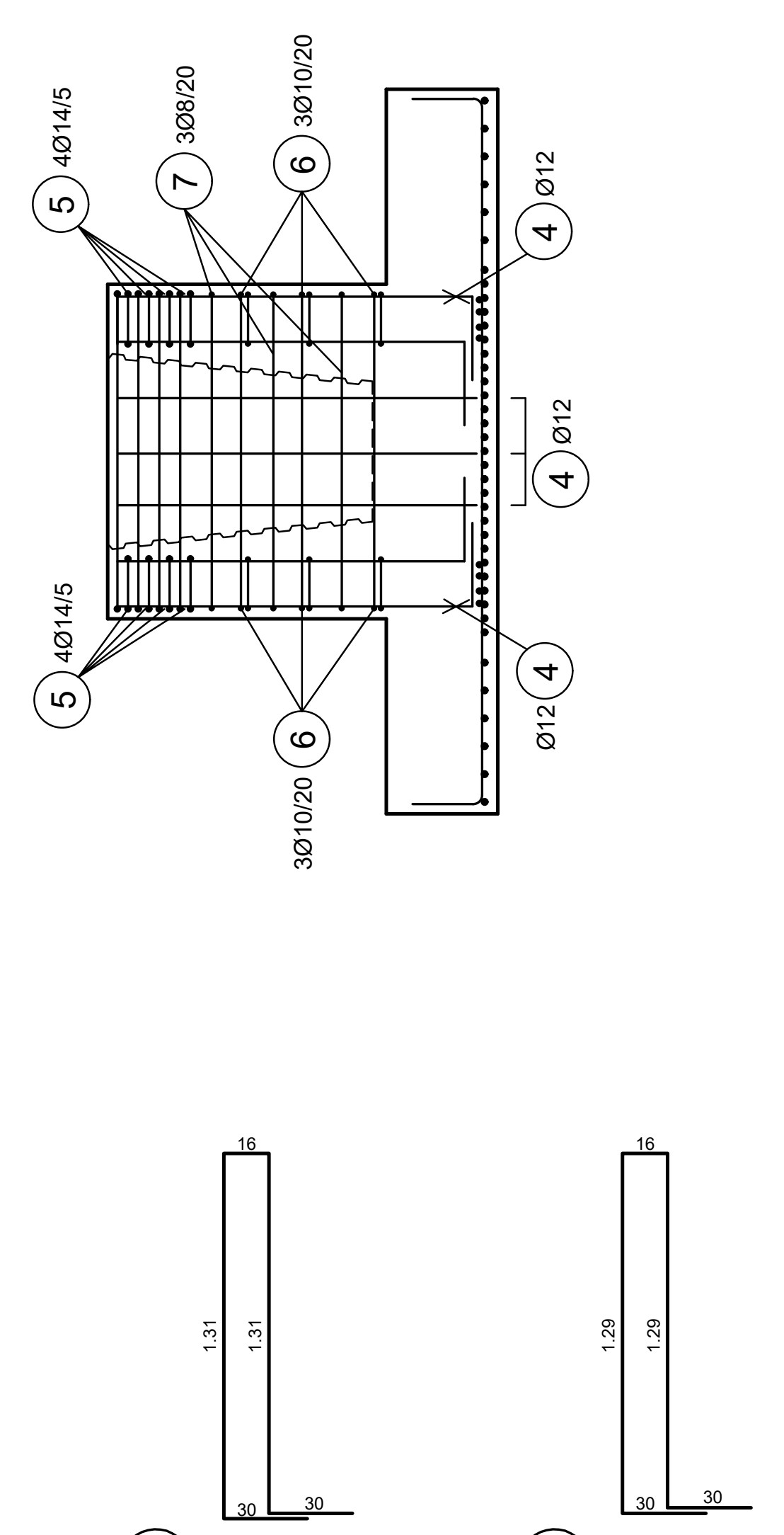

(3) $16 \varnothing 14 \quad L=3.38 m$ (4) $24 \varnothing 12 L=3.34 m$
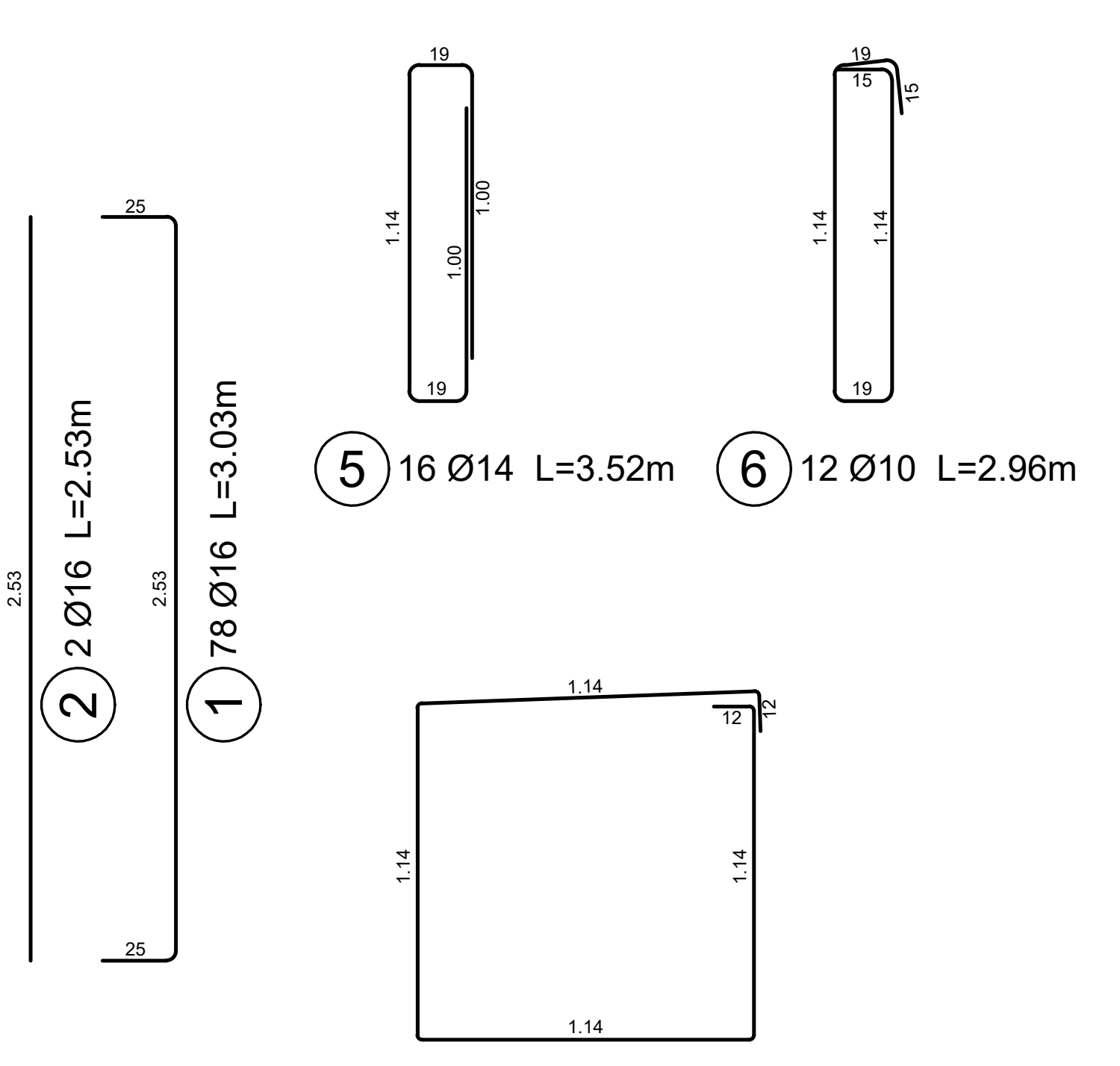

(7) $3.88 \mathrm{~L}=4.80 \mathrm{~m}$

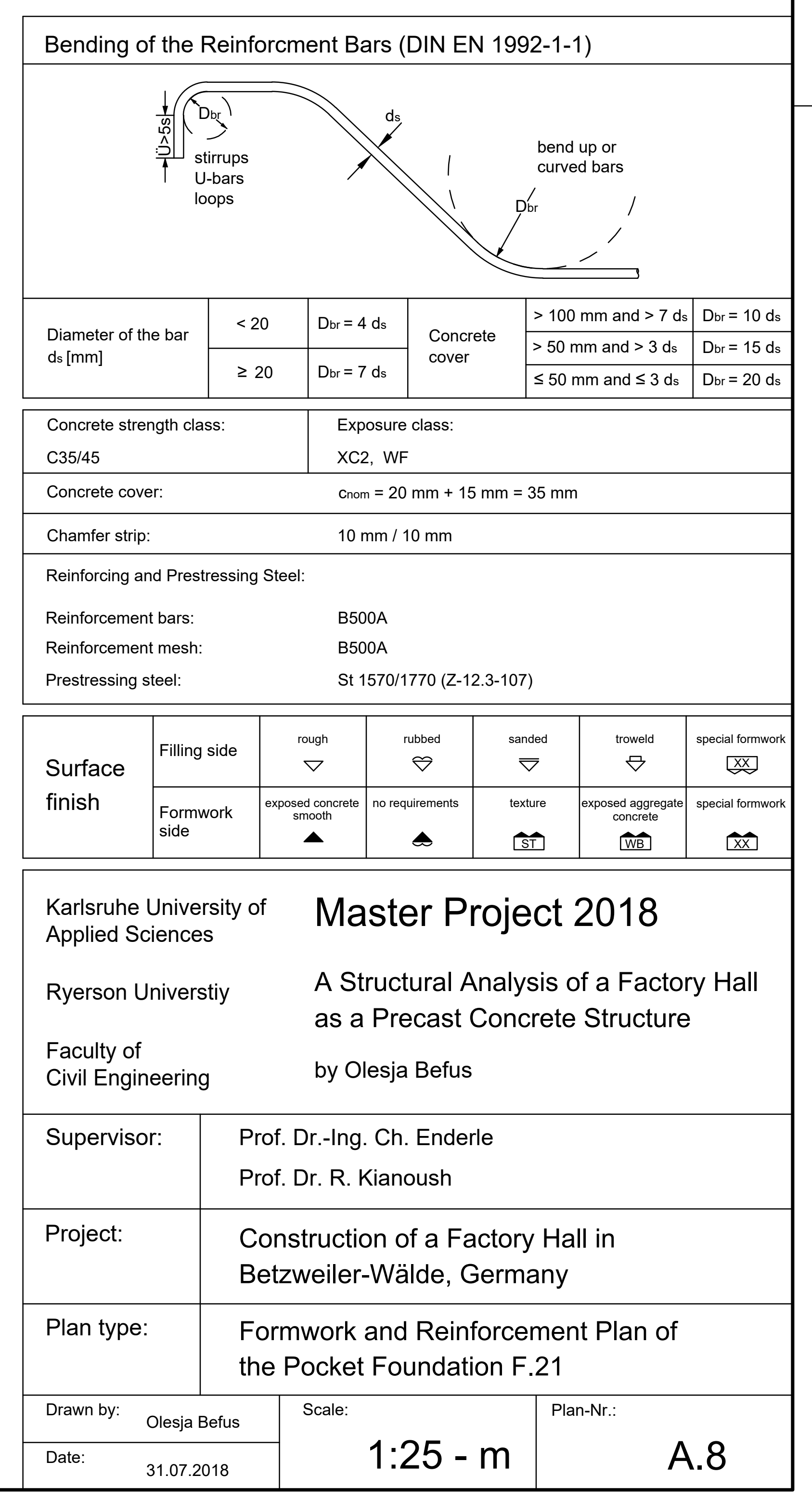




\section{APPENDIX B SOFTWARE CALCULATIONS}

\section{B.1 DESIGN RESULTS FROM 'NEMETSCHEK FRILO'}

B.1.1. Design Calculations POS. P.02 - Prestressed Concrete Purlin ........................................196

B.1.2. Design Calculations POS. P.03 - Prestressed Concrete Truss ........................................207

B.1.3. Design Calculations POS. T.10 - Precast Staircase …......................................................217

B.1.4. Design Calculations POS. C.04 - Precast Concrete Column with Biaxial Wind Loads ... 222

B.1.5. Design Calculations POS. C.05 - Precast Concrete Column ............................................225

B.1.6. Design Calculations POS. S.11 - Semi-Precast Double-Tee Slab .....................................228

B.1.7. Design Calculations POS. S.12 - Semi-Precast Concrete Slab ..........................................233

B.1.8. Design Calculations POS. B.13 - Semi-Precast Concrete Beam .......................................270

B.1.9. Design Calculations POS. W.14a - Load Bearing Concrete Wall ......................................279

B.1.10. Design Calculations POS. V.31 - Steel Canopy................................................................. 304 
B.1.1. Design Calculations POS. P.02 - Prestressed Concrete Purlin 
Demo Frilo

Stuttgarter Straße 40

70469 Stuttgart
Tel.: 0711810020

Fax: 0711858020
Projekt: Design Calculations

Position: P.02 - Prestressed Concrete Purlin

26.07.2018
Seite: 1

\section{Position: P.02 - Prestressed Concrete Purlin}

Spannbettbinder B8 01/2018 (Frilo R-2018-1/P12)
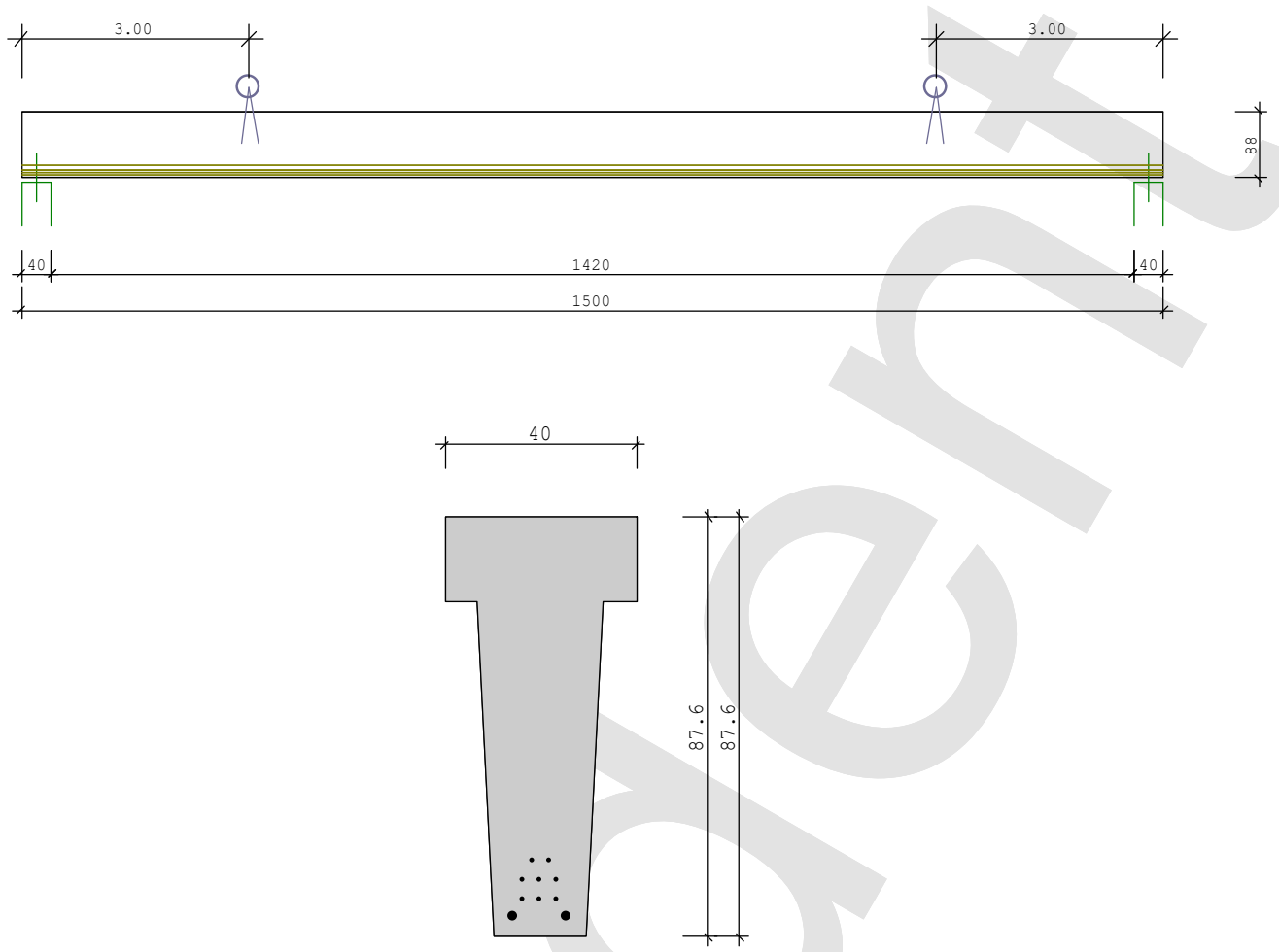

$+19 \neq$

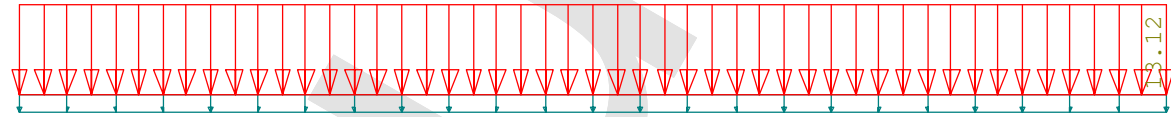

Eigengewicht Fertigteil nicht dargestellt

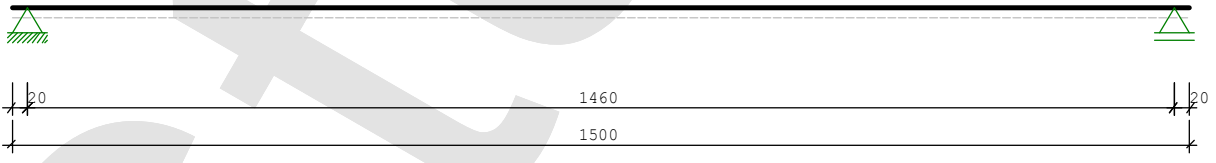

\section{Advices:}

No continuous reinforcement to $10 \mathrm{~cm}$ below UE found!

\section{System:}

\section{Parallel flange beam}

Basics:

Load combinatorics: DIN EN 1990/NA:2010-12 + EN 1990:2002/AC:2010

ULS: Structural safety checks(STR)

permanent/variable design situation with equation 6.10

Design code:

DIN EN 1992-1-1/NA/A1:2015-12 + EN 1992-1-1:2004 /AC:2010

Prestressing for pretensioning 
Demo Frilo

Stuttgarter Straße 40

70469
Tel.: 0711810020

Fax: 0711858020
Projekt: Design Calculations

Position: P.02 - Prestressed Concrete Purlin

26.07.2018

\section{System Geometry:}

Total

$\mathrm{L}=15.00 \mathrm{~m}$

Effective $\mathrm{L} 1=14.60 \mathrm{~m}$

Outstand left

$\mathrm{LO}=0.20 \mathrm{~m}$

right

$\mathrm{L} 2=0.20 \mathrm{~m}$

Height beam : $\quad \mathrm{H} 2=87.6 \mathrm{~cm}$

Relation eff.span to height of beam:

$$
\mathrm{L} 1 / \mathrm{H} 2=16.67
$$

Erection attachment, distance from the beginning resp. end of beam:
Hook
$\mathrm{L} 8=3.00 \mathrm{~m}$
right
$\mathrm{L} 9=3.00 \mathrm{~m}$

\section{Cross-section Precast :}

\begin{tabular}{|l|r|r|r|}
\hline \multicolumn{2}{|l|}{ Layer of cross-section from top to bottom } & & Remarks \\
\hline $\mathrm{Nr}$ & $\begin{array}{r}\text { Width } \\
\text { [cm] }\end{array}$ & $\begin{array}{r}\text { Distance } \\
\text { [cm] }\end{array}$ & Web begin \\
\hline 1 & 40.0 & 0.0 & \\
2 & 40.0 & 17.6 & Web end \\
3 & 26.0 & 17.7 & 87.6 \\
4
\end{tabular}

\section{Material:}

\section{Prestressing steel}

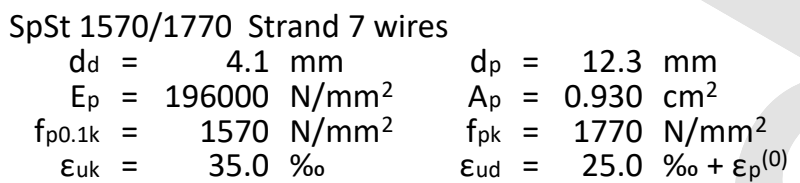

Partial safety factor : $\gamma_{\mathrm{s}}=1.15$

Coeff. prestress:

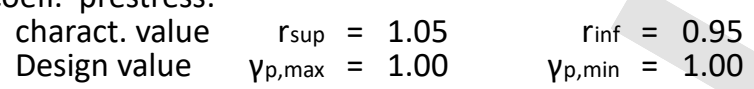

Proof of crack width

Equ. diameter $d_{p v}=7.20 \mathrm{~mm} \quad \xi=0.60$ (Tab. 6.2)

\begin{tabular}{|r|r|r|r|r|}
\hline Relaxation (from approval) & & & \\
\hline$\sigma_{\mathrm{p} 0} / \mathrm{f}_{\mathrm{pk}}$ & $10 \mathrm{~h}$ & $200 \mathrm{~h}$ & $1000 \mathrm{~h}$ & $1000000 \mathrm{~h}$ \\
\hline 0.60 & 0.3 & 0.6 & 0.8 & 2.8 \\
0.70 & 0.8 & 1.6 & 2.0 & 7.0 \\
0.80 & 2.0 & 4.0 & 5.0 & 14.0 \\
\hline
\end{tabular}

$\begin{array}{llll}\begin{array}{l}\text { Permitted stresses: } \\ \text { in formwork }\end{array} & \sigma_{p} \leq 1413.0 \mathrm{~N} / \mathrm{mm}^{2} & \left(0.90^{*} \mathrm{f}_{\mathrm{p} 0.1 \mathrm{k})}\right. \\ \text { after anchor. release } & \sigma_{\mathrm{p}} \leq 1327.5 \mathrm{~N} / \mathrm{mm}^{2} & \left(0.75^{*} \mathrm{fpk}_{\mathrm{pk}}\right. \\ \text { char. Lc } & \sigma_{\mathrm{p}} \leq 1413.0 \mathrm{~N} / \mathrm{mm}^{2} & \left(0.90^{*} \mathrm{f}_{\mathrm{p} 0.1 \mathrm{k}}\right) \\ \text { q.perm.Lc } & \sigma_{\mathrm{p}} \leq 1150.5 \mathrm{~N} / \mathrm{mm}^{2} & \left(0.65^{*} \mathrm{fpk}\right)\end{array}$

Transmission length

$$
\begin{array}{rlrl}
\eta_{\mathrm{p} 1} & =2.85 & \eta_{1} & =1.00 \\
\alpha_{1} & =1.25 & \alpha_{2} & =0.19 \\
\sigma_{\mathrm{pm} 0} & =945 \mathrm{~N} / \mathrm{mm}^{2} & & \\
\mathrm{f}_{\mathrm{ctdt}} & =1.02 \mathrm{~N} / \mathrm{mm}^{2} & \mathrm{fbpt}_{\mathrm{bpt}}=2.91 \mathrm{~N} / \mathrm{mm}^{2} \\
I_{\mathrm{pt}}=0.95 \mathrm{~m} & &
\end{array}
$$

PT: $\quad \mathrm{f}_{\mathrm{ctdt}}=1.02 \mathrm{~N} / \mathrm{mm}^{2}$

Dispersion_length:

$$
\mathrm{d}=0 . \overline{8} 3 \mathrm{~m} \quad \mathrm{I}_{\text {disp }}=1.26 \mathrm{~m}
$$


Reinforcing steel:

\begin{tabular}{|c|c|c|c|c|c|}
\hline & $\begin{array}{r}\text { Longitudinal } \\
\text { B500B }\end{array}$ & & & $\begin{array}{l}\text { Stirrup } \\
\text { B500B }\end{array}$ & \\
\hline & 200000 & $\mathrm{~N} / \mathrm{mm}^{2}$ & $E_{s}$ & 200000 & $\mathrm{~N} / \mathrm{mm}^{2}$ \\
\hline & 500 & $\mathrm{~N} / \mathrm{mm}^{2}$ & $f_{y k}$ & 500 & $\mathrm{~N} / \mathrm{mm}^{2}$ \\
\hline$f_{t k}$ & 540 & $\mathrm{~N} / \mathrm{mm}^{2}$ & $f_{t k}$ & 540 & $\mathrm{~N} / \mathrm{mm}^{2}$ \\
\hline Euk & 50.0 & $\%$ & Euk & 50.0 & $\%$ \\
\hline Eud & 25.0 & \%о & $\varepsilon_{\text {ud }}$ & 25.0 & \%о \\
\hline
\end{tabular}

Partial safety factor :
$\gamma_{\mathrm{s}}=$
1.15
$\gamma_{\mathrm{s}}=$
1.15

permitted stresses in SLS :
$\sigma_{\mathrm{s}} \leq$
$400 \mathrm{~N} / \mathrm{mm}^{2}$
$\sigma_{\mathrm{s}} \leq$
$400 \mathrm{~N} / \mathrm{mm}^{2} \quad\left(0.80 * \mathrm{f}_{\mathrm{yk}}\right)$

\section{Requirements durability:}

attack on concrete attack on reinforc.

min. concrete class stirrup

long. reinforcement prestressed steel allowance in design stirrup concrete coverage longitudinal bars concrete coverage prestressing steel : concrete coverage laying dist. link all. crack width decompression

*1: with cmin,l

*5: bond decisive

WO

XC1

C $16 / 20$

$\phi, I=10 \mathrm{~mm}$

$\phi, \mathrm{m}=16 \mathrm{~mm}$

$\mathrm{d}_{\mathrm{p}}=12.3 \mathrm{~mm}$ strand, $\mathrm{s}>=2.5^{*} \mathrm{dp}, \sigma_{\mathrm{p}}{ }^{(0)}<=1000 \mathrm{~N} / \mathrm{mm} 2$

$\Delta \mathrm{cdev}=10 \mathrm{~mm}$

$\mathrm{C}_{\min , \mathrm{l}}=10 \mathrm{~mm} * 5$

$\mathrm{C}_{\text {nom }, \mathrm{l}}=20 \mathrm{~mm} * 5$

$\mathrm{Cmin}, \mathrm{m}=16 \mathrm{~mm} * 5$

$\mathrm{Cnom}_{\mathrm{m}} \mathrm{m}=30 \mathrm{~mm} * 1$

$\mathrm{C}_{\text {min, }, \mathrm{p}}=25 \mathrm{~mm} * 5$

$\mathrm{Cnom}, \mathrm{p}=35 \mathrm{~mm}$

$\mathrm{C}, \mathrm{I}=20 \mathrm{~mm}$

$W_{\max }=0.20 \mathrm{~mm}$ not req.

\section{Concrete:}

Precast

$$
\begin{aligned}
& \text { C } 30 / 37 \\
& \mathrm{f}_{\mathrm{ck}}=30.00 \mathrm{~N} / \mathrm{mm}^{2} \\
& \alpha_{\mathrm{cc}}=0.85 \\
& \mathrm{f}_{\mathrm{ctk} 0.05}=2.03 \mathrm{~N} / \mathrm{mm}^{2} \\
& \alpha_{\mathrm{ct}}=0.85 \\
& y=25.00 \mathrm{kN} / \mathrm{m}^{3} \text { Unit } \\
& \mathrm{E}_{\mathrm{cm}}=33000 \mathrm{~N} / \mathrm{mm}^{2} \\
& \alpha_{\mathrm{E}}=1.00 \quad \text { Coeff. E-module } \\
& \mathrm{G}_{\mathrm{cm}}=13200 \mathrm{~N} / \mathrm{mm}^{2} \\
& \text { char. LC } \\
& \sigma_{c} \geq-18.00 \mathrm{~N} / \mathrm{mm}^{2} \\
& \text { q.perm.Lc } \\
& \sigma_{c} \geq-13.50 \mathrm{~N} / \mathrm{mm}^{2} \\
& \text { Removal the anchor } \mathrm{t}=\mathrm{tOT} \text { (sto) } \\
& f_{\mathrm{cm}(\mathrm{t})}=25.18 \mathrm{~N} / \mathrm{mm}^{2} \\
& f_{c k}(t)=17.18 \mathrm{~N} / \mathrm{mm}^{2} \\
& \text { linear creep } \quad \sigma_{c} \geq-7.73 \mathrm{~N} / \mathrm{mm}^{2} \quad(k 2=0.45) \\
& \text { maximum } \\
& \sigma_{c} \geq-10.31 \mathrm{~N} / \mathrm{mm}^{2} \quad(k 6=0.60)
\end{aligned}
$$

\section{Creep modulus \& shrinkage strain}

no heat treatment, t0T $=$ t0

CementStrenght class 42,5R;52,5

$\rho=0.5$ (Aging coefficient)

Reference point for $t 0$ is the start of the concreting of the precast

\begin{tabular}{|l|r|r|}
\hline Creep & $\begin{array}{r}\text { t0 } \\
\text { Days }\end{array}$ & $\begin{array}{r}\text { RH } \\
\%\end{array}$ \\
\hline Storage & 3 & 70 \\
Utilization precast & 180 & 50 \\
\hline
\end{tabular}


Demo Frilo

Stuttgarter Straße 40

70469 Stuttgart
Tel.: 0711810020

Fax: 0711858020
Projekt: Design Calculations

Position: P.02 - Prestressed Concrete Purlin

26.07.2018

Seite: 4

\begin{tabular}{|c|c|c|c|c|c|c|c|c|c|c|c|c|c|c|c|}
\hline L. & \multicolumn{2}{|c|}{ Segment } & \multicolumn{2}{|c|}{$\begin{array}{r}\text { Part- } \\
\text { cross-section }\end{array}$} & to & $\mathrm{t}$ & $\alpha$ & $\begin{array}{r}\text { to,eff } \\
\text { B.9 }\end{array}$ & $\begin{array}{l}\beta \text { to } \\
\text { B.5 }\end{array}$ & \multicolumn{2}{|c|}{$\begin{array}{r}\beta_{H} \\
\text { B. } 8\end{array}$} & $\begin{array}{r}\beta c(t, t 0) \\
\text { B.7 }\end{array}$ & $\begin{array}{l}\text { фRH } \\
\text { B.3 }\end{array}$ & $\begin{array}{r}\beta \mathrm{fcm} \\
\text { B.4 }\end{array}$ & $\begin{array}{r}\phi(t, t 0) \\
\text { B. } 1\end{array}$ \\
\hline $\begin{array}{l}1 \\
2\end{array}$ & 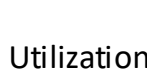 & & & & $\begin{array}{r}3.0 \\
180.0\end{array}$ & & 1 & $\begin{array}{l}7.7 \\
7.7\end{array}$ & $\begin{array}{l}0.62 \\
0.62\end{array}$ & 515 & & & $\begin{array}{l}1.47 \\
1.80\end{array}$ & & \\
\hline L. & $\begin{array}{r}\mathrm{A} \\
{\left[\mathrm{cm}^{2}\right]}\end{array}$ & $\begin{array}{r}U \\
{[\mathrm{~cm}]}\end{array}$ & $\begin{array}{r}\text { ho } \\
{[\mathrm{cm}]}\end{array}$ & & $\mathrm{ds}(\mathrm{t} 0, \mathrm{ts})$ & $\begin{array}{r}\text { ßds }(t, t \\
3.1\end{array}$ & & $\begin{array}{r}\text { BRH } \\
\text { B.12 }\end{array}$ & & $\begin{array}{c}\text { ed, } 0 \\
11\end{array}$ & & & $\begin{array}{r}\varepsilon_{\mathrm{ca}} / 10 \\
3 .\end{array}$ & & $\begin{array}{r}\varepsilon c s(t, t 0) \\
{[\% o]}\end{array}$ \\
\hline $\begin{array}{l}1 \\
2\end{array}$ & & & & & $\begin{array}{l}0.000 \\
0.640\end{array}$ & $\begin{array}{l}0.64 \\
0.99\end{array}$ & & $\begin{array}{l}1.02 \\
1.36\end{array}$ & & $\begin{array}{l}1.5 \\
7.9\end{array}$ & 0.9 & & & & $\begin{array}{l}0.327 \\
0.212\end{array}$ \\
\hline
\end{tabular}

\section{Loads:}

\section{Self weight}

Beam beginning g11 $=5.70 \mathrm{kN} / \mathrm{m}$

Beam end $\quad \mathrm{g} 12=5.70 \mathrm{kN} / \mathrm{m}$

Total $\quad \mathrm{G}=85.5 \mathrm{kN}$

Volume $\quad \mathrm{V}=3.42 \mathrm{~m}^{3}$

Surf. $\quad A=31.68 \mathrm{~m}^{2}$

Live loads

\begin{tabular}{|c|c|c|c|c|c|c|c|c|c|c|c|}
\hline \multicolumn{12}{|c|}{ Units: Single load[kN] Single moment $[k N m]$ line load $[k N / m]$} \\
\hline span & type & gle & qle & $\begin{array}{r}\text { Dist. a } \\
{[\mathrm{m}]}\end{array}$ & gri & qri & $\begin{array}{r}\text { Length } \\
{[\mathrm{m}]}\end{array}$ & Fact & Act. & Sim. & Pos. \\
\hline 1 & 1 & 2.59 & 13.12 & 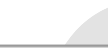 & & 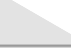 & & 1.00 & 10 & 0 & \\
\hline
\end{tabular}

Actions:

\begin{tabular}{|r|r|r|r|r|r|r|r|}
\hline Act. & $Y \mathrm{VQ}$ & $\psi_{0}$ & $\psi_{1}$ & $\psi_{2}$ & Dep. & Cat. & Description \\
\hline 10 & 1.50 & 0.50 & 0.20 & 0.00 & 0 & $\mathrm{~S}$ & Schnee bis NN $+1000 \mathrm{~m}$ \\
\hline
\end{tabular}

\section{Tendons:}

$\operatorname{Dist}(\mathrm{LE})>4.8 \mathrm{~cm}$ axis horizontal $>4.3 \mathrm{~cm}$ vertical $>3.7 \mathrm{~cm}$

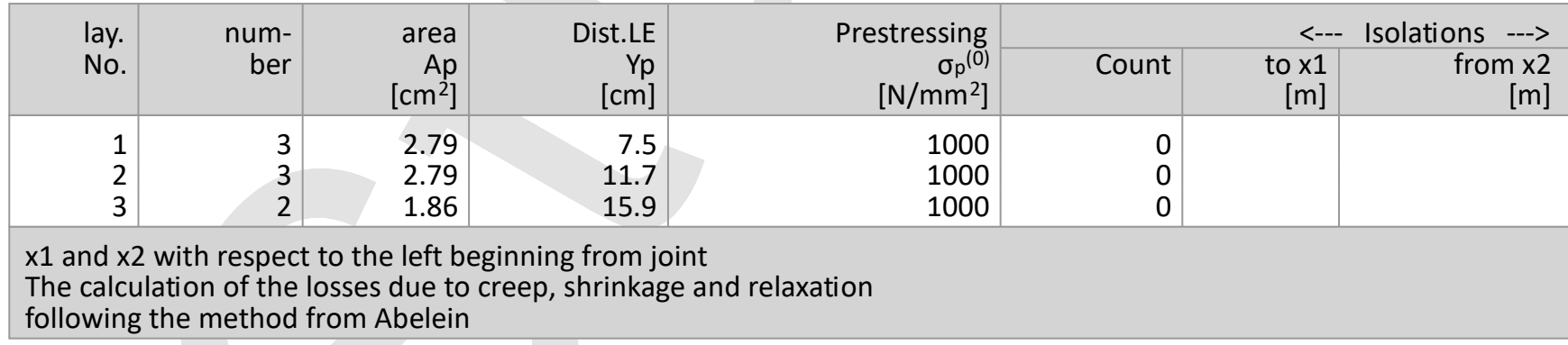

\section{Untensioned reinforcement:}

\begin{tabular}{|r|r|r|r|r|r|r|r|}
\hline $\begin{array}{r}\text { Layer } \\
\text { No. }\end{array}$ & $\begin{array}{r}\text { num- } \\
\text { ber }\end{array}$ & $\begin{array}{r}\text { diam. } \\
\Phi s, I \\
{[\mathrm{~mm}]}\end{array}$ & $\begin{array}{r}\text { area } \\
\text { As } \\
{\left[\mathrm{cm}^{2}\right]}\end{array}$ & $\begin{array}{r}\text { Dist.LE } \\
\text { Ys } \\
{[\mathrm{cm}]}\end{array}$ & $\begin{array}{r}\text { effective range } \\
\text { to } \mathrm{XE} \\
{[\mathrm{m}]}\end{array}$ & $\begin{array}{r}\text { from } \times A \\
{[\mathrm{~m}]}\end{array}$ & $\begin{array}{r}15.00 \\
\hline\end{array}$ \\
\hline 1 & 2 & 16 & 4.02 & 4.0 & 0.00 & 15.00 \\
\hline
\end{tabular}

\section{Surface reinforcement acc.to Tab. NA.J.41 (B0 < D0) :}

Web (Z1/S3)

Edge top (Z2/S3)
AsS $=1.21 \mathrm{~cm}^{2} / \mathrm{m}$

AsO $=0.00 \mathrm{~cm}^{2} / \mathrm{m}$
(UwkS <= XC4) (per side)

(UwkS <= XC4) 
Demo Frilo

Stuttgarter Straße 40

70469 Stuttgart
Tel.: 0711810020

Fax: 0711858020
Projekt: Design Calculations

Position: P.02 - Prestressed Concrete Purlin

26.07.2018
Seite: 5

\section{Settings for shear resistance check}

Bearing width, distance bearing edge, effective height of the bearing line

left $\mathrm{bAl}=0.40 \mathrm{~m} \quad \mathrm{al}=0.20 \mathrm{~m} \quad \mathrm{dAl}=0.83 \mathrm{~m}$

right $b A r=0.40 \mathrm{~m} \quad$ ar $=0.20 \mathrm{~m} \quad \mathrm{dAR}=0.83 \mathrm{~m}$

For shear reinforcement not decisive ranges over support $A$ and $B$ :

$x a R e=1.03 \mathrm{~m}$ direct bearing (width of bearing $/ 2+$ eff. depth)

$x b L i=1.03 \mathrm{~m}$ direct bearing (width of bearing/ $2+$ eff. depth)

\section{Check the limit deformation:}

Total sagging $\quad f \leq L / 250$

Cantilever left $f \leq 0.2 \mathrm{~cm}$

Span f $\leq 5.8 \mathrm{~cm}$

Cantilever right $f \leq 0.2 \mathrm{~cm}$

quasi- permanent combination and eff. char. prestress

Deflection due to shrinkage considered

Tension stiffening: Member rigidity, Characteristic combination

RESULTS ( summary)

\section{Reaction forces ( $t$ = infinitely):}

\begin{tabular}{|l|r|r|r|r|r|}
\hline Units: all [kN] G:perm., Q:variable. ,V: Sum & \multicolumn{3}{l|}{} \\
\hline \multirow{2}{*}{ Support point } & G & min Q & max Q & min V & max V \\
\hline A (left) & 61.66 & 0.00 & 95.78 & 61.66 & 226.90 \\
B (right) & 61.66 & 0.00 & 95.78 & 61.66 & 226.90 \\
\hline
\end{tabular}

max. bending moment in erection state(char. value):

$$
\mathrm{MF}==570.36 \mathrm{kNm} \quad \text { at } \mathrm{x}==7.50 \mathrm{~m}
$$

Checks are not complied with:

\begin{tabular}{|l|r|r|l|r|r|}
\hline Checkvalue & Extrem & Utilisation & $x[\mathrm{~m}]$ \\
\hline Resisting tens force bot & $\eta=$ & 0.98 & & 1.02 & 14.80 \\
Crack MinAs+AsDuc top & AsMin $=$ & $* * * *$ & $\mathrm{~cm}^{2}$ & 0.02 \\
Prc.:Compr.stress t0(sto) & $\sigma c=$ & -28.39 & $\mathrm{~N} / \mathrm{mm}^{2}$ & 2.75 & 3.00 \\
\hline
\end{tabular}

\section{Warning}

Prc.:lin. creep t0(Sto) $\sigma c=-15.73 \mathrm{~N} / \mathrm{mm} 2 \quad x=0.79 \mathrm{~m}$ $\sigma c<0.45 * \mathrm{fck}(\mathrm{t})=-7.73 \mathrm{~N} / \mathrm{mm} 2$

_disproportional creeping by increased creep modulus considered(fk= 2.01)

\section{Required shear reinforcement:}

Column A: asw $=2.73 \mathrm{~cm}^{2} / \mathrm{m}$

Column B: $\quad$ asw $=2.73 \mathrm{~cm}^{2} / \mathrm{m}$

\section{Bursting reinforcement}

left Laying length $=0.95 \mathrm{~m}$

$$
\text { from } x=0.00 \mathrm{~m} \quad \text { As }=3.8 \mathrm{~cm}^{2}
$$

right Laying length $=0.95 \mathrm{~m}$

$$
\text { from } x=15.00 \mathrm{~m} \quad \text { As }=3.8 \mathrm{~cm}^{2}
$$

\section{Check of anchorage}

left: Tensile force resistance in anchoring area additional reinforcement necessary

right: Tensile force resistance in anchoring area additional reinforcement necessary

Overview crit. sections

Selected basic grid: 20 Sections
Util $=1.02$

Util $=1.02$

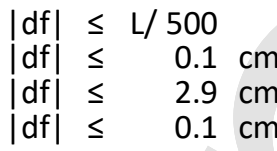


Demo Frilo

Stuttgarter Straße 40

70469

Stuttgart
Tel.: 0711810020

Fax: 0711858020
Projekt: Design Calculations

Position: P.02 - Prestressed Concrete Purlin

26.07.2018
Seite: 6

\begin{tabular}{|c|c|c|c|c|c|}
\hline Checkvalue & & Extrem & & Utilisation & $x[\mathrm{~m}]$ \\
\hline $\begin{array}{l}\text { Flexural capacity bottom } \\
\text { Flexural capacity top } \\
\text { Resisting tens force bot } \\
\text { Resisting tens force top } \\
\text { Prc.:Compr.stress t0(sto) } \\
\text { Prc.:Compr.stress Cc } \\
\text { Tension prestress. steel } \\
\text { Stress in prestress.steel } \\
\text { Stress in rebars } \\
\text { Crack MinAs+AsDuc bottom } \\
\text { Crack MinAs+AsDuc top } \\
\text { Crack width bottom } \\
\text { Crack width top } \\
\text { Sagging top } \\
\text { Sagging bottom } \\
\text { Incr.-deflection(Util) } \\
\text { Prc.:Shear reinf (web) } \\
\text { Concrete strut capacity }\end{array}$ & $\begin{aligned} \eta & = \\
\eta & = \\
\eta & = \\
\eta & = \\
\sigma c & = \\
\sigma c & = \\
\sigma p, Q c & = \\
\sigma p, C c & = \\
\sigma s & = \\
\text { AsMin } & = \\
\text { AsMin } & = \\
w k & = \\
w k & = \\
f o & = \\
\text { fu } & = \\
|d f| & = \\
\text { asw } & = \\
\eta & =\end{aligned}$ & $\begin{array}{r}1.02 \\
* * * * \\
0.98 \\
* * * * \\
-28.39 \\
-17.20 \\
963.2 \\
1110.9 \\
156.8 \\
2.4 \\
* * * * \\
0.00 \\
* * * * \\
-1.0 \\
0.1 \\
0.7 \\
2.73 \\
2.72\end{array}$ & $\begin{array}{l}\mathrm{N} / \mathrm{mm}^{2} \\
\mathrm{~N} / \mathrm{mm}^{2} \\
\mathrm{~N} / \mathrm{mm}^{2} \\
\mathrm{~N} / \mathrm{mm}^{2} \\
\mathrm{~N} / \mathrm{mm}^{2} \\
\mathrm{~cm}^{2} \\
\mathrm{~cm}^{2} \\
\mathrm{~mm} \\
\mathrm{~mm} \\
\mathrm{~cm} \\
\mathrm{~cm} \\
\mathrm{~cm} \\
\mathrm{~cm}^{2} / \mathrm{m}\end{array}$ & $\begin{array}{r}0.98 \\
* * * * \\
1.02 \\
* * * * \\
2.75 \\
0.96 \\
0.84 \\
0.79 \\
0.39 \\
0.37 \\
* * * * \\
0.01 \\
* * * * \\
0.17 \\
0.01 \\
0.24 \\
1.00 \\
0.37\end{array}$ & $\begin{array}{r}7.50 \\
0.59 \\
14.80 \\
0.59 \\
3.00 \\
3.00 \\
7.50 \\
7.50 \\
7.50 \\
0.02 \\
0.02 \\
7.50 \\
0.47 \\
7.11 \\
0.00 \\
7.89 \\
1.23 \\
0.40\end{array}$ \\
\hline
\end{tabular}

\begin{tabular}{|l|r|r|r|r|}
\hline Linear creep limit, informative: & & Extrem & Utilisation & $x$ [m] \\
\hline Prc.:lin. creep tO(Sto) & $\sigma c=$ & -15.73 & $\mathrm{~N} / \mathrm{mm}^{2}$ & 2.03 \\
Prc.:Compression quasi-permanent Lc & $\sigma c=$ & -7.07 & $\mathrm{~N} / \mathrm{mm}^{2}$ & 0.79 \\
\hline
\end{tabular}

\begin{tabular}{|l|r|r|r|r|r|}
\hline Tensile stress state I, informative: & & Extrem & Utilisation & $x[\mathrm{~m}]$ \\
\hline Prc.:Tens.stress (IS) & $\sigma \mathrm{t}=$ & $9.34 \mathrm{~N} / \mathrm{mm}^{2}$ & 3.23 & 7.50 \\
Prc.:Tens.stress (SC) & $\sigma \mathrm{t}=$ & 3.17 & $\mathrm{~N} / \mathrm{mm}^{2}$ & 1.76 & 0.79 \\
\hline
\end{tabular}


Demo Frilo

Stuttgarter Straße 40

70469

Stuttgart
Projekt: Design Calculations

Position: P.02 - Prestressed Concrete Purlin

26.07.2018
Seite: 7

Internal forces

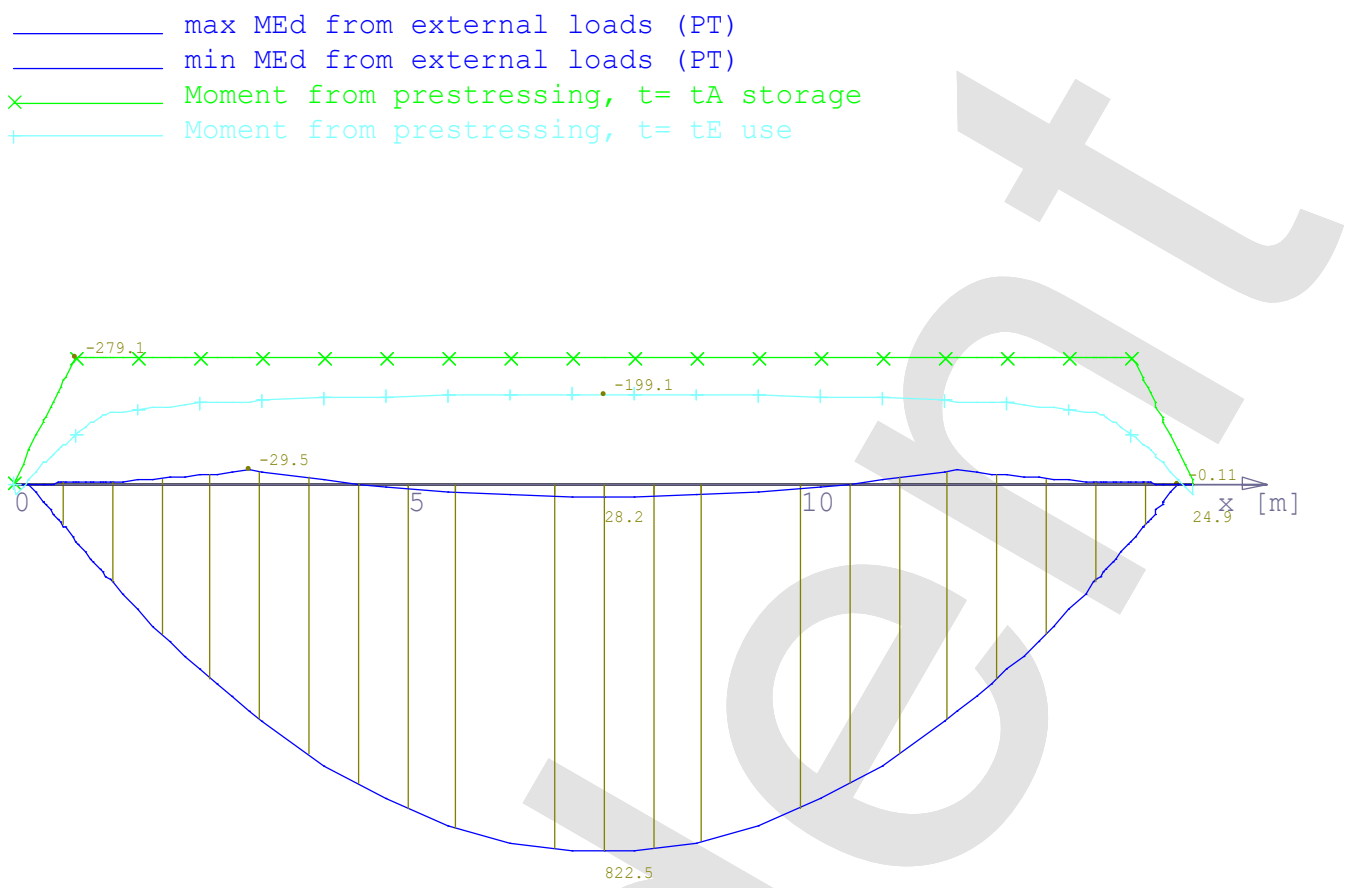

max VEd from external loads (PT)

min VEd from external loads (PT)

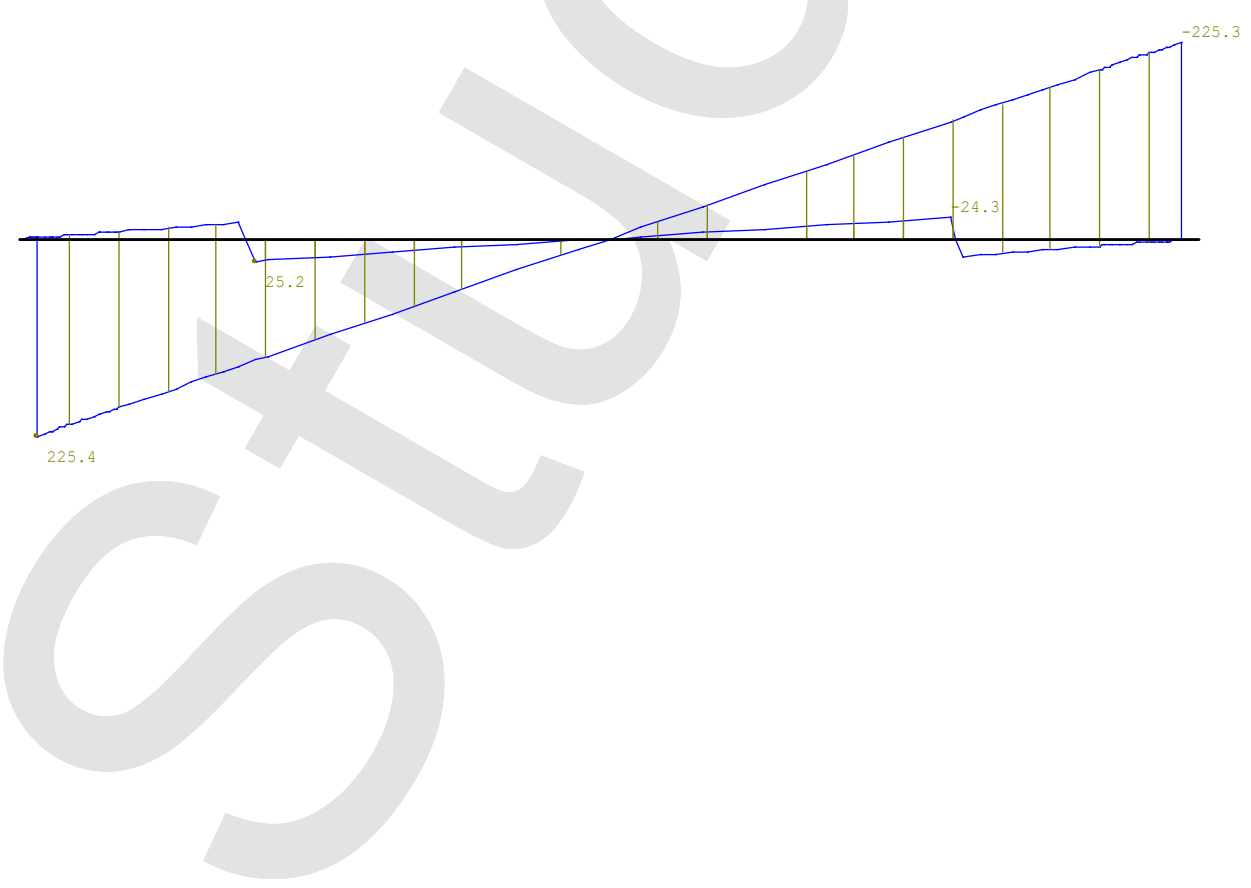


Demo Frilo

Stuttgarter Straße 40

70469

Stuttgart

Tel.: 0711810020

Fax: 0711858020
Projekt: Design Calculations

Position: P.02 - Prestressed Concrete Purlin

26.07.2018

Bending resitance (failure safety)

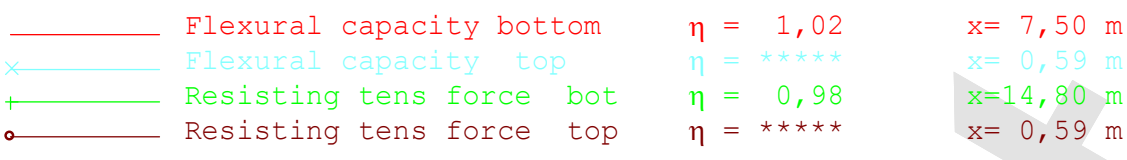

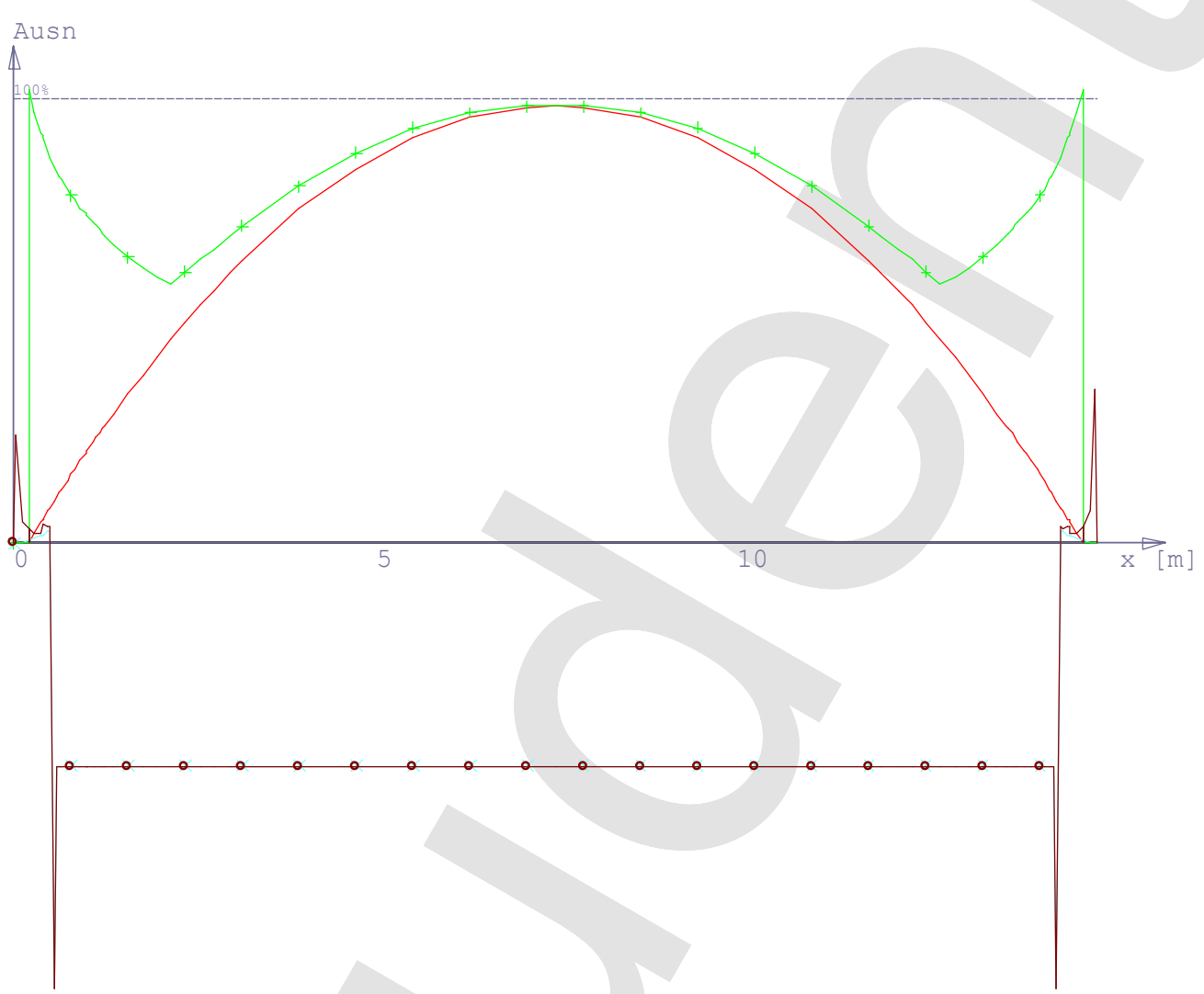

Deformation

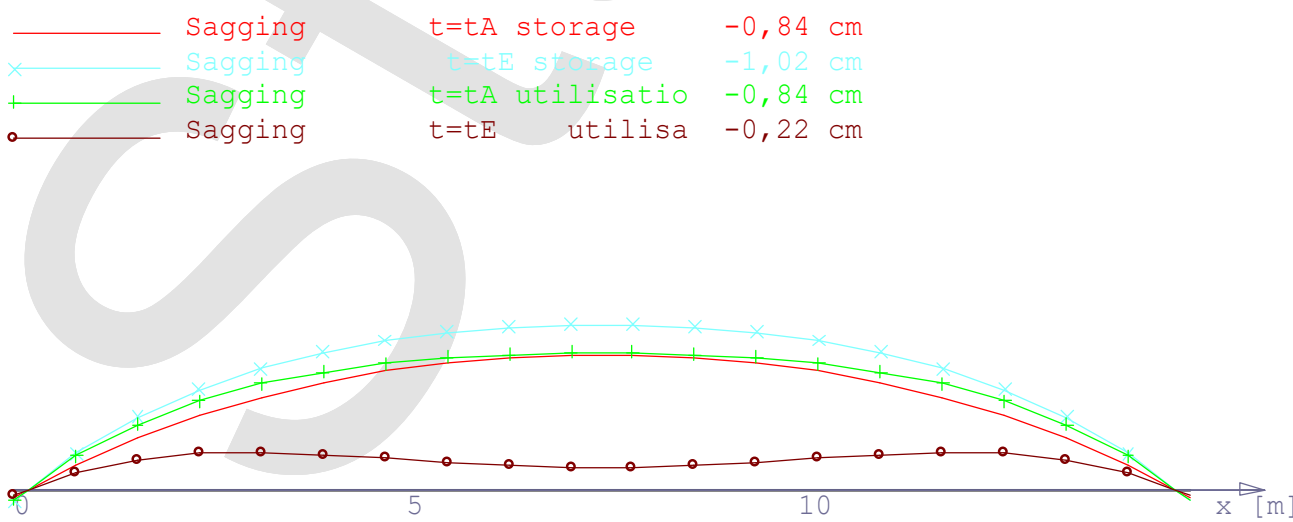


Demo Frilo

Stuttgarter Straße 40

70469 Stuttgart
Tel.: 0711810020

Fax: 0711858020
Projekt: Design Calculations

Position: P.02 - Prestressed Concrete Purlin

26.07.2018
Seite: 9

Shear force resistance (shear covering)

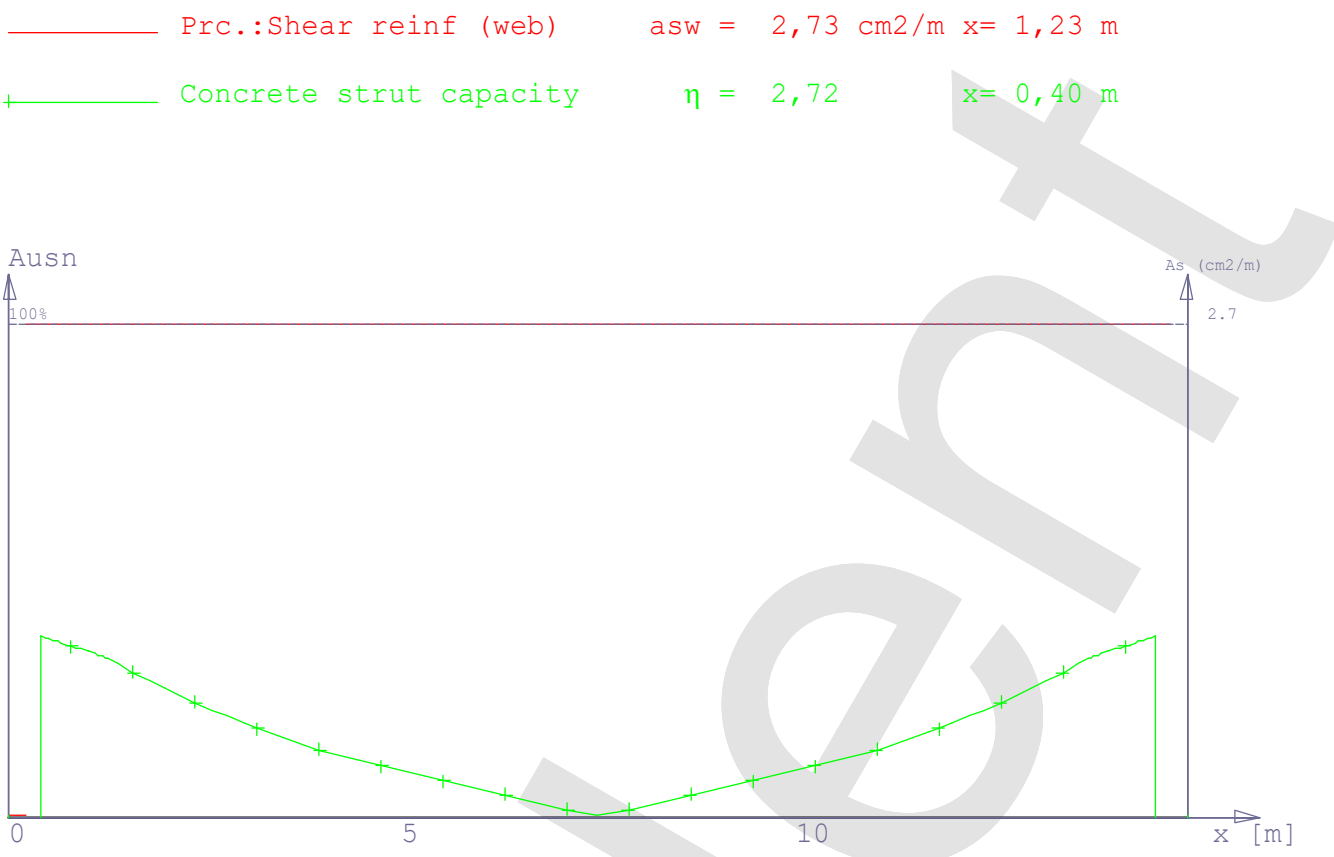

\section{Anchorage by bond ( over the left bearing)}

$$
\begin{aligned}
I_{\mathrm{pt} 2} & =1.14 \mathrm{~m} \\
\mathrm{fctd}_{\mathrm{ct}}(\mathrm{t}) & =1.02 \mathrm{~N} / \mathrm{mm}^{2} \\
\mathrm{fbpt} & =2.91 \mathrm{~N} / \mathrm{mm}^{2} \\
\eta \mathrm{P} 2 & =1.40
\end{aligned}
$$

\begin{tabular}{|c|c|c|c|c|c|}
\hline $\begin{array}{r}x \\
{[\mathrm{~m}]}\end{array}$ & $\begin{array}{r}\mathrm{Zp} \\
{[\mathrm{kN}]}\end{array}$ & $\begin{array}{r}\mathrm{Zs}_{\mathrm{s}} \\
{[\mathrm{kN}]}\end{array}$ & $\begin{array}{r}\text { TEd } \\
{[\mathrm{kN}]}\end{array}$ & $\begin{array}{r}\eta= \\
\left(Z_{p}+Z_{s}\right) / T_{E d}\end{array}$ & Util \\
\hline $\begin{array}{l}0.20 \\
0.39 \\
0.59 \\
0.79 \\
0.99 \\
1.18 \\
1.38 \\
1.58 \\
1.78 \\
1.97 \\
2.17 \\
2.37 \\
2.57 \\
2.76\end{array}$ & $\begin{array}{r}92.5 \\
179.3 \\
271.3 \\
363.3 \\
455.2 \\
544.6 \\
646.7 \\
748.8 \\
850.9 \\
947.9 \\
1044.8 \\
1044.9 \\
1045.0 \\
1045.1\end{array}$ & $\begin{array}{l}176.8 \\
176.7 \\
176.6 \\
176.5 \\
176.4 \\
176.4 \\
176.4 \\
176.5 \\
176.5 \\
176.5 \\
176.5 \\
176.5 \\
176.5 \\
176.5\end{array}$ & $\begin{array}{l}273.7 \\
325.8 \\
374.0 \\
421.1 \\
466.8 \\
509.0 \\
551.9 \\
593.4 \\
633.6 \\
670.5 \\
708.0 \\
744.4 \\
778.8 \\
808.4\end{array}$ & $\begin{array}{l}0.98 \\
1.09 \\
1.20 \\
1.28 \\
1.35 \\
1.42 \\
1.49 \\
1.56 \\
1.62 \\
1.68 \\
1.73 \\
1.64 \\
1.57 \\
1.51\end{array}$ & $\begin{array}{l}1.02 \\
0.92 \\
0.84 \\
0.78 \\
0.74 \\
0.71 \\
0.67 \\
0.64 \\
0.62 \\
0.60 \\
0.58 \\
0.61 \\
0.64 \\
0.66\end{array}$ \\
\hline
\end{tabular}

\begin{tabular}{|c|c|c|c|c|c|c|c|c|c|c|c|}
\hline $\begin{array}{l}\text { No. } \\
\text { Lay. }\end{array}$ & $\begin{array}{r}\text { Dist.LE } \\
{[\mathrm{cm}]}\end{array}$ & $\begin{array}{r}X A \\
{[\mathrm{~m}]}\end{array}$ & $\begin{array}{r}\sigma_{p} \\
{\left[\mathrm{~N} / \mathrm{mm}^{2}\right]}\end{array}$ & Eq. & $\begin{array}{l}\text { lopd } \\
{[\mathrm{m}]}\end{array}$ & $\begin{array}{r}x k \\
{\left[\mathrm{~cm}^{2}\right]}\end{array}$ & $\begin{array}{r}\Sigma Z_{p} \\
{[k N]}\end{array}$ & $\begin{array}{r}\Sigma Z_{s} \\
{[k N]}\end{array}$ & $\begin{array}{r}T_{E d} \\
{[k N]}\end{array}$ & $\begin{array}{r}\text { add. As } \\
{\left[\mathrm{cm}^{2}\right]}\end{array}$ & \\
\hline 1 & 7.5 & 0.00 & 693.42 & 8.21 & 2.37 & 0.20 & 92.5 & 176.8 & 273.7 & 0.1 & (PT) \\
\hline 2 & 11.7 & 0.00 & 706.41 & 8.21 & 2.35 & 0.20 & 92.5 & 176.8 & 273.7 & 0.1 & (PT) \\
\hline 3 & 15.9 & 0.00 & 719.33 & 8.21 & 2.34 & 0.20 & 92.5 & 176.8 & 273.7 & 0.1 & (PT) \\
\hline
\end{tabular}

XA: Beginning of the anchoring area of the steel layer (dist. from the corresp. binder side)

Eq. 8.21.1: Anchorage area uncracked, op acc.to fig. 8.17DE (b)

Eq. 8.21.1: Anchorage area cracked, op acc.to fig. 8.17DE (b)

$\mathrm{xk}$ : decisive section in the anchoring area of the layer (distance from the beginning of the binder)

add. As: Additional sagging reinforcement required for anchorage 
Demo Frilo

Stuttgarter Straße 40

70469
Tel.: 0711810020

Fax: 0711858020
Projekt: Design Calculations

Position: P.02 - Prestressed Concrete Purlin

26.07.2018

\section{Anchorage by bond ( over the right bearing)}

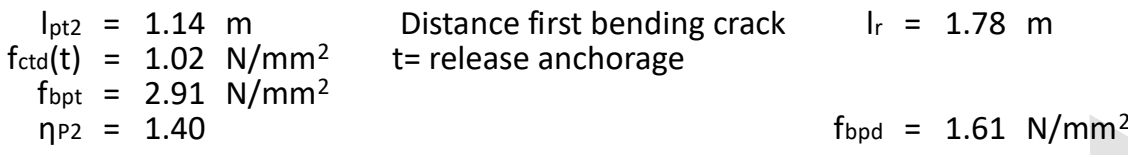

\begin{tabular}{|c|c|c|c|c|c|}
\hline $\begin{array}{r}x \\
{[\mathrm{~m}]}\end{array}$ & $\begin{array}{r}Z_{p} \\
{[k N]}\end{array}$ & $\begin{array}{r}Z_{s} \\
{[k N]}\end{array}$ & $\begin{array}{r}\text { TEd } \\
{[\mathrm{kN}]}\end{array}$ & $\begin{array}{r}\eta= \\
\left(Z_{p}+Z_{s}\right) / T_{E d}\end{array}$ & Uti \\
\hline 12.24 & 1045.1 & 176.5 & 808.4 & 1.51 & 0.66 \\
\hline 12.43 & 1045.0 & 176.5 & 778.8 & 1.57 & 0.64 \\
\hline 12.63 & 1044.9 & 176.5 & 744.4 & 1.64 & 0.61 \\
\hline 12.83 & 1044.8 & 176.5 & 708.0 & 1.73 & 0.58 \\
\hline 13.03 & 947.9 & 176.5 & 670.5 & 1.68 & 0.60 \\
\hline 13.22 & 850.9 & 176.5 & 633.6 & 1.62 & 0.62 \\
\hline 13.42 & 748.8 & 176.5 & 593.4 & 1.56 & 0.64 \\
\hline 13.62 & 646.7 & 176.4 & 551.9 & 1.49 & 0.67 \\
\hline 13.82 & 544.6 & 176.4 & 509.0 & 1.42 & 0.71 \\
\hline 14.01 & 455.2 & 176.4 & 466.8 & 1.35 & 0.74 \\
\hline 14.21 & 363.3 & 176.5 & 421.1 & 1.28 & 0.78 \\
\hline 14.41 & 271.3 & 176.6 & 374.0 & 1.20 & 0.84 \\
\hline 14.61 & 179.3 & 176.7 & 325.8 & 1.09 & 0.92 \\
\hline 14.80 & 92.5 & 176.8 & 273.7 & 0.98 & 1.02 \\
\hline
\end{tabular}

$Z_{p}$ : resisting tensile force by the prestressed steel

$Z_{s}$ : resisting tensile force by the rebars

TEd: tensile force to be anchored

\begin{tabular}{|c|c|c|c|c|c|c|c|c|c|c|c|}
\hline $\begin{array}{l}\text { No. } \\
\text { Lay. }\end{array}$ & $\begin{array}{r}\text { Dist.LE } \\
{[\mathrm{cm}]}\end{array}$ & $\begin{array}{r}X A \\
{[\mathrm{~m}]}\end{array}$ & $\begin{array}{r}\sigma_{p} \\
{\left[\mathrm{~N} / \mathrm{mm}^{2}\right]}\end{array}$ & Eq. & $\begin{array}{l}\text { lbpd } \\
{[\mathrm{m}]}\end{array}$ & $\begin{array}{r}x k \\
{\left[\mathrm{~cm}^{2}\right]}\end{array}$ & $\begin{array}{r}\Sigma Z p \\
{[k N]}\end{array}$ & $\begin{array}{r}\Sigma Z_{s} \\
{[k N]}\end{array}$ & $\begin{array}{r}\text { TEd } \\
{[\mathrm{kN}]}\end{array}$ & $\begin{array}{r}\text { add. As } \\
{\left[\mathrm{cm}^{2}\right]}\end{array}$ & \\
\hline $\begin{array}{l}1 \\
2 \\
3\end{array}$ & $\begin{array}{r}7.5 \\
11.7 \\
15.9\end{array}$ & $\begin{array}{l}0.00 \\
0.00 \\
0.00\end{array}$ & $\begin{array}{l}693.42 \\
706.41 \\
719.33\end{array}$ & $\begin{array}{l}8.21 \\
8.21 \\
8.21\end{array}$ & $\begin{array}{l}2.37 \\
2.35 \\
2.34\end{array}$ & $\begin{array}{l}14.80 \\
14.80 \\
14.80\end{array}$ & $\begin{array}{l}92.5 \\
92.5 \\
92.5\end{array}$ & $\begin{array}{l}176.8 \\
176.8 \\
176.8\end{array}$ & $\begin{array}{l}273.7 \\
273.7 \\
273.7\end{array}$ & $\begin{array}{l}0.1 \\
0.1 \\
0.1\end{array}$ & $\begin{array}{l}\text { (PT) } \\
\text { (PT) } \\
\text { (PT) }\end{array}$ \\
\hline
\end{tabular}

XA: Beginning of the anchoring area of the steel layer (dist. from the corresp. binder side)

Eq. 8.21.1: Anchorage area uncracked, $\sigma p$ acc.to fig. 8.17DE (b)

Eq. 8.21.1: Anchorage area cracked, op acc.to fig. 8.17DE (b)

$x \mathrm{k}$ : decisive section in the anchoring area of the layer (distance from the beginning of the binder)

add. As: Additional sagging reinforcement required for anchorage

\section{Bursting Reinforcement at beginning of beam}

$\gamma_{p, \text { unfav }}=1.35 \quad I_{\text {disp }}=1.26 \mathrm{~m}$

\begin{tabular}{|l|r|r|r|r|r|r|r|r|}
\hline \multicolumn{1}{|c|}{ Initiation zone } & \multicolumn{4}{|r|}{ Section over the last effective position of tension. member } \\
\hline No. & $\begin{array}{r}\text { from } \\
{[\mathrm{m}]}\end{array}$ & $\begin{array}{r}\text { to } \\
{[\mathrm{m}]}\end{array}$ & $\begin{array}{r}\text { Dist.LE } \\
{[\mathrm{cm}]}\end{array}$ & $\begin{array}{r}\mathrm{Nc} \\
{[\mathrm{kN}]}\end{array}$ & $\begin{array}{r}\mathrm{Np} \\
{[\mathrm{kN}]}\end{array}$ & $\begin{array}{r}\mathrm{Tp} \\
{[\mathrm{kN}]}\end{array}$ & $\begin{array}{r}\text { Factor } \\
\text { Interpolation }\end{array}$ & $\begin{array}{r}\text { req. As } \\
{\left[\mathrm{cm}^{2}\right]}\end{array}$ \\
\hline 1 & 0.00 & 1.26 & 16.9 & -369.9 & 699.3 & 329.4 & 0.376 & 3.8 \\
\hline
\end{tabular}

The bursting reinforcement must be arranged in zone of reduced dispersion length.

red. dispersion length indented wire w.o. strand $3 / 4 * 1$ disp $=0.95 \mathrm{~m}$

\section{Burstring Reinforcement at end of beam}

\begin{tabular}{|c|c|c|c|c|c|c|c|c|}
\hline \multicolumn{3}{|c|}{ Initiation zone } & \multicolumn{6}{|c|}{ Section over the last effective position of tension. member } \\
\hline No. & $\begin{array}{r}\text { from } \\
{[\mathrm{m}]}\end{array}$ & $\begin{array}{r}\text { to } \\
{[\mathrm{m}]}\end{array}$ & $\begin{array}{r}\text { Dist.LE } \\
{[\mathrm{cm}]}\end{array}$ & $\begin{array}{r}\mathrm{N}_{\mathrm{c}} \\
{[\mathrm{kN}]}\end{array}$ & $\begin{array}{r}\mathrm{Np} \\
{[\mathrm{kN}]}\end{array}$ & $\begin{array}{r}T_{p} \\
{[k N]}\end{array}$ & $\begin{array}{r}\text { Factor } \\
\text { Interpolation }\end{array}$ & $\begin{array}{r}\text { req. As } \\
{\left[\mathrm{cm}^{2}\right]}\end{array}$ \\
\hline 1 & 15.00 & 13.74 & 16.9 & -369.9 & 699.3 & 329.4 & 0.376 & 3.8 \\
\hline
\end{tabular}

The bursting reinforcement must be arranged in zone of reduced dispersion length.

red. dispersion length indented wire w.o. strand $3 / 4 * 1$ disp $=0.95 \mathrm{~m}$ 
B.1.2. Design Calculations POS. P.03 - Prestressed Concrete Truss 
Demo Frilo

Stuttgarter Straße 40

70469

Stuttgart
Tel.: 0711810020

Fax: 0711858020
Projekt: Design Calculations

Position: P.03 - Prestressed Concrete Truss

26.07.2018

\section{Position: P.03 - Prestressed Concrete Truss}

Spannbettbinder B8 01/2018 (Frilo R-2018-1/P12)
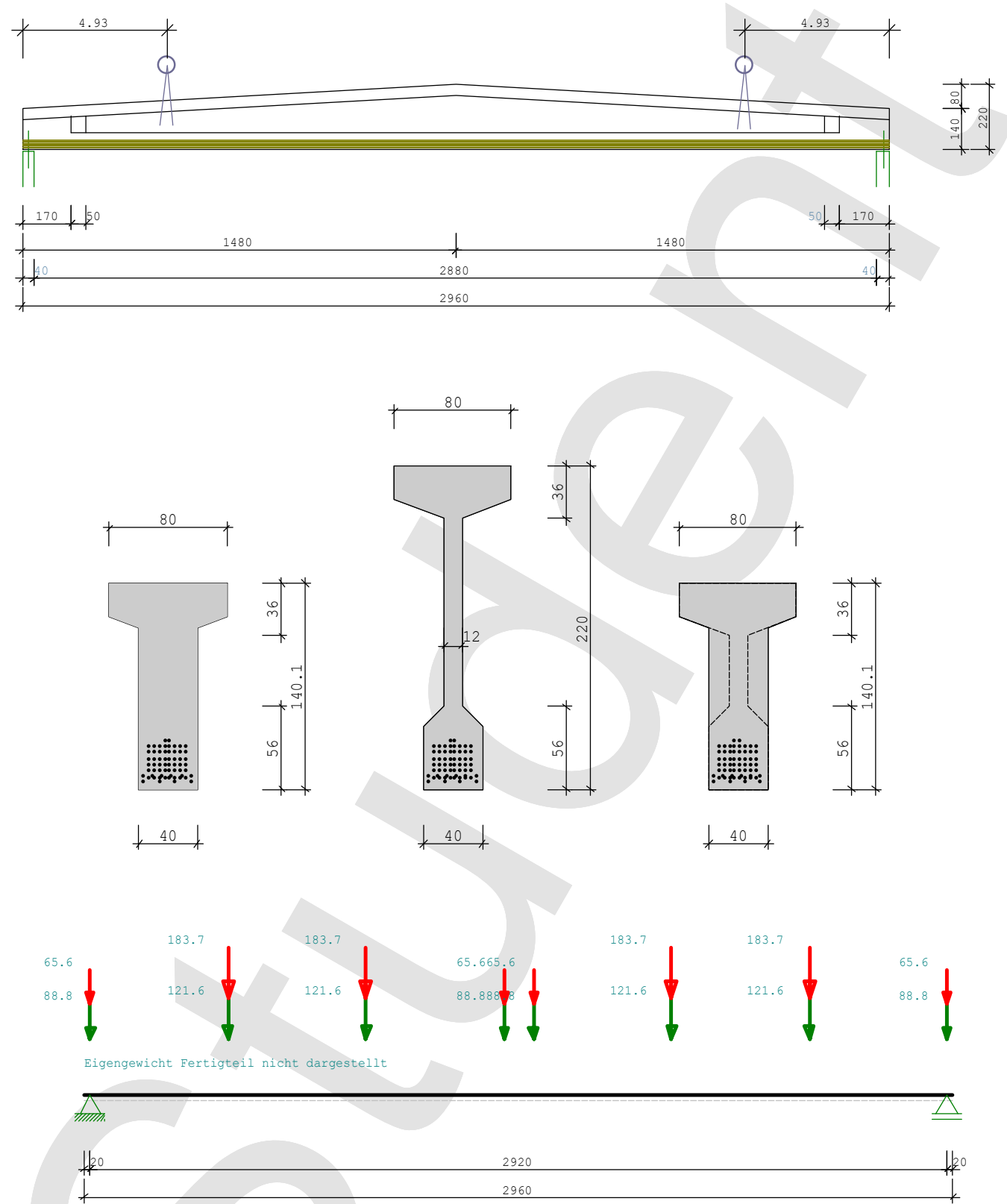

\section{Advices:}

No continuous reinforcement to $10 \mathrm{~cm}$ below UE found!

\section{System:}

\section{Double-pitch roof}

Basics:

Load combinatorics: DIN EN 1990/NA:2010-12 + EN 1990:2002/AC:2010

ULS: Structural safety checks(STR)

permanent/variable design situation with equation 6.10

Design code:

DIN EN 1992-1-1/NA C1:2012-06 + EN 1992-1-1:2004 /AC:2010

Prestressing for pretensioning 
Demo Frilo

Stuttgarter Straße 40

70469
Tel.: 0711810020

Fax: 0711858020
Projekt: Design Calculations

Position: P.03 - Prestressed Concrete Truss

26.07.2018

\section{System Geometry:}

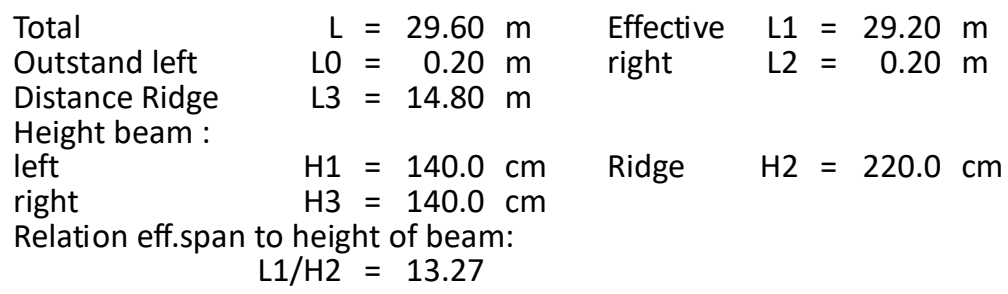

Erection attachment, distance from the beginning resp. end of beam: Hook $\quad L 8=4.93 \mathrm{~m}$ right $L 9=4.93 \mathrm{~m}$

Cross-section Precast :

\begin{tabular}{|l|r|r|r|}
\hline \multicolumn{2}{|l|}{ Layer of cross-section from top to bottom } \\
\hline $\mathrm{Nr}$ & $\begin{array}{r}\text { Width } \\
\text { [cm] }\end{array}$ & $\begin{array}{r}\text { Distance } \\
\text { [cm] }\end{array}$ & Remarks \\
\hline 1 & 80.0 & 0.0 & \\
2 & 80.0 & 22.4 & Web begin \\
3 & 12.0 & 36.0 & Web end \\
4 & 12.0 & 164.0 & \\
5 & 40.0 & 178.0 & \\
6 & 40.0 & 220.0 & \\
\hline \multicolumn{2}{|l|}{ Web height over beam length constant } \\
\hline
\end{tabular}

Bearing strengthening:

\begin{tabular}{|l|r|r|r|}
\hline & $\begin{array}{r}\text { Length } \\
{[\mathrm{cm}]}\end{array}$ & $\begin{array}{r}\text { Haunch } \\
{[\mathrm{cm}]}\end{array}$ & $\begin{array}{r}\text { Width } \\
{[\mathrm{cm}]}\end{array}$ \\
\hline left & 170.0 & 50.0 & 40.0 \\
right & 170.0 & 50.0 & 40.0 \\
\hline
\end{tabular}

\section{Material:}

\section{Prestressing steel}

$$
\begin{aligned}
& \text { SpSt 1500/1770 Strand } 7 \text { wires } \\
& \mathrm{d}_{\mathrm{d}}=\quad 4.1 \mathrm{~mm} \quad \mathrm{~d}_{\mathrm{p}}=12.4 \mathrm{~mm} \\
& E_{p}=195000 \mathrm{~N} / \mathrm{mm}^{2} \quad A_{p}=0.934 \mathrm{~cm}^{2} \\
& \mathrm{f}_{\mathrm{p} 0.1 \mathrm{k}}=1500 \mathrm{~N} / \mathrm{mm}^{2} \quad \mathrm{f}_{\mathrm{pk}}=1770 \mathrm{~N} / \mathrm{mm}^{2} \\
& \varepsilon_{\text {uk }}=35.0 \% \text { o } \quad \varepsilon_{\text {ud }}=25.0 \% \text { o }+\varepsilon_{p}(0)
\end{aligned}
$$

Partial safety factor : $\gamma_{\mathrm{s}}=1.15$

Coeff. prestress:

$$
\begin{array}{lrlrl}
\text { charact. value } & r_{\text {sup }} & =1.05 & r_{\text {inf }} & =0.95 \\
\text { Design value } & \gamma_{p, \max } & =1.00 & \gamma_{p}, \min & =1.00
\end{array}
$$

Proof of crack width

Equ. diameter $d_{p v}=7.21 \mathrm{~mm} \quad \xi=0.60$ (Tab. 6.2)

\begin{tabular}{|r|r|r|r|r|}
\hline Relaxation (from approval) & & & \\
\hline$\sigma_{\mathrm{p} 0} / \mathrm{f}_{\mathrm{pk}}$ & $10 \mathrm{~h}$ & $200 \mathrm{~h}$ & $1000 \mathrm{~h}$ & $1000000 \mathrm{~h}$ \\
\hline 0.60 & 0.3 & 0.6 & 0.8 & 2.8 \\
0.70 & 0.8 & 1.6 & 2.0 & 7.0 \\
0.80 & 2.0 & 4.0 & 5.0 & 14.0 \\
\hline
\end{tabular}
Permitted stresses:
in formwork
$\sigma_{p} \leq 1350.0 \mathrm{~N} / \mathrm{mm}^{2} \quad\left(0.90 * \mathrm{f}_{\mathrm{p} 0.1 \mathrm{k}}\right)$
after anchor. release
char. LC
$\sigma_{\mathrm{p}} \leq 1275.0 \mathrm{~N} / \mathrm{mm}^{2} \quad\left(0.85^{*} \mathrm{f}_{\mathrm{p} 0.1 \mathrm{k}}\right)$
q.perm.Lc
$\sigma_{p} \leq 1350.0 \mathrm{~N} / \mathrm{mm}^{2} \quad\left(0.90 * \mathrm{f}_{\mathrm{p} 0.1 \mathrm{k}}\right)$
$\sigma_{p} \leq 1150.5 \mathrm{~N} / \mathrm{mm}^{2} \quad(0.65 * \mathrm{fpk})$ 
Transmission length

$$
\begin{aligned}
\eta_{p 1} & =2.85 & \eta_{1} & =1.00 \\
\alpha_{1} & =1.25 & \alpha_{2} & =0.19 \\
\sigma_{p m 0} & =917 \mathrm{~N} / \mathrm{mm}^{2} & & \\
\text { PT: } \quad f_{c t d t} & =1.36 \mathrm{~N} / \mathrm{mm}^{2} & \mathrm{fbpt}_{\mathrm{bp}} & =3.86 \mathrm{~N} / \mathrm{mm}^{2} \\
\mathrm{I}_{\mathrm{pt}} & =0.70 \mathrm{~m} & &
\end{aligned}
$$

\begin{tabular}{|c|c|c|c|c|c|}
\hline & $\begin{array}{r}\text { Longitudinal } \\
\text { B } 500 \text { B }\end{array}$ & & & $\begin{array}{l}\text { Stirrup } \\
\text { B } 500 \text { B }\end{array}$ & \\
\hline & 200000 & $\mathrm{~N} / \mathrm{mm}^{2}$ & $E_{s}$ & 200000 & $\mathrm{~N} / \mathrm{mm}^{2}$ \\
\hline & 500 & $\mathrm{~N} / \mathrm{mm}^{2}$ & $f_{y k}$ & 500 & $\mathrm{~N} / \mathrm{mm}^{2}$ \\
\hline$f_{t k}$ & 540 & $\mathrm{~N} / \mathrm{mm}^{2}$ & $f_{t k}$ & 540 & $\mathrm{~N} / \mathrm{mm}^{2}$ \\
\hline Euk & 50.0 & $\%$ & Euk & 50.0 & $\%$ \\
\hline Eud & 25.0 & $\%$ & $\varepsilon_{\text {ud }}$ & 25.0 & $\%$ \\
\hline
\end{tabular}

Dispersion length:

$$
\mathrm{d}=1 . \overline{3} 4 \mathrm{~m} \quad l_{\text {disp }}=1.51 \mathrm{~m}
$$

Reinforcing steel:

Partial safety factor
$\gamma_{\mathrm{s}}=$
1.15
$\gamma_{\mathrm{s}}=$
1.15

permitted stresses in SLS :
$\sigma_{\mathrm{s}} \leq$
$400 \mathrm{~N} / \mathrm{mm}^{2}$
$\sigma_{s} \leq$
$400 \mathrm{~N} / \mathrm{mm}^{2} \quad\left(0.80 * f_{\mathrm{yk}}\right)$

\section{Requirements durability:}

attack on concrete
attack on reinforc.
min. concrete class
stirrup
long. reinforcement
prestressed steel
allowance in design
stirrup
concrete coverage
longitudinal bars
concrete coverage
prestressing steel :
concrete coverage
laying dist. link
all. crack width
decompression
$* 1:$ with cmin,l
$* 5$ : bond decisive

top

wo

XC1

C $16 / 20$

$\phi, I=10 \mathrm{~mm}$

$\phi, \mathrm{m}=16 \mathrm{~mm}$

$\mathrm{dp}_{\mathrm{p}}=12.4 \mathrm{~mm}$ strand, $\sigma_{\mathrm{p}}{ }^{(0)}<=1000 \mathrm{~N} / \mathrm{mm} 2$

$\Delta c_{\text {dev }}=10 \mathrm{~mm}$

$\mathrm{C}_{\text {min, }, \mathrm{l}}=10 \mathrm{~mm} * 5$

Cnom, $1=20 \mathrm{~mm} * 5 * 5$

$\mathrm{Cmin}, \mathrm{m}=16 \mathrm{~mm} * 5$

Cnom, $\mathrm{m}=30 \mathrm{~mm} * 1$

$C_{\text {min, } p}=31 \mathrm{~mm} * 5$

$\mathrm{Cnom}, \mathrm{p}=41 \mathrm{~mm}$

$\mathrm{C}, \mathrm{I}=20 \mathrm{~mm}$

$\mathrm{W}_{\max }=0.20 \mathrm{~mm}$ not req.

$$
\begin{aligned}
& \text { bottom } \\
& \text { W0 } \\
& \text { XC1 } \\
& \text { C } 16 / 20
\end{aligned}
$$

$\phi, \mathrm{m}=20 \mathrm{~mm}$

$\Delta \mathrm{c}_{\text {dev }}=10 \mathrm{~mm}$

$\mathrm{C}_{\text {min, }, 1}=10 \mathrm{~mm} * 5 * 5$

Cnom, $1=20 \mathrm{~mm} * 5 * 5$

$\mathrm{Cmin}, \mathrm{m}=20 \mathrm{~mm} \quad *_{5}$

Cnom, $\mathrm{m}=30 \mathrm{~mm}$

$C_{\text {min, } p}=31 \mathrm{~mm}$

Cnom, $p=41 \mathrm{~mm}$

$\mathrm{C}, \mathrm{I}=20 \mathrm{~mm}$

$\mathrm{W}_{\max }=0.20 \mathrm{~mm}$ not req.

\section{Concrete:}

Precast

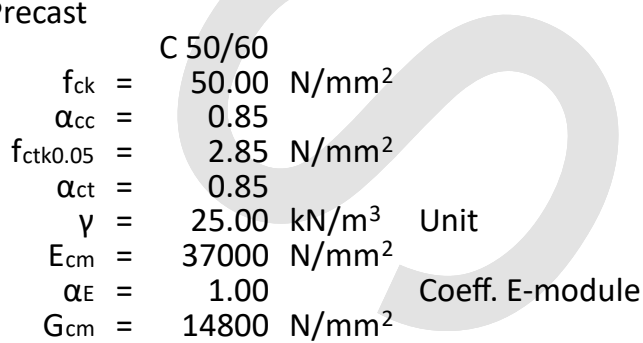

Partial safety factor :

$\gamma_{c}=1.50$

permitted stresses in SLS :
char. LC
$\sigma_{c} \geq-30.00 \mathrm{~N} / \mathrm{mm}^{2}$
q.perm.Lc
$\sigma_{c} \geq-22.50 \mathrm{~N} / \mathrm{mm}^{2}$
Removal the anchor $\mathrm{t}=\mathrm{tOT}$ (sto)
$f_{\mathrm{cm}(\mathrm{t})}=38.43 \mathrm{~N} / \mathrm{mm}^{2}$
$f_{c k}(t)=30.43 \mathrm{~N} / \mathrm{mm}^{2}$
linear creep $\quad \sigma_{c} \geq-13.69 \mathrm{~N} / \mathrm{mm}^{2} \quad(k 2=0.45)$
maximum $\quad \sigma_{c} \geq-18.26 \mathrm{~N} / \mathrm{mm}^{2} \quad(k 6=0.60)$ 
Demo Frilo

Stuttgarter Straße 40

70469
Tel.: 0711810020

Fax: 0711858020
Projekt: Design Calculations

Position: P.03 - Prestressed Concrete Truss

26.07.2018
Seite: 4

\section{Creep modulus \& shrinkage strain}

no heat treatment, t0T $=$ t0

CementStrenght class $42,5 \mathrm{R} ; 52,5$

$\rho=0.5$ (Aging coefficient)

Reference point for t0 is the start of the concreting of the precast

\begin{tabular}{|l|r|r|}
\hline Creep & t0 & RH \\
Days & $\%$ \\
\hline Storage & 3 & 70 \\
Utilization precast & 180 & 50 \\
\hline
\end{tabular}

\begin{tabular}{|c|c|c|c|c|c|c|c|c|c|c|c|c|}
\hline L. & Segment & $\begin{array}{r}\text { Part- } \\
\text { cross-section }\end{array}$ & to & $\mathrm{t}$ & $\alpha$ & $\begin{array}{r}\text { to,eff } \\
\text { B.9 }\end{array}$ & $\begin{array}{l}\beta \text { to } \\
\text { B.5 }\end{array}$ & $\begin{array}{r}\text { ßH } \\
\text { B.8 }\end{array}$ & $\begin{array}{r}\beta_{c(t, t 0)} \\
\text { B.7 }\end{array}$ & $\begin{array}{l}\phi R H \\
\text { B.3 }\end{array}$ & $\begin{array}{r}\beta \mathrm{fcm} \\
\text { B.4 }\end{array}$ & $\begin{array}{r}\phi(t, t 0) \\
\text { B.1 }\end{array}$ \\
\hline$\frac{1}{2}$ & $\begin{array}{r}\text { Storage } \\
\text { Utilization precast }\end{array}$ & $\mathrm{PcC}$ & $\begin{array}{r}3.0 \\
1800\end{array}$ & $\begin{array}{r}180.0 \\
26000.0\end{array}$ & 1 & $\begin{array}{l}7.7 \\
77\end{array}$ & & 498.2 & & 1.23 & 2.21 & 1.13 \\
\hline
\end{tabular}

\begin{tabular}{|r|r|r|r|r|r|r|r|r|r|r|r|}
\hline L. & $\begin{array}{r}\mathrm{A} \\
{\left[\mathrm{cm}^{2}\right]}\end{array}$ & $\begin{array}{r}\mathrm{U} \\
{[\mathrm{cm}]}\end{array}$ & $\begin{array}{r}\mathrm{h0} \\
{[\mathrm{cm}]}\end{array}$ & ßds $(\mathrm{t0}, \mathrm{ts})$ & $\begin{array}{r}\beta_{\mathrm{ds}}(\mathrm{t}, \mathrm{ts}) \\
3.10\end{array}$ & $\begin{array}{r}\beta_{\mathrm{RH}} \\
\text { B.12 }\end{array}$ & $\begin{array}{r}\varepsilon_{\mathrm{cd}, 0} \\
\text { B.11 }\end{array}$ & $\begin{array}{r}\beta_{\mathrm{as}} \\
3.13\end{array}$ & $\begin{array}{r}\varepsilon_{\mathrm{ca}} / 10 \mathrm{e} 6 \\
3.12\end{array}$ & $\begin{array}{r}\varepsilon_{\mathrm{cs}}(\mathrm{t}, \mathrm{t0}) \\
{[\% \mathrm{o}]}\end{array}$ \\
\hline 1 & 5997.60 & 617.6 & 194.2 & 0.000 & 0.620 & 1.02 & 402.5 & 0.93 & 100.00 & 0.308 \\
\hline 2 & 5997.60 & 617.6 & 194.2 & 0.620 & 0.996 & 1.36 & 536.0 & 1.00 & 100.00 & 0.180 \\
\hline
\end{tabular}

\section{Loads:}

\section{Self weight}

Beam beginning

Support reinforcement end left

Support reinforcement haunch left

Ridge

Support reinforcement haunch right

Support reinforcement end right

Beam end

Total

Volume

Surf.

\section{Live loads}

\begin{tabular}{|c|c|c|c|c|c|c|c|c|c|c|c|}
\hline \multicolumn{12}{|c|}{ Units: Single load[kN] Single moment $[k N m]$ line load[kN/m] } \\
\hline span & type & gle & qle & $\begin{array}{r}\text { Dist. a } \\
{[\mathrm{m}]}\end{array}$ & gri & qri & $\begin{array}{r}\text { Length } \\
{[\mathrm{m}]}\end{array}$ & Fact & Act. & Sim. & Pos. \\
\hline 1 & 2 & 88.80 & 65.60 & 0.00 & & & & 1.00 & 10 & 0 & \\
\hline 1 & 2 & 88.80 & 65.60 & 14.10 & & & & 1.00 & 10 & 0 & \\
\hline 1 & 2 & 88.80 & 65.60 & 15.10 & & & & 1.00 & 10 & 0 & \\
\hline 1 & 2 & 88.80 & 65.60 & 29.20 & & & & 1.00 & 10 & 0 & \\
\hline 1 & 2 & 121.60 & 183.70 & 4.70 & & & & 1.00 & 10 & 0 & \\
\hline $\begin{array}{l}1 \\
1\end{array}$ & 2 & 121.60 & 183.70 & 9.40 & & & & 1.00 & 10 & 0 & \\
\hline 1 & 2 & 121.60 & 183.70 & 19.80 & & & & 1.00 & 10 & 0 & \\
\hline 1 & 2 & 121.60 & 183.70 & 24.50 & & & & 1.00 & 10 & 0 & \\
\hline
\end{tabular}

\section{Actions:}

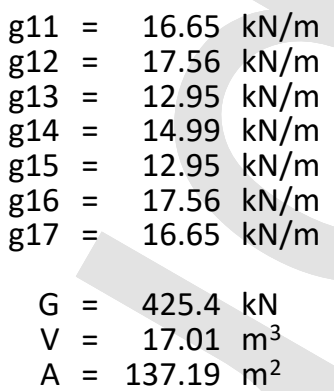

\begin{tabular}{|r|r|r|r|r|r|r|r|}
\hline Act. & $\gamma_{Q}$ & $\psi_{0}$ & $\psi_{1}$ & $\psi_{2}$ & Dep. & Cat. & Description \\
\hline 10 & 1.50 & 0.50 & 0.20 & 0.00 & 0 & $\mathrm{~S}$ & Schnee bis NN $+1000 \mathrm{~m}$ \\
\hline
\end{tabular}

\section{Tendons:}

$\operatorname{Dist}($ LE) $>5.4 \mathrm{~cm}$ axis horizontal $>3.7 \mathrm{~cm}$ vertical $>3.7 \mathrm{~cm}$ 


\begin{tabular}{|c|c|c|c|c|c|c|c|c|}
\hline \multirow{2}{*}{$\begin{array}{l}\text { lay. } \\
\text { No. }\end{array}$} & \multirow{2}{*}{$\begin{array}{r}\text { num- } \\
\text { ber }\end{array}$} & \multirow{2}{*}{$\begin{array}{r}\text { area } \\
\mathrm{Ap} \\
{\left[\mathrm{cm}^{2}\right]}\end{array}$} & \multirow{2}{*}{$\begin{array}{r}\text { Dist. LE } \\
Y p \\
{[\mathrm{~cm}]}\end{array}$} & \multirow{2}{*}{$\begin{array}{r}\text { Prestressing } \\
\sigma_{\mathrm{p}}(0) \\
{\left[\mathrm{N} / \mathrm{mm}^{2}\right]}\end{array}$} & \multicolumn{3}{|c|}{ <--- Isolations ---> } & \multirow[b]{2}{*}{$\begin{array}{c}\text { Type } \\
\mid 1\end{array}$} \\
\hline & & & & & Count & $\begin{array}{r}\text { to } \times 1 \\
{[\mathrm{~m}]}\end{array}$ & $\begin{array}{r}\text { from } \times 2 \\
{[\mathrm{~m}]}\end{array}$ & \\
\hline 1 & 8 & 7.47 & 7.5 & 1000 & 0 & & & LE \\
\hline 2 & 8 & 7.47 & 11.7 & 1000 & 0 & & & LE \\
\hline 3 & 8 & 7.47 & 15.9 & 1000 & 0 & & & LE \\
\hline 4 & 8 & 7.47 & 20.1 & 1000 & 0 & & & LE \\
\hline 5 & 8 & 7.47 & 24.3 & 1000 & 0 & & & LE \\
\hline 6 & 8 & 7.47 & 28.5 & 1000 & 0 & & & LE \\
\hline 7 & 2 & 1.87 & 32.7 & 1000 & 0 & & & LE \\
\hline
\end{tabular}

$\mathrm{x} 1$ and $\mathrm{x} 2$ with respect to the left beginning from joint

$\mathrm{LE}=$ parallel lower edge, $\mathrm{UE}=$ parallel upper edge

The calculation of the losses due to creep, shrinkage and relaxation

following the method from Abelein

Untensioned reinforcement:

\begin{tabular}{|c|c|c|c|c|c|c|c|}
\hline \multirow{2}{*}{$\begin{array}{l}\text { Layer } \\
\text { No. }\end{array}$} & \multirow{2}{*}{$\begin{array}{r}\text { num- } \\
\text { ber }\end{array}$} & \multirow{2}{*}{$\begin{array}{r}\text { diam. } \\
\text { Фs,l } \\
{[\mathrm{mm}]}\end{array}$} & \multirow{2}{*}{$\begin{array}{r}\text { area } \\
\text { As } \\
{\left[\mathrm{cm}^{2}\right]}\end{array}$} & Dist.LE & \multicolumn{2}{|c|}{ effective range } & \multirow[b]{2}{*}{$\begin{array}{r}\text { Type } \\
\text { II }\end{array}$} \\
\hline & & & & $\begin{array}{r}Y s \\
{[\mathrm{~cm}]}\end{array}$ & $\begin{array}{r}\text { from } \times A \\
{[\mathrm{~m}]}\end{array}$ & $\begin{array}{r}\text { to } x E \\
{[\mathrm{~m}]}\end{array}$ & \\
\hline $\begin{array}{l}1 \\
2\end{array}$ & $\begin{array}{l}4 \\
6\end{array}$ & $\begin{array}{l}20 \\
16\end{array}$ & $\begin{array}{l}12.57 \\
12.06\end{array}$ & $\begin{array}{l}4.0 \\
8.0\end{array}$ & $\begin{array}{l}0.00 \\
0.00\end{array}$ & $\begin{array}{l}29.60 \\
29.60\end{array}$ & $\begin{array}{l}\text { LE } \\
\text { LE }\end{array}$ \\
\hline
\end{tabular}

$X A$ and $x E$ with respect to the left beginning from joint

$\mathrm{LE}=$ parallel lower edge, $\mathrm{UE}=$ parallel upper edge

Min. reinforcement width of crack not required

(user defined)

Surface reinforcement acc.to Tab. NA.J.41 (BO < D0) :
Web (Z1/S3)
AsS $=0.78 \mathrm{~cm}^{2} / \mathrm{m}$
(UwkS $<=$ XC4) (per side)
Top flange $(\mathrm{Z} 3 / \mathrm{S} 1) \quad$ AsO $=0.00 \mathrm{~cm}^{2} / \mathrm{m}$
(UwkS $<=$ XC4)

\section{Settings for shear resistance check}

Bearing width, distance bearing edge, effective height of the bearing line

left $\mathrm{bAl}=0.40 \mathrm{~m} \quad \mathrm{al}=0.20 \mathrm{~m} \quad \mathrm{dAl}=1.35 \mathrm{~m}$

right $b A r=0.40 \mathrm{~m} \quad$ ar $=0.20 \mathrm{~m} \quad \mathrm{dAR}=1.35 \mathrm{~m}$

For shear reinforcement not decisive ranges over support $A$ and $B$ :

$x a R e=1.55 \mathrm{~m}$ direct bearing (width of bearing $/ 2+$ eff. depth)

$x b L i=1.55 \mathrm{~m}$ direct bearing (width of bearing/ $2+$ eff. depth)

\section{Check the limit deformation:}

Total sagging $\quad f \leq L / 250 \quad$ Increase deflection $|d f| \leq L / 500$

$\begin{array}{llrl}\text { Cantilever left } & f \leq 0.2 \mathrm{~cm} & |d \mathrm{df}| \leq 0.1 \mathrm{~cm} \\ \text { Span } & \mathrm{f} \leq 11.7 \mathrm{~cm} & |\mathrm{df}| \leq 5.8 \mathrm{~cm}\end{array}$

Cantilever right $f \leq 0.2 \mathrm{~cm}$

quasi- permanent combination and eff. char. prestress

Deflection due to shrinkage considered

Tension stiffening: Member rigidity, Characteristic combination

\section{RESULTS ( summary)}

\section{Reaction forces ( $t$ = infinitely):}

\begin{tabular}{|l|r|r|r|r|r|}
\hline Units: all [kN] G:perm., Q:variable. ,V: Sum & \multicolumn{3}{l|}{} \\
\cline { 2 - 6 } Support point & G & $\min$ Q & $\max$ Q & $\min$ V & <--ULS(PT)----> \\
\hline A (left) & 633.57 & 0.00 & 498.60 & 633.57 & 1603.23 \\
B (right) & 633.57 & 0.00 & 433.00 & 633.57 & 1603.23 \\
\hline
\end{tabular}

max. bending moment in erection state(char. value):
$\mathrm{MF}==8002.46 \mathrm{kNm}$
at $\mathrm{x}=14.80 \mathrm{~m}$

Checks are not complied with:

\begin{tabular}{|l|r|r|r|r|}
\hline Checkvalue & & Extrem & Utilisation & $x[\mathrm{~m}]$ \\
\hline Prc.:Compr.stress t0(sto) & $\sigma c=$ & -41.26 & $\mathrm{~N} / \mathrm{mm}^{2}$ & 2.26 \\
\hline
\end{tabular}


Demo Frilo

Stuttgarter Straße 40

70469
Tel.: 0711810020

Fax: 0711858020
Projekt: Design Calculations

Position: P.03 - Prestressed Concrete Truss

26.07.2018
Seite: 6

\section{Warning}

Prc.:lin. creep t0(Sto) $\sigma c=-30.13 \mathrm{~N} / \mathrm{mm} 2 \quad \mathrm{x}=28.96 \mathrm{~m}$

$\sigma c<0.45^{*} \mathrm{fck}(\mathrm{t})=-13.69 \mathrm{~N} / \mathrm{mm} 2$

_disproportional creeping by increased creep modulus considered(fk= 2.25)

\section{Required shear reinforcement:}

Column A: asw $=14.61 \mathrm{~cm}^{2} / \mathrm{m}$

Column B: asw $=14.61 \mathrm{~cm}^{2} / \mathrm{m}$

\section{Bursting reinforcement}

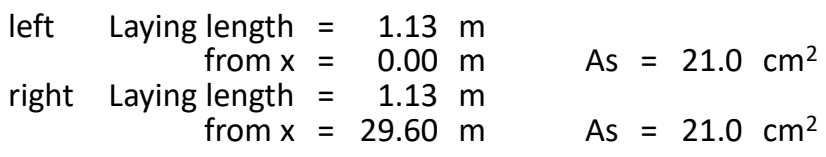

\section{Check of anchorage}

left: Tensile force resistance in anchoring area right: Tensile force resistance in anchoring area

Overview crit. sections
Util $=0.51$

Util $=0.51$

Selected basic grid: 10 Sections

\begin{tabular}{|c|c|c|c|c|c|}
\hline Checkvalue & & Extrem & & Utilisation & $x[\mathrm{~m}]$ \\
\hline $\begin{array}{l}\text { Flexural capacity bottom } \\
\text { Flexural capacity top } \\
\text { Resisting tens force bot } \\
\text { Resisting tens force top } \\
\text { Prc.:Compr.stress t0(sto) } \\
\text { Prc.:Compr.stress Cc } \\
\text { Tension prestress. steel } \\
\text { Stress in prestress.steel } \\
\text { Stress in rebars } \\
\text { Crack MinAs+AsDuc bottom } \\
\text { Crack MinAs+AsDuc top } \\
\text { Crack width bottom } \\
\text { Crack width top } \\
\text { Sagging top } \\
\text { Sagging bottom } \\
\text { Incr.-deflection(Util) } \\
\text { Prc.:Shear reinf (web) } \\
\text { Concrete strut capacity }\end{array}$ & $\begin{aligned} \eta & = \\
\eta & = \\
\eta & = \\
\eta & = \\
\sigma c & = \\
\sigma c & = \\
\sigma p, Q c & = \\
\sigma p, C c & = \\
\sigma s & = \\
\text { AsMin } & = \\
\text { AsMin } & = \\
w k & = \\
w k & = \\
\text { fo } & = \\
\text { fu } & = \\
|d f| & = \\
\text { asw } & = \\
\eta & =\end{aligned}$ & \begin{tabular}{r|}
1.15 \\
$* * * *$ \\
1.15 \\
$* * * *$ \\
-41.26 \\
-20.54 \\
936.2 \\
1003.9 \\
35.5 \\
13.5 \\
--- \\
0.00 \\
$* * * *$ \\
-3.4 \\
0.6 \\
2.0 \\
14.61 \\
1.13
\end{tabular} & $\begin{array}{l} \\
\mathrm{N} / \mathrm{mm}^{2} \\
\mathrm{~N} / \mathrm{mm}^{2} \\
\mathrm{~N} / \mathrm{mm}^{2} \\
\mathrm{~N} / \mathrm{mm}^{2} \\
\mathrm{~N} / \mathrm{mm}^{2} \\
\mathrm{~cm}^{2} \\
\mathrm{~cm}^{2} \\
\mathrm{~mm} \\
\mathrm{~mm} \\
\mathrm{~cm} \\
\mathrm{~cm} \\
\mathrm{~cm} \\
\mathrm{~cm}^{2} / \mathrm{m}\end{array}$ & $\begin{array}{r}0.87 \\
* * * * \\
0.87 \\
* * * * \\
2.26 \\
0.68 \\
0.81 \\
0.74 \\
0.09 \\
0.34 \\
--- \\
0.01 \\
* * * * \\
0.29 \\
0.05 \\
0.35 \\
1.00 \\
0.89\end{array}$ & $\begin{array}{r}9.60 \\
0.50 \\
9.56 \\
0.50 \\
4.93 \\
4.93 \\
1.70 \\
9.60 \\
9.60 \\
14.80 \\
---- \\
9.60 \\
0.33 \\
16.44 \\
13.16 \\
13.16 \\
2.20 \\
2.20\end{array}$ \\
\hline
\end{tabular}

\begin{tabular}{|l|r|r|r|r|}
\hline Linear creep limit, informative: & & Extrem & Utilisation & $x$ [m] \\
\hline Prc.:lin. creep t0(Sto) & $\sigma c=$ & -30.13 & $\mathrm{~N} / \mathrm{mm}^{2}$ & 2.20 \\
Prc.:Compression quasi-permanent Lc & $\sigma c=$ & -14.35 & 28.96 \\
\hline
\end{tabular}

\begin{tabular}{|l|r|r|r|r|r|}
\hline Tensile stress state I, informative: & & Extrem & Utilisation & $x[\mathrm{~m}]$ \\
\hline Prc.:Tens.stress (IS) & $\sigma \mathrm{t}=$ & 10.80 & $\mathrm{~N} / \mathrm{mm}^{2}$ & 2.65 & 9.60 \\
Prc.:Tens.stress (SC) & $\sigma \mathrm{t}=$ & 6.01 & $\mathrm{~N} / \mathrm{mm}^{2}$ & 2.38 & 28.96 \\
\hline
\end{tabular}


Demo Frilo

Stuttgarter Straße 40

70469

Stuttgart
Projekt: Design Calculations

Position: P.03 - Prestressed Concrete Truss

26.07.2018
Seite: 7

Internal forces

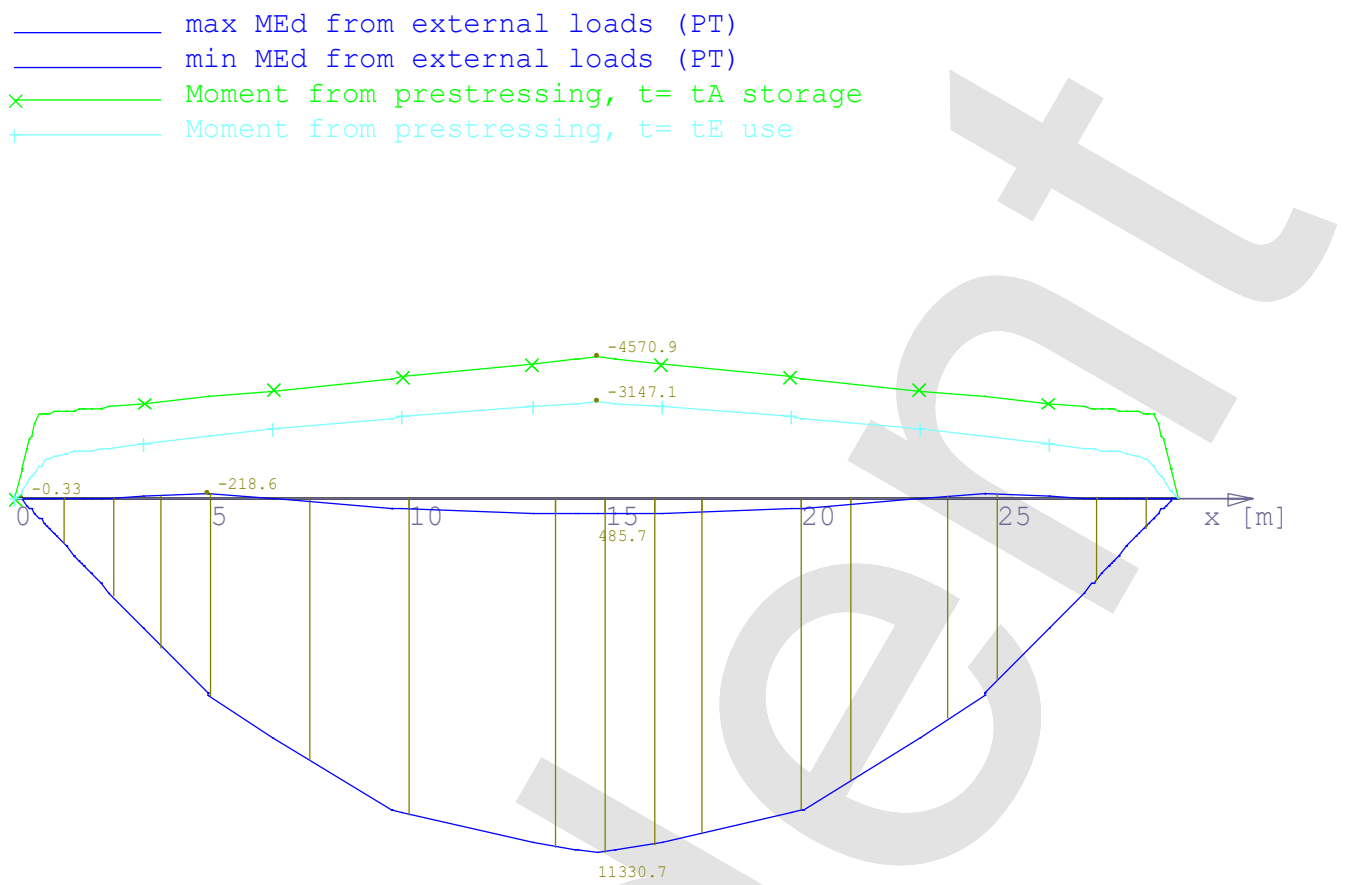

max VEd from external loads (PT) min VEd from external loads (PT)

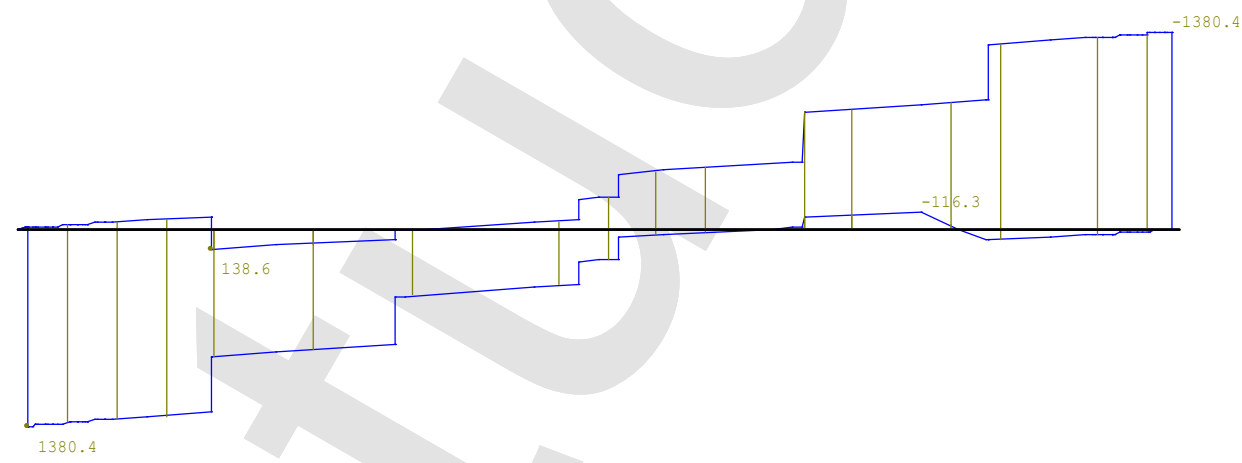

Deformation
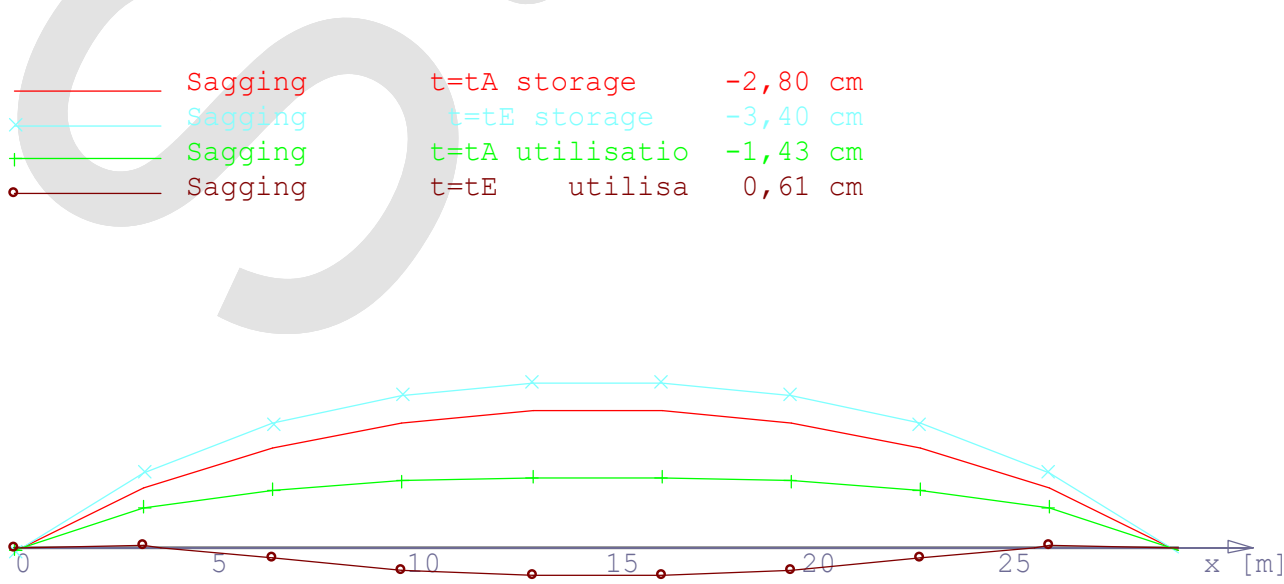
Demo Frilo

Stuttgarter Straße 40

70469
Tel.: 0711810020

Fax: 0711858020
Projekt: Design Calculations

Position: P.03 - Prestressed Concrete Truss

26.07.2018

Seite: 8

\section{Anchorage by bond ( over the left bearing)}

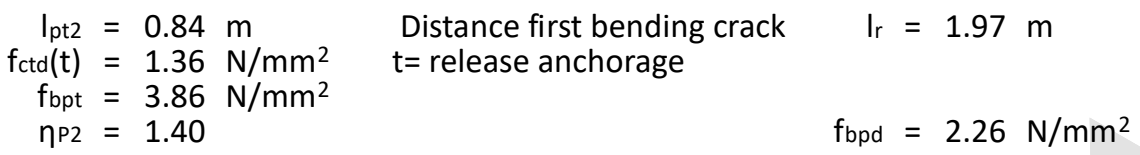

\begin{tabular}{|c|c|c|c|c|c|}
\hline $\begin{array}{r}x \\
{[\mathrm{~m}]}\end{array}$ & $\begin{array}{r}Z_{p} \\
{[k N]}\end{array}$ & $\begin{array}{r}\mathrm{Zs}_{\mathrm{s}} \\
{[\mathrm{kN}]}\end{array}$ & $\begin{array}{r}T_{E d} \\
{[k N]}\end{array}$ & $\begin{array}{r}\eta= \\
\left(Z_{p}+Z_{s}\right) / T_{E d}\end{array}$ & Util \\
\hline 0.20 & 1727.6 & 1078.7 & 1440.7 & 1.95 & 0.51 \\
\hline 0.33 & 2834.9 & 1077.6 & 1597.6 & 2.45 & 0.41 \\
\hline 0.66 & 5669.9 & 1076.3 & 1825.6 & 3.70 & 0.27 \\
\hline 0.99 & 6233.4 & 1076.3 & 2174.4 & 3.36 & 0.30 \\
\hline 1.32 & 6237.2 & 1076.5 & 2484.4 & 2.94 & 0.34 \\
\hline 1.64 & 6258.3 & 1077.3 & 2810.0 & 2.61 & 0.38 \\
\hline 1.97 & 6223.2 & 1075.9 & 3144.0 & 2.32 & 0.43 \\
\hline
\end{tabular}

$\mathrm{Zp}$ : resisting tensile force by the prestressed steel

$Z_{s}$ : resisting tensile force by the rebars

TEd: tensile force to be anchored

\begin{tabular}{|c|c|c|c|c|c|c|c|c|c|c|c|}
\hline $\begin{array}{l}\text { No. } \\
\text { Lay. }\end{array}$ & $\begin{array}{r}\text { Dist.LE } \\
{[\mathrm{cm}]}\end{array}$ & $\begin{array}{r}X A \\
{[\mathrm{~m}]}\end{array}$ & $\begin{array}{r}\sigma_{p} \\
{\left[\mathrm{~N} / \mathrm{mm}^{2}\right]}\end{array}$ & Eq. & $\begin{array}{l}\text { lbpd } \\
{[\mathrm{m}]}\end{array}$ & $\begin{array}{r}x k \\
{\left[\mathrm{~cm}^{2}\right]}\end{array}$ & $\begin{array}{r}\Sigma \mathrm{Zp} \\
{[\mathrm{kN}]}\end{array}$ & $\begin{array}{r}\Sigma Z_{s} \\
{[k N]}\end{array}$ & $\begin{array}{r}\text { TEd } \\
{[\mathrm{kN}]}\end{array}$ & $\begin{array}{r}\text { add. As } \\
{\left[\mathrm{cm}^{2}\right]}\end{array}$ & \\
\hline 1 & 7.5 & 0.00 & 621.60 & 8.21 & 1.79 & & & \multirow{7}{*}{\multicolumn{3}{|c|}{$\begin{array}{l}\text { Anchorage range uncracked } \\
\text { Anchorage range uncracked } \\
\text { Anchorage range uncracked } \\
\text { Anchorage range uncracked } \\
\text { Anchorage range uncracked } \\
\text { Anchorage range uncracked } \\
\text { Anchorage range uncracked }\end{array}$}} & (PT) \\
\hline 2 & 11.7 & 0.00 & 632.42 & 8.21 & 1.78 & & & & & & (PT) \\
\hline 3 & 15.9 & 0.00 & 643.25 & 8.21 & 1.77 & & & & & & (PT) \\
\hline 4 & 20.1 & 0.00 & 654.07 & 8.21 & 1.76 & & & & & & (PT) \\
\hline 5 & 24.3 & 0.00 & 664.90 & 8.21 & 1.75 & & & & & & (PT) \\
\hline 6 & 28.5 & 0.00 & 675.72 & 8.21 & 1.73 & & & & & & (PT) \\
\hline 7 & 32.7 & 0.00 & 686.55 & 8.21 & 1.82 & & & & & & (PT) \\
\hline
\end{tabular}

XA: Beginning of the anchoring area of the steel layer (dist. from the corresp. binder side)

Eq. 8.21.1: Anchorage area uncracked, $\sigma p$ acc.to fig. 8.17DE (b)

Eq. 8.21.1: Anchorage area cracked, op acc.to fig. 8.17DE (b)

$\mathrm{xk}$ : decisive section in the anchoring area of the layer (distance from the beginning of the binder)

add. As: Additional sagging reinforcement required for anchorage

\section{Anchorage by bond ( over the right bearing)}

$\mathrm{l}_{\mathrm{pt} 2}=0.84 \mathrm{~m}$

Distance first bending crack

$\mathrm{lr}=1.97 \mathrm{~m}$

$f_{c t d}(t)=1.36 \mathrm{~N} / \mathrm{mm}^{2}$

$f_{b p t}=3.86 \mathrm{~N} / \mathrm{mm}^{2}$

$\eta \mathrm{P} 2=1.40$

$\mathrm{fbpd}=2.26 \mathrm{~N} / \mathrm{mm}^{2}$

\begin{tabular}{|c|c|c|c|c|c|}
\hline $\begin{array}{r}x \\
{[\mathrm{~m}]}\end{array}$ & $\begin{array}{r}\mathrm{Zp} \\
{[\mathrm{kN}]}\end{array}$ & $\begin{array}{r}\mathrm{Zs} \\
{[\mathrm{kN}]}\end{array}$ & $\begin{array}{r}\text { TEd } \\
{[\mathrm{kN}]}\end{array}$ & $\begin{array}{r}\eta= \\
\left(Z_{p}+Z_{s}\right) / T_{E d}\end{array}$ & Util \\
\hline 27.63 & 6223.2 & 1075.9 & 3144.0 & 2.32 & 0.43 \\
\hline 27.96 & 6258.3 & 1077.3 & 2810.0 & 2.61 & 0.38 \\
\hline 28.28 & 6237.2 & 1076.5 & 2484.4 & 2.94 & 0.34 \\
\hline 28.61 & 6233.4 & 1076.3 & 2174.4 & 3.36 & 0.30 \\
\hline 28.94 & 5669.9 & 1076.3 & 1825.6 & 3.70 & 0.27 \\
\hline 29.27 & 2834.9 & 1077.6 & 1597.6 & 2.45 & 0.41 \\
\hline 29.40 & 1727.6 & 1078.7 & 1440.7 & 1.95 & 0.51 \\
\hline
\end{tabular}

$\mathrm{Zp}$ : resisting tensile force by the prestressed steel

$Z_{s}$ : resisting tensile force by the rebars

$T_{E d}$ : tensile force to be anchored

\begin{tabular}{|c|c|c|c|c|c|c|c|c|c|c|c|}
\hline $\begin{array}{l}\text { No. } \\
\text { Lay. }\end{array}$ & $\begin{array}{r}\text { Dist.LE } \\
{[\mathrm{cm}]}\end{array}$ & $\begin{array}{r}X A \\
{[\mathrm{~m}]}\end{array}$ & $\begin{array}{r}\sigma p \\
{\left[\mathrm{~N} / \mathrm{mm}^{2}\right]}\end{array}$ & Eq. & $\begin{array}{l}\text { lbpd } \\
{[\mathrm{m}]}\end{array}$ & $\begin{array}{r}x k \\
{\left[\mathrm{~cm}^{2}\right]}\end{array}$ & $\begin{array}{r}\Sigma \mathrm{Zp} \\
{[\mathrm{kN}]}\end{array}$ & $\begin{array}{r}\Sigma Z \mathrm{~s} \\
{[\mathrm{kN}]}\end{array}$ & $\begin{array}{r}\text { TEd } \\
{[\mathrm{kN}]}\end{array}$ & $\begin{array}{r}\text { add. As } \\
{\left[\mathrm{cm}^{2}\right]}\end{array}$ & \\
\hline 1 & 7.5 & 0.00 & 621.60 & 8.21 & 1.79 & & \multirow{2}{*}{\multicolumn{4}{|c|}{ Anchorage range uncracked }} & (PT) \\
\hline 2 & 11.7 & 0.00 & 632.42 & 8.21 & 1.78 & & & & & & (PT) \\
\hline 3 & 15.9 & 0.00 & 643.25 & 8.21 & 1.77 & & \multicolumn{4}{|c|}{$\begin{array}{l}\text { Anchorage range uncracked } \\
\text { Anchorage range uncracked }\end{array}$} & (PT) \\
\hline 4 & 20.1 & 0.00 & 654.07 & 8.21 & 1.76 & & \multicolumn{4}{|c|}{ Anchorage range uncracked } & (PT) \\
\hline 5 & 24.3 & 0.00 & 664.90 & 8.21 & 1.75 & & \multicolumn{4}{|c|}{ Anchorage range uncracked } & (PT) \\
\hline 6 & 28.5 & 0.00 & 675.72 & 8.21 & 1.73 & & \multicolumn{4}{|c|}{ Anchorage range uncracked } & (PT) \\
\hline 7 & 32.7 & 0.00 & 686.55 & 8.21 & 1.82 & & \multicolumn{4}{|c|}{ Anchorage range uncracked } & (PT) \\
\hline
\end{tabular}


Demo Frilo

Stuttgarter Straße 40

70469 Stuttgart
Tel.: 0711810020

Fax: 0711858020
Projekt: Design Calculations

Position: P.03 - Prestressed Concrete Truss

26.07.2018
Seite: 9

XA: Beginning of the anchoring area of the steel layer (dist. from the corresp. binder side)

Eq. 8.21.1: Anchorage area uncracked, $\sigma p$ acc.to fig. 8.17DE (b)

Eq. 8.21.1: Anchorage area cracked, op acc.to fig. 8.17DE (b)

$\mathrm{xk}$ : decisive section in the anchoring area of the layer (distance from the beginning of the binder) add. As: Additional sagging reinforcement required for anchorage

\section{Bursting Reinforcement at beginning of beam}

$\gamma_{p, \text { unfav }}=1.35 \quad$ Idisp $=1.51 \mathrm{~m}$

\begin{tabular}{|l|r|r|r|r|r|r|r|r|}
\hline & \multicolumn{4}{|c|}{ Initiation zone } & \multicolumn{4}{|c|}{ Section over the last effective position of tension. member } \\
\hline No. & $\begin{array}{r}\text { from } \\
{[\mathrm{m}]}\end{array}$ & $\begin{array}{r}\text { to } \\
{[\mathrm{m}]}\end{array}$ & $\begin{array}{r}\text { Dist.LE } \\
{[\mathrm{cm}]}\end{array}$ & $\begin{array}{r}\mathrm{N}_{\mathrm{c}} \\
{[\mathrm{kN}]}\end{array}$ & $\begin{array}{r}\mathrm{N}_{\mathrm{p}} \\
{[\mathrm{kN}]}\end{array}$ & $\begin{array}{r}\mathrm{T}_{\mathrm{p}} \\
{[\mathrm{kN}]}\end{array}$ & $\begin{array}{r}\text { Factor } \\
\text { Interpolation }\end{array}$ & $\begin{array}{r}\text { req. As } \\
{\left[\mathrm{cm}^{2}\right]}\end{array}$ \\
\hline 1 & 0.00 & 1.51 & 33.7 & -2434.6 & 4221.8 & 1787.2 & 0.378 & 21.0 \\
\hline
\end{tabular}

The bursting reinforcement must be arranged in zone of reduced dispersion length.

red. dispersion length indented wire w.o. strand $3 / 4 * 1$ disp $=1.13 \mathrm{~m}$

\section{Burstring Reinforcement at end of beam}

$\gamma_{p, \text { unfav }}=1.35 \quad I_{\text {disp }}=1.51 \mathrm{~m}$

\begin{tabular}{|c|c|c|c|c|c|c|c|c|}
\hline \multicolumn{3}{|c|}{ Initiation zone } & \multicolumn{6}{|c|}{ Section over the last effective position of tension. member } \\
\hline No. & $\begin{array}{r}\text { from } \\
{[\mathrm{m}]}\end{array}$ & $\begin{array}{r}\text { to } \\
\text { [m] }\end{array}$ & $\begin{array}{r}\text { Dist.LE } \\
{[\mathrm{cm}]}\end{array}$ & $\begin{array}{r}\mathrm{N}_{\mathrm{c}} \\
{[\mathrm{kN}]}\end{array}$ & $\begin{array}{r}\mathrm{N}_{\mathrm{p}} \\
{[\mathrm{kN}]}\end{array}$ & $\begin{array}{r}T_{p} \\
{[k N]}\end{array}$ & $\begin{array}{r}\text { Factor } \\
\text { Interpolation }\end{array}$ & $\begin{array}{r}\text { req. As } \\
{\left[\mathrm{cm}^{2}\right]}\end{array}$ \\
\hline 1 & 29.60 & 28.09 & 33.7 & -2434.6 & 4221.8 & 1787.2 & 0.378 & 21.0 \\
\hline
\end{tabular}

The bursting reinforcement must be arranged in zone of reduced dispersion length.

red. dispersion length indented wire w.o. strand $3 / 4 * 1$ disp $=1.13 \mathrm{~m}$ 
B.1.3. Design Calculations POS. T.10 - Precast Staircase 
Demo Frilo

Stuttgarter Straße 40

70469

Stuttgart

Tel.: 0711810020

Position: T.10 - Precast Staircase

Fax: 0711858020

\section{Position: T.10 - Precast Staircase}

Treppenlauf (neu) B7+ 01/18A (Frilo R-2018-1/P12)

\section{System}

\section{System graphics}

Scale $1: 38.1$

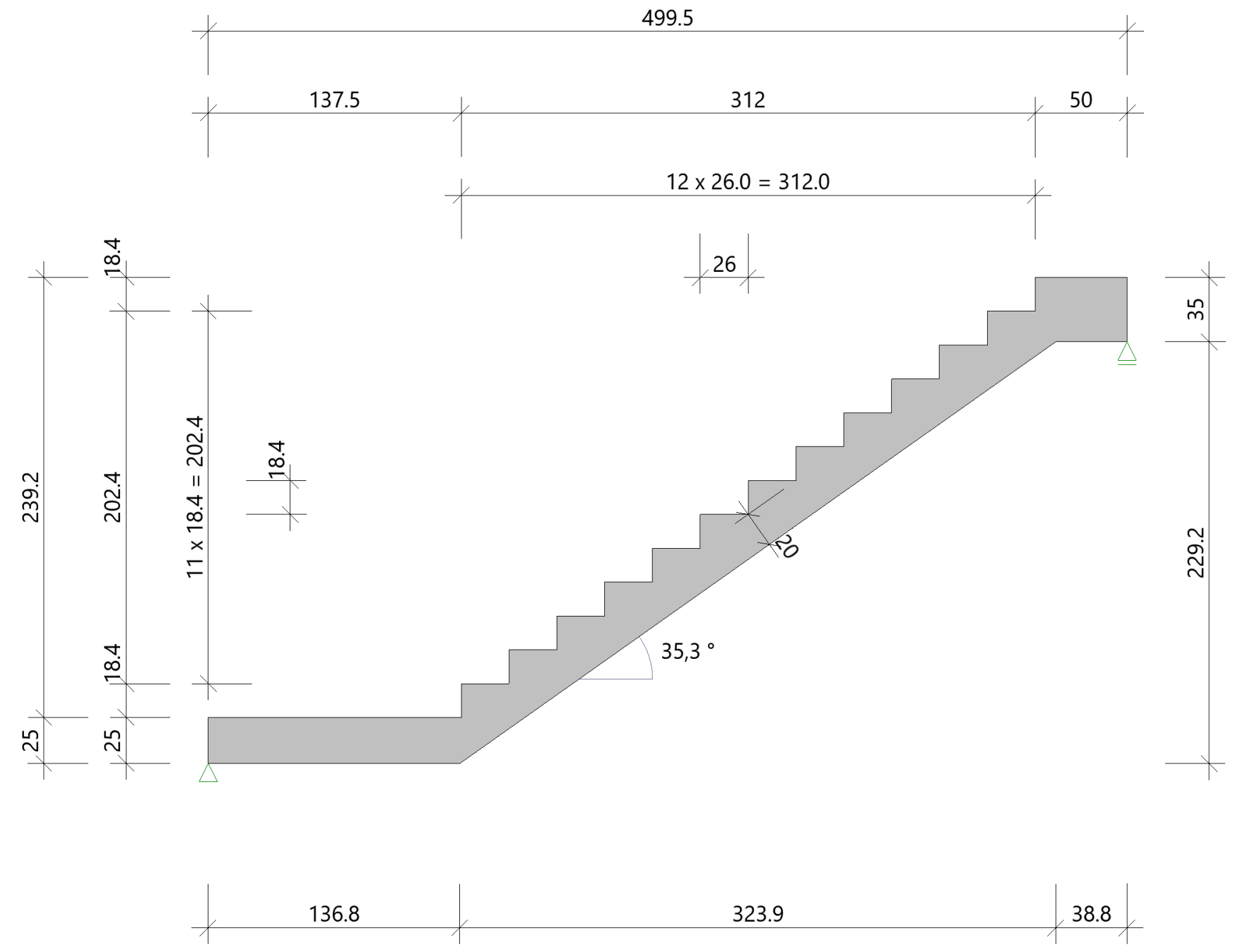

\section{Geometry}

Rfbfloor landing top - Rfb floor landing bottom Length from 1-st to to last step tread Length lower landing to FE support Length upper landing to FE support width of flight Width of cover Live load width Number of rises Height of step bottom Height of step top

Stair steps

Thickness of staircase

Thickness lower landing

Thickness upper landing

Length of the staircase bottom view in plan distance 1 st step to inflexion point bottom

$\begin{array}{rlrl}\mathrm{H}_{1} & = & 239.2 \mathrm{~cm} \\ \mathrm{~L}_{1}= & 312.0 \mathrm{~cm} \\ \mathrm{~L}_{2}= & 137.5 \mathrm{~cm} \\ \mathrm{~L}_{3}= & 50.0 \mathrm{~cm} \\ \mathrm{~B}_{1}= & 100.0 \mathrm{~cm} \\ \mathrm{~B}_{2}= & 100.0 \mathrm{~cm} \\ \mathrm{~B}_{3}= & 100.0 \mathrm{~cm} \\ \mathrm{n}_{5}= & 13 \\ \mathrm{H}_{1}= & 18.4 \mathrm{~cm} \\ \mathrm{H}_{1}= & 18.4 \mathrm{~cm} \\ / \mathrm{L}_{5}= & 18.4 \quad 26.0 \mathrm{~cm} \\ \mathrm{D}_{1}= & 20.0 \mathrm{~cm} \\ \mathrm{D}_{2}= & 25.0 \mathrm{~cm} \\ \mathrm{D}_{3}= & 35.0 \mathrm{~cm} \\ \mathrm{~L}_{4}= & 323.9 \mathrm{~cm} \\ \mathrm{~L}_{5}= & -0.7 \mathrm{~cm}\end{array}$


Demo Frilo

Stuttgarter Straße 40

70469

Stuttgart
Tel.: 0711810020

Fax: 0711858020
Projekt: Design Calculations

Position: T.10 - Precast Staircase

26.07.2018
Seite: 2

\section{bearing}

bttm: hinged without console

top: hinged without console

\section{Support}

\begin{tabular}{|c|c|c|c|}
\hline $\begin{array}{l}\text { Location } \\
\text { - }\end{array}$ & $\begin{array}{r}\text { horizontal } \\
\mathrm{kN} / \mathrm{m}\end{array}$ & $\begin{array}{r}\text { vertical } \\
\mathrm{kN} / \mathrm{m}\end{array}$ & $\begin{array}{r}\text { turning } \\
\mathrm{kNm} / \mathrm{rad}\end{array}$ \\
\hline $\begin{array}{l}\text { left } \\
\text { right }\end{array}$ & $\begin{array}{l}\text { rigid } \\
\text { free }\end{array}$ & $\begin{array}{l}\text { rigid } \\
\text { rigid }\end{array}$ & $\begin{array}{l}\text { free } \\
\text { free }\end{array}$ \\
\hline
\end{tabular}

\section{Durability}

\section{Requirements durability}

attack on concrete attack on reinforc.

min. concrete class

long. reinforcement allowance in design longitudinal bars concrete coverage

laying dist. link

all. crack width

*5: bond decisive

$\begin{aligned} & X 0 \\ X C 1 & \\ & C 16 / 20 \\ \phi, m & =10 \mathrm{~mm} \\ \Delta \mathrm{Cdev} & =10 \mathrm{~mm} \\ C_{\min , \mathrm{m}} & =10 \mathrm{~mm} \\ C_{\text {nom,m }} & =20 \mathrm{~mm} \\ \mathrm{C}, \mathrm{l} & =20 \mathrm{~mm} \\ W_{\max } & =0.40 \mathrm{~mm}\end{aligned}$

\section{Loads}

safety and combination factors

\begin{tabular}{|c|c|c|c|c|c|}
\hline Action group & YG & YQ & $\psi_{0}$ & $\psi_{1}$ & $\psi_{2}$ \\
\hline Kat. B: Bürogebäude & 1,35 & 1,5 & 0,7 & 0,5 & 0,3 \\
\hline
\end{tabular}

\section{Load}

\begin{tabular}{|l|l|r|r|}
\hline Location & Type & $\mathrm{g}$ & $\mathrm{kN} / \mathrm{m}^{2}$ \\
- & - & $\mathrm{q}$ \\
\hline Stairway & Covering & 1.50 & - \\
Landing/console bottom & Live load & 1.50 & - \\
& Covering & - & 3.00 \\
Landing/console top & Live load & - \\
& Covering & 3.00 \\
\hline
\end{tabular}

\section{Resulting loading (relative to the horizontal surface)}

\begin{tabular}{|c|c|c|c|}
\hline $\begin{array}{l}\text { Location } \\
\text { - }\end{array}$ & $\begin{array}{l}\text { Type } \\
-\end{array}$ & $\mathrm{kN} / \mathrm{m}^{2}$ & $\mathrm{kN} / \mathrm{m}^{\mathrm{q}}$ \\
\hline Stairway & $\begin{array}{l}\text { Self weight } \\
\text { Covering } \\
\text { Live load } \\
\text { Total }\end{array}$ & $\begin{array}{r}8.43 \\
1.50 \\
- \\
9.93\end{array}$ & $\begin{array}{r}- \\
- \\
3.00 \\
3.00\end{array}$ \\
\hline Landing/console bottom & $\begin{array}{l}\text { Self weight } \\
\text { Covering } \\
\text { Live load } \\
\text { Total }\end{array}$ & $\begin{array}{r}6.25 \\
1.50 \\
- \\
7.75\end{array}$ & $\begin{array}{r}- \\
- \\
3.00 \\
3.00\end{array}$ \\
\hline Landing/console top & $\begin{array}{l}\text { Self weight } \\
\text { Covering } \\
\text { Live load } \\
\text { Total }\end{array}$ & $\begin{array}{r}8.75 \\
1.50 \\
- \\
10.25\end{array}$ & $\begin{array}{r}- \\
- \\
3.00 \\
3.00\end{array}$ \\
\hline
\end{tabular}

The dead weight is with gamma $=25.00 \mathrm{kN} / \mathrm{m}^{3}$ considered . 


\section{Standard, Materials und Reinforcement layer}

Design acc.to DIN EN 1992-1-1/NA/A1:2015-12

Construction materials: Concrete C30/37

$$
\begin{aligned}
\gamma_{c} & =1.50 \\
f_{c k} & =30.0 \mathrm{~N} / \mathrm{mm}^{2}
\end{aligned}
$$

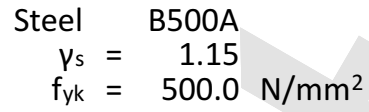

Individual lengths

\begin{tabular}{|l|c|c|c|}
\hline & Lower landing & Stairway & Upper landing \\
\hline dimension & $1.37 \mathrm{~m}$ & $4.05 \mathrm{~m}$ (Ltot) & $0.31 \mathrm{~m}$ \\
& & $3.31 \mathrm{~m}$ (Lhor) & \\
\hline
\end{tabular}

Reinforcement layer bottom $\quad \mathrm{d}_{1}=3.0 \mathrm{~cm}$

Reinforcement upper layer $\quad \mathrm{d}_{2}=3.0 \mathrm{~cm}$

\section{Results}

\section{Bending design}

All design results per $m$ stair width!

\section{Flexural reinforcement}

\begin{tabular}{|c|c|c|c|c|c|c|}
\hline $\begin{array}{l}\text { Location } \\
\text { - }\end{array}$ & $\begin{array}{r}\mathrm{d} \\
\mathrm{cm}\end{array}$ & $\begin{array}{r}\text { MEd } \\
\mathrm{kNm} / \mathrm{m}\end{array}$ & $\begin{array}{r}N_{E d} \\
k N / m\end{array}$ & $\begin{array}{l}\text { req. asb } \\
\mathrm{cm}^{2} / \mathrm{m}\end{array}$ & $\begin{array}{l}\text { req. ast } \\
\mathrm{cm}^{2} / \mathrm{m}\end{array}$ & $\begin{array}{c}\text { \&About } \\
-\end{array}$ \\
\hline $\begin{array}{l}\text { lower landing, span reinforcement } \\
\text { Stairway, span reinforcement } \\
\text { upper landing, span reinforcement }\end{array}$ & $\begin{array}{l}25.0 \\
20.0 \\
35.0\end{array}$ & $\begin{array}{r}42.48 \\
54.46 \\
6.73\end{array}$ & $\begin{array}{l}0.0 \\
0.3 \\
0.0\end{array}$ & $\begin{array}{l}4.4 \\
7.5 \\
4.1\end{array}$ & $\begin{array}{l}0.0 \\
0.0 \\
0.0\end{array}$ & *) \\
\hline
\end{tabular}

\section{exist. reinforcement}

Stairway, span reinforcement exist. a sbtm

$7 \varnothing 12 / 15.0 \mathrm{~cm}$ $=7.54 \mathrm{~cm}^{2} / \mathrm{m}$

\section{(Suggestion from program for number $\varnothing$ )}

\section{Shear design}

\section{Shear reinforcement B500A}

\begin{tabular}{|c|c|c|c|c|c|c|c|c|c|}
\hline Location & $\begin{array}{r}V_{\text {Ed }} \\
\mathrm{kN} / \mathrm{m}\end{array}$ & $\begin{array}{r}\text { Ned } \\
\mathrm{kN} / \mathrm{m}\end{array}$ & kz & Degree & $\begin{array}{r}\text { ast } \\
\mathrm{cm}^{2} / \mathrm{m}\end{array}$ & $\begin{array}{l}V_{R d, c} \\
k N / m\end{array}$ & $\begin{array}{l}V_{R d, \max } \\
\mathrm{kN} / \mathrm{m}\end{array}$ & $\begin{array}{r}\text { req. asstir } \\
\mathrm{cm}^{2} / \mathrm{m}^{2}\end{array}$ & $\begin{array}{c}\text { \&About } \\
-\end{array}$ \\
\hline lower landing left & 41.2 & 0.0 & 0.82 & 18.4 & 0.0 & 115.1 & 688.5 & 9.3 & *) \\
\hline lower landing right & 20.7 & 0.0 & 0.82 & 18.4 & 4.4 & 115.1 & 688.5 & 9.3 & $\left.*{ }^{\prime}\right)$ \\
\hline Stairway left & 16.9 & -12.0 & 0.76 & 18.4 & 5.6 & 93.4 & 497.3 & 0.0 & \\
\hline Stairway right & -31.4 & 22.3 & 0.76 & 18.4 & 2.9 & 89.9 & 497.3 & 0.0 & \\
\hline upper landing left & -38.5 & 0.0 & 0.88 & 18.4 & 4.1 & 147.0 & 1071.0 & 9.3 & *) \\
\hline upper landing right & -44.3 & 0.0 & 0.88 & 18.4 & 0.0 & 147.0 & 1071.0 & 9.3 & $*)$ \\
\hline
\end{tabular}

\section{crack width verification}

The check is carried out with the quasi-permanent action combination

\section{Crack width limitation stairs:}

\begin{tabular}{|l|r|r|r|r|r|r|r|r|r|r|}
\hline Location & $\begin{array}{r}\mathrm{d} \\
-\end{array}$ & $\begin{array}{r}\text { MEd } \\
\mathrm{kNm}\end{array}$ & $\begin{array}{r}\mathrm{NEd}_{\mathrm{kN}} \\
\mathrm{kNm}\end{array}$ & $\begin{array}{r}\text { exist. Asb } \\
\mathrm{cm}^{2}\end{array}$ & $\begin{array}{r}\text { exist. Ast } \\
\mathrm{cm}^{2}\end{array}$ & $\begin{array}{r}\text { Env.Cl } \\
-\end{array}$ & $\begin{array}{r}\mathrm{d}_{\text {s, exist }} \\
\mathrm{mm}\end{array}$ & $\begin{array}{r}\mathrm{d}_{\text {s, limit }} \\
\mathrm{mm}\end{array}$ & $\begin{array}{r}\text { exist. W } \\
\mathrm{mm}\end{array}$ & $\begin{array}{r}\text { perm. W } \\
\mathrm{mm}\end{array}$ \\
\hline Stairway, bottom side & 20.0 & 32.75 & 0.1 & 7.9 & 0.0 & $\mathrm{XC1}$ & 12 & 24 & 0.20 & 0.40 \\
\hline
\end{tabular}

\section{Deformation}

The calculation will be done with quasi permanent Action combination at state I $\left(E_{c m}=33000 \mathrm{~N} / \mathrm{mm}^{2}\right)$.

$\max . \mathrm{f}=0.4 \mathrm{~cm}$ (in staircase at $\mathrm{x}=1.45 \mathrm{~m}$ )

Note: The deflection value is to be understood perpendicular to the corresponding member axis. The $x$-value refers to the beginning of the member (beginning lower platform, staircase or upper platform) and runs in the direction of the member axis. 
Demo Frilo

Stuttgarter Straße 40

70469

Stuttgart
Tel.: 0711810020

Fax: 0711858020
Projekt: Design Calculations

Position: T.10 - Precast Staircase

26.07.2018
Seite: 4

\section{Support reactions}

\section{Definition supporting forces}
(A) support left
(v) vertical supporting force
(B) support right
(v) horizontal supporting force

\section{Support reactions per $\mathrm{m}$ stair width}

\begin{tabular}{|c|c|c|c|c|}
\hline & $\begin{array}{r}A_{v} \\
\mathrm{kN} / \mathrm{m}\end{array}$ & $\begin{array}{r}A_{h} \\
\mathrm{kN} / \mathrm{m}\end{array}$ & $\begin{array}{r}B v \\
k N / m\end{array}$ & $\begin{array}{r}B h \\
k N / m\end{array}$ \\
\hline$\gamma=1.0$ & & & & \\
\hline Total & 29.7 & 0.0 & 32.0 & 0.0 \\
\hline from g & 22.2 & 0.0 & 24.5 & 0.0 \\
\hline from q & 7.5 & 0.0 & 7.5 & 0.0 \\
\hline $\begin{array}{l}\text { p-times } \\
\text { Total }\end{array}$ & 191? & ח & 11? & 00 \\
\hline from $g$ & $\begin{array}{l}41.2 \\
30.0\end{array}$ & 0.0 & $\begin{array}{l}44.5 \\
33.0\end{array}$ & 0.0 \\
\hline from q & 11.2 & 0.0 & 11.2 & 0.0 \\
\hline
\end{tabular}

\section{Self weight of stairs}

The self-weight of the stair (without covering) $\mathrm{G} k$ is $39.2 \mathrm{kN}$ 
B.1.4. Design Calculations POS. C.04 - Precast Concrete Column with Biaxial Wind Loads 
Demo Frilo

Stuttgarter Straße 40

70469

Stuttgart
Tel.: 0711810020

Fax: 0711858020
Projekt: Design Calculations

Position: C.04 - Precast Concrete Column

26.07.2018
Seite: 1

\section{Position: C.04 - Precast Concrete Column}

Reinforced Concrete Column B5 01/2018 (Frilo R-2018-1/P12)

\section{CANTILEVER COLUMN, Rectangle, 2-axial strained}

Calculation base: DIN EN 1992-1-1/NA/A1:2015-12

$\mathrm{E}=34000 \mathrm{~N} / \mathrm{mm} 2 \rho=2500 \mathrm{~kg} / \mathrm{m} 3$
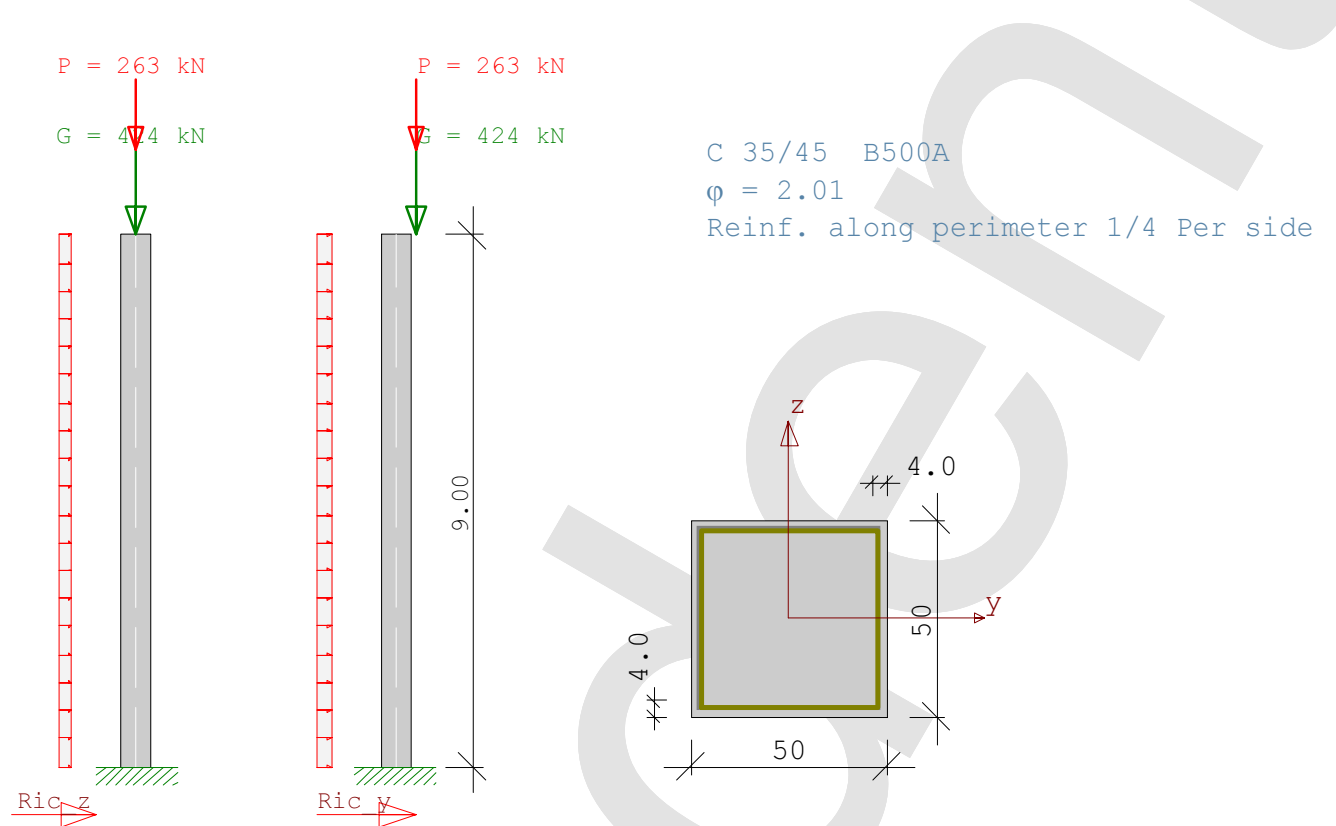

$1 \quad$ Mcry $=$

$66.87 \mathrm{kNm}$

Mcrz =

$66.87 \mathrm{kNm}$

\begin{tabular}{|c|c|c|c|c|c|c|c|c|}
\hline \multicolumn{9}{|c|}{ NODES - LOADS : } \\
\hline LcNo. KNo. & $\begin{array}{c}V \\
(k N)\end{array}$ & $\begin{array}{c}\text { ey } \\
(\mathrm{cm})\end{array}$ & $\begin{array}{c}\mathrm{ez} \\
(\mathrm{cm})\end{array}$ & $\begin{array}{c}\text { Py } \\
(\mathrm{kN})\end{array}$ & $\begin{array}{c}\mathrm{Pz} \\
(\mathrm{kN})\end{array}$ & $\begin{array}{c}\mathrm{My} \\
(\mathrm{kNm})\end{array}$ & $\begin{array}{l}\text { Mz act con alt } \\
(\mathrm{kNm})\end{array}$ & \\
\hline 12 & $\begin{array}{r}423.60 \\
263.00 \\
56.25\end{array}$ & $\begin{array}{r}35.0 \\
35.0 \\
\text { dead }\end{array}$ & & & & & $\cdot$ & $\begin{array}{l}g \\
p\end{array}$ \\
\hline
\end{tabular}

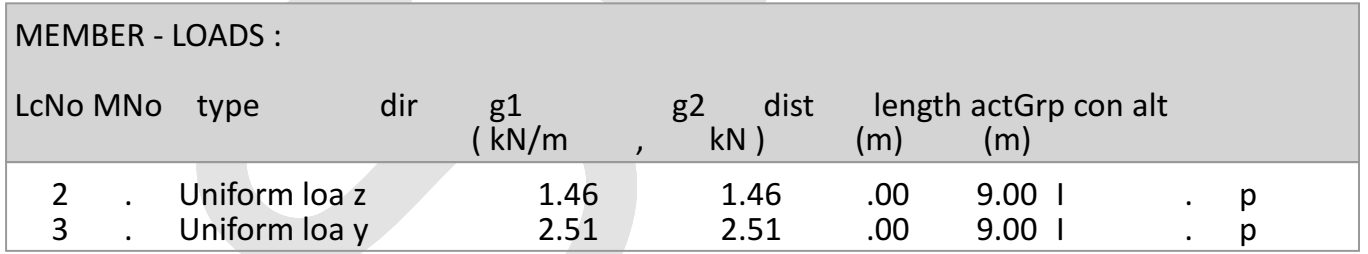

\section{Actions:}

No. Cl Name

$\psi 0 \quad \psi 1 \quad \psi 2 \quad \gamma$

I 4 Wind loads

J 3 Snow loads $<1000 m$

$\begin{array}{llll}0.60 & 0.20 & 0.00 & 1.50\end{array}$

$\begin{array}{llll}0.50 & 0.20 & 0.00 & 1.50\end{array}$

All actions are use like independent. 
Demo Frilo

Stuttgarter Straße 40

70469 Stuttgart
Tel.: 0711810020

Fax: 0711858020
Projekt: Design Calculations

Position: C.04 - Precast Concrete Column

26.07.2018
Seite: 2

\section{Further design fundamentals:}

Accuracy Gkn $=6.14 \mathrm{e}-5$

Number of sub-element per member section: 6

Stress-strain-curve of concr. for deform. analy. EN 1992-1-1 3.1.5

Calc. of compr. force in concr.without deduction of reinf

If $\mathrm{n}>-0.10$ : eff El acc.toEN2 7.4.2 (7.19)

Creep effects are considered by modified stress-strain-

\section{curve.}

$\phi$ eff $=\phi 0 * \mathrm{M0} / \mathrm{Med}$ ( $\mathrm{M0}$ By permanent combination with ei)

consequency class acc. EN 1990 tab b.1CC2 -> KFi = 1.0 (Tab B.3)

FLBemBn.DLL: version9.0.1.121

$\mathrm{NKi} / \mathrm{N}=5.18 \mathrm{dir} \_\mathrm{y} \mathrm{NKi} / \mathrm{N}=5.18 \mathrm{dir} \_\mathrm{z}$ cross sect. of concr. only

\section{CALCULATED COMBINATIONS by 3 Loads Kombi_D}

$\begin{array}{ccccccccc}\text { Lc- Comb } & \text { K1 } & \text { K2 } & \text { K3 } & \text { K4 } & \text { K5 } & \text { K6 } & \text { K7 } & \text { K8 } \\ & \text { g } & \text { g } & \text { g } & \text { g } & \text { g } & \text { g } & \text { g } & \text { g } \\ & \text { J } & \text { I } & \text { I } & \text { J } & \text { I } & \text { I } & & \text { I } \\ 1 & \text { x } & . & \text { x } & \text { x } & \text { x } & \text { x } & \text { x } & \text {. } \\ 2 & \text { x } & \text {. } & \text { x } & \text {. } & \text { x } & \text {. } & \text { x } & \text { x } \\ 3 & \text { x } & \text { x } & \text { x } & \text { x } & \text {. } & \text { x } & \text { x } & \text {. }\end{array}$

Partial safety factor $\quad \gamma C=1.50 \quad \gamma S=1.15 \quad \gamma G=1.35 / 1.00$

Proof according DIN EN 1992-1-1/NA/A1:2015-12

$\nu C=1.50 \nu S=1.15$ фeff $=1.37$

Design values $\mathrm{LcCom}=1$ in : $\mathrm{y}$-direction $\mathrm{z}$-direction

\section{System}

Buckling length

Slenderness

Normal force

Specific normal force

Inter. moment

Methodical eccentr.

Related eccentr.

Unintentional eccentrici

Displacement Th.2.Ord.

Design moment

Reinforcement

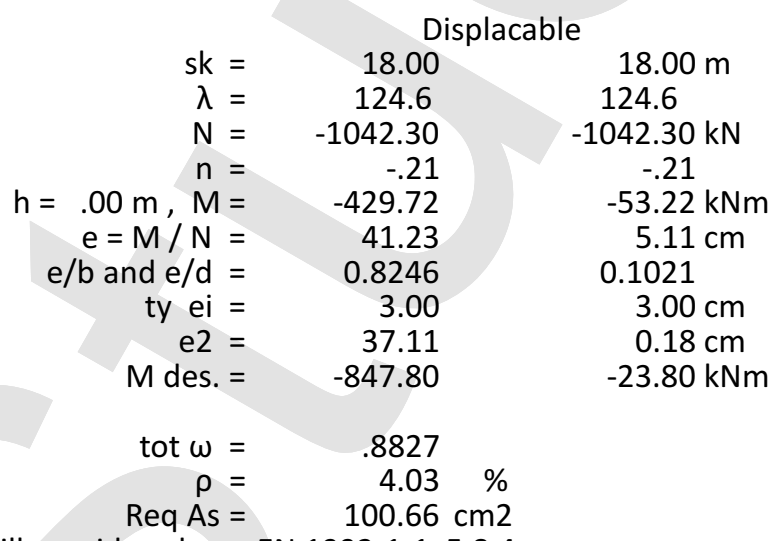


B.1.5. Design Calculations POS. C.05 - Precast Concrete Column 
Demo Frilo

Stuttgarter Straße 40

70469

Stuttgart
Tel.: 0711810020

Fax: 0711858020
Projekt: Design Calculations

Position: C.05 - Precast Concrete Column

26.07.2018
Seite: 1

\section{Position: C.05 - Precast Concrete Column}

Reinforced Concrete Column B5 01/2018 (Frilo R-2018-1/P12)

\section{CANTILEVER COLUMN, Rectangle, 2-axial strained}

Calculation base: DIN EN 1992-1-1/NA/A1:2015-12

$\mathrm{E}=34000 \mathrm{~N} / \mathrm{mm} 2 \rho=2500 \mathrm{~kg} / \mathrm{m} 3$
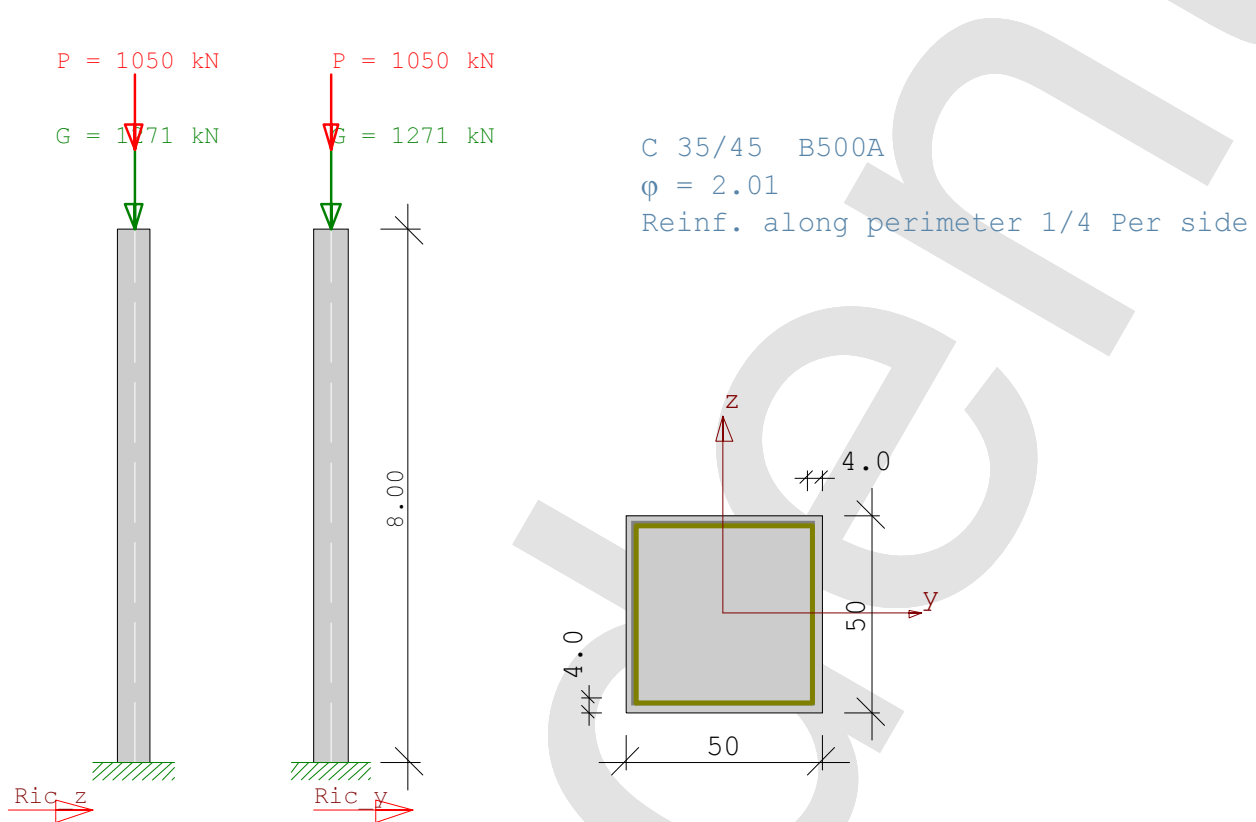

1

Mcry =

$66.87 \mathrm{kNm}$

Mcrz =

$66.87 \mathrm{kNm}$

\begin{tabular}{|c|c|c|c|c|c|c|c|c|}
\hline \multicolumn{9}{|c|}{ NODES - LOADS : } \\
\hline LcNo. KNo. & $\begin{array}{c}V \\
(k N)\end{array}$ & $\begin{array}{c}\text { ey } \\
(\mathrm{cm})\end{array}$ & $\begin{array}{c}\mathrm{ez} \\
(\mathrm{cm})\end{array}$ & $\begin{array}{c}\text { Py } \\
(\mathrm{kN})\end{array}$ & $\begin{array}{c}\mathrm{Pz} \\
(\mathrm{kN})\end{array}$ & $\begin{array}{c}\mathrm{My} \\
(\mathrm{kNm})\end{array}$ & $\begin{array}{l}\text { Mz act con alt } \\
(\mathrm{kNm})\end{array}$ & \\
\hline 12 & $\begin{array}{r}1271.0 \\
1050.0 \\
50.00\end{array}$ & & & & & & $\cdot$ & $\begin{array}{l}g \\
p\end{array}$ \\
\hline
\end{tabular}

Actions:

No. Cl Name

$\psi 0 \quad \psi 1 \quad \psi 2 \quad \gamma$

J 3 Snow loads $<1000 \mathrm{~m}$

$\begin{array}{llll}0.50 & 0.20 & 0.00 & 1.50\end{array}$

Further design fundamentals:

Accuracy Gkn $=8.48 \mathrm{e}-5$

Number of sub-element per member section: 6

Stress-strain-curve of concr. for deform. analy. EN 1992-1-1 3.1.5

Calc. of compr. force in concr.without deduction of reinf.

If $n>-0.10$ : eff El acc.toEN2 7.4.2 (7.19)

Creep effects are considered by modified stress-strain-

curve.

фeff $=\phi 0 * \mathrm{M0} / \mathrm{Med}$ (M0 By permanent combination with ei)

consequency class acc. EN 1990 tab b.1CC2 $->\mathrm{KFi}=1.0$ (Tab B.3)

FLBemBn.DLL: version9.0.1.121

mininmum moments acc. to 6.1 (4) are not considered. 
Demo Frilo

Stuttgarter Straße 40

70469

Stuttgart
Tel.: 0711810020

Fax: 0711858020
Projekt: Design Calculations

Position: C.05 - Precast Concrete Column

26.07.2018

$\mathrm{NKi} / \mathrm{N}=2.03$ dir_y NKi/N $=2.03$ dir_z cross sect. of concr. only

CALCULATED COMBINATIONS by 1 Loads Kombi_D

LC-Comb K1 K2

$1 \times$.

Partial safety factor $\gamma C=1.50 \quad \gamma S=1.15 \quad \gamma G=1.35 / 1.00$

Proof according DIN EN 1992-1-1/NA/A1:2015-12

$\gamma C=1.50 \nu \mathrm{S}=1.15$ фeff $=.80$

Design values $\mathrm{LcCom}=1$ in : $\mathrm{y}$-direction $\mathrm{z}$-direction

System

Buckling length

Slenderness

Normal force

Specific normal force

Inter. moment

Methodical eccentr.

Related eccentr.

Unintentional eccentrici

Displacement Th.2.Ord.

Design moment

Reinforcement

\section{Displacable}

sk $=\quad 16.00 \quad 16.00 \mathrm{~m}$

$\begin{array}{lll}\lambda= & 110.7 & 110.7\end{array}$

$\mathrm{N}=-\mathbf{3 3 5 8 . 3 5} \quad-3358.35 \mathrm{kN}$

$\begin{array}{lll}\mathrm{n}= & -.68 & -.68\end{array}$

$\mathrm{h}=.00 \mathrm{~m}, \mathrm{M}=\quad 0.00$

0.00

0.0000

2.83

10.06

$0.00 \mathrm{kNm}$

$0.00 \mathrm{~cm}$

0.0000

$2.83 \mathrm{~cm}$

$10.06 \mathrm{~cm}$

$-432.75 \mathrm{kNm}$

$\begin{array}{rr}M \text { des. }= & -432.74 \\ \text { tot } \omega= & .9541\end{array}$

$\begin{array}{rrr}\rho & = & 4.35 \quad \% \\ \text { Req As } & =\quad 108.81 \mathrm{~cm} 2\end{array}$

The influence of creep will considered acc. EN 1992-1-1 5.8.4 curve. 
B.1.6. Design Calculations POS. S.11 - Semi-Precast Double-Tee Slab 


\section{Demo Frilo}

Stuttgarter Straße 40

70469

Stuttgart
Tel.: 0711810020

Fax: 0711858020
Projekt: Design Calculations

Position: S.11 - Semi-Precast Double-Tee Slab

26.07.2018
Seite: 1

\section{Position: S.11 - Semi-Precast Double-Tee Slab}

Durchlaufträger DLT10 01/2018 (Frilo R-2018-1/P12)

Scale $1: 100$
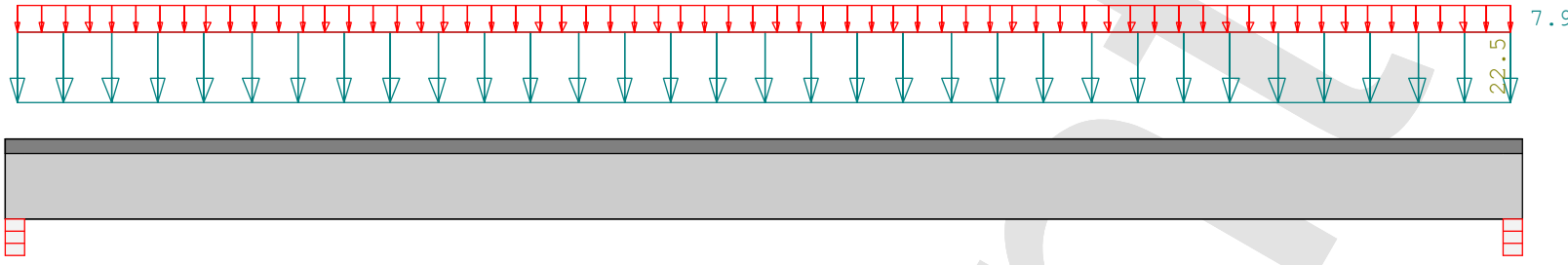

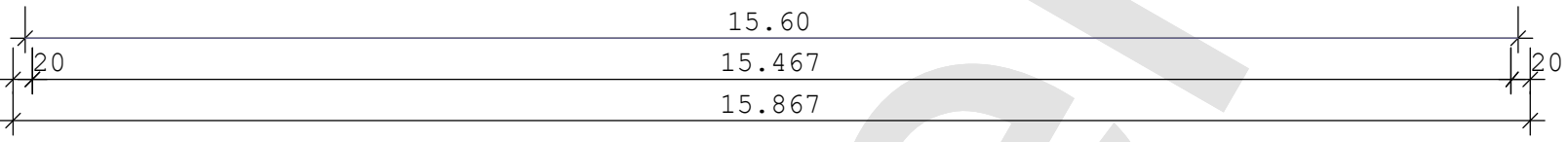

effective breadth and design

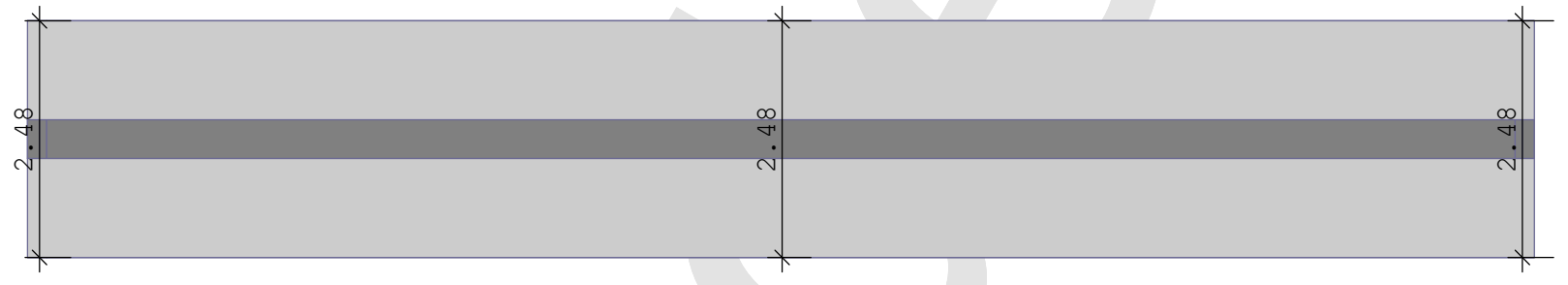

Reinforced concrete girder C35/45 E = 34000 N/mm2 DIN EN 1992-1-1/NA/A1:2015-12

System length cross-section values

\begin{tabular}{|c|c|c|c|}
\hline Span & $I(\mathrm{~m})$ & $\mathrm{bb}$ & $\mathrm{hb}$ \\
\hline 1 & 15.60 & & \\
\hline
\end{tabular}

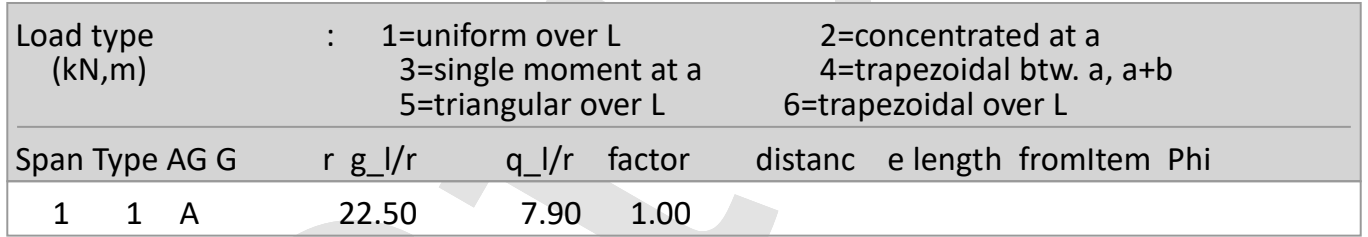

\section{Actions:}

No. Cl Name

$\psi 0 \quad \psi 1 \quad \psi 2 \quad \gamma$

A 1 Cat A-domestic

$\begin{array}{llll}0.70 & 0.50 & 0.30 & 1.50\end{array}$

Consequency class CC 2 acc. EN 1990 Tab. B1 $>\mathrm{K}_{\mathrm{Fi}}=1.0$ Tab. B3

In following tables the last cell the row is a reference to

the number of the related superposition ( see below).

In tables with internal forces multiplied by Gamma is additionally

a reference to the main action.

Results for 1-times loads

Span moments maximum

\begin{tabular}{|c|c|c|c|c|c|c|c|c|}
\hline Span & & & Mf & M le & M ri & V le & V ri & comb \\
\hline 1 & $x 0=$ & 7.80 & 924.77 & 0.00 & 0.00 & 237.12 & -237.12 & 2 \\
\hline
\end{tabular}


Demo Frilo

Stuttgarter Straße 40

$70469 \quad$ Stuttgart
Tel.: 0711810020

Fax: 0711858020
Projekt: Design Calculations

Position: S.11 - Semi-Precast Double-Tee Slab

26.07.2018

\begin{tabular}{|cccccccc|}
\hline \multicolumn{2}{l}{ Support moments maximum } & \multicolumn{3}{c}{ ( kNm,kN) } \\
\hline Column & M le & M ri & V le & V ri & $\max F$ & $\min F$ & comb \\
\hline 1 & 0.00 & 0.00 & 0.00 & 237.12 & 237.12 & 175.50 & 2 \\
2 & 0.00 & 0.00 & -237.12 & 0.00 & 237.12 & 175.50 & 2 \\
\hline
\end{tabular}

\begin{tabular}{|c|c|c|c|c|c|c|c|c|c|c|c|}
\hline \multicolumn{12}{|c|}{ Moment boundary diagram } \\
\hline \multicolumn{2}{|c|}{$\begin{array}{l}\mathrm{x} / \mathrm{L}=.0 \\
\text { Span }\end{array}$} & .1 & .2 & .3 & .4 & .5 & .6 & .7 & .8 & .9 & 1.0 \\
\hline 1 & 0.00 & 246 & 438 & 575 & 657 & 684 & 657 & 575 & 438 & 246 & 0.00 \\
\hline 1 & 0.00 & 333 & 592 & 777 & 888 & 925 & 888 & 777 & 592 & 333 & 0.00 \\
\hline
\end{tabular}

\begin{tabular}{|ccrrrrrl|}
\hline \multicolumn{2}{l}{ Support reactions } \\
\hline Column & by & $\mathrm{g}$ & $\max \mathrm{q}$ & $\min \mathrm{q}$ & Fulload & $\max$ & $\min$ \\
\hline 1 & 175.50 & 61.62 & 0.00 & 237.12 & 237.12 & 175.50 \\
2 & 175.50 & 61.62 & 0.00 & 237.12 & 237.12 & 175.50 \\
Total: & 351.00 & 123.24 & 0.00 & 474.24 & 474.24 & 351.00 \\
\hline
\end{tabular}

\begin{tabular}{|c|c|c|c|c|c|}
\hline \multicolumn{5}{|c|}{ Support reactions } & \multirow[t]{2}{*}{$(\mathrm{kN})$} \\
\hline CA & $\begin{array}{l}\text { Column } 1 \\
\max \end{array}$ & $\min$ & $\begin{array}{l}\text { Column } 2 \\
\max \end{array}$ & $\min$ & \\
\hline $\begin{array}{l}\mathrm{g} \\
\mathrm{A}\end{array}$ & $\begin{array}{r}175.5 \\
61.6\end{array}$ & $\begin{array}{r}175.5 \\
0.0 \\
\end{array}$ & $\begin{array}{r}175.5 \\
61.6\end{array}$ & $\begin{array}{r}175.5 \\
0.0 \\
\end{array}$ & \\
\hline tot & 237.1 & 175.5 & 237.1 & 175.5 & \\
\hline
\end{tabular}

\begin{tabular}{|c|c|c|c|c|}
\hline \multicolumn{5}{|c|}{ Deflections calculated according to uncracked concrete! } \\
\hline \multirow{2}{*}{$\begin{array}{l}\text { Deflections } \\
\text { Span No. }\end{array}$} & \multicolumn{2}{|c|}{ maximum } & \multicolumn{2}{|c|}{ minimum } \\
\hline & $x(m)$ & $\mathrm{f}(\mathrm{cm})$ comb & $x(m)$ & $\mathrm{f}(\mathrm{cm}) \mathrm{comb}$ \\
\hline 1 & 7.80 & 1.68 & 0.00 & 0.00 \\
\hline
\end{tabular}

Results for $\gamma$-times loads

Partial safety factor $\gamma \mathrm{G} * \mathrm{~K}_{\mathrm{Fi}}=1.35$ constant over whole girder length

\begin{tabular}{|c|c|c|c|c|c|c|c|c|}
\hline \multicolumn{7}{|c|}{ Span moments maximum } & \multicolumn{2}{|c|}{$(\mathrm{kNm}, \mathrm{kN})$} \\
\hline Span & & & Mfd & Mdle & Mdri & V le & V ri & comb \\
\hline 1 & $x 0=$ & 7.80 & 1284.48 & 0.00 & 0.00 & 329.35 & -329.36 & $A$ \\
\hline
\end{tabular}

\begin{tabular}{|cccrrrrrr|}
\hline \multicolumn{2}{l}{ Support moments maximum } & \multicolumn{3}{c}{ ( kNm,kN ) } \\
\hline Support & Mdle & Mdri & Vdle & Vdri & $\max F$ & $\min F$ & comb \\
\hline 1 & 0.00 & 0.00 & 0.00 & 329.35 & 329.35 & 175.50 & A & 2 \\
2 & 0.00 & 0.00 & -329.35 & 0.00 & 329.36 & 175.50 & A 2 \\
\hline
\end{tabular}

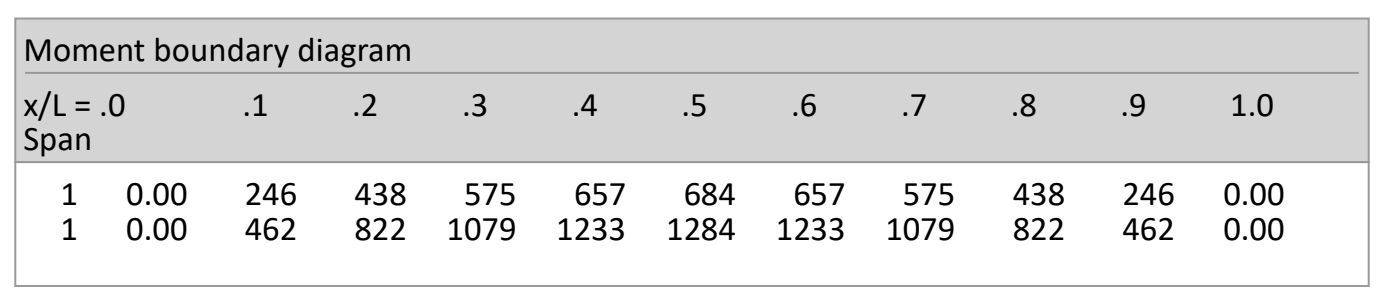


Demo Frilo

Stuttgarter Straße 40

70469 Stuttgart
Tel.: 0711810020

Fax: 0711858020
Projekt: Design Calculations

Position: S.11 - Semi-Precast Double-Tee Slab

26.07.2018

\begin{tabular}{|c|c|c|c|c|c|c|c|c|c|c|}
\hline \multicolumn{11}{|c|}{ shoulder shear } \\
\hline \multirow{2}{*}{$\begin{array}{r}\text { Span } \\
1\end{array}$} & \multirow{2}{*}{$\begin{array}{c}\begin{array}{c}x a \\
(\mathrm{~cm})\end{array} \\
0\end{array}$} & \multirow{2}{*}{$\begin{array}{c}\begin{array}{c}x e \\
(\mathrm{~cm})\end{array} \\
390\end{array}$} & \multirow{2}{*}{$\begin{array}{r}\begin{array}{c}\mathrm{mle} \\
(\mathrm{kNm})\end{array} \\
0.0\end{array}$} & \multirow{2}{*}{$\begin{array}{c}\begin{array}{c}\text { mri } \\
(\mathrm{kNm})\end{array} \\
963.4\end{array}$} & \multirow{2}{*}{ 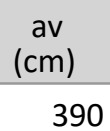 } & \multirow{2}{*}{$\begin{array}{l}\begin{array}{l}\text { beff } \\
(\mathrm{cm})\end{array} \\
248\end{array}$} & \multicolumn{4}{|c|}{$\begin{array}{l}\text { dFcd vEd vEd,perm. asf } \\
\begin{array}{ll}(\mathrm{kN}) & (\mathrm{kN} / \mathrm{m} 2)(\mathrm{cm} 2 / \mathrm{m})\end{array}\end{array}$} \\
\hline & & & & & & & 554 & 947 & 7325 & 2.7 \\
\hline 1 & 390 & 780 & 963.4 & 1284.5 & 390 & 248 & 185 & 316 & 7325 & 0.9 \\
\hline 1 & 780 & 1170 & 1284.5 & 963.4 & 390 & 248 & 185 & 316 & 7325 & 0.9 \\
\hline 1 & 1170 & 1560 & 963.4 & 3.3 & 390 & 248 & 552 & 944 & 7325 & 2.7 \\
\hline
\end{tabular}

At the following table the loads are specified by their internal numeration.

The following table of calculated combinations referenced.

to these numbers

\begin{tabular}{|c|c|c|}
\hline $\begin{array}{l}\text { Load type } \\
\qquad(k N, m)\end{array}$ & $\begin{array}{l}\text { 1= uniform over } \mathrm{L} \\
3=\text { single moment at a } \\
5=\text { triangular over } \mathrm{L}\end{array}$ & $\begin{aligned} 2 & =\text { concentrated at } a \\
4 & =\text { trapezoidal btw. } a, a+b \\
6= & \text { trapezoidal over } L\end{aligned}$ \\
\hline
\end{tabular}

No. span Type Grp $\quad$ g1 $\quad$ q1 $\quad$ g2 $\quad$ q2 factor distance length

$\begin{array}{llllllll}1 & 1 & 1 & A & 1 & 22.50 & 7.90 & 1.00\end{array}$

$$
\begin{aligned}
& \text { Calculated combinations from } 1 \text { Loads } \\
& \text { IC } \quad \text { K1 } \quad \text { K2 }
\end{aligned}
$$

$\begin{array}{ll}\text { g } & \mathrm{g} \\ \mathrm{x} & \mathrm{X}\end{array}$

The combinations above will be managed as followed :

Calculating ULS the dead loads will be exceeded

all at once alternating by GammaG $=1,00 / 1,35$.

If in one combination live-loads from different actions

exists, then will be investigated, which action is

the dominating one.

The effect of the duration of action will be checked

too. 
B.1.7. Design Calculations POS. S.12 - Semi-Precast Concrete Slab 
Demo Frilo

Stuttgarter Straße 40

70469

Stuttgart

Position: S.12 - Semi-Precast Concrete Slab

26.07.2018

Seite: 1

\section{Position: S.12 - Semi-Precast Concrete Slab}

Plates by Finite Elements PLT 01/2018A (Frilo R-2018-1/P12)

System: Floor Plan

-> Siehe Anhang Pläne

System: Floor Plan with FE Mesh

-> Siehe Anhang Pläne 
Demo Frilo

Stuttgarter Straße 40

70469

Stuttgart
Projekt: Design Calculations

Position: S.12 - Semi-Precast Concrete Slab

26.07.2018
Seite: 2

\section{LOADCASE 1 "Self-Weight"}

Type:

Dead loads due to

Plate, beams and parapets are included:

Action:

Partial safety factor action:

Partial safety factor concrete:

Partial safety factor steel:

Load points:

Point loads:

Line loads:

Area loads:

Temperature loads:

Total of input loads:

(portion on the plate)

Dead loads due to

plate and beams:

Fax: 0711858020 permanent

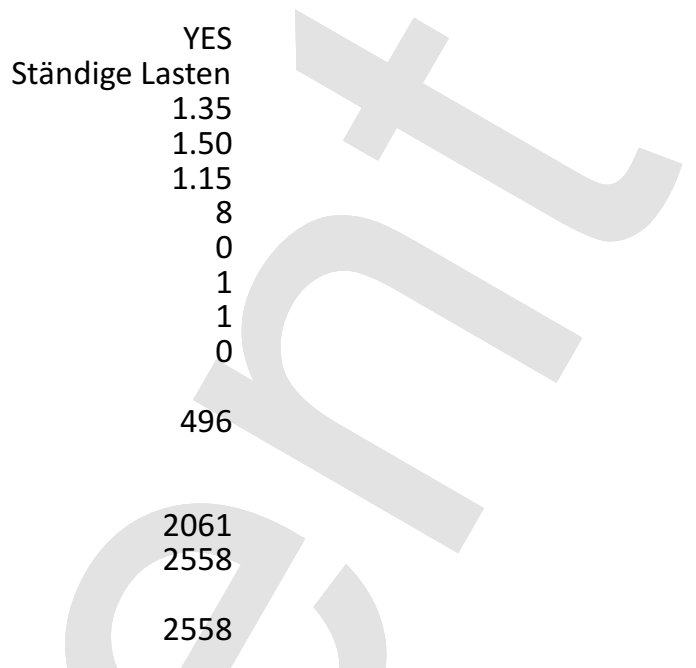

\section{NOTE}

All effects of actions (like moments, shear forces,

support reactions, deflections, etc.) of an individual load case are, unlike the results of a superposition of load cases,

plain, i.e. characteristic values.

Design results are based on design quantities including the partial safety coefficients.

Load case 1 "Self-Weight"

Area Loads

-> Siehe Anhang Pläne 
Demo Frilo

Stuttgarter Straße 40

70469

Stuttgart
Projekt: Design Calculations

Position: S.12 - Semi-Precast Concrete Slab

26.07.2018
Seite: 3

LOADCASE 2 "Live Load"

Type:

Dead loads due to

Plate, beams and parapets are included:

Action:

Partial safety factor action:

Partial safety factor concrete:

Partial safety factor steel:

Load points:

Point loads:

Line loads:

Area loads:

Temperature loads:

Total of input loads:

(portion on the plate)

Total of all support reactions:

\section{NOTE}

All effects of actions (like moments, shear forces,

support reactions, deflections, etc.) of an individual load case

are, unlike the results of a superposition of load cases,

plain, i.e. characteristic values.

Design results are based on design quantities including the partial safety coefficients.

Load case 2 "Live Load"

Area Loads

-> Siehe Anhang Pläne

non permanent

NO
Büros
1.50
1.50
1.15
8
0
1
1
0

747


Demo Frilo

Stuttgarter Straße 40

70469

Stuttgart
Tel.: 0711810020

Fax: 0711858020
Projekt: Design Calculations

Position: S.12 - Semi-Precast Concrete Slab

26.07.2018

\section{SUPERPOSITION 1 "Characteristic"}

\begin{tabular}{|c|c|c|c|c|}
\hline \multicolumn{5}{|l|}{ Load Cases Involved } \\
\hline Number Load case & Type & $\begin{array}{r}\text { Dead } \\
\text { Loads } \\
\text { included }\end{array}$ & $\begin{array}{l}\text { Action } \\
\text { Shrt Name } \\
\text { Hnd }\end{array}$ & $\begin{array}{l}\text { Alter- } \\
\text { native } \\
\quad \text { Group }\end{array}$ \\
\hline $\begin{array}{l}1 \text { Self-Weight } \\
2 \text { Live Load }\end{array}$ & $\begin{array}{l}\text { permanent } \\
\text { variable }\end{array}$ & $\begin{array}{c}\text { yes } \\
\text { no }\end{array}$ & $\begin{array}{l}\text { g Ständige ... } \\
2 \text { Büros }\end{array}$ & 0 \\
\hline
\end{tabular}

\section{Actions Concerned}

Number Shrt Name

Type Hnd

1 g Ständige Lasten permanent

2 Büros variable

Superposition 1 "Characteristic"

Deflection [mm] MAX

-> Siehe Anhang Pläne

Superposition 1 "Characteristic"

Deflection [mm] MIN

-> Siehe Anhang Pläne 
Demo Frilo

Stuttgarter Straße 40
Tel.: 0711810020

Fax: 0711858020
Projekt: Design Calculations

Position: S.12 - Semi-Precast Concrete Slab 26.07.2018
Seite: 5

Superposition 1 "Characteristic"

Beam B1

-> Siehe Anhang Pläne

Superposition 1 "Characteristic"

\begin{tabular}{|c|c|c|c|c|c|c|c|c|}
\hline \multicolumn{3}{|c|}{$\begin{array}{l}\text { Beam B1 } \\
\text { Start: } 16(0.000 / 2.365)\end{array}$} & \multicolumn{6}{|c|}{ End: 17 (19.000 / 2.365$)$} \\
\hline \multirow{2}{*}{$\begin{array}{r}x \\
{[\mathrm{~m}]}\end{array}$} & \multicolumn{2}{|c|}{ Bending Moment } & \multicolumn{2}{|c|}{ Shear Force } & \multirow[b]{2}{*}{$\begin{array}{r}\mathrm{MAX} \\
{[\mathrm{kNm}]}\end{array}$} & \multirow{2}{*}{$\begin{array}{r}\text { Torsion } \\
\text { moment } \\
\text { MIN } \\
{[\mathrm{kNm}]}\end{array}$} & \multirow{2}{*}{\multicolumn{2}{|c|}{$\begin{array}{r}\text { Deflec } \\
\text { tion } \\
\text { IAX MIN } \\
\text { Im] }[\mathrm{mm}]\end{array}$}} \\
\hline & $\begin{array}{r}\mathrm{MAX} \\
{[\mathrm{kNm}]}\end{array}$ & $\begin{array}{r}\mathrm{MIN} \\
{[\mathrm{kNm}]}\end{array}$ & $\begin{array}{r}\mathrm{MAX} \\
{[\mathrm{kN}]}\end{array}$ & $\begin{array}{l}\mathrm{MIN} \\
{[\mathrm{kN}]}\end{array}$ & & & & \\
\hline 0.00 & -0.5 & -0.6 & 87.2 & 67.8 & 1.2 & 0.9 & 0.0 & 0.0 \\
\hline 0.52 & 44.8 & 34.8 & 87.2 & 67.8 & 1.2 & 0.9 & 0.3 & 0.2 \\
\hline 0.52 & 43.2 & 33.6 & 72.7 & 56.5 & 1.3 & 1.0 & 0.3 & 0.2 \\
\hline 1.04 & 81.0 & 63.0 & 72.7 & 56.5 & 1.3 & 1.0 & 0.5 & 0.4 \\
\hline 1.04 & 79.4 & 61.8 & 55.1 & 42.7 & 1.3 & 1.0 & 0.5 & 0.4 \\
\hline 1.56 & 108.1 & 84.0 & 55.1 & 42.7 & 1.3 & 1.0 & 0.7 & 0.6 \\
\hline 1.56 & 106.5 & 82.8 & 36.6 & 28.3 & 1.3 & 1.0 & 0.7 & 0.6 \\
\hline 2.08 & 125.5 & 97.5 & 36.6 & 28.3 & 1.3 & 1.0 & 0.9 & 0.7 \\
\hline 2.08 & 123.7 & 96.2 & 17.8 & 13.7 & 1.3 & 1.0 & 0.9 & 0.7 \\
\hline 2.60 & 133.0 & 103.3 & 17.8 & 13.7 & 1.3 & 1.0 & 0.9 & 0.7 \\
\hline 2.60 & 130.9 & 101.7 & -1.3 & -1.5 & 1.3 & 1.0 & 0.9 & 0.7 \\
\hline 3.12 & 130.1 & 101.0 & -1.3 & -1.5 & 1.3 & 1.0 & 0.9 & 0.7 \\
\hline 3.12 & 127.5 & 99.0 & -17.1 & -21.8 & 1.3 & 1.0 & 0.9 & 0.7 \\
\hline 3.65 & 116.2 & 90.1 & -17.1 & -21.8 & 1.3 & 1.0 & 0.9 & 0.7 \\
\hline 3.65 & 112.9 & 87.6 & -34.5 & -44.4 & 1.3 & 1.0 & 0.9 & 0.7 \\
\hline 4.17 & 89.8 & 69.6 & -34.5 & -44.4 & 1.3 & 1.0 & 0.7 & 0.6 \\
\hline 4.17 & 85.6 & 66.4 & -55.9 & -71.9 & 1.3 & 1.0 & 0.7 & 0.6 \\
\hline 4.69 & 48.2 & 37.3 & -55.9 & -71.9 & 1.3 & 1.0 & 0.5 & 0.4 \\
\hline 4.69 & 43.4 & 33.6 & -85.0 & -109.4 & 1.3 & 1.0 & 0.5 & 0.4 \\
\hline 5.21 & -10.7 & -13.6 & -85.0 & -109.4 & 1.3 & 1.0 & 0.3 & 0.3 \\
\hline 5.21 & -14.6 & -18.7 & -130.8 & -168.4 & 1.3 & 1.0 & 0.3 & 0.3 \\
\hline 5.73 & -82.7 & -106.4 & -130.8 & -168.4 & 1.3 & 1.0 & 0.1 & 0.1 \\
\hline 5.73 & -83.4 & -107.2 & -232.0 & -299.0 & 1.4 & 1.0 & 0.1 & 0.1 \\
\hline 6.25 & -204.2 & -262.9 & -232.0 & -299.0 & 1.4 & 1.0 & 0.0 & 0.0 \\
\hline 6.25 & -204.1 & -262.8 & 264.8 & 205.8 & 1.3 & 1.0 & 0.0 & 0.0 \\
\hline 6.75 & -101.2 & -130.4 & 264.8 & 205.8 & 1.3 & 1.0 & -0.0 & -0.0 \\
\hline 6.75 & -99.3 & -127.9 & 140.3 & 109.1 & 1.2 & 0.9 & -0.0 & -0.0 \\
\hline 7.25 & -44.8 & -57.8 & 140.3 & 109.1 & 1.2 & 0.9 & 0.1 & 0.0 \\
\hline 7.25 & -40.4 & -52.2 & 90.7 & 70.5 & 0.9 & 0.7 & 0.1 & 0.0 \\
\hline 7.75 & -5.2 & -6.8 & 90.7 & 70.5 & 0.9 & 0.7 & 0.1 & 0.1 \\
\hline 7.75 & -1.9 & -2.6 & 64.5 & 50.1 & 0.5 & 0.4 & 0.1 & 0.1 \\
\hline 8.25 & 29.6 & 23.1 & 64.5 & 50.1 & 0.5 & 0.4 & 0.2 & 0.2 \\
\hline 8.25 & 32.1 & 25.1 & 47.0 & 36.5 & 0.2 & 0.1 & 0.2 & 0.2 \\
\hline 8.75 & 55.7 & 43.3 & 47.0 & 36.5 & 0.2 & 0.1 & 0.3 & 0.2 \\
\hline 8.75 & 56.8 & 44.2 & 31.0 & 24.0 & -0.1 & -0.1 & 0.3 & 0.2 \\
\hline 9.25 & 72.3 & 56.2 & 31.0 & 24.0 & -0.1 & -0.1 & 0.4 & 0.3 \\
\hline 9.25 & 72.3 & 56.3 & 13.2 & 10.1 & -0.1 & -0.2 & 0.4 & 0.3 \\
\hline 9.75 & 78.9 & 61.3 & 13.2 & 10.1 & -0.1 & -0.2 & 0.4 & 0.3 \\
\hline 9.75 & 78.1 & 60.7 & -5.8 & -7.4 & -0.1 & -0.2 & 0.4 & 0.3 \\
\hline 10.25 & 74.4 & 57.8 & -5.8 & -7.4 & -0.1 & -0.2 & 0.4 & 0.3 \\
\hline 10.25 & 73.0 & 56.7 & -24.3 & -31.2 & -0.1 & -0.1 & 0.4 & 0.3 \\
\hline 10.75 & 57.4 & 44.6 & -24.3 & -31.2 & -0.1 & -0.1 & 0.3 & 0.2 \\
\hline 10.75 & 55.3 & 42.9 & -46.0 & -59.2 & 0.0 & 0.0 & 0.3 & 0.2 \\
\hline 11.25 & 25.7 & 19.9 & -46.0 & -59.2 & 0.0 & 0.0 & 0.2 & 0.2 \\
\hline 11.25 & 22.6 & 17.5 & -73.7 & -94.9 & 0.1 & 0.1 & 0.2 & 0.2 \\
\hline 11.75 & -19.3 & -24.8 & -73.7 & -94.9 & 0.1 & 0.1 & 0.1 & 0.1 \\
\hline 11.75 & -22.2 & -28.5 & -115.0 & -148.0 & 0.2 & 0.1 & 0.1 & 0.1 \\
\hline 12.25 & -79.7 & -102.5 & -115.0 & -148.0 & 0.2 & 0.1 & 0.0 & 0.0 \\
\hline 12.25 & -80.2 & -103.2 & -203.3 & -261.7 & 0.1 & 0.1 & 0.0 & 0.0 \\
\hline 12.75 & -181.9 & -234.0 & -203.3 & -261.7 & 0.1 & 0.1 & 0.0 & 0.0 \\
\hline 12.75 & -180.9 & -232.8 & 238.7 & 185.6 & -0.1 & -0.1 & 0.0 & 0.0 \\
\hline 13.27 & -84.3 & -108.5 & 238.7 & 185.6 & -0.1 & -0.1 & 0.1 & 0.1 \\
\hline 13.27 & -83.1 & -106.9 & 133.1 & 103.4 & -0.4 & -0.5 & 0.1 & 0.1 \\
\hline 13.79 & -29.2 & -37.6 & 133.1 & 103.4 & -0.4 & -0.5 & 0.2 & 0.2 \\
\hline 13.79 & -25.6 & -33.0 & 93.7 & 72.8 & -0.8 & -1.1 & 0.2 & 0.2 \\
\hline
\end{tabular}


Demo Frilo

Stuttgarter Straße 40

70469
Tel.: 0711810020

Fax: 0711858020
Projekt: Design Calculations

Position: S.12 - Semi-Precast Concrete Slab

26.07.2018
Seite: 6

\begin{tabular}{|c|c|c|c|c|c|c|c|c|}
\hline $\begin{array}{l}\text { Beam B1 } \\
\text { Start: } 16\end{array}$ & $00 / 2.3$ & & End: 17 & $000 / 2$ & & & & \\
\hline$x$ & Bendin & loment & & Force & & & & eflec- \\
\hline$[\mathrm{m}]$ & $\begin{array}{r}\mathrm{MAX} \\
{[\mathrm{kNm}]}\end{array}$ & $\begin{array}{r}\mathrm{MIN} \\
{[\mathrm{kNm}]}\end{array}$ & $\begin{array}{r}\mathrm{MAX} \\
{[\mathrm{kN}]}\end{array}$ & $\begin{array}{l}\mathrm{MIN} \\
{[\mathrm{kN}]}\end{array}$ & $\begin{array}{r}\mathrm{MAX} \\
{[\mathrm{kNm}]}\end{array}$ & $\begin{array}{r}\mathrm{MIN} \\
{[\mathrm{kNm}]}\end{array}$ & $\begin{array}{r}\operatorname{MAX} \\
{[\mathrm{mm}]}\end{array}$ & $\begin{array}{r}\mathrm{MIN} \\
{[\mathrm{mm}]}\end{array}$ \\
\hline 14.31 & 15.9 & 12.4 & 93.7 & 72.8 & -0.8 & -1.1 & 0.4 & 0.3 \\
\hline 14.31 & 19.6 & 15.3 & 71.9 & 55.8 & -1.2 & -1.6 & 0.4 & 0.3 \\
\hline 14.83 & 57.1 & 44.3 & 71.9 & 55.8 & -1.2 & -1.6 & 0.5 & 0.4 \\
\hline 14.83 & 59.7 & 46.4 & 53.3 & 41.4 & -1.5 & -2.0 & 0.5 & 0.4 \\
\hline 15.35 & 87.5 & 68.0 & 53.3 & 41.4 & -1.5 & -2.0 & 0.7 & 0.5 \\
\hline 15.35 & 89.4 & 69.4 & 33.7 & 26.2 & -1.6 & -2.1 & 0.7 & 0.5 \\
\hline 15.87 & 106.9 & 83.1 & 33.7 & 26.2 & -1.6 & -2.1 & 0.7 & 0.6 \\
\hline 15.87 & 108.4 & 84.2 & 12.9 & 10.1 & -1.7 & -2.2 & 0.7 & 0.6 \\
\hline 16.40 & 115.2 & 89.5 & 12.9 & 10.1 & -1.7 & -2.2 & 0.8 & 0.6 \\
\hline 16.40 & 116.6 & 90.6 & -6.3 & -8.2 & -1.6 & -2.1 & 0.8 & 0.6 \\
\hline 16.92 & 112.3 & 87.3 & -6.3 & -8.2 & -1.6 & -2.1 & 0.7 & 0.6 \\
\hline 16.92 & 113.7 & 88.3 & -22.5 & -29.1 & -1.6 & -2.0 & 0.7 & 0.6 \\
\hline 17.44 & 98.6 & 76.6 & -22.5 & -29.1 & -1.6 & -2.0 & 0.6 & 0.5 \\
\hline 17.44 & 100.1 & 77.8 & -38.2 & -49.2 & -1.5 & -2.0 & 0.6 & 0.5 \\
\hline 17.96 & 74.5 & 57.9 & -38.2 & -49.2 & -1.5 & -2.0 & 0.4 & 0.3 \\
\hline 17.96 & 76.2 & 59.2 & -52.7 & -68.0 & -1.5 & -1.9 & 0.4 & 0.3 \\
\hline 18.48 & 40.8 & 31.7 & -52.7 & -68.0 & -1.5 & -1.9 & 0.2 & 0.2 \\
\hline 18.48 & 42.5 & 33.0 & -64.5 & -82.9 & -1.3 & -1.7 & 0.2 & 0.2 \\
\hline 19.00 & -0.5 & -0.7 & -64.5 & -82.9 & -1.3 & -1.7 & 0.0 & 0.0 \\
\hline
\end{tabular}


Demo Frilo

Stuttgarter Straße 40

70469

Stuttgart
Tel.: 0711810020

Fax: 0711858020
Projekt: Design Calculations

Position: S.12 - Semi-Precast Concrete Slab

26.07.2018
Seite: 7

\section{SUPERPOSITION 2 "USL Permanent/Transient"}

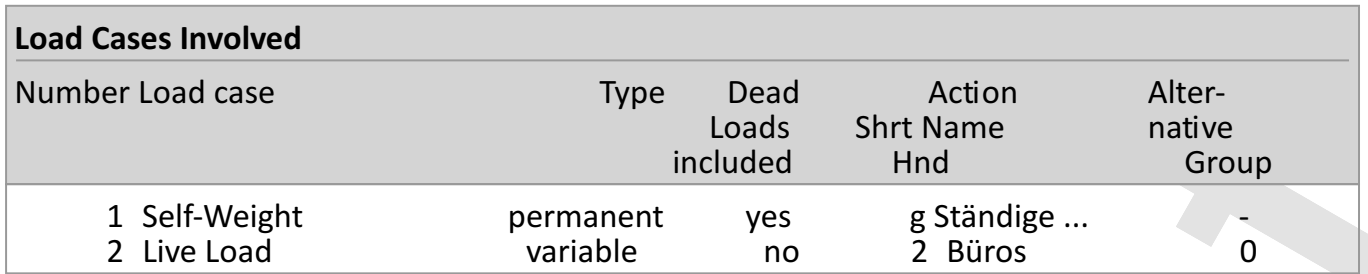

\begin{tabular}{|c|c|c|c|c|}
\hline \multicolumn{5}{|l|}{ Actions Concerned } \\
\hline $\begin{array}{c}\text { Number Shrt Name } \\
\text { Hnd }\end{array}$ & Type & $\begin{array}{l}\text { iafety } \\
\text { sup }\end{array}$ & inf & $\begin{array}{l}\text { Combination } \\
\text { dom ndom }\end{array}$ \\
\hline $\begin{array}{ll}1 & \text { g Ständige Lasten } \\
2 & 2 \text { Büros }\end{array}$ & $\begin{array}{l}\text { permanent } \\
\text { variable }\end{array}$ & $\begin{array}{l}1.35 \\
1.50\end{array}$ & $\begin{array}{l}1.00 \\
0.00\end{array}$ & $\begin{array}{ll}1.00 & 1.00 \\
1.00 & 0.70\end{array}$ \\
\hline
\end{tabular}

Partial safety factor concrete:

Partial safety factor steel:

\section{NOTE: Design Values}

All results of a superposition of load cases include both using both the partial safety

and combination factors.: DIN EN 1990/NA:2010-12

\section{NOTE: Combination Factors}

With the combination of independent, variable actions the individual main action is determined for both each location and for each action quantity. In geneneral, the main actions differ with each location and each quantity. the leading effect.

The individually found main action receives the conbination factor 1.00 . In case of only one variable action this action is considered the main action.

Superposition 2 "USL Permanent/Transient" Moment $\mathrm{m}-1[\mathrm{kNm} / \mathrm{m}] \mathrm{MAX}$

Design Values (x Gamma)

-> Siehe Anhang Pläne

Superposition 2 "USL Permanent/Transient" Moment $\mathrm{m}-1[\mathrm{kNm} / \mathrm{m}] \mathrm{MIN}$

Design Values (x Gamma)

-> Siehe Anhang Pläne

Superposition 2 "USL Permanent/Transient" Moment $\mathrm{m}-2[\mathrm{kNm} / \mathrm{m}] \mathrm{MAX}$

Design Values (x Gamma)

-> Siehe Anhang Pläne

Superposition 2 "USL Permanent/Transient" Moment m-2 [kNm/m] MIN

Design Values (x Gamma)

-> Siehe Anhang Pläne

Superposition 2 "USL Permanent/Transient" Moment $\mathrm{m}-12[\mathrm{kNm} / \mathrm{m}] \mathrm{MAX}$

Design Values (x Gamma)

-> Siehe Anhang Pläne

Superposition 2 "USL Permanent/Transient" Moment m-12 [kNm/m] MIN

Design Values (x Gamma) 
Demo Frilo

Stuttgarter Straße 40

70469

Stuttgart

Tel.: 0711810020

Fax: 0711858020
Projekt: Design Calculations

Position: S.12 - Semi-Precast Concrete Slab

26.07.2018
Seite: 8

-> Siehe Anhang Pläne

Superposition 2 "USL Permanent/Transient" Design Moment, bottom mB-1 [kNm/m]

Design Values (x Gamma)

-> Siehe Anhang Pläne

Superposition 2 "USL Permanent/Transient" Design Moment, bottom mB-2 [kNm/m]

Design Values (x Gamma)

-> Siehe Anhang Pläne

Superposition 2 "USL Permanent/Transient" Design Moment, top mB-1 [kNm/m]

Design Values (x Gamma)

-> Siehe Anhang Pläne

Superposition 2 "USL Permanent/Transient" Design Moment, top mB-2 [kNm/m]

Design Values (x Gamma)

-> Siehe Anhang Pläne

Superposition 2 "USL Permanent/Transient" Reinforcement, bottom aS-1 $\left[\mathrm{cm}^{2} / \mathrm{m}\right]$

-> Siehe Anhang Pläne

Superposition 2 "USL Permanent/Transient" Reinforcement, bottom aS-2 $\left[\mathrm{cm}^{2} / \mathrm{m}\right]$

-> Siehe Anhang Pläne

Superposition 2 "USL Permanent/Transient" Reinforcement, top aS-1 $\left[\mathrm{cm}^{2} / \mathrm{m}\right]$

-> Siehe Anhang Pläne

Superposition 2 "USL Permanent/Transient" Reinforcement, top aS-2 $\left[\mathrm{cm}^{2} / \mathrm{m}\right]$

-> Siehe Anhang Pläne

Superposition 2 "USL Permanent/Transient" Shear Force q-1z [kN/m]

Design Values (x Gamma)

-> Siehe Anhang Pläne

Superposition 2 "USL Permanent/Transient" Shear Force q-2z [kN/m]

Design Values (x Gamma)

-> Siehe Anhang Pläne

Superposition 2 "USL Permanent/Transient" Shear reinforcement $\left[\mathrm{cm}^{2} / \mathrm{m}^{2}\right]$

-> Siehe Anhang Pläne 
Demo Frilo

Stuttgarter Straße 40

Superposition 2 "USL Permanent/Transient"

Beam B1

-> Siehe Anhang Pläne

Superposition 2 "USL Permanent/Transient"

\section{Beam B1}

Start: 16 (0.000 /2.365)

End: 17 (19.000 /2.365)

\begin{tabular}{|c|c|c|c|c|c|c|c|}
\hline \multirow{3}{*}{$\begin{array}{r}x \\
{[\mathrm{~m}]}\end{array}$} & \multicolumn{2}{|c|}{ Bending Moment } & \multirow{3}{*}{$\begin{array}{r}\text { As } \\
\text { bottom } \\
{\left[\mathrm{cm}^{2}\right][\mathrm{cm}}\end{array}$} & \multirow{2}{*}{$\begin{array}{l}\text { As } \\
\text { top }\end{array}$} & \multicolumn{2}{|c|}{ Torsional Moment } & \multirow{3}{*}{$\begin{array}{r}\text { As } \\
\text { Longitudinal } \\
\text { Torsion }\end{array}$} \\
\hline & MAX & MIN & & & & $\begin{array}{ll}X & \text { MIN }\end{array}$ & \\
\hline & {$[\mathrm{kNm}]$} & {$[\mathrm{kNm}]$} & & 2] & $\mathrm{kNm}]$ & {$[\mathrm{kNm}]\left[\mathrm{cm}^{2}\right]$} & \\
\hline 0.00 & -0.5 & -0.9 & 0.00 & 5.54 & 1.7 & 0.9 & 0.71 \\
\hline 0.52 & 61.9 & 34.8 & 3.19 & 0.00 & 1.7 & 0.9 & 0.71 \\
\hline 0.52 & 59.7 & 33.6 & 3.19 & 0.00 & 1.8 & 1.0 & 0.77 \\
\hline 1.04 & 112.1 & 63.0 & 3.77 & 0.00 & 1.8 & 1.0 & 0.77 \\
\hline 1.04 & 109.8 & 61.8 & 3.69 & 0.00 & 1.8 & 1.0 & 0.77 \\
\hline 1.56 & 149.5 & 84.0 & 5.04 & 0.00 & 1.8 & 1.0 & 0.77 \\
\hline 1.56 & 147.3 & 82.8 & 4.96 & 0.00 & 1.8 & 1.0 & 0.77 \\
\hline 2.08 & 173.7 & 97.5 & 5.86 & 0.00 & 1.8 & 1.0 & 0.77 \\
\hline 2.08 & 171.2 & 96.2 & 5.78 & 0.00 & 1.8 & 1.0 & 0.77 \\
\hline 2.60 & 184.0 & 103.3 & 6.22 & 0.00 & 1.8 & 1.0 & 0.77 \\
\hline 2.60 & 181.1 & 101.7 & 6.12 & 0.00 & 1.8 & 1.0 & 0.76 \\
\hline 3.12 & 180.0 & 101.0 & 6.08 & 0.00 & 1.8 & 1.0 & 0.76 \\
\hline 3.12 & 176.4 & 99.0 & 5.96 & 0.00 & 1.8 & 1.0 & 0.00 \\
\hline 3.65 & 160.7 & 90.1 & 5.42 & 0.00 & 1.8 & 1.0 & 0.00 \\
\hline 3.65 & 156.1 & 87.6 & 5.27 & 0.00 & 1.8 & 1.0 & 0.75 \\
\hline 4.17 & 124.2 & 69.6 & 4.18 & 0.00 & 1.8 & 1.0 & 0.75 \\
\hline 4.17 & 118.5 & 66.4 & 3.99 & 0.00 & 1.8 & 1.0 & 0.75 \\
\hline 4.69 & 66.7 & 37.3 & 3.19 & 0.00 & 1.8 & 1.0 & 0.75 \\
\hline 4.69 & 60.1 & 33.6 & 3.19 & 0.00 & 1.8 & 1.0 & 0.76 \\
\hline 5.21 & -10.7 & -18.8 & 0.00 & 5.54 & 1.8 & 1.0 & 0.76 \\
\hline 5.21 & -14.6 & -25.8 & 0.00 & 5.54 & 1.8 & 1.0 & 0.70 \\
\hline 5.73 & -82.7 & -147.2 & 0.00 & 5.54 & 1.8 & 1.0 & 0.70 \\
\hline 5.73 & -83.4 & -148.4 & 0.00 & 5.54 & 1.9 & 1.0 & 0.47 \\
\hline 6.25 & -204.2 & -363.8 & 0.00 & 13.67 & 1.9 & 1.0 & 0.46 \\
\hline 6.25 & -204.1 & -363.6 & 0.00 & 13.66 & 1.9 & 1.0 & 0.49 \\
\hline 6.75 & -101.2 & -180.4 & 0.00 & 6.26 & 1.9 & 1.0 & 0.49 \\
\hline 6.75 & -99.3 & -176.9 & 0.00 & 6.14 & 1.7 & 0.9 & 0.70 \\
\hline 7.25 & -44.8 & -79.9 & 0.00 & 5.54 & 1.7 & 0.9 & 0.70 \\
\hline 7.25 & -40.4 & -72.2 & 0.00 & 5.54 & 1.3 & 0.7 & 0.54 \\
\hline 7.75 & -5.2 & -9.4 & 0.00 & 5.54 & 1.3 & 0.7 & 0.54 \\
\hline 7.75 & -1.9 & -3.7 & 0.00 & 5.54 & 0.8 & 0.4 & 0.32 \\
\hline 8.25 & 41.0 & 23.1 & 3.19 & 0.00 & 0.8 & 0.4 & 0.32 \\
\hline 8.25 & 44.4 & 25.1 & 3.19 & 0.00 & 0.3 & 0.1 & 0.11 \\
\hline 8.75 & 77.0 & 43.3 & 3.19 & 0.00 & 0.3 & 0.1 & 0.11 \\
\hline 8.75 & 78.5 & 44.2 & 3.19 & 0.00 & -0.1 & -0.1 & 0.00 \\
\hline 9.25 & 100.0 & 56.2 & 3.36 & 0.00 & -0.1 & -0.1 & 0.00 \\
\hline 9.25 & 100.0 & 56.3 & 3.36 & 0.00 & -0.1 & -0.3 & 0.00 \\
\hline 9.75 & 109.2 & 61.3 & 3.67 & 0.00 & -0.1 & -0.3 & 0.00 \\
\hline 9.75 & 108.1 & 60.7 & 3.63 & 0.00 & -0.1 & -0.3 & 0.00 \\
\hline 10.25 & 103.0 & 57.8 & 3.46 & 0.00 & -0.1 & -0.3 & 0.00 \\
\hline 10.25 & 101.0 & 56.7 & 3.39 & 0.00 & -0.1 & -0.1 & 0.00 \\
\hline 10.75 & 79.4 & 44.6 & 3.19 & 0.00 & -0.1 & -0.1 & 0.00 \\
\hline 10.75 & 76.5 & 42.9 & 3.19 & 0.00 & 0.0 & 0.0 & 0.01 \\
\hline 11.25 & 35.5 & 19.9 & 3.19 & 0.00 & 0.0 & 0.0 & 0.01 \\
\hline 11.25 & 31.3 & 17.5 & 3.19 & 0.00 & 0.2 & 0.1 & 0.07 \\
\hline 11.75 & -19.3 & -34.3 & 0.00 & 5.54 & 0.2 & 0.1 & 0.07 \\
\hline 11.75 & -22.2 & -39.4 & 0.00 & 5.54 & 0.3 & 0.1 & 0.11 \\
\hline 12.25 & -79.7 & -141.8 & 0.00 & 5.54 & 0.3 & 0.1 & 0.11 \\
\hline 12.25 & -80.2 & -142.7 & 0.00 & 5.54 & 0.2 & 0.1 & 0.05 \\
\hline 12.75 & -181.9 & -323.7 & 0.00 & 11.96 & 0.2 & 0.1 & 0.05 \\
\hline 12.75 & -180.9 & -322.1 & 0.00 & 11.90 & -0.1 & -0.2 & 0.04 \\
\hline 13.27 & -84.3 & -150.1 & 0.00 & 5.54 & -0.1 & -0.2 & 0.04 \\
\hline 13.27 & -83.1 & -147.9 & 0.00 & 5.54 & -0.4 & -0.7 & 0.31 \\
\hline 13.79 & -29.2 & -52.1 & 0.00 & 5.54 & -0.4 & -0.7 & 0.31 \\
\hline 13.79 & -25.6 & -45.6 & 0.00 & 5.54 & -0.8 & -1.5 & 0.64 \\
\hline
\end{tabular}


Demo Frilo

Stuttgarter Straße 40
Tel.: 0711810020

Fax: 0711858020
Projekt: Design Calculations

Position: S.12 - Semi-Precast Concrete Slab 26.07.2018

\begin{tabular}{|c|c|c|c|c|c|c|c|}
\hline $\begin{array}{l}\text { Beam } \\
\text { Start: } 1\end{array}$ & $00 / 2.36$ & & End: 17 & $9.000 / 2$ & 65) & & \\
\hline$x$ & Bendi & 1oment & & As & Torsio & onal Moment & \\
\hline & MAX & MIN & bottom & top & MAX & MIN & Longitudinal \\
\hline$[\mathrm{m}]$ & {$[\mathrm{kNm}]$} & {$[\mathrm{kNm}]$} & {$\left[\mathrm{cm}^{2}\right][\mathrm{cm}$} & 2] [ & $\mathrm{kNm}]$ & $\mathrm{kNm}]\left[\mathrm{cm}^{2}\right]$ & \\
\hline 14.31 & 21.9 & 12.4 & 3.19 & 0.00 & -0.8 & -1.5 & 0.64 \\
\hline 14.31 & 27.1 & 15.3 & 3.19 & 0.00 & -1.2 & -2 & 0.95 \\
\hline 14.83 & 78.9 & 44.3 & 3.19 & 0.00 & -1.2 & -2.2 & 0.95 \\
\hline 14.83 & 82.6 & 46.4 & 3.19 & 0.00 & -1.5 & -2.7 & 1.16 \\
\hline 15.35 & 121.0 & 68.0 & 4.07 & 0.00 & -1.5 & -2.7 & 1.16 \\
\hline 15.35 & 123.6 & 69.4 & 4.16 & 0.00 & -1.6 & -2.9 & 1.25 \\
\hline 15.87 & 147.9 & 83.1 & 4.99 & 0.00 & -1.6 & -2.9 & 1.25 \\
\hline 15.87 & 150.0 & 84.2 & 5.06 & 0.00 & -1.7 & -3.0 & 1.27 \\
\hline 16.40 & 159.4 & 89.5 & 5.38 & 0.00 & -1.7 & -3.0 & 1.27 \\
\hline 16.40 & 161.3 & 90.6 & 5.44 & 0.00 & -1.6 & -2.9 & 1.24 \\
\hline 16.92 & 155.4 & 87.3 & 5.24 & 0.00 & -1.6 & -2.9 & 1.24 \\
\hline 16.92 & 157.3 & 88.3 & 5.31 & 0.00 & -1.6 & -2.8 & 1.20 \\
\hline 17.44 & 136.4 & 76.6 & 4.59 & 0.00 & -1.6 & -2.8 & 1.20 \\
\hline 17.44 & 138.5 & 77.8 & 4.67 & 0.00 & -1.5 & -2.7 & 1.16 \\
\hline 17.96 & 103.0 & 57.9 & 3.46 & 0.00 & -1.5 & -2.7 & 1.16 \\
\hline 17.96 & 105.4 & 59.2 & 3.54 & 0.00 & -1.5 & -2.7 & 1.13 \\
\hline 18.48 & 56.4 & 31.7 & 3.19 & 0.00 & -1.5 & -2.7 & 1.13 \\
\hline 18.48 & 58.8 & 33.0 & 3.19 & 0.00 & -1.3 & -2.4 & 1.03 \\
\hline 19.00 & -0.5 & -1.0 & 0.00 & 5.54 & -1.3 & -2.4 & 1.03 \\
\hline
\end{tabular}

Beam B1

Start: 16 (0.000 /2.365)

End: 17 (19.000 /2.365)

\begin{tabular}{|c|c|c|c|c|c|c|}
\hline $\begin{array}{r}x \\
{[m]}\end{array}$ & $\begin{array}{l}\text { MAX } \\
{[k N]}\end{array}$ & $\begin{array}{r}\text { Shear Force } \\
\text { MIN } \\
{[\mathrm{kN}]}\end{array}$ & $\begin{array}{r}\text { Compress } \\
\text { strut } \\
\cot [1]\end{array}$ & $\begin{array}{r}\text { VEd / } \\
\text { VRd,c } \\
{[1]}\end{array}$ & $\begin{array}{r}\text { VEd / } \\
\text { VRd, } \max \\
{[1]}\end{array}$ & $\begin{array}{l}\text { As } \\
\text { tirrup } \\
2 / m]\end{array}$ \\
\hline 0.00 & 120.7 & 67.8 & 3.00 & 1.80 & 0.17 & 2.78 \\
\hline 0.52 & 120.7 & 67.8 & 3.00 & 1.80 & 0.17 & 2.78 \\
\hline 0.52 & 100.6 & 56.5 & 3.00 & 1.50 & 0.14 & 2.78 \\
\hline 1.04 & 100.6 & 56.5 & 3.00 & 1.50 & 0.14 & 2.78 \\
\hline 1.04 & 76.2 & 42.7 & 3.00 & 1.14 & 0.11 & 2.78 \\
\hline 1.56 & 76.2 & 42.7 & 3.00 & 1.14 & 0.11 & 2.78 \\
\hline 1.56 & 50.6 & 28.3 & 3.00 & 0.76 & 0.07 & 2.78 \\
\hline 2.08 & 50.6 & 28.3 & 3.00 & 0.76 & 0.07 & 2.78 \\
\hline 2.08 & 24.6 & 13.7 & 3.00 & 0.37 & 0.04 & 2.78 \\
\hline 2.60 & 24.6 & 13.7 & 3.00 & 0.37 & 0.04 & 2.78 \\
\hline 2.60 & -1.3 & -2.1 & 3.00 & 0.03 & 0.00 & 2.78 \\
\hline 3.12 & -1.3 & -2.1 & 3.00 & 0.03 & 0.00 & 2.78 \\
\hline 3.12 & -17.1 & -30.2 & 3.00 & 0.45 & 0.04 & 2.78 \\
\hline 3.65 & -17.1 & -30.2 & 3.00 & 0.45 & 0.04 & 2.78 \\
\hline 3.65 & -34.5 & -61.4 & 3.00 & 0.92 & 0.09 & 2.78 \\
\hline 4.17 & -34.5 & -61.4 & 3.00 & 0.92 & 0.09 & 2.78 \\
\hline 4.17 & -55.9 & -99.5 & 3.00 & 1.48 & 0.14 & 2.78 \\
\hline 4.69 & -55.9 & -99.5 & 3.00 & 1.48 & 0.14 & 2.78 \\
\hline 4.69 & -85.0 & -151.4 & 3.00 & 2.26 & 0.22 & 2.78 \\
\hline 5.21 & -85.0 & -151.4 & 3.00 & 2.26 & 0.22 & 2.78 \\
\hline 5.21 & -130.8 & -233.1 & 2.67 & 3.48 & 0.30 & 3.40 \\
\hline 5.73 & -130.8 & -233.1 & 2.67 & 3.48 & 0.30 & 3.40 \\
\hline 5.73 & -232.0 & -413.6 & 1.76 & 6.17 & 0.41 & 9.00 \\
\hline 6.25 & -232.0 & -413.6 & 1.74 & 4.91 & 0.42 & 9.29 \\
\hline 6.25 & 366.3 & 205.8 & 1.85 & 4.35 & 0.38 & 7.79 \\
\hline 6.75 & 366.3 & 205.8 & 1.87 & 5.47 & 0.38 & 7.53 \\
\hline 6.75 & 194.0 & 109.1 & 3.00 & 2.90 & 0.28 & 2.78 \\
\hline 7.25 & 194.0 & 109.1 & 3.00 & 2.90 & 0.28 & 2.78 \\
\hline 7.25 & 125.5 & 70.5 & 3.00 & 1.87 & 0.18 & 2.78 \\
\hline 7.75 & 125.5 & 70.5 & 3.00 & 1.87 & 0.18 & 2.78 \\
\hline 7.75 & 89.2 & 50.1 & 3.00 & 1.33 & 0.13 & 2.78 \\
\hline 8.25 & 89.2 & 50.1 & 3.00 & 1.33 & 0.13 & 2.78 \\
\hline 8.25 & 65.1 & 36.5 & 3.00 & 0.97 & 0.09 & 2.78 \\
\hline 8.75 & 65.1 & 36.5 & 3.00 & 0.97 & 0.09 & 2.78 \\
\hline 8.75 & 42.8 & 24.0 & 3.00 & 0.64 & 0.06 & 2.78 \\
\hline
\end{tabular}


Demo Frilo

Stuttgarter Straße 40

70469
Tel.: 0711810020

Fax: 0711858020
Projekt: Design Calculations

Position: S.12 - Semi-Precast Concrete Slab

26.07.2018
Seite: 11

\begin{tabular}{|c|c|c|c|c|c|c|}
\hline \multicolumn{3}{|c|}{$\begin{array}{l}\text { Beam B1 } \\
\text { Start: } 16(0.000 / 2.365)\end{array}$} & \multicolumn{4}{|c|}{ End: 17 (19.000 / 2.365) } \\
\hline $\begin{array}{r}x \\
{[\mathrm{~m}]}\end{array}$ & $\begin{array}{r}\mathrm{MAX} \\
{[\mathrm{kN}]}\end{array}$ & $\begin{array}{r}\text { Shear Force } \\
\text { MIN } \\
{[k N]}\end{array}$ & $\begin{array}{r}\text { Compress } \\
\text { strut } \\
\text { cot [1] }\end{array}$ & $\begin{array}{r}\text { VEd / } \\
\text { VRd,c } \\
{[1]}\end{array}$ & $\begin{array}{r}\text { VEd / } \\
\text { VRd,max } \\
{[1][}\end{array}$ & $\begin{array}{r}\text { As } \\
\text { Stirrup } \\
\left.\mathrm{m}^{2} / \mathrm{m}\right]\end{array}$ \\
\hline 9.25 & 42.8 & 24.0 & 3.00 & 0.64 & 0.06 & 2.78 \\
\hline 9.25 & 18.2 & 10.1 & 3.00 & 0.27 & 0.03 & 2.78 \\
\hline 9.75 & 18.2 & 10.1 & 3.00 & 0.27 & 0.03 & 2.78 \\
\hline 9.75 & -5.8 & -10.2 & 3.00 & 0.15 & 0.01 & 2.78 \\
\hline 10.25 & -5.8 & -10.2 & 3.00 & 0.15 & 0.01 & 2.78 \\
\hline 10.25 & -24.3 & -43.1 & 3.00 & 0.64 & 0.06 & 2.78 \\
\hline 10.75 & -24.3 & -43.1 & 3.00 & 0.64 & 0.06 & 2.78 \\
\hline 10.75 & -46.0 & -81.9 & 3.00 & 1.22 & 0.12 & 2.78 \\
\hline 11.25 & -46.0 & -81.9 & 3.00 & 1.22 & 0.12 & 2.78 \\
\hline 11.25 & -73.7 & -131.3 & 3.00 & 1.96 & 0.19 & 2.78 \\
\hline 11.75 & -73.7 & -131.3 & 3.00 & 1.96 & 0.19 & 2.78 \\
\hline 11.75 & -115.0 & -204.8 & 3.00 & 3.06 & 0.29 & 2.78 \\
\hline 12.25 & -115.0 & -204.8 & 3.00 & 3.06 & 0.29 & 2.78 \\
\hline 12.25 & -203.3 & -362.0 & 1.92 & 5.40 & 0.38 & 7.09 \\
\hline 12.75 & -203.3 & -362.0 & 1.91 & 4.49 & 0.38 & 7.19 \\
\hline 12.75 & 330.2 & 185.6 & 2.03 & 4.10 & 0.36 & 6.18 \\
\hline 13.27 & 330.2 & 185.6 & 2.04 & 4.93 & 0.36 & 6.08 \\
\hline 13.27 & 184.1 & 103.4 & 3.00 & 2.75 & 0.26 & 2.78 \\
\hline 13.79 & 184.1 & 103.4 & 3.00 & 2.75 & 0.26 & 2.78 \\
\hline 13.79 & 129.7 & 72.8 & 3.00 & 1.94 & 0.18 & 2.78 \\
\hline 14.31 & 129.7 & 72.8 & 3.00 & 1.94 & 0.18 & 2.78 \\
\hline 14.31 & 99.4 & 55.8 & 3.00 & 1.48 & 0.14 & 2.78 \\
\hline 14.83 & 99.4 & 55.8 & 3.00 & 1.48 & 0.14 & 2.78 \\
\hline 14.83 & 73.8 & 41.4 & 3.00 & 1.10 & 0.11 & 2.78 \\
\hline 15.35 & 73.8 & 41.4 & 3.00 & 1.10 & 0.11 & 2.78 \\
\hline 15.35 & 46.6 & 26.2 & 3.00 & 0.70 & 0.07 & 2.78 \\
\hline 15.87 & 46.6 & 26.2 & 3.00 & 0.70 & 0.07 & 2.78 \\
\hline 15.87 & 17.9 & 10.1 & 3.00 & 0.27 & 0.03 & 2.78 \\
\hline 16.40 & 17.9 & 10.1 & 3.00 & 0.27 & 0.03 & 2.78 \\
\hline 16.40 & -6.3 & -11.4 & 3.00 & 0.17 & 0.02 & 2.78 \\
\hline 16.92 & -6.3 & -11.4 & 3.00 & 0.17 & 0.02 & 2.78 \\
\hline 16.92 & -22.5 & -40.2 & 3.00 & 0.60 & 0.06 & 2.78 \\
\hline 17.44 & -22.5 & -40.2 & 3.00 & 0.60 & 0.06 & 2.78 \\
\hline 17.44 & -38.2 & -68.1 & 3.00 & 1.02 & 0.10 & 2.78 \\
\hline 17.96 & -38.2 & -68.1 & 3.00 & 1.02 & 0.10 & 2.78 \\
\hline 17.96 & -52.7 & -94.0 & 3.00 & 1.40 & 0.13 & 2.78 \\
\hline 18.48 & -52.7 & -94.0 & 3.00 & 1.40 & 0.13 & 2.78 \\
\hline 18.48 & -64.5 & -114.7 & 3.00 & 1.71 & 0.16 & 2.78 \\
\hline 19.00 & -64.5 & -114.7 & 3.00 & 1.71 & 0.16 & 2.78 \\
\hline
\end{tabular}




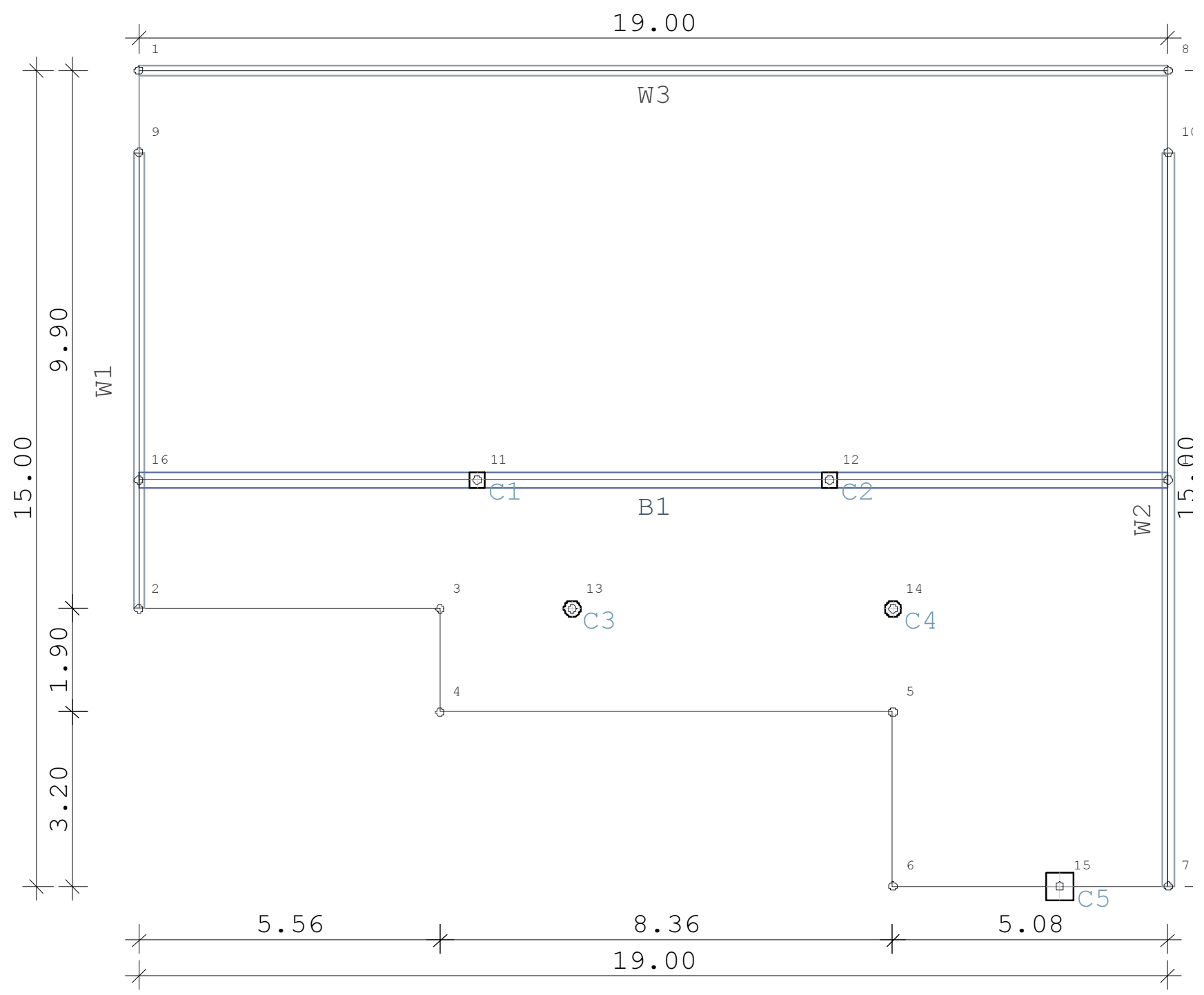

Projekt: Design Calculations

Position: S.12 - Semi-Precast Concrete Slab

System: Floor Plan

\section{Demo Frilo}

Stuttgarter Straße 40

70469 Stuttgart

Tel.: 0711810020

Email: info@frilo.de
Prj.Nr.:

Seite: 1

$1: 125$

26.07.2018 


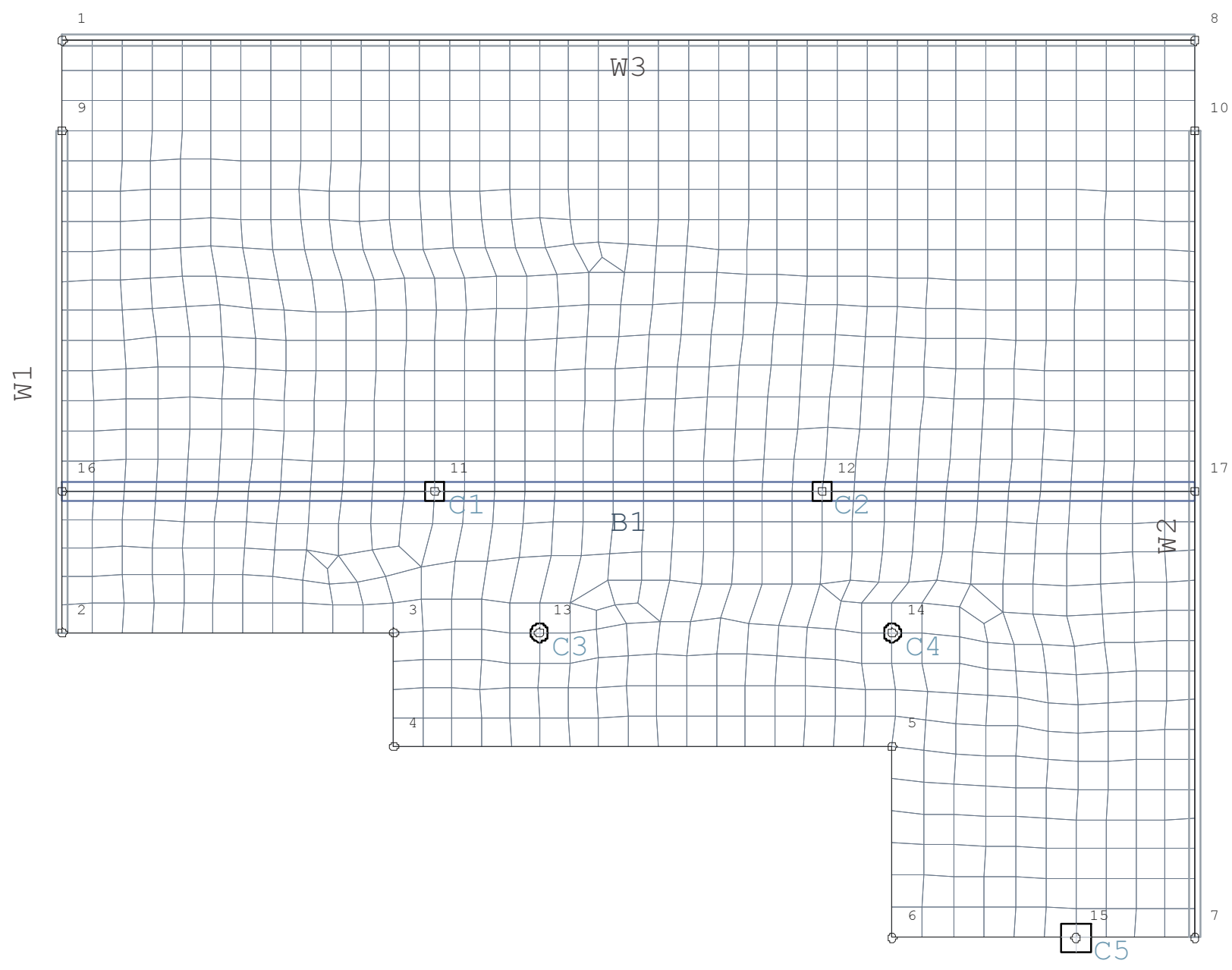

Projekt: Design Calculations

Position: S.12 - Semi-Precast Concrete Slab

System: Floor Plan with FE Mesh

\section{Demo Frilo}

Stuttgarter Straße 40

70469 Stuttgart

Tel.: 0711810020

Email: info@frilo.de
Prj.Nr.:

Seite: 2

$1: 125$

26.07 .2018 


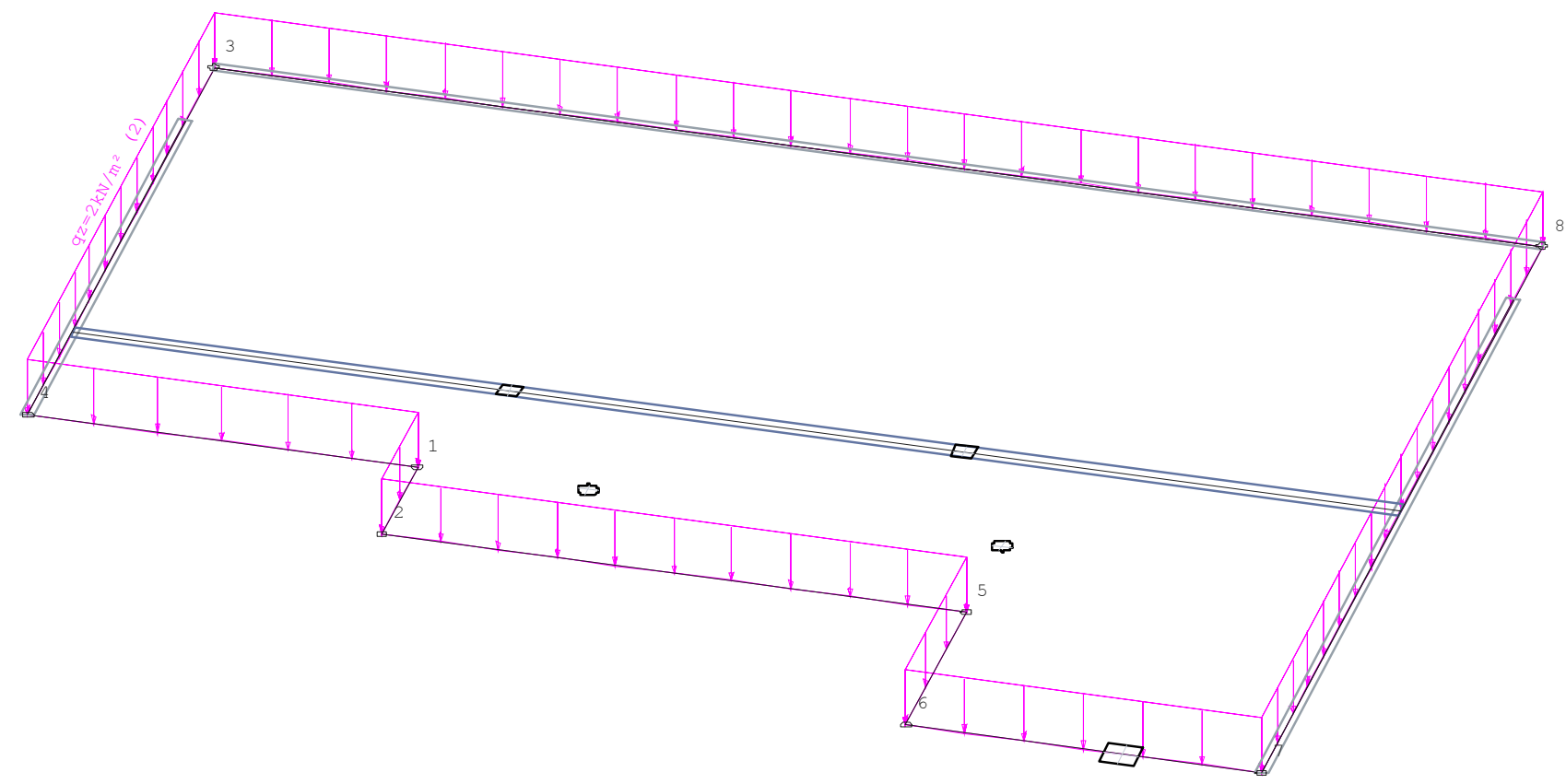

Projekt: Design Calculations

Position: S.12 - Semi-Precast Concrete Slab

Load case 1 "Self-Weight"

Area Loads

Demo Frilo

Stuttgarter Straße 40

70469 Stuttgart

Tel.: 0711810020

Email: info@frilo.de
Prj.Nr.:

Seite: 3

$1: 125$

26.07 .2018 


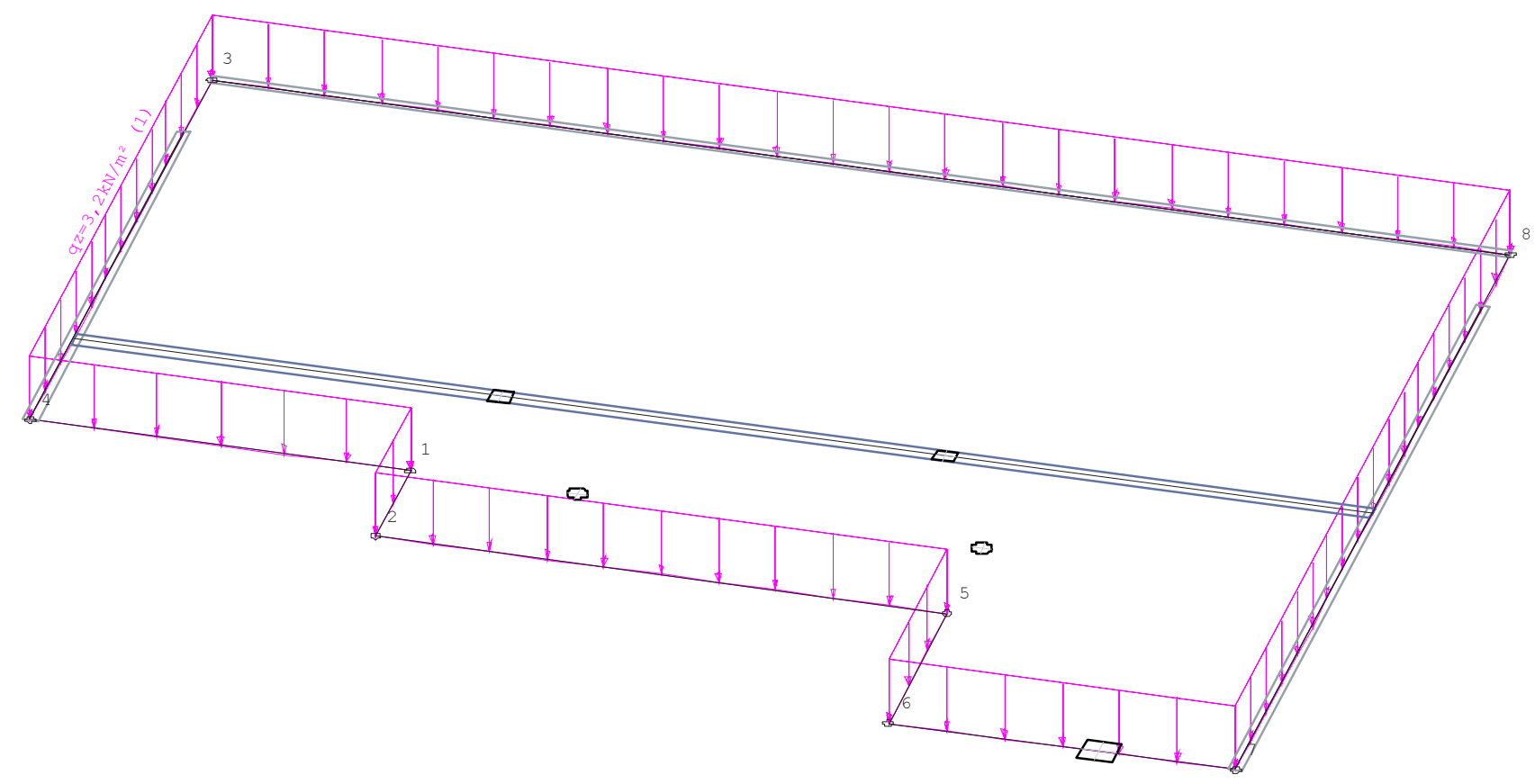

Projekt: Design Calculations

Position: S.12 - Semi-Precast Concrete Slab

Load case 2 "Live Load"

Area Loads

Demo Frilo

Stuttgarter Straße 40

70469 Stuttgart

Tel.: 0711810020

Email: info@frilo.de
Prj.Nr.:

Seite: 4

$1: 125$

26.07 .2018 

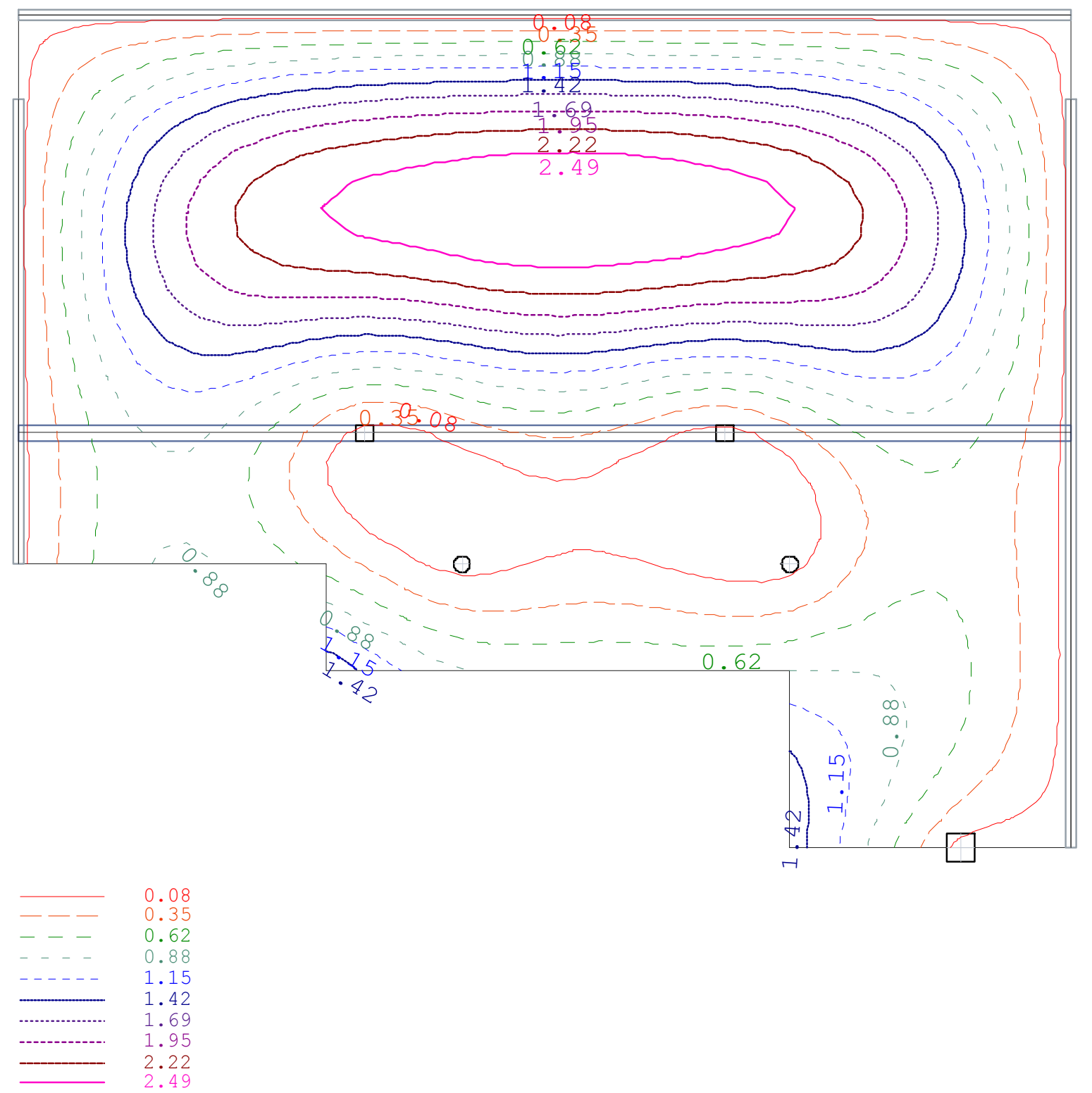

Projekt: Design Calculations

Position: S.12 - Semi-Precast Concrete Slab

Superposition 1 "Characteristic"

Deflection [mm] MAX

\section{Demo Frilo}

Stuttgarter Straße 40

70469 Stuttgart

Tel.: 0711810020

Email: info@frilo.de
Prj.Nr.:

Seite: 5

$1: 125$

26.07.2018 

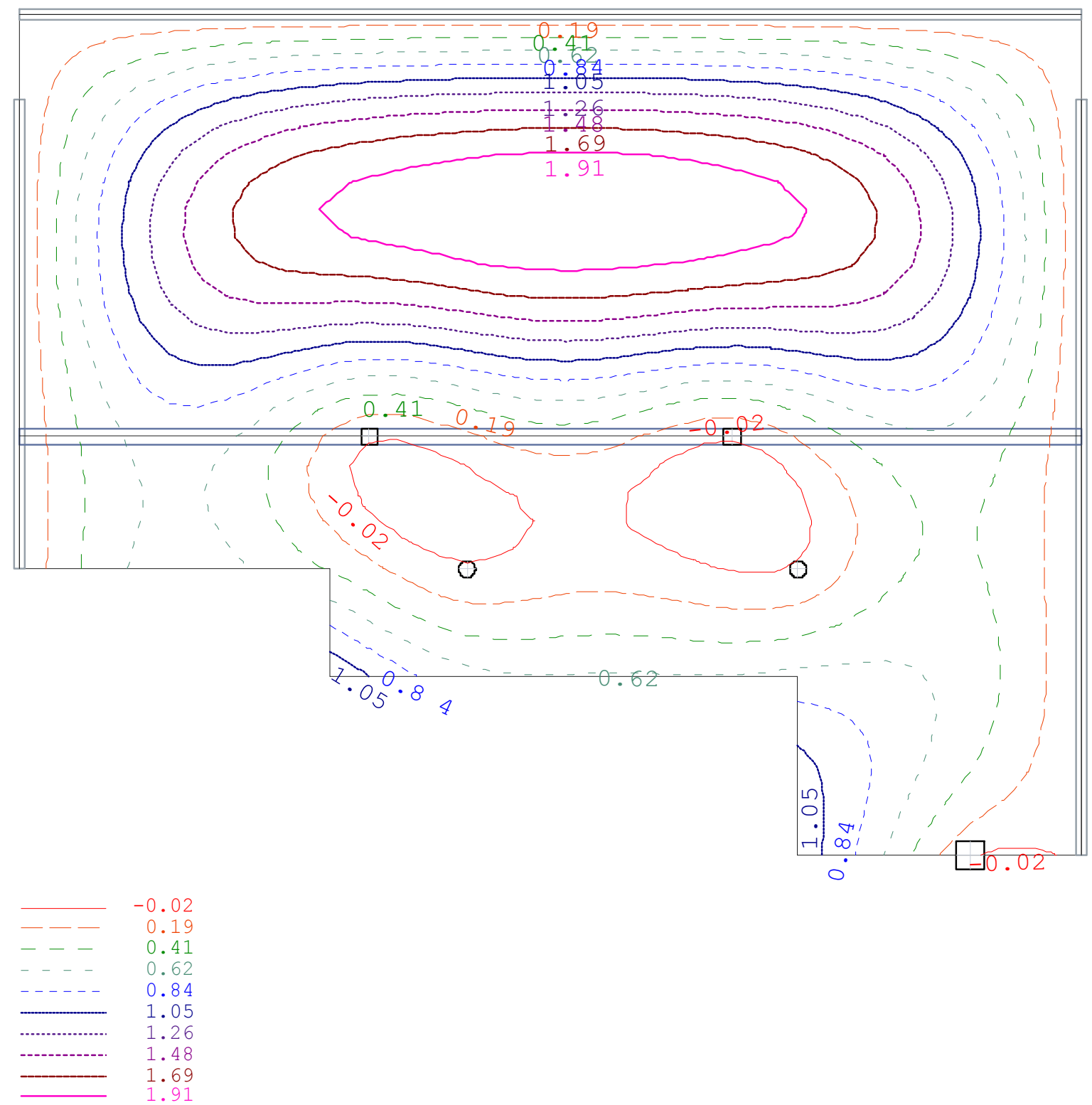

Projekt: Design Calculations

Position: S.12 - Semi-Precast Concrete Slab

Superposition 1 "Characteristic"

Deflection [mm] MIN

\section{Demo Frilo}

Stuttgarter Straße 40

70469 Stuttgart

Tel.: 0711810020

Email: info@frilo.de
Prj.Nr.:

Seite: 6

$1: 125$

26.07.2018 
Beam B1: Deflection [mm]

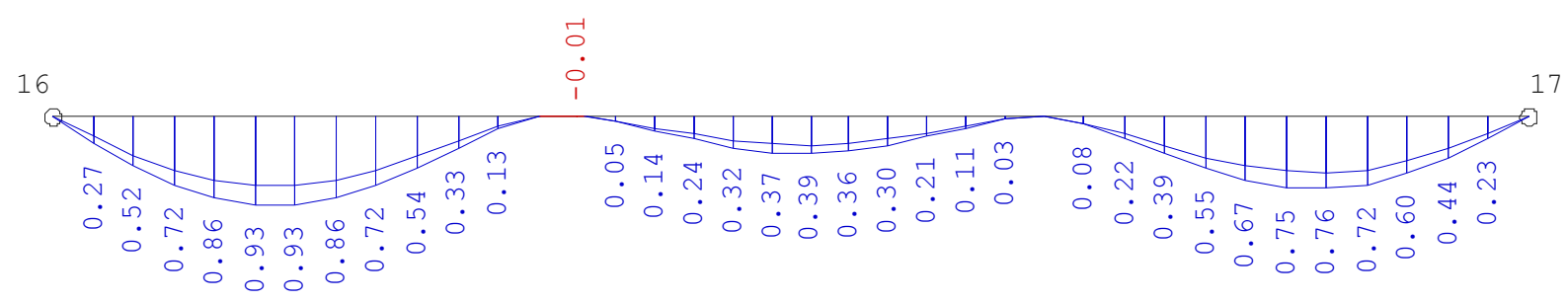

Projekt: Design Calculations

Position: S.12 - Semi-Precast Concrete Slab

Superposition 1 "Characteristic"

Beam B1

\section{Demo Frilo}

Stuttgarter Straße 40

70469 Stuttgart

Tel.: 0711810020

Email: info@frilo.de
Prj.Nr.:

Seite: 7

$1: 125$

26.07.2018 


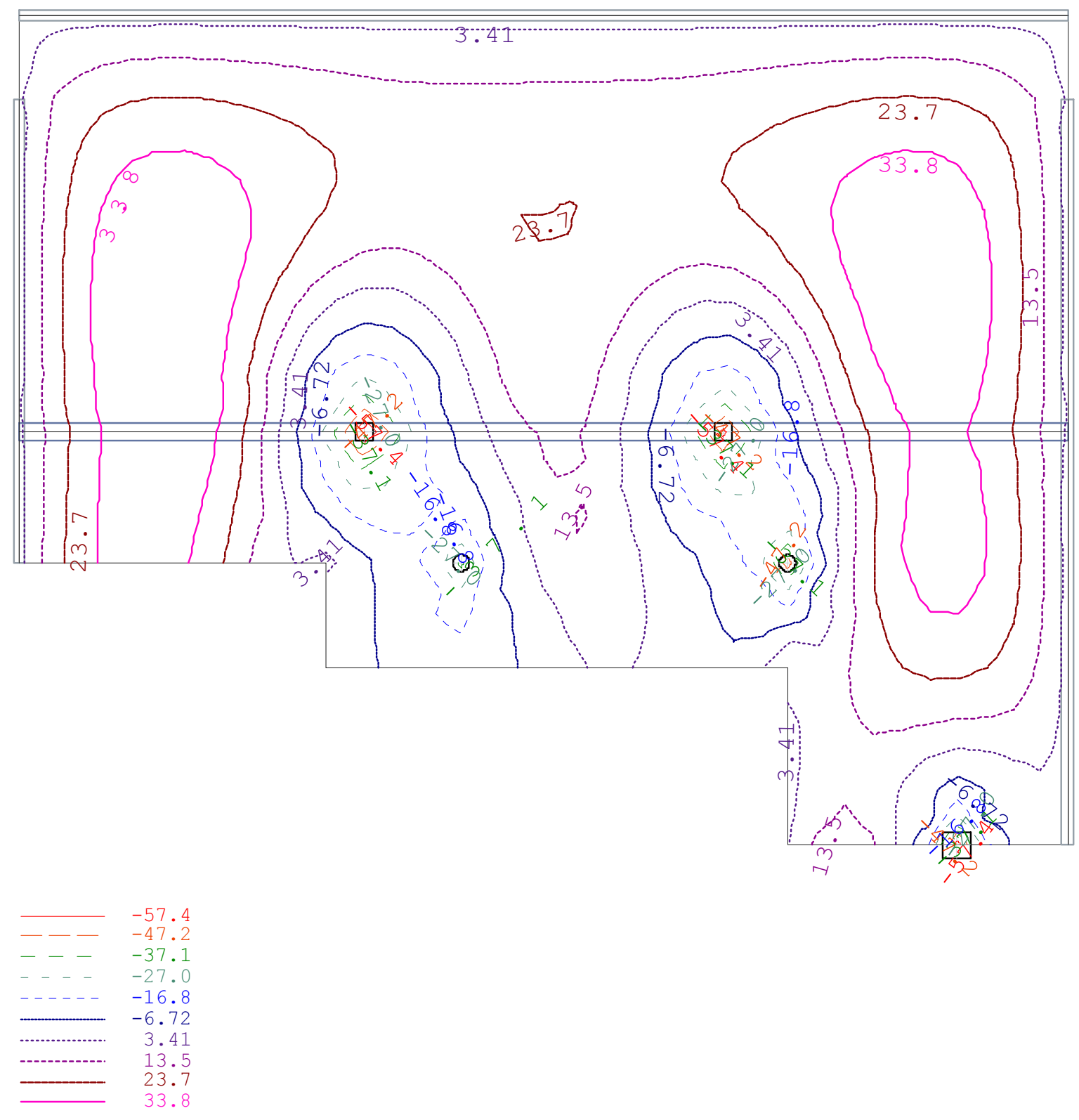

Projekt: Design Calculations

Position: S.12 - Semi-Precast Concrete Slab

Superposition 2 "USL Permanent/Transient"

Moment m-1 [kNm/m] MAX

\section{Demo Frilo}

Stuttgarter Straße 40

70469 Stuttgart

Tel.: 0711810020

Email: info@frilo.de
Prj.Nr.:

Seite: 8

$1: 125$

26.07.2018 


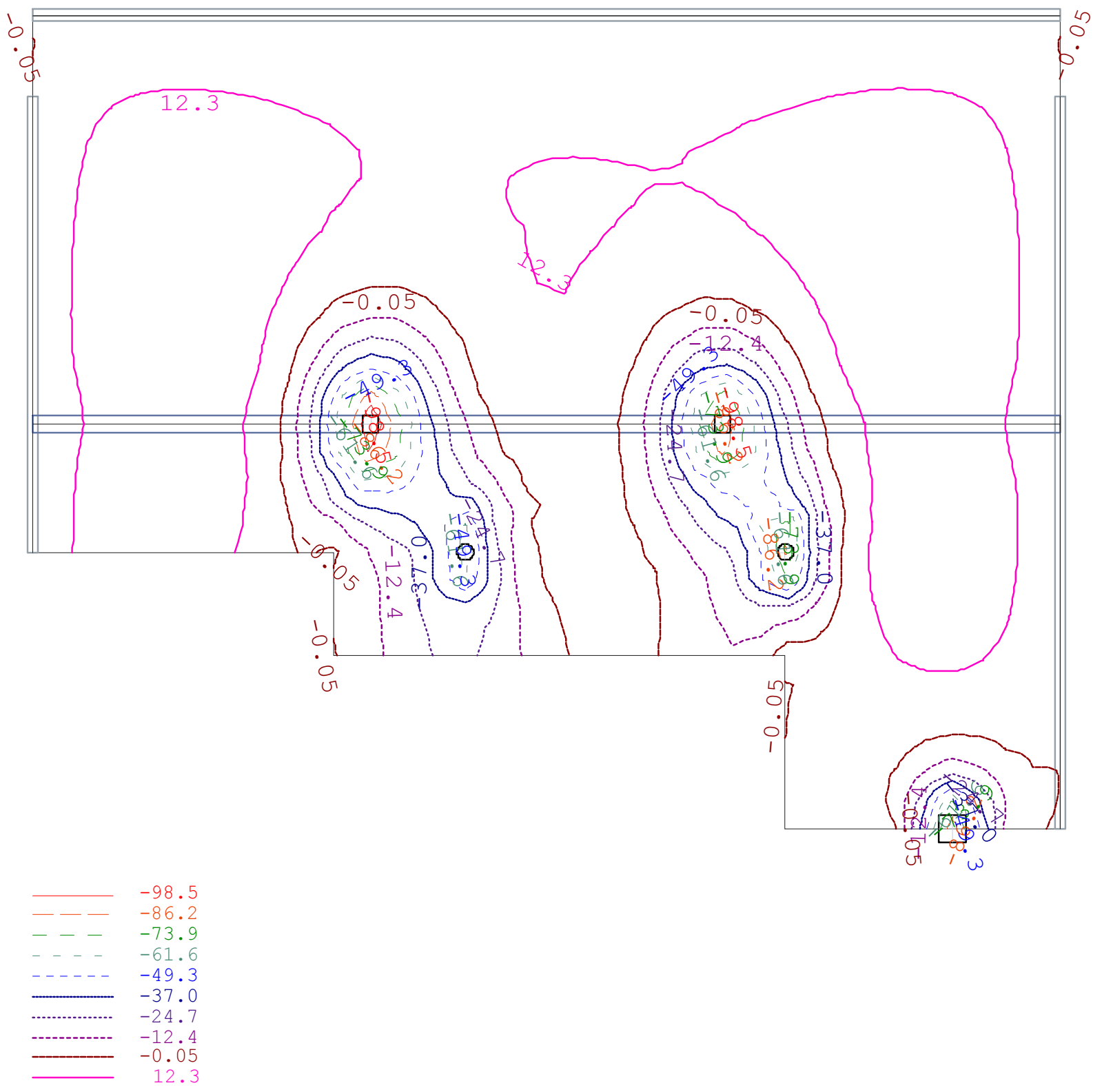

Projekt: Design Calculations

Position: S.12 - Semi-Precast Concrete Slab

Superposition 2 "USL Permanent/Transient"

Moment m-1 [kNm/m] MIN

Demo Frilo

Stuttgarter Straße 40

70469 Stuttgart

Tel.: 0711810020

Email: info@frilo.de
Prj.Nr.:

Seite: 9

$1: 125$

26.07 .2018 

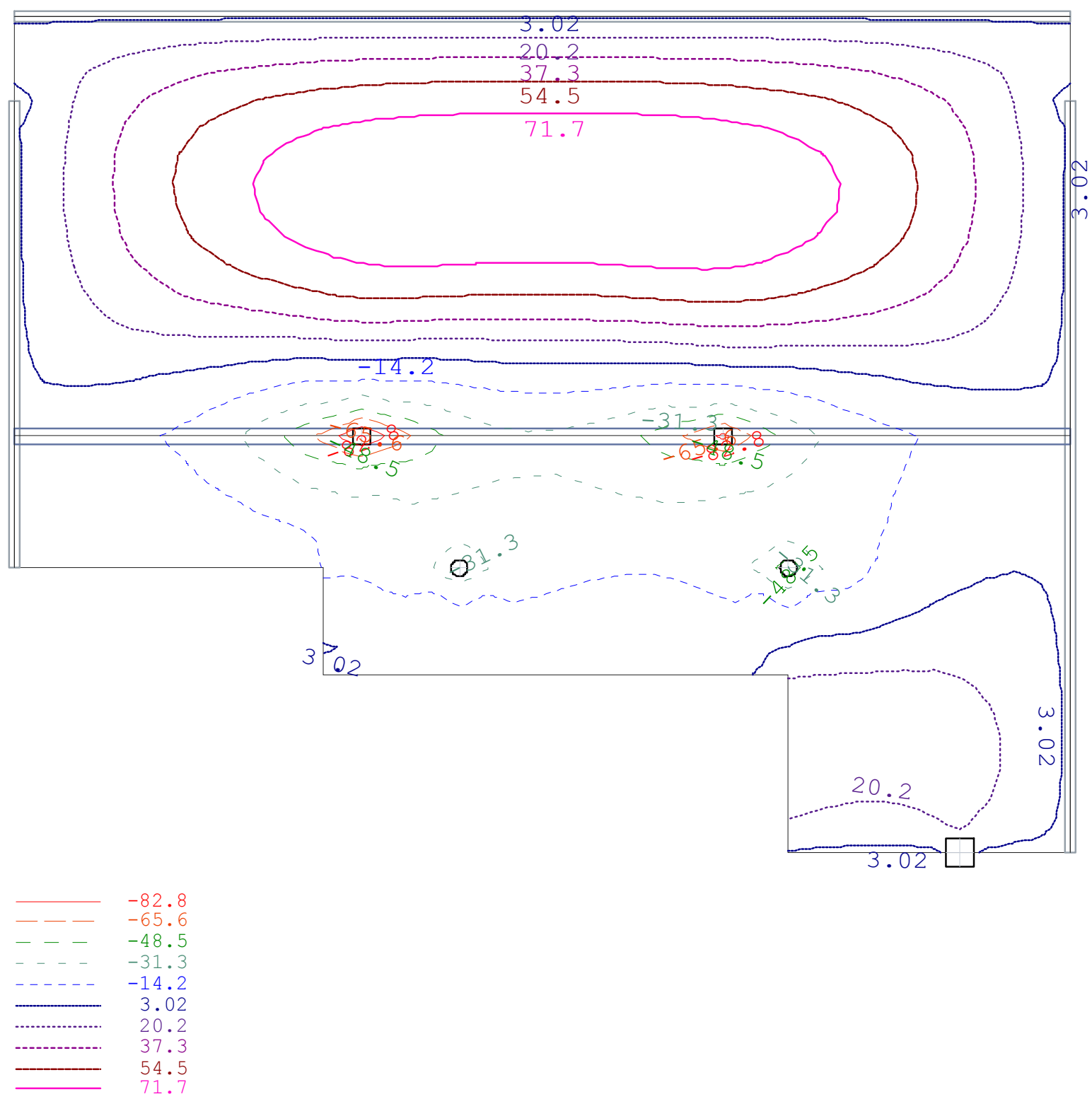

Projekt: Design Calculations

Position: S.12 - Semi-Precast Concrete Slab

Superposition 2 "USL Permanent/Transient"

Moment m-2 [kNm/m] MAX

\section{Demo Frilo}

Stuttgarter Straße 40

70469 Stuttgart

Tel.: 0711810020

Email: info@frilo.de

\begin{tabular}{|l|}
\hline \begin{tabular}{l} 
Prj.Nr.: \\
Seite: 10 \\
\hline $1: 125$ \\
26.07 .2018 \\
\end{tabular} \\
\\
\\
\end{tabular}



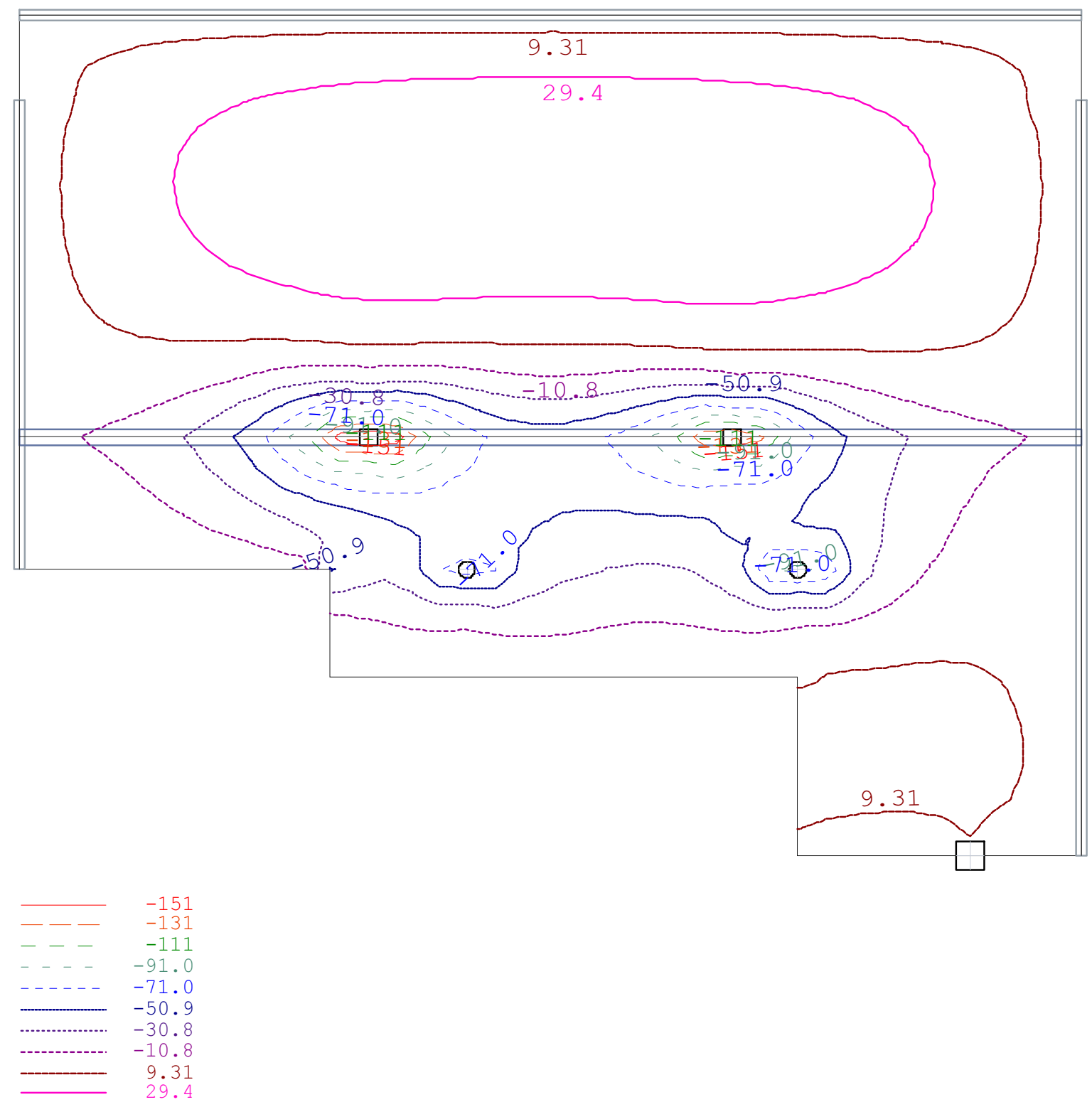

Projekt: Design Calculations

Position: S.12 - Semi-Precast Concrete Slab

Superposition 2 "USL Permanent/Transient"

Moment m-2 [kNm/m] MIN

Demo Frilo

Stuttgarter Straße 40

70469 Stuttgart

Tel.: 0711810020

Email: info@frilo.de
Prj.Nr.:

Seite: 11

$1: 125$

26.07 .2018 


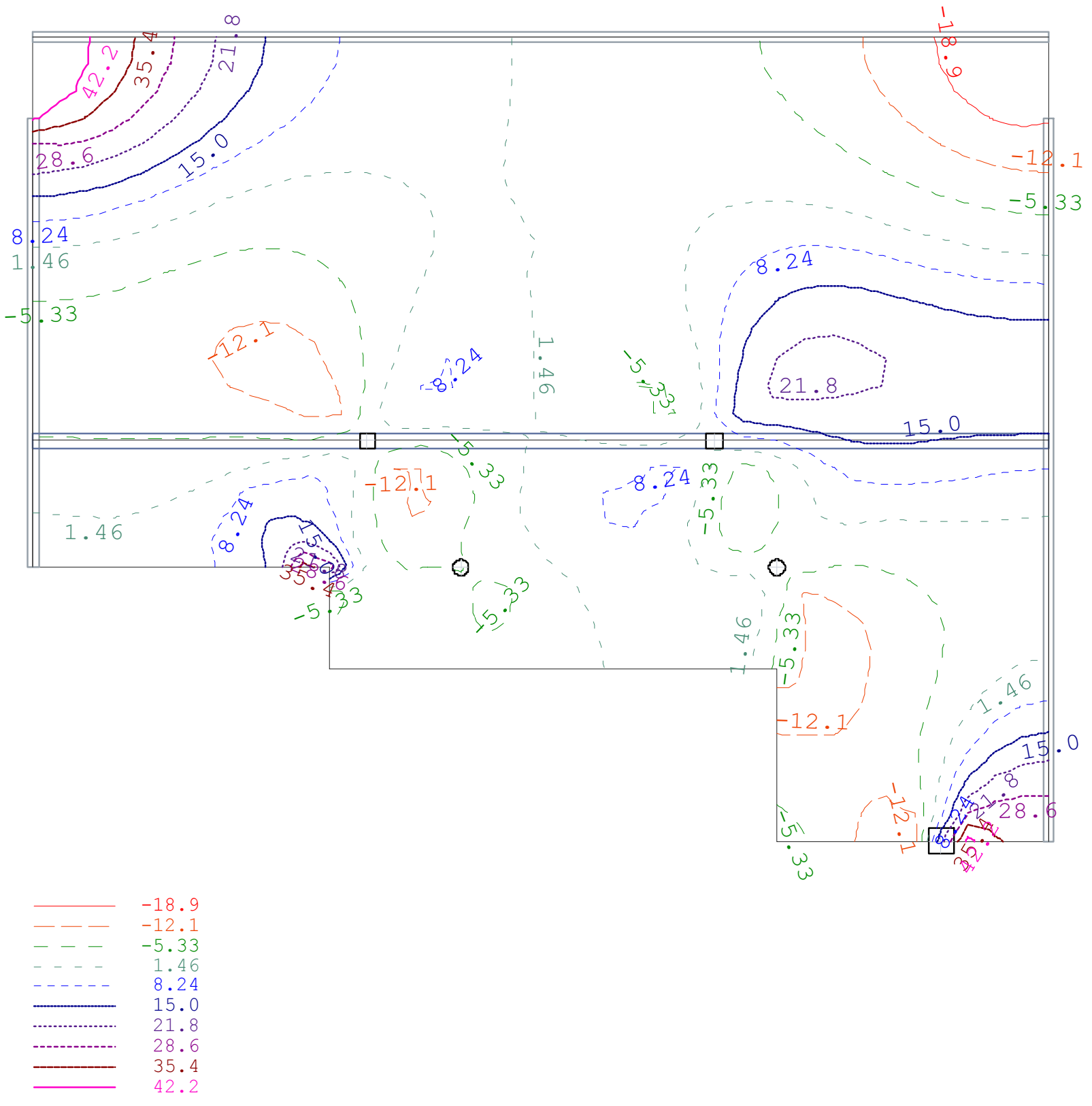

Projekt: Design Calculations

Position: S.12 - Semi-Precast Concrete Slab

Superposition 2 "USL Permanent/Transient"

Moment m-12 [kNm/m] MAX

\section{Demo Frilo}

Stuttgarter Straße 40

70469 Stuttgart

Tel.: 0711810020

Email: info@frilo.de
Prj.Nr.:

Seite: 12

$1: 125$

26.07.2018 


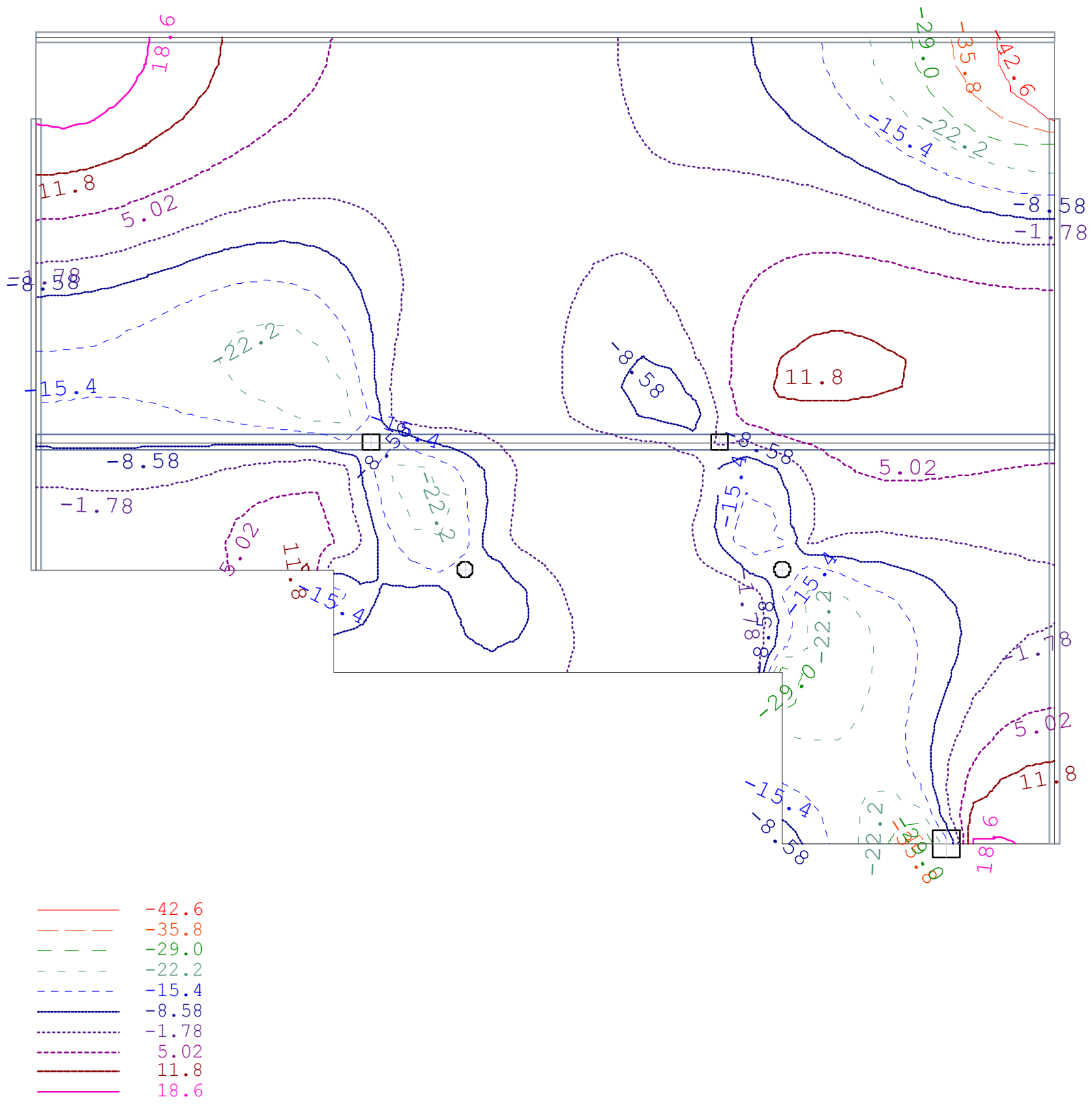

Projekt: Design Calculations

Position: S.12 - Semi-Precast Concrete Slab

Superposition 2 "USL Permanent/Transient"

Moment m-12 [kNm/m] MIN

\section{Demo Frilo}

Stuttgarter Straße 40

70469 Stuttgart

Tel.: 0711810020

Email: info@frilo.de
Prj.Nr.:

Seite: 13

$1: 125$

26.07.2018 

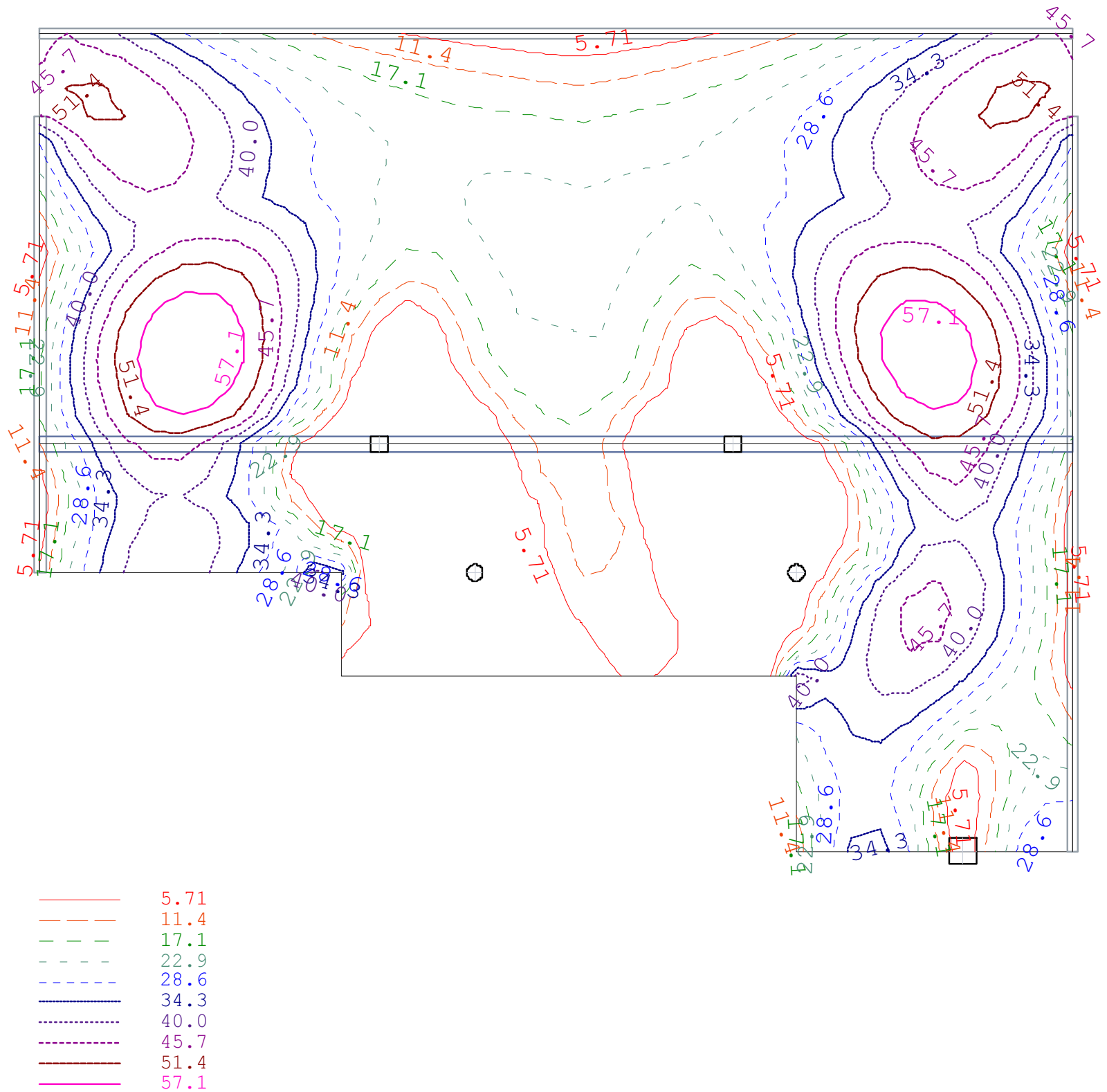

Projekt: Design Calculations

Position: S.12 - Semi-Precast Concrete Slab

Superposition 2 "USL Permanent/Transient"

Design Moment, bottom mB-1 [kNm/m]

\section{Demo Frilo}

Stuttgarter Straße 40

70469 Stuttgart

Tel.: 0711810020

Email: info@frilo.de

\begin{tabular}{|l|}
\hline \begin{tabular}{l} 
Prj.Nr.: \\
Seite: 14 \\
\hline $1: 125$ \\
26.07 .2018 \\
\end{tabular} \\
\\
\\
\end{tabular}



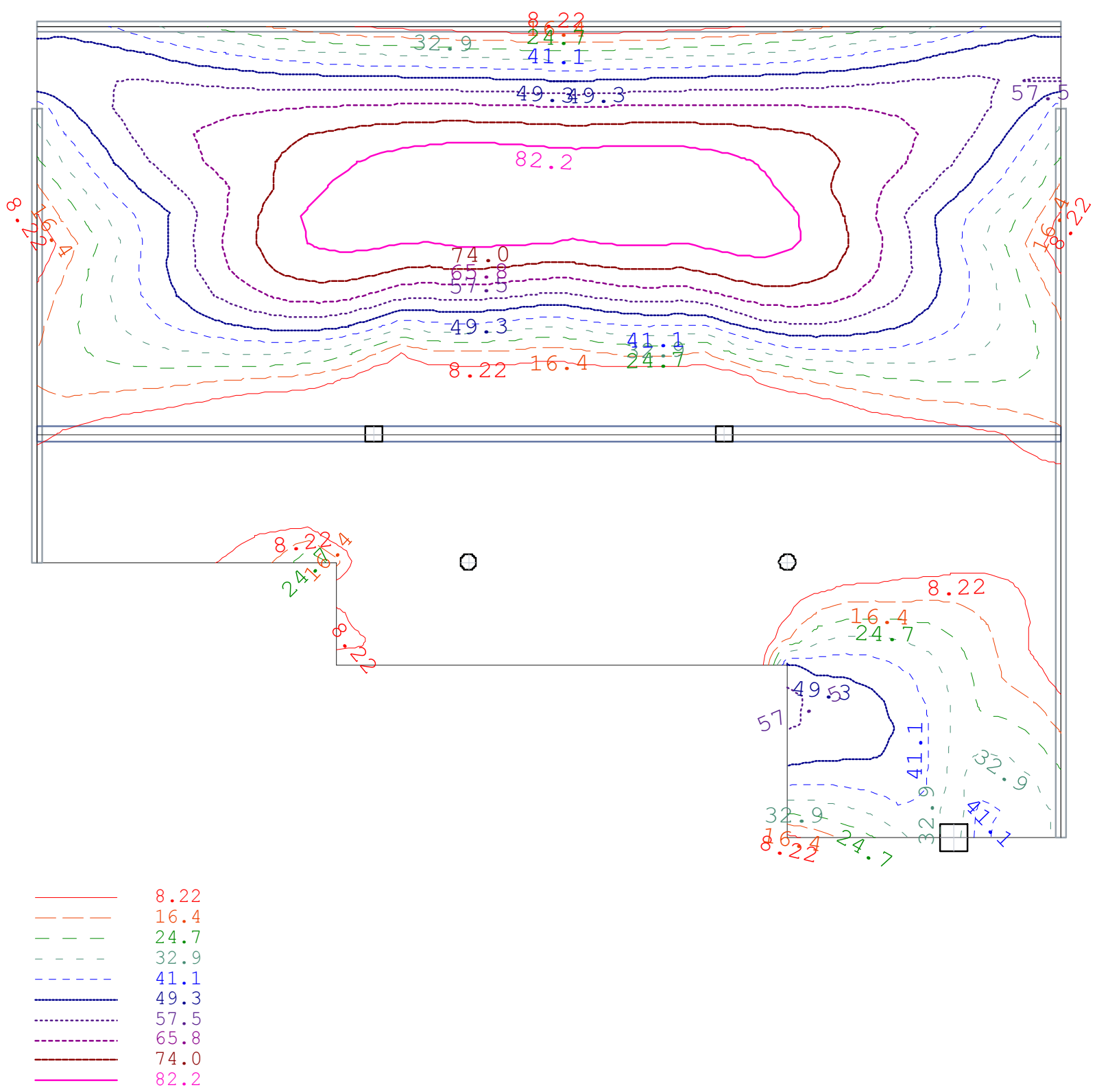

Projekt: Design Calculations

Position: S.12 - Semi-Precast Concrete Slab

Superposition 2 "USL Permanent/Transient"

Design Moment, bottom mB-2 [kNm/m]

\section{Demo Frilo}

Stuttgarter Straße 40

70469 Stuttgart

Tel.: 0711810020

Email: info@frilo.de
Prj.Nr.:

Seite: 15

$1: 125$

26.07 .2018 


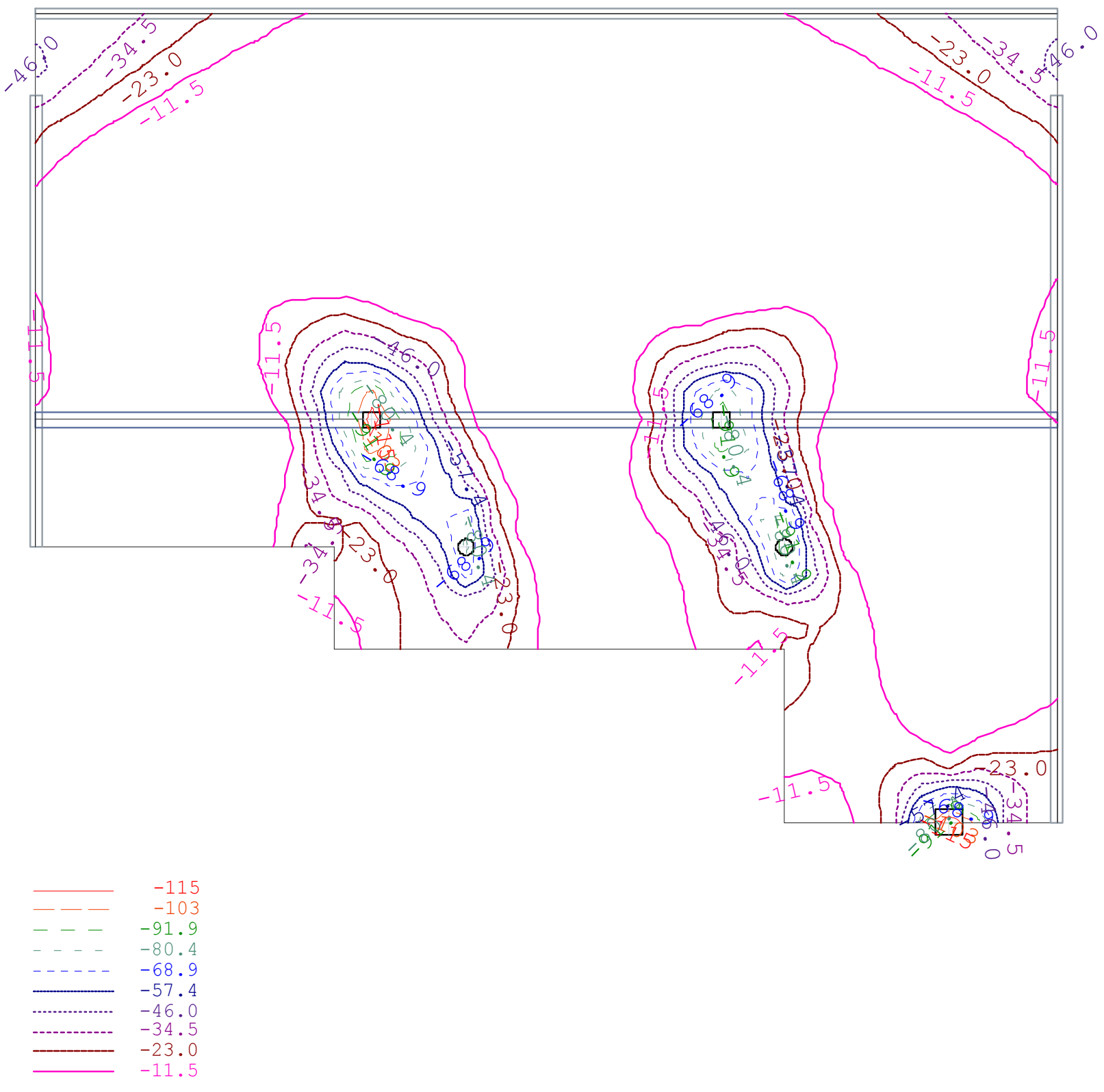

Projekt: Design Calculations

Position: S.12 - Semi-Precast Concrete Slab

Superposition 2 "USL Permanent/Transient"

Design Moment, top mB-1 [kNm/m]

\section{Demo Frilo}

Stuttgarter Straße 40

70469 Stuttgart

Tel.: 0711810020

Email: info@frilo.de
Prj.Nr.:

Seite: 16

$1: 125$

26.07 .2018 


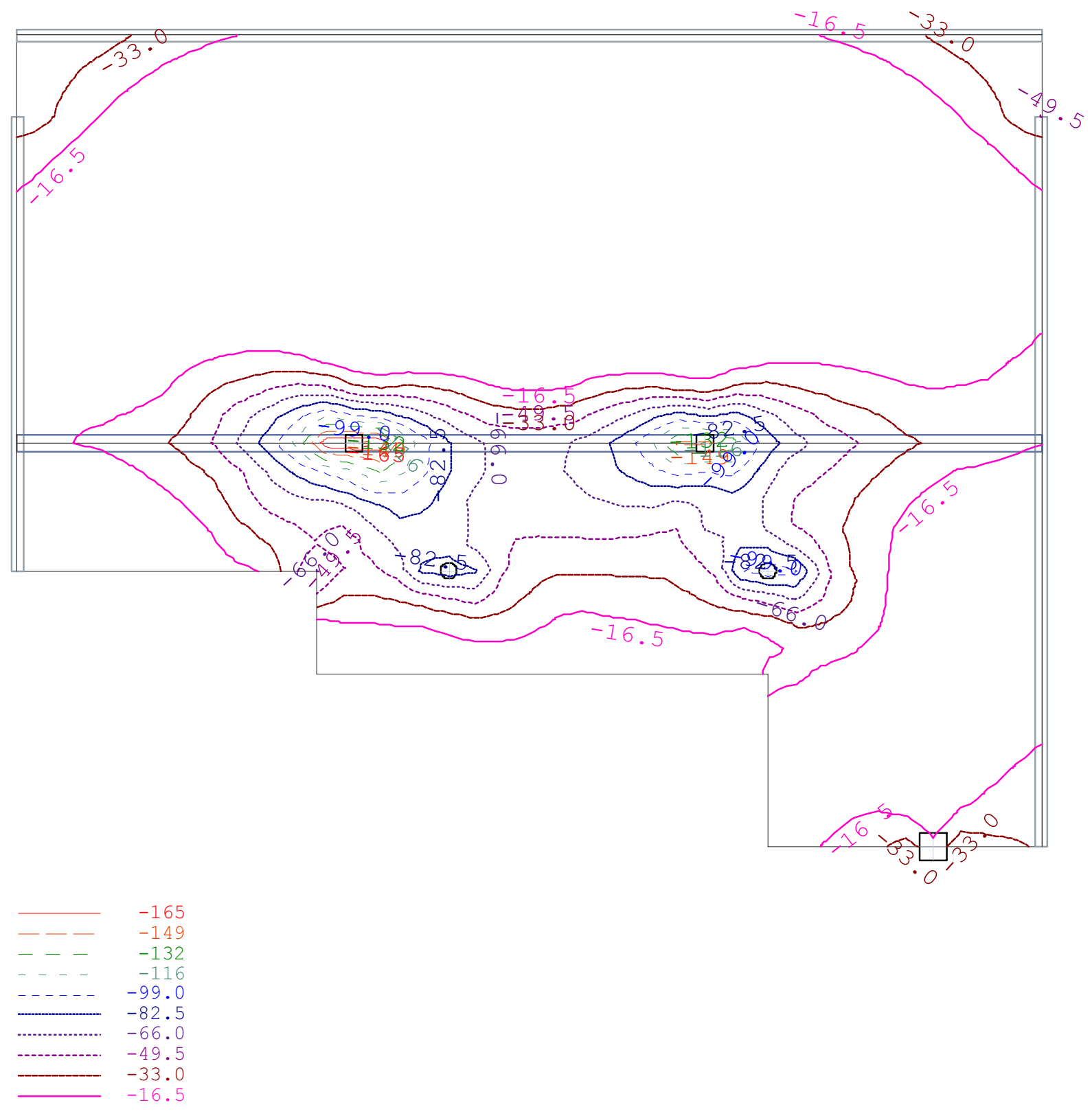

Projekt: Design Calculations

Position: S.12 - Semi-Precast Concrete Slab

Superposition 2 "USL Permanent/Transient"

Design Moment, top mB-2 [kNm/m]

\section{Demo Frilo}

Stuttgarter Straße 40

70469 Stuttgart

Tel.: 0711810020

Email: info@frilo.de

\begin{tabular}{|l|}
\hline $\begin{array}{l}\text { Prj.Nr.: } \\
\text { Seite: } 17\end{array}$ \\
\hline $1: 125$ \\
26.07 .2018 \\
\end{tabular}




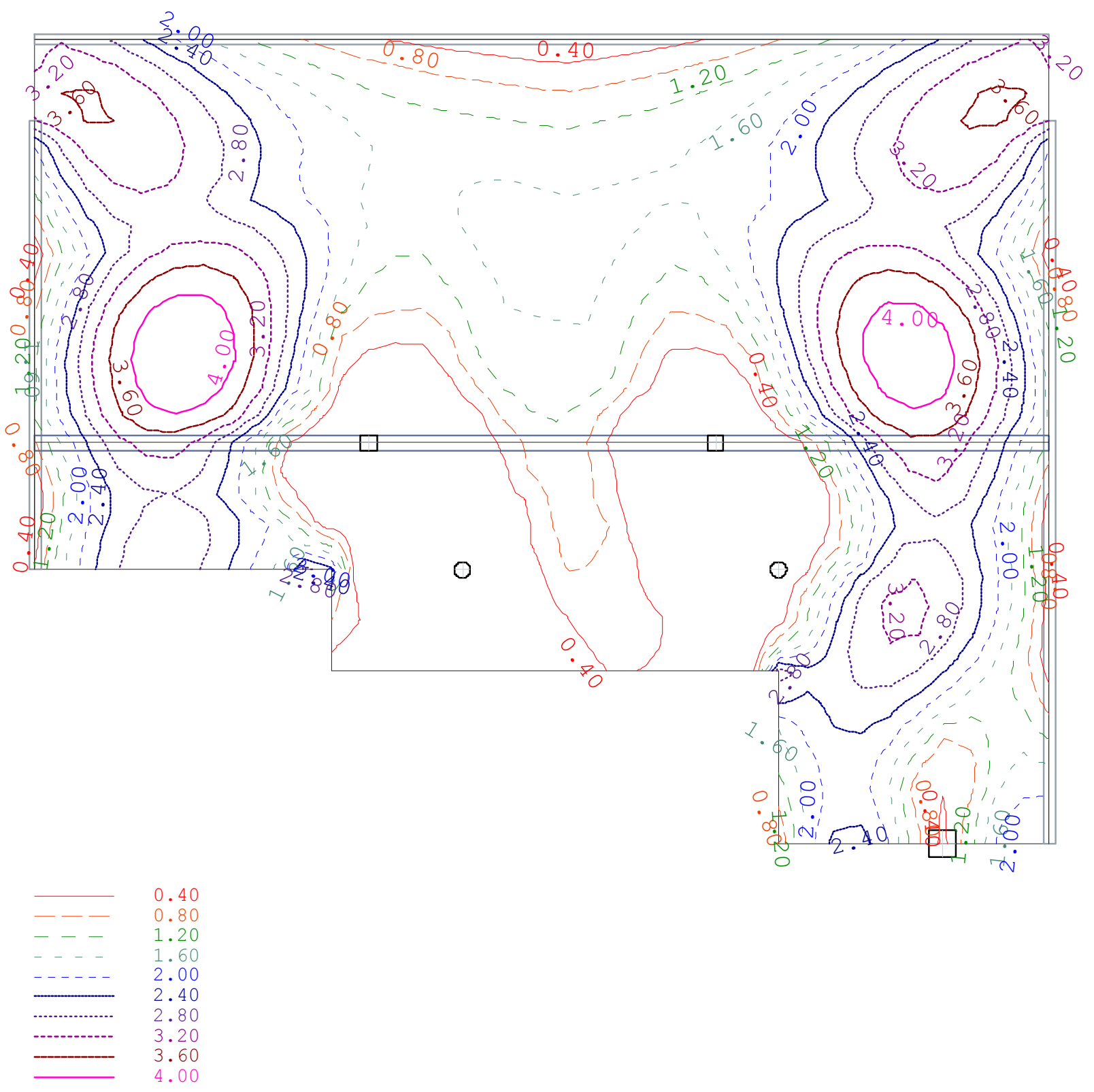

Projekt: Design Calculations

Position: S.12 - Semi-Precast Concrete Slab

Superposition 2 "USL Permanent/Transient"

Reinforcement, bottom aS-1 $\left[\mathrm{cm}^{2} / \mathrm{m}\right]$

\section{Demo Frilo}

Stuttgarter Straße 40

70469 Stuttgart

Tel.: 0711810020

Email: info@frilo.de
Prj.Nr.:

Seite: 18

$1: 125$

26.07.2018 

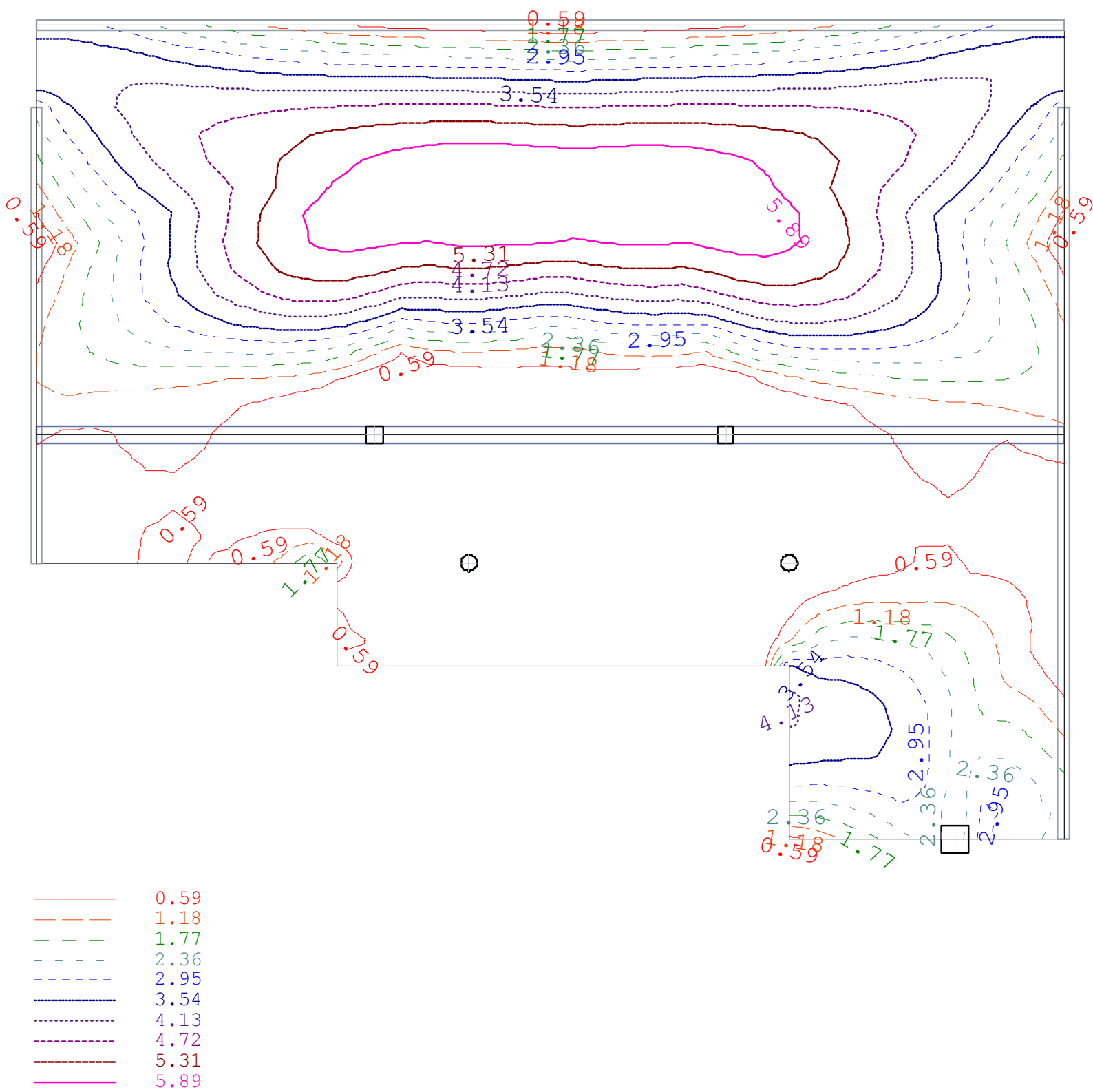

Projekt: Design Calculations

Position: S.12 - Semi-Precast Concrete Slab

Superposition 2 "USL Permanent/Transient"

Reinforcement, bottom aS-2 $\left[\mathrm{cm}^{2} / \mathrm{m}\right]$

\section{Demo Frilo}

Stuttgarter Straße 40

70469 Stuttgart

Tel.: 0711810020

Email: info@frilo.de
Prj.Nr.:

Seite: 19

$1: 125$

26.07.2018 

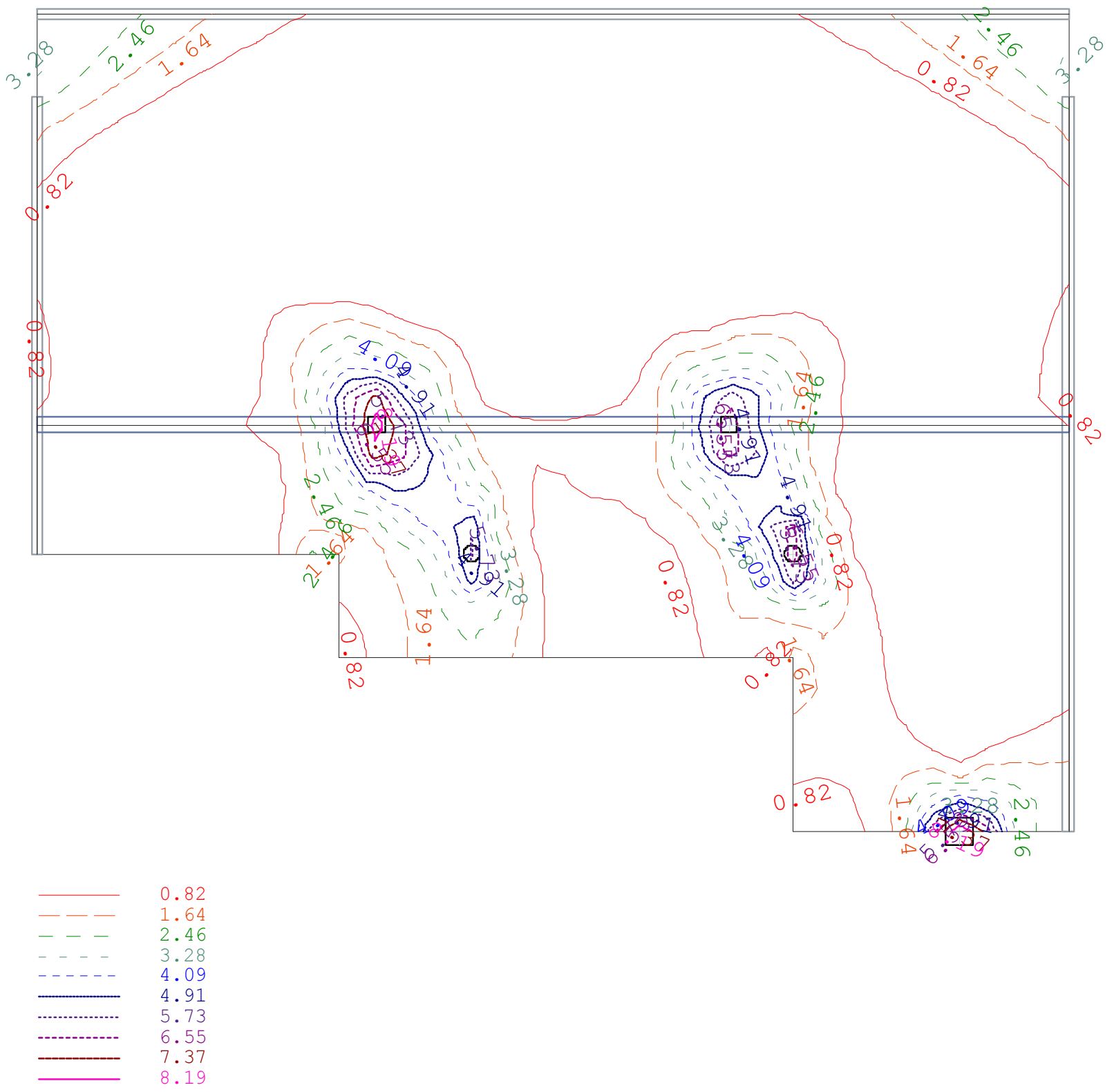

Projekt: Design Calculations

Position: S.12 - Semi-Precast Concrete Slab

Superposition 2 "USL Permanent/Transient"

Reinforcement, top aS-1 $\left[\mathrm{cm}^{2} / \mathrm{m}\right]$

Demo Frilo

Stuttgarter Straße 40

70469 Stuttgart

Tel.: 0711810020

Email: info@frilo.de
Prj.Nr.:

Seite: 20

$1: 125$

26.07.2018 

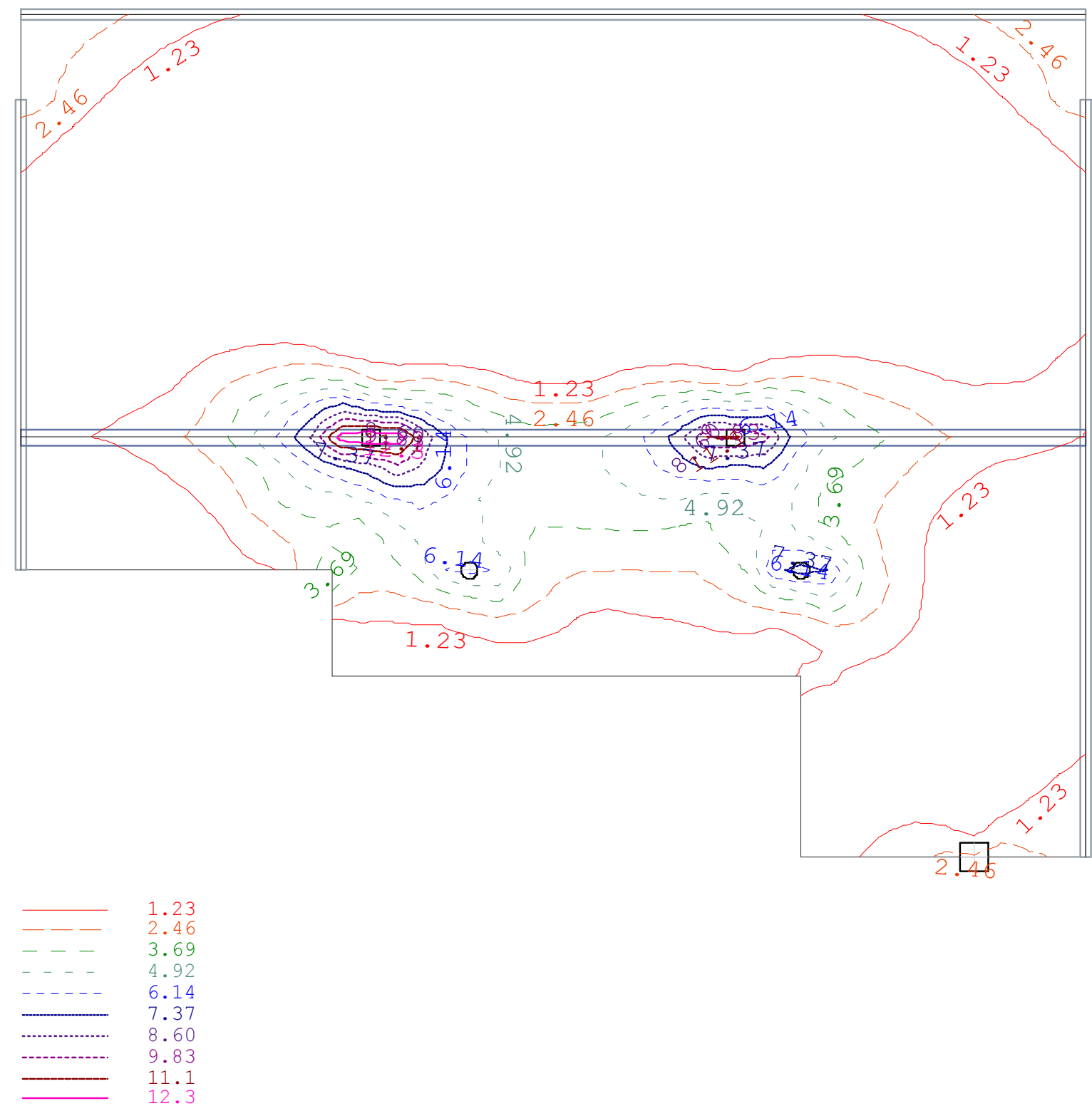

Projekt: Design Calculations

Position: S.12 - Semi-Precast Concrete Slab

Superposition 2 "USL Permanent/Transient"

Reinforcement, top aS-2 $\left[\mathrm{cm}^{2} / \mathrm{m}\right]$

Demo Frilo

Stuttgarter Straße 40

70469 Stuttgart

Tel.: 0711810020

Email: info@frilo.de
Prj.Nr.:

Seite: 21

$1: 125$

26.07 .2018 


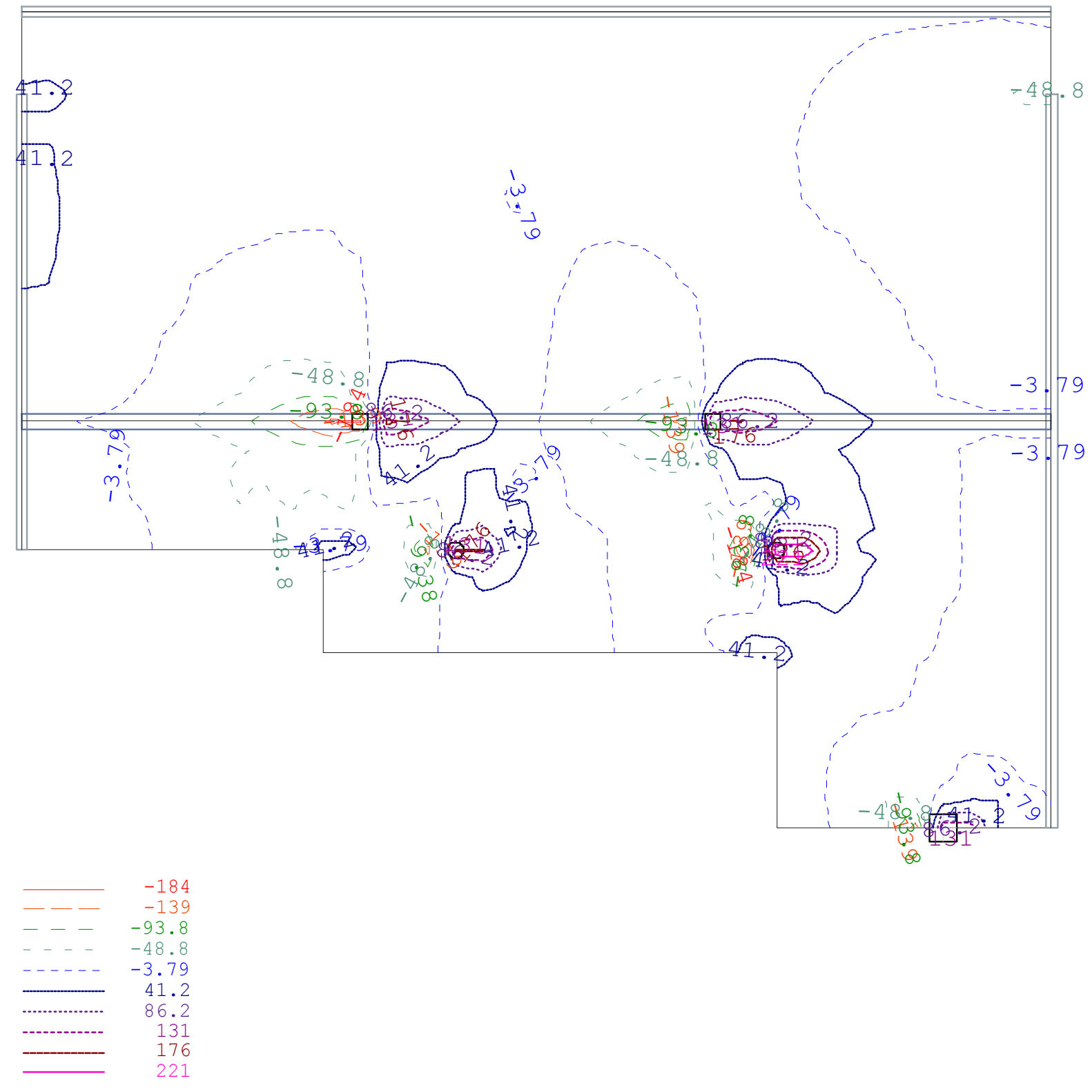

Projekt: Design Calculations

Position: S.12 - Semi-Precast Concrete Slab

Superposition 2 "USL Permanent/Transient"

Shear Force q-1z [kN/m]

\section{Demo Frilo}

Stuttgarter Straße 40

70469 Stuttgart

Tel.: 0711810020

Email: info@frilo.de
Prj.Nr.:

Seite: 22

$1: 125$

26.07.2018 

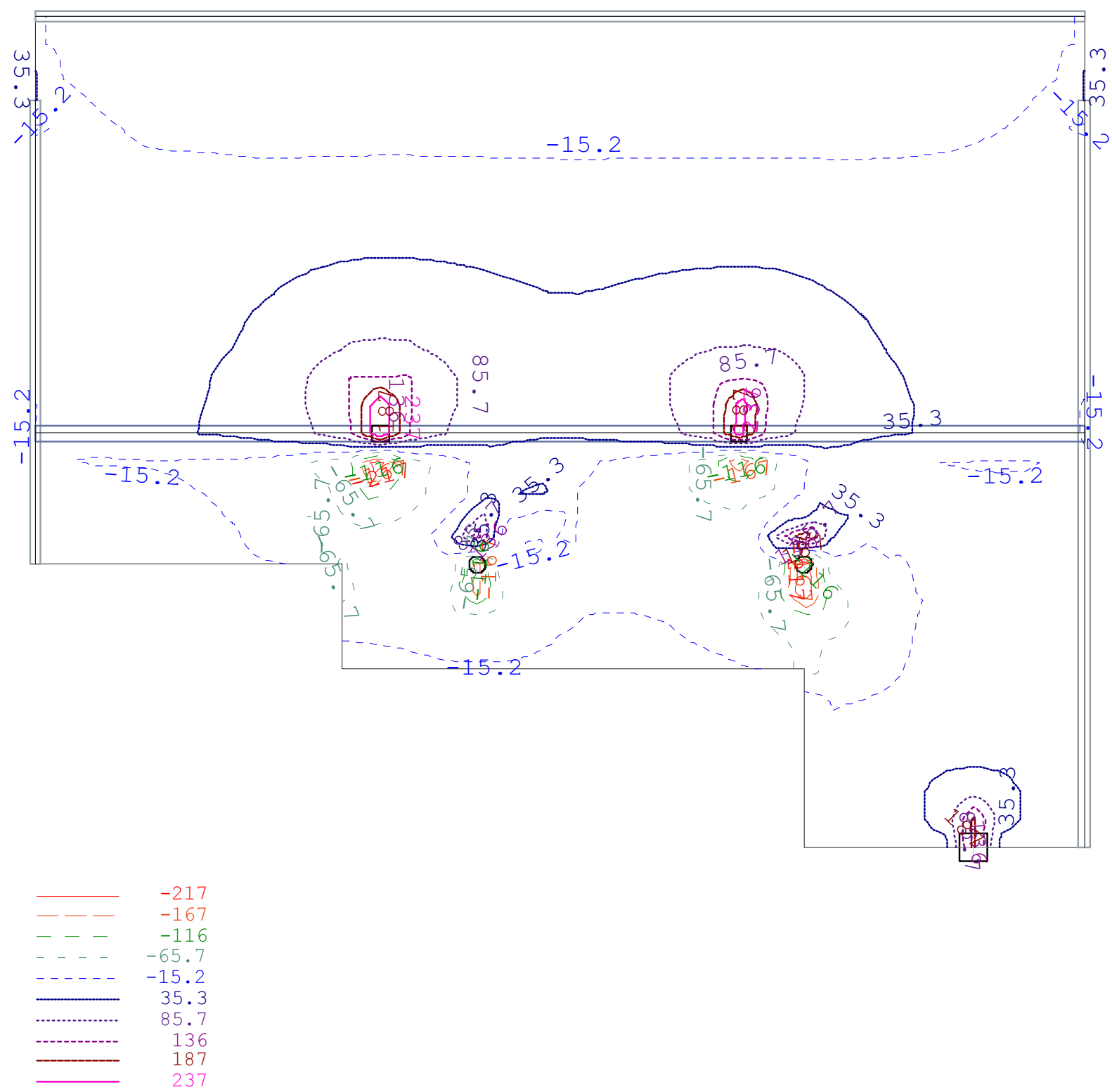

Projekt: Design Calculations

Position: S.12 - Semi-Precast Concrete Slab

Superposition 2 "USL Permanent/Transient"

Shear Force $q-2 z[k N / m]$

Demo Frilo

Stuttgarter Straße 40

70469 Stuttgart

Tel.: 0711810020

Email: info@frilo.de
Prj.Nr.:

Seite: 23

$1: 125$

26.07 .2018 


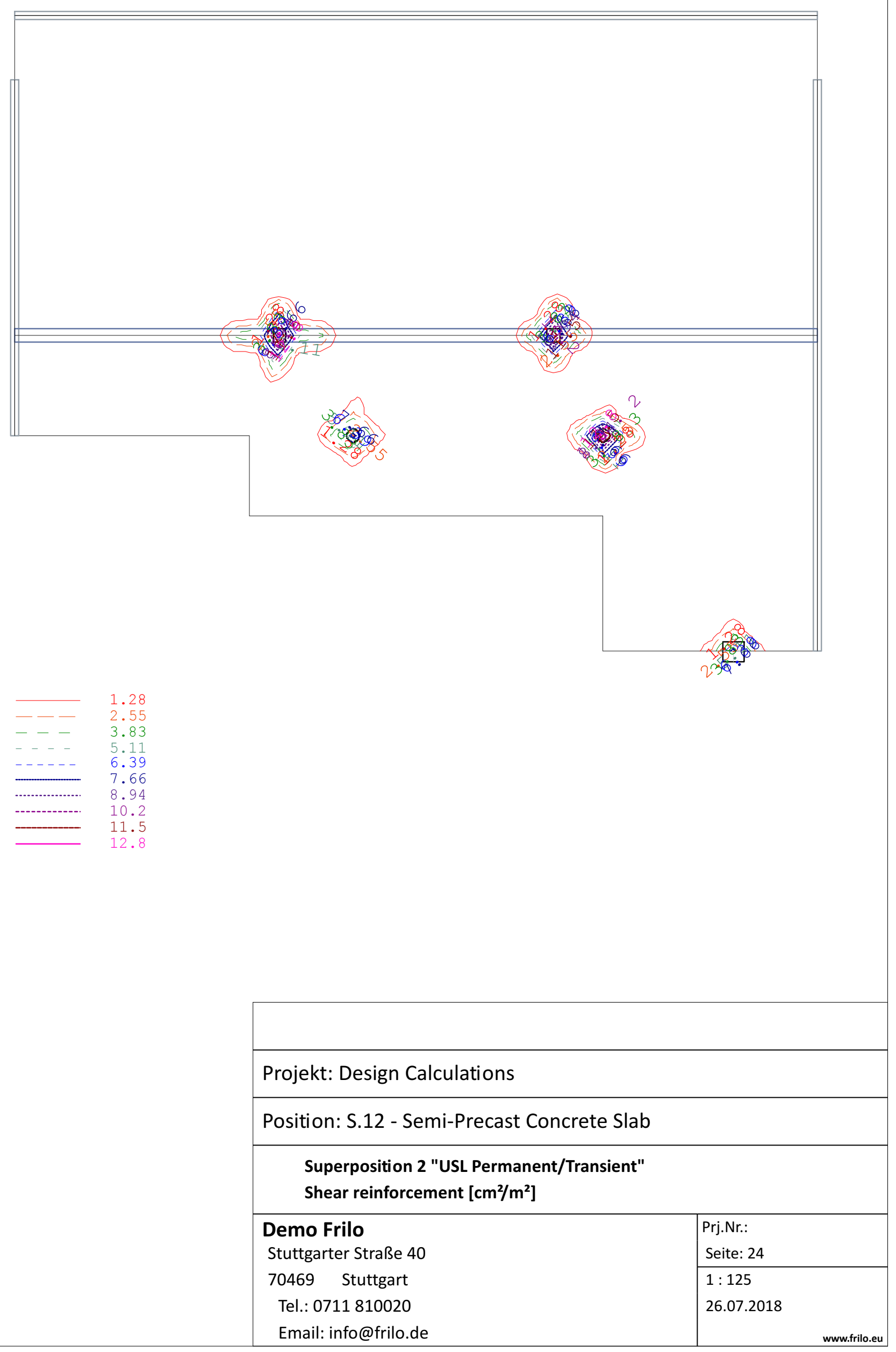


Beam B1: Bending Moment [ $\mathrm{kNm}]$

Design Values (x Gamma)

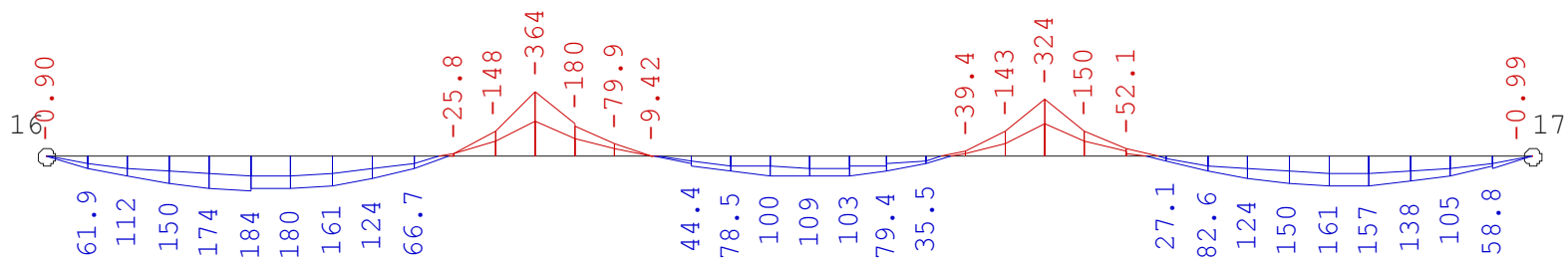

Beam B1: Flexural Reinforcement $\left[\mathrm{cm}^{2}\right]$

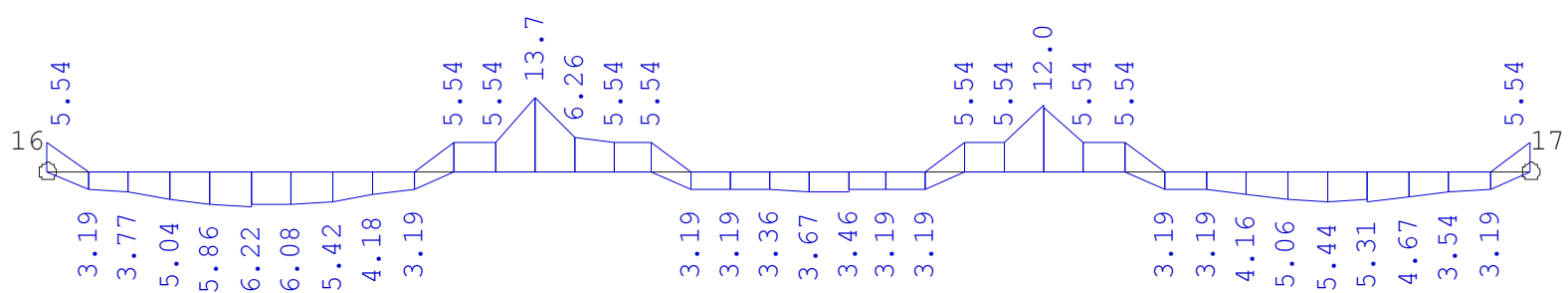

Beam B1: Shear Force $[\mathrm{kN}]$

Design Values ( $x$ Gamma)

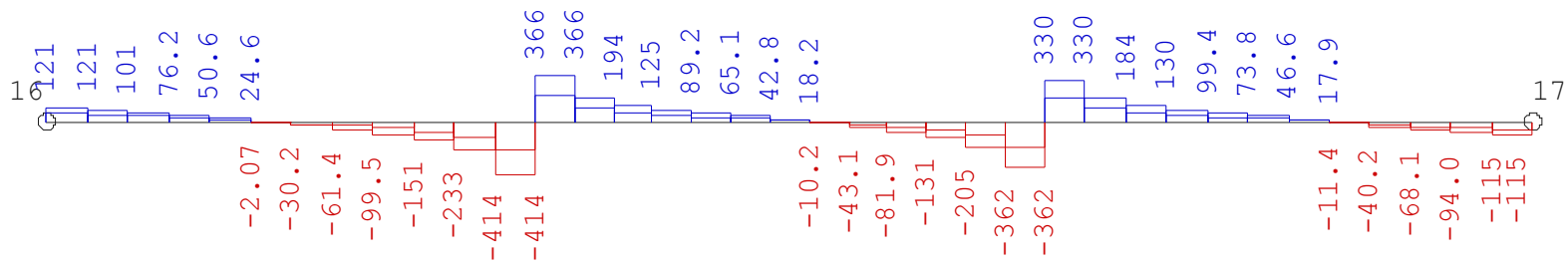

Beam B1: Shear reinforcement $\left[\mathrm{cm}^{2} / \mathrm{m}\right]$ due to shear force and torsion

$$
\begin{aligned}
& \circ \\
& \circ \\
& \circ
\end{aligned}
$$

ค.

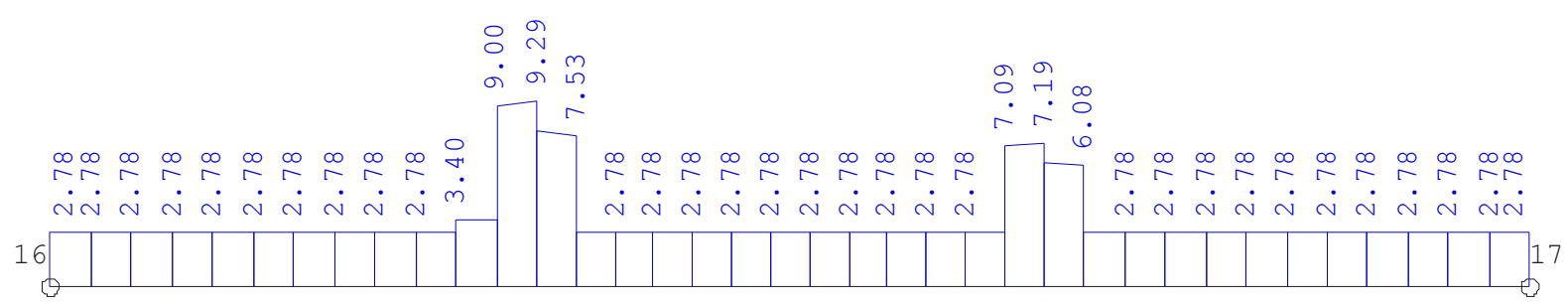

Projekt: Design Calculations

Position: S.12 - Semi-Precast Concrete Slab

Superposition 2 "USL Permanent/Transient"

Beam B1

\section{Demo Frilo}

Stuttgarter Straße 40

70469 Stuttgart

Tel.: 0711810020

Email: info@frilo.de
Prj.Nr.:

Seite: 25

$1: 125$

26.07.2018 
B.1.8. Design Calculations POS. B.13 - Semi-Precast Concrete Beam 
Demo Frilo

Stuttgarter Straße 40

70469 Stuttgart
Tel.: 0711810020

Fax: 0711858020
Projekt: Design Calculations

Position: B.13 - Semi-Precast Concrete Beam

26.07.2018
Seite: 1

\section{Position: B.13 - Semi-Precast Concrete Beam}

Durchlaufträger DLT10 01/2018 (Frilo R-2018-1/P12)

Scale $1: 125$

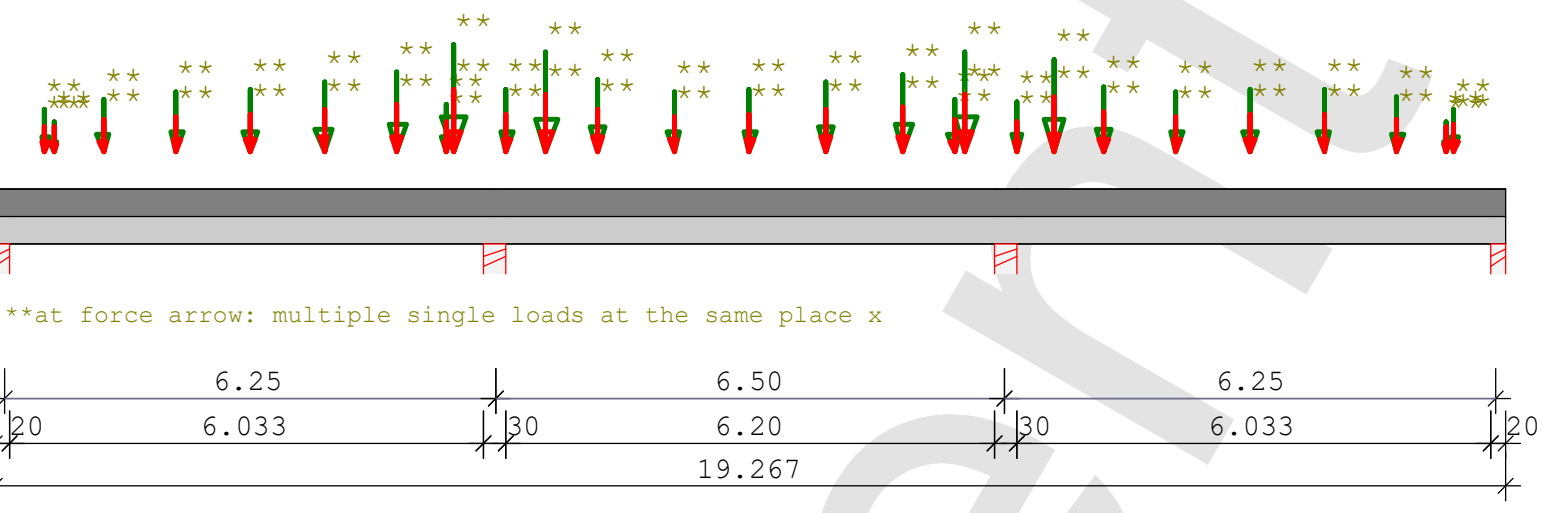

effective breadth and design

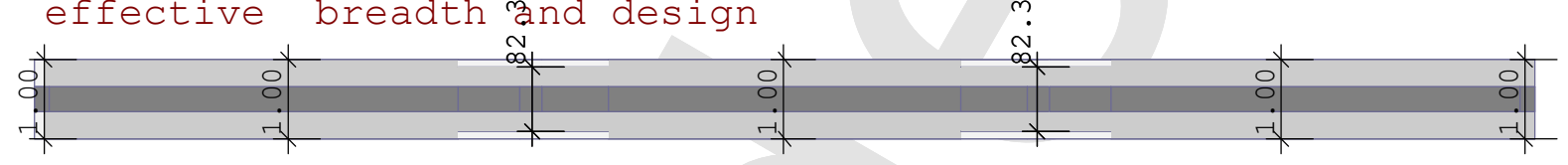

Reinforced concrete girder over 3 Spans C30/37 E $=33000 \mathrm{~N} / \mathrm{mm} 2$

DIN EN 1992-1-1/NA/A1:2015-12

Decke über NG (Normalgeschoss) von Gebäudemodell

\begin{tabular}{|cccccccccc|}
\hline System & length & \multicolumn{9}{c|}{ cross-section values } & & & \\
\hline Span & $\mathrm{I}(\mathrm{m})$ & & bt & ht & b0 & h0 & bb & hb \\
\hline 1 & 6.25 & constant & 100.0 & 35.0 & 30.0 & 70.0 & & \\
2 & 6.50 & constant & 100.0 & 35.0 & 30.0 & 70.0 & & \\
3 & 6.25 & constant & 100.0 & 35.0 & 30.0 & 70.0 & & \\
\hline
\end{tabular}

Cross-sections with eff. active breadth

\begin{tabular}{crccccccc|}
\hline $\begin{array}{c}\mathrm{x} \\
(\mathrm{m})\end{array}$ & $\begin{array}{c}\mathrm{bt} \\
(\mathrm{cm})\end{array}$ & $\begin{array}{c}\mathrm{ht} \\
(\mathrm{cm})\end{array}$ & $\begin{array}{c}\mathrm{b0} \\
(\mathrm{cm})\end{array}$ & $\begin{array}{c}\mathrm{h} 0 \\
(\mathrm{~cm})\end{array}$ & $\begin{array}{c}\mathrm{bb} \\
(\mathrm{cm})\end{array}$ & $\begin{array}{c}\mathrm{hb} \\
(\mathrm{cm})\end{array}$ & $\begin{array}{c}\text { Wyb } \\
(\mathrm{m} 3)\end{array}$ & $\begin{array}{c}\text { Wyt } \\
(\mathrm{m} 3)\end{array}$ \\
\hline 0.00 & 100.0 & 35.0 & 30.0 & 70.0 & & & 0.0327 & 0.0568 \\
5.31 & 100.0 & 35.0 & 30.0 & 70.0 & & 0.0327 & 0.0568 \\
5.31 & 82.3 & 35.0 & 30.0 & 70.0 & & 0.0311 & 0.0500 \\
6.25 & 82.3 & 35.0 & 30.0 & 70.0 & & 0.0311 & 0.0500 \\
7.22 & 82.3 & 35.0 & 30.0 & 70.0 & 0.0311 & 0.0500 \\
7.23 & 100.0 & 35.0 & 30.0 & 70.0 & & 0.0327 & 0.0568 \\
11.77 & 100.0 & 35.0 & 30.0 & 70.0 & & 0.0327 & 0.0568 \\
11.78 & 82.3 & 35.0 & 30.0 & 70.0 & & 0.0311 & 0.0500 \\
12.75 & 82.3 & 35.0 & 30.0 & 70.0 & & 0.0311 & 0.0500 \\
13.69 & 82.3 & 35.0 & 30.0 & 70.0 & & 0.0327 & 0.0568 \\
13.69 & 100.0 & 35.0 & 30.0 & 70.0 & & 0.0327 & 0.0568 \\
19.00 & 100.0 & 35.0 & 30.0 & 70.0 & & & & \\
\hline
\end{tabular}


Demo Frilo

Stuttgarter Straße 40

70469

Stuttgart
Tel.: 0711810020

Fax: 0711858020
Projekt: Design Calculations

Position: B.13 - Semi-Precast Concrete Beam

26.07.2018
Seite: 2

\begin{tabular}{|c|c|c|c|c|c|c|c|}
\hline \multicolumn{8}{|c|}{ Beam-related loads $(\mathrm{kN}, \mathrm{m})$} \\
\hline \multicolumn{2}{|c|}{$\begin{array}{l}\text { Load type } \\
\qquad(k N, m)\end{array}$} & : & \multicolumn{3}{|c|}{$\begin{array}{l}1=\text { uniform over } \mathrm{L} \\
3=\text { single moment at a } \\
5=\text { triangular over } \mathrm{L}\end{array}$} & \multicolumn{2}{|c|}{$\begin{array}{l}\text { 2=concentrated at } a \\
\text { 4=trapezoidal btw. } a, a+b \\
\text { 6=trapezoidal over } L\end{array}$} \\
\hline \multicolumn{2}{|c|}{ Type EG Gr } & \multirow{2}{*}{$\begin{array}{l}\text { VK } \\
0.00\end{array}$} & \multirow{2}{*}{$\begin{array}{l}\text { g_l/r } \\
11.37\end{array}$} & \multirow{2}{*}{$\begin{array}{l}q \_l / r \\
0.00\end{array}$} & \multirow{2}{*}{$\begin{array}{l}\text { fac. } \\
1.00\end{array}$} & \multirow{2}{*}{$\begin{array}{c}\text { dist. Lb/Lc } \\
0.52\end{array}$} & fromltem Phi \\
\hline 2 & A & & & & & & G0_LF1 \\
\hline 2 & A & 0.00 & 4.58 & 0.00 & 1.00 & 0.63 & G0_LF1 \\
\hline 2 & $A$ & 0.00 & 20.40 & 0.00 & 1.00 & 1.25 & G0_LF1 \\
\hline 2 & $A$ & 0.00 & 26.10 & 0.00 & 1.00 & 2.19 & G0_LF1 \\
\hline 2 & $A$ & 0.00 & 30.21 & 0.00 & 1.00 & 3.13 & G0_LF1 \\
\hline 2 & $A$ & 0.00 & 38.41 & 0.00 & 1.00 & 4.06 & G0_LF1 \\
\hline 2 & A & 0.00 & 52.30 & 0.00 & 1.00 & 5.00 & G0_LF1 \\
\hline 2 & $A$ & 0.00 & 15.23 & 0.00 & 1.00 & 5.63 & G0_LF1 \\
\hline 2 & A & 0.00 & 101.28 & 0.00 & 1.00 & 5.73 & $\mathrm{G} 0^{-} \mathrm{LF} 1$ \\
\hline 2 & A & 0.00 & 29.01 & 0.00 & 1.00 & 6.40 & G0_LF1 \\
\hline 2 & $A$ & 0.00 & 85.47 & 0.00 & 1.00 & 6.90 & G0_LF1 \\
\hline 2 & $A$ & 0.00 & 40.81 & 0.00 & 1.00 & 7.55 & G0_LF1 \\
\hline 2 & $A$ & 0.00 & 27.16 & 0.00 & 1.00 & 8.53 & G0_LF1 \\
\hline 2 & $A$ & 0.00 & 29.30 & 0.00 & 1.00 & 9.50 & G0_LF1 \\
\hline 2 & A & 0.00 & 39.59 & 0.00 & 1.00 & 10.48 & G0_LF1 \\
\hline 2 & $A$ & 0.00 & 50.36 & 0.00 & 1.00 & 11.45 & G0_LF1 \\
\hline 2 & A & 0.00 & 19.03 & 0.00 & 1.00 & 12.10 & G0_LF1 \\
\hline 2 & $A$ & 0.00 & 88.31 & 0.00 & 1.00 & 12.25 & G0_LF1 \\
\hline 2 & A & 0.00 & 17.98 & 0.00 & 1.00 & 12.90 & G0_LF1 \\
\hline 2 & A & 0.00 & 74.32 & 0.00 & 1.00 & 13.38 & G0_LF1 \\
\hline 2 & $A$ & 0.00 & 33.38 & 0.00 & 1.00 & 14.00 & G0_LF1 \\
\hline 2 & $A$ & 0.00 & 26.94 & 0.00 & 1.00 & 14.94 & G0_LF1 \\
\hline 2 & $A$ & 0.00 & 30.16 & 0.00 & 1.00 & 15.88 & G0_LF1 \\
\hline 2 & $A$ & 0.00 & 28.71 & 0.00 & 1.00 & 16.81 & G0_LF1 \\
\hline 2 & $A$ & 0.00 & 21.95 & 0.00 & 1.00 & 17.75 & G0_LF1 \\
\hline 2 & A & 0.00 & 4.85 & 0.00 & 1.00 & 18.38 & $\mathrm{GO}^{-} \mathrm{LF} 1$ \\
\hline 2 & A & 0.00 & 11.75 & 0.00 & 1.00 & 18.48 & G0- LF1 \\
\hline 2 & B 1 & 0.00 & 0.00 & 3.10 & 1.00 & 0.52 & G0_LF2 \\
\hline 2 & B 1 & 0.00 & 0.00 & 1.30 & 1.00 & 0.63 & G0_LF2 \\
\hline 2 & B 1 & 0.00 & 0.00 & 5.81 & 1.00 & 1.25 & G0_LF2 \\
\hline 2 & B 1 & 0.00 & 0.00 & 7.48 & 1.00 & 2.19 & G0_LF2 \\
\hline 2 & B 1 & 0.00 & 0.00 & 8.70 & 1.00 & 3.13 & G0_LF2 \\
\hline 2 & B 1 & 0.00 & 0.00 & 11.11 & 1.00 & 4.06 & G0_LF2 \\
\hline 2 & B 1 & 0.00 & 0.00 & 15.15 & 1.00 & 5.00 & G0_LF2 \\
\hline 2 & B 1 & 0.00 & 0.00 & 4.41 & 1.00 & 5.63 & G0_LF2 \\
\hline 2 & B 1 & 0.00 & 0.00 & 29.23 & 1.00 & 5.73 & G0_LF2 \\
\hline 2 & B 1 & 0.00 & 0.00 & 8.35 & 1.00 & 6.40 & G0_LF2 \\
\hline 2 & B 1 & 0.00 & 0.00 & 24.58 & 1.00 & 6.90 & G0_LF2 \\
\hline 2 & B 1 & 0.00 & 0.00 & 11.63 & 1.00 & 7.55 & G0_LF2 \\
\hline 2 & B 1 & 0.00 & 0.00 & 7.69 & 1.00 & 8.53 & G0_LF2 \\
\hline 2 & B 1 & 0.00 & 0.00 & 8.41 & 1.00 & 9.50 & G0_LF2 \\
\hline 2 & B 1 & 0.00 & 0.00 & 11.43 & 1.00 & 10.48 & $\mathrm{GO}^{-} \mathrm{LF} 2$ \\
\hline 2 & B 1 & 0.00 & 0.00 & 14.52 & 1.00 & 11.45 & G0_LF2 \\
\hline 2 & B 1 & 0.00 & 0.00 & 5.48 & 1.00 & 12.10 & G0_LF2 \\
\hline 2 & B 1 & 0.00 & 0.00 & 25.36 & 1.00 & 12.25 & G0_LF2 \\
\hline 2 & B 1 & 0.00 & 0.00 & 5.15 & 1.00 & 12.90 & G0_LF2 \\
\hline 2 & B 1 & 0.00 & 0.00 & 21.28 & 1.00 & 13.38 & G0_LF2 \\
\hline 2 & B 1 & 0.00 & 0.00 & 9.51 & 1.00 & 14.00 & G0_LF2 \\
\hline 2 & B 1 & 0.00 & 0.00 & 7.72 & 1.00 & 14.94 & G0_LF2 \\
\hline 2 & B 1 & 0.00 & 0.00 & 8.73 & 1.00 & 15.88 & G0_LF2 \\
\hline 2 & B 1 & 0.00 & 0.00 & 8.31 & 1.00 & 16.81 & $\mathrm{GO}-\mathrm{LF} 2$ \\
\hline 2 & B 1 & 0.00 & 0.00 & 6.30 & 1.00 & 17.75 & G0_LF2 \\
\hline 2 & B 1 & 0.00 & 0.00 & 1.38 & 1.00 & 18.38 & G0_LF2 \\
\hline 2 & B 1 & 0.00 & 0.00 & 3.22 & 1.00 & 18.48 & G0_LF2 \\
\hline Total & & & 958.96 & 275.34 & & & \\
\hline
\end{tabular}

\section{Actions:}

No. Cl Name

$\psi 0 \quad \psi 1 \quad \psi 2 \quad \psi$

A 1 Cat A - domestic

$\begin{array}{llll}0.70 & 0.50 & 0.30 & 1.50\end{array}$

B 1 Cat B-offices

$\begin{array}{llll}0.70 & 0.50 & 0.30 & 1.50\end{array}$




\section{Demo Frilo}

Stuttgarter Straße 40

70469

Stuttgart
Tel.: 0711810020

Fax: 0711858020
Projekt: Design Calculations

Position: B.13 - Semi-Precast Concrete Beam

26.07.2018

Consequency class CC 2 acc. EN 1990 Tab. B1 $->K_{F i}=1.0$ Tab. B3

In following tables the last cell the row is a reference to

the number of the related superposition (see below).

In tables with internal forces multiplied by Gamma is additionally

a reference to the main action.

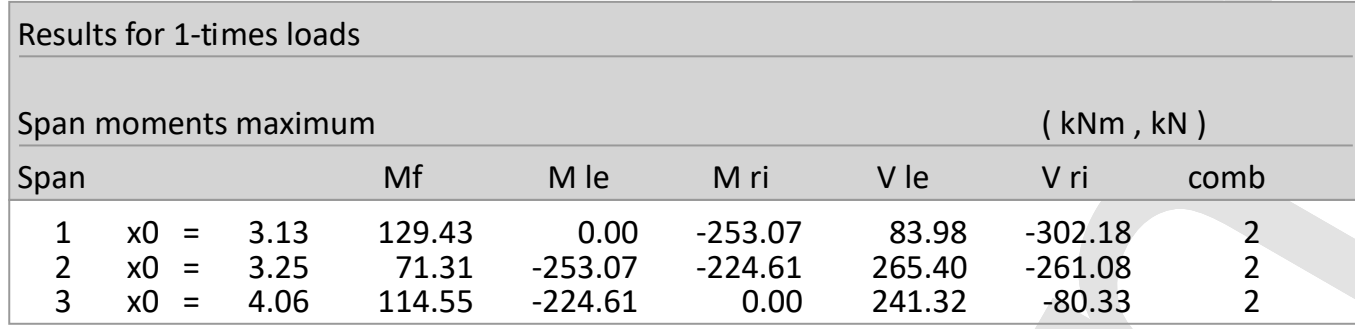

\begin{tabular}{|crrrrrrr|}
\hline \multicolumn{2}{l}{ Support moments maximum } \\
\hline \multicolumn{1}{l}{ Column } & M le & M ri & V le & \multicolumn{1}{c|}{ V ri } & $\max F$ & $\min F$ & \multicolumn{1}{c|}{ comb } \\
\hline 1 & 0.00 & 0.00 & 0.00 & 83.98 & 83.98 & 65.36 & 2 \\
2 & -253.07 & -253.07 & -302.18 & 265.40 & 567.59 & 440.74 & 2 \\
3 & -224.61 & -224.61 & -261.08 & 241.32 & 502.39 & 390.37 & 2 \\
4 & 0.00 & 0.00 & -80.33 & 0.00 & 80.33 & 62.48 & 2 \\
\hline
\end{tabular}

\begin{tabular}{|c|c|c|c|c|c|c|c|c|c|c|c|}
\hline \multicolumn{12}{|c|}{ Moment boundary diagram } \\
\hline \multicolumn{2}{|c|}{$\begin{array}{l}\mathrm{x} / \mathrm{L}=.0 \\
\text { Span }\end{array}$} & .1 & .2 & .3 & .4 & .5 & .6 & .7 & .8 & .9 & 1.0 \\
\hline $\begin{array}{l}1 \\
1\end{array}$ & $\begin{array}{l}0.00 \\
0.00\end{array}$ & $\begin{array}{l}39.7 \\
51.0\end{array}$ & $\begin{array}{l}70.5 \\
90.8\end{array}$ & $\begin{array}{r}88.7 \\
114\end{array}$ & $\begin{array}{r}98.6 \\
127\end{array}$ & $\begin{array}{l}100 \\
129\end{array}$ & $\begin{array}{r}83.4 \\
108\end{array}$ & $\begin{array}{l}54.3 \\
70.1\end{array}$ & $\begin{array}{l}13.2 \\
17.2\end{array}$ & $\begin{array}{l}-77.8 \\
-60.5\end{array}$ & $\begin{array}{l}-253 \\
-197\end{array}$ \\
\hline $\begin{array}{l}2 \\
2\end{array}$ & $\begin{array}{l}-253 \\
-197\end{array}$ & $\begin{array}{l}-99.2 \\
-77.0\end{array}$ & $\begin{array}{l}-22.5 \\
-17.4\end{array}$ & $\begin{array}{l}15.7 \\
20.1\end{array}$ & $\begin{array}{l}40.0 \\
51.4\end{array}$ & $\begin{array}{l}55.5 \\
71.3\end{array}$ & $\begin{array}{l}51.9 \\
66.8\end{array}$ & $\begin{array}{l}35.4 \\
45.6\end{array}$ & $\begin{array}{l}6.11 \\
7.92\end{array}$ & $\begin{array}{l}-72.0 \\
-55.9\end{array}$ & $\begin{array}{l}-225 \\
-175\end{array}$ \\
\hline $\begin{array}{l}3 \\
3\end{array}$ & $\begin{array}{l}-225 \\
-175\end{array}$ & $\begin{array}{l}-84.8 \\
-65.8\end{array}$ & $\begin{array}{l}-8.16 \\
-6.31\end{array}$ & $\begin{array}{l}32.4 \\
41.7\end{array}$ & $\begin{array}{l}62.6 \\
80.6\end{array}$ & $\begin{array}{r}84.5 \\
109\end{array}$ & $\begin{array}{r}87.4 \\
113\end{array}$ & $\begin{array}{r}81.5 \\
105\end{array}$ & $\begin{array}{l}66.5 \\
85.6\end{array}$ & $\begin{array}{l}37.8 \\
48.7\end{array}$ & $\begin{array}{l}0.00 \\
0.00\end{array}$ \\
\hline
\end{tabular}

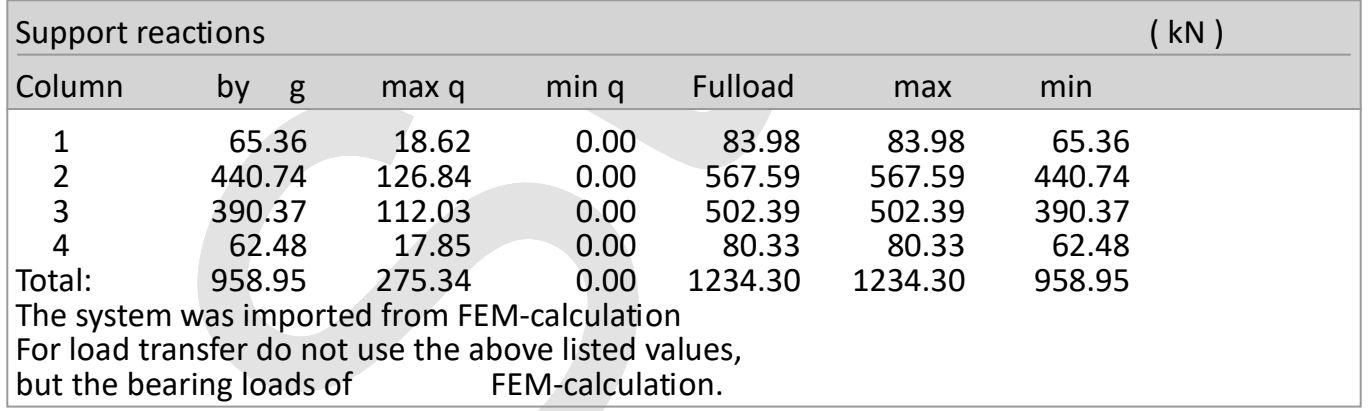

\begin{tabular}{|c|c|c|c|c|c|c|c|c|}
\hline \multicolumn{7}{|c|}{ Support reactions } & \multicolumn{2}{|c|}{$(\mathrm{kN})$} \\
\hline CA & $\underset{\max }{\text { Column } 1}$ & $\min$ & $\begin{array}{l}\text { Column } 2 \\
\max \end{array}$ & $\min$ & $\begin{array}{l}\text { Column } 3 \\
\text { ax }\end{array}$ & $\min$ & $\underset{\max }{\text { Column } 4}$ & $\min$ \\
\hline$g$ & 65.4 & 65.4 & 440.7 & 440.7 & 390.4 & 390.4 & 62.5 & 62.5 \\
\hline A & 0.0 & 0.0 & 0.0 & 0.0 & 0.0 & 0.0 & 0.0 & 0.0 \\
\hline B & 18.6 & 0.0 & 126.8 & 0.0 & 112.0 & 0.0 & 17.9 & 0.0 \\
\hline tot & 84.0 & 65.4 & 567.6 & 440.7 & 502.4 & 390.4 & 80.3 & 62.5 \\
\hline
\end{tabular}


Demo Frilo

Stuttgarter Straße 40

70469

Stuttgart
Tel.: 0711810020

Fax: 0711858020
Projekt: Design Calculations

Position: B.13 - Semi-Precast Concrete Beam

26.07.2018

Seite: 4

Results for $\gamma$-times loads

Partial safety factor $\gamma \mathrm{G} * \mathrm{~K}_{\mathrm{Fi}}=1.35$ spanwise constant

\begin{tabular}{|c|c|c|c|c|c|c|c|c|}
\hline Span & nomen & maxin & & & & & ( $\mathrm{kNm}$, & N) \\
\hline Span & & & Mfd & Mdle & Mdri & V le & V ri & comb \\
\hline $\begin{array}{l}1 \\
2 \\
3\end{array}$ & $\begin{array}{l}x 0= \\
x 0= \\
x 0=\end{array}$ & $\begin{array}{l}3.13 \\
3.25 \\
4.06\end{array}$ & $\begin{array}{l}197.27 \\
126.56 \\
171.67\end{array}$ & $\begin{array}{r}0.00 \\
-317.72 \\
-273.05\end{array}$ & $\begin{array}{r}-313.74 \\
-287.33 \\
0.00\end{array}$ & $\begin{array}{l}121.99 \\
365.78 \\
327.81\end{array}$ & $\begin{array}{l}-412.28 \\
-362.58 \\
-117.16\end{array}$ & $\begin{array}{ll}\text { B } & 2 \\
\text { B } & 2 \\
\text { B } & 2\end{array}$ \\
\hline
\end{tabular}

\begin{tabular}{|crrrrrrrr|}
\hline \multicolumn{2}{l}{ Support moments maximum } \\
\multicolumn{1}{l}{ Support } & Mdle & Mdri & Vdle & Vdri & $\max F$ & $\min F$ & comb \\
\hline 1 & 0.00 & 0.00 & 0.00 & 121.99 & 121.99 & 59.54 & B & 2 \\
2 & -358.75 & -358.75 & -419.48 & 373.71 & 793.19 & 432.82 & B 2 \\
3 & -321.19 & -321.19 & -369.12 & 335.52 & 704.63 & 380.77 & B & 2 \\
4 & 0.00 & 0.00 & -117.16 & 0.00 & 117.16 & 56.46 & B & 2 \\
\hline
\end{tabular}

\begin{tabular}{|c|c|c|c|c|c|c|c|c|c|c|c|}
\hline \multicolumn{12}{|c|}{ Moment boundary diagram } \\
\hline \multicolumn{2}{|c|}{$\begin{array}{l}x / L=.0 \\
\text { Span }\end{array}$} & .1 & .2 & .3 & .4 & .5 & .6 & .7 & .8 & .9 & 1.0 \\
\hline 1 & 0.00 & 36.0 & 63.3 & 77.8 & 84.1 & 82.3 & 61.6 & 28.8 & -15.9 & -127 & -359 \\
\hline 1 & 0.00 & 74.2 & 133 & 169 & 190 & 197 & 171 & 122 & 53.0 & -40.7 & -188 \\
\hline 2 & -359 & -154 & -55.9 & -14.0 & 11.2 & 27.6 & 24.9 & 9.32 & -19.3 & -117 & -321 \\
\hline 2 & -188 & -60.0 & 7.30 & 57.5 & 99.8 & 127 & 119 & 89.2 & 36.3 & -38.4 & -164 \\
\hline 3 & -321 & -136 & -39.3 & 5.98 & 40.0 & 65.6 & 72.4 & 70.2 & 59.0 & 34.1 & 0.00 \\
\hline 3 & -164 & -47.1 & 21.7 & 84.0 & 134 & 169 & 171 & 156 & 126 & 71.1 & 0.00 \\
\hline
\end{tabular}

Scale $1: 200$

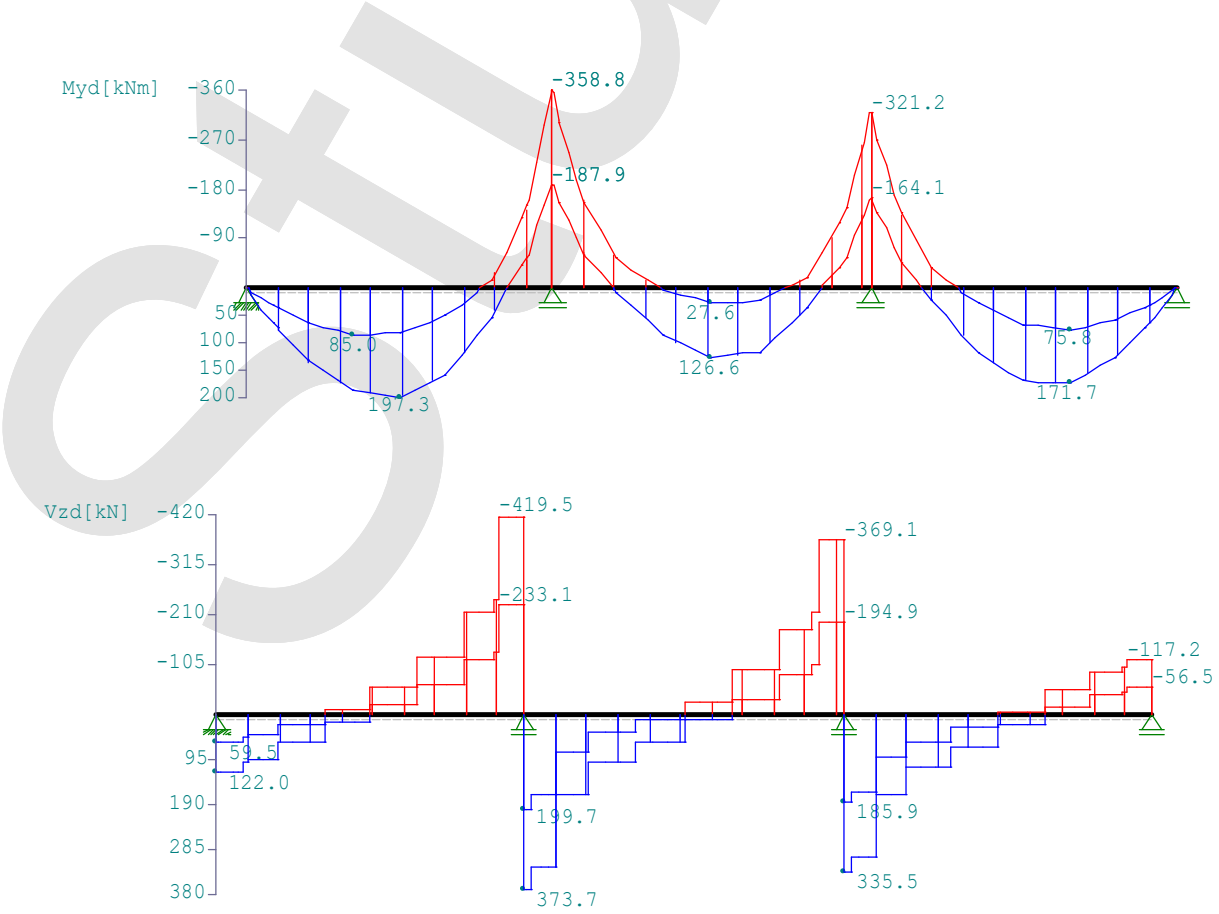


Demo Frilo

Stuttgarter Straße 40

70469 Stuttgart
Tel.: 0711810020

Fax: 0711858020
Projekt: Design Calculations

Position: B.13 - Semi-Precast Concrete Beam

26.07.2018
Seite: 5

Design DIN EN 1992-1-1/NA/A1:2015-12

FLBemBn.DLL: Version 9.0.1.121 (1)

C30/37 B500A normaly ductil

Concrete cover:

Reinforcement location:

$\mathrm{cv}=3.0 \mathrm{~cm} \quad>=$ req. $\mathrm{cv}$

$\mathrm{dt}=4.0 \mathrm{~cm} \quad \mathrm{~dB}=8 \mathrm{dS}=14$

$\mathrm{Db}=4.0 \mathrm{~cm} \quad \mathrm{~dB}=8 \quad \mathrm{dS}=14$

In Spans with kx > 0.45 is EN 19925.5 (5)to be considered.

Span reinforcement is not curtailed.

The ductility reinforcement by 9.2.1.1 is contained in the required reinforcement.

Creep factor $\phi=2.58 \quad \varepsilon \mathrm{cs}=0.39 \% \mathrm{~h} 0=22.50 \mathrm{~cm}$

\section{Support conditions}

support width $(\mathrm{cm})$ support type

$\begin{array}{llll}1 & 20.0 & \text { Concrete } & \text { direct } \\ 2 & 30.0 & \text { Concrete } & \text { direct } \\ 3 & 30.0 & \text { Concrete } & \text { direct } \\ 4 & 20.0 & \text { Concrete } & \text { direct }\end{array}$

Reduction of the moments of supports $<=15 \%$

Minimum reinforcement EN2 9.2.1.1 (9.1) fctm $=2.90 \mathrm{~N} / \mathrm{mm} 2$

Calculating Wy, breadth of slab is limited to $2 * \mathrm{~b} 0$.

\begin{tabular}{|cccccc|} 
Q.No. & $\begin{array}{c}\min \mathrm{Mb} \\
(\mathrm{kNm})\end{array}$ & $\begin{array}{c}\text { req As } \\
(\mathrm{cm} 2)\end{array}$ & $\begin{array}{r}\min \mathrm{Mo} \\
(\mathrm{kNm})\end{array}$ & $\begin{array}{r}\text { req } \mathrm{As} \\
(\mathrm{cm} 2)\end{array}$ & \\
\hline 1 & 94.80 & 3.19 & -153.75 & 5.18 & $90.0 / 35.0 / 30.0 / 70.0$ \\
\hline
\end{tabular}

\section{Span reinforcement}

\begin{tabular}{|c|c|c|c|c|c|c|c|c|c|}
\hline $\begin{array}{l}\text { Span } \\
\text { No. }\end{array}$ & $\begin{array}{c}x \\
(m)\end{array}$ & $\begin{array}{c}\text { Myd } \\
(\mathrm{kNm})\end{array}$ & $\begin{array}{r}\min \text { Myd } \\
(k N m)\end{array}$ & $\begin{array}{c}\mathrm{d} \\
(\mathrm{cm})\end{array}$ & $k x$ & \multicolumn{2}{|c|}{ 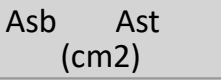 } & & \\
\hline \multirow[t]{2}{*}{1} & 3.13 & 197.3 & & 66.0 & 0.05 & 6.7 & 0.0 & B & \\
\hline & 5.73 & -150.7 & -150.7 & 66.0 & 0.09 & 0. & 5.2 & B & \\
\hline \multirow[t]{2}{*}{2} & 3.25 & 126.6 & & 66.0 & 0.04 & 4. & 0.0 & B & \\
\hline & 0.65 & -154.5 & -154.5 & 66.0 & 0.10 & 0. & 5.3 & B & \\
\hline \multirow[t]{2}{*}{3} & 4.06 & 171.7 & & 66.0 & 0.05 & 5.8 & 0.0 & B & \\
\hline & 0.94 & -86.1 & -86.1 & 66.0 & 0.06 & 0.0 & $5.2 *$ & B & \\
\hline
\end{tabular}

* Minimum reinforcement acc.to DIN EN 1992-1 9.2.1.1 (1)

On first support are at least $4.2 \mathrm{~cm} 2$ to be anchored.

On last support are at least $4.0 \mathrm{~cm} 2$ to be anchored.

Shear force VK-support is with $\mathrm{F}=\mathrm{V}, \mathrm{Ed} * \operatorname{Cot}($ Theta) $/ 2$ considered.

\begin{tabular}{|c|c|c|c|c|c|c|c|c|c|}
\hline \multicolumn{10}{|c|}{ Support reinforcement DIN EN 1992:2015 5.5} \\
\hline $\begin{array}{l}\text { Column } \\
\text { No. }\end{array}$ & $\begin{array}{c}x \\
(m)\end{array}$ & $\begin{array}{c}\text { Myd } \\
(\mathrm{kNm})\end{array}$ & $\begin{array}{c}\text { des.. Myd } \\
\text { (kNm) }\end{array}$ & $\begin{array}{c}d \\
(\mathrm{~cm})\end{array}$ & kx & $\begin{array}{l}\text { Asb } \\
\quad(\mathrm{cm} 2)\end{array}$ & 2) & \multicolumn{2}{|c|}{ comb } \\
\hline $1 \mathrm{ri}$ & 0.00 & 0.0 & & & & & & & 1 \\
\hline $2 \mathrm{le}$ & 0.15 & -350.1 & -236.2 & 66.0 & 0.14 & 0.0 & 8.4 & B & 2 \\
\hline $2 \mathrm{ri}$ & 0.15 & -350.1 & -244.5 & 66.0 & 0.14 & 0.0 & 8.7 & $\mathrm{~B}$ & 2 \\
\hline $3 \mathrm{le}$ & 0.15 & -310.7 & -211.7 & 66.0 & 0.12 & 0.0 & 7.4 & $\mathrm{~B}$ & 2 \\
\hline $3 \mathrm{ri}$ & 0.15 & -310.7 & -215.2 & 66.0 & 0.13 & 0.0 & 7.5 & $\mathrm{~B}$ & 2 \\
\hline
\end{tabular}




\section{Demo Frilo}

Stuttgarter Straße 40

$70469 \quad$ Stuttgart
Tel.: 0711810020

Fax: 0711858020
Projekt: Design Calculations

Position: B.13 - Semi-Precast Concrete Beam

26.07.2018

Seite: 6

\begin{tabular}{|c|c|c|c|c|c|c|c|}
\hline \multicolumn{8}{|c|}{ Support reinforcement DIN EN 1992:2015 5.5} \\
\hline $\begin{array}{l}\text { Column } \\
\text { No. }\end{array}$ & $\begin{array}{c}x \\
(m)\end{array}$ & $\begin{array}{c}\text { Myd } \\
(\mathrm{kNm})\end{array}$ & $\begin{array}{c}\text { des.. Myd } \\
\text { (kNm) }\end{array}$ & $\begin{array}{c}d \\
(\mathrm{~cm})\end{array}$ & kx & $\begin{array}{l}\text { Asb Ast } \\
(\mathrm{cm} 2)\end{array}$ & comb \\
\hline 4 le & 0.00 & 0.0 & & & & & 1 \\
\hline
\end{tabular}

\begin{tabular}{|c|c|c|c|c|c|c|c|c|c|c|}
\hline \multirow{2}{*}{$\begin{array}{c}\text { column } \\
\text { No. } \\
1 \mathrm{ri}\end{array}$} & \multirow{2}{*}{$\begin{array}{l}\text { dist } \\
(\mathrm{m}) \\
0.52\end{array}$} & \multirow{2}{*}{$\begin{array}{l}\text { kz } \\
0.90\end{array}$} & \multirow{2}{*}{$\begin{array}{c}\begin{array}{l}\text { VEd } \\
(\mathrm{kN})\end{array} \\
122.0\end{array}$} & \multirow{2}{*}{$\begin{array}{l}\Theta \\
\left({ }^{\circ}\right) \\
18.4\end{array}$} & \multirow{2}{*}{$\begin{array}{r}\begin{array}{r}\text { VRd,c } \\
(\mathrm{kN})\end{array} \\
67.0\end{array}$} & \multicolumn{5}{|c|}{$\begin{array}{l}\text { VRd,max a_max asw } \\
(\mathrm{kN}) \quad(\mathrm{cm})(\mathrm{cm} 2 / \mathrm{m}) \text { comb }\end{array}$} \\
\hline & & & & & & 685.1 & 30.0 & $2.8^{\sim}$ & B & 2 \\
\hline $1 \mathrm{ri}$ & 0.73 & 0.90 & 94.5 & 18.4 & 67.0 & 685.1 & 30.0 & $2.8^{\sim}$ & B & 2 \\
\hline $1 *$ & 1.39 & 0.90 & 60.0 & 18.4 & 67.0 & 685.1 & 30.0 & $2.8^{\sim}$ & B & 2 \\
\hline $2 \mathrm{le}$ & 0.52 & 0.90 & -419.5 & 29.6 & 71.6 & 980.7 & 30.0 & 9.2 & $B$ & 2 \\
\hline $2 \mathrm{le}$ & 0.81 & 0.90 & -213.6 & 29.6 & 71.6 & 980.7 & 30.0 & 4.7 & $B$ & 2 \\
\hline $2^{*}$ & 1.47 & 0.90 & -122.6 & 29.6 & 67.0 & 980.7 & 30.0 & $2.8^{\sim}$ & B & 2 \\
\hline 2 ri & 0.65 & 0.90 & 322.2 & 26.0 & 72.6 & 899.9 & 30.0 & 6.1 & B & 2 \\
\hline $2 \mathrm{ri}$ & 0.81 & 0.90 & 172.2 & 26.0 & 72.6 & 899.9 & 30.0 & 3.2 & B & 2 \\
\hline $2 *$ & 1.47 & 0.90 & 102.0 & 26.0 & 72.6 & 899.9 & 30.0 & $2.8^{\sim}$ & B & 2 \\
\hline 3 le & 0.50 & 0.90 & -369.1 & 28.0 & 68.8 & 946.7 & 30.0 & 7.6 & B & 2 \\
\hline 3 le & 0.81 & 0.90 & -180.2 & 28.0 & 68.8 & 946.7 & 30.0 & 3.7 & B & 2 \\
\hline $3 *$ & 1.47 & 0.90 & -93.4 & 28.0 & 67.0 & 946.7 & 30.0 & $2.8^{\sim}$ & B & 2 \\
\hline $3 \mathrm{ri}$ & 0.62 & 0.90 & 303.6 & 25.0 & 69.2 & 875.1 & 30.0 & 5.5 & B & 2 \\
\hline $3 \mathrm{ri}$ & 0.81 & 0.90 & 172.8 & 25.0 & 69.2 & 875.1 & 30.0 & 3.1 & B & 2 \\
\hline $3 *$ & 1.47 & 0.90 & 114.9 & 25.0 & 69.2 & 875.1 & 30.0 & $2.8^{\sim}$ & B & 2 \\
\hline $4 \mathrm{le}$ & 0.52 & 0.90 & -117.2 & 18.4 & 67.0 & 685.1 & 30.0 & $2.8^{\sim}$ & B & 2 \\
\hline $4 \mathrm{le}$ & 0.73 & 0.90 & -88.5 & 18.4 & 67.0 & 685.1 & 30.0 & $2.8^{\sim}$ & B & 2 \\
\hline $4^{*}$ & 1.39 & 0.90 & -51.3 & 18.4 & 67.0 & 685.1 & 30.0 & $2.8^{\sim}$ & B & 2 \\
\hline
\end{tabular}

\begin{tabular}{|c|c|c|c|c|c|c|c|c|c|c|}
\hline \multicolumn{11}{|c|}{ shoulder shear } \\
\hline \multirow{2}{*}{$\begin{array}{r}\text { Span } \\
1\end{array}$} & \multirow{2}{*}{$\begin{array}{c}\begin{array}{c}x a \\
(\mathrm{~cm})\end{array} \\
0\end{array}$} & \multirow{2}{*}{$\begin{array}{c}\begin{array}{c}x e \\
(\mathrm{~cm})\end{array} \\
156\end{array}$} & \multirow{2}{*}{$\begin{array}{r}\begin{array}{c}\mathrm{mle} \\
(\mathrm{kNm})\end{array} \\
0.0\end{array}$} & \multirow{2}{*}{$\begin{array}{r}\frac{\begin{array}{c}\text { mri } \\
(\mathrm{kNm})\end{array}}{150.8}\end{array}$} & \multirow{2}{*}{$\begin{array}{r}\begin{array}{c}\text { av } \\
(\mathrm{cm})\end{array} \\
156\end{array}$} & \multirow{2}{*}{$\begin{array}{l}\begin{array}{l}\text { beff } \\
(\mathrm{cm})\end{array} \\
100\end{array}$} & \multicolumn{4}{|c|}{$\begin{array}{l}\text { dFcd vEd vEd,perm. asf } \\
(\mathrm{kN}) \quad(\mathrm{kN} / \mathrm{m} 2)(\mathrm{cm} 2 / \mathrm{m})\end{array}$} \\
\hline & & & & & & & 89 & 162 & 6278 & 1.1 \\
\hline 1 & 156 & 313 & 150.8 & 197.3 & 156 & 100 & 27 & 50 & 6278 & 0.3 \\
\hline 1 & 313 & 422 & 197.3 & 139.3 & 110 & 100 & 34 & 89 & 6278 & 0.6 \\
\hline 1 & 422 & 532 & 139.3 & -0.1 & 110 & 100 & 82 & 214 & 6278 & 1.4 \\
\hline 2 & 125 & 225 & 1.1 & 84.0 & 100 & 100 & 49 & 139 & 6278 & 0.9 \\
\hline 2 & 225 & 325 & 84.0 & 126.6 & 100 & 100 & 25 & 71 & 6278 & 0.5 \\
\hline 2 & 325 & 437 & 126.6 & 103.9 & 112 & 100 & 13 & 34 & 6278 & 0.2 \\
\hline 2 & 437 & 549 & 103.9 & -0.2 & 112 & 100 & 61 & 156 & 6278 & 1.0 \\
\hline 3 & 107 & 257 & 0.2 & 137.8 & 150 & 100 & 81 & 155 & 6278 & 1.0 \\
\hline 3 & 257 & 406 & 137.8 & 171.7 & 150 & 100 & 20 & 38 & 6278 & 0.3 \\
\hline 3 & 406 & 516 & 171.7 & 112.2 & 109 & 100 & 35 & 91 & 6278 & 0.6 \\
\hline 3 & 516 & 625 & 112.2 & 1.2 & 109 & 100 & 65 & 171 & 6278 & 1.1 \\
\hline 3 & 516 & 625 & 112.2 & 1.2 & 109 & 100 & 65 & 171 & 6278 & 1.1 \\
\hline 3 & 516 & 625 & 112.2 & 1.2 & 109 & 100 & 65 & 171 & 6278 & 1.1 \\
\hline
\end{tabular}

At the following table the loads are specified by their internal numeration.

The following table of calculated combinations referenced.

to these numbers

\begin{tabular}{|c|c|c|}
\hline $\begin{array}{l}\text { Load type } \\
\qquad(k N, m)\end{array}$ & $\begin{array}{l}\text { 1=uniform over } \mathrm{L} \\
3=\text { single moment at a } \\
5=\text { triangular over } \mathrm{L}\end{array}$ & $\begin{array}{l}2=\text { concentrated at } a \\
4=\text { trapezoidal btw. } a, a+b \\
6=\text { trapezoidal over } L\end{array}$ \\
\hline
\end{tabular}

No. span Type Grp $\quad$ g1 $\quad$ q1 $\quad$ g2 $\quad$ q2 factor distance length

\begin{tabular}{|c|c|c|c|c|c|}
\hline 1 & 2 & A 2 & 11.37 & 0.00 & 1.00 \\
\hline 2 & 2 & A 2 & 4.58 & 0.00 & 1.00 \\
\hline 3 & 2 & A 2 & 20.40 & 0.00 & 1.00 \\
\hline 4 & 2 & A 2 & 26.10 & 0.00 & 1.00 \\
\hline 5 & 2 & A 2 & 30.21 & 0.00 & 1.00 \\
\hline & 2 & A 2 & 38.41 & 0.00 & 1.00 \\
\hline
\end{tabular}


Demo Frilo

Stuttgarter Straße 40

70469
Tel.: 0711810020

Fax: 0711858020
Projekt: Design Calculations

Position: B.13 - Semi-Precast Concrete Beam

26.07.2018
Seite: 7

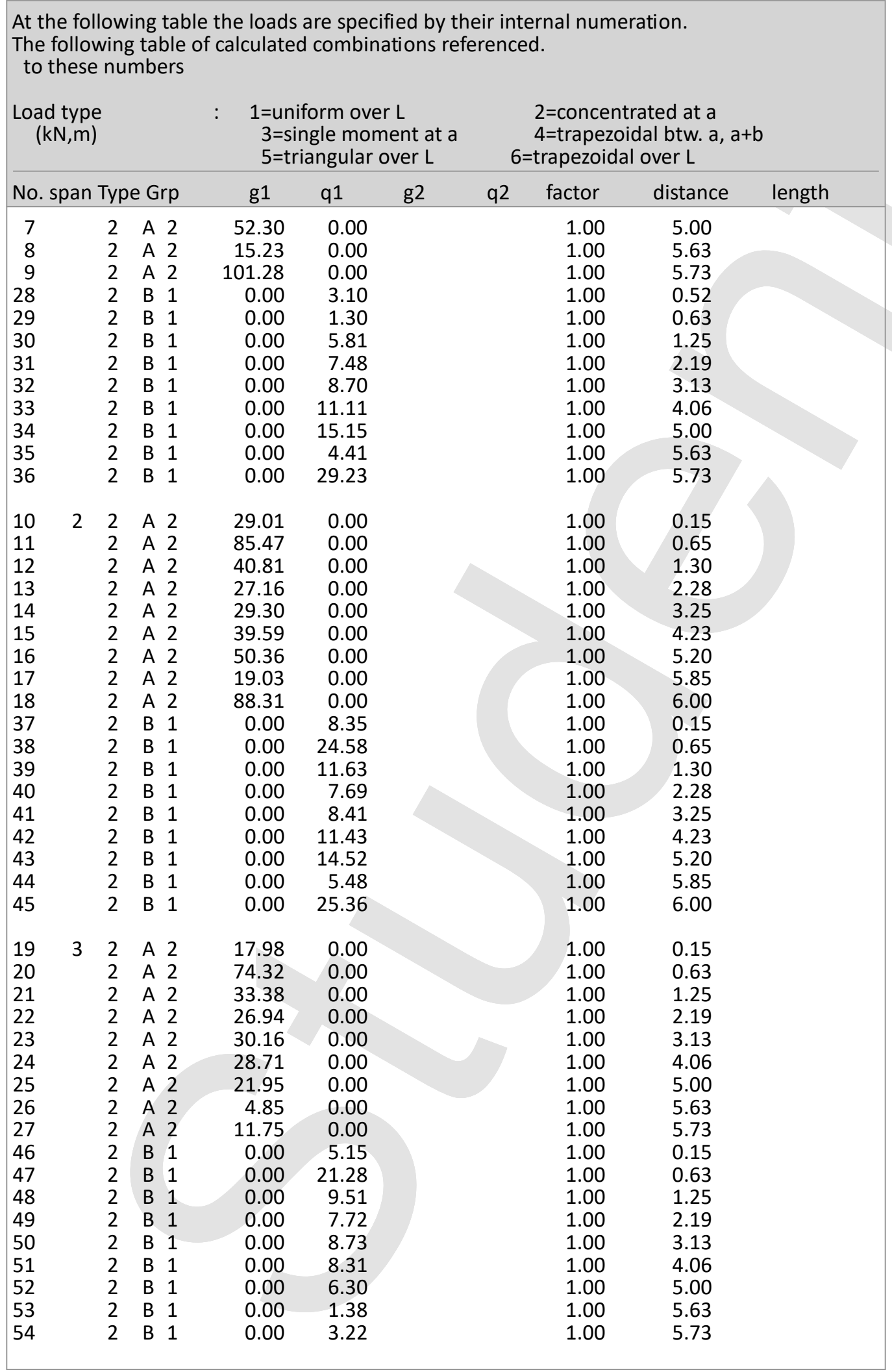


Demo Frilo

Stuttgarter Straße 40

70469

Stuttgart

Tel.: 0711810020

Fax: 0711858020
Projekt: Design Calculations

Position: B.13 - Semi-Precast Concrete Beam

26.07.2018
Seite: 8

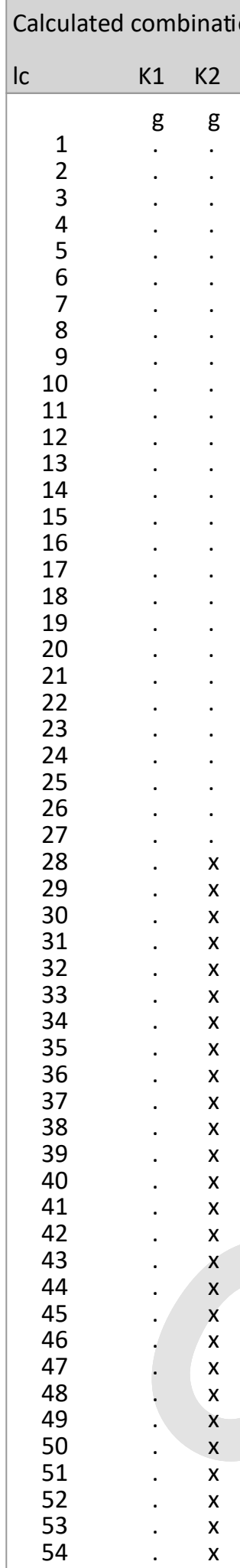

The combinations above will be managed as followed:

Calculating ULS the dead loads will be exceeded

one by one alternating by GammaG $=1,00 / 1,35$.

If in one combination live-loads from different actions

exists, then will be investigated, which action is

the dominating one.

The effect of the

duration of action will be checked

too. 
B.1.9. Design Calculations POS. W.14a - Load Bearing Concrete Wall 
Demo Frilo

Stuttgarter Straße 40

70469

Stuttgart
Tel.: 0711810020

Fax: 0711858020
Projekt: Design Calculations

Position: W.14a - Load Bearing Concrete

Wall Single

26.07.2018
Seite: 1

\section{Position: W.14a - Load Bearing Concrete Wall_Single}

Panels by Finite Elements SCN 01/2018 (Frilo R-2018-1/P12)

\section{System}

Scale $1: 75$

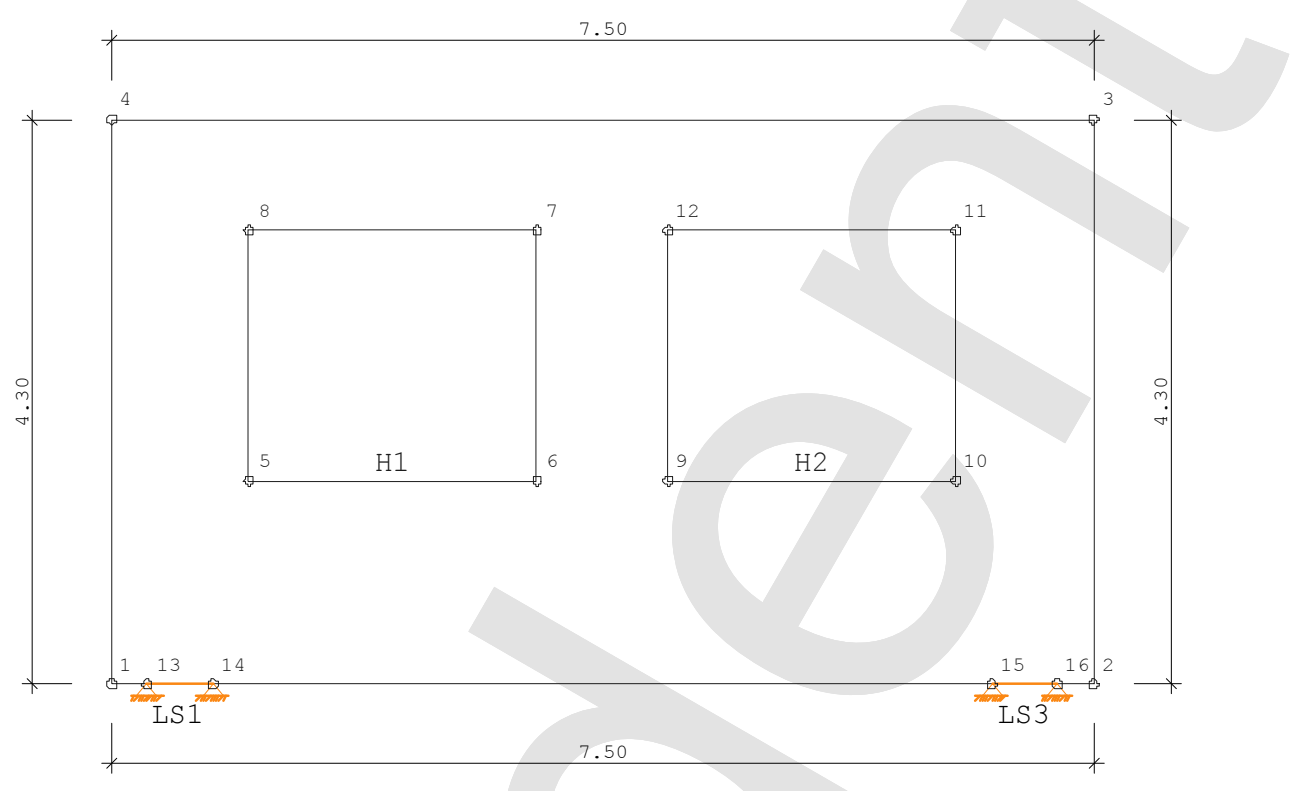


Demo Frilo

Stuttgarter Straße 40

70469

Stuttgart

LOADCASE 1 "POS. S.11 - Dead Load"

Type:

Self-weight of the slab is included:

Action:

Partial safety factor:

Load points:

Point loads:

\section{Forces, vertical}

Total of input loads: (portion on the slab)

Self-weight of the slab:

Total of all loads:

Total of all support reactions:

\section{Forces, horizontal}

Total of input loads: (portion on the slab)

Total of all support reactions:

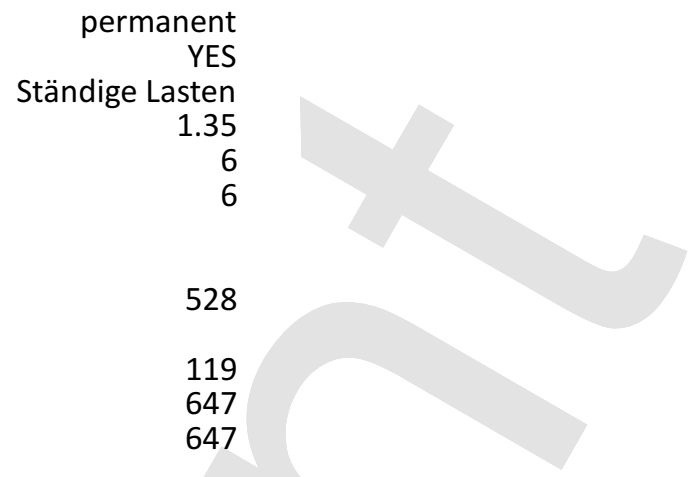

\section{NOTE}

All effects of actions (like internal forces, support reactions, displacements, etc.) of an individual load case are, unlike the results of a superposition of load cases, simple, i.e. characteristic, values

Design results are based on design quantities including the partial safety coefficients.

Load case 1 "POS. S.11 - Dead Load"

Point loads

Scale $1: 75$

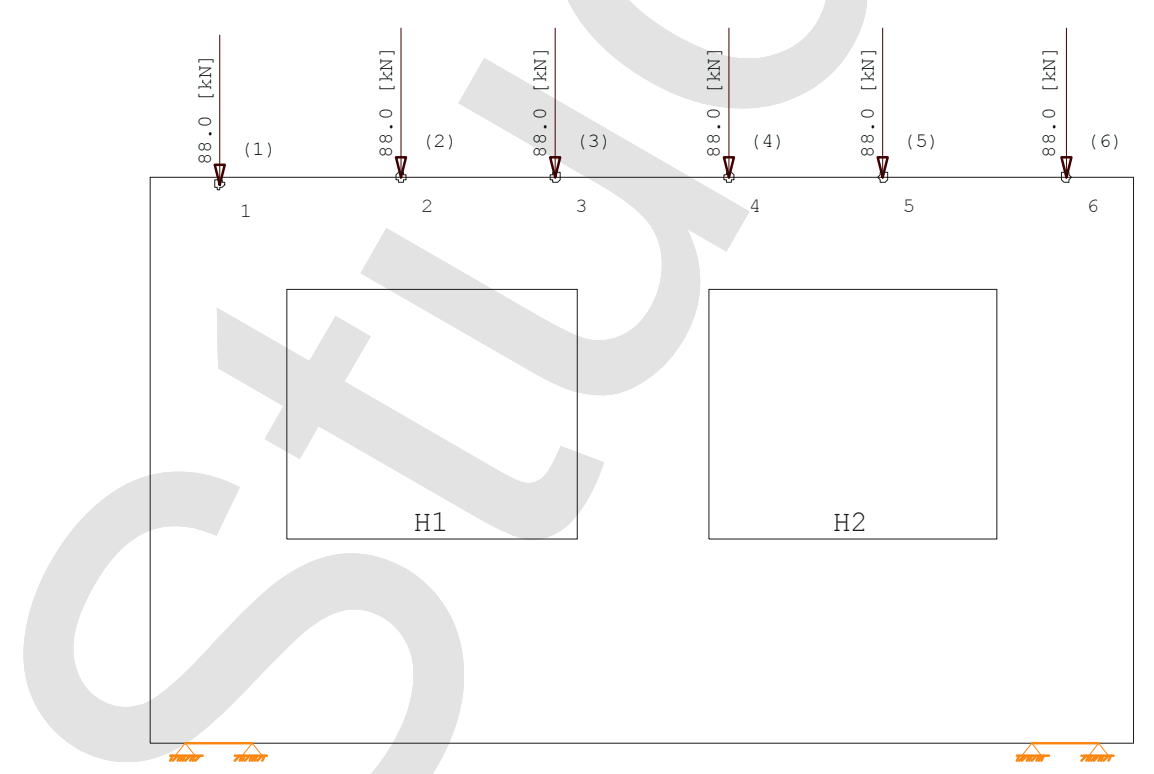


Demo Frilo

Stuttgarter Straße 40

70469
Tel.: 0711810020

Fax: 0711858020
Projekt: Design Calculations

Position: W.14a - Load Bearing Concrete

Wall Single

26.07.2018
Seite: 3

Load case 1 "POS. S.11 - Dead Load"

Principal Forces [kN/m]

Scale $1: 50$

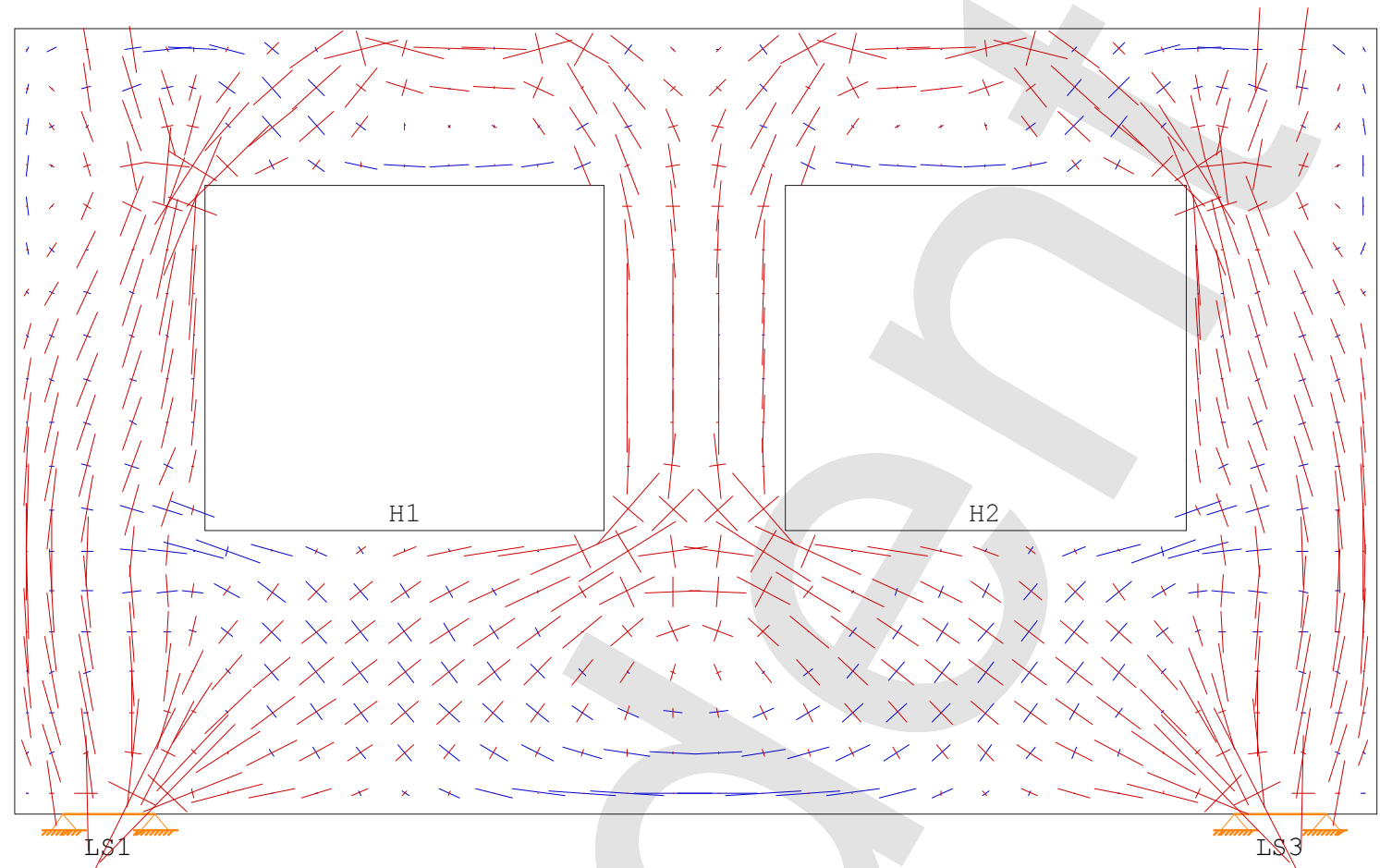


Demo Frilo

Stuttgarter Straße 40

70469

Stuttgart

LOADCASE 2 "POS. S.11 - Live Load"

Type:

Self-weight of the slab is included:

Action:

Partial safety factor:

Load points:

Point loads:

\section{Forces, vertical}

Total of input loads: (portion on the slab)

Total of all support reactions:

\section{Forces, horizontal}

Total of input loads: (portion on the slab)

Total of all support reactions:

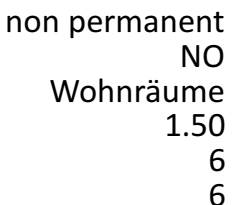

non permanent

NO

Wohnräume

1.50

6

186

186

\section{0}

0

\section{NOTE}

All effects of actions (like internal forces, support reactions,

displacements, etc.) of an individual load case are, unlike the results of a superposition of load cases, simple, i.e. characteristic, values

Design results are based on design quantities including the partial safety coefficients.

Load case 2 "POS. S.11 - Live Load"

Point loads

Scale $1: 75$ 
Demo Frilo

Stuttgarter Straße 40

70469
Tel.: 0711810020

Fax: 0711858020
Projekt: Design Calculations

Position: W.14a - Load Bearing Concrete

Wall Single

26.07.2018
Seite: 5

Load case 2 "POS. S.11 - Live Load"

Principal Forces [kN/m]

Scale $1: 50$

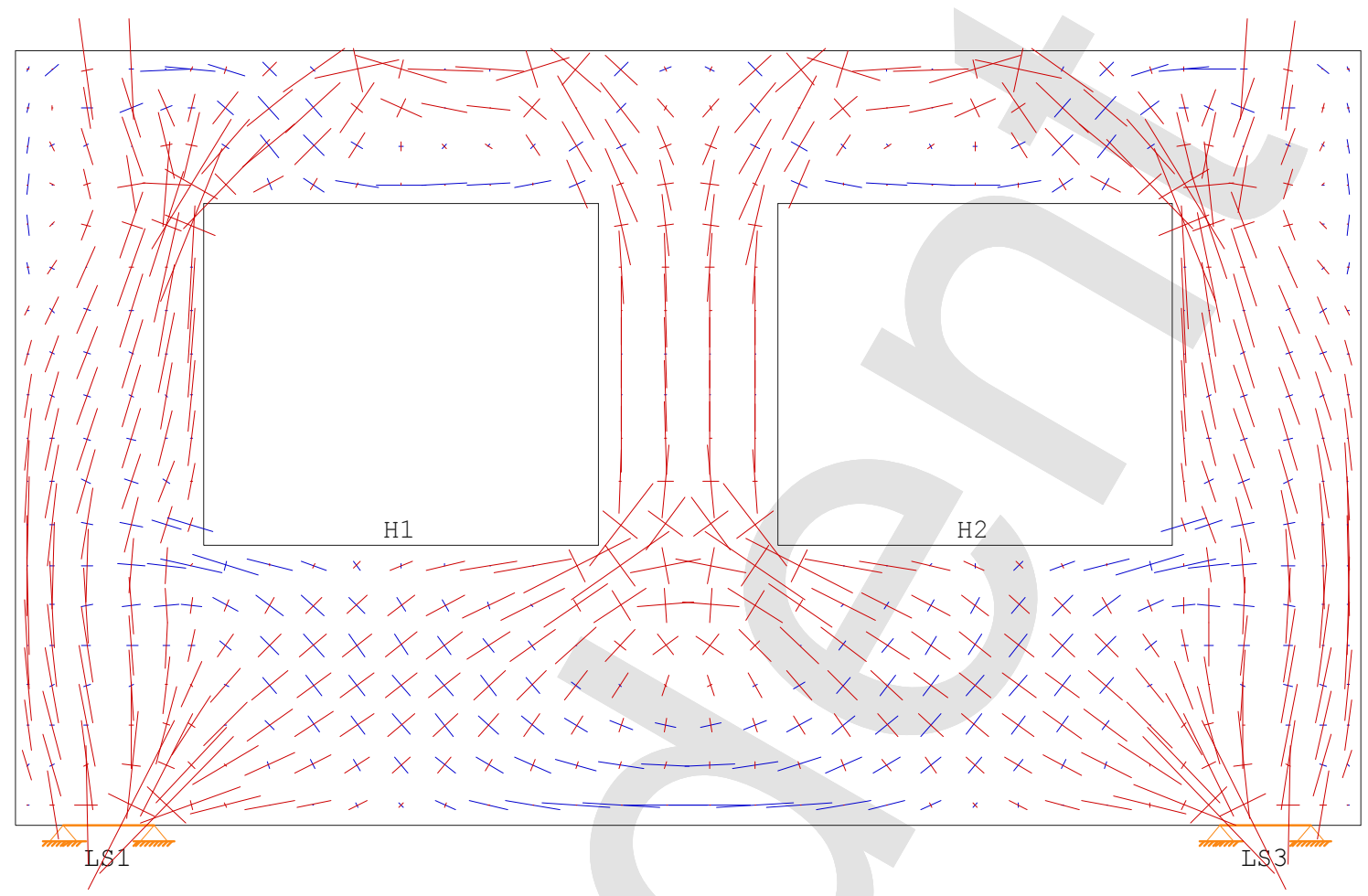


Demo Frilo

Stuttgarter Straße 40

70469

Stuttgart
Tel.: 0711810020

Fax: 0711858020
Projekt: Design Calculations

Position: W.14a - Load Bearing Concrete

Wall Single

26.07.2018

\section{SUPERPOSITION 1 "Characteristic"}

\begin{tabular}{|c|c|c|c|c|c|}
\hline \multicolumn{6}{|c|}{ Load Cases Involved } \\
\hline Number & Load case & Type & $\begin{array}{r}\text { Self } \\
\text { Self- } \\
\text { Weight }\end{array}$ & $\begin{array}{l}\text { Action } \\
\text { Shrt Name } \\
\text { Hnd }\end{array}$ & $\begin{array}{l}\text { Alter- } \\
\text { native } \\
\text { Group }\end{array}$ \\
\hline $\begin{array}{l}1 \\
2\end{array}$ & $\begin{array}{l}11 \text { - Dea... } \\
11 \text { - Liv... }\end{array}$ & $\begin{array}{l}\text { permanent } \\
\text { non perm }\end{array}$ & $\begin{array}{c}\text { yes } \\
\text { no }\end{array}$ & $\begin{array}{l}\text { g Ständige ... } \\
1 \text { Wohnräume }\end{array}$ & \\
\hline
\end{tabular}

\begin{tabular}{|rcr|}
\hline Action & & \\
\hline Number & $\begin{array}{c}\text { Shrt Name } \\
\text { Hnd }\end{array}$ & Type \\
\hline 1 & g Ständige Lasten & $\begin{array}{c}\text { permanent } \\
\text { non perm }\end{array}$ \\
\hline 2 & 1 Wohnräume & \\
\hline
\end{tabular}


Demo Frilo

Stuttgarter Straße 40

70469 Stuttgart
Tel.: 0711810020

Fax: 0711858020
Projekt: Design Calculations

Position: W.14a - Load Bearing Concrete

Wall Single

26.07.2018
Seite: 7

\section{SUPERPOSITION 2 "ULS Permanent/Transient"}

\section{Load Cases Involved}

\begin{tabular}{|c|c|c|c|c|c|}
\hline Number & Load case & Type & $\begin{array}{r}\text { Self } \\
\text { Self- } \\
\text { Weight }\end{array}$ & $\begin{array}{l}\text { Action } \\
\text { Shrt Name } \\
\text { Hnd }\end{array}$ & $\begin{array}{l}\text { Alter- } \\
\text { native } \\
\text { Group }\end{array}$ \\
\hline $\begin{array}{l}1 \\
2\end{array}$ & $\begin{array}{l}11 \text { - Dea... } \\
11 \text { - Liv... }\end{array}$ & $\begin{array}{r}\text { permanent } \\
\text { non perm }\end{array}$ & $\begin{array}{c}\text { yes } \\
\text { no }\end{array}$ & $\begin{array}{l}\text { g Ständige ... } \\
1 \text { Wohnräume }\end{array}$ & $\overline{0}$ \\
\hline
\end{tabular}

\begin{tabular}{|ccccccc|}
\hline \multicolumn{1}{|l|}{ Action } & & & & \\
\hline $\begin{array}{c}\text { Number Shrt Name } \\
\text { Hnd }\end{array}$ & Type Partial Safety & \multicolumn{2}{c|}{$\begin{array}{c}\text { Combination } \\
\text { dom ndo }\end{array}$} \\
\hline 1 & g Ständige Lasten & permanent & 1.35 & 1.00 & 1.00 & 1.00 \\
2 & 1 Wohnräume & non perm & 1.50 & 0.00 & 1.00 & 0.70 \\
\hline
\end{tabular}

\section{NOTE: Design Values}

All results of a superposition of load cases

Include both partial safety and combination

factors: DIN EN 1990/NA:2010-12

\section{NOTE: Combination Factors}

With the combination of independent, variable actions

the individual dominant action is determined for both each location and for each action quantity.

In geneneral, the dominant actions differ with each location and each quantity

The individually found dominant action receives the conbination factor 1.00 . In case of only one variable action this action is considered dominant.

\section{Superposition 2 "ULS Permanent/Transient"} Concrete Load (Compressive) uC-1, uC-2 [\%]

Scale $1: 50$

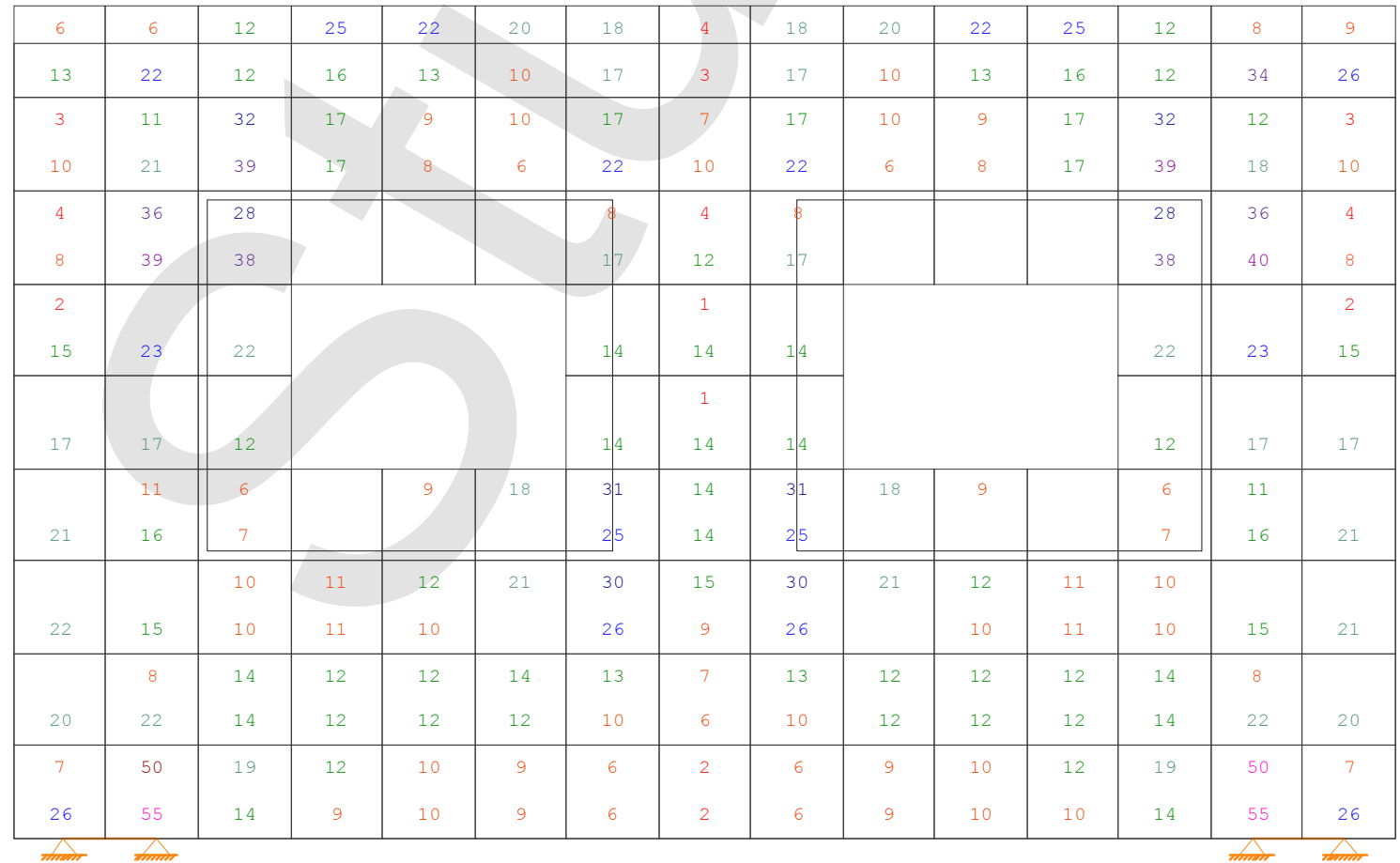

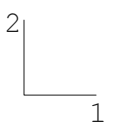


Demo Frilo

Stuttgarter Straße 40

70469

Stuttgart
Tel.: 0711810020

Fax: 0711858020
Projekt: Design Calculations

Position: W.14a - Load Bearing Concrete

Wall Single

26.07.2018
Seite: 8

Superposition 2 "ULS Permanent/Transient"

Reinforcement, Total aS-1, aS-2 $\left[\mathrm{cm}^{2} / \mathrm{m}\right]$ Total

Scale $1: 50$

\begin{tabular}{|c|c|c|c|c|c|c|c|c|c|c|c|c|c|c|}
\hline 3.76 & 12.2 & 7.54 & 1.74 & & & 1.58 & 2.05 & 1.58 & & & 1.73 & 7.52 & 12.9 & 4.26 \\
\hline 1.99 & 2.43 & 4.30 & 2.66 & 0.21 & 0.28 & 3.67 & 0.73 & 3.67 & 0.28 & 0.21 & 2.66 & 4.30 & 3.24 & 3.23 \\
\hline 1.18 & 3.40 & 3.88 & 4.41 & 6.05 & 5.40 & 4.50 & 0.26 & 4.52 & 5.42 & 6.07 & 4.43 & 3.88 & 3.70 & 1.25 \\
\hline 4.25 & 0.68 & 4.93 & 5.34 & 1.21 & 1.08 & 0.90 & 0.37 & 0.90 & 1.08 & 1.21 & 5.34 & 4.93 & 0.74 & 4.21 \\
\hline 0.89 & 0.54 & 0.79 & 7.49 & 8.97 & 8.17 & 1.98 & & 2.00 & 8.19 & 9.00 & 7.52 & 0.79 & 0.56 & 0.89 \\
\hline 4.21 & 0.11 & 1.00 & 1.50 & 1.79 & 1.63 & 0.40 & & 0.40 & 1.64 & 1.80 & 1.50 & 1.00 & 0.11 & 4.20 \\
\hline 1.33 & 1.25 & & & & & & 0.12 & & & & & & 1.24 & 1.32 \\
\hline 0.92 & 0.25 & & & & & & & & & & & & 0.25 & 0.92 \\
\hline 1.20 & 1.47 & & & & & & 0.12 & & & & & & 1.47 & 1.20 \\
\hline 0.24 & 0.29 & & & & & & & & & & & & 0.29 & 0.24 \\
\hline 1.58 & 13.7 & 14.1 & 3.59 & & 0.14 & 0.94 & & 0.94 & 0.14 & & 3.66 & 14.1 & 13.8 & 1.61 \\
\hline 0.32 & 5.36 & 4.81 & 0.72 & & 0.72 & 0.19 & & 0.19 & 0.72 & & 0.73 & 4.80 & 5.38 & 0.32 \\
\hline 1.56 & 6.00 & 11.5 & 4.83 & 1.34 & 0.52 & & & & 0.53 & 1.37 & 4.89 & 11.6 & 6.06 & 1.60 \\
\hline 0.31 & 1.20 & 5.59 & 4.20 & 3.95 & 2.60 & & & & 2.63 & 3.96 & 4.20 & 5.57 & 1.21 & 0.32 \\
\hline 0.97 & 1.47 & 3.21 & 3.45 & 3.03 & 4.05 & 3.94 & 3.01 & 3.95 & 4.06 & 3.04 & 3.48 & 3.23 & 1.50 & 0.99 \\
\hline 0.19 & 0.29 & 3.44 & 4.74 & 4.64 & 4.19 & 2.18 & 0.60 & 2.19 & 4.21 & 4.66 & 4.75 & 3.41 & 0.30 & 0.20 \\
\hline 2.09 & 0.32 & 0.41 & 1.10 & 3.64 & 8.66 & 12.3 & 13.1 & 12.3 & 8.64 & 3.64 & 1.08 & 0.41 & 0.31 & 2.11 \\
\hline 2.18 & 1.60 & 2.07 & 3.64 & 3.67 & 3.23 & 2.46 & 2.62 & 2.46 & 3.24 & 3.69 & 3.66 & 2.06 & 1.57 & 2.19 \\
\hline
\end{tabular}

\footnotetext{
$\max a s-1: 14.1\left[\mathrm{~cm}^{2} / \mathrm{m}\right]$ (Total)

$2 \max a s-2: 5.59\left[\mathrm{~cm}^{2} / \mathrm{m}\right]$ (Total)
} 
Demo Frilo

Stuttgarter Straße 40

70469

Stuttgart
Tel.: 0711810020

Fax: 0711858020
Projekt: Design Calculations

Position: W.14a - Load Bearing Concrete Wall Double

26.07.2018

\section{Position: W.14a - Load Bearing Concrete Wall_Double}

Panels by Finite Elements SCN 01/2018 (Frilo R-2018-1/P12)

\section{System}

Scale $1: 75$

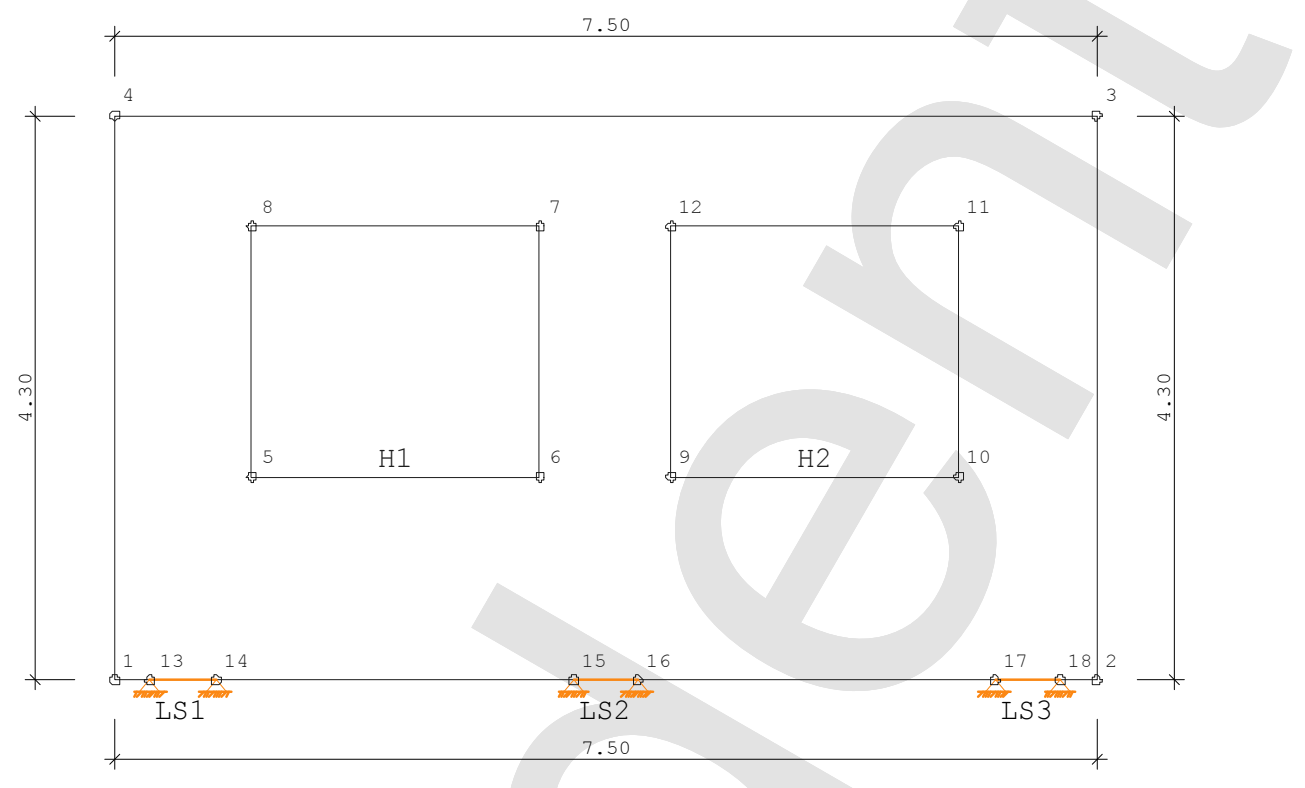


Demo Frilo

Stuttgarter Straße 40

70469

Stuttgart

LOADCASE 1 "POS. S.11 - Dead Load"

Type:

Self-weight of the slab is included:

Action:

Partial safety factor:

Load points:

Point loads:

\section{Forces, vertical}

Total of input loads: (portion on the slab)

Self-weight of the slab:

Total of all loads:

Total of all support reactions:

\section{Forces, horizontal}

Total of input loads: (portion on the slab)

Total of all support reactions:

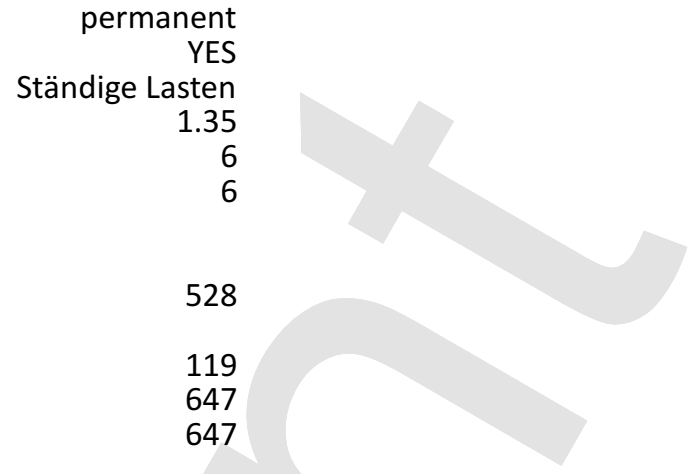

0

\section{NOTE}

All effects of actions (like internal forces, support reactions, displacements, etc.) of an individual load case are, unlike the results of a superposition of load cases, simple, i.e. characteristic, values

Design results are based on design quantities including the partial safety coefficients.

Load case 1 "POS. S.11 - Dead Load"

Point loads

Scale $1: 75$ 
Demo Frilo

Stuttgarter Straße 40

70469
Tel.: 0711810020

Fax: 0711858020
Projekt: Design Calculations

Position: W.14a - Load Bearing Concrete

Wall Double

26.07.2018
Seite: 3

Load case 1 "POS. S.11 - Dead Load"

Principal Forces [kN/m]

Scale $1: 50$

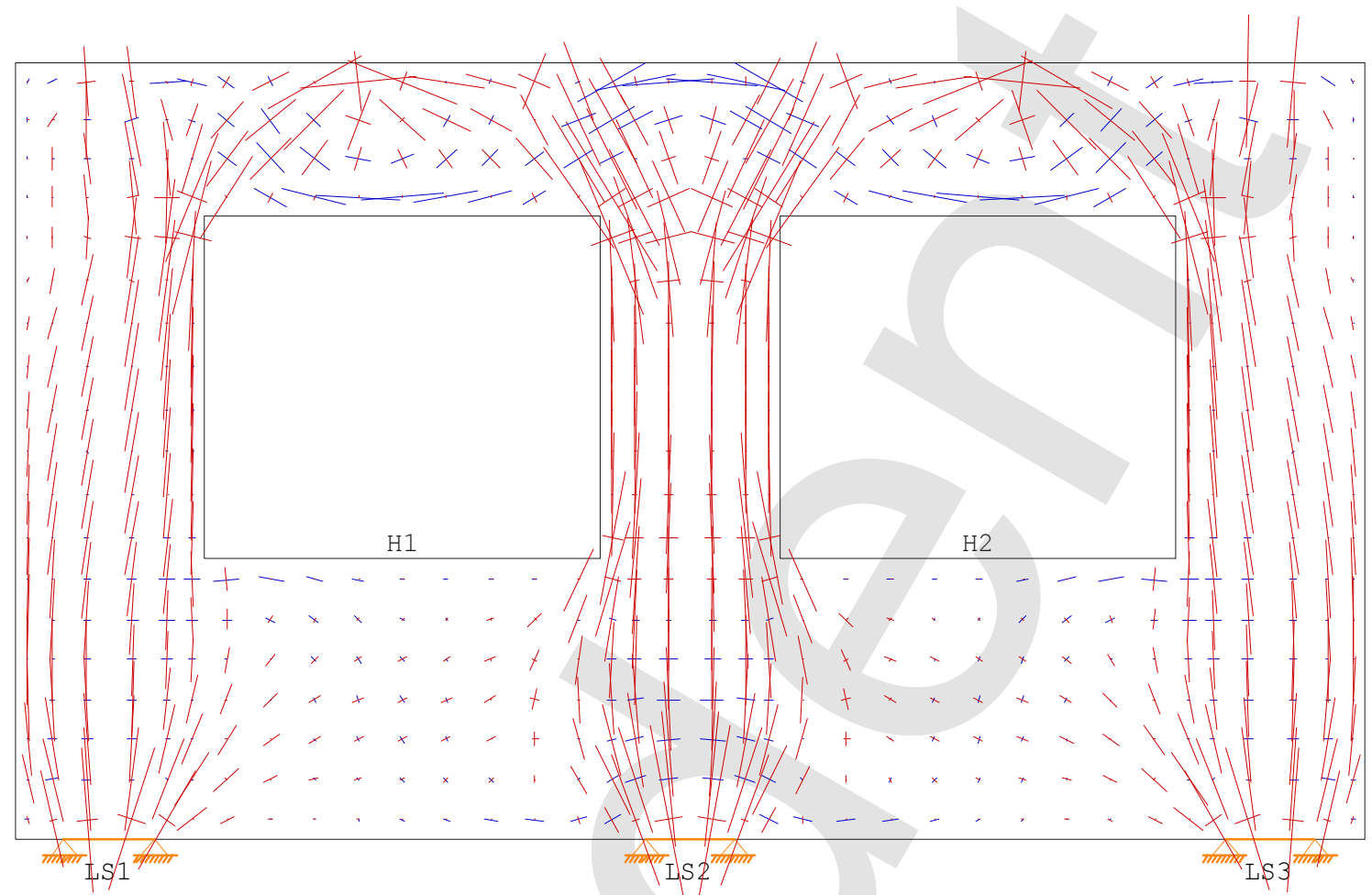


Demo Frilo

Stuttgarter Straße 40

70469

Stuttgart

LOADCASE 2 "POS. S.11 - Live Load"

Type:

Self-weight of the slab is included:

Action:

Partial safety factor:

Load points:

Point loads:

\section{Forces, vertical}

Total of input loads: (portion on the slab)

Total of all support reactions:

\section{Forces, horizontal}

Total of input loads: (portion on the slab)

Total of all support reactions:

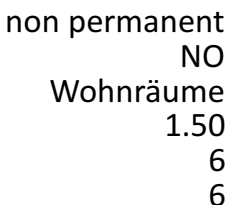

non permanent

NO

Wohnräume

1.50

6

186

186

\section{0}

0

\section{NOTE}

All effects of actions (like internal forces, support reactions,

displacements, etc.) of an individual load case are, unlike the results of a superposition of load cases, simple, i.e. characteristic, values

Design results are based on design quantities including the partial safety coefficients.

Load case 2 "POS. S.11 - Live Load"

Point loads

Scale $1: 75$ 
Demo Frilo

Stuttgarter Straße 40

70469

Stuttgart
Tel.: 0711810020

Fax: 0711858020
Projekt: Design Calculations

Position: W.14a - Load Bearing Concrete

Wall Double

26.07.2018
Seite: 5

Load case 2 "POS. S.11 - Live Load"

Principal Forces [kN/m]

Scale $1: 50$

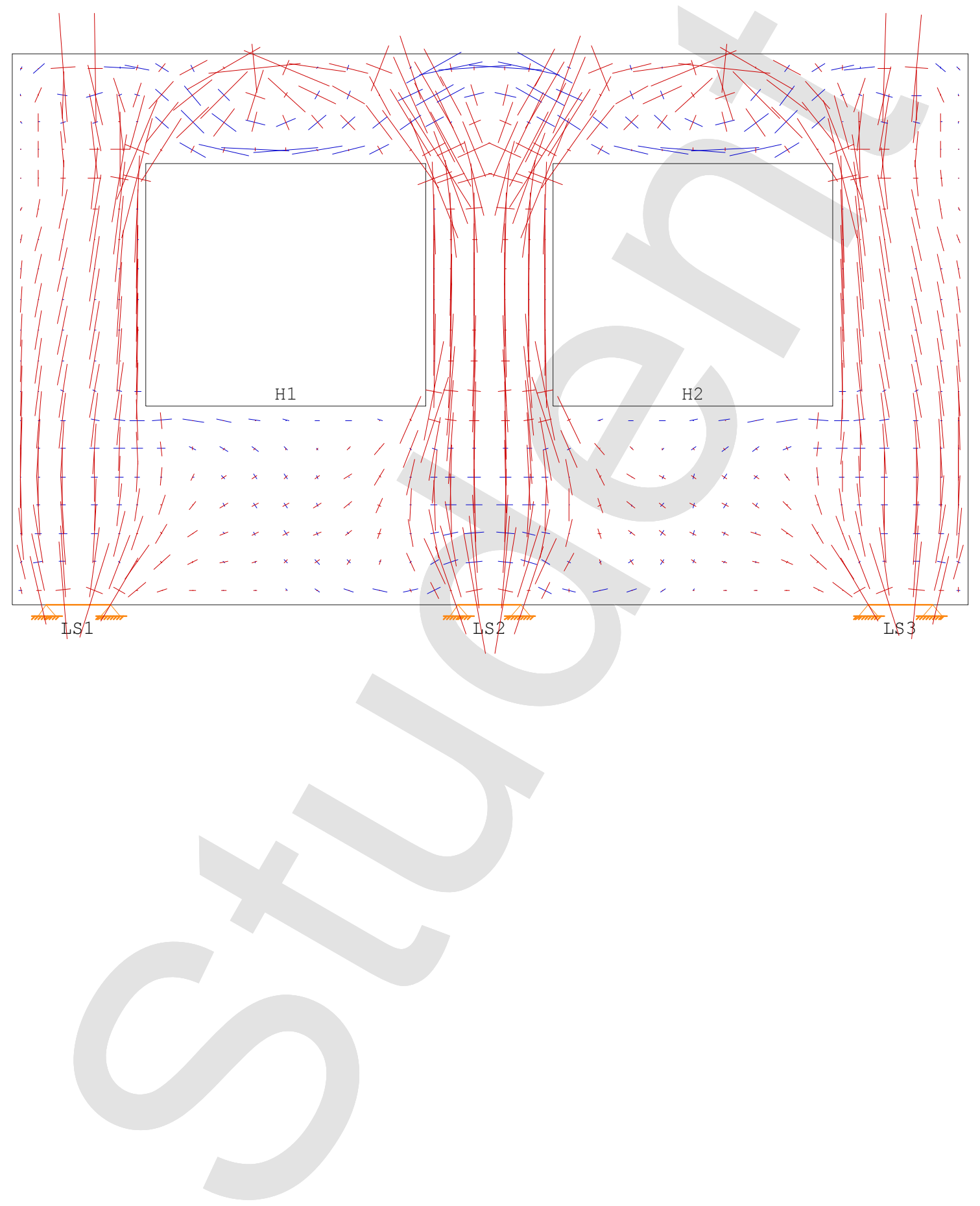


Demo Frilo

Stuttgarter Straße 40

70469

Stuttgart
Tel.: 0711810020

Fax: 0711858020
Projekt: Design Calculations

Position: W.14a - Load Bearing Concrete Wall Double

26.07.2018

\section{SUPERPOSITION 1 "Characteristic"}

\begin{tabular}{|c|c|c|c|c|c|}
\hline \multicolumn{6}{|c|}{ Load Cases Involved } \\
\hline Number & Load case & Type & $\begin{array}{r}\text { Self } \\
\text { Self- } \\
\text { Weight }\end{array}$ & $\begin{array}{l}\text { Action } \\
\text { Shrt Name } \\
\text { Hnd }\end{array}$ & $\begin{array}{l}\text { Alter- } \\
\text { native } \\
\text { Group }\end{array}$ \\
\hline $\begin{array}{l}1 \\
2\end{array}$ & $\begin{array}{l}11 \text { - Dea... } \\
11 \text { - Liv... }\end{array}$ & $\begin{array}{l}\text { permanent } \\
\text { non perm }\end{array}$ & $\begin{array}{c}\text { yes } \\
\text { no }\end{array}$ & $\begin{array}{l}\text { g Ständige ... } \\
1 \text { Wohnräume }\end{array}$ & \\
\hline
\end{tabular}

\begin{tabular}{|rcr|}
\hline Action & & \\
\hline Number & $\begin{array}{c}\text { Shrt Name } \\
\text { Hnd }\end{array}$ & Type \\
\hline 1 & $\begin{array}{l}1 \text { Wohnräume } \\
\text { g Ständige Lasten }\end{array}$ & $\begin{array}{c}\text { non perm } \\
\text { permanent }\end{array}$ \\
\hline
\end{tabular}


Demo Frilo

Stuttgarter Straße 40

$70469 \quad$ Stuttgart
Tel.: 0711810020

Fax: 0711858020
Projekt: Design Calculations

Position: W.14a - Load Bearing Concrete

Wall Double

26.07.2018

Seite: 7

SUPERPOSITION 2 "ULS Permanent/Transient"

\section{Load Cases Involved}

\begin{tabular}{|c|c|c|c|c|c|}
\hline Number & Load case & Type & $\begin{array}{r}\text { Self } \\
\text { Self- } \\
\text { Weight }\end{array}$ & $\begin{array}{l}\text { Action } \\
\text { Shrt Name } \\
\text { Hnd }\end{array}$ & $\begin{array}{l}\text { Alter- } \\
\text { native } \\
\text { Group }\end{array}$ \\
\hline $\begin{array}{l}1 \\
2\end{array}$ & $\begin{array}{l}\text { POS. S.11 - Dea... } \\
\text { POS. S.11 - Liv... }\end{array}$ & $\begin{array}{r}\text { permanent } \\
\text { non perm }\end{array}$ & $\begin{array}{c}\text { yes } \\
\text { no }\end{array}$ & $\begin{array}{l}\text { g Ständige ... } \\
1 \text { Wohnräume }\end{array}$ & 0 \\
\hline
\end{tabular}

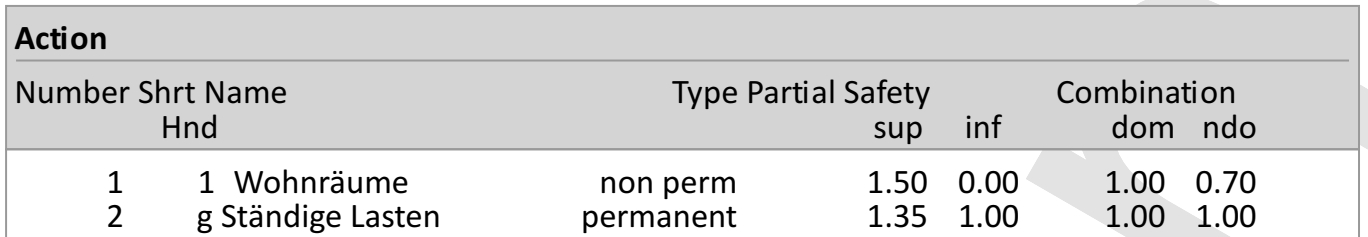

\section{NOTE: Design Values}

All results of a superposition of load cases

Include both partial safety and combination

factors: DIN EN 1990/NA:2010-12

\section{NOTE: Combination Factors}

With the combination of independent, variable actions

the individual dominant action is determined for both each location and for each action quantity.

In geneneral, the dominant actions differ with each

location and each quantity

The individually found dominant action receives the conbination factor 1.00 . In case of only one variable action this action is considered dominant.

\section{Superposition 2 "ULS Permanent/Transient"} Concrete Load (Compressive) uC-1, uC-2 [\%]

Scale $1: 50$

\begin{tabular}{|c|c|c|c|c|c|c|c|c|c|c|c|c|c|c|}
\hline 8 & 8 & 9 & 21 & 21 & 10 & 20 & 3 & 20 & 10 & 21 & 21 & 9 & 10 & 11 \\
\hline 13 & 21 & 9 & 14 & 14 & 10 & 20 & 3 & 20 & 10 & 14 & 14 & 9 & 33 & 26 \\
\hline 3 & 4 & 15 & 11 & 10 & 9 & 21 & 8 & 21 & 9 & 10 & 11 & 15 & 4 & \\
\hline 8 & 19 & 24 & 16 & 10 & 9 & 29 & 12 & 29 & 9 & 10 & 16 & 24 & 15 & 9 \\
\hline 2 & 16 & 11 & T & 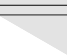 & 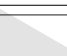 & 24 & 9 & 24 & & & & 12 & 16 & 2 \\
\hline 6 & 24 & 25 & & & 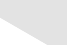 & 28 & 16 & 28 & & & & 25 & 24 & 6 \\
\hline 2 & 2 & & & & & 1 & 1 & 1 & & & & & 2 & 2 \\
\hline 10 & 19 & 19 & & & & 17 & 17 & 17 & & & & 19 & 19 & 10 \\
\hline 11 & 16 & 16 & & & & 17 & $\begin{array}{c}1 \\
17\end{array}$ & 17 & & & & 16 & 16 & 11 \\
\hline 12 & $\begin{array}{c}1 \\
12\end{array}$ & 13 & & 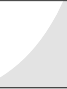 & $\begin{array}{l}1 \\
1\end{array}$ & \begin{tabular}{l|l}
1 & 0 \\
1 & 9
\end{tabular} & $\begin{array}{c}3 \\
15\end{array}$ & $\begin{array}{l}10 \\
20\end{array}$ & $\begin{array}{l}1 \\
1\end{array}$ & & & 13 & $\begin{array}{c}1 \\
12\end{array}$ & 12 \\
\hline & & 1 & 2 & 1 & 2 & 9 & 2 & 9 & 2 & 1 & 2 & 1 & & \\
\hline 12 & 12 & 8 & 2 & 1 & 3 & 18 & 14 & 18 & 3 & 1 & 2 & 8 & 11 & 12 \\
\hline & & 3 & 3 & 2 & 2 & 2 & & 2 & 2 & 2 & 3 & 3 & & \\
\hline 14 & 13 & 6 & 2 & 2 & 3 & 14 & 17 & 14 & 3 & 2 & 2 & 6 & 13 & 14 \\
\hline 7 & 14 & 5 & 2 & 2 & 2 & 9 & 13 & 9 & 2 & 2 & 2 & 5 & 14 & 7 \\
\hline 23 & 24 & 5 & & 2 & 2 & 20 & 31 & 20 & 2 & 2 & & 6 & 24 & 23 \\
\hline
\end{tabular}

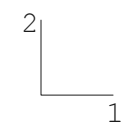


Demo Frilo

Stuttgarter Straße 40

70469

Stuttgart
Tel.: 0711810020

Fax: 0711858020
Projekt: Design Calculations

Position: W.14a - Load Bearing Concrete

Wall Double

26.07.2018
Seite: 8

\section{Superposition 2 "ULS Permanent/Transient"}

Reinforcement, Total aS-1, aS-2 $\left[\mathrm{cm}^{2} / \mathrm{m}\right]$ Total

Scale $1: 50$

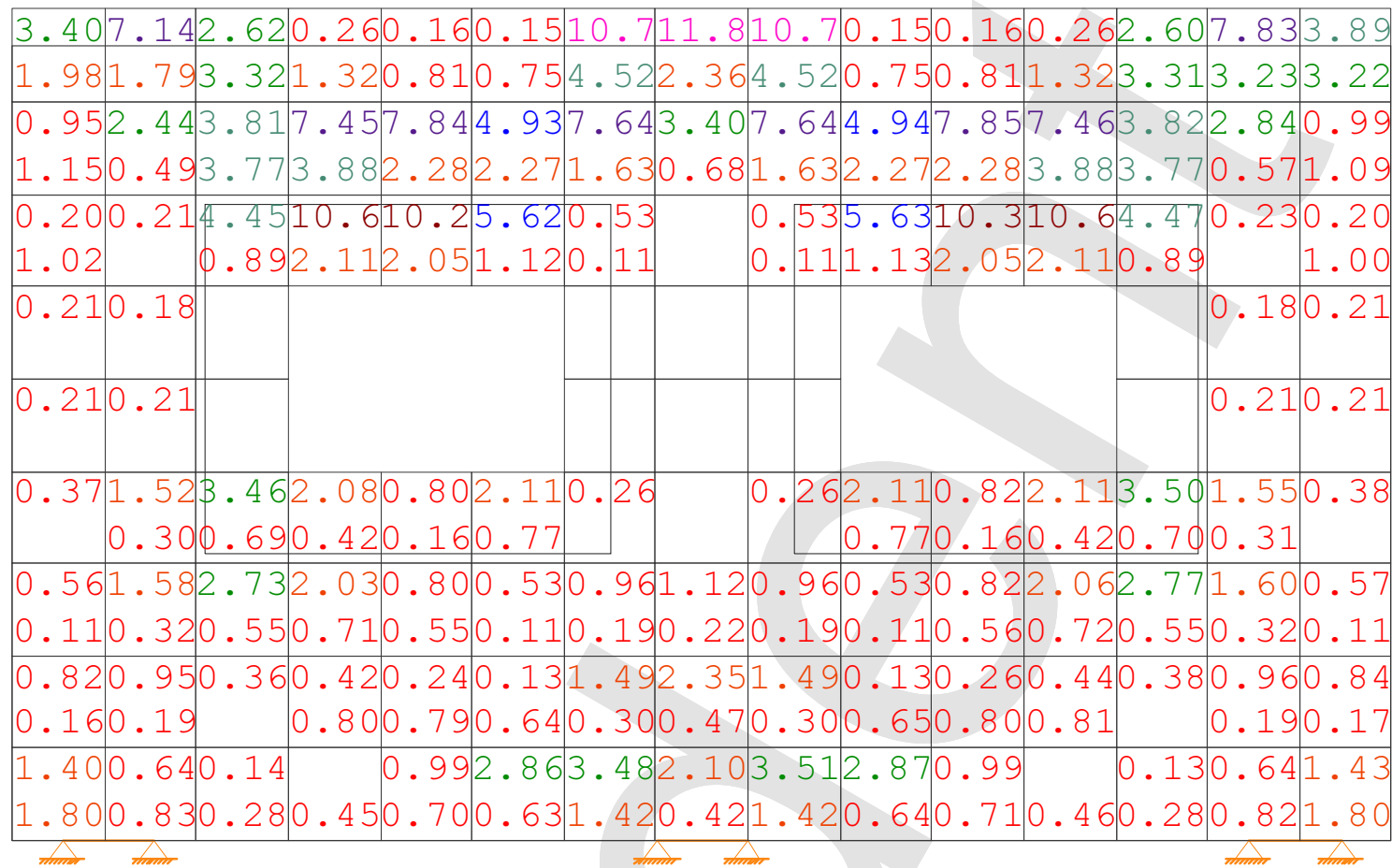

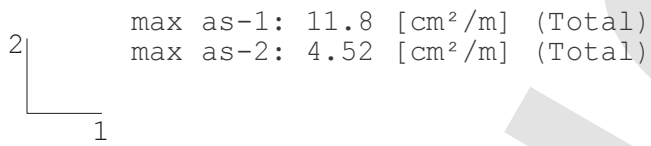


Demo Frilo

Stuttgarter Straße 40

70469

Stuttgart
Tel.: 0711810020

Fax: 0711858020
Projekt: Design Calculations

Position: W.14a - Load Bearing Concrete

Wall Contin

26.07.2018

\section{Position: W.14a - Load Bearing Concrete Wall_Contin}

Panels by Finite Elements SCN 01/2018 (Frilo R-2018-1/P12)

\section{System}

Scale $1: 75$

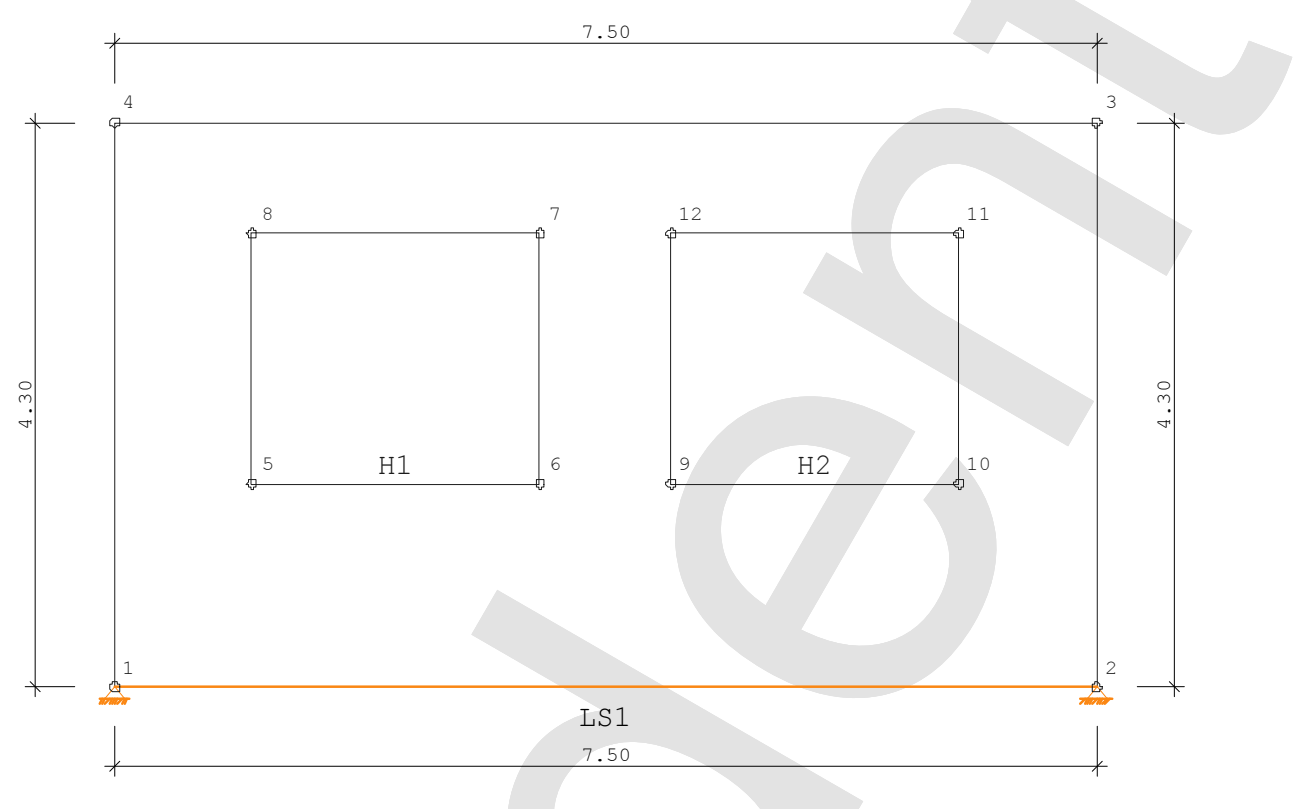


Demo Frilo

Stuttgarter Straße 40

70469

Stuttgart

LOADCASE 1 "POS. S.11 - Dead Load"

Type:

Self-weight of the slab is included:

Action:

Partial safety factor:

Load points:

Point loads:

\section{Forces, vertical}

Total of input loads: (portion on the slab)

Self-weight of the slab:

Total of all loads:

Total of all support reactions:

\section{Forces, horizontal}

Total of input loads: (portion on the slab)

Total of all support reactions:

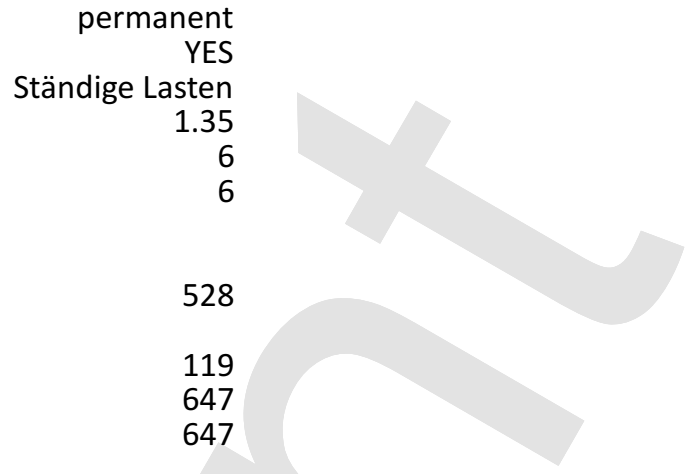

\section{NOTE}

All effects of actions (like internal forces, support reactions, displacements, etc.) of an individual load case are, unlike the results of a superposition of load cases, simple, i.e. characteristic, values

Design results are based on design quantities including the partial safety coefficients.

Load case 1 "POS. S.11 - Dead Load"

Point loads

Scale $1: 75$

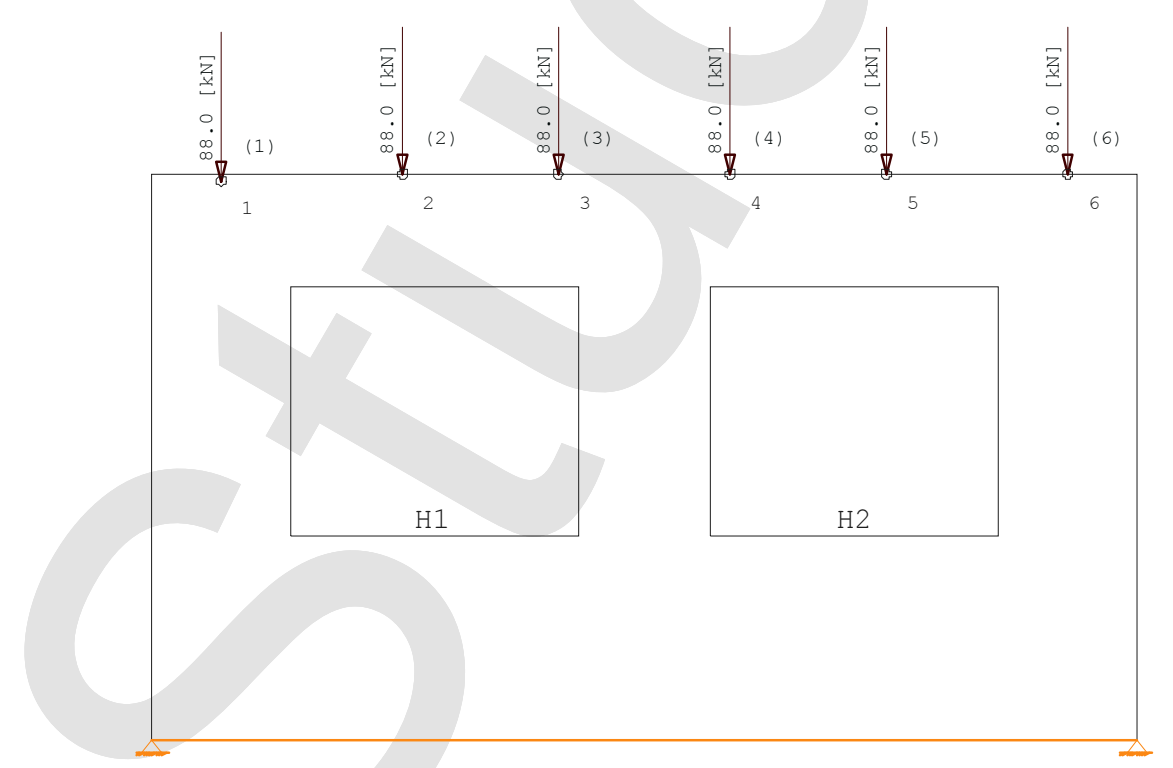


Demo Frilo

Stuttgarter Straße 40

70469
Tel.: 0711810020

Fax: 0711858020
Projekt: Design Calculations

Position: W.14a - Load Bearing Concrete

Wall Contin

26.07.2018
Seite: 3

Load case 1 "POS. S.11 - Dead Load"

Principal Forces [kN/m]

Scale $1: 50$

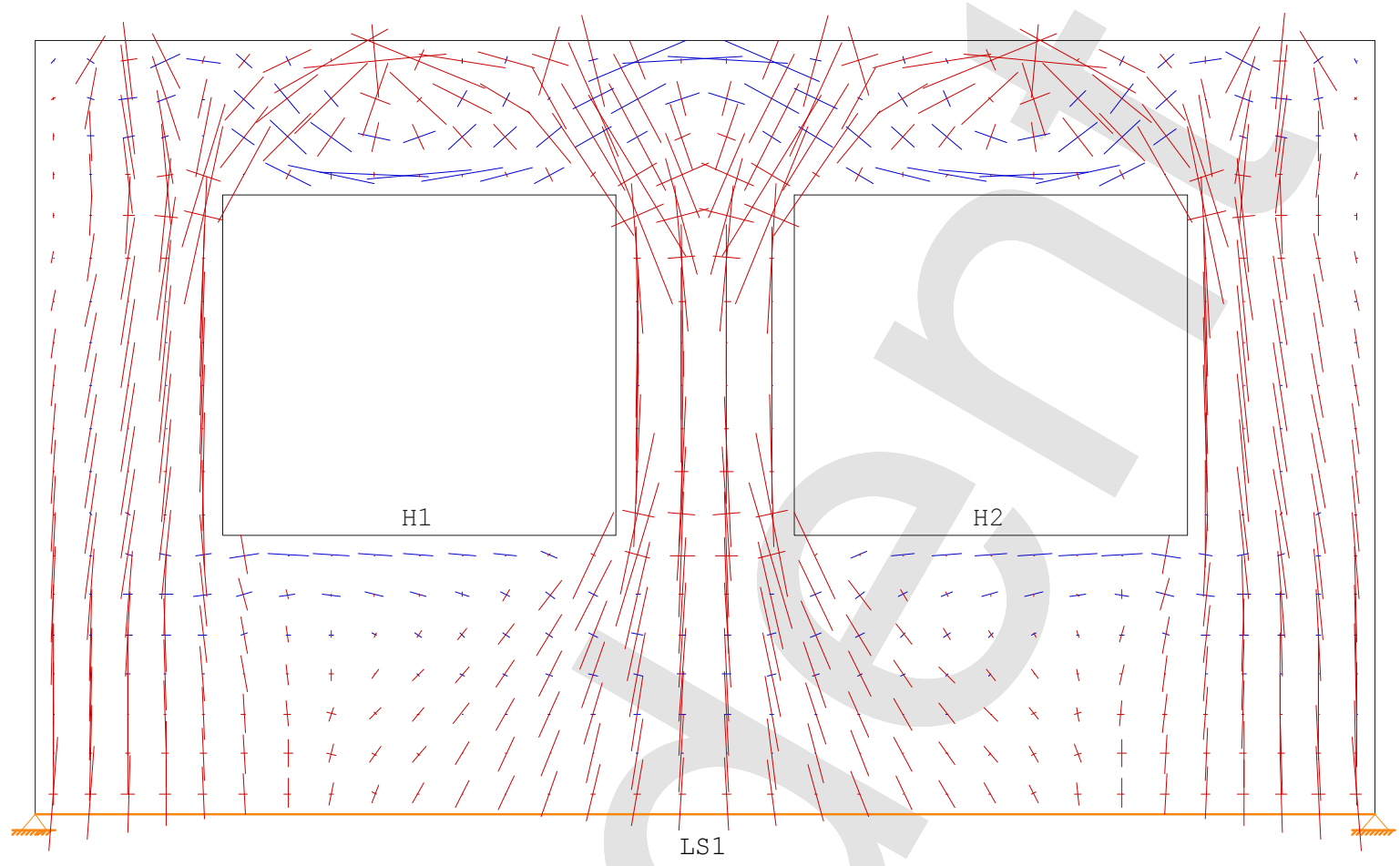


Demo Frilo

Stuttgarter Straße 40

70469

Stuttgart

LOADCASE 2 "POS. S.11 - Live Load"

Type:

Self-weight of the slab is included:

Action:

Partial safety factor:

Load points:

Point loads:

\section{Forces, vertical}

Total of input loads: (portion on the slab)

Total of all support reactions:

\section{Forces, horizontal}

Total of input loads: (portion on the slab)

Total of all support reactions:

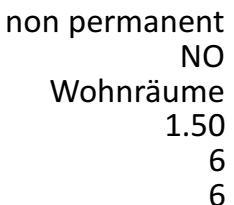

non permanent

NO

Wohnräume

1.50

6

186

186

\section{0}

0

\section{NOTE}

All effects of actions (like internal forces, support reactions,

displacements, etc.) of an individual load case are, unlike the results of a superposition of load cases, simple, i.e. characteristic, values

Design results are based on design quantities including the partial safety coefficients.

Load case 2 "POS. S.11 - Live Load"

Point loads

Scale $1: 75$ 
Demo Frilo

Stuttgarter Straße 40

70469

Stuttgart

Load case 2 "POS. S.11 - Live Load"

Principal Forces [kN/m]

Scale $1: 50$

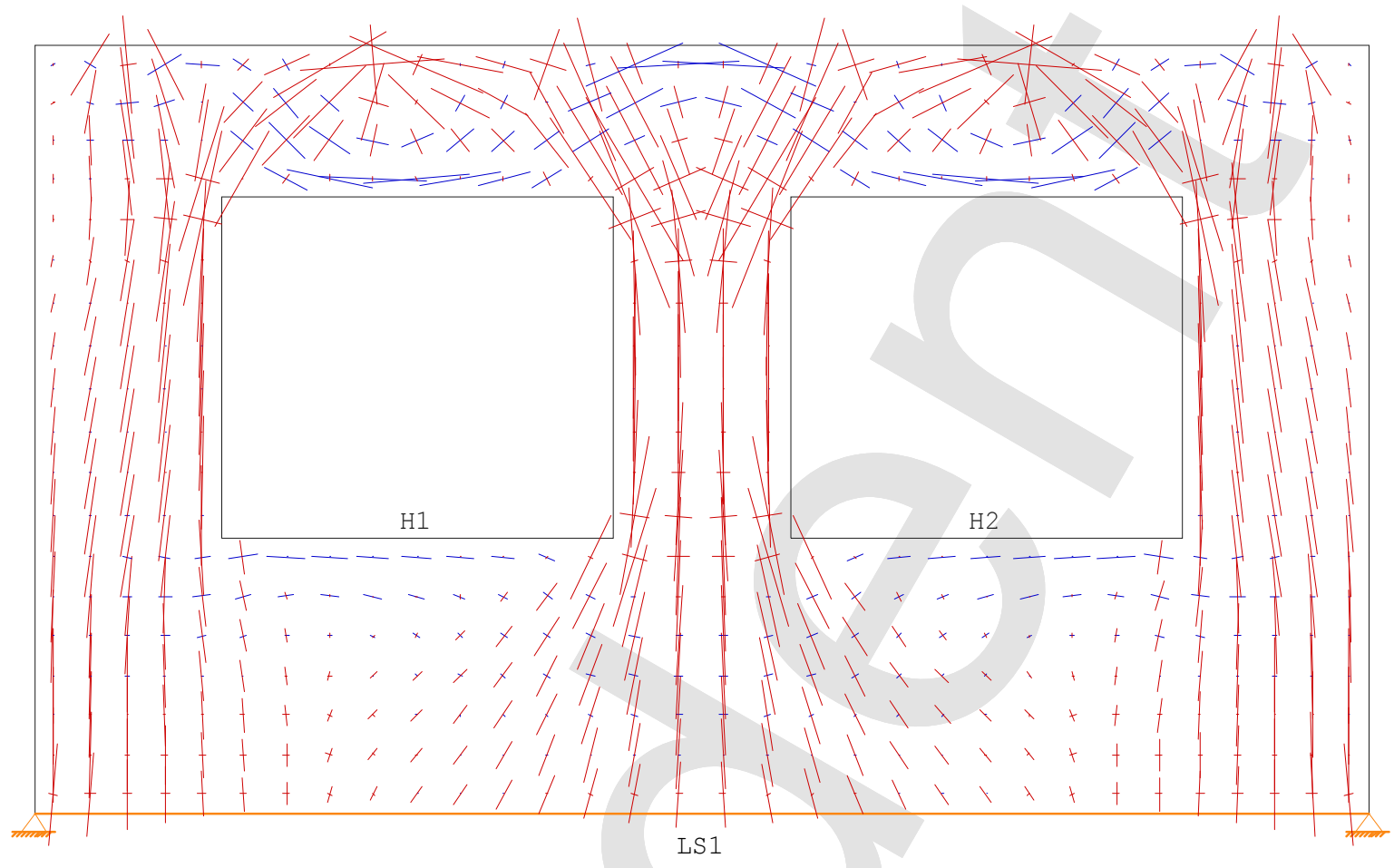


Demo Frilo

Stuttgarter Straße 40

70469

Stuttgart
Tel.: 0711810020

Fax: 0711858020
Projekt: Design Calculations

Position: W.14a - Load Bearing Concrete

Wall Contin

26.07.2018

\section{SUPERPOSITION 1 "Characteristic"}

\begin{tabular}{|c|c|c|c|c|c|}
\hline \multicolumn{6}{|c|}{ Load Cases Involved } \\
\hline Number & Load case & Type & $\begin{array}{r}\text { Self } \\
\text { Self- } \\
\text { Weight }\end{array}$ & $\begin{array}{l}\text { Action } \\
\text { Shrt Name } \\
\text { Hnd }\end{array}$ & $\begin{array}{l}\text { Alter- } \\
\text { native } \\
\text { Group }\end{array}$ \\
\hline $\begin{array}{l}1 \\
2\end{array}$ & $\begin{array}{l}11 \text { - Dea... } \\
11 \text { - Liv... }\end{array}$ & $\begin{array}{l}\text { permanent } \\
\text { non perm }\end{array}$ & $\begin{array}{c}\text { yes } \\
\text { no }\end{array}$ & $\begin{array}{l}\text { g Ständige ... } \\
1 \text { Wohnräume }\end{array}$ & \\
\hline
\end{tabular}

\begin{tabular}{|rcr|}
\hline Action & & \\
\hline Number & $\begin{array}{c}\text { Shrt Name } \\
\text { Hnd }\end{array}$ & Type \\
\hline 1 & g Ständige Lasten & $\begin{array}{c}\text { permanent } \\
\text { non perm }\end{array}$ \\
\hline 2 & 1 Wohnräume & \\
\hline
\end{tabular}


Demo Frilo

Stuttgarter Straße 40

70469 Stuttgart
Tel.: 0711810020

Fax: 0711858020
Projekt: Design Calculations

Position: W.14a - Load Bearing Concrete

Wall_Contin

26.07.2018

Seite: 7

SUPERPOSITION 2 "ULS Permanent/Transient"

\section{Load Cases Involved}

\begin{tabular}{|cccccc|}
\hline Number & Load case & Type & $\begin{array}{r}\text { Self } \\
\text { Self- } \\
\text { Weight }\end{array}$ & $\begin{array}{l}\text { Action } \\
\text { Shrt Name } \\
\text { Hnd }\end{array}$ & $\begin{array}{c}\text { Alter- } \\
\text { native } \\
\text { Group }\end{array}$ \\
\hline 1 & POS. S.11 - Dea... & $\begin{array}{r}\text { permanent } \\
\text { non perm }\end{array}$ & $\begin{array}{c}\text { yes } \\
\text { no }\end{array}$ & $\begin{array}{c}\text { g Ständige ... } \\
1 \text { Wohnräume }\end{array}$ & - \\
\hline
\end{tabular}

\begin{tabular}{|ccccccc|}
\hline \multicolumn{1}{|l|}{ Action } & & & & \\
\hline $\begin{array}{c}\text { Number Shrt Name } \\
\text { Hnd }\end{array}$ & Type Partial Safety & \multicolumn{2}{c|}{$\begin{array}{c}\text { Combination } \\
\text { dom ndo }\end{array}$} \\
\hline 1 & g Ständige Lasten & permanent & 1.35 & 1.00 & 1.00 & 1.00 \\
2 & 1 Wohnräume & non perm & 1.50 & 0.00 & 1.00 & 0.70 \\
\hline
\end{tabular}

\section{NOTE: Design Values}

All results of a superposition of load cases

Include both partial safety and combination

factors: DIN EN 1990/NA:2010-12

\section{NOTE: Combination Factors}

With the combination of independent, variable actions

the individual dominant action is determined for both each location and for each action quantity.

In geneneral, the dominant actions differ with each

location and each quantity

The individually found dominant action receives the conbination factor 1.00 . In case of only one variable action this action is considered dominant.

\section{Superposition 2 "ULS Permanent/Transient"} Concrete Load (Compressive) uC-1, uC-2 [\%]

Scale $1: 50$

\begin{tabular}{|c|c|c|c|c|c|c|c|c|c|c|c|c|c|c|}
\hline 7 & 7 & 9 & 21 & 21 & 10 & 17 & 3 & 17 & 10 & 21 & 21 & 9 & 10 & 9 \\
\hline 16 & 18 & 9 & 14 & 14 & 10 & 19 & 3 & 19 & 10 & 14 & 14 & 9 & 21 & 19 \\
\hline 1 & 3 & 13 & 11 & 10 & 9 & 19 & 8 & 19 & 9 & 10 & 11 & 13 & 3 & 1 \\
\hline 14 & 18 & 22 & 15 & 11 & 9 & 29 & 11 & 29 & 9 & 11 & 15 & 22 & 18 & 14 \\
\hline 1 & 13 & 10 & 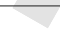 & 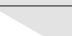 & 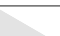 & 20 & 9 & 20 & & & & 10 & 13 & 1 \\
\hline 7 & 21 & 24 & & & 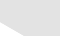 & 26 & 16 & 26 & & & & 24 & 21 & 7 \\
\hline 1 & 2 & & & 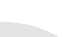 & & $t$ & 1 & $t$ & & & & & 2 & 1 \\
\hline 10 & 17 & 19 & & & & 17 & 17 & 17 & & & & 19 & 17 & 10 \\
\hline 10 & 16 & 16 & & & & 17 & 17 & 17 & & & & 16 & 16 & 10 \\
\hline 11 & 1 & 14 & & & & $2{ }_{1}$ & 15 & 21 & & & & 14 & 12 & 11 \\
\hline 11 & 11 & 11 & 1 & 2 & 6 & $\begin{array}{l}10 \\
20\end{array}$ & 13 & 10 & $\begin{array}{l}4 \\
6\end{array}$ & 2 & 1 & 11 & 11 & 11 \\
\hline 12 & 9 & $\begin{array}{l}1 \\
6\end{array}$ & $\begin{array}{l}1 \\
2\end{array}$ & $\begin{array}{l}2 \\
3\end{array}$ & $\begin{array}{l}4 \\
7\end{array}$ & 13 & 13 & 13 & $\begin{array}{l}4 \\
7\end{array}$ & 2 & $\begin{array}{l}1 \\
2\end{array}$ & 1 & 9 & 12 \\
\hline 3 & 2 & 1 & 1 & 2 & 2 & 2 & 2 & 2 & 2 & 2 & 1 & 1 & 2 & 3 \\
\hline 12 & 7 & 4 & 2 & 4 & 7 & 9 & 9 & 9 & 7 & 4 & 2 & 4 & 7 & 12 \\
\hline
\end{tabular}

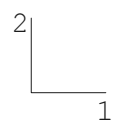


Demo Frilo

Stuttgarter Straße 40

70469

Stuttgart
Tel.: 0711810020

Fax: 0711858020
Projekt: Design Calculations

Position: W.14a - Load Bearing Concrete

Wall Contin

26.07.2018
Seite: 8

\section{Superposition 2 "ULS Permanent/Transient"}

Reinforcement, Total aS-1, aS-2 $\left[\mathrm{cm}^{2} / \mathrm{m}\right]$ Total

Scale $1: 50$

\begin{tabular}{|c|c|c|c|c|c|c|c|c|c|c|c|c|c|c|}
\hline 1.81 & 4.82 & 2.42 & 0.24 & 0.18 & 0.18 & 9.90 & 12.5 & 9.90 & 0.18 & 0.18 & 0.24 & 2.39 & 5.27 & 2.60 \\
\hline 1.36 & 1.71 & 3.25 & 1.19 & 0.92 & 0.88 & 3.84 & 2.50 & 3.84 & 0.88 & 0.92 & 1.18 & 3.24 & 1.57 & 1.22 \\
\hline 1.27 & 2.22 & 3.93 & 7.78 & 8.14 & 5.02 & 7.76 & 3.58 & 7.76 & 5.03 & 8.15 & 7.79 & 3.94 & 2.27 & 1.39 \\
\hline 1.14 & 0.44 & 3.69 & 3.78 & 2.41 & 2.36 & 1.69 & 0.72 & 1.69 & 2.37 & 2.41 & 3.78 & 3.69 & 0.45 & 1.07 \\
\hline 0.18 & & 5.04 & 10.9 & 10.5 & 5.59 & 0.61 & & 0.61 & 5.59 & 10.5 & 10.9 & 5.05 & 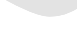 & 0.18 \\
\hline 0.91 & & 1.01 & 2.18 & 2.09 & 1.12 & 0.12 & & 0.12 & 1.12 & 2.09 & 2.18 & 1.01 & & 0.89 \\
\hline 0.14 & 0.12 & & & & & & & & & & & & 0.12 & 0.14 \\
\hline 0.15 & 0.12 & & & & & & & & & & & & 0.12 & 0.14 \\
\hline 0.22 & 0.48 & 4.27 & 3.43 & 2.74 & 2.83 & 0.31 & & 0.31 & 2.83 & 2.74 & 3.43 & 4.27 & 0.48 & 0.22 \\
\hline & & 0.85 & 0.69 & 0.55 & 0.82 & & & & 0.82 & 0.55 & 0.69 & 0.85 & & \\
\hline 0.40 & 1.57 & 2.89 & 2.79 & 2.30 & 1.85 & 1.39 & 0.29 & 1.39 & 1.85 & 2.30 & 2.79 & 2.89 & 1.57 & 0.40 \\
\hline & 0.31 & 0.58 & 0.56 & 0.46 & 0.76 & 0.59 & & 0.59 & 0.76 & 0.46 & 0.56 & 0.58 & 0.31 & \\
\hline \multirow[t]{3}{*}{0.32} & 0.72 & 0.63 & 0.39 & 0.60 & 0.96 & 1.09 & 0.67 & 1.09 & 0.96 & 0.60 & 0.39 & 0.63 & 0.72 & 0.32 \\
\hline & 0.14 & 0.13 & & 0.30 & 0.27 & 0.22 & 0.13 & 0.22 & 0.27 & 0.30 & & 0.13 & 0.14 & \\
\hline & & & & 0.12 & 0.10 & 0.22 & 0.32 & 0.22 & 0.10 & 0.12 & & & & \\
\hline
\end{tabular}

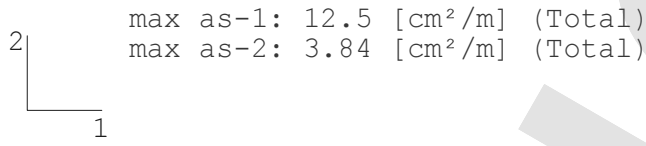


B.1.10. Design Calculations POS. V.31 - Steel Canopy 
Demo Frilo

Stuttgarter Straße 40

70469 Stuttgart
Tel.: 0711810020

Fax: 0711858020
Projekt: Design Calculations

Position: V.31.1 - Steel Canopy

28.07.2018
Seite: 1

\section{Position: V.31.1 - Steel Canopy}

Durchlaufträger DLT10 01/2018 (Frilo R-2018-1/P12)

Scale $1: 25$

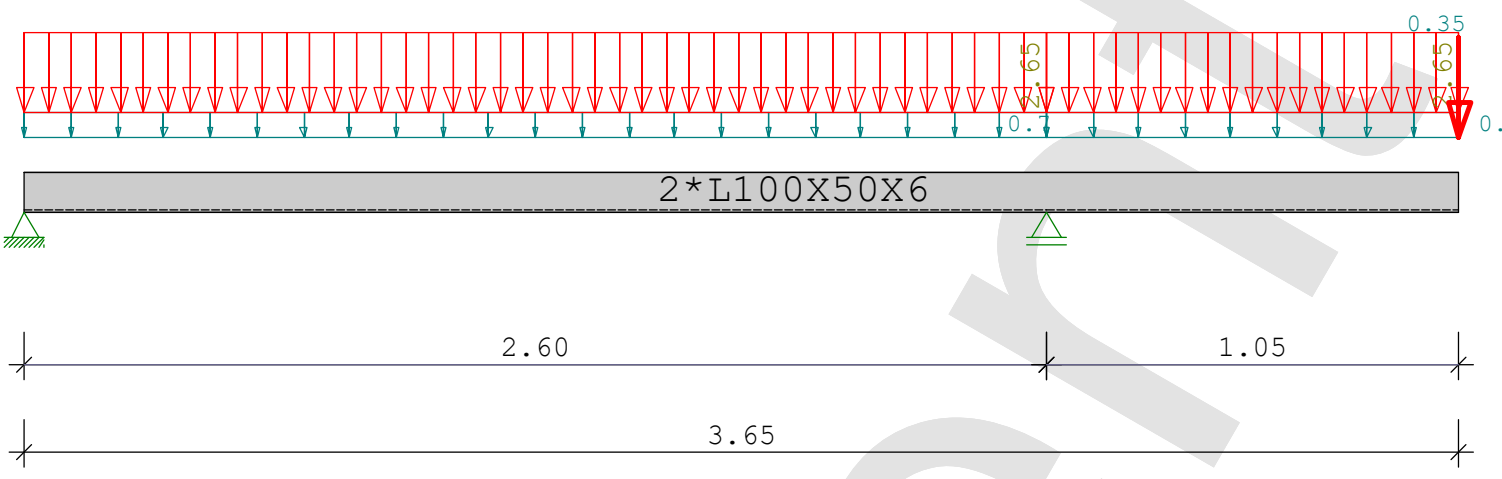

Steel girder S235 DIN EN 1993-1-1/NA:2010-12

E-modulus $E=210000 \mathrm{~N} / \mathrm{mm} 2$

\begin{tabular}{|llllllll|}
\hline System & length & \multicolumn{5}{c|}{ cross-section values } \\
\hline Span & $\mathrm{L}(\mathrm{m})$ & & CsNo. & $\mathrm{I}(\mathrm{cm} 4)$ & $\mathrm{St}(\mathrm{cm} 3)$ & $\mathrm{Sb}(\mathrm{cm} 3)$ \\
\hline $\begin{array}{l}1 \\
\begin{array}{l}\text { Cantilever } \\
\text { right }\end{array}\end{array}$ & 2.600 & constant & 1 & 179.4 & 27.6 & 2 & L100X50X6 \\
\hline
\end{tabular}

\begin{tabular}{|c|c|c|c|c|}
\hline \multirow{2}{*}{$\begin{array}{l}\text { Load type } \\
\qquad(\mathrm{kN}, \mathrm{m}) \\
\text { Span Type AG G }\end{array}$} & \multicolumn{2}{|c|}{$\begin{array}{l}\text { 1=uniform over } \mathrm{L} \\
\text { 3=single moment at a } \\
\text { 5=triangular over } \mathrm{L}\end{array}$} & \multicolumn{2}{|c|}{$\begin{array}{l}\text { 2=concentrated at } a \\
\text { 4=trapezoidal btw. } a, a+b \\
6=\text { trapezoidal over } L\end{array}$} \\
\hline & $r$ g_ll/r & factor & distanc & e length fromltem Phi \\
\hline $\begin{array}{cc}1 & 1 \\
\text { Cantilever }\end{array}$ & 0.700 & 2.650 & & \\
\hline $\begin{array}{lll}\mathrm{CaRi} & 1 & \mathrm{~J} \\
& 2 & \mathrm{~J}\end{array}$ & $\begin{array}{l}0.700 \\
0.000\end{array}$ & $\begin{array}{ll}2.650 & 1.000 \\
0.350 & 1.000\end{array}$ & 1.050 & \\
\hline
\end{tabular}

Dead load of girder is considered with Gamma

$=78.5 \mathrm{kN} / \mathrm{m} 3$.

\section{Actions:}

No. Cl Name

$\psi 0 \quad \psi 1 \quad \psi 2 \quad \gamma$

J 3 Snow loads $<1000$ m

$\begin{array}{llll}0.50 & 0.20 & 0.00 & 1.50\end{array}$

Consequency class CC 2 acc. EN 1990 Tab. B1 $\rightarrow \mathrm{K}_{\mathrm{Fi}}=1.0$ Tab. B3

In following tables the last cell the row is a reference to

the number of the related superposition ( see below).

In tables with internal forces multiplied by Gamma is additionally

a reference to the main action.

\begin{tabular}{|c|c|c|c|c|c|c|c|}
\hline \multicolumn{8}{|c|}{ Results for 1 -times loads } \\
\hline \multicolumn{6}{|c|}{ Span moments maximum } & \multicolumn{2}{|c|}{$(\mathrm{kNm}, \mathrm{kN})$} \\
\hline Span & & Mf & M le & M ri & V le & V ri & comb \\
\hline 1 & $x 0=1.250$ & 2.72 & 0.00 & -0.46 & 4.36 & -4.71 & 2 \\
\hline
\end{tabular}


Demo Frilo

Stuttgarter Straße 40

70469
Tel.: 0711810020

Fax: 0711858020
Projekt: Design Calculations

Position: V.31.1 - Steel Canopy

28.07.2018

\begin{tabular}{|cccccccc|}
\hline \multicolumn{2}{l}{ Support moments maximum } & \multicolumn{3}{c|}{$(\mathrm{kNm}, \mathrm{kN})$} \\
\hline Column & M le & M ri & V le & V ri & $\max F$ & $\min F$ & comb \\
\hline 1 & 0.00 & 0.00 & 0.00 & 4.36 & 4.36 & 0.21 & 2 \\
2 & -2.29 & -2.29 & -5.41 & 4.01 & 9.43 & 2.14 & 3 \\
\hline
\end{tabular}

\begin{tabular}{|c|c|c|c|c|c|c|}
\hline \multicolumn{6}{|c|}{ Support reactions } & \multirow[t]{2}{*}{$(\mathrm{kN})$} \\
\hline Column & by & $\max q$ & $\min q$ & Fulload & $\max$ & \\
\hline 1 & 0.91 & 3.45 & -0.70 & 3.65 & 4.36 & 0.21 \\
\hline 2 & 2.14 & 7.28 & 0.00 & 9.43 & 9.43 & 2.14 \\
\hline Total: & 3.06 & 10.73 & -0.70 & 13.08 & 13.78 & 2.35 \\
\hline
\end{tabular}

Support reactions

$(\mathrm{kN})$

\begin{tabular}{|ccccc|} 
& Column 1 & \multicolumn{3}{c}{ Column 2} \\
CA & $\max$ & $\min$ & $\max$ & $\min$ \\
$\mathrm{g}$ & 0.9 & 0.9 & 2.1 & 2.1 \\
$\mathrm{~J}$ & 3.4 & -0.7 & 7.3 & 0.0 \\
\hline tot & 4.4 & 0.2 & 9.4 & 2.1 \\
\hline
\end{tabular}

Results for $\gamma$-times loads

Partial safety factor $\gamma \mathrm{G} * \mathrm{~K}_{\mathrm{Fi}}=1.35$ constant over whole girder length

\begin{tabular}{|c|c|c|c|c|c|c|c|}
\hline \multicolumn{5}{|c|}{ Span moments maximum } & \multicolumn{3}{|c|}{$(\mathrm{kNm}, \mathrm{kN})$} \\
\hline Span & & Mfd & Mdle & Mdri & V le & V ri & comb \\
\hline 1 & $x 0=1.250$ & 4.01 & 0.00 & -0.62 & 6.40 & -6.88 & $\mathrm{~J}$ \\
\hline
\end{tabular}

\begin{tabular}{|cccccrrrr|}
\hline \multicolumn{2}{l}{ Support moments maximum } & \multicolumn{3}{c}{ (kNm, kN ) } \\
\hline Support & Mdle & Mdri & Vdle & Vdri & $\max F$ & $\min F$ & comb \\
\hline 1 & 0.00 & 0.00 & 0.00 & 6.40 & 6.40 & -0.14 & $\mathrm{~J}$ & 2 \\
2 & -3.37 & -3.37 & -7.93 & 5.89 & 13.82 & 2.14 & $\mathrm{~J}$ & 3 \\
\hline
\end{tabular}

Scale $1: 50$

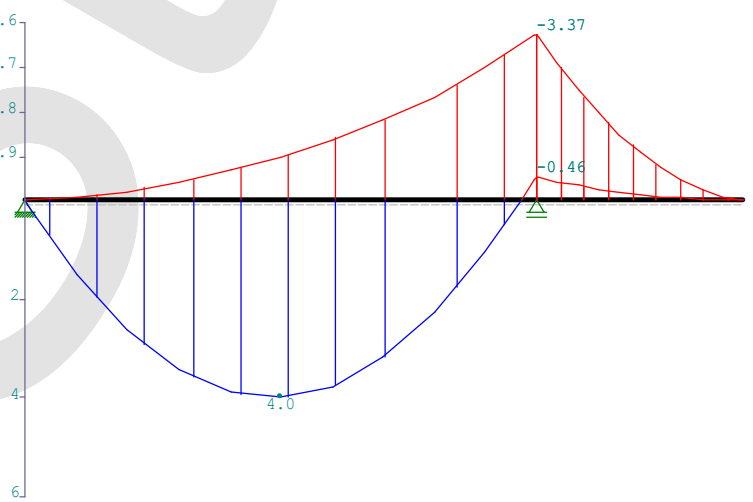


Demo Frilo

Stuttgarter Straße 40
Tel.: 0711810020

Fax: 0711858020
Projekt: Design Calculations

Position: V.31.1 - Steel Canopy

28.07.2018

Seite: 3
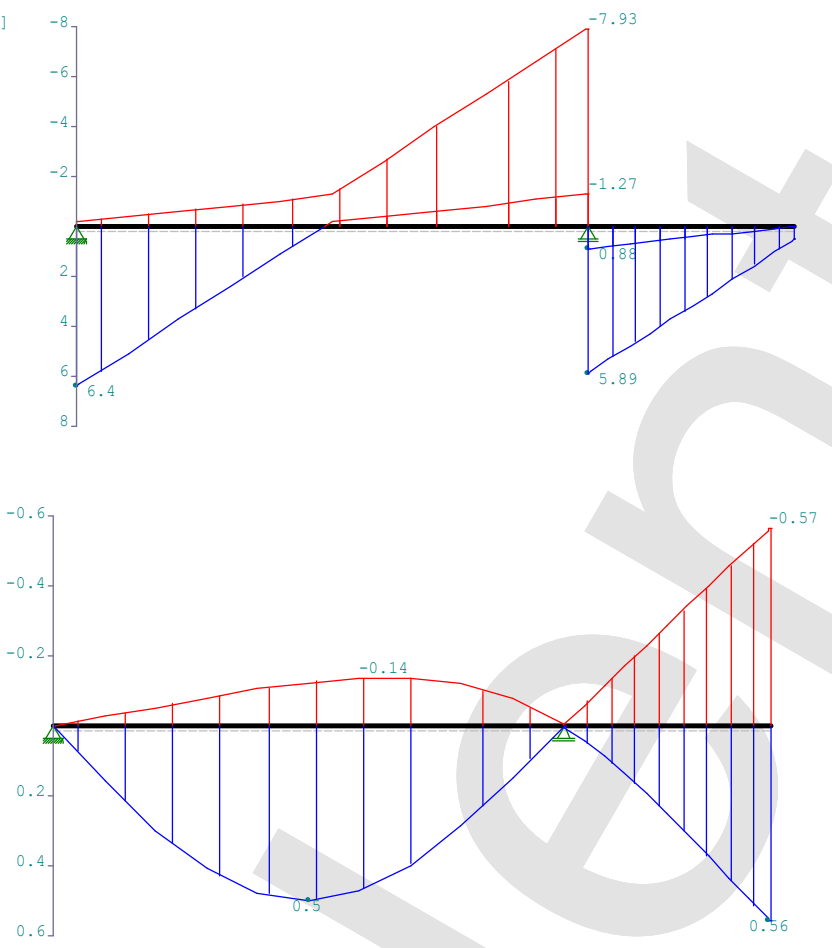

\begin{tabular}{|crrrrr|}
\hline Cross sectionsS235 & fyk $=$ & $235 \mathrm{~N} / \mathrm{mm} 2$ & & \\
type name & $\mathrm{Npl}$ & Mplyd & Vplzd & Mplzd & Vplyd \\
\hline $10 \quad$ L100X50X6 & 205 & 6 & 66 & 2 & 32 \\
\hline
\end{tabular}

proof acc. DIN EN 1993-1-1/NA:2010-12 6.2.1 (6.1)

$\gamma \mathrm{MO}=1.00$

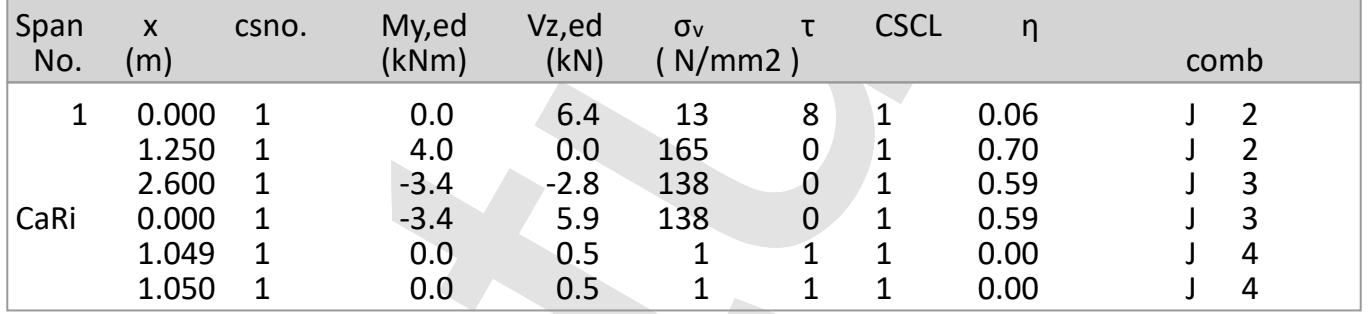

proof acc. DIN EN 1993-1-1/NA:2010-12 6.2.1 (6.2)

$\gamma \mathrm{MO}=1.00$

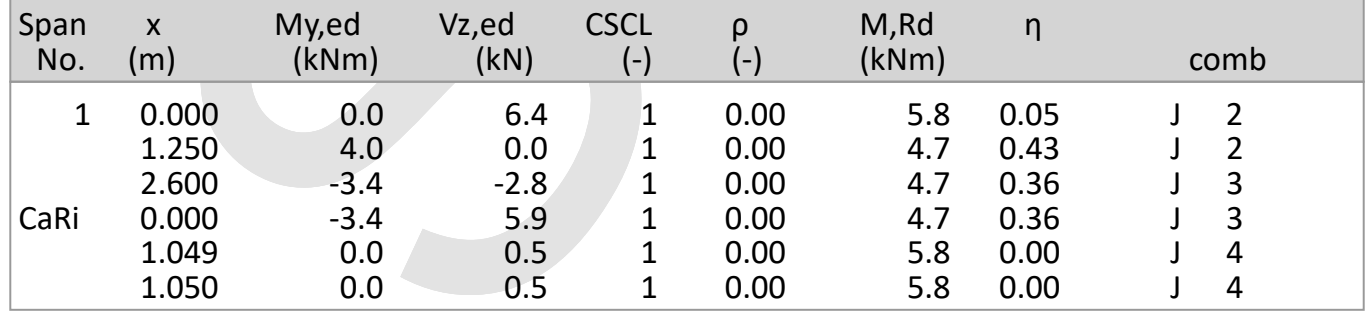

Compression flange is supported continuously.

Proof of torsional-flexural buckling is not necessary. 
Demo Frilo

Stuttgarter Straße 40

70469

Stuttgart
Tel.: 0711810020

Fax: 0711858020
Projekt: Design Calculations

Position: V.31.1 - Steel Canopy

28.07.2018

\begin{tabular}{|c|c|c|c|c|c|c|c|}
\hline \multicolumn{8}{|c|}{$\begin{array}{l}\text { Permissible deflection : in span perm. } f=L / 300 \\
\text { characteristic combination Cantilever } \quad L / 150\end{array}$} \\
\hline $\begin{array}{l}\text { Span } \\
\text { No. }\end{array}$ & $\begin{array}{c}x \\
(m)\end{array}$ & $\begin{array}{c}\mathrm{fg} \\
(\mathrm{cm})\end{array}$ & $\begin{array}{l}\text { ftot } \\
(\mathrm{cm})\end{array}$ & $\begin{array}{c}f \\
(\mathrm{~cm})\end{array}$ & $\begin{array}{l}\text { perm.f } \\
(\mathrm{cm})\end{array}$ & $\eta$ & comb \\
\hline CaRi & $\begin{array}{l}1.300 \\
1.049\end{array}$ & $\begin{array}{r}0.08 \\
-0.03\end{array}$ & $\begin{array}{r}0.50 \\
-0.57\end{array}$ & $\begin{array}{r}0.499 \\
-0.567\end{array}$ & $\begin{array}{l}0.867 \\
0.700\end{array}$ & $\begin{array}{l}0.58 \\
0.81\end{array}$ & $\begin{array}{l}2 \\
2\end{array}$ \\
\hline
\end{tabular}

At the following table the loads are specified by their internal numeration.

The following table of calculated combinations referenced.

to these numbers

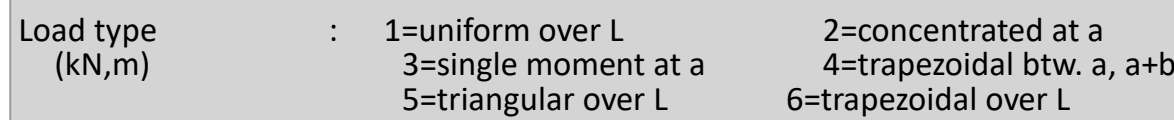

No. span Type Grp $\quad$ g1 $\quad$ q1 $\quad$ g2 $\quad$ q2 factor distance length
$\begin{array}{lllll}1 & 1 & 1 & \mathrm{~J} & 1\end{array}$
0.70
2.65
1.00

Cantilever

$\begin{array}{lllllllll}2 & \mathrm{CaRi} & 1 & \mathrm{~J} & 2 & 0.70 & 2.65 & 1.00 & \\ 3 & 2 & \mathrm{~J} & 2 & 0.00 & 0.35 & 1.00 & 1.05\end{array}$

Calculated combinations from 3 Loads

\begin{tabular}{rrrrrr} 
Ic & & K1 & K2 & K3 & K4 \\
\hline & & g & g & g & g \\
1 & . & x &. & x \\
2 & . &. & x & x \\
3 & . & . & x & x
\end{tabular}

The combinations above will be managed as followed :

Calculating ULS the dead loads will be exceeded

all at once alternating by GammaG $\quad=1,00 / 1,35$.

If in one combination live-loads from different actions

exists, then will be investigated, which action is

the dominating one.

The effect of the duration of action will be checked

too. 


\section{Position: V.31.2 - Steel Canopy}

Durchlaufträger DLT10 01/2018 (Frilo R-2018-1/P12)

Scale $1: 75$

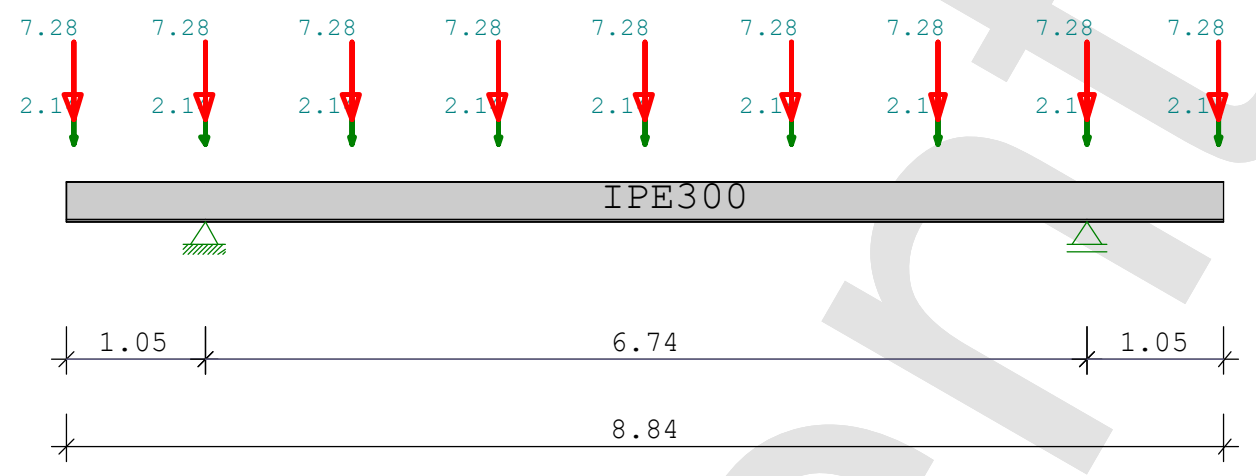

Steel girder S235 DIN EN 1993-1-1/NA:2010-12

E-modulus $E=210000 \mathrm{~N} / \mathrm{mm} 2$

\begin{tabular}{|c|c|c|c|c|c|c|}
\hline \multirow{2}{*}{$\begin{array}{l}\text { System } \\
\text { Span }\end{array}$} & \multirow{2}{*}{\multicolumn{2}{|c|}{$\begin{array}{l}\text { length } \\
L(m)\end{array}$}} & \multicolumn{4}{|c|}{ cross-section values } \\
\hline & & & CsNo. & $\mathrm{I}(\mathrm{cm} 4)$ & $\mathrm{St}(\mathrm{cm} 3) \mathrm{Sb}(\mathrm{cm} 3)$ & \\
\hline 1 & 6.740 & constant & 1 & 8360.0 & 557.0 & IPE300 \\
\hline $\begin{array}{l}\text { Cantilever } \\
\text { left } \\
\text { right }\end{array}$ & $\begin{array}{l}1.050 \\
1.050\end{array}$ & $\begin{array}{l}\text { constant } \\
\text { constant }\end{array}$ & $\begin{array}{l}1 \\
1\end{array}$ & $\begin{array}{l}8360.0 \\
8360.0\end{array}$ & $\begin{array}{l}557.0 \\
557.0\end{array}$ & $\begin{array}{l}\text { IPE300 } \\
\text { IPE300 }\end{array}$ \\
\hline
\end{tabular}

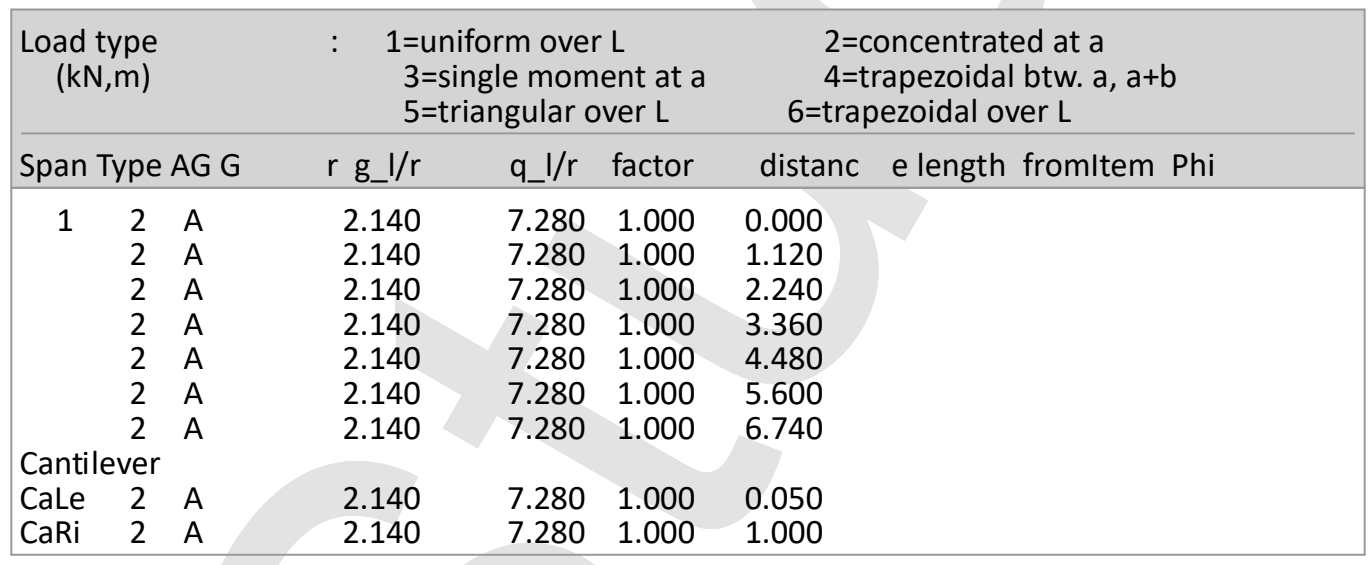

\section{Actions:}

No. Cl Name

$\psi 0 \quad \psi 1 \quad \psi 2 \quad \gamma$

A 1 Cat A - domestic

$\begin{array}{llll}0.70 & 0.50 & 0.30 & 1.50\end{array}$

Consequency class CC 2 acc. EN 1990 Tab. B1 $\rightarrow \mathrm{K}_{\mathrm{Fi}}=1.0$ Tab. B3

In following tables the last cell the row is a reference to

the number of the related superposition ( see below).

In tables with internal forces multiplied by Gamma is additionally

a reference to the main action. 
Demo Frilo

Stuttgarter Straße 40

70469 Stuttgart
Tel.: 0711810020

Fax: 0711858020
Projekt: Design Calculations

Position: V.31.2 - Steel Canopy

28.07.2018

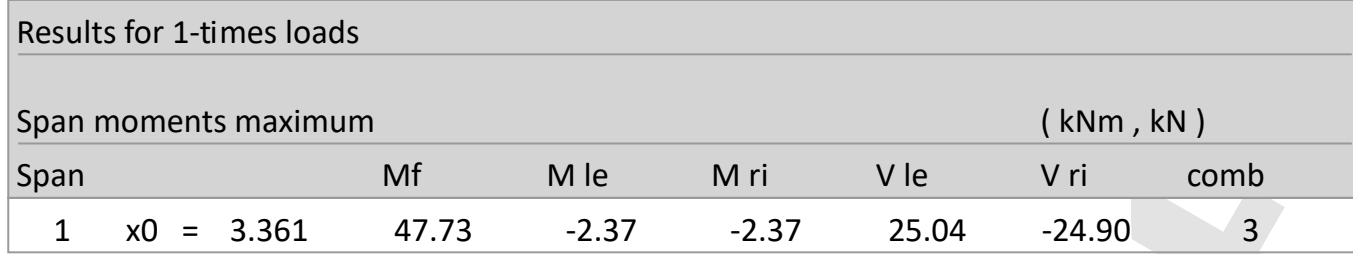

\begin{tabular}{|cccccccc|}
\hline \multicolumn{2}{l}{ Support moments maximum } & \multicolumn{3}{c}{ ( $\mathrm{kNm}, \mathrm{kN})$} \\
\hline Column & M le & M ri & V le & V ri & $\max F$ & $\min F$ & comb \\
\hline 1 & -9.65 & -9.65 & -9.86 & 26.12 & 45.41 & 10.43 & 2 \\
2 & -9.65 & -9.65 & -25.98 & 9.86 & 45.27 & 10.40 & 2 \\
\hline
\end{tabular}

\begin{tabular}{|cccccccc|}
\hline \multicolumn{7}{l}{ Support reactions } \\
\hline Column & by $\mathrm{g}$ & $\max \mathrm{q}$ & $\min \mathrm{q}$ & Fulload & $\max$ & \multicolumn{2}{c|}{$\min$} \\
\hline 1 & 11.51 & 33.89 & -1.08 & 44.33 & 45.41 & 10.43 \\
2 & 11.48 & 33.79 & -1.08 & 44.19 & 45.27 & 10.40 \\
Total: & 22.99 & 67.68 & -2.16 & 88.51 & 90.67 & 20.83 \\
\hline
\end{tabular}

Support reactions

$(\mathrm{kN})$

\begin{tabular}{|ccccc}
\hline & Column 1 & \multicolumn{3}{c}{ Column 2} \\
CA & $\max$ & $\min$ & $\max$ & $\min$ \\
\hline $\mathrm{g}$ & 11.5 & 11.5 & 11.5 & 11.5 \\
$\mathrm{~A}$ & 33.9 & -1.1 & 33.8 & -1.1 \\
\hline tot & 45.4 & 10.4 & 45.3 & 10.4 \\
\hline
\end{tabular}

Results for $\gamma$-times loads

Partial safety factor $\gamma \mathrm{G} * \mathrm{~K}_{\mathrm{Fi}}=1.35$ constant over whole girder length

\begin{tabular}{|c|c|c|c|c|c|c|c|c|}
\hline \multicolumn{6}{|c|}{ Span moments maximum } & \multicolumn{3}{|c|}{$(\mathrm{kNm}, \mathrm{kN})$} \\
\hline Span & & & Mfd & Mdle & Mdri & V le & V ri & comb \\
\hline 1 & $x 0$ & $=3.361$ & 69.97 & -3.20 & -3.20 & 36.55 & -36.34 & $A$ \\
\hline
\end{tabular}

\begin{tabular}{|c|c|c|c|c|c|c|c|c|}
\hline \multicolumn{5}{|c|}{ Support moments maximum } & \multicolumn{4}{|c|}{$(\mathrm{kNm}, \mathrm{kN})$} \\
\hline Support & Mdle & Mdri & Vdle & Vdri & $\max F$ & $\min F$ & com & \\
\hline $\begin{array}{l}1 \\
2\end{array}$ & $\begin{array}{l}-14.12 \\
-14.12\end{array}$ & $\begin{array}{l}-14.12 \\
-14.12\end{array}$ & $\begin{array}{l}-14.41 \\
-37.96\end{array}$ & $\begin{array}{l}38.17 \\
14.41\end{array}$ & $\begin{array}{l}66.38^{*} \\
66.18^{*}\end{array}$ & $\begin{array}{l}9.89 * \\
9.86 *\end{array}$ & $\begin{array}{l}A \\
A\end{array}$ & $\begin{array}{l}2 \\
2\end{array}$ \\
\hline
\end{tabular}

Scale $1: 100$

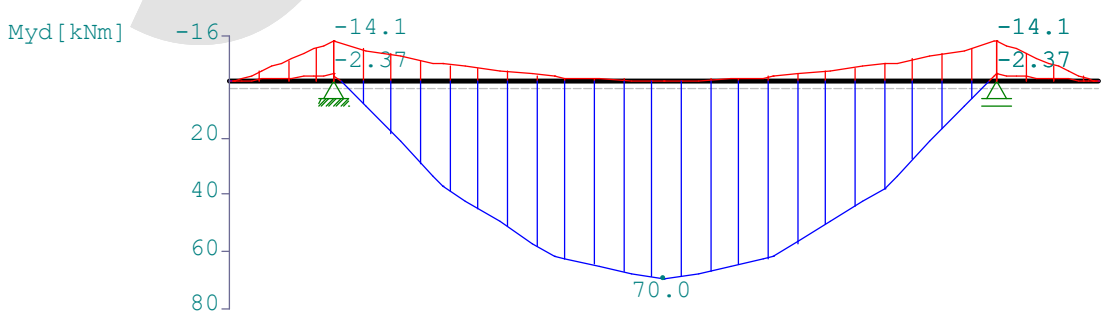


Demo Frilo

Stuttgarter Straße 40

70469
Tel.: 0711810020

Fax: 0711858020
Projekt: Design Calculations

Position: V.31.2 - Steel Canopy

28.07.2018
Seite: 3

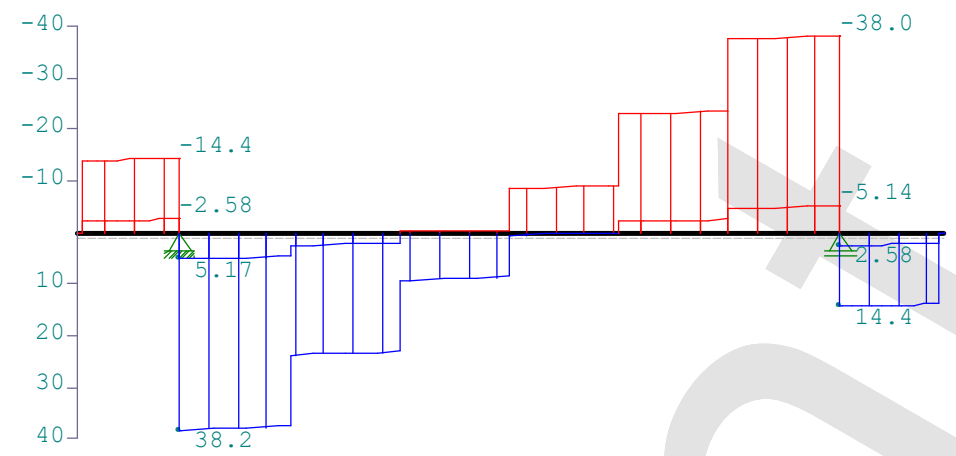

\begin{tabular}{|crrrrr|}
\hline $\begin{array}{l}\text { Cross sectionsS235 } \\
\text { type name }\end{array}$ & fyk $=$ & $235 \mathrm{~N} / \mathrm{mm} 2$ & & & \\
\hline 2 & $\mathrm{Npl}$ & Mplyd & Vplzd & Mplzd & Vplyd \\
\hline IPE300 & 1264 & 148 & 348 & 29 & 436 \\
\hline
\end{tabular}

\begin{tabular}{|c|c|c|c|c|c|c|c|c|c|c|}
\hline \multicolumn{6}{|c|}{ proof acc. DIN EN 1993-1-1/NA:2010-12 6.2.1 (6.1) } & \multicolumn{5}{|c|}{$\gamma \mathrm{MO}=1.00$} \\
\hline $\begin{array}{c}\text { Span } \\
\text { No. }\end{array}$ & $\begin{array}{c}x \\
(m)\end{array}$ & csno. & $\begin{array}{l}\text { My,ed } \\
(\mathrm{kNm})\end{array}$ & $\begin{array}{r}\text { Vz,ed } \\
(\mathrm{kN})\end{array}$ & $\begin{array}{l}\sigma_{\mathrm{v}} \\
(\mathrm{N} / \mathrm{mm} 2\end{array}$ & )$^{\tau}$ & $\mathrm{CSCL}$ & $\eta$ & \multicolumn{2}{|c|}{ comb } \\
\hline \multirow[t]{4}{*}{ CaLe } & 0.000 & 1 & 0.0 & 0.0 & 0 & 0 & 1 & 0.00 & & 1 \\
\hline & 0.049 & 1 & 0.0 & 0.0 & 0 & 0 & 1 & 0.00 & & 1 \\
\hline & 0.051 & 1 & 0.0 & -13.8 & 12 & 7 & 1 & 0.05 & A & 2 \\
\hline & 1.050 & 1 & -14.1 & -14.4 & 26 & 2 & 1 & 0.11 & $A$ & 2 \\
\hline \multirow[t]{14}{*}{1} & 0.000 & 1 & -14.1 & 38.2 & 35 & 16 & 1 & 0.15 & $A$ & 5 \\
\hline & 0.001 & 1 & -14.1 & 38.2 & 35 & 16 & 1 & 0.15 & $A$ & 5 \\
\hline & 1.119 & 1 & 37.3 & 35.9 & 67 & 5 & 1 & 0.29 & $A$ & 3 \\
\hline & 1.121 & 1 & 37.4 & 22.1 & 67 & 3 & 1 & 0.29 & $A$ & 3 \\
\hline & 2.239 & 1 & 61.8 & 21.5 & 111 & 3 & 1 & 0.47 & $A$ & 3 \\
\hline & 2.241 & 1 & 61.8 & 7.7 & 111 & 1 & 1 & 0.47 & $A$ & 3 \\
\hline & 3.359 & 1 & 70.0 & 7.0 & 126 & 1 & 1 & 0.53 & $A$ & 3 \\
\hline & 3.361 & 1 & 70.0 & -6.8 & 126 & 1 & 1 & 0.53 & $A$ & 3 \\
\hline & 4.479 & 1 & 62.0 & -7.4 & 111 & 1 & 1 & 0.47 & $A$ & 3 \\
\hline & 4.481 & 1 & 62.0 & -21.2 & 111 & 3 & 1 & 0.47 & $A$ & 3 \\
\hline & 5.599 & 1 & 37.9 & -21.9 & 68 & 3 & 1 & 0.29 & A & 3 \\
\hline & 5.601 & 1 & 37.8 & -35.7 & 68 & 5 & 1 & 0.29 & A & 3 \\
\hline & 6.739 & 1 & -14.1 & -38.0 & 35 & 16 & 1 & 0.15 & A & 7 \\
\hline & 6.740 & 1 & -14.1 & -38.0 & 35 & 16 & 1 & 0.15 & A & 7 \\
\hline \multirow[t]{4}{*}{ CaRi } & 0.000 & 1 & -14.1 & 14.4 & 26 & 2 & 1 & 0.11 & A & 6 \\
\hline & 0.999 & 1 & 0.0 & 13.8 & 12 & 7 & 1 & 0.05 & A & 7 \\
\hline & 1.001 & 1 & 0.0 & 0.0 & 0 & 0 & 1 & 0.00 & A & 7 \\
\hline & 1.050 & 1 & 0.0 & 0.0 & 0 & 0 & 1 & 0.00 & A & 7 \\
\hline
\end{tabular}

\begin{tabular}{|c|c|c|c|c|c|c|c|c|}
\hline proof & acc. DIN & | 1993-1-1 & JA:2010-1 & 6.2 .1 & & & $\gamma \mathrm{MO}=$ & \\
\hline $\begin{array}{l}\text { Span } \\
\text { No. }\end{array}$ & $\begin{array}{c}x \\
(m)\end{array}$ & $\begin{array}{l}\text { My,ed } \\
(\text { (kNm) }\end{array}$ & $\begin{array}{l}\mathrm{Vz}, \mathrm{ed} \\
(\mathrm{kN})\end{array}$ & $\begin{array}{r}\mathrm{CSCL} \\
(-)\end{array}$ & $\begin{array}{l}\rho \\
(-)\end{array}$ & $\begin{array}{l}\text { M,Rd } \\
(\mathrm{kNm})\end{array}$ & $\eta$ & comb \\
\hline CaLe & 0.000 & 0.0 & 0.0 & 1 & 0.00 & 148.0 & 0.00 & 1 \\
\hline & 0.049 & 0.0 & 0.0 & 1 & 0.00 & 148.0 & 0.00 & 1 \\
\hline & 0.051 & 0.0 & -13.8 & 1 & 0.00 & 148.0 & 0.04 & A \\
\hline & 1.050 & -14.1 & -14.4 & 1 & 0.00 & 148.0 & 0.10 & A \\
\hline 1 & 0.000 & -14.1 & 38.2 & 1 & 0.00 & 148.0 & 0.11 & $A$ \\
\hline & 0.001 & -14.1 & 38.2 & 1 & 0.00 & 148.0 & 0.11 & A \\
\hline & 1.119 & 37.3 & 35.9 & 1 & 0.00 & 148.0 & 0.25 & $A$ \\
\hline & 1.121 & 37.4 & 22.1 & 1 & 0.00 & 148.0 & 0.25 & $A$ \\
\hline & 2.239 & 61.8 & 21.5 & 1 & 0.00 & 148.0 & 0.42 & A \\
\hline & 2.241 & 61.8 & 7.7 & 1 & 0.00 & 148.0 & 0.42 & A \\
\hline & 3.359 & 70.0 & 7.0 & 1 & 0.00 & 148.0 & 0.47 & A \\
\hline & 3.361 & 70.0 & -6.8 & 1 & 0.00 & 148.0 & 0.47 & $A$ \\
\hline & 4.479 & 62.0 & -7.4 & 1 & 0.00 & 148.0 & 0.42 & $A$ \\
\hline & 4.481 & 62.0 & -21.2 & 1 & 0.00 & 148.0 & 0.42 & A \\
\hline & 5.599 & 37.9 & -21.9 & 1 & 0.00 & 148.0 & 0.26 & A \\
\hline
\end{tabular}


Demo Frilo

Stuttgarter Straße 40

70469

Stuttgart
Tel.: 0711810020

Fax: 0711858020
Projekt: Design Calculations

Position: V.31.2 - Steel Canopy

28.07.2018

\begin{tabular}{|c|c|c|c|c|c|c|c|c|}
\hline \multicolumn{7}{|c|}{ proof acc. DIN EN 1993-1-1/NA:2010-12 6.2.1 (6.2) } & \multicolumn{2}{|c|}{$\gamma \mathrm{MO}=1.00$} \\
\hline $\begin{array}{l}\text { Span } \\
\text { No. }\end{array}$ & $\begin{array}{c}x \\
(m)\end{array}$ & $\begin{array}{l}\text { My,ed } \\
\text { (kNm) }\end{array}$ & $\begin{array}{l}\text { Vz,ed } \\
\quad(k N)\end{array}$ & $\begin{array}{r}\mathrm{CSCL} \\
(-)\end{array}$ & $\begin{array}{l}\rho \\
(-)\end{array}$ & $\begin{array}{l}\text { M,Rd } \\
(\mathrm{kNm})\end{array}$ & $\eta$ & comb \\
\hline \multirow{7}{*}{ CaRi } & 5.601 & 37.8 & -35.7 & 1 & 0.00 & 148.0 & 0.26 & A \\
\hline & 6.739 & -14.1 & -38.0 & 1 & 0.00 & 148.0 & 0.11 & A 7 \\
\hline & 6.740 & -14.1 & -38.0 & 1 & 0.00 & 148.0 & 0.11 & A 7 \\
\hline & 0.000 & -14.1 & 14.4 & 1 & 0.00 & 148.0 & 0.10 & A \\
\hline & 0.999 & 0.0 & 13.8 & 1 & 0.00 & 148.0 & 0.04 & A 7 \\
\hline & 1.001 & 0.0 & 0.0 & 1 & 0.00 & 148.0 & 0.00 & A 7 \\
\hline & 1.050 & 0.0 & 0.0 & 1 & 0.00 & 148.0 & 0.00 & A 7 \\
\hline
\end{tabular}

Compression flange is supported continuously.

Proof of torsional-flexural buckling is not necessary.

\begin{tabular}{|c|c|c|c|c|c|c|c|}
\hline \multicolumn{8}{|c|}{$\begin{array}{l}\text { Permissible deflection : in span perm. } f=L / 300 \\
\text { characteristic combination Cantilever } L / 150\end{array}$} \\
\hline $\begin{array}{r}\text { Span } \\
\text { No. }\end{array}$ & $\begin{array}{c}x \\
(m)\end{array}$ & $\begin{array}{c}\mathrm{fg} \\
(\mathrm{cm})\end{array}$ & $\begin{array}{l}\text { ftot } \\
(\mathrm{cm})\end{array}$ & $\begin{array}{c}f \\
(\mathrm{~cm})\end{array}$ & $\begin{array}{l}\text { perm.f } \\
(\mathrm{cm})\end{array}$ & $\eta$ & comb \\
\hline CaLe & 0.000 & -0.12 & -0.60 & -0.603 & 0.700 & 0.86 & 3 \\
\hline & 3.370 & 0.27 & 1.25 & 1.245 & 2.247 & 0.55 & 3 \\
\hline CaRi & 1.050 & -0.12 & -0.60 & -0.603 & 0.700 & 0.86 & 3 \\
\hline
\end{tabular}

At the following table the loads are specified by their internal numeration.

The following table of calculated combinations referenced.

to these numbers

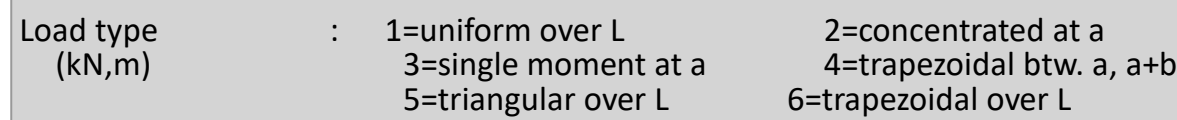

No. span Type Grp $\quad$ g1 $q 1 \quad$ g2 $\quad$ q2 factor distance length

$\begin{array}{lllllllll}2 & 1 & 2 & \text { A } & 2 & 2.14 & 7.28 & 1.00 & 0.00 \\ 3 & & 2 & \text { A } & 2 & 2.14 & 7.28 & 1.00 & 1.12 \\ 4 & & 2 & \text { A } & 2 & 2.14 & 7.28 & 1.00 & 2.24 \\ 5 & & 2 & \text { A } & 2 & 2.14 & 7.28 & 1.00 & 3.36 \\ 6 & & 2 & \text { A } & 2 & 2.14 & 7.28 & 1.00 & 4.48 \\ 7 & & 2 & \text { A } & 2 & 2.14 & 7.28 & 1.00 & 5.60 \\ 8 & & 2 & \text { A } & 2 & 2.14 & 7.28 & 1.00 & 6.74\end{array}$

Cantilever

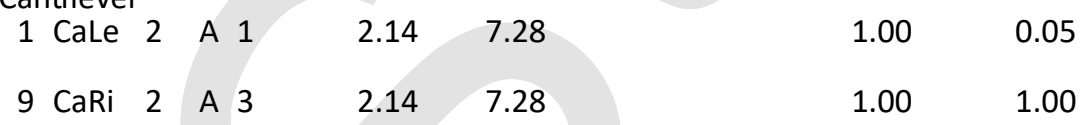

Calculated combinations from 9 Loads

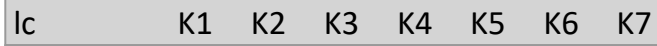

\begin{tabular}{|c|c|c|c|c|c|c|c|}
\hline & g & g & $\mathrm{g}$ & $\mathrm{g}$ & g & g & $\mathrm{g}$ \\
\hline 1 & . & $x$ & . & $x$ & $x$ & . & . \\
\hline 2 & . & . & $x$ & . & $x$ & . & $x$ \\
\hline 3 & . & . & $x$ & . & $x$ & . & $x$ \\
\hline 4 & . & . & $x$ & . & $x$ & . & $x$ \\
\hline 5 & . & . & $x$ & . & $x$ & . & $x$ \\
\hline 6 & . & . & $x$ & . & $x$ & . & $x$ \\
\hline 7 & . & . & $x$ & . & $x$ & . & $x$ \\
\hline 8 & . & . & $x$ & . & $x$ & . & $x$ \\
\hline 9 & . & $x$ & & . & . & $x$ & $x$ \\
\hline
\end{tabular}


Demo Frilo

Stuttgarter Straße 40

70469

Stuttgart

Calculated combinations from 9 Loads

IC

$\begin{array}{lllllll}\text { K1 } & \text { K2 } & \text { K3 } & \text { K4 } & \text { K5 } & \text { K6 } & \text { K7 }\end{array}$

The combinations above will be managed as followed :

Calculating ULS the dead loads will be exceeded

all at once alternating by GammaG $\quad=1,00 / 1,35$

If in one combination live-loads from different actions

exists, then will be investigated, which action is

the dominating one.

The effect of the duration of action will be checked

too. 
Demo Frilo

Stuttgarter Straße 40

70469 Stuttgart
Tel.: 0711810020

Fax: 0711858020
Projekt: Design Calculations

Position: V.31.4 - Steel Canopy

28.07.2018

\section{Position: V.31.4 - Steel Canopy}

Durchlaufträger DLT10 01/2018 (Frilo R-2018-1/P12)

Scale $1: 75$

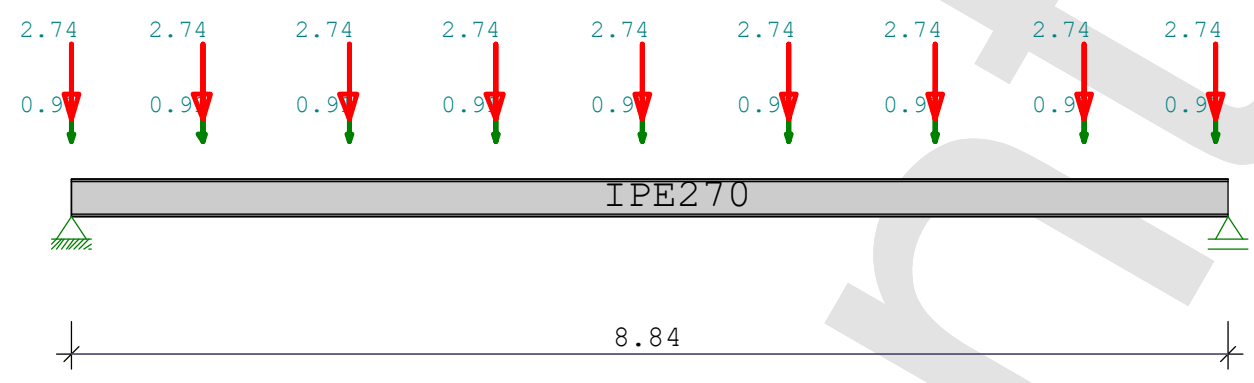

Steel girder S235 DIN EN 1993-1-1/NA:2010-12

E-modulus $E=210000 \mathrm{~N} / \mathrm{mm} 2$

\begin{tabular}{|clllllll|}
\hline System & length & \multicolumn{5}{c|}{ cross-section values } \\
\hline Span & $\mathrm{L}(\mathrm{m})$ & & CsNo. & $\mathrm{I}(\mathrm{cm} 4)$ & $\mathrm{St}(\mathrm{cm} 3)$ & $\mathrm{Sb}(\mathrm{cm} 3)$ & \\
\hline 1 & 8.840 & constant & 1 & 5790.0 & 429.0 & 429.0 & IPE270 \\
\hline
\end{tabular}

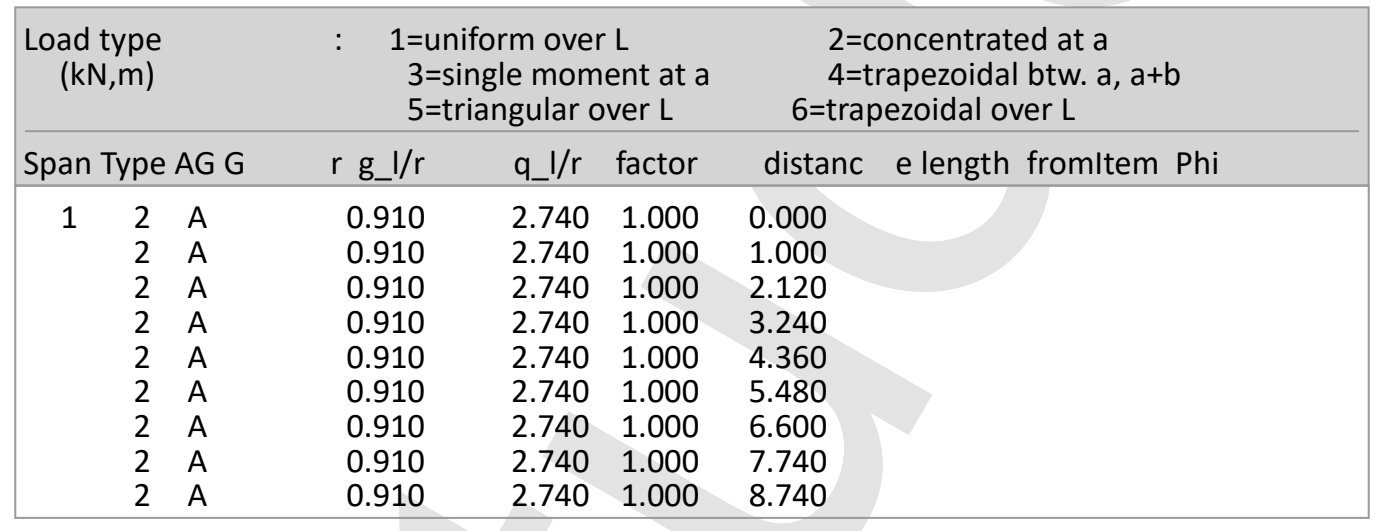

Dead load of girder is considered with Gamma

$=78.5 \mathrm{kN} / \mathrm{m} 3$.

Actions:

No. Cl Name

$\psi 0 \quad \psi 1 \quad \psi 2 \quad \psi$

A 1 Cat A-domestic

$\begin{array}{llll}0.70 & 0.50 & 0.30 & 1.50\end{array}$

Consequency class CC 2 acc. EN 1990 Tab. B1 -> K Fi $=1.0$ Tab. B3

In following tables the last cell the row is a reference to

the number of the related superposition (see below).

In tables with internal forces multiplied by Gamma is additionally

a reference to the main action.

\begin{tabular}{|c|c|c|c|c|c|c|c|}
\hline \multicolumn{8}{|c|}{ Results for 1-times loads } \\
\hline \multicolumn{5}{|c|}{ Span moments maximum } & \multicolumn{3}{|c|}{$(\mathrm{kNm}, \mathrm{kN})$} \\
\hline Span & & Mf & M le & M ri & V le & V ri & comb \\
\hline 1 & $x 0=4.361$ & 35.59 & 0.00 & 0.00 & 14.57 & -17.81 & 2 \\
\hline
\end{tabular}


Demo Frilo

Stuttgarter Straße 40

70469

Stuttgart
Tel.: 0711810020

Fax: 0711858020
Projekt: Design Calculations

Position: V.31.4 - Steel Canopy

28.07.2018

\begin{tabular}{cccccccc}
\multicolumn{2}{l}{ Support moments maximum } & \multicolumn{3}{c}{$(\mathrm{kNm}, \mathrm{kN})$} \\
\hline Column & $\mathrm{M} \mathrm{le}$ & $\mathrm{M} \mathrm{ri}$ & $\mathrm{V} \mathrm{le}$ & $\mathrm{V} \mathrm{ri}$ & $\max \mathrm{F}$ & $\min \mathrm{F}$ & $\mathrm{comb}$ \\
\hline 1 & 0.00 & 0.00 & 0.00 & 14.57 & 18.22 & 5.74 & 2 \\
2 & 0.00 & 0.00 & -17.81 & 0.00 & 17.81 & 5.64 & 2 \\
\hline
\end{tabular}

\begin{tabular}{|c|c|c|c|c|c|c|}
\hline \multicolumn{6}{|c|}{ Support reactions } & \multirow[t]{2}{*}{$(\mathrm{kN})$} \\
\hline Column & by & $\max q$ & $\min q$ & Fulload & $\max$ & \\
\hline 1 & 5.74 & 12.48 & 0.00 & 18.22 & 18.22 & 5.74 \\
\hline 2 & 5.64 & 12.18 & 0.00 & 17.81 & 17.81 & 5.64 \\
\hline Total: & 11.38 & 24.66 & 0.00 & 36.04 & 36.04 & 11.38 \\
\hline
\end{tabular}

Support reactions

$(\mathrm{kN})$

\begin{tabular}{|ccccc|}
\hline & Column 1 & \multicolumn{3}{c}{ Column 2} \\
CA & $\max$ & $\min$ & $\max$ & $\min$ \\
\hline $\mathrm{g}$ & 5.7 & 5.7 & 5.6 & 5.6 \\
$\mathrm{~A}$ & 12.5 & 0.0 & 12.2 & 0.0 \\
\hline tot & 18.2 & 5.7 & 17.8 & 5.6 \\
\hline
\end{tabular}

Results for $\gamma$-times loads

Partial safety factor $\gamma \mathrm{G} * \mathrm{~K}_{\mathrm{Fi}}=1.35$ constant over whole girder length

\begin{tabular}{|c|c|c|c|c|c|c|c|}
\hline \multicolumn{5}{|c|}{ Span moments maximum } & \multicolumn{3}{|c|}{$(\mathrm{kNm}, \mathrm{kN})$} \\
\hline Span & & Mfd & Mdle & Mdri & V le & V ri & comb \\
\hline 1 & $x 0=4.361$ & 51.66 & 0.00 & 0.00 & 21.14 & -25.87 & A 2 \\
\hline
\end{tabular}

\begin{tabular}{|c|c|c|c|c|c|c|c|}
\hline \multicolumn{5}{|c|}{ Support moments maximum } & \multicolumn{3}{|c|}{$(\mathrm{kNm}, \mathrm{kN})$} \\
\hline Support & Mdle & Mdri & Vdle & Vdri & $\max F$ & $\min F$ & comk \\
\hline $\begin{array}{l}1 \\
2\end{array}$ & $\begin{array}{l}0.00 \\
0.00\end{array}$ & $\begin{array}{l}0.00 \\
0.00\end{array}$ & $\begin{array}{r}0.00 \\
-25.87\end{array}$ & $\begin{array}{r}21.14 \\
0.00\end{array}$ & $\begin{array}{c}26.48^{*} \\
25.87\end{array}$ & $\begin{array}{c}5.74^{*} \\
5.64\end{array}$ & $\begin{array}{ll}A & 2 \\
A & 2\end{array}$ \\
\hline
\end{tabular}

Scale $1: 100$

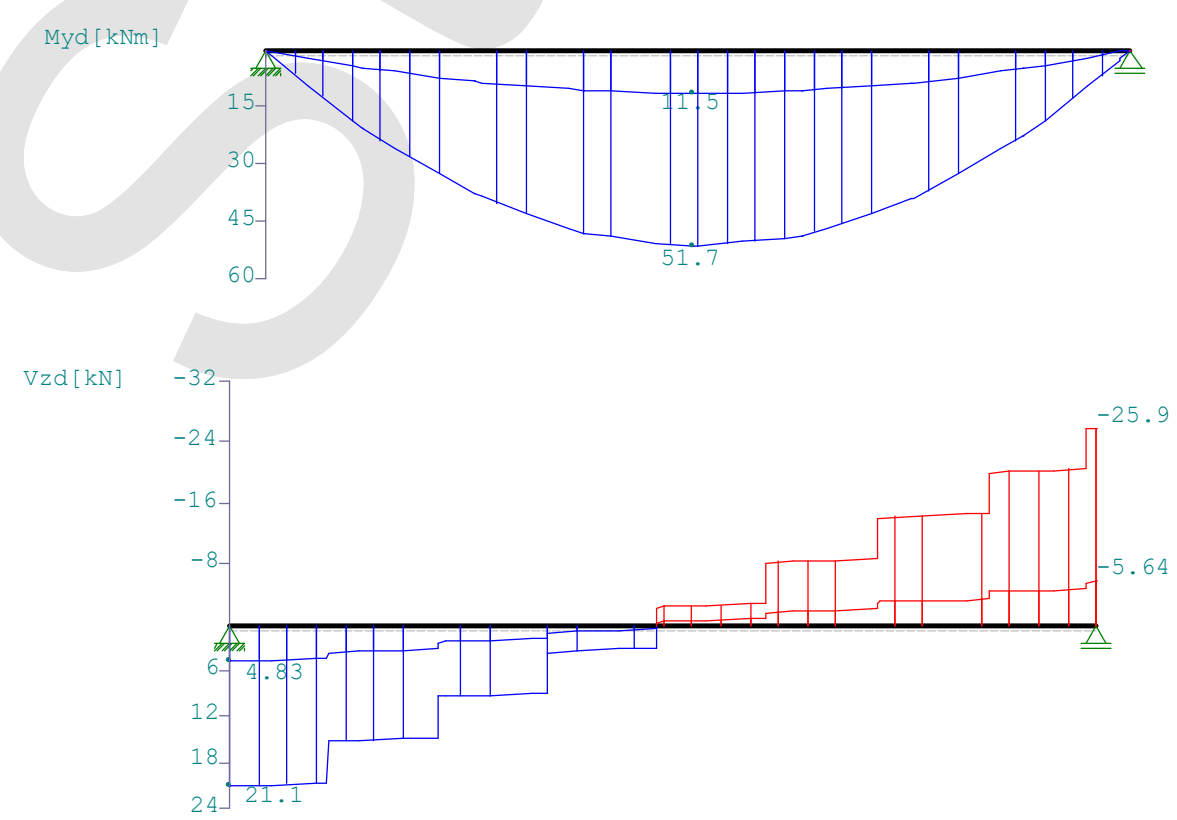


Demo Frilo

Stuttgarter Straße 40

70469
Tel.: 0711810020

Fax: 0711858020
Projekt: Design Calculations

Position: V.31.4 - Steel Canopy

28.07.2018
Seite: 3

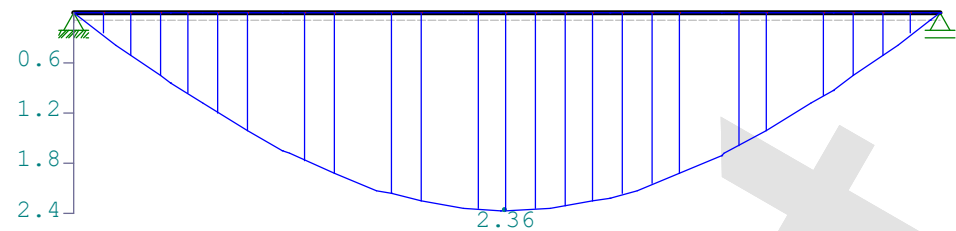

\begin{tabular}{|crrrrr|}
\hline $\begin{array}{l}\text { Cross sectionsS235 } \\
\text { type name }\end{array}$ & fyk $=$ & $235 \mathrm{~N} / \mathrm{mm} 2$ & & & \\
& $\mathrm{Npl}$ & Mplyd & Vplzd & Mplzd & Vplyd \\
\hline 2 IPE270 & 1079 & 114 & 300 & 23 & 374 \\
\hline
\end{tabular}

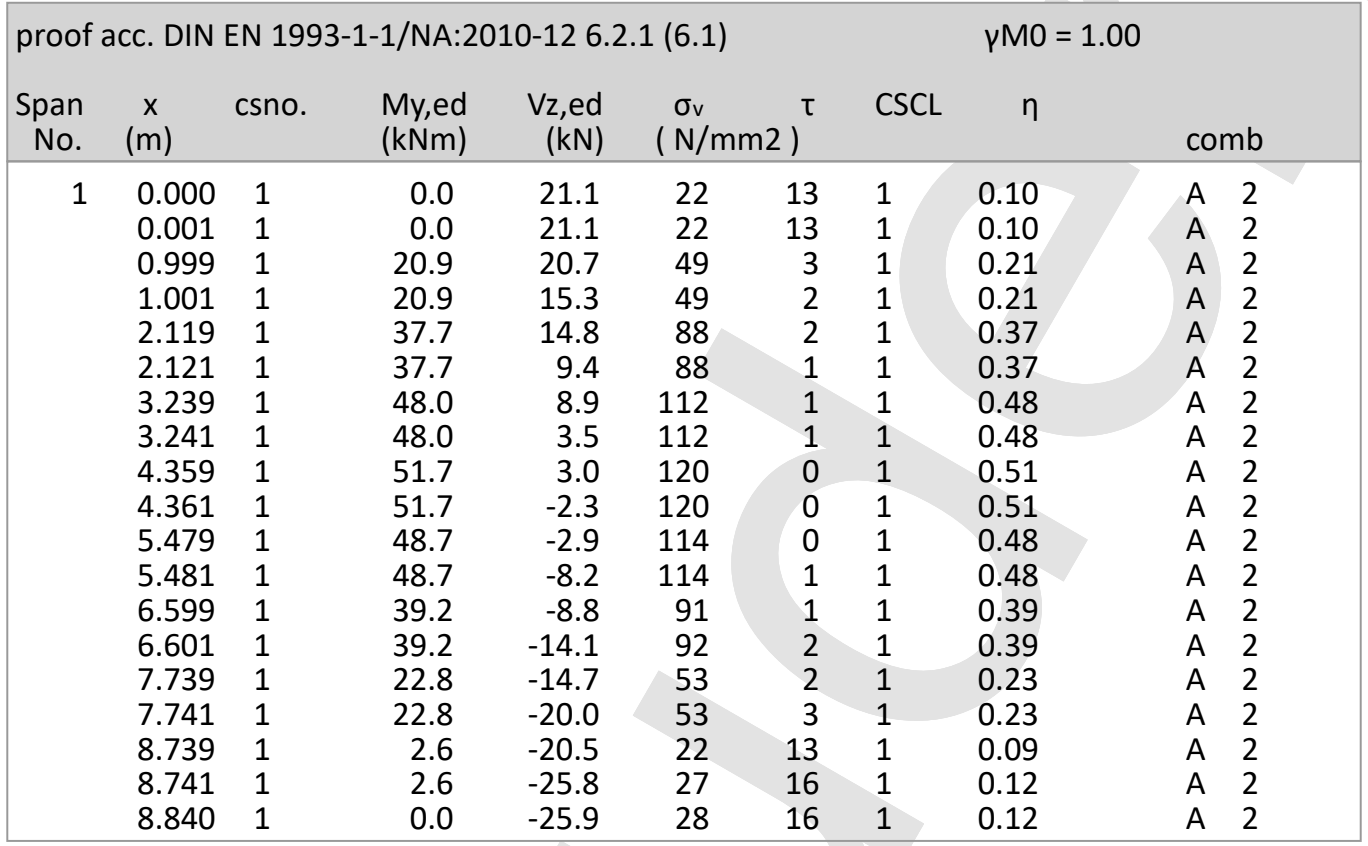

\begin{tabular}{|c|c|c|c|c|c|c|c|c|c|}
\hline \multicolumn{7}{|c|}{ proof acc. DIN EN 1993-1-1/NA:2010-12 6.2.1 (6.2) } & \multicolumn{3}{|c|}{$\gamma M 0=1.00$} \\
\hline $\begin{array}{c}\text { Span } \\
\text { No. }\end{array}$ & $\begin{array}{c}x \\
(m)\end{array}$ & $\begin{array}{l}\text { My,ed } \\
(\mathrm{kNm})\end{array}$ & $\begin{array}{l}\text { Vz,ed } \\
\quad(k N)\end{array}$ & $\begin{array}{r}\mathrm{CSCL} \\
(-)\end{array}$ & $\begin{array}{l}\rho \\
(-)\end{array}$ & $\begin{array}{l}\text { M,Rd } \\
(\mathrm{kNm})\end{array}$ & $\eta$ & \multicolumn{2}{|c|}{ comb } \\
\hline 1 & 0.000 & 0.0 & 21.1 & 1 & 0.00 & 114.1 & 0.07 & A & 2 \\
\hline & 0.001 & 0.0 & 21.1 & 1 & 0.00 & 114.1 & 0.07 & $A$ & 2 \\
\hline & 0.999 & 20.9 & 20.7 & 1 & 0.00 & 114.1 & 0.18 & $A$ & 2 \\
\hline & 1.001 & 20.9 & 15.3 & 1 & 0.00 & 114.1 & 0.18 & $A$ & 2 \\
\hline & 2.119 & 37.7 & 14.8 & 1 & 0.00 & 114.1 & 0.33 & $A$ & 2 \\
\hline & 2.121 & 37.7 & 9.4 & 1 & 0.00 & 114.1 & 0.33 & $A$ & 2 \\
\hline & 3.239 & 48.0 & 8.9 & 1 & 0.00 & 114.1 & 0.42 & $A$ & 2 \\
\hline & 3.241 & 48.0 & 3.5 & 1 & 0.00 & 114.1 & 0.42 & $A$ & 2 \\
\hline & 4.359 & 51.7 & 3.0 & 1 & 0.00 & 114.1 & 0.45 & $A$ & 2 \\
\hline & 4.361 & 51.7 & -2.3 & 1 & 0.00 & 114.1 & 0.45 & $A$ & 2 \\
\hline & 5.479 & 48.7 & -2.9 & 1 & 0.00 & 114.1 & 0.43 & $A$ & 2 \\
\hline & 5.481 & 48.7 & -8.2 & 1 & 0.00 & 114.1 & 0.43 & $A$ & 2 \\
\hline & 6.599 & 39.2 & -8.8 & 1 & 0.00 & 114.1 & 0.34 & $A$ & 2 \\
\hline & 6.601 & 39.2 & -14.1 & 1 & 0.00 & 114.1 & 0.34 & $A$ & 2 \\
\hline & 7.739 & 22.8 & -14.7 & 1 & 0.00 & 114.1 & 0.20 & $A$ & 2 \\
\hline & 7.741 & 22.8 & -20.0 & 1 & 0.00 & 114.1 & 0.20 & $A$ & 2 \\
\hline & 8.739 & 2.6 & -20.5 & 1 & 0.00 & 114.1 & 0.07 & A & 2 \\
\hline & 8.741 & 2.6 & -25.8 & 1 & 0.00 & 114.1 & 0.09 & $A$ & 2 \\
\hline & 8.840 & 0.0 & -25.9 & 1 & 0.00 & 114.1 & 0.09 & $A$ & 2 \\
\hline
\end{tabular}


Demo Frilo

Stuttgarter Straße 40

70469

Stuttgart
Tel.: 0711810020

Fax: 0711858020
Projekt: Design Calculations

Position: V.31.4 - Steel Canopy

28.07.2018

\begin{tabular}{|c|c|c|c|c|c|c|c|}
\hline \multicolumn{8}{|c|}{$\begin{array}{l}\text { Permissible deflection: in span perm. } f=L / 300 \\
\text { characteristic combination }\end{array}$} \\
\hline $\begin{array}{c}\text { Span } \\
\text { No. }\end{array}$ & $\begin{array}{c}x \\
(m)\end{array}$ & $\begin{array}{c}\mathrm{fg} \\
(\mathrm{cm})\end{array}$ & $\begin{array}{l}\mathrm{ftot} \\
(\mathrm{cm})\end{array}$ & $\begin{array}{c}f \\
(\mathrm{~cm})\end{array}$ & $\begin{array}{l}\text { perm.f } \\
(\mathrm{cm})\end{array}$ & $\eta$ & comb \\
\hline 1 & 4.420 & 0.77 & 2.36 & 2.359 & 2.947 & 0.80 & 2 \\
\hline
\end{tabular}

At the following table the loads are specified by their internal numeration.

The following table of calculated combinations referenced.

to these numbers

$\begin{array}{ccc}\text { Load type } & : \quad \text { 1=uniform over } L & \text { 2=concentrated at } a \\ (\mathrm{kN}, \mathrm{m}) & \begin{array}{c}\text { 3=single moment at a } \\ \text { 5=triangular over } L\end{array} & \begin{array}{l}\text { 4=trapezoidal btw. } a, a+b \\ \text { 6=trapezoidal over } L\end{array}\end{array}$

No.span Type Grp $\quad$ g1 $q 1 \quad$ g2 $\quad$ q2 factor distance length

$\begin{array}{lllllll}1 & 1 & 2 & \text { A } & 1 & 0.91 & 2.74\end{array}$

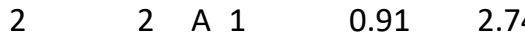

$\begin{array}{llllll}3 & 2 & \text { A } & 1 & 0.91 & 2.74\end{array}$

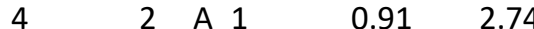

$\begin{array}{llllll}5 & 2 & \text { A } & 1 & 0.91 & 2.74\end{array}$

$\begin{array}{lllll}6 & 2 & \text { A } 1 & 0.91 & 2.74\end{array}$

$\begin{array}{llllll}7 & 2 & \text { A } & 1 & 0.91 & 2.74\end{array}$

$\begin{array}{llllll}8 & 2 & \text { A } & 1 & 0.91 & 2.74\end{array}$

$\begin{array}{llllll}9 & 2 & A & 1 & 0.91 & 2.74\end{array}$

.74
.74
.74
.74
.74
74
74
.74
.74

$\begin{array}{ll}1.00 & 0.00 \\ 1.00 & 1.00 \\ 1.00 & 2.12 \\ 1.00 & 3.24 \\ 1.00 & 4.36 \\ 1.00 & 5.48 \\ 1.00 & 6.60 \\ 1.00 & 7.74 \\ 1.00 & 8.74\end{array}$

\section{Calculated combinations from 9 Loads}

$\begin{array}{rrrr}\text { Ic } & & \text { K1 } & \text { K2 } \\ & & \text { g } & \mathrm{g} \\ 1 & . & \mathrm{x} \\ 2 & . & \mathrm{x} \\ 3 & . & \mathrm{x} \\ 4 & . & \mathrm{x} \\ 5 & . & \mathrm{x} \\ 6 & . & \mathrm{x} \\ 7 & . & \mathrm{x} \\ 8 & . & \mathrm{x} \\ 9 & . & \mathrm{x}\end{array}$

The combinations above will be managed as followed:

Calculating ULS the dead loads will be exceeded

all at once alternating by GammaG $=1,00 / 1,35$.

If in one combination live-loads from different actions

exists, then will be investigated, which action is

the dominating one.

The effect of the 


\section{B.2 INTERNAL FORCES FROM 'DLUBAL RSTAB'}

B.2.1. Internal Forces POS. Z.01 - Trapezoidal Sheet Covering .................................................319

B.2.2. Internal Forces POS. P.03 - Prestressed Concrete Truss................................................ 329

B.2.3. Internal Forces POS. T.10 - Precast Concrete Staircase ….................................................. 337

B.2.4. Internal Forces POS. W.14a - Strut-and-Tie Model Load Bearing Wall ............................ 342

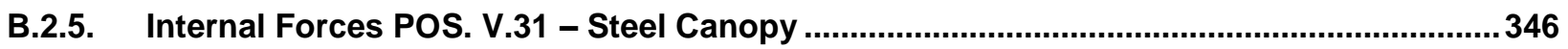


B.2.1. Internal Forces POS. Z.01 - Trapezoidal Sheet Covering 


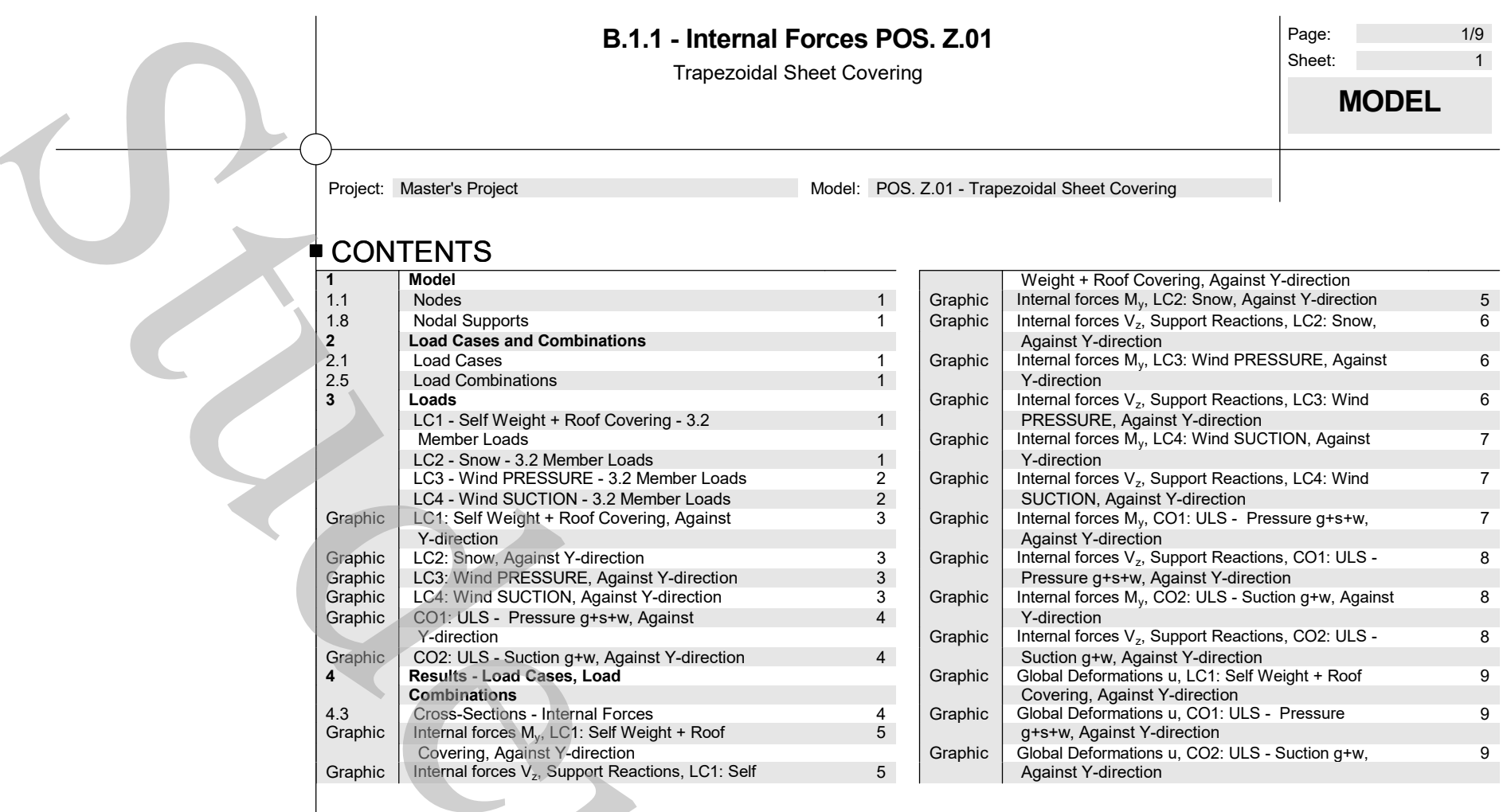

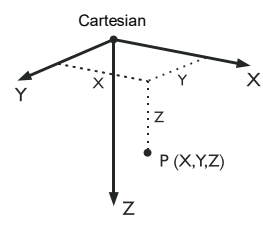

- 1.1 NODES

\begin{tabular}{|c|c|l|r|r|r|}
\hline $\begin{array}{c}\text { Node } \\
\text { No. }\end{array}$ & Reference & Coordinate & \multicolumn{2}{|c|}{ Node Coordinates } & Comment \\
\hline 1 & - & System & $X[\mathrm{~m}]$ & \multicolumn{1}{c|}{$\mathrm{Z}[\mathrm{m}]$} & \\
2 & - & Cartesian & 0.000 & 0.000 & \\
3 & - & Cartesian & 4.700 & 0.000 & \\
4 & - & Cartesian & 9.400 & 0.000 & \\
& & 14.100 & 0.000 & \\
\hline
\end{tabular}

- 1.8 NODAL SUPPORTS

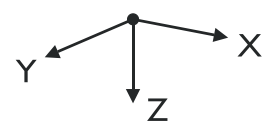

\begin{tabular}{|c|c|c|c|c|c|c|}
\hline \multirow{2}{*}{$\begin{array}{c}\text { Support } \\
\text { No. }\end{array}$} & \multirow[b]{2}{*}{ Nodes No. } & \multirow{2}{*}{$\begin{array}{c}\text { Rotation }\left[{ }^{\circ}\right] \\
\text { about } \mathrm{Y}\end{array}$} & \multicolumn{3}{|c|}{ Support or Spring $[\mathrm{kN} / \mathrm{m}][\mathrm{kNm} / \mathrm{rad}]$} & \multirow[b]{2}{*}{ Comment } \\
\hline & & & $u_{x^{\prime}}$ & $\mathrm{u}_{\mathrm{Z}^{\prime}}$ & $\varphi Y^{\prime}$ & \\
\hline 1 & 1 & 0.00 & 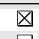 & $\underline{\nabla}$ & $\square$ & \\
\hline 2 & $2-4$ & 0.00 & $\square$ & $\Delta$ & $\square$ & \\
\hline
\end{tabular}

\subsection{LOAD CASES}

\begin{tabular}{|c|c|c|c|c|c|c|}
\hline Load & Load Case & EN $1990 \mid$ DIN & & Wei & & \\
\hline Case & Description & Action Category & Active & $x$ & Y & Z \\
\hline LC1 & Self Weight + Roof Covering & Permanent & $\sqsupset$ & & & \\
\hline LC2 & Snow & Snow $(H \leq 1000 m$ a.s.I. $)$ & $\sqsupset$ & & & \\
\hline LC3 & Wind PRESSURE & Wind & $\sqsupset$ & & & \\
\hline LC4 & Wind SUCTION & Wind & $\sqsupset$ & & & \\
\hline
\end{tabular}

\subsection{LOAD COMBINATIONS}

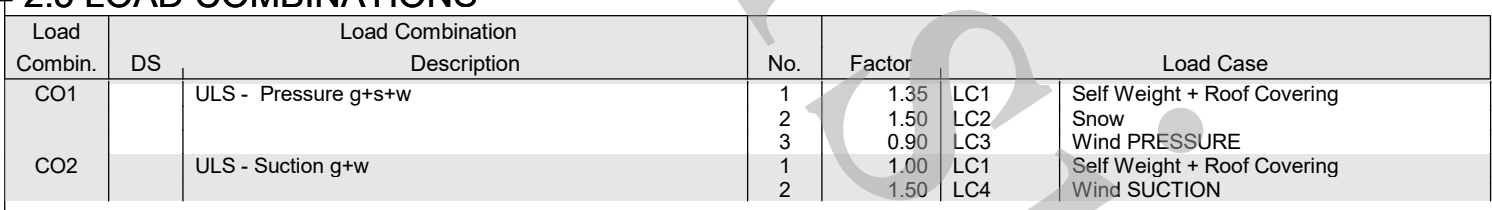

LC1

Self Weight + Roof Covering

LC2

Snow

\subsection{MEMBER LOADS}

\begin{tabular}{|c|c|c|c|c|c|c|c|c|c|}
\hline No. & $\begin{array}{c}\text { Reference } \\
\text { to }\end{array}$ & $\begin{array}{c}\text { On Members } \\
\text { No. }\end{array}$ & $\begin{array}{c}\text { Load } \\
\text { Type }\end{array}$ & $\begin{array}{c}\text { Load } \\
\text { Distribution }\end{array}$ & $\begin{array}{c}\text { Load } \\
\text { Direction }\end{array}$ & $\begin{array}{c}\text { Reference } \\
\text { Length }\end{array}$ & $\begin{array}{c}\text { Load Parameters } \\
\text { Symbol }\end{array}$ \\
\hline 1 & Members & $1-3$ & Force & Uniform & $\mathrm{Z}$ & True Length & $\mathrm{p}$ & 0.500 & $\mathrm{kN} / \mathrm{m}$ \\
\hline
\end{tabular}

3.2 MEMBER LOADS

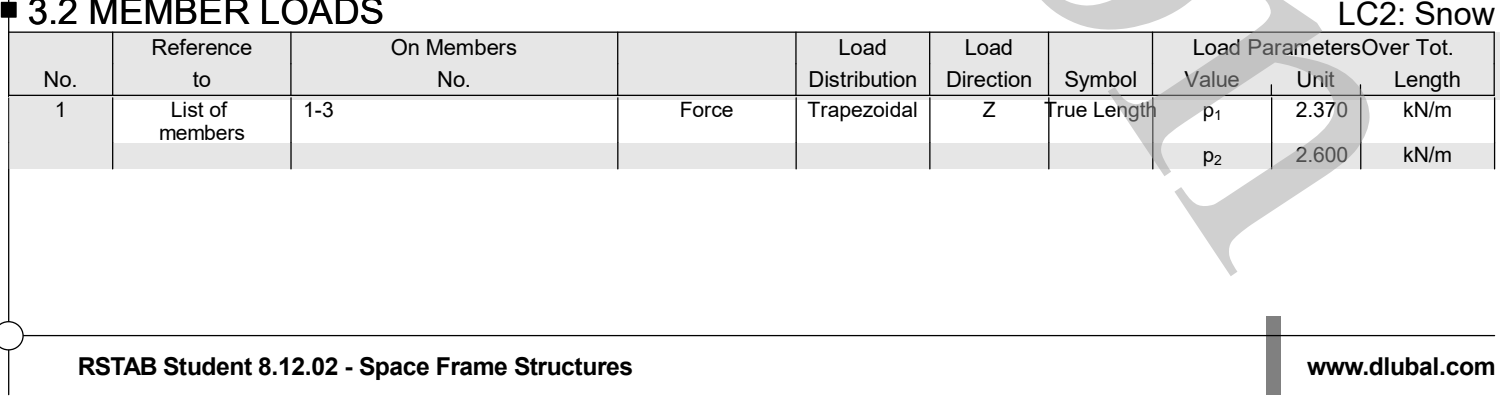




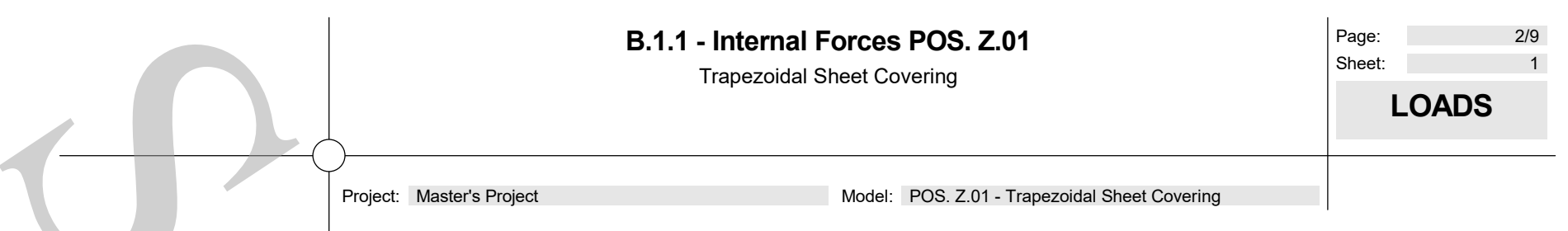

LC3

3.2 MEMBER LOADS

LC3: Wind PRESSURE

Wind PRESSURE

LC4

\begin{tabular}{|c|c|c|c|c|c|c|c|c|c|}
\hline & Reference & On Members & Load & Load & Load & Reference & \multicolumn{3}{|c|}{ Load Parameters } \\
\hline No. & to & No. & Type & Distribution & Direction & Length & Symbol & Value & Unit \\
\hline 1 & Members & $1-3$ & Force & Uniform & $\mathrm{Z}$ & True Length & $p$ & 0.110 & $\mathrm{kN} / \mathrm{m}$ \\
\hline
\end{tabular}

- 3.2 MEMBER LOADS

\begin{tabular}{|c|c|c|c|c|c|c|c|c|c|}
\hline 3.2 & $=\mathrm{MBE}$ & OADS & & & & & \multicolumn{3}{|c|}{ LC4: Wind SUCTION } \\
\hline & Reference & On Members & Load & Load & Load & Reference & & d Paramet & \\
\hline No. & to & No. & Type & Distribution & Direction & Length & Symbol & Value & Unit \\
\hline 1 & Members & 1 & Force & Uniform & Z & True Length & $p$ & -1.000 & $\mathrm{kN} / \mathrm{m}$ \\
\hline 2 & Members & 2,3 & Force & Uniform & Z & True Length & $\mathrm{p}$ & -0.670 & $\mathrm{kN} / \mathrm{m}$ \\
\hline
\end{tabular}



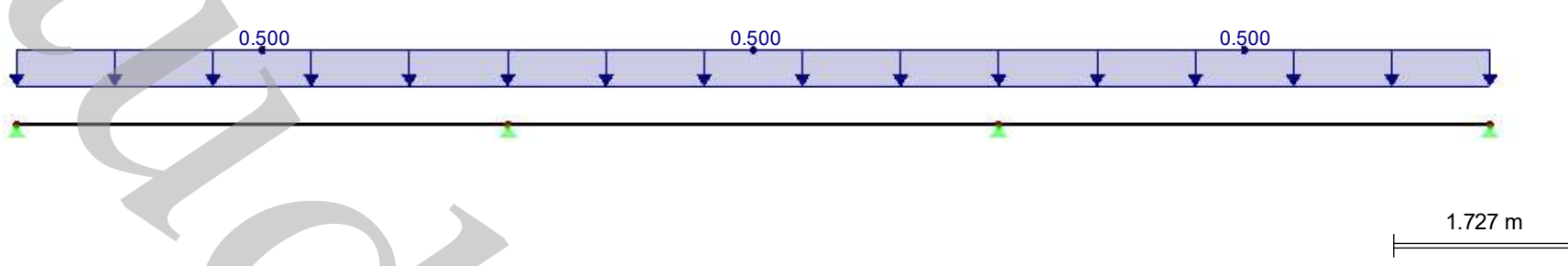

- LC2: SNOW

LC 2: Snow

Loads $[\mathrm{kN} / \mathrm{m}]$

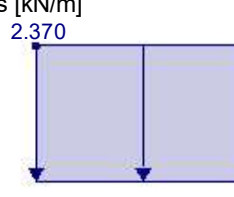

Against Y-direction
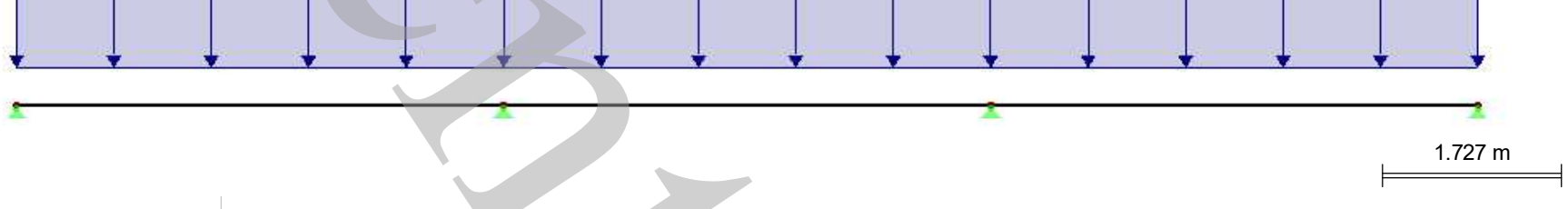

- LC3: WIND PRESSURE

LC 3: Wind PRESSURE Loads $[\mathrm{kN} / \mathrm{m}$ ]

Against $Y$-direction
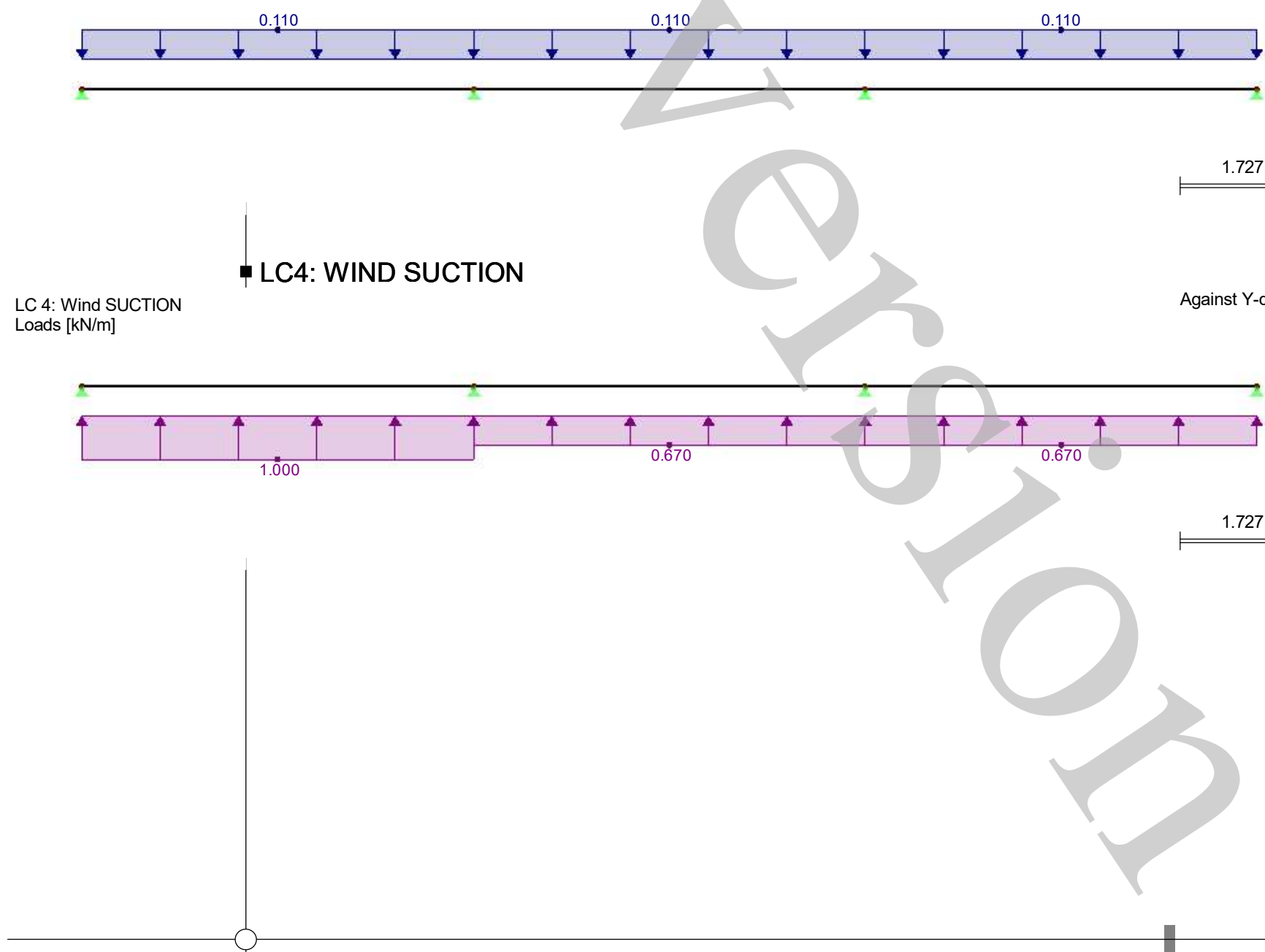


\section{CO1: ULS - PRESSURE G+S+W}

CO 1: ULS - Pressure $\mathrm{g}+\mathrm{s}+\mathrm{w}$

Loads $[\mathrm{kN} / \mathrm{m}]$

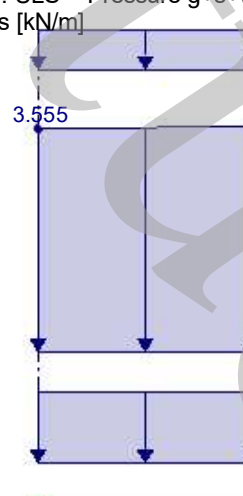




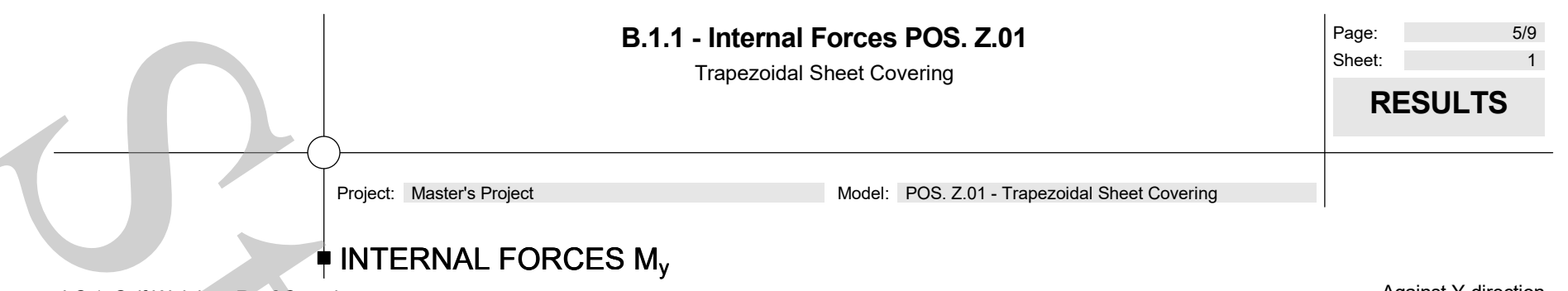

LC 1: Self Weight + Roof Covering

Internal Forces M-y

Against Y-direction

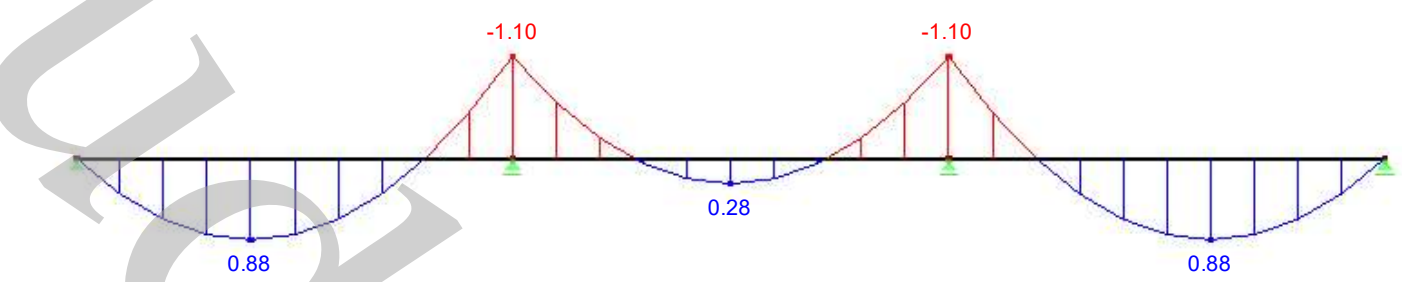

Max M-y: 0.88, Min M-y: $-1.10[k N m]$

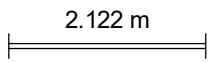

\section{INTERNAL FORCES $V_{z}$, SUPPORT REACTIONS}

LC 1: Self Weight + Roof Covering

Internal Forces $\mathrm{V}-\mathrm{z}$

Support Reactions $[\mathrm{kN}]$

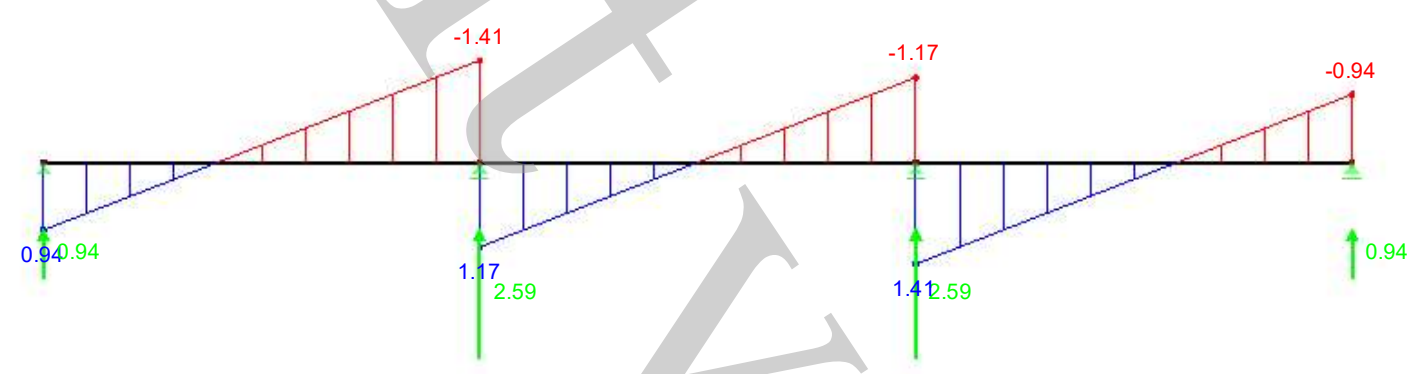

Max V-z: 1.41, Min V-z: $-1.41[\mathrm{kN}]$

Max P-X': 0.00 , Min P-X': $0.00 \mathrm{kN}$

Max P-Z': 2.59, Min P-Z': $0.94 \mathrm{kN}$

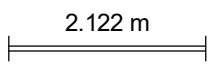

LC 2: Snow

INTERNAL FORCES $\mathrm{M}_{\mathrm{y}}$

Internal Forces M-y

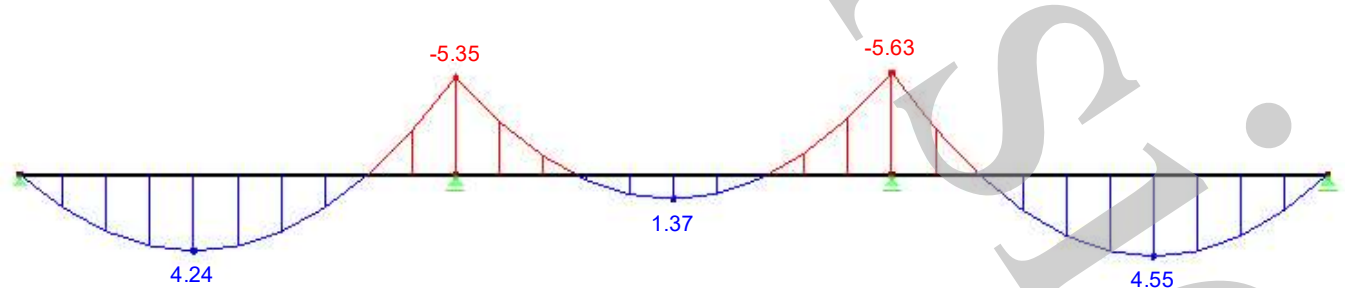

Max M-y: 4.55, Min M-y: -5.63 [kNm]

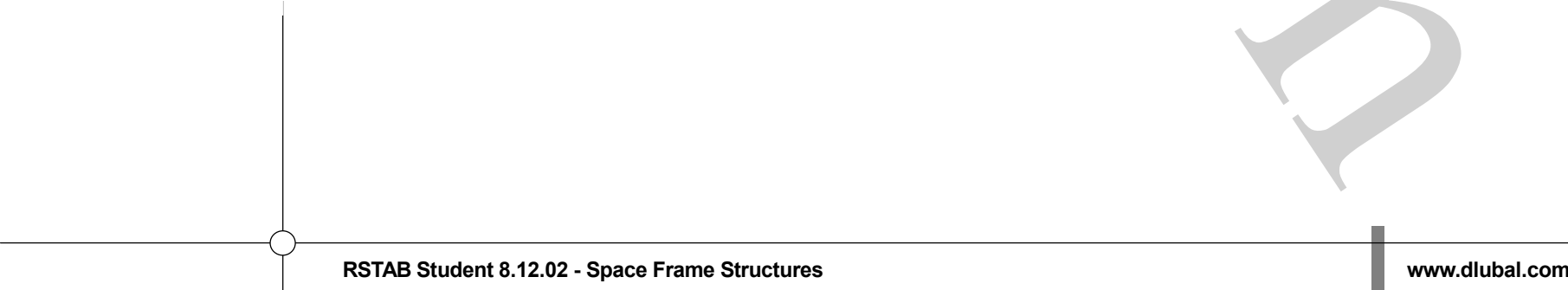




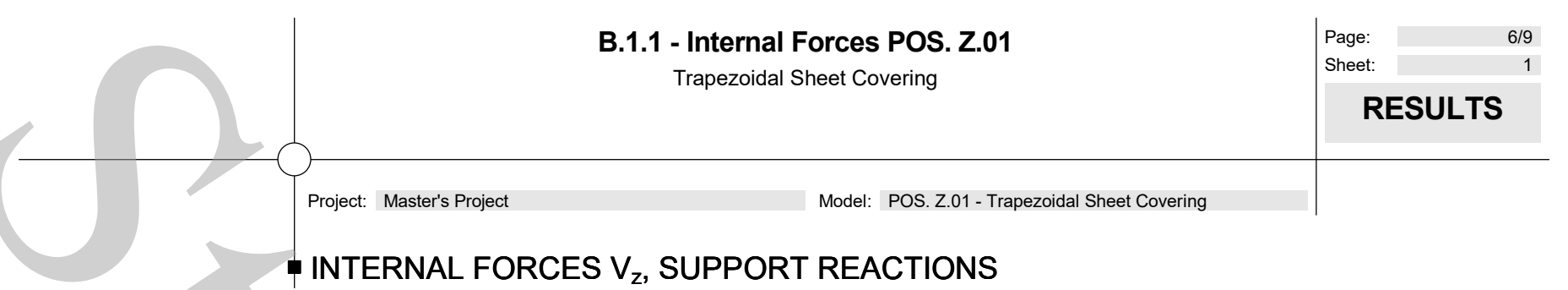

LC 2: Snow

INTERNAL FORCES $V_{z}$, SUPPORT REACTIONS

Internal Forces $\mathrm{V}$-z

Support Reactions[kN]

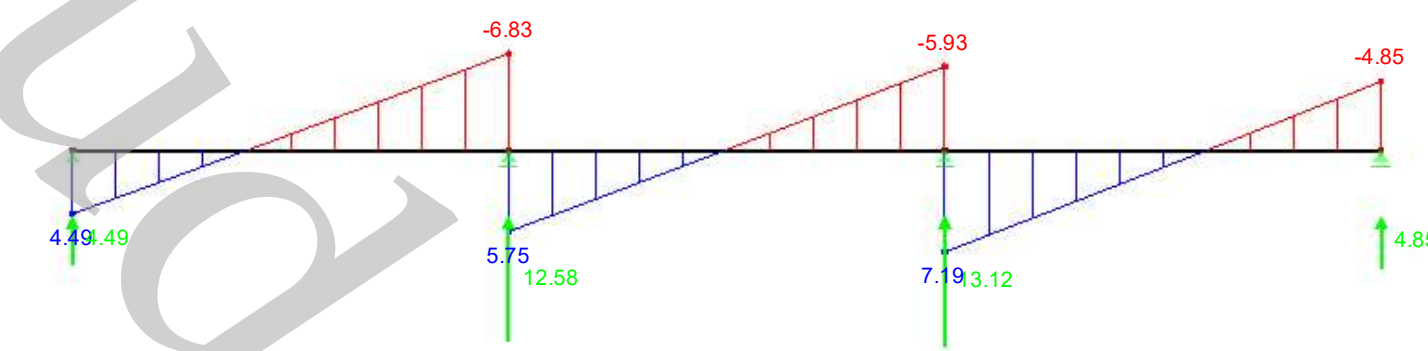

Max V-z: 7.19, Min V-z: $-6.83[\mathrm{kN}]$

Max P-X': 0.00 , Min P-X': $0.00 \mathrm{kN}$

Max P-Z': 13.12, Min P-Z': $4.49 \mathrm{kN}$

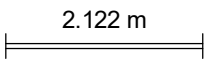

INTERNAL FORCES $M_{y}$

LC 3: Wind PRESSURE

Internal Forces M-y

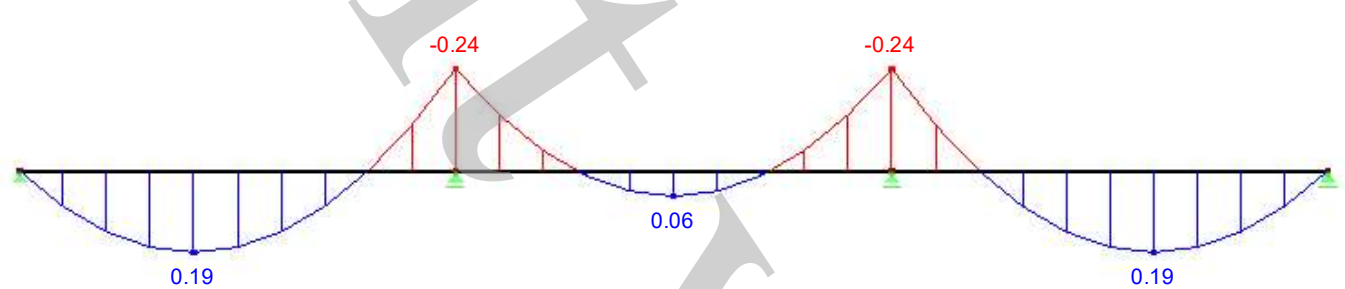

Max M-y: 0.19, Min M-y: $-0.24[\mathrm{kNm}]$

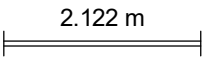

INTERNAL FORCES $V_{z}$, SUPPORT REACTIONS

LC 3: Wind PRESSURE

Internal Forces $V-z$

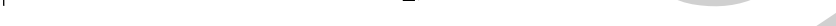

Support Reactions[kN]
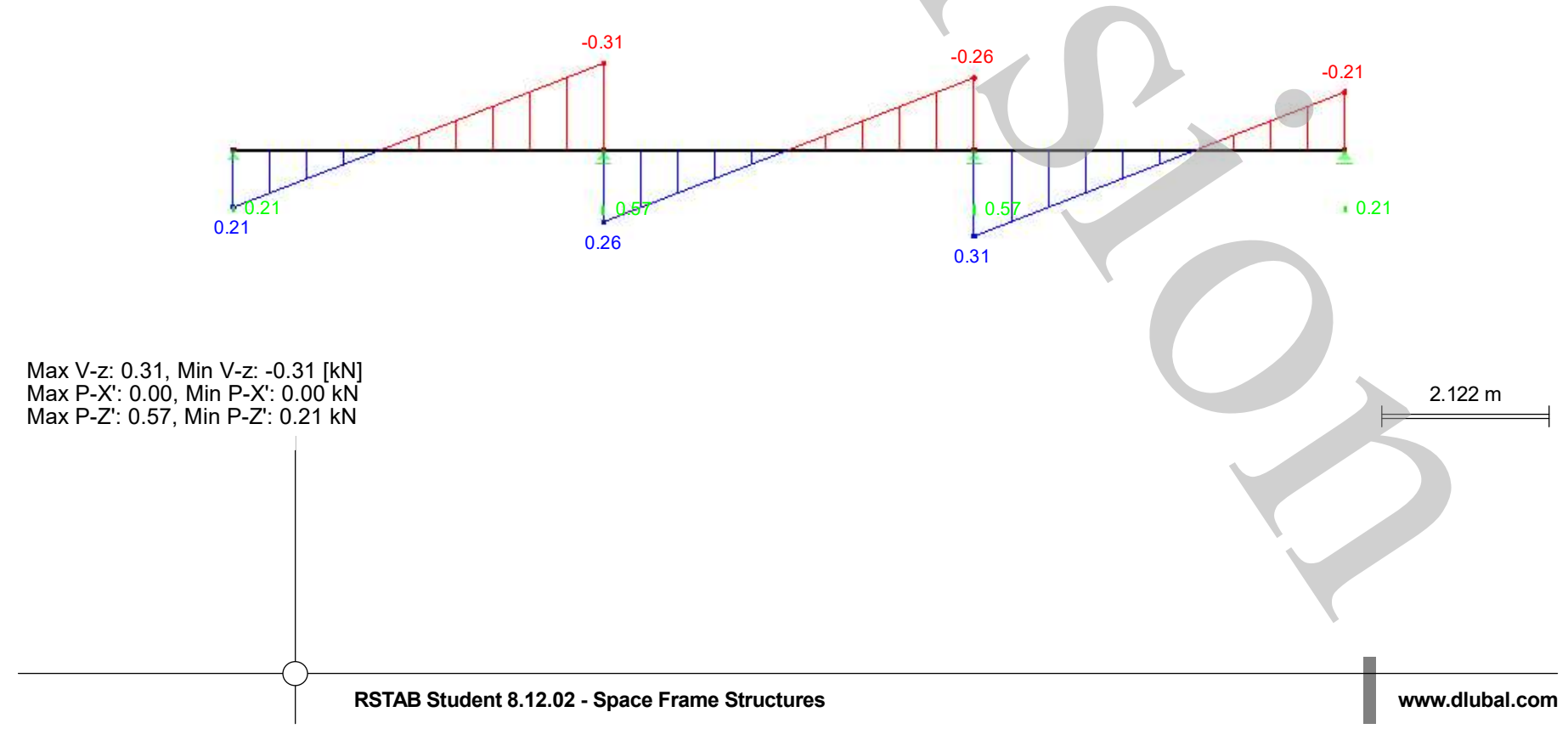


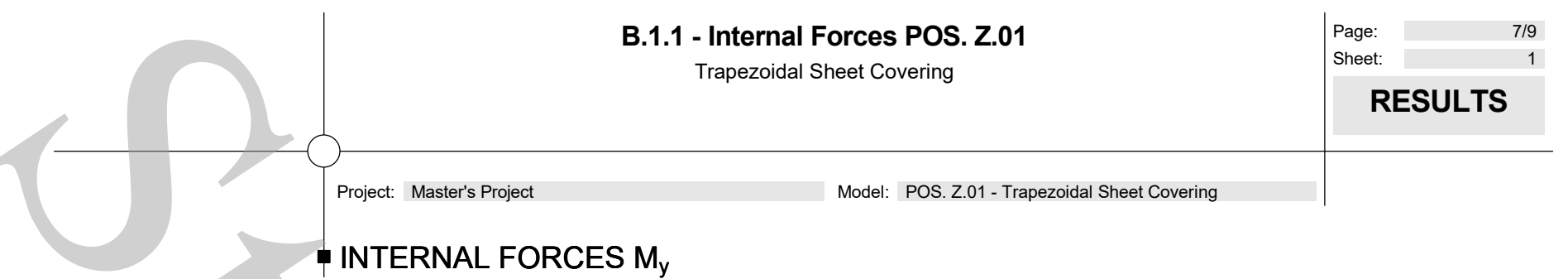

LC 4: Wind SUCTION

- INTERNAL FORCES $\mathrm{M}_{\mathrm{y}}$

Internal Forces $\mathrm{M}-\mathrm{y}$

Against Y-direction

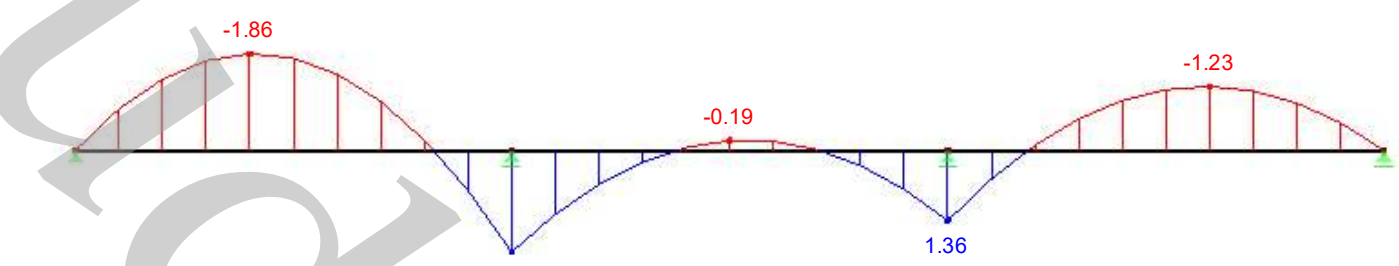

Max M-y: 1.97, Min M-y: -1.86 [kNm]

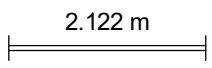

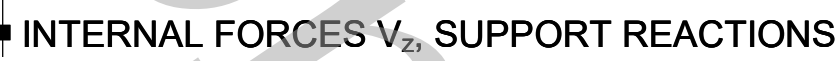

LC 4: Wind SUCTION

Internal Forces $\mathrm{V}-\mathrm{z}$

Support Reactions[kN]

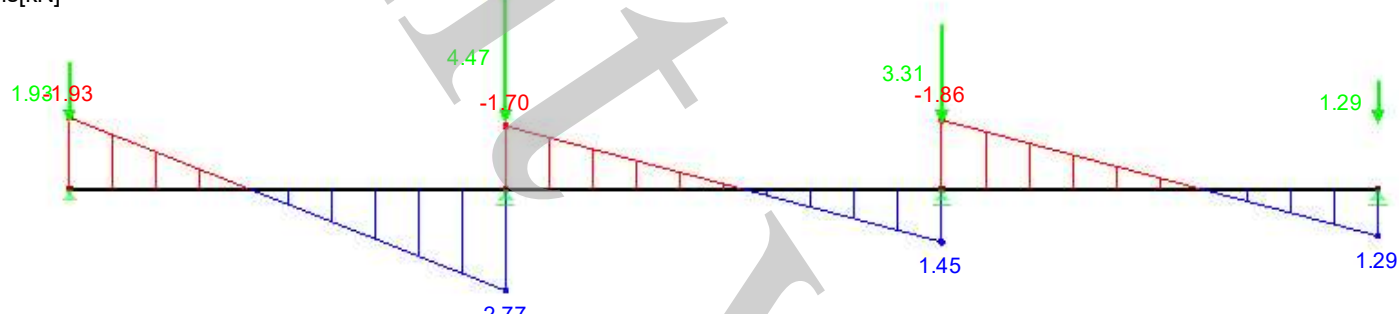

Max V-z: 2.77, Min V-z: $-1.93[\mathrm{kN}]$

Max P-X': 0.00 , Min P-X': $0.00 \mathrm{kN}$

Max P-Z': -1.29 , Min P-Z': $-4.47 \mathrm{kN}$

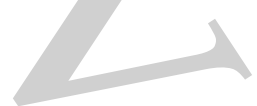

\section{INTERNAL FORCES $\mathrm{M}_{\mathrm{y}}$}

CO 1: ULS - Pressure $\mathrm{g}+\mathrm{s}+\mathrm{w}$

Internal Forces M-y

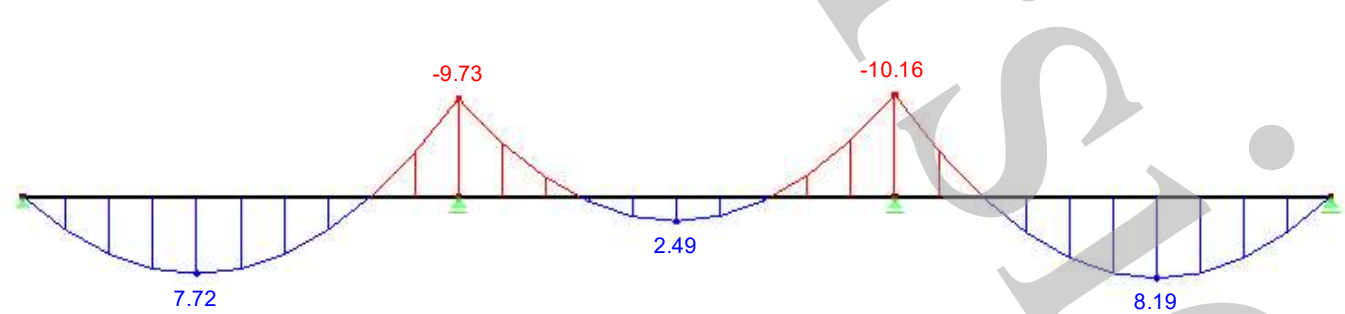

Max M-y: 8.19, Min M-y: -10.16 [kNm]

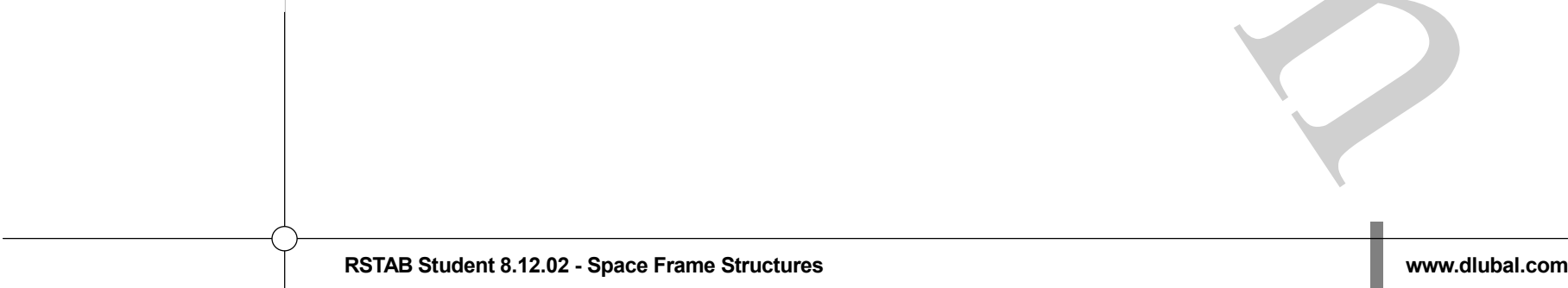


Support Reactions[kN]

\section{INTERNAL FORCES $V_{z}$, SUPPORT REACTIONS}

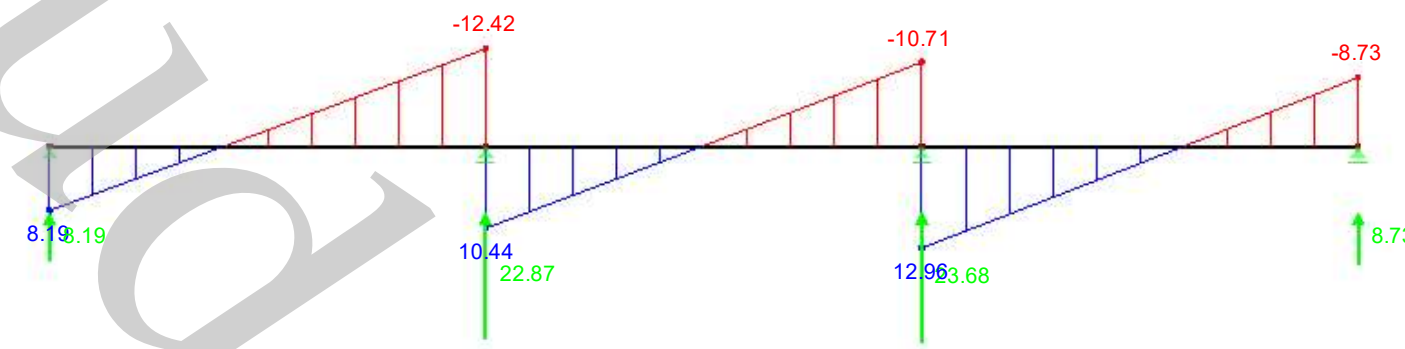

Max V-z: 12.96, Min V-z: -12.42 [kN]

Max P-X': 0.00 , Min P-X': $0.00 \mathrm{kN}$

Max P-Z': 23.68, Min P-Z': $8.19 \mathrm{kN}$

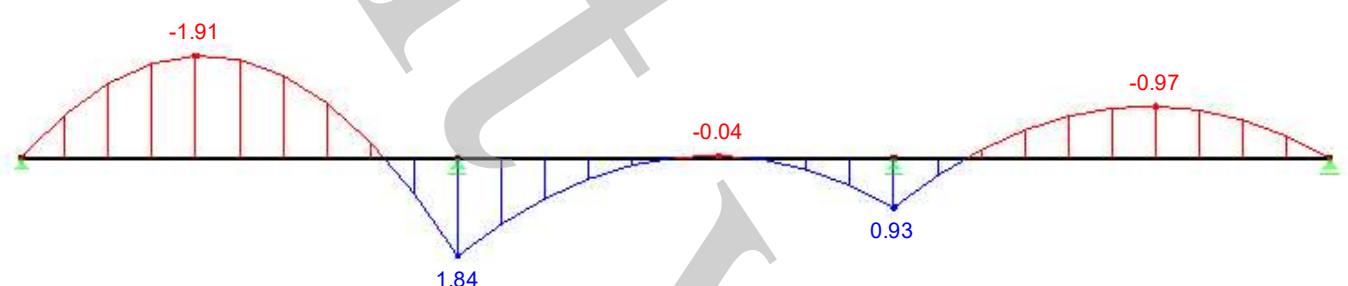

\section{- INTERNAL FORCES V $V_{z}$, SUPPORT REACTIONS}

CO 2: ULS - Suction g+w

Internal Forces $\mathrm{V}-\mathrm{z}$

Support Reactions[kN]
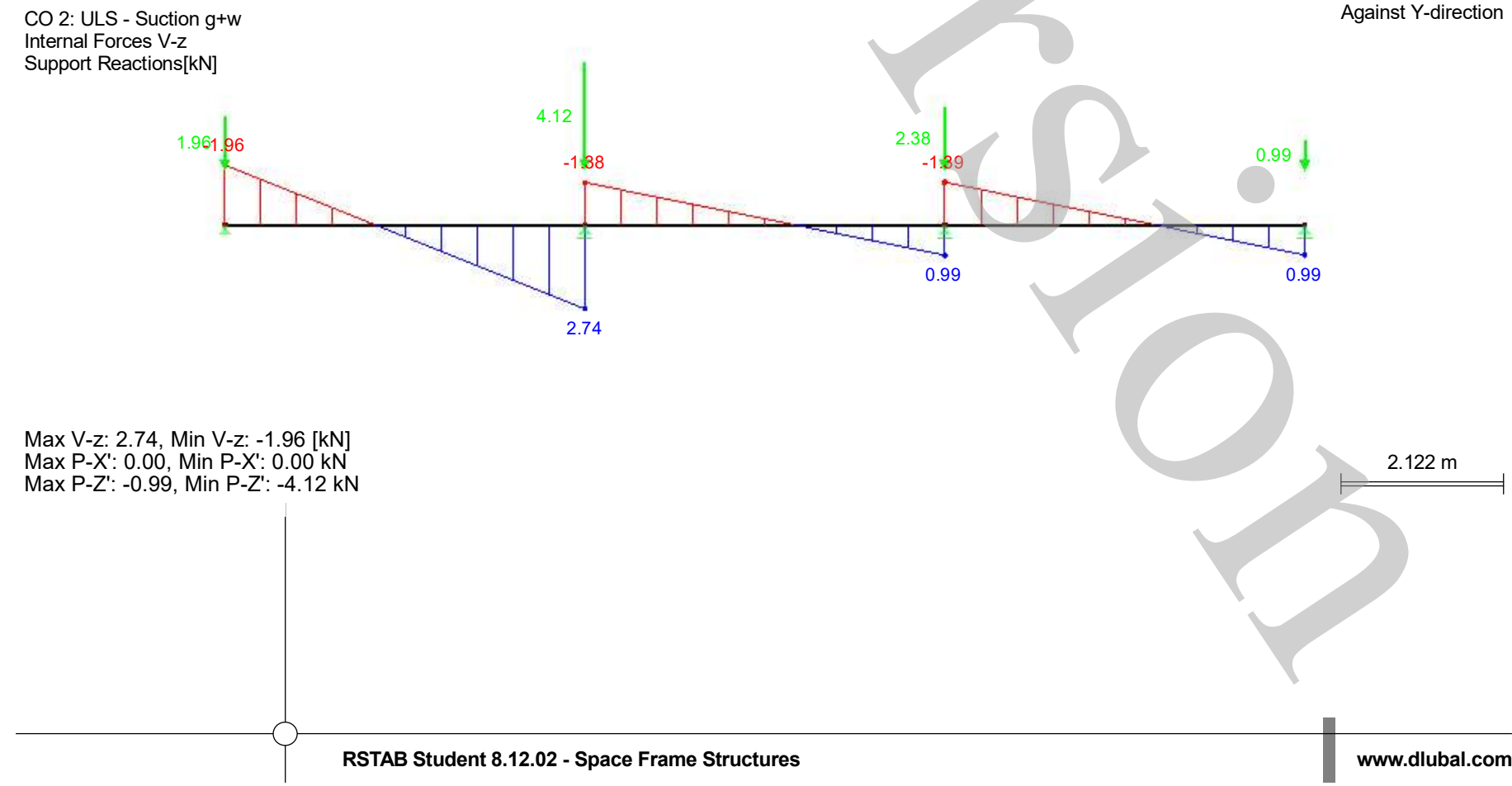


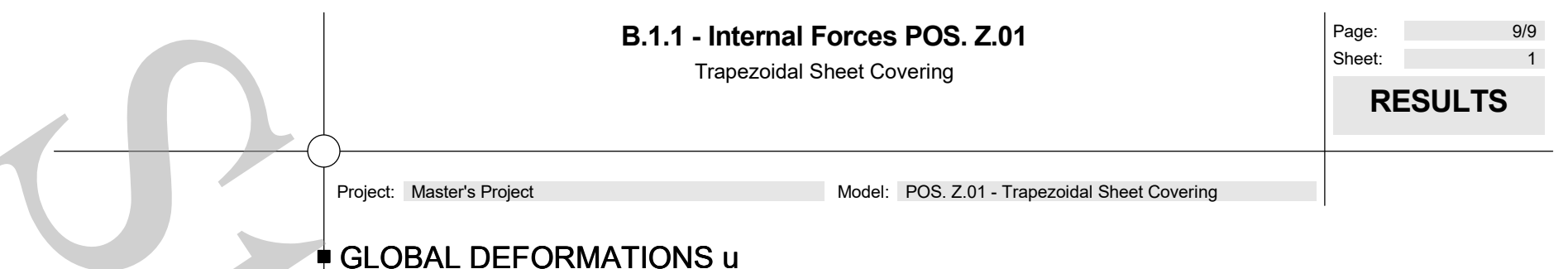

LC 1: Self Weight + Roof Covering Global Deformations u

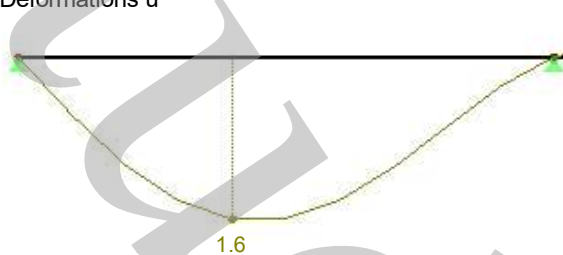

Max u: 1.6, Min u: 0.0 [mm]

Factor of deformations: 880.00

$1.727 \mathrm{~m}$

- GLOBAL DEFORMATIONS u

CO 1: ULS - Pressure $\mathrm{g}+\mathrm{s}+\mathrm{w}$

Global Deformations u

Against Y-direction

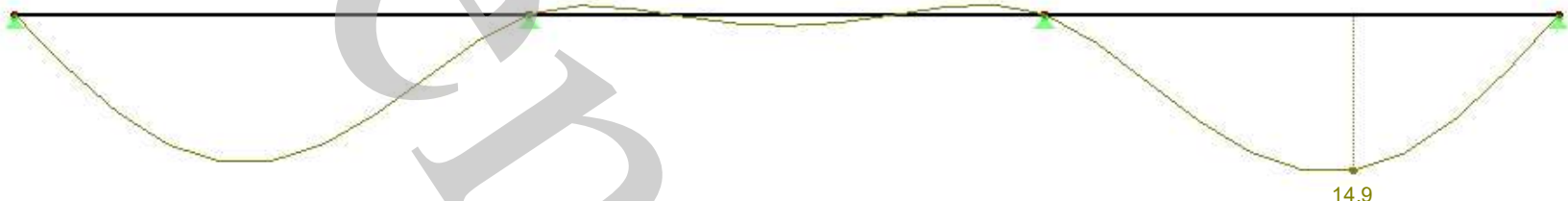

Max u: 14.9, Min u: $0.0[\mathrm{~mm}]$

Factor of deformations: 95.00

$1.727 \mathrm{~m}$

GLOBAL DEFORMATIONS u

CO 2: ULS - Suction $\mathrm{g}+\mathrm{w}$

Global Deformations u

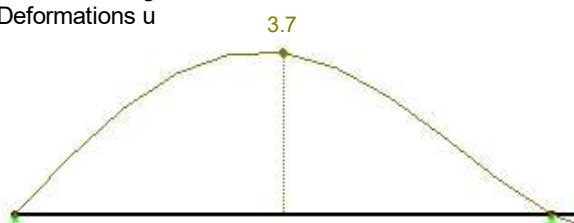

Against Y-direction

Max u: 3.7, Min u: $0.0[\mathrm{~mm}]$

Factor of deformations: 380.00
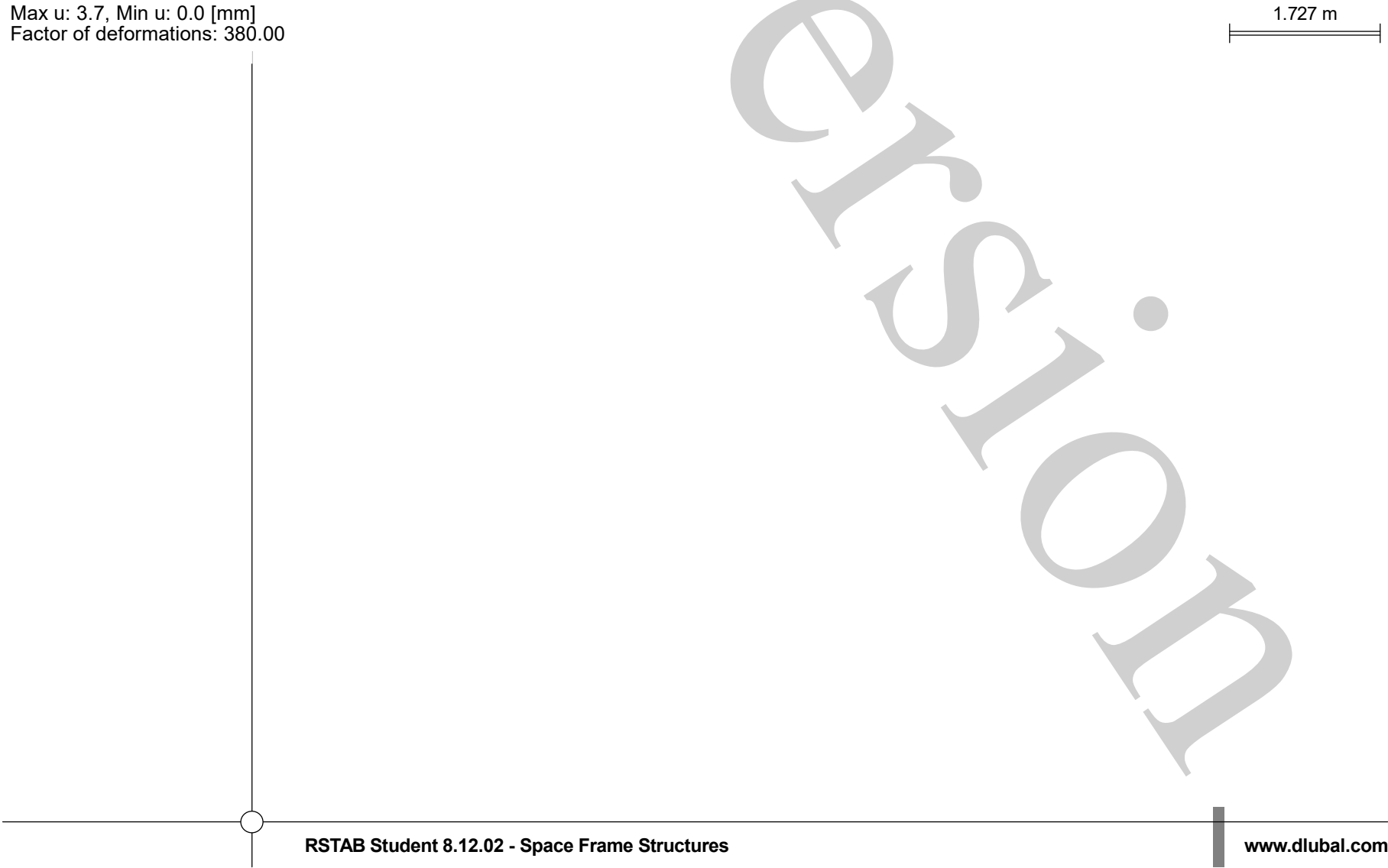
B.2.2. Internal Forces POS. P.03 - Prestressed Concrete Truss 


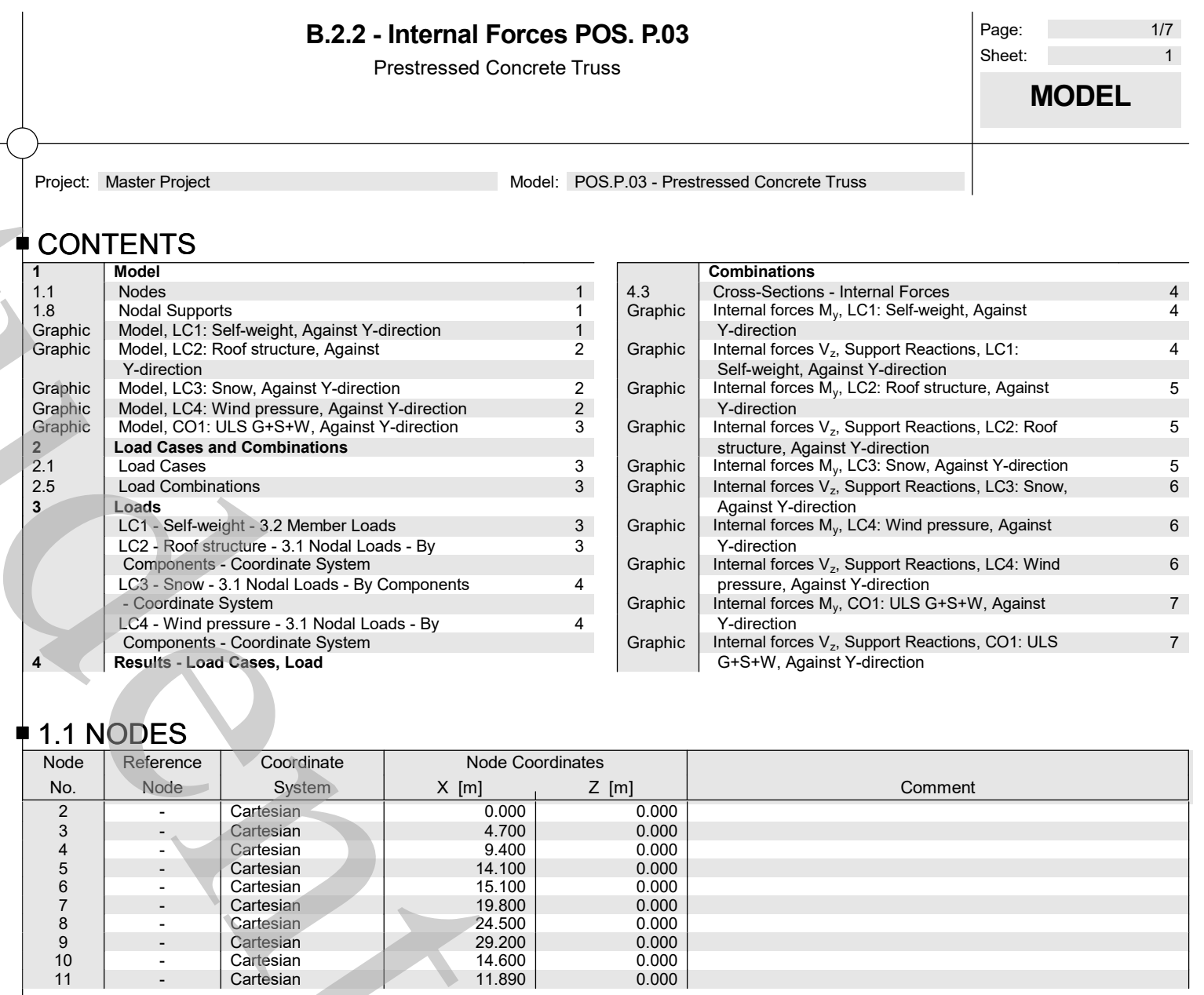

- 1.8 NODAL SUPPORTS

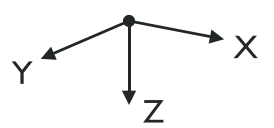

\begin{tabular}{|c|c|c|c|c|c|c|}
\hline Support & & Rotation [ $\left.{ }^{\circ}\right]$ & & $\mathrm{ng}[\mathrm{k}$ & & \\
\hline No. & Nodes No. & about $Y$ & $u_{x^{\prime}}$ & $\mathrm{u}_{\mathrm{Z}^{\prime}}$ & $\varphi Y^{\prime}$ & Comment \\
\hline 1 & 2 & 0.00 & $\nabla$ & 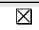 & $\square$ & \\
\hline 2 & 9 & 0.00 & $\square$ & $\vec{\nabla}$ & $\vec{\square}$ & \\
\hline
\end{tabular}

MODEL

LC 1: Self-weight Loads [kN/m]
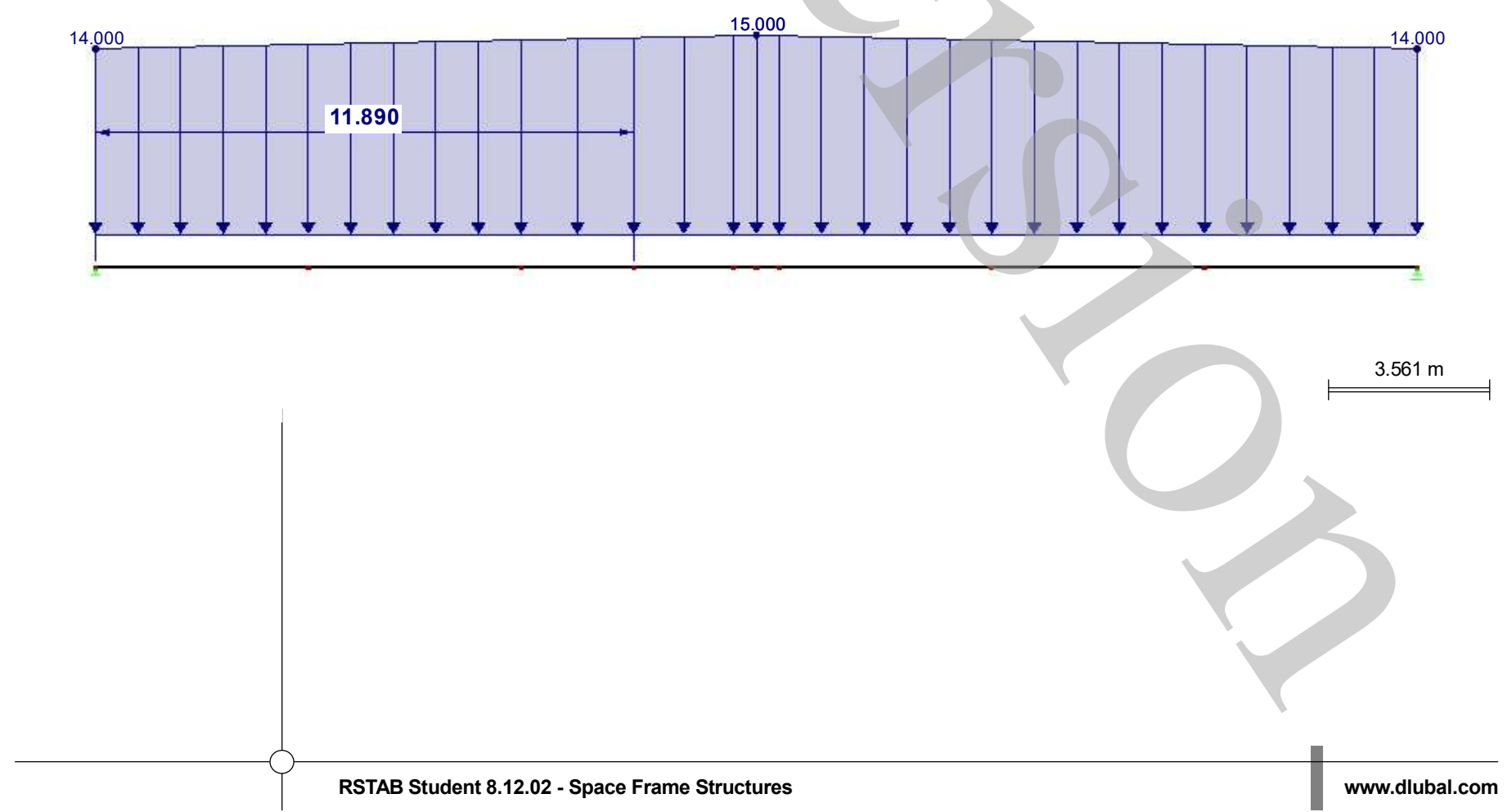


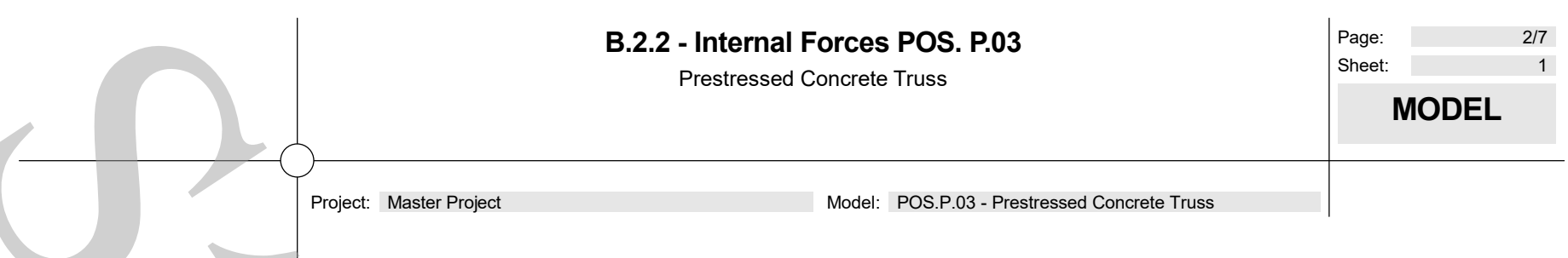

MODEL

LC 2: Roof structure

Against Y-direction Loads $[\mathrm{kN}]$
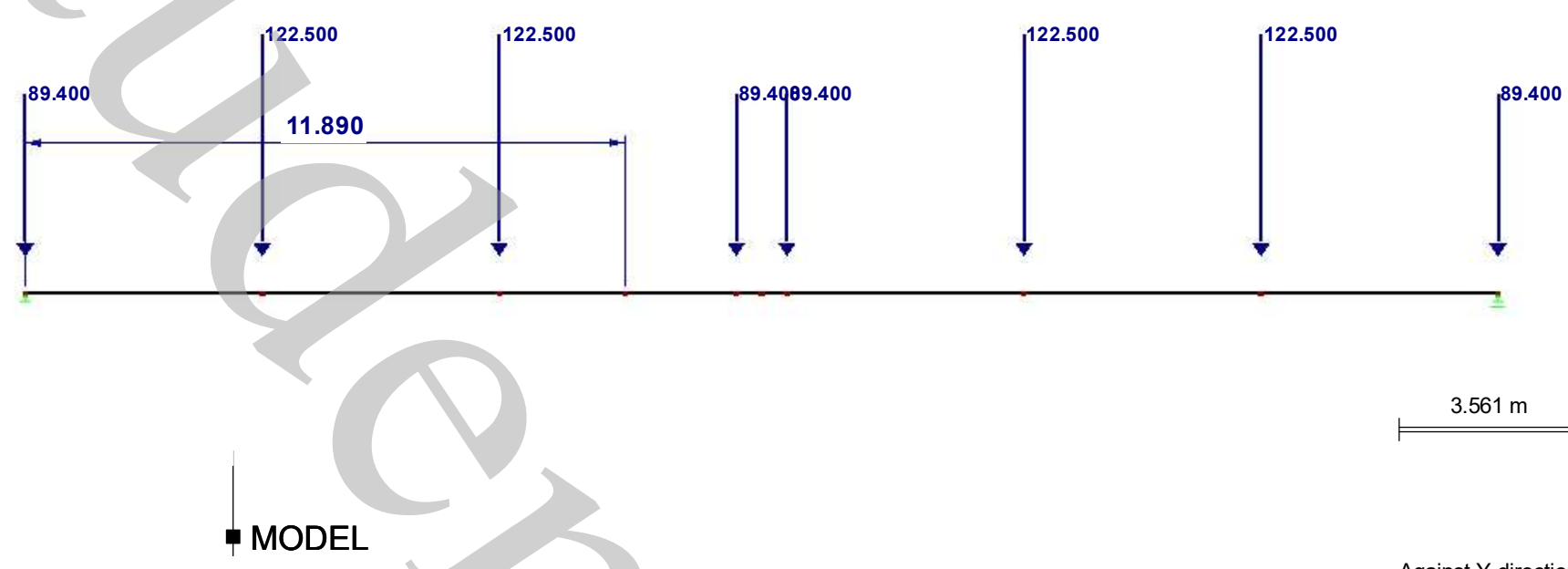

LC 3: Snow Loads [kN]
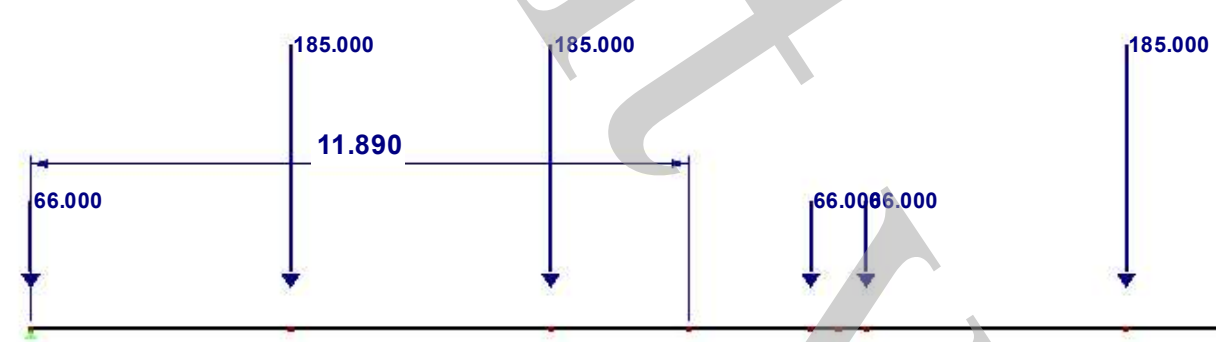

Against Y-direction
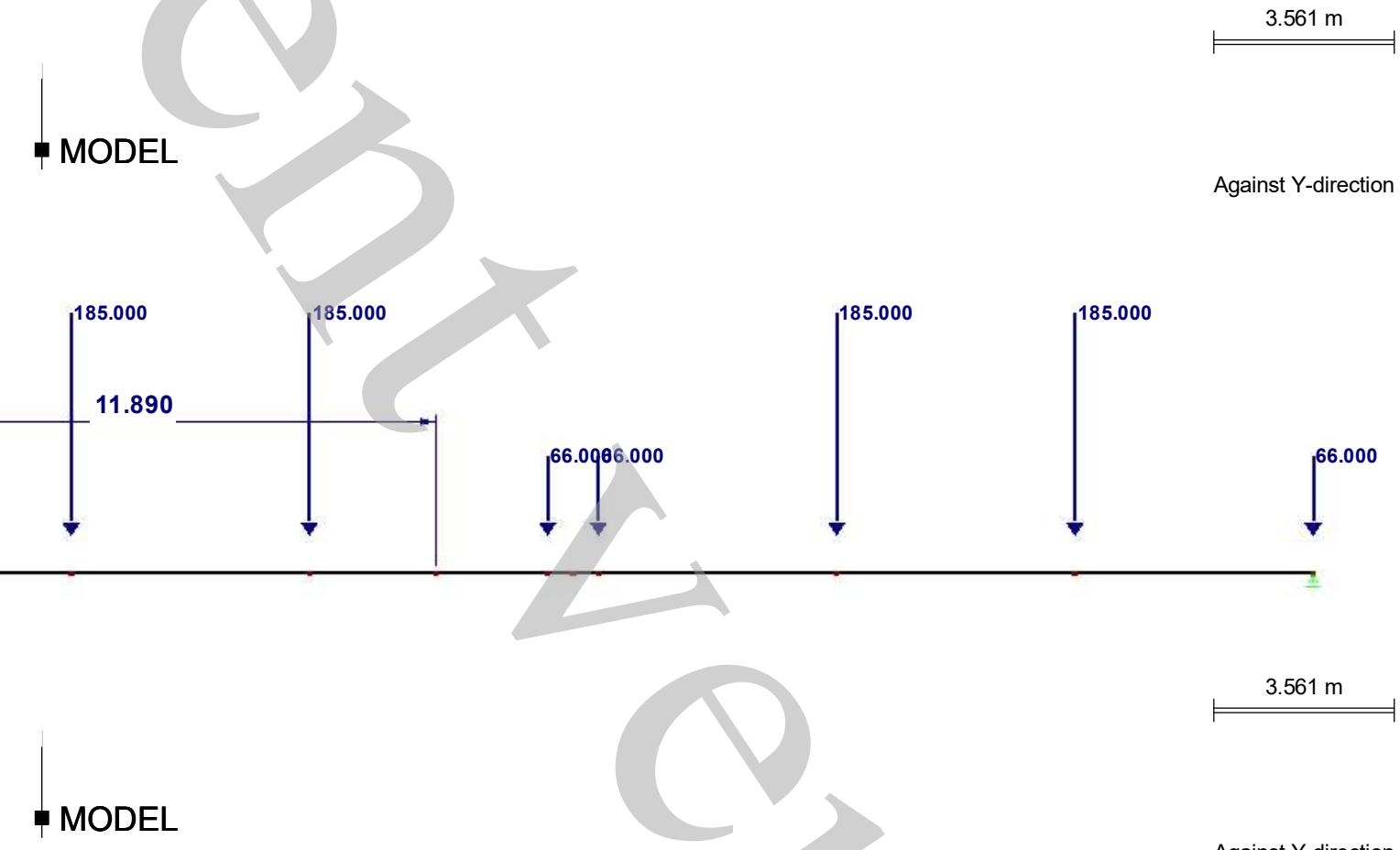

LC 4: Wind pressure Loads [kN]

Against Y-direction

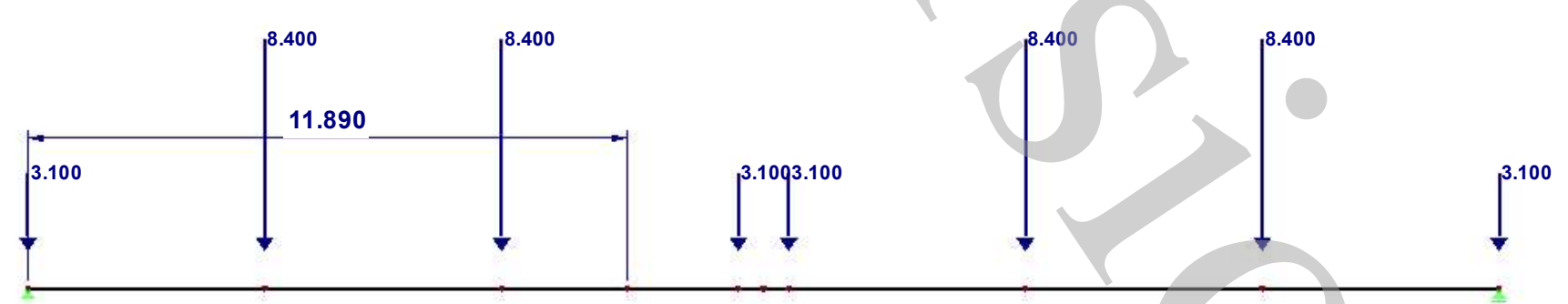



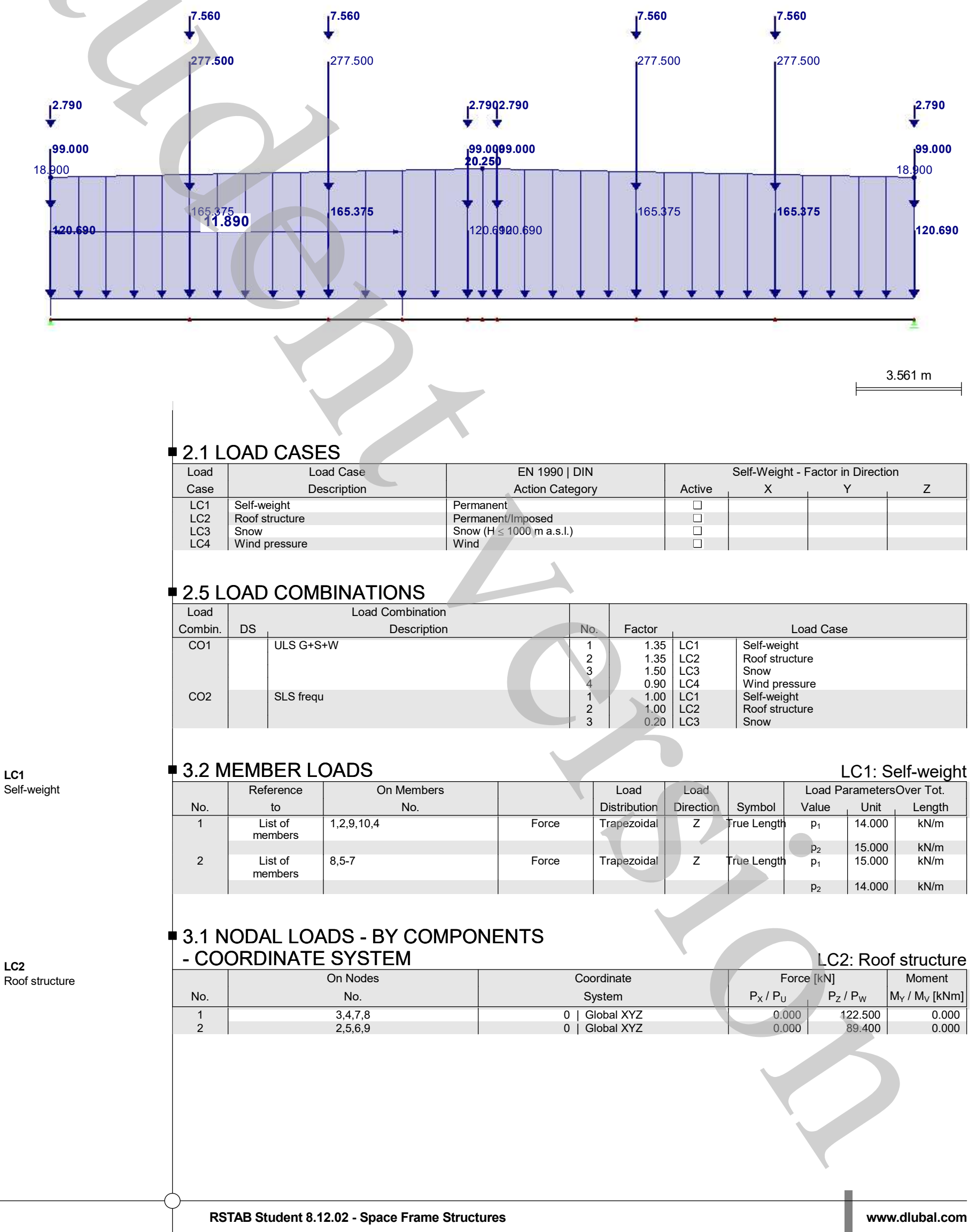


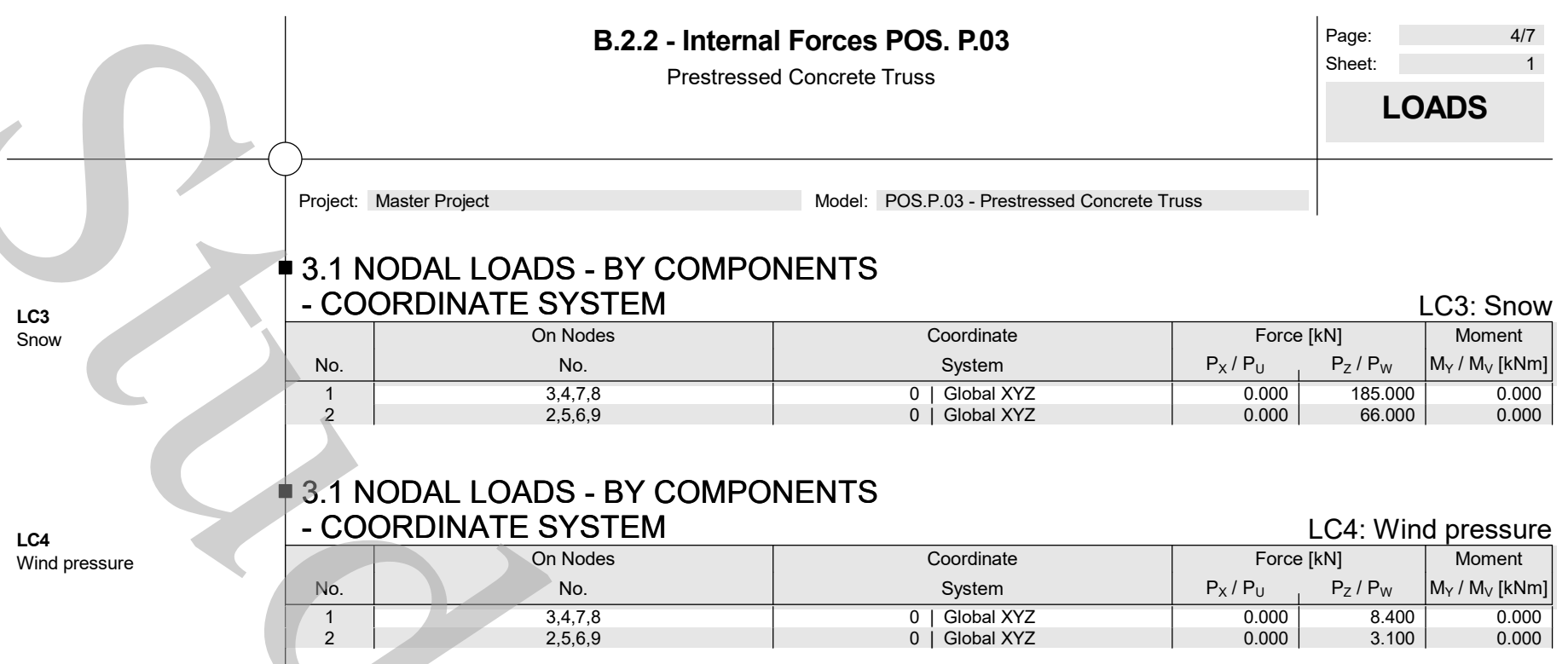

- 4.3 CROSS-SECTIONS - INTERNAL FORCES

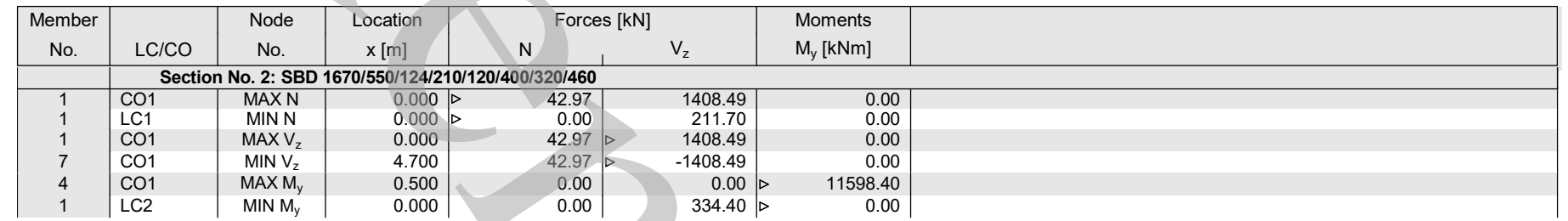

- INTERNAL FORCES M

LC 1: Self-weight

Internal Forces M-y

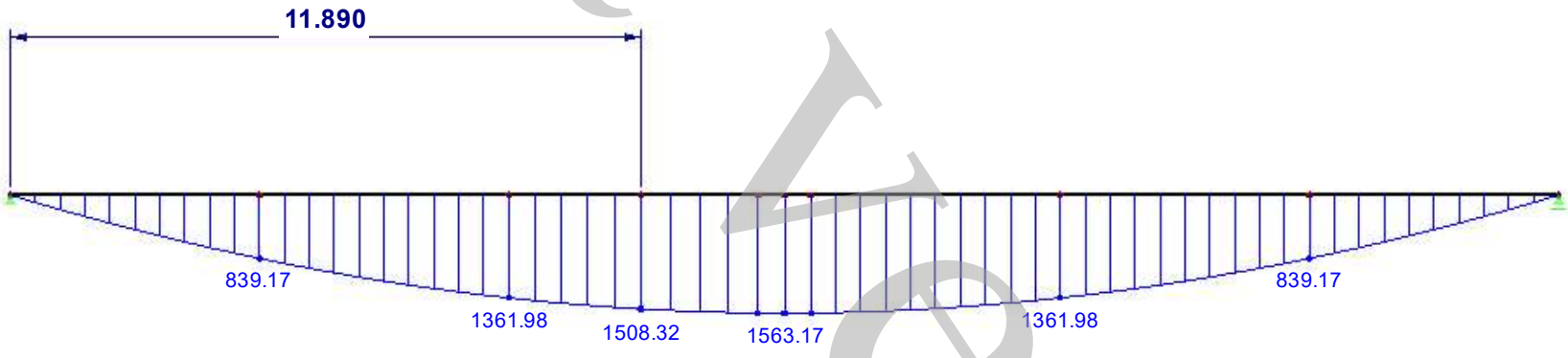

Max M-y: 1563.17, Min M-y: 0.00 [kNm]

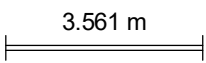

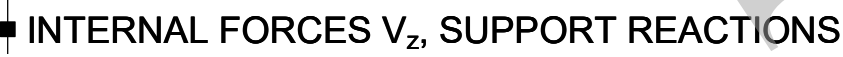

LC 1: Self-weight

Internal Forces V-z

Support Reactions[kN]

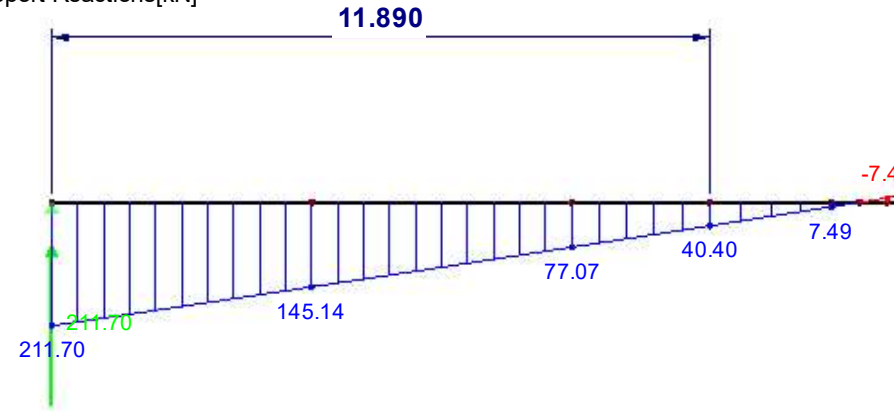

Against Y-direction

Max V-z: 211.70, Min V-z: -211.70 [kN]

Max P-X': 0.00, Min P-X': $0.00 \mathrm{kN}$

Max P-Z': 211.70, Min P-Z': $211.70 \mathrm{kN}$

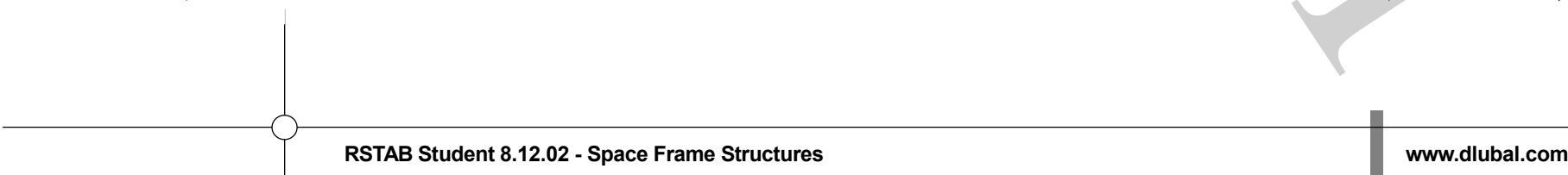




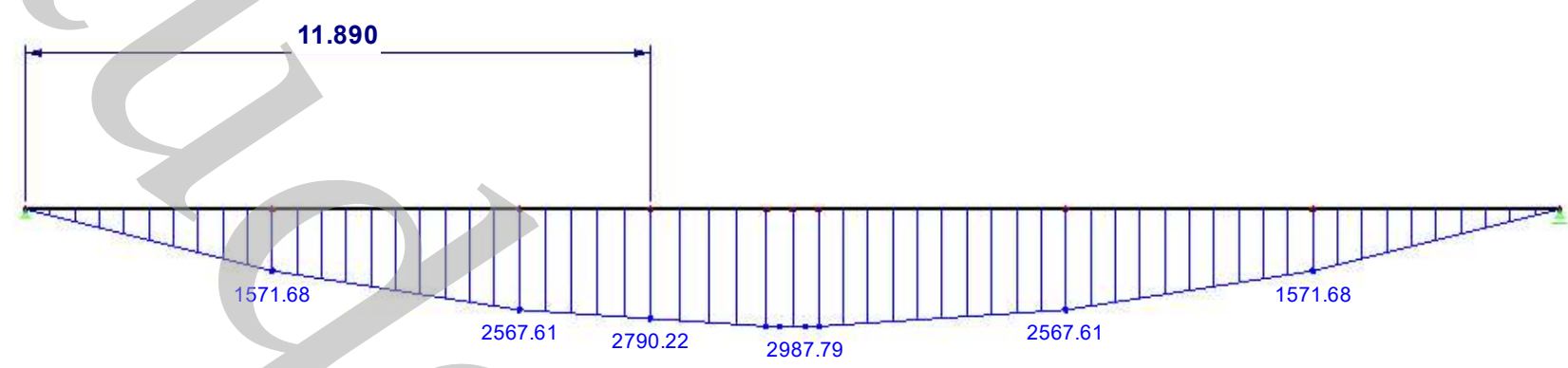

Max M-y: 2987.79, Min M-y: 0.00 [kNm]

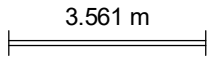

- INTERNAL FORCES V $V_{z}$, SUPPORT REACTIONS

LC 2: Roof structure

Internal Forces V-z

Support Reactions[kN]

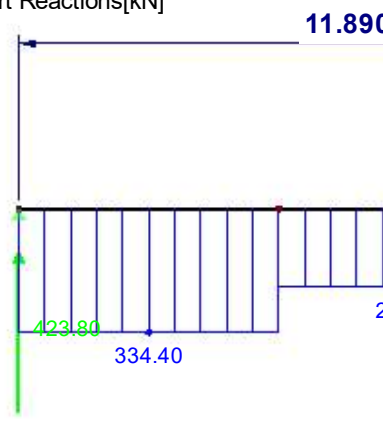

Max V-z: 334.40, Min V-z: $-334.40[\mathrm{kN}]$

Max P-X': 0.00 , Min P-X': $0.00 \mathrm{kN}$

Max P-Z': 423.80, Min P-Z': $423.80 \mathrm{kN}$

11.890

\section{- INTERNAL FORCES My}

LC 3: Snow

Internal Forces M-y

11.890

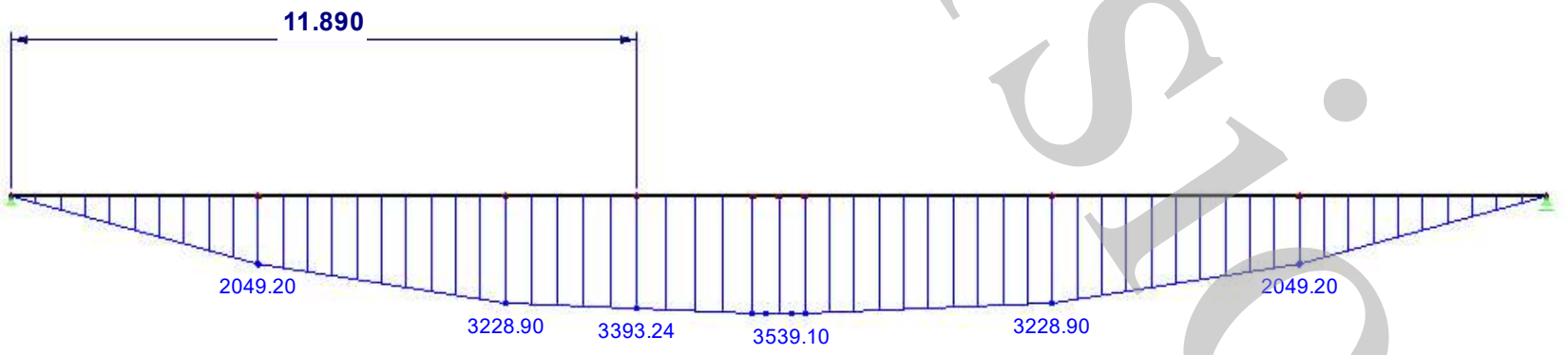

Max M-y: 3539.10, Min M-y: 0.00 [kNm]

$3.561 \mathrm{~m}$

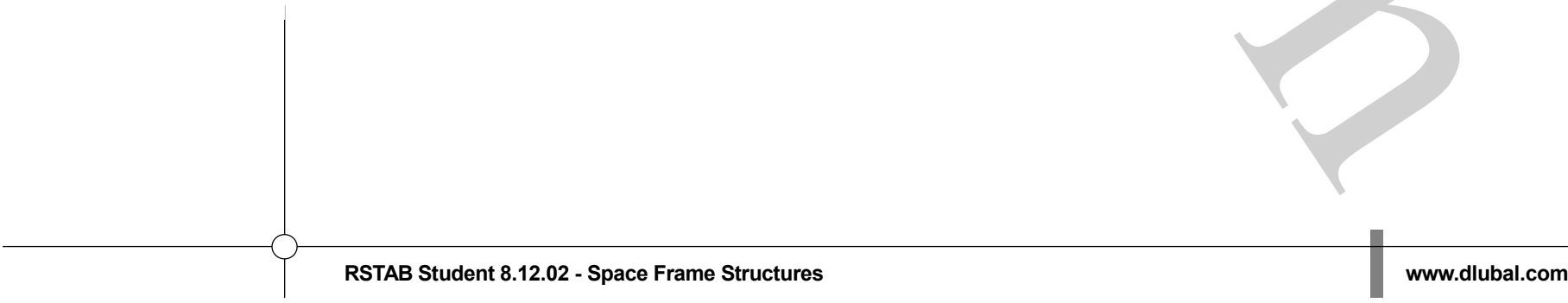




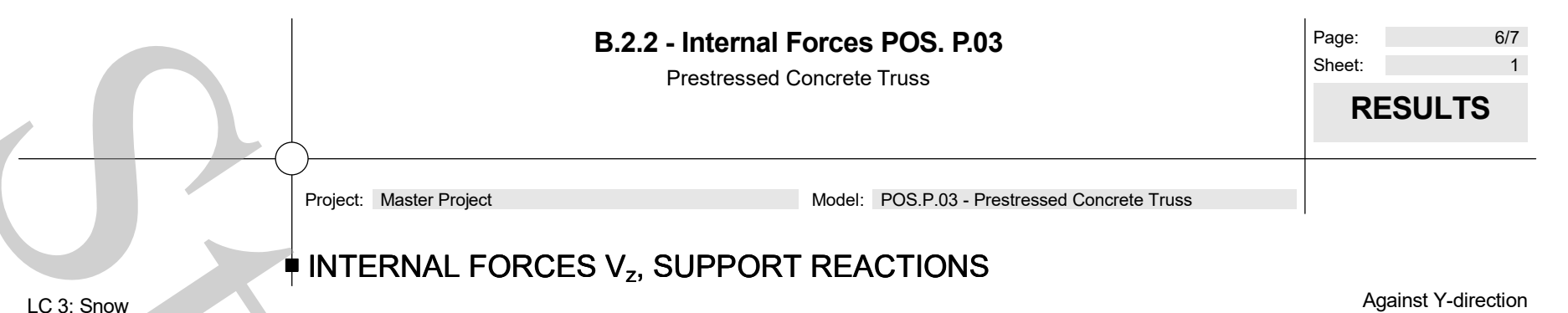

LC 3: Snow

Internal Forces $\mathrm{V}-\mathrm{z}$

Support Reactions[kN]

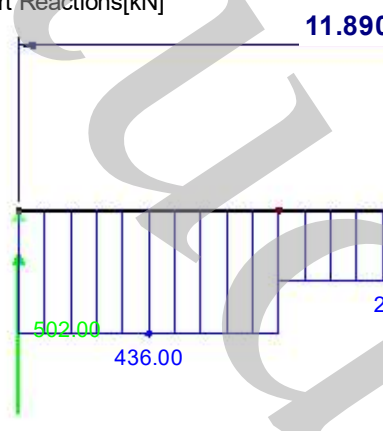

Max V-z: 436.00, Min V-z: $-436.00[\mathrm{kN}]$

Max P-X': 0.00, Min P-X': $0.00 \mathrm{kN}$

Max P-Z': 502.00 , Min P-Z': 502.00 kN

Against Y-direction .890

\section{INTERNAL FORCES M}

LC 4: Wind pressure Internal Forces M-y

11.890

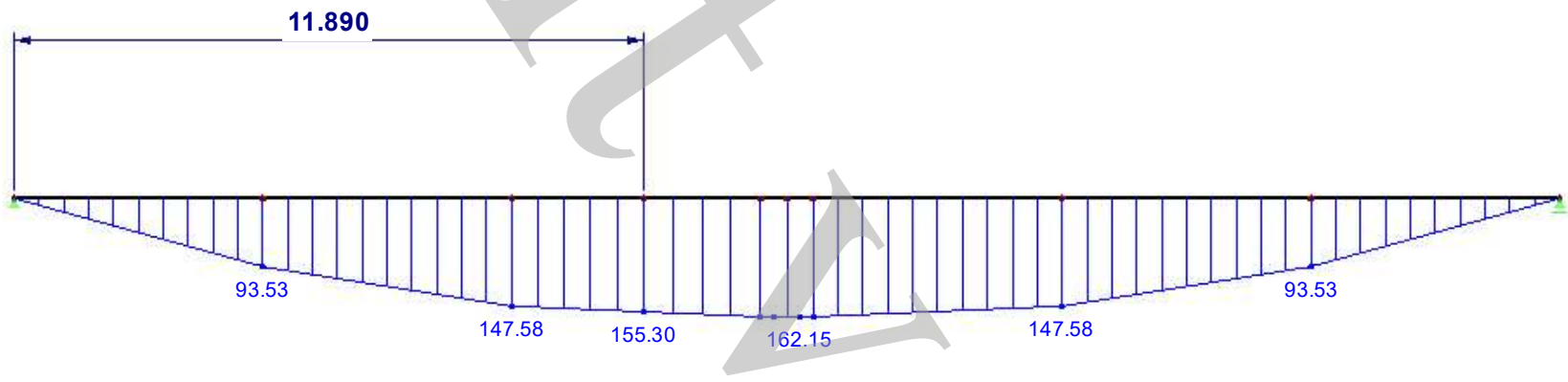

Max M-y: 162.15, Min M-y: 0.00 [kNm]

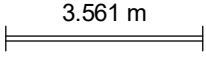

INTERNAL FORCES $V_{z}$, SUPPORT REACTIONS

LC 4: Wind pressure

Internal Forces $\mathrm{V}-\mathrm{z}$

Support Reactions[kN]

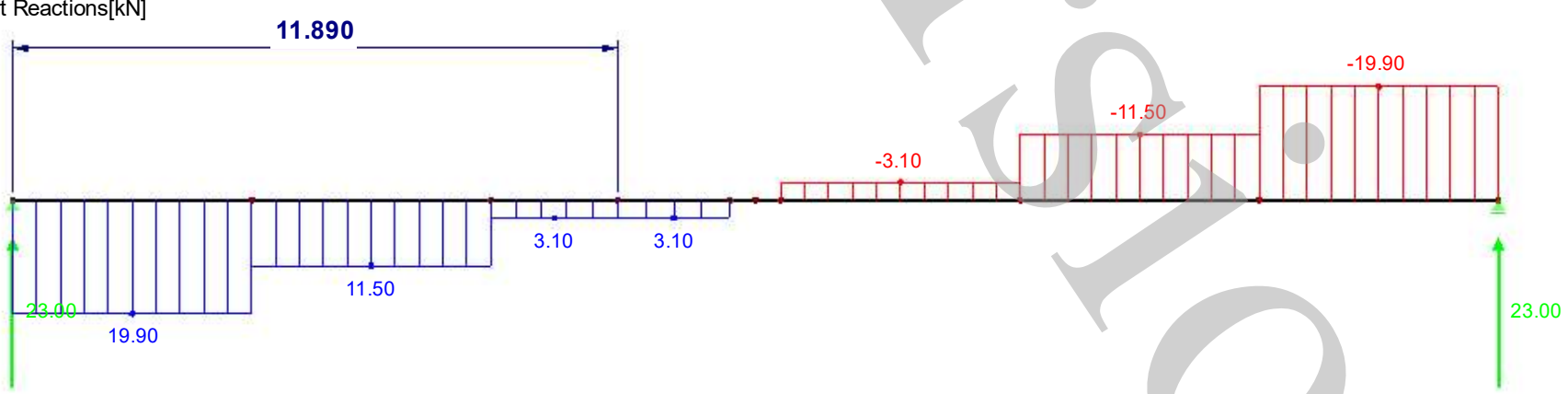

Max V-z: 19.90, Min V-z: -19.90 [kN]

Max P-X': 0.00, Min P-X': $0.00 \mathrm{kN}$

Max P-Z': 23.00, Min P-Z': 23.00 kN

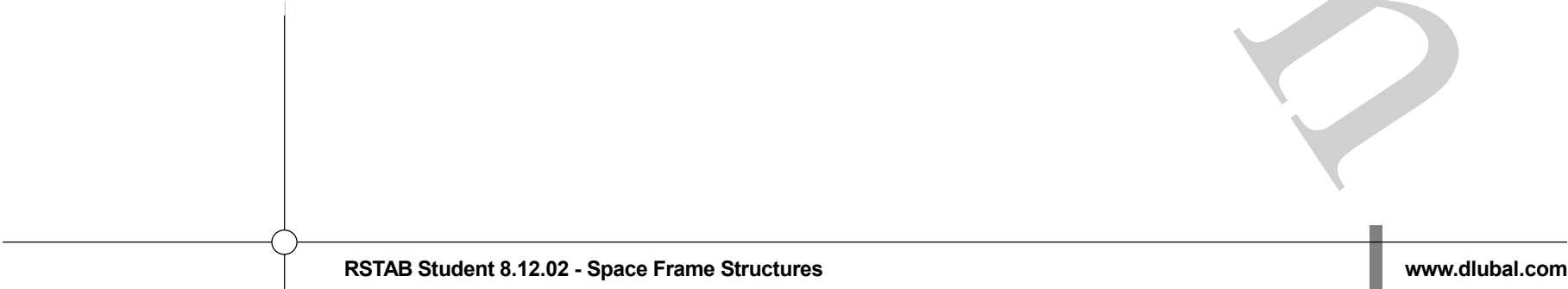




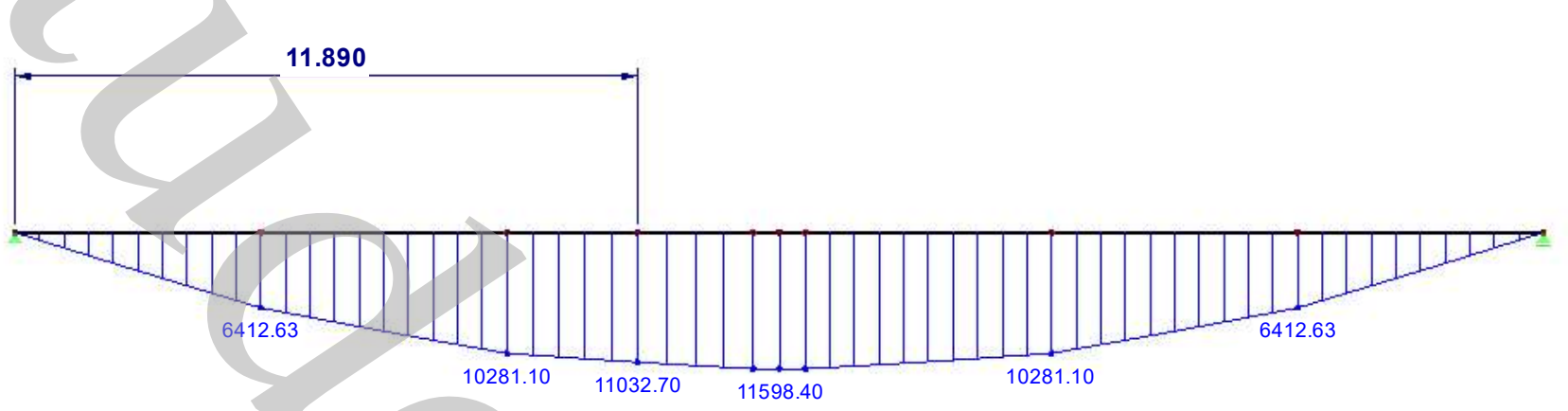

Max M-y: 11598.38, Min M-y: 0.00 [kNm]

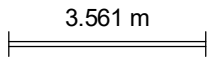

CO 1: ULS G+S+W

INTERNAL FORCES $V_{z}$, SUPPORT REACTIONS

Internal Forces $\mathrm{V}-\mathrm{Z}$

Support Reactions[kN]

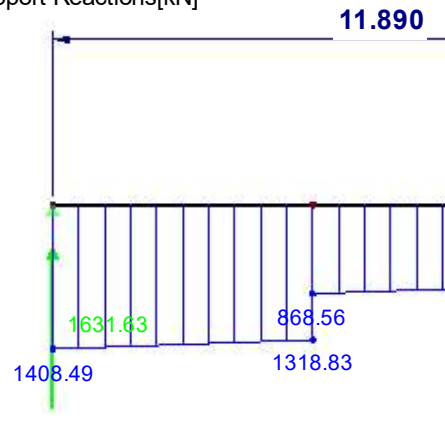

Max V-z: 1408.49, Min V-z: -1408.49 [kN]

Max P-X': 0.00, Min P-X': $0.00 \mathrm{kN}$

Max P-Z': 1631.63, Min P-Z': 1631.63 kN

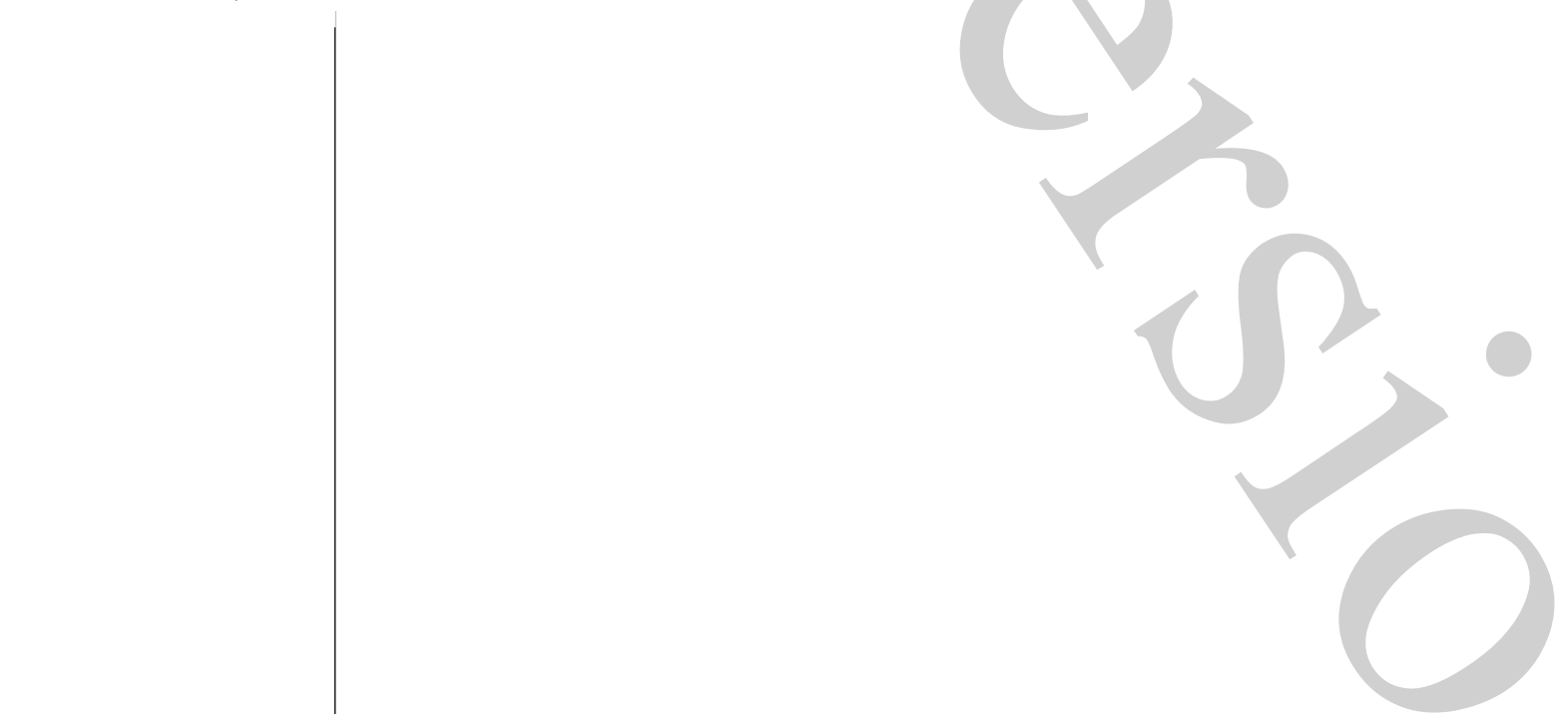


B.2.3. Internal Forces POS. T.10 - Precast Concrete Staircase 


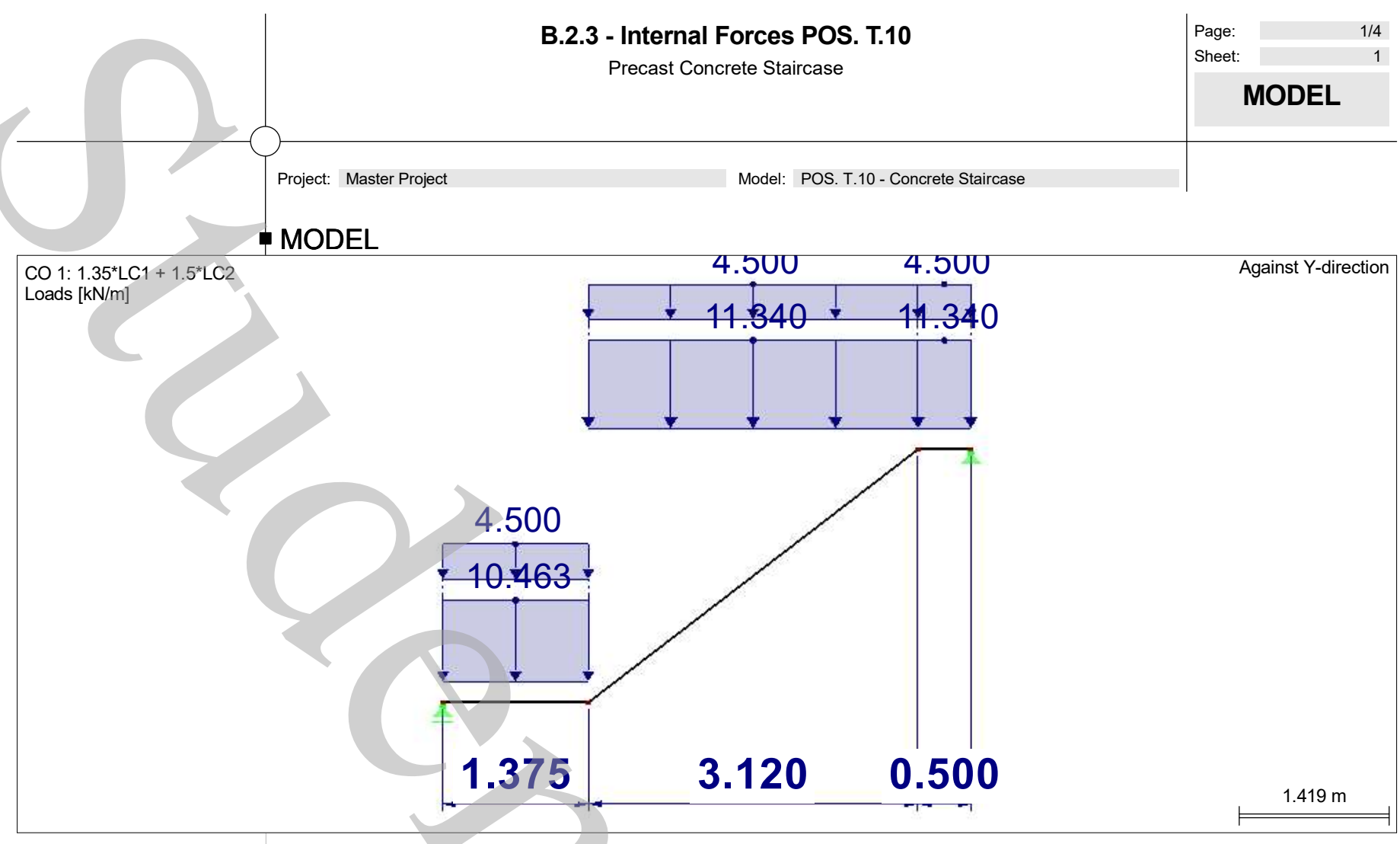

1.2 MATERIALS

\begin{tabular}{|c|c|c|c|c|c|c|}
\hline $\begin{array}{l}\text { Matl. } \\
\text { No. }\end{array}$ & $\begin{array}{c}\text { Modulus } \\
\mathrm{E}\left[\mathrm{kN} / \mathrm{cm}^{2}\right]\end{array}$ & $\begin{array}{l}\text { Modulus } \\
\mathrm{G}\left[\mathrm{kN} / \mathrm{cm}^{2}\right]\end{array}$ & $\begin{array}{c}\text { Spec. Weight } \\
\gamma\left[\mathrm{kN} / \mathrm{m}^{3}\right]\end{array}$ & $\begin{array}{c}\text { Coeff. of Th. Exp. } \\
\alpha\left[1 /{ }^{\circ} \mathrm{C}\right]\end{array}$ & $\begin{array}{c}\text { Partial Factor } \\
\gamma_{M}[-]\end{array}$ & $\begin{array}{c}\text { Material } \\
\text { Model }\end{array}$ \\
\hline 1 & $\begin{array}{r}\text { Steel S 235 | EN 1993-1 } \\
21000.00\end{array}$ & 8076.92 & 78.50 & $1.20 \mathrm{E}-05$ & 1.00 & $\begin{array}{l}\text { Isotropic Linear } \\
\text { Elastic }\end{array}$ \\
\hline 2 & $\begin{array}{r}\text { Concrete C30/37 | DIN } 1 \\
2830.00\end{array}$ & $\begin{array}{r}5-1: 2008-08 \\
1179.17\end{array}$ & 25.00 & $1.00 \mathrm{E}-05$ & 1.00 & $\begin{array}{l}\text { Isotropic Linear } \\
\text { Elastic }\end{array}$ \\
\hline 3 & $\begin{array}{r}\text { Concrete C30/37 | DIN } 1 \\
2830.00\end{array}$ & $\begin{array}{r}5-1: 2008-08 \\
1179.17\end{array}$ & 25.00 & $1.00 \mathrm{E}-05$ & 1.00 & $\begin{array}{l}\text { Isotropic Linear } \\
\text { Elastic }\end{array}$ \\
\hline
\end{tabular}

1.3 CROSS-SECTIONS

\begin{tabular}{|c|c|c|c|c|c|c|c|c|}
\hline Section & Matl. & $\mathrm{J}\left[\mathrm{cm}^{4}\right]$ & $\mathrm{I}_{\mathrm{y}}\left[\mathrm{cm}^{4}\right]$ & $\mathrm{I}_{\mathrm{z}}\left[\mathrm{cm}^{4}\right]$ & Principal Axes & Rotation & Overall Dime & ons [mm] \\
\hline No. & No. & $\mathrm{A}\left[\mathrm{cm}^{2}\right]$ & $\mathrm{A}_{\mathrm{y}}\left[\mathrm{cm}^{2}\right]$ & $\mathrm{A}_{\mathrm{z}}\left[\mathrm{cm}^{2}\right]$ & $\alpha\left[^{\circ}\right]$ & $\alpha^{\prime}\left[{ }^{\circ}\right]$ & Width b & Height $\mathrm{h}$ \\
\hline 1 & $\begin{array}{l}\text { Rectangl } \\
2\end{array}$ & 20.00 & 166.67 & 16.67 & 0.00 & 0.00 & 20.0 & 100.0 \\
\hline 2 & $\begin{array}{l}\text { Rectangl } \\
2\end{array}$ & 25.00 & 13.02 & 20.83 & 0.00 & 0.00 & 100.0 & 25.0 \\
\hline 3 & $\begin{array}{l}\text { Rectangl } \\
2\end{array}$ & $\begin{array}{l}0 / 200 \\
2000.00\end{array}$ & 66666.67 & 1666.67 & 0.00 & 0.00 & 1000.0 & 200.0 \\
\hline
\end{tabular}

\subsection{LOAD CASES}

\begin{tabular}{|c|c|c|c|c|c|c|}
\hline Load & Load Case & EN 1990 | DIN & & Neic & in & \\
\hline Case & Description & Action Category & Active & $x$ & $\mathrm{Y}$ & Z \\
\hline $\begin{array}{l}\text { LC1 } \\
\text { LC2 }\end{array}$ & $\begin{array}{l}\text { Self-weight } \\
\text { Imposed load }\end{array}$ & $\begin{array}{l}\text { Permanent } \\
\text { Imposed - Category B: office area }\end{array}$ & $\vec{\sqsupset}$ & & & \\
\hline
\end{tabular}




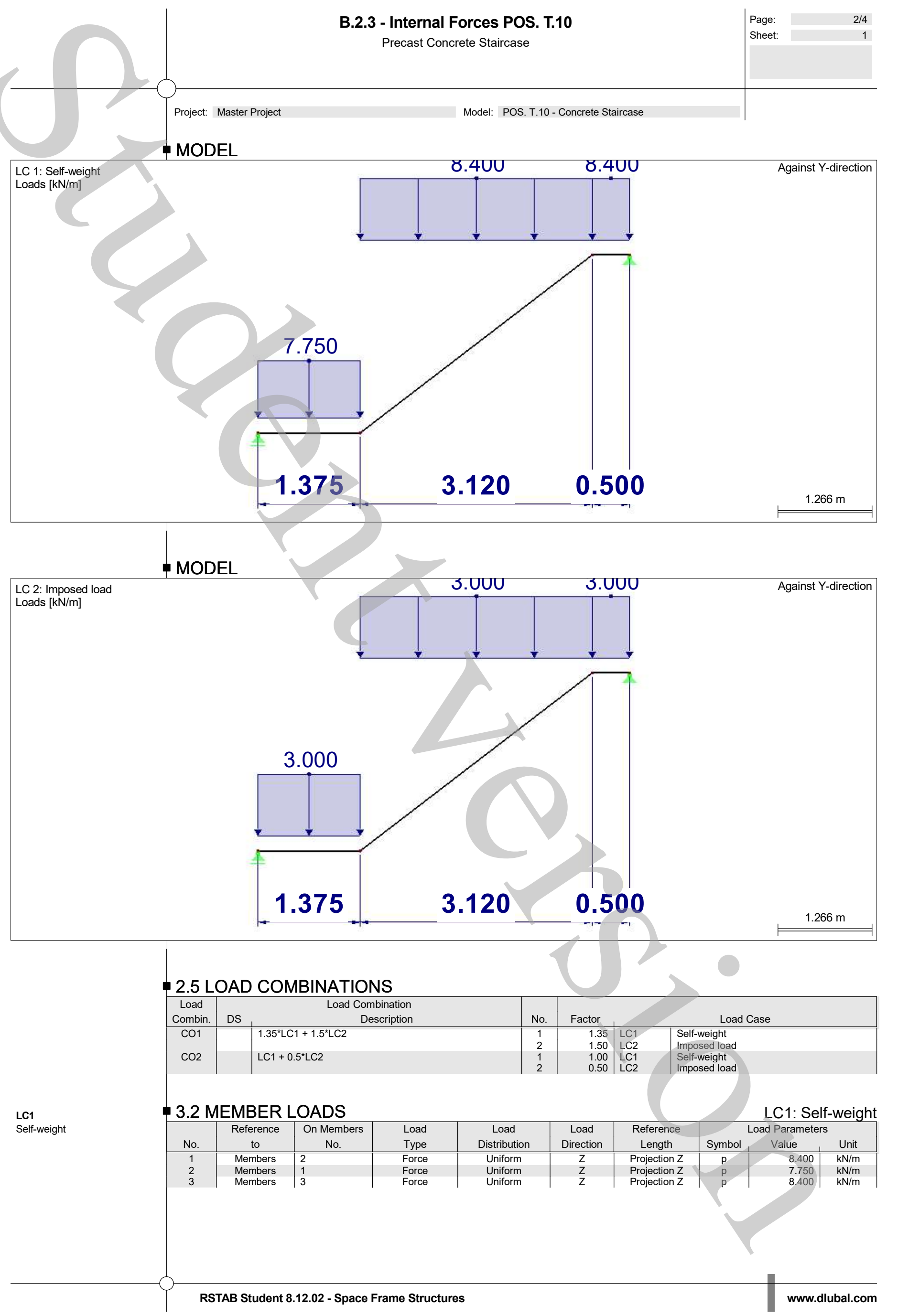




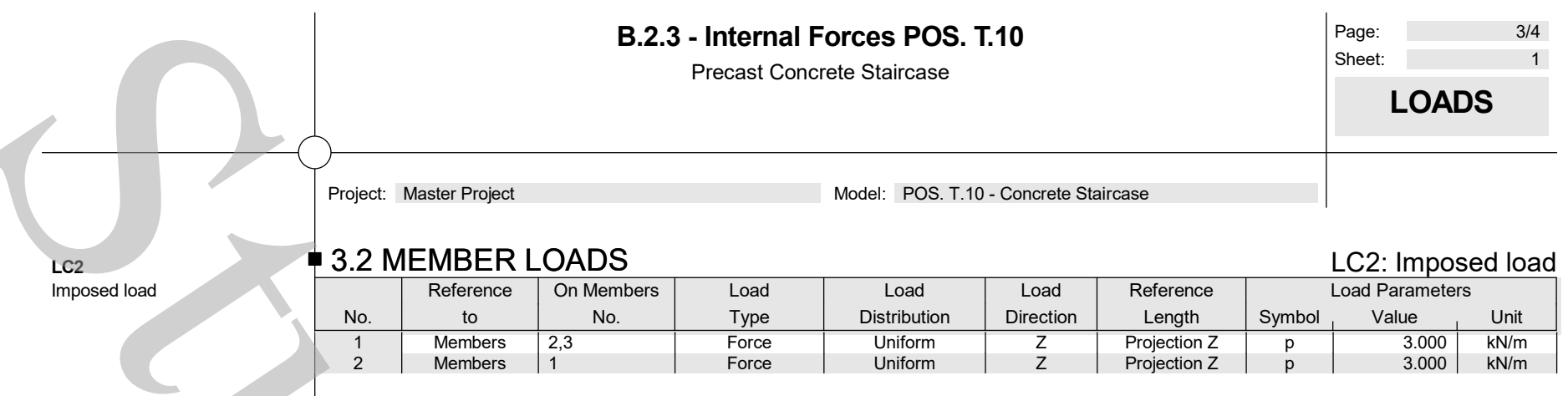

- 4.3 CROSS-SECTIONS - INTERNAL FORCES

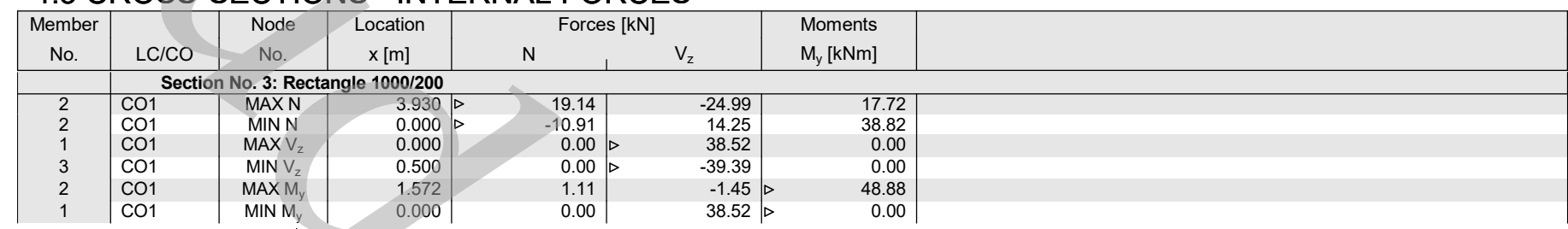

- INTERNAL FORCES N

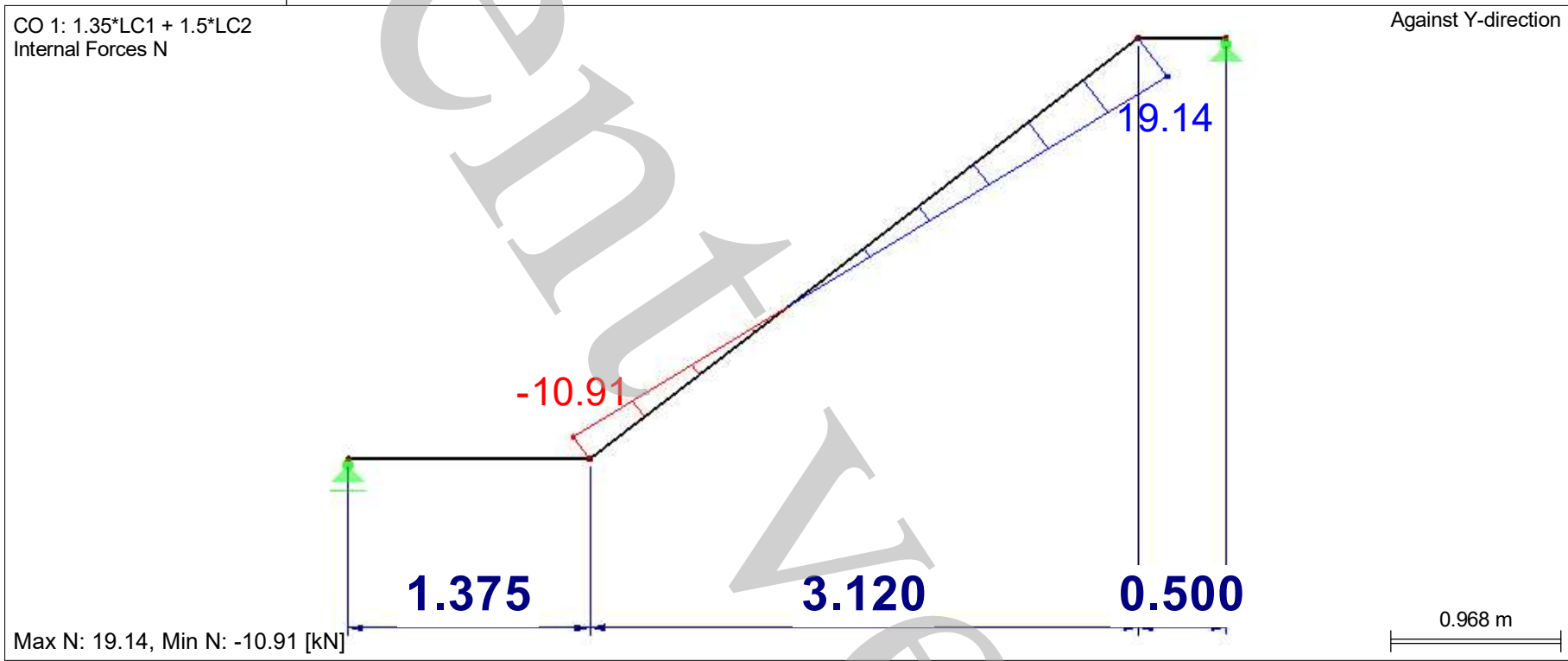

- INTERNAL FORCES $V_{z}$

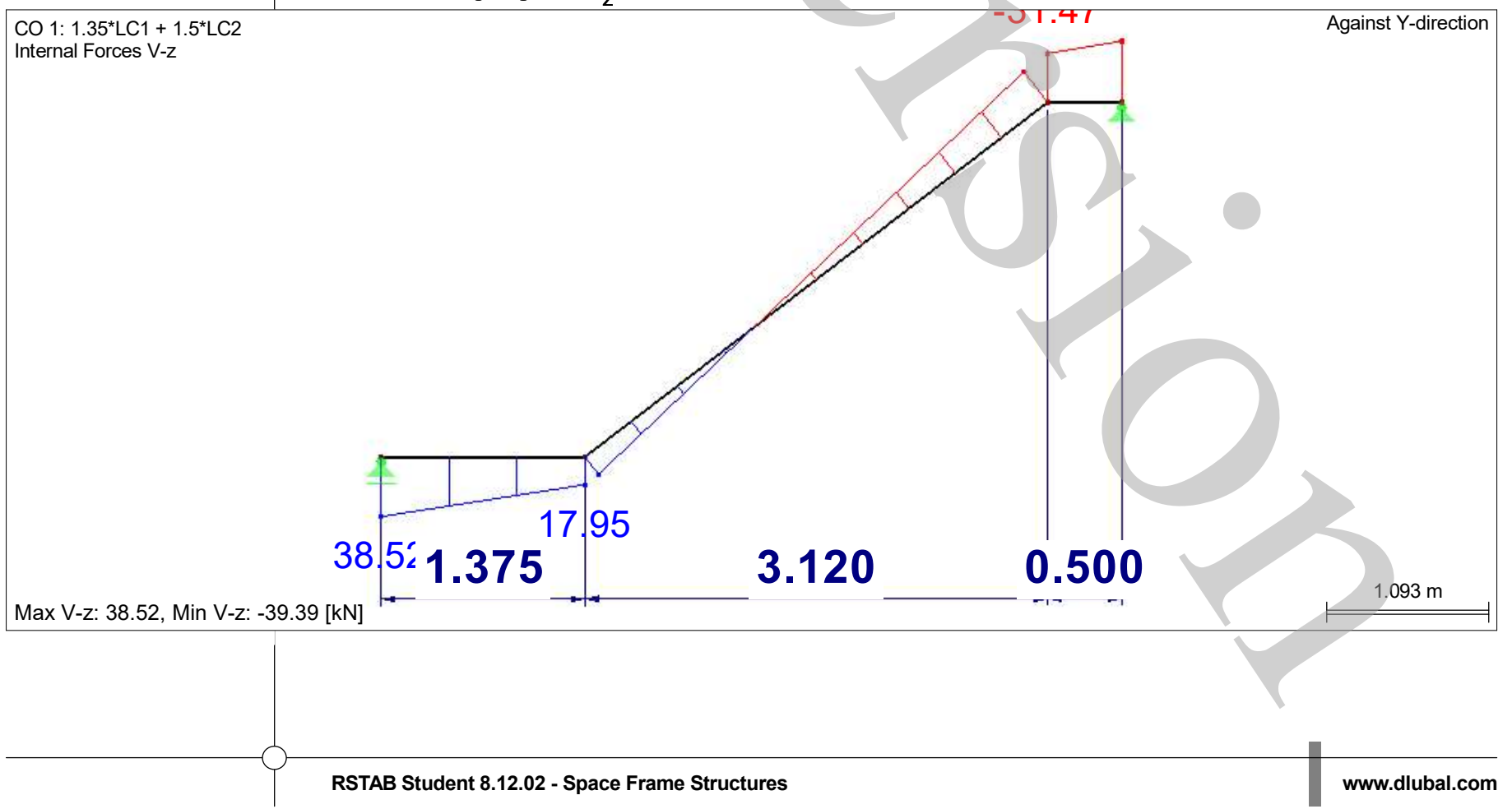



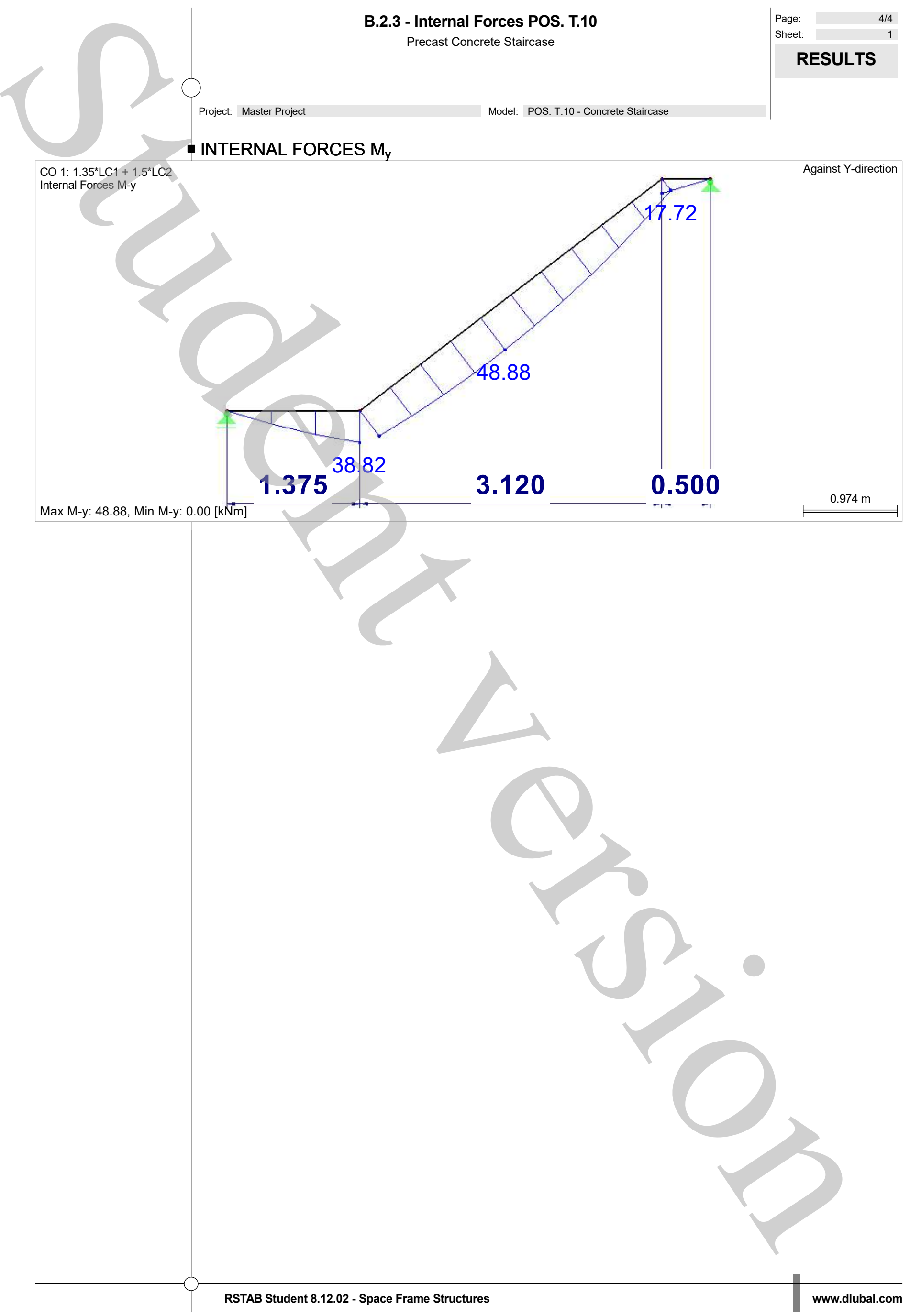
B.2.4. Internal Forces POS. W.14a - Strut-and-Tie Model Load Bearing Wall 


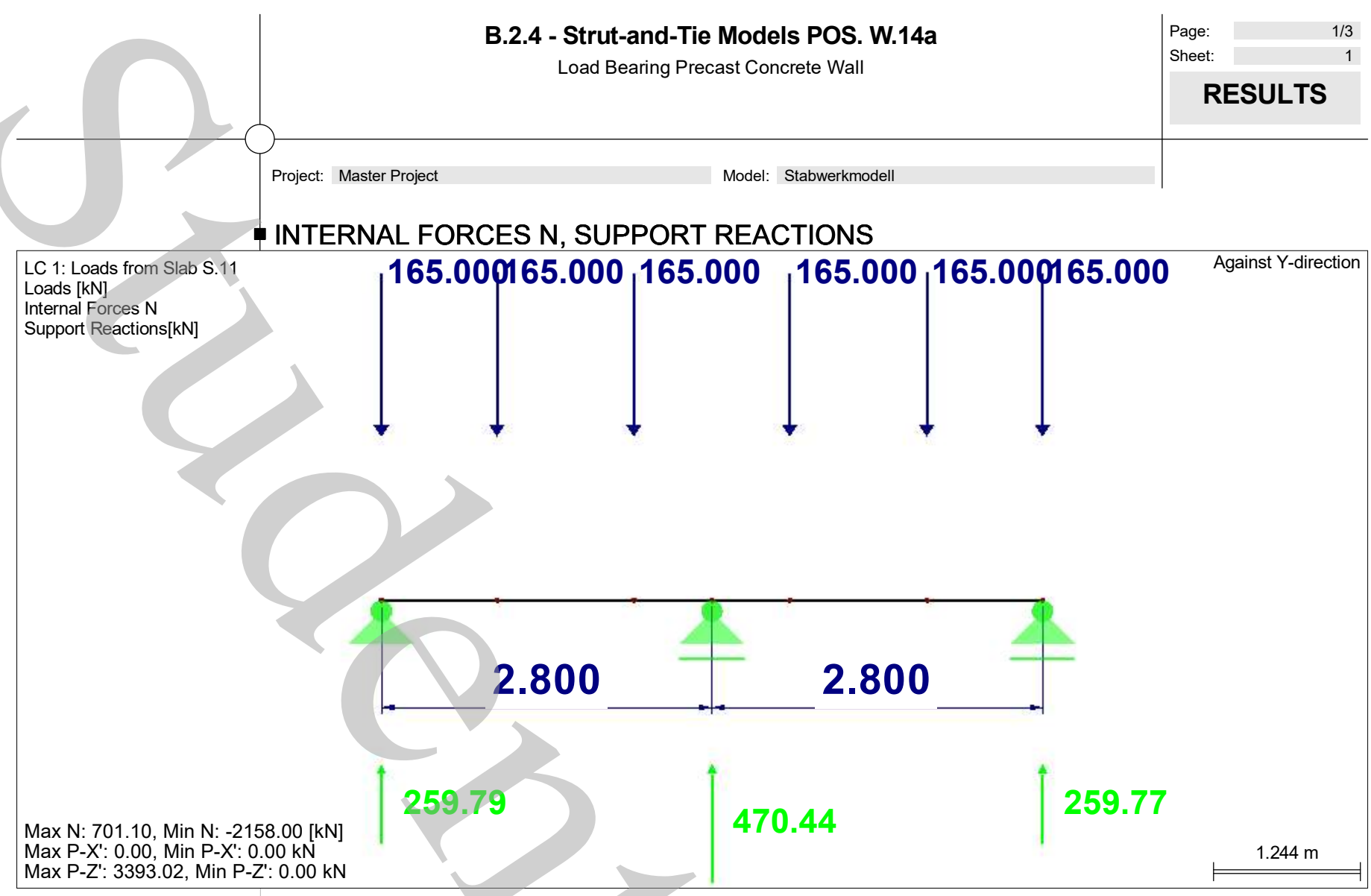

- INTERNAL FORCES N, SUPPORT REACTIONS

LC 1: Loads from Slab S.11

Loads [kN]

$165.000165 .000,165.000165 .000$

Against Y-direction

orces $\mathrm{N}$

Support Reactions[kN]

Max P-X': 0.00, Min P-X': $0.00 \mathrm{kN}$

Max P-Z': 3393.02, Min P-Z': 0.00 kN

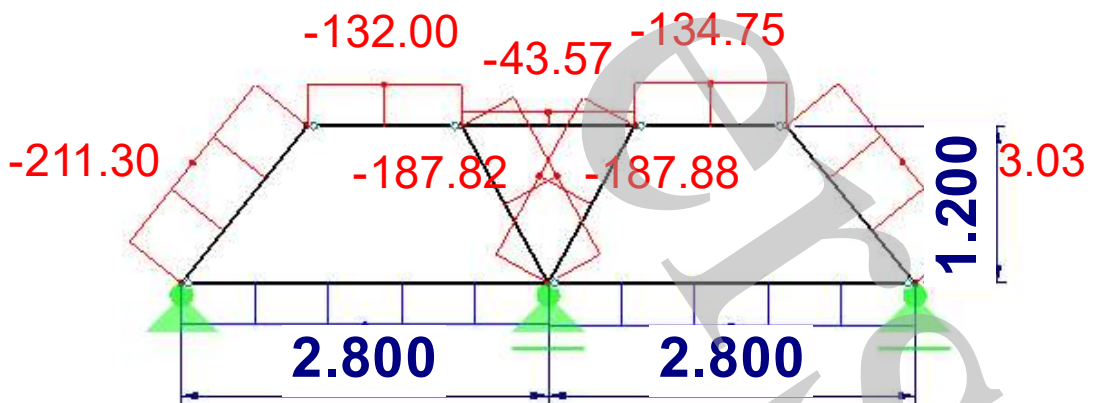

166.18 


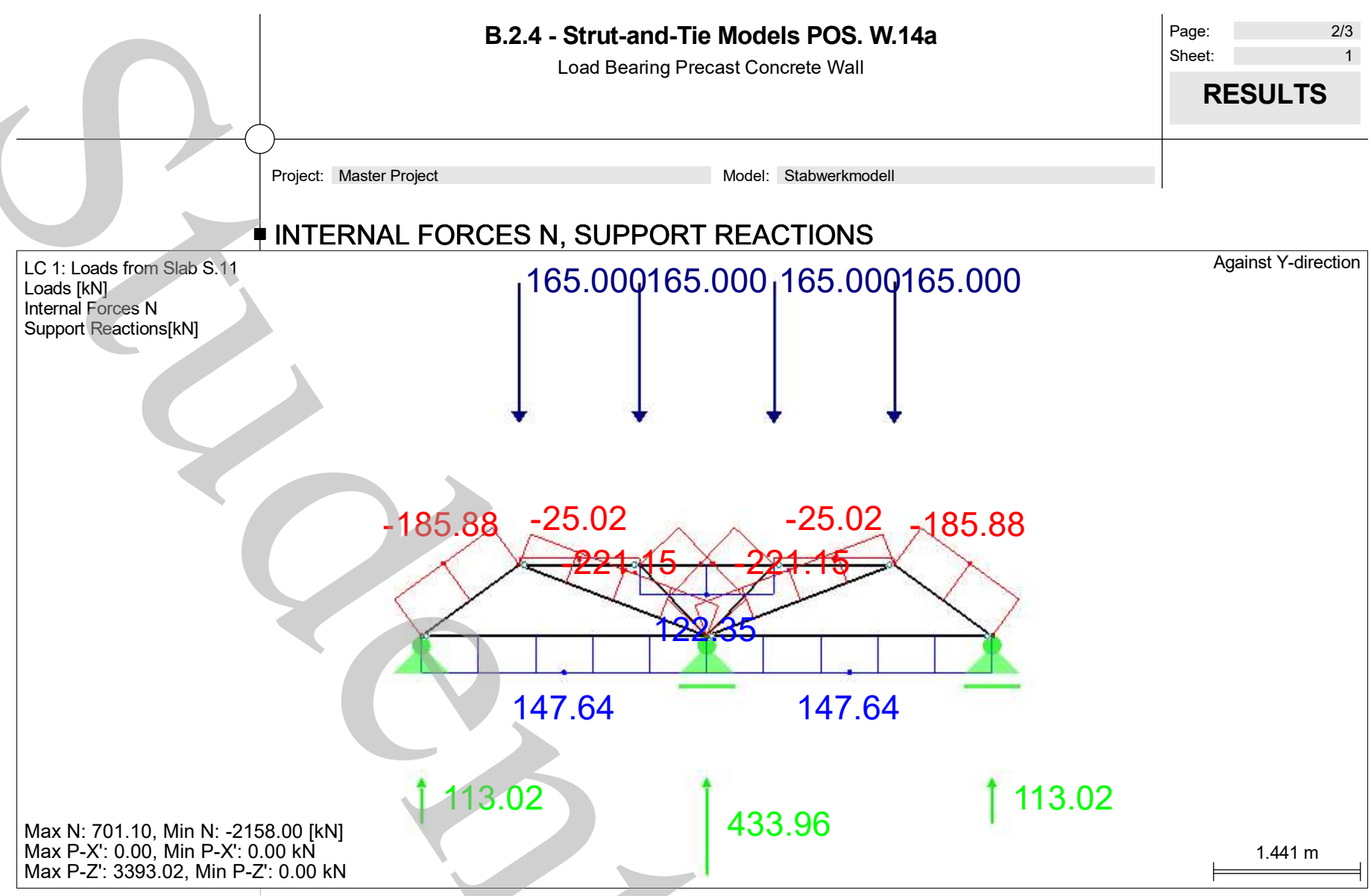

- INTERNAL FORCES N, SUPPORT REACTIONS

LC 1: Loads from Slab S.11

Loads [kN]

Internal Forces $\mathrm{N}$

Support Reactions[kN]

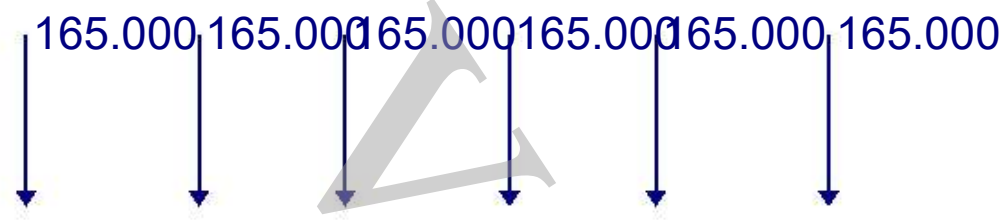

Against Y-direction

Max N.721.10NAin N: -2158.00 [kN]

Ma* \$.50.60, 14 In P-X': $0.00 \mathrm{kN}$

Max P-Z': 3393.02, Min P-Z': 0.00 kN

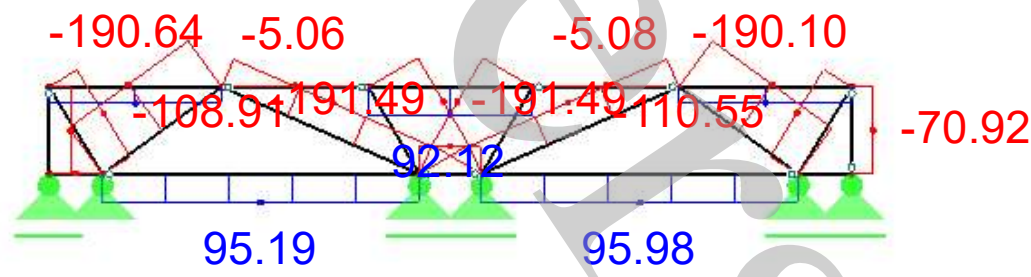

1 62.263 .81

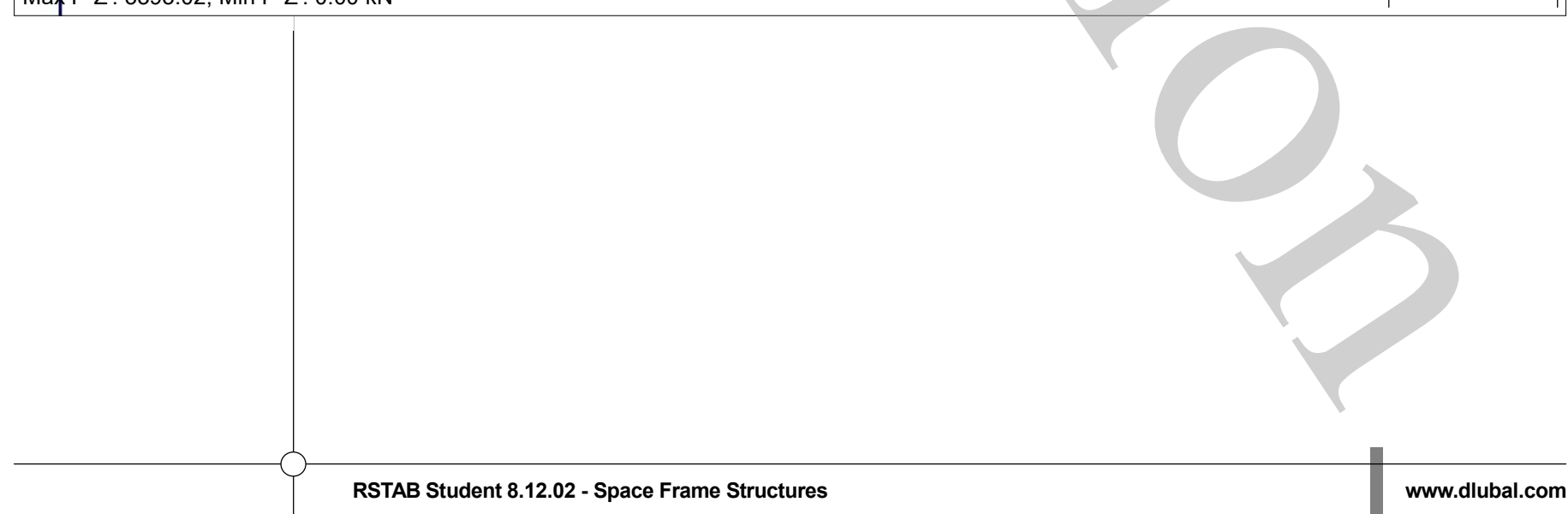




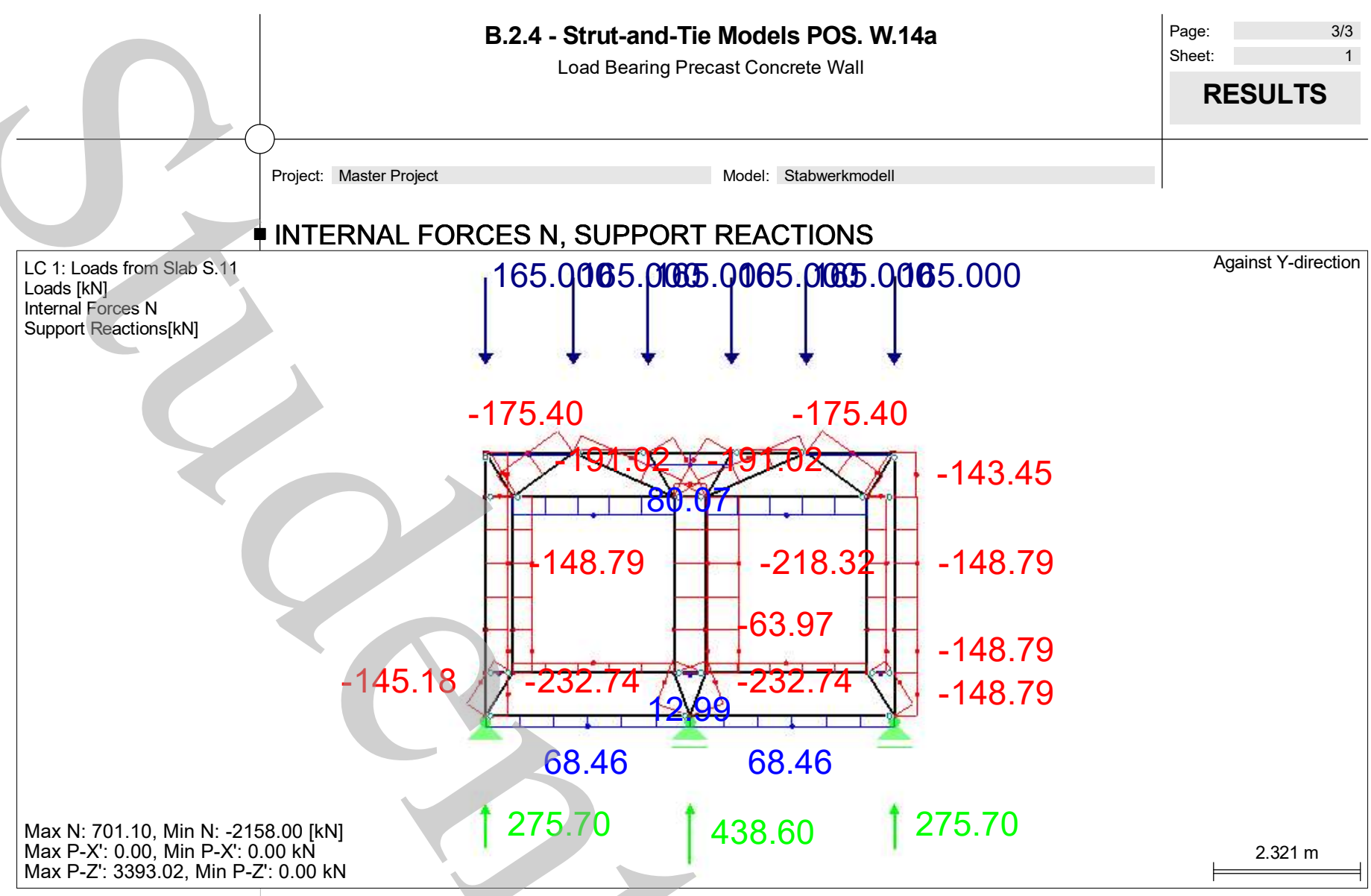

- INTERNAL FORCES N, SUPPORT REACTIONS

LC 1: Loads from Slab S.11

Loads [kN]

Internal Forces $\mathrm{N}$

Support Reactions[kN]

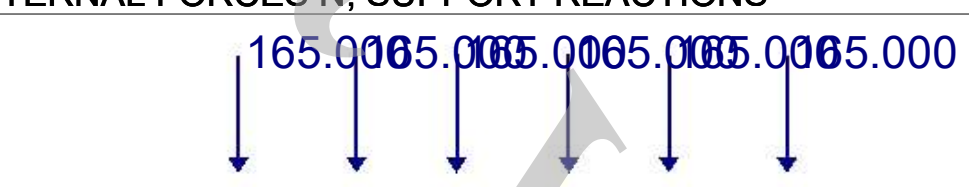

Against Y-direction

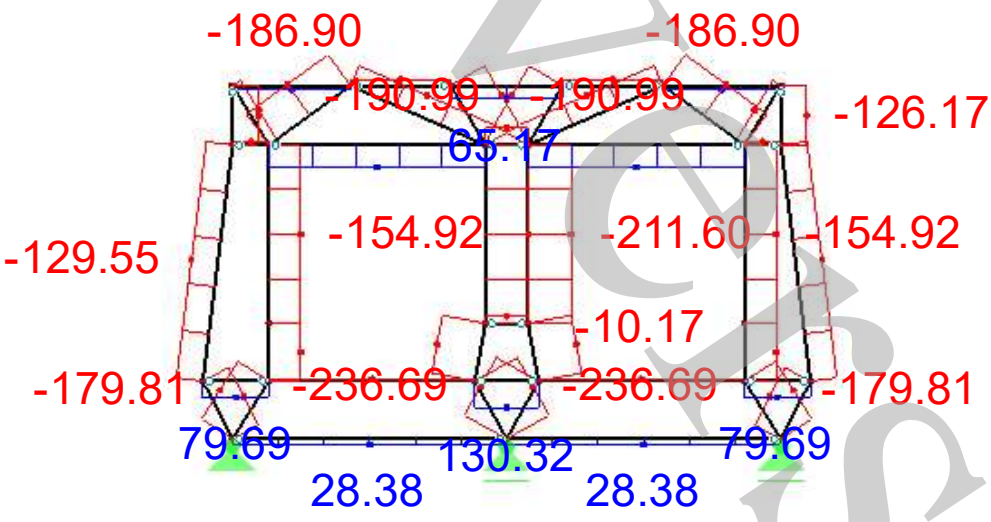

$\operatorname{Max} N$ : 701.10, Min N: $-2158.00[\mathrm{kN}]$ 
B.2.5. Internal Forces POS. V.31 - Steel Canopy 


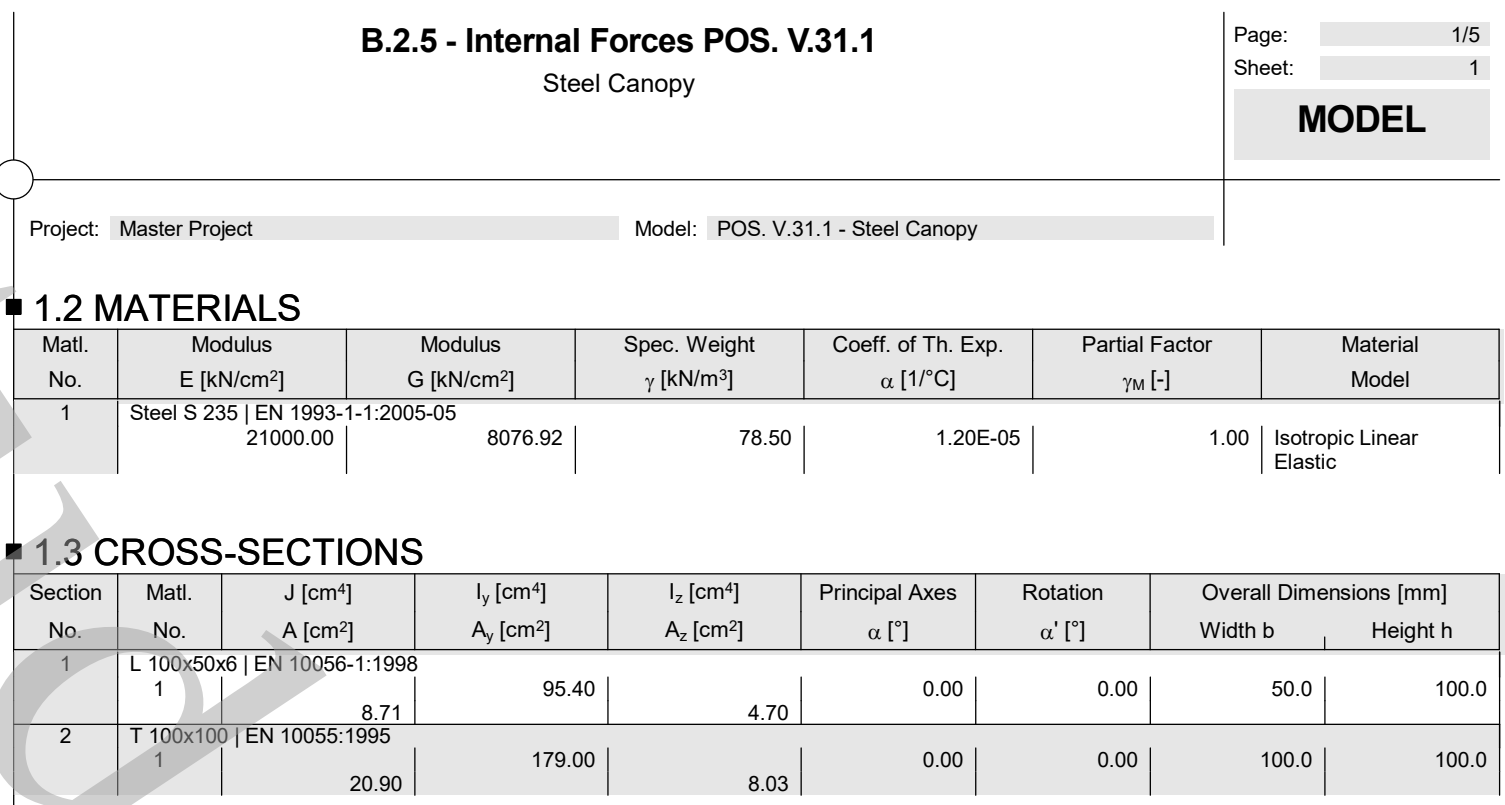

\section{- 2.5 LOAD COMBINATIONS}

\begin{tabular}{|c|c|c|c|c|c|c|}
\hline $\begin{array}{l}\text { Load } \\
\text { Combin. }\end{array}$ & DS & $\begin{array}{r}\text { Load Combination } \\
\text { Description }\end{array}$ & No. & Factor & & Load Case \\
\hline \multirow[t]{3}{*}{$\mathrm{CO} 1$} & & $1.35^{*} \mathrm{LC} 1+1.35^{*} \mathrm{LC} 2+1.5^{*} \mathrm{LC} 3+1.5^{*} \mathrm{LC} 4$ & 1 & 1.35 & LC1 & Self-weight Span \\
\hline & & $\longrightarrow$ & 2 & 1.35 & LC2 & Self-weight Cantilever \\
\hline & & & $\begin{array}{l}3 \\
4\end{array}$ & $\begin{array}{l}1.50 \\
1.50\end{array}$ & LC3 & Snow Span \\
\hline \multirow{2}{*}{$\mathrm{CO} 2$} & & 1.35*LC1 + LC2 + 1.5*LC3 & 1 & 1.35 & LC1 & Self-weight Span \\
\hline & & & 2 & 1.00 & LC2 & Self-weight Cantilever \\
\hline \multirow{4}{*}{$\mathrm{CO} 3$} & & Self-weight & 1 & 1.00 & LC3 & Snow Span \\
\hline & & & 2 & 1.00 & LC2 & Self-weight Cantilever \\
\hline & & Snow & 1 & 1.00 & LC3 & Snow Span \\
\hline & & & 2 & 1.00 & LC4 & Snow Cantilever \\
\hline
\end{tabular}

\section{- 2.6 RESULT COMBINATIONS}

\begin{tabular}{|c|l|l|}
\hline $\begin{array}{c}\text { Result } \\
\text { Combin }\end{array}$ & Description & \\
\hline RC1 & & CO1 or $\mathrm{CO} 2$ \\
\hline
\end{tabular}

SUPPORT REACTIONS

CO 1: $1.35^{*} \mathrm{LC} 1+1.35^{*} \mathrm{LC} 2+1.5^{*} \mathrm{LC} 3+1.5^{*} \mathrm{LC} 4$

Loads $[\mathrm{kN} / \mathrm{m}],[\mathrm{kN}]$

Support Reactions[kN]

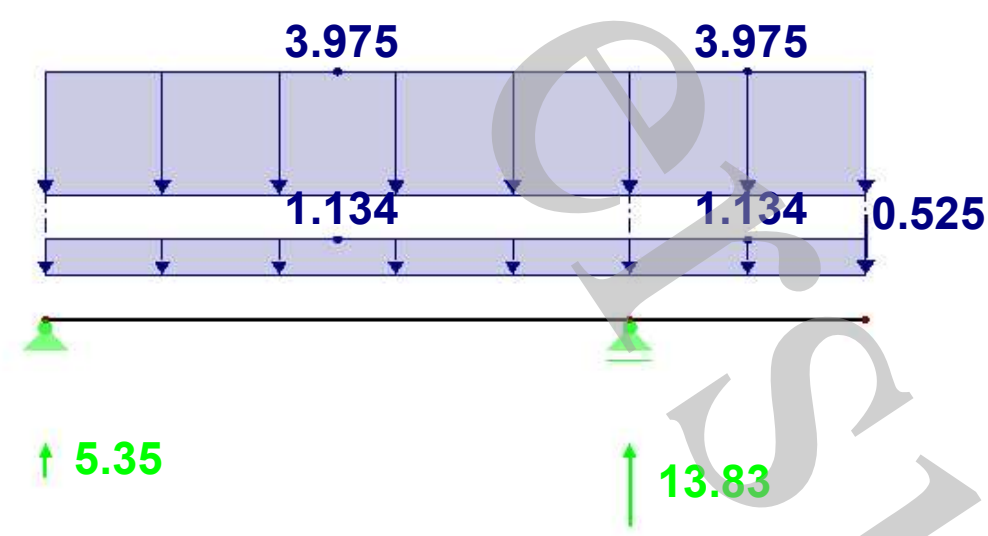

Against Y-direction

Max P-X': 0.00 , Min P-X': $0.00 \mathrm{kN}$ 


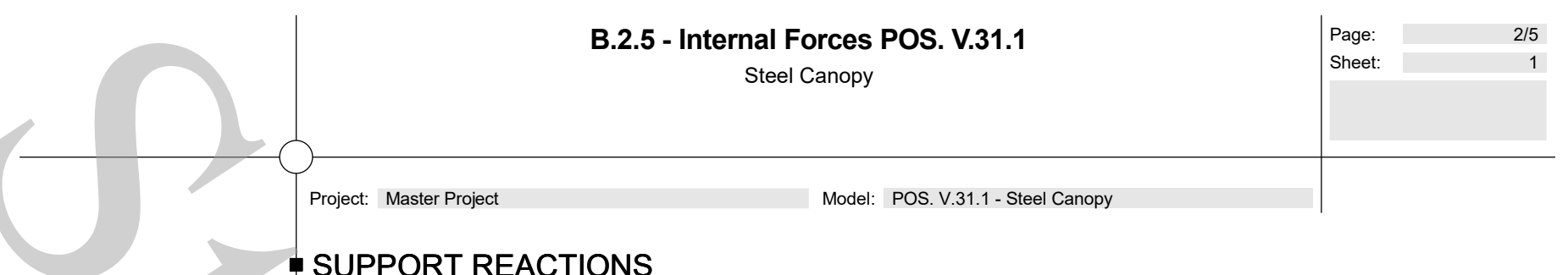

CO 2: $1.35^{*} \mathrm{LC} 1+\mathrm{LC} 2+1.5^{*} \mathrm{LC} 3$

Loads $[\mathrm{kN} / \mathrm{m}]$

Support Reactions[kN]

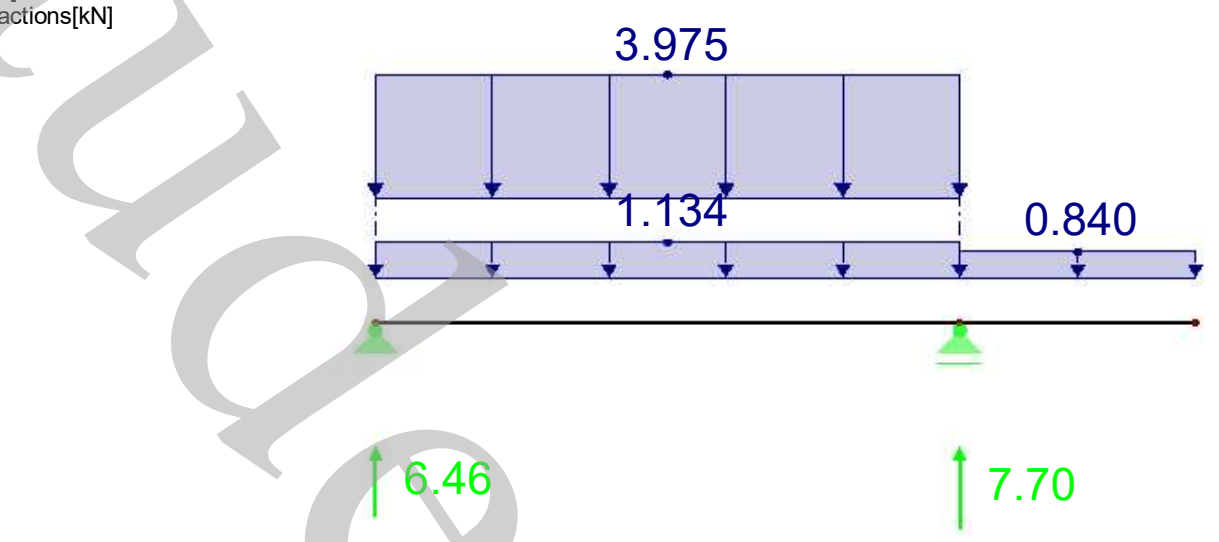

Max P-X': 0.00, Min P-X': $0.00 \mathrm{kN}$

Max P-Z': 7.70, Min P-Z': 6.46 kN

$0.877 \mathrm{~m}$

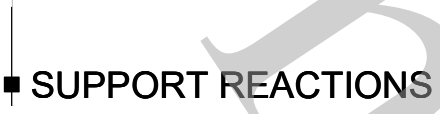

CO 4: Snow

Loads [kN/m], [kN]

Support Reactions[kN]

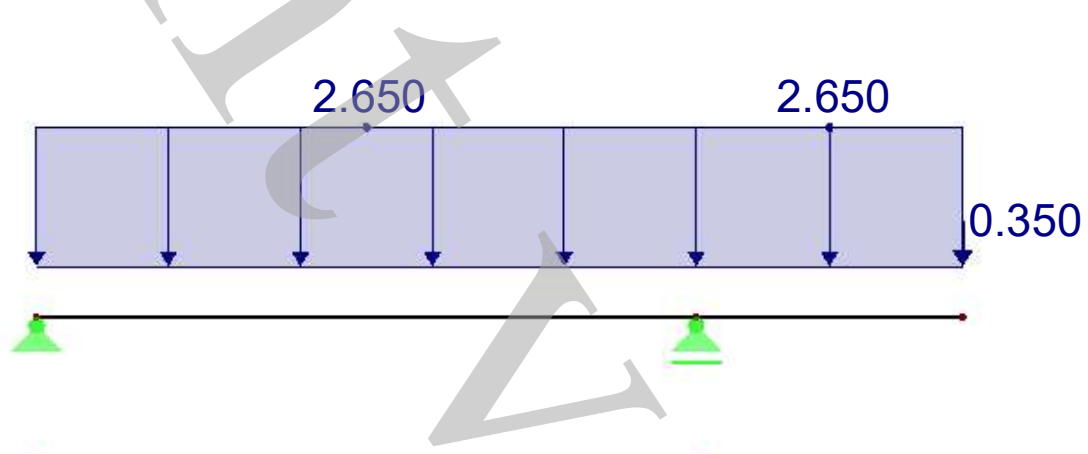

\section{$\uparrow 2.74$}

Max P-X': 0.00, Min P-X': $0.00 \mathrm{kN}$

Max P-Z': 7.28, Min P-Z': 2.74 kN

\subsection{8}

Against Y-direction

\section{- SUPPORT REACTIONS}

CO 4: Snow

Loads $[\mathrm{kN} / \mathrm{m}]$, [kN]

Support Reactions[kN]

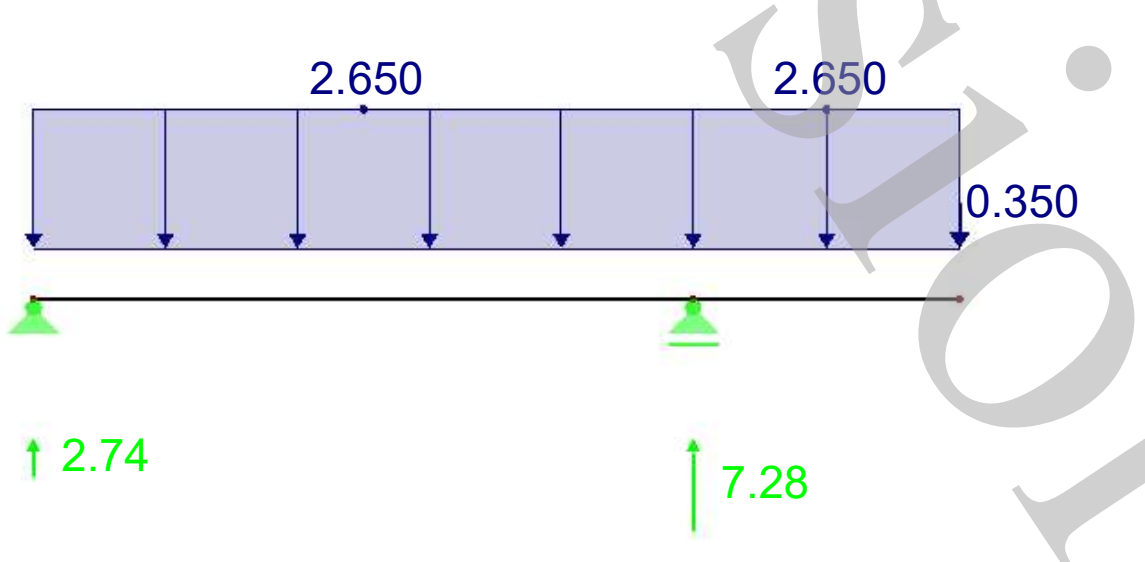




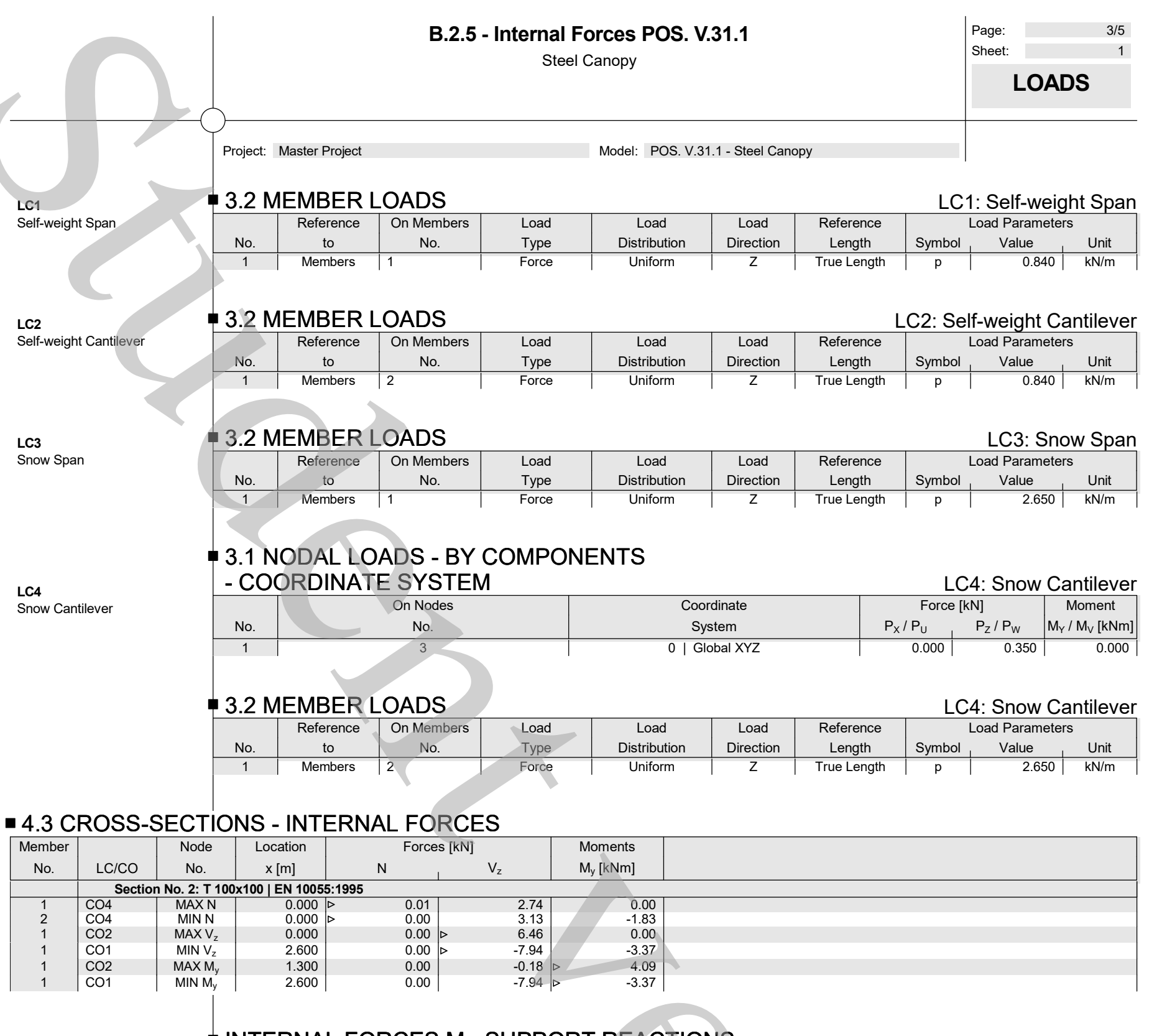

CO 1: $1.35^{*} \mathrm{LC} 1+1.35^{*} \mathrm{LC} 2+1.5^{*} \mathrm{LC} 3+1.5^{*} \mathrm{LC} 4$

Internal Forces M-y

Support Reactions[kN]

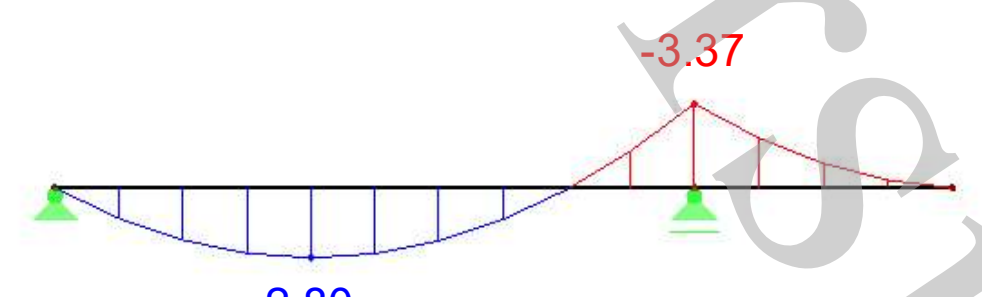

$\uparrow 5.35 \quad 2.80$

13.83

Max M-y: 2.80, Min M-y: -3.37 [kNm]

Max P-X': 0.00, Min P-X': $0.00 \mathrm{kN}$

Max P-Z': 13.83, Min P-Z': 5.35 kN 
Support Reactions[kN]

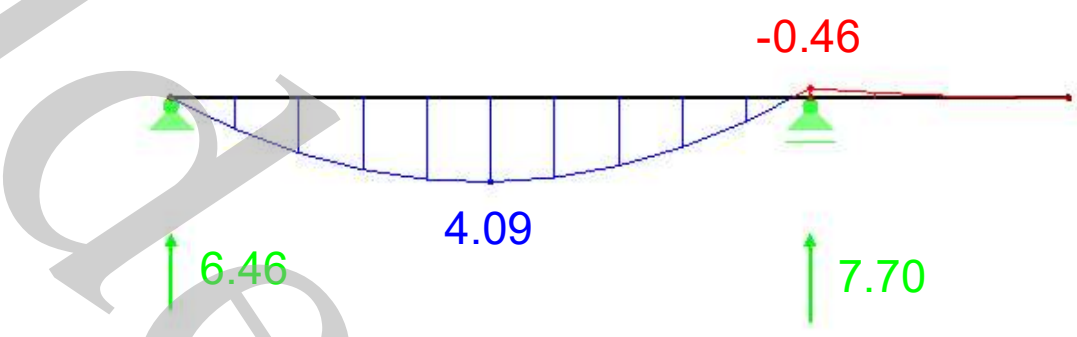

Max M-y: 4.09, Min M-y: $-0.46[\mathrm{kNm}]$

Max P-X': 0.00, Min P-X': 0.00 kN

Max P-Z': 7.70, Min P-Z': 6.46 kN

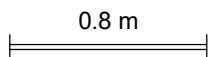

- INTERNAL FORCES $V_{z}$, SUPPORT REACTIONS

CO 1: $1.35^{*} \mathrm{LC} 1+1.35^{*} \mathrm{LC} 2+1.5^{*} \mathrm{LC} 3+1.5^{*} \mathrm{LC} 4$

Internal Forces $\mathrm{V}-\mathrm{z}$

Support Reactions[kN]

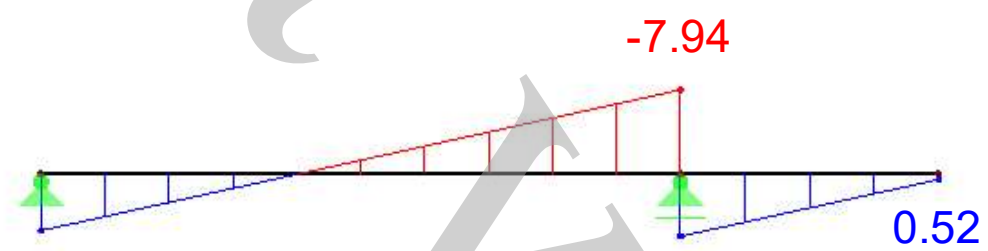

\subsection{5}

5.89

\section{$\uparrow 5.35$}

Max V-z: 5.89, Min V-z: -7.94 [kN]

Max P-X': 0.00 , Min P-X': $0.00 \mathrm{kN}$

Max P-Z': 13.83, Min P-Z': 5.35 kN

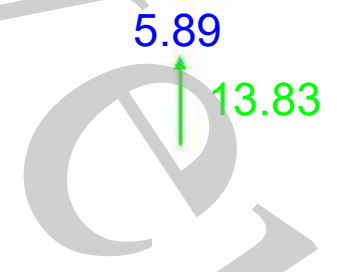

\section{INTERNAL FORCES $V_{z}$, SUPPORT REACTIONS}

CO 2: $1.35^{*} L C 1+L C 2+1.5^{*} L C 3$

Internal Forces $\mathrm{V}-\mathrm{z}$

Support Reactions[kN]

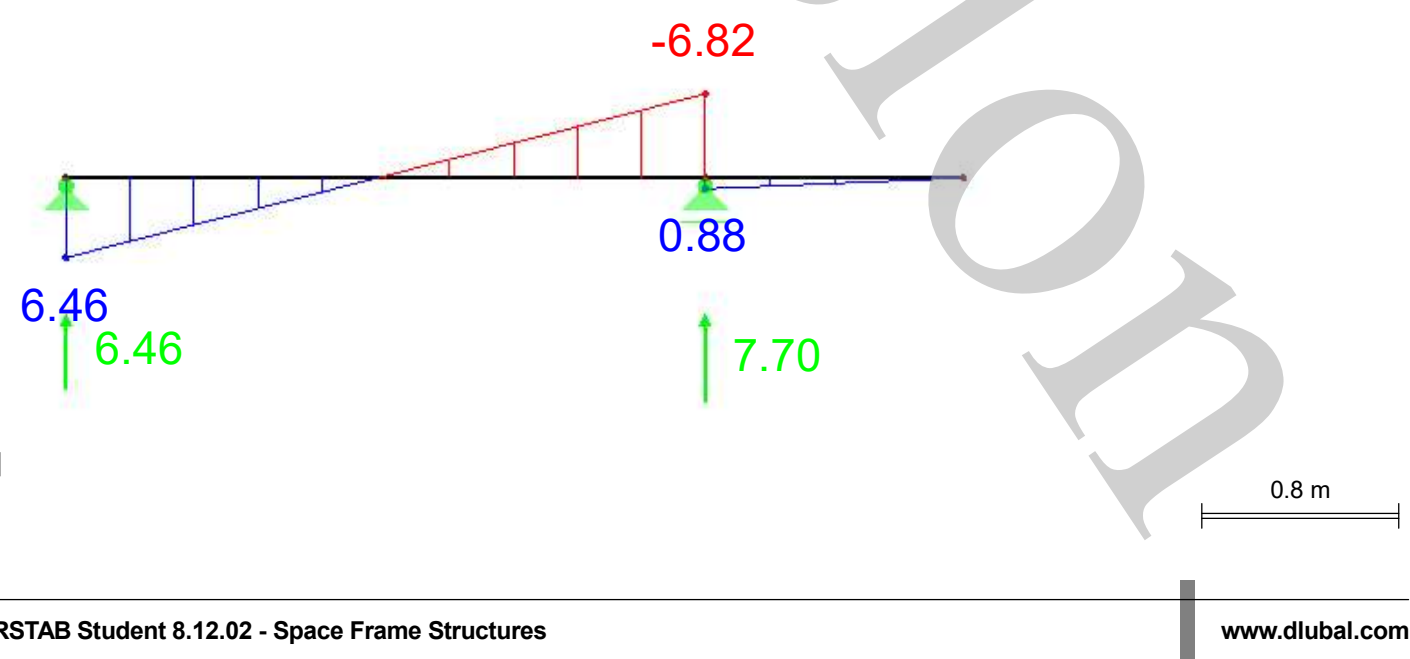


4.3 CROSS-SECTIONS - INTERNAL FORCES

\begin{tabular}{|c|c|c|c|c|c|c|c|c|c|}
\hline $\begin{array}{c}\text { Member } \\
\text { No. }\end{array}$ & $\mathrm{RC}$ & $\begin{array}{l}\text { Node } \\
\text { No. }\end{array}$ & $\begin{array}{l}\text { Location } \\
x[\mathrm{~m}]\end{array}$ & & & Forces & s [kN] & $\begin{array}{l}\text { Moments } \\
\mathrm{M}_{\mathrm{y}}[\mathrm{kNm}]\end{array}$ & $\begin{array}{l}\text { Corresponding } \\
\text { Load Cases }\end{array}$ \\
\hline & & lo. 2: T1 & $100 \mid$ EN 100 & 1995 & & & & & \\
\hline 1 & $\mathrm{RC1}$ & & 0.000 & MAXN & $\triangleright$ & 0.00 & 0.00 & 0.00 & \\
\hline 1 & RC1 & & 0.000 & MIN N & $\triangleright$ & 0.00 & 0.00 & 0.00 & \\
\hline 1 & $\mathrm{RC} 1$ & & 0.000 & $\operatorname{MAX} V_{z}$ & & 0.00 & 6.46 & 0.00 & $\mathrm{CO} 2$ \\
\hline 1 & $\mathrm{RC} 1$ & & 2.600 & $\mathrm{MIN} \mathrm{V}_{\mathrm{z}}$ & & 0.00 & -7.94 & -3.37 & CO 1 \\
\hline 1 & $\mathrm{RC} 1$ & & 1.300 & MAX My & & 0.00 & -0.18 & 4.09 & $\mathrm{CO} 2$ \\
\hline 1 & $\mathrm{RC} 1$ & & 2.600 & MIN My & & 0.00 & -7.94 & -3.37 & CO 1 \\
\hline
\end{tabular}

\section{INTERNAL FORCES $M_{y}$, SUPPORT REACTIONS}

$\mathrm{RC}$ 1: $\mathrm{CO} 1$ or $\mathrm{CO} 2$

Internal Forces M-y

Support Reactions[kN]

Result Combinations: Max and Min Values

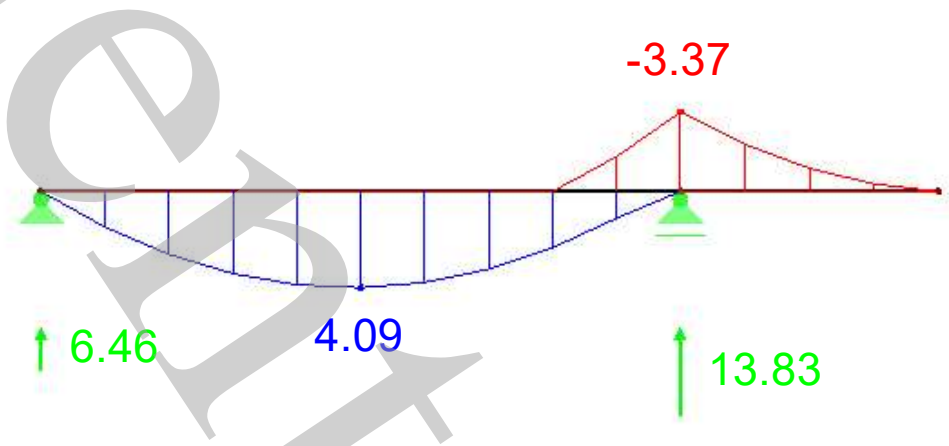

Max M-y: 4.09, Min M-y: -3.37 [kNm]

Max P-X': 0.00 , Min P-X': $0.00 \mathrm{kN}$

Max P-Z': 13.83, Min P-Z': $0.00 \mathrm{kN}$

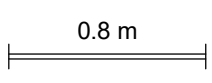

INTERNAL FORCES $V_{z}$, SUPPORT REACTIONS

$\mathrm{RC}$ 1: $\mathrm{CO} 1$ or $\mathrm{CO} 2$

Internal Forces $\mathrm{V}$ - $\mathrm{z}$

Support Reactions $[\mathrm{kN}]$

Result Combinations: Max and Min Values

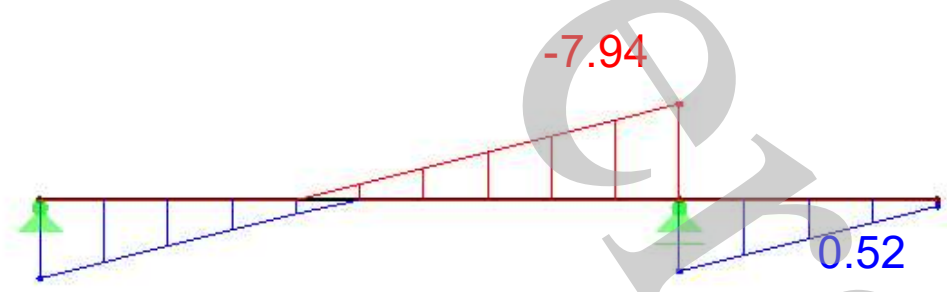

6.46

5.89

Max V-z: 6.46, Min V-z: $-7.94[k N]$

Max P-X': 0.00 , Min P-X': $0.00 \mathrm{kN}$

Max P-Z': 13.83, Min P-Z': $0.00 \mathrm{kN}$ 


\section{MODEL}

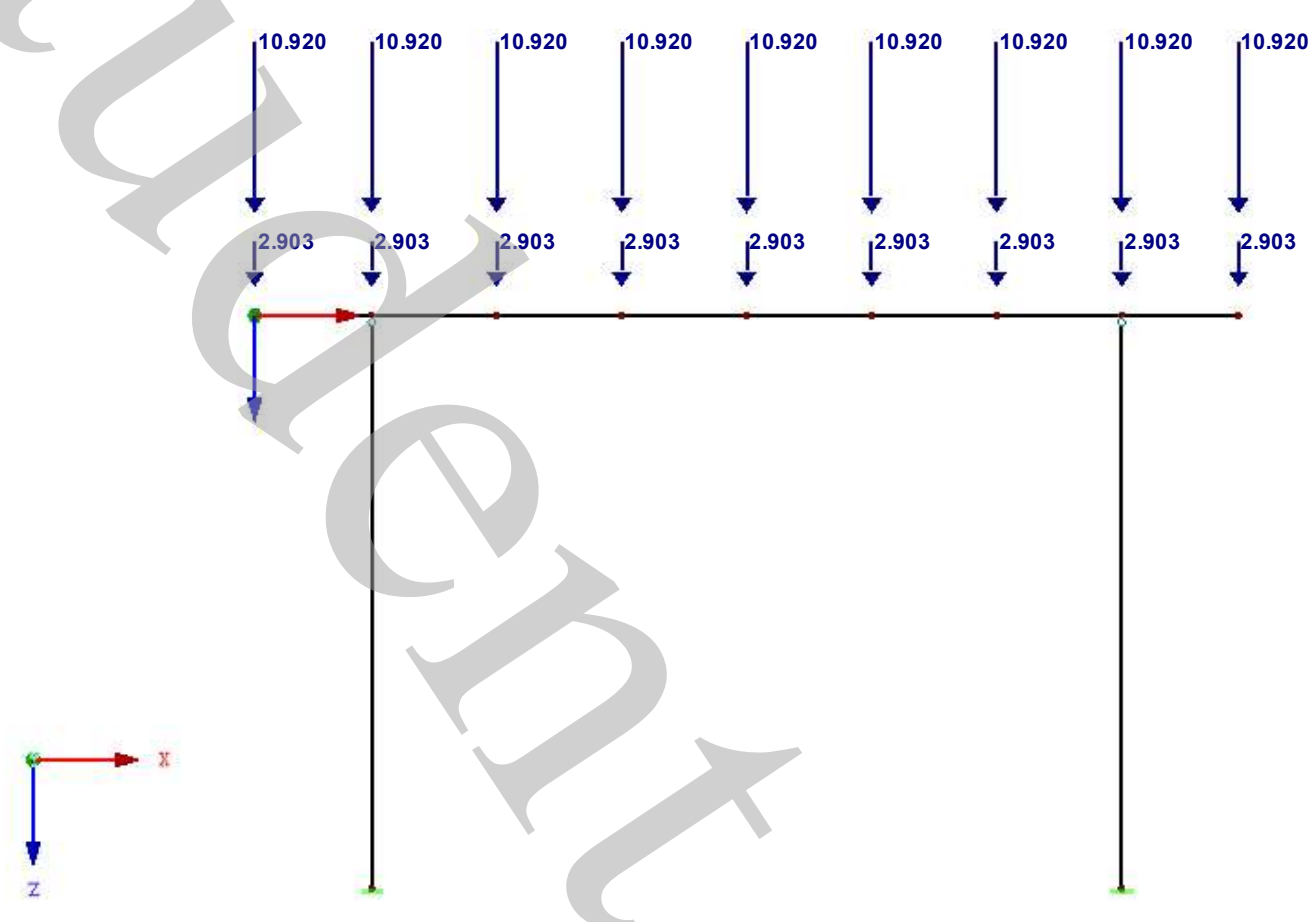

1.2 MATERIALS

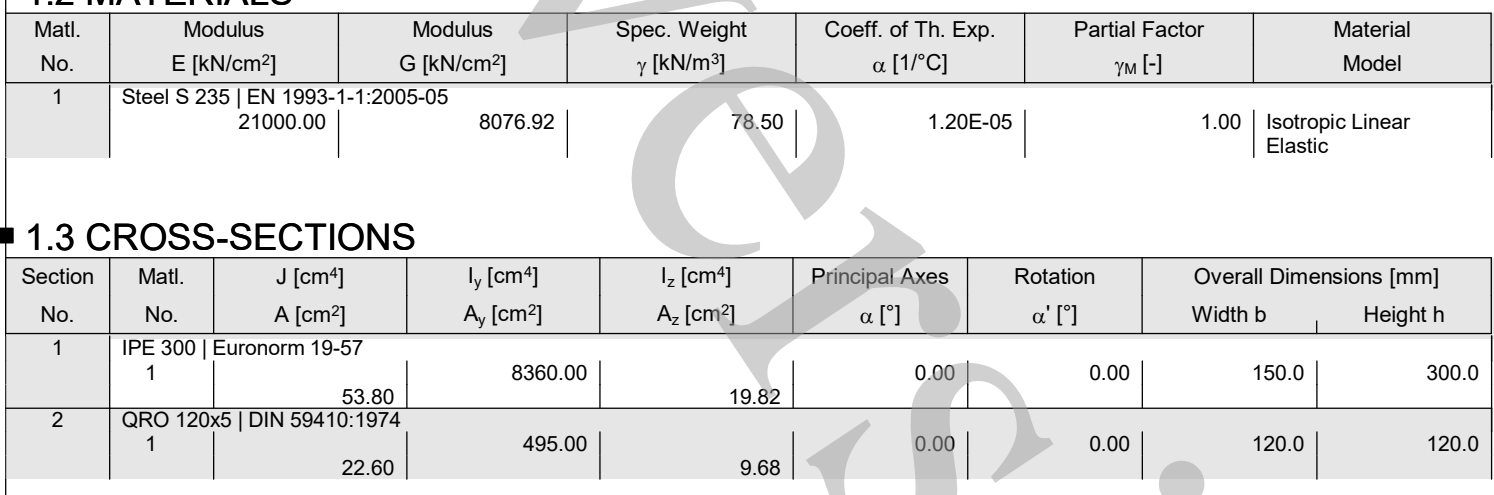

- 1.8 NODAL SUPPORTS<smiles>[Y]C([Y])[Z]</smiles>

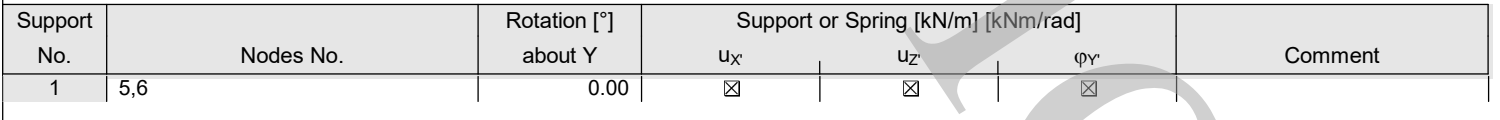

\subsection{LOAD CASES}

\begin{tabular}{|c|c|c|c|c|c|}
\hline Load & Load Case & EN 1990 | DIN & & Weight - Factor in $D$ & \\
\hline Case & Description & Action Category & Active & $\begin{array}{lll}X & Y & Y\end{array}$ & Z \\
\hline LC1 & Permanent & Permanent & 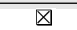 & 0.000 & 1.000 \\
\hline LC2 & Snow & Snow $(H \leq 1000 m$ a.s.l. $)$ & $\vec{\sqsupset}$ & & \\
\hline
\end{tabular}


- 2.5 LOAD COMBINATIONS

\begin{tabular}{|c|c|c|c|c|c|c|}
\hline Load & & Load Combination & & & & \\
\hline Combin. & DS & Description & No. & Factor & & Load Case \\
\hline $\mathrm{CO} 1$ & & $1.35^{*} \mathrm{LC} 1+1.5^{*} \mathrm{LC} 2$ & $\begin{array}{l}1 \\
2\end{array}$ & $\begin{array}{l}1.35 \\
1.50\end{array}$ & $\begin{array}{l}\text { LC1 } \\
\text { LC2 }\end{array}$ & $\begin{array}{l}\text { Permanent } \\
\text { Snow }\end{array}$ \\
\hline
\end{tabular}

- 3.1 NODAL LOADS - BY COMPONENTS

LC1 - COORDINATE SYSTEM

\begin{tabular}{|c|c|c|c|c|c|}
\multicolumn{3}{|c|}{ LC1: Permanent } \\
\hline No. & On Nodes & Coordinate & \multicolumn{3}{|c|}{ Force $[\mathrm{kN}]$} \\
$\mathrm{P}_{\mathrm{X}} / \mathrm{P}_{\mathrm{U}}$ & $\mathrm{P}_{\mathrm{Z}} / \mathrm{P}_{\mathrm{W}}$ & $\mathrm{M}_{\mathrm{Y}} / \mathrm{M}_{\mathrm{V}}[\mathrm{kNm}]$ \\
\hline 1 & No. & System & 0.000 & 2.150 & 0.000
\end{tabular}

LC2

\subsection{NODAL LOADS - BY COMPONENTS} - COORDINATE SYSTEM

Snow

\begin{tabular}{|c|c|c|c|c|c|}
\hline \multicolumn{6}{|c|}{ - COORDINATE SYSTEM } \\
\hline & On Nodes & Coordinate & Forc & & Moment \\
\hline No. & No. & System & $\mathrm{P}_{\mathrm{X}} / \mathrm{P}_{\mathrm{U}}$ & $\mathrm{P}_{\mathrm{Z}} / \mathrm{P}_{\mathrm{w}}$ & $\mathrm{M}_{\mathrm{Y}} / \mathrm{M}_{\mathrm{V}}[\mathrm{kNm}]$ \\
\hline 1 & $1-4,7-11$ & \begin{tabular}{l|l}
0 & Global XYZ
\end{tabular} & 0.000 & 7.280 & 0.000 \\
\hline
\end{tabular}

- 4.3 CROSS-SECTIONS - INTERNAL FORCES

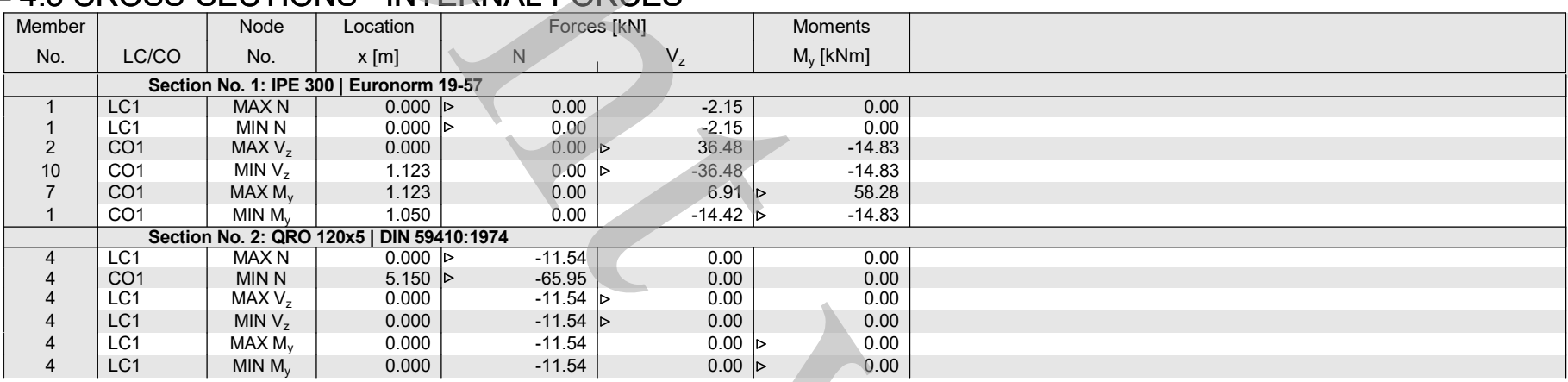

INTERNAL FORCES My

LC 1: Permanent Internal Forces M-y

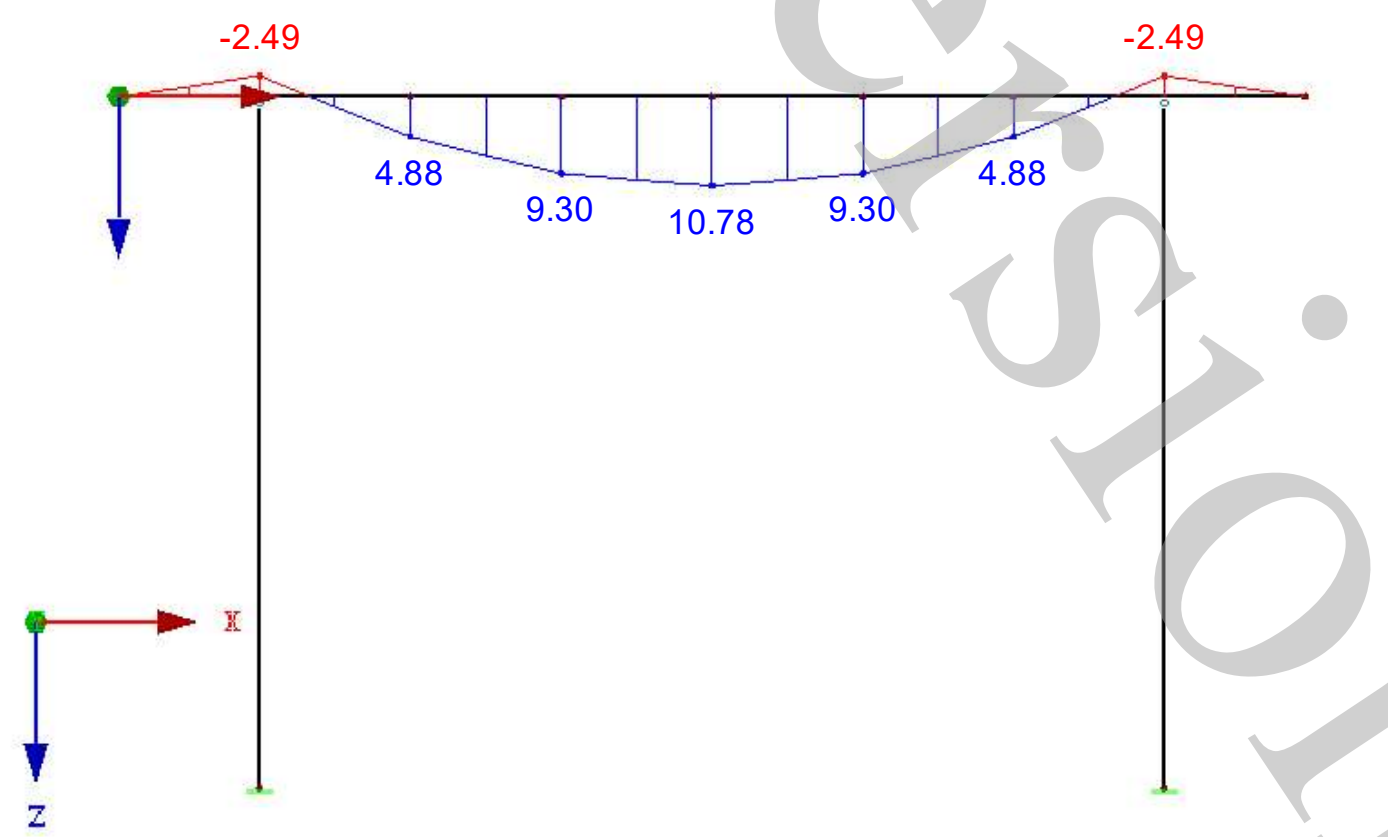




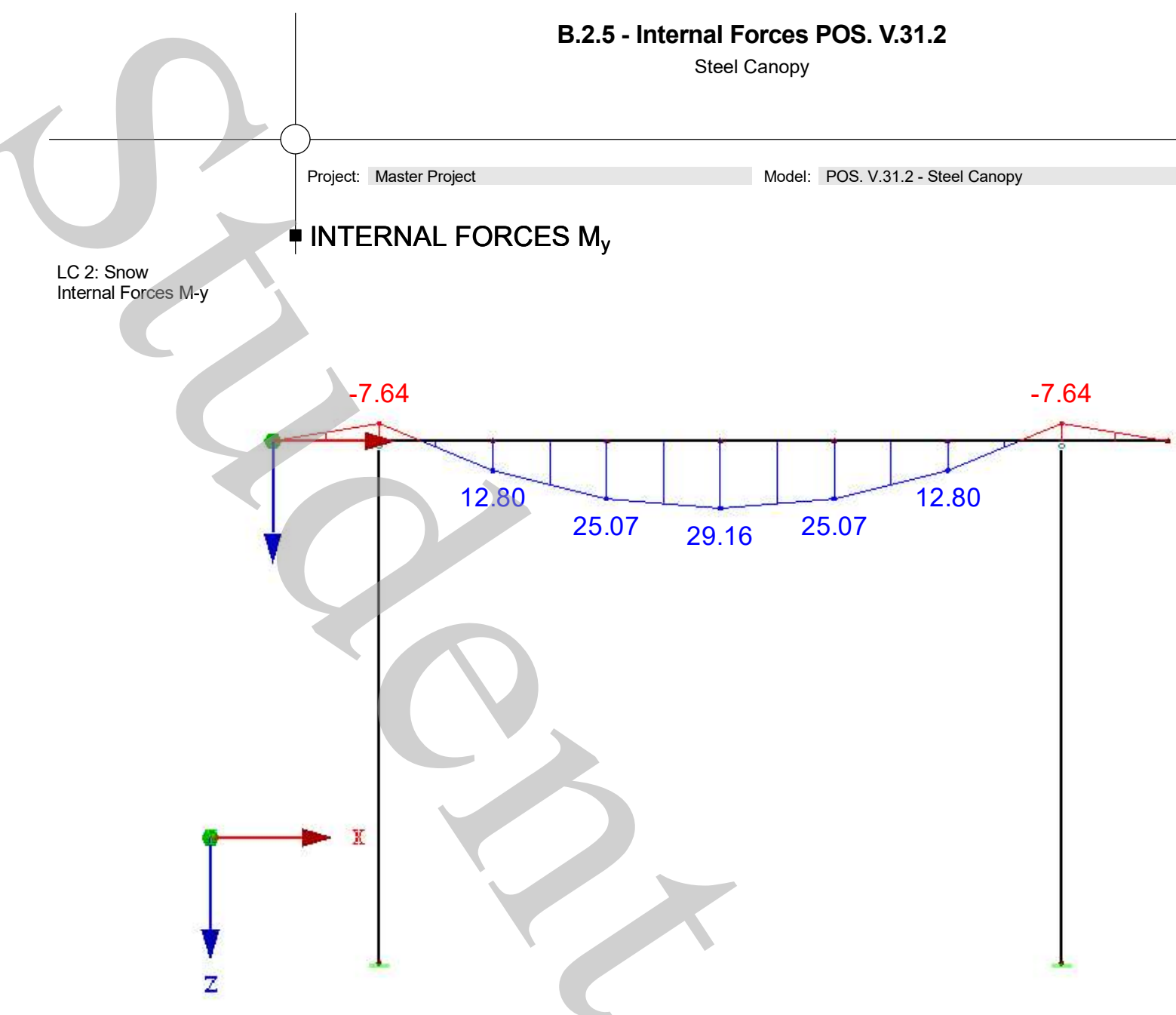

Max M-y: 29.16, Min M-y: -7.64 [kNm]
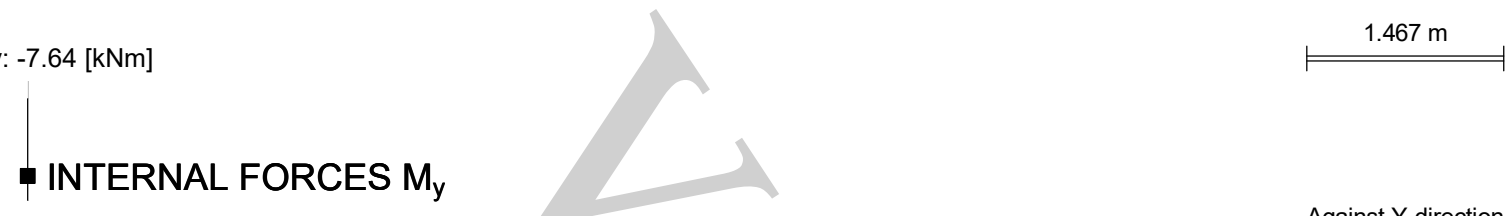

Against Y-direction

\section{RESULTS}

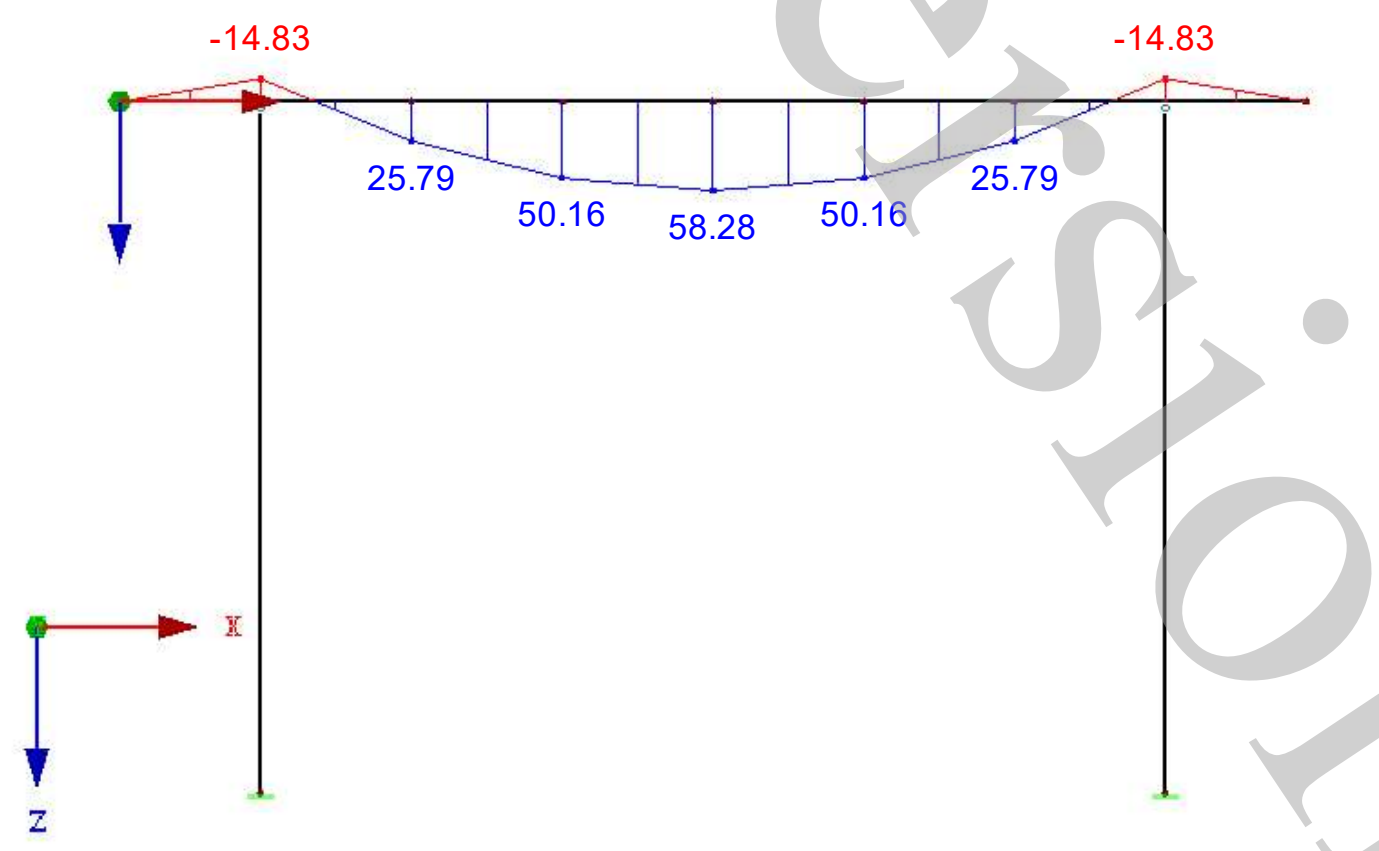




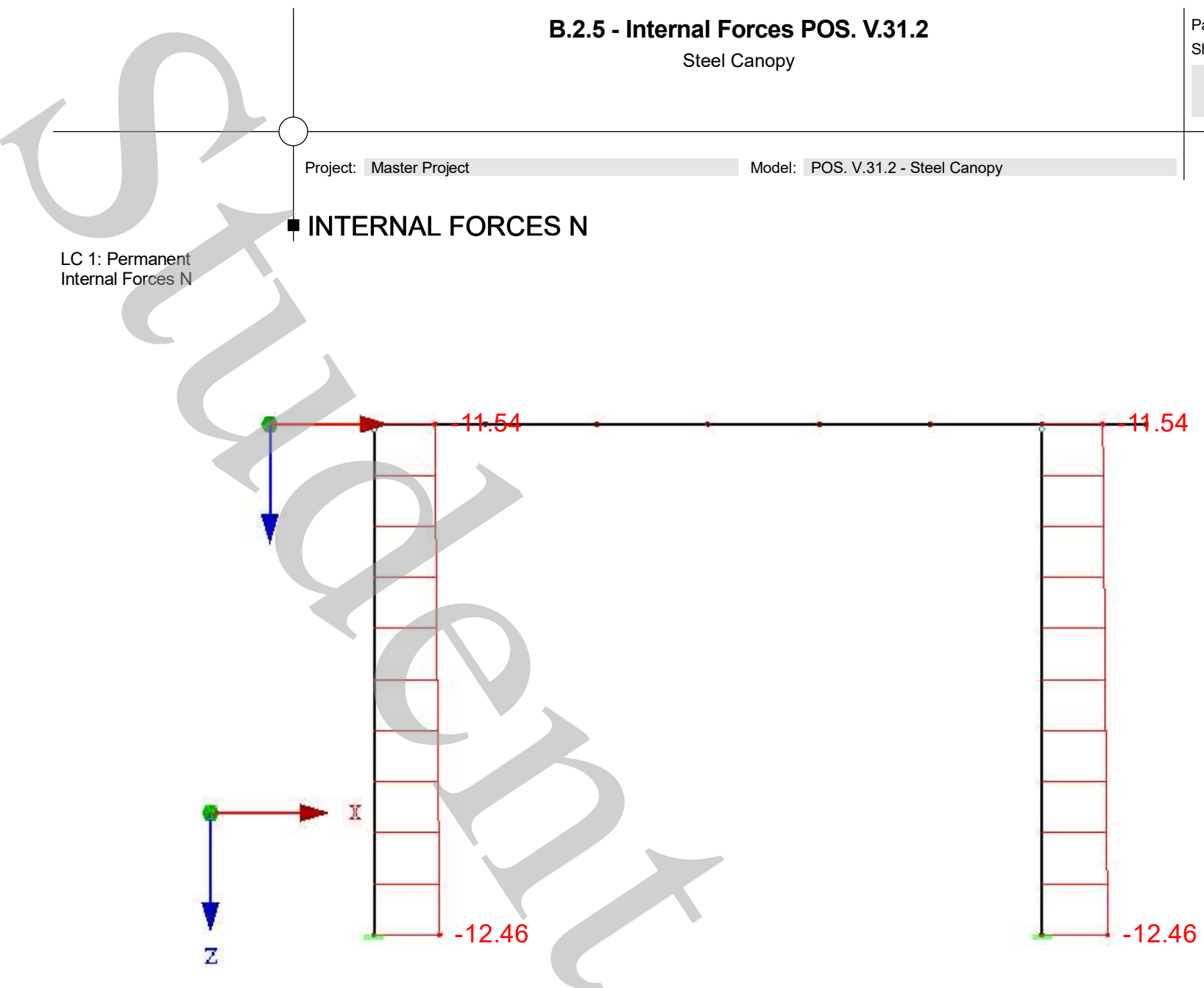

$\operatorname{Max} N: 0.00, \operatorname{Min} N:-12.46[\mathrm{kN}]$

\section{RESULTS}

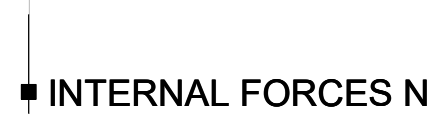

LC 2: Snow

Internal Forces $\mathrm{N}$

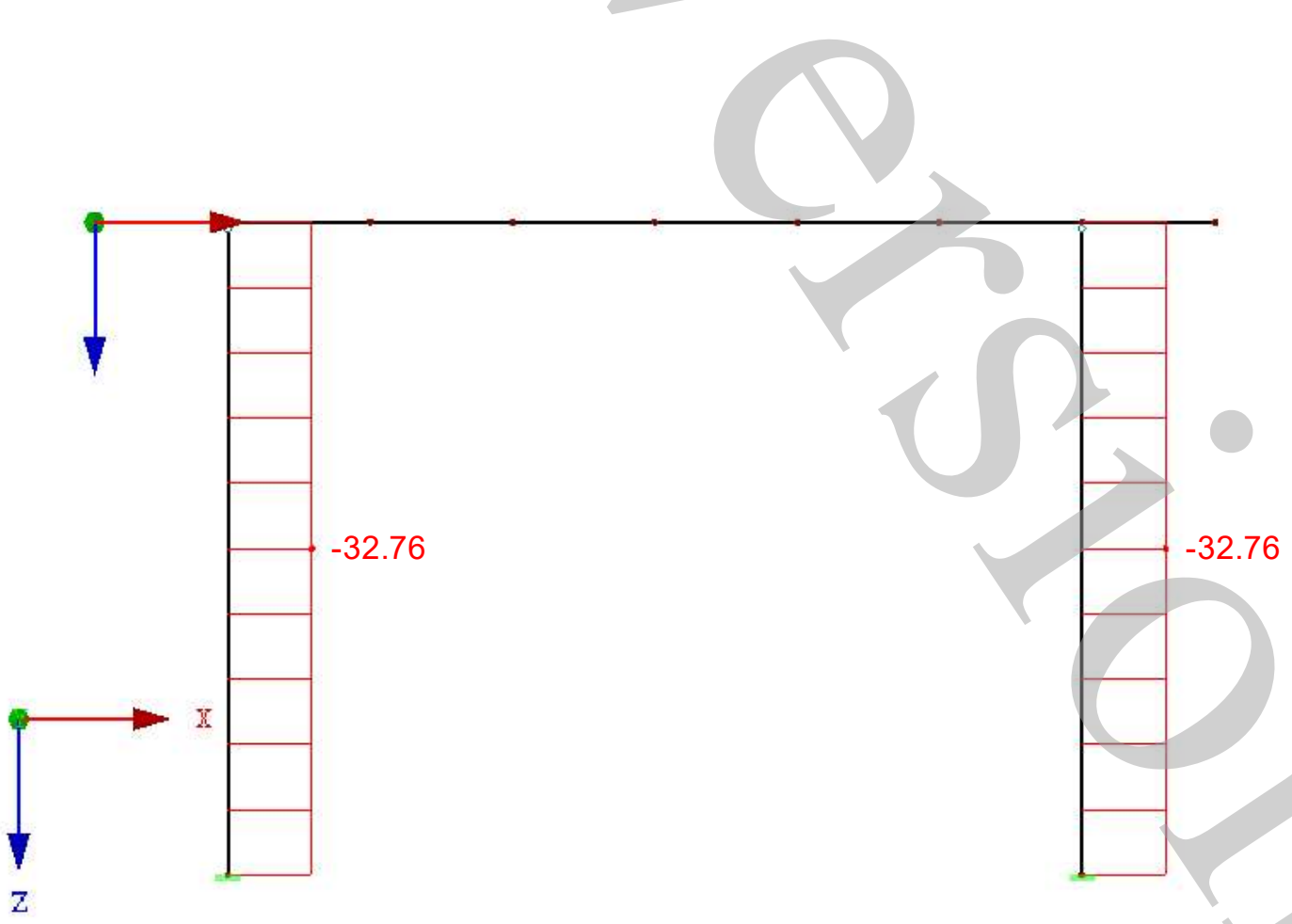




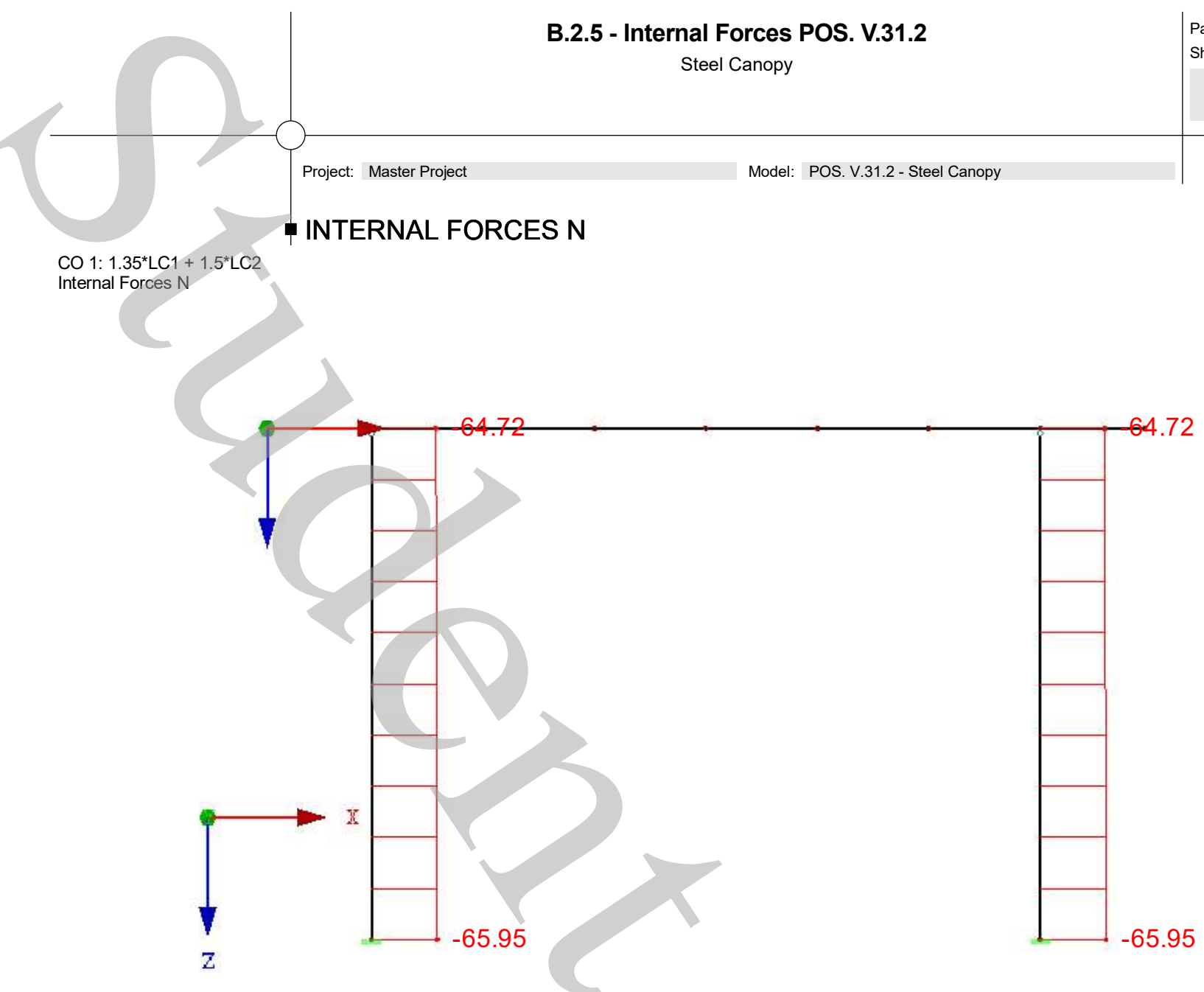

$\operatorname{Max} N: 0.00, \operatorname{Min} N:-65.95[\mathrm{kN}]$

\section{RESULTS}

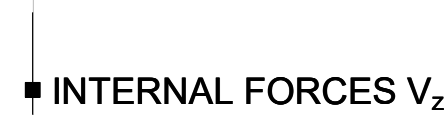

LC 1: Permanent Internal Forces V-z

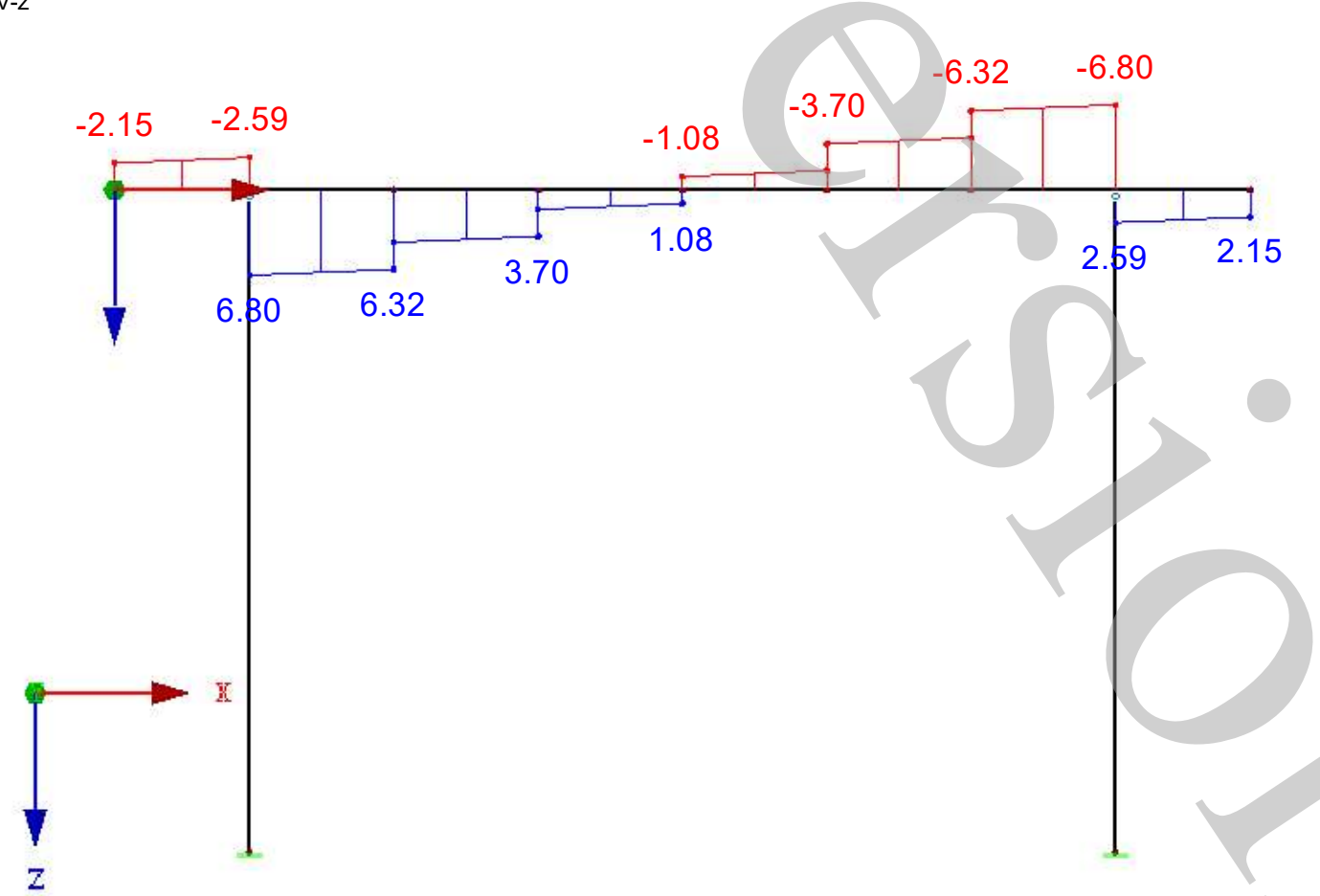




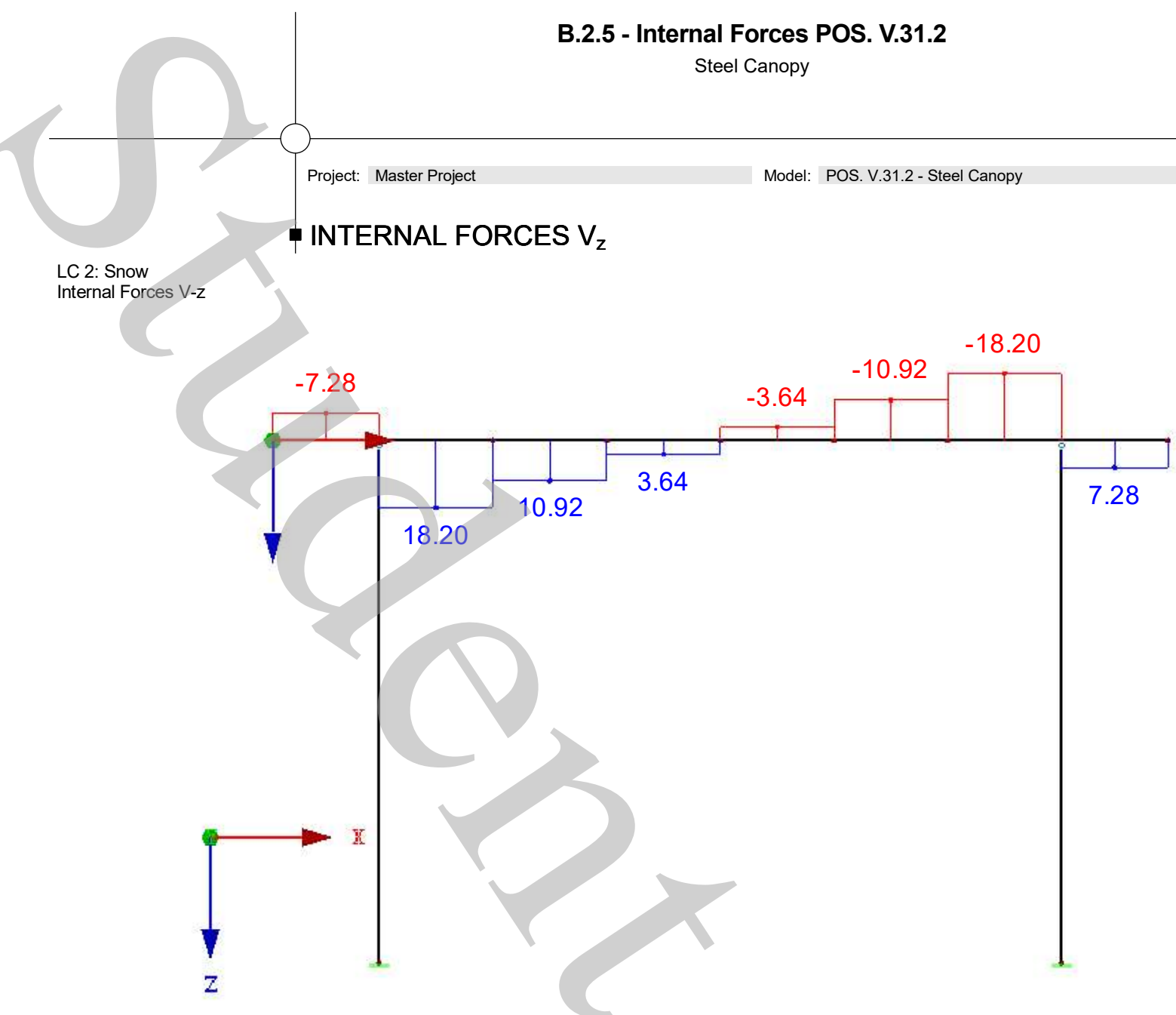

Max V-z: 18.20, Min V-z: -18.20 [kN]
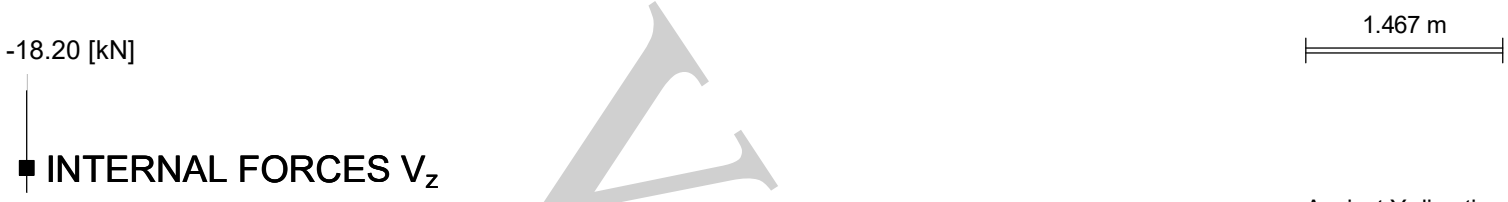

CO 1: $1.35^{*} \mathrm{LC} 1+1.5^{\star} \mathrm{LC} 2$

Internal Forces $\mathrm{V}$-z

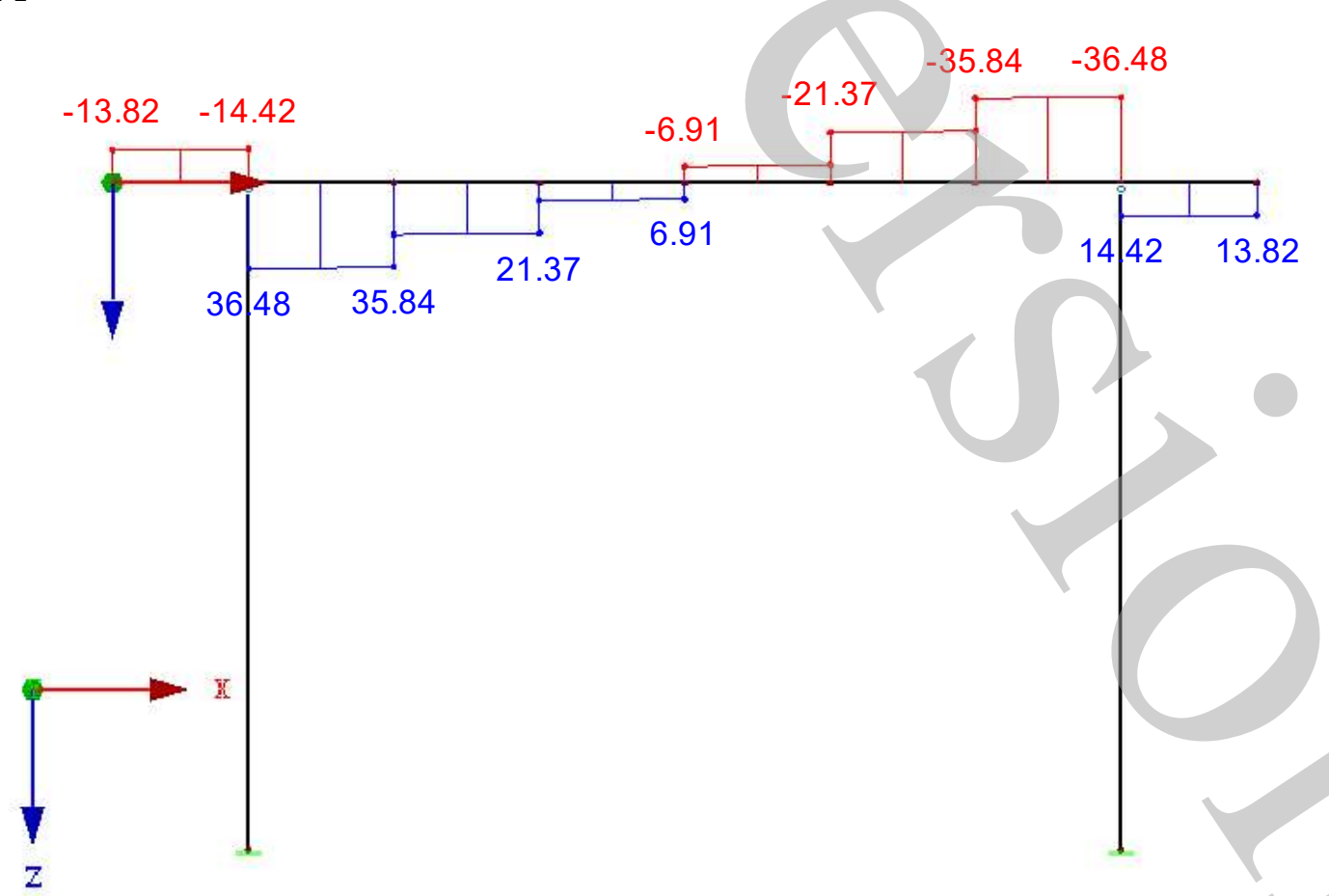




\subsection{MATERIALS}

\begin{tabular}{|c|c|c|c|c|c|c|}
\hline $\begin{array}{l}\text { Matl. } \\
\text { No. }\end{array}$ & $\begin{array}{l}\text { Modulus } \\
\mathrm{E}\left[\mathrm{kN} / \mathrm{cm}^{2}\right]\end{array}$ & $\begin{array}{l}\text { Modulus } \\
\mathrm{G}\left[\mathrm{kN} / \mathrm{cm}^{2}\right]\end{array}$ & $\begin{array}{c}\text { Spec. Weight } \\
\gamma\left[\mathrm{kN} / \mathrm{m}^{3}\right]\end{array}$ & $\begin{array}{c}\text { Coeff. of Th. Exp. } \\
\alpha\left[1 /{ }^{\circ} \mathrm{C}\right]\end{array}$ & $\begin{array}{c}\text { Partial Factor } \\
\gamma_{M}[-]\end{array}$ & $\begin{array}{c}\text { Material } \\
\text { Model }\end{array}$ \\
\hline 1 & $\begin{array}{r}\text { Steel S 235 | EN 1993- } \\
21000.00\end{array}$ & 8076.92 & 78.50 & 1.20E-05 & 1.00 & $\begin{array}{l}\text { Isotropic Linear } \\
\text { Elastic }\end{array}$ \\
\hline
\end{tabular}

\subsection{CROSS-SECTIONS}

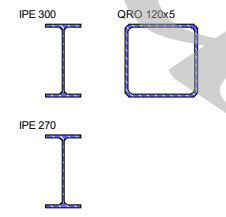

\begin{tabular}{|c|c|c|c|c|c|c|c|c|}
\hline \multirow{2}{*}{$\begin{array}{c}\text { Section } \\
\text { No. }\end{array}$} & \multirow{2}{*}{$\begin{array}{c}\text { Matl. } \\
\text { No. }\end{array}$} & \multirow{2}{*}{$\begin{array}{l}\mathrm{J}\left[\mathrm{cm}^{4}\right] \\
\mathrm{A}\left[\mathrm{cm}^{2}\right]\end{array}$} & \multirow{2}{*}{$\begin{array}{l}\mathrm{I}_{\mathrm{y}}\left[\mathrm{cm}^{4}\right] \\
\mathrm{A}_{\mathrm{y}}\left[\mathrm{cm}^{2}\right]\end{array}$} & \multirow{2}{*}{$\begin{array}{l}\mathrm{I}_{z}\left[\mathrm{~cm}^{4}\right] \\
\mathrm{A}_{z}\left[\mathrm{~cm}^{2}\right]\end{array}$} & \multirow{2}{*}{$\begin{array}{c}\text { Principal Axes } \\
\alpha\left[^{\circ}\right]\end{array}$} & \multirow{2}{*}{$\begin{array}{c}\text { Rotation } \\
\alpha^{\prime}\left[{ }^{\circ}\right]\end{array}$} & \multicolumn{2}{|c|}{ Overall Dimensions [mm] } \\
\hline & & & & & & & Width $b$ & Height $h$ \\
\hline & $\begin{array}{c}\mathrm{IPE} \\
1\end{array}$ & $\begin{array}{r}\text { onorm 19-57 } \\
53.80 \\
\end{array}$ & 8360.00 & 19.82 & 0.00 & 0.00 & 150.0 & 300.0 \\
\hline 2 & $\begin{array}{c}\text { QRO } 12 \\
1\end{array}$ & $\begin{array}{r}\text { DIN 59410:1974 } \\
22.60 \\
\end{array}$ & 495.00 & 9.68 & 0.00 & 0.00 & 120.0 & 120.0 \\
\hline & $\begin{array}{c}\text { IPE } 270 \\
1\end{array}$ & $\begin{array}{r}\text { onorm 19-57 } \\
45.90\end{array}$ & 5790.00 & 16.57 & 0.00 & 0.00 & 135.0 & 270.0 \\
\hline
\end{tabular}

CO 1: $1.35^{\star} \mathrm{LC} 1+1.5^{\star} \mathrm{LC} 2$

MODEL Loads $[\mathrm{kN}]$

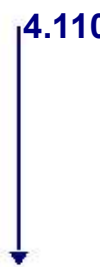

1.229

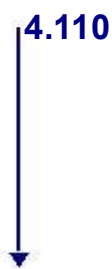

1.229

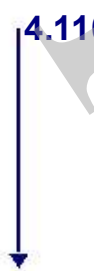

1.229

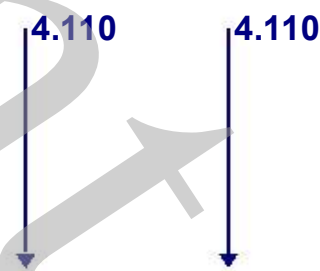

1.229
1.229

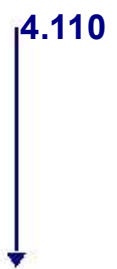

1.229

Against Y-direction
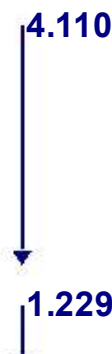

4.110

4.110

2.1 LOAD CASES

\begin{tabular}{|c|c|c|c|c|c|c|}
\hline Load & Load Case & EN 1990 | DIN & \multicolumn{4}{|c|}{ Self-Weight - Factor in Direction } \\
\hline Case & Description & Action Category & Active & $x$ & $\mathrm{Y}$ & Z \\
\hline LC1 & Permanent & Permanent & $\underline{\nabla}$ & 0.000 & & 1.000 \\
\hline LC2 & Snow & Snow $(H \leq 1000 m$ a.s.l. $)$ & $\vec{\exists}$ & & & \\
\hline
\end{tabular}

2.1.1 LOAD CASES - CALCULATION PARAMETERS

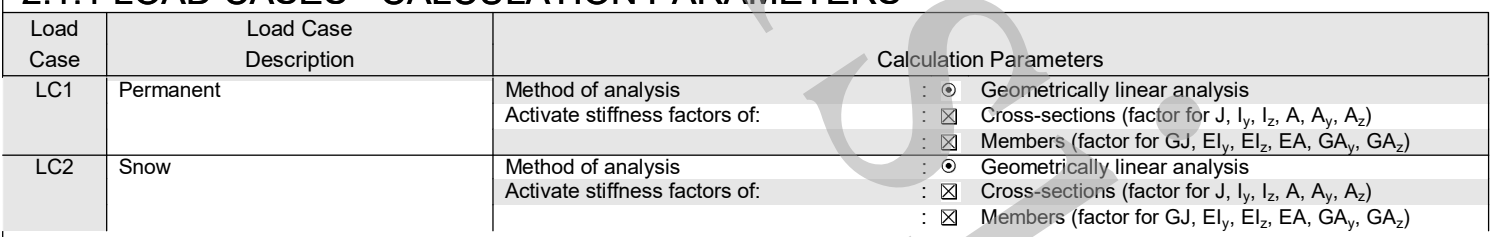

2.5 LOAD COMBINATIONS

\begin{tabular}{|c|c|c|c|c|c|c|}
\hline Load & & Load Combination & & & & \\
\hline Combin. & DS & Description & No. & Factor & & Load Case \\
\hline $\mathrm{CO} 1$ & & $1.35^{*} \mathrm{LC} 1+1.5^{\star} \mathrm{LC} 2$ & $\begin{array}{l}1 \\
2\end{array}$ & $\begin{array}{l}1.35 \\
1.50\end{array}$ & $\begin{array}{l}\text { LC1 } \\
\text { LC2 }\end{array}$ & $\begin{array}{l}\text { Permanent } \\
\text { Snow }\end{array}$ \\
\hline $\mathrm{CO} 2$ & & LC1 + LC2 & $\begin{array}{l}1 \\
2\end{array}$ & $\begin{array}{l}1.00 \\
1.00\end{array}$ & $\begin{array}{l}\text { LC1 } \\
\text { LC2 }\end{array}$ & $\begin{array}{l}\text { Permanent } \\
\text { Snow }\end{array}$ \\
\hline
\end{tabular}




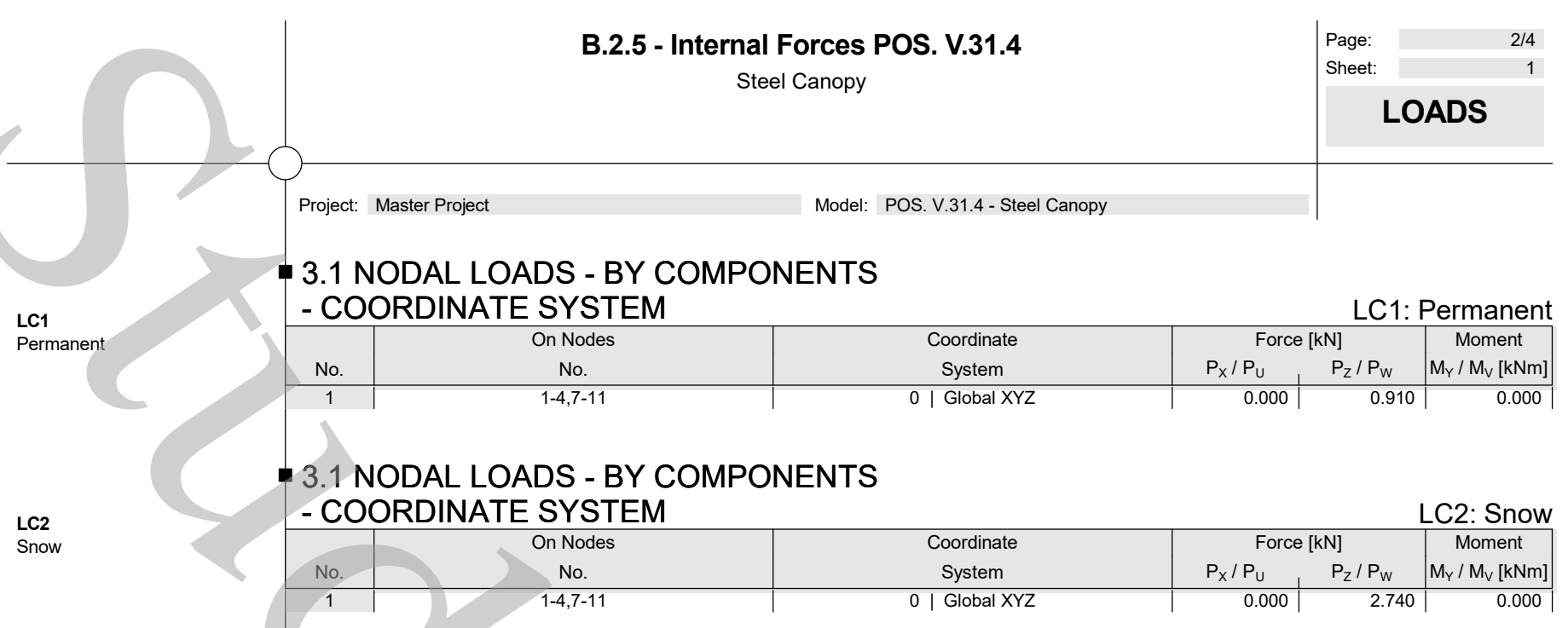

- 4.3 CROSS-SECTIONS - INTERNAL FORCES

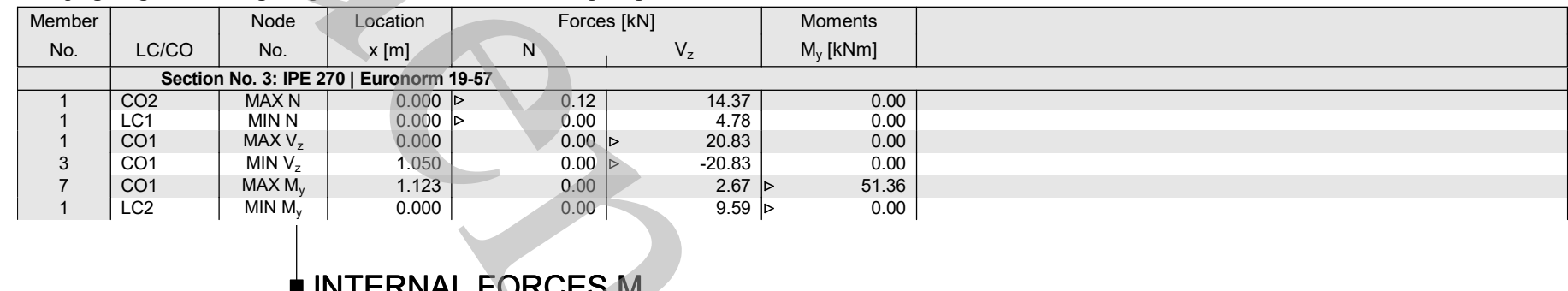

LC 1: Permanent

Internal Forces M-y

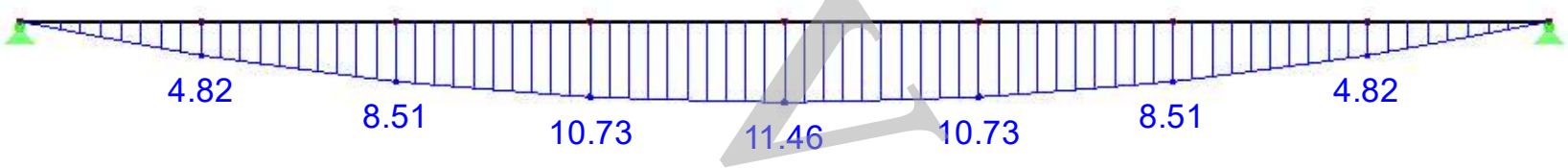

Max M-y: 11.46, Min M-y: $0.00[\mathrm{kNm}]$

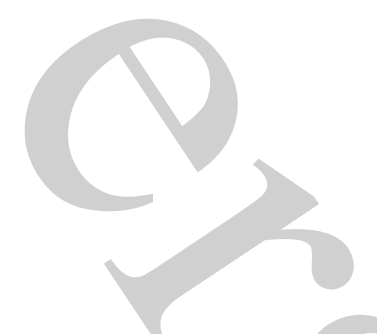

LC 2: Snow

INTERNAL FORCES My

Internal Forces M-y

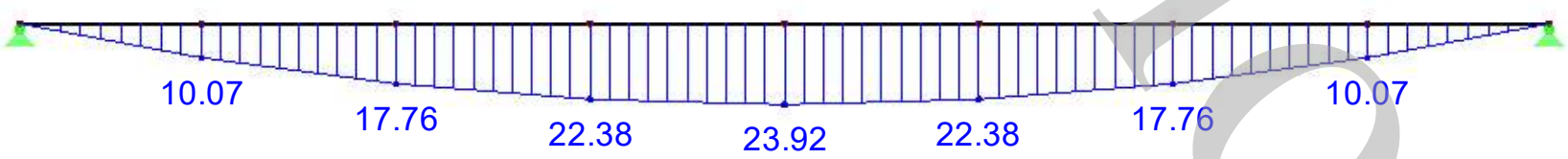

Max M-y: 23.92, Min M-y: 0.00 [kNm] 


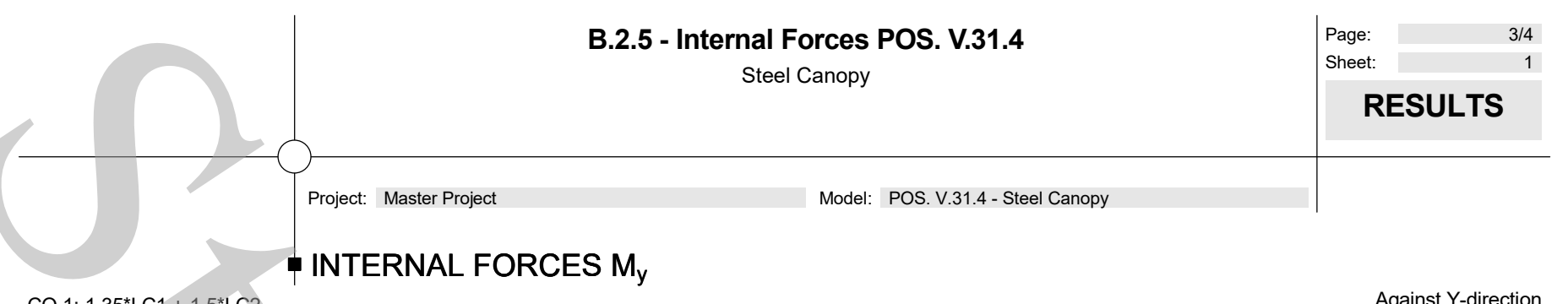

CO 1: $1.35^{\star} \mathrm{LC} 1+1.5^{*} \mathrm{LC} 2$

Internal Forces M-y

Against Y-direction
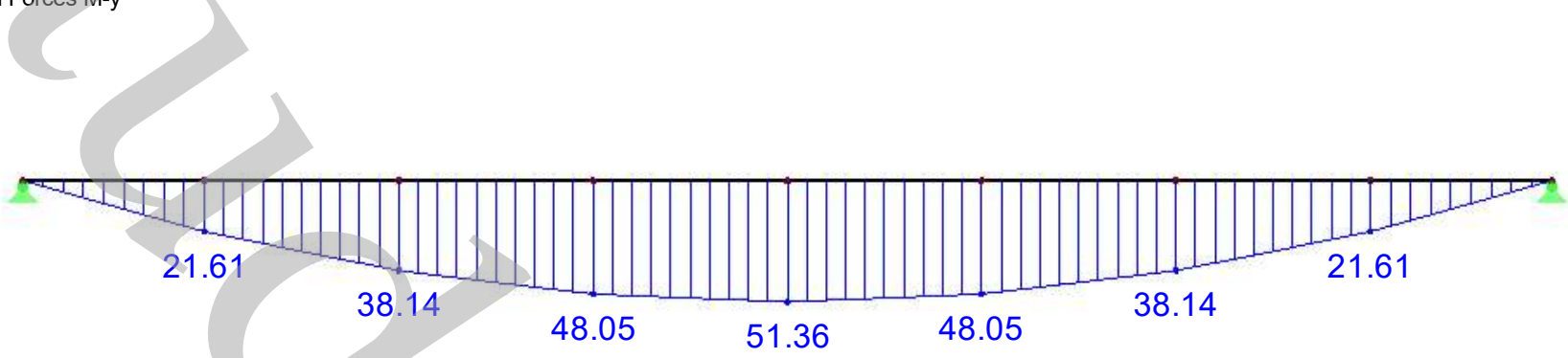

Max M-y: 51.36, Min M-y: 0.00 [kNm]

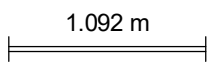

INTERNAL FORCES V

LC 1: Permanent

Internal Forces $\mathrm{V}-\mathrm{z}$

Against Y-direction

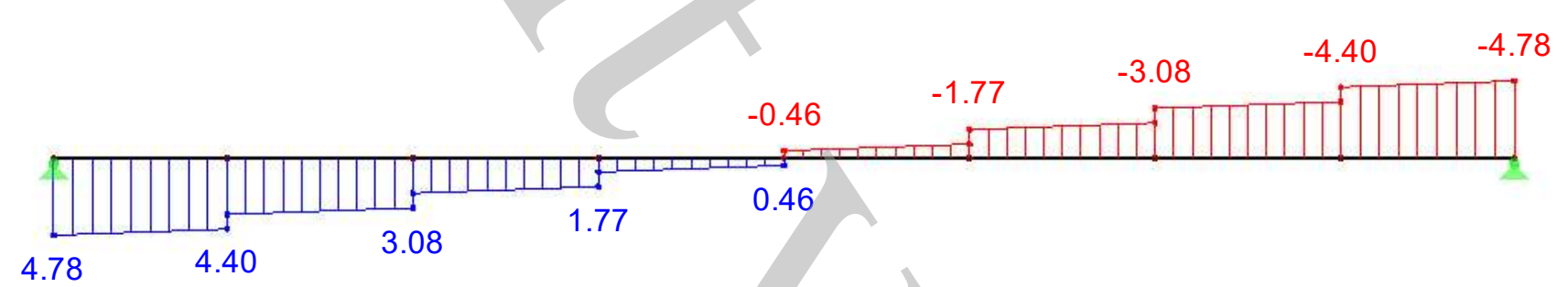

Max V-z: 4.78, Min V-z: -4.78 [kN]

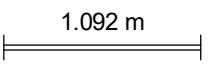

INTERNAL FORCES $V_{z}$

LC 2: Snow

Internal Forces V-z

Against Y-direction

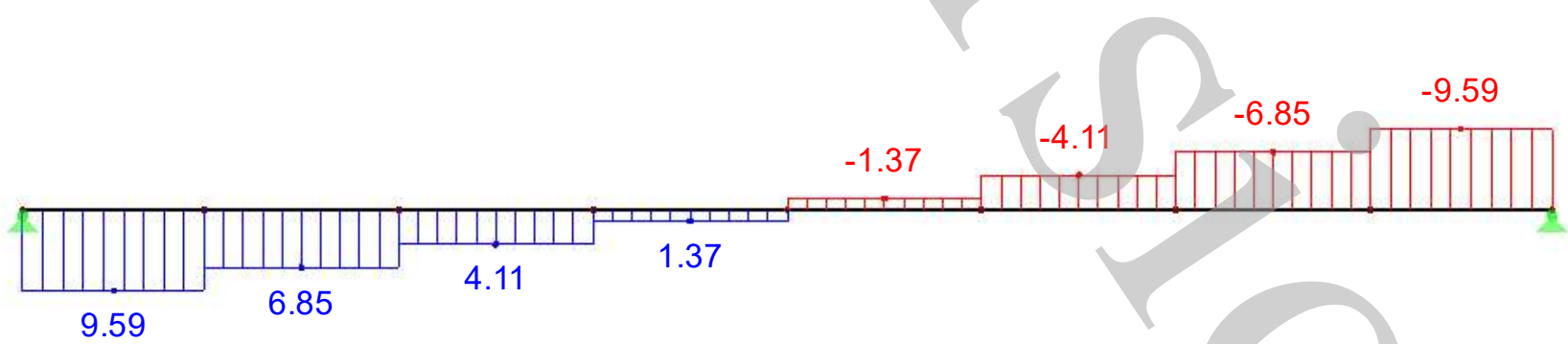

Max V-z: 9.59, Min V-z: -9.59 [kN]

$1.092 \mathrm{~m}$

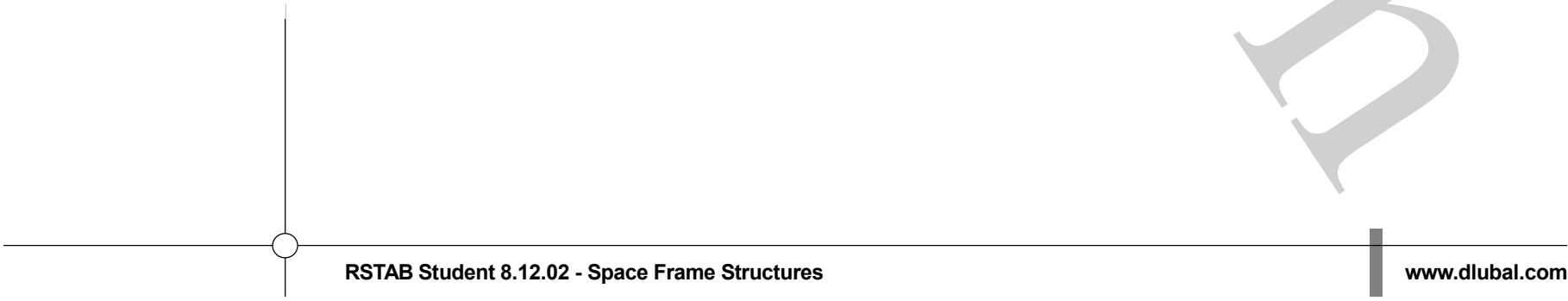




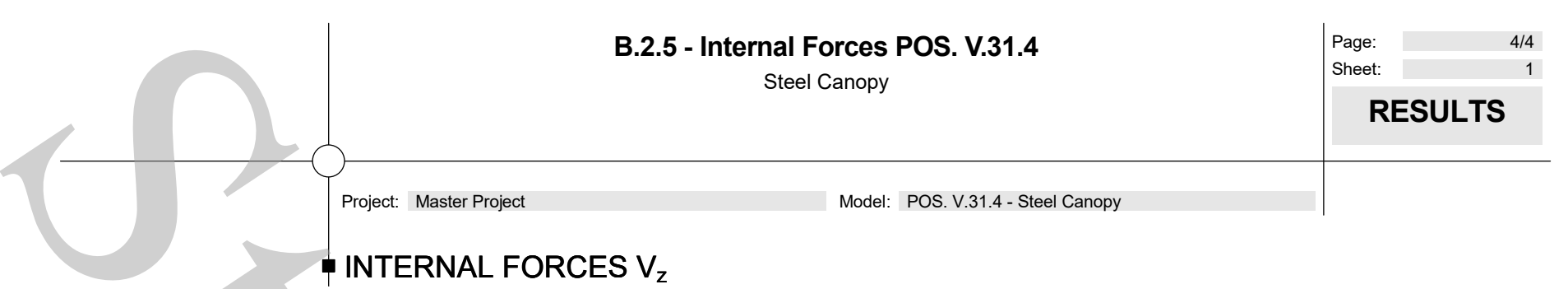

CO 1: $1.35^{*} \mathrm{LC} 1+1.5^{*} \mathrm{LC} 2$

Against Y-direction Internal Forces $\mathrm{V}-\mathrm{z}$

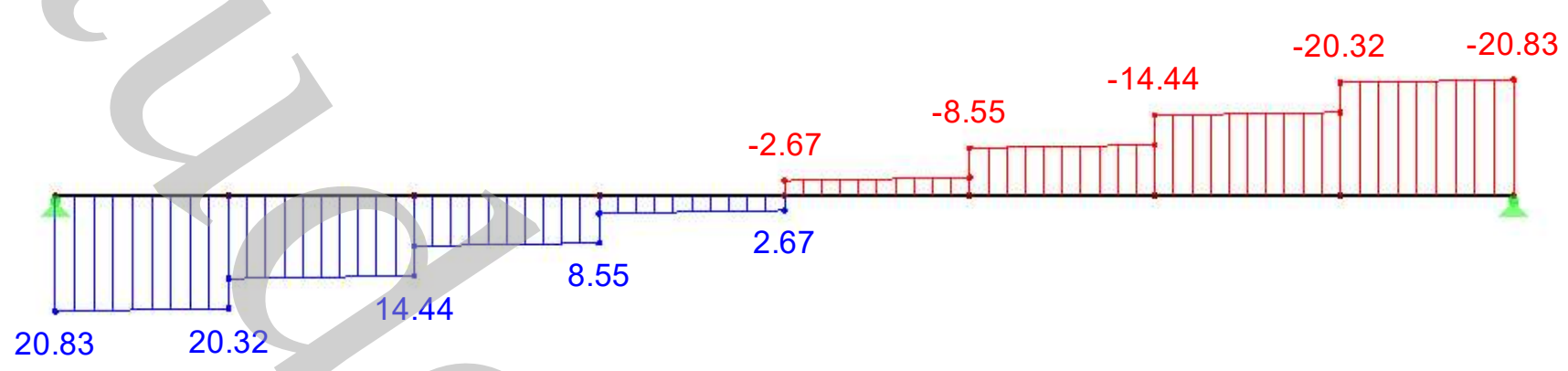

Max V-z: 20.83, Min V-z: -20.83 [kN] 


\section{REFERENCES}

[1] P. Schmidt, „Schneelasten,“ in Bautabellen für Ingenieure 20. Auflage, Köln, Werner Verlag, 2012, pp. 3.46-3.51.

[2] G. C. Lohmeyer, K. Ebeling und H. Bergmann, Stahlbetonbau. Bemessung - Konstruktion Ausführung. 8. Auflage, Wiesbaden: Vieweg+Teubner Verlag, 2010.

[3] A. Goris and U. P. Schmitz, Bemessungstafeln nach Eurocode 2: Normalbeton, hochfester Beton, Leichtbeton., Köln: Bundesanzeiger-Verag, 2014.

[4] A. Steinle, H. Bachmann und M. Tillmann, Bauen mit Betonfertigteilen im Hochbau, Berlin: Ernst \& Sohn, 2015.

[5] F. D. B. e.V., "Merkblatt Nr. 6 - Toleranzen und Passungsberechnungen für Betonfertigteile," Bonn, 2015.

[6] Calenberg Ingenieure, "Standard Elastomerlager. Übersicht und Bemessungshilfe," Calenberg Ingenieure, Salzhemmendorf, 2009.

[7] R. Avak, R. Conchon and M. Aldejohann, Stahlbetonbau in Beispielen Teil 2, Köln: Bundesanzeiger Verlag, 2017.

[8] C. Enderle, Lecture notes "Stahlbetonfertigteilbau", Karlsruhe: Hochschule Karlsruhe, 2017.

[9] R. Avak und K. Meiss, Spannbetonbau. Theorie, Praxis, Berechnungsbeispiele nach Eurocode 2., Berlin: Bauwerk - Beuth Verlag, 2015. 


\section{STANDARDS AND DATA SHEETS}

\section{STANDARDS}

DIN EN 1990:2010-12 Eurocode: Basis of structural design and DIN EN 1990/NA:2010-12 National Annex - Nationally determined paramteers for DIN EN 1990

DIN EN 1991-1-1:2010-12 Eurocode 1: Actions on structures - Part 1-1: General actions - Densities, selfweight, imposed loads for buildings; and DIN EN 1991-1-1/NA:2010-12 National Annex - Nationally determined paramteers for DIN EN 1991-1-1

DIN EN 1991-1-3:2010-12 Eurocode 1: Actions on structures - Part 1-3: General actions - Snow loads; and DIN EN 1991-1-3/NA:2010-12 National Annex - Nationally determined paramteers for DIN EN 1991 - 1- 3

DIN EN 1991-1-4:2010-12 Eurocode 1: Actions on structures - Part 1-4: General actions - Wind actions; and DIN EN 1991-1-4/NA:2010-12 National Annex - Nationally determined paramteers for DIN EN 1991 - 1- 4

DIN EN 1992-1-1:2011-01 Eurocode 2: Design of concrete structures - Part 1-1: General rules and rules for buildings; and DIN EN 1992-1-1/NA:2011-01 National Annex - Nationally determined paramteers for DIN EN 1992 - 1- 1

\section{DATA SHEETS}

- HOESCH Sizing Tabele for Trapezoidal Sheets

- HOESCH Cross-Section Properties Table for Trapezoidal Sheets

- Technical Product Information for HALFEN Transport Anchor System

- Calenberg Ingenieure Sizing Tables for Elastomeric Bearings

- $\quad$ Sizing Table for SCHÖCK Tronsole Type T

- $\quad$ Building Control Certification for Prestressing Steel Z-12.3-107 